\title{
EPDIC 11
}

\author{
Proceedings of the \\ Eleventh European Powder Diffraction Conference
}

held

September 19-22, 2008

in

Warsaw, Poland

VOLUME I

Editors

Bogdan Palosz ${ }^{1}$, Jordi Rius ${ }^{2}$

and Udo Welzel ${ }^{3}$

${ }^{1}$ Institute of High Pressure Physics, Polish Academy of Sciences, Warsaw, Poland

${ }^{2}$ Institut de Ciència de Materials de Barcelona (CSIC), Catalonia, Spain

${ }^{3}$ Max Planck Institute for Metals Research, Stuttgart, Germany

Supplement No. 30 of Zeitschrift für Kristallographie

Oldenbourg Verlag 



\section{EPDIC 11}

Eleventh European Powder Diffraction Conference

\section{Warsaw, Poland, September $19-22,2008$}

Conference location:

Conference chairman:

Organising committee:
Warsaw University of Technology, Warsaw, Poland

Bogdan Palosz

Stanisław Gierlotka

Piotr Jaśkiewicz

Zbigniew Kaszkur

Jan Kozubowski ↔

Henryk Morawiec

Wojciech Paszkowicz

Adam Pietraszko

Janusz Zachara
Ewa Grzanka

Mariusz Jaskólski

Andrzej Katrusiak

Wieslaw Lasocha

Andrzej Orlowski

Jerzy Pielaszek

Marek Wolcyrz
Andrzej Zięba 
Scientific programme committee: Bogdan Palosz, Warsaw, Poland (Chair) Jordi Rius, Barcelona, Spain (Chair) Nathalie Audebrand, Rennes, France Robert J. Cernik, Daresbury, UK Radovan Cerný, Genève, Switzerland. William I.F. David, Oxon, $U K$ Robert Delhez, Delft, The Netherlands Andrew Fitch, Grenoble, France Hartmut Fuess, Darmstadt, Germany Herbert E. Göbel, München, Germany Alessandro F. Gualtieri, Modena, Italy Torbjörn Gustafsson, Uppsala, Sweden Sergey A. Ivanov, Moscow, Russia Arnt Kern, Karlsruhe, Germany Radomir Kužel, Prague, Czech Republic Christian Lengauer, Vienna, Austria Ho-kwang (David) Mao, Washington, USA Anton Meden, Ljubljana, Slovenia Stanko Popovič, Zagreb, Croatia Thomas Proffen, Los Alamos, USA Paolo Scardi, Trento, Italy Osamu Shimomura, Tsukuba, Japan Kenny Ståhl, Lyngby, Denmark Hideo Toraya, Tokyo, Japan Tamás Ungár, Budapest, Hungary Arnold C. Vermeulen, Almelo, The Netherlands Udo Welzel, Stuttgart, Germany 


\section{EPDIC11 has been sponsored by:}

- Bruker AXS GmbH

- PANalytical B.V.

- International Centre for Diffraction Data ICDD

- International Union of Crystallography IUCR

- European Crystallographic Association ECA

- Committee on Crystallography of the Polish Academy of Sciences 



\section{Preface}

\section{The History of the European Powder Diffraction Conference}

As the Chair of the Organizing Committee of the first European Powder Diffraction Conference, EPDIC 1, I was invited by the Editors of the current Proceedings, on the occasion of my retirement from the EPDIC Committee, to write the Preface for the current Proceedings with a retrospective view back in time to the beginnings and the early-history of a common European Conference on Powder Diffraction.

Before the initiation of the EPDIC Conferences in 1991, exchange of knowledge and new developments in the field of powder diffraction was mainly taking place during dedicated sessions of the annual meetings of the national crystallographic associations, including also the European Crystallographic Associaton (ECA). Also the International Union of Crystallography (IUCr) organized a dedicated satellite meeting on powder diffraction every three years.

As some neighbouring countries in Western and Eastern Europe started organizing joined annual meetings, an increasing community of powder diffractionists proposed establishing a common meeting on powder diffraction in the late $80 \mathrm{~s}$. A view to the USA and the success of the "Denver X-ray Conference" (this conference series was initiated already in 1951 by the University of Denver with a one-day symposium on the application of X-rays to the study of materials) encouraged a group of European members of the "Joint Committee on Powder Diffraction Standards" (today International Centre for Diffraction Data - ICDD) in 1988 to give a start signal to organize an independent gathering of powder diffractionists in Europe. Important names in this context were Walter Eysel (Heidelberg, main initiator), Jan Visser (Delft, Scientific Chair of EPDIC 1), Daniel Louër (Rennes), Zbignew Bojarski (Katovice), Vaclav Valvoda (Prague) and Herbert Göbel (Munich, Chair of the Organizing Committee of EPDIC 1). The opportunity to start was the offer of the German Crystallographic Association (DGK) of a combined meeting at the University of Munich planned for March 1991. The first circular was distributed in 1989, mainly through the channels of the national crystallographic associations and via the manufacturers of diffraction instrumentation.

Many details had to be determined during the organization of EPDIC 1: First of all the name of the Meeting/Conference and a suitable abbreviation. We received critical remarks from the ECA for naming the event a 'Conference', whereas the ECM held just a 'Meeting'. However, Jan Visser insisted on the "C" in the abbreviation of EPDIC, because it was better to pronounce. The scientific scope of EPDIC and the procedures for the following EPDIC's had to be clearly defined, to avoid conflicts with other conferences/meetings in the field of diffraction, but also to guarantee the continued success of this new forum.

During the organization of EPDIC 1 the "Iron Curtain" was taken down, so that also participants from all Eastern European countries could attend. Their contributions were an important enrichment both with respect to scientific developments and practical applications of powder diffraction. Many contacts between scientists from East and West were formed, 
that resulted in common projects and sometimes partnerships and cooperations still existing today.

In total, EPDIC 1 was attended by 370 participants. More than half of them came from the unified parts of Germany and about 60 from Eastern Europe. The social event, an evening in one of Munich's famous beer halls during the "strong - beer" season of Lent, certainly is an unforgettable experience for all participants...

The large number of participants, the fact that most European Countries were represented by leading scientists in the field and the broad spectrum of applications of powder diffraction covered by the Conference attracted also the entire industry of equipment manufacturers in Europe and the world: A comprehensive exhibition of X-ray instrumentation took place during the conference.

The scientific program consisted of two and a half days of working sessions, comprising a honorary lecture of Professor H. Jagodzinski on "The Role of Munich for X-ray Powder Crystallography and the History of X-ray Powder Diffraction", 16 main lectures ("X-ray Diffraction Profiles Due to Real Polycrystals" by P. Klimanek, "Quo Vadis Quantitative Powder Diffraction Analysis" by J. Fiala, "Crystal Structure Analysis and Refinement by the Two-Step Method" by G. Will, "Neutron Powder Diffraction and Oxide Superconductors" by A.W. Hewat, "Applied Crystallography in Advanced Ceramics" by R.L. Snyder, "New Instrumentation in Powder Diffraction" by J. Ihringer, "New Detectors in X-ray Diffraction" by P. Tucker, "Energy Dispersive XRPD at High Pressure" by L. Gerward, "Glory and Misery of the Structure Analysis of Thin Polycrystalline Films" by V. Valvoda, "Characterization of Epitaxial Thin Films by X-ray Diffraction" by A. Segmüller, "Powder Diffraction Using Synchrotron Radiation" by M. Hart, "X-ray Absorption and Reflection in Materials Science" by B. Lengeler, "Preferred Orientation in Powder Diffraction" by H.-J. Bunge, "X-ray Stress Analysis" by J.M. Sprauel, "On the Use of Rietveld Refinements for Structural Studies" by P.-E. Werner, "Indexing of Powder Diffraction Patterns" by D. Louër), 120 poster presentations and 54 oral contributions. Two parallel sessions were held in order to accommodate a large number of talks.

Since the first EPDIC Conference, scientific progress reported during the Conferences has been registered in Conference Proceedings. Concerning the Proceedings the EPDIC community is indebted to Rob Delhez and Eric J. Mittemeijer for having taken care of the editorial work for the Proceedings for many years. Since EPDIC 9, the Proceedings are published 'open access' as supplement issues of the 'Zeitschrift für Kristallographie': The free on-line accessibility in combination with the traditional publication of the Proceedings in the form of printed volumes strengthens the importance of these Proceedings as a medium for the publication of cutting-edge developments and compact state-of-the art overviews in the field of powder diffraction.

The last EPDIC conference. EPDIC 11, was attended by about 350 participants (nearly 60 coming from outside Europe) and, as for previous EPDIC editions, current hot research topics and the newest developments of powder diffraction were presented. Particular emphasis was laid on direct and reciprocal space methods in powder diffraction structure 
determination, the 'charge flipping' algorithm, nanomaterials and total scattering analysis, to mention only a few of the topics treated in the conference.

Another important aspect was the search for new or improved forms of organization of the conference to make it more effective and attractive. Following the general trend towards the organization of small focused meetings, EPDIC workshops either preceding or following the main meeting have been organized during the last conferences. There is an increasing interest in this form of education and exchange of knowledge and expertise that also helps to promote scientific collaboration through the formation of personal links. At EPDIC 9 in Prague there was only one workshop, already three took place in Geneva at EPDIC 10, and now six workshops took place in Warsaw at EPDIC 11. For the future, a point deserving further attention would be the analysis of the circumstances that recommend treating a topic as a workshop rather than as a normal microsymposium.

Since EPDIC 3 in Vienna, it is a tradition to reward one young scientist, who contributed significantly to the field of powder diffraction, with the Young Scientist Award.

It was an unpleasant situation for the EPDIC Committee during each selection procedure of award candidates to exclude candidates not meeting the age limit (35 years) but having remarkable contributions to the field. The EPDIC committee therefore welcomes with great satisfaction the introduction of the EPDIC Award for Distinguished Scientist, which was awarded to Juan Rodríguez-Carvajal during EPDIC 11.

The next EPDIC Conference - EPDIC 12 - will be held in Darmstadt in 2010 as a joint meeting with the 26th European Crystallographic Meeting, ECM 26.

Looking back to the eleven EPDIC Conferences (see listing of previous conferences on the following page) and their impact on the field it can be concluded that the EPDIC Conference has developed as the leading conference dedicated exclusively to all aspects of powder diffraction.

Herbert Göbel

Munich

May 2009 


\section{EPDIC Conferences: The History and the Future}

Previous EPDIC Conferences

EPDIC 1, Munich, Germany. 14-16 March 1991. Chairman: Dr. H.E. Göbel

EPDIC 2, Enschede, The Netherlands. 30 July-1 August 1992. Chairman: Dr. T.W. Ryan

EPDIC 3, Vienna, Austria. 25-28 September 1994. Chairman: Prof. A. Preisinger

EPDIC 4, Chester, UK. 10-14 July 1995. Chairman: Dr. R.J. Cernik

EPDIC 5, Parma, Italy. 25-28 May 1997. Chairman: Prof. G. Artioli

EPDIC 6, Budapest, Hungary. 22-25 August 1998. Chairman: Prof. T. Ungár

EPDIC 7, Barcelona, Spain. 20-23 May 2000. Chairman: Prof. J. Rius

EPDIC 8, Uppsala, Sweden. 23-26 May 2002. Chairman: Dr. I.G.R. Tellgren

EPDIC 9, Prague, Czech Republic. 2-5 September 2004. Chairman: Prof. R. Kužel

EPDIC 10, Geneva, Switzerland. 1-4 September 2006. Chairman: Dr. R. Cerný

EPDIC 11, Warsaw, Poland. 19-22 September 2008. Chairman: Prof. B. Palosz

Forthcoming EPDIC Conference

EPDIC 12, Darmstadt, Germany. 27-30 August 2010. Chairman: Prof. H. Fuess 


\section{Editorial Notes}

The number of papers in these Proceedings is 73. The total number of papers published in the Proceedings of the preceding EPDIC conferences ranges from 88 to about 190.

The subdivision of the papers over the sections has been largely maintained as for preceding EPDIC proceedings. Only very minor adjustments, to adapt the subsections to the submitted papers, have been performed.

Reviewing the eleven editions of the EPDIC Proceedings, the ratios of the numbers of papers on developments in the methods and techniques of powder diffraction and those on applications of powder diffraction methods to specific classes of materials are found to be $1.0,0.7,0.5,1.0,0.9,0.5,0.7,0.7,0.8,0.5$ and, for the current proceedings, 0.8 .

As for the EPDIC10 Proceedings, a strict refereeing procedure was adopted for the Proceedings of EPDIC11. Each contribution was considered by at least one referee. The referees were, to a large extent, participants of EPDIC11. A few (in this sense) external referees were contacted as well. We thank all referees for their efforts and time spent on the manuscripts. We did not correct the English used, apart from minor corrections in a few papers.

We also thank Mrs Hilda David and Mrs Maritta Dudek (Max Planck Institute for Metals Research, Dept of Prof. Dr Ir. E.J. Mittemeijer, Stuttgart, Germany) for final technical corrections and invaluable help during the preparation of the required material for the publisher.

We sincerely hope that these Proceedings will be a useful collection of papers outlining the newest developments in the field of Powder Diffraction.

B. Palosz

Warsaw
J. Rius

Barcelona
U. Welzel

Stuttgart

May 2009 



\section{European Powder Diffraction Conference Award for Young Scientists}

\section{Sponsored by PANalytical B.V. (Almelo, The Netherlands)}

The EPDIC Award for Young Scientists is assigned at each EPDIC Conference and honours outstanding scientific achievement by young scientists in the field of powder diffraction. The award winner is invited to present a plenary talk at the next European Powder Diffraction Conference. The award has a value of 1,000 Euro.

The EPDIC Committee of each EPDIC Conference decides who is the winner. Short proposals of candidates containing descriptions of the scientific contributions to be assessed should be addressed to any member of the EPDIC Committee who is asked to make names public within this committee. As a rule, an age limit of 35 applies to the candidates. 



\section{European Powder Diffraction Conference Award for Distinguished Powder Diffractionists}

\section{Sponsored by Bruker AXS (Karlsruhe, Germany)}

The EPDIC Award for Distinguished Powder Diffractionists was assigned for the first time during the EPDIC-11 Conference for outstanding results and/or continued, important contributions to the field of powder diffraction. The award winner is invited to present a plenary talk at the next European Powder Diffraction Conference. The award has a value of 1,000 Euro.

The EPDIC Committee of each EPDIC Conference decides who is the winner. Short proposals of candidates containing descriptions of the scientific contributions to be assessed should be addressed to any member of the EPDIC Committee who is asked to make names public within this committee. 



\section{EPDIC Award for "Distinguished Powder Diffractionists"}

\section{Introductory speech by the Chairman of the EPDIC Committee}

On behalf of the EPDIC Committee, I am pleased to announce Dr. Juan Rodriguez-Carvajal as recipient of the Award for "Distinguished Powder Diffractionists" in its EPDIC 11 edition.

Dr. Rodriguez-Carvajal, simply Juan for his friends, was born in Sevilla (1953) and studied Physics first at the University of Sevilla and later at the University of Barcelona where he performed his doctorate. This was at the end of the seventies, beginning of the eighties. At that time I was also beginning my scientific career at the Crystallography Department and still remember the friendly and sometimes not so friendly discussions between the Director of the Department (the late Prof. Manuel Font-Altaba) and Juan regarding the possibilities of powder diffraction and the Rietveld method in relation to single-crystal diffraction. In retrospective, I think that both were right in many of their arguments. After several contracts in different University Faculties and Engineer Schools in Barcelona, Juan joined the CSIC (Institut de Cièneia de Materials de Barcelona) but soon moved to the ILL (Institut LaueLangevin) where he worked as a physicist from 1988 to 1994. There, he was co-responsible for different diffractometers. Later on, he worked as "Physicien Chercheur" at "Laboratoire Léon Brillouin, Centre d'Etudes de Saclay" from 1994 to 2006, and recently, again at ILL, he was appointed Leader of the Diffraction Group of that Institute.

Juan has been recognised with this Award mainly because of three major merits clearly demonstrated during his 28 years of professional activity in the field of powder diffraction and crystallography.

(1) The first merit is that by means of powder diffraction techniques, he has made an exceptional contribution to the study of structural and magnetic aspects of strongly correlated oxides. His scientific activity has represented a decisive step forward for the advanced analysis of both structural and magnetic diffracted intensities of complex materials. His ability to extract information from diffraction patterns of complex materials has led him to collaborate with an extraordinarily large number of groups all around the world, investigating a large variety of materials through profuse collaborations with many different groups and diffraction scientists.

(2) There is no doubt that the most outstanding contribution of Juan to the scientific and diffraction communities is the "FullProf computer program for crystallographic and magnetic studies". From 1988, the successive developments of this software package have allowed the crystallographic community to address an increasing number of features, aspects and problems related with the information included in X-ray and neutron diffraction patterns. At present, FullProf is a world-wide used program going from laboratory to synchrotron Xray diffraction, and from neutron reactors to spallation sources and constitutes an 
extraordinary tool with a huge impact on the scientific community, and also on the instrumental development.

(3) A third important characteristic of Juan is his willingness to share his scientific expertise with everybody asking him for help and advice, either about scientific matters or on questions related to FullProf. In this way, an extraordinary number of powder diffractionists have benefited from his expertise.

His CV can be summarised as follows:

1) Research and Teaching experience in Condensed Matter Physics and Crystallography for 28 years.

2) Expertise in:

(i) Powder and single crystal X-ray and neutron seattering;

(ii) Symmetry analysis, crystallography and magnetism;

(iii) Oxides presenting remarkable properties: superconductivity, giant magnetoresistance, charge, spin \& orbital ordering;

(iv) Computer programming and data analysis;

(v) Neutron diffraction instrumentation.

Due to his expertise he has also been member of many scientific institutions and committees.

3) Publications \& computer programs:

209 regular papers in journals (36 in Physical Review B, 5 Rapid Comm.; 5 PRLs), 103 papers in journals resulting from proceedings,

$59 \mathrm{reports} / \mathrm{book} /$ proceedings contributions and about 220 communications in meetings.

Total number of article citations exceeds 6900 . The current h-index is 38 .

Author of FULLPROF, one of the most frequently used powder diffraction computer programs in the world. (currently 2040 citations of the article Physica B 192, 55 (1993) plus 2795 direct citations of the use of the program)

4) Supervisor of 8 doctoral theses, 18 stages and post-docs and invited to more than 60 international events (courses and conferences).

To finish this short introduction, the members of the EPDIC Committee want to express their congratulations to Juan on receiving this award as well as their sincere appreciation for his contribution to the development of powder diffraction.

J. Rius

Barcelona

September 2008 


\section{Table of Contents}

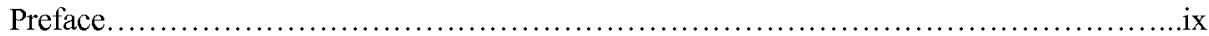

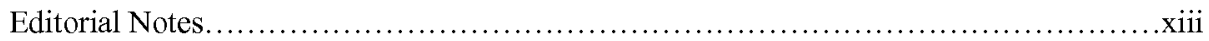

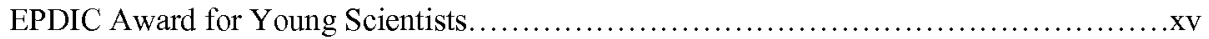

EPDIC Award for Distinguished Powder Diffractionists. .............................

EPDIC Award for Distinguished Powder Diffractionists - Introductory Speech..............xix

\section{VOLUME I}

\section{The EPDIC11 Award for Young Scientists Lecture}

\section{A. L. Goodwin}

The crystallography of flexibility: Local structure and dynamics in framework materials.

\section{METHOD DEVELOPMENT AND APPLICATION}

\section{I.1 Determination of Crystal Structure}

R. Oishi, M. Yonemura, A. Hoshikawa, T. Ishigaki, K. Mori, S. Torii, T. Morishima,

T. Kamiyama

New approach to the indexing of powder diffraction patterns using topographs....

Y. I. Yakimov, E. S. Semenkin, I. S. Yakimov

Two-level genetic algorithm for a full-profile fitting of X-ray powder patterns.

R. B. von Dreele

Characterization of proteins by powder diffraction.

J. A. Doebbler, R. B. von Dreele

Macromolecular powder diffraction: Structure solution via molecular replacement.

A. S. Wills

Indexing magnetic structures and crystallographic distortions from powder diffraction:

Brillouin zone indexing. 


\section{I.2 Qualitative and Quantitative Phase Analysis}

R. Kleeberg

State-of-the-art and trends in quantitative phase analysis of geological and

raw materials.

P. S. Whitfield, L. D. Mitchell

The effects of particle statistics on Rietveld analysis of cement. ...

B. Peplinski, A. N. Fitch, A. Evans, R. M. Ibberson, D. M. Többens, L. M. D. Cranswick,

I. Dörfel, F. Emmerling, R. Matschat

Structural characterization of a coarse-grained transparent silicon carbide powder by a combination of powder diffraction techniques.

\section{I.3 Analysis of Microstructure and Macrostress}

\section{I.3.1 Residual Stresses}

I. A. Denks, T. Manns, Ch. Genzel, B. Scholtes

An experimental approach to the problem of transforming stress distributions from

the LAPLACE- into real space.

\section{I.3.2 Line Broadening Analysis}

R. Delhez, A. C. Vermeulen

A practical vision on Line Profile Analysis today and in the years to come....

K. Beyerlein, A. Cervellino, M. Leoni, R. L. Snyder, P. Scardi

Debye equation versus Whole Powder Pattern Modelling: Real versus reciprocal space modelling of nanomaterials.

J. Martinez-Garcia, M. Leoni, P. Scardi

Diffraction contrast factor of dislocations: The case of scheelite $\mathrm{CaWO}_{4}$

A. Leineweber, E. J. Mittemeijer, A. C. Lawson, J. A. Roberts, J. A. Valdez, W. S. Kreher

Thermally induced microstrain broadening in polycrystalline hexagonal zinc.

A. Leineweber, T. Gressmann, M. Nikolussi, E. J. Mittemeijer

The $h k l$ dependences of microstrain and of macrostress-induced macrostrain; a comparison for intrinsically extremely anisotropic cementite, $\mathrm{Fe}_{3} \mathrm{C}$ 


\section{I.4 Texture}

A. Roatta, A. Fourty, R. E. Bolmaro

A primer on whole through processing simulation understanding of deformation and annealing textures in low carbon steels.

R. E. Bolmaro, R. A. Renzetti, M. J. R. Sandim, H. R. Z. Sandim, J. W. Signorelli,

M. Ferrante

ECAP of Fe. Experiments and simulations of the in-elbow textures.

A. Fourty, H.-G. Brokmeier, R. Martino, R. E. Bolmaro

Experiments and simulation evaluation in quartz veins textures in the

Guamanes shear belt, Córdoba Pampean Ranges, Central Argentina.

T. Kryshtab, A. Kryvko

The use of extinction phenomenon for investigation of textured thin film microstructure.

\section{I.5 In-situ and Non-ambient Measurements}

\section{B. Hinrichsen, R. E. Dinnebier, M. Jansen}

On the intensity distribution within Debye-Scherrer rings. What is different in

high pressure experiments? Part I: Theory....

B. Hinrichsen, R. E. Dinnebier, M. Jansen

On the intensity distribution within Debye-Scherrer rings. What is different in

high pressure experiments? Part II: Practical Application.

\section{INSTRUMENTAL}

\section{Z. Matěj, L. Nichtová, R. Kužel}

Coplanar grazing exit X-ray diffraction on thin polyerystalline films. ...

J. te Nijenhuis, M. Gateshki, M. J. Fransen

Possibilities and limitations of X-ray diffraction using high-energy X-rays on a laboratory system

G. Kimmel, D. Mogilyanski

Advantages and disadvantages of fast XRPD measurement by using image-plate and rotating anode source.... 


\section{H. Toraya}

Diffracted-beam analyzer with multiple single crystals for high resolution parallel-beam X-ray diffraction.

M. A. Kojdecki, W. Mielcarek, K. Prociów, J. Warycha

Comparison of two standards for powder X-ray diffractometry

V. Saengsuwan, W. Klysubun, T. Bovornratanaraks, S. Rugmai

Synchrotron beamline optics for X-ray powder diffraction under high-pressure conditions at the Siam Photon Laboratory

S. Vratislav, M. Dlouhá, L. Kalvoda, M. Dráb

Texture research of metals and rocks on the KSN-2 neutron diffractometer.

M. J. Styles, D. P. Riley, J. Christoforidis, S. Olsen

Modular in-situ reaction chamber design for time resolved diffraction. 201

\section{SOFTWARE}

P. S. Dubinin, I. S. Yakimov, O. E. Piksina, Y. I. Yakimov, A. N. Zaloga

RETRIEVE - a system for XRPD phase and structure analysis.

A. J. Florence, J. Bardin, B. Johnston, N. Shankland, T. A. N. Griffin, K. Shankland

Structure determination from powder data: Mogul and CASTEP...

H. Xu, Ch. M. Weeks, R. H. Blessing

Powder Shake-and-Bake Method

K. Shankland, T. A. N. Griffin, J. van de Streek, J. C. Cole, N. Shankland, A. J. Florence, W. I. F. David

Large-scale distributed computing for accelerated structure solution. 


\section{VOLUME II}

\section{MATERIALS}

\section{IV.1 Thin Layers}

\section{Nichtová, R. Kužel, Z. Matěj, J. Šicha, J. Musil}

Time and thickness dependence of crystallization of amorphous magnetron deposited $\mathrm{TiO}_{2}$ thin films.

M. Quaas, H. Wulff, O. Ivanova, C. A. Helm

Formation of nickel hydrides in reactive plasmas.

J. Sheng, U. Welzel, E. J. Mittemeijer

Interdiffusion and stress development in $\mathrm{Ni}-\mathrm{Cu}$ thin film diffusion couples.

\section{IV.2 Nanocrystalline and Amorphous Materials}

G. Dercz, L. Pajak, K. Prusik, R. Pielaszek, J. J. Malinowski

Nanocrystalline $\mathrm{MgO}$ powder materials prepared by sol-gel studied by

$\mathrm{X}$-ray diffraction and electron microscopy.....

\section{IV.3 Metals and Alloys}

\section{IV.3.1 General}

E. Yu. Filatov, K. V. Yusenko, E. S. Vikulova, P. E. Plyusnin, Yu. V. Shubin XRD investigation and thermal properties of $\left[\mathrm{Ir}\left(\mathrm{NH}_{3}\right)_{6}\right]\left[\mathrm{Co}\left(\mathrm{C}_{2} \mathrm{O}_{4}\right)_{3}\right] \cdot \mathrm{H}_{2} \mathrm{O}$ and $\left[\mathrm{Co}\left(\mathrm{NH}_{3}\right)_{6}\right]\left[\operatorname{Ir}\left(\mathrm{C}_{2} \mathrm{O}_{4}\right)_{3}\right]$ - precursors for $\mathrm{Co}_{0.50} \mathrm{Ir}_{0.50} \ldots$

K. V. Yusenko, I. V. Korolkov, S. A. Martynova, S. A. Gromilov $\mathrm{Ru}-\mathrm{Re}, \mathrm{Ru}-\mathrm{Os}$, and $\mathrm{Re}-\mathrm{Os}$ solid solutions - preparation under mild conditions, powder XRD investigation and phase diagram analysis.

P. Ari-Gur, G. Kimmel, J. Wi Richardson, A. Huq

Application of neutron powder diffraction for the study of non-stoichiometric $\mathrm{Ni}_{2} \mathrm{MnGa}$ based alloys. .... 


\section{IV.3.2 Microstructure, Stress and Texture}

V. E. Danilchenko, V. E. Iakovlev

Structural - phase state of maraging alloys.

H. J. Krzton, W. Pilarczyk

Rietveld quantitative phase analyses in iron alloys processed by mechanical

alloying method.

A.V. Druker; C. Sobrero, J. Malarria, U. Garbe,H.-G. Brokmeier, R. E. Bolmaro

Effect of texture heterogeneities on the shape memory properties of

rolled Fe-Mn-Si SMA

T: Goryczka

Texture and structure of grain boundary in Ni-Ti strip produced by twin

roll casting technique.

S. Popovic, Ž. Skoko, G. Štefanić

Temperature dependence of microstructure of Al-Ag-Zn alloys

A. M. Venter, C. P. la Grange, F. Hofmann, T-S. Jun, J. Belnoue, P. R. van Heerden,

A. Evans, A. M. Korsunsky

Synchrotron investigations of non-uniformly shaped shot-peened samples

\section{IV.4 Minerals and Inorganics}

\section{IV.4.1 Structural Changes, In-situ and Non-Ambient Investigations}

H. Boysen, I. Kaiser-Bischoff, M. Lerch, S. Berendts, A. Börger, D. M. Trots,

M. Hoelzel, A. Senyshyn

Structures and properties of variously doped Mayenite investigated by neutron

and synchrotron powder diffraction

O. A. Bulavchenko, S. V. Cherepanova, S. V. Tsybulya

In situ XRD investigation of $\mathrm{CO}_{3} \mathrm{O}_{4}$ reduction.

J. Darul

Thermal instability of the tetragonally distorted structure of copper-iron materials.

A. Senyshyn, J. M. Engel, I. D. H. Oswald, L. Vasylechko, M. Berkowski

Powder diffraction studies of pressure-induced instabilities in orthorhombic $\mathrm{LnGaO}_{3} \ldots \ldots 341$ 
J. Zabicky, G. Kimmel, E. Goncharov, F. Guirado

Magnesium titanate phases from xerogels by hot stage X-ray powder diffractometry.......

A. F. Gualtieri, M. Lassinantti Gualtieri, C. Meneghini

In situ synchrotron powder diffraction study of the thermal decomposition of cement-

asbestos: Preliminary results.

D. Jansen, F. Goetz-Neunhoeffer, J. Neubauer, W.-D. Hergeth, R. Haerzschel In-situ XRD investigations of the influence of PDADMAC on ettringite formation in cement systems.

\section{IV.4.2 Determination of Crystal Structure; Structure Refinement}

A. Dobrowolska, E. Zych

Structural and spectroscopic properties of $\mathrm{BaHfO}_{3}: \mathrm{Eu}$ - the issue of the dopant location in the host lattice.

N. V. Tarakina, T. A. Denisova, L. G. Maksimova, Y. V. Baklanova, A. P. Tyutyunnik, I. F. Berger, V. G. Zubkov, G. van Tendeloo

Investigation of stacking disorder in $\mathrm{Li}_{2} \mathrm{SnO}_{3}$.

B. Gawel, W. Surga, W. Lasocha

$\mathrm{X}$-ray diffraction studies of transition metal molybdates. ...

W. Easocha, M. Grzywa, M. Oszajca

Molybdates of p-bromoanilinium - synthesis and crystal structure of new catalytic materials

R. A. Snellings, A. F. Gualtieri, J. Elsen

The Rietveld structure refinement of an exceptionally pure sample of clinoptilolite from Ecuador and its Na-, K-, and Ca-exchanged forms....

N. V. Tarakina, V. G. Zubkov, I. I. Leonidov, A. P. Tyutyunnik, L. L. Surat, J. Hadermann, G. van Tendeloo

Crystal structure of the group of optical materials $\mathrm{Ln}_{2} \mathrm{MeGe}_{4} \mathrm{O}_{12}(\mathrm{Me}=\mathrm{Ca}, \mathrm{Mn})$

H. Wang, H. Ehrenberg, A. Senyshyn, R. Schierholz, J.-C. Jaud, H. Fuess

Structural investigation on the $(1-\mathrm{x}) \mathrm{Pb}\left(\mathrm{Mg}_{1 / 3} \mathrm{Nb}_{2 / 3}\right) \mathrm{O}_{3}-\mathrm{xPbTiO}_{3}(\mathrm{x}=0 ; 0.21)$

solid solution using powder diffraction. 407 


\section{IV.4.3 Determination of Magnetic Structure, Magnetic Materials}

W. Nowicki

Synthesis, structure and magnetic properties of Fe-doped tetragonal $\mathrm{Li}_{0.95} \mathrm{Mn}_{2.05} \mathrm{O}_{4}$

\section{IV.4.4 Microstructure, Phase Analysis}

\section{A. Leineweber, M. Leoni}

Simulation of layer-faulting in $\mathrm{Nb}_{2} \mathrm{Co}_{7}$ intermetallic compound using DIFFaX+

A. Argüelles, M. Leoni, J. A. Blanco, C. Marcos

Structure and microstructure of $\mathrm{Mg}$-vermiculite.

A. Sanz, J. Bastida, M. A. Kojdecki, A. Caballero, F. J. Serrano

Evolution of size and shape of mullite crystallites in triaxial porcelains

G. Cozzi, J. Bastida, A. Álvarez-Larena, M. A. Kojdecki, P. Pardo

Crystallite size of kaolinites as indicator of different geochemical types of bauxite

S. Seufert, C. Hesse, F. Goetz-Neunhoeffer, J. Neubauer

Discrimination of bassanite and anhydrite III dehydrated from gypsum at

different temperatures

P. Piszora, J. Nawrocki, J. Darul, W. Nowicki, A. Evans

High resolution X-ray powder diffraction measurements of the wet rust from the drinking water distribution system...

B. Peplinski, C. Adam, M. Michaelis, G. Kley, F. Emmerling, F.-G. Simon

Reaction sequences in the thermo-chemical treatment of sewage sludge ashes revealed by X-ray powder diffraction - A contribution to the European project SUSAN

J. A.G. Carrió, I. C. A. Dutra, M. C. Terence, R. Toledo, D. R. dos Santos

Quantitative analysis of clay materials and thermal treated bricks

B. Simionescu, M. Aflori, M. Olaru

Mineral composition and stone conservation of cultural heritage building materials studied by PXRD

T. Zaremba

Anisotropic grain growth of bismuth titanate in molten salt fluxes 


\section{IV.5 Organic Materials}

D. Jehnichen, D. Pospiech, S. Ptacek, K. Eckstein, P. Friedel, A. Janke,

C. M. Papadakis

Nanophase-separated diblock copolymers: Structure investigations on

PPMA- $b$-PMMA using X-ray scattering methods.

J. B. van Mechelen, R. Peschar, H. Schenk

Structure and polymorphism of trans mono-unsaturated triacylglycerols.

Author Index

xxxiii 

The EPDIC Award for

Young Scientists Lecture 2008 



\title{
The crystallography of flexibility: Local structure and dynamics in framework materials
}

\section{A. L. Goodwin}

Department of Earth Sciences, Downing Street, Cambridge CB2 3EQ, UK alg44@cam.ac.uk

Keywords: total scattering, framework materials, pair distribution function

\begin{abstract}
This paper reviews some recent diffraction-based studies of highly-flexible framework materials. Emphasis is placed on characterisation of the variations in local structure and dynamical behaviour that give rise to the unusual physical properties of these materials - negative thermal expansion, negative linear compressibility and pressure-induced amorphisation. The relevance of total scattering as a probe of local structure is discussed and a number of general protocols for analysing reverse Monte Carlo (RMC) atomistic refinements of total scattering data are described. Such protocols include both real- and reciprocalspace tools for quantifying correlated processes, including localised ferroelectric displacements and soft-mode lattice dynamics. These techniques are presented in the context of specific case studies of a number of important framework systems, such as $\mathrm{ZrW}_{2} \mathrm{O}_{8}, \mathrm{Zn}(\mathrm{CN})_{2}$, $\mathrm{Ag}_{3}\left[\mathrm{Co}(\mathrm{CN})_{6}\right]$ and $\mathrm{SrTiO}_{3}$. The paper concludes with a discussion of spin flexibility in magnetic systems and their relevance to local magnetic structure determination.
\end{abstract}

\section{Introduction}

Framework materials are network solids in which transition-metal centres are connected via single-atom or molecular "bridges" to form an extended covalent lattice. $\mathrm{ZrW}_{2} \mathrm{O}_{8}$, for example, is assembled from a network of $\mathrm{Zr}-\mathrm{O}-\mathrm{W}$ linkages [1], $\mathrm{Zn}(\mathrm{CN})_{2}$ from $\mathrm{Zn}-\mathrm{CN}-\mathrm{Zn}$ bridges [2], and metal-organic frameworks (MOFs) from extended $\mathrm{M}$-[organic]-M motifs [3]. Effectively constrained only by the distance to their metal-atom "tethers", these bridging atoms or groups are relatively free to vary their position and/or orientation with minimal energy cost. What this means is that local flexibility is inherent to most framework materials. Their structures are characterised by an excess configurational entropy in that they can adopt a number of different configurations with equivalent energies. The low-energy dynamics that results then defines a different set of thermodynamic properties to those of "normal" materials, and this difference presents both opportunities and challenges to the crystallography community. Opportunities, because crystallography offers a readily accessible method of discovering and quantifying unusual thermodynamic behaviour. The archetypal example is the discovery of negative thermal expansion (NTE, contraction on heating) in materials such as $\mathrm{ZrW}_{2} \mathrm{O}_{8}$ [1]. 
NTE has its own practical applications, and is of interest in its own right. But it is an important effect to characterise for the additional reason that it signals the existence of atypical lattice dynamics $[4,5]$. Materials that exhibit NTE are almost universally found to undergo series of interesting temperature and/or pressure-dependent phase transitions [6], often including phenomena such as pressure-induced amorphisation (PIA) [7]. Their elastic properties are bizarre [8]; their phonon spectra are strongly temperature-dependent $[9,10]$; their crystal lattices are often capable of both the local and long-range deformations required to support guest sorption/desorption [11], ion-conduction [12] and/or crystal-crystal transitions [13]. Crystallographic measurements of structure variation with temperature, pressure and sorbate concentration are becoming increasingly routine, making diffraction the method of choice for characterising the unusual mechanics of flexible framework materials.

Perhaps the key difficulty in studying these systems involves translating this experimental characterisation into an understanding of the underlying physics responsible for the effects. Local flexibility gives rise to disorder and to symmetry breaking, and both complicate the physical interpretation of average structure determinations. The perovskite community, for example, has faced these same issues for decades in the form of unphysical shortening of metal-oxygen bond lengths and divergence of oxygen displacement parameters near displacive phase transitions [14]. Crystallographic anomalies such as these require an appreciation of local structure in order to be understood. Consequently there is a strong drive to add to "traditional" crystallographic approaches a complementary picture of local structure and dynamics, and this is where "modern" crystallographic techniques of total seattering and pair distribution function (PDF) analysis are proving invaluable $[15,16]$. These two aspects of "the crystallography of flexibility" - characterisation of unusual physics via average structure analysis and subsequent explanation in terms of local structure and dynamics-form the basis of the research summarised in this paper.

Addressed first is the recent discovery of very large NTE effects in new families of flexible framework systems with linear bridging motifs. The focus then shifts to the extraction of local structure and dynamical information for these, and similar, systems from total scattering data, with the discussion centring around the reverse Monte Carlo (RMC) method. A number of specific case studies are then given in the subsequent section. The paper concludes with a discussion of magnetic flexibility in magnetic materials and associated crystallographic strategies for the characterisation of local magnetic structure.

\section{Linearly-bridged frameworks}

Since the discovery of large NTE in $\mathrm{ZrW}_{2} \mathrm{O}_{8}$ in 1996 [1], there has been a strong community effort to identify increasingly extreme NTE behaviour in related materials. NTE itself is measured in terms of the linear coefficient of thermal expansion $\alpha=\mathrm{d}(\ln l) / \mathrm{d} T$ (or its volume equivalent $\left.\alpha_{V}=\mathrm{d}(\ln V) / \mathrm{d} T\right)$; the value $\alpha=-9.1 \mathrm{MK}^{-1}$ for $\mathrm{ZrW}_{2} \mathrm{O}_{8}$ is about as negative as values for typical engineering materials are positive [4,5]. The stronger the NTE, the smaller the quantity required to produce composites with zero thermal expansion - the most often cited application of NTE. Despite a large body of research in the area, the focus on materials assembled from metal-oxygen-metal linkages (like $\mathrm{ZrW}_{2} \mathrm{O}_{8}$ itself) has failed to produce materials with any stronger overall NTE behaviour [5]. In all these systems, NTE could almost always be thought of in simple terms as arising from volume-reducing transverse displace- 
ments of $\mathrm{O}$ atoms in metal-oxygen-metal linkages (figure $1(\mathrm{a})$ ) $[4,5] . \mathrm{ZrW}_{2} \mathrm{O}_{8}$ shows the strongest effect because these displacements can be correlated throughout the crystal lattice via a continuum of low-energy phonons that preserve metal coordination geometries [17].

(a)

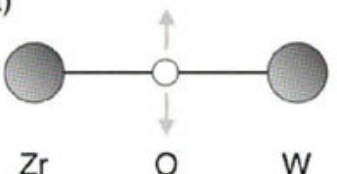

(b)

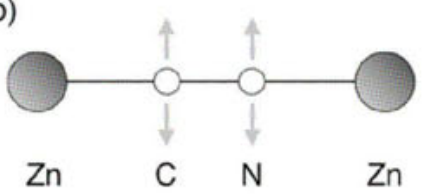

(c)

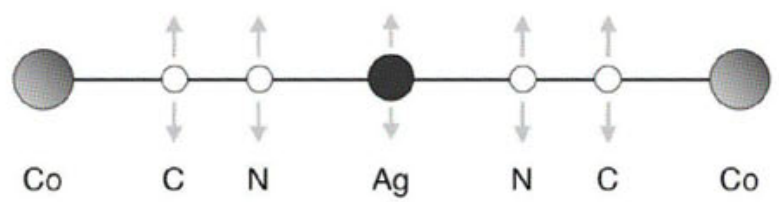

Figure 1. Underconstrained bridging motifs found in (a) $\mathrm{ZrW}_{2} \mathrm{O}_{8}$, (b) $\mathrm{Zn}(\mathrm{CN})_{2}$ and (c) $\mathrm{Ag}_{3}\left[\mathrm{Co}(\mathrm{CN})_{6}\right]$.

A relatively recent strategy for producing materials of superior NTE properties has been to replace single-atom metal-oxygen-metal linkages by diatomic metal-cyanide-metal (M$\mathrm{CN}-\mathrm{M}$ ) linkages [18,19], as found in many transition metal cyanides [20]. This circumvents any problems associated with correlation, since the $\mathrm{C}$ and $\mathrm{N}$ atoms can move in different directions, and so displacements around one metal centre can occur essentially independently of those around its neighbours (figure 1(b)) [18]. Cyanide-containing frameworks should then be fundamentally more flexible than their oxide-containing counterparts, and it was reasoned that NTE is likely to be a general property of these materials [19].

This was indeed found to be the case, and following on from an initial report of NTE in $\mathrm{Zn}(\mathrm{CN})_{2}[21]$ it was shown that the entire compositional family $\mathrm{Zn}_{x} \mathrm{Cd}_{1-x}(\mathrm{CN})_{2}$ exhibits isotropic NTE that is more than twice as strong as that in $\mathrm{ZrW}_{2} \mathrm{O}_{8}$ [18]. Analysis of the transverse displacement parameters of the $\mathrm{C}$ and $\mathrm{N}$ atoms in these materials supported the general picture of flexibility described above, and it was possible even to correlate thermal changes in these parameters with the observed coefficients of thermal expansion [18].

Further support for the generality of NTE in these families came from a study of the family $\operatorname{MPt}(\mathrm{CN})_{6}(\mathrm{M}=\mathrm{Zn}, \mathrm{Cd})[22]$. This second family of compounds is isostructural to the perovskites, except that in this case the octahedral metal centres are separated by rigid rods rather than by individual atoms. While NTE is exceptionally rare amongst perovskites (including in "empty" members such as $\mathrm{ReO}_{3}$ and $\mathrm{WO}_{3}[23,24]$ ), strong NTE was found for both $\mathrm{ZnPt}(\mathrm{CN})_{6}$ and $\mathrm{CdPt}(\mathrm{CN})_{6}\left(\alpha=-3.4\right.$ and $-6.7 \mathrm{MK}^{-1}$, respectively) [22]. Moreover, these materials were shown to exhibit zeolitic behaviour, and it was possible to tune the thermal expansion behaviour of $\mathrm{ZnPt}(\mathrm{CN})_{6}$ between $\alpha=-3.4$ and $+1.8 \mathrm{MK}^{-1}$ by adding or removing guest water molecules within the framework lattice [22]. Similar behaviour has been demonstrated even more recently for single-network $\mathrm{Cd}(\mathrm{CN})_{2}$, whose thermal expansion varies between $\alpha=-33.5$ and $+10.0 \mathrm{MK}^{-1}$ for different concentrations of included $\mathrm{CCl}_{4}$ [25].

Other groups have now measured NTE in a number of different cyanide-containing frameworks [26-31], so that the effect appears to be a general property of the family. 
Perhaps the most recent development in the field has been to produce "ultra-flexible frameworks" by replacing the diatomic cyanide bridges by penta-atomic dicyanometallate linkages [32]. Transition metal centres $\mathrm{M}$ are then connected via $\mathrm{M}-(\mathrm{CN})-\mathrm{A}-(\mathrm{CN})-\mathrm{M}(\mathrm{A}=\mathrm{Cu}, \mathrm{Ag}$, $\mathrm{Au}$ ) bridging motifs (figure 1(c)). There are no known examples with cubic crystal symmetry, so thermal expansion in these systems is necessariliy anisotropic. The first such material to be studied for its thermal expansion behaviour was silver hexacyanocobaltate, $\mathrm{Ag}_{3}\left[\mathrm{Co}(\mathrm{CN})_{6}\right][32,33]$. The crystal structure of this material can be considered as a threedimensional equivalent of a sheet of "garden lattice fencing". In particular, there is essentially no enthalpy cost associated with a combined expansion of the lattice in one direction and contraction in a perpendicular direction [34] - just as garden lattice itself can adopt a variety of different geometries (figure 2). This leaves the actual dimensions of the material to be determined by very weak non-covalent interactions: in this case, van der Waals-like $\mathrm{Ag}$... Ag "argentophilic" interactions. The extreme flexibility of the framework confers the thermodynamics of a van der Waals solid onto a covalent crystalline material.

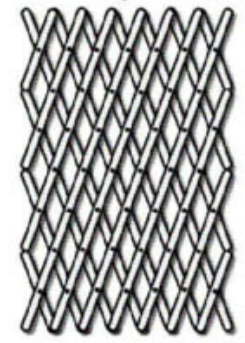

low $V$

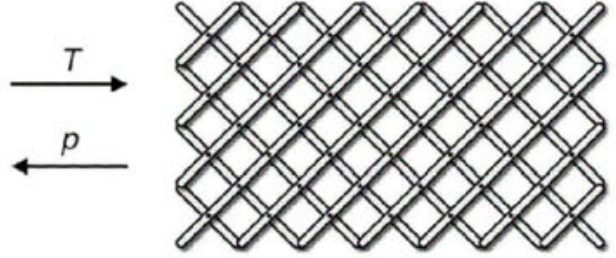

high $V$

Figure 2. The framework structure of $\mathrm{Ag}_{3}\left[\mathrm{Co}(\mathrm{CN})_{6}\right]$ behaves very much like lattice fencing: a contraction in one direction is coupled to expansion in a perpendicular direction.

In terms of thermal expansion, what was found was that the crystal expands enormously with increasing temperature along some directions $\left(\alpha=+144 \mathrm{MK}^{-1}\right)$ and exhibits similarly strong NTE along its trigonal axis $\left(\alpha=-126 \mathrm{MK}^{-1}\right)$ [32]. Throughout this process, the actual change in bond lengths along the $\mathrm{Co}-\mathrm{C}-\mathrm{N}-\mathrm{Ag}-\mathrm{N}-\mathrm{C}-\mathrm{Co}$ linkage is all but insignificant by comparison [32]. The process is thought to be driven by a van der Waals-like expansion of $\mathrm{Ag}$... Ag contact; coefficients of thermal expansion greater than $100 \mathrm{MK}^{-1}$ (the threshold for so-called "colossal" thermal expansion) have really only ever been observed for crystalline materials in van der Waals solids such as low-temperature Xe [35]. Further support for the role of $\mathrm{Ag}$... Ag contacts came via measuring thermal expansion properties of two compositional variants with the same covalent connectivity: namely, $\mathrm{Ag}_{3}\left[\mathrm{Fe}(\mathrm{CN})_{6}\right]$ and $\mathrm{D}_{3}\left[\mathrm{Co}(\mathrm{CN})_{6}\right]$. What was found was that the former is also a "colossal" thermal expansion material, but the latter exhibits more conservative values of $\alpha$ that are not atypical for framework materials [36]. Characterisation of the pressure-dependent behaviour of $\mathrm{Ag}_{3}\left[\mathrm{Co}(\mathrm{CN})_{6}\right]$ revealed similarly atypical phenomena. On increasing hydrostatic pressure, the framework lattice contracts along some directions but actually expands along the NTE axis, exhibiting the incredibly rare phenomenon of negative linear compressibility (NLC). In the few instances where NLC has been observed previously [37], the effect has only ever been very modest: the strongest effect 
reported was for $\mathrm{BAsO}_{4}$, for which the isothermal compressibility $K=-\mathrm{d}(\ln V) / \mathrm{d} P$ is $-2 \mathrm{GPa}^{-1}$ [38]. By contrast, $\mathrm{NLC}$ in $\mathrm{Ag}_{3}\left[\mathrm{Co}(\mathrm{CN})_{6}\right]$ is nearly 40 times stronger, with $K=-76 \mathrm{GPa}^{-1}$ [39]. Expansion along this axis is so rapid that a first-order collapse occurs at $0.19 \mathrm{GPa}$ to give a monoclinic high-pressure phase $\mathrm{Ag}_{3}\left[\mathrm{Co}(\mathrm{CN})_{6}\right]-\mathrm{II}$ that, remarkably, also shows NLC, albeit with a more conservative coefficient of $K=5.3 \mathrm{GPa}^{-1}$ measured up to $7.65 \mathrm{GPa}[39]$.

$\mathrm{Ag}_{3}\left[\mathrm{Co}(\mathrm{CN})_{6}\right]$ appears not to be alone amongst these flexible frameworks in exhibiting unusual pressure-dependent behaviour. Research elsewhere has shown the NTE behaviour of $\mathrm{Zn}(\mathrm{CN})_{2}$ is enhanced on increasing pressure [40]; then, at higher pressures, the material undergoes a series of phase transitions before eventually becoming amorphous at hydrostatic pressures of approximately $11 \mathrm{GPa}$ [41]. It seems reasonable to expect other reports of unusual pressure dependencies in related materials as the field is allowed to mature.

\section{Total scattering and reverse Monte Carlo}

The principal motivation for applying total scattering techniques to the study of flexible framework materials is to extract information about the correlated processes responsible for behaviour such as NTE. Total scattering experiments, a form of powder diffraction, aim to measure both the Bragg (elastic) and diffuse (inelastic) contributions to the scattering function $S(Q)[15,16]$; Despite the additional experimental demands associated with obtaining absolutely normalised scattering intensities, the approach has a key advantage over "traditional" crystallographic methods in that one obtains information about both one-particle and two-particle correlations [42]. The one-particle correlations define the average structure of the material, while the two-particle correlations characterise the local deviations from this average structure-the correlated displacements implicated in e.g. NTE.

There are a number of different approaches for extracting local structure information from total scattering data, and most focus at some point on the "pair distribution function" (PDF), or one of its variants [43]. The PDF, denoted by $g(r)$, represents the local distribution of atoms around an "average" atom in the material: each peak corresponds to a particular pair of atoms, with its position in $r$ denoting the average interatomic separation, and its width representing the spread of distances due to vibrational motion. The relevance to total scattering is that $g(r)$ and $S(Q)$ are related by sine Fourier transform [43]. As $S(Q)$ is measured to increasingly large $Q$ (possible using high-energy $\mathrm{X}$-ray or spallation neutron sources) one obtains a measure of the PDF to increasingly fine real-space resolution. The process of translating this dense mélange of real-space and reciprocal-space information into a physical understanding of the correlated processes present is perhaps the major challenge in the field.

The reverse Monte Carlo (RMC) approach [44,45] tackles this problem by using the total scattering data to produce large atomistic structural models - the advantage being that it reduces the problem of analysing total scattering data to the problem of analysing a threedimensional box of atoms, a task which is generally more intuitive and for which many tools already exist. The refinement process is data-driven, so that the atomistic models produced are at once consistent with the average structure information contained within the Bragg intensities on the one hand, and the pair correlations represented in the PDF on the other hand. There is also the option to constrain the model with chemical or physical intuition - by constraining bond angles or polyhedral geometries, for example [46] just as "rigid body" 
constraints might be used in "traditional" crystallographic refinement.

The process of RMC refinement begins with solution and Rietveld structure refinement of the average structure via standard Bragg profile methods. The unit cell so obtained is then used to generate a large atomistic supercell. The number of atoms in this supercell will depend on the computational resources at hand, and the nature of the problem to be solved. A typical RMC configuration might contain between 10000 and 100000 atoms. From this atomistic configuration it is possible to calculate the scattering function $S(Q)$, the PDF and also the Bragg intensity profile as modelled in e.g. GSAS [47]. These three functions can be compared directly against the experimental data, and the difference between the calculated and experimental functions, together with any bond-length or bond-angle constraints, is used to drive the Monte Carlo refinement. First, a randomly-selected atom is moved by a random distance in a random direction, and the new seattering functions calculated. If the fits to data improve (i.e. the Monte Carlo energy decreases) then the move is accepted; if not then the move is accepted only with a finite probability that depends on how greatly the fits have deteriorated. A new move is proposed, checked, accepted or rejected, and the process repeated until the configuration is capable of reproducing the experimental data as closely as possible. Great care is taken to ensure that the resultant configuration provides as physically realistic a description of material structure as possible: to this end, one can calculate EXAFS spectra [48], TEM images [49], bond-valence sums [49] and even phonon dispersion curves [50] and check these against the respective experimental functions. Many independent RMC configurations are produced, and from this collection of "snapshots" of material structure it becomes possible to quantify the correlated processes of interest.

\section{Correlations and dynamics from powder diffraction}

The particular correlations of interest will almost certainly differ from one material to the next, and indeed for some systems very little may be known a priori to help target a search for correlated behaviour. At the very simplest extreme, bond-length and bond-angle distributions can be extracted routinely from the RMC configurations [46], and often these are important in their own right. Progressing towards higher-order correlations, one can calculate (e.g.) distributions of polyhedral distortions, tilts and translations [51]; relevant examples include quantification of the role of so-called rigid unit modes (RUMs) in the NTE [52] and PIA [53] behaviour of $\mathrm{ZrW}_{2} \mathrm{O}_{8}$, and the characterisation of an octahedral-tilting transition in $\mathrm{SrSnO}_{3}$ perovskite [54].

Calculation of TEM images is a useful technique for identifying key correlation behaviour whose form might not be known a priori [49,55]. Any structured diffuse seattering present in these images will reflect the periodicity of the correlations, and by masking different atoms in the configuration it is straightforward to identify the particular atom types from which the diffuse intensity arises. One can then calculate real-space correlation functions $\langle\mathbf{u}(0) . \mathbf{u}(r)\rangle$ to determine the range and distribution of the corresponding features. This technique was applied in the study of $\mathrm{SrSnO}_{3}$ to identify polar nano-domains: a form of localised ferroelectric instability where Sn atoms across small two-dimensional islands of the crystal lattice undergo concerted displacements from their average positions [54].

Over the past four years there has been an effort to extend this approach to the quantitative determination of phonon dispersion curves [50]. Doing so asks very subtle questions of the 
data, as it takes the technique of powder diffraction into the domain of triple-axis spectroscopy. There had been some discussion in the literature regarding the possibility (or otherwise) of extracting phonons from PDF peak widths [56-58]. The hope had always been that these widths would reflect the energetics of interactions between pairs of atoms - stronglybound pairs giving sharp peaks in the PDF and weakly-bound pairs giving broader peaks (figure 3) [59]. What RMC provided was a model-independent approach of assessing what phonon information survived the orientational averaging and energy integration implicit in powder diffraction, and what information was lost $[50,60]$.

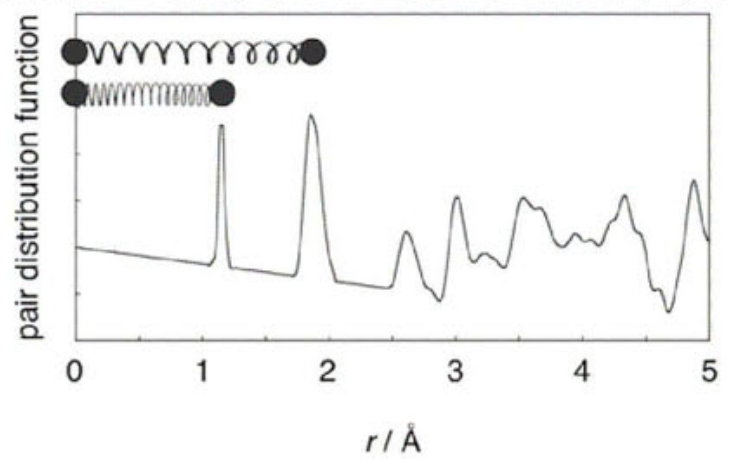

Figure 3. Peak widths in the pair distribution function contain information regarding the energy of interatomic interactions: in general terms, broad peaks correspond to low-energy interactions and sharp peaks to high-energy interactions.

The calculations involve Fourier transform of the atomic displacements observed in RMC configurations into phonon mode coordinates. The magnitude of correlated displacement calculated for a given mode coordinate is then inversely proportional to the corresponding phonon mode energy [61]. The greater the displacement, the lower the energy - essentially the same concept as that which links broad peaks in the PDF to low-energy interactions. Assembling all phonon mode energies for different wave-vectors $\mathbf{k}$, one can piece together the diffraction-based phonon dispersion curves.

By comparing these total scattering phonon dispersion curves for $\mathrm{MgO}[50]$ and for $\mathrm{SrTiO}_{3}$ [60] with those obtained independently in spectroscopic experiments, what became obvious was that total scattering performed well in the low-energy region of the phonon spectrum but that much of the information about high-frequency modes was lost. This is perhaps an unsurprising result in that one expects the real-space signature of high-frequency modes to be subtle indeed $[60,62]$. Nevertheless it is usually the low-frequency modes that are of interest in functional materials: these are most easily populated with temperature and most likely to be involved in phase transitions or NTE behaviour. The archetypal example is the soft-mode transition in $\mathrm{SrTiO}_{3}$ at $105 \mathrm{~K}$ [63]: the temperature dependence of the relevant zoneboundary mode was entirely recoverable using total scattering and RMC [60].

Applying these techniques to the linearly-bridged frameworks discussed above, it was possible to verify the role of transverse vibrational motion of $\mathrm{Co}-\mathrm{C}-\mathrm{N}-\mathrm{Ag}-\mathrm{N}-\mathrm{C}-\mathrm{Co}$ linkages in the low-energy dynamics of $\mathrm{Ag}_{3}\left[\mathrm{Co}(\mathrm{CN})_{6}\right]$, particularly in the form of RUM-type vibrations that preserved $\left[\mathrm{CoC}_{6}\right]$ octahedral geometries [33]. Perhaps the most important result, how- 
ever, came from the calculation of mean atomic vibrational frequencies. As the lattice undergoes its enormous changes in dimension, these energies did not change substantially for the $\mathrm{Co}$ or $\mathrm{C}$ or $\mathrm{N}$ atoms, but at higher temperatures there was a significant decrease in the $\mathrm{Ag}$ vibrational energies $[32,33]$. This energy decrease could be mapped quantitatively onto the increase in lattice enthalpy associated with the expansion and contraction of the framework. This confirmed that anharmonicity of Ag vibrations was the source of the small, but important, energy contribution needed to bring about the "colossal" variation in lattice parameters.

\section{Magnetic flexibility}

The availability of high-quality neutron total scattering instruments, such as GEM at ISIS [64], raises the additional possibility of extracting information about local fluctuations in magnetic structure in magnetic materials. Diffuse magnetic scattering is sometimes the only available handle on magnetic structure in disordered or frustrated systems: the most highprofile example is that of the "spin ice" pyrochlores, where the magnetic ground state has no long-range periodicity, and hence does not produce magnetic Bragg peaks [65].

To this end, the RMC technique has recently been extended to allow for refinement of magnetic structures [45]. Associated with the RMC atomistic supercell is another supercell of equivalent dimensions, containing individual classical spin vectors for each magnetic atom in the original RMC configuration. The refinement process then involves both atom translations and spin reorientations, so that in practice the procedure is actually very similar. A study of antiferromagnetic ordering in $\mathrm{MnO}$ showed that magnetic structure solution and refinement was possible even when starting from random spin configurations [66].

In the present context of flexibility, one might even consider the spin ice systems to be an example of a magnetically-flexible system. The analogy lies in their residual configurational entropy: in these materials there are many ways of ordering the spins (subject to the characteristic 2-in-2-out tetrahedral cluster rules) with equivalent energies. Indeed, the crystallography is complicated for this very reason - the absence of any Bragg component to the scattering means that traditional structural refinement approaches fail entirely. An RMC approach, by contrast, quickly recovers the correct local structure from the powder diffuse scattering, even when started from random spin configurations [67].

The protocols for extracting quantitative lattice dynamical information from RMC configurations can even be extended to these magnetic refinements, where the interest now is in characterising the spin-wave dispersion curves [68]. Despite the overlap between spin-wave and phonon contributions to the inelastic scattering in $\mathrm{MnO}$, the spin-wave dispersion could indeed be recovered, offering a possible method of charactering spin waves in materials for which single-crystal samples cannot easily be obtained [68].

\section{Concluding remarks}

This paper has aimed to review the author's approaches to studying physical properties of flexible framework materials using powder diffraction techniques. A comprehensive review of the state of such a broad field is unfortunately beyond the scope of this manuscript; nevertheless, in the work of other groups, as in the work described here, two key applications of powder diffraction emerge. On the one hand, powder diffraction is ideally suited to charac- 
terising the unusual physical behaviour of framework materials, such as NTE, NLC and temperature- or pressure-induced phase transitions. On the other hand, there is a drive to extend powder diffraction techniques, principally total scattering, to probe dynamical processes in these same materials. The extraction of quantitative local structure and dynamical information from total scattering data is surely a nascent field, but one of sufficiently widespread importance to merit the patience and care needed to develop a rigourous and general protocol for use by the broader crystallography community.

\section{References}

1. Mary, T.A., Evans, J.S.O., Vogt, T. \& Sleight, A.W., 1996, Science, 272, 90.

2. Hoskins, B.F. \& Robson, R., 1990, J. Am. Chem. Soc., 112, 1546.

3. Cheetham, A.K. \& Rao, C.N.R., 2007, Science, 318, 59.

4. Evans, J.S.O., 1999, J. Chem. Soc., Dalton Trans., 3317.

5. Barrera, G.D., Bruno, J.A.O., Barron, T.H.K. \& Allan, N.L., 2005, J. Phys.: Condens. Matt., 17, R217.

6. Evans, J.S.O., Hu, Z., Jorgensen, J.D., Argyriou, D.N., Short, S. \& Sleight, A.W., 1997, Science, 275, 61 .

7. Perottoni, C.A. \& da Jornada, J.A.H., 1998, Science, 280, 886.

8. Pantea, C., Migliori, A., Littlewood, P., Zhao, Y., Ledbetter, H., Lashley, J.C., Kimura, T., Van Duijn, J. \& Kowach, G.R., 2006, Phys. Rev. B, 73, 214118.

9. Ernst, G., Broholm, C., Kowach, G.R. \& Ramirez, A.P., 1998, Nature, 396, 147.

10. Chapman, K.W., Hagen, M., Kepert C.J. \& Manuel, P., 2006, Physica B, 385-6, 60.

11. Hammonds, K.D, Deng, H., Heine, V. \& Dove, M.T. 1997, Phys. Rev. Lett., 78, 3701.

12. Sartbaeva, A., Wells, S.A. \& Redfern, S.A.T., 2004, J. Phys.: Condens. Matt., 16, 8173.

13. Kepert, C.J., 2006, Chem. Commun., 695.

14. Vogt, T. \& Schmall, W.W., 1993, Europhys. Lett., 24, 281.

15. Nield, V.M. \& Keen, D.A., 2000, Diffuse Neutron Scattering from Crystalline Materials (Oxford: Oxford University).

16. Egami, T. \& Billinge, S.J.L., 2003, Underneath the Bragg Peaks: Structural Analysis of Complex Materials (Oxford: Pergamon).

17. Pryde, A.K.A., Hammonds, K.D., Dove, M.T., Heine, V., Gale, J.D. \& Warren, M.C., 1996, J. Phys.: Condens. Matt., 8, 10973.

18. Goodwin, A.L. \& Kepert, C.J., 2005, Phys. Rev. B, 71, 140301(R).

19. Goodwin, A.L., 2006, Phys. Rev. B, 74, 134302.

20. Sharpe, A.G., 1976, The Chemistry of Cyano Complexes of the Transition Metals (London: Academic).

21. Williams, D., Partin, D.E., Lincoln, F.J., Kouvetakis, J. \& O’Keeffe, M., 1997, J. 
Solid State Chem., 134, 164.

22. Goodwin, A.L., Chapman, K.W. \& Kepert, C.J., 2005, J. Am. Chem. Soc., 127, 17980 .

23. Mastuno, N., Yoshimi, M., Ohtake, S., Akahane, T. \& Tsuda, N., 1978, J. Phys. Soc. Jpn., 45, 1542.

24. Crichton, W.A., Bouvier, P. \& Grzechnik, A., 2003, Mater. Res. Bull., 38, 289.

25. Phillips, A.E., Goodwin, A.L., Halder, G.J., Southon, P.D. \& Kepert, C.J., 2008, Angew. Chem. Int. Ed., 47, 1396.

26. Hibble, S.J., Cheyne, S.M., Hannon, A.C. \& Eversfield, S.G., 2002, Inorg. Chem., $\mathbf{4 1 ,} 4990$.

27. Hibble, S.J., Hannon, A.C. \& Cheyne, S.M., 2003, Inorg. Chem., 42, 4724.

28. Margadonna, S., Prassides, K. \& Fitch, A.N., 2004, J. Am. Chem. Soc, 126, 15390.

29. Pretsch, T., Chapman, K.W, Halder, G.J. \& Kepert, C.J., 2006, Chem. Commun., 1857.

30. Chapman, K.W., Chupas, P.J. \& Kepert, C.J., 2006, J. Am. Chem. Soc., 128, 7009.

31. Hibble, S.J., Chippindale, A.M., Poh1, A.H. \& Hannon, A.C., 2007, Angew. Chem. Int. Ed., 46, 7116.

32. Goodwin, A.L., Calleja, M., Conterio, M.J., Dove, M.T., Evans, J.S.O., Keen, D.A., Peters, L. \& Tucker, M.G., 2008, Science, 319, 794.

33. Conterio, M.J., Goodwin, A.L., Tucker, M.G., Keen, D.A., Dove, M.T., Peters, L. \& Evans, J.S.O., 2008, J. Phys.: Condens. Matt., 20, 255225.

34. Calleja, M., Goodwin, A.L. \& Dove, M.T., 2008, J. Phys.: Condens. Matt., 20, 255226 .

35. Sears, D.R. \& Klug, H.P., 1962, J. Chem. Phys., 37, 3002.

36. Goodwin, A.L., Keen, D.A., Tucker, M.G., Dove, M.T., Peters, L. \& Evans, J.S.O., 2008, J. Am. Chem. Soc., 130, 9660.

37. Baughman, R.H., Stafström, S., Cui, C. \& Dantas, J.O., 1998, Science, 279, 1522.

38. Haines, J., Chateau, C., Léger, J.M., Bogicevic, C., Hull, S., Klug, D.D. \& Tse, J.S., 2003, Phys. Rev. Lett., 91, 015503.

39. Goodwin, A.L., Keen, D.A. \& Tucker, M.G., 2008, Proc. Natl. Acad. Sci. U.S.A., in press.

40. Chapman, K.W. \& Chupas, P.J., 2007, J. Am. Chem. Soc., 129, 10090.

41. Poswal, H.K., Tyagi, A.K., Lausi, A., Deb, S.K. \& Sharma, S.M., 2008, J. Solid State Chem., in press.

42. Dove, M.T., 2002, Eur. J. Miner., 14, 331.

43. Keen, D.A., 2001, J. Appl. Cryst., 34, 172.

44. McGreevy, R.L. \& Pusztai, L., 1988, Mol. Simul., 1, 359.

45. Tucker, M.G., Keen, D.A., Dove, M.T., Goodwin, A.L. \& Hui, Q., 2007, J. Phys.: Condens. Matt., 19, 334218. 
46. Tucker, M.G., Dove, M.T. \& Keen, D.A., 2001. J. Appl. Cryst., 34, 630.

47. Clarson, A. \& Von Dreele, R.B., 1994, General Structure Analysis System (GSAS), Los Alamos National Laboratory Report LAUR 86-748.

48. Tucker, M.G., Keen, D.A., Evans, J.S.O. \& Dove, M.T., 2007, J. Phys.: Condens. Matt., 19, 335215.

49. Goodwin, A.L., Withers, R.L. \& Nguyen H.-B., 2007, J. Phys.: Condens. Matt., 19, 335216.

50. Goodwin, A.L., Tucker, M.G., Dove, M.T. \& Keen, D.A., 2004, Phys. Rev. Lett., 93, 075502 .

51. Wells, S.A., Dove, M.T. \& Tucker, M.G. 2002, J. Phys.: Condens. Matt., 14, 4567.

52. Tucker, M.G., Goodwin, A.L., Dove, M.T., Keen, D.A., Wells, S.A. \& Evans, J.S.O., Phys. Rev. Lett., 95, 255501.

53. Keen, D.A., Goodwin, A.L., Tucker, M.G., Dove, M.T., Evans, J.S.O., Crichton, W. A. \& Brunelli, M., 2007, Phys. Rev. Lett., 98, 225501.

54. Goodwin, A.L., Redfern. S.A.T., Dove, M.T., Keen, D.A. \& Tucker, M.T., Phys. Rev. $B, 76,174114$.

55. Withers, R.L., 2005, Z. Kristallogr., 220, 1027.

56. Dimitrov, D.A., Louca, D. \& Röder, H., 1999, Phys. Rev. B, 60, 6204.

57. Reichardt, W. \& Pintschovius, L., 2001, Phys. Rev. B, 63, 174302.

58. Graf, M.J., Jeong, I.-K., Starr, D.L. \& Heffner, R.H., 2003, Phys. Rev. B, 68, 064305 .

59. Chung, J.S. \& Thorpe, M.F., 1997, Phys. Rev. B, 55, 1545.

60. Goodwin, A.L., Tucker, M.G., Cope, E.R., Dove, M.T. \& Keen, D.A., 2005, Phys. Rev. B, 72, 214304.

61. Dove, M.T., 1993, Introduction to Lattice Dynamics (Cambridge: Cambridge University).

62. Cope, E.R. \& Dove, M.T., 2007 J. Appl. Cryst., 40, 589.

63. Cowley, R.A., 1962, Phys. Rev. Lett., 9, 159.

64. Hannon, A.C., 2005, Nucl. Instrum. Meth. Phys. Res., Sect. A, 551, 88.

65. Bramwell, S.T. \& Gringas, M.J.P., 2001, Science, 294, 1495.

66. Goodwin, A.L., Tucker, M.G., Dove, M.T. \& Keen, D.A., 2006, Phys. Rev. Lett., 96, 047209.

67. Goodwin, A.L., manuscript in preparation.

68. Goodwin, A.L., Dove, M.T., Tucker, M.G. \& Keen, D.A., 2007, Phys. Rev. B, 75, 075423.

Acknowledgements. The author gratefully acknowledges many valuable discussions with M.T. Dove (Cambridge), M.G. Tucker and D.A. Keen (RAL), and J.S.O. Evans (Durham). 

I.

METHOD DEVELOPMENT AND APPLICATION

I.1 Determination of Crystal Structure 



\title{
New approach to the indexing of powder diffraction patterns using topographs
}

\section{R. Oishi ${ }^{1, *}$, M. Yonemura ${ }^{2}$, A. Hoshikawa ${ }^{2}$, T. Ishigaki ${ }^{2}$, K. Mori ${ }^{3}$, S. Torii ${ }^{1}$, T. Morishima ${ }^{1}$, T. Kamiyama ${ }^{1}$}

${ }^{1}$ High Energy Accelerator Research Organization (KEK), 1-1, Oho, Tsukuba-city, Ibaraki, Japan

2 Ibaraki University, 4-12-1, Nakanarisawa, Hitachi-city, Japan

${ }^{3}$ Kyoto University, Kumatori-cho Sennan-gun, Osaka, Japan

"ryoko.oishi@kek.jp

Keywords: powder indexing, quadratic form, algorithm, powder ab-initio cell determination

\begin{abstract}
A new indexing algorithm is proposed that searches solutions thoroughly and quickly. The algorithm is well established for poor quality data e.g. for data containing some false peaks, or for those cases that peak positions coincide with two or more different lattices. It has succeeded for any lattice symmetry by carrying out the thorough search in several minutes at most. A graph structure called topograph, first introduced by the mathematician Conway, plays an important role in our method. In this paper, we introduce the algorithm and give some preliminary results.
\end{abstract}

\section{Introduction}

At present, there are many algorithms and software for the indexing of powder patterns e.g. Ito's method [7,9], the trial-and-error method in TREOR [4], the dichotomy method in Dicvol [2], the Monte-Carlo method in McMaille [1], a genetic algorithm in GAMATCH [6] etc. Nevertheless, there are still some limitations and room for the improvement of indexing methods. Especially, the following difficulties have been discussed for a long time:

a) Users have no way to judge if the solution supplied by the indexing algorithm is the real one or if other proper lattices exist.

b) The indexing results are highly sensitive to data noise; wrong solutions are often caused by errors in peak search.

Although it is true that these problems are difficult to solve, the method proposed in this paper may help Crystallographers to struggle these problems. In the sequel, we explain how the above problems are essential to this field and related to thorough search.

Regarding point a), even for a complete pattern, there might be more than one indexing solution (see e.g. [8] for a 2-dimensional example of different lattices with perfectly coincident peak positions. From this, we can easily construct infinitely many pairs of 3-dimensional 
lattices with identical peak positions, although the indexing solution is always finite for a pattern.)

Point b) includes the situation of diffraction patterns with false peaks due to the presence of impurities. Since these false peaks might increase the number of the possible solutions, thorough search is also required in this case. Statistical treatment is also effective to resolve the sensitiveness. Using a property of the general lattices, a new criterion different from the figures of merit in [10] can be defined for the diffraction pattern, i.e. an integer is associated with the lattice, and it is bigger if it corresponds to the real solution or to a lattice very near to it.

In this paper, we introduce this new criterion and the algorithm based on it. The criterion is defined as the number of edges of a graph structure. For 2-dimensional lattices, the graph structure is corresponds to Conway's topograph, which is also used in this method, since 2dimensional sublattices are first constructed. In the next section, we explain how to use Conway's topographs for powder indexing; the basic idea is explained in the 2-dimensional case, since it is easier to draw figures. The details of the indexing algorithm are described in section "Indexing algorithms". The algorithm is implemented in a $\mathrm{C}++$ program. Although it just finishes the first development stage, it already supplies the candidate unit-cell solutions. We also comment the present software status and some results in section "Preliminary results".

\section{Conway's topographs}

In the sequel, for a reciprocal vector $\vec{K}$ that corresponds to the Miller index $\vec{h}$, we denote $Q(\vec{h}):=\|\vec{K}\|^{2}$, where $\|\vec{K}\|$ means the vector length. It is called the Q-value of $\vec{h}$. Historically, the following equation, introduced by Runge, Ito, and Wolff [5, 9], is widely used for powder indexing:

$$
Q\left(\vec{h}_{1}+\vec{h}_{2}\right)+Q\left(\vec{h}_{1}-\vec{h}_{2}\right)=2\left(Q\left(\vec{h}_{1}\right) \quad Q\left(\vec{h}_{2}\right)\right)
$$

These authors also introduced several other formulae written as a linear sum of the Q-values,

$$
\begin{aligned}
& Q(m \vec{h})=m^{2} Q(\vec{h}),
\end{aligned}
$$

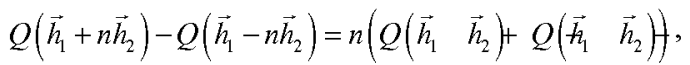

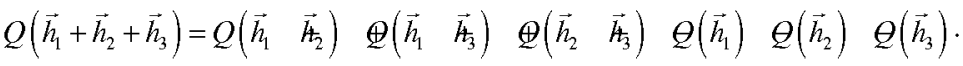

The new method only treats the first two types and ignores the rest, because all the general linear equations of Q-values including (3), (4) are linear sums of (1) and (2). This allows simplifying the algorithm. Especially, we will concentrate on (1), because there is not so much to do with (2). Due to the errors in peak positions, such equations among Q-values often hold simply by accident. To avoid this, a new method using Conway's topograph is proposed that will tell us how the equations are related to each other. 
Conway's topograph consists of many equations of type (1), and the following graph (figure 1) is associated with this equation,

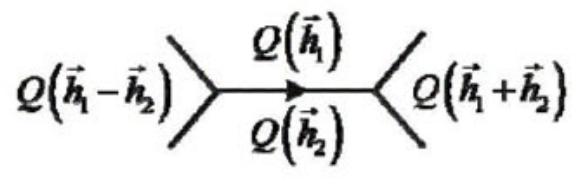

Figure 1. The graph for equation (1).

The arrow in the diagram pointing to the right indicates $Q\left(\vec{h}_{1}-\vec{h}_{2}\right)<Q\left(\vec{h}_{1}+\vec{h}_{2}\right)$. Otherwise, the arrow points to the left. Conway's topograph is a tree consisting of infinite directed edges as represented in figure 2 . It shows the topograph for a 2-dimensional lattice with basis vectors $\vec{h}_{1}, \vec{h}_{2}$.

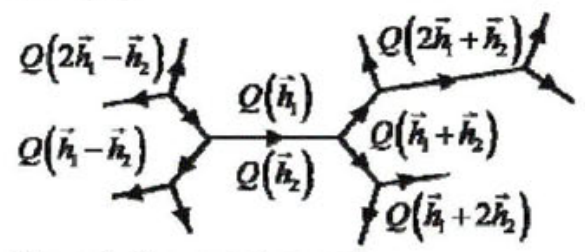

Figure 2. Conway's topograph.

It is a system connecting equations of type (1). Besides the graph of figure 1, we can see in figure 2 three additional subgraphs corresponding to the equations:

$$
\begin{aligned}
& Q\left(2 \vec{h}_{1}+\vec{h}_{2}\right)+Q\left(\vec{h}_{2}\right)=2\left(Q\left(\vec{h}_{1}\right) \quad Q\left(\vec{h}_{4} \vec{h}_{2}\right)\right)+ \\
& Q\left(\vec{h}_{1}+2 \vec{h}_{2}\right)+Q\left(\vec{h}_{2}\right)=2\left(Q\left(\vec{h}_{2}\right) \quad Q\left(\vec{h}_{1} \vec{h}_{2}\right)\right), \\
& Q\left(2 \vec{h}_{1}-\vec{h}_{2}\right)+Q\left(\vec{h}_{2}\right)=2\left(Q\left(\vec{h}_{1}\right) \quad Q\left(\vec{h}_{4} \vec{h}_{2}\right)\right)-
\end{aligned}
$$

All of them are obtained simply by replacing the variables in equation (1). According to Ito's method, if we have a quadruple $\left(Q_{1}, Q_{2}, Q_{3}, Q_{4}\right)$ fulfilling the condition $2\left(Q_{1}+Q_{2}\right)=Q_{3} \quad Q_{4}$, we may expect that there are two indices $\vec{h}_{1}, \vec{h}_{2}$, such that

$$
Q_{1}=Q\left(\vec{h}_{1}\right), Q_{2}=Q\left(\vec{h}_{2}\right), Q_{3}=Q\left(\vec{h}_{1} \vec{h}_{2}\right),-Q_{3}=Q\left(\begin{array}{ll}
\vec{h}_{1} & \vec{h}_{2}
\end{array}\right)+
$$

Then, the values of $Q\left(2 \vec{h}_{1}+\vec{h}_{2}\right), Q\left(\vec{h}_{1}+2 \vec{h}_{2}\right), Q\left(2 \vec{h}_{1}+\vec{h}_{2}\right)$ can be calculated using equations (5), (6), (7). These things can be figured using Conway's topograph; first, start with the state of figure 1 . If there is a peak at the position predicted for $Q\left(2 \vec{h}_{1}+\vec{h}_{2}\right)$ by (5), then, extend the corresponding edge to the direction. If there really exist Miller indices $\vec{h}_{1}, \vec{h}_{2}$ such that (8) holds, the topograph will have many edges. Otherwise, it will stop growing soon, 
because it is quite rare to always find a different irrelevant peak at the expected position, accidentally.

By counting the number of edges in the graph, we can know how the lattice expanded by $\vec{h}_{1}, \vec{h}_{2}$ has more possibility to be contained in the original lattice. A graph structure for the 3dimensional lattice is a combination of Conway's topograph, and it is also possible to count the edges. To evaluate the number effectively, our basic algorithm is similar to Ito's method, although in our method many graphs are constructed.

Figure 3 describes the topograph for a 2-dimensional sublattice of the diamond lattice. Num-

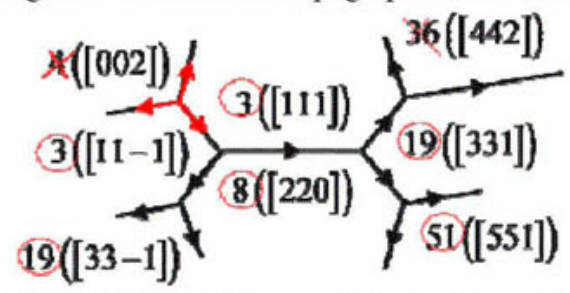

Figure 3. Conway's topograph for the diamond lattice.

bers $3,8,19, \ldots$ are the Q-values of the diamond lattice with unit-edge, and $[\ldots]$ are the Miller indices. It can be seen that the topograph has infinitely many edges despite of the extinction rule. This seems to hold also for general cases. In addition, among the proper sublattices, the criterion becomes more reliable for smaller unit-cells because the number of peaks is finite. As a result, the real solution always obtains the highest probabilistic score.

\section{Indexing algorithm}

At present, the parameters that the program requires are the peak positions, the peak widths (used instead of the errors of the Q-values), the adjusting number to multiply the Q-errors by, the conversion parameters from TOF/dispersion-angle to Q-values, the number of the peaks and the topographs to use, and the number of data of the candidates to output.

The errors of the Q-values are used to define the permitted regions for each peak. As mentioned above, it would be better to use the peak widths instead of the errors of the real peak positions, because the errors by the peak widths have some desirable properties e.g. they are easy to estimate, have a smaller values for lower peaks, and can represent naturally how the peaks overlap. Since the test results show that the algorithm is not sensitive to small differences of the peak widths or the conversion parameters including the zero-point shift, very accurate values for the parameters should be not necessary.

The indexing procedure may be described as follows:

(1) Search for the peaks in the diffraction pattern. Prepare an input file containing the positions and the widths of the peaks.

(2) Calculate the $\mathrm{Q}$-values from the peak positions.

(3) Detect 4-tuples $\left(Q_{1}, Q_{2}, Q_{3}, Q_{4}\right)$ of the Q-values fulfilling the equation $Q_{3}+Q_{4}=2\left(Q_{1} \Theta_{2}\right)$. If $Q_{3}<Q_{4}$, this is supposed to correspond to the directed graph: 


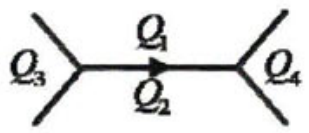

Otherwise, the arrow points in the opposite direction. Moreover, if there exists a $Q_{5}$ such that $Q_{2}+Q_{5}=2\left(\begin{array}{ll}Q_{1} & Q_{4}\end{array}\right)$, the graph is extended as follows,

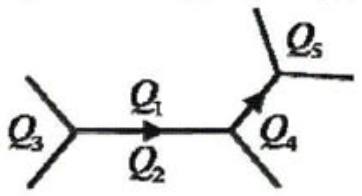

By repeating the procedure, Conway's topographs are constructed.

(4) The 4-tuples of Q-values detected in step (3), are supposed equal to the square lengths of the edges and the diagonals of a parallelogram in the lattice. (figure at the right.) If it is false, the number of the edges is less because peaks are rarely detected at the predicted position. Therefore, topographs with many edges have more possibility to correspond to the cell of the reciprocal lattice of the crystal.

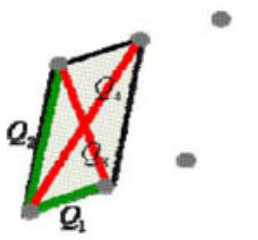

(5) If some topographs contain a common Q-value, they can be combined by considering that the Q-value corresponds to the same reciprocal lattice-vector; by doing so, a 3-dimensional lattice is gained that contains all the 2-dimensional lattices associated with the original topographs. Hence, from the unit-cell parameters of these sublattices, the parameters of the 3-dimensional lattices are also calculated. There is also a graph for the 3-dimensional lattice constructed by combining topographs; each node corresponds to a basis of the 3-dimensional lattice, and the edges given by Ito's formula connect the nodes, as for the 2-dimensional case. Similarly, the graphs with more edges have more possibility to correspond to the original lattice. The candidates for the solution are chosen in this way.

(6) Let $\Lambda_{0}$ be the solution. Then, for any sublattice $\Lambda$ derived from the previous steps, there exist an integer $n$ such that $\Lambda_{0} \subset 1 / n \Lambda$. Hence, we can estimate $\Lambda_{0}$ from $\Lambda$. The diffraction peaks are indexed using the obtained unit-cell parameters. (Although the original lattice is already detected in step (5) for all the test data in section 4 , this process might be necessary in some cases.)

\section{Preliminary results}

We report here the status of the software and some preliminary results. More results and the details will be separately shown. (Some functions necessary to output parameters properly are not yet ready.) The program is written in $\mathrm{C}++$. It already supplies tens of candidate lattices including the correct answer for the test data: a) Diamond lattice ( $F d 3 m$, Silicon), b) Tetragonal ( $I 4_{1} /$ amd, Anatase), c) Orthogonal (Fdd2, PrBaSuCrO), d) Triclinic (P1, protein). 
These powder data were chosen randomly by the first author among several data sets proposed by the collaborators. The peak positions were found by our original peak search program.

Although it depends on the number of peaks, the calculation takes only a few minutes at most. The detailed calculation time will be reported with the test results. Our indexing algorithm works well for general lattices and in the presence of false peaks due to impurities, e.g. in the low angle region for silicon or in the high angle region for PrBaSuCrO. These results show that the program has the expected capacity. Due to the several new characteristics of our algorithm, there seems to be a lot of interesting aspects to investigate in the future.

\section{References}

1. Le Bail, A., 2002, McMaille program, http:/www.cristal.org/McMaille/.

2. Boultif, A. \& Louër, D., 2004, Powder pattern indexing with the dichotomy method, J. Appl. Cryst., 37, 724.

3. Conway, J.H. \& Fung, F.Y.C., 1997, The Sensual (Quadratic) Form, The Mathematical Association of America.

4. Kohlbeck, F. \& Horl, E.M., 1978, Trial and error indexing program for powder patterns of monoclinic substances, J. Appl. Cryst., 11, 60.

5. Runge, C., 1917, Die Bestimmung eines Kristallsystems durch Röntgenstrahlen, $Z$. Physik, 18, 509.

6. Tam, K.Y. \& Compton, R.G., 1995, GAMATCH- a genetic algorithm-based program for indexing crystal faces, J. Appl. Cryst., 28, 640.

7. Visser, J.W., 1969, A Fully Automatic Program for Finding the Unit Cell from Powder Data, J. Appl. Cryst., 2, 89.

8. Watson, G.L., 1979, Determination of a binary quadratic form by its values at integer points, Mathematica, 26, (1), 72 .

9. Wolff, P.M., 1958, Detection of simultaneous zone relations among powder diffraction lines, J. Appl. Cryst., 2, 89.

10. Wolff, P.M., 1968, A simplified criterion for the reliability of a powder pattern indexing, J. Appl. Cryst., 1, 108. 


\title{
Two-level genetic algorithm for a full- profile fitting of X-ray powder patterns
}

\author{
Y. I. Yakimov", E. S. Semenkin, I. S. Yakimov
}

Siberian Federal University, Krasnoyarsk, Russia

"yar_yakimov@mail.ru

Keywords: genetic algorithm, global optimisation, structure solution, full-profile fitting

Abstract. A new approach for automated crystal structure solution and refinement from powder diffraction data based on two-level Genetic Algorithm combined with Rietveld-like DDM method is reported. Application of this method is demonstrated on two samples with previously known crystal structures, and the performance is discussed.

\section{Introduction}

Over the past few years, many new strategies, called Direct Space Techniques, have been developed for solving crystal structures [1]. These real-space methods approach structure solution by the generation of test crystal structures and assessment of the quality (fitness) of each structure by comparison between the calculated diffraction pattern for that structure and the experimental powder pattern. The key to the success of this approach is the application of an effective global optimisation procedure used to find the global minimum of the fitness hypersurface corresponding to the best structure solution. Genetic algorithms (GA) are such efficient global optimisation techniques in which the fittest individuals (encoded test structures) of a population survive and spawn next improved generations [2]. GA already has proven to be useful in crystal structure solution $[1,3]$ but structure determination from powder diffraction data in common is still not a routine.

This work is dedicated to GA spread-out to Rietveld-like derivative difference minimization (DDM) method [4] including full-profile fitting and refinement of crystal structure models. A two-level hybrid genetic algorithm has been developed for this purpose. The algorithm is implemented as a shell over the full-profile analysis program DDM [4] and tested on some powder patterns of single and multi-phase samples with known stable crystal structures.

\section{Methodology}

The proposed algorithm is based on composition of the dual GA with the least-squares method (LSM) designed as follows (figure 1). First-level GA individuals comprise values of profile and structure parameters used in the Rietveld method. Each second-level GA individual is a bit string containing one bit per parameter, where bit values define parameters to be refined with the LSM on a current iteration (thus specifying a refinement strategy). Thereby stochastic and deterministic search procedures are combined each benefiting from another. 
During generation change, better and better solutions arise gradually tending to optimum.

\section{Level one algorithm}

First level algorithm is similar to conventional GA $[2,3]$. GA chromosomes consist of parameter values in binary form with encoding accuracy defined by the user. Initial population is generated randomly, i.e. a priori known general atomic coordinates are not required. GA fitness function is based on the weighted profile $\mathrm{R}$-factor $\left(\mathrm{R}_{\mathrm{DDM}}\right.$, see below). Fitness determines the chances of individual to become a parent and pass its genes (parameter values) to offspring through processes of recombination and mutation [2]. In addition, the best and some better offsprings undergo local minimisation with DDM (see below). Lamarckian concept of evolution is used [2,3], i.e. parameter values found by local search replace old ones.

\section{Level two algorithm}

A population consists of a number of bit strings $-B$-individuals (initially could be generated randomly or by special schemata) and parameter strings transferred from above level $-P$ individuals. Each $B$-individual bit set in ' $T$ ' specifies a corresponding parameter of $P$ individual to be refined on a current generation. In whole, $B$-type individuals define DDM search subspace. The better the refining has been, the higher $B$-type fitness is assigned. $B$ individuals are recombined and mutated without $P$-individuals altering. Thus genetic operations over $B$-individuals generate strategies of $P$-individuals refinement.

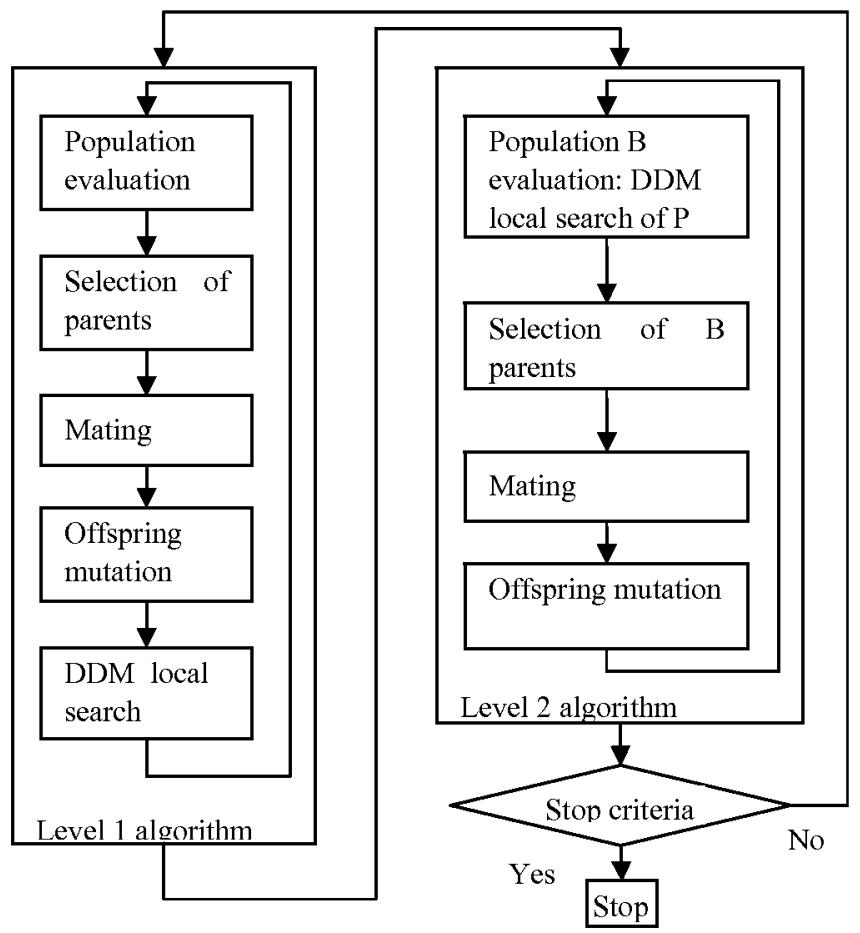

Figure 1. Flow-chart for dual-level GA algorithm. 
Both levels are executed alternately with transferring the best individual and some randomly chosen individuals to another level. Evolution of trial structures at the first-level GA is helpful in searching parameter values suitable for the DDM minimisation on the second GA level and overcoming local minima. The procedure stops when the GA evolution converges to an acceptable $R$ value.

\section{DDM minimization}

A derivative difference minimization (DDM) method [4] is used for structure refinement instead of the classic Rietveld procedure. It involves no background modeling due to leastsquares minimization of difference curve derivatives:

$$
M F=\sum w_{1}\left(\frac{\partial}{\partial \theta}(Y o-Y c)\right)^{2}+\sum w_{2}\left(\frac{\partial^{2}}{\partial \theta^{2}}(Y o-Y c)\right)^{2},
$$

where $Y_{o}$ and $Y_{C}-$ observed and calculated profile intensities, $\theta$ - diffraction angle, $w_{i}-$ weights.

The refinement residual $R_{D D M}$ is a modification of the usual $R_{\mathrm{wp}}$ value and is calculated as follows:

$$
R_{D D M}=50 \sqrt{\frac{\sum_{k=1}^{2} \sum_{i=m+1}^{N-m} w_{i}^{k}\left[\sum_{j=-m}^{m} s_{j}^{k}\left(Y o_{i+j}-Y c_{i+j}\right)\right]^{2}}{\sum_{k=1}^{2} \sum_{i=m+1}^{N-m} w_{i}^{k}\left[\sum_{j=-m}^{m} s_{j}^{k} Y o_{i+j}\right]^{2}}+\frac{\sum_{i=m+1}^{N-m} w_{i}\left[Y o_{i}-\sum_{j=-m}^{m} s_{j}^{0}\left(Y o_{i+j}\right)\right]^{2}}{\sum_{i=m+1}^{N-m} w_{i} Y o_{i}^{2}}},
$$

$s_{j}^{k}-$ Savitzky-Golay coefficients for the derivative of order $\mathrm{k}$ with the profile convolution interval $[-m, m], N$ is the number of points in the profile, $w_{i}^{k}$ are the weights (see [4]).

$\mathrm{R}_{\mathrm{DDM}}(2)$ is used as the first-level GA fitness function.

The variable structural and profile parameters $v_{r}$ are refined by iterative solving of a linearized system:

$$
\sum_{k=1}^{2} \sum_{i=m+1}^{N-m} w_{i}^{k}\left[\sum_{j=-m}^{m} s_{j}^{k}\left(Y o_{i+j}-Y c_{i+j}\right)\right] \cdot\left[\sum_{j=-m}^{m} s_{j}^{k} \frac{\partial Y c_{i+j}}{\partial v_{r}}\right]=0
$$

(adapted Newton-Raphson algorithm).

\section{Program implementation and results}

The algorithm is implemented as a shell over the DDM program [4] that carries out fullprofile fitting as described above. The interface comprises several tabs with the following information:

- Settings tab allows to setup the GA with a number of controls,

- Patterns tab displays true (if known), initial (if given) and best found values of optimised parameters and corresponding experimental and calculated patterns (figure 2),

- Progress tab shows how effective and fruitful the last GA stage was (figure 3). 
The algorithm performance is illustrated here on two powder patterns of single and multiphase samples with previously known crystal structures. The task was to find the atomic structure, whereas profile parameters were known from prior analysis (Rietveld refinement).

\section{Sample 1}

The crystal structure of $\mathrm{Pd}\left(\mathrm{NH}_{3}\right)_{2}\left(\mathrm{NO}_{2}\right)_{2}$ (see table 1) initially had been solved from powder diffraction data [5] by Patterson search. This sample shows how the structure model is found and refined automatically from X-ray powder diffraction data by applying the proposed GA method. All general atomic coordinates and thermal vibration parameters B (excluding those of $H$ ) were searched with GA over the asymmetric unit cell part.

Table 1. Fractional atomic coordinates: previously known (true) and found by GA (calc).

\begin{tabular}{|c|c|c|c|c|c|c|c|c|}
\hline \multicolumn{9}{|c|}{$\begin{array}{l}\mathrm{Pd}\left(\mathrm{NH}_{3}\right)_{2}\left(\mathrm{NO}_{2}\right)_{2}, \quad \text { Space group } P-1(\text { no. } 2) \\
\text { Unit cell: } a=5.4251(1) \AA, b=6.3209(1) \AA, c=5.0031(1) \AA, \\
\alpha=111.87(0)^{\circ}, \beta=100.4(0)^{\circ}, \gamma=91.37(0)^{\circ}\end{array}$} \\
\hline Atom & $\mathrm{X}$ true & $\mathrm{X}$ calc & Y true & Y calc & $\mathrm{Z}$ true & $\mathrm{Z}$ calc & B true & B calc \\
\hline $\mathrm{Pd}$ & 0.5000 & - & 0.5000 & - & 0.5000 & $\begin{array}{l}- \\
-\end{array}$ & 0.4840 & 0.4850 \\
\hline $\mathrm{N}$ & 0.3440 & 0.34480 & 0.6970 & 0.69737 & 0.3000 & 0.29992 & 1.2300 & 1.2350 \\
\hline $\mathrm{O} 1$ & 0.1200 & 0.12050 & 0.7270 & 0.72687 & 0.2840 & 0.28350 & 3.2520 & 3.2622 \\
\hline $\mathrm{O} 2$ & 0.4690 & 0.46878 & 0.7910 & 0.79121 & 0.1810 & 0.18030 & 1.6650 & 1.6679 \\
\hline N1 & 0.2090 & 0.20847 & 0.2450 & 0.24489 & 0.2680 & 0.26647 & 1.0860 & 1.0741 \\
\hline $\mathrm{H1}$ & 0.1000 & 0.10247 & 0.2220 & 0.21747 & 0.3670 & 0.37555 & 5.0000 & - \\
\hline $\mathrm{H} 2$ & 0.2850 & 0.27740 & 0.1260 & 0.12336 & 0.2190 & 0.21450 & 5.0000 & - \\
\hline $\mathrm{H3}$ & 0.0750 & 0.07745 & 0.2770 & 0.27781 & 0.0990 & 0.11603 & 5.0000 & - \\
\hline
\end{tabular}

Setings Potemt | Progess | Populetion | Papuision II |

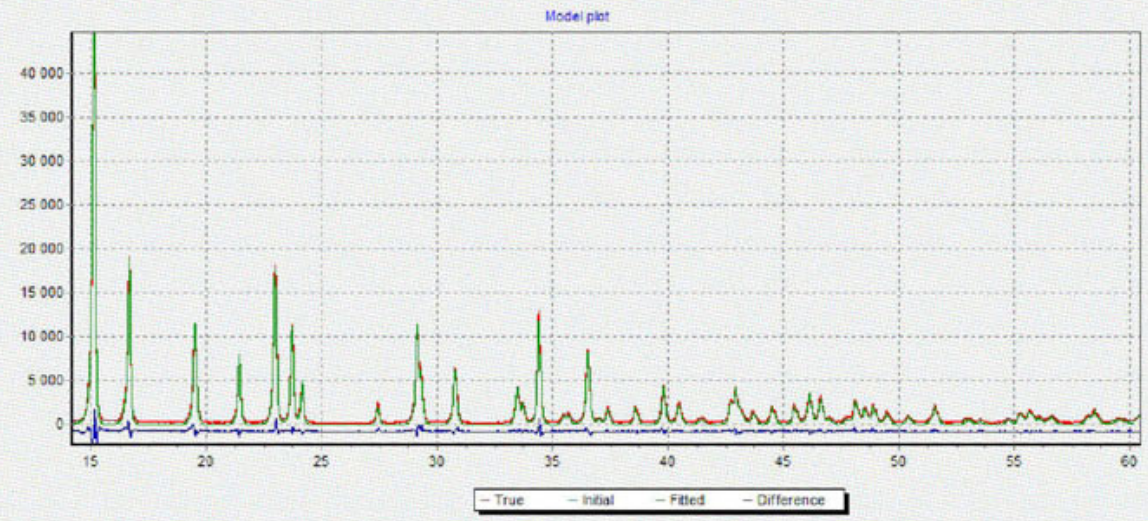

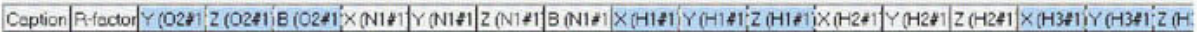
\begin{tabular}{l|l|lllllllllllllllllll}
\hline Best & 8,200 & 0,79121 & 0,18030 & 1,66790 & 0,20847 & 0,24489 & 0,26547 & 1,07406 & 0,10247 & 0,21747 & 0,37555 & 0,27740 & 0,12336 & 0,21450 & 0,07745 & 0,27781 & 0,111
\end{tabular} Figure 2. Observed (red), calculated (green) and difference (blue) XRD powder profiles for sample lón GA last stage. Table holds reference (previously known, not used in calculations) and best found atomic coordinates with corresponding $R_{D D M}$ values. 
Settings | Patterns Progress | Population I | Population II |
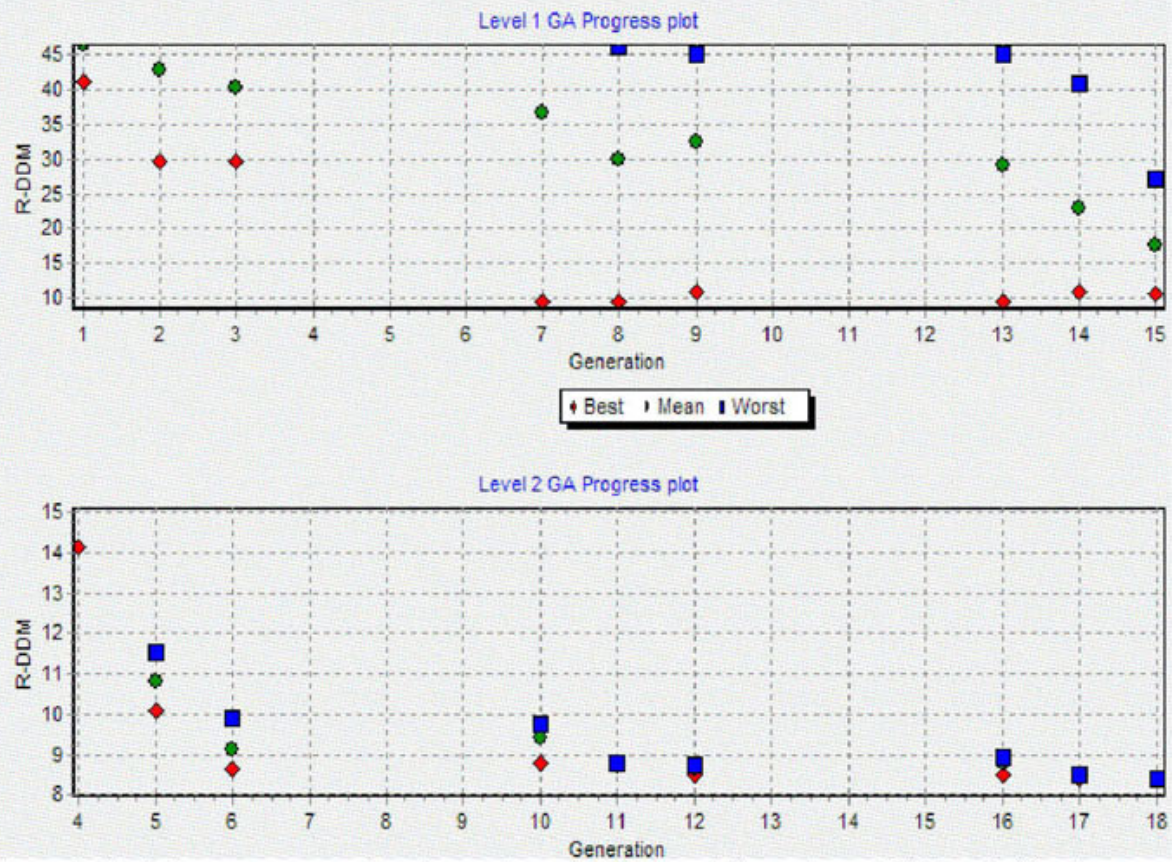

Figure 3. Evolution progress on first (upper image) and second (lower image) GA levels ( $R$ value over generations is shown). Diamonds correspond to best found solution fitness $\left(R_{D D M}\right)$, circles-average $R_{D D M}$ over the whole generation, squares - worst structure fitness.

Solution including all $\mathrm{H}$ atomic coordinates was found at the end of third GA cycle. Note that problem dimension ( 26 parameters being optimised) is comparable to population sizes used: 3 generations of 20 first-level individuals along with 3 generations of 10 second-level individuals per two-level algorithm cycle. Time elapsed: $4 \mathrm{~min} 21 \mathrm{sec}$ (on CPU AMD X2 $4400+)$.

\section{Sample 2}

A well-known four-phase sample CPD-2 [6] from Round Robin on QPA was chosen to assess the performance of the described GA for quantitative phase analysis. True and calculated CPD-2 phase compositions are given in table 2 below. All general atomic coordinates and B's (13 structural parameters in total, except B for $H$ ) were searched with the GA. At the final stage the phase concentrations $W_{j}$ were calculated from their scale factors $S_{j}$ as usual for QPA by Rietveld method:

$W_{j}=S_{j} \rho_{j} / \sum_{i} S_{i} \rho_{i}$,

where $\rho_{j}$ is the density of phase $j$. 
Table 2. Composition of sample CPD2.

\begin{tabular}{|l|l|l|l|l|}
\hline Mineral & Formula & True & Calculated & Error \\
\hline Corundum & $\mathrm{Al}_{2} \mathrm{O}_{3}$ & 21.27 & 21.24 & 0.03 \\
\hline Fluorite & $\mathrm{CaF}_{2}$ & 22.53 & 22.58 & 0.05 \\
\hline Zincite & $\mathrm{ZnO}$ & 19.94 & 19.68 & $\mathbf{0 . 2 6}$ \\
\hline Brucite & $\mathrm{Mg}(\mathrm{OH})_{2}$ & 36.26 & 36.50 & 0.24 \\
\hline
\end{tabular}

This solution was obtained at the end of the first algorithm cycle with $\mathrm{R}_{\mathrm{DDM}}$ decreasing from $39.6 \%$ for the initial generation to $8.8 \%$ for the last generation of the second GA level. Evolution of 4 generations of the first-level population of 10 individuals and 5 generations of the second-level population of 5 individuals was involved. Time spent: $54 \mathrm{sec}$.

\section{Concluding remarks}

Two-level genetic algorithm has shown satisfactory performance and efficiency. The combination of stochastic and deterministic approaches has proven rather fruitful and profitable.

The capability of successful hydrogen coordinates search owing to DDM-GA combining could be noticed. It should be emphasized that crystal structure solution and refinement were carried out automatically here without any user intervention.

Since presented multi-level GA is the issue of on-going research, further improvements are expected. Method is being tested on more complex structures.

\section{References}

1. Rizzi, R. (Ed.), 2007, CPD IUCr Newsletter, 35.

2. Michalewicz, Z., 1996, Genetic Algorithms + Data Structures = Evolution Programs (Berlin: Springer-Verlag).

3. Turner, G.W., Tedesco, E., Harris, K.D.M., Johnston, R.L. \& Kariuki, B.M., 2000, Chem. Phys. Lett., 321, 183.

4. Solovyov, L.A., 2004, J. Appl. Cryst., 37, 743.

5. Blokhin, A.I., Solovyov, L.A., Blokhina, M.L., Yakimov, I.S. \& Kirik, S.D., 1996, Russ. J. Coord. Chem., 22, 185.

6. Scarlett, N.V.Y., Madsen, I.C., Cranswick, L.M.D., Lwin, T., Groleau, E., Stephenson, G., Aylmore, M. \& Agron-Olshina, N., 2002, J. Appl. Cryst., 35, 383.

Acknowledgements. We are grateful to L.A. Solovyov for providing the opportunity to employ DDM method in this project. 


\title{
Characterization of proteins by powder diffraction
}

\section{R. B. von Dreele}

XSD, Argonne National Laboratory, 9700 S. Cass Ave., Argonne, IL, USA, 60439 vondreele@anl.gov

Keywords: protein powder diffraction, Rietveld refinement, structure solution

\begin{abstract}
A simulation of a protein powder diffraction pattern was stunning in the apparent amount of information that was seen. A subsequent experiment on metmyoglobin gave a powder diffraction pattern that showed very little sample broadening; the peak widths were essentially limited by the instrument resolution. The challenge is to make use of this in protein structure analysis. This talk will recall some of those early experiments and data analyses as well as an overview of current progress and future possibilities.
\end{abstract}

\section{Introduction}

In a Presidential Address to the Physical Society in 1921, W. H. Bragg described the "Second Method" (i.e. powder diffraction) as one in which "All the spectra of the different planes are thrown together on the same diagram, and must be disentangled." [1]. A powder sample consists of many $\left(-10^{9}\right.$ within $\left.1 \mu 1\right)$ small crystals that fill $\sim 50 \%$ of the volume; the remainder filled with air, mother liquor or other (usually) non-Bragg scattering material. The resulting diffraction pattern is then the aggregate of all the individual crystallite diffraction patterns and thus appears as rings (figure 1).

Besides using 2D imaging detectors, powder diffraction data can be collected at high resolution (i.e. sharp peaks) with multidetector analyzer systems (figure 2) [2] to give data such as found for the green tea component catechin (figure 3). Modern methods of analysis for this data (structure solution [3] and Rietveld refinement [4]) have become more powerful and readily applied to small organic molecules (eg. catechin tetrahydrate; figure 4) [5].

\section{Proteins and powders}

Experience has shown that obtaining single crystals for protein structure determination is rarely successful; investigations that begin with a promising sequence in any genome yield a protein structure deposited with the RCSB PDB only $5-10 \%$ of the time. A substantial fraction of the losses $(\sim 30 \%)$ occur at the step in producing single crystals of sufficient size (e.g. $>20 \mu \mathrm{m}$ on all sides) and of suitable quality for diffraction. In fact, polycrystalline material is obtained in perhaps as much as $\sim 50 \%$ of the cases [6]. Powder diffraction provides an attractive alternative because it removes the requirement for large crystals; the more readily avail- 


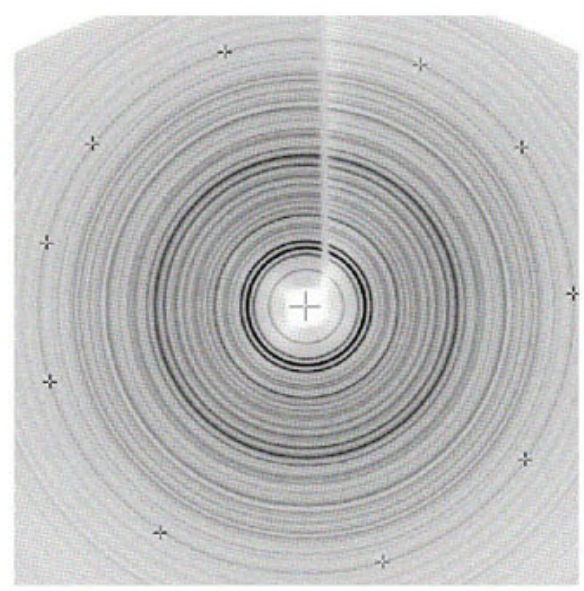

Figure 1. An X-ray powder diffraction pattern of hen egg white lysozyme (HEWL) obtained on a MAR345 image plate at $20 \mathrm{keV}$.

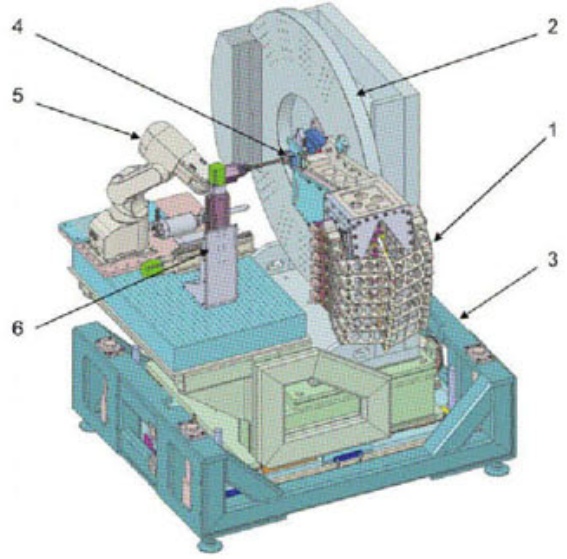

Figure 2. A 12 detector/analyzer diffractometer installed on beam line $11 B M B$ at APS, Argonne National Laboratory. Items noted are 1) detector assembly, 2) main goniometer circle, 3) frame, 4) sample position, 5) sample changing robot and 6) beam stop.

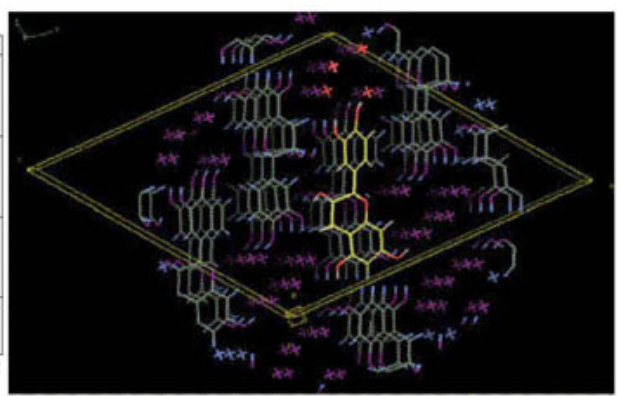

Figure 4. Crystal structure of catechin tetrahydrate determined from high resolution powder diffraction data.

able $\sim 1 \mu \mathrm{m}$ crystallite size is optimal. Moreover, powder diffraction provides views of phase transitions and polyphasic behaviour of potential pharmokinetic significance as well as more general protein materials science interest.

\section{Initial studies}

The potential for protein powder diffraction was not understood until a pattern simulation of a small ( $\sim 400$ atoms) protein revealed the wealth of information that was possibly available. A subsequent experiment on metmyoglobin [7] showed a protein powder pattern (figure 5) to be extremely rich in very sharp diffraction lines consistent with $\sim 1 \mu \mathrm{m}$ crystallites that are 


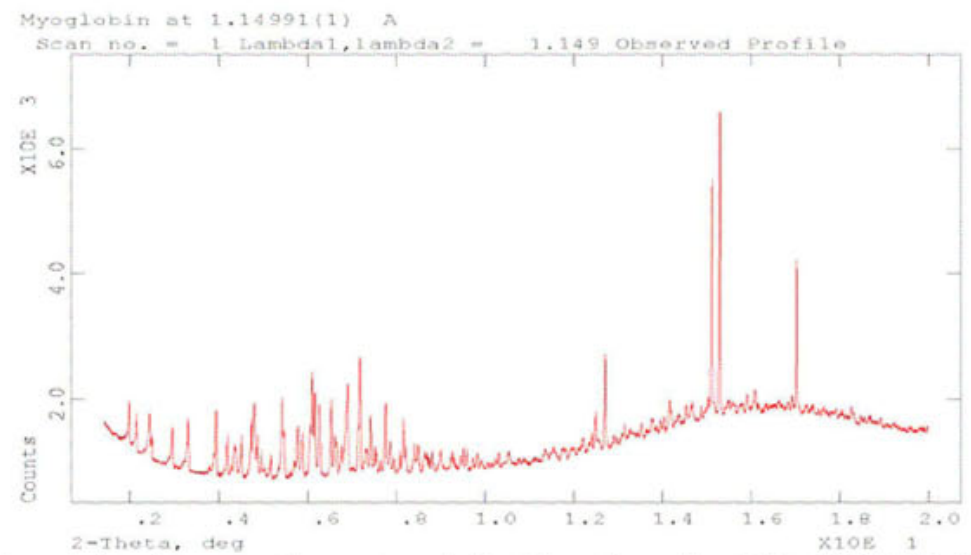

Figure 5. Powder pattern from metmyoglobin taken at beam line X3b1 of NSLS. Five intense lines at $\sim 12.5^{\circ}, \sim 15^{\circ}$ and $\sim 17^{\circ}$ are from ammonium sulfate; the rest are from metmyoglobin. Pattern extends to $3.3 \AA$ d-spacing.

homogeneous in composition and virtually defect free, i.e. "perfect" powders for diffraction [8]. After extensive modification to GSAS [9] this data was used in a successful demonstration of a restrained Rietveld refinement of this $\sim 1260$ atom protein structure [7].

A subsequent examination of the $\mathrm{T}_{3} \mathrm{R}_{3}{ }^{\mathrm{f}}$ structural variant of human $\mathrm{Zn}$-insulin showed that grinding of single crystals induces a phase change where the $R 3$ rhombohedral unit cell was doubled along the $c$-axis which then reverts back to the previously known cell in a day or two. A very simple molecular replacement model allowed a solution to be found from the powder data for the doubled $c$-axis variant; subsequent restrained Rietveld refinement gave a structure (figure 6) [10] for this $\sim 1600$ atom protein that was later found in a cryocooled $\mathrm{T}_{3} \mathrm{R}_{3}{ }^{\mathrm{f}}$ single crystal. Apparently the same phase transition was induced by pressure from the thermally contracting cryoprotectant [11]. A more recent study has expanded on the use of molecular replacement for solving protein structures from powder data; Margiolaki, et al. [12] solved the structure of the 67 residue 2 nd SH3 ponsin domain from powder data using a $38 \%$ homologue. This structure was confirmed by a subsequent single crystal study. The agreement between the two results was $0.52 \AA$ RMS difference with the main chain and $1.20 \AA$ for all atoms. As a further exploration of the possibilities of protein powder diffraction, studies were made of binding of $\mathrm{N}$-acetyglucosamine and its oligomers on hen egg white lysozyme (HEWL) [13]. The diffraction patterns readily showed changes upon complex formation and extracted structure factors revealed the location and orientation of the bound ligand (figure 7) and subsequent restrained Rietveld refinement yielded satisfactory structure models in each case (figure 8 ).

\section{Subsequent studies}

Powder diffraction affords an exploration of the "materials science" aspects of proteins not readily accessible by other methods. For example, the subtle changes in protein lattice parameters in response to sample temperature and preparation conditions are most easily studied 


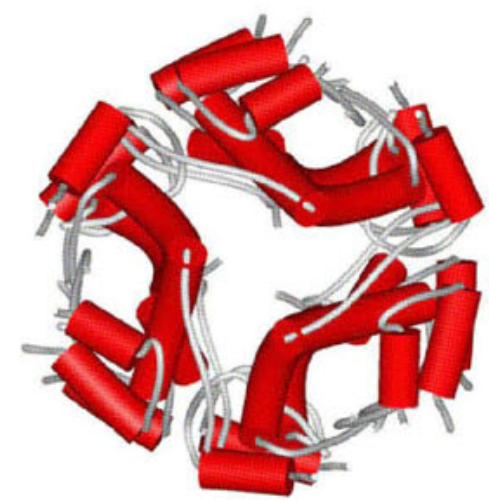

Figure 6. Schematic model of the doubled c-axis variant of $T_{3} R_{3}^{f}$ human $Z$ n-insulin found from powder diffraction. One $T_{3} R f$ unit is turned $\sim 9^{\circ}$ about the c-axis with respect to the other thus effecting the cell doubling.

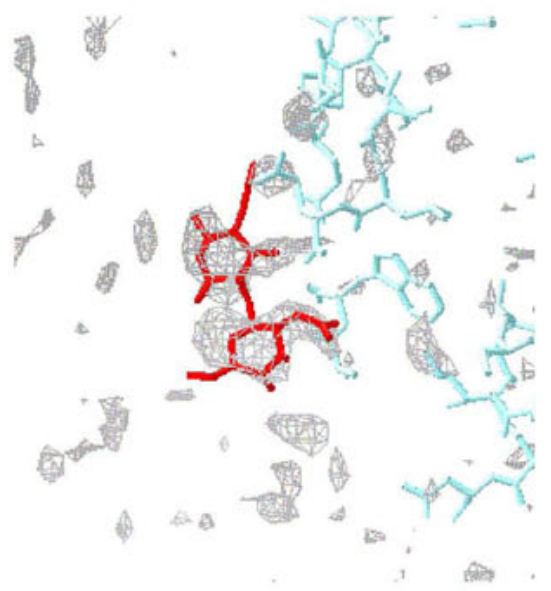

Figure 7. Electron density $\triangle F$ map for $N, N^{\prime}-$ diacetylchitobiose $\left(\mathrm{NAG}_{2}\right)$ complexed with HEWL.

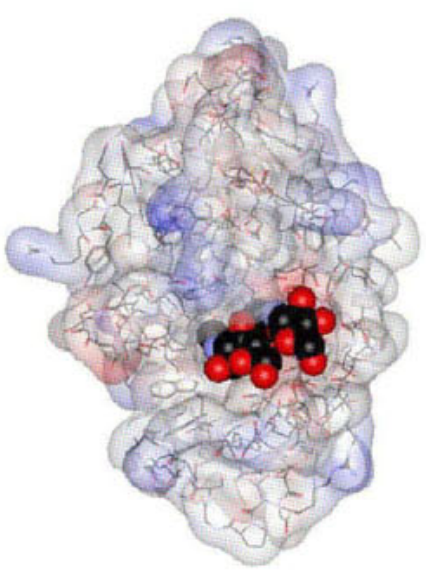

Figure 8. A view of the structure of the HEWL-NAG $\mathrm{H}_{2}$ complex.

at high precision by powder diffraction. In a study of the effect of changing $\mathrm{pH}$ of an acetate buffer on crystalline HEWL, Basso, et al. [14] could readily identify the phaseboundaries (figure 9) for this material as well as see detailed changes within each phase domain. The relatively small changes in lattice parameters of tetragonal HEWL with $\mathrm{pH}$, salt concentration and even radiation exposure could be exploited to improve the structural results by using multiple powder patterns that exhibit these changes [14,15]; the relative shift in Bragg peak positions reveals additional information not available from a single powder pattern. Because 
of the requirement that a powder sample consist of a very large number of microcrystals, sample preparation frequently requires very high protein concentrations. Thus,

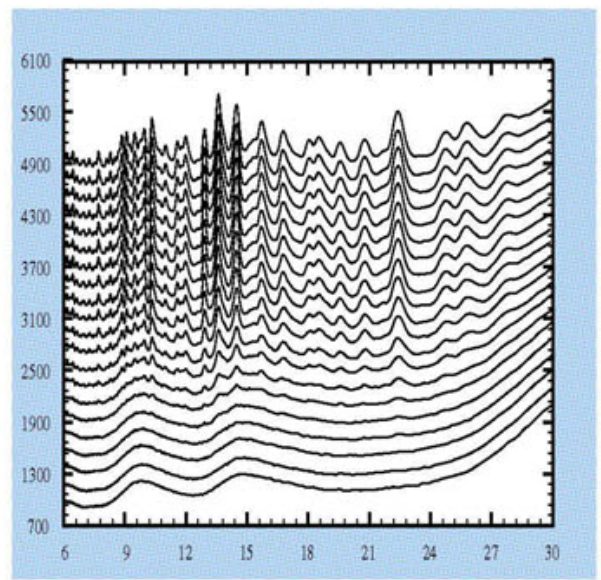

Figure 9. In situ crystallization of HEWL from amorphous precipitate in $1.5 \mathrm{M} \mathrm{NaCl}$ pH 5phthalate buffer. Patterns taken $5 \mathrm{~min}$ apart on beamline 1BM at APS and are offset vertically for clarity; horizontal scale is $d$ spacing in $A$.

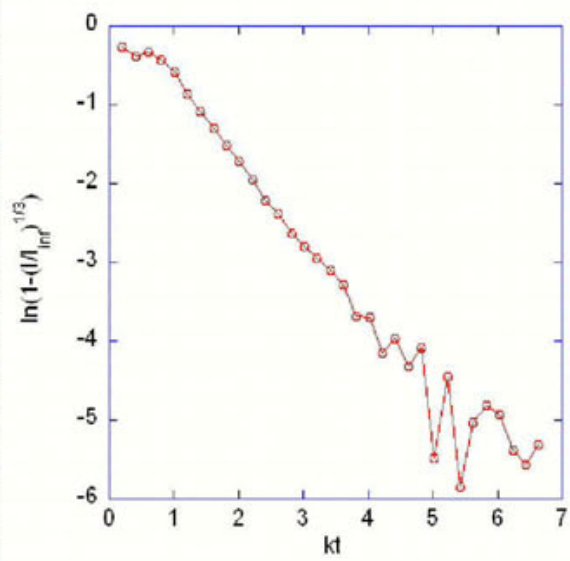

Figure 10. Universal 1st order kinetics plot $\left(k=4.8 h r^{-1}\right)$ for HEWL crystallization.

the initial precipitation may be amorphous (figure 10) and show no Bragg diffraction but crystallizes soon after (e.g. figure 1). This afforded the possibility of following the crystallization in situ (figure 9) and determining the crystallization kinetics (figure 10). The 1st order rate constant $\left(4.8 \mathrm{hr}^{-1}\right)$ obtained here was $\sim 1000$ times faster than previously observed for HEWL crystallization from supersaturated solutions [16]. A determination of the time evolution of the HEWL lattice parameters during crystal growth suggested, although with high uncertainties, that there was a slight compression $(\sim 1.5 \%)$ of the HEWL $a$-axis at the beginning of the crystallization (e.g. for crystallite size $<1000 \AA$ ). The $c$-axis showed no discernable compression. Interestingly, a similar compression of tetragonal HEWL was found under hydrostatic pressure of $\sim 0.2 \mathrm{GPa}$ [17]. Larger crystallites showed no compression of the lattice parameters. This may be a surface tension effect for the smaller crystallites. It was also clear from this work that protein nucleation is inhibited by X-ray radiation; very frequently no in situ crystallization was observed but did occur just outside the beam path through the sample.

\section{Concluding remarks}

This brief survey shows the scope of protein powder diffraction encompassing protein structural and materials science studies including structure determinations via molecular replacement, protein-ligand structures, in situ crystallization, phase transitions and solvent effects. A challenge is the de novo solution of a protein crystal structure from powder dif- 
fraction data. An additional challenge is the development of powder data collection methods that are most effective for proteins; they exhibit extremely sharp Bragg peaks which are at or near the limits afforded by the instrumentation. However, radiation damage imposes severe time limits on data collection so new methods are needed to give better data quality needed for structure solution.

\section{References}

1. Bragg, W.H., 1921, Structure of Organic Crystals, Proc. Phys. Soc. London, 34, 33.

2. Lee, P.L., Shu, D., Ramanathan, M., Preissner, C., Wang, J., Beno, M.A., Von Dreele, R.B., Ribaud, L., Kurtz, C., Antao, S.M., Jiao, X. \& Toby, B.H., 2008, J. Synchr. Rad., 15, 427.

3. Caliandro, R., Giacovazzo, C., \& Rizzi, R., 2008, in Powder Diffraction Theory and Practice, edited by R.E. Dinnebier \& S.J.L. Billinge (Cambridge: The Royal Society of Chemistry), pp. 227-265.

4. Rietveld, H.M., 1969, J. Appl. Crystallogr., 22, 151-2; Von Dreele, R.B. 2008, in Powder Diffraction Theory and Practice, edited by R.E. Dinnebier \& S.J.L. Billinge (Cambridge: The Royal Society of Chemistry), pp. 266-281.

5. Doebbler, J., Harper, J., \& Von Dreele, R.B. (in preparation).

6. Chayen, N.E., 2002, Trends in Biotechnology, 20, 98; Kim, S. \& Gillilan, R.E., CHESS Users' Meeting, Cornell University, Ithaca, NY, June 10-11, 2008.

7. Von Dreele, R.B., 1998, J. Appl. Crystallogr., 32, 1084.

8. Von Dreele, R.B. 2001, Acta Crystallogr., D57, 1836.

9. Larson, A.C. \& Von Dreele, R.B., 1986-2004, General Structure Analysis System (GSAS), Los Alamos National Laboratory Report LAUR 86-748.

10. Von Dreele, R.B., Stephens, P.W., Blessing, R.H., \& Smith, G.D., 2000, Acta Crystallogr., D56, 1549.

11. Smith, G.D., Panghorn, W. \& Blessing, R.H., 2001, Acta Crystallogr., D57, 1091.

12. Margiolaki, I., Wright, J.P., Wilmanns, M., Fitch, A.N. \& Pinotsis, N., 2007, J. Am. Chem. Soc., 129, 11865.

13. Von Dreele, R.B., 2001, Acta Cryst., D57, 1836; Von Dreele, R.B., 2005, Acta Crystallogr., D61, 22.

14. Basso, S., Fitch, A.N., Fox, G.C., Margiolaki, I. \&Wright, J.P., 2005, Acta Crystallogr., D61, 1612.

15. Von Dreele, R.B., 2007, J. Appl. Crystallogr., 40, 133.

16. Kim, Y.W., Barlow, D.A., Caraballo, K.G. \& Baird, J.K., 2003, Mol. Phys., 101, 2677.

17. Fourme, R., Kahn, R., Mezouar, M., Girard, E., Hoerentrup, C., Prange, T. \& Ascone, I., 2001, J. Synchr. Rad., 8, 1149.

Acknowledgements. Supported by US Department of Energy/Office of Science/Basic Energy Sciences under Contract No. DE-AC-02-06CH11357. 


\section{Macromolecular powder diffraction: Structure solution via molecular replacement}

\section{J. A. Doebbler, R. B. von Dreele*}

Advanced Photon Source, Argonne National Laboratory

"vondreele@anl.gov

Keywords: molecular replacement, protein, powder diffraction

Abstract. Macromolecular powder diffraction is a burgeoning technique for protein structure solution - ideally suited for cases where no suitable single crystals are available. Over the past seven years, pioneering work by Von Dreele et al. [1,2] and Margiolaki et al. [3,4] has demonstrated the viability of this approach for several protein structures. Among these initial powder studies, molecular replacement solutions of insulin and turkey lysozyme into alternate space groups were accomplished. Pressing the technique further, Margiolaki et al. [5] executed the first molecular replacement of an unknown protein structure: the SH3 domain of ponsin, using data from a multianalyzer diffractometer.

To demonstrate that cross-species molecular replacement using image plate data is also possible, we present the solution of hen egg white lysozyme using the $60 \%$ identical human lysozyme (PDB code: 1LZ1) as the search model. Due to the high incidence of overlaps in powder patterns, especially in more complex structures, we have used extracted intensities from five data sets taken at different salt concentrations in a multi-pattern Pawley refinement. The use of image plates severely increases the overlap problem due to lower detector resolution, but radiation damage effects are minimized with shorter exposure times and the fact that the entire pattern is obtained in a single exposure. This image plate solution establishes the robustness of powder molecular replacement resulting from different data collection techniques.

\section{Introduction}

X-ray powder diffraction techniques were developed in the 1940s and soon became an invaluable tool for phase identification and, more recently, determination of small-molecule crystal structures. At the turn of the $2 \mathrm{I}^{\text {st }}$ century, Von Dreele addressed the question of how to interpret the structure of a protein if suitable single crystals were not available [6]. The answer was macromolecular powder diffraction. Over the following eight years, the technique has emerged as a viable counterpart to single-crystal diffraction studies. In 2000, Von Dreele et al. demonstrated molecular replacement of $\mathrm{T}_{3} \mathrm{R}_{3}{ }^{\mathrm{f}}$ human insulin into a new crystalline phase [6]. This work was important because it demonstrated that if the structure of one 
protein derivative was known from single-crystal studies, then the structural changes in a series of protein derivatives could be determined with powder data. Later, in 2005, Margiolaki et al. published a molecular replacement and subsequent refinement from powder data of the structure of turkey egg white lysozyme in the hexagonal phase [3]. These two studies affirmed that molecular replacement could work for powder data and established that powder diffraction is a useful probe for protein polymorphs. Most recently, Margiolaki et al. solved the structure of the 67-residue SH3 domain of ponsin by molecular replacement [5]. This previously unknown structure was solved using a model with $40 \%$ sequence identity, demonstrating that powder diffraction could be used not only for phase and derivative identification but for novel structural studies as well.

In this analysis, we survey a $14 \mathrm{kDa}$ molecular weight structure and determine the robustness of molecular replacement solutions using powder data. The hen egg white lysozyme (HEWL) structure was solved by this method using the $60 \%$ identical human lysozyme (PDB code: 1LZ1) as the starting model. Data were collected on an image plate, which achieves high d-spacing resolution with a single image, thus virtually eliminating any changes resulting from radiation damage on a per-shot basis. However, the peaks are distinctly broader on an image plate than with the best multianalyzer diffractometers available, due to the resolution of detector, and it is therefore more difficult to resolve the peaks into individual observable intensities [7]. For large structures and high-symmetry space groups, the overlaps become even more problematic. Unlike multianalyzer diffractometers, the extracted intensities from image plates in the low $\mathrm{d}$-spacing regime cannot be treated as if they were traditional single crystal diffraction data.

\section{Materials and methods}

The raw data used in this analysis are the same as used in a previous study, obtained on beamline 1-BM at the Advanced Photon Source, Argonne National Laboratory [8]. Radiation at $20 \mathrm{keV}$ was focused to the surface of a MAR345 detector positioned $\sim 715 \mathrm{~mm}$ away from and $6.5 \mathrm{~cm}$ vertically offset in relation to the sample. The macromolecular powder slurry in mother liquor was exposed for $30 \mathrm{~s}$ while spinning at $60 \mathrm{~Hz}$. For this multi-pattern analysis, we focused on the samples prepared with $0.05 \mathrm{M}$ potassium hydrogen phthalate $(\mathrm{pH}$ 4.0 ) and $1.25,1.00,0.75,0.50$, and $0.25 \mathrm{M} \mathrm{NaCl}$, respectively.

A multi-pattern Pawley refinement [9] was carried out in GSAS [10] to obtain a set of structure factors for each of the five data sets. The Pawley refinement spanned 0.6-14.0 $2 \theta$, which corresponds to a d-spacing range of 56-2.5 $\AA$. Initially an 18-term Chebyshev function in EXPGUI [11] was used to describe the background and the preliminary profile coefficients were obtained from the analysis of $\mathrm{LaB}_{6}$ standard. After the Pawley intensities stabilized, the background was modified to a log linear interpolation function, which uses more points in the low $2 \theta$ range.

A combined refinement of data sets with differing salt concentrations offers a much better assignment of reflection intensities, because the resulting small anisotropic changes in lattice parameters alters the relative reflection positions, thus changing their overlaps. The 5 data sets were merged with the overlp [8] routine in GSAS which also adjusts the reflection fullwidth-at-half-maximum (FWHM) values subsequently required by the MonteCarlo/Simulated Annealing (MC/SA) program (PSSP) described below. These adjustments 
in FWHM reveal the additional information provided by the changes in reflection overlap from one pattern to another. In all, 4380 unique reflections were obtained from each profile; for the five data sets, this gave a total of 21,900 reflections and a merged R-value of $7.23 \%$. The human lysozyme model was altered with the CCP4 program chainsaw [12] in preparation for the molecular replacement trials. This algorithm took information from a Jalview [13] pair-wise sequence alignment of the human and hen egg white lysozymes and truncated the human model. One extra residue was deleted and 51 residues were truncated to their last common atom. This ensured that the model retained minimal bias toward inaccurate side chain information.

We initially attempted to solve the molecular replacement problem using the extracted intensities and conventional single-crystal diffraction software in the CCP4 suite [12]. Trials involving the amore [14] and phaser [15] algorithms either rendered nonsensical solutions (replete with severe symmetry mate clashes) or simply failed to converge. A single trial using the beast [16] program ran for two days without converging. Conversely, the molrep [17] function ran successfully, with several seemingly worthwhile solutions of rotation and translation functions - contrast values were around 95 , where a contrast above 2.5 is reported to be definitely a solution. Upon further analysis, most were found to be false positives. The solutions displayed severe clashes with crystallographic symmetry mates, which is geometrically unfeasible. Occasionally molrep would produce the correct solution, but with such high contrast scores for all solutions, there was no discrimination between correct and incorrect solutions.

Thus, it was determined that the single-crystal diffraction software was not equipped to deal with intensities, which become increasingly ambiguous with increasing $2 \theta$ (resulting from the overlaps inherent to powder data). This was in contrast to previous studies utilizing multianalzyer diffractometer data, which can be better treated as single-crystal data at low resolution. Therefore, we shifted focus to a locally modifed version of the PSSP program [18], which employs a cost function to account for the poor intensity assignments.

\section{Results and discussion}

PSSP was run using the first 150 reflections $(56>d>8 \AA)$ from the multi-pattern Pawley refinement and the 934-atom result from the chainsaw truncation of the human model. Six parameters were varied over the course of the simulated annealing protocol-three Euler angles and three coordinates. Rotations were performed over the z-axis $\left(0-360^{\circ}\right)$, the $\mathrm{x}$-axis $\left(0-180^{\circ}\right)$, and the $z$-axis again $\left(0-360^{\circ}\right)$ corresponding to the Eulerian angles $\omega, \chi$, and $\phi$, respectively. A restricted asymmetric unit was chosen to preferentially define an origin point in the tetragonal space group. The parameters were allowed to vary from $-1 / 8$ to $1 / 8,0$ to $1 / 2$, and $1 / 4$ to $3 / 4$ of the unit cell dimensions for the $x, y$, and $z$ axes, respectively. The simulated annealing began with the "temperature" at 50 and "cooled" in steps of $20 \%$ to a final temperature of $0.001^{\circ}$, with 5000 cycles at each temperature. The results of the five trials are shown in table 1.

Similar $\mathrm{S}$ values were observed for all five runs, though trial 2 exhibited the lowest $\mathrm{S}$ value, and therefore the best solution. Trials $2-5$ superimposed nicely with the previously published 194L structure, and all four displayed similar orientations in the unit cell (see figure 1). The first solution only differed from the $194 \mathrm{~L}$ structure by a $180^{\circ}$ rotation. This signified 
a different choice of origin (due to the full angular range searched) rather than an erroneous solution, since symmetry contacts remained the same.

Table 1. Human to Hen PSSP results. The S-value is the score for the individual trial. The $\omega, \chi$, and $\phi$ are the Euler angles in degrees and the $x, y$, and $z$ are the coordinates of the center of mass of the model with the highest score for the trial.

\begin{tabular}{|c|c|c|c|c|c|c|c|}
\hline Trial & S value & $\boldsymbol{\omega}$ & $\chi$ & $\phi$ & $\mathbf{x}$ & $\mathbf{y}$ & $\mathbf{z}$ \\
\hline $\mathbf{1}$ & 0.4704 & 179.25 & 174.32 & 350.37 & 0.0021 & 0.2802 & 0.7266 \\
\hline $\mathbf{2}$ & 0.4587 & 151.29 & 5.27 & 200.95 & -0.0048 & 0.2782 & 0.5223 \\
\hline $\mathbf{3}$ & 0.4588 & 153.23 & 5.21 & 199.21 & -0.0061 & 0.2782 & 0.5234 \\
\hline $\mathbf{4}$ & 0.4624 & 128.23 & 4.70 & 224.76 & -0.0061 & 0.2762 & 0.5221 \\
\hline $\mathbf{5}$ & 0.4600 & 135.66 & 4.83 & 217.35 & -0.0060 & 0.2769 & 0.5226 \\
\hline
\end{tabular}

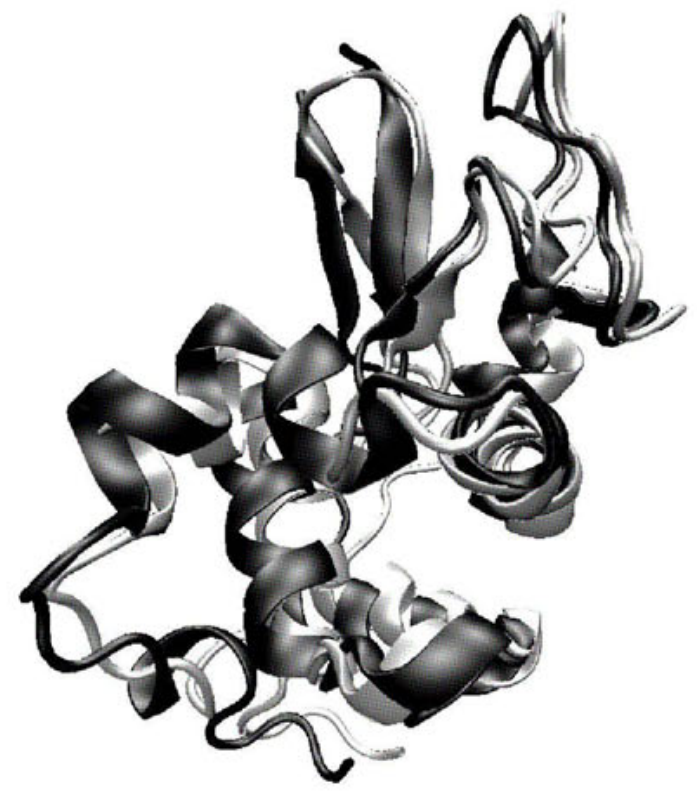

Figure 1. Superposition of the result of PSSP trial two (black) and the previously published HEWL structure (grey). 


\section{Conclusion}

The use of the PSSP algorithm combined with the overlap intensity extraction protocol of GSAS allowed us to extend the powder diffraction molecular replacement technique to larger proteins and enabled the use of image plate data. PSSP rendered five out of five correct solutions and provided a robust method for molecular replacement of proteins using powder diffraction data. Further research into larger molecular weight proteins is ongoing.

\section{References}

1. Von Dreele, R.B., et al., 2000, Acta Cryst, D56, 1549.

2. Von Dreele, R.B., 2003, Methods Enzym, 368, 254.

3. Margiolaki, I., et al., 2005, Acta Cryst., D61, 423.

4. Margiolaki, I. \& Wright, J.P., 2008, Acta Cryst., A64, 169.

5. Margiolaki, I., et al., 2007, J. Am. Chem. Soc., 129, 11865.

6. Von Dreele, R.B., 1999, J. Appl. Cryst., 32, 1084.

7. Margiolaki, I., et al., 2007, Z. Kristallogr. Suppl., 26, 1.

8. Von Dreele, R.B., 2007, J. Appl. Cryst., 40, 133.

9. Pawley, G.S., 1981, J. Appl. Cryst., 14, 357.

10. Larson, A.C. \& Von Dreele, R.B., 1994, General Structure Analysis System (GSAS), Los Alamos National Laboratory Report, 86-748.

11. Toby, B.H., 2001, J. Appl. Cryst., 34, 210.

12. Bailey, S., 1994, Acta Cryst., D50, 760.

13. Clamp, M.,Cuff, et al., 2004, Bioinformatics, 20, 426.

14. Navaza, J., 1994, Acta Cryst., A50, 157.

15. McCoy, A.J.,et al., 2007, J. Appl. Cryst., 40, 658.

16. Read, R.J., 2001, Acta Cryst., D55, 1759.

17. Vagin, A. \& Teplyakov, A., 1997, J. Appl. Cryst., 30, 1022.

18. Pagola, S. \& Stephens, P.W., 2000, Mat. Sci. Forum, 321-324, 40.

Acknowledgements. Use of the APS was supported by the DOE/OS/BES under contract number W-31-109-ENG-38. 



\title{
Indexing magnetic structures and crystallographic distortions from powder diffraction: Brillouin zone indexing
}

\author{
A. S. Wills ${ }^{1,2}$ \\ ${ }^{1}$ UCL, Department of Chemistry, 20 Gordon Street, London, UK, WC1H 0AJ \\ ${ }^{2}$ London Centre for Nanotechnology, 17-19 Gordon Street, London, WC1H 0AH \\ a.s.wills@ucl.ac.uk
}

Keywords: magnetic structure, powder, indexing, $\mathrm{k}$ vector

\begin{abstract}
A considerable challenge is faced by researchers wishing to identify the propagation vector(s) associated with a magnetic structure or a lattice distortion from powder diffraction data, due to the severe destruction of information by powder averaging and search algorithms based on extracted peak positions. In this article a new method is introduced that is based on the points, lines and planes of the Brillouin zone of the crystal structure before the transition. These correspond to different classifications of the translational symmetry of the resultant order and of the Bloch wave that is used to describe the magnetic structure or phonon mode.

An automated search algorithm for the study of magnetic structures is described based on reverse-Monte Carlo refinement of moment orientations for a given $\mathrm{k}$ vector that markedly reduces the number of false-positives and allows the straight forward analysis of systems that contain contributions from several unrelated $\mathrm{k}$ vectors.
\end{abstract}

\section{Introduction}

The determination of the propagation vector(s) associated with a magnetic structure or a lattice distortion from powder diffraction data is a major challenge in powder diffraction, as much information is destroyed by powder averaging. An additional difficulty arises from the way that procedures to index the relevant diffraction peaks are decoupled from the nature of the physical processes that drives the phase transition: indexing is often carried out as a mathematical problem based on extracted peak positions and the calculation of the positions of reflections for simple trial cells. Other methods based on the formalism of a propagation vector, $\mathrm{k}$ vector, enable the exploration of both commensurate and incommensurate trial structures, either following grid searches or non-linear procedures but have difficulties with systems that involve several $\mathrm{k}$ vectors or poor data. [1]

Despite the high symmetry points of the Brillouin zone (BZ) being one of the basic concepts in solid state physics [2], they have not been systematically applied before to the indexing of 
magnetic structures and the propagation vectors associated with a displacive crystallographic phase transition. Their applicability arises from the restriction of magnetic structures and phonons to following the equation of a plane wave in accordance with Bloch's theorem. The $\mathrm{k}$ vector then defines the different classes of the translational symmetry of the resultant structure and in many cases the observed propagation vectors correspond to the different symmetry points, lines and planes in the Brillouin zone of the crystal structure before the ordering transition.

In this article we demonstrate how the Brillouin zone can be used to construct trial $\mathrm{k}$ vectors, and introduce a procedure for the determination of the $\mathrm{k}$ vector of a magnetic powder diffraction spectrum based on the reverse Monte-Carlo refinement of suitably located magnetic moments. Together these techniques enable the characterisation of complex systems, such as those with several propagation vectors which are not symmetry related, that may otherwise have been incorrectly characterised by a general point in the Brillouin zone.

\section{The reciprocal lattice}

Consider a lattice in direct space defined by the primitive translations $\mathbf{a}_{1}, \mathbf{a}_{2}, \mathbf{a}_{3}$. The position vector of any lattice point of that Bravais lattice is given by

$$
\mathbf{T}_{n_{1} n_{2} n_{3}}=n_{1} \mathbf{a}_{1}+n_{2} \mathbf{a}_{2}+n_{3} \mathbf{a}_{3},
$$

where $n_{1}-n_{3}$ are integers. The associated reciprocal lattice vectors are given by

$$
\begin{aligned}
& \mathbf{b}_{1}=2 \pi \frac{\mathbf{a}_{2} \times \mathbf{a}_{3}}{\mathbf{a}_{1} \cdot\left(\mathbf{a}_{2} \times \mathbf{a}_{3}\right)} \\
& \mathbf{b}_{2}=2 \pi \frac{\mathbf{a}_{3} \times \mathbf{a}_{1}}{\mathbf{a}_{1} \cdot\left(\mathbf{a}_{2} \times \mathbf{a}_{3}\right)} \\
& \mathbf{b}_{3}=2 \pi \frac{\mathbf{a}_{1} \times \mathbf{a}_{2}}{\mathbf{a}_{1} \cdot\left(\mathbf{a}_{2} \times \mathbf{a}_{3}\right)}
\end{aligned}
$$

and are the basic vectors of the Bravais lattice in reciprocal space, or $\mathbf{k}$ space. The reciprocal lattice may then be defined as the set of wave vectors $\mathbf{k}$ that satisfies the equation

$$
e^{i \mathbf{k} \mathbf{T}}=1
$$

where $\mathbf{T}$ is a real space lattice vector defined in equation (1).

\section{The Brillouin zone}

The concept of the Brillouin zones was developed from Bloch's theory. Ignoring the correlations between electrons, this is equivalent to the 'independent particle approximation' of Hartree-Fock, where every electron has a separate wave function that satisfies the Schrödinger equation: 


$$
-\frac{\hbar^{2}}{2 m}\left(\frac{\partial^{2}}{\partial x^{2}}+\frac{\partial^{2}}{\partial y^{2}}+\frac{\partial^{2}}{\partial z^{2}}\right) \psi+V \psi=E \psi,
$$

where the potential $V$ has the symmetry of the lattice. Whereas in atoms and molecules the Eigenvalues of (1) are well separated, in crystalline solids they form a continuous manifold and must be characterised by continuously varying parameters as well as discrete points. [2]

The allowed solutions of (6) have the form of a Bloch wave:

$$
\Psi_{\mathbf{k}}(\mathbf{r})=u_{\mathbf{k}}(\mathbf{r}) \exp (i \mathbf{k r}),
$$

where $\mathbf{k}$ is a reciprocal vector that may be defined using the basis vectors $\mathbf{a}_{1}-\mathbf{a}_{3}$ of the Bravais lattice. $u_{\mathbf{k}}(\mathbf{r})$ is a periodic function with the same periodic properties as $V(\mathrm{r})$. The solution $\psi_{\mathrm{k}}(\mathbf{r})$ has the form of a plane wave that is modulated by the periodic function $u_{\mathbf{k}}(\mathbf{r})$. Moving away from electronic wavefunctions, per se, magnetic structures or phonons are also the Eigenfuncitons of periodic Hamiltonians and are characterised by a sum over different Bloch waves

$$
\Psi_{\mathbf{k}}\left(\mathbf{r}_{i}\right)=\sum_{k, \alpha} \psi_{\mathbf{k}}^{\alpha}\left(\mathbf{r}_{j}\right) \exp \left(i \mathbf{k} \mathbf{t}_{i j}\right),
$$

where $\mathbf{t}_{i j}=\mathbf{r}_{j}-\mathbf{r}_{i}, \mathbf{k}$ is the wavevector, and $\alpha$ is the index of the Bloch wave. [3-5]

The first BZ then defines a set of unique $\mathrm{k}$ vectors that satisfy (7) and cannot be made shorter by adding translation vectors of the reciprocal lattice. The different possible $\mathrm{k}$ vectors of the BZ may then be characterised into different points, lines and planes based on their small representations of the group of the wave vector, and the symmetry elements that leave the $\mathrm{k}$ vector invariant. [2] If the wave vector lies in a general position, the group of the wave vector will contain only the identity operation. For other values, the group of $\mathrm{k}$ contains more than the identity, for example in a tetragonal crystal where the $\mathrm{k}$ vector lies along the fourfold axis.

\section{Application of Brillouin zone indexing to magnetic powder diffraction}

The method is based on making the refinement process as physical as possible in order to counter the loss of information from powder averaging. Introducing physics at even a basic level greatly reduces the parameter space to be searched and makes tractable the analysis of systems that cannot be achieved by other methods, and follows the philosophy that we developed of refining magnetic structures in terms of the mixing coefficients of basis vectors in SARA $h[6]$. When applied to magnetic structures there are two main features of this process: the generation of the trial $\mathrm{k}$ vectors following the symmetry points of the Brillouin zone, and their rigorous testing using moment vectors and a reverse-Monte Carlo-Rietveld algorithm. 
The technique was originally developed for the analysis of the magnetic scattering in the frustrated magnets $\beta-\mathrm{Mn}[7]$ and gadolinium gallium garnet (GGG) [8], as the competition between the magnetic interactions make the $\mathrm{k}$ vector impossible to guess, without extremely difficult theoretical calculations. Typically, such calculations are not possible as the magnetic structure provides the necessary starting information.

\section{Generation of $k$ vectors}

There are several conventions that are used to define the high symmetry points, lines and planes of the Brillouin zone. For simplicity we use that of Kovalev [9] as it allows direct translation of the observed ordering wavevector to the representational analysis calculations of the basis vectors of the different possible magnetic structures (a technique that we have applied to several systems, including $\mathrm{Gd}_{2} \mathrm{Sn}_{2} \mathrm{O}_{7}[10]$ and $\left.\mathrm{CuB}_{2} \mathrm{O}_{4}[11]\right)$. The search procedure is based on trying the high symmetry points, then the lines and then the planes. This search order prevents the true symmetry from being misunderstood when the high symmetry points are coincident with lower symmetry lines.

There are typically only a few commensurate symmetry points and these are searched sequentially. If the diffraction spectrum cannot be fitted, grid searches can be used following the lines and the planes of the BZ. It should be notes that it is possible for 'symmetric' $\mathrm{k}$ vectors to occur within the degrees of freedom allowed for a given line or plane, by this we mean the presence of commensurate or related components rather the defined high symmetry points, and the restriction of possible planes may be appropriate if the components of the determined $\mathrm{k}$ vector from a grid search are close to a particular value or relationship.

The generation of the $\mathrm{k}$ vectors based on the $\mathrm{BZ}$ and subsequent conversion to the conventional unit cell chosen for the crystal structure also prevents mistakes. Firstly, the special nature of particular $\mathrm{k}$ vectors becomes apparent. Following this, the nature of an ordering wave vector, and its degrees of freedom become recognised as important physical parameters that distinguish a simple $\mathrm{k}$ vector based on a symmetry point from a locked-in $\mathrm{k}$ vector (based on an incommensurate line or plane in the BZ). The conversion to the conventional direct space cell, also prevents mistakes over the limits of possible $\mathrm{k}$ vectors that are frequent in centred cells, as the upper limit on the $\mathrm{k}$ vector along particular directions may be greater than unity.

\section{Trialling of a given k vector}

In order to reduce the susceptibility to false-positives that is intrinsic to conventional $\mathrm{k}$ vector search algorithms, the trialling of a given $\mathrm{k}$ vector is based on the physical scattering process. The orientations of moments that are located at the crystallographic sites are refined against the experimental data using reverse-Monte Carlo (RMC) techniques. It is important to note that intensities are not extracted, but the full profile is used. This technique was first developed by us and released in SARA $h[6]$ and prevents information loss due to extraction of intensities. 
The number of RMC cycles that are required depends on the precise problem under investigation, though $50-100$ are typically enough to determine whether a $\mathrm{k}$ vector is able to fit the observed diffraction pattern.

\section{Systems with several unrelated propagation vectors}

One of the benefits of this methodology is that magnetic intensity will only be calculated at $\mathrm{d}$-spacings suitable for moments at a given set of positions, with a particular k-vector. This creates a robust refinement strategy that can cope easily with difficult systems, such as those that contain several unrelated propagation vectors. Traditional methods, based on d-spacings alone do not discriminate the contributions of the different phases to the observed diffraction spectrum. In many cases the loss of information due to powder averaging and the use of only reflection positions makes the separation of different contributions untenable.

Using this more physical method, the presence of several unrelated propagation vectors becomes apparent and is signalled by the ability of a particular $\mathrm{k}$ vector to fit well a subset of the magnetic diffraction peaks and not others. An additional phase can then be introduced and used to determine the $\mathrm{k}$ vector of the next set of unfitted peaks. This procedure may be repeated as many times as required, and we have ourselves used this process to determine the propagation vectors of the magnetic field-induced ordering of the frustrated magnet GGG, where 2 commensurate and 1 incommensurate propagation vectors are simultaneously observed [8].

\section{Systems with magnetic or non-magnetic impurity phases}

Similarly, a dataset that has a small subset of peaks that cannot be refined may possess an impurity phase. ${ }^{1}$ It should be remembered that with neutron seattering it is possible that magnetic impurity peaks may only be seen at low temperature, as the magnetic scattering may be stronger than the nuclear, depending on the details of the structure factors involved.

\section{Brillouin zone indexing and $k$ vector searches with SARA $h$}

In the program SARA $h$ the above procedure is implemented though a metaprogram structure with the Rietveld refinement program FullProf (FP) [12]. SARA $h$ sets up the magnetic phase in the orientation matrix format based on the nuclear phase. The user may then automatically go through the different points, lines and planes of the BZ with SARA $h$ automatically editing the FP input (pcr) file, launching the FP refinements, and performing RMC refinement of the moment orientations. The values of the $\mathrm{k}$ vector with the best fits, as characterised by the Rietveld $\chi^{2}$, are then listed. Particular $\mathrm{k}$ vectors chosen by the user may be automatically substituted into the FP input file and tested manually. Commensurate and incommensurate $\mathrm{k}$ vectors can be determined in this manner as SARA $h$ automatically makes all relevant changes to the FP input file. Additional phases to describe additional $\mathrm{k}$ vectors can be added automatically as required.

\footnotetext{
${ }^{1}$ The observation of a small set of peaks suggests that untested propagation vectors, such as those missed in a general grid search of the Brillouin zone are not involved as these low symmetry k vectors typically have many reflections.
} 


\section{References}

1. Wilkinson, C., Lautenschlager, G., Hock, R. \& Weitzel, H., 1991, Appl. Cryst., 24, 365.

2. Bouckaert, L. P., Smoluchowski, R. \& Wigner, E., 1936, Phys. Rev., 50, 58.

3. Wills, A. S., 2005, J. Mater. Chem., 15, 245.

4. Wills, A. S., 2007, Z. Kristallogr., 26, 53.

5. Bradley, C. J. \& Cracknell, A. P., 1972, The Mathematical Theory of Symmetry in Solids (Oxford: Clarendon).

6. Wills, A. S., 2000, Physica B, 276, 680 .

Program available from www.ccp14.ac.uk

7. Stewart, J. R., Wills, A. S., Leavey, C., Rainford, B. \& Ritter, C., 2007, J. Phys.: Condens. Matter., 19, 145291.

8. Petrenko, O. A., Balakrishnan, G., Paul, D. M $^{\mathrm{c}}$ K., Yethira, M., McIntyre, G. J. \& Wills, A. S., 2009, J. Phys.: Conf. Ser., 145, 012026.

9. Kovalev, 1993, Representations of the Crystallographic Space Groups: Irreducible representations, Induced representations and Corepresentations (2nd Ed) (Amsterdamn: Gordon \& Breach Science Publishers).

10. Wills, A. S., Zhitomirsky, M., Canals, B., Sanchez, J. P., Bonville, P., Damlas de Réotier, P. \& Yaouanc, A., 2006, J. Phys.: Condens. Matter, 18, L37.

11. Boehm, M., Roessli, B., Schefer, J., Wills, A. S., Ouladdiaf, B., Lelièvre-Berna, E., Staub, U., Petrakovskii, G., 2003, Phys. Rev., 68, 024405.

12. Rodriguez-Carvajal, J., FullProf, program available from www ccp14.ac uk.

Acknowledgements. ASW would like to thank the Royal Society for funding. 
I.2 Qualitative and Quantitative Phase Analysis 



\title{
State-of-the-art and trends in quantitative phase analysis of geological and raw materials
}

\section{R. Kleeberg}

Technical University Bergakademie Freiberg, Mineralogical Institute, Brennhausgasse 14, D-09596 Freiberg, Germany

kleeberg@mineral.tu-freiberg.de

Keywords: quantitative phase analysis, Rietveld analysis, clay minerals

\begin{abstract}
The accuracy of X-ray powder diffraction based techniques in quantitative phase analysis of geological materials is discussed. Based on the outcomes of recent Round Robin projects, the general quality of routinely applied methods must be estimated to be much lower than claimed in some individual publications. Estimated standard deviations for simple mixtures and intermediate mass fractions are typically below 3\% (absolute) and can reach $1 \%$ if outliers are eliminated. In case of more complex compositions like clay-bearing rocks, the top laboratories are able to match the true phase abundances of most minerals with a similar accuracy, but the bias for clays or high absorbing minerals is mostly found to be higher. The majority of the laboratories have significant problems with the correct qualitative identification of minerals in complex mixtures. The Rietveld method tends to become the most popular technique for quantification, even for disordered structures like clay minerals. The general level of QPA could be enhanced by teaching the basics of X-ray diffraction physics and crystallography, and by providing user-friendly software with stable mathematic algorithms and structure data optimized for quantification.
\end{abstract}

\section{Introduction}

Quantitative phase analysis (QPA) as a classical application of X-ray powder diffraction develops as a tool for scientific work and research as well as in product or process control and troubleshooting in mining and processing industry. Despite the long history and early publications related to principles and physics, the accuracy of QPA is often discussed critically. Since personal computers became available, whole powder pattern fitting methods like the Rietveld method $[1,2]$ or techniques based on the summation of experimental patterns $[3$, 4] were adapted to the problem of phase analysis and gave rise to a big number of publications showing the performance of such methods in the analysis of rock material, e.g. $[5,6]$. Modifications of the Rietveld method [7] were made in order to tackle the problem of partially unknown structures and to calibrate the method for routine applications like in the cement industry [8]. The accuracy of the methods proposed is usually demonstrated either by 
comparison of the results obtained with independent methods like microscopy or by recalculation of the phase analysis into chemical composition $[6,8]$, sometimes from the agreement of the results with the known compositions of a number of reference mixtures. Very high accuracy and precision is claimed for optimized procedures, e.g. $\pm 0.3 \mathrm{wt} \%$ for minor components in Portland cement [9]. On the other hand, big differences between the chemical composition calculated from the Rietveld phase analysis and the measured chemistry indicate big systematic errors, e.g. in [10]. Even if the data do fit the chemistry closely [11], the accuracy of phase quantification obtained from a priori unknown samples is hardly proved. Reputable methodical evaluation of QPA by Rietveld analysis and reference intensity ratio (RIR) methods on test mixtures representing sandstones [12] showed absolute errors of up to $\pm 5 \mathrm{wt} \%$ for clay minerals of percentage $10-20 \mathrm{wt} \%$, and a general accuracy within $=3 \mathrm{wt} \%$ at the $95 \%$ confidence level was estimated for routine analysis of geo-materials.

However, such individual studies can reflect only the complexity of the special problem discussed as well as the experience of the authors. Thus, general trends can be evaluated much better by a higher number of data obtained from inter-laboratory Round Robin projects. Even if the group of participants in any Round Robin will never be representative for the whole number of laboratories practising XRD-based QPA methods around the globe, the outcomes are expected to be less influenced by personal experience and preferences.

\section{Round Robin projects}

The limited number of inter-laboratory Round Robin projects focusing on the QPA of rocks and related materials can not provide information about all methodical aspects. Nevertheless, it is impossible to summarize and compare all their published data. This is simply why the projects have been designed to attain different goals, and their complexity and task setting are not comparable. Therefore the paper focuses on general aspects of previously published projects and on some unpublished details of the "Reynolds Cup" [20, 21].

\section{METEFA '96 [13]}

Two mixtures containing quartz, calcite, and corundum in intermediate percentage (within 16.6-50.6\%) had to be analyzed, and two similar mixtures of known mass fractions were provided for calibration purposes. Fifteen participants provided "preferred method" results additionally to the obligatory method based on single line intensities. The majority of the participants used Bragg-Brentano geometry, some results based on Debye-Scherrer measurements, and Guinier setups were also reported. In the data analysis Rietveld methods dominated (8 participants), but single line methods in Chung style [14] or pattern addition from calculated diffractograms were also applied. All absolute deviations were in a $\pm 10 \mathrm{wt} \%$ interval, and estimated standard deviation e.s.d. (without outlier elimination) was about $3 \%$ for the whole series. No systematic errors were identified. The small number of participants did not allow the identification of any methodical source of errors. The only tendency observed was that Rietveld techniques seem to provide lower absolute differences from the true values. However, the uncertainties of results that can be attributed to the influence of the operators, even for such a rather simple and well-defined system, seem to be comparable or higher than those who have been reported by an experienced analyst for a more complex system like sandstones [12]. 


\section{IUCr CPD Round Robin [15, 16]}

This comprehensive project was designed to determine the level of accuracy and precision of the QPA for different systems and selected problems, e.g. simple mixtures with different ranges of mass fractions, preferred orientation of platy minerals, highly absorbing material, amorphous content, and more complex compositions like a natural granodiorite and a synthetic bauxite. The qualitative composition was generally known. Additionally, the organizers provided own measuring data, in order to check the impact of operator strategies in data analysis. Just a short survey on these very valuable results can be given here.

The majority of the participants contributing results to the more complex samples ( 81 out of 84 , i.e. $96 \%$ ) decided to analyze the CPD provided data by the Rietveld method [16]. The participant collected data $(\mathrm{N}=198)$ were analyzed also mainly by the Rietveld method $(81 \%)$, by single line RIR techniques (14\%), by full-pattern matching procedures $(3 \%)$ and some other particular methods. Thus, the outcomes represent mainly Rietveld techniques.

For simple mixtures (corundum, fluorite, zincite) the estimated standard deviations (calculated from 42-72 data sets) were found to be of the same magnitude as in [13]. The slightly higher errors for participant collected date indicate some uncertainties in sample preparation and measurement. Some outliers could be identified to originate from corrupted structure models, inappropriate parameter setting, and wrong refinement strategy [15].

The problem of preferred orientation (PO) was demonstrated for a rather simple mixture (platy brucite with corundum, zincite and fluorite). After the elimination of outliers (accepting the $50 \%$ percentile of the absolute Kullback-Liebler distances only), the e.s.d. for brucite percentage was in the same magnitude of $1 \%$ like for the non-platy minerals, and the true and analyzed values agreed at a 95\% confidence level. This indicates that the PO correction procedures as usually applied in Rietveld techniques do work effectively.

The common problem of microabsorption was highlighted by providing CPD measured data from coarse (21-36 $\mu \mathrm{m})$ high absorbing minerals (magnetite and zircon) in the mixture. As expected, no reliable results could be obtained from these data, although different correction procedures were applied. Even if the sample preparation was done by the participants, the spread of the results was found to be extreme, and the $50 \%$ percentile has still an error of about $2.5 \%$, much higher than in the other mixtures. Thus, the microabsorption problem was identified to be crucial in the analysis by laboratory X-ray sources.

A natural granodiorite with roughly known qualitative composition was chosen as an example for a more complex system. The results showed big standard deviations (absolute 4.3$7.4 \%$ for the full set of 10 resp. 23 participants analyzing their own resp. CPD supplied data) for a main component (about 30\%) like quartz. This is much worse than the results obtained by the organizers of the Round Robin by analyzing 12 sets of data from 12 sub-samples prepared with different milling time and analyzed by 2 independent operators (absolute e.s.d. $1.5 \%$ ). This finding highlights again the impact of operator's experience on the QPA results.

\section{Quantitative clay mineral analysis [17]}

The special problem of clay mineral analysis is worth to be treated more in detail, simply because of the phase analysis of clay minerals by XRD methods is a traditional field, and an alternative method for identification and quantification is lacking, due to the specifics of layer silicates. In the inter-laboratory comparison organized primarily for the German Clay Group (DTTG), two natural clays of "common" composition had to be analyzed. Nineteen 
laboratories submitted results, mostly obtained from classical single-line methods from powders and oriented samples of the separated clay fraction. Only 3 participants tried to apply the Rietveld method to the bulk powder pattern or the coarser fraction of the samples. The majority of the participants did apply supporting methods like chemical analysis, ionexchange measurements and other methods as well. The traditional procedure in clay analysis is typically divided in two steps: (1) the analysis of the bulk sample without differentiation of the clay minerals, phyllosilicates as a sum, and (2) the determination of the ratio of the clay minerals from the $<2 \mu \mathrm{m}$ fraction from intensities of the basal reflections from oriented samples. The outcomes illustrated impressively the limitations of this approach. The percentages of the non-clay minerals in the bulk samples could be analyzed with about the same precision like in other investigations (3-4 wt\% error interval at the $95 \%$ level for quartz in intermediate mass fractions), but the reported abundances (at $95 \%$ level) of clay minerals in the $<2 \mu \mathrm{m}$ fraction were found to vary extremely, e.g. between 14 and $33 \%$ for kaolinite, and between 33 and $50 \%$ for illite in one sample. This finding was interpreted to be caused mainly by the application of different RIR values (or Mineral Intensity Factors) for the basal clay mineral peaks, but other reasons like errors in sample preparation, different degree of orientation of the minerals, or instrumental artefacts like beam overflow could not be excluded. Such an outcome supports the suggestion of several authors that the quantification of clay minerals should be done primarily from random powders [e.g. 12, 18]. Additionally, the Round Robin highlighted the fact that there are serious uncertainties in the qualitative analysis of clay minerals. E.g., the identification of the mixed-layered minerals and the differentiation of vermiculite and smectite were far from being consistent between the laboratories.

\section{Reynolds Cup [19, 20, 21]}

The Reynolds Cup was initiated in 2001 by Douglas McCarty, Jan Srodon (Chevron Texaco), and Dennis Eberl (US Geological Survey), and is hosted by the Clay Minerals Society. The project focuses on the evaluation of the complete procedure of QPA of clay-bearing rocks, including sample preparation and phase identification. Since 2002, every second year 3 mixtures of reference minerals representing clay-bearing rocks were prepared by a principal organizer and offered for any type of identification and quantification procedure to interested laboratories around the world. In order to enhance the number of participants (and the fun factor) a contest-like judging was chosen, simply by ranking the participants according to the lowest sum of absolute bias between reported and true mass fractions. Only the first to third rank finishers are named and honoured by a trophy. The growing number of participants delivering results ( 15 in 2002, 45 in 2008) demonstrates the success of the project.

Despite of the lack of a detailed statistical analysis of all data, the reports $[19,20,21]$ as well as the data supplied to the participants allow identification of some trends. First, it can be stated that all participants used any type of laboratory powder diffraction as primary tool for identification and quantification. In contrast to the Round Robin project described in the last section, the Rietveld method and full-pattern summation procedures applied to randomly oriented samples dominated the field. Single line techniques were less frequently used (figure 1). Until now, the most accurate results were always reported by laboratories applying full-profile fitting procedures (summation of experimental reference patterns or Rietveld techniques). No single-line based technique was able to deliver top data, and in general laboratories applying such methods were concentrated at the end of the standing $[20,21]$. An 
interesting observation can be made if the names of the top finishers applying pattern summation techniques are put into the context of the program used: All these persons are either the author or member of the development team of the program used, have extensive experience in clay analysis, and have collected a comprehensive database of reference patterns of pure standards. Despite of two of these successful programs (RockJock and FULLPAT) are freely available, until now no "non-insider" was able to come close to the top of the standing with such a technique. This may indicate that the correct application of "simple" linear fitting procedures is not straightforward.

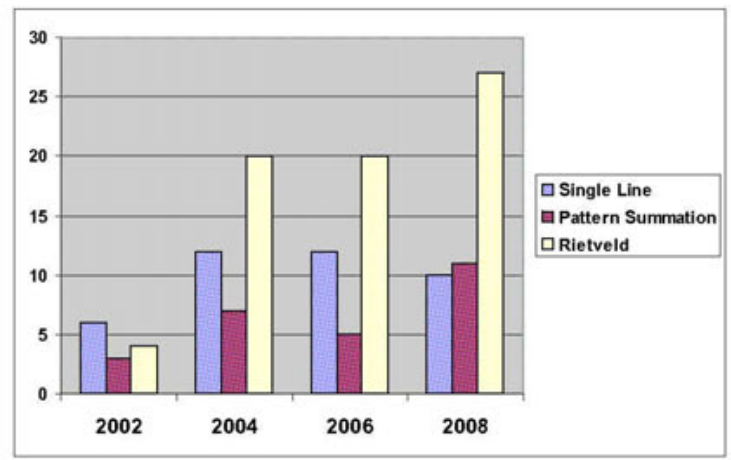

Figure 1. Distribution of primary quantification methods used in previous Reynolds Cup contests.

Within the big group of laboratories applying Rietveld programs, not only the authors/coauthors of certain Rietveld software provided accurate data. Users without inside knowledge of the program used, but skilled in crystallography and XRD methods also reported top results. Indeed one person was always one of the top finishers, and he used 3 different Rietveld programs for his analysis. As a tendency it can be seen that more analysts prefer a program coming with a ready-to-use structure database of minerals, and these laboratories are also tending to perform better than those using a program without such a database. This indicates that the preparation of crystal structure data for the use in QPA may be a source of errors. The top results show that very accurate phase analysis of complicated mixtures, including clay minerals, is possible. For example, in the 2 nd contest in 2004 , the top 4 reached a total bias below $29 \%$. Because 29 minerals or mineral groups had to be analyzed (9-10 per sample), these laboratories reported results better than $\pm 1 \mathrm{wt} \%$ of the true values in average, and the winner presented a total bias of $14 \%$. This is much better than expected for such complicated compositions. On the other hand, the average quality of the results submitted is far from this accuracy, and a significant improvement of the general level seems to be necessary.

\section{Conclusions}

Accurate QPA results for complex samples can be achieved by the careful application of XRD full-pattern fitting techniques like Rietveld refinement or pattern summation. The limiting factors are mainly user skills in phase identification and in the appropriate use of existing software. Thus, the general level of accuracy can be raised by (i) teaching the basics of XRD quantification techniques like the Rietveld method and (ii) by supporting the operators by 
user-friendly software optimized for QPA, inclusive the preparation of ready-to-use compilations of structure data for common minerals.

\section{References}

1. Hill, R.J. \& Howard, C.J., 1987, J. Appl. Cryst., $20,467$.

2. Bish, D.L. \& Howard, S. J., 1988, J. Appl. Cryst., 21, 86.

3. Smith D.K., Johnson G.G. Jr, Scheible A.., Wims A.M., Johnson J.L. \& Ullmann G. 1987, Powder Diffr., 2, 73.

4. Cressey, G. \& Schofield, P.F., 1996, Powder Diffr., 11, 35.

5. Bish, D.L. \& Post, J.E., 1993, Amer. Mineralogist, 78, 932.

6. Hill, R.J., Tsambourakis, G. \& Madsen, I.C., 1993, J. Petrol., 34, 867.

7. Taylor, J.C. \& Rui, Z., 1992, Powder Diffr., 7, 152.

8. Scarlett, N.V.Y., Madsen, I.C., Manias, C. \& Retallack, D., 2001, Powder Diffr., 16,71 .

9. Füllmann, T. \& Walenta, G., 2003, ZKG international, 56, (5), 45.

10. Ruan, C.D. \& Ward, C.R., 2002, Appl. Clay Sci., 21, 227.

11. Monecke, T., Köhler, S., Kleeberg, R. \& Herzig, P.M., 2001, Canad. Mineralogist, 39, 1617.

12. Hillier, S., 2001, Clay Miner., 35, 291.

13. Rafaja, D. \& Valvoda, V., 1996, in Round Robin on the Quantitative Phase Analysis (Praha: Krystalograficka spolecnost, p. 32.

14. Chung, F.H., 1974,. J. Appl. Cryst., 7, 519.

15. Madsen, C., Scarlett, N.V.Y., Cranswick, L.M.D. \& Lwin, T., 2001, J. Appl. Cryst., 34, 409 .

16. Scarlett, N.V.Y., Madsen, I.C., Cranswick, L.M.D., Lwin, T., Groleau, E., Stephenson, G., Aylmore, M. \& Agron-Olshina, N., 2002, J. Appl. Cryst., 35, 383.

17. Ottner, F., Gier, S., Kuderna, M. \& Schwaighofer, B., 2000, Appl. Clay Sci., 17, 223.

18. Środoń, J., Drits, V.A., McCarty, D.K., Hsieh, J.C.C. \& Eberl, D.D., 2001, Clays Clay Miner., 49, 514.

19. MeCarty, D.K., 2002, IUCr Comm. Powder Diffr. Newsl., 27, 12.

20. Kleeberg, R., 2004, IUCr Comm. Powder Diffr. Newsl., 30, 22.

21. Omotoso, O., McCarty, D.K., Hillier, S. \& Kleeberg, R., 2006, Clays Clay Miner., $\mathbf{5 4},(6), 751$.

Acknowledgements. The author is grateful to all the colleagues involved in the Round Robin projects, and to two anonymous referees for constructive reviews of the manuscript. 


\title{
The effects of particle statistics on Rietveld analysis of cement
}

\section{P. S. Whitfield", L. D. Mitchell}

National Research Council Canada, 1200 Montreal Road, Ottawa ON K1A 0R6 CANADA "pamela.whitfield@nrc.gc.ca

Keywords: quantitative analysis, cement, Rietveld analysis

\begin{abstract}
Quantitative Rietveld analysis of cements is now a common tool in both industry and research. Many papers analyse cements without any additional sample preparation, over and above the grinding carried out by the manufacturer. The particle sizes in cements are usually in the range of $25-40 \mu \mathrm{m}$, which is much coarser than recommended in texts dealing with particle statistics in diffraction. A systematic study has been undertaken to examine the effect of particle statistics on the results from Rietveld analysis of cements, and the influence of reducing the particle size by micronizing. In addition, the effects of changing divergence slit and use of sample rotation have been examined.
\end{abstract}

\section{Introduction}

Although it may seem obvious, one requirement for powder diffraction is that the sample is a real, statistical powder. The random orientation of crystallites in the sample is vital to obtain reliable relative intensities in a one dimensional 'slice' through the Debye-Scherrer cones, so this is more than an academic, mathematical issue. This begs the question "when is a powder really a powder?" This question was examined in the context of X-ray powder diffraction in an important recent paper by the late Deane Smith [1]. Where the sample consists of ideal spherical particles, whether a random distribution of crystallites has been achieved depends on both the particle size, and the volume of sample being sampled by the instrument. The volume can depend on both the diffractometer geometry and the absorption of the sample.

For a single phase sample, crystallites need to be 1 micron or smaller to produce a sufficient number of crystallites to approximate random powder [1]. Most samples analyzed do not meet these standards, which limits the accuracy and reproducibility of any quantitative analysis. Spinning the sample does not improve the statistics greatly. Achieving a 1 micron particle size requires some effort in sample preparation, with some kind of milling or grinding required. Milling can damage the microstructure of some materials (clays are particularly vulnerable) and dehydrate some phases (e.g. gypsum) so care must be taken in choosing an appropriate mill and conditions. Workers analyzing challenging samples frequently use a special mill produced by McCrone that was designed for sample preparation. Poor particle statistics can have consequences for the relative intensities, and can be a source of significant error [2-4]. 
The effects of poor particle statistics become more obvious using microdiffraction techniques. Figure 1 demonstrates this, in a comparison between the data obtained from an asreceived cement sample and one that was 'micronized' for 15 minutes in isopropyl alcohol. The cement shown in figure 1(a) has a particle size of around 25 microns. Although 20 microns (600 mesh) is often thought of as being sufficient for X-ray powder diffraction, the 2D frame shows the sample to be very grainy from the spotty nature of the Debye-Scherrer rings. The micronized sample has particles of mostly 2 microns and under, and shows nice, even intensities along the Debye-Scherrer rings as seen in figure 1(b). It should be obvious that good particle statistics become increasingly difficult to obtain for quantitative analysis of increasingly complex mixtures, as theoretically, each component of the mixture must have sufficient crystallites to form a random powder. Cements are complex, multi-phase systems, so figure 1 shows that satisfactory statistics are achievable from a practical viewpoint.

Quantitative X-ray analysis in any form requires reliable peak intensities to obtain accurate results. Consequently, for years it has been recommended to reduce the particle size to the order of a micron or two. Besides particle statistics, coarse particles can have adverse effects in the form of microabsorption and preferential orientation. Some efforts can be made to improve the particle statistics by varying the experimental data collection strategy. Using a larger divergence slit is an easy way to sample a larger volume of material, although care must be taken to avoid beam-overspill. Sample rotation is often used as the main method for improving sample statistics, but in the form usually present on laboratory X-ray diffractometers it is not as effective [5] as people often believe. Rotating a 30-50 $\mu \mathrm{m}$ sample improves the statistics by $5-8$ times $[4,6]$, but the improvement in terms of diffracting crystallites needs to be orders of magnitude [1].

Rietveld analysis [7] is now the most common technique for QXRD of cements as the addition of a standard is not required [8]. Crystal structures are known for the major cement phases, so it is readily applied to most circumstances. The different cement phase polymorphs are not often distinguishable in laboratory data, although they should be considered with high resolution, especially synchrotron data. Although techniques exist for reducing preferential orientation (back-loading, etc) and correcting microabsorption [9], particle statistics have to be addressed at the data collection stage. Specialised approaches such as the use of capillaries are not widely used in the laboratory and are often not practical in industry despite the excellent results that can be obtained [10]. Rietveld software usually produces estimated errors in the form of estimated standard deviations (ESDs). Although these errors are only estimates, they are often used in the place of statistically derived errors.

This piece of work aims to gauge the effect that particle statistics have on the analysis of cements. Both micronized and raw materials were analysed. The repeatability in mounting and re-mounting samples was studied, as well as the effect of changing divergence slit size and use of rotation. The use of multiple repeats means that real errors with confidence limits can be calculated instead of using the software-derived ESDs. 


\section{Experimental}

A freshly opened sample of NIST SRM 637 Pink Cap Portland cement was split into two fractions, the first was used without processing. The second was processed with a McCrone micronizing mill. The cement was transferred to the milling vessel, containing corundum grinding elements, and isopropyl alcohol (IPA) was added as a liquid phase. The sample was milled for 10 minutes; after which the slurry was removed to a Petri dish and allowed to dry in a fume cupboard. After drying the micronized cement was transferred to a sealed sample bottle. Both fractions were analysed using a Bruker D8 Advance X-ray diffractometer with

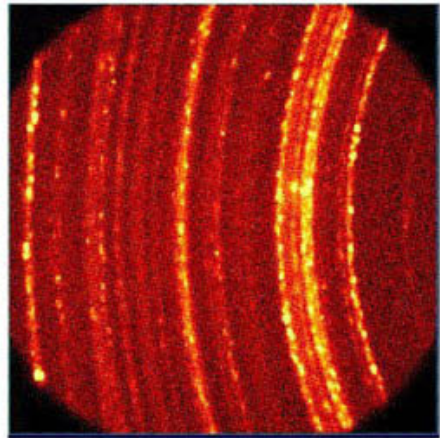

(a)

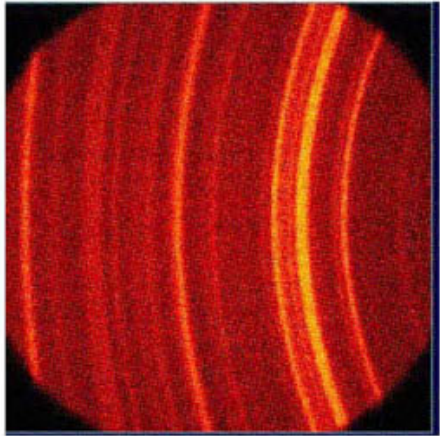

(b)

Figure 1. 2D frames from (a) as-received cement and (b) micronized cement. The data were taken on a CoK $\alpha$ GADDS system with a $1 \mathrm{~mm}$ monocapillary optic.

$\mathrm{CuK} \alpha$. The machine was configured to a Bragg-Brentano setting with a Vantec PSD detector, motorised divergence slit and a rotating sample stage.

To study the reproducibility, each cement sample was mounted, run and re-mounted twice (a total of 3 mountings per sample). Four data sets per sample mount were collected with varying strategies (see table 1) to affect the particle statistics through changing the sample volume illumination and use of sample rotation. Repeating the data collection after remounting allows an actual standard deviation to be calculated, in addition to the estimated standard deviations that the Rietveld software produces.

Count times were chosen such that the peak intensities were as close as possible to each other, despite the different slit settings. Additionally all the data sets were normalised before analysis to minimise the effect of differing intensities on the final calculated errors. The $R_{w p}$ residual is sensitive to the signal-to-noise ratio, and the intention was that the errors from the analysis should be due to the differences in particle statistics rather than other experimental factors.

Data analysis was done with a beta version of TOPAS 4 from Bruker-AXS [11]. Quantitative analysis was undertaken using the standard structures of cement phases. The possible use of calcium silicate polymorphs was explored, but didn't significantly affect the result, probably due to the lower resolution of laboratory data versus that obtained with a synchrotron. Fundamental parameters were used to derive the instrument function including the effect of the PSD. The zero point error was determined using SRM $660 \mathrm{a}\left(\mathrm{LaB}_{6}\right)$ and subsequently fixed. 
In line with common practice the March-Dollase preferential orientation correction [12] was used on the tricalcium silicate $\left(\mathrm{C}_{3} \mathrm{~S}\right)$ phase. Refined parameters of each phase included lattice parameters and Lorentzian size broadening. No attempt was made to correct for

Table 1. Data collection conditions for each sample mounting.

\begin{tabular}{|l|c|c|c|c|}
\hline Run number & 1 & 2 & 3 & 4 \\
\hline Divergence slit & 0.3 & 0.1 & 0.3 & 0.1 \\
\hline Rotated & No & No & Yes & Yes \\
\hline
\end{tabular}

microabsorption using the Brindley correction as the unmicronized material is far from the required monodisperse particle distribution [9].

\section{Results}

Figure 2 shows overlays of what in each case should be identical patterns using the same diffractometer settings, but three different sample mountings for as-received and micronized samples. The un-micronized sample shown in figure 2(a) shows significant variation in relative peak intensities. This is a matter for concern, as variability in the relative intensities will increase the errors in the derived phase fractions. Also note in figure 2(a) that one of the repeats shows a noticeable periclase reflection at 43 degrees. The relative periclase peak intensity increased greatly on decreasing the divergence slit, from 0.3 to 0.1 degrees, leading to the conclusion that a single large grain was present somewhere near the centre of the sample. Analysis of the other two repeats yielded negligible periclase. Figure 2(b) shows a corresponding plot for the micronized sample. There is a marked improvement in the reproducibility, with no sign of a distinct periclase reflection. Figure 3 shows the refined values for periclase for 3 repeats each for micronized and un-micronized material using the same diffractometer settings. It is immediately apparent that the micronized sample gave practically identical results in each case, whilst the un-micronized sample showed variability much greater than the calculated estimated standard deviations produced by TOPAS. The NIST certificate for SRM637 gives a certified Mg content of $0.6 \%$, which is agreement with the results from the micronized material. Consequently, micronizing can have dramatic effects on the results from the minor phases. An additional benefit from improved particle statistics is a reduction in the residuals from Rietveld refinements.

Figure 4 shows the mean and 95\% confidence limits with both the micronized and unmicronized samples for (a) tricalcium silicate $\left(\mathrm{C}_{3} \mathrm{~S}\right)$ and $(\mathrm{b})$ dicalcium silicate $\left(\mathrm{C}_{2} \mathrm{~S}\right)$. The only statistically significant difference in $\mathrm{C}_{3} \mathrm{~S}$ between micronized and un-micronized samples occurred in the 0.1 degree un-rotated sets, which theoretically should have the worst particle statistics. There is a distinct trend for lower alite values and reduced errors in the micronized samples. March- Dollase coefficients for the un-micronized $\mathrm{C}_{3} \mathrm{~S}$ were approximately 0.93 whilst those of the micronized samples were around 0.98 . This is very close to the value for a perfect powder of 1.0 [12]. Together with the slight peak broadening evident in the patterns from the smaller crystallites in the micronized samples, it demonstrates the better quality of the powder after micronizing. There are no statistically significant differences for the $\mathrm{C}_{2} \mathrm{~S}$ phase between micronized and un-micronized samples, although in the case of the unrotated $0.1^{\circ}$ set this is mainly due to a greatly increased error. Once again the 
errors are generally reduced in the micronized samples, but for $\mathrm{C}_{2} \mathrm{~S}$ the values tend to be higher.

Figure 5 shows quantitative results for (a) calcium aluminoferrite $\left(\mathrm{C}_{4} \mathrm{AF}\right)$ and (b) tricalcium aluminate $\left(\mathrm{C}_{3} \mathrm{~A}\right)$. For $\mathrm{C}_{4} \mathrm{AF}$ there is significantly more scatter in the mean of the unmicronized samples, but the differences are not statistically significant. This is in part due to the large errors associated with the un-micronized results with a $0.1^{\circ}$ divergence slit. The quantitative data for $\mathrm{C}_{3} \mathrm{~A}$ in figure $5(\mathrm{~b})$ is the only case where the micronized and un-micronized samples show statistically different results with all of the diffractometer settings. The micronized samples consistently show approximately $0.8 \%$ lower $\mathrm{C}_{3} \mathrm{~A}$ values than the unmicronized samples.

The systematic shifts in phase contents are consistent for many of the phases even if not statistically significant. Particle statistics in themselves should not introduce a systematic shift, but other effects are related to crystallite sizes, one of them being microabsorption. The initial crystallite size of each phase is unknown, so the relative changes in microabsorption effects on milling are not necessarily predictable.
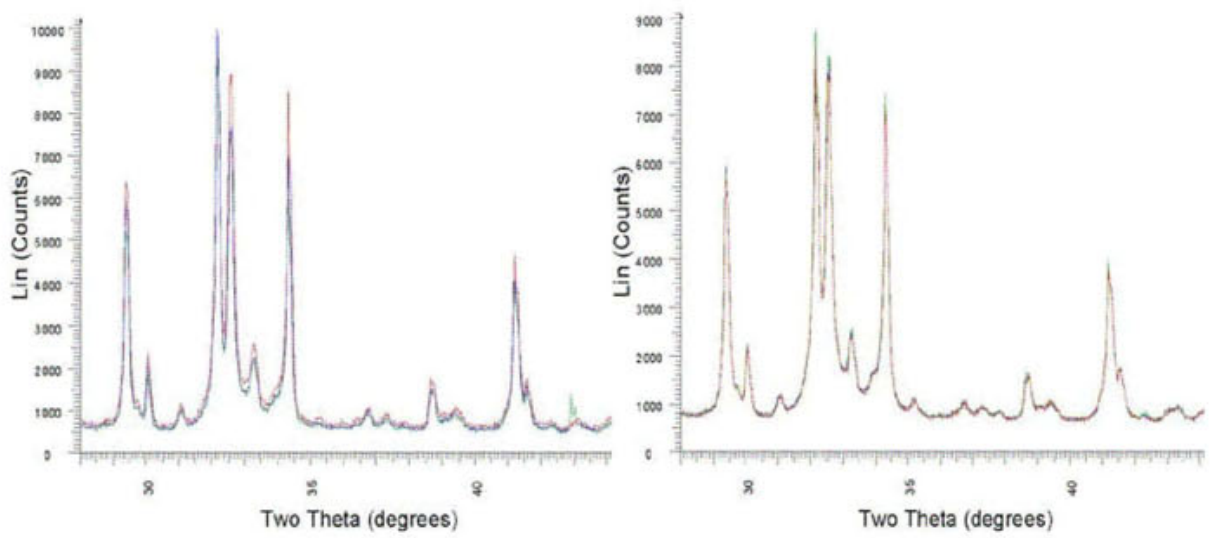

Figure 2. Overlay of 3 repeats of (a) un-micronized and (b) micronized material using 0.3 degree divergence slit without rotation. Note the presence of a periclase reflection at around 43 degrees two theta in only one of the un-micronized datasets.

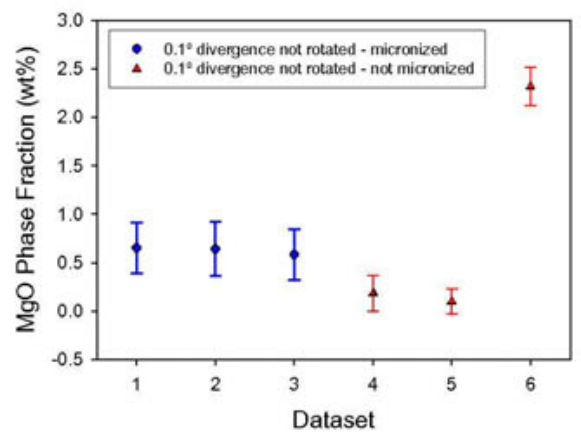

Figure 3. Quantitative results for periclase. Three identical repeats of micronized and unmicronized samples. The errors shown correspond to $2 \sigma$ of the ESDs. 


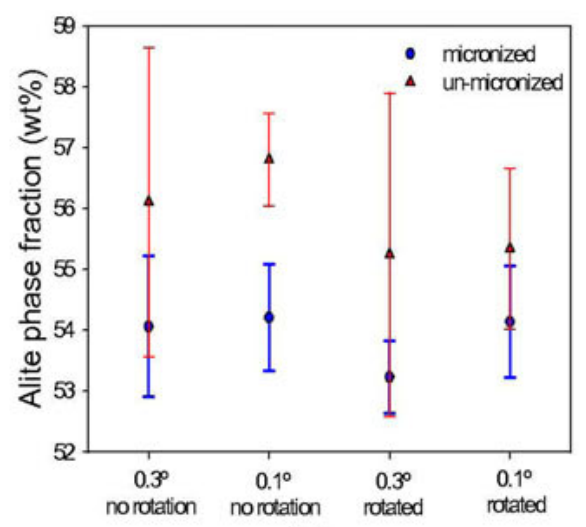

(a)

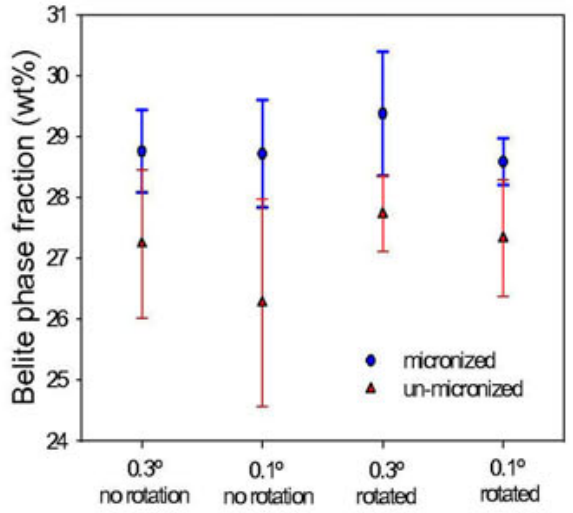

(b)

Figure 4. Quantitative results showing the mean and $95 \%$ confidence limits for (a) $C_{3} S$ - alite and (b) $C_{2} \mathrm{~S}$ - belite, using different experimental procedures. The standard deviations are statistical as opposed to ESDs.

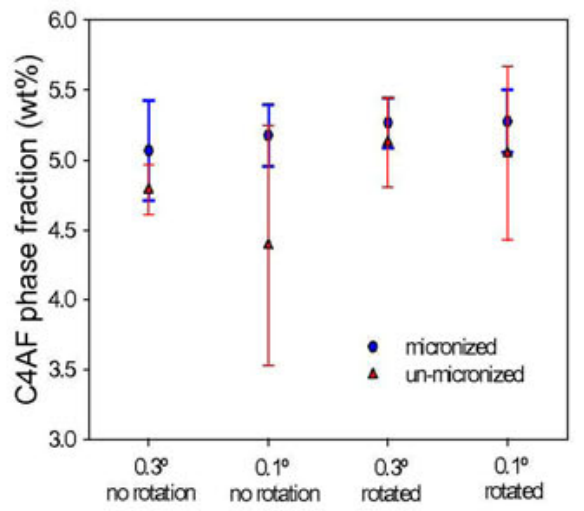

(a)

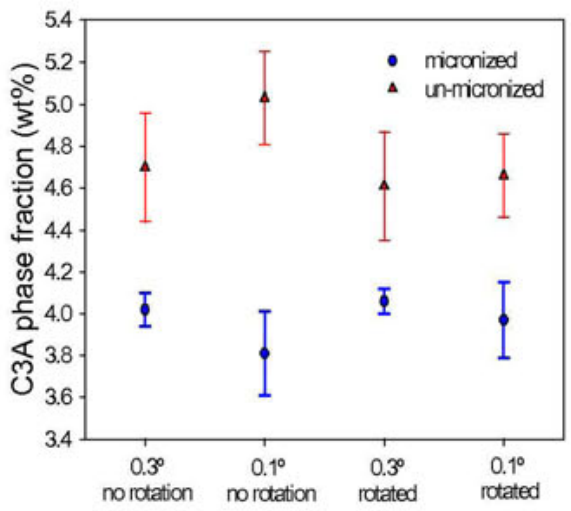

(b)

Figure 5. Quantitative results showing the mean and $95 \%$ confidence limits for (a) $C_{4} A F$ and (b) $C_{3} A$ using different experimental procedures. The standard deviations are statistical as opposed to ESDs.

\section{Discussion and conclusions}

In terms of the major cement phases, the only one that consistently gave statistically different results between the two sample preparation methods was the $\mathrm{C}_{3} \mathrm{~A}$. In many instances this was only because of the large errors in the un-micronized results. The $\mathrm{C}_{3} \mathrm{~S}$ did yield statistically different results where a 0.1 degree divergence slit was used without rotation. This particular combination should theoretically produce the poorest statistics, so the result is no 
surprising. Sample rotation improves the reproducibility but is far less effective than micronizing. For $\mathrm{C}_{3} \mathrm{~S}$, the micronized samples have consistently lower mean values whilst the $\mathrm{C}_{2} \mathrm{~S}$ values were higher. Dramatic differences can occur in the minor phases. It appears as though large grains of periclase were present in the SRM. Micronizing successfully homogenized the sample, producing consistent results for periclase, despite its low abundance. However, the un-micronized samples have wildly varying results, depending on whether a grain was present in the area illuminated by the $\mathrm{X}$-rays. In many instances the standard deviations calculated from three repeats of the micronized sample were lower than the ESDs calculated by TOPAS. This suggests that ESDs are not always a good indicator of the accuracy that can be obtained using Rietveld analysis for quantification of cements. In conclusion, it would appear as though Rietveld analysis of un-micronized cements will give results for the two major phases that are the same within $95 \%$ confidence limits of a micronized sample. However, the errors are significantly higher and the divergence slit can have an additional effect on the errors. The results for the $C_{3} A$ repeats show a statistically significant difference between the micronized and un-micronized sample. Micronizing can have a major affect on the results of the minor phases, as demonstrated by the periclase in this paper. The micronized sample produced consistent results, whilst the un-micronized sample produced different results when remounted. It can be noted that the calculated ESDs for the periclase in the un-micronized sample were relatively small despite the result being obviously wrong.

\section{References}

1. Smith, D.K., 2001, Powder Diffr., 16, 186.

2. Alexander, L.E., Klug, H.P. \& Kummer, E., 1948, J. Appl.Phys., 19, 742.

3. Klug, H.P. \& Alexander, L.E., 1974, X-ray diffraction procedures for polycrystalline and amorphous materials (New York: Wiley).

4. Elton, N.J. \& Salt, P.D., 1996, Powder Diffr., 11, 218.

5. Hill, R.J. \& Madsen, I.C., 2002, in Structure Determination from Powder Diffraction Data, edited by W.I.F. David, K. Shankland, L.B. McCusker, \& Ch. Baerlocher (Oxford, Oxford University Press), pp. 98-117.

6. De Wolff, P.M., Taylor, J.M. \& Parrish, W., 1959, J. Appl.Phys., 30, 63.

7. Rietveld, H.M., 1967, Acta Cryst., 22, 151.

8. Hill, R.J. \& Howard, C.J., 1987, J. Appl.Crystallogr., 20, 467.

9. Brindley, G.W., 1945, Philos. Mag., 3, 347.

10. Mitchell, L.D., Margeson, J. \& Whitfield, P.S., 2006, Powder Diffr., 21, 111.

11. Bruker-AXS GmbH, 2008, DIFFRACplus TOPAS.

12. Dollase, W.A., 1986, J. Appl.Crystallogr., 19, 267. 



\section{Structural characterization of a coarse- grained transparent silicon carbide powder by a combination of powder diffraction techniques}

\section{B. Peplinski ${ }^{1,{ }^{*}}$, A. N. Fitch $^{2}$, A. Evans ${ }^{2}$, R. M. Ibberson ${ }^{3}$, D. M. Többens ${ }^{4}$, L. M. D. Cranswick ${ }^{5}$, I. Dörfel ${ }^{1}$, F. Emmerling ${ }^{1}$, R. Matschat ${ }^{1}$}

${ }^{1}$ BAM Federal Institute for Materials Research and Testing

Richard-Willstätter-Str. 11, D-12489 Berlin, Germany

${ }^{2}$ European Synchrotron Radiation Facility (ESRF), BP 220, F-38043 Grenoble, France

${ }^{3}$ ISIS Facility, Rutherford Appleton Laboratory (RAL), Chilton, Didcot, OX11 0QX, UK

${ }^{4}$ Hahn-Meitner-Institute (HMI), SF2, Berlin, Germany, now Institute of Mineralogy and Petrography, University of Innsbruck, Innrain 52, A-6020 Innsbruck, Austria

${ }^{5}$ National Research Council of Canada, Canada, Chalk River ON, Canada, K0J $1 \mathrm{JO}$

*burkhard.peplinski@bam.de

Keywords: silicon carbide, polytypes, phase quantification, reference material

Abstract. Diffraction of hard synchrotron radiation as well as constant-wavelength and time-of-flight neutron diffraction were used for the structural characterization of a silicon carbide powder having extremely low levels of chemical impurities, high perfection of the crystalline lattice and a grain size of up to $150 \mu \mathrm{m}$. The presence of three polytypes was ascertained and the ratios of their mass fractions were determined to be $\mathrm{w}_{15 \mathrm{R}}: \mathrm{w}_{6} \mathrm{H}$ $=0.002,3(8)$ and $\mathrm{w}_{4 \mathrm{H}}: \mathrm{w}_{6 \mathrm{H}}=0.000,6(2)$.

\section{Introduction}

Silicon carbide powders having chemical impurity levels as low as a few $\mathrm{mg}$ per $\mathrm{kg}$ or even less, high perfection of the crystalline lattice and a crystallite size exceeding several tens or even hundred $\mu \mathrm{m}$ show not only high hardness but also exceptional chemical resistance (insolubility in acids), as well as unique electronic and optical properties. One of the main challenges to a reliable structural characterization of such a material is its rather large crystallite size considerably exceeding the value acceptable for reliable $\mathrm{X}$-ray powder diffraction measurements. Any grinding bears a high risk of changing/destroying the original real structure characteristics of the sample, e.g. the degree of stacking disorder and their polytype composition. Thus, grinding can significantly distort the outcome of the structural investigation and, 
therefore, should be avoided. Consequently, highly penetrating radiation has to be used for any quantitative diffraction analysis and measures have to be taken to reduce the unfavourable influence of insufficient crystal orientation statistics as much as possible.

\section{Sample}

The element composition of the sample was extensively examined mainly by atomic absorption spectrometry (AAS) and by inductively coupled plasma optical emission spectrometry with electrothermal evaporation of the sample (ETV-ICP OES). The content of the following trace elements were determined: $\mathrm{Al}, \mathrm{B}, \mathrm{Ca}, \mathrm{Cr}, \mathrm{Cu}, \mathrm{Fe}, \mathrm{Mg}, \mathrm{Na}, \mathrm{Ni}, \mathrm{Ti}, \mathrm{V}, \mathrm{Zr}$. The mass fraction of the $\mathrm{Al}$ component incorporated into the bulk of the material is about $44 \mathrm{mg} / \mathrm{kg}$, and that of B about $4 \mathrm{mg} / \mathrm{kg}$. The other trace metals are mainly adhered to the surface of the particles. For that part of their mass fractions that is dissolved in the silicon carbide crystals values below $1 \mathrm{mg} / \mathrm{kg}$ were determined.

According to SEM the most frequently occurring particle size is about $20 \mu \mathrm{m}$. The smallest particles are ca. $8 \mu \mathrm{m}$ while a considerable number of particles have dimensions between 100 and $180 \mu \mathrm{m}$ (see figure 1). TEM in combination with the FIB preparation technique showed that the powder particles are quite perfect single crystals. Dislocations were found only in a surface layer less than $1 \mu \mathrm{m}$ thick but not in the interior of crystals.
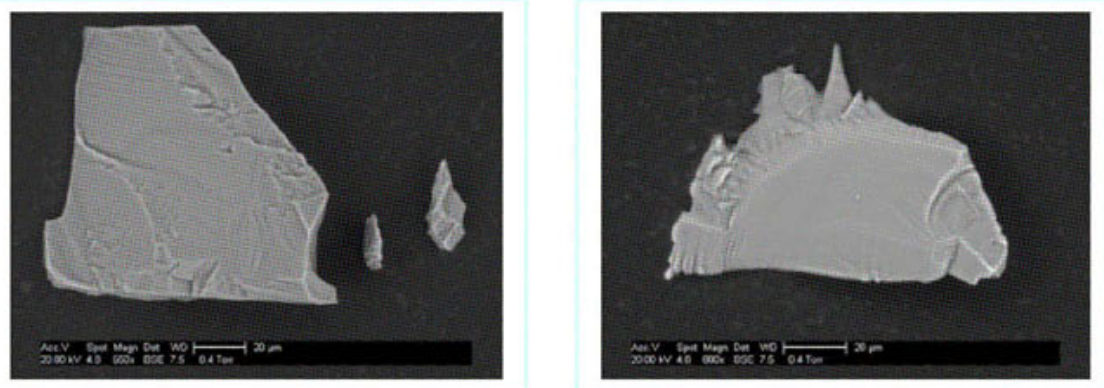

Figure 1. SEM pictures of individual crystallites of the silicon carbide powder sample. The largest dimensions in the 2D-projection of the displayed crystallites are ca. $140 \mu \mathrm{m}$ (left) and $110 \mu \mathrm{m}$ (right).

\section{Data collection and data analysis}

Instrumentation and conditions of data collection used in the present diffraction investigation as well as computer programs applied for data evaluation are summarized in table 1.

\section{Results and discussion}

All three diffraction patterns have FWHM values very close to the instrument contributions to the line profiles, see figure 2 . The three patterns were evaluated by the Rietveld method [1-2] describing the sample as $6 \mathrm{H}-\mathrm{SiC}$, either completely pure or with traces of other $\mathrm{SiC}$ 
Table 1. Characteristics of the three diffraction patterns of the silicon carbide material.

\begin{tabular}{|c|c|c|c|}
\hline & pattern \#1 & pattern \#2 & pattern \#3 \\
\hline diffracted radiation & $\begin{array}{c}\text { monochromatic } \\
\text { neutrons } \\
\lambda \approx 1.7967 \AA\end{array}$ & $\begin{array}{l}\text { pulsed neutrons } \\
\text { from spallation } \\
\text { source }\end{array}$ & $\begin{array}{c}\text { monochromatized } \\
\text { synchrotron radiation } \\
\lambda \approx 0.8003 \AA\end{array}$ \\
\hline instrument; facility & E9; HMI, BENSC & HRPD $^{1}$; ISIS. RAL & ID31; ESRF \\
\hline type of pattern & $\begin{array}{c}\text { from a } \\
\text { single specimen }\end{array}$ & $\begin{array}{c}\text { from a } \\
\text { single specimen }\end{array}$ & $\begin{array}{l}\text { synthesized from the } \\
\text { patterns of } 10 \text { specimen }\end{array}$ \\
\hline type of specimen & $\begin{array}{l}\text { capillary, } \emptyset 16 \mathrm{~mm} \text {, } \\
\text { vanadium }\end{array}$ & $\begin{array}{l}\text { capillary, } \varnothing 8 \mathrm{~mm} \text {, } \\
\text { vanadium }\end{array}$ & $\begin{array}{l}\text { capillary, } \varnothing 1 \mathrm{~mm} \text {, } \\
\text { borosilicate }\end{array}$ \\
\hline specimen rotation & none & none & $3000 \mathrm{rpm}$ \\
\hline mass of specimen & $12 \mathrm{~g}$ & $4 \mathrm{~g}$ & $80 \mathrm{mg}(=10 \cdot 8 \mathrm{mg})$ \\
\hline total measuring time & $17 \mathrm{~h}$ & $5 \mathrm{~h}$ & $10 \mathrm{~h}=(10 \cdot 1 \mathrm{~h})$ \\
\hline range of d-values & $34.0 \AA-0.916 \AA$ & $2.4 \AA-0.674 \AA$ & $22.9 \AA-0.825 \AA$ \\
\hline FWHM $^{2}$ & $0.016 \AA$ & $0.002 \AA$ & $0.001 \AA$ \\
\hline signal-to-Bkg ${ }^{2}$ & 33 & 350 & 650 \\
\hline noise-to-Bkg ${ }^{3}$ & \pm 0.125 & \pm 1 & \pm 0.05 \\
\hline $\begin{array}{l}\text { Rietveld } \\
\text { programs used }\end{array}$ & $\begin{array}{l}\text { FullProf.2k V.4.30 } \\
\text { (TOPAS V.2.1) }\end{array}$ & TOPAS V.4.1 & $\begin{array}{l}\text { TOPAS V.2.1 } \\
\text { TOPAS V.4.1 }\end{array}$ \\
\hline \multicolumn{4}{|c|}{$1 \quad$ pattern \#2 was collected before the major upgrade of the neutron guide in 2007} \\
\hline \multicolumn{4}{|c|}{$\begin{array}{l}{ }^{2,3} \text { FWHM and signal-to-background ratio for the } 103 \text { reflection of } 6 \mathrm{H}-\mathrm{SiC} \text { at } \sim 2.36 \AA \text {; } \\
\text { noise-to-background ratio near that line. }\end{array}$} \\
\hline
\end{tabular}

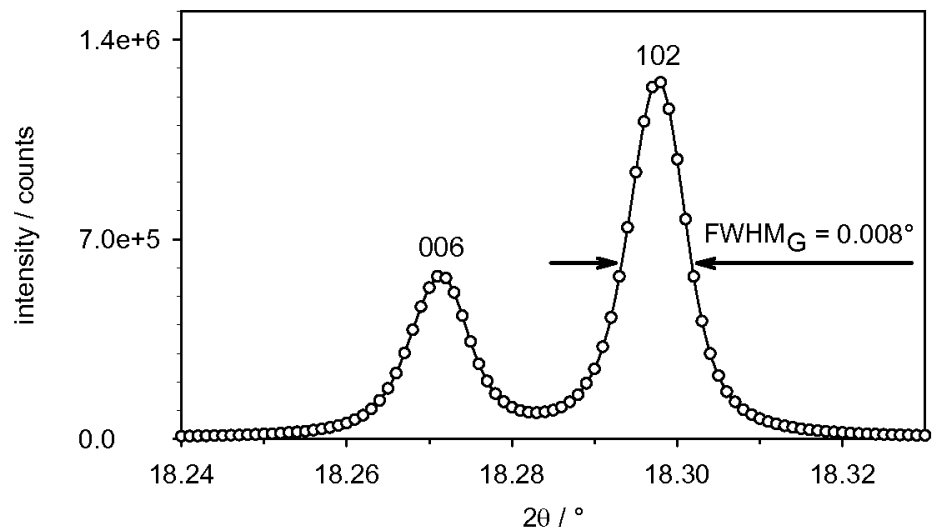

Figure 2. Section of diffraction pattern $\# 3$ displaying two reflections of $6 H$-SiC illustrating the exceptional high angular resolution.

polytypes, see figures 3 and 4 . The values of the goodness-of-fit $(\mathrm{GoF})$ achieved with each of these three observed $\mathrm{SiC}$ patterns are significantly higher than those resulting from the refinements of a standard silicon powder measured under identical instrument conditions. This discrepancy between the agreement indices might partly be caused by a very low, but nonzero content of planar disorder (stacking faults) that is known to cause (hkl)-dependant line 
shifts as well as distortions of line profiles and of the background [3]. Another factor to be considered is extinction, as the goodness-of-fit for the two neutron diffraction patterns improves when changing from Rietveld to Pawley refinements (from 2.55 to 2.29 and from 1.57 to 1.47 for pattern \#1 and \#2, respectively).

Pattern \#3 has superior angular resolution and signal-to-background ratio. Comparing it to the calculated diffractions patterns of the pure $\mathrm{SiC}$ polytypes it clearly shows that there are - besides the reflections of the main $6 \mathrm{H}-\mathrm{SiC}$ component - well discernible 21 isolated reflections of $15 \mathrm{R}-\mathrm{SiC}$ and 6 isolated reflections of $4 \mathrm{H}-\mathrm{SiC}$, see figure 4 . This proves that the sample does not consist of completely pure $6 \mathrm{H} \mathrm{SiC}$, but contains traces of $15 \mathrm{R}$ and $4 \mathrm{H} \mathrm{SiC}$.

Phase quantification should consider two main specifics of the present sample: the occurrence of systematic coincidences of diffraction lines of symmetry-related polytypes and the extremely different phase abundance of the main and minor components. Consequently, for reliable phase quantification only isolated diffraction lines should be evaluated. This was done by applying the Rietveld method to several angular ranges of pattern \#3 that include all isolated reflections of the two minor phases as well as 23 isolated and intense reflections of

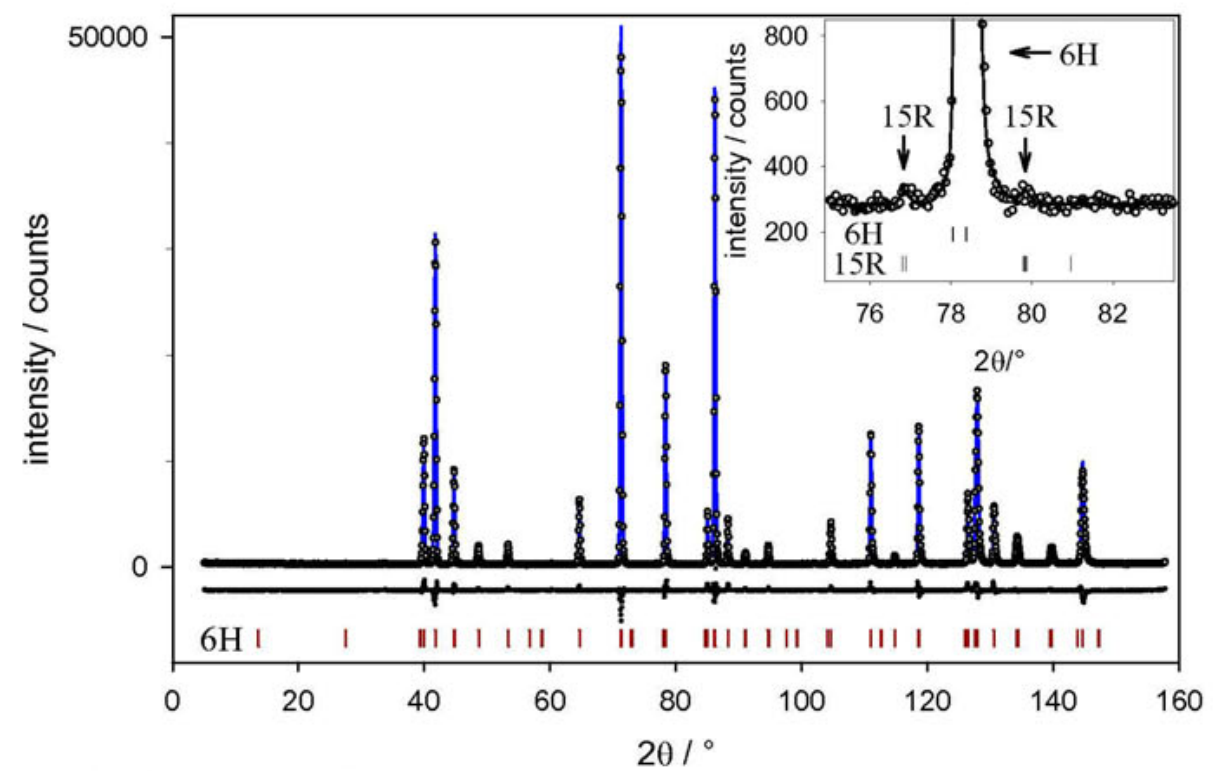

Figure 3. Rietveld plot for diffraction pattern \#1;

whole angular range: $R w p=6.6$ Rexp $=3.2 \quad$ GoF $=2.03$;

section $73.0^{\circ}-84.6^{\circ}: \quad R w p=4.47$ Rexp $=3.51$ GoF $=1.27$ (see also table 2).

Table 2. Results of the Rietveld analysis of two selected sections of diffraction pattern \#1.

\begin{tabular}{|c|c|c|c|c|c|}
\hline \multicolumn{2}{|c|}{ angular and d-value ranges } & \multicolumn{2}{|c|}{ GoF } & \multirow{2}{*}{$W_{15 \mathrm{R}}: W_{6 \mathrm{H}}$} & \multirow{2}{*}{$W_{4 \mathrm{H}}: W_{6 \mathrm{H}}$} \\
\hline $2 \theta /{ }^{\circ}$ & $\mathrm{d} / \AA$ & only $6 \mathrm{H}$ & $4 \mathrm{H}+6 \mathrm{H}+15 \mathrm{R}$ & $0.002,7(19)$ & $0.001,7(12)$ \\
\hline $38.0-44.0$ & $2.40-2.90$ & 2.41 & 2.35 & $0.002,9(6)$ & - \\
\hline $73.0-84.6$ & $1.36-1.48$ & 1.35 & 1.27 & 0.002 & - \\
\hline
\end{tabular}



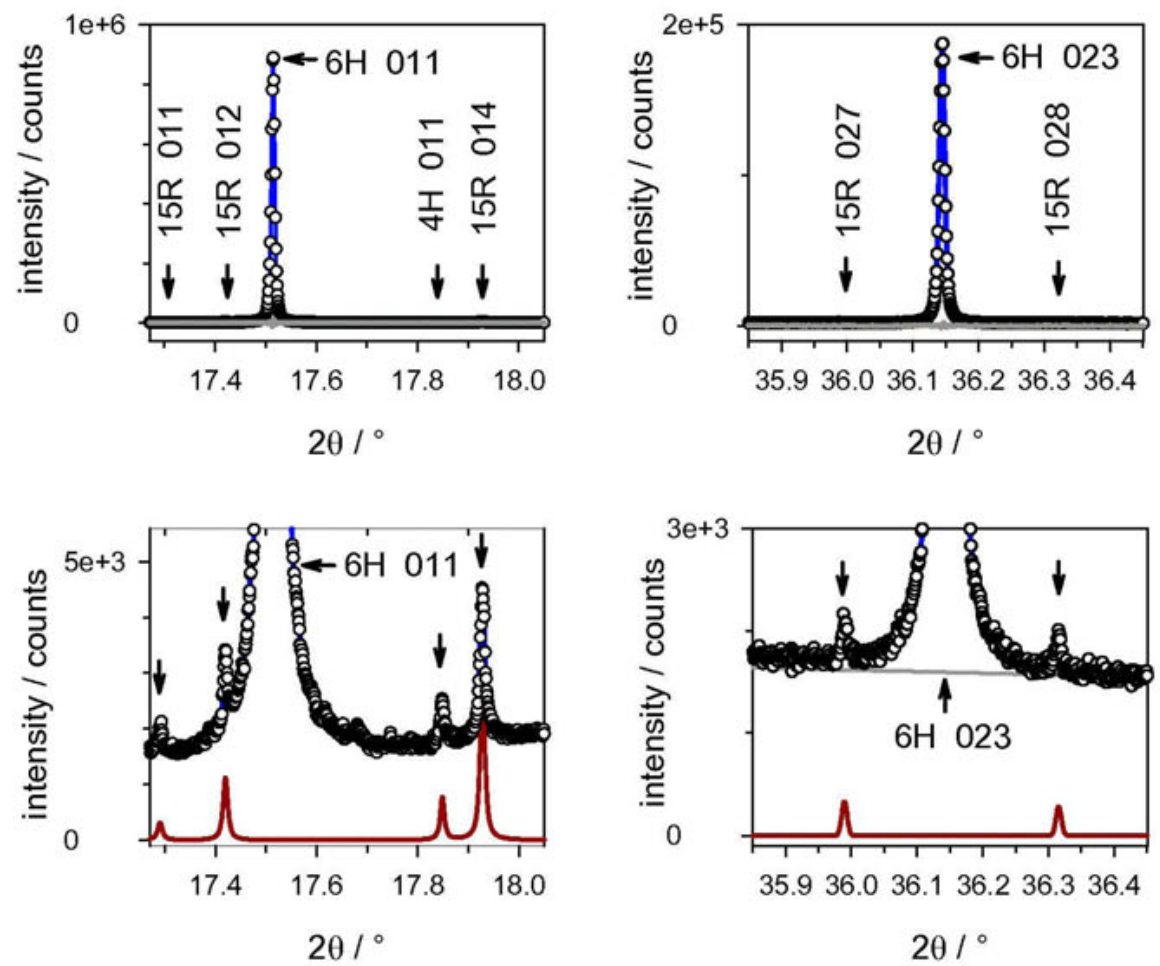

Figure 4. Rietveld plots for the two displayed angular ranges of diffraction pattern \#3;

top left: $\quad$ full intensity scale, $R_{w p}=1.93, R_{\text {exp }}=0.84, G o F=2.30$;

bottom left: $\quad$ Same as previous but reduced intensity scale. The lower curve shows the calculated contributions of the 15R-and $4 \mathrm{H}-\mathrm{SiC}$ components, i.e. of the two minor phases.

Ratios of the mass fractions from this refinement $w_{15 R}: w_{6 H}=0.003,88(20)$ and $w_{4 H}: w_{6 H}=0.000,643(58)$;

top right: $\quad$ full intensity scale, $R_{w p}=1.66, R_{\text {exp }}=1.39, G o F=1.19$;

bottom right: Same as previous but reduced intensity scale. The lower curve is the calculated contribution of the 15R-SiC component, i.e. of one of the minor phases. Ratio of the mass fractions from this refinement $w_{I S R}: w_{6 H}=0.003,0(3)$.

the $6 \mathrm{H}-\mathrm{SiC}$ main component. In the result the following values for the ratios of the mass fractions were determined (numbers in parentheses are estimated standard deviations): $\mathrm{w}_{15 \mathrm{R}}: \mathrm{w}_{6 \mathrm{H}}=0.002,3(8)$ and $\mathrm{w}_{4 \mathrm{H}}: \mathrm{w}_{6 \mathrm{H}}=0.000,6(2)$.

The angular resolution in pattern \#1 is considerably lower than in pattern \#3, see FWHM values in line 10 of table 1 , and there are only two narrow angular ranges in pattern \#1 where 
isolated reflections of the minor components might be detected if their phase abundance is sufficiently high. Visual inspection, search/match routines and Rietveld analyses carried out on these two sections as well as on the whole pattern \#1 support the finding derived from pattern \#3 by showing that the sample is an unusually pure $6 \mathrm{H}$ silicon carbide and that the mass fractions of possible traces of the 15R- and 4H-polytypes of silicon carbide are far below the $1 \%$ level, see table 2 and figure 3 . It is this huge difference in the phase abundance of the major and minor polytypes - together with the extreme size and lattice perfection of the $\mathrm{SiC}$ crystallites - that distinguishes the $\mathrm{SiC}$ material investigated in the present work from those analyzed in previous powder diffraction investigations, see e.g. [4], which had reported in detail on the phase quantification of micron-sized, commercial SiC powders by applying the Rietveld method to diffraction patterns collected with conventional X-ray powder diffraction instrumentation - an analytical technique rather unsuited for the given $\mathrm{SiC}$ material.

\section{Summary}

A coarse-grained silicon carbide powder was analysed 'as received' by two neutron powder diffractometers and one synchrotron radiation powder diffractometer. These three investigations complete each other very nicely as the mass of the analysed specimen and the representativeness of the results of the diffraction analysis for the whole material are very large in the case of the two neutron diffraction data sets while the synchrotron radiation data provide superior values of the background level, the angular resolution and of the detection limit for minor constituents. The outcome of these three diffraction experiments concurrently confirmed the exceptional high phase and polytype purity of the investigated $6 \mathrm{H}$ silicon carbide powder. In addition to this important finding, the outstanding performance of the ESRF instrument allowed to detect in the diffraction pattern of this silicon carbide powder 27 well discernible, isolated and indexed reflections of the $15 \mathrm{R}$ and $4 \mathrm{H}$ polytypes of silicon carbide thus proving that this material contains very minor mass fractions of the $15 \mathrm{R}$ and $4 \mathrm{H} \mathrm{SiC}$ polytypes. The ratio of the mass fractions of these polytypes to the mass fraction of the main polytype was determined to be $\mathrm{w}_{15 \mathrm{R}}: \mathrm{w}_{6 \mathrm{H}}=0.002,3(8)$ and $\mathrm{w}_{4 \mathrm{H}}: \mathrm{w}_{6 \mathrm{H}}=0.000,6(2)$.

\section{References}

1. Rietveld, H.M., 1969, J. Appl. Cryst., 2, 65.

2. Young, R.A., 1995, The Rietveld Method, International Union of Crystallography, Monographs on Crystallography (Oxford University Press).

3. Ustinov, A.I., 1999, in Defect and Microstructure Analysis by Diffraction, edited by Snyder, R.L., Fiala, J. \& Bunge, H.J. (Oxford: Oxford University Press), IUCr Monographs on Crystallography, 10, 264-317.

4. Ortiz, A.L., Cumberra, F.L, Sanchez-Bajo, F., Guiberteau, F., Xu, H. \& Padture, N.P., 2000, J. Am. Ceram. Soc., 83, 2282.

\section{Acknowledgments}

One of the authors (B.P.) would like to thank D. Schulz, Leibniz Institute for Crystal Growth (IKZ), Berlin, for helpful discussions, as well as G. Kley and H.-E. Maneck, both BAM. 
I.3 Analysis of Microstructure and Macrostress

I.3.1 Residual Stresses 



\title{
An experimental approach to the problem of transforming stress distributions from the LAPLACE- into real space
}

\section{A. Denks ${ }^{1, *}$, T. Manns ${ }^{2}$, Ch. Genzel ${ }^{1}$, B. Scholtes ${ }^{2}$}

${ }^{1}$ Helmholtz-Centre Berlin, Glienicker Straße 100, 14109 Berlin, Germany

${ }^{2}$ University Kassel, Mönchebergstraße 3, 34125 Kassel, Germany

*denks@helmholtz-berlin.de

Keywords: diffraction, residual stress analysis, stress gradients, energy dispersive diffraction

\begin{abstract}
The mathematical transformation of stress depth distributions $\sigma(\tau)$ in the LAPLACE-space into distributions $\sigma(z)$ in real space is difficult owing to the scattering of: experimental data. The assessment of the currently used techniques is obtained by an experimental approach based on the analysis of the same depth region by means of different diffraction techniques delivering distinct data points $\sigma\left(\tau_{\mathrm{k}}\right)$ and $\sigma\left(\mathrm{z}_{\mathrm{k}}\right)$, respectively. The comparison of the results shows that the (inverse) LAPLACE transform can only be applied under restrictive assumptions. Improvements may be achieved by using more complex models in the "universal plot" evaluation procedure.
\end{abstract}

\section{Introduction}

Regarding those cases where the stress distribution has to be linked with materials depth properties (e.g. features on micrographs) the real space depth distribution is of importance rather than average stress values. When dealing with steep stress gradients in the surface, however, real space (strain scanning) techniques in most cases do not exhibit a sufficient surface sensitivity and thus diffraction techniques in reflection mode are applied which draw their depth resolution from the exponential beam attenuation. The penetration depth $\tau$ can be considered as the centroid of the diffraction information or likewise the depth region from which $63 \%$ of the total diffraction power derives from. For samples of thickness $D \gg \tau$ it is given by

$$
\tau=\frac{1}{\mu(E) \cdot m}
$$

where $\mu(\mathrm{E})$ is the energy dependent linear absorption coefficient and $\mathrm{m}$ is a geometrical factor.

Any diffraction method in reflection mode based on the $\sin ^{2} \psi$-technique delivers a profile $\sigma(\tau)$ which is the LAPLACE-transform (LT) of the real space profile $\sigma(\mathrm{z})$ according to $\sigma(\tau)=\frac{1}{\tau} L\left[\sigma(z), \frac{1}{\tau}\right]=\frac{1}{\tau} \int_{0}^{\infty} \sigma(z) e^{-\frac{z}{\tau}} d z$ 
Thus, in order to obtain absolute stress values $\sigma(\mathrm{z})$ in real depths $\mathrm{z}$ the inverse LAPLACEtransform (ILT) has to be applied on the evaluated distributions from the experiment.

Efforts to transform the data points $\sigma\left(\tau_{\mathrm{k}}\right)$ obtained from the experiment into distributions $\sigma(\mathrm{z})$ face serious problems. In any case the reason is the scattering in the experimental data which generates high uncertainties in the calculated distribution $\sigma(z)$. Consequently, the numerical inverse LAPLACE-transform (NILT) is rarely used though different approaches exist [1].

In practice, a more stable method is used based on the idea of fitting a function to the data points on the $2 \theta^{\mathrm{hkl}}\left(\sin ^{2} \psi\right)$ - scale $[2,3]$ or the $\sigma(\tau)$ - scale [4] and transforming the function by means of the ILT.

The aim of this paper is to asses the mathematical procedure by means of experimental data $\sigma\left(\tau_{\mathrm{k}}\right)$ and $\sigma\left(\mathrm{z}_{\mathrm{k}}\right)$ covering the same depth region. This is realized by high energy white beam diffraction [5] on the one hand and the sublayer removal technique using fine steps as well as a strain scanning based technique [6] on the other hand.

\section{Experimental}

\section{Sample}

The sample material is the roller bearing steel $100 \mathrm{Cr} 6$ in a hardened and tempered state. The heat treatment yields a fine grained microstructure of martensite, highly dispersed carbides $\left(\mathrm{M}_{23} \mathrm{C}_{6}\right)$ and retained austenite. From micrographs and phase analyses can be concluded that the grain size is in the sub-micrometer range and the volume fractions of austenite and carbides, respectively, do not exceed $10 \mathrm{vol} \%$.

The sample was ground and subsequently shot peened with an Almen intensity of approx. $0.16 \mathrm{mmA}$ and coverage of $200 \%$. Investigation of the topography reveals an average roughness of $0.52 \mu \mathrm{m}$.

\section{Residual stress analysis}

The measurements to obtain surface parallel stress component $\sigma_{\|}\left(\tau_{\mathrm{k}}\right)$ in the LAPLACE-space were performed at the synchrotron beamline for energy dispersive diffraction, EDDI, at BESSY [5]. The diffraction angle was $16 \mathrm{deg}$. and in order to prevent any thermal loading an aluminium filter of $2 \mathrm{~mm}$ in thickness was placed in the primary beam. The tilting angle $\psi$ was increased in 32 steps up to $89 \mathrm{deg}$. with gradually decreasing step sizes. The evaluated diffraction lines are $111,200,211,220,310,222,321,411,420$ and 431 . Considering the white beam spectrum used from $22 \mathrm{keV}(110)$ to $79 \mathrm{keV}$ (431) a depth region from approx. $0.1 \mu \mathrm{m}$ to $135 \mu \mathrm{m}$ could be covered.

The data were analyzed using the universal plot method [4] assuming a biaxial stress state. It is based on plotting the surface parallel stress $\sigma_{\|}(\tau)$ given by

$\sigma_{\|}(\tau)=\frac{\frac{1}{2}\left[\varepsilon_{0, \psi}^{k h t}(\tau)+\varepsilon_{00, \psi}^{h k t}(\tau)\right]}{1 / 2 s_{2}^{h k t} \sin ^{2} \psi+2 s_{1}^{h k t}}$

for all evaluated reflections hkl into one "master plot" versus $\tau$. 
The elastic behaviour of any lattice plane is taken into account by the diffraction elastic constants $s_{1}^{\text {hkl }}$ and $1 / 2 s_{2}^{\text {hkl }}$.

The strain free lattice spacings $d_{0}^{\text {hkl }}$ needed for strain calculation are obtained in the stress free direction $\psi^{* h k t}$ in the rotation symmetric case given by

$\sin ^{2} \psi^{* h k l}=\frac{-2 s_{1}^{h k l}}{1 / 2} s_{2}^{h k l}$.

Experimental stress values $\sigma_{\|}\left(\mathrm{z}_{\mathrm{k}}\right)$ in real space were evaluated after stepwise removal of the surface region by electrolytic polishing and subsequent $\sin ^{2} \psi$ measurements using CrK $\alpha$ radiation and the diffraction lines 110 and 211 . The distribution $\sigma_{\|}\left(\mathrm{z}_{\mathrm{k}}\right)$ was corrected regarding the average penetration depth of $\mathrm{CrK} \alpha$ radiation and the Moore and Evans correction [7] was applied taking account for the macro residual stresses released by the layer removal. The other technique applied is based on a recently developed "stress scanning" technique [6] allowing $\sin ^{2} \psi$ measurements within a flat gauge volume orientated parallel to the sample surface and subsequently scanned through the sample. The spatial resolution is given by the gauge volume height of approx. $10 \mu \mathrm{m}$.

The evaluation of the depth distributions $\sigma_{\|}(\mathrm{z})$ and $\sigma_{\|}(\tau)$ from the data points $\sigma_{\|}\left(\tau_{\mathrm{k}}\right)$ and $\sigma_{\|}\left(z_{k}\right)$, respectively, is performed by fitting the experimental data by means of an exponentially damped polynom as successfully used in [2]. The functions are shown in table 1 and denoted $\mathrm{P}_{\mathrm{N}}$ regarding the order of the damped polynom for $\sigma(\mathrm{z})$.

Table 1. Fitting functions used to describe the stress depth distribution in the shot peened steel sample.

\begin{tabular}{|c|c|}
\hline$\sigma(\mathrm{z})$ & $\sigma(\tau)$ \\
\hline$\left[\sum_{n=0}^{N} a_{n} z^{n}\right] e^{-a_{N+1} z}$ & $\frac{1}{\tau} \sum_{n=0}^{N} a_{n} \frac{n !}{\left(a_{N+1}+\frac{1}{\tau}\right)^{n+1}}$ \\
\hline
\end{tabular}

\section{Results and discussion}

Figure 1a shows the residual stress profile $\sigma\left(\tau_{\mathrm{k}}\right)$ obtained from the universal plot evaluation of the $\sin ^{2} \psi$ measurement. Owing to the ten diffraction lines included and the 32 steps in the tilting angle $\psi$ from $\psi=0^{\circ}$ to $\psi=89^{\circ}$ the plot contains 320 data points leading to a detailed stress distribution in the LAPLACE-space especially in the near surface region up to $30 \mu \mathrm{m}$. In order to highlight the origin of scattering as discussed later, the data points are separately marked in the figure for different $\sin ^{2} \psi$ intervals. All fits of exponentially damped functions with increasing number of parameters fit satisfactorily and do not reveal any significant differences apart from the surface down to $1 \mu \mathrm{m}$ and the deeper regions from $60 \mu \mathrm{m}$. The d- $\sin ^{2} \psi$-plots also do not give any hints on the best fit and thus are not presented here.

However, figure $1 \mathrm{~b}$ attests significant aberration of the distributions $\sigma(\mathrm{z})$ obtained by taking the parameters from figure la. It seems that $\mathrm{P}_{1}$ and $\mathrm{P}_{3}$ show feasible distributions, although slightly different. Starting from function $\mathrm{P}_{5}$ an overshooting leads to oscillating distributions which certainly do not represent a realistic residual stress depth distribution in shot peened 
samples. Thus, following the formalism outlined in [3] a practical way of handling the problem might be given by increasing the number of fitting parameters until the overshooting occurs and claim the last fit to be the most realistic. Certainly, the transformation procedure still has to be regarded somehow arbitrary.

The only way of getting a more stable procedure is to perform a more effective fitting, by means of two criteria: less scattering $\Delta \sigma\left(\tau_{k}\right)$ in the data points and use of a more applicable fitting function.

The scattering of the data is not mainly ascribed to poor diffraction signal intensity as the relatively small error bars in figure la show, which derive from the average error in the determination of the diffraction line positions. Only those diffraction lines which appear at higher energies from approx $65 \mathrm{keV}$ tend to exhibit higher scattering due to the decreasing intensity supplied from the photon source. As these diffraction lines deliver the information of higher depths they are indispensable.

The more essential reason for the scattering might be found in the near surface region. Figure 1a shows that the scatter band is in the range of $100 \mathrm{MPa}$ leading to different "interpretation" of the stress distribution using different fitting functions (approx. -500 MPa for $\mathrm{P}_{1}$ and approx. -300 for $\mathrm{P}_{7}$ ). The scattering in this region may certainly be reduced when considering the anisotropic plastic behaviour of the individual diffraction lines hkl in the highly plastically deformed near surface region.

The other reason is based on the fact that all data points near to the stress free direction $\psi^{* * k l}$ (marked as " $x$ " in figure 1a) exhibit high uncertainties as the calculated stresses result from small strains and a large multiplicity factor (insertion of equation (5) into equation (3) give division by zero), i.e. any experimental errors (e.g. misalignment, poor grain statistics) lead to large errors for these data points.

The second criterion is demonstrated by figure 2 showing that the chosen fitting functions in table 1 are generally being suitable to describe the depth distribution $\sigma(z)$ down to $300 \mu \mathrm{m}$ in depth. The approximation of the data points $\sigma\left(\mathrm{z}_{\mathrm{k}}\right)$ increases with increasing number of parameters and is satisfactory from $\mathbf{P}_{5}$. Comparison of the inverse LAPLACE-transformed function $P_{5}$ in figure $1 b$, however, reveals already overshooting. $P_{3}$ shows reasonable results in figure $1 \mathrm{~b}$ but has not enough parameters to describe $\sigma(\mathrm{z})$ satisfactorily as shown in figure 2 .

\section{Conclusions}

The scattering of the experimental data $\sigma\left(\tau_{k}\right)$ leads to unstable mathematical conditions if the fitting function has too many parameters but might be unsuitable when having too little. In any case the comparison of the experimentally obtained distributions $\sigma\left(\tau_{\mathrm{k}}\right)$ and $\sigma\left(\mathrm{z}_{\mathrm{k}}\right)$ shows that even if applicable functions are found which can describe the depth distribution $\sigma(\tau)$ satisfactorily the calculated $\sigma(z)$ distribution differ significantly, especially in deeper sample regions.

Improvements can only be achieved by reduction of data scattering. Applying the universal plot method the first measure is to increase counting statistics and decrease misalignment which is a more sensitive criterion than using the simple $\sin ^{2} \psi$ evaluation procedure. Secondly, seattering may be reduced when regarding the multiplication of any possible sources of errors near to the strain free direction. Thirdly, the anisotropic behaviour in the plastically deformed surface region may be taken into account for any individual lattice plane hkl. 


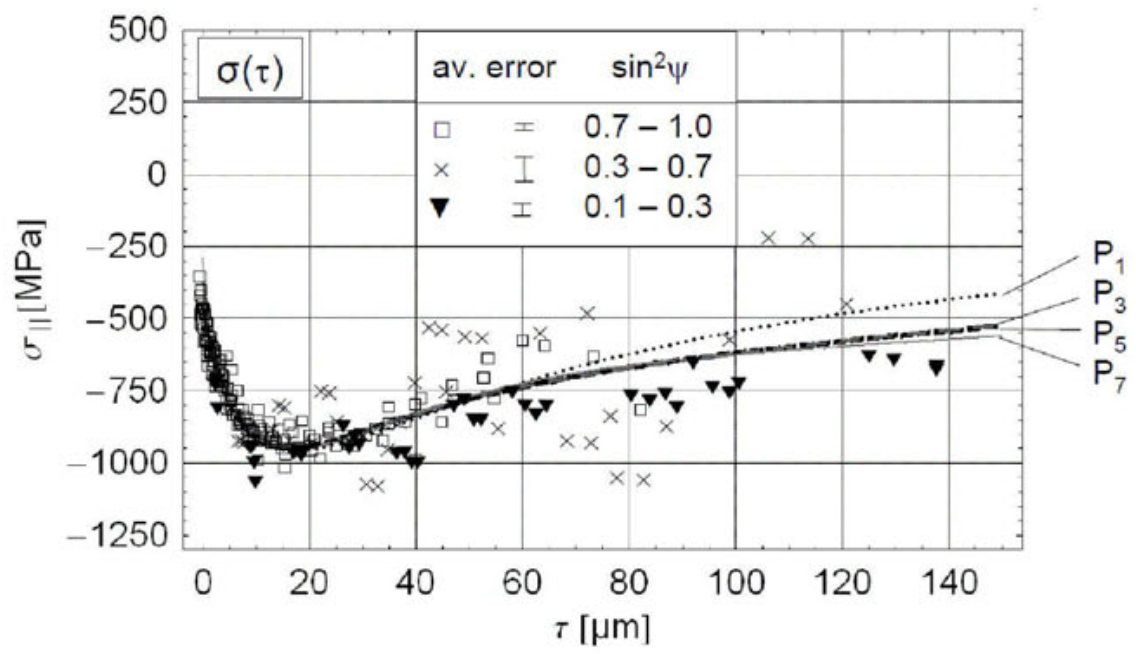

(a)

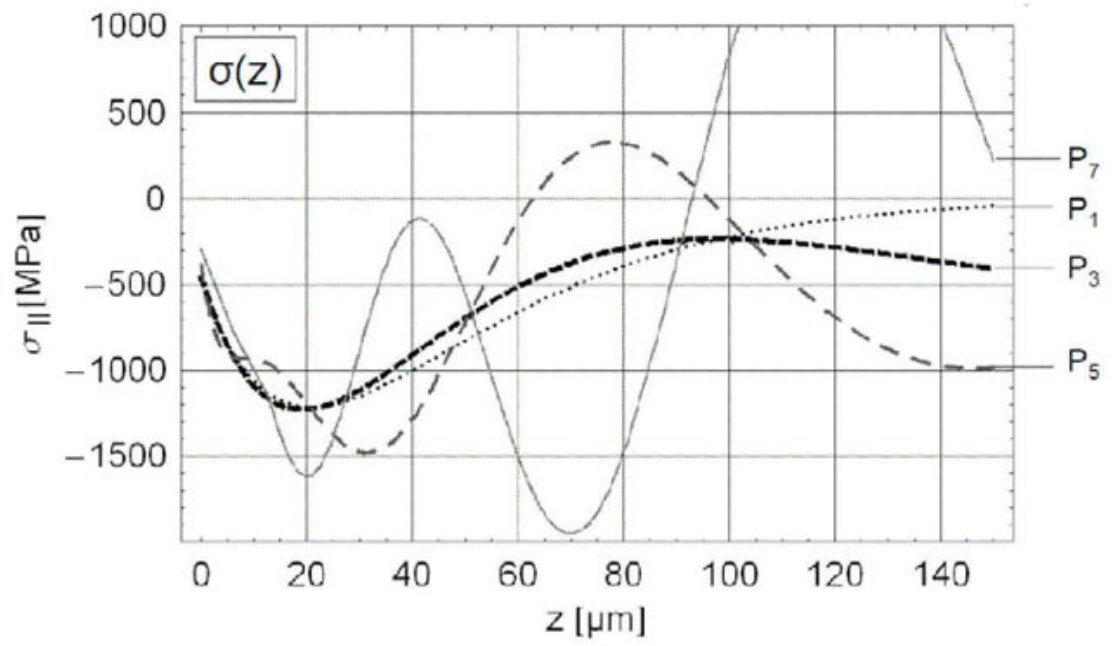

(b)

Figure 1. Experimental data points $\sigma\left(\tau_{k}\right)$ fitted with damped polynoms of different order given in table 1 (a) and calculated $\sigma(z)$ distributions by ILT (b). 


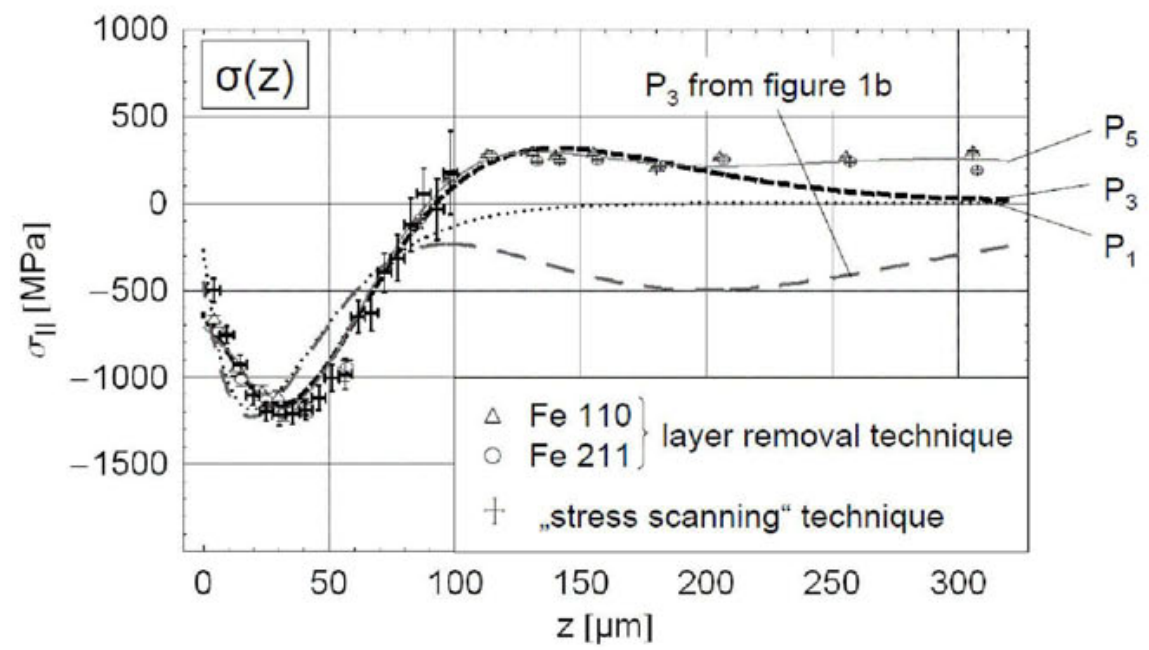

Figure 2. Experimental data points $\sigma\left(z_{k}\right)$ fitted with the functions given in table 1 and for comparison $P_{3}$ from figure $1 b$.

\section{References}

1. Zhu, X., Predecki, P. \& Ballard, B., 1995, Adv. X-ray Anal., 38, 263.

2. Hauk, V. \& Krug, K., 1984, HTM, 39, 273

3. Ruppersberg, H., Detemple, I. \& Krier, J., 1989, Phys. Stat. Sol. (A), 116, 681.

4. Eigenmann, B., Scholtes, B. \& Macherauch, E., 1990, Mat.-Wiss. u. Werkstofftech., 21, 257

5. Genzel, Ch., Denks, I.A., Gibmeier, J., Klaus, M. \& Wagener, G., 2007, Nucl. Instr. Meth. A, 578, 23.

6. Denks, I.A., Thesis, University Kassel, 2008.

7. Moore, M.G. \& Evans, W.P., 1948, Trans. SAE, 66.

Acknowledgements. The authors whish to thank the German Research Foundation (DFG) for financial support. 
I.3.2 Line Broadening Analysis 



\title{
A practical vision on Line Profile Analysis today and in the years to come
}

\author{
R. Delhez ${ }^{1, *}$, A. C. Vermeulen ${ }^{2}$
}

${ }^{1}$ Dept. of Materials Science and Engineering, Delft University of Technology, Mekelweg 2, 2628 CD Delft, The Netherlands

${ }^{2}$ PANalytical BV, Lelyweg 1, 7602 EA Almelo, The Netherlands

*R.Delhez@TUDelft.nl

Keywords: Line Profile Analysis, line broadening, microstructure, lattice defects, micro strain, crystallite size, dislocations, stacking faults, twin faults, anti-phase boundaries

Abstract. Line Profile Analysis [LPA] is a useful tool for diffraction pattern analysis to determine the microstructure of polycrystalline materials. LPA is not difficult and therefore it is tempting to think that it is simple, but it is complicated and that requires careful operation. Some microstructural phenomena may influence other branches of diffraction analysis like the determination of crystal structures, of residual stresses and of crystallographic texture. In today's practice simple procedures (e.g. Williamson Hall plots) are used widely, and often without justification to determine crystallite sizes and lattice strains (size-strain analysis) instead of dedicated, rather complicated methods of Line Profile Analysis. It will be pointed out (i) when the use of simple methods is acceptable, (ii) why and when existing rather complicated methods shall be used, and which requirements / conditions hold for their use, and (iii) what is the desirable direction of developments.

\section{Introduction}

Line Profile Analysis [LPA] is the quantitative interpretation of the breadths and shapes and sometimes also the positions - of individual, or a set of HKL reflections. Numerous reviews have been written, also recently $[1,2]$.

Table 1 shows when LPA is worthwhile to apply and reliable in daily practice.

Table 1. Reliable application of Line Profile Analysis in practice.

\begin{tabular}{|l|l|}
\hline Line Profile Analysis yields quantitative results \\
\hline Routinely & $\begin{array}{l}\text { averaged lattice distortion (i.e. microstrain, cause undefined); averaged crystallite } \\
\text { sizes }\end{array}$ \\
\hline Non-routinely & $\begin{array}{l}\text { crystallite size distributions; dislocation types and their densities; types, } \\
\text { orientations and densities of stacking and twin faults; nano-materials }\end{array}$ \\
\hline $\begin{array}{l}\text { Specially or } \\
\text { incidentally }\end{array}$ & $\begin{array}{l}\text { high (non-equilibrium) vacancy concentrations; spatial arrangements, types, den- } \\
\text { sities, \& orientations of dislocations; spatial arrangements, types, densities, and } \\
\text { orientations of planar faults, e.g. stacking and twin faults; anti-phase boundaries; } \\
\text { concentration gradients e.g. caused by sintering; materials containing particles. }\end{array}$ \\
\hline
\end{tabular}




\section{Line Profile Analysis is not difficult, but it is complicated}

Line Profile Analysis [LPA] seems to be not difficult, because it is simple to learn the basics and therefore it is tempting to think that it is simple and easy to apply. A few basic, simple equations were deduced about a century ago. Of course they are still valid and useful. But the practical application of LPA is complicated (see below) and therefore one should be careful and profit from existing expertise. It is a pity that diffractionists (including the authors) are often too lazy to search for expertise - induced by the general, daily hurry. Further, there is a lack of software containing explicit expertise. Often software contains some expertise, but by not making it explicit - there is no dissemination of this expertise and it may be lost.

\section{The Basics of Line Profile Analysis methods}

Figure 1 shows an example of some related sharp and broadened line profiles. It is common practice to characterize a line profile with its position (e.g. top, centroid - yielding a lattice spacing), its intensity (e.g. integrated intensity - yielding its structure factor), its breadth (e.g. full width at half maximum [FWHM] and integral breadth - yielding "size" and "strain", cf. section "Breadth methods"), and "shape" - a rather vague term, often characterized by a single parameter, but in fact much more complicated (i.e. less understood) as will become clearer in what follows.

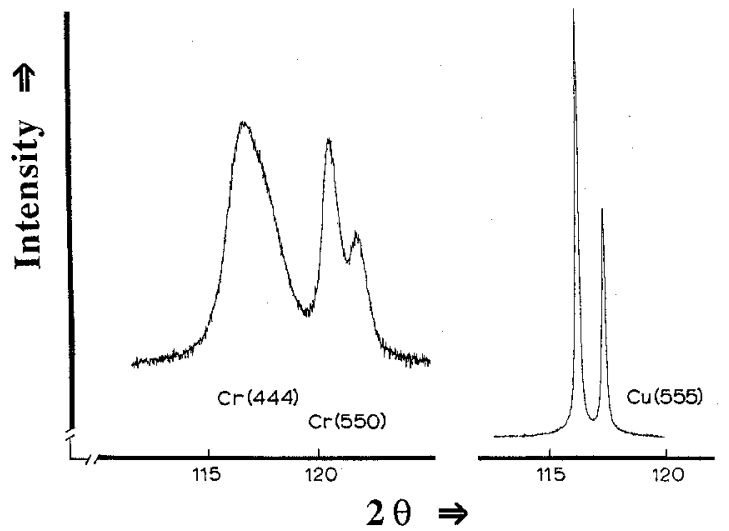

Figure 1. The adjacent, strongly broadened $\{444\}$ and $\{550\}$ reflections of an electrolytic Cr layer (left) and the sharp \{555\} reflection of a well annealed Cu powder (right) using MoK $\alpha$ radiation. The $\mathrm{Cu}$ reflection shows the so-called instrumental broadening. The \{550\} reflection of the $\mathrm{Cr}$ layer is narrower than the $\{444\}$, although one would expect the opposite, because $2 \theta$ for the $\{550\}$ is larger than for the $\{444\}$. The HKL dependent broadening of the $\{444\}$ and $\{550\}$ reflections was generated by crystallites with different growth directions implying the perfection of growth depended on crystallographic direction. [Intensity in arbitrary units; diffraction angle $2 \theta$ in degrees.]

The profile of a reflection from an ideal (perfect) crystal can be calculated and it turns out that its breadth and shape can be neglected in comparison with the contributions of almost all diffractometers, radiation sources, and specimens used in practice. Therefore diffraction-line breadth and shape consist of the contributions listed in table 2. All contributions occur simultaneously and therefore their influences on diffraction interact and this interaction is 
Table 2. Constitution of diffraction-line profiles.

\begin{tabular}{|c|c|}
\hline Contribution & Constitution of contribution \\
\hline Instrument geometry & $\begin{array}{l}\text { Bragg Brentano (para-focusing), parallel beam, specimen } \\
\text { tilting, mechanical aspects of the instrument (eucentricity), ... }\end{array}$ \\
\hline Optical components & $\begin{array}{l}\text { X-ray spectrum, source, collimators, slits, mirrors, } \\
\text { monochromators, } \ldots\end{array}$ \\
\hline $\begin{array}{l}\text { Crystal structure and micro- } \\
\text { structure of the compounds present }\end{array}$ & $\begin{array}{l}\text { space group, lattice dimensions, crystallite size, } \\
\text { microstrain, lattice defects }\end{array}$ \\
\hline Physical properties of the specimen & transparency, surface condition, fluorescence \\
\hline Specimen (shape and production) & texture, macrostresses, composition of phases present. \\
\hline \multicolumn{2}{|l|}{$h(y)=\int_{-\infty}^{\infty} f(x) \cdot g(y-x) d x$} \\
\hline \multicolumn{2}{|c|}{$\begin{array}{l}\text { It is obvious that the term deconvolution means the inverse of the convolution process. } \\
\text { If } \mathrm{f}(\mathrm{x}), \mathrm{g}(\mathrm{z}) \text {, and } \mathrm{h}(\mathrm{y}) \text {, with } \mathrm{z}=\mathrm{y}-\mathrm{x} \text {, are written as Fourier series } \mathrm{F}(\mathrm{n}), \mathrm{G}(\mathrm{n}) \text {, and } \mathrm{H}(\mathrm{n}) \text { and these are } \\
\text { substituted in equation (1a) it is found that }\end{array}$} \\
\hline$H(n)=G(n) \cdot F(n)$ & ( \\
\hline
\end{tabular}

accurately - and in most cases exactly - described by (repeated) convolution: see table 2. Convolution and deconvolution are exploited implicitly or explicitly in practice: section "Line Profile Analysis methods in practice".

\section{Line Profile Analysis methods in practice}

The main types of LPA method applied in practice are listed in table 3, roughly in order of increasing mathematical complexity. This is also the order in which they appeared in literature and laboratory practice. The steps from Breadth methods to Fourier methods to Profile Fitting methods were important steps forward, but the simpler the method the more simplification it implies and simplification always means sacrificing trueness, reliability, precision and details in results. Moreover, it was realized that these methods lacked physical

Table 3. Types of LPA method and their merits.

\begin{tabular}{|c|c|c|c|l|}
\hline LPA method & $\begin{array}{c}\text { Quality of } \\
\text { its results in } \\
\text { general }\end{array}$ & $\begin{array}{c}\text { Sensitivity to } \\
\text { quality of } \\
\text { measurement }\end{array}$ & $\begin{array}{c}\text { Sensitivity to } \\
\text { specific types } \\
\text { of defect }\end{array}$ & $\begin{array}{c}\text { Examples } \\
\text { of methods }\end{array}$ \\
\hline $\begin{array}{c}\text { Breadth methods } \\
\text { (simple; few lines })\end{array}$ & qualitative & isolated lines & "Size" \& \\
\hline $\begin{array}{c}\text { Fourier methods } \\
(D F T s ; \text { few lines })\end{array}$ & $\begin{array}{c}\text { somewhat } \\
\text { detailed }\end{array}$ & $\begin{array}{c}\text { isolated lines, } \\
\text { low background }\end{array}$ & "Size" \& \\
"Strain" & WA plots \\
\hline $\begin{array}{c}\text { Profile Fitting methods } \\
\text { (analytical fit functions })\end{array}$ & $\begin{array}{c}\text { usually } \\
\text { qualitative }\end{array}$ & little & sometimes & $\begin{array}{l}\text { Popa, } \\
\text { Stephens }\end{array}$ \\
\hline $\begin{array}{c}\text { Powder Pattern } \\
\text { Calculation methods } \\
\text { (physical fit functions })\end{array}$ & quantitative & almost none & yes, all types & WPPM \\
\hline
\end{tabular}

$D F T=$ Discrete Fourier Transform

*) not taking into account the counting statistics 
background and/or detailed input from diffraction and therefore the interest in physical descriptions of line-broadening effects started to rise leading to Powder Pattern Calculation methods. At present some adequate algorithms and software exist to accommodate the rather complex mathematics required for the physical models.

Fourier methods will not be discussed in this paper, because of their complicated nature and their limited routine use in practice. An example is the Warren Averbach analysis [3].

Profile Fitting methods using analytical functions will not be discussed here, because they have practical and theoretical shortcomings compared to Powder Pattern Calculation methods using physical fit functions, e.g. [4].

Exploiting deconvolutions to accomplish LPA of individual line profiles:

Breadth methods use deconvolution implicitly as they assume that all line profiles concerned are simple functions (e.g. all Lorentz, or Gauss). Then simple formulas for the breadths hold, e.g. Williamson-Hall plots (see table 4 that uses pure Lorentzian profiles). Exploiting convolutions to accomplish LPA of $a$ set of line profiles from a compound:

LPA is done by calculating line profiles through convolutions of contributions described by physical profile functions directly or through Fourier synthesis, followed by matching to a recorded powder pattern, cf. section "Powder Pattern Calculation ...".

Table 4. Basics of Williamson-Hall plots.

\begin{tabular}{|c|l|l|}
\hline Step & & Basic equation \\
\hline i & $\beta$ due to instrument and microstructure & $\beta_{\text {obs }}=\beta_{\text {instrum }}+\beta_{\text {struct }}$ \\
\hline ii & $\beta$ due to microstructure & $\beta_{\text {struct }}=\beta_{\text {size }}+\beta_{\text {stain }}$ \\
\hline iii & $\beta$ due to crystallite size (Scherrer) & $\beta_{\text {size }}=\mathrm{K} \lambda / \mathrm{D} \cos \theta$ \\
\hline iv & $\beta$ due to lattice deformation (Wilson) & $\beta_{\text {strain }}=4 \mathrm{e} \tan \theta$ \\
\hline $\mathrm{v}$ & $\beta$ due to instrument and microstructure & $\beta_{\text {obs }}-\beta_{\text {instrum }}=\mathrm{K} \lambda / \mathrm{D} \cos \theta+4 \mathrm{e} \tan \theta$ \\
\hline \multirow{2}{*}{ vi } & $\begin{array}{c}\text { Relation of breadths } \\
\text { in real space and in reciprocal space }\end{array}$ & $\left(\beta_{\text {struct }} \cos \theta\right) / \lambda=\beta_{\text {struct, rec }}$ \\
\hline
\end{tabular}

Step $i=$ removal of instrumental broadening if the broadening is purely Lorentzian.

Step $i i=$ splits structural broadening into "size"and "strain" broadening using Lorentz functions If the broadening is purely Gaussian or Voigtian, analogous equations hold that are a little more complicated than equations (2a) and (2b).

Steps iii, $i v, v$, vi and formula (2a) hold for integral breadths $\beta$ in radians; to convert radians to ${ }^{\circ} 2 \theta$ multiply by $180 / \pi$. The unit of $\beta_{\text {struct, rec }}$ in (2b) is the reciprocal of the unit used for $D$ and $d$.

\section{Breadth methods}

The most widely used breadth method is the Williamson-Hall plot (WH plot). WH plots are based usually on one of the two simple equations (see table 4):

Williamson Hall plot in real space: $\quad\left(\beta_{o b s}-\beta_{\text {instrum }}\right) \cos \theta=K \lambda / D+4 e \sin \theta$. (2a)

Williamson Hall plot in reciprocal space: $\quad \beta_{\text {struct, }, \text { ec }}=K / D+2 e / d$.

WH plots are used widely because they are simple, look nice and suggest useful and usable results: see the example in figures $2 a$ and $2 b$. The important questions that arise are:

- Is the kind of interpretation like the one given in figure $2 \mathrm{~b}$ a correct interpretation?

Maybe. Nowadays one shall test these results either by synthesizing the pattern using the model parameters or by using better physical modeling and up-to-date software. 
- Is the kind of interpretation like the one given in figure $2 \mathrm{~b}$ allowed?

Only if one knows for sure that the only effects present are size effects plus dislocations of the type and orientation used in the actual calculation.

One should be aware (i) that the effort to generate the interpretation given in figure $2 b$ is considerable and (ii) that this interpretation can be applied only to the material investigated because it uses its elastic constants and their HKL dependence. So this is not a simple (quick) interpretation, although it uses a simple plot.

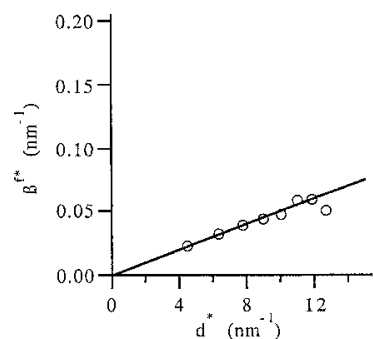

ist. dens. $=10 \times 10^{14} \mathrm{~m}^{-2}$ only edge, 12 slip systems

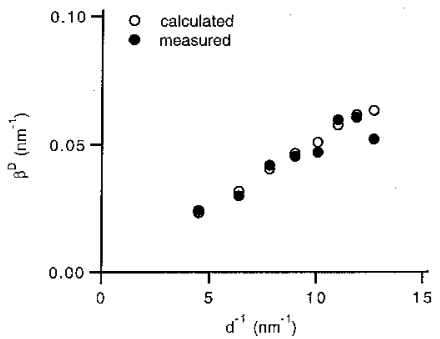

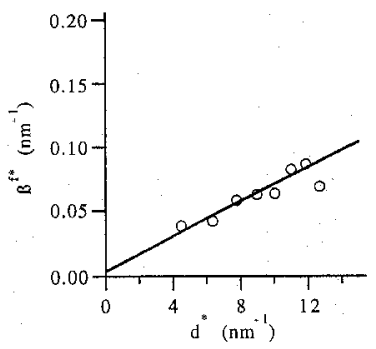

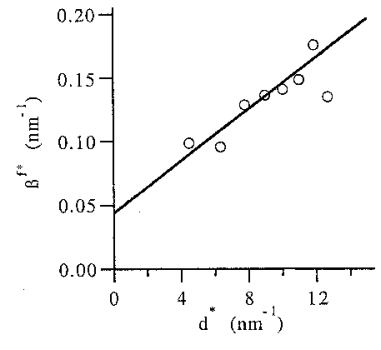

disi. dens $=10 \times 10^{14} \mathrm{~m}^{-2}$ only screw, 12 slip systems

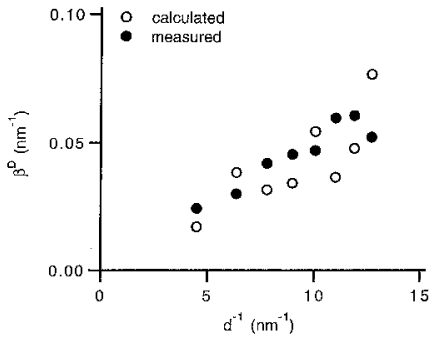

Figure 2. An example from practice: WH plots for Mo powders ball milled for 0.25, 1 and 4 hours. The diffraction lines show broadening and, as a consequence, their tails overlap. Instrumental broadening was removed from the measured breadths by simple subtraction (see Step i of table 4). Clearly broadening increases with diffraction angle and milling time - and overlap increases in the same way (see Steps iii and iv of table 4). The WH plots in figure $2 a$ show that the breadths depend on lattice spacing, i.e. on HKL. Outliers appear to be absent. Consideration of the three WH plots supports this. Clear, increasing trends are visible: (a) in the magnitude and "scatter" of the data and (b) in cut-off from the ordinate and in the slope of the WH plots.

It is well known that ball milling reduces (average) crystallite size and increases lattice deformation. Therefore it was tempting - and carried out - to fit the breadths with breadths calculated for various densities of dislocations with edge or line character. The best agreements are shown in figure $2 b$.

It is quite correct that WH plots should be used widely:

$\circ$ if one wants to inspect powder patterns, because they are very simple and very helpful.

- if one wants to observe trends in a single powder pattern, but they are not quantitative.

- if one wants to monitor trends in series of experiments, but they are not quantitative.

o they should therefore be implemented in commercial software for these purposes.

All use of these methods for other purposes is misuse - understandable, but objectionable. 
In today's practice (parts of) powder patterns are unravelled into individual line profiles by Profile Fitting with analytical functions, and the breadths found for these line profiles are used frequently as input for breadth methods, but usually they yield wrong (or at least inaccurate) results, because the convolutions of most mathematical functions can not be translated into sums of manageable functions of integral breadths, like equations $(2 a, b)$.

\section{Powder Pattern Calculation methods for parafocusing and parallel beam geometry}

Mainly during the last decade important physical-mathematical developments have occurred:

- Diffraction instruments can be described using their geometry and the physical dimensions of its components, e.g. the "Fundamental Parameters Approach" [5]

- A general description of diffraction by dislocations of all types has been found for all crystal systems, i.c. a general expression for the so-called Contrast Factor [6].

- A description of diffraction by planar defects for all crystal systems [7].

- Debye equation and Pair Distribution Function applied to diffraction for (a distribution of) finite crystallite sizes.

- First step to algorithms to verify the model used through calculation of its probability [8]. These developments enable microstructural analysis of polycrystalline specimens from $\theta / \theta$ and $\theta / 2 \theta$ scans in parafocusing or parallel beam geometry [5]. Such analyses interpret socalled "anisotropic broadening", i.e. the dependence of broadening on crystallographic direction $(=\mathrm{HKL})$ and - in solid objects - on the direction in the object, see table 5.

Table 5. Influences of types of defect on line profiles of the matrix of the material investigated.

\begin{tabular}{|l|l|c|}
\hline Defect & Significant influence on & HKL dependence \\
\hline Vacancies & lattice parameters, occasionally breadth & No \\
\hline Dislocations & breadth, shape & Always \\
\hline Stacking and Twin Faults & breadth, shape, shift & Always \\
\hline Crystallite Sizes \& Shapes & breadth, shape & Often \\
\hline Precipitates and Inclusions & breadth, shape, shift, lattice parameters & Always \\
\hline Anti-phase Boundaries & breadth, shape, extra HKL & Always \\
\hline
\end{tabular}

In addition: in solid objects the amounts, types, and dimensions of defects are related often to position and direction in the object.

It is a pity that these recent methods can not be applied like the Breadth methods, i.e. without knowledge of the material investigated, because input parameters are required that describe the crystal structure and the microstructure of that material. Another concern is that $\theta / 2 \theta$ and $\theta / \theta$ scans often contain insufficient information for a correct analysis of the microstructure.

\section{Developments required to create more robust methods for microstructure analysis}

Because $\theta / 2 \theta$ and $\theta / \theta$ scans often contain insufficient information for a correct analysis of the microstructure, measurements in more directions are required by applying (i) specimen tilting - $\omega$ tilt, i.e. around the $\theta$ axis and/or $\chi$ tilt, i.e. around the intersection of the specimen surface and the plane of diffraction - and specimen rotation around the surface normal $(\phi)$ and/or (ii) using area detectors.

Most line profiles thus recorded will differ from those of $\theta / 2 \theta$ and $\theta / \theta$ scans. Such scans may imply changes in the choice of radiation source, beam shape and diffraction geometry and therefore Whole Powder Pattern Calculation methods will have to develop into new methods 
Table 6. The four areas of complexity in LPA methods and their levels.

Optimum combinations are: $(A 1, B 1, C 1),(A 2, B 2, C 2),(A 3$ or $A 4, B 3, C 3)$ irrespective of $D$.

\begin{tabular}{|l|l|}
\hline \multicolumn{2}{|l|}{ Mathematical complexity } \\
\hline A1. & $\begin{array}{l}\text { line-profile characteristics from either direct calculation from recorded intensities or from } \\
\text { fitting with a analytical functions }\end{array}$ \\
\hline A2. & simple functions to model (contributions to) line profiles, e.g. Lorentzians, Gaussians \\
\hline A3. & complicated functions to model microstructural and physical contributions to line profiles \\
\hline A4. & detailed mathematical modeling in real space, e.g. Finite Element Methods) \\
\hline Modeling the instrument - mechanical and optical aspects \\
\hline B1. & by measurement that yields a non-continuous function of $2 \theta^{*}$ ) \\
\hline B2. & by assumption one can create a $2 \theta$ dependent analytical functions *) \\
\hline B3. & $\begin{array}{l}\text { by detailed calculation that contains the (i) diffraction geometry*), (ii) mechanical aspects } \\
\text { (e.g. axes and their misalignments), (iii) spectral distribution, (iv) transmission functions for } \\
\text { optical components, e.g. slits, mirrors, monochromators, (v) geometry and transparency of } \\
\text { the specimen, (vi) others. }\end{array}$ \\
\hline Modeling the microstructure - crystallite size, defects, et cetera \\
\hline C1. & by assumption of one and the same analytical function for all defects \\
\hline C2. & by assumption of different analytical functions for different defects \\
\hline C3. & $\begin{array}{l}\text { by convolution of contributions of (i) crystallite size and crystallite shape along with their } \\
\text { distributions, (ii) dislocations, (iii) planar defects, e.g. stacking and twin faults, (iv) anti-phase } \\
\text { boundaries, (v) others using either mathematical functions or physical models. **) }\end{array}$ \\
\hline Modeling materials and actual objects \\
\hline D1. & $\begin{array}{l}\text { a material is (i) an ideal single phase powder, or (ii) a single phase with various defects, (iii) a } \\
\text { mixture of phases and various defects, or (iv) as (iii) but related to e.g. texture and/or macro- } \\
\text { stress, or (v) as (iv) but structured, e.g. containing inclusions / precipitates }\end{array}$ \\
\hline D2. & $\begin{array}{l}\text { an object with or without internal structure, e.g. layered, which can be modeled } \\
\text { mathematically e.g. by Finite Element modeling. }\end{array}$ \\
\hline
\end{tabular}

*) including tilting according to $\omega$ and/or $\chi$, and rotation, $\phi(\omega, \chi$, and $\phi$ explained in section "Developments required ...")

**) appropriate physical models exist for C3 (i), (ii), (iii), and (iv), but how do we obtain the input parameters? At present this prevents implementation in commercial software.

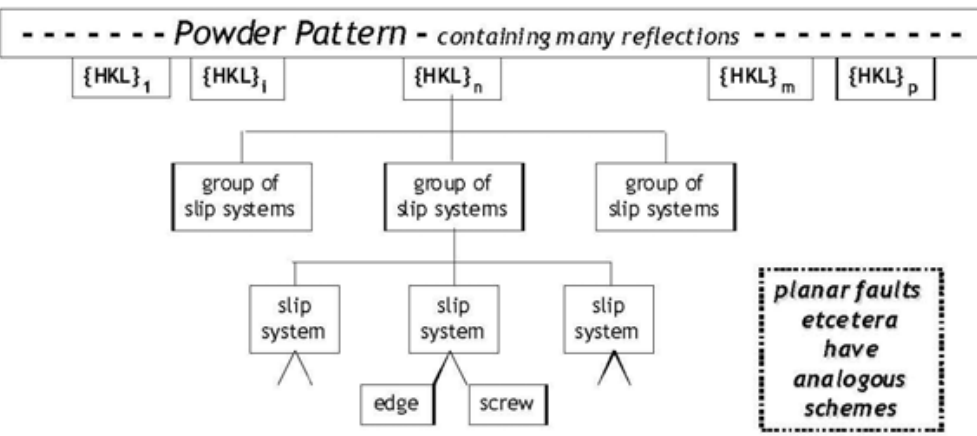

Figure 3. Scheme for combining (by convolution) the contributions to the broadening of the reflection $(H K L)_{n}$ by the various types and orientations of dislocations in a specimen. Other HKL's shall be treated analogously. For other types of defect analogous schemes hold. 
that we will denote here as Whole Reciprocal Space Calculation methods. These methods are of general use, and required for textured powders and solid objects. For their development synchrotron facilities will lead the way with the evaluation of their diffraction experiments.

\section{The structured complexity in Line Profile Analysis methods}

To understand and develop LPA methods (e.g. Whole Reciprocal Space Calculation methods see previous section) their complexity has to be considered. A good representation of the complexity of and the relations within and between Structure Determination and LPA methods are the figures 1 and 2 of [9]. Here we propose to go into more detail by introducing four "areas and their levels of complexity" in LPA methods: see table 6 .

All contributions from these areas interact (cf. figure 3) and must be convoluted successively to profiles that can be fitted to the recorded pattern to obtain their "weights"/amounts.

\section{Conclusions}

- At present

- WH plots shall only be used for data inspection and for monitoring trends

- For the determination of structural defects no sound quantitative method exists that requires no material specific information

- Powder Pattern Calculation methods are the best methods

- Microstructure analysis can be integrated further in crystal structure determination

- In the years to come it will be necessary to exploit / develop:

- Measurements in multiple directions by $\omega$ and/or $\chi$ tilting and/or area detectors. This requires significant software development.

- Simultaneous determination of microstructure, texture, macrostress for solid objects

- Methods and software for model verification, which requires as a routine the calculation of errors in data and results

\section{References}

1. Snyder, R.L., Fiala, J. \& Bunge, H.J., (Editors), 1999, Defects and Microstructure analysis by Diffraction (Oxford, UK: Oxford University Press).

2. Mittemeijer, E.J. \& Welzel, U., 2008, Z. Kristallogr., 223, 552.

3. Warren, B.E., 1969, X-ray Diffraction (Reading (Ma), USA: Addison-Wesley).

4. Snyder, R.L., 1993, in The Rietveld Method, edited by R.A. Young (Oxford, UK: Oxford University Press), pp. 111-131.

5. Mittemeijer, E.J. \& Scardi, P. (Editors), 2004, Diffraction Analysis of the Microstructure of Materials (Berlin: Springer Verlag).

6. Leoni, M., Martinez-Garcia, J. \& Scardi, P., 2007, J. Appl. Cryst., 40, 719.

7. Leoni, M., 2008, Z. Kristallogr., 223, 561.

8. Armstrong, N., Oral presentation at EPDIC-9, Prague.

9. Dinnebier, R.E. \& Billinge, S.J.L., 2008, Powder Diffraction: Theory and Practice (Cambridge, UK: Royal Soc. Chemistry), Preface, pp. vi, ix. 


\title{
Debye equation versus Whole Powder Pattern Modelling: Real versus reciprocal space modelling of nanomaterials
}

\author{
K. Beyerlein ${ }^{1,2,{ }^{*}}$, A. Cervellino ${ }^{3}$, M. Leoni ${ }^{1}$, \\ R. L. Snyder ${ }^{2}$, P. Scardi ${ }^{1}$
}

${ }^{1}$ University of Trento, Department of Materials Engineering and Industrial Technologies, via Mesiano, 77 - 38100 Trento - Italy

${ }^{2}$ Georgia Institute of Technology, Department of Materials Science and Engineering, 771 Ferst Dr. - Atlanta, GA 30332 - USA

${ }^{3}$ Swiss Light Source, Paul Scherrer Institute, Villigen PSI, 5232 - Switzerland * beyerle@ing.unitn.it

Keywords: Whole Powder Pattern Modelling, Debye Equation, nanoscale materials

\begin{abstract}
Real space methods like the Debye equation are increasingly being employed as an alternative to traditional Line Profile Analysis (LPA) techniques for the study of size and strain effects in nanomaterials. Until recently, the use of this technique in modelling was hindered by the time necessary to complete a calculation. This limitation encouraged development of the alternative, reciprocal-space Whole Powder Pattern Modelling, which on the other hand lacks physical validation when applied to the study of very small atomic clusters $(<5 \mathrm{~nm})$. A necessary proper comparison between these two approaches is proposed here to highlight the limits and potentials of each method.
\end{abstract}

\section{Introduction}

Line Profile Analysis (LPA) is traditionally based on a modelling of diffraction peak profiles, which analyzes how diffracted intensity is distributed about reciprocal space points. As a rule, crystalline domains are considered as continuous and ideal solids, a model that may not be entirely accurate at the nanoscale where the discrete nature of matter is more evident. As a consequence, the growing interest in nanomaterials has driven research towards a rediscovery of the Debye equation. This method obtains the diffraction pattern from systems of randomly oriented small crystals by considering the interference of waves scattered by atom pairs: distributions of crystals with a variety of shapes and novel morphologies, have been extensively discussed in the recent literature [1-3].

The Debye equation, although rather effective in describing diffraction from small crystals, has some disadvantages with respect to traditional LPA methods. Methods for dealing with 
statistically described crystal defects, small deformations, and ways to include an averaging of the lattice orientation with respect to the particle shape are all in their infancy. It follows that line broadening effects, and the simultaneous inclusion of different kinds of defects is not yet feasible using the Debye equation. The possibility of simultaneously modelling several broadening contributions is instead a prominent feature of advanced LPA methods. It is therefore important to explore differences between the Debye - real space - approach and the traditional - reciprocal space - approach to LPA. This is particularly interesting to assess the compatibility of the results obtained for nanocrystalline systems with domains in the order of 3 to $10 \mathrm{~nm}$, where both approaches are frequently used.

To this purpose, diffraction patterns simulated using a recently devised Debye algorithm have been here modelled using the WPPM (Whole Powder Pattern Modelling) approach $[4,5]$. Results show a good compatibility between the methods and point out the key role of noise (i.e., data quality) in limiting our possibility to unveil the finest details of the nanostructure.

\section{Simulations and modelling}

In this study, simulations are focussed on spherical gold crystals with a lattice parameter of $4.081 \AA$. Spheres were chosen because the shape function has an analytical expression that can be treated more strictly by WPPM. The Debye approach utilized four unique lognormal size distributions in simulating the patterns. These distributions were chosen to create sets of patterns defined by a common Scherrer size. This allowed for the study of any differences in the peak profiles calculated by the two approaches due to the effect of the distribution shape while preserving the integral peak breadth. Two sets of diffraction patterns were simulated to have Scherrer sizes $\left(\left\langle\mathrm{D}>_{\mathrm{V}}\right)\right.$ of $2.7 \mathrm{~nm}$ and $4.0 \mathrm{~nm}$, respectively; while the standard deviations of the natural logarithm $(\sigma)$ used were 0.15 and 0.40 , resulting in a narrow and wide distribution for each set. (See ref. 6 , and references therein, for a precise definition of $\angle \mathrm{D}\rangle_{V}$ and $\sigma$.) In order to perform the Debye function simulation [3,7], a series of increasingly large spherical Au clusters with fec structure were created. The diameter that related to less than .1 percent of the assumed volume weighted size distribution defined the maximum sphere size used in the simulations. Since in these constructions the diameter was not a continuous variable, two rules to govern the step size have been assumed: i) that the radius difference $\Delta r$ between consecutive clusters is constant, ii) that the volume increment between consecutive clusters is an integer multiple of the Wigner-Seitz unit cell volume. Using these two assumptions it is simple to see that $\Delta \mathrm{r}=a(3 / 2 \pi)^{1 / 3} / 2=0.39 a$. This discrete spacing is arbitrary but also sensible, as confirmed by our results. Clusters have been processed in order to obtain sets of interatomic pair distances and multiplicities. If we imagine that each unique distance is a Dirac delta weighted by its multiplicity, we can see that the pair distance density distribution is a Dirac comb. Immediately, though, the generated set of unique distances is fed into the sampling routine, which convolutes the distance Dirac comb with a suitable Gaussian profile [7], sampling the resulting continuous distance density. As the sampling step is inversely related to the maximum diffraction vector length Qmax, the sampling is performed simultaneously with different steps ranging from 0.03 to $0.96 \AA$, covering quite all possible experimental conditions. The sampled pseudodistance sets were then used to calculate diffraction patterns via a suitably adapted Debye formula, which is amenable to a fast trans- 
form. More details on the fast Debye simulation algorithms can be found elsewhere $[3,7]$.

Three levels of Poisson noise were then added to the simulated intensity to obtain patterns with signal-to-noise ratios ( $\mathrm{SNR}=\mathrm{VI}_{\max }$ ) of $316.2,100$, and 31.6 (max noise added). Then simulated patterns were modelled with the WPPM approach [4,5,8], refining the parameters of the $f c c$ lattice, size distribution, small angle scattering contribution and a Chebyshev polynomial background. A range of trial size distribution forms was assumed including: continuous and discrete lognormal distributions, and a continuous gamma distribution. The results of these analyses for each distribution are given in the following sections.

\section{Continuous lognormal distribution}

The patterns calculated by the Debye approach were first modelled assuming a continuous lognormal distribution of spheres in the WPPM framework. The obtained size distributions matched exactly the expected Debye distribution for all studied patterns. Even at the small particle size range of $1-10 \mathrm{~nm}$, the WPPM method was able to accurately distinguish the different lognormal parameters of two size distributions with the same Scherrer size (integral breadth). The exact match between the discrete distribution used in the Debye simulations and the continuous curve employed in WPPM was beyond expectations (cf. figure 2).

The lower residual and weighted sum of squares (wss $\left.\equiv \sum\left[\left(\mathrm{I}_{\text {Debye }}-\mathrm{I}_{\mathrm{WPPM}}\right) / \mathrm{I}_{\text {Debye }}\right]^{2}\right)$ for the distributions with a larger Scherrer size of $4.0 \mathrm{~nm}$ was an expected result (cf. figure 1). As the size increases the differences between the discrete Debye crystal, and the spatially averaged reciprocal space method, become less influential. Furthermore, at larger sizes the shape of the particle created in the Debye approach is increasingly well represented by a sphere.

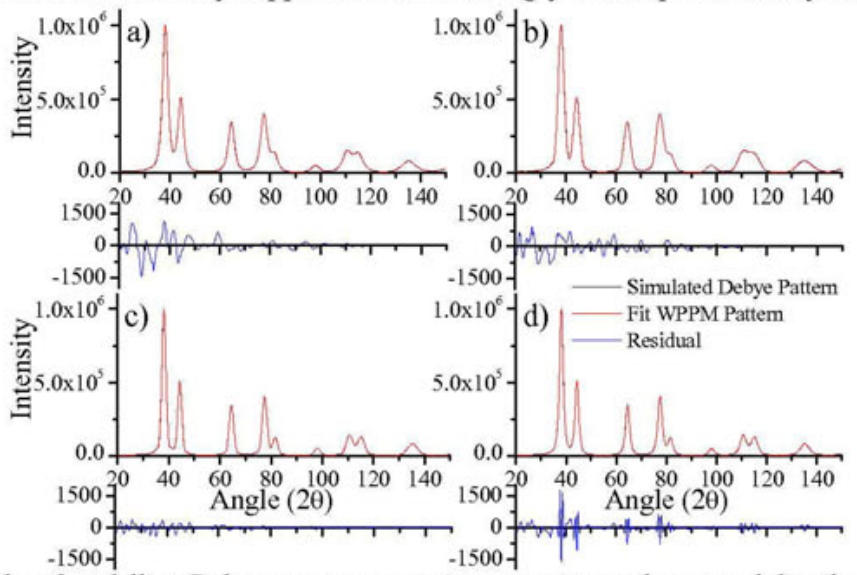

Figure 1. Results of modelling Debye patterns assuming a continuous lognormal distribution

a) $\angle D>v=2.7 \mathrm{~nm} \sigma=0.1491$ wss $=3072.61 ; b)<D>v=2.7 \mathrm{~nm} \sigma=0.3975$ wss $=4088.86$;

c) $\langle D>v=4.0 \mathrm{~nm} \sigma=0.1494$ wss $=766.35 ; d)<D>v=4.0 \mathrm{~nm} \sigma=0.3946$ wss $=990.89$;

The agreement of the obtained WPPM distribution with that used in the Debye simulations remained excellent as the SNR was decreased in the patterns. There was only a very slight deviation from the expected distribution at the highest investigated noise level: in particular the obtained distribution tended to broaden and shift to sizes smaller than expected. 


\section{Discrete lognormal distribution}

A discretely sampled lognormal size distribution was also employed in WPPM to model the simulated patterns (i.e. a set of weighted delta functions placed at regular steps $\Delta \mathrm{r}=$ $a(3 / 2 \pi)^{1 / 3} / 2$, where $a=$ lattice parameter and $\Delta r=$ spherical radius step, same as in the Debye simulation). This form of the distribution was chosen in order to make the WPPM hypotheses more consistent with the discreteness of the Debye approach, and give a reference point to the continuous distribution assumption. The weight of each delta function was governed by the value of a lognormal distribution, and the parameters of this governing function were then refined in the WPPM framework, keeping the number of free parameters the same as in the continuous case.

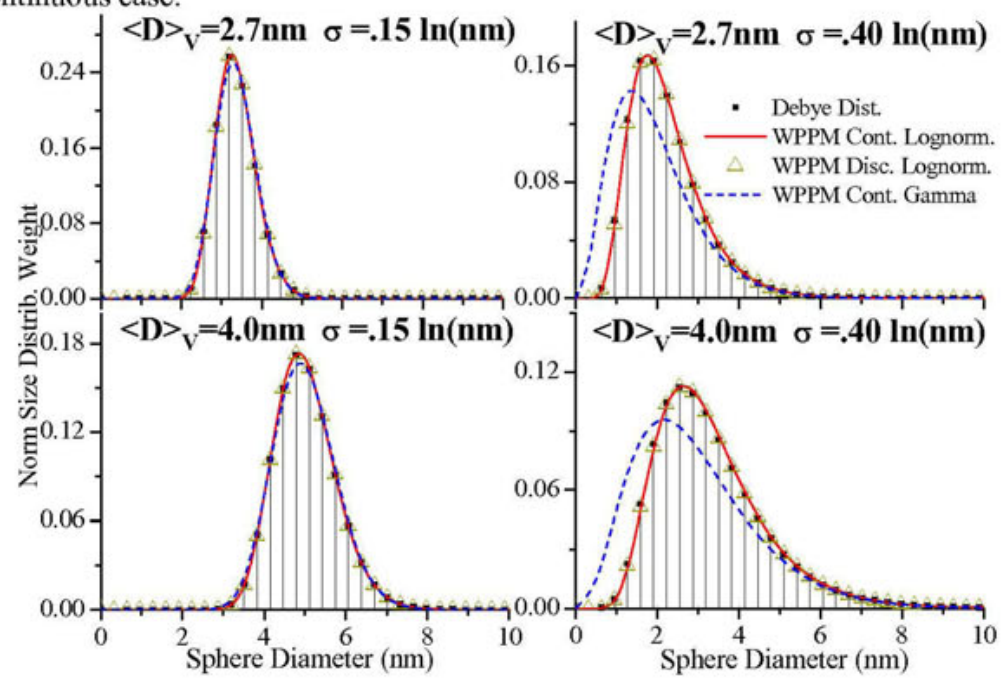

Figure 2. Comparison of distributions obtained from modelling of patterns before the addition of noise.

The discrete lognormal size distribution parameters found had the same agreement with the expected values as those found for the continuous case (see figure 2). The weighted sum of squares, and residual intensity, did not show any significant improvement from the continuous case. Furthermore, the background obtained when utilizing this distribution was found to be very similar to that obtained when assuming the continuous lognormal distribution. Therefore, it is not accounting for unseen effects on the pattern due to the discreteness of the size distribution. It is then our conclusion that the discrete size distribution utilized in the described Debye approach does not result in noticeable effects in the simulated pattern. As noise was added to the data the agreement of the discrete distribution followed the same general trend as the continuous lognormal distribution.

\section{Continuous gamma distribution}

To investigate the sensitivity of these methods to the functional form of the size distribution, the patterns were also modelled assuming a gamma distribution (a correct choice in the case of self-constrained polycrystal grain growth) [6]. The inaccurate assumption of a gamma distribution was only noticeable in the modelling of the patterns from the broader and 
skewed size distributions (cf. figure 2). In these cases the character of the distribution used in modelling becomes evident, and the gamma distribution was in better agreement at larger volumes due to the larger influence of this size range on the diffraction pattern.

The ability of WPPM to recover a good representation of the expected distribution in the small size range became worse as the SNR was decreased. However, modelling of the patterns with increased noise obtained Scherrer sizes that were still in good agreement with the expected values. Therefore, even when the assumed distribution is not representative of the actual particle sizes, such as in the case of the broader size distributions, the correct Scherrer size was preserved by WPPM.

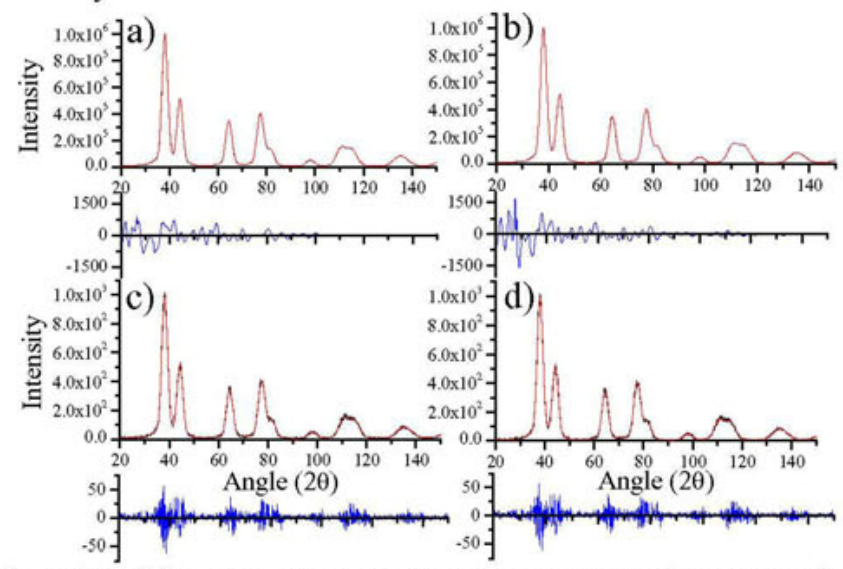

Figure 3. Best fit models of the $\langle D>v=2.7 \mathrm{~nm} \sigma=.15$ pattern assuming a) continuous lognormal dist. no noise, b) continuous gamma dist., no noise, c) continuous lognormal dist. with $S N R=31.6, b)$ contimuous gamma dist., $S N R=31.6$.

Large differences between the two distribution assumptions were observed only in the noisefree patterns, where the weighted sum of squares was 5-10 times lower when assuming a lognormal versus a gamma function. The addition of noise as the residual and weighted sum of squares became nearly equivalent masking the ability to discern which size distribution form was a better model (see figure 3). Therefore, while the mean (Scherrer) size was always matched, reliable details of the size distribution form could not be obtained from the noisy data. In this case it seems hopeless to distinguish the two distributions from diffraction alone; the growth conditions and other observations must be taken into consideration.

\section{Histogram distribution}

An alternative to assuming a size distribution shape is implementing a free histogram model, as proposed previously in the literature [9]. Histograms with a range of step sizes and initial starting conditions were studied: good agreement was found when the bin size was set to $\Delta r$ and when the expected distribution was narrow. Correlation between the weights of bins at small sizes and the background was especially observed as the bin size was decreased. As noise was added to the pattern, the obtained histogram, tended to overweight the small size bins, as the agreement with the size distribution diminished. These trends are in agreement with what was observed on experimentally measured ceria diffraction patterns in a previous publication [9]. 


\section{Concluding remarks}

The Whole Powder Pattern Modelling of the lognormal size distributions from the patterns simulated by the Debye equation has shown to be effective in reconstructing the model parameters even from noisy data. By WPPM, an accurate description of spherical crystallite size distributions in the $3-10 \mathrm{~nm}$ range has been obtained. This degree of agreement is not obvious considering the different assumptions of the two techniques. However, the discrete description of the size distribution in the Debye approach has been shown to be consistent with the continuous function used in reciprocal space methods.

An important conclusion of this study is that the correct description of the particle, or shape function, is increasingly significant as the size reduces. In reciprocal space methods the shape function describes a continuous body; while this attribute is strictly dependant on all assumptions made when constructing the particle in the Debye method. The higher residual intensities presented in figure 1 for the modelled patterns of smaller Scherrer sizes show that this difference in the techniques is more evident as the size of the crystal decreases. Thus, it is most important to decide if the underlying averaging of reciprocal space methods, or the specificity of the Debye method, is more appropriate before applying them to a nanocrystalline system.

In all cases the size distributions which best modelled the simulated patterns where in good agreement with the expected distribution in the larger size range, even in the modelling of the simulated patterns assuming a gamma distribution. The existence of noise in the diffraction pattern has been shown to accentuate this effect as the weight of the small sizes becomes increasingly overestimated with the decrease of the pattern quality (SNR). It has also been shown that the existence of noise in the pattern hides any measure of whether an assumed distribution function is appropriate. This difficulty illustrates a general limitation of diffraction that the fine details of a microstructure obtained by the line profile analysis of noisy powder diffraction data are not reliable.

\section{References}

1. Kumpf, C., Neder, R.B., Niederdraenk, F., Luczak, P., Stahl, A., Scheuerman, M., Joshi, M., Kulkami, S.K., Barglik-Chory, C., Heske, C., Umbach, E., 2005, J. Chem. Phys., 125, 224707.

2. Oddershede, J., Christiansen, T.L., Ståhl, K., 2008, J. Appl. Cryst., 41, 537.

3. Cervellino, A., Giannini, C., Guagliardi, A., Ladisa, M., 2005, Phys. Rev. B, 72, 035412.

4. Scardi, P. \& Leoni, M., 2002, Acta Cryst., A58, 190.

5. Scardi, P. \& Leoni, M., 2004, in Diffraction Analysis of the Microstructure of Materials, edited by E.J. Mittemeijer \& P. Scardi (Berlin: Springer), pp. 51-91.

6. Scardi, P. \& Leoni, M., 2001, Acta Cryst., A57, 604.

7. Cervellino, A., Giannini, C., Guagliardi, A., 2006, J. Comput. Chem., 27, 995.

8. Leoni, M., Confente, T. \& Scardi, P., 2006, Z. Kristallogr. Suppl., 23, 249.

9. Leoni, M. \& Scardi, P., 2004, J. Appl. Cryst., 37, 629. 


\section{Diffraction contrast factor of dislocations: The case of scheelite $\mathrm{CaWO}_{4}$}

\section{J. Martinez-Garcia ${ }^{1,2,}$, M. Leoni ${ }^{2}$, P. Scardi ${ }^{2}$}

${ }^{1}$ École Polytechnique Fédérale de Lausanne, Laboratoire de Cristallographie, BSP, CH-1015 Lausanne, Switzerland

${ }^{2}$ Department of Materials Engineering and Industrial Technologies, University of Trento, via Mesiano 77, Trento 38100, Italy

"jorge.martinezgarcia@epfl.ch

Keywords: dislocations, contrast factors, metric, line profile

Abstract. Dislocations are known for their peculiar effects on the diffraction profiles. Each given dislocation type, produces specific anisotropic line broadening basically determined by the so-called contrast factor (CF). Owing to the lack of a suitable mathematical frame to deal with dislocations in crystal of any symmetry, CFs have been so far determined for high symmetry materials. In this work we present the main points of a general approach for determining $\mathrm{CF}$, as well as a specific application to scheelite $\mathrm{CaWO}_{4}$.

\section{Introduction}

The long range strain field of dislocations is responsible for the local loss of translational symmetry in the atomic arrangement. This leads to peculiar effects in diffraction: peak profiles are broadened in such a way that the line widths and shapes depend on the anisotropic nature of both the dislocation strain field and the elastic medium [1-5].

Relations between profile parameters quantifying the dislocation-induced line broadening and microstructural parameters describing the features of the dislocation arrangement have been well established within the frame of the kinematical diffraction theory. Existing models interpret the dislocation broadening in terms of an average dislocation density ( $\rho$ Burgers vector modulus $\left(b_{v}\right)$, a correlation parameter $\left(R_{e}\right)$, and the dislocation contrast (or orientation) factor $\left(C_{h k l}\right)$ [1-4]. In the case of a "restrictedly random dislocation distribution" [3], for example, the integral breadth can be expressed as

$$
\beta_{h k l} \propto b_{v} d_{h k l}^{*} \sqrt{\rho C_{h k l}}
$$

where $d^{*}$ is the modulus of the diffraction vector $d^{*}$ in Bragg condition.

In contrast with $\rho, b_{\mathrm{v}}$ and $R_{e}$, which depend only on the nature and distribution of the dislocations ensemble, the contrast factor also depends on the orientation of the dislocations with 


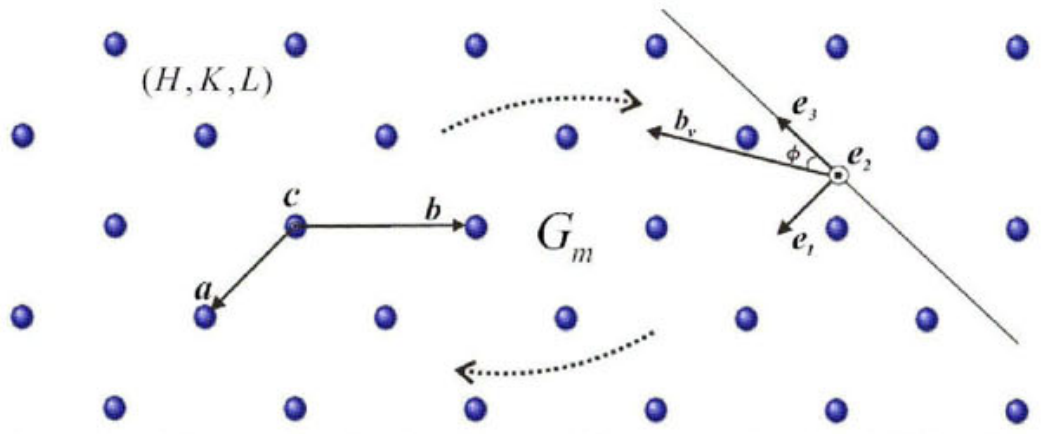

Figure 1. A dislocation line $\left(b_{v}, e_{3}\right)$ of character $\phi$ lying on its slip plane $(H K L)$ is shown in a general crystal system with lattice vectors $(a, b, c)$ and metric tensor $G_{m \cdot}\left\{e_{1}, e_{2}, e_{3}\right\}$ denote the basis of the dislocation slip-coordinate system.

respect to the diffraction vector and to the crystallographic axes, and on the elastic properties of the medium. As such, the contrast factor is the main responsible for the anisotropy of the line broadening (cf. equation (1)), and may allow for a clear separation between domain size and dislocation strain effects in diffraction.

In this work we summarize the main points of a general approach recently proposed for determining the dislocation contrast factor [6]. As a typical case of study, results are reported for scheelite $\mathrm{CaWO}_{4}$.

\section{Contrast factor calculation: A general approach}

According to [7], the contrast factor for a single slip system can be conveniently written as

$$
C_{h k l}=\sum_{i, m}^{3} \sum_{n, j}^{2} E_{i j m n} G_{i j m n}
$$

where $E_{i j m n}$ and $G_{i j m n}$ are 4-rank symmetrical tensors, respectively known as elastic and geometrical parts of the contrast factor. The elastic part takes into account the crystal elastic properties and the displacement field $\boldsymbol{u}$ of a dislocation with character $\phi$ the geometrical part, instead, accounts for the orientation of the diffraction vector with respect to the slip- coordinate system $\left\{\boldsymbol{e}_{i}, i=1,2,3\right\}$, placed on the dislocation line. Tensor elements can be calculated as

$$
\begin{array}{ccc}
G_{i j m n}=\tau_{i} \tau_{j} \tau_{m} \tau_{n}, & \tau_{\mathrm{i}}=\left(\boldsymbol{d}^{*} / d^{*}\right) \cdot \boldsymbol{e}_{i}, & (i, m)=1,2,3 \\
E_{i j m n}=(1 / \pi) \int_{0}^{\pi} \beta_{i j}(\varphi) \beta_{m n}(\varphi) d \varphi, & \beta_{i j}(\varphi)=\left(2 \pi r / b_{v}\right)\left(\partial u_{i} / \partial x_{j}\right),(j, n)=1,2
\end{array}
$$

where $\tau_{i}$ are direction cosines between the $i$-axis of the slip coordinate system and the diffraction vector, $u_{i}$ is the component of $\boldsymbol{u}$ along $\boldsymbol{e}_{i}$, and $\varphi$ is the polar angle of a position vector $\boldsymbol{r}$ in the $\left\{\boldsymbol{e}_{1}, \boldsymbol{e}_{2}\right\}$ plane: $\boldsymbol{r}=\cos (\varphi) \boldsymbol{e}_{1}+\sin (\varphi) \boldsymbol{e}_{2}$. In the case of untextured polycrystals, equation (1) has to be averaged over all crystallographically equivalent slip-coordinate systems. As can be seen from the above equations, quantitative calculations of $C_{h k l}$ require, as first step, the definition of the slip-system frame $\left\{\boldsymbol{e}_{i}, i=1,2,3\right\}$, describing the geometrical features of the dislocation (i.e. Burgers vector, slip plane, dislocation character, etc). At the same time 
the unit vectors $e_{i}$ are embedded in a given crystal coordinate system (figure (1)), so that any transformation of the elastic tensor or of the diffraction vector components on $\left\{\boldsymbol{e}_{i}, i=1,2,3\right\}$, involves the metric properties of the corresponding crystal system. In this way the influence of the lattice geometry and elastic properties on $C_{k k l}$, and therefore on the diffraction profiles, can be found.

General expressions valid for any crystal system have recently been reported for the direction cosines $\tau_{i}$, which fully determine the geometrical tensor $G_{i j m n}[6]$ :

$$
\begin{aligned}
& \tau_{2}=\frac{[h, k, l] \cdot G_{m}^{*} \cdot[H, K, L]^{T}}{\sqrt{[h, k, l] \cdot G_{m}^{*} \cdot[h, k, l]^{T}} \sqrt{[H, K, L] \cdot G_{m}^{*} \cdot[H, K, L]^{T}}} \\
& \tau_{3}=\frac{[h, k, l] \cdot M^{T} \cdot R\left(\phi, \boldsymbol{e}_{2}\right) \cdot\left[M^{t}\right]^{-1} \cdot[U, V, W]^{T}}{\sqrt{[U, V, W] \cdot G_{m} \cdot[U, V, W]^{T}} \sqrt{[h, k, l] \cdot G_{m}^{*} \cdot[h, k, l]^{T}}} \\
& \tau_{1}=\sqrt{1-\tau_{2}^{2}-\tau_{3}^{2}} \cdot
\end{aligned}
$$

In the above equations $T$ stands for the transpose operator, $\phi$ is the character of the dislocation, $[U, V, W]$ denotes the coordinates of the Burgers vector in the crystal basis, $H K L$ the Miller indices of the slip plane, and $h k l$ are general Miller indices. The metric tensor $G_{m}$ (from which its reciprocal $G^{*}$ ), and the matrices $M$ and $R$ can be determined as

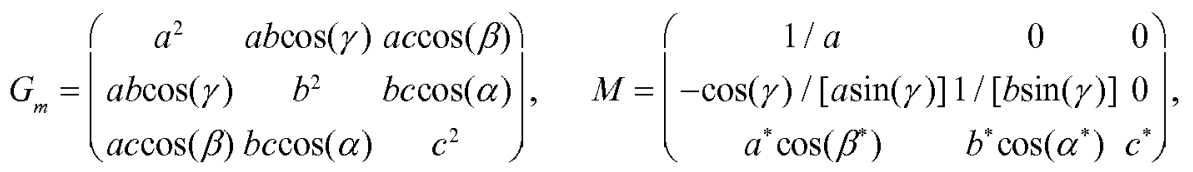

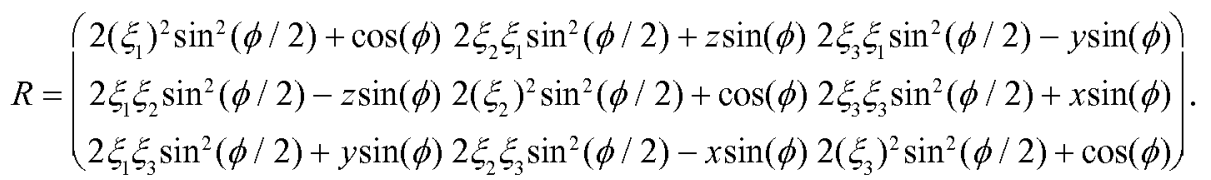

where $\xi_{1}, \xi_{2}, \xi_{3}$ in $R$ are the orthogonal coordinates of the diffraction vector given by

$$
\left[\xi_{1}, \xi_{2}, \xi_{3}\right]^{T}=\left(1 / d_{h k l}\right) M \cdot[h, k, l]^{T}, \quad d_{h k l}=\sqrt{[h, k, l] \cdot G_{m}^{*} \cdot[h, k, l]^{T}},
$$

$\{a, b, c, \alpha, \beta, \gamma\}$ are the unit-cell parameters.

For the elastic part, $E_{i j m n}$, it has been shown that $\beta_{i j} \varphi$ in equation (2) can be written as [6]:

$$
\beta_{i j}(\varphi)=\operatorname{Im}\left[\sum_{q}^{3} \frac{A_{i j} D_{m} p_{q}^{(n-1)}}{\operatorname{Cos}(\varphi)+p_{q} \operatorname{Sin}(\varphi)}\right], \quad D_{q}=\frac{\boldsymbol{L}_{q} \cdot \boldsymbol{b}_{v}}{\boldsymbol{A}_{q} \cdot \boldsymbol{L}_{q}}
$$


where $p_{q}$ and $\left(\boldsymbol{A}_{q} \boldsymbol{L}_{q}\right)$ are the eigensolutions of the fundamental elasticity matrix $N$ [8], obeying the eigenrelation

$$
N\left(\begin{array}{l}
\boldsymbol{A}_{q} \\
\boldsymbol{L}_{q}
\end{array}\right)=p_{q}\left(\begin{array}{l}
\boldsymbol{A}_{q} \\
\boldsymbol{L}_{q}
\end{array}\right), \quad q=(1,2,3)
$$

Equations (4) and (6) contain all (metric and elastic) information for a straight dislocation in a general crystal system; when inserted into equation (2), they render equation (1) readily usable for any crystal symmetry and elastic properties.

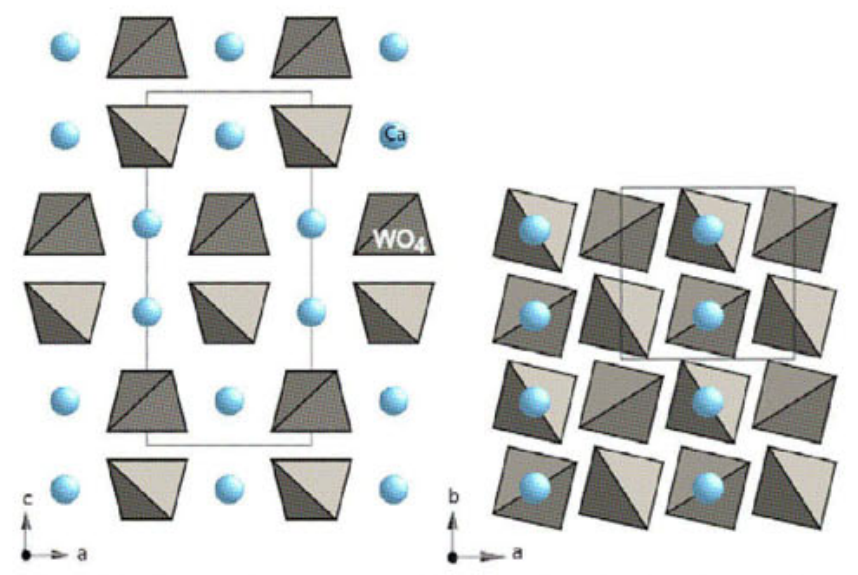

Figure 2. The crystal structure of $\mathrm{CaWO}_{4}$ projected along the $\boldsymbol{b}$ and $c$ axes.

\section{The case of polycrystalline scheelite $\mathrm{CaWO}_{4}$}

Scheelite $\mathrm{CaWO}_{4}$ is a member of the family of compounds with general chemical formula $\mathrm{A}\left[\mathrm{XO}_{4}\right]$. This family of structures has been widely investigated owing to their optical and elastoplastic properties, leading to interesting applications. In particular, $\mathrm{CaWO}_{4}$ is of special interest for use as fiber-matrix intelayers in ceramic composites [9].

$\mathrm{CaWO}_{4}$ has a tetragonal body centred lattice with space group $\mathrm{I}_{1} / a$ and unit cell parameters $a=0.5243$ and $c=11.376 \mathrm{~nm}$ (figure (2)) [10]. Dislocations have been observed lying on $\langle 110\rangle\{001\}$ and $1 / 2\langle 111\rangle\{11 \overline{2}\}$ slip systems [9], whereas single crystal elastic constants are: $C_{11}=141, C_{33}=125, C_{44}=33.7, C_{66}=40.7, C_{12}=61, C_{13}=41$ and $C_{16}=-17 \mathrm{Gpa}$ [11]. Taking into account the above information, average contrast factors $\left(\bar{C}_{h k l}\right)$ for $\mathrm{CaWO}_{4}$ were calculated by using equations (1-7) for screw and edge orientations. Final results are shown 
a)

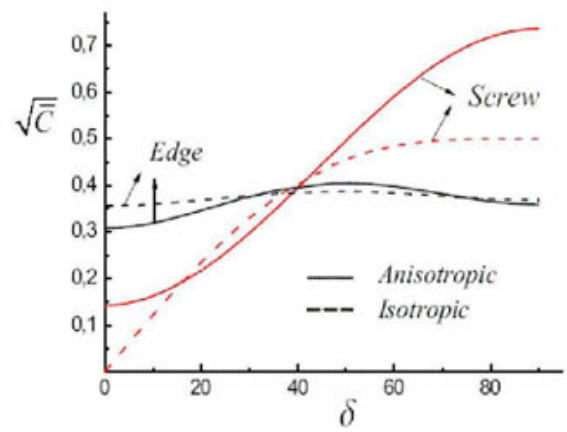

b)

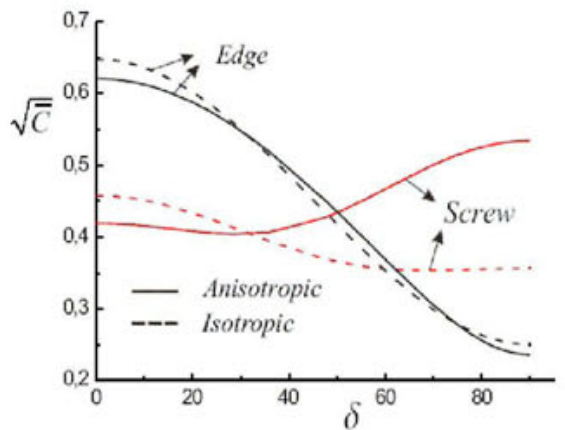

Figure 3. Dependence of the square root of $C_{h k l}$ on the angle $\delta$ between $d^{*}$ and the $c$ axis of the tetragonal unit cell of $\mathrm{CaWO}_{4}:(a)<110>\{001\}$ slip system, (b) $<111>\{11-2\}$ slip system. Dashed lines correspond to calculations using the elastic isotropy approximation.

in parametric form in equation (8), expressing $\bar{C}_{h k l}$ as a fourth order crystallographic invariant of the Miller indices, and in graphic form in figure (3). Values of the coefficients $E_{\mathrm{i}}$ are reported in table (1) for each slip system and dislocation orientation. As expected, equation (8) is proportional to the mean square strain invariant for the Laue class $4 / \mathrm{m}[12,13]$.

$$
\bar{C}_{h k l}=\frac{E_{1}\left(h^{4}+k^{4}\right)+E_{2} l^{4}+2 E_{3} h^{2} k^{2}+2 E_{4} l^{2}\left(h^{2}+k^{2}\right)+4 E_{5} h k\left(h^{2}-k^{2}\right)}{\left[h^{2}+k^{2}+\left(\frac{a}{c}\right)^{2} l^{2}\right]^{2}}
$$

Table 1. Calculated coefficients $E_{i}$ of equation (8) for each slip system and dislocation orientation.

\begin{tabular}{|c|c|c|c|c|c|c|}
\hline Slip-System & Character & $E_{1}$ & $E_{2}$ & $E_{3}$ & $E_{4}$ & $E_{5}$ \\
\hline$\langle 110\rangle\{001\}$ & Screw & 0.5415 & 0.0009 & -0.4009 & 0.0244 & 0.1190 \\
\hline$\langle 110\rangle\{001\}$ & Edge & 0.1289 & 0.0043 & 0.3867 & 0.0452 & -0.0012 \\
\hline $1 / 2\langle 111\rangle\{11 \overline{2}\}$ & Screw & 0.2853 & 0.0079 & -0.0813 & 0.0263 & 0.0649 \\
\hline $1 / 2\langle 111\rangle\{11 \overline{2}\}$ & Edge & 0.0558 & 0.0174 & 0.1675 & 0.0456 & -0.0016 \\
\hline
\end{tabular}

Figure (3) shows the dependence of $\bar{C}_{h k l}^{1 / 2}$, which is proportional to the integral breath of the diffraction profiles (see equation (1)) on the angle $\delta$ between the diffraction vector $\boldsymbol{d}^{*}$ and the $c$ axis of the tetragonal lattice. As expected, there are large differences in the shapes of the functions associated with different slip systems and/or dislocation characters (i.e. edge and 
screw orientation). Moreover, elastic anisotropy is quite strong and the effect, especially for screw dislocation, cannot in general be neglected.

\section{Concluding remarks}

The evaluation of the dislocation CFs have been so far only possible for materials showing cubic and hexagonal symmetry $[14,15]$. In this work the main points of a general approach recently proposed for determining $C_{h k l}$ have been presented and used for determining the average contrast factors in scheelite $\mathrm{CaWO}_{4}$. The new approach extends the applicability of the Klimanek-Kuzel procedure [7] to any material, independently of its symmetry and elastic behaviour. The new results presented in this work for $\mathrm{CaWO}_{4}$ are just an example of a general procedure, that can be used for any materials to extract microstructural information from $\mathrm{X}$-ray powder diffractions experiments. The validity of the approach has already been tested experimentally in $\mathrm{Cu}_{2} \mathrm{O}$ and $\mathrm{SnO}_{2}$ powders $[13,15]$.

\section{References}

1. Krivoglaz, M.A. \& Ryaboshapka, K.P., 1963, Fiz. Metal. Metalloved, 15, 18.

2. Krivoglaz, M.A.,1996, X-ray and Neutron diffraction in Nonideal Crystals (Berlin: Springer-Verlag).

3. Wilkens, M., 1970, in Fundamental Aspects of Dislocation Theory, edited by J.A. Simmons, R. de Witt \& R. Bullough (Washington DC: NBS Spec. Publ. 317), pp.1195-1221.

4. Groma, I., Ungàr, T. \& Wilkens, M., 1988, J. Appl. Cryst., 21, 47.

5. Martinez-Garcia, J., Leoni, M. \& Scardi, P., 2008, Phil. Mag. Lett., 88, (6), 443.

6. Martinez-Garcia, J., Leoni, M. \& Scardi, P., 2009, Acta. Cryst. A, 65.

7. Klimanek, P. \& Kužel, R., 1988, J. Appl. Cryst., 21, 59.

8. Ting, T.C.T., 1996, Anisotropic Elasticity: Theory and Applications (NY: Oxford University Press).

9. Mogilevsky, P., 2005, Phil. Mag., 85, (30), 3511.

10. Kay, M.I., Frazer, B.C. \& Almodovar, I., 1964, J. Chem. Phys., 40, 504.

11. Bass, J.D., in Mineral Physics and Crystallography: A Handbook of Physical Constants, edited by T.J. Ahrens (Washington, DC: A.G.U). pp. 45-63.

12. Popa, N., 1998, J. Appl. Cryst., 31, 176.

13. Leoni, M., Martinez-Garcia, J. \& Scardi, P., 2007, J. Appl. Cryst., 40, 719.

14. Ungàr, T. \& Tichy, G., 1999, Phys. Stat. Sol. (a), 171, 425.

15. Martinez-Garcia, J., Leoni, M. \& Scardi. P., 2007, Phys. Rev. B., 76, 174117.

Acknowledgements. One of authors (JMG) would like to acknowledge the support from the EPDIC-11 organizing committee and the École Polytechnique Fédérale de Lausanne (EPFL). 


\title{
Thermally induced microstrain broadening in polycrystalline hexagonal zinc
}

\author{
A. Leineweber ${ }^{1, *}$, E. J. Mittemeijer ${ }^{1}$, A. C. Lawson ${ }^{2}$, \\ J. A. Roberts ${ }^{2}$, J. A. Valdez ${ }^{2}$, W. S. Kreher ${ }^{3}$ \\ ${ }^{1}$ Max Planck Institute for Metals Research, Stuttgart, Germany \\ ${ }^{2}$ Los Alamos National Laboratory, Los Alamos, New Mexico, USA \\ ${ }^{3}$ Institute for Materials Science, Dresden University of Technology, Dresden, Germany \\ *a.leineweber@mf.mpg.de
}

Keywords: thermal microstresses, line-broadening analysis, zinc, plastic deformation

\begin{abstract}
Neutron powder-diffraction experiments on polycrystalline hexagonal zinc (powder specimen with internally polycrystalline powder particles) show considerable temperature-dependent line broadening. Whereas as-received zinc exhibits narrow reflections at 300 $\mathrm{K}$, during cooling to a minimum temperature of $10 \mathrm{~K}$ considerable line-broadening appears, which largely disappears during reheating. The line broadening can be ascribed to microstrains induced by thermal microstresses due to the intrinsic anisotropy of the thermal expansion (shrinkage) of hexagonal zinc. Differences between the observed microstrains and theoretical predictions considering elastic deformation of the thermally strained grains can be explained by additional effects as plastic deformation and surface relaxation.
\end{abstract}

\section{Introduction}

In non-cubic, single-phase polycrystalline materials, dependence of the thermal-expansion coefficient on orientation in the crystal frame of reference induces, upon temperature change, locally varying microstresses. Thus even local plastic deformation or grain-boundary cracking can occur. Understanding and experimental analysis of such thermally-induced microstresses, during production and during service, can be decisive for accurately estimating the endurance of materials.

The thermal stresses and strains as well as their distributions occurring in such polycrystals can be estimated by two types of methods. The first approach is based on finite-element calculations evaluating the stress-strain state in model polycrystals (e.g. [1]). However, the simulation of three-dimensional polycrystals of sufficient size is numerically very laborious and is also strongly hindered by the usually incomplete microstructual information available. The second approach is based on statistical and internal-energy considerations [2, 3], combined with the assumption of "maximum-entropy" (information theory) for the resulting microstress-microstrain distributions. Besides these approaches also further works exist deal- 
ing with estimation of thermal microstresses using the Eshelby-Kröner approach (e.g. [4]). However, in these works no systematic efforts were made to assess the stress-strain distributions.

In the present contribution polycrystalline hexagonal zinc, of intrinsically anisotropic thermal expansion, is considered. It is shown how the thermally induced microstrains occurring upon cooling strongly influence the broadening observed in the powder-diffraction patterns. The experimental results are compared with theoretical estimations based on information theory.

\section{Estimation of microstrain distribution parameters from materials constants $[2,3]$}

In this section the results of an information-theory based approach are presented for estimation of the first and second moments of the thermal stresses and strains occurring in an infinte random massive polycrystal of a hexagonal material. Input informations are only the thermoelastic constants, i.e. the anisotropic thermal expansion coefficients and the singlecrystal elastic constants, where all tensorial quantities are defined in the (Cartesian) crystal frame of reference. The approach assumes only elastic deformation according to Hooke's law.

The stresses and strains build up if the polycrystal experiences a temperature change (usually drop) from a stress-free state at a temperature $T_{0}$ (e.g. the manufacturing temperature) to a temperature $T_{1}$. During this temperature change a free-standing single crystal would experience a strain $\varepsilon_{i j}^{T}=\int_{T_{0}}^{T_{1}} \alpha_{i j} d T$ where $\alpha_{i j}$ are the components of the temperature-dependent thermal expansion tensor, for which $\alpha_{11}=\alpha_{22}$ and $\alpha_{i j}=0$ for $i \neq j$ hold, as imposed by the hexagonal symmetry. Information theory $[2,3]$ estimates for the average stress $\langle\sigma\rangle$ (again symmetry invariant-second-rank tensor in the above-mentioned Cartesian crystal frame of reference) are:

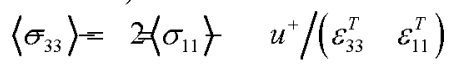

with $u^{+}$being an upper bound for the stored strain energy in the polycrystal:

$u^{+}=\frac{\left(C_{1111}+C_{1122}\right) C_{3333}-2 C_{1133}^{2}}{2 C_{1111}+C_{3333}+2 C_{1122}+4 C_{1133}}\left(\varepsilon_{33}^{T}-\varepsilon_{11}^{T}\right)^{2}$.

Note that eq. (1) implies that the stored strain energy $u^{*}$ occurring in a more general formulation $[2,3]$ amounts here $u^{*}=u^{+} / 2$, corresponding to the state of maximum entropy. Using Hooke's law, from eq. (1) also the first moments of the microstrain distribution (symmetry invariant $\langle\varepsilon\rangle$ ) can be calculated:

$$
\begin{aligned}
& \left\langle\varepsilon_{11}\right\rangle=\left\langle\varepsilon_{22}\right\rangle \quad \varepsilon_{1 \bar{\Gamma}}^{T} \frac{u^{+}}{\left(\varepsilon_{33}^{T}-\varepsilon_{11}^{T}\right)} \frac{\left(S_{1111}+S_{1122}-2 S_{1133}\right)}{2} \\
& \left\langle\varepsilon_{33}\right\rangle=\varepsilon_{33}^{T} \frac{u^{+}}{\left(\varepsilon_{33}^{T}-\varepsilon_{11}^{T}\right)}\left(S_{3333}-S_{1133}\right),
\end{aligned}
$$

where $S_{i j p q}$ are the components of the compliance tensor. 
The second moments of the Gaussian microstress-microstrain distributions can be formulated as fourth-rank tensors with the components $\left\langle\left(\varepsilon_{i j}-\left\langle\varepsilon_{i j}\right\rangle\right)\left(\varepsilon_{p q}-\left\langle\varepsilon_{p q}\right\rangle\right)\right\rangle=\left\langle\Delta \varepsilon_{i j} \Delta \varepsilon_{p q}\right\rangle$ and $\left\langle\left(\sigma_{i j}-\left\langle\sigma_{i j}\right\rangle\right)\left(\sigma_{p q}-\left\langle\sigma_{p q}\right\rangle\right)\right\rangle=\left\langle\Delta \sigma_{i j} \Delta \sigma_{p q}\right\rangle$ with

$\left\langle\Delta X_{i j} \Delta X_{p q}\right\rangle=\frac{1}{12} u^{+} Y_{i j p q}$,

where for $X_{i j}=\varepsilon_{i j}$ one obtains $Y_{i j p q}=S_{i j p q}$ and for $X_{i j}=\sigma_{i j}$ one obtains $Y_{i j p q}=C_{i j p q}$. Note that thermal microstresses are absent, if the thermal expansion/shrinkage of the hexagonal material under consideration would accidentally be isotropic, i.e. $\varepsilon_{33}^{T}=\varepsilon_{11}^{T}$.

\section{Microstrain in powder-diffraction patterns [5]}

Within the Stokes-Wilson approximation [6] (strain constant in each coherently diffracting grain)_the microstrain distribution shows up in powder-diffraction patterns as the projection of the microstrain distribution along the direction diffraction vector. If the microstrain along the diffraction vector $\mathbf{g}_{h k l}\left(h k l\right.$ : Miller indices) is $\varepsilon_{h k l}$ for a certain crystallite, the microstrain distribution along the diffraction vector can be quantified in terms of the moments of $\varepsilon_{h k l}$, which can be related with the moments of the microstrain distribution. The first moment is

$\left\langle\varepsilon_{h k l}\right\rangle=\left\langle\varepsilon_{i j}\right\rangle x_{i} x_{j}$

and the second central moment is

$$
\left.\left\langle\left(\Delta \varepsilon_{h k i}\right)^{2}\right\rangle=\left\langle\left(\varepsilon_{h k i}-\left\langle\varepsilon_{h k i}\right\rangle\right)^{2}\right\rangle=\phi \text { क } \varepsilon_{p q}\right\rangle x_{i} x_{j} x_{p} x_{q},
$$

which is given here in terms of the unit vector $\mathbf{x}$. Moreover, higher moments can be considered, on the basis of which the line broadening contribution due to microstrain can be described by an Edgeworth series for each reflection [7]. On an experimental diffraction scale (diffraction angle, or $d$-spacing for a time-of-flight neutron diffraction experiment) the first moments exhibit as peak shifts

$\left\langle\Delta 2 \theta_{\overline{h k t}}\right\rangle=2 \tan \left\langle\theta_{h k l}\right\rangle\left\langle\varepsilon_{h k i}\right\rangle$ or $\left\langle\Delta d_{h k i}\right\rangle=\left\langle d_{h k t}\right\rangle\left\langle\varepsilon_{h k i}\right\rangle$

whereas the second (and the higher moments) show up as line broadening, e.g.

$\left\langle\left(\Delta 2 \theta_{h k l}\right)^{2}\right\rangle=4 \tan ^{2}\left\langle\theta_{h k l}\right\rangle\left\langle\varepsilon_{h k l}^{2}\right\rangle$ or $\left\langle\Delta d_{h k l}^{2}\right\rangle=\left\langle d_{h k l}\right\rangle^{2}\left\langle\left(\varepsilon_{h k l}\right\rangle^{2}\right\rangle$.

On the basis of line-broadening data and eq. (9) not all components of $\left\langle\Delta \varepsilon_{i j} \Delta \varepsilon_{p q}\right\rangle$ can be determined independently [5]. This leads to the introduction of fitting parameters $Z_{i j p q}$ which are equal for an arbitrary permutation of the indices:

$\left\langle\left(\Delta \varepsilon_{h k l}\right)^{2}\right\rangle=Z_{i j p q} x_{i} x_{j} x_{p} x_{q}$.

For the hexagonal case of interest only $Z_{1111}=\left\langle\left(\Delta \varepsilon_{11}\right)^{2}\right\rangle, Z_{3333}=\left\langle\left(\Delta \varepsilon_{33}\right)^{2}\right\rangle$ and $Z_{1133}=$ $\frac{1}{3}\left[2\left\langle\left(\Delta \varepsilon_{13}\right)^{2}\right\rangle+\left\langle\Delta \varepsilon_{11}\right\rangle\left\langle\Delta \varepsilon_{33}\right\rangle\right]$ are obtained as independent parameters, where $\left\langle\left(\Delta \varepsilon_{13}\right)^{2}\right\rangle$ and $\left\langle\Delta \varepsilon_{11}\right\rangle\left\langle\Delta \varepsilon_{33}\right\rangle$ cannot be determined independently.

The first and second moments of the microstrain distribution derived in section "Estimation of microstrain..." can thus be used as physical basis for the phenomenological description for the effect of microstrain on a powder-diffraction pattern. 


\section{Thermal microstresses in zinc below room temperature}

Zinc is known to exhibit a strong intrinsically anisotropic thermal expansion [8], where below room temperature the relative change of the lattice parameter $c$ is about 10 times larger than that of the lattice parameter $a$. Further, the single-crystal elastic constants indicate that zinc is softest along the $c$ direction $\left(C_{1111}=164 \mathrm{GPa}\right.$ vs. $C_{3333}=63 \mathrm{GPa}$ [9] at ambient temperature). The non-zero $C_{i j p q}$ 's increase by $3-9 \%$ upon cooling from $300 \mathrm{~K}$ to $10 \mathrm{~K}$; these effects are neglected in the following.

Neutron powder-diffraction experiments were performed on the time-of-flight diffractometer NPD at Los Alamos National Laboratory (USA). The sample was zinc powder (figure 1), in which the powder particles consist of many differently oriented crystallites. Neutron powderdiffraction data were first collected at $300 \mathrm{~K}$, then at different temperatures during cooling down to a minimum temperature of $10 \mathrm{~K}$ and during subsequent reheating to a final temperature of $225 \mathrm{~K}$. Whereas at the starting temperature of $300 \mathrm{~K}$ the reflections are quite narrow, strong broadening occurs at $10 \mathrm{~K}$ (figure 2), which reduces during reheating, i.e. the linebroadening effects are largely reversible (but not completely; see what follows).

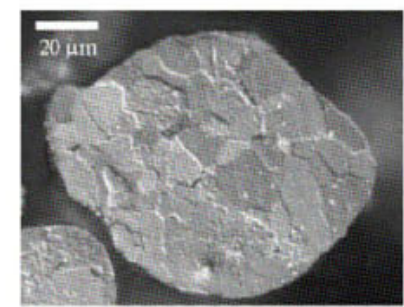

Figure 1. (top). Metallographic cross-section of the employed zinc powder after etching.

Figure 2. (right). Comparsion of a part of the neutron powder-diffraction patterns of zinc powder at $300 \mathrm{~K}$ and $10 \mathrm{~K}$. Note the hkl (indicated) dependence of the peak shifts and of the line broadenings.

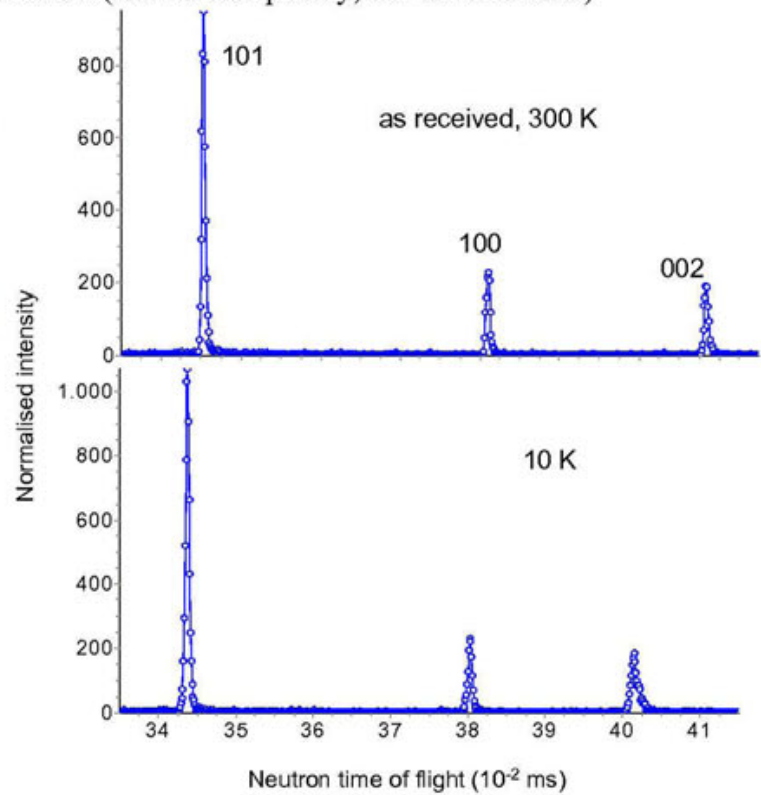

The data were quantitatively evaluated using Pawley fits (Rietveld-like full-pattern fits without structure model; the integrated intensity were refined as parameters) using the TOPAS software [10]. The narrow line-profiles of the $300 \mathrm{~K}$ data were adopted as describing the instrumental resolution, and the additional line-broadening occurring in the following measurements was considered as thermally induced microstrain broadening, which was described by an anisotropic Edgeworth-series approach (modified Gaussian peak-shape; to be described in detail elsewhere [7]) which allowed extraction of information on the $2^{\text {nd }}\left(Z_{1111}\right.$, $Z_{3333}$, and $Z_{1133}$ ) and of higher moments of the microstrain distribution. The first moments of 
the microstrain distribution were obtained from the refined lattice parameters, i.e. $\left\langle\varepsilon_{11}\right\rangle=$ $\left(a-a_{0}\right) / a_{0}$ and $\left\langle\varepsilon_{33}\right\rangle=\left(c-c_{0}\right) / c_{0}$, where $a_{0}, c_{0}$ are the lattice parameters pertaining to the initial $300 \mathrm{~K}$ measurement. These experimental results can be compared with the predictions from information theory (see section "Estimation of microstrain..." eq. (3-5)).

For the first moments theory predicts that the thermal microstresses in a polycrystal lead due to eqs. (3-4) to an of course less anisotropic average thermal shrinkage than for a single crystal (given by $\varepsilon_{11}^{T}$ and $\varepsilon_{33}^{T}$ ) as shown in figure 3a. However, the experimentally determined values for the first strain moments for the polycrystal, $\left\langle\varepsilon_{11}\right\rangle,\left\langle\varepsilon_{33}\right\rangle$ (calculated from the lattice parameters, see above) largely correspond to $\varepsilon_{11}^{T}$ and $\varepsilon_{33}^{T}$, which is surprising.

Theory further predicts that the tensor components $\left\langle\Delta \varepsilon_{i j} \Delta \varepsilon_{p q}\right\rangle$ are proportional to the compliance tensor components $S_{i j p q}$. In fact, $Z_{3333}=\left\langle\left(\Delta \varepsilon_{33}\right)^{2}\right\rangle$ is found as largest $Z_{i j p q}$ parameter, which is in agreement with the SECs (see above). For this reason $Z_{3333}=\left\langle\left(\Delta \varepsilon_{33}\right)^{2}\right\rangle$ is the most reliably determined $Z_{i j p q}$ parameter (compared to $Z_{1111}$ and $Z_{1133}$ ). Therefore, the following discussion focuses on $Z_{3333}$. The expected temperature-dependent evolution of $\left\langle\left(\Delta \varepsilon_{33}\right)^{2}\right\rangle^{1 / 2}$ according to eq. (5) is shown in figure $3 \mathrm{~b}$. The experimentally determined values for $\left\langle\left(\Delta \varepsilon_{33}\right)^{2}\right\rangle^{1 / 2}$ are of the same order of magnitude as the theoretically predicted ones, but are approximately a factor of 2 smaller. Strikingly, the experimentally observed values are not a simple function of temperature, but there appears to be some hysteresis: Upon reheating from the lowest temperature of $10 \mathrm{~K}$, the experimental values for $\left\langle\left(\Delta \varepsilon_{33}\right)^{2}\right\rangle^{1 / 2}$ decrease faster with increasing temperature than they first increased during cooling with decreasing temperature. Furthermore, the observed line broadening is asymmetric (figure 1). The latter observation is incompatible with the theory (section "Estimation of microstrain..."), which predicts a Gaussian microstrain distribution $[2,3]$.

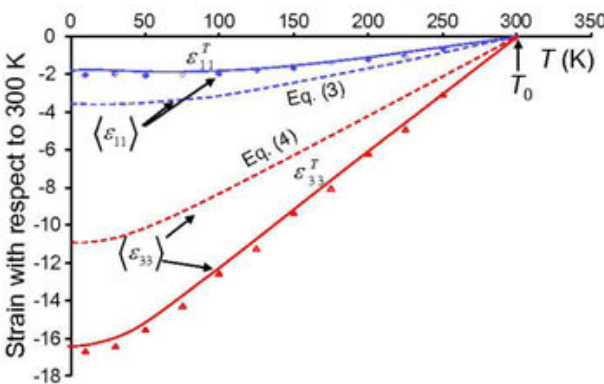

(a)

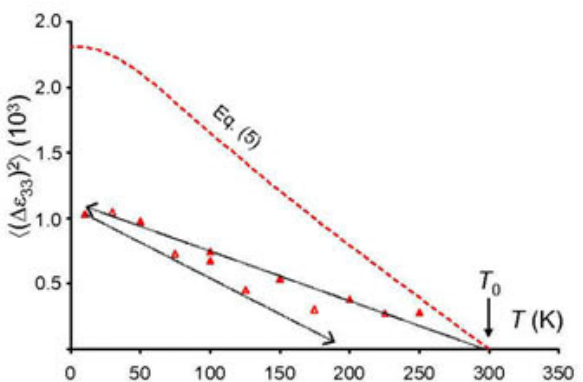

(b)

Figure 3. Evolution as a function of temperature of the (a) first moments and (b) second moments (only for $\varepsilon_{33}$ parallel to [001]) of the microstrain distribution. Continuous lines (only (a)): $\varepsilon_{11}^{T}$ and $\varepsilon_{33}^{T}$ from single-crystal thermal-expansion data; dashed lines: evolutions calculated according to section "Estimation of microstrain..."; data points (solid for cooling, empty for reheating): experimental values. In (b) the dotted arrows indicate the hysteresis of the line widths.

The discrepancies between experiment and theory can be explained by two effects not considered in the theory discussed in section "Estimation of microstrain...": local plastic defor- 
mation and surface relaxation, the latter allowing relief of elastic stresses at the surface of the powder particles. Both effects would cause the first moments $\left\langle\varepsilon_{11}\right\rangle$ and $\left\langle\varepsilon_{33}\right\rangle$ to become closer to $\varepsilon_{11}^{T}$ and $\varepsilon_{33}^{T}$ than expected and that the occurring line broadening is reduced. Indeed, plastic deformation has been reported to occur during thermal cycling of zinc between 30 and $150^{\circ} \mathrm{C}[11]$.

The observed hysteresis of $Z_{3333}$ (figure $3 b$ ) can thus also be explained by the occurrence of plastic deformation. During cooling elastic strains are built up, which are considerably released by plastic deformation. Then, upon reheating less temperature increase than temperature decrease upon cooling is needed to achieve a strain-free state. In fact, a close inspection of the experimental $\left\langle\varepsilon_{11}\right\rangle$ and $\left\langle\varepsilon_{33}\right\rangle$ values given in figure $3 \mathrm{a}$ also indicates a small hysteresis not visible for the scale in figure $3 \mathrm{a}$.

\section{Conclusions}

Upon cooling below room temperature polycrystalline zine (internally polycrystalline powder particles) shows considerable microstrain broadening, which largely disappears upon reheating. This microstrain broadening is due to stress fields varying from grain to grain (eigenstresses of $2^{\text {nd }}$ kind). Analysis of the first moments (peak shifts) and second moments (peak broadening) of the microstrain distribution reveals that the thermal misfit is partially accommodated by surface relaxation and local plastic deformation.

The effects observed here for zinc should in general affect quite frequently the powderdiffraction patterns of non-cubic polycrystalline materials, if the lattice parameters change anisotropically with temperature.

\section{References}

1. Zimmermann, A., Fuller Jr., E.R. \& Rödel, J., 1999, J. Am. Ceram. Soc., 82, 3155.

2. Kreher, W. \& Pompe, W., 1989, Internal Stresses in Heterogeneous Solids (Berlin: Akademie-Verlag).

3. Kreher, W.S., 1996, Comp. Mater. Sci., 7, 147.

4. Fréour, S., Gloaguen, D., François, M. \& Guillén, R., 2004, Phys. Stat. Sol. (a), 201, 59.

5. Leineweber, A., 2006, J. Appl. Crystallogr., 39, 509.

6. Wilson, A.J.C., 1955, Nuovo Cimento, 1, 277.

7. Leineweber, A., in preparation.

8. Meyerhoff, R.W. \& Smith, J.F., 1962, J. Appl. Phys., 33, 219.

9. Alers, G.A. \& Neighbours, J.R., 1958, J. Phys. Chem. Solids, 7, 58.

10. TOPAS. General Profile and Structure Analysis Software for Powder Diffraction Data (Karlsruhe, Germany: Bruker AXS GmbH).

11. Boas, W. \& Honeycombe, R.W.K., 1946, Proc. Roy. Soc., 186, 57.

Acknowledgements. The help by Prof. J.S.O. Evans (Durham University, UK) for providing macros used to perform the refinements on the basis of the time-of-flight neutron data as employed in TOPAS is gratefully acknowledged. 


\title{
The $h k l$ dependences of microstrain and of macrostress-induced macrostrain; a comparison for intrinsically extremely anisotropic cementite, $\mathrm{Fe}_{3} \mathrm{C}$
}

\section{A. Leineweber ${ }^{*}$, T. Gressmann, M. Nikolussi, E. J. Mittemeijer}

Max Planck Institute for Metals Research, Heisenbergstr. 3, 70569 Stuttgart, Germany *a.leineweber@mf.mpg.de

Keywords: residual stress analysis, macrostrain, microstrain, elastic anisotropy, line-profile analysis

\begin{abstract}
On the basis of X-ray diffraction analysis performed on polycrystalline cementite $\left(\mathrm{Fe}_{3} \mathrm{C}\right)$ layers grown on $\alpha$-iron substrates, a distinctly positive correlation of the macrostrain (as revealed by the sample-tilting angle dependent peak positions) and of the microstrain (as revealed by the corresponding peak broadening) pertaining to different $h k l$, respectively, was observed. In fact, the $h k l$ dependences of both quantities are similar. This can be understood by the strong dependences of both macro- and microstrain on some average values of the (anisotropic) elastic constants along the diffraction vector.
\end{abstract}

\section{Introduction}

Macrostressed polycrystalline thin surface layers (i.e. subjected to mechanical stress) will in case of intrinsic elastic anisotropy and variable grain orientation also exhibit microstrain. Whereas the macrostrain leads to typical sample-orientation peak shifting, the microstrain distribution will lead to broadening of the diffraction peaks. Macrostrain and microstrain may provide valuable information about the overall state of stress/strain in the layer, i.e. the average values and the distributions, see e.g. [1]. However, most macrostress studies by diffraction techniques, e.g. using the $\sin ^{2} \psi$-method [2], do not consider the line broadening of the diffraction peaks. That may be partly due to the often relatively large instrumental broadening of the diffractometers dedicated to (macro)stress analysis, masking the structural line broadening.

In the course of X-ray diffraction (macro)stress measurements performed on cementite $\left(\mathrm{Fe}_{3} \mathrm{C}\right.$ ) layers grown on $\alpha$-iron substrates using gaseous nitrocarburising [3], the instrumental configuration was chosen such to allow combining the possibility for specimen tilting with high instrumental resolution. The results of the macrostress analysis were published previously [4]. The present work addresses the state of microstrain in the $\mathrm{Fe}_{3} \mathrm{C}$ layer, in particular 
with reference to the state of (elastic) macrostrain. At first, in section "Macrostrains and microstrains", the effect of single-crystal elastic anisotropy both on the anisotropic macrostrain and on the anisotropic microstrain is discussed. This serves as basis for the data evaluation in section "Results and discussion".

\section{Macrostrains and microstrains}

Consider a rigid substrate with a thin surface layer, which exhibits in the plane parallel to the surface isotropic residual macrostress $\sigma_{\|}$. For a given $h k l$ the macrostress leads to a $\psi$ dependent peak(-centroid) shift with respect to the position pertaining to the strain-free state (index 0). From this shift the corresponding (average) $h k l$ - and $\psi$-dependent elastic macrostrain $\left\langle\Delta \varepsilon_{h k}(\psi)\right\rangle$ can be calculated according to:

$\left\langle\Delta\left(2 \theta_{h k l}(\psi)\right)\right\rangle=\left\langle 2 \theta_{h k l}(\psi)\right\rangle-2 \theta_{0, h k l}=-2\left\langle\Delta \varepsilon_{h k l}(\psi)\right\rangle \tan \theta_{0, h k l}$.

Neglecting the occurrence of macroscopic elastic anisotropy (in the specimen frame of reference; e.g. due to texture, anisotropic grain interaction $[2], \ldots$.$) , the h k l$ - and $\psi$-dependent elastic macrostrain measured according to eq. (1) is related with the macrostress $\sigma_{\|}$by [2]

$\left\langle\varepsilon_{h k l}(\psi)\right\rangle=2 S_{1}^{h k t} \sigma_{\|} \frac{1}{2} S_{2}^{h+k} \sigma_{\|} \sin ^{2} \psi=\left\langle\varepsilon_{h k l}(0)\right\rangle\left\langle\left\langle\varepsilon_{\text {chkl }}(90)\right\rangle\left\langle\varepsilon_{h k l}(0)\right\rangle\right) \sin ^{2} \psi$,

with $S_{1}^{h k l}$ and $1 / 2 S_{2}^{h k l}$ as the $h k l$-dependent X-ray elastic constants (XECs). These XECs can be regarded as averages of certain single-crystal elastic constants (SECs) along the direction of the diffraction vector (in the crystal's frame of reference) according to $\left.S_{1}^{h k t}=\langle-v / E)\right\rangle^{h k l}$ and $\left.1 / 2 S_{2}^{h k l}=\langle(1+v) / E)\right\rangle^{h k l}$ (with $E$ being the Young's modulus, and with $v$ being the Poisson ratio), where the type of averaging depends on the type of grain interaction. $S_{1}^{h k i}$ and $1 / 2 S_{2}^{h k t}$ can straightforwardly be calculated for certain types of (here isotropic) grain interaction from the single-crystal elastic constants [2], in particular for the extreme cases of Voigt- and Reuss-type grain interactions. For the Voigt case the XECs $S_{1}^{\mathrm{V}}$ and $\frac{1}{2} S_{2}^{\mathrm{V}}$ are constants independent from $h k l$. For the Reuss case the XECs $S_{1}^{\mathrm{R}, h k l}$ and $\frac{1}{2} S_{2}^{\mathrm{R}, h k l}$ can be expressed as $4^{\text {th }}$ order polynomials in the components $x_{1}, x_{2}$ and $x_{3}$ of a unit vector $\mathbf{x}(h k l)$ parallel to the diffraction vector of the peak $h k l$ (expressed with respect to the crystal frame of reference, a Cartesian coordinate system defined in a given way with respect to the crystallographic coordinate system with the basis vectors $\mathbf{a}, \mathbf{b}$ and $\mathbf{c}$ ):

$S_{1}^{\mathrm{R}, h k l}=R_{1, j p q}^{\mathrm{R}} x_{i} x_{j} x_{p} x_{q}$ and $S_{2}^{\mathrm{R}, h k l}=R_{2, j p q}^{\mathrm{R}} x_{i} x_{j} x_{p} x_{q}$,

applying the Einstein summation convention. In eq. (3) both polynomials are symmetry invariant with respect to the crystal class, and the coefficients $R_{(1,2), y p q}^{\mathrm{R}}$ present combinations of SECs. The $R_{(1,2), y p q}^{\mathrm{R}}$ coefficients are equal for an arbitrary permutation of $i, j, p$ and $q$.

It has been suggested that for an intermediate character of grain interaction, one may employ some weighted averages of the Reuss type and Voigt type XECs (formulas given in ref. [5]), $S_{1,2}^{\text {hkl }}=w S_{1,2}^{\mathrm{V}} \quad\left(1+w+S_{1,2}^{\mathrm{R}, h k l}\right.$,

with an $h k l$-independent weighing factor $w(0 \leq w \leq 1)$, which may be determined by fitting on the basis of data pertaining to different $h k l$. Due to the $h k l$-independent $S_{1}^{\mathrm{V}}$ and $\frac{1}{2} S_{2}^{\mathrm{V}}$, and 
in view of eq. (3), also the overall $S_{1,2}^{\text {hkl }}$ in eq. (4) can be expressed as $4^{\text {th }}$-order polynomials in $x_{1}, x_{2}$, and $x_{3}$, e.g. with the polynomial coefficients $R_{1, i p q}$ and $R_{2, i j p q}$, analogously to eq. (3): $S_{1}^{h k l}=R_{1, i j p q} x_{i} x_{j} x_{p} x_{q}$ and $S_{2}^{h k l}=R_{2, j i p q} x_{i} x_{j} x_{p} x_{q}$.

Irrespective of the relatively complex formulas leading to eq. (5), it is obviously expected that the change in macrostrain with $\psi$ varying from $0^{\circ}$ to $90^{\circ}$ amounting $\left\langle\varepsilon_{h k l}\left(90^{\circ}\right)\right\rangle-\left\langle\varepsilon_{h k l}\left(0^{\circ}\right)\right\rangle=\frac{1}{2} S_{2}^{h k l} \sigma_{\| l}$ (this quantity is called for simplicity in the following the macrostrain) will be large if the direction of the diffraction vector given by $\mathbf{x}(h k l)$ in the crystal frame of reference corresponds to an elastically compliant direction of the crystal and small if a stiff direction is concerned.

The strain in the specimen along the diffraction vector is usually not constant but is distributed. The variance of the microstrain distribution along the diffraction vector, $\left\langle\left(\varepsilon_{h k l}-\left\langle\varepsilon_{h k}\right\rangle\right)^{2}\right\rangle$ $=\left\langle\left(\Delta \varepsilon_{k k l}\right)^{2}\right\rangle$ (a possible $\psi$ dependence of the variance is not indicated here, e.g. by $\left\langle\left(\Delta \varepsilon_{h k l}(\psi\rangle\right)^{2}\right\rangle$ because no systemetic variation with $\psi$ was found), appears as microstrain broadening on the diffraction scale as (strain is assumed to be constant in each coherently diffracting crystallite)

$\left\langle\left(\Delta 2 \theta_{h k l}\right)^{2}\right\rangle=4 \tan ^{2} \theta_{0, h k}\left\langle\left(\Delta \varepsilon_{h k l}\right)^{2}\right\rangle$.

Like it is the case for $S_{1,2}^{h k l}$ under the above-mentioned assumptions (cf. eq. (5)), also the variance $\left\langle\left(\Delta \varepsilon_{h k l}\right)^{2}\right\rangle$ can be expressed as a symmetry-invariant $4^{\text {th }}$-order polynomial in $x_{1}, x_{2}$, and $x_{3}$ :

$\left\langle\left(\Delta \varepsilon_{h k l}\right)^{2}\right\rangle=Z_{i j p q} x_{i} x_{j} x_{p} x_{q}$,

where, again, like in eq. (3) and eq. (5), the $Z_{i j p q}$ coefficients are equal for an arbitrary permutation of $i, j, p$, and $q$. These $Z_{i j p q}$ coefficients are connected with the variances and/or the covariances of the distribution of the strain-tensor components over the diffracting grains formulated with respect to the crystal frame of reference as used for the vector $\mathbf{x}(h k l)$ [6]. In the course of a phenomenological assessment of the microstrain broadening like in the course of a Rietveld refinement, the $Z_{i j p q}$ coefficients can be refined as parameters.

It is noted that in practice, the microstrain broadening often exhibits a pseudo-Voigt like shape, which has no finite variance. In order to avoid this complication instead of the variance of the line broadening or of the microstrain along the diffraction vector in eq. (6) and eq. (7), the corresponding squared Full-Width at Half Maxima (FWHMs), $B_{\Delta 2 \theta_{k h t}}^{2}$ and $B_{\Delta \varepsilon_{h l l}}^{2}$, will be employed. The corresponding coefficients in eq. (7) then are indicated by $Z_{i j p q}^{B}$.

Values for the $Z_{i j p q}$ or the $Z_{i j p q}^{B}$ parameters can be derived for different types of models for the microstrain distribution pertaining to various physical origins: different types of isotropic field-tensor variations connected with strain via anisotropic property tensors [7], microstrains due to dislocations $[8,9]$, or thermal microstrain estimated employing maximum entropy method [10]. Refs. [7-10] deal, in particular, with cases of microstress distributions and corresponding microstrain distributions connected with each other via the intrinsic elastic anisotropy. In all these cases the elastic anisotropy in some way affects the $Z_{i j p q}$ coefficients and determines their values.

In general, without adopting a particular physical model for the microstrain distribution, like [7-10], one may expect that peaks $h k l$ show in particular large microstrain broadening, if the crystal direction parallel to the diffraction vector is in particular elastically compliant. This 
statement is analogous to that below eq. (5) concerning the $\mathbf{x}(h k l)$ dependence of $1 / 2 S_{2}^{h k l} \sigma_{\|}$. Thus, if anisotropy of the microstrain broadening is determined by the anisotropy of the single-crystal elastic compliance, the $\mathbf{x}(h k l)$ dependency of $B_{\Delta s_{h b l}}^{2}$ should be similar to that of $1 / 2 S_{2}^{h k i} \sigma_{\|}$.

\section{Experimental}

A massive, polycrystalline $\mathrm{Fe}_{3} \mathrm{C}$ layer (thickness $4.6 \mu \mathrm{m}$ ) was grown on a plate-like $\alpha$-iron substrate (thickness $1 \mathrm{~mm}$ ) by a dedicated nitrocarburisation procedure $[3,4]$. High-resolution X-ray powder-diffraction experiments on this layer were performed at HASYLAB, Hamburg, Germany. Station B2 was equipped with a Eulerian cradle and operated with a wavelength of $0.79323 \AA$, which is sufficiently small to ensure negligible absorption of radiation within the $\mathrm{Fe}_{3} \mathrm{C}$ layer. An analyser crystal in the diffracted beam ensured narrow instrumental profiles, which were determined by measuring peaks of a $\mathrm{LaB}_{6}$ (NIST SRM660a) standard sample. During the measurements both specimens $\left(\mathrm{Fe}_{3} \mathrm{C}\right.$ and $\left.\mathrm{LaB}_{6}\right)$ were rotated around their surface normal to achieve better crystallite statistics. A selected number of peaks were measured at different sample tilting angles $\psi$ for both specimens.

Data evaluation was carried out with the TOPAS [11] software. The instrumental profile was determined by fitting of split-Pseudo Voigt functions to the $\mathrm{LaB}_{6}$ peaks. The instrumental profile shape and width parameters pertaining to different Bragg angles were interpolated by means of polynomials to determine values at arbitrary Bragg angles. On this basis the peak profiles recorded from the $\mathrm{Fe}_{3} \mathrm{C}$ layer were evaluated by convoluting the instrumental-profile function at the respective Bragg angle with a split pseudo-Voigt function, representing the physical line broadening, with fitted shape and width parameters. The peak positions as well as the FWHMs of the physical line broadening, $B_{\Delta 2 \theta_{k+k}}$, for various $h k l$ were the basis for the further evaluations.

\section{Results and discussion}

The $\psi$-dependent peak shifts had been used in order to evaluate the $\mathrm{Fe}_{3} \mathrm{C}$ layer's state of macrostrain and macrostress [4]. To this end SECs from first-principles calculations had been used for calculation of XECs according to eq. (4), yielding a compressive (mechanical) macrostress of $\sigma_{\|}=-440 \mathrm{MPa}$. The macrostrains $1 / 2 S_{2}^{h k l} \sigma_{\|}=\left\langle\varepsilon_{h k l}\left(90^{\circ}\right)\right\rangle-\left\langle\varepsilon_{h k l}\left(0^{\circ}\right)\right\rangle$ in the $\sin ^{2} \psi$ plots (macrostrain) according to eq. (2) vary significantly with $h k l$, as expected for $h k l$ dependent XECs. This reflects the extreme elastic anisotropy indicated by the SECs (very small $C_{2323}$ vs. $10 \times \operatorname{larger} C_{1313}$ and $\left.C_{1212}\right)$, which leads to the largest peak shifts from ( $\psi=$ $0^{\circ}$ to $90^{\circ}$ ) for peaks with small $h$ and simultaneously large $k$ and $l$ (indices and tensors refer to the Pnma setting of the cementite's crystal structure).

The line widths vary primarily (also) with $h k l$; slight variations with $\psi$ occur, too, but no systematic $\psi$ dependency can be discerned. Therefore, in the following the averaged values of the line widths recorded at $\psi=25^{\circ}$ and $37^{\circ}$ are used. Further, it is assumed that the complete physical line broadening is of microstrain type. Thus, using eq. (6), using the squared FWHMs instead of the variances, one can calculate $B_{\Delta \varepsilon_{k l t}}^{2}$. The $B_{\Delta \varepsilon_{k+k}}^{2}$ values have been plot- 
ted versus the $1 / 2 S_{2}^{h k l} \sigma_{\|}=\left\langle\varepsilon_{h k l}(90)\right\rangle\left\langle\varepsilon_{k k l}\left(0^{\circ}\right)\right\rangle$ in figure 1. Evidently, a clear positive correlation occurs: correlation factor of 0.80 , although a few systematic outliers occur (e.g. 002). Note that this analysis is possible simply on the basis of the bare experimental data, without knowledge of values for the XECs.

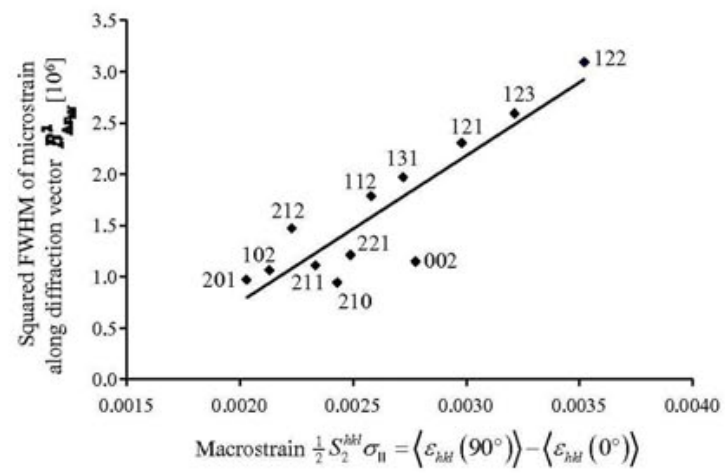

Figure 1. Comparison of the squared FWHM values of the microstrain distribution along the diffraction vector with the slopes of $\sin ^{2} \psi$ plots (macrostrain) according to eq. (2) for various hkl.

a)

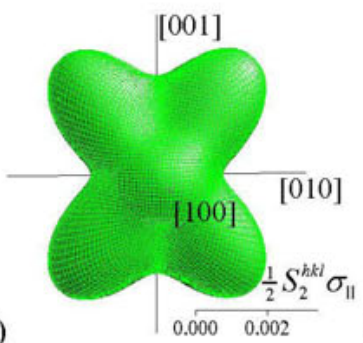

b)

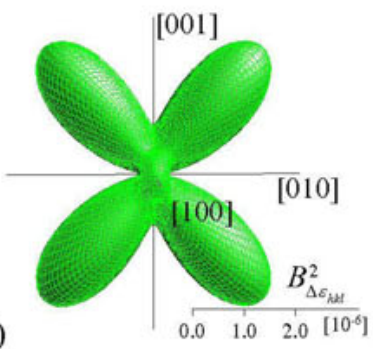

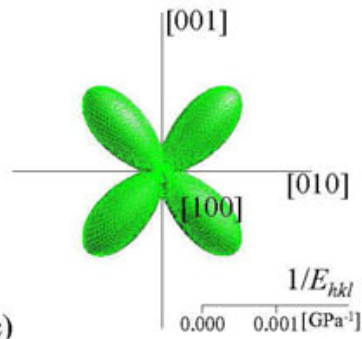

Figure 2. (a) and (b): Tensor surfaces (drawn with Wintensor [12]) representing microstructural properties of the cementite layers as a function of the direction $\boldsymbol{x}(\mathrm{hkl})$ of the diffraction vector in the crystal coordinates: (a) slopes of the $\sin ^{2} \psi$ plots (macrostrain) and (b) squared FWHM of the microstrain distribution along the diffraction vector. The tensor surface in (c) illustrates the direction dependence of the reciprocal Young's modulus [4].

Moreover, the similar anisotropies of the macrostrain $\left(1 / 2 S_{2}^{k k l} \sigma_{\| l}=\left\langle\varepsilon_{h k l}\left(90^{\circ}\right)\right\rangle-\left\langle\varepsilon_{h k l}\left(0^{\circ}\right)\right\rangle\right)$ and of the microstrain ( $B_{\Delta \sigma_{S H}}^{2}$ ) in the crystal frame of reference can be illustrated on the basis of tensor surfaces representing the direction dependencies of these quantities: see figure 2 . For that purpose, on the basis of the experimental data for different $h k l, 6$ different coefficients $1 / 2 R_{2, j p q}^{\mathrm{R}} \sigma_{\|}$representing the macrostrain were fitted on the basis of the $1 / 2 S_{2}^{k k l} \sigma_{\|}$data (eq. (5)) and analogously 6 different coefficients $Z_{l j p q}^{B}$ representing the microstrain were fitted on the basis of the $B_{\triangle \alpha_{S H}}^{2}$ data (eq. (7); using the squared FWHMs instead of the vari- 
ances). The number of 6 coefficients follows from the invariance with respect to the orthorhombic symmetry of $\mathrm{Fe}_{3} \mathrm{C}$ [6]. Indeed, the surfaces look qualitatively similar. They both also resemble the tensor surface describing the direction dependence of the reciprocal Young's modulus $1 / E_{h k l}=S_{i j p q} x_{i} x_{j} x_{p} x_{q}$ (also shown in figure 2), which demonstrates the softness of directions intermediate between [010] and [001]. This softness is a direct consequence of the small shear modulus $C_{2323}$ mentioned above.

The remaining differences in the precise shapes of the tensor surfaces are e.g. related with the averaging necessary for going from the SECs (fig. 2c) to the XECs (contained in fig. 2b). Moreover, different models exist (and more are imaginable) for how elastic anisotropy may show up microstrain [7-10].

\section{Conclusion}

The intrinsic anisotropic elastic properties of a solid may be exhibited by the anisotropy of the observed elastic macrostrain and of elastic microstrain: Elastically compliant directions exhibit larger values of macrostrain and of microstrain than stiff directions.

\section{References}

1. Sayers, C.M., 1984, Phil. Mag. A, 49, 243.

2. Welzel, U., Ligot, J., Lamparter, P., Vermeulen, A.C. \& Mittemeijer, E.J., 2005, $J$. Appl. Cryst., 38, 1.

3. Gressmann, T., Nikolussi, M., Leineweber, A. \& Mittemeijer, E.J., 2006, Scr. Mater., 55, 723.

4. Nikolussi, M., Shang, S.L., Gressmann, T., Leineweber, A. \& Mittemeijer, E.J., Wang, Y. \& Liu, Z.-K., 2008, Scr. Mater., 59, 814.

5. Howard, C.J. \& Kisi, E.H., 1999, J. Appl. Cryst., 32, 624.

6. Leineweber, A., 2006, J. Appl. Cryst., 39, 509.

7. Leineweber, A., 2007, J. Appl. Cryst., 40, 362.

8. Ungar, T. \& Tichy, G., 1999, Appl. Phys. Lett., 69, 425.

9. Leoni, M., Martinez-Garcia, J. \& Scardi, P., 2007, J. Appl. Cryst., 40, 790.

10. Kreher, W. \& Pompe, W., 1989, Internal Stresses in Heterogeneous Solids (Berlin: Akademie-Verlag).

11. TOPAS. General Profile and Structure Analysis Software for Powder Diffraction Data (Karlsruhe, Germany: Bruker AXS GmbH).

12. Kaminsky, W., 2004, Wintensor Version 1.1, Cologne, Seattle, Oxford.

Acknowledgements. The authors thank Dr. D. Trots and M. Hinterstein (TU Darmstadt/HASYLAB) for their support with the synchrotron measurements. 
I.4 Texture 



\title{
A primer on whole through processing simulation understanding of deformation and annealing textures in low carbon steels
}

\author{
A. Roatta, A. Fourty, R. E. Bolmaro*
}

Instituto de Física Rosario, CONICET-UNR. Bv. 27 de febrero 210 bis, 2000, Rosario, Argentina

*bolmaro@ifir-conicet.gov.ar

Keywords: steel, rolling test, transformation texture, recrystallization

\begin{abstract}
Processing steels to achieve particular useful properties is a science as well as an art. Many of the properties of modern steels are a successful combination of empiric and scientific knowledge. Deep drawing low carbon steels have been used and improved through many decades of research and technological advance. However the micro mechanisms involved in the development of particular microstructures and properties are still under discussion. The current paper shows an integrated attempt to obtain consistent microscopic data from micromechanical simulations coupled with recrystallization and phase transformation codes. The simulations are performed in a way such that the information obtained from certain temperatures, levels or processes are used in the next steps to proceed further. The goal is not avoiding experiments but having a whole through scale and time integration for judging the validity of similar parameters and assumptions during the different processing steps. The simulations include high and low temperature deformation and recrystallization in the austenite region, phase transformation to room temperature, low temperature deformation and recrystallization in the ferrite phase region. The results are compared with experiments available in the literature.
\end{abstract}

\section{Introduction}

The final texture of steel sheets dominates the formability properties of materials. It is well known that the presence of strong gamma fibre [1] foretells good properties for deep drawing. The development of crystallographic texture is due to a complex interaction between, at least, three processes: deformation, recrystallization and phase transformation. There is a large empiric knowledge applied to industrial processes to obtain the necessary microstructures and textures for different features. Nevertheless, a complete understanding of recrystallization and phase transformation phenomena would allow to control and improve the specific properties of industrial materials. 
In polycrystals, while plastic deformation changes grain orientations by slip and twinning, recrystallization cleans up dislocations created during plastic deformation and low energetic grains grow at the expenses of old high energetic grains. On the other hand, phase transformations set up specific crystallographic relationships between product and parent orientations. Bain, Kurdjumov-Sachs and Nishiyama-Wassermann proposed the first models, widely employed in steels [2-5]. While phase transformation phenomena are easily measurable, although sometimes you lack original or parent data, recrystallisation occurrence is judged by indirect measurements. Shape and size of grains as well as calorimetric methods are used to investigate the progress of recrystallisation. Recently some methods associated with EBSD technique have been implemented understanding that the lower the confidence index for crystal indexing the higher the stored energy on that particular region [6-7].

In the present paper we simulated, in different stages, three typical thermomechanical processes for low carbon steels: rolling, phase transformation by cooling and recrystallization. Rolling deformation is simulated with a viscoplastic selfconsistent code (VPSC) [8], phase transformation is described through the Kurdjumov-Sachs transformation [3] and recrystallization is calculated with a code that includes nucleation and grain growth [9]. In this way, two typical whole processes of material are described:

Process A: hot rolling in austenite no-recrystallization range, phase transformation on cooling, cold rolling and recrystallization in ferrite.

Process B: hot rolling in austenite recrystallization range, static recrystallization, phase transformation on cooling, cold rolling and recrystallization in ferrite.

After the end of each stage we access to computational crystallographic texture and other micromechanical variables needed as input data for next stage. In this way, it is possible to simulate the whole process from a random texture without other additional data than intrinsic material properties (crystal symmetry, strain rate sensitivity, critical stress on slip systems, etc.) to a final ferrite texture.

\section{Results and discussion}

We consider an initial random texture with slip as the principal mode of plastic deformation. Reported values for $\alpha$-iron stacking fault energy, of about $75 \mathrm{~mJ} / \mathrm{m}^{2}$ [10], closer to high stacking fault energy (HSFE) metals $\left(80 \sim 200 \mathrm{~mJ} / \mathrm{m}^{2}\right)$ and previous results [11] for global and local texture simulations in comparison with experimental data, validate the hypothesis of considering that twinning is not a main deformation mechanism.

\section{Process A}

Rolling tests simulations, until a von Mises strain of 2.0 (incremental steps equal to 0.025 ), have been performed on fec materials mimicking HSFE materials. We adopted typical $\{111\}$ $<110>$ slip systems. The constitutive law is a viscoplastic one with $n=100$ (inverse of the strain rate sensitivity). Interaction coefficient was fixed to $\mathrm{n}^{\text {effective }}=2$, modelling a stiff interaction between grains and the homogenous equivalent medium [12]. It was considered a hardening law with self-hardening equal to 1 and latent hardening, equal to 1.4. The interaction matrix is also function of the strain rate of each slip system. Grain fragmentation was allowed by co-spinning $[12,13]$ and a misorientation between fragments is generated during the deformation process [14]. The out coming micromechanical data comprehends a set of 10000 crystallite orientations, representative of the grains, their volume fractions, critical resolved shear stresses, individual strains and stresses and misorientations among fragments and neighbours. 
The partial $\varphi_{2}$-sections of the orientation distribution function (ODF) corresponding to hot rolled austenite in no-recrystallization range, calculated by MTM-FHM software [15], are shown in fig. 1. The texture is described quantitatively in terms of a small number of major components. The orientations with misorientations larger than $15^{\circ}$ around the ideal rolling components are classified as 'Others'. The volume fractions of selected components are: $18.6 \% \mathrm{Cu}, 8.9 \% \mathrm{Bs}, 4.6 \% \mathrm{G}, 29.7 \% \mathrm{~S}$ and $2.5 \% \mathrm{Cube}$. They show a pronounced beta-fibre.

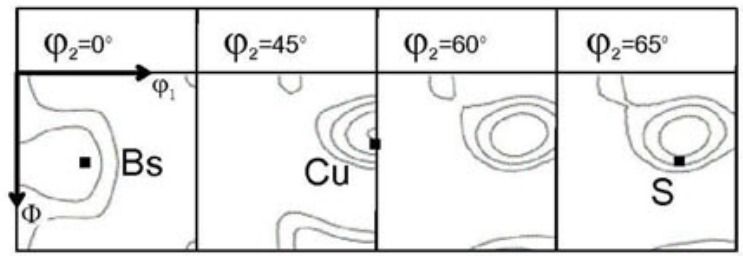

Figure 1. Partial simulated $O \mathrm{ODF}^{\prime} \varphi_{2}$-sections for hot rolled material at von Mises strain $=2.0$. Isolevels: $1,2,4,8$.

Figure 2 a) shows the $\varphi_{2}=45^{\circ}$ section of the ODF resulting from fec-bce phase transformation of texture displayed in figure 1. The code, based in Kurdjumov-Sachs model, transforms each original orientation in a set of 24 product orientations obtained by $90^{\circ}$ rotations about a common $<112>$ axis. The results show a good description of principal texture components although their intensities are lower than the literature experimental data [16-18], especially on the $\gamma$-fibre. The preceding results are used as input data for a cold rolling test simulation until a von Mises strain of 1.0. We employed the usually $\{110\}<111>$ and $\{112\}<111>$ bcc slip systems, a viscoplastic constitutive law with $\mathrm{n}=50$ and $\mathrm{n}^{\text {effective }}=1$, a potential hardening law with coefficients $\mathrm{H}_{0}=1.4$ y N=0.23 [19]. Both, latent and self hardenings are assumed equal to 1. Grain fragmentation was allowed. Figure $2 \mathrm{~b}$ ) shows the resulting $\varphi_{2}=45^{\circ}$ section of the ODF. $\alpha$ and $\gamma$ fibres are present, nevertheless it will be important to enhance the simulation of the previous stage to obtain a higher $\gamma$-fibre intensity.

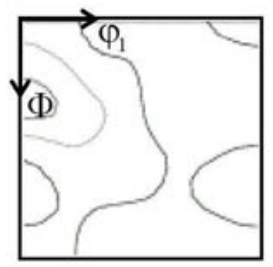

a)

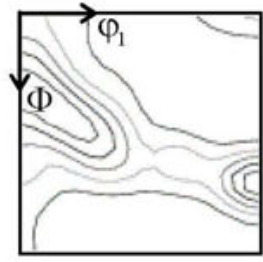

b)

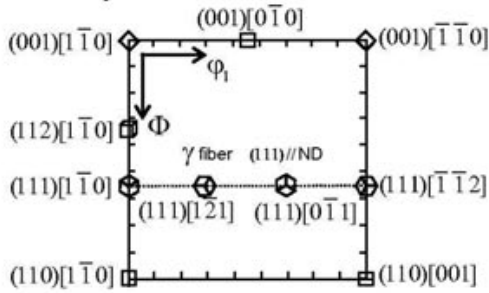

c)

Figure 2. Partial simulated $O D F ' s, \varphi_{2}=45^{\circ}:$ a) phase transformation from austenitic hot rolled steel, b)later cold rolling deformation of product material, c) ideal components. Iso-levels: 1, 2, 3, ..

For simulating the next recrystallization stage, we analysed resulting stored deformation energies (E), which are calculated as functions of CRSS of active slip systems. Figure 3 a) describes stored energy distribution for orientations with $42.5^{\circ}<\varphi_{2}<47.5^{\circ}$. The size of circles is proportional to the value of $\mathrm{E}$ in each orientation. Figure $3 \mathrm{~b}$ ) shows average energy values on $\alpha$ and $\gamma$ fibres with a tolerance of $\pm 5^{\circ}$. We can see on the $\alpha$ fibre that $\mathrm{E}(100)<$ $\mathrm{E}(211)<\mathrm{E}(111)<\mathrm{E}(110)$. Meanwhile, on the $\gamma$ fibre the stored energy distribution is uni- 
form and larger than $\mathrm{E}(100)$ and $\mathrm{E}(211)$. The experimental stored energies presented by Wauthier et al. and Rajmohan et al. [20-21], using neutron diffraction line broadening, confirm the tendency of our results.

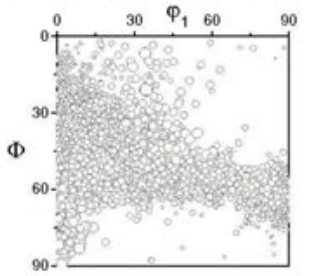

a)

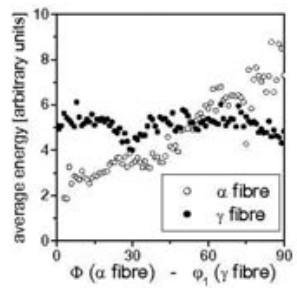

b)

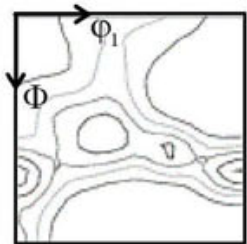

c)

Figure 3. a) Stored energy distribution, at $\varphi_{2}=45^{\circ}$; b) average energy on $\alpha$ and $\gamma$ fibres, both at the end of cold rolling step of process $A$.c) Final recrystallization texture, at $\varphi_{2}=45^{\circ}$. Isolevels: $1,2,3, \ldots$

Meanwhile deformation stored energies drive nucleation, grain growth depends statistically on grain boundary energy which mainly depends on misorientation. Both phenomena are modelled by a previous developed code [9], where nucleation happens in grains with a probability depending on the stored energy, measured as a function of the CRSS. The criteria used to regulate the growing process results from the competition between the surface and volume energies of pairs of grains and their misorientation. For growing we considered a product probability, with a factor taking into account the larger mobility of HAB (High Angle Boundaries) comparing with LAB (Low Angle Boundaries) and the other one representing the driving pressure dependence of the pair stored energy difference. The larger the energy difference the larger the driving force.

\section{Process B}

The micromechanical variables obtained at the end of hot rolling simulation with the same parameters as process A are used as input data in recrystallization simulation. Representative sections of resulting ODF at constant $\varphi_{2}$ are shown in figure 4, where the recrystallization cubic component ( $19.6 \%$ Cube), characteristic of HSFE fcc metals, is present.

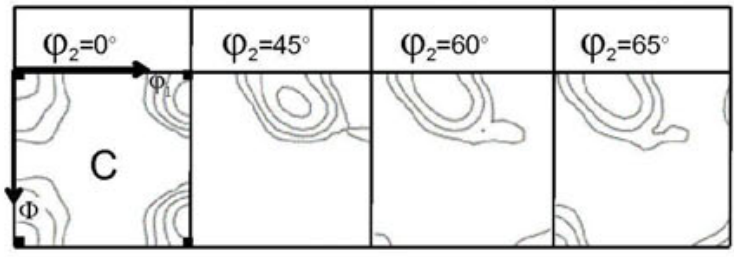

Figure 4. Simulated partial ODF's $\varphi_{2}$-sections for hot rolled material at von Mises strain $=2.0$ in recrystallization range. Iso-levels: $1,2,4,8$.

Later on, we calculated the transformation texture (figure 5 a) and simulated the ferrite cold rolled test with identical parameters to the corresponding to process A (figure $5 \mathrm{~b}$ ). This texture, although less sharp, behaves as the experimental ones [22] except for the presence of orientations close to $<110>\{110\}$. 
Figures 6 a) and b) show stored energy distributions for orientations with $42.5^{\circ}<\varphi_{2}<47.5^{\circ}$ and average energy values on $\alpha$ and $\gamma$ fibres with a tolerance of $\pm 5^{\circ}$, respectively. Experimental results presented by Samajdar et al. [22], using X-ray diffraction and measuring substructure and misorientations validates the trend found on the $\alpha$ fibre. On the $\gamma$ fibre, we observe a slight drop on the calculated intensity for $\mathrm{E}(112)$ respect to $\mathrm{E}(111)$. This result does not agree with the trend published by Samajdar et al.[22]. It is important to notice that their experimental spread increases from $\mathrm{E}(111)$ to $\mathrm{E}(112)$. Similar simulated results have been obtained, for this stage of the processing, by Wierzbanowski et al. [23], who also have found that the stored energy in the $\gamma$ fibre is favourable for further nucleation and presents advantages for strengthening after recrystallization.

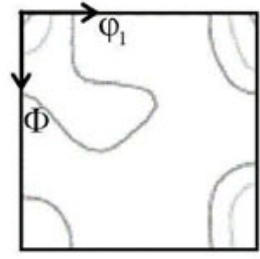

a)

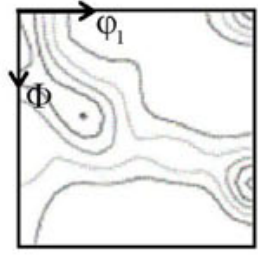

b)

Figure 5. Simulated partial $O D F ' s, \varphi_{2}=45^{\circ}:$ a) phase transformation from austenitic recrystallized hot rolled steel, b) later cold rolling deformation of product material. Iso-levels: 1, 2, 3, ..

Finally, we proceeded to calculate the recrystallization final ferrite texture (figure $6 \mathrm{c}$ ) from the microscopic data of previous steps. It is observed again the presence of undesirable orientations about $\langle 110>\{110\}$ inherited from high energetic grains.

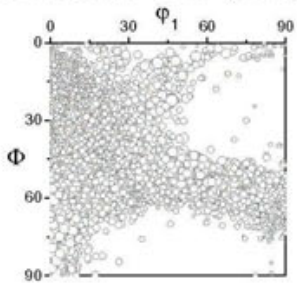

a)

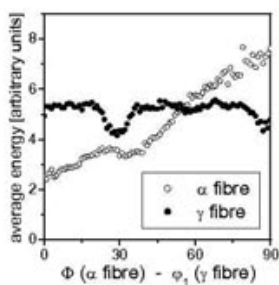

b)

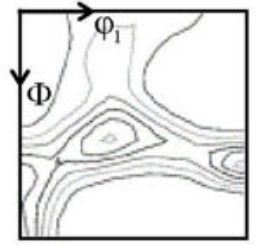

c)

Figure 6. a) Stored energy distribution at $\varphi_{2}=45^{\circ}$; b) average energy on $\alpha$ and $\gamma$ fibers, both at the end of cold rolling step of process $B$. c) Final recrystallization texture at $\varphi_{2}=45^{\circ}$. Isolevels: $1,2,3, \ldots$

\section{Concluding remarks}

In this contribution, we have shown that detailed microscopic physical information, resulting from VPSC micromechanical models, and results from a computational recrystallization model can be used as input values for transformation texture predictions with fairly reasonable success. An interesting feature of the present work is the simulation of complete processes from a random initial texture to a final product. This process involves texture development in hot rolling condition, possible recrystallization, depending on temperature range, phase transformation, cold rolling in ferrite phase and recrystallization. 
Orientations along $\gamma$ fibre in fcc-bcc transformation texture are necessary to obtain a good description of cold rolled texture of this material.

Average energy distribution on $\alpha$ and $\gamma$ fibres agree to a large extent with published experimental results. Calculations of stored energy for all grain orientations, which are very difficult to measure, allowed us to obtain ferrite recrystallization textures with a well defined $\gamma$ fibre.

\section{References}

1. Ray, R.K., Jonas, J.J. \& Hook, R.E., 1994, Int. Mat. Reviews, 39, 129.

2. Bain, E.C., 1924, Trans. AIME, 70, 25.

3. Kurdjumov, G. \& Sachs, G., 1930, Z. Phys., 64, 225.

4. Nishiyama, Z., 1934, Sci. Rep. Res. Inst., Tôhoku Univ., 23, 637.

5. Wassermann, G., 1933, Arch. Eisenhüttenwesen, 16, 647.

6. Tarasiuk J., Gerber Ph. \& Bacroix B., 2002, Acta Mater., 50, (6), 1467.

7. Gerber Ph., Tarasiuk J., Chauveau Th., \& Bacroix B. 2003, Acta Mater., 51, 6359.

8. Lebensohn, R.A. \& Tomé, C.N., 1993, Acta Metall. Mater., 41, 2611.

9. Bolmaro R., Roatta A, Fourty A. \& Signorelli J., 2005, Scripta Mater., 53, 147.

10. Charnock, W. \& Nutting, J., 1967, Metal Sci. J. 1, 123.

11. Roatta, A., Fourty, A. \& Bolmaro, R., 2008, to be published.

12. Bolmaro, R.E., Brokmeier, H.-G., Signorelli, J.W., Fourty, A. \& Bertinetti, M.A, 2004, in Diffraction Analysis of the Microstructure of Materials, edited by E.J. Mittemeijer \& P. Scardi ( Germany: Springer Series in Mat. Sci.), pp. 393-412.

13. Bolmaro R.E., Lebensohn R.A. \& Brokmeier H.-G., 1997, Comp. Mater. Sci., 9, 237.

14. Bolmaro, R.E., Fourty, A., Roatta, A., Bertinetti, M.A., Turner, P.A. \& Signorelli, J.W., 2000, Scripta Mater., 43, 553.

15. Manual "MTM-FHM" software system, 2004, P. Van Houtte, Department of Metallurgy and Materials Engineering, Katholieke Universiteit, Leuven, Belgium.

16. Savoie, J., Ray, R.K., Butrón-Guillén, M.P. \& Jonas, J.J., 1994, Acta Metall. Mater., 42, 2511.

17. Gardiola, B., Humbert, M., Esling, C., Flemming, G. \& Hensger, K.E, 2001, Mat. Sci. Eng. A, 303, 60.

18. Urabe, T. \& Jonas, J.J., 1994, ISIJ Int., 34, 435.

19. Zhou, Y. \& Neale, W.K., 1995, Int. J. Mech. Sci., 7, 1.

20. Wauthier, A., Bacroix, B., Chauveau, T., Castelnau, O. \& Régle, H., 2008, Proc. XV International Conference of Texture of Materials, Pittsburg, USA.

21. Rajmohan, N., Hayakawa, Y., Szpunar, J.A. \& Root, J.H., 1997, Acta Mater., 45, 2485

22. Samajdar, I., Verlinden, B., Van Houtte, P. \& Vanderschueren, D., 1997, Mat. Sci. Eng. A, 238, 343.

23. Wierzbanowski, K., Tarasiuk, J., Bacroix, B., Sztwiertnia, K. \& Gerber, P.. 2003, Metals Mater. Int., 9, (1), 9. 


\title{
ECAP of Fe. Experiments and simulations of the in-elbow textures
}

\author{
R. E. Bolmaro 1,", R. A. Renzetti ${ }^{2}$, M. J. R. Sandim ${ }^{2}$, \\ H. R. Z. Sandim ${ }^{2}$, J. W. Signorelli ${ }^{1}$, M. Ferrante ${ }^{3}$
}

${ }^{1}$ Instituto de Física Rosario, CONICET-UNR, Bv. 27 de febrero 210 bis, 2000, Rosario, Argentina

${ }^{2}$ Departamento de Engenharia de Materiais - Escola de Engenharia de Lorena - EEL, Universidade de São Paulo, 12600-970, Lorena, Brazil

3 Departamento de Engenharia de Materiais - Universidade Federal de São Carlos, São Carlos (SP) Brasil, 13.565-905

*bolmaro@ifir-conicet.gov.ar

Keywords: ECAP, steel, deformation texture, micromechanical simulations

\begin{abstract}
The study of deformation properties of low carbon steels is of particular interest because of their many technological applications. Obtaining fine grained Fe based materials can be approached by one of the several available Severe Plastic Deformation (SPD) techniques. The current paper shows experimental data and simulations of the deformation process of iron samples by Equal Channel Angular Extrusion (ECAE). The samples were extruded in a $120^{\circ}$ channel die either by one or a few passes. The heterogeneity and local development of the deformation on the elbow of the channel has been studied by X-ray measuring and simulation of the texture evolution. The Self Consistent models used for simulation allowed the calculation of the spin of the main texture components which agreed pretty well with the experiments.
\end{abstract}

\section{Introduction}

Besides alloying modifications different ways of thermomechanical processing of steels are designed for the obtainment of novel microstructures and properties. Recently the technique of applying SPD to metals and alloys allows microstructural changes based mainly on the capacity to reduce grain sizes and engineer grain boundaries [1-4]. Large accumulated energies, due to the SPD process, can be further used, either partially or in total, to obtain recovered and recrystallized structures with new properties [5]. Among the most studied materials we find the FCC metals and alloys. However, lower symmetry materials are rarer. BCC materials have been studied but some details of their behaviour and particularly the technological consequences of their SPD processing are far from being understood. 


\section{Experiments}

A hot-forged iron slab containing 40 wt-ppm C, 940 wt-ppm Mn, and 160 wt-ppm Si was used in this study. The slab was machined into a $14 \times 14 \times 70 \mathrm{~mm}^{3}$ billet and annealed at $600^{\circ} \mathrm{C}$ for $2 \mathrm{~h}$ before ECAE processing to get a full-recrystallized structure. The annealed material displayed a coarse-grained structure with grain sizes in the range 100-500 $\mu \mathrm{m}$. The billet was partially processed by ECAE (half of its total length) at room temperature using an extrusion speed of $5 \mathrm{~mm} / \mathrm{min}$. The ECAE die has $\phi=120^{\circ}, \psi=0^{\circ}$, and $\mathrm{R}=8 \mathrm{~mm}$, as shown in fig. 1 (a). Five slices were cut from the billet making approximately $12^{\circ}$ to each other, as depicted in fig. 1 (b). Textures were determined in the five slices using an X-ray Philips MPD goniometer $\left(\mathrm{CuK}_{\alpha}\right.$ radiation). The results were analyzed using the popLA software package by which a quite good compatibility of pole figures could be obtained. Recalculation of the measured pole figures allowed the obtainment of the different pole figures either on the ND, TD or ED planes of the sample.

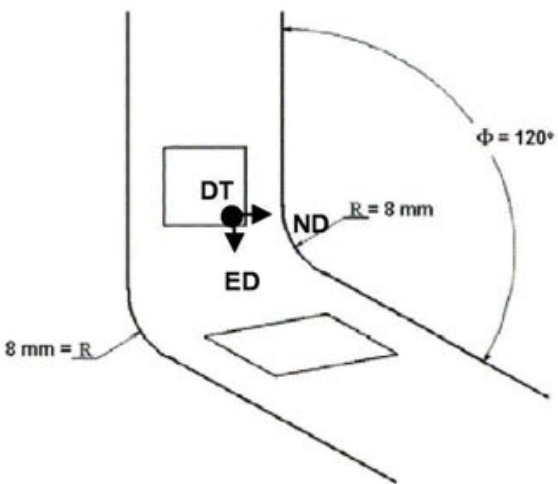

(a)

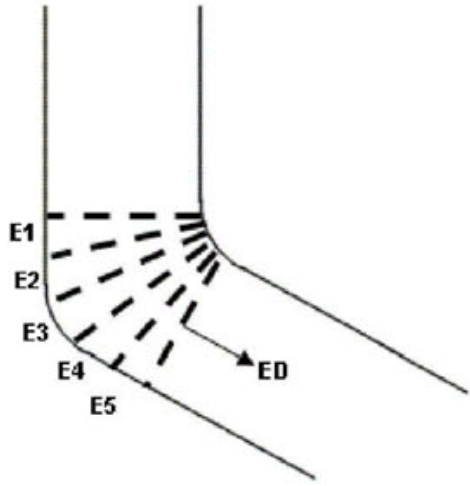

(b)

Figure 1. Schematic drawing showing the cross section of the ECAE die: (a) die geometry; and (b) five slices of billet after partial extrusion. The extrusion direction (ED), the normal direction (ND) and the transverse direction (TD) are indicated.

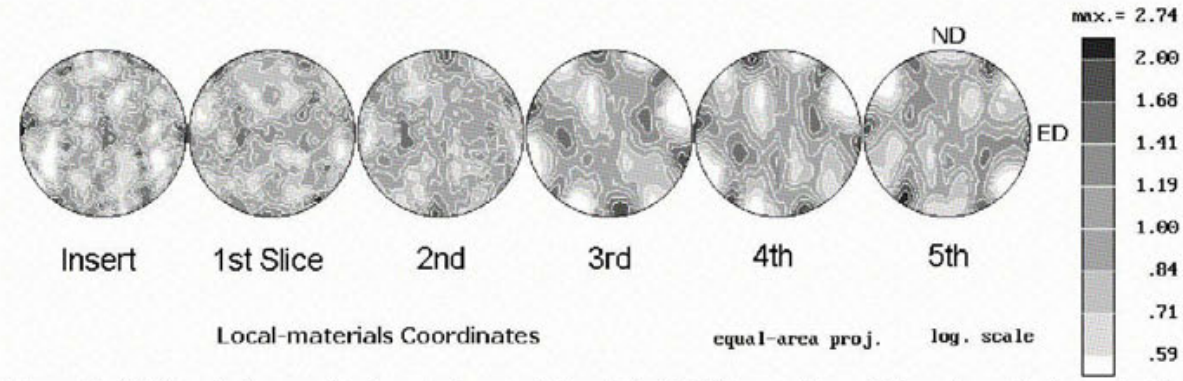

Figure 2. (100) pole figures for inserted material and the 5 different slices. Taken from the lateral side. 
Figure 2 shows the (100) pole figures, as observed from the TD direction, for the different slices cut every $12^{\circ}$ on the ECAE tool elbow. What is called the ED is the local ED direction. It means that the ED direction is rotated $12^{\circ}$ around the TD for each slice until it reaches the orientation of the outgoing ED. The pole figures show the typical swirling aspect of every simple shear deformation. The texture sharpness strengthens until the slice number 3 but stays almost on the same severity from there on. One of the components can be used to calculate the relative spin of the patterns with respect to the local sample axes to be later on compared with the simulations. The rest of the pole figures are not shown for the sake of compactness of the presentation.

A careful metallographic observation was made on the side faces of the samples after complete deformation. Samples were polished and etched by using OP-CHEM polishing cloth and OP-S (Struers) polishing suspension and later on chemically etched during 5-10 seconds with a solution of $50 \mathrm{ml}$ of ethylic alcohol and $1.5 \mathrm{ml}$ of nitric acid. Two of the metallographies are shown in figure 3 . They show that some special slip directions develop in different grains following approximately two different directions on the sample: a) direction along the main shearing plane on the channel elbow and b) direction along the extrusion direction of the channel. As it has been shown by Signorelli et al. [6] the contribution of each grain to the average deformation in ECAE may be achieved through a limited number of slip systems for each crystal. That feature is shared by almost any deformation process including fragmentation and it has important implications for any simulation attempt by micromechanical modelling.
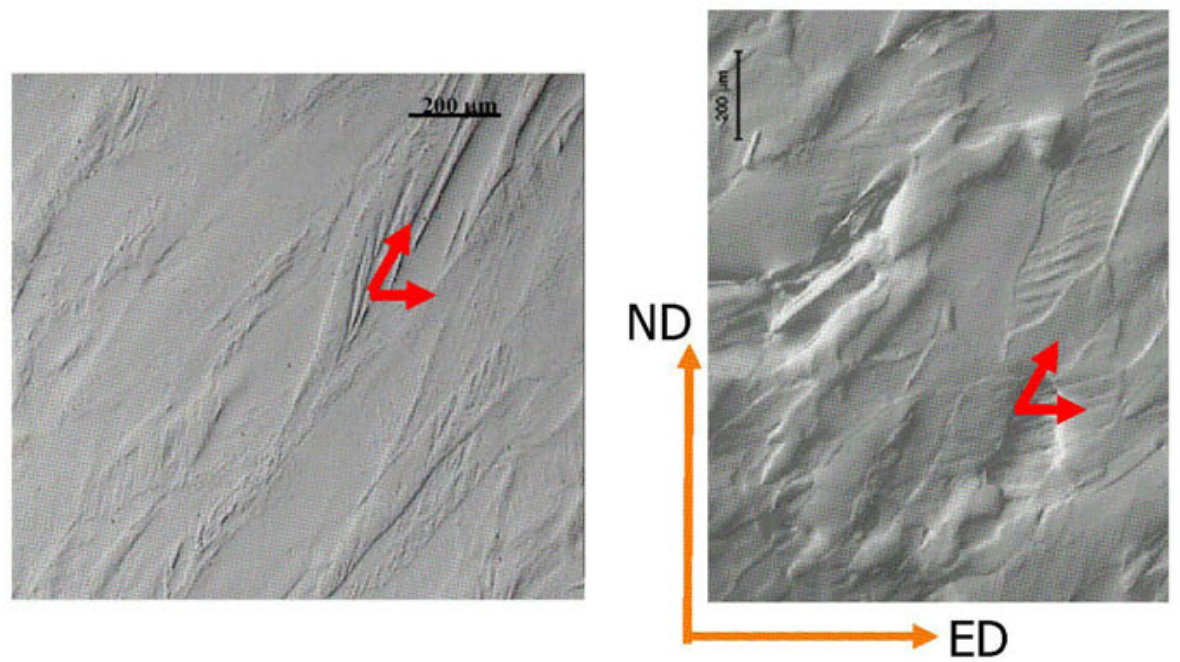

Figure 3. Metallographies showing different grains with the most active directions along main shear planes contained on the elbow shear plane and the extrusion direction (Nomarski contrast). 


\section{Texture modelling}

Texture simulations, to be compared with the experimental results, were performed using a rate-dependent Viscoplastic Self-Consistent model (VPSC), with capabilities to take in account the heterogeneity and anisotropy of each crystal. Each grain deforms homogeneously following a viscoplastic constitutive law [7]. The stresses and strains in each grain are unknowns related by a viscoplastic law and connected with their macroscopic counterparts by a Self Consistent homogenization micro-macro transition. This scheme allows each grain to deform somehow different from the rest depending on its orientation, anisotropy and previous deformation. The interaction between the grain and the matrix is solved by the Eshelby formalism [8,9] by following the approach of Lebensohn and Tomé [10].

The starting material comprised 1000 randomly oriented grains with the same weight for each one. The slip systems were chosen as usual for BCC materials as: $\{110\}<111>$, $\{112\}<111>,\{123\}<111>$ with Critical Resolved Shear Stresses (CRSS) given by 0.058 , 0.056 and $0.060 \mathrm{MPa}$, respectively, and no hardening due to deformation. The code was run on the assumption of a tangent interaction between micro and macro behaviour and in steps of $1 \%$ von Mises deformation. Considering the well established phenomenon of activation of very few slip systems in each grain or fragment, quite far from the 5 slip systems required by Taylor models, the VPSC model was set with a stress exponent $n=50$ which allows the crystals to deform by using an average of 2-3 slip systems. The calculations were performed assuming two scenarios: a) homogeneous deformation along the shear plane b) heterogeneous deformation along that plane.

For the second case a varying velocity gradient was calculated by using Finite Elements Methods (FEM) numerical simulations. The program was FORGE2005 $\mathbb{R}$, commercial FEM software, specialized in materials forming [11]. It solves thermal and/or mechanical boundary value problems using an updated Lagrangean scheme. The FEM is based on a mixed velocity-pressure formulation with an enhanced $(\mathrm{P} 1+/ \mathrm{P} 1)$ 4-node tetrahedral element.

\section{Texture simulation results and comparisons}

Fig. 4 shows the results obtained assuming an ideal velocity gradient applied uniformly distributed along the elbow and developing at equal intervals like if it were stopped every $12^{\circ}$ to simulate the texture of every slice. The textures show the typical swirling effect given by shear deformation overlapped by the rigid rotation of $12^{\circ}$ given by the reorientation of the extrusion direction. The severity of the texture is due mainly to the fact that none of the heterogeneities, neither the macroscopic nor the microscopic ones, developed during deformation are included on the simulation. Self Consistent models allow for the consideration of some minor heterogeneity among crystals but the main source of microseopic heterogeneity, grain fragmentation, has to be included by different approaches. Considering that the purpose of the research was only to understand the evolution of textures on the very shear plane, we will not include this kind of heterogeneity, either on this approach or in the hybrid FEM model, but only a Gaussian smoothing of $5^{\circ}$ as a further randomizing effect.

The macroscopic heterogeneity, the one stemming from the forming process and boundary conditions imposed by the tools, is approached by using the velocity gradients calculated with FEM. They were calculated assuming that the flow is so restricted by the ECAE test 
that the evolution of textures does not modify the velocity gradient itself in the different locations. So the velocity gradient can be calculated assuming a regular isotropic plastic material and later on it can be used in independent simulations of texture. The results of each simulation can be added in a single file representing the average resultant texture. Figure 5 shows the simulations obtained by applying 6 different velocity gradients calculated by FEM, at equidistant intervals from the corner of the elbow along the shear plane, and adding the results of the 6 velocity gradients at intervals of time equivalents to the $12^{\circ}$ apart slices. The same swirling and rotational effects are overlapped. The results are less sharp but still $30 \%$ sharper than the experimental texture.

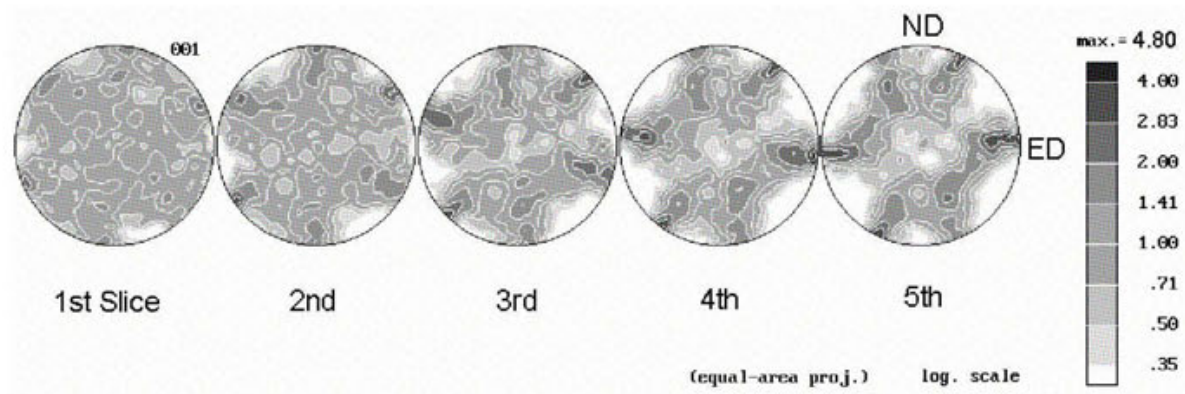

Figure 4. (100) PF simulated by assuming ideal shear on elbow plane for slices 1 through 5 and further $5^{\circ}$ Gaussian smoothing.

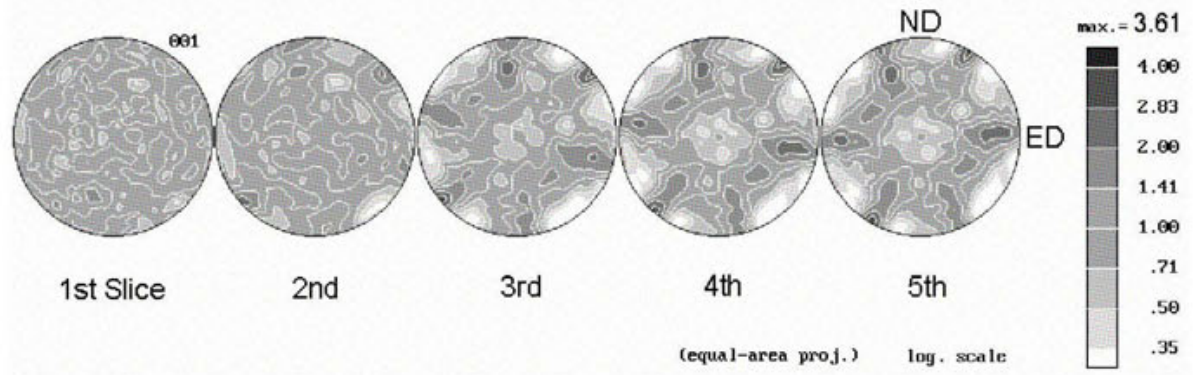

Figure 5. (100) PF simulated by considering 6 different velocity gradients calculated by FEM, adding the resultant textures and further $5^{\circ}$ Gaussian smoothing. Slices 1 through 5 .

\section{Analysis and conclusions}

The development of the main components is due to the combined effect of both shearing effect and the sample axes rotation. By analysing the experimental results and the simulations we can calculate the rotation angle of the main components on the three cases. Table 1 shows the angles of rotation of the spinning components (measured by just one of them, the first one to appear on the right-top quadrant) with respect to a hypothetical horizontal reference plane. Except for the first slices, where the maxima are difficult to determine, some resemblance between the measured and simulated pole figures can be observed. In some 
slices FEM simulations seem to perform better than the ideal shear approach. However we must keep in mind that textures are measured every $5^{\circ} \times 5^{\circ}$ and that errors for sample positioning and mathematical analysis can reach around half of that interval. The superior capabilities of FEM based models for simulations of textures in heterogeneous deformation processes, because of the possibility of taking in account macroscopic heterogeneities, are apparently more on matching the intensities than on matching the location of the components. Generation of dislocation microstructures, by allowing crystallites to co-spin and fragment, should also be included in the simulations, as it was done in [11]. However, it was not the purpose of the current paper to evaluate lower levels of heterogeneity but only the influence of in-elbow macroscopic heterogeneity.

Table 1. Angle formed by the first maxima on the right-top quadrant, measured c.c.w. from the horizontal line.

\begin{tabular}{|c|c|c|c|c|c|}
\hline Slices $\rightarrow$ & 1 & 2 & 3 & 4 & 5 \\
\hline Experimental & 19 & 23 & 48 & 55 & 57 \\
\hline $\begin{array}{c}\text { Simulation } \\
\text { Ideal shear }\end{array}$ & $23 ?$ & 34 & 44 & 55 & 64 \\
\hline $\begin{array}{c}\text { Simulation } \\
\text { FEM }\end{array}$ & $\iota ?$ & 42 & 42 & 50 & 60 \\
\hline
\end{tabular}

\section{References}

1. Aida, T., Matsuki, K., Horita, Z. \& Langdon, T.G., 2001, Scripta Mater., 5, 575.

2. Iwahashi, Y., Horita, Z., Nemoto, M. \& Langdon, T.G., 1998, Acta Mater., 46, 3317.

3. Valiev, R.Z., 2004, Nature Materials, 3, 511.

4. Zhu, Y.T. \& Lowe, T.C., 2000, Mater. Sci. Eng. A, 291, 46.

5. Prangnell, P.B., Hayes, J.S., Bowen, J.R., Apps, P.J. \& Bate, P.S., 2004, Acta Mater, 52, 3193.

6. Signorelli, J.W., Turner, P.A., Sordi, V., Ferrante, M., Vieira, E.A. \& Bolmaro, R.E., 2006, Scripta Materialia, 55, 1099.

7. Molinari, A., Canova, G.R. \& Ahzi, S., 1987, Acta Metall., 35, 2983.

8. Eshelby, J.D., 1957, Proc. Roy. Soc. London A, 241, 376.

9. Mura, T, 2001, Micromechanics of Defects in Solids, 2nd ed. (Dordrecht: MartinusNijhoff).

10. Lebensohn, R.A.\& Tomé, C.N., 1993, Acta Metall. Mater., 41, 2611.

11. Mocellin K., Fourment L., Coupez T. \& Chenot J.-L., 2001, Int. J. Num. Meth. Eng., 2, 473-488.

Acknowledgements. This work has been partially funded by the Project PICT-12306. ANPCyT, (Argentina). Authors are also grateful to CNPq (Brazil) for supporting this work. 


\title{
Experiments and simulation evaluation in quartz veins textures in the Guamanes shear belt, Córdoba Pampean Ranges, Central Argentina
}

\author{
A. Fourty ${ }^{1}$, H.-G. Brokmeier², R. Martino ${ }^{3}$, \\ R. E. Bolmaro ${ }^{1, *}$
}

${ }^{1}$ Instituto de Física Rosario, CONICET-UNR., Bv. 27 de febrero 210 bis, 2000 Rosario, Argentina

${ }^{2}$ Institute of Materials Research, GKSS-Research Centre, Max-Planckstr. 3, D-21502 Geesthacht, Germany

3 CONICET and Cátedra de Geología Tectónica, F.C.E.F. y N. Universidad Nacional de Córdoba, Av. Velez Sarsfield 1611, X 5016 GCA-Córdoba, Argentina

*Bolmaro@ifir-conicet.gov.ar

Keywords: quartz texture, VPSC micromechanical simulation, microstructural geology

Abstract. Texture characterization of deformed rocks allows the confirmation of kinematically determined deformation processes occurring during geologic events at plate margins and in intraplate settings. The understanding of such processes is of both basic and economical importance to decipher deformation regimes, mechanisms and PresureTemperature (PT) conditions.

The Guamanes Deformation Belt (GDB) is a shear zone at Sierras de Córdoba (Argentina) which extends approximately 45 kilometers parallel to the $64^{\circ} 50^{\prime} 00^{\prime}$ ' meridian. Its width varies in between 1 and 4 kilometers and can be subdivided in a northern and southern tracts. The southern part has been already quite well studied with a complete description of its mylonitic rocks and the kinematic. Phyllonites in the northern tract and mylonites with phyllonites and non deformed rocks in the southern tract apparently separate two well defined domains of deformation. Two different deformation events, one ductile followed by a brittle stage, have been identified. We will present ductile textures developed in quartz veins included in strongly deformed granitic pegmatites which were intruded in the shear belt during the deformation. The data will be analyzed by regular texture analysis and viscoplastic self consitent simulations.

The kinematic inferred from the simulations indicates a main reverse sense of displacement with a minor sinistral component developed in a simple shear regime. 


\section{Introduction}

The GDB is a shear zone located at the Sierras de Córdoba (Argentina) extending parallel, by ca. $45 \mathrm{Km}$, to the $64^{\circ} 50^{\prime} 00^{\prime \prime}$ meridian. Its width varies along its strike in between $1-4 \mathrm{Km}$ and it is possible to separate a Northern and a Southern tract. Martino [1,2] defined the GDB shear zone and studied it with certain detail in its Southern tract, describing the mylonitic rocks that lie along it and establishing its kinematics. This last author recognized the prevalence of phyllonites in the north and of mylonites with intercalations of phyllonites and undeformed rocks in the south of the Southern tract. These rocks, according to Martino [1, 2], separate two regional domains of deformation named A and B. Recently Lyons et al. [3] studied the GDB in its Northern tract defining rocks of the series of the mylonites lying inside it. They dated the last event that affected the belt in $360 \mathrm{Ma}$ (Devonian) and proposed that the tectonic activity would have begun in the Early Cambrian. This belt would separate here two different metamorphic complexes named Pichanas (Domain B) and Cruz del Eje (Domain A) Complexes, that are respectively to the west and east of the shear zone.

Two events of deformation have been identified in the GDB shear zone, in which a ductile stage is continued by another fragile one. The most conspicuous feature is a fanning mylonitic foliation, being low angle to the west and subvertical to the east, with an average foliation plane of $\mathrm{N} 354^{\circ} / 70^{\circ} \mathrm{E}$. On this plane, clusters of quartz, forming stripe and stretching lineations, with average values of $\mathrm{N} 30^{\circ}-80^{\circ}$ of strike and $20^{\circ}-80^{\circ}$ of dip, are recognized. The sense of displacement using diverse kinematic indicators recognized in different lithologies show that the Guamanes Shear Zone is sinistral with a strong reverse component and it is supposed that it has been formed by a simple shear mechanism.

One of the several lithologies deformed by the shear zone are granitic pegmatites of which the sample 1162, that will mainly be investigated in the current presentation, is representative. Quartz veins, deformed jointly with the pegmatites, also cut through those pegmatites. The GDB shear zone does not affect the Achala batholith dated in $368 \pm 2 \mathrm{Ma}$ (U-Pb zircon, Dorais et al. [4]), for what it is inferred that their age would date from the Early Palaeozoic. It should be noted that auriferous veins were also emplaced along the strike of the shear zone.

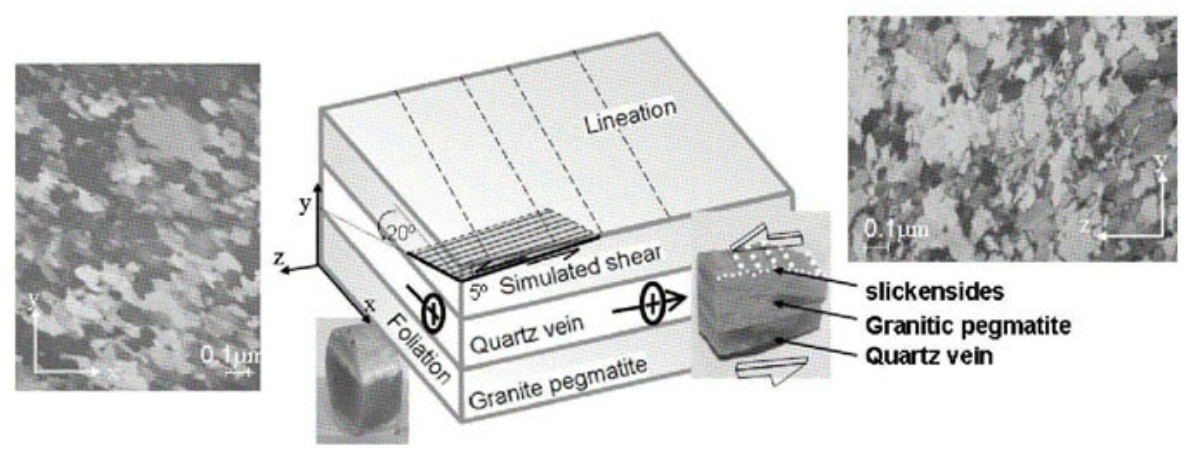

Figure 1. Scheme and photographs of the quartzite sample and coordinates used for the analysis. Micrograph of section XZ (perpendicular to foliation and parallel to lineation), and micrograph of section YZ (perpendicular to foliation and to lineation). 
The Guamanes shear zone was probably developed when the Laurentian Precordillera Terrain docked against the pacific margin of Gondwana during Ordovician - Silurian times. After that a new terrain accretion occurred when the Chilenia Terrain was emplaced against the sutured Precordillera-pacific margin of Gondwana producing the Devonian reactivation of several shear zones including Guamanes Shear Zone.

\section{Experiments}

Several samples were collected from the described region. A photograph of the sample that will occupy the main part of the paper, named as 1162, is shown in figure 1 . The reason to focus in just one sample is two-fold: scarcity of space and the need for testing compatibility between symmetry assumptions and external markers and simulation procedures. The quartz vein is well separated from the granitic pegmatite region. Textures for the quartz vein were measured by both X-ray diffraction on a Philips MPD texture goniometer and by neutron diffraction on Tex-2 machine, GKSS, Geesthacht, Germany. Results are shown in figure 2 for the $(0001)$ and $(10 \overline{1} 0)$ pole figures reconstructed from the experimental pole figures $(10 \overline{1} 0),(10 \overline{1} 1),(11 \overline{2} 0)$ and $(11 \overline{2} 2)$. The recalculation was made by usual tools of texture analysis (popLA and Beartex based softwares) including Orientation Distribution Function determination. Neutron textures were in principle used to allow a better statistical data recollection but the close match between X-rays and neutron results showed that there was no heterogeneity on the vein and that X-rays were a perfect tool for texture evaluation. Samples for X-ray measurements were grind polished until 2000 grit paper and later on polished with colloidal silica. Neutron textures are not shown because of the lack of available space.

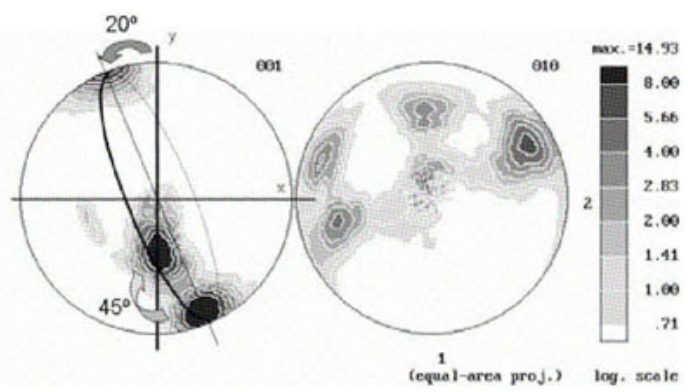

Figure 2. Texture measured by X-ray diffraction in a Philips MPD equipment with Eulerean cradle, $X$-ray lens and parallel beam geometry.

\section{Simulations}

There is always a lack of experimental data on the simulation of the development of textures in rocks. External markers, as foliation and lineation, together with grain shape determination should suffice to construct a trustable model of the cinematic of the deformation. However, conspiring against it are the many entangled thermomechanical processes through which 
each particular region has gone. A superposition of processes may erase precious information, like shear plane and shear direction usually reflected on foliation or lineation of the rocks. Sometimes it might also be that external markers are contradictory or misleading like, for instance, grain shapes and morphological textures, that can be modified at intermediate stages by recrystallization processes. In those cases, when the exact occurrence of the phenomena is unknown, the orientation of the main axes of the grains does not convey information than can be used on the analysis.

One of the main purely geometrical characteristics provided by pole figures are the symmetries associated with the crystal symmetry together with the deformation symmetry. They are reflected on the symmetry of the pole figures and they should be respected and should be compatible with the deformation process imposed by any model [5]. The current paper shows an application of that principle on the deduction of the shearing plane and direction to be used on the development of a prospect scenario of the geological processes in the area.

On figure 2 we have included the main symmetry elements of the pole figure. It is clear that because of the lack of a proper symmetry the pole figures can not be understood as a simple shear contained on the plane of the paper, which would be the first choice given the lineation direction observed on the rock. The shear direction suggested by the symmetry seems to be contradictory with the lineation direction. On figure 1 the traces of what are called lineation marks are evident. However we have drawn arrows indicating the shear direction perpendicular to those lines, on the interpretation that those marks are actually a consequence of fragmentation processes happening at approximately $90^{\circ}$ of the main shearing stress [6]. Those marks would be more of the kind revealed by Paterson [6] as happening at low confining pressures and called slickensides. Those grooves could be interpreted as showing an easy gliding direction along the slippery direction, with the steps on one wall facing the direction towards which the other wall moves [7]. However, when the failure is somehow cataclastic instead of a gliding character they run perpendicularly to the shear direction. Assuming that the symmetry elements of the pole figures are to be followed as main markers still we need a model able to produce a splitting of the $\langle\mathrm{c}\rangle$ axes to produce two main poles (actually 4 because of the axes crystal multiplicity). That phenomenon would happen only in the case of having crystals reorienting with high sensitivity to the initial orientation. That means that only very few, probably 1 or 2, slip systems should be preferentially active at each moment. A polycrystalline Self Consistent rate dependent model, with capabilities to take in account the heterogeneity and anisotropy of each crystal, is used to simulate the behavior of the quartz vein. Each grain deforms homogeneously following a viscoplastic constitutive law (VPSC) [8]. The stresses and strains in each grain are unknowns related by a viscoplastic law and connected with their macroscopic counterparts by a Self Consistent homogenization micro-macro transition. This scheme allows each grain to deform somehow different from the rest depending on its orientation, anisotropy and previous deformation. The interaction between the grain and the matrix is solved by the Eshelby formalism $[9,10]$ by following the approach of Lebensohn and Tomé [11].

The crystal behaviour follows a potential law relating the applied shear stress $\tau^{\mathrm{s}}$ in each plane and the Critical Resolved Shear Stress (CRSS) of that system $\tau_{\mathrm{c}}^{\mathrm{s}}$. The total strain rate $\mathbf{d}$ in each grain is obtained adding the shear strain rates of each active slip system s: 


$$
\mathbf{d}=\dot{\gamma}_{0} \sum_{s} \frac{\mathbf{m}^{s} \otimes \mathbf{m}^{s}}{\tau_{\mathrm{c}}^{s}}\left|\frac{\mathbf{m}^{s}: \mathbf{s}}{\tau_{\mathrm{c}}^{s}}\right|^{\mathrm{n}-1}: \mathbf{s}=\mathbf{M}: \mathbf{s}
$$

were $\mathbf{S}$ is the microscopic deviatoric stress, $\mathbf{m}^{\mathbf{s}}$ is the Schmid tensor describing the geometry of the slip system $s$ in the single crystal, $\dot{\gamma}_{0}$ is a reference strain rate velocity, $\mathrm{n}$ is the inverse of the strain rate sensitivity, $\mathbf{M}$ is the secant viscoplastic compliance crystal modulus and $\otimes$ means the tensor product of involved quantities. The material behaviour on viscoplastic regime is described through the following expression:

$$
\overline{\mathbf{d}}=\overline{\mathbf{M}}(\overline{\mathbf{s}}): \overline{\mathbf{s}}
$$

where $\overline{\mathbf{M}}$ is the overall secant viscoplastic compliance modulus.

The interaction equation relates micro (grain-ellipsoid) and macro $\overline{\mathbf{d}}$ (polycrystal-HEM) strain rate differences, with micro and macro $(\overline{\mathbf{S}})$ deviatoric stresses differences:

$$
\text { d } \overline{\mathbf{d}}=-\tilde{\mathbf{M}}(\mathbf{s}-\overline{\mathbf{s}})
$$

where $\widetilde{\mathbf{M}}$ is the interaction tensor, which is a function of the overall modulus and the shape and orientation of the ellipsoid that represents the embedded grain. The macroscopic (overall) secant modulus can be adjusted iteratively using the following self-consistent equation:

$$
\begin{aligned}
& \overline{\mathbf{M}}=\left\langle\mathbf{M}:(\mathbf{M}+\tilde{\mathbf{M}})^{-1}:(\overline{\mathbf{M}}+\tilde{\mathbf{M}})\right\rangle \\
& \overline{\mathbf{d}}=\overline{\mathbf{M}}: \overline{\mathbf{s}}
\end{aligned}
$$

We considered 1000 initially spherical and random oriented grains. Quartz crystal is treated as hexagonal. We assumed that strain is accommodated by slip on basal $\langle a\rangle$, prismatic $\langle a\rangle$, pyramidal $\langle a\rangle$ and pyramidal $\langle\mathrm{c}+\mathrm{a}\rangle$ with the corresponding relative critical resolved shear stresses, 1, 6, 3, and 6, following values reported by Wenk et al. [12]. The stress exponent was taken equal to 30 .

In the present study, the strain hardening between slip systems is taken into account by adopting an isotropic hardening. Then, the evolution of the critical shear stresses is given by:

$$
\dot{\tau}_{\mathrm{c}}=\sum_{s} \mathbf{h}^{s}\left|\dot{\gamma}^{s}\right|
$$

where $\mathbf{h}^{s}$ are the hardening moduli which are taken equal to 3 .

We performed a shear test over a plane tilted $5^{\circ}$ around $\mathbf{\underline { }}$ axis and $20^{\circ}$ around $\underline{\mathbf{z}}$ axis, as it was suggested by de pole figure symmetry. The resulting pole figures are shown in figure 3 . The patterns are more laterally spread than the experimental ones. The difference in severity can be due to at least two phenomena. First, there are clues on the samples suggesting the presence of a second event of deformation that has not been considered. Second, quartz easily recrystallizes after certain amount of deformation. Recrystallization might be able to concentrate the components already developed by deformation. 


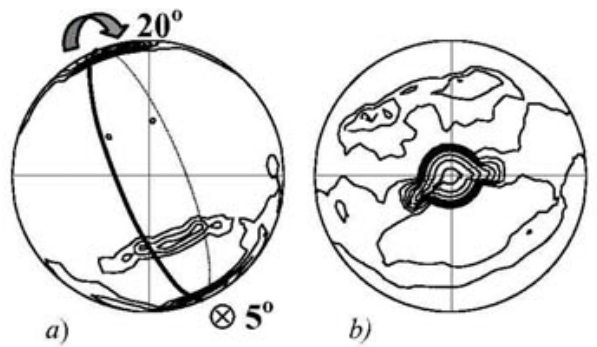

Figure 3. Simulated pole figures. a) Basal poles, isolevels: 2, 4, 6, 8, 10, 12. b) Prismatic poles, isolevels: $1,2,3,4,5,7,9,11$.

\section{Conclusions}

The analysis of the symmetries during deformation of polycrystals is frequently oversimplified on the assumptions about the main deformation axes imposed during the process. On rocks, and generally speaking, on structural geology there are many external markers that have to be carefully analyzed to deduce the external stress and strain fields that have been acting on the region of interest. We have emphasized here one of those markers, that usually goes unattended on simulations, and its symmetry. The symmetry of the texture itself is a powerful information even when it seems to be in contradiction with external markers as grain shape, lineation and/or foliation. In the current case we were able to simulate textures very close to the experimental results in a case that presented rather difficult to interpret patterns. The slickensides concept allows, once the symmetry of the pole figure is followed as the main marker, to interpret the lineation marks as the direction of occurrence of cataclastic failure at approximately $90^{\circ}$ with respect to the shearing direction. The textures are reconciled with external markers information once the symmetry of the texture is taken as the main track of the deformation process. The simulations allow elucidating the influence of the different tilting and rotation angles on the actual symmetry of the pole figures. The sharpnesses of the simulated components are low comparing with the experimental results and we propose to interpret that inconsistency as a consequence of recrystallization phenomena.

\section{References}

1. Martino, R.D., 1988, Geología y petrología del basamento metamórfico de la región situada al norte de Cuchilla Nevada, Sierra Grande de Córdoba, PhD Thesis, Facultad de Ciencias Exactas, Físicas y Naturales, Universidad Nacional de Córdoba, $\mathrm{N}^{\circ}$ 276, 174 p., 29 fig., 10 maps (unpublished).

2. Martino, R.D., 1993, La faja de deformación Guamanes: petrografía, estructura interna y significado tectónico, Sierra Grande de Córdoba, Revista de la Asociación Geológica Argentina, 48, (4), 417.

3. Lyons, P., Skirrow, R.G. \& Stuart-Smith, P.G., 1997, Geology and Metalogeny of the "Sierras Septentrionales de Córdoba", Argentine-Australian Cooper Project, Australian Geological Survey Org. Subsec. de Minería de la Nación, Argentina, 132 p. 
4. Dorais, M.J., Lira, R., Chen, Y. \& Tingey, D., 1997, Origin of biotite-apatite-rich enclaves, Achala batholith, Argentina, Contrib. to Miner. Petrology, 130, 31.

Paterson, M.S. \& Weiss, L.E., 1961, Bull. Geolog. Soc. America, 72, 841.

5. Paterson, M.S., 1958, Bull. Geolog. Soc. America, 69, 465.

6. Hills, E.S., 1953, Outlines of Structural Geology (London: Methuen \& Co. Ltd.).

7. Molinari, A., Canova, G.R. \& Ahzi, S., 1987, Acta Metall., 35, 2983.

8. Eshelby, J.D., 1957, Proc. Roy. Soc. London, A241, 376.

9. Mura, T., 1991, Micromechanics of Defects in Solids, 2nd ed. (Dordrecht: MartinusNijhoff).

10. Lebensohn, R.A.\& Tomé, C.N., 1993, Acta Metall. Mater., 41, 2611.

11. Wenk, H.-R, Canova, G., Molinari, A. \& Kocks, U.F., 1989, J. Geophys. Res., 94, B12, 17895. 



\title{
The use of extinction phenomenon for investigation of textured thin film microstructure
}

\author{
T. Kryshtab ${ }^{1, *}$, A. Kryvko ${ }^{2}$
}

${ }^{1}$ Instituto Politecnico Nacional-ESFM, Av. IPN, Ed. 9 U.P.A.L.M., 07738, Mexico D.F.

${ }^{2}$ Instituto Politecnico Nacional-ESIME, Av. IPN, Ed. 4 U.P.A.L.M., 07738, Mexico D.F.

"tkrysh@esfm.ipn.mx

Keywords: X-ray diffraction, extinction, thin film, texture, microstructure

\begin{abstract}
Evaluation of microstructure in films with large and perfect grains by the conventional X-rays diffraction methods is impossible when the shape of the diffracted peak reaches the instrumental one. In this case the dynamic seattering processes take place and the extinction phenomenon, related to the crystal microstructure, is observed. In textured films the diffracted intensity is affected both by grains orientation (pole density) and extinction phenomenon. The results of the microstructure investigation of the annealed and co-doped with $\mathrm{Cu}$, $\mathrm{Cl}$ and $\mathrm{Ga} \mathrm{ZnS}: \mathrm{Cu}$ thin films using extinction phenomenon are presented. D8 Bruker X-ray diffractometer with an Euler cradle and two non-polarized $\mathrm{Cu}$ and $\mathrm{Co}$ radiations was used. Low and high index reflections were measured. The evaluation of thin film microstructure was performed by separation of the extinction phenomenon in pole density and determination of the extinction coefficients. The domain thickness was evaluated from the values of the primary extinction and the extinction length. The average domain disorientation angle was determined from the value of the secondary extinction. The dislocation density at domain boundaries was calculated from average domain disorientation angle and domain thickness. The influence of film microstructure on the incorporation of $\mathrm{Cu}$ and $\mathrm{Cl}$ dopants into $\mathrm{ZnS}$ lattice and as the result a decrease of the phase transition temperature are demonstrated.
\end{abstract}

\section{Introduction}

Polycrystalline thin films prepared by different deposition techniques commonly have texture. Due to a highly dynamic process of film deposition it is impossible to predict the texture and microstructure. The X-ray diffraction (XRD) technique for texture investigation provides information on the volume fraction of the sample with grains orientation in accepted format as a pole figure (PF) [1]. But PF, obtained from the integrated intensity of diffracted X-rays, does not contain information about the microstructure characteristics. The problems of the microstructure analysis of imperfect crystals, based on XRD peak broadening, have been elaborated in the framework of the kinematical scattering theory $[2,3]$. However, the analysis of distortion fields associated with dislocations by means of evaluation of peak profile broadening does not give any reasonable results applied to real crystals with 
dislocation densities less than $10^{8} \mathrm{~cm}^{-2}$, in which dynamical scattering processes take place. An increase of grain size and an improvement of grain crystalline quality as a consequence of post-deposition treatments lead to a decrease of the diffracted integrated intensity owing to extinction phenomenon and reaching the full-width at half maximum (FWHM) of the diffracted peak to the instrumental one. The integrated intensity from textured thin films contained large and perfect grains is affected both by the grains orientation such as a pole density (PD) and by the extinction phenomenon. At the same time the characteristics of extinction are related to the crystal microstructural feature [4] and can be used for its evaluation. The conceptions of the primary and the secondary extinctions were introduced by Darwin and characterize two different phenomena [5]. The primary extinction concerns with the dynamical scattering and occurs in perfect domains with size larger than $10^{-4}-10^{-5} \mathrm{~cm}$, while the secondary extinction depends on domains disorientation and takes place when the domains are sufficiently similarly oriented [5]. In our previous publication [4] the use of extinction coefficients for microstructure evaluation of textured aluminum samples was demonstrated. The proposed technique is based on some assumptions and thin films have their peculiarities as compound composition and thickness, so the application of this technique for thin films studied must be developed.

$\mathrm{ZnS}$ with $\mathrm{Cu}$ doping can be used as green and blue emitting active layer in various optoelectronics devices. The operating characteristics of the devices, based on thin films, strongly depend on the thin film texture and microstructure (domain size, dislocation density, concentration of dopants, and effect of their incorporation into the lattice), which are determined by deposition technique and post-deposition treatment. Stabilization of $\mathrm{Cu}$ monovalent cation in bivalent $\mathrm{Zn}$ sublattice can be obtained by the use of $\mathrm{Cl}$ monovalent anion or Ga trivalent cation. It was shown in our previous work [6] that the phase transition of $\mathrm{ZnS}$ : $\mathrm{Cu}$ thin film from cubic to hexagonal structure can be observed at lower temperature by the use of $\mathrm{Cl}$ as co-doping element. In order to explain this effect it was proposed a model, based on thin film microstructure and dopants incorporation into $\mathrm{ZnS}$ lattice.

The aim of the work is to investigate $\mathrm{ZnS}: \mathrm{Cu}$ thin films microstructure after a special annealing and co-doping with $\mathrm{Cl}$ and $\mathrm{Ga}$ by the use of extinction phenomenon and to clarify the influence of dopants distribution in $\mathrm{ZnS}$ lattice on phase transition temperature on the base of microstructure characteristics, obtained from the coefficients of the primary and secondary extinction. As far as we know, the primary and the secondary extinction phenomena have not been applied simultaneously for microstructure determination of textured thin films.

\section{Experimental details}

The samples used for investigation consist of $\mathrm{ZnS}: \mathrm{Cu}$ thin films deposited by electron beam evaporation method onto $\mathrm{BaTiO}_{3}$ substrates with thickness about $1 \mu \mathrm{m}$ after a special annealing at the atmospheric pressure. The annealing and co-doping of $\mathrm{ZnS}: \mathrm{Cu}$ films were carried out at $600-800{ }^{\circ} \mathrm{C}$ by embedding the samples in the blend of $\mathrm{ZnS}$ powder with the compounds of $\mathrm{Cu}$ and $\mathrm{Cl}(\mathrm{Cu}$ concentration was equal to 0.3 at \% and $\mathrm{Cl}$ concentration was equal to 2 at. \%). The $\mathrm{ZnS}:[\mathrm{Cu}, \mathrm{Cl}]$ films obtained after the annealing in the blend at $650^{\circ} \mathrm{C}$ were also co-doped by $\mathrm{Ga}$ at $800{ }^{\circ} \mathrm{C}$ and $950^{\circ} \mathrm{C}$. More details about the samples preparation can be seen in [6]. The measurements of PFs, profiles and integrated intensities of diffracted peaks in the maxima of PFs were carried out using D8 Bruker X-ray diffractometer with an 
Euler cradle with $\mathrm{Cu}$ and $\mathrm{Co}$ non-polarized radiations. Integrated intensities for the films and $\mathrm{ZnS}$ powder standard samples with cubic or hexagonal structure were measured for low and high index reflections.

\section{Results and discussion}

Structural investigation realized in our previous work [6] showed that as-deposited $\mathrm{ZnS}: \mathrm{Cu}$ films had only a cubic structure and very strong texture in $\langle 111\rangle$ direction. The annealing of $\mathrm{ZnS}: \mathrm{Cu}$ films in the blend at $600{ }^{\circ} \mathrm{C}$ and in the $\mathrm{ZnS}$ powder with and without $\mathrm{Ga}$ vapors at $1000{ }^{\circ} \mathrm{C}$ did not lead to any modifications of crystal structure and texture ( $\mathrm{ZnS}$ phase transition temperature is $1020^{\circ} \mathrm{C}$ ). It was concluded that $\mathrm{Ga}$ with $\mathrm{Cu}$ act only as strong activators of recrystallization processes. After the annealing of $\mathrm{ZnS}: \mathrm{Cu}$ films in the blend at $800{ }^{\circ} \mathrm{C}$ a strong phase transition from cubic to hexagonal structure for $\mathrm{ZnS}:[\mathrm{Cu}, \mathrm{Cl}]$ film was observed. An additional annealing in the $\mathrm{ZnS}$ powder with $\mathrm{Ga}$ vapors of $\mathrm{ZnS}:[\mathrm{Cu}, \mathrm{Cl}]$ films, obtained after the first annealing in the blend at $650{ }^{\circ} \mathrm{C}$, leaded to a phase transition and the fraction of grains with hexagonal structure depended on the temperature of the additional annealing. The additional annealing at $800{ }^{\circ} \mathrm{C}$ leaded to a small fraction of the film volume with hexagonal structure and the additional annealing at $950{ }^{\circ} \mathrm{C}$ resulted in the phase transition almost in whole volume of the film. The model that explains such result was proposed in [6] and it is based on the influence of microstructure (size of perfect domains and dislocation density) on homogeneity of $\mathrm{Cu}$ and $\mathrm{Cl}$ distribution in $\mathrm{ZnS}$ lattice and the variation of the amount of $\mathrm{Cu}^{+}-\mathrm{Cl}^{-}$ionic binding. Therefore, these microstructure characteristics must be evaluated for confirmation of the proposed model.

We measured direct pole figures for $\mathrm{ZnS}:[\mathrm{Cu}, \mathrm{Cl}]$ and $\mathrm{ZnS}$ : $[\mathrm{Cu}, \mathrm{Cl}, \mathrm{Ga}]$ films in order to determine the space position of the texture maximum. The PFs were measured for the first order reflection with Co-radiation and are shown in figure 1.

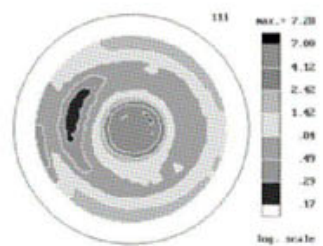

a

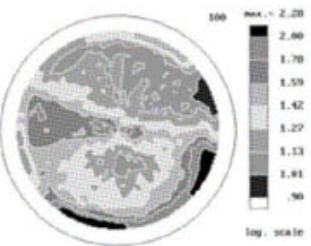

b

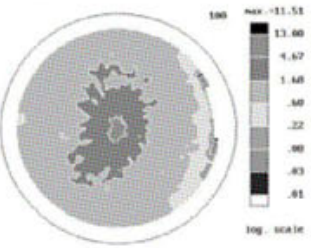

C

Figure 1. Direct pole figures for: $\langle 111\rangle$ crystallographic direction for $\mathrm{ZnS}: \mathrm{Cu}, \mathrm{Cl}$ film obtained after the annealing at $600^{\circ} \mathrm{C}(\mathrm{a}) ;<100>$ crystallographic direction for $\mathrm{ZnS}: \mathrm{Cu}, \mathrm{Cl}$, Ga films obtained after the additional annealing with Ga vapors at $800^{\circ} \mathrm{C}$ (b) and $950^{\circ} \mathrm{C}(\mathrm{c})$, respectively.

The PF for $\mathrm{ZnS}$ : $[\mathrm{Cu}, \mathrm{Cl}]$ film with cubic crystal structure was measured for $<111>$ crystallographic direction. As the diffraction peak position for 111 reflection from $\mathrm{ZnS}$ with cubic structure almost coincides with peak position for 002 reflection from $\mathrm{ZnS}$ with hexagonal structure, it is impossible to evaluate the volume fraction of the film in which structure phase transition took place according to 002 reflection. In order to obtain the information for sure from the hexagonal phase of $\mathrm{ZnS}:[\mathrm{Cu}, \mathrm{Cl}, \mathrm{Ga}]$ films, obtained after the second annealing with Ga vapors at $800{ }^{\circ} \mathrm{C}$ and $950^{\circ} \mathrm{C}$, the PFs for these films were measured for $<100>$ crys- 
tallographic direction. A strongly marked texture is observed for $\mathrm{ZnS}$ : $[\mathrm{Cu}, \mathrm{Cl}]$ film with cubic crystal structure and $\mathrm{ZnS}:[\mathrm{Cu}, \mathrm{Cl}, \mathrm{Ga}]$ films after the additional annealing at $950^{\circ} \mathrm{C}$.

The XRD peak profiles measured in the texture maxima showed that they were unsuited for microstructure evaluation. The measurements for the films and powder samples were carried out with Co-radiation at the same space position. The FWHM of the peak for 111 reflection from $\mathrm{ZnS}$ : $[\mathrm{Cu}, \mathrm{Cl}]$ film, obtained after the annealing at $600{ }^{\circ} \mathrm{C}$ was 0.16 degree, while for $\mathrm{ZnS}$ powder sample with cubic structure it was 0.15 degree. The calculated FWHM of diffracted peaks for 100 reflection for $\mathrm{ZnS}$ : $[\mathrm{Cu}, \mathrm{Cl}, \mathrm{Ga}]$ films, obtained after the second annealing with Ga vapors at $800^{\circ} \mathrm{C}$ was 0.16 degree and 0.15 degree for the film annealed at 950 ${ }^{\circ} \mathrm{C}$, and for $\mathrm{ZnS}$ powder sample with hexagonal structure it was 0.17 degree.

For the application of extinction phenomenon for microstructure determination of $\mathrm{ZnS}$ thin films it is necessary to evaluate the changes of the primary extinction coefficient for different wavelengths used and for the first and second order reflections. The primary extinction coefficient can be explained within the terms of domain thickness $l$ and extinction length $\Lambda$ [7]:

$$
\varepsilon=\tanh (l / \Lambda)(l / \Lambda)^{-1} \text {. }
$$

The extinction length for the symmetrical Bragg case and nonpolarized radiation can be presented by the equation [4]:

$$
\Lambda=v\left(2 d\left|C_{d, m}\right| r_{0} F_{H}\right)^{-1},
$$

where $v$ is the unit cell volume, $r_{0}$ is the electron orbit radius, $F_{H}$ is the structure factor, $d$ is the spacing of the lattice planes, and $C_{d y n}$ is the polarization factor for dynamical scattering. The difference in the primary extinction coefficient for different wavelengths will appear according to the structure factor $F_{H}$ and $C_{d y n}$. The thin films studied were $\mathrm{ZnS}$ compound. The wavelength of the $\mathrm{K}$-absorption edge for $\mathrm{S}$ is $0.50184 \mathrm{~nm}$ and is distant from wavelengths used (Co- and $\mathrm{Cu}$ - radiations), but for $\mathrm{Zn}$ it is $0.12833 \mathrm{~nm}$ and is very close to these wavelengths [8]. So, the dispersion corrections for the atomic scattering amplitudes in the structure factor must be taken into account. The calculated values of structure factors and extinction lengths for different reflections and radiations used are presented in table 1 .

Table 1. Calculated values of the structure factor $F_{H}$ and the extinction length $A$ for different reflections from $\mathrm{ZnS}$ with $\mathrm{Cu} \mathrm{K}_{\alpha^{-}}$and $\mathrm{Co} K_{\alpha^{-}}$radiations.

\begin{tabular}{|c|c|c|c|c|}
\hline Reflection $h k l$ & $\mathrm{~F}(\mathrm{Cu})$ & $\mathrm{F}(\mathrm{Co})$ & $\Lambda(\mathrm{Cu})(\mu \mathrm{m})$ & $\Lambda(\mathrm{Co})(\mu \mathrm{m})$ \\
\hline 111 & 103.37 & 104.45 & 0.963 & 0.976 \\
\hline 222 & 31.68 & 32.48 & 8.41 & 9.23 \\
\hline 100 & 35.34 & 35.84 & 1.04 & 1.08 \\
\hline 200 & 27.37 & 27.66 & 3.1 & 3.4 \\
\hline
\end{tabular}

The obtained results show that the structure factor $F_{H}$ and the extinction length $\Lambda$ differ negligibly for the first order reflections and different wavelengths. For the second order reflections the extinction length increases by a few times. Therefore, the approximation [4] that claims that for the low index reflections the primary extinction coefficient does not depend on wavelength used and for the second order reflection the primary extinction can be neglected and the primary extinction coefficient is equal to unity are valid for the films studied. The conceptions and equations from the work [4] can be applied to the correction of PD, 
determination of the coefficients of the primary and secondary extinction in $\mathrm{ZnS}$ textured thin films, and evaluation of the microstructure of textured annealed $\mathrm{ZnS}$ thin films.

The PD in textured sample $\left(P_{m}\right)$ is defined as $P_{m}=I_{m}^{T} / I_{m}^{P}$, where $I_{m}^{T}$ and $I_{m}^{P}$ are the integrated intensities diffracted from the textured and powder standard samples. As the integrated intensity is measured for "infinite" powder standard samples, the integrated intensity diffracted from textured thin film with the thickness $t$ must be corrected to "infinite" thick layer as:

$$
I_{c o r}^{T}=I_{m}^{T} / 1-\exp (-2 \mu t / \sin \theta \cos \varphi),
$$

where $\mu$ is the linear absorption coefficient, $\theta$ is the Bragg angle and $\varphi$ is the angle of thin film inclination. The value of PD was determined using the the corrected integrated intensity of XRD peak for the film and integrated intensity of XRD peak for $\mathrm{ZnS}$ powder standard sample with cubic or hexagonal structure measured under the same space conditions. The determined PDs for different order of reflections and wavelengths, as well as the corrected ones by the proposed in [4] method are presented in table 2 .

Table 2. The values of determined PDs $P_{C}^{\text {hit }}$ and $P_{C u}^{\text {hhd }}$ for different reflection with $C o$ - and Cu-radiations and the corrected ones $P_{\text {cor }}^{\text {kd }}$.

\begin{tabular}{|l|c|c|c|c|}
\hline \multicolumn{1}{|c|}{ Film type (annealing T) } & $P_{C_{o}}^{\text {hkl }} ;\{h k l\}$ & $P_{C_{o}}^{\text {(hkl) }} ;\{h k l\}$ & $P_{c c}^{\text {hllk }} ;\{h k l\}$ & $P_{c o r}^{\text {hkl }} ;\{h k l\}$ \\
\hline $\mathrm{ZnS}: \mathrm{Cu}, \mathrm{Cl}\left(600^{\circ} \mathrm{C}\right)$ & $9.7 ;\{111\}$ & $18.6 ;\{222\}$ & $9.2 ;\{111\}$ & $19.5 ;\{111\}$ \\
\hline $\mathrm{ZnS}: \mathrm{Cu}, \mathrm{Cl}, \mathrm{Ga},\left(800^{\circ} \mathrm{C}\right)$ & $2.9 ;\{100\}$ & $6.1 ;\{200\}$ & $3.0 ;\{100\}$ & $7.2 ;\{100\}$ \\
\hline $\mathrm{ZnS}: \mathrm{Cu}, \mathrm{Cl}, \mathrm{Ga},\left(950^{\circ} \mathrm{C}\right)$ & $10.9 ;\{100\}$ & $19.6 ;\{200\}$ & $10.6 ;\{100\}$ & $27.6 ;\{100\}$ \\
\hline
\end{tabular}

The values of directly calculated PDs, which must be constant for a given direction, are in fact different for the first and second order reflections. The values of PDs for the first order reflections are smaller than those for the second order reflections due to a strong influence of the primary and secondary extinction. A small difference in PDs for the first order reflections with different wavelengths is evidently due to the secondary extinction. The directly obtained PDs are also smaller than the corresponding corrected PDs for all the films.

The solution of the system of three equations for two PDs measured for the first order reflection with two radiations and one PD for the second order reflection gives the coefficients of the primary and secondary extinction [4]. The domain thickness $l$ was calculated from the values of the primary extinction coefficient $\varepsilon$ and the extinction length $\Lambda$ [8]. The coefficient of the secondary extinction $g$ was used for the determination of the average domain disorientation angle $\bar{u}$. The calculation of the dislocation density $N_{D}$ at domain boundaries within the framework of the mosaic crystal model, using the values of $l$ and $\bar{u}$ was performed as in [9]:

$$
N_{D}=\sqrt{2 / \pi}(\bar{u} / 3 l b)
$$

where $b$ is the magnitude of the Burgers vector for $\mathrm{ZnS}$ with cubic or hexagonal structure, respectively. The results of the calculations are presented in table 3 .

Table 3. Calculated values of the extinction parameters and values of the domain thickness $l$, the average angle of domain disorientation $\bar{u}$ and the dislocation density $N_{D}$ in the domain boundaries.

\begin{tabular}{|l|c|c|c|c|c|}
\hline Film type (annealing T) & $\varepsilon$ & $g$ & $l^{\text {hkl }}(\mu \mathrm{m})$ & $\bar{u}^{(\text {hkl) }}(\mathrm{Rad})$ & $N_{D}^{k, k l} \cdot 10^{-7}\left(\mathrm{~cm}^{-2}\right)$ \\
\hline $\mathrm{ZnS}: \mathrm{Cu}, \mathrm{Cl}\left(600^{\circ} \mathrm{C}\right)$ & 0.929 & 1009 & 0.26 & $3.5 \cdot 10^{-4}$ & 9.4 \\
\hline $\mathrm{ZnS}: \mathrm{Cu}, \mathrm{Cl}, \mathrm{Ga}\left(800^{\circ} \mathrm{C}\right)$ & 0.575 & 730 & 1.67 & $4.9 \cdot 10^{-4}$ & 6.1 \\
\hline $\mathrm{ZnS}: \mathrm{Cu}, \mathrm{Cl}, \mathrm{Ga}\left(950^{\circ} \mathrm{C}\right)$ & 0.508 & 1463 & 2.05 & $2.4 \cdot 10^{-4}$ & 2.4 \\
\hline
\end{tabular}


The obtained results show that the domain size increased and the dislocation density decreased in $\mathrm{ZnS}:[\mathrm{Cu}, \mathrm{Cl}, \mathrm{Ga}]$ film with strong phase transition noticeably with respect to $\mathrm{ZnS}$ : $[\mathrm{Cu}, \mathrm{Cl}]$ with cubic structure and $\mathrm{ZnS}$ : $[\mathrm{Cu}, \mathrm{Cl}, \mathrm{Ga}]$ film with small fraction of hexagonal structure. It is important to note that these films have almost the same concentration of $\mathrm{Cu}$ and $\mathrm{Cl}$ dopants after the thermal diffusion at the annealing in the blend.

Such microstructure modification leads to more homogeneous distribution of dopants in $\mathrm{ZnS}$ lattice owing to the decrease of extended defects as gatherers of impurities. These results confirm the proposed model [6] of the effect of $\mathrm{Cu}$ and co-doping element ( $\mathrm{Ga}$, and $\mathrm{Cl}$ ) incorporation into $\mathrm{Zn}$ and $\mathrm{S}$ sublattices on the phase transition temperature.

\section{Conclusions}

An original XRD technique for evaluation of microstructure of textured $\mathrm{ZnS}$ thin films was applied in the case when the extinction phenomenon was observed. The data obtained showed that the primary and secondary extinction were present in the films after the special annealing. The application of this technique confirms the influence of the domain size and dislocation densities on distribution of $\mathrm{Cu}$ and $\mathrm{Cl}$ atoms in $\mathrm{ZnS}$ lattice and decrease of the phase transition temperature owing to the increase of the amount of $\mathrm{Cu}^{+}-\mathrm{Cl}^{-}$ionic binding.

\section{References}

1. Randle, V. \& Engler, O., 2000, Introduction to Texture Analysis Macrotexture, Microtexture and Orientation Mapping (Amsterdam: Gordon and Beach Science Publisher).

2. Krivoglaz, M.A., 1969, Theory of X-ray and Thermal Neutron Scattering by Real Crystals (New York: Plenum Press).

3. Krivoglaz, M.A., 1996, X-ray and Neutron Scattering in Nonideal Crystals (Berlin Heidelberg-New York: Springer-Verlag).

4. Kryshtab, T., Palacios-Gomez, J., Mazin, M. \& Gomez-Gasga, G., 2004, Acta Mater., 52, 3027.

5. James, R.W., 1965, The Optical Principles of the Diffraction of X-rays, $3^{\text {rd }}$ ed. (London: G. Bell).

6. Kryshtab, T., Palacios Gómez, J. \& Mazin, M., 2006, Z. Kristallogr. Suppl., 23, 287.

7. Pinsker, Z.G., 1978, Dynamical Scattering of X-rays in Crystals (Berlin- Heidelberg - New York, Springer-Verlag).

8. International Tables for X-ray Crystallography, 1992 (Dordrecht, Boston, London: Kluwer Acad. Publ.).

9. Larson, I.A., \& Corey, C.L., 1969, J. Appl. Phys., 40, 2708.

Acknowledgements. The authors would like to thank the CONACyT of Mexico for financial support of this work by the projects N 83425 and Dr. J. A. Andraca-Adame and Dr. G. Gómez Gasga ESFM-IPN, Mexico D.F. for their X-ray diffraction measurement. 
I.5 In-situ and Non-ambient Measurements 



\title{
On the intensity distribution within Debye-Scherrer rings. What is different in high pressure experiments? Part I: Theory
}

\section{B. Hinrichsen ${ }^{1}$, R. E. Dinnebier ${ }^{2, *}$, M. Jansen ${ }^{2}$}

${ }^{1}$ Bruker AXS GmbH, Östliche Rheinbrückenstrasse 49, 76187 Karlsruhe, Germany

${ }^{2}$ Max Planck Institute for Solid State Research, Heisenbergstraße 1, 70569 Stuttgart, Germany

"r.dinnebier@fkf.mpg.de

Keywords: Pareto distribution, high pressure powder diffraction, fractile filter, intensity distribution

\begin{abstract}
Generally, high pressure powder diffraction using diamond anvil cells (DAC's) fails to produce intensity data in the traditional powder diffraction sense as a result of experimental constraints. This often makes the raw data unsuited for standard Rietveld refinements. In a study based on data collected using image plates at state of the art beamlines the intensity distribution has been closely scrutinized. It was found not to follow the crystallographically well known i.e. Poisson, gamma, normal or lognormal distributions. The intensities are far better described by the Pareto distribution originally developed for economical statistics. This distribution model is reconcilable with the experimental features of high pressure powder diffraction. An advantageous aspect of this distribution is the ability to extract meaningful normally distributed intensities, which present a sound basis for least squares minimization within structural refinement.
\end{abstract}

\section{Introduction}

The domain of high pressure powder diffraction has led to an astonishing amount of fundamental discoveries covering many fields from the geosciences over chemistry to physics $[1,2]$. Diamond anvil cells have been use since the 50's [3] to produce the extreme pressures reached in these experiments. In addition to their supreme hardness, the diamonds transparency to X-rays enables the diffracted X-ray light to be scattered through the diamond over a large angular range. The scattered rays are detected by area detectors, from which the intensities belonging to discrete diffraction angles are averaged to produce a conventional powder diffraction pattern. 
Several properties of this experiment make the analysis a rather challenging undertaking. The sample volume is minute, generally only filling a fraction of the volume of the available cavity (in the order of $1 \mathrm{E}-3 \mathrm{~mm}^{3}$ ). To ensure hydrostatic conditions and reduce inter-granular stress the majority of the cavity is filled with a pressure medium, leading to a further substantial reduction of the scattering volume. To increase the strength of the scattered signal a high brilliance X-ray source is required, resulting in most of the high pressure powder diffraction experiments being performed at $3^{\text {rd }}$ generation synchrotron sources.

Conventional powder diffraction experiments involve rotating the sample to ensure that as many crystallites as possible are brought into a diffraction position. This is rarely done in high pressure experiments. It is a precaution to reduce the possibility of gasket diffraction contaminating the signal, but also to avoid the single crystal diamonds from moving into a diffracting position.

A further precaution against accidental gasket diffraction is the reduction of the beam size. The synchrotron beam is reduced to a diameter of a few micrometers. This leads to a further reduction of the illuminated volume to ca. $1 \mathrm{E}-5 \mathrm{~mm}^{3}$.

This small sample volume causes the signal to be miniscule in comparison to standard powder diffraction experiments that generally have an illuminated sample volume in the order of $0.1 \mathrm{~mm}^{3}$.

These constraints result in a powder diffraction experiment that is very far removed from an ideal setup. The resulting diffraction data are equally troublesome. The intensities are very difficult to interpret, even after the use of filters. Single extremely intense spikes, originating from grains in an ideal diffraction position tend to dominate the diffraction pattern. The great majority of the grains is not in an ideal diffraction position, thereby leading to very many weak intensities. These intensities have been found not to follow any common crystallographic distribution model. It should be noted, that the strong deviations of the distribution are not due to detector effects as has been described by [4]. It will be demonstrated that a phenomenological model that describes the experimental data sufficiently well is the Pareto distribution.

\section{Distribution models in counting statistics}

The binomial distribution is generally regarded as the model of choice for the analysis of counting statisties. The discrete binomial probability distribution [5] is given by equation (1). This returns the probability $P_{B}$ of exactly $n$ successes out $N$ trials where each trial has the probability of success $p$ and probability of failure $q=1-p$. In two-dimensional powder diffraction success would represent an intensity count at a pixel failure would represent no intensity.

$$
\begin{aligned}
P_{B}(n \mid N) & =\left(\begin{array}{l}
N \\
n
\end{array}\right) p^{n} q^{N-n} \\
& =\frac{N !}{n !(N-n) !} p^{n}(1-p)^{N-n}
\end{aligned}
$$


The limits of this distribution are represented by the normal- and the Poisson-distribution [6]. When $n \rightarrow \infty$ and $p$ remains unchanged the continuous normal (Gaussian) estimation (2) is valid [7].

$$
P_{N}(n)=\frac{1}{\sigma \sqrt{2 \pi}} \exp \left[-\frac{(n-N p)^{2}}{2 \sigma^{2}}\right]
$$

Here $\sigma^{2}$ is the variance. The discrete Poisson estimation is valid if $n \rightarrow \infty$ and $p \rightarrow 0$, with $N p=v>0$.

$$
P_{P}(n)=\frac{v^{n} e^{-v}}{n !}
$$

The Poisson estimation holds well for low counts and the normal estimation holds well for higher mean values. From a computational point of view testing the Poisson estimation is limited to $n$ less than 170 as the factorial for IEEE standard floating point variables cannot be computed for higher values. On the other hand the Poisson estimation offers a reduced formalism for the distribution function, containing only one free parameter $v$ which represents the mean and the variance. Two free parameters are used to describe the normal distribution - the variance $\sigma^{2}$ is free as is the mean $N p$.

\section{Arithmetic statistical values}

Fitting the population of each bin* to a distribution function is computationally unfeasible. This is due to instabilities when dealing with small populations and to the high computational cost involved with nonlinear fitting routines. For standard integration computations arithmetic means, medians and variances are calculated. The arithmetic mean $\bar{x}$ of $N$ number of observations $x$ is given by equation (4).

$$
\bar{x} \equiv \frac{1}{N} \sum_{i=1}^{N} x_{i}
$$

The variance is defined by equation (5).

$$
\sigma^{2}=\frac{1}{N} \sum_{i=1}^{N}\left(x_{i} \bar{x}\right)^{2} \quad-
$$

The median $\tilde{x}$ is given by equation (6) for a population $\mathrm{Y}$ of the size $\mathrm{N}$ that has been prevously sorted.

$$
\tilde{x} \equiv \begin{cases}Y_{(N-1) / 2} & \text { if } N \text { is odd } \\ \frac{1}{2}\left(Y_{N / 2}+Y_{1+N / 2}\right) & \text { if } N \text { is even }\end{cases}
$$

\footnotetext{
"A bin is a container into which pixels are grouped. It spans a $2 \mathrm{D}$ region of $2 \theta$ that is identical to the $2 \theta$ step width of the integrated pattern. The intensities of the pixels within a bin determine the corresponding intensity of a step in the integrated pattern.
} 
In evaluating the filter performance we will compare the values returned by the arithmetic functions with those returned by fitting the model functions to the histograms.

\section{Model testing on ideal data}

To determine the statistical characteristics of the data an ideal image was selected. This image is a high quality calibration image of a well-defined standard substance, in our case $\mathrm{LaB}_{6}$. Contrary to what might be expected the distribution of intensities within a Bragg peak is not ideally suited for the statistical characterization. Although polarization and Lorentz effects can be corrected a substantial azimuthal intensity deviation probably stemming from sample absorption mars a rational model selection. Background intensity caused by air scattering is however better suited for statistical analysis. Polarization correction results in a perfectly flat intensity distribution along the azimuth. A histogram of such intensities contributing to a $0.02^{\circ}$ bin is shown in figure 1 .

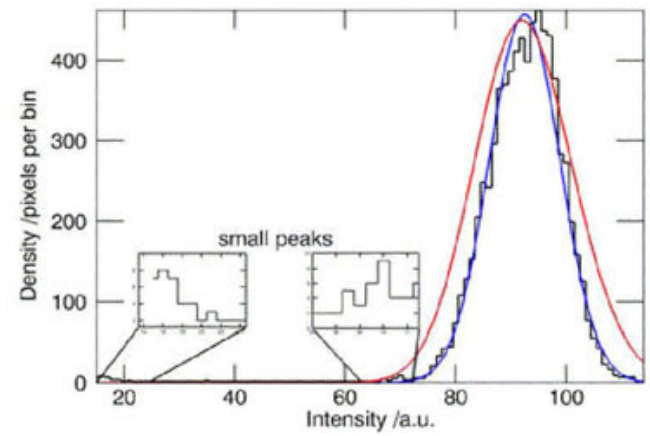

Figure 1. The black line shows a histogram of the intensities contributing to one bin of $0.02^{\circ}$ in $2 \theta$ of the final integrated background intensity. Two distribution models have been overlaid, in blue the normal (Gaussian) probability distribution function and in red the Poisson probability mass function. The Poisson distribution is not continuous as might be suggested by the line but discrete.

Two small peaks can be seen to the lower intensities; these originate from the primary beam holder and the capillary shadowing as can be seen in the azimuthal plot in figure 2 .

Two solid lines representing the statistical models are overlaid, the blue line represents the normal distribution and the red line corresponds to the Poisson distribution. Contrary to common belief [8] the Poisson distribution overestimates the variance of the data sample.

A normal distribution is clearly the most convincing model describing the signal statistics of a very small diffraction angle range and the complete azimuthal range. This is the typical data entering a single bin when integrating the $2 \mathrm{D}$ image into a conventional $1 \mathrm{D}$ powder diffractogram. Intensity distributions fitting a Poisson distribution were not observed. 


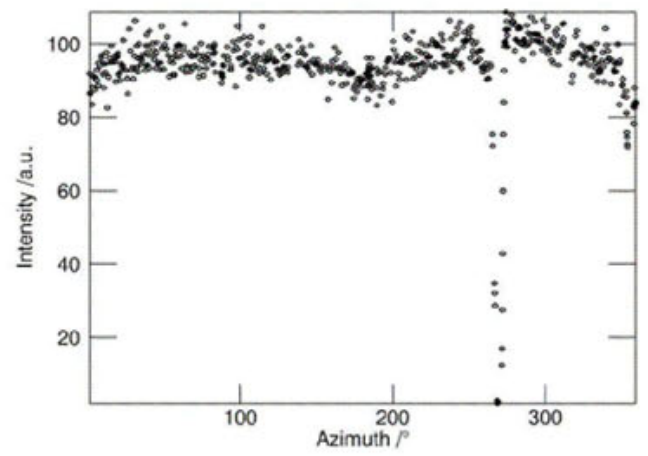

Figure 2. The distribution of the intensity contained in the histogram in figure 1 is shown as a function of the azimuth. The intensity is the background between the first two Bragg reflections of the standard $\mathrm{LaB}_{6}$ calibration sample. Air scattering is the main source of the background radiation. The black symbols represent the intensity of a single pixel. The main drop in intensity at $270^{\circ}$ is due to shadowing by the primary beam stop arm. The intensity reduction at $0 \% 360^{\circ}$ and $180^{\circ}$ is due to shadowing by the sample capillary.

\section{High pressure data intensity distribution}

As already mentioned the quality of high-pressure data poses probably the greatest challenge to analysis. An example of such data can be seen in figure 3 .

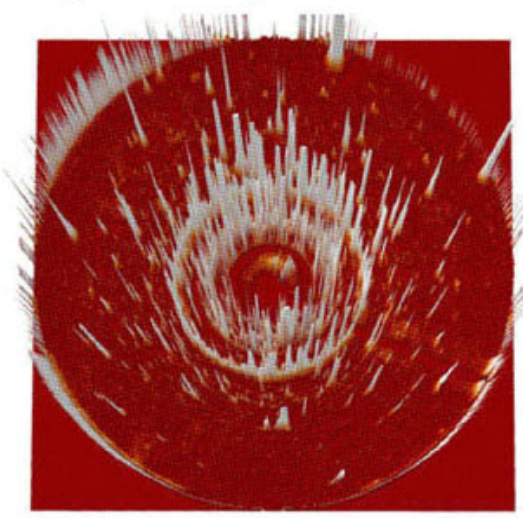

Figure 3. A rendered image of a high pressure powder diffraction data set collected by a twodimensional image plate detector $\left(\mathrm{SnSO}_{4}\right.$ at $\mathrm{P}=16 \mathrm{GPa}$ [9]). The white spikes are high intensity peaks. The low intensity pixels contribute the light red rings visible at their base.

The number of high intensity pixels tends to be a couple of orders of magnitude lower than the number of low intensity pixels. On account of the small number of intense spots they inevitably fail to ensure a 'statistical' distribution. Due to their intensity that often lies orders of magnitude above the low intensity pixels, they have a very strong and unpredictable effect on the integrated pattern, falsifying the intensities considerably. This effect is not alleviated 
by a mere integration. Incorrectly filtered images can result in peaks that cannot be fitted by conventional peak profile models as well as error bars that are completely meaningless.We have observed this effect in a number of high pressure experiments performed with different diamond anvil cells, different pressure media and at different beamlines at the APS, ESRF, and SLS. As a typical example, we have chosen high-pressure powder diffraction data on $\mathrm{SnSO}_{4}[9]$. To obtain an acceptably resolved histogram from our experimental data of $\mathrm{SnSO}_{4}$ at $\mathrm{P}=16 \mathrm{GPa}$ an integration bin of highly dispersed intensities had to be first filtered. An initial fractile filter of the upper $5 \%$ was required (figure 4 ).

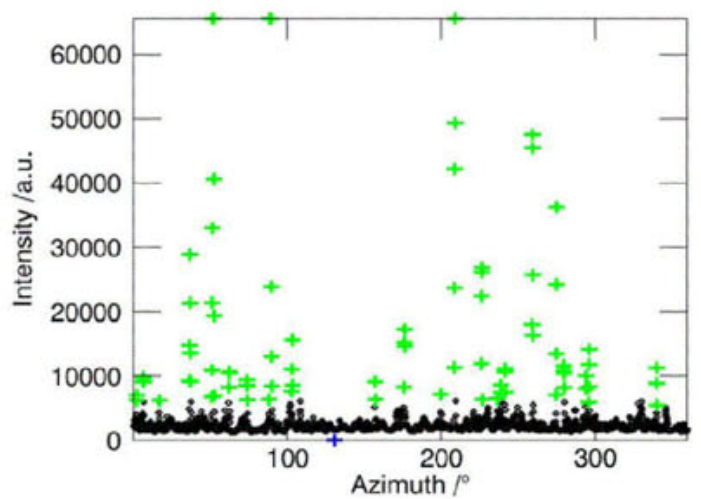

Figure 4. The intensity is shown as a function of the azimuth. The displayed intensity originates from a typical high pressure experiment $\left(\mathrm{SnSO}_{4}\right.$ at a pressure of $\mathrm{P}=16 \mathrm{GPa}$ [9]). The filtered pixels have been coloured, green the high intensities, blue the low intensities.

The resulting histogram can be seen in figure 5 .

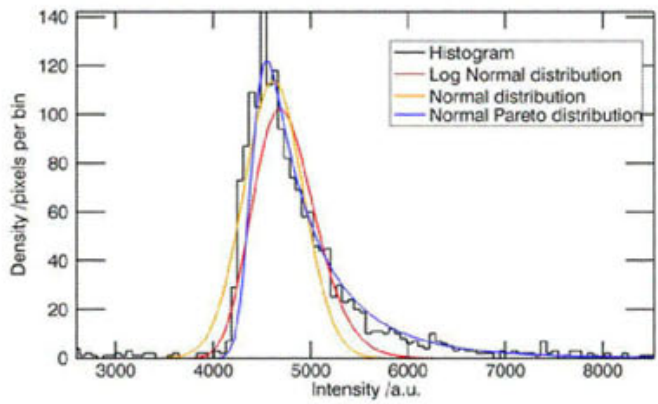

Figure 5. The intensity distribution of a filtered bin from a high-pressure experiment ( $\mathrm{SnSO}_{4}$ at a pressure of $P=16 \mathrm{GPa}$ [9]) is displayed. The best fit of intensity distributions of the normal, the log normal and proposed normal Pareto distributions are overlaid in colour. The best correspondence is given by the normal Pareto distribution.

The distribution of the intensities is markedly different to those distributions studied before. Fitting a normal distribution, which has been so successful describing ideal intensity distributions, to the HP data set leads to a most unconvincing result (the orange line in figure 5 and the $\mathrm{Chi}^{2}$ value in table 1). 
The intensity distribution is exclusively a sample characteristic - no sample environment contamination affects them. As the distribution has many low intensity and few high pixels the power law distributions seem the appropriate descriptive choice. Of these the most promising is the Pareto distribution [10] (7) which was initially devised to model the wealth distribution among individuals of a society. This has become generally known as the 80/20 rule. Eighty percent of the wealth is owned by twenty percent of the population. Wide applications of this distribution in the fields of biology, geology and physics [11] have been found to date.

$$
P_{\text {Pareto }}(x)= \begin{cases}0 & \text { for } x<b \\ \frac{a b^{a}}{x^{a+1}} & \text { for } x \geq b\end{cases}
$$

The two parameters of the Pareto distribution (7) describe the smallest possible value of $x$ which is $b$, and a form parameter $a$. The Pareto probability density function is sharp containing a singularity at $x=b$. This is not compatible to the experimental data in which mainly the detector causes a 'Gaussian blur' of the Pareto distribution (see figure 5). To make allowance for this effect the Pareto distribution is folded with a normal distribution function leading to what will be called a normal Pareto function (8). This convolution gives the best $\mathrm{Chi}^{2}$ of any distribution model tested (table 1).

$$
P_{N P}(x)=P_{\text {normal }}(x) \quad P_{\text {Pareto }}(x \varnothing
$$

This formalism for the intensity distribution is most attractive as when the parameter $a$ in (7) tends toward infinity the Pareto distribution tends toward a Dirac delta function, thereby reducing the convoluted function to the normal distribution of monodisperse intensities. The influence of the parameter $a$ especially on the tail of the function can be seen in figure 6 .

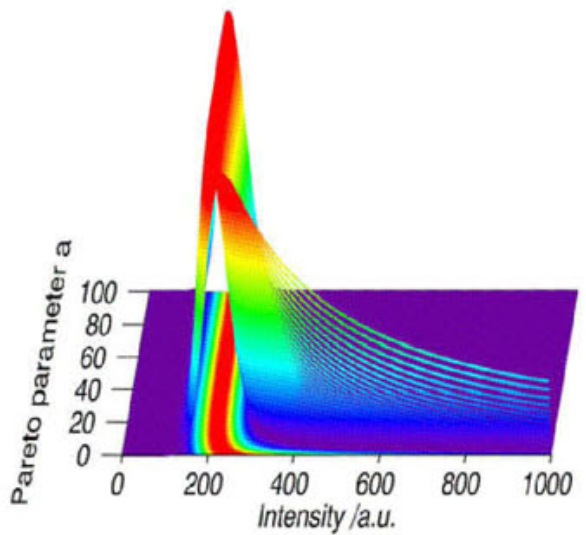

Figure 6. The effect of parameter a is shown on the convoluted Pareto probability density function. At values of a below 20 a shift of the peak to higher intensities can be seen. The slower falloff of the distribution function to higher intensities is the most pronounced characteristic of small a-values in the distribution function.

Here one can quite clearly see that 'infinity' is effectively reached at the modest value of $a=20$. 
Table 1. The table displays the chi-squared statistical test results (goodness of fit) of the distribution models to the filtered intensities contained in a single bin from a high pressure experiment. The best fit is given by the Normal Pareto distribution.

\begin{tabular}{|l|l|}
\hline Model & $\mathrm{Chi}^{2}$ \\
\hline Normal distribution & 991 \\
\hline Lognormal distribution & 357 \\
\hline Normal Pareto distribution & 117 \\
\hline
\end{tabular}

\section{Discussion}

In this first part, we derived a phenomenological description of the intensity distribution in powder diffraction data from high pressure experiments in DAC's. Its relation to the normal distribution making it suitable for the developments of intensity filters is shown. The practical application and its software implementation are the focus of the second part.

\section{References}

1. Lundegaard, L.F., Weck, G., McMahon, M.I., Desgreniers, S. \& Loubeyre, P., 2006, Nature, 443, 201.

2. Murakami, M., Hirose, K., Kawamura, K., Sata, N. \& Ohishi, Y., 2004, Science, 304, 855 .

3. Weir, C.E., Lippincott, E.R., Valkenburg, A.V. \& Bunting, E.N., 1959, J. Res. Natl. Bur. Stand., 63A, 55.

4. Amann, U. \& Ihringer, J., 2002, Z. Kristallogr. Suppl., 20, 79.

5. Abdi, H., 2007, in Binomial Distribution: Binomial and Sign Tests., (Ed. N.J. Salkind), Sage, Thousand Oaks, pp. 87-89.

6. Poisson, S.-D., Recherches sur la probabilité des jugements en matières criminelles et matière civile, $1838, \mathrm{p}$.

7. De Moivre, A., 1969, The Doctrine of Chances, $1738 \mathrm{p}$.

8. Chall, M., Knorr, K., Ehm, L. \& Depmeier, W., 2000, High Pressure Res., 17, 315.

9. Hinrichsen, B., Dinnebier, R. \& Jansen, M., 2008, Z. Kristallogr., 223, 195.

10. Pareto, V., Cours d'economie Politique, Droz, Geneva, 1896 p.

11. Newman, M.E.J., 2005, Contemp. Phys., 46, 323.

Acknowledgements. Financial support by the Bundesministerium für Bildung und Forschung (BMBF) and the Fonds der Chemischen Industrie (FCI) is gratefully acknowledged. 


\section{On the intensity distribution within Debye-Scherrer rings. What is different in high pressure experiments? Part II: Practical application}

\section{B. Hinrichsen ${ }^{1}$, R. E. Dinnebier, ${ }^{2, *}$, M. Jansen ${ }^{2}$}

${ }^{1}$ Bruker AXS GmbH, Östliche Rheinbrückenstrasse 49, 76187 Karlsruhe, Germany

${ }^{2}$ Max Planck Institute for Solid State Research, Heisenbergstraße 1, 70569 Stuttgart, Germany

"r.dinnebier@fkf.mpg.de

Keywords: Pareto distribution, high pressure powder diffraction, fractile filter, intensity distribution

Abstract. In part I, a phenomenological approach describing the intensity distribution in high pressure powder diffraction experiments using diamond anvil cells and two dimensional detectors was developed. This part deals with the practical application in filtering this type of data.

\section{Extracting normally distributed intensities}

A convolution of the presumably normally distributed data and a Pareto like function, representing the asymmetric contribution of larger grains in reflection condition was found. The focus of this paper is the application of this distribution for the development of optimized intensity filters and their influence on parameters in Rietveld refinement.

On the search for effective filtering algorithms, the simple fractile filters [1] proved to be very efficient. In this method, the highest and/or the lowest fraction Frac of discrete intensity data $I$ within one bin are removed.

$$
I_{\text {obs }} \geq \frac{I_{\max }-I_{\min }}{100} *\left(100-\text { Frac }_{h i}\right), \quad I_{\text {obs }} \leq \frac{I_{\max }-I_{\min }}{100} *\left(\text { Frac }_{\text {low }}\right)
$$

The challenge is to determine a Frac value which removes outliers while preserving the normally distributed intensity data (fig.1). 


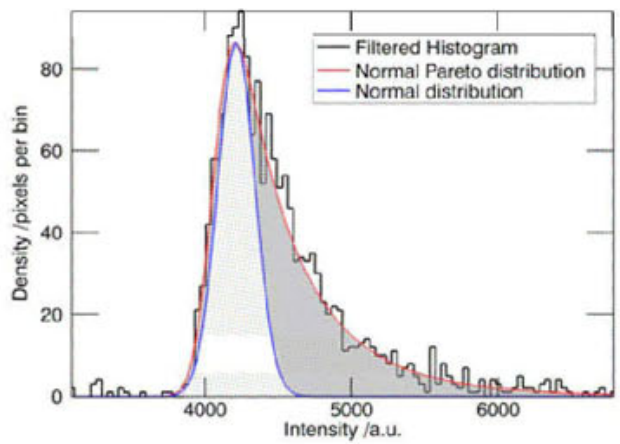

Figure 1. A normal-Pareto distribution is compared to a normal distribution of an ideal sample. To the low intensity side of the functions an almost perfect correspondence of the two functions is observed. To higher intensities the greyed area represents the additional high intensity pixels of a normal Pareto distributed signal which needs to be filtered.

A normal distribution fits very snugly under the main peak of the normal Pareto distribution. This distribution describes the largest and weakest portion of the intensities. To estimate arithmetically the values of this distribution the following steps were taken. Both the integrals of ideal normal and the Pareto distribution were computed. Their difference is an estimation of the normal fraction to the entire distribution according to (2). This is equivalent to estimating the high intensity fraction to be filtered, under the approximation that the high intensity slope of the normal distribution is infinite and the low intensity slopes of both distributions are equal.

$$
\int P_{N}(x) d x-\int P_{N P}(x) d x=1 \quad \text { Frac }_{h i}
$$

$\mathrm{Frac}_{h i}$ from equation (2) is then the high intensity filtering fraction to be set. In the tin sulphate data it leads to a high intensity filter fraction of 0.43 . As the histogram already has been filtered by $5 \%$ of the high intensities, these have to be added. This leads us to a high intensity filter setting of almost $50 \%$. The effect on the arithmetic statistical values can be seen in table 1. The arithmetic standard deviation and the mean correspond excellently to the model values. In general the fraction to be filtered is not merely dependent on the parameter $a$ of the Pareto distribution but also on the width of the normal distribution $P_{n o r m a l}$ with which it is convolved. The effect of both parameters is shown in figure 2 .

It is quite surprising that the removal of such a substantial amount of the data leads to such a dramatic improvement of the data quality. This underlines once more the absolute necessity of large 2D detectors for the success of these types of experiments.

Table 1. The table displays the fitted mean of the normal Pareto distribution and the arithmetic mean from the filtered data, and the associated errors. The values are identical within error.

\begin{tabular}{|l|l|}
\hline Mean(model) & $4014(103)$ \\
\hline Mean(filtered) & $4244(124)$ \\
\hline
\end{tabular}




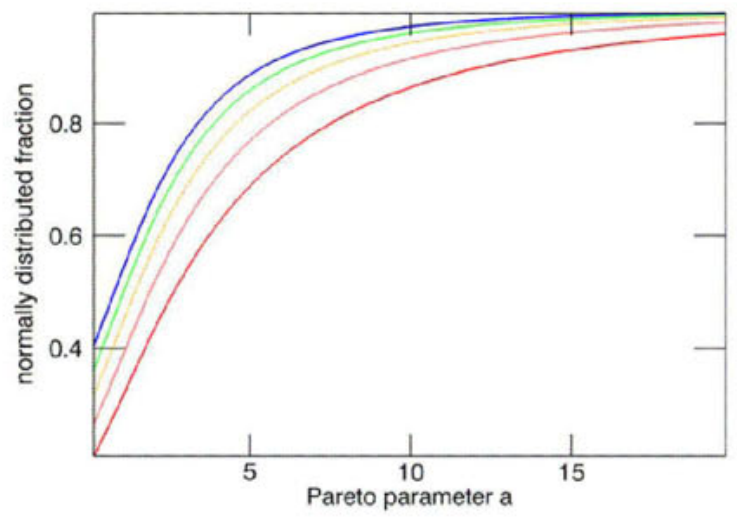

Figure 2. As the high intensity tail is directly related to the a parameter of the Pareto function, the fraction of the integral of the normal probability density function to the integral of the entire normal Pareto function can be plotted as a function of parameter $a$. Different plots have been made to show the influence of varying the sigma of $P_{\text {normal }}$. The lines from the red through to the blue represent the results for sigma values of $0.5,0.7,0.9,1.1,1.3$ times $\sqrt{I}$ respectively. For high values of parameter a the normal fraction approaches 1 asymptotically. For a filter setting which would reduce the Pareto distribution to a roughly normal distribution 1-normal fraction of the highest intensities should be removed.

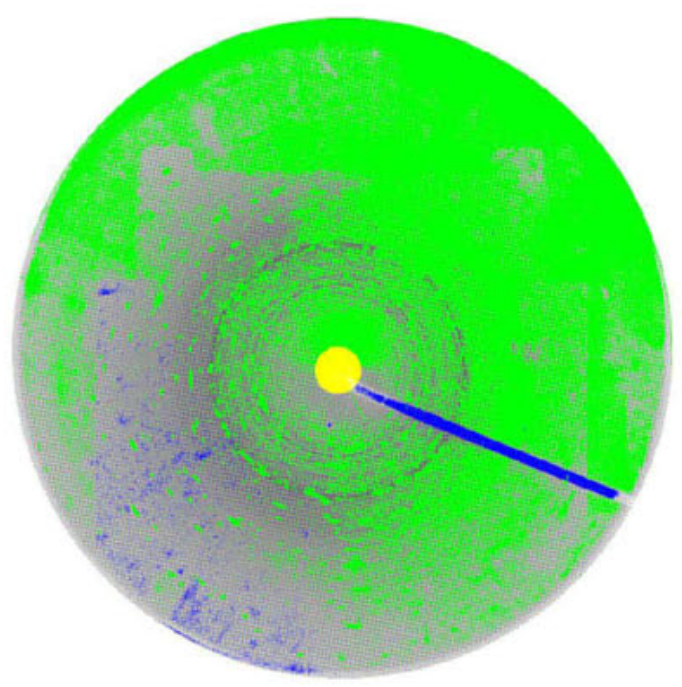

Figure 3. The effect of filtering on the diffraction image is shown in this figure. The green mask represents the pixels which belong to the top $48 \%$ of the intensities per integration bin. The blue mask shows the pixels which belong to the bottom $2 \%$ of the intensities per integration bin. The yellow mask is the beam stop mask filtering the first $2^{\circ} 2 \Theta$ of the diffraction image. Only the grey region of the image is used for the integration to a $1 D$ diffractogram. 
Once applied to the entire image (figure 3 ) the effect on the final diffraction pattern can be seen in figure 4 (top). Even more pronounced is the effect of the filtering on the standard deviation of the integrated pattern (figure 4 bottom).
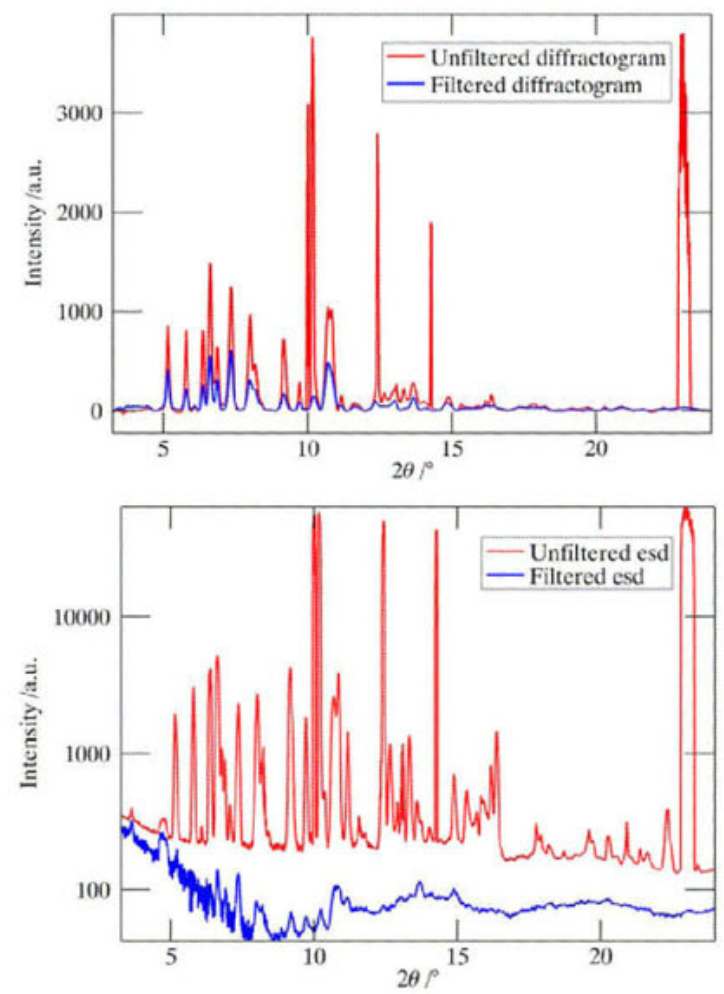

Figure 4. The effect of filtering on the final integrated and background corrected pattern (top) as well as on the on the standard deviation (bottom) is shown. In red the pattern of the unfiltered image is shown. In blue the diffractogram of the image is shown that had $48 \%$ of the highest intensities and $2 \%$ of the lowest intensities removed. It should be noted that the unfiltered esd's are larger than the corresponding intensity values due to the extreme outliers in the intensity distribution.

The method has been successfully applied to data collected from ID9 (ESRF) and Station 9.5HP (SRS). In both cases the normal Pareto distribution has been shown to describe the intensity distribution more precisely than the normal or log normal models.

Results of a systematic investigation on the dependence of Rietveld-refined parameters on Frachi (1) using 2D image plate data of $\mathrm{SnSO}_{4}$ in a DAC slightly above ambient pressure are visualized in fig. 5. It is obvious, that different types of parameters respond differently with increasing Frac $_{h i}$. While the positional parameters of strong scatterers seem to be insensitive, those of light scatterers vary by up to $2 \%$. Realistic temperature factors are reached at $\mathrm{Frac}_{h i}$ $>30 \%$. In general, the most reliable results are obtained at $30 \leq \mathrm{Frac}_{h i} \leq 50 \%$ as can be de- 
duced from the hatched areas in fig. 5. This also holds for the lattice parameters which vary the least in this range. On the downside, over-filtering leads to an increase of the FWHM as indicated by a decrease of the average domain size.
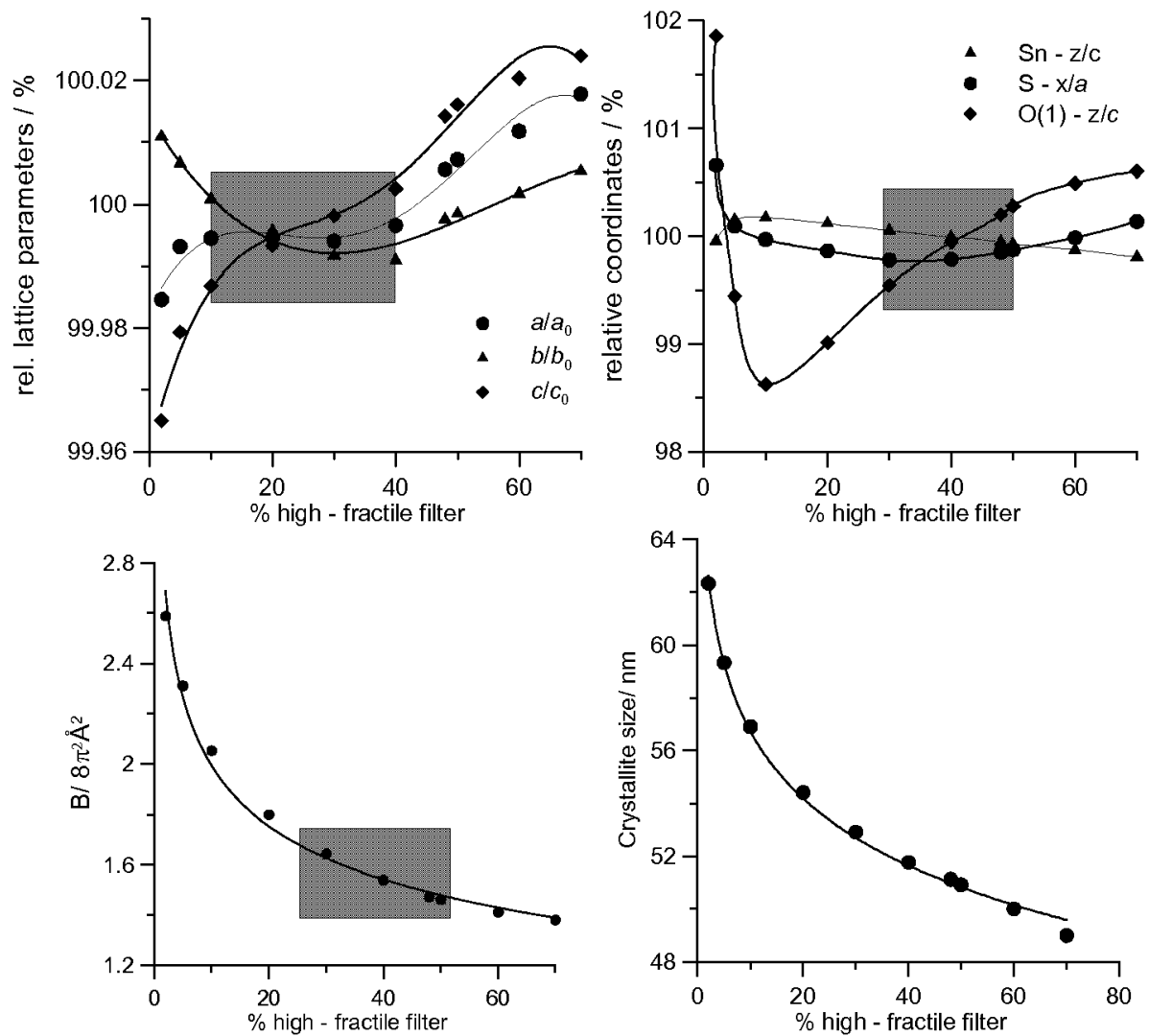

Figure 5. The effect of filtering the highest intensities in the range from $2 \%$ to $70 \%$ on the relative parameters of the Rietveld refinement of $\mathrm{SnSO}_{4}$ in a $\mathrm{DAC}$ slightly above ambient pressure. The most reliable results are typically obtained around the top $40 \%$ of the highest intensities filtered off as marked by the hatched area.

So far, the method has been successfully applied to several high pressure data sets [2-4]. A recent example of the new high pressure phase of the $\mathrm{NLO}$ compound $\mathrm{BiB}_{3} \mathrm{O}_{6}$ is given in fig. 6. 

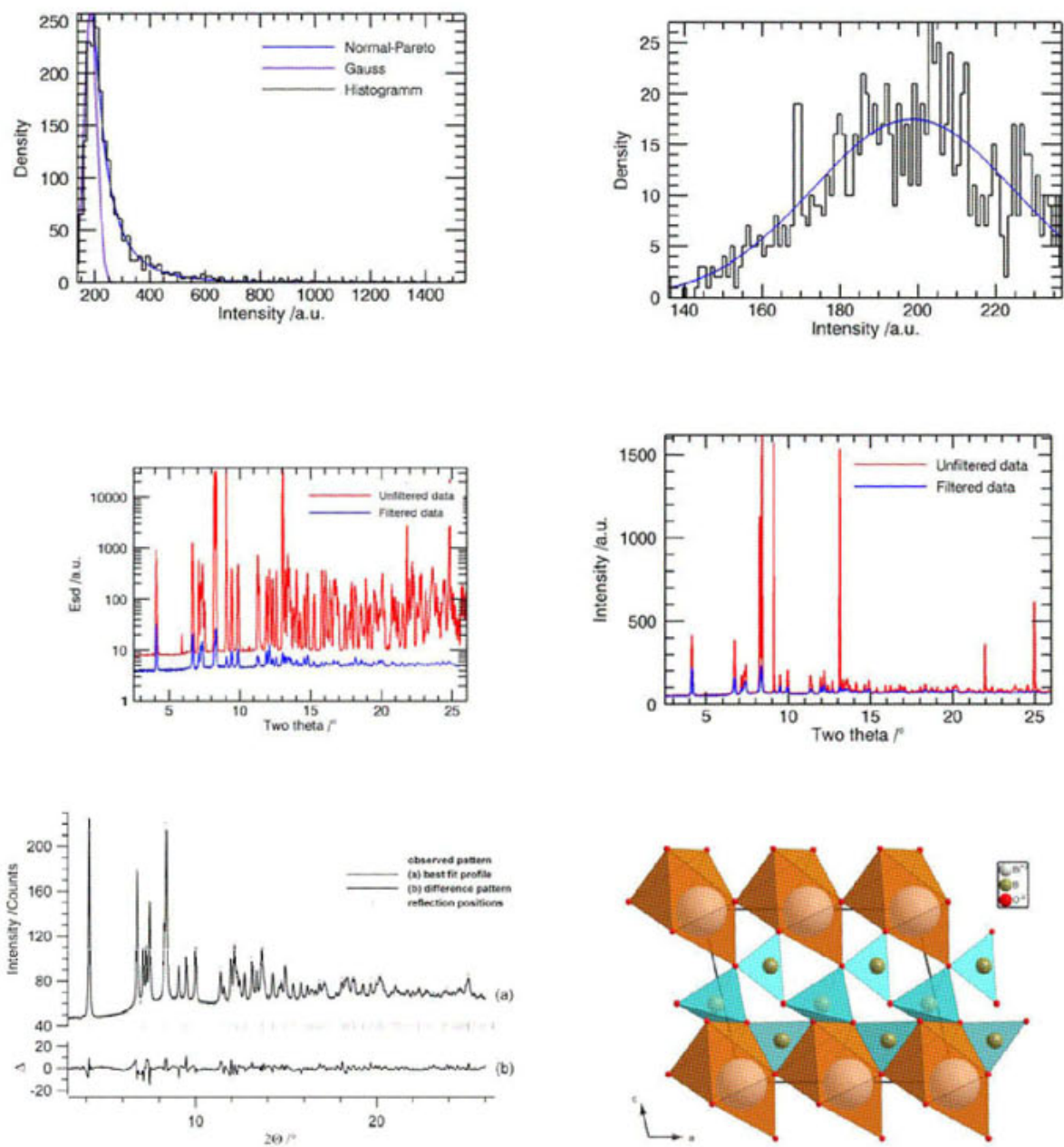

Figure 6. Powder diffraction data of $\varepsilon-\mathrm{BiB}_{3} \mathrm{O}_{4}$ at $\mathrm{P}=8.35 \mathrm{GPa}$, collected by a two-dimensional image plate detector. The top $48 \%$ and the bottom $2 \%$ of the intensities per integration bin were filtered) (a) $a$ bin is displayed showing fits to the expected normal and the proposed normal-Pareto distribution. The effect of filtering is further visualized on the final integrated and background corrected pattern (e) and on the standard deviations (f) (In red the integrated pattern of the unfiltered image, in blue the diffractogram of the filtered image). The Rietveld refinement plot of the the triclinic e-phase of $\mathrm{BiB}_{3} \mathrm{O}_{6}$ at $\mathrm{P}=8.34 \mathrm{GPa}$ (bottom) (d) and the corresponding projection of the crystal structure exhibiting $\mathrm{BO}_{3}$ triangles, $\mathrm{BO}_{4}$ tetrahedra and $\mathrm{BiO}_{x}$ polyhedra are drawn. 


\section{Discussion and conclusion}

High-pressure powder diffraction data often cannot be described using crystallographically common distribution models. They follow far more closely a smooth Pareto distribution. To achieve this, a normal distribution is folded with a Pareto distribution. A great advantage of this description is the ability to extract normally distributed data by deducing a fractile filter setting from the fitted model parameters.

It should be stressed that many publications exist that deal in great detail with the effect of crystallite size distribution on the intensity distribution and that again on the peak profile of the final one-dimensional diffraction pattern [5-7]. A few papers even dealing with the analysis of two-dimensional images in this respect can be found $[8,9]$. The difference between those works and the one presented here is that the intensity distribution seen in high pressure experiments does not primarily originate from size differences - but from the experimental constraints. In addition the effect of the intensity distribution on the diffraction pattern is far greater, and cannot unlike size distribution effects be modelled by a profile function.

There are at present methods of dealing with such aberrant data. A Bayesian approach to the Rietveld fitting procedure reduces the impact of too high intensities but can lead to spectacularly wrong results. There remains no substitution for good data with normally distributed intensities.

Finally this paper presented a method for achieving this goal, based on a sound description of the measured intensities. This has been applied successfully to data from various experimental sources [2-4]. All functions presented in this paper are integrated into the freely available software Powder3D-IP.

\section{References}

1. Georgii, H.-O., 2004, Stochastik (Berlin: de Gruyter), p. 225.

2. Dinnebier, R.E., Hinrichsen, B., Lennie, A. \& Jansen, M., 2009, Acta Crystallogr., Sect. $B, \mathbf{6 5}, 1$.

3. Hinrichsen, B., Dinnebier, R. \& Jansen, M., 2008, Z. Kristallogr., 223, 195.

4. Hinrichsen, B., Dinnebier, R.E., Rajiv, P., Hanfland, M., Grzechnik, A. \& Jansen, M., 2006, J. Phys.: Condens. Matter, 18, S1021.

5. Deb, A.K., Chatterjee, P. \& Sen Gupta, S.P., 2007, J. Appl. Crystallogr., 40, 33.

6. Langford, J.I., Louër, D. \& Scardi, P., 2000, J. Appl. Crystallogr., 33, 964.

7. Ida, T., Shimazaki, S., Hibino, H. \& Toraya, H., 2003, J. Appl. Crystallogr., 36, 1107.

8. Hirsch, P.B., 1954, Br. J. Appl. Phys., 5, 257.

9. Rodriguez-Navarro, A.B., Alvarez-Lloret, P., Ortega-Huertas, M. \& RodriguezGallego, M., 2006, J. Am. Ceramic Soc., 89, 2232.

Acknowledgements. Financial support by the Bundesministerium für Bildung und Forschung (BMBF) and the Fonds der Chemischen Industrie (FCI) is gratefully acknowledged. 

II. INSTRUMENTAL 



\title{
Coplanar grazing exit X-ray diffraction on thin polycrystalline films
}

\section{Z. Matěj , L. Nichtová, R. Kužel}

Department of Condensed Matter Physics, Faculty of Mathematics and Physics, Charles University in Prague, Ke Karlovu 5, 12116 Praha 2, Czech Republic *matej@karlov.mff.cuni.cz

Keywords: thin films, powder diffraction, grazing-exit, depth profiling

\begin{abstract}
X-ray powder diffraction analysis in the coplanar grazing exit (GE) parallel beam geometry was tested on thin polycrystalline $\mathrm{TiO}_{2}$ films with the aid of a laboratory diffractometer. Peak position, width and intensity were simulated by a model accounting for the effects of refraction, scattering in the layered sample, absorption and instrumental effects related to the grazing exit geometry. A model of absorption-induced peak broadening close to the critical angle was created. Measured data for a series of samples with different thicknesses were successfully fitted by the model. It is shown that the grazing exit coplanar diffraction is a suitable technique for thin films analysis in particular because of its flexible spatial and angular resolution.
\end{abstract}

\section{Introduction}

In comparison with the common coplanar grazing incidence (GI) diffraction geometry [1], the grazing exit (GE) diffraction technique [2-3] is not so common method for analysis of: thin polycrystalline films. In the coplanar GE geometry the incidence beam impinges on the sample surface at a high angle and the diffracted beam is detected at a low exit angle [2-3] (figure 1 in [2]). It was demonstrated [2-3] that in conjunction with synchrotron microbeam, spatial resolution (irradiated sample area) $10 \mu \mathrm{m} \times 10 \mu \mathrm{m}$ can be achieved retaining surface sensitivity about few nanometers. The GE diffraction was also shown [4] to be less sensitive on the sample displacement, for the stress evaluation using a Bragg-Brentano type diffractometer. In addition to diffraction, some small angle scattering and coherent scattering techniques also utilize GE beam condition [5-6]. When a PSD detector is used, angular resolution in the GE geometry is a function of the exit angle and the irradiated sample area, which is small compared to the GI case. Hence 2D maps of scattered intensity with sufficient resolution (because of narrow diffracted beam) can be obtained with a laboratory diffractometer. One disadvantage mainly for large sources and in-situ experiments has to be mentioned: contrary to the GI case, in the GE geometry beam source and sample relative position is changing.

In this contribution, results of laboratory measurements in the GE geometry with a PSD detector are presented and parameters of diffraction lines are analysed. Besides necessary 
position and intensity corrections, which were discussed elsewhere [2-3, 7], more attention is given to the description of the line width including line broadening close to the critical angle.

\section{Experimental conditions}

Well crystalline $\mathrm{TiO}_{2}$ single phase (anatase) thin films prepared by magnetron sputtering on the Si single crystal substrates were studied. Details of the sample preparation are described by the authors elsewhere [8]. Four samples of nominal thickness $48 \mathrm{~nm}, 180 \mathrm{~nm}, 630 \mathrm{~nm}$ and $800 \mathrm{~nm}$, respectively, were chosen for the present GE measurements.

The GE XRD measurements were done by using the PANalytical MPD diffractometer with a laboratory $\mathrm{Cu}-\mathrm{K} \alpha$ tube and a graded parabolic mirror in the incident beam and the PIXcel PSD detector with variable anti-scatter slits in the diffracted beam. The whole range $\left(\sim 3^{\circ}\right)$ of the PSD detector was used and hence the anti-scatter slits were fully opened $\left(4^{\circ}\right)^{*}$. The axial divergence was controlled by Soller slits $(0.04 \mathrm{rad})$ and the incident beam axial width by a beam mask. The irradiated sample area was about $(1-2 \mathrm{~mm} \times 15 \mathrm{~mm})^{* * *}$. * Peak-to-background ratio can be improved significantly with decreasing the PSD active length and the opening of the anti-scatter slits. ${ }^{* *}$ In the GE geometry spatial resolution is about few millimetres in the case of a laboratory diffractometer. It is believable that a number of irradiated crystallites (in our samples $>10^{8}$ ) is high enough to ensure sufficient grain statistics (which does not have to be the case of the synchrotron micro-beam [3]).

\section{Data analysis}

Measurements were performed in terms of $\omega$ (angle of incidence) vs. $2 \Theta$ intensity distribution maps around anatase Bragg reflections (figure 1). The PSD is suitable for detection of a $2 \Theta$ range with a constant incidence angle $\omega$. Physical interpretation of such a scan is complicated by the fact that intensity distribution in the $2 \Theta$ scan corresponds to a different small exit angle $\alpha_{f}=2 \Theta-\omega$ for each value of the diffraction angle $2 \Theta$. Hence the raw data were numerically transformed by the interpolation procedure into the $\left(\alpha_{f} \times 2 \Theta\right)$-space. Individual $2 \Theta$ scans were then fitted by the pseudo-Voigt function and obtained dependences of the peaks position, width and intensity on the exit angle $\alpha_{f}$ were analysed. Only measurements for the strongest anatase 101 reflection are presented.

\section{Peak position}

Dependences of the $2 \Theta$ anatase 101 reflection position on the exit angle $\alpha_{f}$ for all samples are depicted in figure 2 . It is clearly visible that $2 \Theta$ peak position correction for the refraction effect $[2-3,7]$ is necessary. The complex dielectric susceptibility $\chi_{0}$ and the critical angle $\alpha_{c}$ for $\mathrm{TiO}_{2}$ with a density $\rho_{T i O 2}$ and the used X-ray wavelength were calculated by [9]:

$$
\chi_{0}=-0.2410910^{-4}+\mathrm{i} 0.1199910^{-5}, \quad \not z_{c} 0.28, \rho_{T i O 2}=3.92 \mathrm{~g} \mathrm{~cm}^{-3} \text {. }
$$

The refraction effect for the incidence $\left(\alpha_{i}^{\text {film }}\right)$ and exit $\left(\alpha_{f}^{\text {film }}\right)$ angles of the beam inside the film can be easily calculated using the Snell law, the real part of the complex refraction index and the fact that inside the film the Bragg angle is the "true" Bragg angle $2 \Theta_{\mathrm{B}}$ :

$n_{r e}^{\text {fim }} \cos \left(\alpha_{i, f}^{f i m}\right)=\cos \left(\alpha_{i, f}^{v a c}\right), n^{\text {film }} \approx 1+\chi_{0}^{\text {fim }} / 2,2{ }_{B} \Theta \alpha_{i}^{\text {fim }}+\alpha_{f}^{\text {fim }}$.

The refinable model parameters are the "true" Bragg angle $2 \Theta_{\mathrm{B}}$ and the relative film density $n_{r}\left(\rho^{f i l m}=n_{r} \cdot \rho_{T i O 2}\right)$, by which the complex dielectric susceptibility $\chi_{0}$ is multiplied in order to obtain the dielectric susceptibility of the film $\chi_{0}^{\text {film }}$. The refined values of the anatase 101 
Bragg angle and the relative density $n_{r}$ for all samples are given in table 1 . The calculated $2 \Theta_{\mathrm{B}}\left(\alpha_{f}\right)$ dependences are depicted in figure 2 .

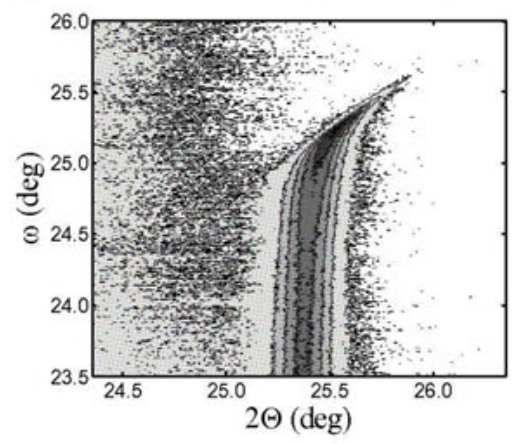

Figure 1. Part of the measured map of intensity distribution around the anatase 101 reflection (48 $\mathrm{nm}$ thin film sample).

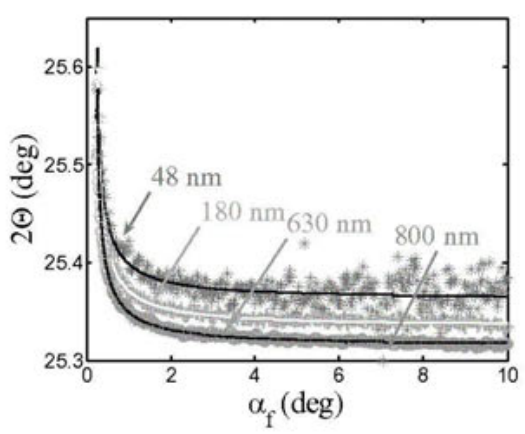

Figure 2. Anatase 101 reflection $2 \Theta$ position as a function of the exit angle: points - data, lines - fits.

\section{Peak width}

Dependences of the anatase 101 reflection FWHMs on the exit angle $\alpha_{f}$ are shown in figure 3. Two main features of all $F W H M\left(\alpha_{f}\right)$ dependences in figure 3 were considered. (i) The increasing peak width with increasing exit angle $\alpha_{f}$ clearly observable for higher $\alpha_{f}$ values, which is a simple consequence of a decreasing angular instrumental resolution and (ii) the huge diffraction line broadening close to the critical refraction angle due to the extremely strong absorption effect.

Calculation of the instrumental effects was based on the concept of the "virtual" slit (width $\delta_{\text {slit }} \sim 0.013^{\circ}$ ) of the PSD detector window observing the irradiated part of the sample by a slightly divergent $\left(\delta_{d i v} \sim 0.055^{\circ}\right)$ beam of height $h_{\mathrm{i}}$ coming from the mirror $\left(l_{m} \sim 50 \mathrm{~mm}\right.$ away from the sample). The beam height $\left(h_{i} \sim 0.67 \mathrm{~mm}\right)$ was assessed from the width of the direct beam $\left(\sim 0.24^{\circ}\right)$. This gives for the instrumental broadening $F W H M_{\mathrm{g}}$ :

FWHM ()$_{g}^{20}=\left(\delta_{d i v}^{2}+\delta_{\text {slit }}^{2}\right)+\left(\frac{h}{R} \frac{180}{\pi} \frac{\sin \left(\alpha_{f}\right)}{\sin \left(\alpha_{i}\right)}\right)^{2}=(\delta)^{2}+\left(\gamma \frac{\sin \left(\alpha_{f}\right)}{\sin \left(\alpha_{i}\right)}\right)^{2}$,

where $h=\left(h_{i}+h_{b}\right)$ is the height of the incident beam on the sample position (including a contribution of the incident beam divergence: $h_{b} \sim l_{m} \bullet \delta_{\text {div }}$ ) and $R$ is the goniometer radius ( $R$ $\sim 240 \mathrm{~mm}$ ). The calculated $\delta$ and $\gamma$ values in equation 3 are $\delta_{\text {calc }}=0.057^{\circ}$ and $\gamma_{\text {calc }}=0.17^{\circ}$. The terms are summed up in squares, as it is characteristic for Gauss-type profiles. This Gauss-like model fits data much better than a simple linear sum of all contributions.

The absorption effect in the film was calculated using the common theory of the X-ray wave propagation in layered samples [10]. According to this theory the imaginary part $Q_{\mathrm{im}, \mathrm{z}}$ of the perpendicular (to the sample surface) component of the scattering vector $\boldsymbol{Q}$ describes the absorption effect (in the sample reference system):

$$
Q_{i m, z}=2 \pi / \lambda \operatorname{Im}\left(\sqrt{\sin ^{2}\left(a_{f}^{f i l m}\right)+\chi_{0}^{f i l m}}+\sqrt{\sin ^{2}\left(a_{i}^{f i l m}\right)+\chi_{0}^{f i m}}\right) .
$$

Its reciprocal value is the penetration depth $T_{p}=1 / Q_{\text {im,z }}$. 
Powder diffraction intensity scattered by a small crystallite is given by the 3D Fourier transform of the crystallite shape function $\Omega_{\mathrm{c}}(\boldsymbol{r})$ projected into the direction of the diffraction vector $\boldsymbol{H}_{\mathrm{c}}$ of the crystallite (integrated over the space $q \perp$ perpendicular to the $\boldsymbol{H}_{\mathrm{c}}$ ):

$$
I_{c} \sim \int d^{2} \boldsymbol{q}_{\perp}\left|\int d^{3} \boldsymbol{r} \Omega_{c}(\boldsymbol{r}) e^{-i q \cdot \boldsymbol{r}}\right|^{2}=\int d r_{\|} e^{-i q r_{\|}} \int d^{3} \boldsymbol{r} \Omega_{c}\left(\boldsymbol{r}-\frac{\boldsymbol{r}_{\|}}{2}\right) \Omega_{c}\left(\boldsymbol{r}+\frac{\boldsymbol{r}_{\|}}{2}\right) e^{2 Q_{i m, z}, \boldsymbol{r}},
$$

where $\boldsymbol{q}=\boldsymbol{Q}-\boldsymbol{H}_{\mathrm{c}}$. The $\boldsymbol{r}_{\mid}$vector is a real space vector parallel to the diffraction vector $\boldsymbol{H}_{\mathrm{c}}$. A simple 2D model of square crystallites with the edge of length $D$ was used to study characteristic properties of the absorption effect. Orientation of edges of individual crystallites with respect to the sample surface as well as the lattice orientation with respect to the sample surface and crystallites edges were assumed to be completely random. Without absorption this model predicts size broadening which is both $h k l$ and $\psi \sim \Theta_{\mathrm{B}}-\omega$ independent, where $\psi$ is the inclination angle of $\boldsymbol{H}_{\mathrm{c}}$ from the sample normal. Fourier coefficients can be calculated using equation (5) for any orientation of the square crystallites with respect to the diffraction vector analytically and then numerically averaged over all such possible orientations. From the Fourier coefficients diffraction profile and its FWHM can be calculated. It can be shown that this model gives for high absorption values $\left(Q_{\mathrm{im}, \mathrm{z}}{ }^{*} D\right)>>1$ an absorption controlled integral breadth $\beta \sim 1 / 2 Q_{\mathrm{im}, \mathrm{z}}$ which is almost independent of the crystallite size $D$ and modified solely by a function of the inclination angle $\psi$.

Table 1. Refined parameters: $2 \Theta_{B}$ - "true" anatase 101 reflection angle, $n_{r}-$ relative film density, $\delta$-constant and $\gamma$-constant beam height angular instrumental resolution parameters.

\begin{tabular}{|l|c|c|c|c|c|}
\hline Sample & $2 \Theta_{\mathrm{B}}\left({ }^{\circ}\right)$ & $n_{r}$ & $\delta\left(^{\circ}\right)$ & $\gamma\left(^{\circ}\right)$ & Thickness $(\mathrm{nm})$ \\
\hline $1-48 \mathrm{~nm}$ & 25.360 & $0.93 \pm 0.02$ & $0.067 \pm 0.001$ & $0.197 \pm 0.007$ & $32 \pm 2$ \\
$4-180 \mathrm{~nm}$ & 25.332 & $0.93 \pm 0.01$ & & & $180 \pm 4$ \\
$7-630 \mathrm{~nm}$ & 25.312 & $0.90 \pm 0.01$ & & & $580 \pm 8$ \\
$8-800 \mathrm{~nm}$ & 25.312 & $0.88 \pm 0.01$ & $0.061 \pm 0.001$ & $0.190 \pm 0.001$ & $840 \pm 12$ \\
\hline
\end{tabular}

Anatase 101 reflection of the thinnest $(48 \mathrm{~nm})$ film is significantly broader than the same reflections of all other (thicker) samples, which are of almost same width (figure 3). Only the thinnest $(48 \mathrm{~nm})$ and the thickest $(800 \mathrm{~nm})$ samples were chosen for peak width analysis. From a size-strain analysis of the whole GI $2 \Theta$ scans done elsewhere [4], the median of the crystallites size distribution was found about $250 \mathrm{~nm}$ for the thicker sample $(800 \mathrm{~nm})$ and it was comparable with the film thickness for the thinner $(48 \mathrm{~nm})$ sample. The refined value of the effective microstrain was $e \sim 0.2 \%$ for both samples. Fixed values $D=50 \mathrm{~nm}$ and $D=250 \mathrm{~nm}$ were chosen for the peak width simulations for the $48 \mathrm{~nm}$ and $800 \mathrm{~nm}$ samples, respectively. The profile corresponding to the size broadening was calculated according to the described model of "square" crystallites and it was convoluted with the Gauss function model (equation 3) for the instrumental broadening with addition of the microdeformation broadening term. FWHM of the obtained profile was calculated. The physical broadening was fixed, the refinable parameters were angular terms $\delta$ and $\gamma$ describing the instrumental function:

$$
F W H M=\left(F W H M_{D}(e=0.2 \%) \otimes F W H M_{g}(\delta, \gamma)\right) \otimes F W H M_{S}\left(D, Q_{i m, z}{ }^{*} D, \psi\right),
$$

where $F W H M_{D}$ accounts for the microdeformation effect and $F W H M_{S}$ for the size effect. 
The results of the refinement procedure are shown in figure 3 and parameter values are given in table 1. Agreement of measured and calculated $F W H M \mathrm{~s}$ is very good. The refinement gives averaged values (from both samples) $\gamma \sim 0.19^{\circ}$ and $\delta \sim 0.064^{\circ}$. The refined $\gamma$ value is equivalent to the beam height $h_{i} \sim 0.75 \mathrm{~mm}$ in equation (3). Both the $\delta$ and $\gamma$ values ${ }^{*}$ are close to the values in equation (3) determined from the direct beam measurement $\left(\gamma_{\text {calc }}=0.17^{\circ}, \delta_{\text {calc }}=0.057^{\circ}\right)$.

- It should be considered that the $\delta$ value is affected also by an uncertainty in the determination of the physical broadening (crystallite size and deformation). E.g. from the Scherrer formula it can be directly deduced that the relative error of $\delta$ introduced in this way is the same as the relative error of the crystallite size $(\sim 5 \%)$. Another systematic error affecting the $\delta$ value can be caused by neglecting some other possible effects: e.g. an effect of a finite spectral width of the used X-ray source.

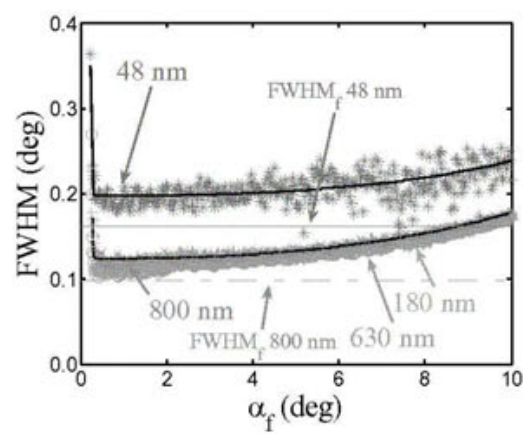

Figure 3. Anatase 101 peak FWHM as a function of the exit angle: points - data, lines - fits (physical broadening as determined from GI $2 \Theta$ scans analysis marked by $\mathrm{FWHM}_{\mathrm{f}}$ lines).

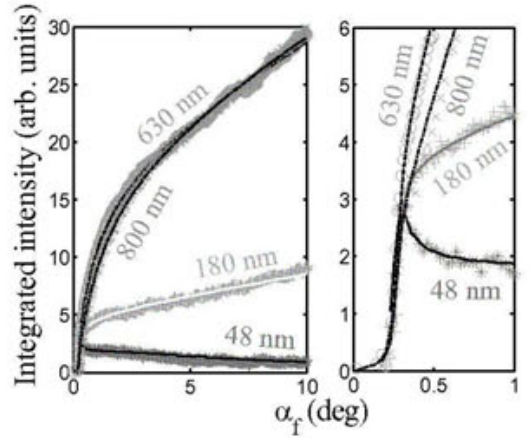

Figure 4. Anatase 101 reflection integrated intensity as a function of the exit angle: points data, lines - fits.

\section{Peak intensity}

Diffracted integral intensity as a function of the exit angle $\alpha_{f}$ is depicted in figure 4. Data were simulated using the common theory [10] as described explicitly for the grazing incidence non-coplanar diffraction $[10,11]$. Only two scattering processes were assumed: simple diffraction of the incident beam and diffraction followed by specular reflection on the interfaces. The second process is weak ( $<3 \%$ of total intensity) and hence it has been neglected in previous considerations. Scattered intensity flux can be expressed as:

$$
I\left(\alpha_{f}\right) \sim T\left\{\left|t_{\text {film }}^{(1)}\left(\alpha_{1, i}\right)\right|^{2}\left|t_{\text {film }}^{(2)}\left(\alpha_{f}\right)\right|^{2} \frac{1-e^{-2 Q_{1,2, m} T}}{2 Q_{1, z, m} T}+\left|t_{\text {film }}^{(1)}\left(\alpha_{2, i}\right)\right|^{2}\left|r_{\text {film }}^{(2)}\left(\alpha_{f}\right)\right|^{2} \ldots\right\},
$$

where e.g. $t^{(l)}$ film $\left(\alpha_{1, i}\right)$ is the amplitude of the transmitted incident wave in the film and e.g. $r^{(2)}$ film $\left(\alpha_{f}\right)$ is the amplitude of specularly reflected outgoing wave in the film. $T$ is the thickness of the film. Wave amplitudes are calculated by the standard theory for multilayer stacks [10] assuming the $\mathrm{TiO}_{2}$ film and the $\mathrm{Si}$ substrate. The instrumental correction accounting for the angular acceptance of the detector ("virtual" slit) was included as a simple factor of equation (7) for higher angles $\left(\alpha_{f}>1^{\circ}\right)$. For smaller angles it was convoluted with the intensity distribution (7) to describe properly the sharp edge intensity dependence close 
to the critical angle. The form of the factor was the same as in equation (3) but the terms were added linearly. Another correction is the $\sin \left(\alpha_{i}\right)$ term accounting for the X-ray flux on the sample. For the thinnest $48 \mathrm{~nm}$ sample also a texture correction was necessary. It was measured for the 101 anatase reflection directly by a texture goniometer and corrected for absorption in the fitting procedure using the model parameters (thickness $T$, relative density $n_{r}$ ). The differences between expected (from the deposition) and refined values of the film thickness (table 1) are lower than 10\% with the exception of the thinnest sample.

\section{Concluding remarks}

Thickness and density of thin polycrystalline $\mathrm{TiO}_{2}$ films were determined by the coplanar GE $X$-ray diffraction with the aid of a laboratory diffractometer with a PSD detector. In this geometry: peak-to-background ratio, spatial and angular resolution are controlled by instrumental factors (incident beam height, detector distance, slits-height etc.) and can be suitably adjusted. The GE geometry offers spatial resolution about few millimetres in the case of a laboratory diffractometer [2-3]. This can be utilized e.g. for testing samples homogeneity. The angular resolution is a function of the exit angle, but can be simulated and included into the models describing diffraction lines features (width, intensity). Anomalous diffraction peak broadening is observed close to the critical angle and this effect can be explained by strong absorption of scattering waves. The coplanar GE geometry is confirmed to be a suitable method for thin films analysis.

\section{References}

1. Lim, G., Parrish, W., Ortiz, C., Bellotto, M. \& Hart, M., 1987, J. Mater. Res., 2, 471 .

2. Noma, T. \& Iida, A., 1998, J. Synchrotron Rad., 5, 902.

3. Noma, T., Takada, K. \& Iida, A., 1999, X-ray Spectrom., 28, 433.

4. Njeh, A., Wieder, T. \& Fuess, H., 2000, Powder Diffr., 15 (4), 211.

5. Zhang, W. \& Robinson, I.K., 2007, Z. Kristallogr., 222, 601.

6. Pfeiffer, F., Zhang, W. \& Robinson, I.K., 2004, Appl. Phys. Lett., 84 (11), 1847.

7. Colombi, P., Zanola, P., Bontempi, E. \& Depero,L.E., 2007, Spectroch. Acta B, 62, 554.

8. Nichtová, L., Kužel, R., Matěj, Z., Šícha, J. \& Musil, J., in these proceedings.

9. Stepanov, S.A., XOh on the web, http://sergey.gmea.aps.anl.gov/x0h.html.

10. Pietsch, U., Holý, V. \& Baumbach T., 2004, High-Resolution X-ray Scattering (Springer).

11. Fewster, P.F., Andrew, N.L., Holý, V. \& Barmak, K., 2005, Phys. Rev. B, 72, 174105 .

Acknowledgements. We kindly acknowledge J. Šícha and J. Musil from ZČU Plzen̆ for providing the samples, J. Drahokoupil (FZU AV ČR) and V. Holý (KFKL MFF UK) for many hints and J.F. Woitok and A. Kharchenko from PANalytical for help with the first experiments in Almelo laboratory. The financial support of the Ministry of Education of the Czech Republic (research program MSM 0021620834) and the Czech Academy of Sciences (KAN400720701) are gratefully acknowledged. 


\title{
Possibilities and limitations of X-ray diffraction using high-energy X-rays on a laboratory system
}

\section{J. te Nijenhuis ${ }^{*}$, M. Gateshki, M. J. Fransen}

PANalytical B.V., Lelyweg 1, 7602 EA Almelo, The Netherlands

"hans.te.nijenhuis@panalytical.com

Keywords: diffraction, pair distribution function, high-energy X-rays, nanocrystalline materials

\begin{abstract}
Analyzing powder diffraction data of nanocrystalline and amorphous materials using the pair distribution function method provides useful information about long and short range ordering in the material investigated. We have developed the application of PDF analysis on a standard laboratory system employing an X-ray tube with a silver anode as Xray source. Meaningful results have been achieved from a range of samples that allowed for comparison with previously reported data, obtained using synchrotron radiation.
\end{abstract}

\section{Introduction}

Recent years have shown an increased interest in nanomaterials due to their specific properties. Structural information about these materials can be derived from broad, not welldefined features in a diffractogram. Analysis of nanomaterials therefore needs a totalscattering approach, including both peaks and diffuse scattering, rather than relying on Bragg peaks only. One of the most promising analytical methods used is atomic pair distribution function (PDF) analysis. Originally, this method was developed to study amorphous and highly disordered materials. More recently, it has been used also for the analysis of nanostructured materials. Since the method requires short wavelengths, traditionally the measurements are performed at synchrotron facilities, making use of both the high photon energies and the high photon flux that these beam lines offer. On the other hand, in-house measurements with various X-ray wavelengths have been reported as well $[1,2]$. We have investigated the possibility to study nanomaterials on a standard laboratory system, using Ag $K \alpha$ radiation. In this paper we describe the first results on nanocrystals, liquids and amorphous materials.

\section{Pair distribution function analysis}

The pair distribution function $\mathrm{G}(r)$ describes the probability of finding two atoms separated by a distance $r$ in the material under investigation. The PDF method extracts structure-related information from powder diffraction data [3]. Since the technique takes both Bragg and 
diffuse scattering into account, it provides information not only about the long-range $(>10 \mathrm{~nm})$ atomic ordering but also about the short-range ordering in materials. The method is performed in the following steps:

( $i$ ) the diffraction pattern is corrected for background (using a separate diffraction measurement of an empty sample container), Compton scattering, detector dead-time, absorption, diffraction geometry and polarization;

(ii) the corrected X-ray diffraction data is scaled into electron units and the reduced structure function [4] is calculated;

(iii) the structure function is Fourier transformed into the atomic pair distribution function:

$$
\mathrm{G}(r)=4 \pi \mathrm{r}\left(\rho(r)-\rho_{0}\right)
$$

in which $\rho(r)$ is the local atom number density, and $\rho_{0}$ is the mean atom number density.

Since the method does not presume a periodicity in the material, it is widely applied for the study of nanocrystalline and amorphous materials. The data can be used for full-profile fitting to refine structural models [5].

\section{Experimental setup}

X-ray diffraction measurements were performed on a PANalytical X'Pert PRO MPD system equipped with a programmable divergence slit, a capillary spinner, programmable antiscatter and receiving slits and a scintillation detector. An X-ray tube with a silver anode was used as X-ray source, delivering $\mathrm{Ag} K \alpha$ radiation with a wavelength of $0.0561 \mathrm{~nm}$. Additional shielding was applied to the optical path in order to achieve a feature-free background. The samples have been prepared in glass capillaries with a diameter of $2 \mathrm{~mm}$. Scans along the $2 \theta$ axis were made up to an angle of 160 degrees corresponding to a scattering vector $Q$ of $22 \AA^{-1}$. The scattering vector is given by $Q=4 \pi \sin (\theta) / \lambda$. Data acquisition times were typically in the order of $20 \mathrm{~h}$. Initial data treatment, including background subtraction and optional $\mathrm{K \alpha}_{2}$ stripping was done using X'Pert HighScore [6]. For PDF analysis and fitting, we used the software RAD [7] and PDFgui [5].

\section{Results and discussion}

Samples of different nature - crystalline, nanocrystalline, amorphous solid and liquid - were selected to test the applicability of PDF analysis on a standard XRD system. The results of these experiments are described below.

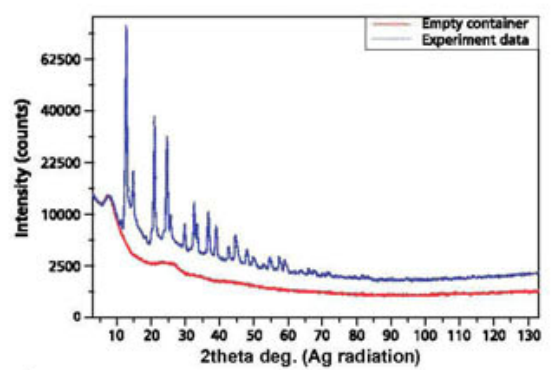

a)

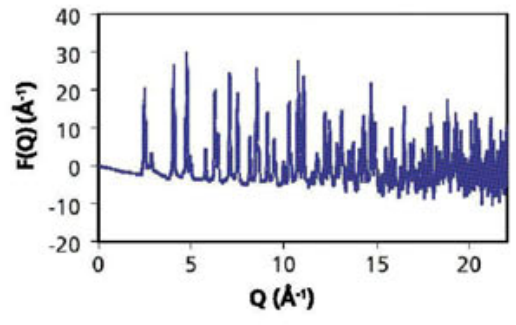

b)

Figure 1. (a) XRD measurement and (b) reduced structure function of silicon carbide. 


\section{Silicon carbide}

Figure la shows a diffraction pattern of silicon carbide powder in a capillary, together with a measurement of an empty capillary. The reduced structure function obtained from the corrected intensity data is shown in figure $1 \mathrm{~b}$. After Fourier transformation the PDF as shown in figure 2 was obtained. The maxima in this graph could be identified as the interatomic distances $\mathrm{Si}-\mathrm{C}, \mathrm{Si}-\mathrm{Si}$ and $\mathrm{C}-\mathrm{C}$, derived from the sphalerite crystal structure of $\mathrm{SiC}$ [8].

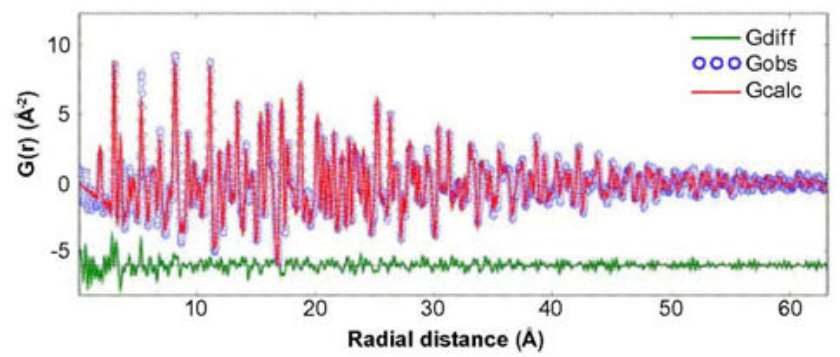

Figure 2. Experimental (circles) and calculated atomic PDF (red line) of SiC.

Table 1. Interatomic distances of $\mathrm{SiC}$ calculated determined from the experimental PDF (fig.2).

\begin{tabular}{|l|c|c|}
\hline Atoms & Orientation & Interatomic distance $(\AA)$ \\
\hline $\mathrm{Si}-\mathrm{C}$ & $1 / 4<111>$ & 1.89 \\
\hline $\mathrm{Si}-\mathrm{Si}, \mathrm{C}-\mathrm{C}$ & $1 / 2<110>$ & 3.08 \\
\hline $\mathrm{Si}-\mathrm{C}$ & $1 / 4<311>$ & 3.61 \\
\hline $\mathrm{Si}-\mathrm{Si}, \mathrm{C}-\mathrm{C}$ & $<100>$ & 4.36 \\
\hline $\mathrm{Si}-\mathrm{C}$ & $1 / 4<331>$ & 4.75 \\
\hline $\mathrm{Si}-\mathrm{Si}, \mathrm{C}-\mathrm{C}$ & $1 / 2<211>$ & 5.34 \\
\hline
\end{tabular}

\section{Anatase}

Measurements performed for PDF analysis typically require long-range scans up to high $2 \theta$ angles, where the diffracted intensities are low. Variable counting time (VCT) methods can be applied to spend longer counting times at the high-angle, low-intensity region of the diffractogram at the cost of time spent on the low-angle region. Schematically the redistribution of measurement times is shown in figure 3 . The total measurement time is the same for both situations.

In order to investigate the improvement of data quality at high Q-values, experiments of typically $20 \mathrm{~h}$ measurement time were performed on nanocrystalline anatase $\left(\mathrm{TiO}_{2}\right)$ with an average particle size of $15 \mathrm{~nm}$ using constant and variable measurement times according to the scheme given in figure 3 . The resulting reduced structure functions are shown in figure 4 . 


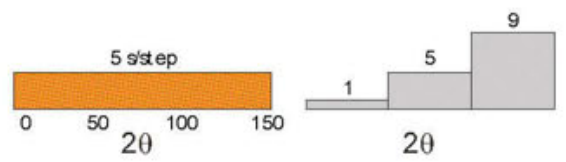

Figure 3. Constant counting time (left) and variable counting time (right) as a function of $2 \theta$ angle.
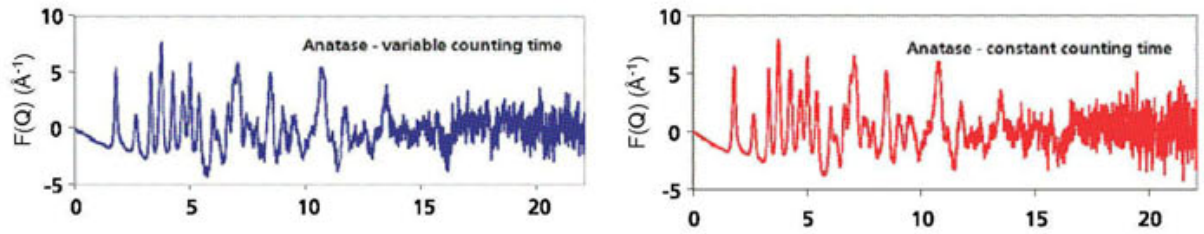

Figure 4. Reduced structure functions of anatase, measured using variable and constant counting times.

The noise level at high Q-values of the variable counting time measurement is improved in comparison with the constant counting time measurement allowing the observation of additional structure-related features. No reduction in data quality has been observed in the low Qrange. The PDF from the VCT experiment and the calculated PDF are in good agreement.

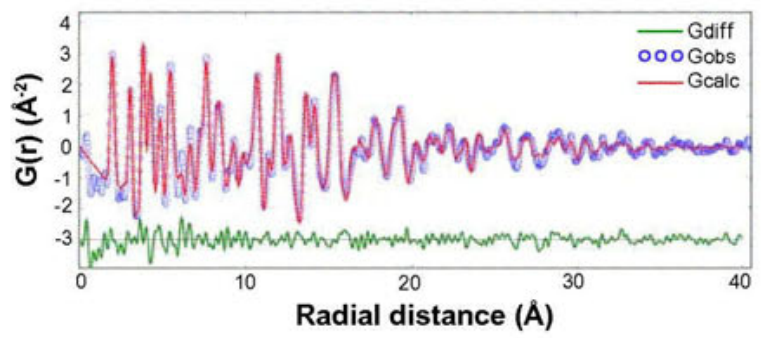

Figure 5. Experimental (circles) and calculated atomic PDF (red line) of nanocrystalline anatase.

\section{Vanadium oxide xerogel}

Vanadium oxide xerogel $\left(\mathrm{V}_{2} \mathrm{O}_{5} \cdot n \mathrm{H}_{2} \mathrm{O}\right)$ does not form crystals, that can be analysed with the use of traditional crystallographic methods. The diffraction pattern (see figure 6) only shows a combination of Bragg-like peaks and broad diffuse features. 


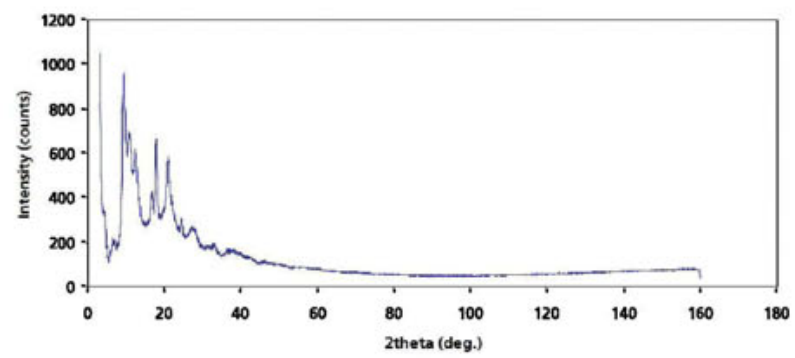

Figure 6. XRD measurement on vanadium oxide xerogel.

The PDF, derived from the measurement was compared to the PDF obtained from a structure model described by Petkov et al. [9]. This model describes the crystallites consisting of bilayers of $\mathrm{V}_{2} \mathrm{O}_{5}$, made of square pyramidal $\mathrm{VO}_{5}$ units and separated by water molecules. PDF analysis in figure 7 shows a good fit at distances in the intralayer region $(r<1.15 \mathrm{~nm})$ and a not so good fit in the interlayer region $(r>1.15 \mathrm{~nm})$. The same observation was made by Petkov et al. [9] based on synchrotron measurements with a wavelength of $0.046 \mathrm{~nm}$. It was proposed that the bilayer slabs are not perfectly stacked, but are turbostratically disordered.

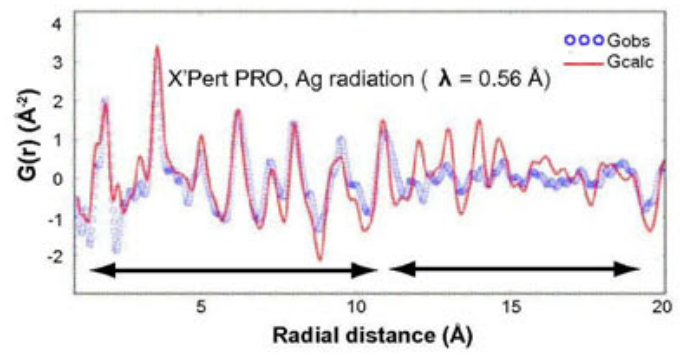

Figure 7. Atomic PDF of vanadium oxide xerogel.

\section{Amorphous solids}

Fumed silica powder has been used as an example of applying PDF analysis to amorphous materials. Traditional structure analysis does not give much information; only a few "humps" can be seen in the scan in figure 8a. After calculating the reduced structure function in figure $8 \mathrm{~b}$ more structure can be observed. PDF analysis of these data helps to reveal the short range order by determining average distances between the nearest neighbouring atoms. 

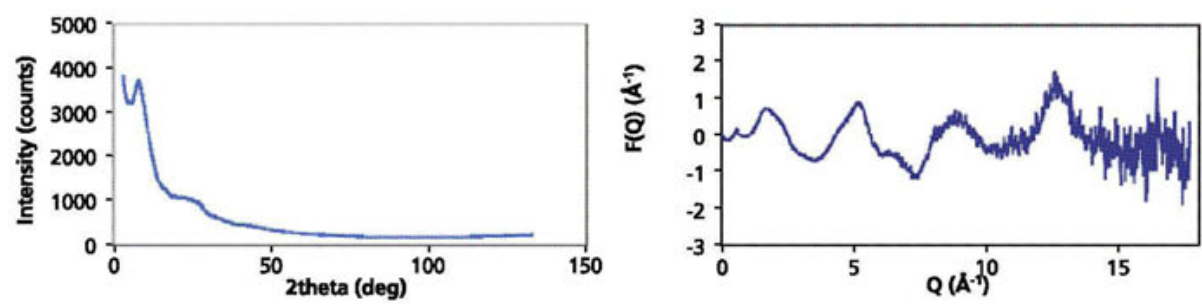

Figure 8. XRD measurement (left) and reduced structure function (right) of silica.

The PDF in figure 9 shows five clear peaks that could be determined as first and second order $\mathrm{Si}-\mathrm{Si}$, O-O or Si-O interatomic distances in silica as given by Mozzi and Warren [1].

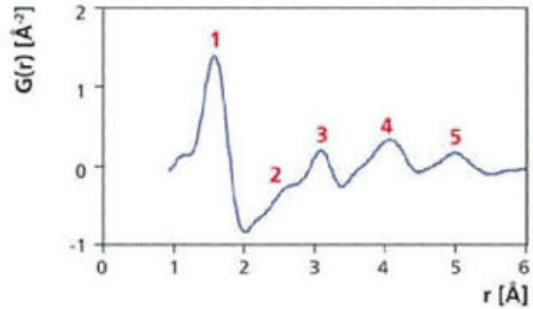

Figure 9. Atomic PDF of fumed silica.

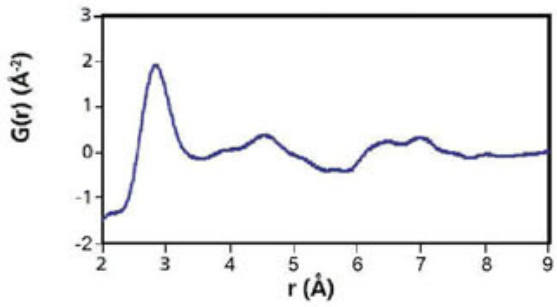

Figure 10. Atomic PDF of water.

\section{Liquids}

As in amorphous materials, liquids do not have a periodic arrangement of the atoms, and therefore no sharp diffraction maxima in the diffractogram. However, with the use of PDF analysis average distances between the atoms can be observed. As an example we show here the results of tap water. The PDF in figure 10 shows a relatively narrow peak for the first O$\mathrm{O}$ distance (ca. $2.8 \AA$ ). The maxima for the second and third coordination spheres are less sharp. These results are in accordance with the synchrotron data, employing photon energies of $10 \mathrm{keV}(\lambda \approx 0.12 \mathrm{~nm})$ reported by Hura et al. [10].

\section{Discussion}

The results achieved from a standard laboratory XRD system show a good agreement with data reported in literature, obtained from both in-house equipment and synchrotron beam lines, despite the different characteristics of synchrotron and tube radiation. The spectrum of the $\mathrm{Ag}$ radiation used contains the $K \alpha_{1}-K \alpha_{2}$ doublet; numerical corrections had to be applied to remove the $\mathrm{KO}_{2}$ component. The spectrum of synchrotron radiation is monochromatic. To our knowledge the resolution of the measurements with open receiving slit are comparable to the resolution of synchrotron data recorded using an imaging plate.

The long $2 \theta$ measurement range allowed for maximum scattering vectors up to $20 \AA^{-1}$. The high-energy X-rays of a synchrotron facility give the possibility to obtain higher Q-vectors, although in practice values larger than $30 \AA^{-1}$ are rarely used for PDF analysis. 
Signal-to-noise ratio of the data depends on the measurement time in both cases. The measurements reported here took 20 to 24 hours typically, compared to a few minutes or even seconds in a synchrotron. On the other hand, the flexibility and accessibility of an inhouse system make it the ideal tool for preparation and pre-screening for valuable beam time.

\section{Conclusions}

Results of PDF analysis on a range of samples measured on a standard laboratory XRD system, equipped with an X-ray tube with a silver anode, have been shown. Meaningful results have been achieved, that allowed for comparison with data reported in literature, even though the attainable Q-range is not as high as with synchrotron radiation. The current results encourage investigating the applicability of the PDF method on other materials with other optical configurations and detectors.

\section{References}

1. Mozzi, R.L. \& Warren, B.E., 1969, J. Appl. Cryst., 2, 164.

2. Brühne, S., Uhrig, E., Luther, K.-D., Assmus, W., Brunelli, M., Masadeh, A.S. \& Billinge, S.J.L., 2005, Z. Kristallogr., 220, 962.

3. Egami, T. \& Billinge, S.J.L., 2003, Underneath the Bragg peaks: Structural Analysis of Complex Materials (Amsterdam, The Netherlands: Elsevier Science B.V.).

4. Klug, H. P. \& Alexander, L. E, 1974, X-ray Diffraction Procedures for Polycrystalline Materials (New York, NY, USA: Wiley).

5. Farrow, C.L., Juhas, P., Liu, J. W., Bryndin, D., Bozin, E. S., Bloch, J., Proffen, Th. \& Billinge, S. J. L., 2007, J. Phys.: Condens. Matter, 19, 335219.

6. X'Pert HighScore, software program for phase identification, www.panalytical.com

7. Petkov, V., 1989, J. Appl. Cryst., 22, 387.

8. Braekken, H., 1930, Z. Kristallogr, 75, 572.

9. Petkov, V., Bozin, E., Billinge, S.J.L., Vogt, T., Trikalitis, P. \& Kanatzidis, M., 2002, J. Am. Chem. Soc., 124, 10157.

10. Hura, G., Sorenson, J., Glaeser, R.M. \& Head-Gordon, T., 2000, J. Chem. Phys., 113,9140 .

Acknowledgements. The authors gratefully acknowledge Prof. V. Petkov, Central Michigan University, Mt. Pleasant, MI, USA and Prof. B. Palosz, Institute of High Pressure Physics, Polish Academy of Sciences, Warsaw, Poland for providing respectively the vanadium oxide xerogel and silicon carbide samples described in this paper. 



\title{
Advantages and disadvantages of fast XRPD measurement by using image-plate and rotating anode source
}

\section{G. Kimmel', D. Mogilyanski}

Institutes for Applied Research, Ben Gurion University of the Negev, Beer-Sheva, Israel *kimmel@bgu.ac.il

Keywords: fast X-ray powder diffraction, image plate detector, Guinier diffractometer, Bragg-Brentano diffractometer, hafnium oxide, Rietveld refinement

\begin{abstract}
An image-plate detector performs simultaneous charge accumulation providing fast data collection. A high resolution Guinier image-plate camera was installed on a rotating anode source. This configuration enables fast measurement with high resolution. There are some differences between the Guinier image-plate camera (GIP) and the conventional Bragg-Brentano diffractometer (BBD). The GIP works in transmission mode with a constant focusing circle. The monochromator is attached to the source providing $\mathrm{K} \alpha_{1}$ radiation. There is no background cut. The range of the scattering angles $2 \theta$ is from 1 to $100^{\circ}$. The BBD works in back reflection mode with multiple focusing circles. The monochromator attached to the detector cuts $\mathrm{K} \beta$ and most of the background but without diminishing of $K \alpha_{2}$. The range of the scattering angles $2 \theta$ is between 0.5 and $158^{\circ}$. In the standard measurement procedure there are also differences in the sample loading and measurement. In GIP a thin layer of powder is loaded and the illuminated area and depth are equal for the entire $2 \theta$ range. In BBD the sample is loaded in a cavity and the illuminated area and depth are changing throughout the $2 \theta$ range. As a final point, the counting principles in GIP and BBD are totally different. Data were collected from several samples in both GIP and BBD. Most of the samples were high quality powders of well known solids like $\mathrm{LaB}_{6}, \mathrm{ZnO}, \alpha \mathrm{Al}_{2} \mathrm{O}_{3}, \mathrm{CeO}$, NIST standards, WC. More complicated structures were studied such as monoclinic $\mathrm{HfO}_{2}$ and $\mathrm{Fe}_{2} \mathrm{O}_{3}$. The crystal structure characteristics including cell parameters and atomic positions were compared. In addition, quantitative analysis was performed for mixtures of corundum and zirconia, in order to compare the results of relative phase amounts in both systems. The main tool for the data processing was the Rietveld method.
\end{abstract}

\section{Introduction}

The Guinier camera is based on a monochromatic beam being transmitted through a powder sample and the diffracted beams being recorded on the focusing circle. The oldest Guinier camera, also called focusing camera [1], used a single crystal that served two purposes: separating the characteristic beam from the white radiation and converging the diffracted beams 
into the focus circle. The diffraction pattern was recorded on an X-ray wet film, after a short exposure time of about $10 \mathrm{~min}$. The diffraction lines were sharp with low background, except when fluorescence occurred. The main disadvantages of the film are poor sensitivity to the $\mathrm{X}$-ray intensity and existence of a top limit to the radiation dose. On the other hand, there is no limit to the flux rate of the X-ray photons. Among the improvements introduced to this detection method system one counts using of high quality crystals in the monochromator, resulting in a pure $K \alpha_{1}$ beam, and replacing the wet film by a counter to measure the scattered intensities while scanning along the focusing circle in steps of about $0.01^{\circ}$ on the $2 \theta$ scale [2]. The counting time for each step is 2 to 10 seconds(s). For a $2 \theta$ range of $90^{\circ}$ and a counting time of $4 \mathrm{~s} / \mathrm{step}$ the measuring time becomes $10 \mathrm{~h}$. A position sensitive detector (PSD) was introduced later on to shorten the measuring time. However, the PSD based on gas ionization or graphite wire suffer from lack of accuracy for diffraction line positions. The efficiency of both counters decreases at high counting rates. The recent imaging plate (IP) detector seems to combine fast measurement with accurate diffraction line positioning. Unlike the wet film, the IP tolerates high exposure doses giving accurate intensity measurements. In addition, the diffraction line positioning on the IP is accurate and maintains linearity at very high counting rates, which is adequate for high intensity X-ray sources, such as high-brilliance synchrotron sources [3-6].

The combination of rotating anode source with Guinier IP Camera (GIPC) is working in BGU from 2003. The following standard reference materials (SRM) of the National Institute for Standards and Technology (NIST), M, have been examined: For intensity the SRM 674a series: $\mathrm{Al}_{2} \mathrm{O}_{3}$ (corundum), $\mathrm{CeO}_{2}, \mathrm{Cr}_{2} \mathrm{O}_{3}, \mathrm{TiO}_{2}$ (rutile) and $\mathrm{ZnO}$ (wurtzite); for accuracy of line position, SRM $640(\mathrm{Si})$ as light element, and $\mathrm{W}, \mathrm{WC}$, and $\mathrm{Ag}$ as heavy elements; and for instrumental line broadening as well as line position accuracy SRM 660: $\mathrm{LaB}_{6}$. [7].

In the present work the GIPC is examined as a tool for crystallographic characterization of powders, compared to conventional Bragg Brentano Diffractometer (BBD), considering cell parameters and atomic positions of $\mathrm{HfO}_{2}$ powder. At room temperature $\mathrm{HfO}_{2}$ is monoclinic, space group P2 $1 / \mathrm{c}$, Pearson Symbol mP12, $\mathrm{ZrO}_{2}$ type. [8].

\section{Experimental}

Material: The material for the XRD test, at room temperature, was a fine powder (particle size $\sim 0.1 \mu \mathrm{m}$ ) of $99.998 \% \mathrm{HfO}_{2}$, (including $100 \mathrm{ppm} \mathrm{Zr}$ ) from Alfa ${ }^{\circledR}$ (Johnson Matthey Company), cat. \#: 235-0132-2.

Instrumental: The new Huber Guinier Camera G670 was installed on an Ultrax 18 - Rigaku $\mathrm{X}$-ray rotating $\mathrm{Cu}$ anode source. The diffraction geometry is of Guinier-type with the sample in transmission-asymmetric geometry, namely, the angle of incidence $45^{\circ}$ to sample normal. In these conditions the sample moves asymptotically to the focal circle (see fig. 1 in [7]). The diameter of the focal circle of the monochromator (B) is $360 \mathrm{~mm}$. The diameter of the focal circle of the camera is $180 \mathrm{~mm}$. The camera covers the $2 \theta$ range from $0^{\circ}$ to $100^{\circ}$. The aluminium camera housing consists of two main parts: (a) The upper inner part of the camera cylinder holds the imaging plate (a Fujifilm ST-Vn stripe), the erasing halogen lamp and the $\mathrm{X}$-ray entrance window; and (b) the lower part contains an electro-mechanical device to actuate the readout unit. The beam of a red $635 \mathrm{~nm}$ diode laser is focused on the surface of the Imaging Plate. The diffusely seattered blue photo-stimulated luminescence (PSL) light enters a photomultiplier tube (PMT) through a blue transmitting edge filter. The step motor 
clock triggers an $\mathrm{A} / \mathrm{D}$-converter, i.e. each step of the driving motor generates a corresponding value of the PMT signal. A complete diffractogram for the $2 \theta$ range from 0 to $100^{\circ}$ consists of 20,000 data points which corresponds to a step resolution of $0.005^{\circ} 2 \theta$. More details about the IP Guinier Camera G670 are given in the website http://www.xhuber.com.

The BBD was a Philips $0 / 2 \theta$ X'PERT diffractometer, powered by PW1710 generator, with reflected beam graphite monochromator and proportional gas detector.

Measuring and analysis procedure: In the GIPC the powders were adhered to a thin flat polyethylene (Mylar) foil attached to the sample holder. The rotating anode was operated at $50 \mathrm{kV}, 80 \mathrm{~mA}(4 \mathrm{~kW})$. The exposure time usually was $2-30 \mathrm{~min}$. The data recording was made by moving the readout system several times and accumulating the data. In most cases three scanning runs of the readout system suffice to obtain a good quality diffractogram; however, additional runs can be made until no residual data remains on the IP. In this work ten readout runs were carried out for all samples. Two different independent XRPD runs of 5 and 10 min respectively, (GIPC-1 and GIPC-2) were made.

In BBD the powders were loaded in a rectangular insert of aluminium, and scanned in a $2 \theta$ range between 10 and $156^{\circ}$ by steps of $0.02^{\circ}$. Two different independent XRPD runs with 6 $\mathrm{s} / \mathrm{step}$ and $1 \mathrm{~s} / \mathrm{step}$, respectively, (BBD-1 and BBD-2) were made.

The scan data were analyzed by the Rietveld method, using FullProf software [9]. Pseudo Voigt profile has been chosen.

\section{Results and discussion}

Signal to background ratio (SBR): It is common not to distinguish between signal-tonoise-ratio (SNR) and signal-to- background-ratio (SBR). Without X-ray excitation, it is known that the IP reads a nearly uniform background which contributes about 80 count/pixel after the halogen lamp operation. This background is observed at all $2 \theta$ angles for each readout-run. It is suggested to call this reading dark background, which should be considered as an instrumental bias. The dark background leads to the impression that the background is uniform. After subtracting the dark counting (800 counts for 10 of readout runs) the background appears as expected for a Guinier camera. The rotating anode provides beam intensity that the dark background can be treated with the normal background. However, the SNR should be considered as the ratio between the background spread (in counts) and the height of the related reflected peak (in counts). Thus, the SNR defines the ability of the instrument to detect very weak diffraction lines.

Results from Rietveld refinement: Fig. 1 shows normalized diffractograms of $\mathrm{HfO}_{2}$, in the $2 \theta$ range between 10 and $90^{\circ}$, obtained with the GIPC and BBD. For the GIPC the exposure time was $120 \mathrm{~s}$ and the source power was $4 \mathrm{~kW}$. The BBD diffractogram was obtained with a $45 \mathrm{kV}, 40 \mathrm{~mA}$ source and step scan width and time of $0.02^{\circ} 20$ and $1 \mathrm{~s}$, respectively. As shown in figure 1 the GIPC has lower SBR but the SNB is more or less the same.

Figure 2 and 3 show the Rietveld refinement results obtained with data from BBD and GIPC, respectively. In both cases an excellent agreement between calculated and observed data was accomplished. The broadening of the diffraction lines was similar in all diffractograms and may be attributed to a grain size of about $50 \mathrm{~nm}$. 


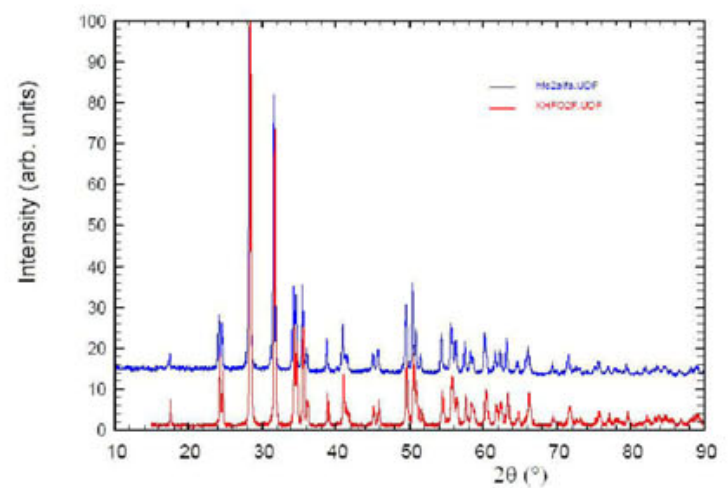

Figure 1. Normalized diffractograms of $\mathrm{HfO}_{2}$ powder from $\mathrm{BBD}$ (bottom) and GIPC (top).

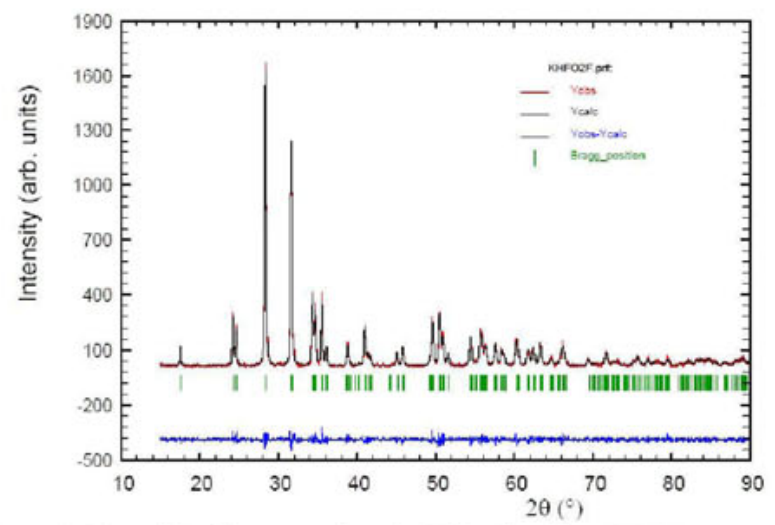

Figure 2. Rietveld refinement plots for $\mathrm{HfO}_{2}$ data from $\mathrm{BBD}-2$.

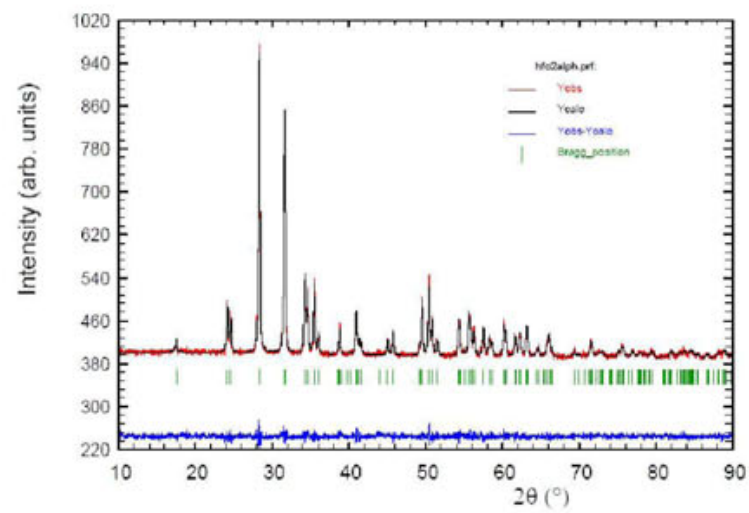

Figure 3. Rietveld refinement plots for $\mathrm{HfO}_{2}$ data from $\mathrm{GIPC}-1$. 
As shown in table 1, the difference in cell parameters between BBD and GIPC is in not more than the difference in two runs using the same equipment. Moreover, differences between the cell parameters from GIPC and BBD are smaller than the existing differences between two sets of published parameters $[8,10]$.

Table 1. Cell parameters in A.

\begin{tabular}{|c|c|c|c|c|c|c|}
\hline Parameter & GIPC-1 & GIPC-2 & BBD-1 & BBD-2 & {$[9]$} & {$[10]$} \\
\hline $\mathrm{a}$ & 5.1181 & 5.1187 & 5.1180 & 5.1143 & 5.1157 & 5.1187 \\
\hline $\mathrm{b}$ & 5.1755 & 5.1763 & 5.1747 & 5.1709 & 5.1819 & 5.1693 \\
\hline $\mathrm{c}$ & 5.2929 & 5.2934 & 5.2923 & 5.2880 & 5.2851 & 5.2970 \\
\hline$\beta$ & 99.2033 & 99.2001 & 99.2000 & 99.2074 & 99.2590 & 99.1800 \\
\hline
\end{tabular}

The atomic positions (table 2) are fairly close for all runs and in good agreement with the published data [8].

Table 3 shows some additional statistic information. As stated in previous work [7] the angular alignment is excellent in GIPC resulting in lower zero shifts. The lower R-Bragg $(\mathrm{Rb})$ in BBD could be attributed to the larger sample volume of these particular samples. Nevertheless, the $\mathrm{Rb}$ from BBD and GIPC are very good values and lower than previous reported [8]. The small value of Chi2 for the GIPC results from the additional background due to the dark counting. If the dark counting is subtracted, Chi2 values change to numbers above 1.0. However, it was chosen to use the raw data scans without modifications in order to emphasise the differences between the systems.

Table 2. Atomic positions from Rietveld refinements.

\begin{tabular}{|c|c|c|c|c|c|c|}
\hline & & GIPC-1 & GIPC-2 & BBD-1 & BBD-2 & Reported [8] \\
\hline \multirow{3}{*}{$\mathrm{Hf}$} & $\mathrm{x} / \mathrm{a}$ & 0.2743 & 0.2743 & 0.2756 & 0.2759 & 0.2764 \\
\hline & $\mathrm{y} / \mathrm{b}$ & 0.0398 & 0.0396 & 0.0400 & 0.0397 & 0.0402 \\
\hline & $\mathrm{z} / \mathrm{c}$ & 0.2078 & 0.2077 & 0.2078 & 0.2079 & 0.2074 \\
\hline \multirow{3}{*}{$\mathrm{O} 1$} & $\mathrm{x} / \mathrm{a}$ & 0.0738 & 0.0747 & 0.0760 & 0.0811 & 0.0709 \\
\hline & $\mathrm{y} / \mathrm{b}$ & 0.3230 & 0.3304 & 0.3338 & 0.3130 & 0.3319 \\
\hline & $\mathrm{z} / \mathrm{c}$ & 0.3468 & 0.3466 & 0.3447 & 0.3443 & 0.3438 \\
\hline \multirow{3}{*}{$\mathrm{O} 2$} & $\mathrm{x} / \mathrm{a}$ & 0.4522 & 0.4554 & 0.4500 & 0.4451 & 0.4464 \\
\hline & $\mathrm{y} / \mathrm{b}$ & 0.7516 & 0.7490 & 0.7561 & 0.7561 & 0.7954 \\
\hline & $\mathrm{z} / \mathrm{c}$ & 0.4742 & 0.4734 & 0.4768 & 0.4762 & 0.4796 \\
\hline
\end{tabular}

Table 3. Figures of merit of the Rietveld refinements.

\begin{tabular}{|c|c|c|c|c|}
\hline & GIPC-1 & GIPC-2 & BBD-1 & BBD-2 \\
\hline $\mathrm{Rp}[\%]$ & 12.9 & 21.3 & 7.7 & 18.1 \\
\hline $\mathrm{Rwp}[\%]$ & 9.75 & 11.4 & 9.5 & 21.9 \\
\hline $\mathrm{Chi} 2$ & 0.0450 & 0.0245 & 1.830 & 1.110 \\
\hline $\mathrm{Rb}[\%]$ & 2.96 & 3.17 & 1.58 & 2.23 \\
\hline Zero correction [ $2 \theta]$ & -0.0260 & -0.0170 & 0.0810 & 0.0800 \\
\hline
\end{tabular}




\section{Summary and conclusions}

The Guinier Imaging Plate Camera is an efficient tool for crystallographic characterization of powders. The reduction of measuring time without affecting the quality is significant. However, there are four drawbacks for the use of this equipment: (a) It only works in transmission mode; (b) there is no cut-off of sample fluorescence; (c) the $2 \theta$ range is limited to $4-100^{\circ}$ (d) the dark counting gives rise to unusual statistic evaluation in Rietveld refinement. It is suggested to adapt the figures of merit of the Rietveld programs to handle this effect.

\section{References}

1. Cohen, J.B., 1966, Diffraction Methods in Materials Science, (New-York: The Macmillan Company).

2. Zevin, L.S. \& Kimmel, G., 1995, Quantitative X-ray Diffractometry, (New-York: Springer).

3. Amemiya, Y., 1995, J. Synch. Rad., 2, 13.

4. Meneghini, C., Atrioli, G., Balerna, A., Gualtieri, A.F., Norby, P. \& Mobilio, S., 2001, J. Synch. Rad., 8, 1162.

5. Stachs, O., Gerber, T. \& Petkov, V., 2000, Rev. Sci. Instr., 71, 4007.

6. Ståhl, K. 2000, J. Appl. Cryst., 33, 394.

7. Gal, J., Mogilyanski, D., Nippus, M., Zabicky, J. \& Kimmel, G., 2005, Nucl. Instrum. Method. Phys. Res. A, 551, 145.

8. Adams, D.M., Leonard, S., Russell, D.R. \& Cernik, R.J., 1991, J. Phys. Chem. Solids, $\mathbf{5 2}, 1181$.

9. Rodriguez-Carvajal, J., 1997,. Fullprof, Program for Rietveld refinement, Laboratories Léon Brillouin ,CEA-CNRS, (Saclay, France).

10. International Centre for Diffraction Data, Powder Diffraction Files, Cards No. 340104 (PA, Newton Square). 


\title{
Diffracted-beam analyzer with multiple single crystals for high resolution parallel- beam X-ray diffraction
}

\section{H. Toraya}

X-ray Research Laboratory, Rigaku Corporation

toraya@rigaku.co.jp

Keywords: crystal analyzer, parallel-beam optics, high-resolution powder diffraction

\begin{abstract}
A new high-performance diffracted-beam analyzer consisting of multiple pieces of perfect crystals in a form of simple and compact device is proposed. Pieces of crystals are set so as to fit their crystal lattice planes with tangent planes of logarithmic spiral, and crystal surfaces of the analyzer form a shape of one-dimensional polygon. Each piece of the crystals works in the same manner as the single crystal analyzer. A diffracted X-ray beam from a specimen is successively reflected by the individual crystals of the analyzer, and reflected Xray beams are detected separately by one-dimensional position sensitive detector. If the analyzer consists of, for example, ten pieces of the crystals, ten diffraction patterns can be obtained in a single diffractometer scan. Thus a resultant intensity becomes greater by one order of magnitude, whereas the angular resolution is the same as that of the single crystal analyzer. Instrumental setup and experimental results are described.
\end{abstract}

\section{Introduction}

A single crystal analyzer (hereafter called as SCA) is one of the best choices as an X-ray optical device for obtaining high-angular resolution and low background intensities in powder and thin-film diffraction experiments [1]. Highest performances regarding the angular resolution and intensities can be obtained when the SCA is coupled with parallel-beam geometry. In synchrotron radiation (SR) experiments, a high brilliance parallel beam can be obtained from undulator or even bending magnet light sources. The SCA has been commonly used for diffractometer scans at many beam-lines in SR facilities worldwide. Intensity gains in high angular-resolution and high-speed experiments in several SR facilities around the world have been further multiplied by using multiple-detector systems, each of which consists of several perfect crystal analyzers [2,3]. With a laboratory X-ray source, diffracted intensities from the SCA are generally lower than those from a SR source. A popular way to improve high diffraction intensities is to use a rotating anode X-ray generator and/or a graded multi-layer mirror. Another way may be the use of a multiple-detector system similar 
to those presently used in several SR facilities. However, the latter choice makes the diffractometer system more complex and also very expensive.

In the present study, a new high-performance diffracted-beam analyzer consisting of several perfect crystals in a form of a simple and compact device is proposed to increase the diffracted intensity by one order of magnitude compared to those of the SCA. An analyzer with high angular resolution has not been used in combination with one- or more-dimensional detectors. In the present study, a new technique using a diffracted-beam analyzer with multiple single crystals together with a one-dimensional silicon strip detector is described.

\section{Instrumentation}

The present device as a diffracted-beam analyzer consists of multi-pieces of perfect single crystals, and it is hereafter called as compact-multi-crystal analyzer (CMCA). Design of the CMCA is based on a quite new concept, and it can be easily understood by a scheme of the CMCA shown in figure 1. The crystal surfaces of the CMCA form a shape of onedimensional concaved polygon, two neighbouring crystals of which are inclined with each other at very small angles. Actual dimensions of the CMCA will, of course, depend on a design scheme. In the present case, the CMCA had a length of about $80 \mathrm{~mm}$, and it can be attached to a detector arm in the same manner as that in the case of an ordinary-type SCA.

With rotating the detector arm, a parallel X-ray beam diffracted from a specimen will be successively reflected on the crystal surfaces of the CMCA (figure 1). If the CMCA consists of, for example, ten perfect single crystals, the diffracted beam from the specimen is reflected on the individual crystal surfaces of the CMCA ten times. These reflected X-ray beams are detected with a position-sensitive detector, which can distinguish from which piece of the crystals the X-ray beam has come. In this example, ten diffraction patterns can be obtained in a single diffractometer scan. Thus the total intensity obtained with the CMCA is

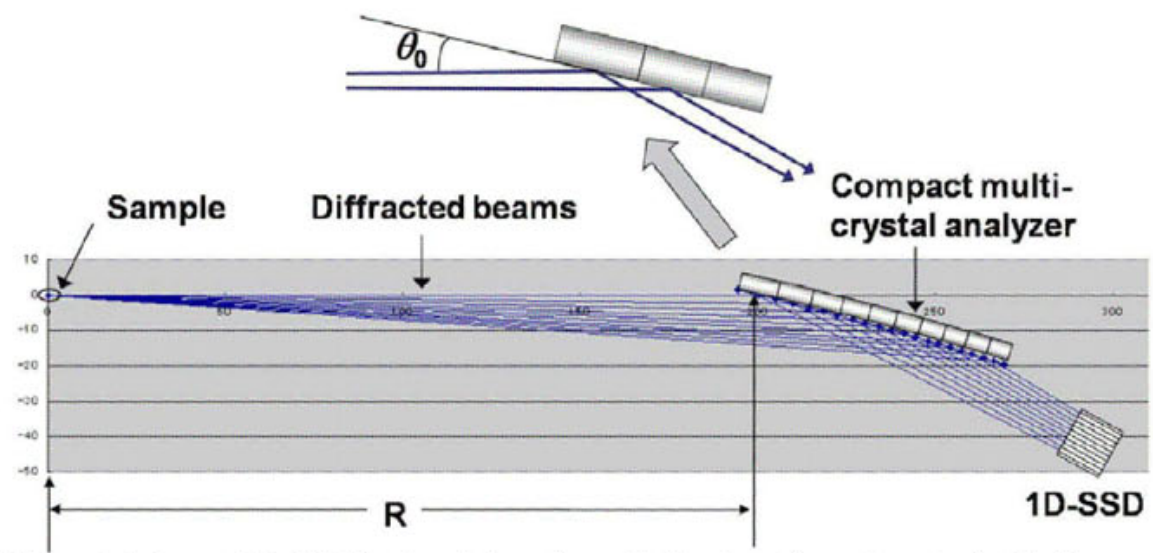

Figure 1. Scheme of the CMCA, viewed along the axial direction of a goniometer. In this diagram, the $X$-ray beam, diffracted from the specimen, is rotated instead of moving the detector arm. Upper diagram shows a portion of the CMCA. 
greater by one order of magnitude than those in the diffractometer scan with the SCA at the same scan speed.

As can be seen from figure 1, crystal lattice planes of individual perfect crystals of the CMCA must satisify the Bragg condition. Then the following equation is derived.

$$
d y / d x=-\tan \left(\theta_{0}-\phi\right)
$$

where $d y / d x$ is a gradient of the crystal lattice plane, $\phi$ is the angle between the diffracted Xray beam and the $x$-axis, and $\theta_{0}$ is the Bragg angle for the crystal lattice plane of CMCA. A shape and dimensions of the CMCA can be derived by solving the differential equation, where mathematical details will be given elsewhere [4]. The shape of the CMCA, shown as a projection along the axial direction in figure 1, can be described by a mathematical function of logarithmic spiral in a form

$$
r=R \exp \left(-\phi / \tan \theta_{0}\right)
$$

where $r$ is the length of the radial axis in a polar coordinate system $(r, \phi), R$ is the $r$ at $\phi=0$. A form of the logarithmic spriral function is dependent on the Bragg angle $\theta_{0}$. Therefore, the shape of the CMCA is determined by a wavelength used in experiments and a $d$-spacing of the crystal lattice plane of the CMCA.

The Bragg reflection condition is satisfied at all points on the logarithmic spiral curve described by eq. (1) for all X-ray beams from the specimen. In order to obtain high-angular resolution for the $\mathrm{X}$-ray beam with a finite beam height, the continuously curved logarithmic spiral surface of the CMCA is replaced with a set of planes, each of which coincides with a tangent plane at a point on the logarithmic spiral. The surface of the CMCA thus obtained has a shape of one-dimensional polygon.

Readers will have an inquiry how can the interference between the neighbouring beams be avoided on the detector surface. Since the parallel-beam geometry is used, the X-ray beam diffracted from the specimen has small divergence. Therefore, as can be seen from figure 1, individual X-ray beams, each of which was diffracted by the crystal of the MCMA, does not intersect with neighbouring beams before reaching the detector surface. Thus Soller collimators are not required to be placed in front of the detector.

In the present study, the CMCA was designed for $\mathrm{CuK \alpha _{1 }}$ radiation, $R=200 \mathrm{~mm}$, and the Bragg angle of $13.64^{\circ}$ for Ge (111). A block of Ge crystal with (111) lattice plane was cut into ten pieces. After polishing individual pieces of the Ge crystals, the CMCA was fabricated by glueing the Ge crystals on a mold made of stainless steel (figure 2). As described on the above, the shape of the CMCA is dependent primarily on the Bragg angle $\theta_{0}$. Thus one piece of the CMCA can be used for one photon energy. It can, however, be easily replaced with a different piece of the MCMA that works for example, for MoKa radiation. 


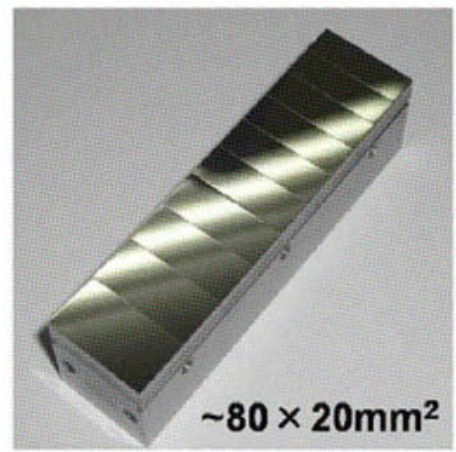

Figure 2. Multi-crystal analyzer.

\section{Experimental}

As shown in figure 3, the CMCA was mounted on the detector arm of the two-axis diffractometer (SmartLab System, Rigaku Corporation). A rotating anode $\mathrm{X}$-ray generator with $\mathrm{Cu}$ target, operated at $9 \mathrm{~kW}(45 \mathrm{kV}, 200 \mathrm{~mA})$, was used as an X-ray source. The $\mathrm{Cu} K \alpha_{1}$ radiation was taken out from a Ge (220) cannel-cut monochromator after collimating the X-ray beam with a parabolic graded multi-layer mirror. Angular apertures of Soller slits on both sides of the specimen were $2.5^{\circ}$. The CMCA was put in a steel box in order to avoid the increase of the background scattering. One-dimensional silicon strip detector (D/teX Ultra, Rigaku Corporation) was used for detecting X-ray beams.

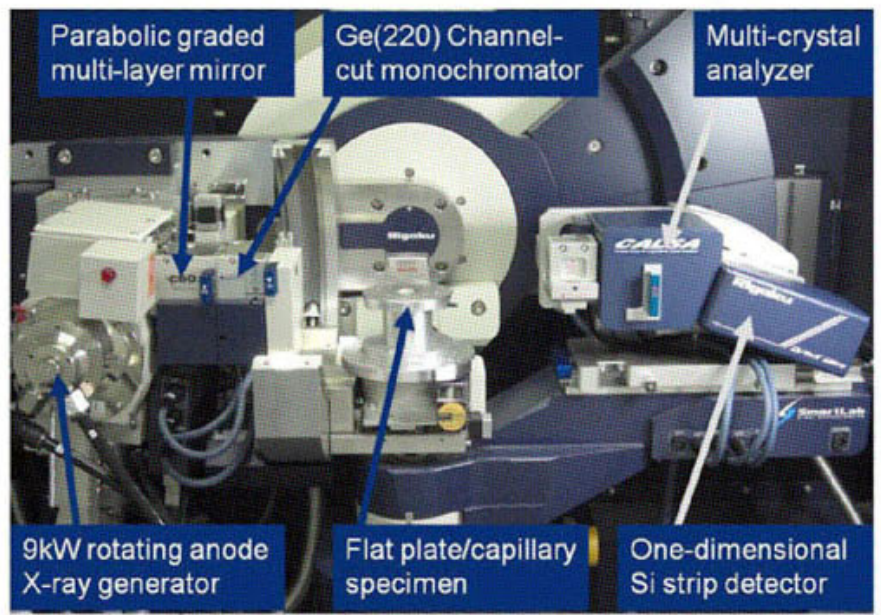

Figure 3. Experimental setup using two-axis diffractometer. 
Both capillary and flat specimens can be measured with the diffractometer system shown in figure 3. In the present study, NIST (National Institute of Standards and Technology) SRM (Standard Reference Material) $660 \mathrm{a} \mathrm{LaB}_{6}$ powder, packed into a glass flat-specimen holder, was used for testing the CMCA. After measuring the diffraction profile of 110 reflection from the $\mathrm{LaB}_{6}$ powder, ten observed diffraction profiles were summed up to integrate them into a single profile.

\section{Results and discussion}

In table 1, full-width at half-maximum (FWHM) values and relative intensities of the four profiles of the 110 reflection from the $\mathrm{LaB}_{6}$ powder are compared, together with experimental conditions. In figure 4, observed diffraction profiles of the same reflection as presented in table 1 are shown. The FWHM of $0.0181^{\circ} / 2 \theta$ for the CMCA is an excellent value as laboratory data, while peak top intensity is more than five times as large as that observed with parallel slit analyzer with an angular aperture of $0.11^{\circ}$. Moreover, very low background intensity has been achieved with the CMCA. The CMCA simply increased the profile intensity by one order of magnitude compared to that obtained with the SCA while it keeps advantages of the crystal analyzer represented by high-angular resolution and low background intensity.

Table 1. FWHM values and relative intensities for four different experimental conditions. PSA: parallel slit analyzer.

\begin{tabular}{|l|l|c|c|c|c|}
\hline Collimation & Monochromator & $\begin{array}{c}\text { Radiation } \\
(\mathrm{Cu})\end{array}$ & Analyzer & $\begin{array}{c}\text { FWHM } \\
(\% / 2 \theta)\end{array}$ & $\begin{array}{c}\text { Relative } \\
\text { intensity }\end{array}$ \\
\hline Parabolic \\
graded mul- & $\begin{array}{l}\text { Ge (220) Chan- } \\
\text { nel-cut mono- } \\
\text { tilayer mir- } \\
\text { ror }\end{array}$ & \multirow{2}{*}{$K \alpha_{1}$} & $\mathrm{CMCA}$ & $0.0181^{\circ}$ & 10 \\
\cline { 4 - 6 } & chromator & SCA & $0.0181^{\circ}$ & 1 \\
\cline { 4 - 6 } & Non & $K \alpha_{1}-K \alpha_{2}$ & PSA & $0.084^{\circ}$ & 2 \\
\hline
\end{tabular}

\section{Concluding remarks}

A high-performance diffracted-beam analyzer has been developed. The analyzer consists of multiple perfect Ge(111) crystals in a form of simple and compact device. Diffracted intensities have been increased by one order of magnitude compared to those of the SCA. Resolution of the CMCA is the same as that obtained with the SCA. A high-resolution crystal analyzer has not been used in combination with one- or more-dimensional detectors. The present CMCA makes possible to be used with the one-dimensional position sensitive detector. 


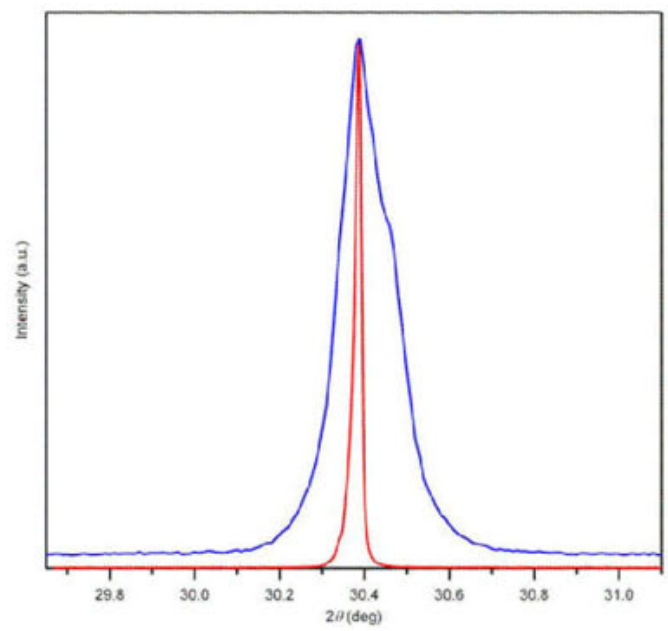

Figure 4. Diffraction profiles of the 110 reflection from $\mathrm{LaB}_{6}$ powder, observed with the CMCA (red line) and the parallel slit analyzer and scintillation counter with $C u K \square_{I}-K \square_{2}$ doublet beam (blue line) without using the channel-cut monochromator. Profile intensities are normalized to have the same value at peak tops.

\section{References}

1. Cox, D.E., 1992. in Synchrotron Radiation Crystallography, edited by D. E. Cox (New York: Academic Press), pp 186-254.

2. Hodeau, J.L., Bordet, P., Anne, M., Prat, A., Fitch, A.N., Dooryhee, E, Vaughan, G. \& Freund, A., 1998, SPIE Proceedings, 3448, 353.

3. Toraya, H., Hibino, H. \& Ohsumi, K., 1996, J. Synchrotron Rad., 3, 75.

4. Toraya, H. (in preparation).

Acknowledgements The author would like to thank to Drs. B. Verman and Y. Platonov of Rigaku Innovative Technology for very fruitful discussion. He also thanks to Dr. H. Konaka of Rigaku Corporation for his experimental and data analysis works and to Dr. M. Nonoguchi for his work of fabricating a proto-type of the multi-crystal analyzer. 


\section{Comparison of two standards for powder X-ray diffractometry}

\section{A. Kojdecki ${ }^{1, *}$, W. Mielcarek ${ }^{2}$, K. Prociów ${ }^{2}$, J. Warycha ${ }^{2}$}

${ }^{1}$ Institute of Mathematics and Cryptology, Military University of Technology, ul. S. Kaliskiego 2, 00-908 Warszawa 49, Poland

${ }^{2}$ Institute of Electrotechnics, ul. M. Skłodowskiej-Curie 55, 50-950 Wrocław

*Marek.Kojdecki@wat.edu.pl

Keywords: X-ray diffraction, instrumental function for powder X-ray diffractometry, crystalline microstructure, powder $\mathrm{X}$-ray diffraction pattern analysis

Abstract. Two methods for obtaining instrumental line profiles for X-ray diffraction (XRD) analysis of polycrystalline materials are studied. Two standards for XRD analysis of zinc oxide, obtained from medium-quality XRD patterns, are compared. The first one is an annealed zinc oxide powder, from which instrumental line profiles were recorded immediately by a diffractometer. The other is a lanthanum hexaboride standard reference material, from which a standard XRD pattern was registered. Based on this pattern, an instrumental function was determined and afterwards instrumental line profiles for zinc oxide were produced by computer simulation. The XRD peaks from zinc oxide occurred a little wider than those produced from lanthanum hexaboride, particularly for larger diffraction angles. A crystalline microstructure of zinc oxide was identified as a possible source of this disagreement. Microstructure characteristics were determined. Discrepancies between line profiles of both XRD patterns, measured and simulated, were reduced by accounting the zinc oxide crystalline microstructure.

\section{Instrumental function and two types of standards for powder $\mathrm{X}$-ray diffractometry}

Experimental line-profiles measured as parts of XRD patterns may be interpreted as convolutions of instrumental line-profiles and pure line-profiles containing information on crystalline microstructure of investigated materials. Instrumental line profiles are important for precision and accuracy of powder XRD analyses [5]. They can be measured from a standard material of same composition as a studied material, that consists of sufficiently big and unstrained crystallites to ensure negligible contribution to line-profiles from crystalline microstructure in comparison to contribution from all instrumental factors. Frequently such a standard can hardly be obtained and some standard reference material should be used instead. This substitution makes further numerical procedure for determining instrumental line-profiles corresponding to Bragg angles for a material under study to be necessary. If the 
axial divergence of $\mathrm{X}$-ray beam can be neglected, then an instrumental function describing instrumental line profiles can be determined from experimental data in a form of the convolution of two functions, $g=s^{*} w[1], s$ originated from a spectral distribution of Xradiation and dependent on Bragg angle, and $w$ originated from a diffractometer geometry and independent of Bragg angle.

\section{Experiment and computations}

\section{Data collection}

Lanthanum hexaboride (NIST SRM 660a) was supported by NIST (with mean crystalline grain size of $8.8 \mu \mathrm{m}$ and cubic unit cell parameter of $4.1569162 \AA \pm 0.0000097 \AA$ [2]). Zinc oxide powder (hexagonal, $a=3.2498 \AA, c=5.2066 \AA$ ) was produced by crystallisation from vapour and annealing. The measurements were performed by using a powder diffractometer (DRON) in the Bragg-Brentano configuration with radius of $180 \mathrm{~mm}$, equipped with a cobalt $K_{\alpha_{1,2}}$ X-ray tube (BWS-29; $\lambda_{K_{\alpha_{1}}}=1.7890 \AA$ ), emission slits of $2 \mathrm{~mm}$, receiving slit of $0.5 \mathrm{~mm}$ and Soller slits of $2^{\circ} 30^{\prime}$. XRD pattern were recorded in range $20^{\circ}-164^{\circ}$ of diffraction angle $(2 \theta)$ in scanning mode with step of $0.01^{\circ}$ and counting time of $3 \mathrm{sec} / \mathrm{step}$. Medium-quality of XRD patterns were chosen to imitate a typical experiment. Line profiles from lanthanum hexaboride were described by using fundamental parameter approach [1] in the form of $g=s^{*} w$ with functions $s$ and $w$ calculated from the recorded diffraction pattern and then the line profiles corresponding to Bragg angles of zinc oxide were simulated. Since the profiles from zinc oxide were a little broader than those from lanthanum hexaboride, characteristics of crystalline microstructure of the first material were found by exploiting the simulated profiles as instrumental ones $[3,4,5]$. Then the profiles resulting from accounting both microstructure and instrumental factors were compared to the measured ones, repeatedly.

\section{Results of measurements and computations}

The instrumental function were determined by using a numerical optimisation procedure. The spectral distribution of X-radiation emitted by a cobalt tube was approximated with a doublet of Lorentzian functions.

Afterwards the instrumental line profiles for lanthanum hexaboride and for zinc oxide were simulated. For lanthanum hexaboride relative discrepancies between the measured and simulated line profiles (about 5\%) were inferior to the triple expected error (in Euclidean norm) estimated with using usual statistical model of registering counts by a detector. The results are illustrated in figure 1 (slight effect of $\mathrm{X}$-ray beam axial divergence is visible in the first peak). For zinc oxide the measured line profiles were broadened in comparison to the simulated ones, as illustrated in figure 2. They could serve as quite reliable standard line profiles only for reflection angles in range of $20^{\circ}-45^{\circ}$. To explain this broadening the crystalline microstructure with the model described below was considered $[3,6,7]$. The microstructure characteristics were determined by taking the measured and simulated line profiles as experimental and instrumental ones, respectively. The best results of modelling crystallites was achieved for hexagonal prism with base in plane (001), with the height to large-base-diagonal-length ratio of 0.48 , and the volume-weighted standardised crystallite 
size distribution and the second-order crystalline lattice strain distribution displayed in figure 4. The line profiles simulated by accounting either the microstructure fitted better the measured ones, as illustrated in figure 3 (although the fit became still not fully satisfactory). A little worse result was achieved for cylinder of revolution as crystallite shape model. The resulting discrepancy between the profiles simulated and measured can be in part a consequence of describing the cobalt X-ray radiation by using a doublet instead of a more accurate quartet of Lorentzian functions. It can be interpreted also in this way, that small but noticeable portion of broadening of the lines from lanthanum hexaboride is caused by the microstructure of mosaic crystalline grains, that reflexes in a little perturbation of calculated pure line profiles and a little inadequacy of the mathematical model of material. This question is difficult to be solved and requires further study.

\section{Model of a polycrystalline material and crystalline microstructure of zinc oxide}

A sample of crystalline powder was considered as a set of crystallites of the same shape, randomly oriented in space. The crystallite shape was modelled as sphere, cylinder or prism $[3,6,7]$. The sizes of crystallites were characterised by the volume-weighted crystallite size distribution $[3,4]$, which might be interpreted as a density of probability of finding a crystallite of assumed shape and size, taken with a weight proportional to its volume, in an analysed sample. The standardised size of a crystallite was defined as the cube root of its volume, while the shape was described by the ratios of its characteristic dimensions. The crystalline lattice strain was assumed to be the second-order strain, homogeneous inside each crystallite and statistically isotropic. The variation of lattice parameters of the crystallites was characterised by the second-order crystalline lattice strain distribution [3], which might be interpreted as a density of probability of finding a crystallite with interplanar distances being different in assumed relation to the distances in the reference lattice (of parameters averaged over all crystallites).

Instrumental function from lanthanum hexaboride and comparison of two standards for zinc oxide in figures
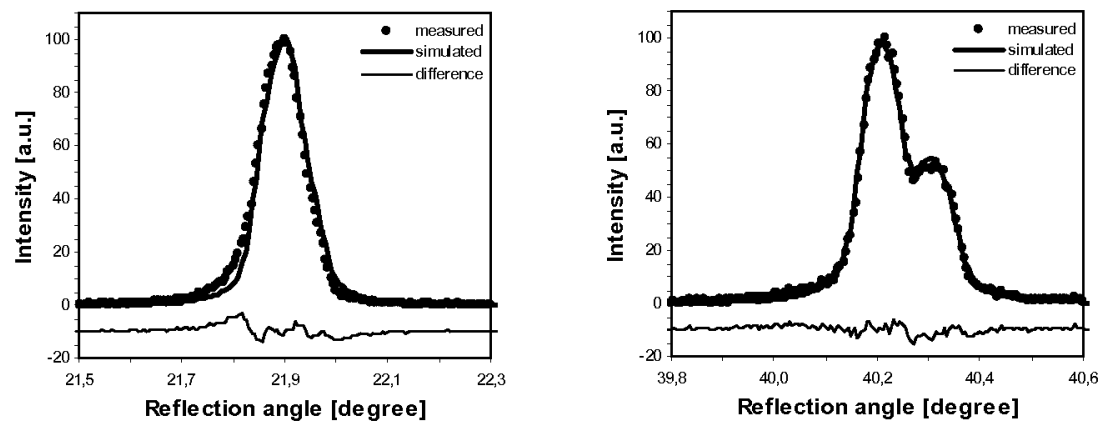

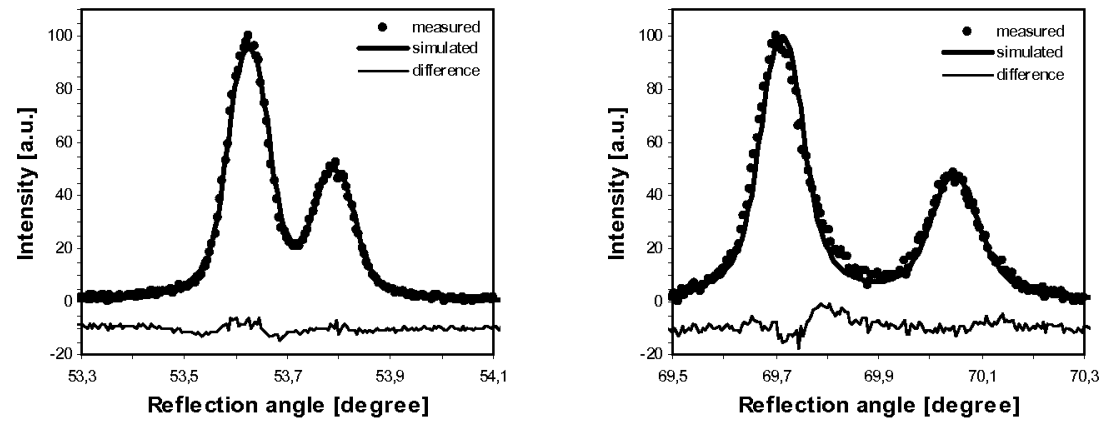

Figure $1 a, b, c, d$. X-ray diffraction line profiles from lanthanum hexaboride measured and simulated by using the instrumental function - reflections $111(R=0.058), 003 / 122(R=0.046), 123(R=0.045)$ and $133(R=0.085)$. Slight effect of $X$-ray beam axial divergence is visible in the first peak.
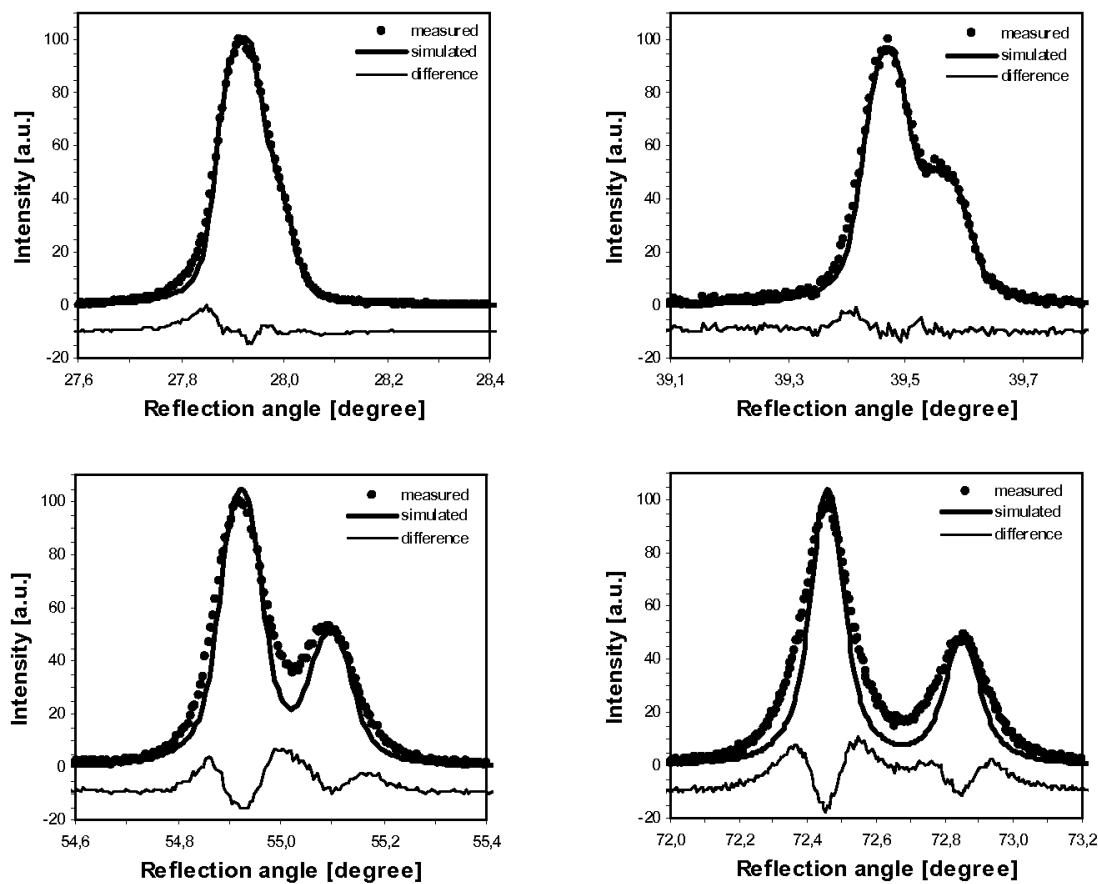

Figure $2 a, b, c, d . X$-ray diffraction line profles measured from zinc oxide and simulated by using the instrumental function from lanthanum hexaboride - reflections $012(R=0.068$; top left), $020(R=0.066)$, $023(R=0.159)$ and $030(R=0.234$; bottom right $)$. Slight broadening caused by crystalline microstructure of zinc oxide is noticeable. 

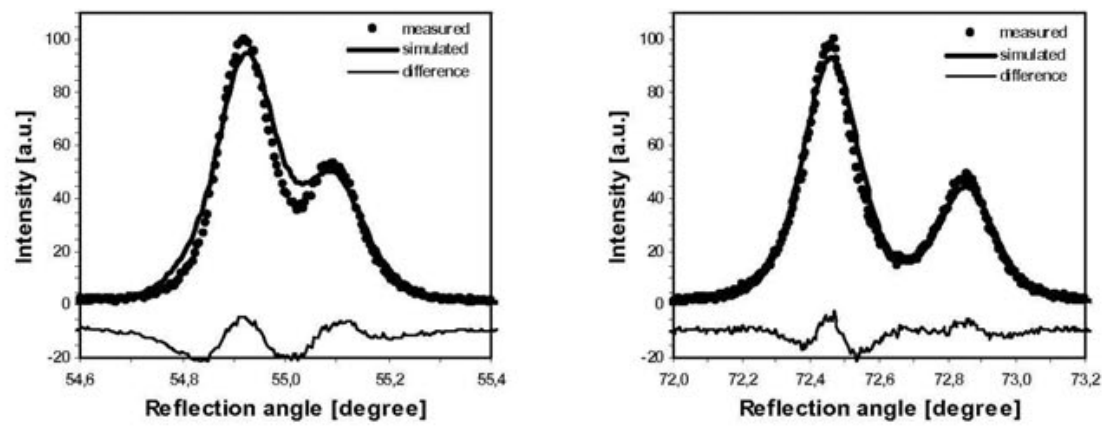

Figure $3 a, b$. X-ray diffraction line profiles measured from zinc oxide and simulated by using the convolution of the instrumental function from lanthamum hexaboride with the function generated by the crystalline microstructure - reflections $023(R=0.120)$ and $030(R=0.080)$.
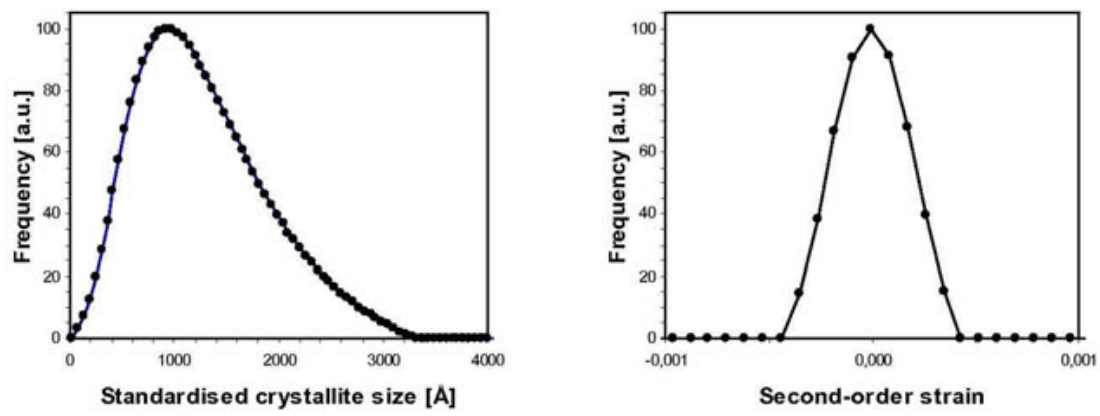

Fig. $4 a, b$. Crystalline microstructure characteristics of zinc oxide sample: the volume-weighted standardised crystallite size distribution and the second-order lattice strain distribution. Crystallites were modelled as prisms with the regular hexagonal base in plane (001) with edges parallel to axes [100], [110] and [010], and with other edges parallel to [001]. The mean volume-weighted crystallite has the largest base diagonal length of $1877 \AA$, the height of $897 \AA$ and the standardised size of $1271 A$. The size distribution with the mean value of $1271 \mathrm{~A}$ and the standard deviation of $614 \mathrm{~A}$ is close to a logarithmic-normal one. The strain distribution has the mean-absolute value of 0.00015 and the standard deviation of 0.00024 .

\section{Conclusions}

The simplified model of instrumental function (with spectral part in the form of a doublet of Lorentzian functions) for a diffractometer equipped with a cobalt X-ray tube is sufficient for reproducing instrumental line profiles with reasonable accuracy. The convolution model of the instrumental function is convenient for calculating easily the instrumental line profiles for any set of Bragg angles. The line profiles from zinc oxide display little broadening which can be mostly attributed to its crystalline microstructure. 


\section{References}

1. Cheary, R.W. \& Coelho, A., 1992, J. Appl. Cryst., 25, 109.

2. Chantler, C.T., Rae, N.A. \& Tran, C.Q., 2007, J. Appl. Cryst., 40, 232.

3. Kojdecki, M.A. \& Mielearek, W., 2000, Materials Sci. Forum, 321-324, 1040.

4. Langford, J.I., Louër, D. \& Scardi, P., 2000, J. Appl. Cryst., 33, 964.

5. Balzar, D., Audebrand, N., Daymond, M.R., Fitch, A., Hewat, A., Langford, J.I., Le Bail, A., Louër, D., Masson, O., MeCowan, C.N., Popa, N.C., Stephens, P.W. \& Toby, B.H., 2004, J. Appl. Cryst., 37, 911.

6. Louër, D., Auffrédic, J.P., Langford, J.I., Ciosmak, D. \& Niepce, J.C., 1983, J. Appl. Cryst., 16, 183.

7. Vargas, R., Louër, D. \& Langford, J.L., 1983, J. Appl. Cryst., 16, 512.

Acknowledgements. This work was supported by the Polish Ministry of Science and Higher Education in years 2008-2010, grant no. NN 510344534. 


\title{
Synchrotron beamline optics for X-ray powder diffraction under high-pressure conditions at the Siam Photon Laboratory
}

\author{
V. Saengsuwan ${ }^{1, *}$, W. Klysubun ${ }^{2}$, T. Bovornratanaraks ${ }^{1}$, \\ S. Rugmai ${ }^{2}$
}

${ }^{1}$ Department of Physics, Chulalongkorn University, Bangkok, Thailand

${ }^{2}$ Synchrotron Light Research Institute, Nakorn Ratchasima, Thailand

varalak.s@student.chula.ac.th

Keywords: X-ray powder diffraction, diamond anvil cell, high-pressure conditions

Abstract. To support various applications for high-pressure research in Thailand, an experimental setup for X-ray powder diffraction under high-pressure conditions using synchrotron radiation, which is based on station 9.1 at the Daresbury Synchrotron Radiation Source, is being developed on the bending magnet beamline BL8 at the Siam Photon Laboratory. Monochromatic X-rays with a photon energy of $9 \mathrm{keV}$ are provided by a fixed-exit double crystal monochromator equipped with $\mathrm{Ge}(220)$ crystals. In a recent experiment, we could record a complete diffraction pattern of the hexagonal phase of $\mathrm{ZnO}$ using an image plate area detector. However, diffraction intensity should be improved by adding focusing optics. In this work, we propose the use of a double multilayer monochromator in combination with a focusing mirror. Results from ray-tracing simulation for the proposed optics will be presented.

\section{Introduction}

Nowadays, the study of structural phase transitions in materials under high-pressure conditions plays an important role in expanding the understanding of physical and chemical properties of materials. To support various applications for high-pressure research in Thailand, an experimental setup for X-ray powder diffraction (XRPD) under high-pressure conditions using synchrotron radiation, which is based on station 9.1 at the Daresbury Synchrotron Radiation Source [1], is being developed on the bending magnet beamline BL8 of the Siam Photon Laboratory (SPL) [2]. Monochromatic X-rays with a photon energy of $9 \mathrm{keV}$ are provided by a fixed-exit double crystal monochromator equipped with $\mathrm{Ge}(220)$ crystals. A diamond anvil cell (DAC) will be used for generating high-pressure conditions in a powder sample. Quasi-hydrostatic pressure in the DAC will be determined by the ruby-fluorescence technique. In our recent work, we can record the complete diffraction pattern of the hexagonal phase of $\mathrm{ZnO}$ at $\mathrm{BL} 8$ using an image plate area detector. However, the diffraction intensity would have been stronger if there were focusing optics to converge the whole X-ray beam onto the sample. In addition, some intensity loss by X-ray absorption in the DAC at $9 \mathrm{keV}$ is 
expected. This can be compensated by higher flux. Here we propose an XRPD beamline to deliver a focused X-ray beam with high photon flux as shown in figure 1. Expected performance of the proposed XRPD beamline was determined by ray-tracing simulation with the aid of SHADOW [3]. The X-ray beam simulated for the bending magnet source was traced through each optic and characterized in terms of photon flux, beam size, beam convergence and energy resolution. As compared to a $\mathrm{Ge}(220)$ double crystal monochromator (DCM) used at BL8 of the SPL [4], higher photon flux can be obtained from a double multilayer monochromator (DMM), which plays a vital role in the high flux applications, for example, XRPD technique, microscopic X-ray fluorescence analysis, small-angle X-ray scattering technique and $\mathrm{X}$-ray topography [5]. We consider a W/B $\mathrm{B}_{4} \mathrm{C}$ DMM [6-9] that is employed in a superconducting bending magnet beamline for high-pressure studies at the Advanced Light Source [10] and a microscopic XRD at HASYLAB [11].

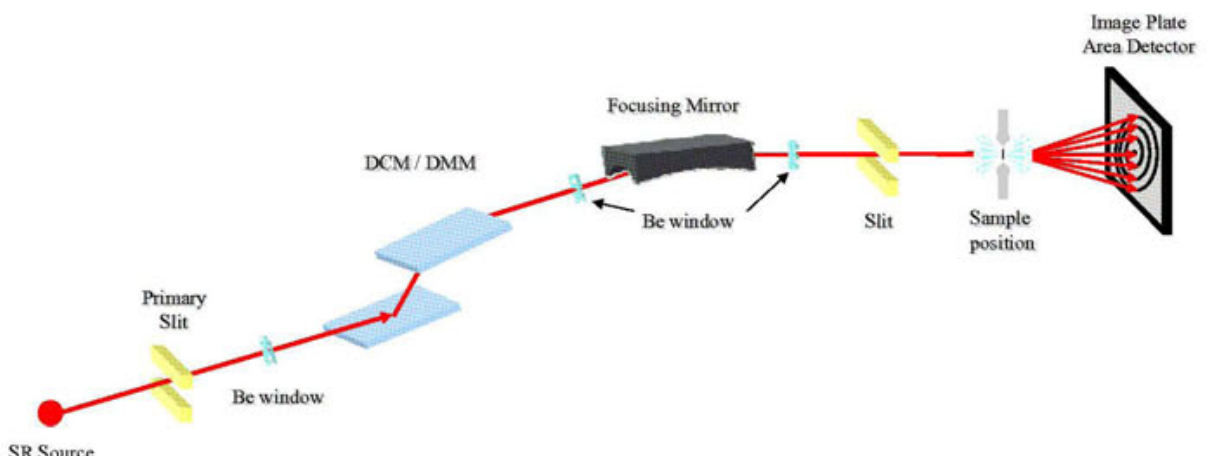

Figure 1. Schematic layout of the proposed XRPD beamline for the SPL.

\section{Beamline Optics}

\section{Double Multilayer Monochromator}

A W/B ${ }_{4} C$ multilayer (ML) with a small double layer period (d-spacing) of $10 \AA, 600$ layer pairs and a ratio of metal to double layer thickness $(\Gamma)$ of 0.5 is selected for this XRPD beamline. The fabrication and characterization of this mirror was reported by $\mathrm{S}$. Braun et al. [12]. Figure 2(a) shows a comparison of the simulated photon flux from the $\mathrm{Ge}(220) \mathrm{DCM}$ and from the W/ $/ \mathrm{B}_{4} \mathrm{C}$ DMM. In overall, the $\mathrm{W} / \mathrm{B}_{4} \mathrm{C}$ DMM provides photon flux about 46 times higher than that of the $\mathrm{Ge}(220) \mathrm{DCM}$. This is mainly attributed to the larger natural bandwidth of the W/ $\mathrm{B}_{4} \mathrm{C}$ DMM. At $9 \mathrm{keV}$, the bandwidth of the W/ $\mathrm{B}_{4} \mathrm{C}$ DMM is 86.0 arcsec while that of the $\mathrm{Ge}(220)$ crystal is only 11.8 arcsec. The opening angle of $3.0(\mathrm{~h}) \times 0.417(\mathrm{v})$ $\mathrm{mrad}^{2}$, which was defined by the primary slit (see figure 1), was selected for calculating the photon flux given by the W/ $\mathrm{B}_{4} \mathrm{C}$ DMM. The vertical angle was set equal to the $\mathrm{W} / \mathrm{B}_{4} \mathrm{C}$ bandwidth and the horizontal angle was selected to match the width of the focusing mirror as described in section "Focusing Mirror". In case of the Ge(220) DCM the opening angle of $1.75(\mathrm{~h}) \times 0.125(\mathrm{v}) \mathrm{mrad}^{2}$ is normally used at the BL8. The reflectivity of the W/B $\mathrm{B}_{4} \mathrm{C}$ ML for different photon energies as a function of glancing angle is presented in figure 2 (b). The rocking curve in figure 3 shows the bandwidth of 86.0 arcsec for $\mathrm{W} / \mathrm{B}_{4} \mathrm{C}$ ML tuned at $9 \mathrm{keV}$. 
This corresponds to an energy resolution $(\triangle \mathrm{E} / \mathrm{E})$ of $6.0 \times 10^{-3}$. It should be noted that the $\mathrm{W} / \mathrm{B}_{4} \mathrm{C}$ DMM yields higher flux than $\mathrm{Ge}(220) \mathrm{DCM}$ at the cost of energy resolution.

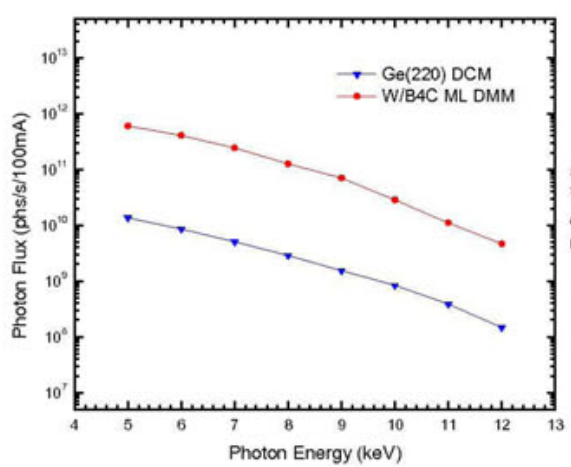

(a)

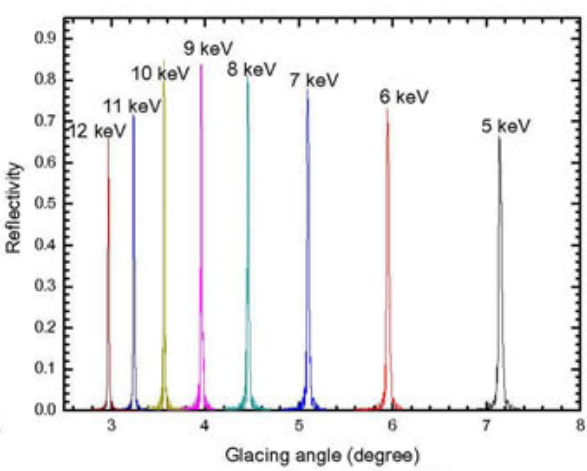

(b)

Figure 2. Comparison of simulated photon flux between the Ge(220) DCM and the $W / B_{4} C D M M$ for photon energies range of $5-12 \mathrm{keV}$ is shown in (a). Reflectivity of the $W / \mathrm{B}_{4} \mathrm{CML}$ with $d$-spacing $10 \mathrm{~A}$, $N=600$ bi-layers and $\Gamma=0.5$ at photon energies of $5,6,7, \ldots, 12 \mathrm{keV}$ is shown in (b).

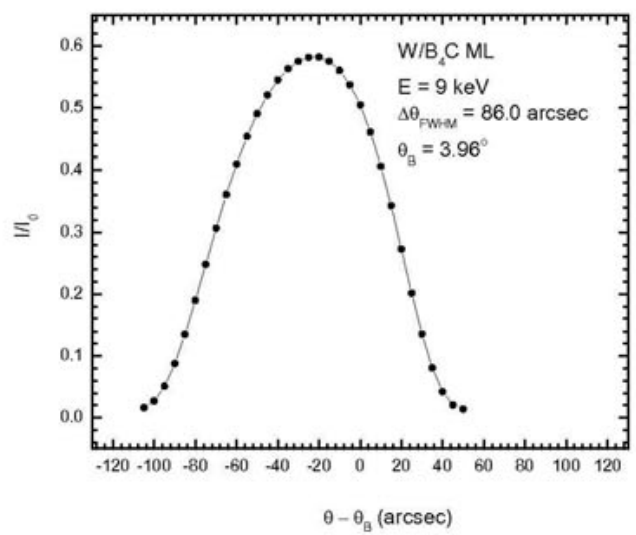

Figure 3. Simulated rocking curve for the DMM with $W / B_{4} C M L$ at $9 \mathrm{keV}$.

\section{Focusing Mirror}

The focusing mirror is a toroidal mirror or a bent cylindrical mirror as illustrated in figure 4(a) with a meridional radius $\mathrm{R}$ for focusing the beam in the horizontal (x) direction and a sagittal radius $\rho$ for focusing the beam in the vertical (z) direction with respect to the optical axis (y) of the $\mathrm{x}$-ray beam. To determine both $\mathrm{R}$ and $\rho$, the optical magnifications are selected such that the focused beam size and beam convergence at the image (sample position) are suitable for XRPD technique. The optical magnifications of the beamline optics are illustrated in figure 4(b). The horizontal magnification and the vertical magnification (M) are given by the ratio of the source and the image plane distances as equivalent to the beam convergence ratio. In addition, the mirror absorption is of considerably importance. We choose 
Rh $5 \mathrm{~nm} / \mathrm{Pt} 25 \mathrm{~nm}$ for optical coating on the mirror. The mirror body may be silicon single crystal or ultra low expansion (ULE) fused silica. The suitable glancing angle $\left(\theta_{\mathrm{c}}\right)$ for the XRPD technique in photon energies range of $5-12 \mathrm{keV}$ is $5 \mathrm{mrad}$. This is shown in figure 5 . For the same length of the mirror, i.e. $1 \mathrm{~m}$, the mirror set at $3 \mathrm{mrad}$ glancing angle accepts $30 \%$ less of the beam than in the case of 5 mrad with slightly higher reflectivity, thus the overall beam intensity throughput of $3 \mathrm{mrad}$ is lower than that of $5 \mathrm{mrad}$.

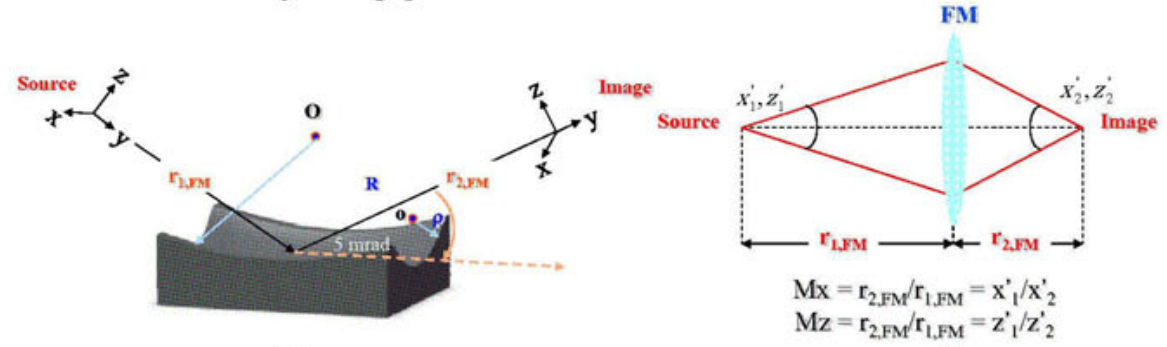

(a)

(b)

Figure 4. Schematic diagram of focusing mirror and the optical magnifications.

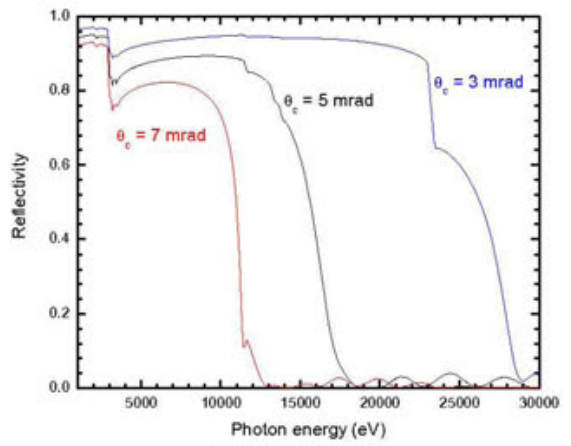

Figure 5. Calculated reflectivity of Rh $5 \mathrm{~nm} /$ Pt $25 \mathrm{~nm}$ coated silicon mirror with $\theta_{c}=3,5$ and 7 mrad.

\section{Results and discussions}

In order to determine the best magnification of the toroidal focusing mirror, ray-tracing simulation was carried out with the aid of SHADOW. A primary X-ray beam from a bending magnet synchrotron source was generated at $2 \%$ bandwidth with energy centred at $9 \mathrm{keV}$. Different mirror magnifications - M0.3, M0.4, M0.5, M0.6 and M0.7 - allowed by the available length of the beamline, $19 \mathrm{~m}$, were tested with the opening angle of $3.0(\mathrm{~h}) \times 0.417(\mathrm{v})$ $\mathrm{mrad}^{2}$. The X-ray beam at the image plane could be characterized by the beam size, beam convergence and beam intensity. The results including a photon flux and the image size at focal plane of the X-ray beam from ray-tracing simulation are presented in figures 6 and 7 . The photon flux at the sample for M0.7 is highest. Moreover, the focused beam size is smallest and spatially symmetric. The expected flux at the sample through a $0.3 \times 0.3 \mathrm{~mm}^{2}$ aperture is $2.7 \times 10^{10}$ photons $/ \mathrm{sec} / 100 \mathrm{~mA}$ and without aperture is $5.9 \times 10^{10}$ photons $/ \mathrm{sec} / 100 \mathrm{~mA}$. For M0.7, the distances from source to the DMM and to the focusing mirror are $9.83 \mathrm{~m}$ and $11.18 \mathrm{~m}$ respectively. 

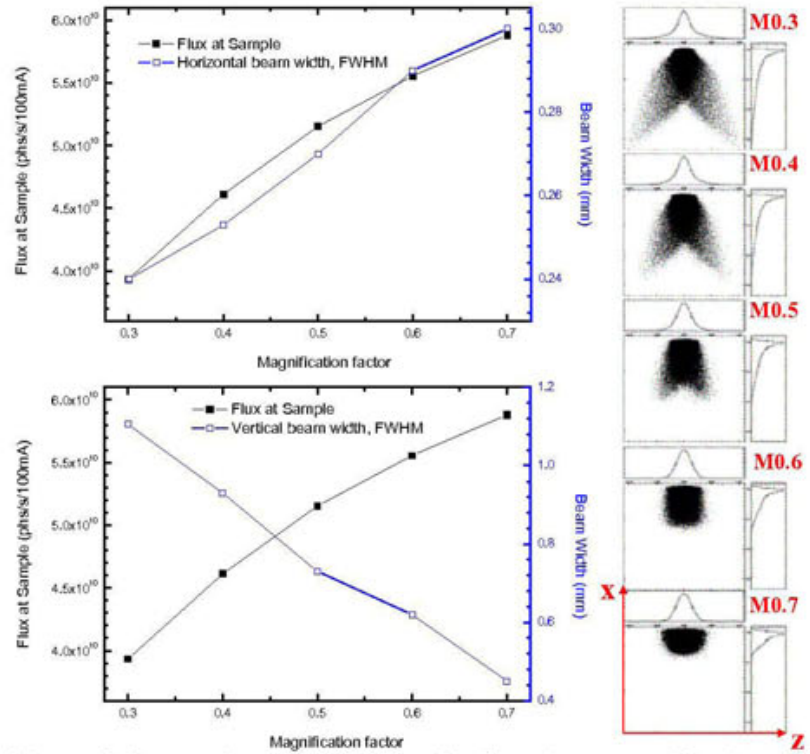
Figure 6. Image size at focal plane for the mirror magnifications M0.3, M0.4, M0.5, M0.6 and M0.7.
Right panel shows spatial distribution (scattered plots) and intensity profiles (histograms) of the focused beams. $x$ and $z$ refer to horizontal and vertical directions of the focused beam with respect to the optical axis.
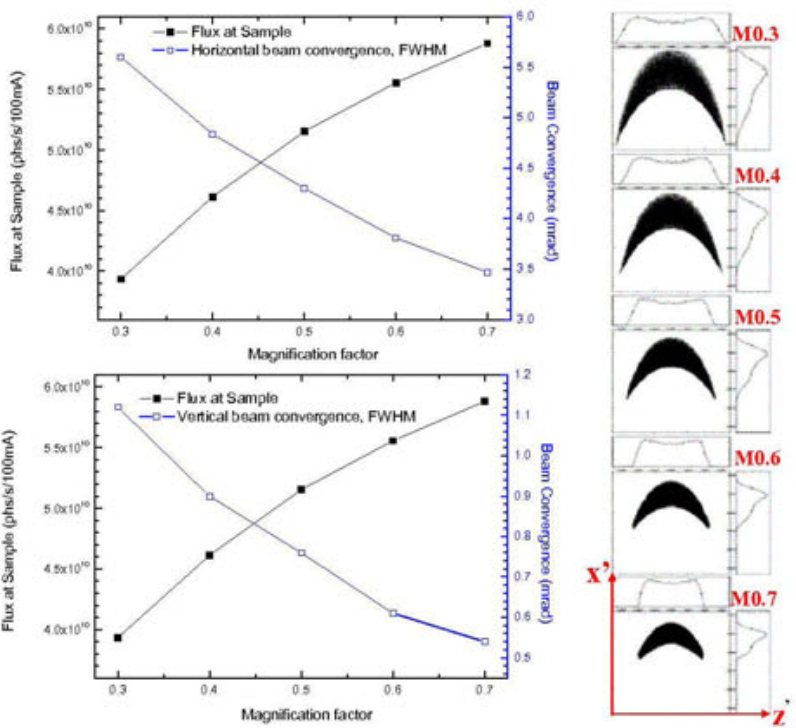

Figure 7. Beam convergence at focal plane for the mirror magnifications M0.3, M0.4, M0.5, M0.6 and M0.7. Right panel shows angular distribution (scattered plots) and intensity profiles (histograms) of the focused beams. $x^{\prime}$ and $z^{\prime}$ refer to convergent angles in sagital and meridional plans of the mirror. 


\section{Conclusions}

At this point, we can conclude that the $\mathrm{W} / \mathrm{B}_{4} \mathrm{C}$ multilayer is appropriate for the $\mathrm{DMM}$ in order to increase the photon flux for the bending magnet beamline at the SPL. The optimal mirror magnification of the toroidal focusing mirror for the limited length of the beamline is M0.7. The expected flux at the sample through a $0.3 \times 0.3 \mathrm{~mm}^{2}$ aperture is about 7500 times higher than that of BL8 currently. In addition, a low level of aberration and a small X-ray beam size are required for the XRPD technique. Therefore, the mirror magnification of M0.7 is a good choice for upgrading the existing beamline or developing a new one.

\section{References}

1. Bovornratanaraks, T., 2001, Ph.D. Thesis (The University of Edinburgh).

2. National Synchrotron Research Center, 2006, Annual Report (Thailand: Gnomes House Co., Ltd.).

3. Welnak, C., Chen, G.J. \& and Cerrina, F., 1994, Nucl. Instrum. Methods Phys. Res. $A, \mathbf{3 4 7}, 344$.

4. Klysubun, W., Sombunchoo, P., Wongprachanukul, P., Tarawarakarn, P., Klinkhieo, S., Chaiprapa, J. \& Songsiriritthigul, P., 2007, Nucl. Instrum. Methods Phys. Res. A, 582, 87.

5. Riesemeier, H., Ecker, K., Görner, W., Müller, B.R., Radtke, M. \& Krumrey, M., 2005, X-Ray Spectrom., 34, 160.

6. Shvyd'ko, Y., 2004, X-Ray Optics: High-Energy-Resolution Applications (Springer Series in Optical Sciences), (Berlin, Heidelberg: Springer-Verlag).

7. A. Kazimirov, A., Smilgies, D.M., Shen, Q., Xiao, X., Hao, Q., Fontes, E., Bilderback, D.H., Gruner, S.M., Platonovb, Y. \& Martynovb, V.V., 2006, J. Synchr. Rad., $13,204$.

8. Morawe, C., Peffen, J.C., Ziegler, E. \& Freund, A.K., 2001, SPIE Proceedings, 4145,61 .

9. Salashchenko, N.N., Platonov, Y.Y. \& Zuev, S.Y., 1995, Nucl. Instrum. Methods Phys. Res. A, 359, 114.

10. Kunz, M., MacDowell, A.A., Caldwell, W.A., Cambie, D., Celestre, R.S., Domning, E.E., Duarte, R.M., Gleason, A.E., Glossinger, J.M., Kelez, N., Plate, D.W., Yu, T., Zaug, J.M., Padmore, H.A., Jeanloz, R., Alivisatos, A.P. \& and Clark, S.M., 2005, J. Synchr. Rad., 12, 650.

11. Falkenberg, G., Clauss, O., Swiderski, A. \& Tschentscher, Th., 2001, X-Ray Spectrom., 30, 170.

12. Braun, S., Gawlitza, P., Menzel, M., Leson, A., Mertin, M. \& Schäfers, F., 2007, AIP Conference Proceedings, 879, 493.

Acknowledgements. The Synchrotron Light Research Institute's Graduate Scholarship under Contract No.GS-48-D05 and Graduate School, Department of Physics and Conference Grant for Ph.D. Student, Chulalongkorn University have supported this work. 


\section{Texture research of metals and rocks on the KSN-2 neutron diffractometer}

\section{S. Vratislav, M. Dlouhá, L. Kalvoda, M. Dráb}

Faculty of Nuclear Science and Physical Engineering, Czech Technical University of Prague, Břehová 7, 11519 Prague 1, Czech Republic

"stanislav.vratislav@fjfi.cvut.cz.

Keywords: neutron diffraction, texture analysis, ODF function, materials science

Abstract. From the industrial point of view the research activities of the Laboratory of Neutron Diffraction (Faculty of Nuclear Sciences and Physical Engineering CTU Prague) are concentrated to the quantitative texture analysis based on the ODF (orientation distribution function). We have developed the experimental and data treatment procedures for this type research. The texture experiments were carried out on the neutron diffractometer KSN-2 using the TG-1 texture goniometer. This diffraction device offers good intensity with wavelengths in the range 0.095 to $0.141 \mathrm{~nm}$. Experimental data in form of the pole figures or the inversion pole figures were treated by means of these codes: popLA, TODF-N, MAUD, GSAS. Quantitative texture analysis by means neutron diffraction data were used on the investigation of the many samples of the technically interesting materials. For example, we have determined the texture parameters on the oriented Si steel sheets and rock materials (limestone test samples).

\section{Introduction}

Texture influences both the behaviour of polycrystalline materials during thermal or mechanical treatment and the anisotropy of various properties in technical materials. The theoretical basis and the mathematical formalism of the description of crystalline structures were worked out deeply by J. Bunge [1] and J. Roe [2]. Such methods of texture description are extensive used in both basic and applied materials research. For example, they engage a important tool in diagnostic analysis of the final properties of different components or they are useful to appoint the texture corrections of the experimental data in stress or phase analysis.

Among these problems we can put nuclear technology (investigations of fuel elements and fuel tubes) and the production of the grain-oriented $\mathrm{Fe}-\mathrm{Si}$ sheet for the transformer cores purposes. During last years the texture analysis methods were used to the analysis of the preferred orientations of a many different rock samples. These fields of research are connected with new approach to the texture analysis, i.e. the Rietveld analysis [3] of the whole range diffraction spectra together with WIMV method $[4,5]$ is used to determined the threedimensional orientation function (ODF). Results of neutron diffraction texture analysis on monomineralic and polymineralic rocks [6] were presented, e.g., on calcite, quartzite, plagio- 
clase, pyrrhotite ores, granite, and orthopyroxene-sillimanite-granulite. The grain orientation distribution (texture) gives important information about this structure and influences the properties of materials.

Neutron texture studies $[7,8]$ are especially interesting because of the low absorption of neutrons in most technically important materials. The high penetration of neutrons through the majority of materials together with a relative large total number of grains in irradiated sample volume [9] is the main advantage for examination of the textures of coarse-grained materials like oriented transformer sheets and hence provide an ideal probe to examine the interior of metals or other materials. Quantitative texture analysis (three-dimensional distribution function - ODF) in connection with neutron diffraction is extensively used in this research field. The utilization of the Rietveld method to the simultaneously refinement of the structure parameters and texture corrections of measured materials is serious advantages for texture research of the low-symmetry compounds with partially overlapped diffraction peaks $[3,4,5]$.

Recent research shows that the correlation between microstructural properties and material parameters defined in the macroscopic models can be obtained by means of material modelling procedures together with experimental research of microstructure and texture parameters of investigated materials (magnetostriction modelling, stress and texture analysis).

Our study deals with the determination of the final texture form on the processing operation stages of oriented magnetic samples and further we can show the preliminary results connecting with the quantitative texture analysis of rocks (limestone samples) by powder neutron diffraction spectra measurements.

\section{Texture analysis by neutron diffraction}

Neutrons are often used in industrial applications of the diffraction methods. The stress and the texture analysis have a very serious place in the basic and the materials research. At present rapid advances in technology are making such investigations more and more useful. The grain orientation distribution - texture - gives important information about this structure and influences the properties of materials. For example, texture influences both the behavior of polycrystalline materials during thermal or mechanical treatment and the anisotropy of various physical properties in technical materials.

\section{Experimental arrangement of the KSN-2 diffractometer}

Double-axis diffractometer KSN-2 $[7,8]$ is performed for neutron diffraction structure and texture analysis and it is located at the HK-2 horizontal channel of the research reactor LVR15 at the INR in Rež. This device is equipped by cryostat with He close circle CP-62-ST/5 (from $8 \mathrm{~K}$, regulation of temperature with precision of $0.1 \mathrm{~K}$, maximal size of the sample : 38 $\mathrm{mm} \times 60 \mathrm{~mm}$ ), furnace up to $1000 \mathrm{~K}$ and the TG-1 texture goniometer [7]. The wavelength of the monochromatic neutron beam can be elected from 0.095 to $0.141 \mathrm{~nm}$ by means of the monochromators: single-crystal $\mathrm{Zn}(0002), \mathrm{Cu}(111)$ and $\mathrm{Cu}(200)$, respectively. Neutron flux at sample position is about $0.7-2 \times 10^{6} \mathrm{n} \mathrm{cm}^{-2} \mathrm{~s}^{-1}$ and the area of the beam cross section is $43 \times 90 \mathrm{~mm}^{2}$. The mean value of the diffractometer resolution is about $\Delta \mathrm{d} / \mathrm{d}=0.0045$. 


\section{Coarse-grained metals}

The set of experimental and calculation methods TODFND (the cubic symmetry of the crystals and orthorhombic symmetry of the specimen) was tested on the orientation magnetic steel sheets and the following steps were done:

- We have examined over 25 the specimens of the Fe-3\%Si sheets [8], which were prepared by the Sheet Rolling plant.

- The experimental data were measured in transmission and reflection arrangement. Each sample consist of the 5 lists (dimension each list: $30 \times 30 \times 0.132 \mathrm{~mm}$ ). The measured data are corrected, normalized and the experimental pole figures are calculated. The texture experiments were carried out on the KSN-2 diffractometer equipped with the texture goniometer TG-1 [7,8]. The neutron monochromatic wavelength was $0.1023 \mathrm{~nm}$. In case of $\mathrm{Fe}-3 \% \mathrm{Si}$ sheets, pole intensity distributions were measured for (110), (200) and (211) reflections and the measured data were processed by the TODFND-codes [10].

- The determined pole figures are used to calculate the coefficients of expansion $C_{1}^{\mu v}$ for expanding ODF into a number of generalized spherical functions. The ODF values leave the software package in the form of sections through the Euler space either for $\phi_{1}$ or $\phi_{2}=$ const (in $5^{\circ}$ steps) and ODF can also be depicted graphically in the individual sections.

-All texture characteristics can be obtained from these TODFND-codes: pole figures, inverse pole figures, ODF $\left(f(\mathrm{~g})\right.$ values in the $5^{\circ}$ steps), parameters of the ideal orientations (HKL) $/ u v w /$, texture index $J$, volume fraction coefficient $f$.

\section{Texture analysis of rock materials}

Limestone belongs to the group of sedimentary rocks with very complicated polycrystalline fabrics. It is widely used in praxis, above all in civil engineering. In spite of the common long-lasting research of limestone microstructure, the mutual relationships among 3D crystal aggregate variations still remain basically unknown. The investigation of the structure parameters and the determination of the preferred orientations of these crystal aggregates could help to give the answer whether the possible structure constrains and features depends on the sedimentary processes or metamorphic stages of the rock structures. Although there is a number of methods available providing separate results sensitive to anisotropy of the aggregates, they were rarely compared either quantitatively or qualitatively. Among these methods belongs neutron diffraction. The first results of which were obtained by neutron diffraction method are briefly summarized here.

The investigated limestone samples [11] were collected near Choteč, Bohemia, from a single limestone fold. Two selected sampling points $(\mathrm{C} 1, \mathrm{C} 2)$ represented a different level of the fold inversion: The sample $\mathrm{C} 1$ was collected from the fold part with the original sedimentation direction (SD) oriented nearly horizontally, in case of the sample C2 the later was turned upside down. Distance between the sampling points was approximately $1 \mathrm{~m}$. Specimen for the experiments were then cut and shaped according to needs of the individual method.

Elemental composition of the material was characterized by instrumental neutron activation analysis (INAA). The measurements were realized at the INP ASCR in Rež. The following elements were found having the mass concentration (c) higher then $0.1 \mathrm{pph}$ : $\mathrm{Ca}(\mathrm{c}=34.72$ pph), $\mathrm{Mg}$ (4733 ppm), Fe(2253 ppm), K (1967 ppm) and Al (1185 ppm).

Neutron diffraction experiments were performed with aim to characterize the bulk crystalline structure and crystallographic preferential orientation (texture) of the selected samples. Two 
types of specimens have been tested: Powders prepared by ball milling and oriented cubes (a $=20 \mathrm{~mm}$ ). The later were cut to have the edges parallel to the three principal directions forming a Cartesian co-ordination system: the SD, the direction perpendicular to the contour lines of the fold (PD), and the contour direction (CD). Diffraction diagrams were collected on the neutron diffractometer $\mathrm{KSN}-2$. The following instrumental settings were employed: Monochromatic parallel primary beam with $\lambda=0.1362 \mathrm{~nm}$ and the resolution of diffractometer in the interval $2 \theta$-range from $4^{\circ}$ to $75^{\circ}$ were about $\Delta \mathrm{d} / \mathrm{d}=0.0045$. The obtained diffraction patterns were corrected for non-linear background and then evaluated using the Rietveld method implemented in software packages GSAS, Material Studio (Accelrys) and MAUD. Texture of the samples was quantified by orientation distribution function (ODF). The first attempt was done to determine the preferred orientation of two calcite samples $C_{1}$ and $C_{2}$ which were descibed above.

\section{Results}

\section{Texture analysis of silicon steel sheets}

As the list of our results is very large the selected texture characteristics of only four specimens (with the processing stages) in table 1 are given. The complete quantitative texture analysis was performed on the four different silicon steel samples [7] and the dependence of the final texture (texture form, level of the $f(g)$ values) on the different processing stages was obtained. Samples were undergone to the different thermal treatment during primary and secondary cold rolling. Results are given in table 1.

The dependence of the final texture form on the temperatures of the (I. and II.) cold rolling was investigated. The texture analysis was performed by means of the ODF function with neutron diffraction experiments. The coordinate system of sheet samples was defined by directions: RD (rolling), ND (normal) and TD (transversal).

Table 1. Parameters of the investigated $\mathrm{Fe}-3 \% \mathrm{Si}$ samples.

\begin{tabular}{|c|c|c|c|c|c|c|c|}
\hline Sample & $\mathrm{T}_{\mathrm{IV}}\left[{ }^{\circ} \mathrm{C}\right]$ & $\mathrm{T}_{\text {IVV }}\left[{ }^{\circ} \mathrm{C}\right]$ & $\mathrm{f}(\mathrm{g})(011)<100>$ & $\mathrm{J}$ & $\mathrm{f}[\%]$ & $\mathrm{Z}_{\mathrm{p}}[\mathrm{W} / \mathrm{kg}]$ & $\mathrm{B}[\mathrm{T}]$ \\
\hline 1 & 20 & 20 & 48.2 & 39 & 36.5 & 1.450 & 1.739 \\
\hline 2 & 200 & 20 & 34.2 & 23 & 29.2 & 1.570 & 1.703 \\
\hline 3 & 20 & 200 & 21.5 & 16 & 27.4 & 1.760 & 1.663 \\
\hline 4 & 200 & 200 & 13.3 & 14 & 24.1 & 1.646 & 1.646 \\
\hline
\end{tabular}

Remarks: $\mathrm{T}_{\mathrm{IV}}\left[{ }^{\circ} \mathrm{C}\right]$ - temperature of the I. cold rolling;

$\mathrm{T}_{\text {IIV }}\left[{ }^{\circ} \mathrm{C}\right]$ - temperature of the II. cold rolling; $\mathrm{f}(\mathrm{g})$ - ODF's values; (HKL) <uvw $>$ - ideal orientation; $\mathrm{J}$ - texture index; $\mathrm{f}[\%]$ - volume fraction; $Z_{\mathrm{p}}[\mathrm{W} / \mathrm{kg}]$ - power losses; $\mathrm{B}[\mathrm{T}]-$ magnetization.

It appears, that the best texture form and $\mathrm{f}(\mathrm{g})$ values were obtained with $\mathrm{T}_{\mathrm{IV}}=\mathrm{T}_{\mathrm{IV}}=20^{\circ} \mathrm{C}$, i.e. for the sample 1 . The power losses $Z_{p}$ are the lowest and the $B$ value is the highest one. The treatment used in this case also leads to very sharp and narrow form of the $\mathrm{f}(\mathrm{g})$ peak values. If the processing temperature is raised up to $200{ }^{\circ} \mathrm{C}$, the texture distribution is becoming 
broader. More detail overview of the characteristic texture parameters and results is given in $[7,8]$.

\section{Texture analysis of limestone}

Powder patterns were collected on the KSN-2 diffractometer equipped by the TG-1 texture goniometer. We have defined an instrument coordinate system $(\mathrm{I}, \mathrm{J}, \mathrm{K})$ and a set of righthanded goniometer angles $(\Omega, X, \Phi)$ after the description given in [3]. The set of the measured patterns is consists the 43 sample diffraction vectors for data analysis of the $C_{1}$ limestone sample and the 29 sample diffraction vectors of the $\mathrm{C}_{2}$ one. Crystalline structure of calcite was refined within the space group R-3c by means of the GSAS software package [3]. Firstly, we refined the powder samples and we determine the complete set of structure parameters (Table 2). In the beginning, the individual scale factors and background coefficient for each powder pattern were determined (table 2). Then the average values of the lattice parameters were used in the refinement process for the spherical harmonic determination. Final refinements for the both samples $\mathrm{C}_{1}$ and $\mathrm{C}_{2}$ were done with $\mathrm{mmm}$ sample symmetry and

Table 2. Crystallographic parameters for calcite samples.

\begin{tabular}{|l|l|l|l|l|}
\hline Sample & $\mathrm{C}_{\text {powder }}$ & $\mathrm{C} 2_{\text {powder }}$ & $\mathrm{Cl}_{\text {aver }}$ & $\mathrm{C}_{\text {aver }}$ \\
\hline space group & $\mathrm{R}-3 \mathrm{c}$ & $\mathrm{R}-3 \mathrm{c}$ & $\mathrm{R}-3 \mathrm{c}$ & $\mathrm{R}-3 \mathrm{c}$ \\
\hline $\mathrm{a} / \AA /$ & $4.9764(5)$ & $4.9769(5)$ & $4.9846(6)$ & $4.9840(6)$ \\
\hline $\mathrm{c} / \AA /$ & $17.013(4)$ & $17.014(4)$ & $17.041(7)$ & $17.046(7)$ \\
\hline $\mathrm{x}_{0} / \AA /$ & $0.2577(5)$ & $0.2580(5)$ & $0.2571(6)$ & $0.2577(7)$ \\
\hline$\mu / \mathrm{cm}^{-1} /$ & $0.298(3)$ & $0.285(3)$ & $0.302(4)$ & $0.297(6)$ \\
\hline $\mathrm{P}$ & - & - & 1.14 & 1.47 \\
\hline $\mathrm{R}_{\mathrm{wp}} / \% /$ & 5.51 & 5.92 & 11.8 & 12.3 \\
\hline
\end{tabular}

Remarks: Samples: $\mathrm{Cl}_{\text {powder }}, \mathrm{C}_{\text {powder }}$ - powder samples, $\mathrm{Cl}_{\text {aver }}$ - average values from 43 powder diffraction diagrams, $\mathrm{C}_{\text {aver }}$ - average values from 29 powder diffraction diagrams; Atomic coordinates: $\mathrm{x}, \mathrm{c}$ and $\mathrm{x}_{0}$ (oxygen parameter); $\mu$ linear absorption coefficient $/ \mathrm{cm}^{-1} / \mathrm{P}$ texture level (maximum) in MRD (multiple of random distribution); GSAS refinement: sample symmetry: $\mathrm{mmm}$, maximum harmonic order $\mathrm{L}=8$.

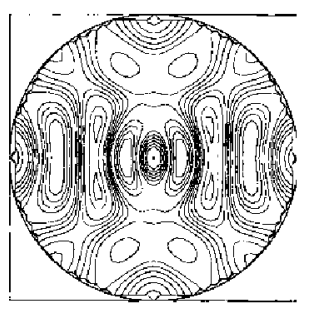

Figure1. The pole figure (006) determined by means of the Rietveld procedure (GSAS package) from the powder patterns of the $C_{I}$ limestone sample. The maximum value is $1.053 \mathrm{MRD}$, contours are in 0.15 MRD steps. 
maximum harmonic order $\mathrm{L}=8$. The next step in the texture analysis used the WIMV $[4,5]$ method and the ODF values were obtained. Results are given in table 2 and figure 1.

Texture of the samples can be characterized as follows: (i) the sample $\mathrm{Cl}$ posses a moderate texture (the calculated ODF sharpness $f=1.14$ ) and the remarkable declination of crystalline c-poles from the SD (ODF maximum occurs within the $\beta$-range $50^{\circ}-70^{\circ} ; \beta$ is the polar angle). (ii) Texture of the sample $\mathrm{C} 2$ is more pronounced $(\mathrm{f}=1.47)$, and the $\mathrm{c}$-poles orientation is closer to the SD $\left(\beta \cong 0^{\circ}-20^{\circ}\right)$. Crystallographic and texture parameters are given in table 2. In figure 1 is showed the (006) pole figure for comparison with results given in [3]. The diffraction patterns gave evidence about traces of additional crystalline phases; their identification is under progress.

\section{Concluding remarks}

Experimental and computing techniques for quantitative texture analysis based on the ODF combined with the diffraction of thermal neutrons were developed and tested. The results show that the procedures can be successfully applied to investigation of oriented transformer sheets and rock materials and can provide the valuable information about the texture of materials and related properties.

\section{References}

1. Bunge, H.J., 1969, Mathematische Methoden der Texturanalyse (Berlin: Akademie Verlag).

2. Roe, R.J., 1965, J. Appl. Phys., 36, 2024.

3. Von Dreele, R.B., 1997, J. Appl. Cryst, 30, 517.

4. Lutterotti, L., Matthies S., Wenk H.R.,1997, J. Appl. Phys., 81, 594.

5. Sitepu, H., O'Connor, B.H., Benmarouane, A., Hansen, T., Ritter \& Brokmeier, G., 2004, Physica B: Condensed Matter, 350, (1-3), Suppl. 1, E573.

6. Schafer, W., 2002, Eur. J. Mineral., 14, 263.

7. Vratislav, S. \& Dlouhá, M., 1995, in Proc. of the Int. Conf. Mathematical Methods of Texture Analysis, Dubna, March $21-24$, Russia, p. 48.

8. Vratislav, S., Dlouhá, M. \& Kalvoda, L., 2005, Solid State Phenomena, 105, 297.

9. Brokmeier H.G., 2006, Physica B: Condensed Matter, 385-386, 623.

10. Dlouhá, M. \& Vratislav, S., 1988, in: Research Report of the CTUT I/88, Prague.

11. Kalvoda, L., Dlouhá, M. \& Vratislav, S., 2008, in: Proc. of the CTU Workshop,12, 215.

Acknowledgements. This work was supported by the MŠMT grant No. MSM6840770021. 


\section{Modular in-situ reaction chamber design for time resolved diffraction}

\section{J. Styles ${ }^{1}$, D. P. Riley ${ }^{1,2,3,}{ }^{*}$, J. Christoforidis ${ }^{4}$, S. Olsen ${ }^{4}$}

${ }^{1}$ Department of Mechanical Engineering, The University of Melbourne, Melbourne, VIC 3010, Australia

2 The Bio21 Institute, The University of Melbourne, Melbourne, VIC 3010, Australia

${ }^{3}$ Australian Institute of Nuclear Science and Engineering, PMB 1 Menai, NSW 2234, Australia

${ }^{4}$ Australian Nuclear Science and Technology Organisation, PMB 1 Menai, NSW 2234, Australia

*DRiley@unimelb.edu.au

Keywords: environment chamber, time resolved diffraction

Abstract. In an effort to mitigate the expense and uncertain performance of customised environment chambers, researchers at the University of Melbourne and the Australian Nuclear Science and Technology Organisation (ANSTO) have designed and are currently constructing a modular reaction chamber, capable of separating the necessities of diffraction methodologies from those of the desired sample environment. The In-Situ Reaction Chamber (ISRC) abstracts many of the details intrinsic to the diffractometer, allowing users to design inexpensive environmental inserts that may be readily customised to their individual needs. Overall, the modularised design aims to reduce the development costs of performing in-situ diffraction experiments, while minimising the experimental setup time and overall uncertainty of ancillary performance.

\section{Introduction}

Progress in many technological fields including energy production, aerospace and biomedicine is increasingly dependent upon novel materials with exceptional mechanical, thermal and chemical properties. Often these materials are produced via complex processing techniques, the traditional optimisation and validation of which have contributed to making them costly both economically and environmentally. However, there is increasing experimental evidence to suggest modern time-resolved diffraction techniques, given sufficient time-resolution, can substantially reduce the time involved in process optimisation. This can be achieved through an examination of the fundamental mechanisms by which a material is synthesised, on a time scale commensurate with the rate limiting kinetic mechanisms [1].

Time resolved in-situ diffraction is a particularly powerful technique in materials research, capable of observing the time evolution of phases as they change in quantity and composition, and correlating these changes with processing parameters such as temperature, pressure 
and time [2]. This provides a vital insight into the kinetics of materials synthesis and processing; information essential to process optimisation [3]. There are however, several challenges inherent in time resolved diffraction techniques, which generally might be categorised into the flux and resolution of modern diffractometers (both of which need to remain high), the development of analysis software capable of handling very large data sets $(>10,000$ diffraction patterns) and the ability to construct sample environments that mirror the processing or operational environments of the materials under investigation.

The time resolution of an in-situ diffraction experiment is determined by the flux of radiation at the sample position and limited by the speed and dynamic range with which detectors might acquire the data. The development of position sensitive detector (PSD) technology for very high flux powder diffractometers (such as D20, GEM and WOMBAT for neutrons and various synchrotrons for X-rays) has enabled diffraction pattern acquisition and data readout times to be reduced to less than a second. This has allowed diffraction studies of rapid material reactions and transformations with the same or better time resolutions as conventional methodologies [4]. A result of these high speed detectors is the accumulation of extremely large data sets, of the order of $>10,000$ diffraction patterns per reaction sequence. To then quantitatively process this data for information such as phase analysis, reaction kinetics and thermal expansion, has traditionally been a laborious and time consuming task, if it can be performed at all. Software with the ability to either sequentially refine patterns or simultaneously fit 2-dimensional data sets (i.e. Rietveld surface fits) have begun to address this issue and make the quantitative analysis of data sets of this size feasible.

Overall, the development of reliable sample environment instrumentation remains a limiting factor in time resolved in-situ studies. If the results of a time resolved in-situ experiment are to be applied to the interpretation of a real process, it is critical that the sample environment be representative of the system under consideration. Often the environment chambers available at major research facilities are optimised for particular instruments and are restricted in their capabilities, or alternately are developed at great expense by user communities for a single, specialised experiment. These approaches often result in the development of chambers customised to the needs of a specific instrument or user and hence restrict the wider application of these techniques. Unfortunately, the difficulty and expense of producing a customised chamber has frequently resulted in poorly performing experiments or has deterred further experimental development.

These issues can be addressed by identifying and separating the experimental aspects associated with a particular instrument or technique, from those associated with sample environment or process simulation. The result is a modular system in which the relatively expensive, instrument specific technology can be developed once by a research institution, while the highly customised environment chambers are developed as needed by individual users. This method significantly reduces the cost of customised chambers and allows users to focus on creating an environment that accurately simulates their system of interest. Furthermore, the user may then have the opportunity to completely characterise the insert off-line, prior to performing their experiment $i n$-situ, thereby minimising the uncertainty of the experiment and hence improving the quality of the data obtained. This directly addresses the increasing over-subscription of proposals to high-flux diffractometers around the world by significantly reducing the time and complexity of experimental setup. 


\section{Design of the in-situ reaction chamber}

Researchers at the University of Melbourne have designed and are currently constructing in collaboration with ANSTO, a modular reaction chamber capable of achieving this separation of technologies. The ISRC (figure 1) has been designed specifically for use on the high intensity powder diffractometer WOMBAT, located at the OPAL Research Reactor (Lucas Heights, Sydney, Australia), and is representative of the instrument specific chamber required by the modular technique.

The ISRC has been designed to address the following issues associated with high-flux neutron powder diffraction:

- Air scatter has been identified as a major contributor to instrumental background and loss of incident neutron flux and as such, the body of the ISRC is to be evacuated to high vacuum (sample atmospheres provided within the environmental insert). While a temporary flight tube from monochromator housing to the sample environment is not envisaged for standard operation, the beam inlet window will allow for the fitting of a continuously evacuated neutron guide. In general, the present design aims to maximise chamber diameter and hence provide the largest sample volume, while removing as much air-scatter as is feasible given the limiting inner dimensions of the curved position sensitive detector.

- Flux attenuation is critical for ultra fast acquisitions, where time resolution is defined by the ultimate flux at the sample position. Aluminium has been used for incident and diffracted beam windows to reduce flux attenuation, the thicknesses of which have been reduced to a minimum. These windows constitute the only component of the ISRC positioned within the incident and diffracted beams.

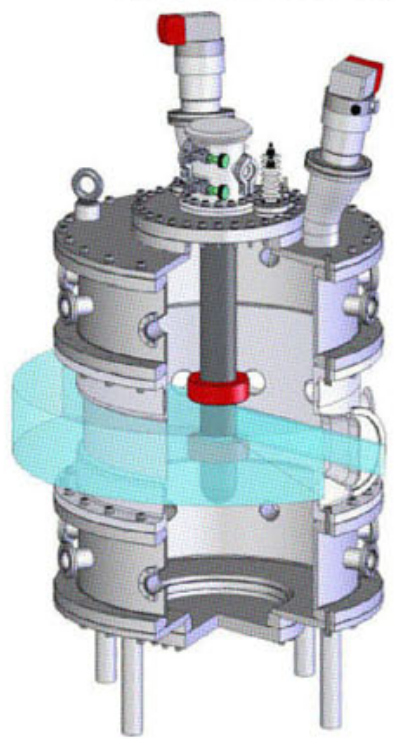

Figure 1. Section view of the assembled ISRC and high temperature insert, showing passage of incident and diffracted beams. 
- Maximum angular resolution, while ultimately defined by the angular acceptance of the PSD, the ISRC can limit the angular range if the beamstop and flight tubes are not correctly integrated. Care has been taken in the design of the ISRC to minimise the impact of these features (figure 2a).

- Diffraction window uniformity has also been determined to be a significant contributor of anomalous background features. High manufacturing tolerances have been established for the window thickness, producing a $+/-0.01 \mathrm{~mm}$ variance over the full $160^{\circ}$ range (figure $2 b$ ).

- Sample alignment is achieved on the ISRC by the use of standard, self-locating vacuum flanges, which are set at a specific height from the centre of the incident beam (figure $2 \mathrm{c}$ ).

- Incident beam definition can minimise divergence from the monochromator, hence improving the full-width half-maximum peak definition. Masking of the incident beam is performed at the flight tube vacuum port, allowing for the use of materials normally sensitive to high temperatures to be applied at an external position (figure 2d).

- Concurrent experimentation can be used to gather more information or verify data while the diffraction measurement is being taken. The ISRC features vacuum ports set at various angles and heights to facilitate these experiments (figure 2e).

- Chamber activation has been addressed in the ISRC by selecting materials that exhibit little if any activation, minimising the down time between experiments/users.

- Thermal loadings have been addressed by incorporating active water cooling within the double wall of the main chamber, permitting the use of very high temperature sample environments (figure $2 \mathrm{f}$ ).

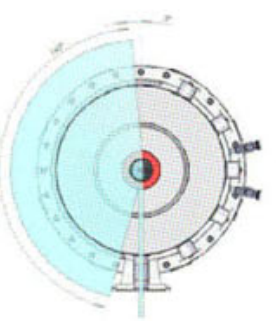

(a)

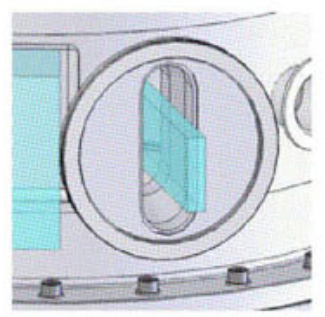

(d)

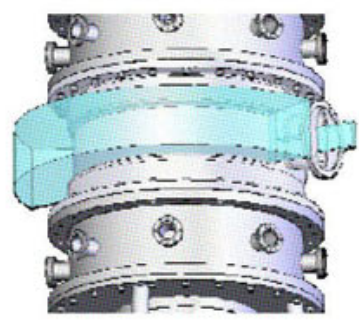

(b)

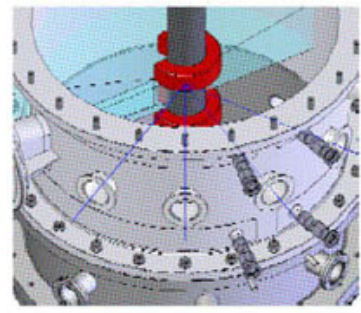

(e)

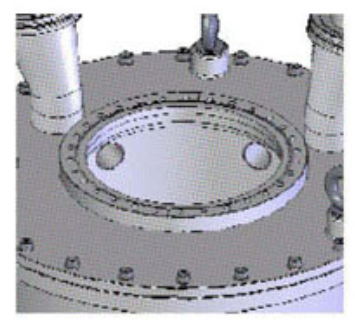

(c)

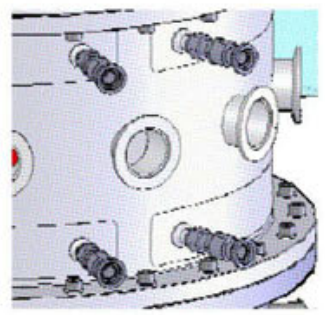

(f)

Figure 2. Features of the In-situ Reaction Chamber (ISRC). 
With the equipment specific to neutron powder diffraction incorporated into the ISRC, customised environmental inserts can be developed with minimal restrictions on design and geometry, provided it conforms to standard dimensions (height, interface flange and volume of chamber). Production costs can be minimised as only the equipment necessary for a specific experiment is required, although the possibilities for multiple applications of the insert may justify substantial development. Careful design may even allow this development to occur in stages, spreading the cost over several experiments.

An example of a customised insert is the high temperature chamber (figure 3) being developed concurrently with the ISRC, which has the following attributes:

- Radiative band heaters mounted external to the sample environment (in the vacuum of the ISRC), above and below the beam height. These heaters allow very high temperatures to be reached accurately and repeatedly without contaminating (or being contaminated by) the sample environment or affecting the diffraction pattern.

- Capacity for independent control of heaters enables users to accurately control thermal gradients in the axial direction.

- High thermal mass of the environment tube assists in thermal stability. This is the only material other than the sample (and sample vessel) to be placed in beam.

- Gas atmospheres are possible with ports for gas flow in and out of chamber. Controlled circulation is achieved via a second internal tube located out of the beam path. Vacuum ports also allow a vacuum to be maintained within the sample environment if required.

- Active cooling of the sample port allows for the use of standard vacuum fittings, facilitating accurate sample alignment.

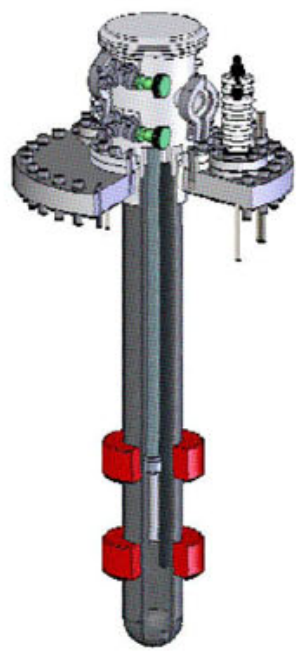

Figure 3. One specific insert device is the high temperature furnace shown here in section view. It has been designed for high temperature assessment of oxide ceramic materials $\left(>1600^{\circ} \mathrm{C}\right)$ under partial oxidising or reducing atmospheres and is intended for use in the investigation of catalytic reactions, crystallographic phase transitions and structural integrity of oxide ceramics as a function of time. 
- Multiple feedthrough ports to allow multiple instrument connections. For example, stationary control thermocouples can be positioned within environment chamber to aid in reproducibility, while sample thermocouples are mounted to the stalk.

- Remote loading of the sample is a possible future extension of the insert.

\section{Discussion}

Traditional methods of performing in-situ diffraction have been limited by the availability of customised reaction chambers. Cost has been a significant factor in limiting this development due to the unique design constraints of each reaction vessel. The present modular design of the ISRC deliberately separates the unique experimental aspects from the generic diffraction requirements; more specifically, customised inserts have been developed to meet the requirements of each reaction, which can then be placed into a diffraction specific chamber. Designed carefully, a single insert may be used on a variety of instruments.

An additional benefit of the modular technique is the ability to easily characterise each insert prior to use, minimising setup time and maximising the likelihood of a successful experiment. For time resolved studies in particular it is essential to minimise the effect of the environment chamber on the passage of the diffracting radiation. Customised inserts allow users to do this via methods most appropriate for their circumstances. Since process optimisation typically involves experimentation using multiple techniques, the ability to use a single, well characterised environment chamber can significantly reduce errors.

\section{Concluding remarks}

High time resolution in-situ diffraction is capable of providing a vital insight into the kinetics of materials processing. The information gathered by this technique can be applied to the optimisation of materials synthesis and the enhancement of operational performance.

Successful material development is dependent upon the quality of the sample environment, which must accurately simulate the conditions of the system under consideration. The feasibility of developing high quality sample environments is increased by separating the technology associated with particular diffraction techniques from the technology necessary for environment simulation.

\section{References}

1. Riley, D.P. \& Kisi, E.H., 2007, J. Am. Ceram. Soc., 90, 2231.

2. Kisi, E.H. \& Riley, D.P., 2002, J. Appl. Crystallogr., 35, 664.

3. Riley, D.P., Kisi, E.H. \& Hansen, T.C., 2008, J. Am. Ceram. Soc., 91, 3207.

4. Riley, D.P., Kisi, E.H., Hansen, T.C. \& Hewat, A.W., 2002, J. Am. Ceram. Soc., 85, 2417.

Acknowledgements. The authors would like to acknowledge the advice and assistance of Dr Thomas Hansen and Dr Paul Henry of the Institut Laue-Langevin (ILL), France and Prof. Erich Kisi of the University of Newcastle, Australia. 
III.

SOFTWARE 



\title{
RETRIEVE - a system for XRPD phase and structure analysis
}

\section{P. S. Dubinin, I. S. Yakimov*, O. E. Piksina, Y. I. Yakimov, A. N. Zaloga}

Siberian Federal University, Krasnoyarsk, Russia

*I-S-Yakimov@yandex.ru

Keywords: X-ray powder diffraction, Standardless Quantitative Phase Analysis, Derivative Difference Minimization, Genetic Algorithm

\begin{abstract}
An integrated retrieval system RETRIEVE oriented on qualitative and standardless quantitative phase and structure XRD analysis of powder patterns has been developed. The system has some original features. A derivative difference minimization (DDM) method and two-level Genetic Algorithm (GA) combined with DDM are intended for direct space searching and prediction of crystal structure models and structure refinement. A search/match based on problem oriented query language was developed for phase identification of complex powder patterns. Three modernized methods of standardless quantitative phase analysis are employed. Applications of these methods are demonstrated on SearchMatch Round Robin - 2002 and Round Robin on Quantitative Phase Analysis - 1998 data.
\end{abstract}

\section{Introduction}

The RETRIEVE was developed historically as a search/match retrieval system [1] intended for phase identification of complex powder patterns. It is ensured by using of a problem oriented query language based on calculus predicate theory instead of simple Boolean logic. Adequately formulated queries provide a high selectivity of phase identification. A model spectrum is constructed from identified reference patterns of PDF2 database (DB) and fitted to experimental powder pattern. Further two automatic methods for standardless quantitative phase analysis (QPA) were developed and integrated into the RETRIEVE. At first, known Reference Intensity Ratio (RIR) method was modified similar to [2] into multi-peak RIR based on using of scale factors from the model spectrum. At second, an enhancement of Rius method [3] was developed for iterative QPA of group of powder patterns (Group QPA) with identical qualitative but different quantitative phase composition. Third interactive standardless method incorporated into the RETRIEVE is DDM method [4]. This method carries out a QPA similar to known Rietveld method as well as crystal structure powder refinement. In order to extend RETRIEVE capabilities to full structure XRPD analysis a genetic algorithm has been developed recently for direct space searching and prediction of crystal structure models [5]. A problem-oriented graphical user-friendly interface (GUI) has been constructed for above methods. 


\section{Methodology}

A search/match method is based on a problem-oriented query language for programming of queries on phase identification. A format of each query is as follows.

$I N=$ «input selection of reference or experimental patterns»;

"sequence of language instructions»;

$O U T=$ «output selection of reference or experimental patterns».

Language instructions have following format:

$\{Y E S \mid N O T\}=I F\{O N E \mid A L L\}$ «binary inclusion relation under terms»;

$L I S T=$ «sequence of terms values»;

where "YES" or "NOT" denotes inclusion or exclusion of an input pattern into output selection, "ONE" or "ALL" denotes fulfillment of conditions for single or all terms included into relation.

Terms of reference or experimental patterns are chemical elements and fragments of formulas, peak positions and intensities, likelihood ratio criteria for adequacy of reference and experimental patterns, etc. Phase identification is treated as making of output selection from true reference patterns. The search/match GUI includes means for automatic programming of queries hidden from a user. A query program is constructed by clicking on reference and experimental patterns and other terms shown on GUI windows (see figure 1).

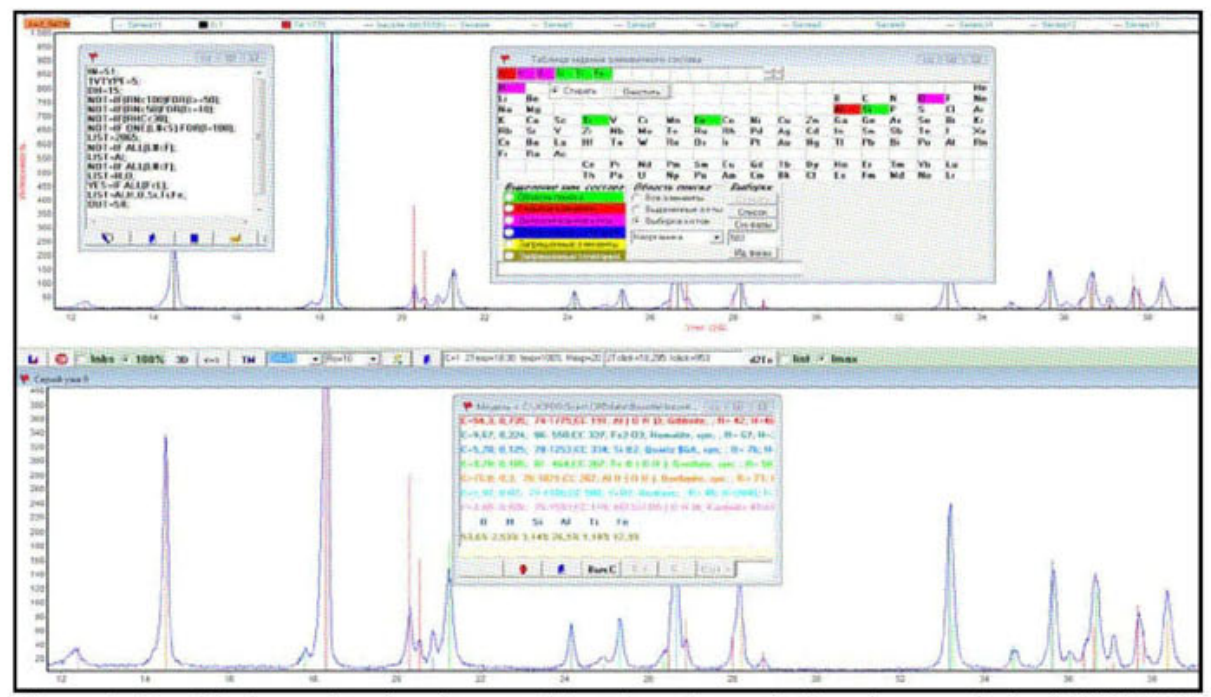

Figure 1. Graphical user interface for search/match and quantitative phase analysis of RETRIEVE; the left top window demonstrates a current query identification program which is usually hidden; the bottom window displays a model spectrum of powder pattern of synthetic bauxite.

Model of experimental powder pattern is constructed as a linear combination of identified reference patterns according to next equation:

$$
I^{M O D}(2,) \Theta \sum_{i}^{m} C_{i} \cdot I^{R E F}\left(2 \Theta_{i j}\right), \quad \mathrm{j}=1, \ldots, \mathrm{n},
$$


where $n$-number of peaks, $m$ - number of phases, $C_{i}$-scale factor coefficients, $I^{R E F}\left(2 \Theta_{i j}\right)-j$-th peak intensity for reference pattern of $i$-th phase.

The model optimization is executed by least square minimization method (LSM) as:

$$
\Phi(\bar{C})=\sum_{j}^{n} \omega_{j}\left[\sum_{i}^{m} C_{i} \cdot I^{R E F}\left(2 \Theta_{i j}\right)-I^{E X P}\left(2 \Theta_{i j}\right)\right]^{2} \longrightarrow 0,
$$

where $w_{i}$-weight coefficients, $I^{E X P}\left(2 \Theta_{i j}\right)$ - intensity of experimental peak $j$ of phase $i$.

An automatic method of standardless multi-peak RIR QPA is applied after the model optimization. The RIR uses scale factor coefficients calculated by (1) with eqs. (2), (3):

$$
W_{i}=\left(C_{i} / K_{i}\right) /\left(\sum_{i}^{m} C_{i} / K_{i}\right),
$$

where $W_{i}$ - weight fraction of phase $i$ in sample, $K_{i}=I_{i} / I_{c o r}$ - corundum ratio of phase $i$ from DB. Or in case of internal standard:

$$
W_{i}=\left(C_{i} / C_{\text {stand }}\right) \cdot\left(K_{\text {stand }} / K_{i}\right) \cdot W_{\text {stand }},
$$

where $C_{\text {stand }}, W_{\text {stand }}, K_{\text {stand }}$ - internal standard data.

The group QPA method is based on iterative refining of phase calibration constants and sample mass absorption coefficients by eqs. (4) - (7). For this method we have a general system of equations combining XRD and chemical data:

$$
\left\{\begin{array}{l}
\sum_{j=1}^{n-n_{a q}}\left[\begin{array}{ll}
P_{k j} & E_{i k}+I_{i j}
\end{array}\right] L_{j} \cdot \sum_{a=1}^{n_{a}}\left[\begin{array}{ll}
P_{k a} & \left.E_{i k}\right)
\end{array}\right] \alpha_{i a}=0 \\
\sum_{j=1}^{n-n_{u}}\left[\begin{array}{ll}
\mu_{j}^{*} & I_{i j}
\end{array}\right] L_{j} \cdot \sum_{a=1}^{n_{a}}\left[\mu_{a}^{*}\right] \alpha_{i a}=1,
\end{array}\right.
$$

where $k=1 . . t, i=1 . . . m, t-$ number of chemical elements; $n_{a}$-number of amorphous phases; $n$-general number of phases; $m$ - number of samples; $E_{i k}$ - concentration of the element $k$ in sample $i ; I_{i j}$ - peak integral intensity of the phase $j$ in sample $i ; P_{k j}$-atomic share of the element $\mathrm{k}$ in phase $j ; L_{j}$ - calibration coefficient for phase $j ; \mu_{j}{ }^{*}, \mu_{a}{ }^{*}$ - mass absorption coefficients of the phases $j$ and $a ; \alpha_{i a}-$ amorphous phase coefficient.

For QPA accuracy increasing the following iterative scheme of alternating refinement of phase calibration constants and sample mass absorption coefficients is designed:

1) Calculation of mass absorption coefficients of the phases;

2) Calculation of initial values of $L_{j}$ and $\alpha_{i a}$ from system of equations (4);

3) Calculation of mass absorption coefficients $M_{i}$ of the sample as

$$
M_{i}=\left(\sum_{j}^{n-n_{a}} I_{i j} \cdot L_{j}+\sum_{a}^{n_{a}} \alpha_{i a}\right)^{-1} .
$$

4) Calculation of $L_{j}$ and $\alpha_{i a}$ from the general system of the balance equations, including mass absorption coefficients as

$$
\left\{\begin{array}{l}
\sum_{j}^{n-n_{a}} P_{k j} I_{i j} M_{i} \cdot L_{j}+\sum_{a}^{n_{a}} P_{k a} M_{i} \quad \alpha_{i a}=E_{i k} \\
\sum_{j}^{n-n_{a}} I_{i j} M_{i} L_{j} \cdot \sum_{a}^{n_{a}}+M_{i} \quad \alpha_{i a}=1 .
\end{array}\right.
$$


5) Repeating of steps 3 - 4 while LSM procedure converges for system (6);

6) Calculation of the phase concentrations $C_{i j}$ as

$$
C_{i j}=\frac{I_{i j} \cdot L_{j}}{\sum_{j}^{n-n_{a}} I_{i j} \cdot L_{j}+\sum_{a}^{n_{a}} \alpha_{i a}}, \quad C_{i a}=\frac{\alpha_{i a}}{\sum_{j}^{n-n_{a}} I_{i j} \cdot L_{j}+\sum_{a}^{n_{a}} \alpha_{i a}} .
$$

The Derivative Difference Minimization method by L.A.Solovyov [4] is used for structure refinement as well as for QPA of multi-phase powder samples. The main advantage of the DDM method is the opportunity of powder diffraction pattern modeling independently of the background curve. The minimization function is chosen as

$$
M F=\sum w_{1}\left[\frac{\partial}{\partial \theta}\left(Y_{0}-Y_{c}\right)\right]^{2}+\sum w_{2}\left[\frac{\partial}{\partial \theta^{2}}\left(Y_{0}-Y_{c}\right)\right]^{2},
$$

where $Y o$ and $Y c$ - observed and calculated profile intensities; $\theta$ - diffraction angle; $w_{i}-$ weights; the sum is over the entire powder profile. A GUI for console DDM program was developed for interactive control of refining of profile and structure parameters (see figure 2). The GUI includes navigation system, patterns and their difference plotting, CIF-file import from structure data bases, phase composition calculation and other features.

A two-level genetic algorithm has been developed for searching of crystal structure models in direct space (see Y.I.Yakimov et al, the same issue). First-level GA chromosomes comprise values of profile and structure parameters used in the DDM method. Second-level GA chromosomes are bit strings which define strategies of parameter refining.

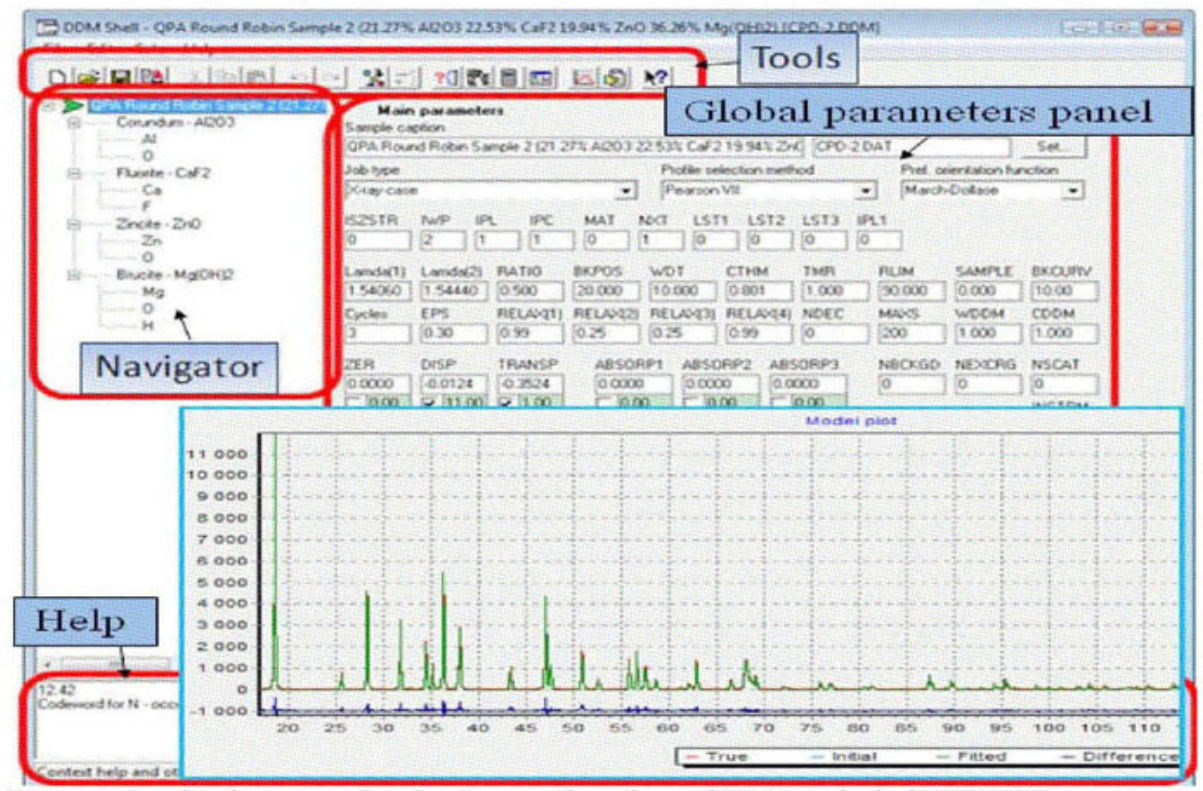

Figure 2. Graphical user interface for Genetic Algorithm and DDM-method of RETRIEVE. 


\section{Results}

The RETRIEVE achieved excellent results on Search-Match Round Robin - 2002 (http://sdpd.univ-lemans.fr/smrr/results ), where this program (participant P19) exactly identified all test samples. The QPA with modified RIR is demonstrated on analysis of synthetic bauxite of Round Robin on QPA [6] (see table 1). Column "RETRIEVE" shows calculated data, column " $\Delta$ " shows difference between "RETRIEVE" and weight fraction data. The last column shows standard deviations of QPA for all participants of the Round Robin.

Table 1. Results of Synthetic Bauxite $Q P A$ with modified RIR.

\begin{tabular}{|c|c|c|c|c|c|}
\hline Formula & Mineral Name & $\begin{array}{c}\text { RETRIEVE } \\
\text { (\% mass) }\end{array}$ & $\begin{array}{c}\text { Weight } \\
\text { (\% mass) }\end{array}$ & $\begin{array}{c}\Delta \\
\text { (\% mass) }\end{array}$ & $\begin{array}{c}\text { RR S.D. } \\
\text { (\% mass) }\end{array}$ \\
\hline $\mathrm{Al}(\mathrm{OH})_{3}$ & Gibbsite & 52.20 & 54.90 & $\mathbf{- 2 . 7 0}$ & 6.59 \\
\hline $\mathrm{AlO}(\mathrm{OH})^{\text {Boehmite }}$ & 15.40 & 14.93 & 0.47 & 2.19 \\
\hline $\mathrm{Fe}_{2} \mathrm{O}_{3}$ & Hematite & 10.10 & 10.00 & 0.10 & 4.67 \\
\hline $\mathrm{FeO}(\mathrm{OH})$ & Goethite & 9.66 & 9.98 & -0.32 & 3.35 \\
\hline $\mathrm{TiO}_{2}$ & Anatase & 2.27 & 2.00 & 0.27 & 0.43 \\
\hline $\mathrm{Al}_{2}\left(\mathrm{Si}_{2} \mathrm{O}_{5}\right)(\mathrm{OH})_{4}$ & Kaolinite & 4.48 & 3.02 & 1.46 & 1.96 \\
\hline $\mathrm{SiO}_{2}$ & Quartz & 5.77 & 5.16 & 0.61 & 1.22 \\
\hline
\end{tabular}

Group QPA was applied to eight CPD1 mixtures from the Round Robin (see tables 2-3).

Table 2. Results of Group QPA with XRD data only (second equation of system (4)).

\begin{tabular}{|c|c|c|c|c|c|c|}
\hline $\begin{array}{c}\mathrm{CPD} 1 \\
\text { patterns }\end{array}$ & $\begin{array}{c}\mathrm{CaF}_{2}, \\
\text { \%mass. }\end{array}$ & $\begin{array}{c}\Delta, \\
\text { \%mass. }\end{array}$ & $\begin{array}{c}\mathrm{ZnO}, \\
\text { \%mass. }\end{array}$ & $\begin{array}{c}\Delta, \\
\text { \%mass. }\end{array}$ & $\begin{array}{c}\mathrm{Al}_{2} \mathrm{O}_{3}, \\
\text { \%mass. }\end{array}$ & $\begin{array}{c}\Delta, \\
\text { \%mass. }\end{array}$ \\
\hline$a$ & 95.49 & 0.68 & 3.54 & -0.50 & 0.96 & -0.19 \\
\hline$b$ & 4.51 & 0.18 & 1.59 & 0.23 & 93.89 & -0.42 \\
\hline$c$ & 1.77 & 0.41 & 92.58 & -1.01 & 5.63 & 0.59 \\
\hline$d$ & 55.26 & $\mathbf{1 . 6 8}$ & 30.81 & -2.08 & 13.91 & 0.38 \\
\hline$e$ & 30.14 & 0.52 & 13.94 & -1.31 & 55.91 & 0.79 \\
\hline$f$ & 18.93 & 1.21 & 52.91 & $\mathbf{- 2 . 3 1}$ & 28.14 & 1.08 \\
\hline$g$ & 35.48 & 1.06 & 32.02 & -2.19 & 32.49 & $\mathbf{1 . 1 2}$ \\
\hline$h$ & 35.98 & 1.29 & 28.22 & -1.97 & 35.78 & 0.66 \\
\hline
\end{tabular}

Table 3. Results of Group QPA with XRD and chemical data (all system (4)).

\begin{tabular}{|c|c|c|c|c|c|c|}
\hline patterns & $\mathrm{CaF}_{2}$ & $\Delta$ & $\mathrm{ZnO}$ & $\Delta$ & $\mathrm{Al}_{2} \mathrm{O}_{3}$ & $\Delta$ \\
\hline$a$ & 95.07 & $\mathbf{0 . 2 6}$ & 3.94 & -0.10 & 0.90 & -0.25 \\
\hline$b$ & 4.42 & 0.09 & 1.74 & $\mathbf{0 . 3 8}$ & 93.83 & $\mathbf{- 0 . 4 8}$ \\
\hline$c$ & 1.60 & 0.24 & 93.22 & -0.37 & 5.17 & 0.13 \\
\hline$d$ & 53.19 & -0.39 & 33.15 & 0.26 & 13.65 & 0.12 \\
\hline$e$ & 29.34 & -0.28 & 15.10 & -0.15 & 55.48 & 0.36 \\
\hline$f$ & 17.73 & 0.01 & 55.39 & 0.17 & 26.87 & -0.19 \\
\hline$g$ & 33.98 & $\mathbf{- 0 . 4 4}$ & 34.28 & 0.07 & 31.72 & 0.35 \\
\hline$h$ & 34.59 & $-\mathbf{0 . 1 0}$ & 30.33 & $\mathbf{0 . 1 4}$ & 35.07 & -0.05 \\
\hline
\end{tabular}


The QPA with DDM method are demonstrated on the CPD1 and CPD2 mixtures of RR on QPA (see table 4). The bottom row shows standard deviations of CPD2 QPA for all participants of the Round Robin.

Table 4. Results of $Q P A$ with DDM method.

\begin{tabular}{|c|c|c|c|c|c|c|c|c|}
\hline \multirow{2}{*}{ Patterns } & \multicolumn{3}{|c|}{ DDM method, \%mass. } & \multicolumn{4}{c|}{$\Delta, \%$ mass. } \\
\cline { 2 - 10 } & $\mathrm{CaF}_{2}$ & $\mathrm{ZnO}$ & $\mathrm{Al}_{2} \mathrm{O}_{3}$ & $\mathrm{Mg}(\mathrm{OH})_{2}$ & $\mathrm{CaF}_{2}$ & $\mathrm{ZnO}$ & $\mathrm{Al}_{2} \mathrm{O}_{3}$ & $\mathrm{Mg}(\mathrm{OH})_{2}$ \\
\hline$a$ & 94.59 & 4.03 & 1.39 & & -0.22 & -0.01 & 0.24 & \\
\hline$b$ & 4.40 & 1.45 & 94.15 & & 0.07 & 0.09 & -0.16 & \\
\hline$c$ & 1.38 & 93.42 & 5.19 & & 0.02 & -0.17 & 0.15 & \\
\hline$g$ & 34.95 & 33.69 & 31.36 & & $\mathbf{0 . 5 3}$ & -0.52 & -0.01 & \\
\hline$h$ & 35.07 & 29.53 & 35.40 & & 0.38 & $\mathbf{- 0 . 6 6}$ & $\mathbf{0 . 2 8}$ & \\
\hline$C P D 2$ & 22.59 & 19.71 & 21.32 & 36.38 & 0.06 & -0.23 & 0.05 & 0.12 \\
\hline RR S.D. & & & & & 2.81 & 5.18 & 2.60 & 7.35 \\
\hline
\end{tabular}

A comparison of RETRIEVE deviations with standard deviations of Round Robin given in tables 1, 4 demonstrates the high accuracy of all standardless RETRIEVE QPA methods.

\section{Conclusions}

The system RETRIEVE for qualitative and standardless quantitative phase and structure XRPD analysis has been described. Problem-oriented query language allows programming and using effective interactive strategies of phase identification of complex powder patterns [7]. The multi-peak RIR, combined with the search-match, provides an automatic QPA. Reference calculated patterns of phases with adequate corundum ratios must be selected from DB for obtaining a good accuracy. The automatic group QPA requires chemical formulas and substantially different quantitative phase composition of samples and a chemical data of samples additionally. However this method is unstable with respect to textures. The DDM QPA is independent of background but needs to know the structural data. The integration of DDM with two-level genetic algorithm can provide a structure analysis. For example, the structure of $\mathrm{Pd}\left(\mathrm{NH}_{3}\right)_{2}\left(\mathrm{NO}_{2}\right)_{2}$ is solved automatically including all hydrogen atomic coordinates (see Y.I.Yakimov et al, same issue). The DDM \& GA method will be tested further.

\section{References}

1. Yakimov, I.S., 1989, J. Nucl. Instr. Meth. 'Phys. Res., A282, 655.

2. Chu, G., Cong, Y.F. \& You, H.J., 2003, Acta metallurgica sinica-English letters, 16, 489.

3. Rius, J., Plana, F. \& Palanques, A., 1987, J. Appl. Cryst., $20,457$.

4. Solovyov, L.A., 2004, J. Appl. Cryst., 37, 743.

5. Harris, K.D.M., Zhou, Z. \& Pan Z., 2007, CPD IUCr Newsletter, 35, 31.

6. Scarlett, N.V.Y., Madsen, I.C. et al, 2002, J. Appl. Cryst., 35, 383.

7. Yakimov, I.S., 2008, Inorganic Materials, 14, 1531. 


\title{
Structure determination from powder data: Mogul and CASTEP
}

\section{A. J. Florence ${ }^{1, *}$, J. Bardin ${ }^{1}$, B. Johnston ${ }^{1}$, N. Shankland ${ }^{1}$, T. A. N. Griffin ${ }^{2}$, K. Shankland ${ }^{2}$}

${ }^{1}$ Strathclyde Institute of Pharmacy and Biomedical Sciences, University of Strathclyde, Glasgow G4 0NR, UK

2 School of Pharmacy, Whiteknights, PO Box 224, Reading RG6 6AD, UK

*alastair.florence@strath.ac.uk

Keywords: structure determination from powder data, simulated annealing, torsion angle constraints, Mogul, DFT, geometry optimisation

\begin{abstract}
When solving the crystal structure of complex molecules from powder data, accurately locating the global minimum can be challenging, particularly where the number of internal degrees of freedom is large. The program Mogul provides a convenient means to access typical torsion angle ranges for fragments related to the molecule of interest. The impact that the application of modal torsion angle constraints has on the structure determination process of two structure solution attempts using DASH is presented. Once solved, accurate refinement of a molecular structure against powder data can also present challenges. Geometry optimisation using density functional theory in CASTEP is shown to be an effective means to locate hydrogen atom positions reliably and return a more accurate description of molecular conformation and intermolecular interactions than global optimisation and Rietveld refinement alone.
\end{abstract}

\section{Introduction}

Structure determination from powder diffraction data (SDPD) using simulated annealing (SA) has become a widely used tool for structural analysis in the absence of single crystals. It remains to be established precisely where the limit of effectiveness of SA actually lies for complex structures. In this context, the term 'complexity' has two connotations, molecular (internal Degrees of Freedom, DoF; torsion angles) and crystallographic (external DoF; position and orientation). Whilst SA successes in dealing with relatively large external DoF have been reported (36 external DoF; [1]) it has also been shown that structures containing 13 internal DoF can pose a significant challenge to SA [2]. It is therefore of interest to explore strategies that maximise the chances of success with structures containing large numbers of internal DoF. Here we demonstrate the application of prior chemical knowledge, in the form of modal torsion angle constraints extracted from the CSD [3] using Mogul [4], to increase the frequency with which the global minimum is located in SDPD using DASH [5]. 
Once the global minimum has been located and the structure solved, Rietveld refinement [6] is typically carried out to obtain a final structure that is both chemically reasonable and gives the best possible fit to the data. When dealing with structures of flexible molecules, it is often necessary to confirm that weakly-scattering hydrogen atoms are correctly located in the SA structure prior to refinement. Although manual location of hydrogen atom positions between nearby donor and acceptor atoms is often facile for rigid groups, this process can be non-trivial for flexible hydrogen-bond donor or acceptor groups. Density functional theory (DFT) can be applied as a complementary tool to SDPD for structure verification [7] and refinement [8] and here we utilise geometry optimisation of the SDPD structure with CASTEP [9] to accurately locate hydrogen atom positions in a molecular crystal structure solved by SDPD.

\section{Mogul-assisted structure determination}

The impact of Mogul-derived torsion angle preferences on the SA approach to structure solution is considered here, noting that the same principles may be applicable to other global optimisation algorithms. The Mogul program has been developed for ease of use in retrieving molecular geometry data from the CSD and is therefore easily implemented within the SDPD process.

\section{Application to verapamil hydrochloride}

Data $\left(2-70^{\circ} 2 \theta\right.$; step size $0.017^{\circ}$; variable count time scheme; $298 \mathrm{~K}$ ) were collected from a capillary-mounted sample using a Bruker-AXS D8 diffractometer $\left(\mathrm{Cu} K \alpha_{1}\right.$; Bruker Lynxeye), yielding $\chi_{\text {Pawiey }}^{2}=1.56$ for a DASH fit to the data range $3-42^{\circ} 2 \theta$. In a previously reported systematic study of the relationship between search space complexity and frequency of success in SDPD [2], verapamil hydrochloride (VHCl; triclinic, $P \overline{1}$, CSD refcode CURHOM, $Z^{\prime}=1$ ) had the highest total number of DoF, with 13 internal (figure 1) plus 9 external DoF. As such, it constitutes a good starting point to assess the impact of Mogul-derived search space restrictions (table 1).

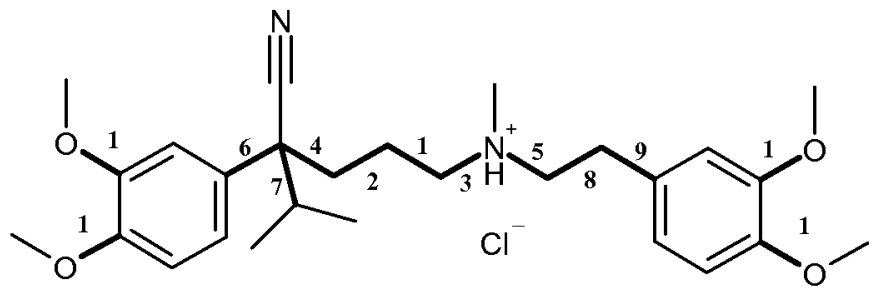

Figure 1. Molecular structure of VHCl, highlighting the 13 internal DoF.

To assess the impact of the restrictions on the stochastic search, a large number of runs are required 'with' and 'without' the restrictions applied. Executions of DASH runs on the Grid MP system at the STFC Rutherford Appleton Laboratory allowed these large numbers of runs to be performed overnight (SA control parameters: $2 \times 10^{7}$ moves, 0.01 cooling rate and 
final simplex refinement). The frequency of success was taken to be the number of SA runs in each batch of 800 reaching the global minimum, defined as $\chi_{\text {Profile }}^{2} / \chi_{\text {Pawley }}^{2} \leq 10$ (table 2).

Table 1. Allowed ranges for the 13 optimisable torsion angles ( $\tau$ ) identified in figure 1.

\begin{tabular}{|l|l|c|l|l|}
\hline$\tau$ & \multicolumn{4}{|c|}{ Allowed torsion angle search space } \\
\hline $1,2,3,4,5,7,8$ & Trimodal & $30^{\circ} \rightarrow 90^{\circ}$ & $150^{\circ} \rightarrow 210^{\circ}$ & $270^{\circ} \rightarrow 330^{\circ}$ \\
\hline 9 & Bimodal & $45^{\circ} \rightarrow 135^{\circ}$ & $225^{\circ} \rightarrow 315^{\circ}$ & \\
\hline 6 & Unimodal & $0^{\circ} \rightarrow 360^{\circ}$ & & \\
\hline $10,11,12,13$ & Unimodal & $-45^{\circ} \rightarrow 45^{\circ}$ & & \\
\hline
\end{tabular}

Table 2. Frequency of success in solving the crystal structure of $\mathrm{VHCl}$.

\begin{tabular}{|c|l|c|}
\hline $\begin{array}{c}\text { Internal } \\
\text { DoF }\end{array}$ & Allowed torsion angle search space & Frequency of success \\
\hline 0 & $\begin{array}{l}\text { All torsions fixed at values in CUR- } \\
\text { HOM }\end{array}$ & $800 / 800$ \\
\hline 13 & $0^{\circ} \rightarrow 360^{\circ}$ (all angles) & $27 / 800$ \\
\hline 13 & As table 1 & $29 / 800$ \\
\hline
\end{tabular}

The complexity of the overall search space is reflected in the greatly reduced frequency of success accompanying the introduction of internal DoF. Interestingly, the torsion angle search space restrictions brought no significant benefit in this particular case. Thus, with $\mathrm{VHCl}$, the implementation of the SA algorithm in DASH is equally effective in full $\left(0^{\circ} \rightarrow\right.$ $360^{\circ}$ ) or reduced (table 1) torsion angle search space.

\section{Application to CSD refcode XELBEV}

When looking for more challenging examples than $\mathrm{VHCl}$, it is logical to look towards structures with $Z>1$. XELBEV (monoclinic, $P 2_{1}$ ), for example, poses a significant increase in complexity, with a total of 18 internal plus 12 external DoF (figure 2).

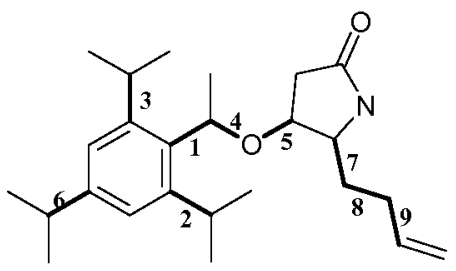

Figure 2. The molecular structure of CSD refcode XELBEV, $Z^{\prime}=2$, highlighting the 9 internal $D o F$.

A DASH Pawley fit to a XELBEV pattern simulated using Mercury CSD $2.0(\lambda=1.54056$ $\AA$; range $5-30^{\circ} 2 \theta$; step size $0.017^{\circ}$ ) yielded $\chi_{\text {Pawley }}^{2}=1.35$. Torsion angle search space restrictions were derived using Mogul (table 3) and frequency of success (table 4) determined as described for $\mathrm{VHCl}$. The frequency of success with fixed conformations is lower than for $\mathrm{VHCl}$, reflecting the increased complexity due to the additional external DoF. In contrast to 
the unrestricted batch ( 0 successes, lowest $\left.\chi_{\text {Profile }}^{2}=27\right)$, the batch that operated within the constrained torsion angle space returned 3 successes. Thus, Mogul plus DASH combined effectively to solve the XELBEV crystal structure. It is worth noting that Mogul-derived search space restrictions only apply during the SA part of the search - they do not apply to the post-SA simplex refinement executed upon reaching (in this case) $2 \times 10^{7}$ moves. Thus, torsion angles that lie beyond the imposed search space can still be accessed at the simplex stage.

Table 3. Allowed ranges for the 9 optimisable torsion angles ( $\tau$ ) identified in figure 2 (identical ranges were imposed on each of the 2 molecules in the asymmetric unit).

\begin{tabular}{|l|l|r|l|l|}
\hline \multicolumn{1}{|c|}{$\tau$} & \multicolumn{4}{|c|}{ Allowed torsion angle search space } \\
\hline $4,7,8$ & Trimodal & $30^{\circ} \rightarrow 90^{\circ}$ & $150^{\circ} \rightarrow 210^{\circ}$ & $270^{\circ} \rightarrow 330^{\circ}$ \\
\hline 1 & Bimodal & $45^{\circ} \rightarrow 135^{\circ}$ & $225^{\circ} \rightarrow 315^{\circ}$ & \\
\hline 9 & Bimodal & $90^{\circ} \rightarrow 135^{\circ}$ & $225^{\circ} \rightarrow 270^{\circ}$ & \\
\hline 6 & Unimodal & $0^{\circ} \rightarrow 360^{\circ}$ & & \\
\hline 5 & Unimodal & $45^{\circ} \rightarrow 315^{\circ}$ & & \\
\hline 2,3 & Unimodal & $190^{\circ} \rightarrow 290^{\circ}$ & & \\
\hline
\end{tabular}

Table 4. Frequency of success in solving the crystal structure of XELBEV.

\begin{tabular}{|c|l|c|}
\hline $\begin{array}{l}\text { Internal } \\
\text { DoF }\end{array}$ & Allowed torsion angle search space & Frequency of success \\
\hline 0 & Torsions fixed at values in XELBEV & $579 / 800$ \\
\hline 18 & $0^{\circ} \rightarrow 360^{\circ}$ (all angles) & $0 / 800$ \\
\hline 18 & As table 3 & $3 / 800$ \\
\hline
\end{tabular}

\section{Geometry optimisation of HCT form II using CASTEP}

In this section, DFT geometry optimisation is applied to determine accurate hydrogen atom positions in the crystal structure of a polymorph (form II) of the thiazide diuretic, hydrochlorothiazide (HCT; figure 3). The crystal structure of form II [10] was solved by SA using DASH from lab powder data collected at $298 \mathrm{~K}$ to $1.76 \AA$ resolution. The positions of hydrogen atoms $\mathrm{H} 1$ and $\mathrm{H} 2$ (figure 3 ) were manually located in the global minimum structure by rotating the $\mathrm{NH}_{2}$ group around the N-S bond to obtain chemically sensible intermolecular contacts. Subsequent Rietveld refinement of this structure yielded an $R_{\text {wp }}$ of $3.75 \%$.<smiles>CCCN(CC)S(=O)(=O)c1cc2c(cc1Cl)NC[NH2+]S2(=O)=O</smiles>

Figure 3. HCT molecule with labelling of $\mathrm{H1}$ and $\mathrm{H} 2$ shown. 
A recent single-crystal determination of form II HCT [11] confirms the accuracy of the SDPD structure with respect to the position of non-hydrogen atom positions. However, the positions of $\mathrm{H} 1$ and $\mathrm{H} 2$ differ significantly in the two structures, as demonstrated by the torsion angle $\tau_{1}$ (H2-N-S-O2) describing the $\mathrm{NH}_{2}$ orientation. The manually located $\mathrm{NH}_{2}$ group is rotated by $40.8^{\circ}$ from the position determined in the single-crystal structure [ $\tau_{1}=$ $8.0^{\circ}(S D P D)$ and $32.7^{\circ}$ (single-crystal); figure $\left.4(\mathrm{a})\right]$.
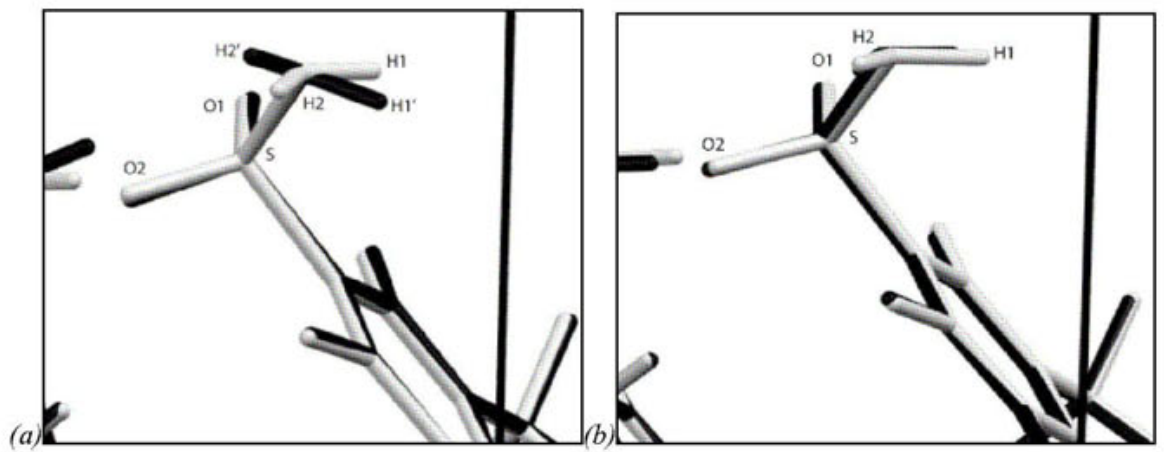

Figure 4. Overlays of (a) HCT form II SDPD (black) and single-crystal (grey) structures with HI and H2 labelled and (b) CASTEP optimised SDPD (grey) and single-crystal (black) structures.

\section{Geometry optimization of crystal structures using CASTEP}

First-principles DFT calculations were performed on the experimental SDPD and singlecrystal structures using CASTEP v.3.2. The unit cell parameters were fixed at their experimental values for all calculations. Preliminary optimizations in space group $P 1$ confirmed the structures were close to minima and subsequent optimizations were carried out in $P 2_{1} / \mathrm{c}$ to facilitate comparison of the optimized structures with the experimental data. A generalised gradient approximation (GGA-PBE) was used to describe the exchange-correlation potential [12] with a plane-wave basis set cut-off of $435 \mathrm{eV}$ and a single k-point for the BZ sampling. MEDIUM convergence criteria were used (energy per atom $2 \times 10^{-5} \mathrm{eV}$, forces $0.05 \mathrm{eV} . \AA^{-1}$, displacement $2 \times 10^{-3} \AA$ ). Calculations were performed on the Scientific Computing Application Resource for Facilities (SCARF) cluster at the SFTC Rutherford Appleton Laboratory and both optimizations converged satisfactorily within the default number of iterations and utilised $2.7 \mathrm{~h}$ of total computing time per structure.

The positions of $\mathrm{H} 1$ and $\mathrm{H} 2$ in the optimized SDPD structure show a much improved correspondence to the experimental single-crystal structure, with $\triangle \tau_{1}$ falling from $40.8^{\circ}$ (SDPD vs. single-crystal structure) to $8.8^{\circ}$ (CASTEP SDPD vs. single-crystal; figure 4(b)). A Rietveld fit of the optimised SDPD structure returned a final $R_{\mathrm{wp}}$ of $3.72 \%$, unchanged from the original published structure. Thus, the combination of SA, quantum chemical geometry optimization and Rietveld refinement has yielded a structure for form II HCT that shows both excellent fit to the experimental data and more accurate hydrogen atom positions than from SA and Rietveld refinement alone. 


\section{Concluding remarks}

Accurate crystal structures are of vital importance to those concerned with studying the basic science underpinning physical form diversity in the organic solid-state. In the absence of suitable single-crystal samples, methods for SDPD are often invaluable. Where there is a requirement to solve crystal structures with large numbers of internal DoF, limiting the search space using experimentally derived torsion angle constraints can be an effective tool for increasing the probability of successful structure solution. These methods add no computational overhead to the global optimization method and, particularly when used in combination with a distributed computing global optimization implementation, can enable structures with significant complexity to be tackled efficiently and with confidence. Once a global minimum structure has been obtained, DFT calculations can be recommended where there is a particular interest in the determination of accurate hydrogen atom positions.

\section{References}

1. Fernandes, P., Shankland, K., Florence, A.J., Shankland, N. \& Johnston, A., 2007, J. Pharm. Sci., 96, 1192.

2. Florence, A.J., Shankland, N., Shankland, K., David, W.I.F., Pidcock, E., Xu, X., Johnston, A., Kennedy, A.R., Cox, P.J., Evans, J.S.O., Steele, G., Cosgrove, S.D. \& Frampton, C.S., 2005, J. Appl. Crystallogr., 38, 249.

3. Allen, F.H., 2002, Acta Crystallogr. Sect. B-Struct. Sci., 58, 380.

4. Bruno, I.J., Cole, J.C., Kessler, M., Luo, J., Motherwell, W.D.S., Purkis, L.H., Smith, B.R., Taylor, R., Cooper, R.I., Harris, S.E. \& Orpen, A.G., 2004, J. Chem. Inf. Comp. Sci., 44, 2133.

5. David, W.I.F., Shankland, K., van de Streek, J., Pidcock, E., Motherwell, W.D.S. \& Cole, J.C., 2006, J. Appl. Crystallogr., 39, 910.

6. Rietveld, H.M., 1969, J. Appl. Crystallogr., 2, 65.

7. Neumann, M.A., Tedesco, C., Destri, S., Ferro, D.R. \& Porzio, W., 2002, J. Appl. Crystallogr., 35, 296.

8. Smrcok, L., Jorik, V., Scholtzova, E. \& Milata, V., 2007, Acta Crystallogr. Sect. BStruct. Sci., 63, 477.

9. Clark, S., Segall, M.D., Pickard, C.J., Hasnip, P.J., Probert, M.I.J., Refson, K. \& Payne, M.C., 2005, Z. Kristallogr., 220, 567.

10. Florence, A., Johnston, A., Fernandes, P., Shankland, K., Stevens, H.N.E., Osmundsen, S. \& Mullen, A.B., 2005, Acta Crystallogr. Sect. E-Struct. Rep. Onl., 61, 02798.

11. Kennedy, A.R., Johnston, A. \& Florence, A.J., 2007, unpublished result.

12. Perdew, J.P., Burke, K. \& Ernzerhof, M., 1996, Phys. Rev. Lett., 77, 3865.

Acknowledgements. The authors thank STFC's e-Science facility for access to computing resources and the University of Strathclyde for funding JB. 


\section{Powder Shake-and-Bake Method}

\section{H. Xu ${ }^{*}$, Ch. M. Weeks, R. H. Blessing}

Hauptman-Woodward Medical Research Institute and Department of Structural Biology, State University of New York at Buffalo, 700 Ellicott Street, Buffalo, NY 14203, USA

*xu@hwi.buffalo.edu

Keywords: powder diffraction, direct methods, Shake-and-Bake, structure determination

Abstract. The dual-space Shake-and-Bake procedure, one of the most successful direct methods for phasing single-crystal diffraction data, has been adapted and modified for structure determination from microcrystalline powder samples. The new method, termed Powder Shake-and-Bake and implemented in the computer program Pow SnB, incorporates extracted and overlapped reflections in the Shake-and-Bake procedure. In each $S n B$ cycle, it performs (i) an overlapped-reflection partition (via partial structural information from the previous cycle), (ii) a reciprocal-space phase refinement (via the reduction of the values of a statistical minimal function), and (iii) a real-space density modification (via peak picking). The successful PowSnB applications with experimental powder diffraction data are described.

\section{Introduction}

The collapse of the three dimensions of reciprocal space to the single dimension of a powder diffraction pattern, with the resultant Bragg-peak overlap becoming particularly severe at shorter $d$-spacings, results in a very substantial loss of information. When powder patterns exhibit clear discrimination between Bragg peaks and background as well as clear resolution of Bragg peaks from one another, individual structure-factor modulus can be reliably obtained by integrated intensity extraction methods such as the Le Bail method [1] or the Pawley method [2]. The extracted reflections are treated as non-overlapped or 'well-determined' reflections. When powder diffraction patterns do not exhibit clear peak-background discrimination and peak-peak resolution, individual intensities obtained from intensity extraction methods must be considered unreliable, and are treated as overlapped reflections. The overlap problem highlights most clearly the loss of information in the powder diffraction measurement and limits the applicability of powder diffraction as a routine tool for determining and refining crystal structures from microcrystalline powders.

Adaptations of traditional direct methods, specifically tailored to deduce the phase-angle information from the non-overlapped reflection intensities, have been particularly encouraging in recent years. The best known direct-methods package is EXPO2004 [3], which has evolved continually and now incorporates indexing, space-group determination, structure solution and refinement capabilities [4]. Recently, the charge flipping method [5] has been adapted to powder diffraction data with some very promising results [6]. Despite these important advances, the overlap problem is not, in general, solved. As a consequence, $a b$ initio 
direct-methods applications to powder diffraction data have limited success in terms of structure size and complexity.

Direct methods work best when large numbers of well-determined reflection intensities have been measured to atomic resolution (better than $1.2 \AA$ ). However, the collapse of the three dimensions of reciprocal space to the single dimension of a powder diffraction results in a very substantial loss of information. For moderately sized crystal structures, even with the highest-resolution powder diffractometers, it is almost impossible to obtain individual integrated intensities at atomic resolution. In this paper, we propose novel constrained global optimization models to tackle simultaneously the overlap problem and the crystallographic phase-angle problem as well as to develop efficient computational algorithms to find the constrained global minimum of the objective function for both modulus extraction and phase-angle estimation.

\section{Powder Shake-and-Bake method}

The Shake-and-Bake algorithm [7], specifically designed for finding the constrained global minimum from single-crystal diffraction data, has been adapted and modified for structure determination from powder diffraction data. The new algorithm, termed Powder Shake-andBake and shown by the flow diagram in figure 1, combines overlapped-reflection partitioning, minimal-function phase refinement and real-space filtering. It is an iterative process that is repeated until a solution is achieved or a designated number of trial structures have been processed. For present purposes, 'solution' does not necessarily imply a complete structure but rather a set of atomic positions that can be readily refined and extended by the standard Rietveld [8] refinement method. With reference to figure 1, the detailed Shake-and-Bake algorithm (Steps 1-3, 5-7) can be found in many publications (e.g., [7]), and only the major differences between Shake-and-Bake and Powder Shake-and-Bake (steps 1 \& 4) are described next.

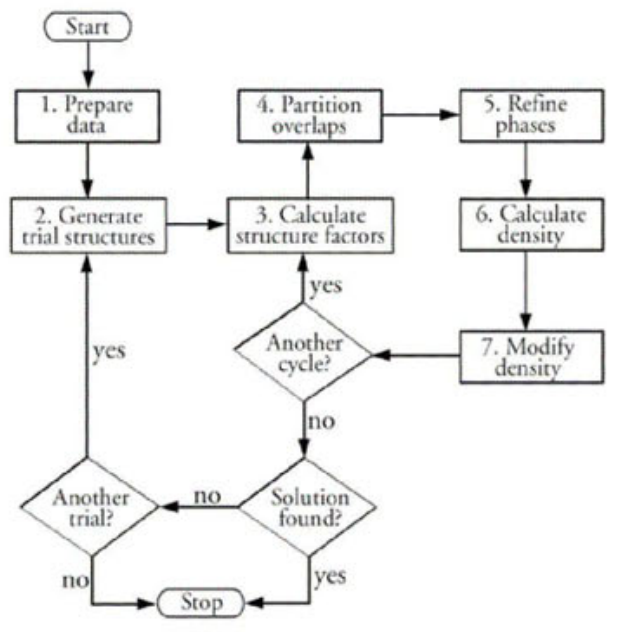

Figure 1. Powder Shake-and-Bake algorithm.

Data preparation. Reflections are divided into a non-overlapped set $G_{0}$ and overlapped sets $G_{j}(j=1,2, \mathrm{~L}, M)$ containing $L_{j}$ reflections with overlap constraints

$$
\left|F_{G_{f}}\right|^{2}=\sum_{l=1}^{L_{f}} m_{i}\left|F_{\mathbf{H}_{i}}\right|^{2}=\sum_{k}[\text { obs }(k)-\operatorname{back}(k)] \text {, }
$$

where $m_{l}$ and $\left|F_{\mathbf{H}_{l}}\right|$ are the $l^{\text {th }}$ reflection multiplicity and structure-factor modulus, respectively, and $o b s(k)-b a c k(k)$ is the background-subtracted peak contribution at the $k^{\text {th }}$ point 
in the diffraction pattern. For reflections $\mathbf{H} \in G_{0}$, the structure-factor moduli $\left|F_{\mathbf{B}}\right|$ are obtained directly from the extracted integrated intensities. For reflections $\mathbf{H} \in G_{j}$, the initial values of the structure-factor moduli are obtained from the overlap-constraint equation (1) with equipartitioning, i.e., $\left|F_{\mathrm{H}}\right|=\left\langle\left|F_{\mathrm{H}}\right|\right\rangle_{\mathbf{H} \in G_{j}}$. Normalized structure-factor moduli $(|E|$ 's) are generated from the structure-factor moduli ( $|F|$ 's) by standard scaling methods such as a Wilson plot. Reflections are then sorted in descending order of $|E|$ values, and a predetermined number of the top reflections is selected to generate a predetermined number of structure invariants $\Phi_{\mathrm{HK}}=\varphi_{\mathrm{H}}+\varphi_{\mathrm{K}}+\varphi_{-\mathbf{H}-\mathbf{K}}$ having the largest values of the modulus factors $A_{\mathrm{HK}}=2 N^{-1 / 2}\left|E_{\mathbf{H}} E_{\mathrm{K}} E_{\mathrm{H}+\mathrm{K}}\right|$.

Overlapped-reflection partition. Let the sum

$$
\sum_{l=1}^{L_{j}} m_{l}\left|E_{\mathbf{H}_{l}}\right|^{2}=\left|E_{G_{j}}\right|^{2}
$$

be the $j^{\text {th }}$ overlap constraint, and the quantities $\left|E_{\mathbf{H}_{l}}^{\text {cal }}\right|\left(1 \leq l \leq L_{j}\right)$ be the individual normalized structure-factor modulus calculated using the trial structure from the previous cycle. Then a new partition for the overlapped reflections is given by

$$
\left|E_{\mathbf{H}_{l}}\right|=\left(\sum_{l=1}^{L_{j}} m_{l}\left|E_{\mathbf{H}_{i}}^{c a l}\right|^{2}\right)^{1 / 2}\left|E_{\mathbf{H}_{i}}^{c a l}\right|\left|E_{G_{j}}\right| .
$$

The newly partitioned reflections are used to generate a new trial structure (via steps 5-7). This iterative re-partitioning of the overlapped reflections is the distinctive feature of the Powder Shake-and-Bake algorithm.

\section{Applications}

The traditional and powder Shake-and-Bake procedures have been tested successfully using experimentally measured atomic-resolution powder diffraction data. Here, we report solution efficacy measured by the success rate (percentage of trial structures that converge to solutions) as well as solution quality measured by (i) the mean phase error (MPE) or average absolute value of the deviations of the phase angles from their values calculated using the final refined coordinates and thermal parameters; (ii) the atom-match rate (AMR) or percentage of atoms whose coordinates are within a $0.5 \AA$ radius of the final refined coordinates; and (iii) the average inter-atomic distance (DIST) in pairs of matched atoms. Unless clearly stated otherwise, the results reported here are based on 1000 trial structures and produced by computer software $S n B$ [9] and PowSnB (traditional and powder Shake-and-Bake procedures, respectively) implementing constrained minimization of the statistical minimal function [ 10 , 11].

Overlap-constraint Protocol P(M). In PowSnB experiment, the extracted intensities are divided into non-overlapped group $\left(\mathrm{d}=\sin \theta / \lambda>\mathrm{d}_{0}\right.$, where $\mathrm{d}_{0}$ is a resolution parameter) and overlapped group $\left(\mathrm{d}_{\max }<\mathrm{d}<\mathrm{d}_{0}\right.$, where $\mathrm{d}_{\max }$ is the highest data resolution). The extracted intensities in the overlapped group are sorted in decreasing order of the $|E|$ values and grouped into subgroups containing $M$ reflections. For each subgroup, the average value of 
the normalized structure factors, $\langle|E|\rangle$, is calculated and assigned to each reflection in the subgroup. The new set of structure factors, which consists of non-overlapped and overlapped reflections having largest initial $|\mathrm{E}|$ values, is subsequently input to PowSnB to test the validity, accuracy and robustness of the Powder Shake-and-Bake procedure.

\section{Cimetidine application}

The crystal structure of the drug molecule cimetidine [12] $\left(\mathrm{C}_{10} \mathrm{H}_{16} \mathrm{~N}_{6} \mathrm{~S}\right)$ is monoclinic, has space group $\mathrm{P} 2_{1} / \mathrm{n}$, and $\mathrm{Z}=4$. The high-resolution, synchrotron $\mathrm{X}$-ray powder pattern was collected on station 8.3 at Daresbury Laboratory with a wavelength of $\lambda=1.4599 \AA$ over a $2 \theta$ range of 6 to $105^{\circ}$. The integrated intensities were extracted from the indexed profile to a resolution of $\mathrm{d}_{\max }=1.13 \AA$ using a modified form of the Pawley pattern decomposition program [2] implemented in the computer software GSAS. [13]. The test results of $S n B$, as well as the results from $P o w \operatorname{Sn} B$ with overlap-constraint protocol $\mathrm{P}(4)$ and a series of resolution parametr $\mathrm{d}_{0}=1.2,1.3, \ldots, 3.0 \AA$, are reported in table 1 . These results demonstrate the following:

- $\quad S n B$ effectively yields high-quality solutions as indicated by $17.0 \%$ success rates, low mean phase errors, high average percentage of matched atoms, and small average distances between matched atoms.

- PowSnB yields an average of $26.2 \%$ success rate using overlap-constraint protocol $\mathrm{P}(4)$ for a series of resolution parameter $\mathrm{d}_{0}=1.2,1.3, \ldots, 3.0 \AA$, which ranges from $16.1 \%$ at $\mathrm{d}_{0}=1.3 \AA$ to $42.0 \%$ at $\mathrm{d}_{0}=2.3 \AA$. The quality of solutions is similar to that from $\operatorname{SnB}$.

- The minimal function histogram is no longer a bimodal distribution for PowSnB as commonly observed in single-crystal applications [9]. This is shown in table 1 by the overlapping figure-of-merit (FOM) ranges for solutions and non-solutions. The unimodal histogram is the result of unavoidable errors in the structure-factor moduli that become more severe as the resolution increases.

Table 1. Comparison of $\mathrm{S} n \mathrm{~B}$ and PowSnB muns for cimetidine powder diffraction data. ${ }^{\dagger}$ Percentage of trial structures having mean phase error $(M P E)<25^{\circ}$. ${ }^{\&}$ Overlap-constraint protocol $\mathrm{P}(4)$ and resolution parameter $d_{0}=2.3 \AA$. * Values of the statistical minimal function.

\begin{tabular}{|c|c|cc|ccc|}
\hline Phasing & Success & \multicolumn{2}{|c|}{ Figure-of-merit Ranges } & \multicolumn{3}{|c|}{ Solution Averages } \\
Method & Rate $^{\dagger}$ & soln & non-solns & $\langle\mathrm{MPE}\rangle$ & $\langle$ AMR $\rangle$ & $\langle\mathrm{DIST}\rangle$ \\
\hline$S n B$ & $17.0^{\%} \%$ & $0.124-0.141$ & $0.161-0.355$ & $4.2^{\circ}$ & $82 \%$ & $0.18 \AA$ \\
$P_{\mathrm{O} w} \mathrm{Sn} B^{\S}$ & $42.0 \%$ & $0.063-0.089$ & $0.072-0.273$ & $9.7^{\circ}$ & $81 \%$ & $0.20 \AA$ \\
\hline
\end{tabular}

For unknown structures, in the absence of bimodal distributions to distinguish solutions from non-solutions, we propose an atom-match-based FOM to identify PowSnB structure solutions:

- The distances between pairs of atoms from two PowSnB trial structures can be calculated after taking the possibility of different origins and enantiomorphs into account [14]. Two atoms are considered matched if their distance is less than $0.5 \AA$.

- The percentage of matched atoms ( $>70 \%$ for solutions and $<50 \%$ for non-solutions) is used as a figure of merit to identify all solution structures from non-solution structures. 
Solutions for cimetidine identified by the atom-match-based FOM were perfectly correlated with solutions identified by low mean phase errors $\left(<25^{\circ}\right)$. Unless clearly stated otherwise, the atom-match-based FOM was used to identify potential solutions discussed later in this paper.

\section{Fluorescein diacetate application}

Fluorescein diacetate [15] $\left(\mathrm{C}_{24} \mathrm{H}_{16} \mathrm{O}_{7}\right)$ crystallizes in the triclinic space group P-1 with two molecules in the unit cell. The high-resolution, synchrotron X-ray powder pattern was measured at the Swiss-Norwegian beam line (BM1) at the European Synchrotron Radiation Facility (ESRF) in Grenoble with a wavelength $\lambda=0.6006 \AA$ over the $2 \theta$ range $2-50^{\circ}$. A total of 2905 individual values of $|F|^{2}$ were extracted from the powder data to $d_{\max }=0.88 \AA$ using the Le Bail pattern decomposition method [1] implemented in the computer software EXTRA [16]. The results of $\operatorname{Sn} B$ applications, reported in the first block of table 2, demonstrate that:

- $\quad S n B$ yielded high quality solutions with fragments of the molecule comprising $79-87 \%$ of the atoms.

- The structure quality yielded at $1.0 \AA$ is better than that at $0.88 \AA$ as indicated by the higher atom-match rate and the shorter distances between matched atoms. This indicates large errors in the extracted intensities from the high-angle part of the powder diffraction pattern.

- $\operatorname{SnB}$ failed to yield any structure solution at a resolution of $1.2 \AA$.

Table 2. Comparative results for the crystal structure of fluorescein diacetate.

\begin{tabular}{|c|c|c|c|ccc|}
\hline Using & Overlap & Data & Success & \multicolumn{3}{|c|}{ Solution Averages } \\
Overlap & Protocol & Resolution & Rate & $\langle\mathrm{MPE}\rangle$ & $\langle$ AMR $\rangle$ & $\langle\mathrm{DIST}\rangle$ \\
\hline No & N/A & $0.88 \AA$ & $2.6 \%$ & $34.2^{\circ}$ & $79 \%$ & $0.21 \AA$ \\
$(\operatorname{Sn} B)$ & & 1.0 & 2.4 & 24.5 & 87 & 0.18 \\
& & 1.2 & 0.0 & N/A & N/A & N/A \\
\hline Yes & P(4) & $1.2 \rightarrow 1.0 \AA$ & 3.1 & 22.7 & 88 & 0.18 \\
$($ Pow $S n B)$ & & $1.3 \rightarrow 1.0 \AA$ & 3.2 & 21.6 & 89 & 0.17 \\
& & $1.4 \rightarrow 1.0 \AA$ & 3.3 & 23.6 & 87 & 0.17 \\
\hline
\end{tabular}

The results of PowSnB applications, reported in the second block of table 2, demonstrate that:

- $\quad P o w S n B$ is a very efficient program for producing high-quality structure solutions from powder diffraction data, as indicated by low mean phase errors $\left(<25^{\circ}\right)$, high atom-match rates $(\geq 87 \%)$, and short matching distances $(\leq 0.18 \AA)$.

- Iterative re-partitioning of overlapped reflections is the key to successful PowSnB applications to powder diffraction data. This is the case when individual reflection intensities can be extracted at lower-than-atomic resolution, provided that signal-to-noise in the powder pattern is sufficient to allow overlap constraints to be reliably obtained up to atomic resolution.

- The change of resolution parameter $\mathrm{d}_{0}=1.2,1.3$ and $1.4 \AA$ has little impact on success rates or the quality of $P o w S n B$ solutions as evidenced by only small changes in all figure-of-merit values. 


\section{Concluding remarks}

Powder Shake-and-Bake is an $a b$ initio direct-phasing method for structure determination from powder diffraction data. It is aimed to improve structure solutions from subatomic resolution data or atomic resolution data with large errors in extracted intensities from powder profile. Pow $S n B$, the computer program implementing the Powder Shake-and-Bake algorithm, is capable of providing high-quality structure models for further structure refinement.

\section{References}

1. Le Bail, A., Duroy, H. \& Fourquet, J.L., 1988, Mat. Res. Bull., 23, 447.

2. Pawley, G.S., 1981, J. Appl. Cryst., 14, 357.

3. Altomare, A., Caliandro, R., Camalli, M., Cuocci, C., Giacovazzo, C., Moliterni, A.G.G. \& Rizzi, R., 2004, J. Appl. Cryst., 37, 1025.

4. Altomare, A., Cuocci, C., Giacovazzo, C., Moliterni, A.G.G. \& Rizzi, R., 2006, $J$. Appl. Cryst., 39, 145.

5. Oszlányi, G. \& Sütö, A., 2008, Acta Cryst., A64, 123.

6. Baerlocher, C., Gramm, F., Massuger, L., McCusker, L.B., He, Z.B., Hovmoller, S. \& Zou, X.D., 2007, Science, 315, 1113.

7. Weeks, C.M., DeTitta, G.T., Hauptman, H.A., Thuman, P. \& Miller, R., 1994, Acta Cryst., A50, 210.

8. Rietveld, H.M., 1969, J. Appl. Cryst., 2, 65.

9. Miller, R., Gallo, S.M., Khalak, H.G. \& Weeks, C.M., 1994, J. Appl. Cryst., 27, 613.

10. Xu, H. \& Hauptman, H.A., 2004, Acta Cryst., A60, 153.

11. Xu, H., Weeks, C.M. \& Hauptman, H.A., 2005, Acta Cryst., D61, 976.

12. Cernik, R.J., Cheetham, A.K., Prout, C.K., Watkin, D.J., Wilkinson, A.P. \& Willis, B.T.M., 1991, J. Appl. Cryst., 24, 222.

13. Larson, A.C. \& Von Dreele, R.B., 2004, Technical Report LAUR 86-748, Los Alamos National Laboratory, Los Alamos, NM.

14. Smith, G.D., 2002, J. Appl. Cryst., 35, 368.

15. Knudsen, K.D., Pattison, P., Fitch, A.N. \& Cernik, R.J., 1998, Angew. Chem. Int. $E d ., 37,2340$.

16. Altomare, A., Burla, M.C., Cascarano, G., Giacovazzo, C., Guagliardi, A., Moliterni, A.G.G. \& Polidori, G., 1995, J. Appl. Cryst., 28, 842.

Acknowledgements. The authors would like to thank Robert Von Dreele (ANL) and Kenneth Knudsen (Institute for Energy Technology, Norway) for the testing data. This research was financially supported by NIH grant GM-72023. 


\title{
Large-scale distributed computing for accelerated structure solution
}

\section{K. Shankland ${ }^{1, *}$, T. A. N. Griffin ${ }^{1}$, J. van de Streek $^{2, \dagger}$, J. C. Cole ${ }^{2}$, N. Shankland ${ }^{3}$, A. J. Florence ${ }^{3}$, W. I. F. David ${ }^{1}$}

${ }^{1}$ ISIS Facility, STFC Rutherford Appleton Laboratory, Oxon OX11 0QX, UK

2 Cambridge Crystallographic Data Centre, Cambridge CB2 1EZ, UK

${ }^{3}$ Strathclyde Institute for Pharmacy \& Biomedical Sciences, University of Strathelyde, Glasgow G4 0NR, UK

$\dagger$ Present address: Avant-Garde Materials Simulation, D-79100 Freiburg, Germany

*kenneth.shankland@stfc.ac.uk

Keywords: structure determination by powder diffraction, distributed computing

\begin{abstract}
Improvements in SDPD methodology have meant that ever more complex structures are being tackled using global optimisation methods. As a very general rule of thumb, the more complex the structure, the more difficult it is to locate the global minimum in the real-space search. This difficulty can, to some extent, be circumvented by running many instances of the search; for stochastic search methods such as simulated annealing, each instance can be run independently of any other. Such search methods are therefore ideally suited to disposition on a distributed grid-type system that makes use of existing networked compute resources. At the Rutherford Appleton Laboratory, the $D A S H$ structure solution code has been adapted to run on a Univa UD GridMP system in order to distribute simulated annealing runs across hundreds of computers simultaneously with excellent scaling. The principles outlined are applicable to other structure solution codes and to other grid-type systems, such as the widely used and freely available CONDOR system.
\end{abstract}

\section{Introduction}

In the last decade, global optimisation methods have come to dominate the field of structure determination from powder diffraction data in respect of molecular organic materials [1]. This dominance is a consequence of proven effectiveness, ease-of-use and computer program availability and one need only look at a small number of recent papers to conclude that their range of applicability is wide. Whilst global optimisation methods cannot match the efficiency of their pure direct-methods competitors, current programs are certainly fast enough to be useable routinely by the average practitioner of powder diffraction. This is down to these programs implementing efficient search algorithms, molecular descriptors and agreement factors. To give some idea of how quickly trial structures can be evaluated, the struc- 
ture of capsaicin (figure $1, P 2_{1} / c, V=1707 \AA^{3}, Z=1,16$ degrees-of-freedom) was solved using the program $D A S H 3.1$, which utilises simulated annealing, a Z-matrix molecular description and a correlated integrated intensities $\chi^{2}$ figure-of-merit [2].

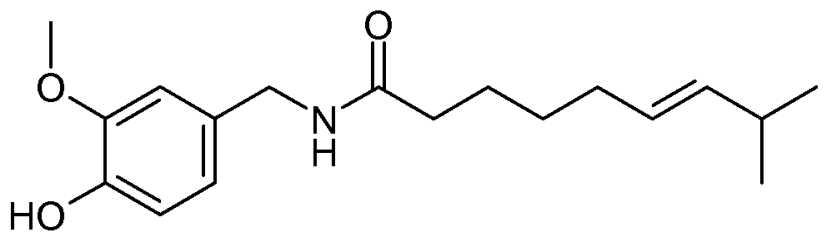

Figure 1. The molecular structure of capsaicin.

Running on a single core of a Core2Quad $2.4 \mathrm{GHz}$ chip, $D A S H$ evaluates some 13,000 trial structures per second to solve the structure in only a few minutes. This can be compared with a rate of $\sim 150$ structures per second for the prototype version of DASH (running on a DEC Alphastation 500/500) that was used to solve the structure in 1998 from the same data . With this level of efficiency, why would one seek to evaluate trial structures faster? First and foremost, because no single optimisation run is guaranteed to locate the global minimum in the agreement factor (e.g. $R_{\mathrm{wp}}, \chi^{2}$ ) space that corresponds to the correct crystal structure and so multiple runs are needed to give a reasonable probability of finding the global minimum. Secondly, as the complexity (defined here as the total number of degrees of freedom being optimised) of the structure under study increases, it is a general rule that the probability of any given run returning the global minimum drops; hence the number of multiple runs must increase to maintain the chances of finding the correct crystal structure. These two factors conspire to increase the total number of agreement factor function evaluations (the most time-consuming part of the structure solution process) that are needed. Furthermore, global optimisation requires that the connectivity of the molecule under study is known. If there are any ambiguities, then multiple models will need to be evaluated. Accordingly, it is desirable to be able to evaluate structures as quickly as possible, to keep overall structure solution times as short as possible.

\section{A strategy for speed}

This section focuses on the widely used simulated annealing (SA) approach to structure solution, though the principles outlined can be applied to many other optimisation algorithms [3]. In order to speed up a SA structure solution, one could adopt the strategy of re-writing the core function evaluation code (which is called a great many times) in order to improve its efficiency. Assuming this code is already well written for a single processor machine, one could envisage re-coding utilising a parallel library such as MPI, to allow an individual function evaluation to be distributed across a number of tightly-coupled CPUs. This requires not only specialist programming expertise but also expensive hardware to run efficiently e.g. Myrinet low-latency interconnects. A much more attractive option is to exploit the fact that the individual SA runs can be performed completely independently of one another, as they do not communicate with one another and differ (in general) only in their random number seeds. Such a CPU-intensive, coarse-grained parallel problem is ideally suited to execution 
on a distributed computing system, where CPU resources are only loosely coupled, typically by a conventional Ethernet network.

\section{Distributed computing and the spare cycles concept}

Consider a typical departmental computer network composed of approximately one hundred powerful PCs. In everyday use, the overall level of CPU usage runs at $\sim 10 \%$ and outside of office hours, this drops to even less. This means that a great many CPU cycles go unused. A prime aim of a system for distributed computing is to harness the spare cycles of these nondedicated PCs for useful work without disrupting normal PC operations. Many systems have been developed for distributed computing: commonly used ones include the academic $C O N$ $D O R$ [4] system, and the commercial Grid MP system [5]. Our work has focussed on the latter, because of established usage in industrial and financial settings. Two servers (wellspecified PCs each with two CPUs, 4Gb RAM and RAID10 disks) share the load of running the main Grid MP software whilst a small piece of software called the mpagent is installed onto every machine in the department that is to participate in 'the grid'. It is the role of the agent to contact the servers to inform them about the capabilities of the machine (client) on which it is running, and to control the execution of any jobs sent to it by the servers. Any jobs that run under its control are executed at the lowest priority afforded by the host operating system, such that those tasks do not interfere with normal PC operations. It is the role of the servers to keep track of the clients, to accept job requests from submitting programs, to allocate those jobs to clients, to accept results from clients and to return those results to the submitting program when requested. All communication is via secure http protocols using web services.

\section{A typical generic workflow}

Imagine a structure solution that consists of four SA runs. A program is written to take this sequence of four runs and split it into individual units, which are then uploaded from a PC onto the servers. On the servers, the units are matched with the requested structure solution program and four workunits (each consisting of the structure solution program and a single SA run) are sent out to four separate client PCs where they are unpacked in a secure disk area and then executed under control of the agent software. Upon completion, the results of the runs are returned to the server and stored. They are then retrieved from the server by the submitting PC for examination. The net outcome is that the structure solution is performed in approximately one-quarter of the time (assuming all $\mathrm{PCs}$ are equal specification) that it would take on a single PC.

\section{The program executable}

In preparing a computer program for execution on a distributed system, it is imperative to remember that the code will be running on client machines over which one has little or no control in terms of their setup. As such, the workunit sent out to the client machines must include all the relevant files, libraries and environment settings that are necessary for successful execution. This is achieved by preparing a program module executable (essentially a ZIP file containing all the aforementioned prerequisites) and placing it on the server, ready for sending out to clients. The executable must be able to run without any user intervention and must not attempt to display anything on the client's display; as such, batch-driven command line executables are ideal. 


\section{The submit / retrieve program}

The submission and retrieval of jobs to and from the server is the responsibility of the application service, which must be written in such as way as to partition the overall workload of a run into manageable workunits. For example, if a sequence of one hundred SA runs each lasting approximately one hour is required, then the application service would most probably divide this into one hundred individual workunits before submitting them to the servers. The application service communicates with the servers using web services and as such, languages such as $\mathrm{C}++$ and Java are commonly used, though any MS.Net language can be utilised.

\section{A grid MP implementation of DASH}

As of $D A S H$ version 3.1, an option exists at the simulated annealing setup stage to execute the SA runs on the single 'host' computer, or to write out a series of files that allow submission of the SA runs to Grid MP servers. The files consist of one 'master' .grd file, plus a number (typically one per SA run) of individual . $d b f$ files; the latter can be interpreted by $D A S H$, operating in its command-line, batch-driven mode to execute a SA run. Once these files are generated, the user can exit $D A S H$ and submit the job to the Grid MP servers using an application service, GDASH, by typing 'gdash filename. $d b f$ ' at a command prompt. It is the task of GDASH to create the data packages that equate to each SA run and to transfer them to the Grid MP servers. Thereafter, these data packages are paired up with a command-line $D A S H$ executable and sent out to available clients. Once the job is submitted, $G D A S H$ returns a job_id to the user and job progress (in terms of the percentage of SA runs completed) can be monitored by the user typing 'gdash $j o b \_i d$ ' at the command prompt. Job progress can also be viewed using the standard Grid MP web interface. If the job is completed, GDASH automatically retrieves the results in the form of a single .dash file that can be read directly into $D A S H$ for examination. If the job is only part-completed, GDASH offers the user the chance to retrieve completed runs, again in the form of a dash file. Should the user find a good solution before the job is complete, they can choose to terminate the job using the Grid MP web interface, thus freeing resources for other jobs.

\section{Performance}

Performance gains achieved using a distributed computing system can be highly significant, conditional upon the size of the system. By way of illustration, table 1 summarises the performance of DASH 3.1 in repeatedly solving the crystal structure of famotidine form B (FFB: $P 2{ }_{1} / c, V=1421 \AA^{3}, Z^{\prime}=1,13$ degrees-of-freedom, synchrotron data to $1.64 \AA$ ) [6].

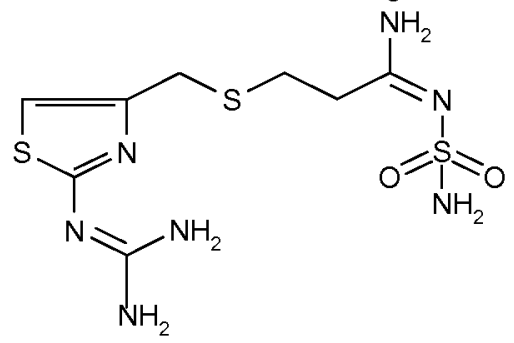

Figure 2. The molecular structure of famotidine. 
Table 1. DASH performance in repeatedly solving FFB. Each $S A$ run consists of $1 \times 10^{7} S A$ moves ( 10 times more than is actually needed to solve FFB) in order to generate easily measureable run times. Note that the batch-driven version of DASH is intrinsically $\sim 10 \%$ faster than the GUI version, as it does not have the overhead of updating the GUI. The full grid consisted of a mixture of Athlon, Duron, P4, Xeon, Core2Duo and Core2Quad CPUs with speeds ranging from $1.2 \mathrm{GHz}$ to $3.6 \mathrm{GHz}$.

\begin{tabular}{|l|l|l|l|}
\hline & Single PC & Test grid & Full grid \\
\hline DASH 3.1 mode & GUI & Batch & Batch \\
\hline Number of SA runs & 64 & 64 & 999 \\
\hline Number of PCs used & 1 & 5 & 163 \\
\hline Core2Quad CPU speed & $2.4 \mathrm{GHz}$ & $\begin{array}{l}\text { Four 2.4GHz } \\
\text { One } 2.8 \mathrm{GHz}\end{array}$ & See caption \\
\hline Number of CPU cores used & 1 & 20 & 317 \\
\hline Total elapsed time & $9 \mathrm{hrs}$ & 24 minutes & 40 minutes \\
\hline Net minutes per single SA run & 8.43 & 0.38 & 0.04 \\
\hline Relative speed & 1 & 22 & 211 \\
\hline
\end{tabular}

As can be seen, a speedup factor of more than 20 can be achieved using only five quad core PCs on the Grid MP system. When tested on the 'full' active Grid MP system in operation at the ISIS Facility, the speedup factor was in excess of 200.

\section{Performance on multi-core CPUs}

The strategy outlined above for running on a distributed computing system can also be applied to standalone computers with multiple CPUs or multi-core CPUs. It is straightforward to create a program that reads the same batch files created for the grid and then starts multiple instances of the structure solution program to fully utilise each CPU or core. In the case of $D A S H$, a small utility program $M D A S H$ permits such operation and returns performance gains of a factor of four on a quad-core CPU using a single-seat licence. Good scaling up to eight cores has been recorded, beyond which point memory limitations on a single computer running a 32-bit operating system may limit performance.

\section{Execution overheads}

There is a small but finite overhead incurred in executing jobs on the grid system. For example, if one SA run takes only $\sim 10$ seconds to complete on a single core, then the overhead of packaging it and sending it to a client PCs starts to become significant. As such, DASH permits the user to specify how many individual SA runs will constitute a single workunit in order to ensure that the grid overhead remains insignificant.

\section{Performance on virtual machines}

Thus far, only the execution of native MS Windows executables on client PCs running MS Windows has been considered. Grid MP also allows computers running the Linux or MacOS. operating systems to be connected in order to run Linux or Mac-based executables. In a 
department dominated by clients running MS Windows, it is still possible to create a significant Linux resource without resort to purchasing new hardware by using virtualisation techniques. By way of example, VMWare Server [7] running under MS Windows has been used to create virtual Linux PCs on many of the ISIS grid client PCs. Each virtual Linux PC functions exactly like a 'real' one in terms of grid operation, providing tremendous flexibility in the programs that can be executed as a result.

\section{Concluding remarks}

Systems for distributed computing offer a scalable, easy-to-implement way of greatly accelerating structure solution by global optimisation. As has been shown, speed gains of more than two orders of magnitude can be achieved on a modest departmental grid of PCs. Such speed gains facilitate calculations and scenarios that could not otherwise be considered, which not only help with complex structure solution cases but also with algorithm development and testing [8]. Distributed grids are unusual in that they utilise existing non-dedicated resources and their power tends to increase with time, as new machines are added and old machines are upgraded. In this regard, the advent of multi-core CPUs is a very valuable trend. However, when setting up such a grid, one must consider not only technical factors but also social ones. The fact that one is executing code on non-dedicated resources means that particular care must be taken not to interfere with normal client PC operations. Our experience is, particularly in the early stages of deployment, that the grid becomes the focus of attention for anyone experiencing any problems with their PC. In reality, the problems rarely have anything to do with the operation of the grid, but one must be prepared to prove this to the satisfaction of the 'owner' of a problematic client PC.

\section{References}

1. David, W.I.F., Shankland, K., McCusker, L.B. \& Baerlocher, Ch. (editors), Structure Determination from Powder Diffraction Data (Oxford: OUP).

2. David, W.I.F., Shankland, K., van de Streek, J., Pidcock, E., Motherwell, W.D.S. \& Cole, J.C., 2006, J. Appl. Crystallogr., 39, 910.

3. Miller, R., Shah, N., Green, M.L., Furey, W. \& Weeks, C.M., 2007, J.Appl. Crystal$\log r ., \mathbf{4 0}, 938$.

4. http://www.es.wisc.edu/condor.

5. Univa UD, Inc., 2008, http://www.univaud.com/hpc/products/grid-mp/.

6. Shankland, K., McBride, L., David, W.I.F., Shankland, N. \& Steele, G., 2002, J. Appl. Crystallogr., 35, 443.

7. VMware, Inc., 2008, http://www.vmware.com.

8. Markvardsen, A.J., Shankland, K., David, W.I.F. \& Didlick, G., 2005, J. Appl. Crystallogr., 38, 107. 


\section{Author Index}

Volume I: pp. 1-232

Volume II: pp. 233-495

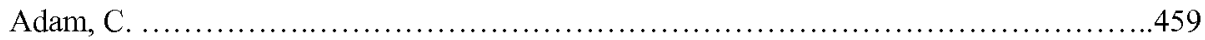

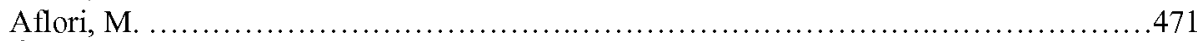

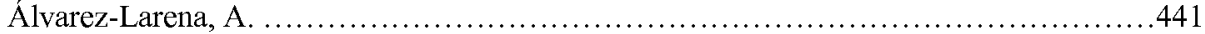

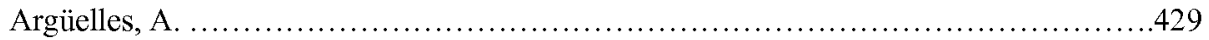

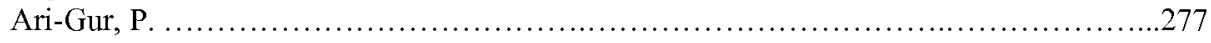

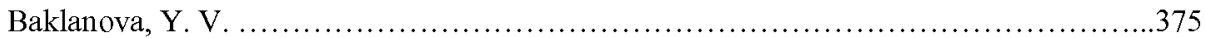

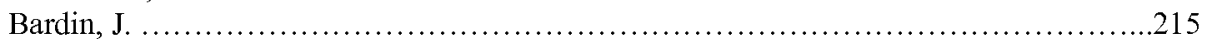

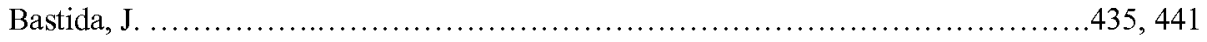

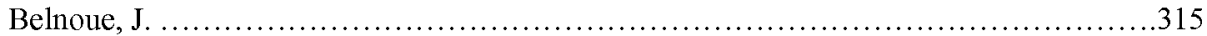

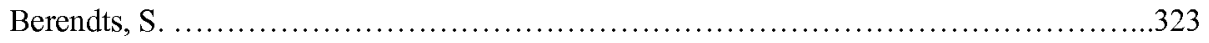

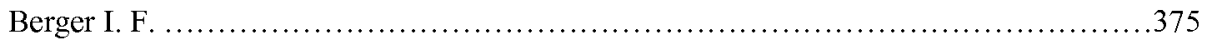

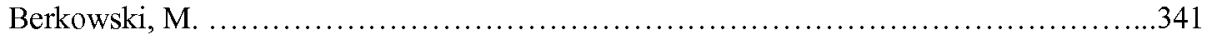

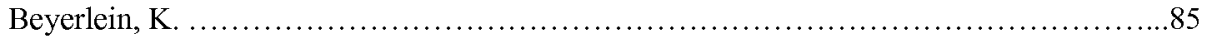

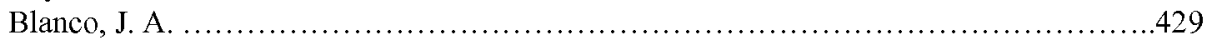

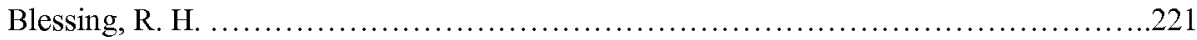

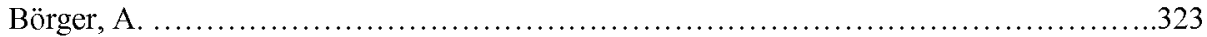

Bolmaro, R. E. ..................................................111, 117, 123, 297

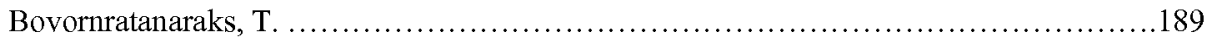

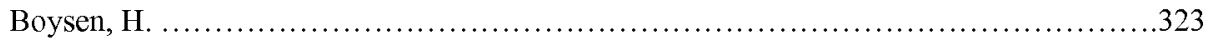

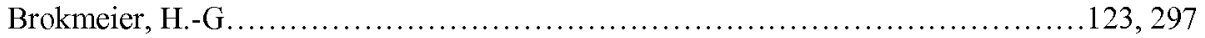

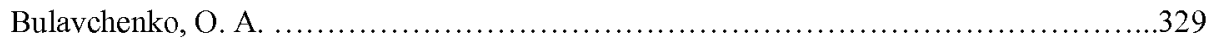

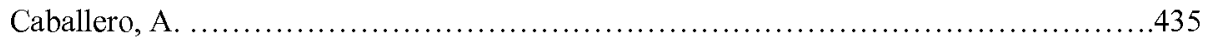

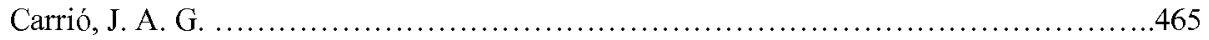

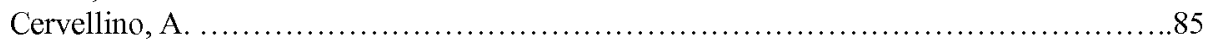

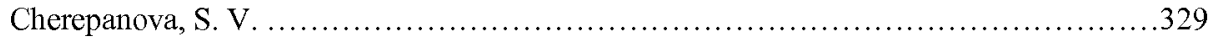

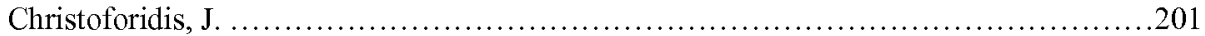

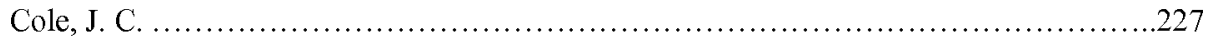

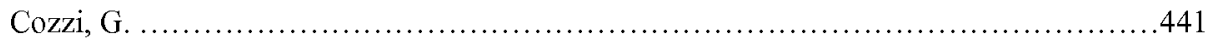

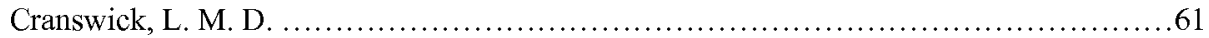

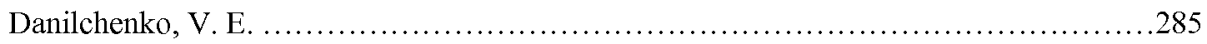

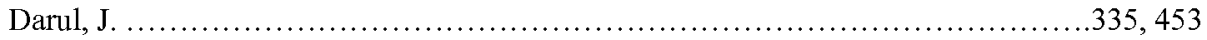

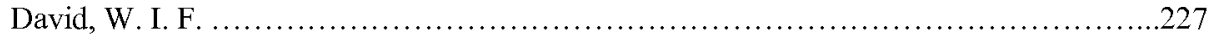

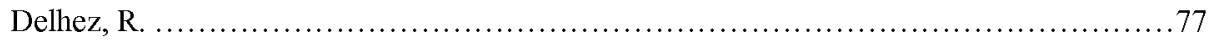

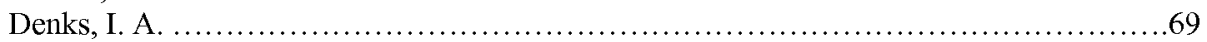

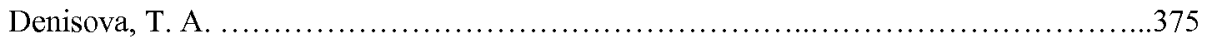

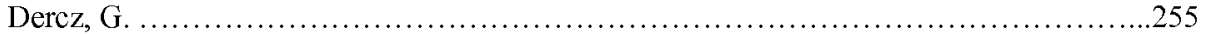

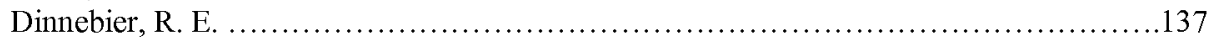




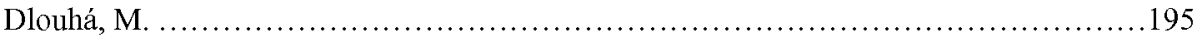

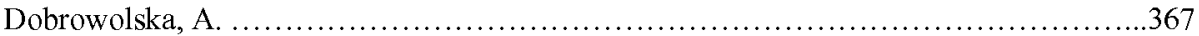

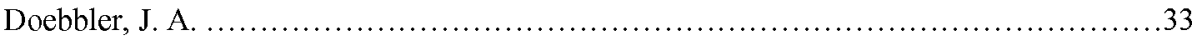

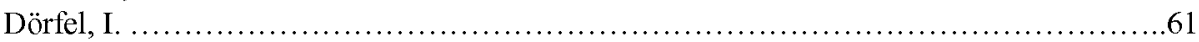

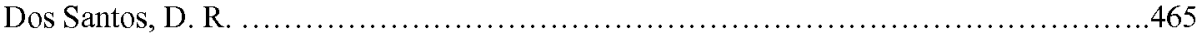

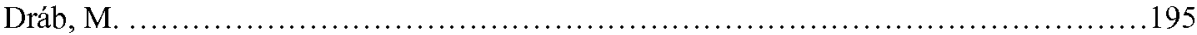

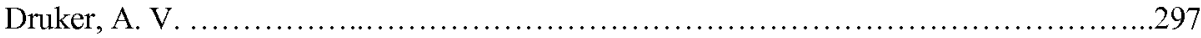

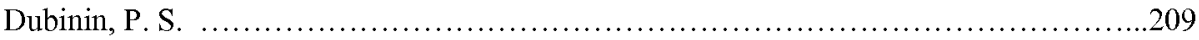

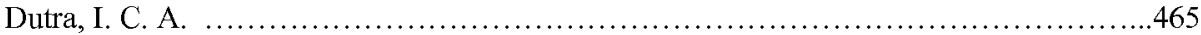

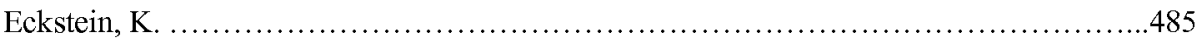

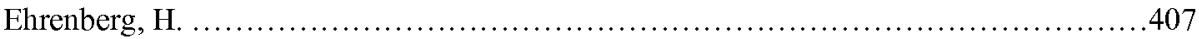

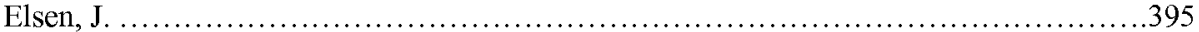

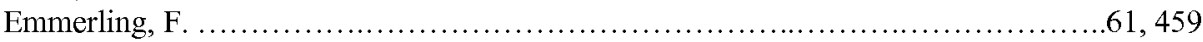

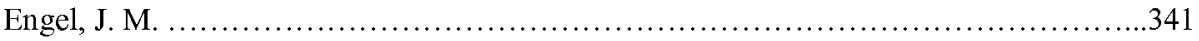

Evans, A. ..................................................... $61,315,453$

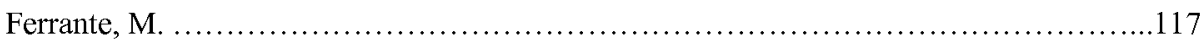

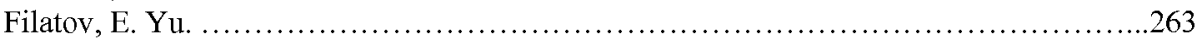

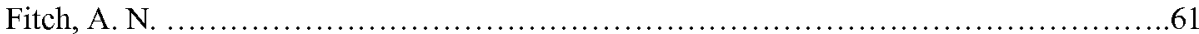

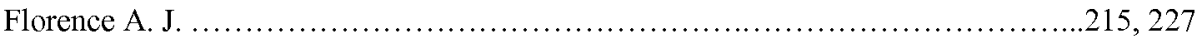

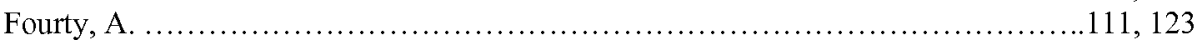

Fransen M. J. ............................................................... 163

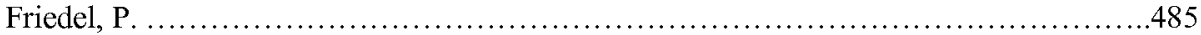

Fuess, H. .............................................................4 407

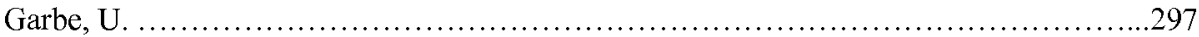

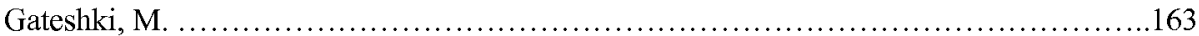

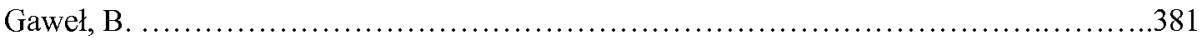

Genzel, Ch. ...................................................................69

Goetz-Neunhoeffer, F. ............................................. 359,447

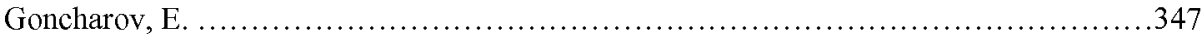

Goodwin, A. L. ...................................................................

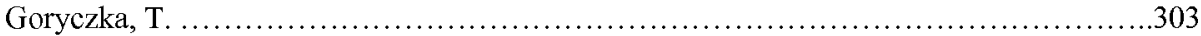

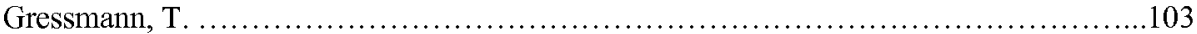

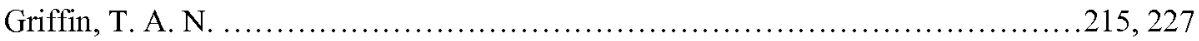

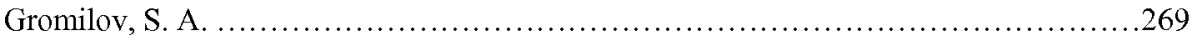

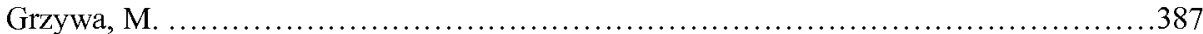

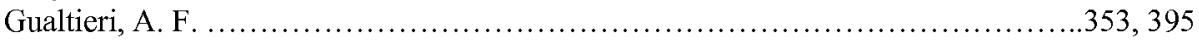

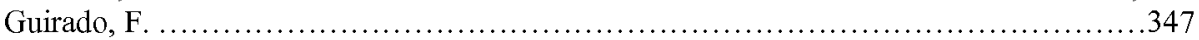

Hadermann, J. ......................................................................401

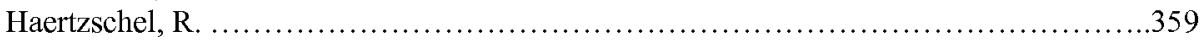

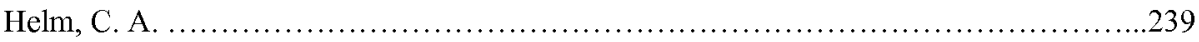

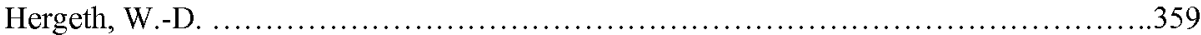

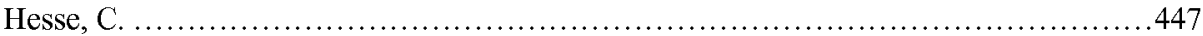

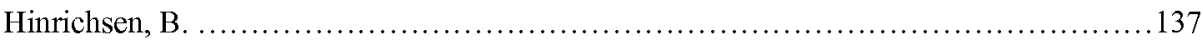




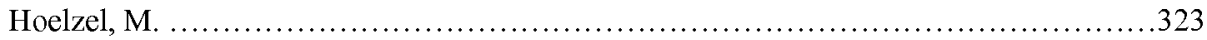

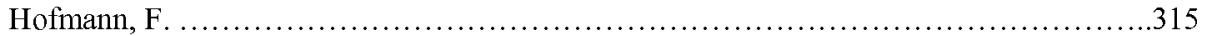

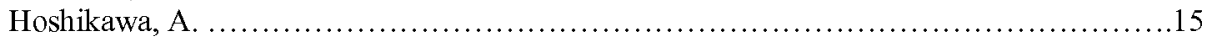

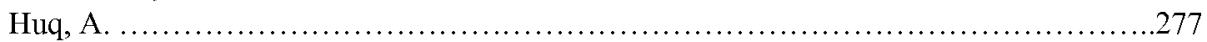

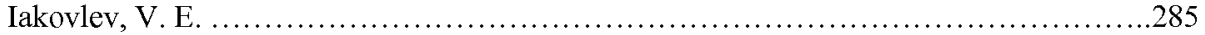

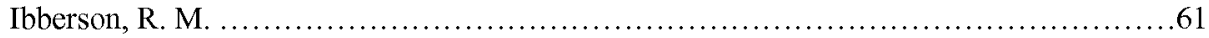

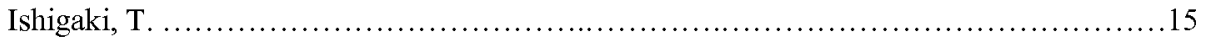

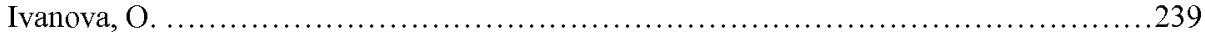

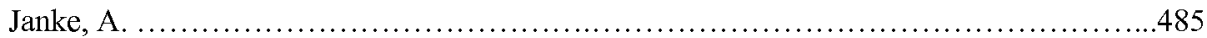

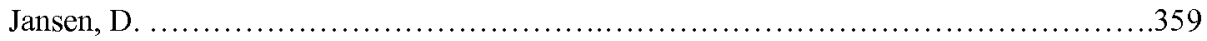

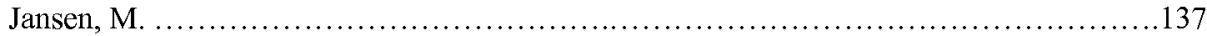

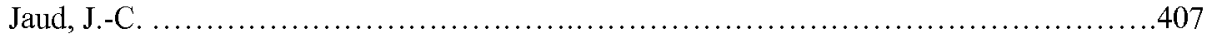

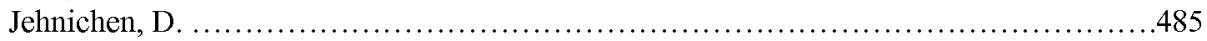

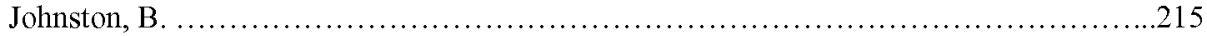

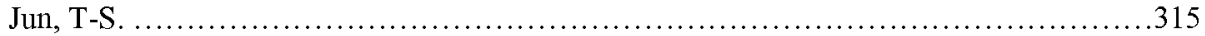

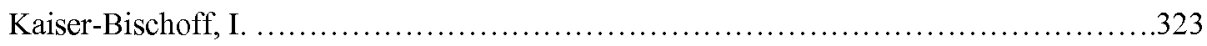

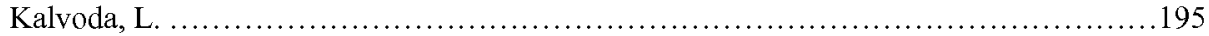

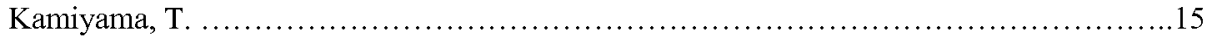

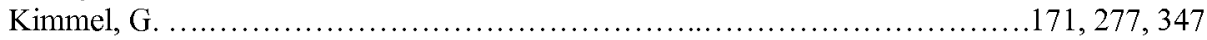

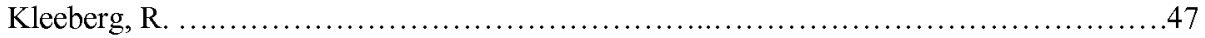

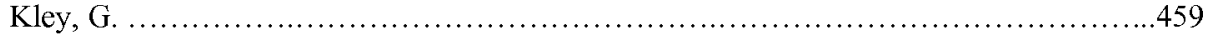

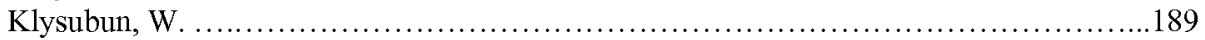

Kojdecki, M. A. .................................................... $183,435,441$

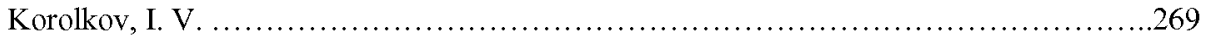

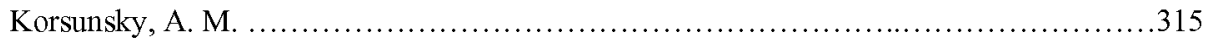

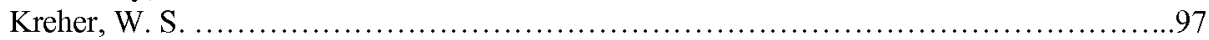

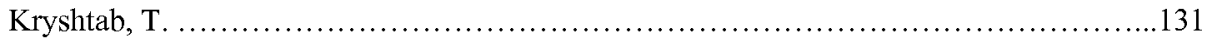

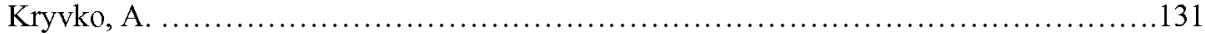

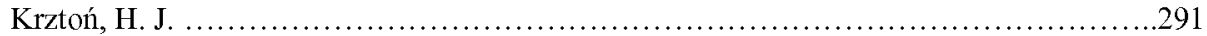

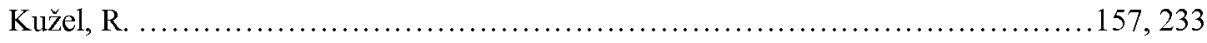

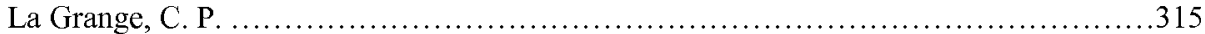

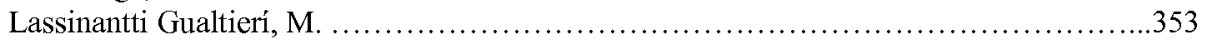

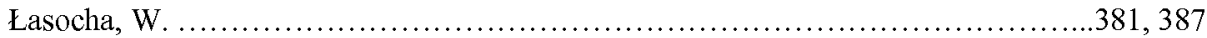

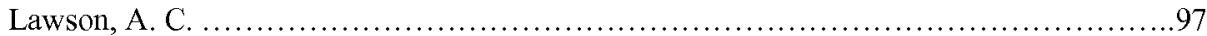

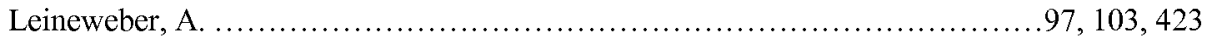

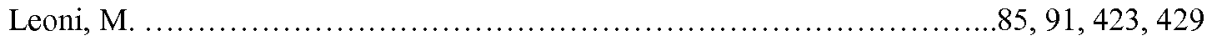

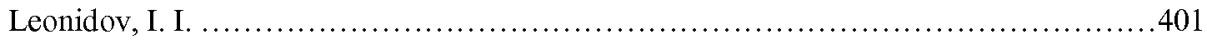

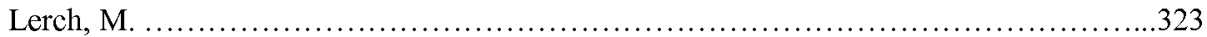

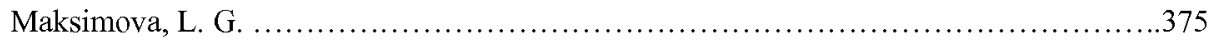

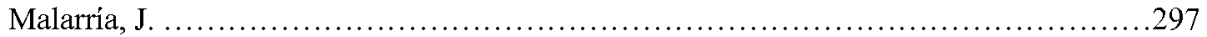

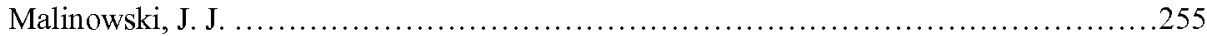

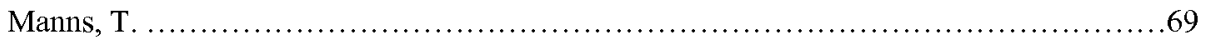




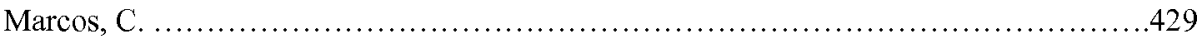

Martinez-Garcia, J. ................................................................. 91

Martino, R. . . . . . . . . . . .

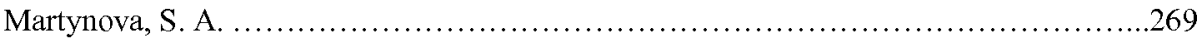

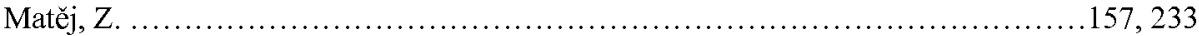

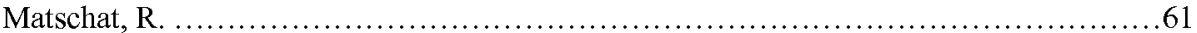

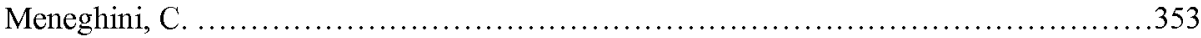

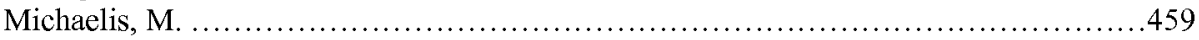

Mielcarek, W. ............................................................... 183

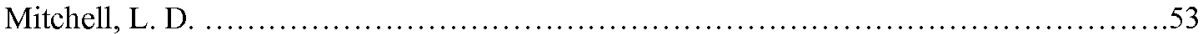

Mittemeijer, E. J. ..............................................97, 103, 245

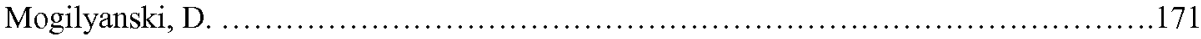

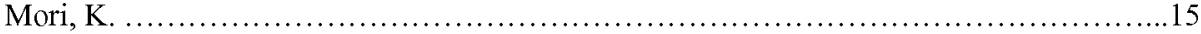

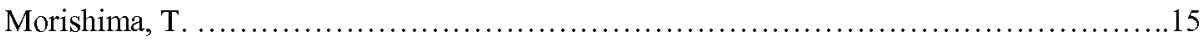

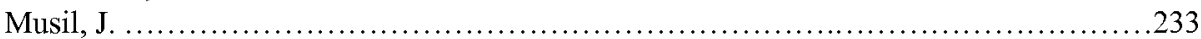

Nawrocki, J. ............................................................ 453

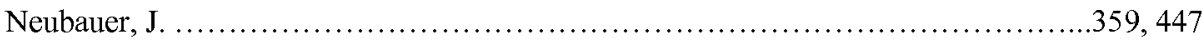

Nichtová, L. ......................................................157, 233

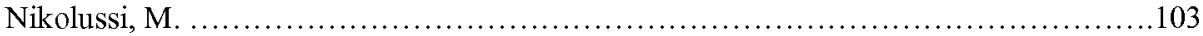

Nowicki, W. ............................................................415, 453

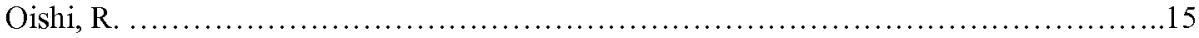

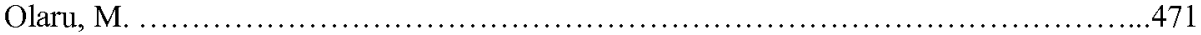

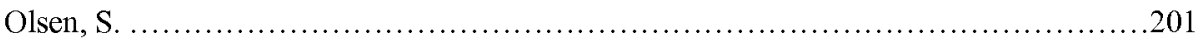

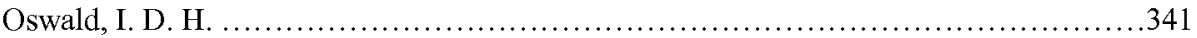

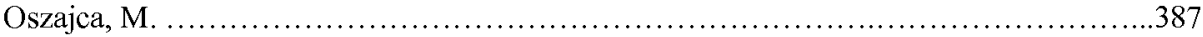

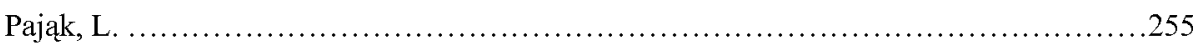

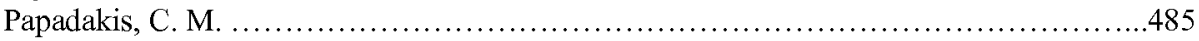

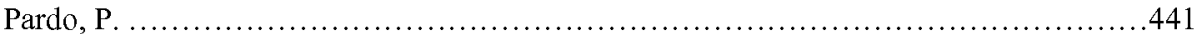

Peplinski, B. ..........................................................61, 459

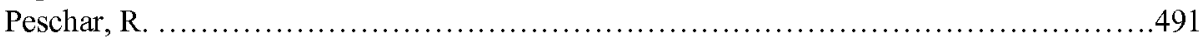

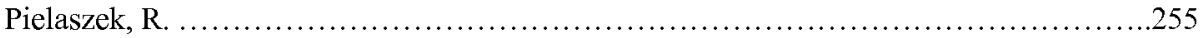

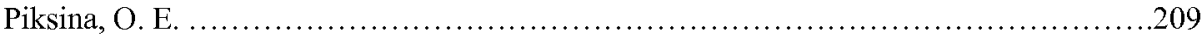

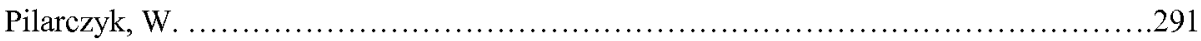

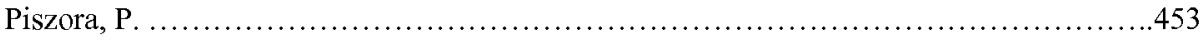

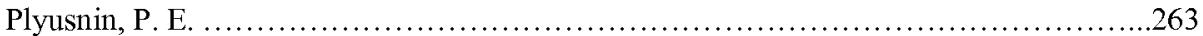

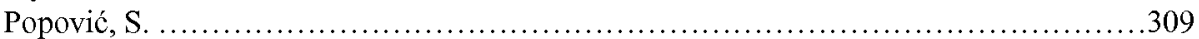

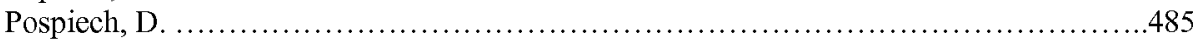

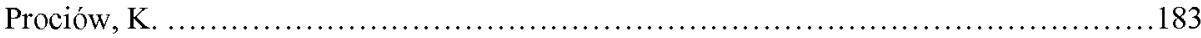

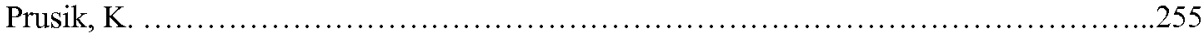

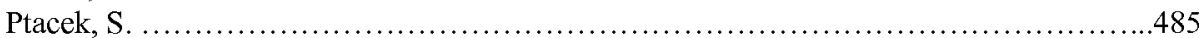

Quaas, M. .....................................................................239 


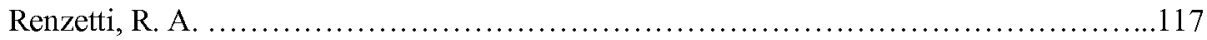

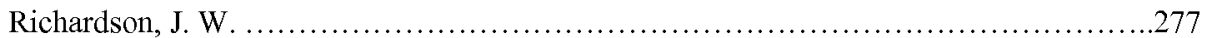

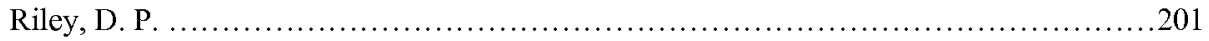

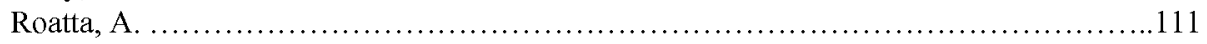

Roberts, J. A. ............................................................... 97

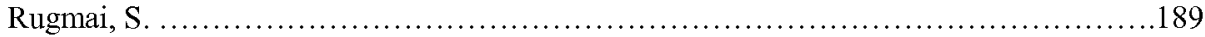

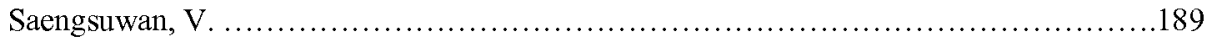

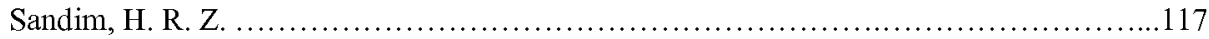

Sandim, M. J. R. ...................................................................... 117

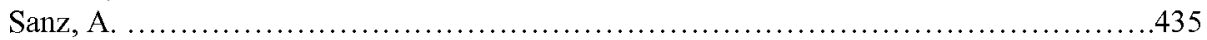

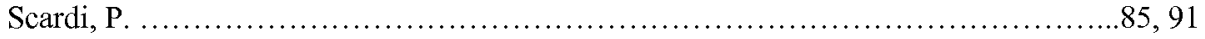

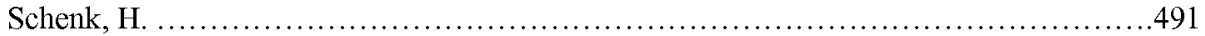

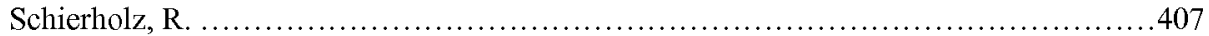

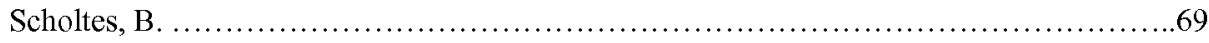

Semenkin, E. S. ............................................................21

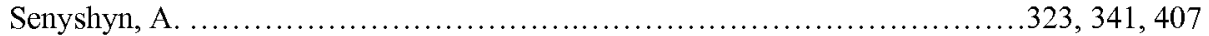

Serrano, F. J. ...................................................................4 435

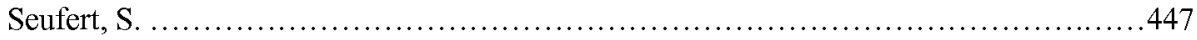

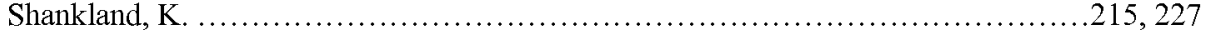

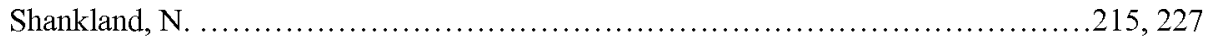

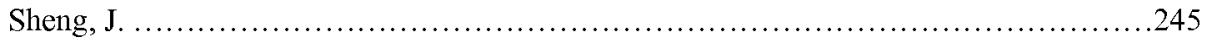

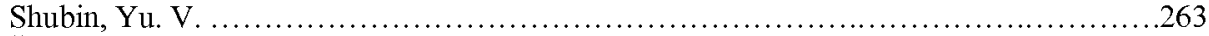

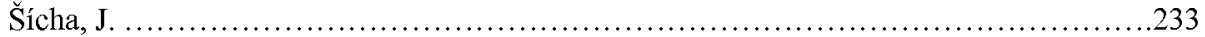

Signorelli, J. W. ........................................................... 117

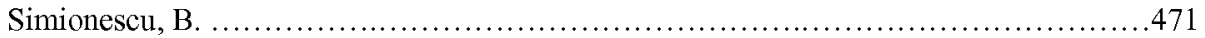

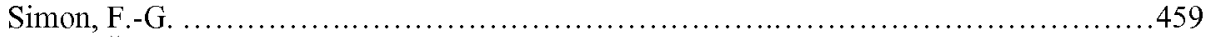

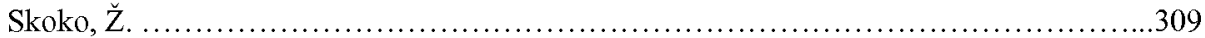

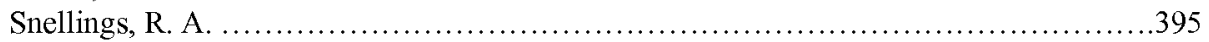

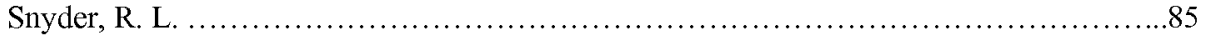

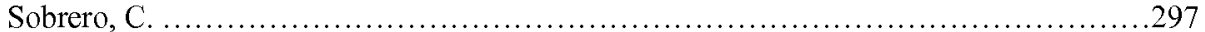

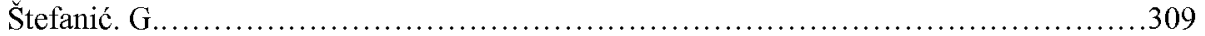

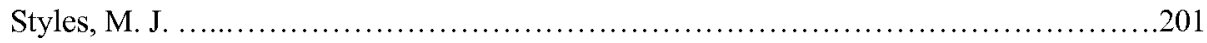

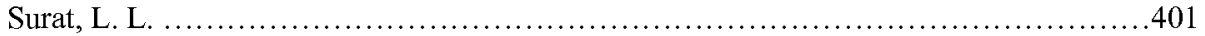

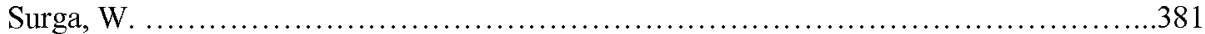

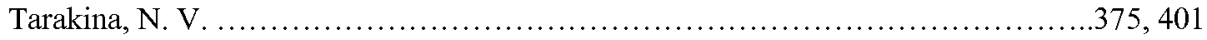

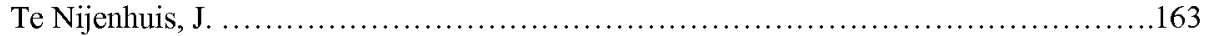

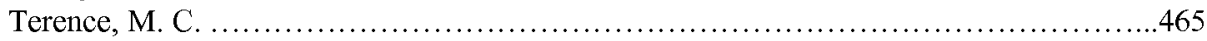

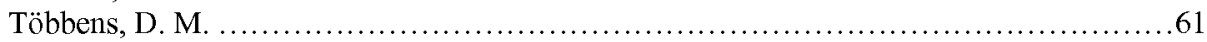

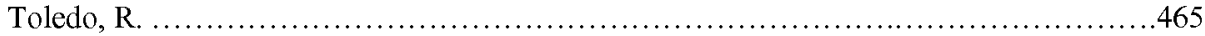

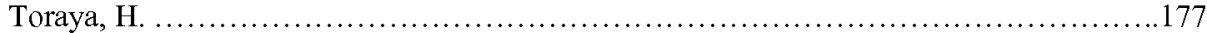

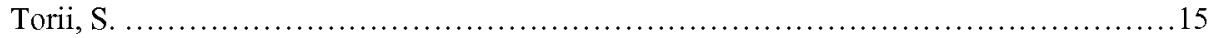

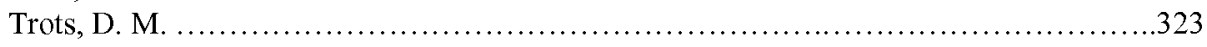

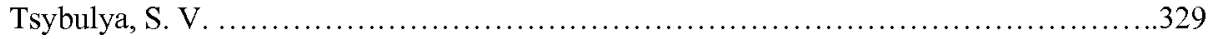

Tyutyunnik, A. P................................................................. 401 


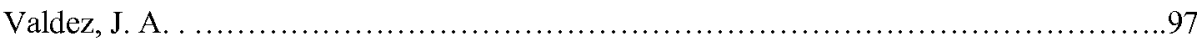

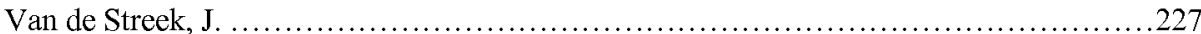

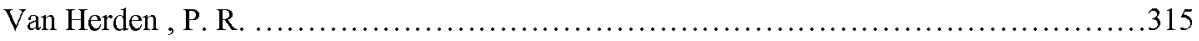

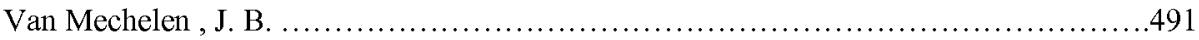

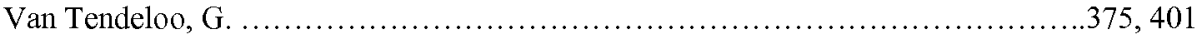

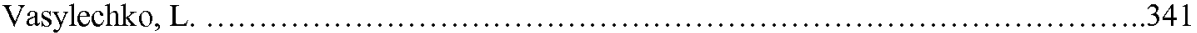

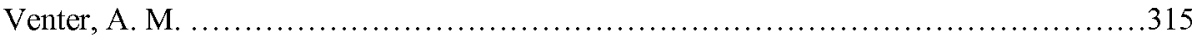

Vermeulen, A. C. .............................................................. 77

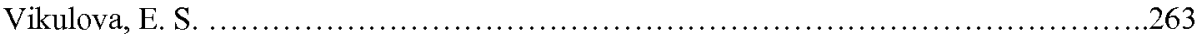

Von Dreele, R. B. ..........................................................27, 33

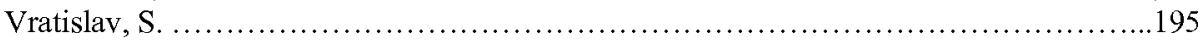

Wang, H. .................................................................. 407

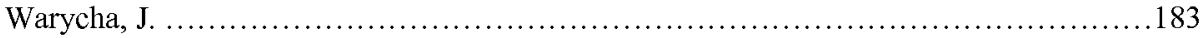

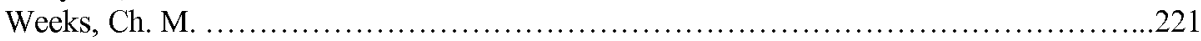

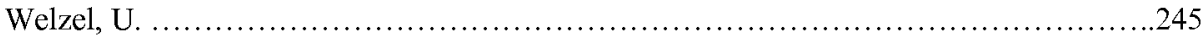

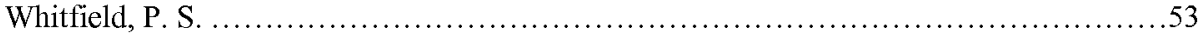

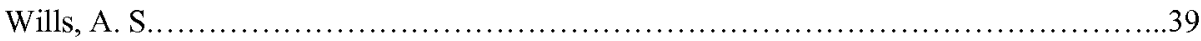

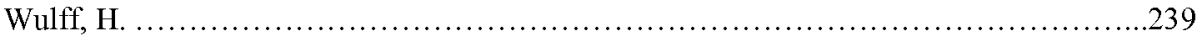

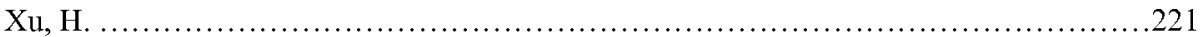

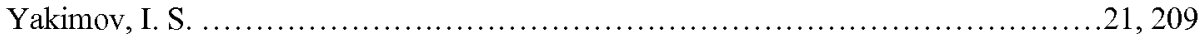

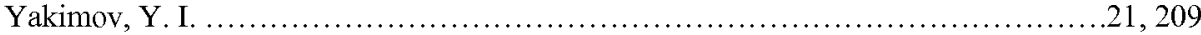

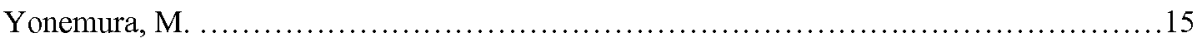

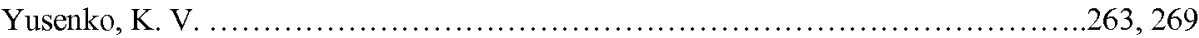

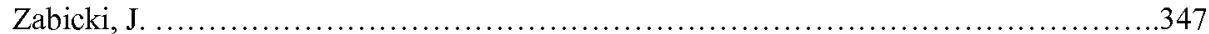

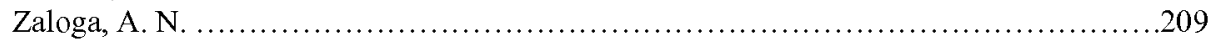

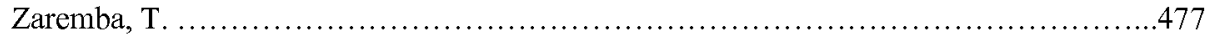

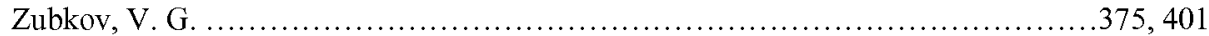

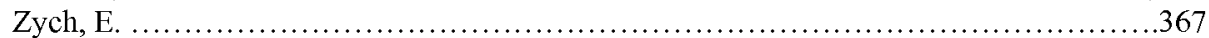




\section{EPDIC 11}

Proceedings of the

Eleventh European Powder Diffraction Conference

held

September 19-22, 2008

in

Warsaw, Poland

\section{VOLUME II}

\section{Editors}

\section{Bogdan Palosz ${ }^{1}$, Jordi Rius ${ }^{2}$ \\ and Udo Welzel ${ }^{3}$}

${ }^{1}$ Institute of High Pressure Physics, Polish Academy of Sciences, Warsaw, Poland

${ }^{2}$ Institut de Ciència de Materials de Barcelona (CSIC), Catalonia, Spain

${ }^{3}$ Max Planck Institute for Metals Research, Stuttgart, Germany

Supplement No. 30 of Zeitschrift für Kristallographie

Oldenbourg Verlag 



\section{EPDIC 11}

Eleventh European Powder Diffraction Conference

\section{Warsaw, Poland, September 19 - 22, 2008}

Conference location:

Conference chairman:

Organising committee:
Warsaw University of Technology, Warsaw, Poland

Bogdan Palosz

Stanislaw Gierlotka

Ewa Grzanka

Piotr Jaśkiewicz

Zbigniew Kaszkur

Jan Kozubowski is

Henryk Morawiec

Wojciech Paszkowicz

Adam Pietraszko

Janusz Zachara
Mariusz Jaskólski

Andrzej Katrusiak

Wieslaw Lasocha Andrzej Orlowski Jerzy Pielaszek Marek Wołcyrz

Andrzej Zięba 
Scientific programme committee: Bogdan Palosz, Warsaw, Poland (Chair) Jordi Rius, Barcelona, Spain (Chair) Nathalie Audebrand, Rennes, France Robert J. Cernik, Daresbury, UK Radovan Cerný, Genève, Switzerland William I.F. David, Oxon, UK Robert Delhez, Delft, The Netherlands Andrew Fitch, Grenoble, France Hartmut Fuess, Darmstadt, Germany Herbert E. Göbel, München, Germany Alessandro F. Gualtieri, Modena, Italy Torbjörn Gustafsson, Uppsala, Sweden Sergey A. Ivanov, Moscow, Russia Arnt Kern, Karlsruhe, Germany Radomir Kužel, Prague, Czech Republic Christian Lengauer, Vienna, Austria Ho-kwang (David) Mao, Washington, USA Anton Meden, Ljubljana, Slovenia Stanko Popovič, Zagreb, Croatia Thomas Proffen, Los Alamos, USA Paolo Scardi, Trento, Italy Osamu Shimomura, Tsukuba, Japan Kenny Ståhl, Lyngby, Denmark Hideo Toraya, Tokyo, Japan Tamás Ungár, Budapest, Hungary Arnold C. Vermeulen, Almelo, The Netherlands Udo Welzel, Stuttgart, Germany 
EPDIC11 has been sponsored by:

- Bruker AXS GmbH

- PANalytical B.V.

- International Centre for Diffraction Data ICDD

- International Union of Crystallography IUCR

- European Crystallographic Association ECA

- Committee on Crystallography of the Polish Academy of Sciences 



\section{Preface}

\section{The History of the European Powder Diffraction Conference}

As the Chair of the Organizing Committee of the first European Powder Diffraction Conference, EPDIC 1, I was invited by the Editors of the current Proceedings, on the occasion of my retirement from the EPDIC Committee, to write the Preface for the current Proceedings with a retrospective view back in time to the beginnings and the early-history of a common European Conference on Powder Diffraction.

Before the initiation of the EPDIC Conferences in 1991, exchange of knowledge and new developments in the field of powder diffraction was mainly taking place during dedicated sessions of the annual meetings of the national crystallographic associations, including also the European Crystallographic Associaton (ECA). Also the International Union of Crystallography (IUCr) organized a dedicated satellite meeting on powder diffraction every three years.

As some neighbouring countries in Western and Eastern Europe started organizing joined annual meetings, an increasing community of powder diffractionists proposed establishing a common meeting on powder diffraction in the late $80 \mathrm{~s}$. A view to the USA and the success of the "Denver X-ray Conference" (this conference series was initiated already in 1951 by the University of Denver with a one-day symposium on the application of X-rays to the study of materials) encouraged a group of European members of the "Joint Committee on Powder Diffraction Standards" (today International Centre for Diffraction Data - ICDD) in 1988 to give a start signal to organize an independent gathering of powder diffractionists in Europe. Important names in this context were Walter Eysel (Heidelberg, main initiator), Jan Visser (Delft, Scientific Chair of EPDIC 1), Daniel Louër (Rennes), Zbignew Bojarski (Katovice), Vaclav Valvoda (Prague) and Herbert Göbel (Munich, Chair of the Organizing Committee of EPDIC 1). The opportunity to start was the offer of the German Crystallographic Association (DGK) of a combined meeting at the University of Munich planned for March 1991. The first circular was distributed in 1989, mainly through the channels of the national crystallographic associations and via the manufacturers of diffraction instrumentation.

Many details had to be determined during the organization of EPDIC 1: First of all the name of the Meeting/Conference and a suitable abbreviation. We received critical remarks from the ECA for naming the event a 'Conference', whereas the ECM held just a 'Meeting'. However, Jan Visser insisted on the " $\mathrm{C}$ " in the abbreviation of EPDIC, because it was better to pronounce. The scientific scope of EPDIC and the procedures for the following EPDIC's had to be clearly defined, to avoid conflicts with other conferences/meetings in the field of diffraction, but also to guarantee the continued success of this new forum.

During the organization of EPDIC 1 the "Iron Curtain" was taken down, so that also participants from all Eastern European countries could attend. Their contributions were an important enrichment both with respect to scientific developments and practical applications of powder diffraction. Many contacts between scientists from East and West were formed, 
that resulted in common projects and sometimes partnerships and cooperations still existing today.

In total, EPDIC 1 was attended by 370 participants. More than half of them came from the unified parts of Germany and about 60 from Eastern Europe. The social event, an evening in one of Munich's famous beer halls during the "strong - beer" season of Lent, certainly is an unforgettable experience for all participants...

The large number of participants, the fact that most European Countries were represented by leading scientists in the field and the broad spectrum of applications of powder diffraction covered by the Conference attracted also the entire industry of equipment manufacturers in Europe and the world: A comprehensive exhibition of X-ray instrumentation took place during the conference.

The scientific program consisted of two and a half days of working sessions, comprising a honorary lecture of Professor H. Jagodzinski on "The Role of Munich for X-ray Powder Crystallography and the History of X-ray Powder Diffraction", 16 main lectures ("X-ray Diffraction Profiles Due to Real Polycrystals" by P. Klimanek, "Quo Vadis Quantitative Powder Diffraction Analysis" by J. Fiala, "Crystal Structure Analysis and Refinement by the Two-Step Method" by G. Will, "Neutron Powder Diffraction and Oxide Superconductors" by A.W. Hewat, "Applied Crystallography in Advanced Ceramics" by R.L. Snyder, "New Instrumentation in Powder Diffraction" by J. Ihringer, "New Detectors in X-ray Diffraction" by P. Tucker, "Energy Dispersive XRPD at High Pressure" by L. Gerward, "Glory and Misery of the Structure Analysis of Thin Polycrystalline Films" by V. Valvoda, "Characterization of Epitaxial Thin Films by X-ray Diffraction" by A. Segmüller, "Powder Diffraction Using Synchrotron Radiation" by M. Hart, "X-ray Absorption and Reflection in Materials Science" by B. Lengeler, "Preferred Orientation in Powder Diffraction" by H.-J. Bunge, "X-ray Stress Analysis" by J.M. Sprauel, "On the Use of Rietveld Refinements for Structural Studies" by P.-E. Werner, "Indexing of Powder Diffraction Patterns" by D. Louër), 120 poster presentations and 54 oral contributions. Two parallel sessions were held in order to accommodate a large number of talks.

Since the first EPDIC Conference, scientific progress reported during the Conferences has been registered in Conference Proceedings. Concerning the Proceedings the EPDIC community is indebted to Rob Delhez and Eric J. Mittemeijer for having taken care of the editorial work for the Proceedings for many years. Since EPDIC 9, the Proceedings are published 'open access' as supplement issues of the 'Zeitschrift für Kristallographie': The free on-line accessibility in combination with the traditional publication of the Proceedings in the form of printed volumes strengthens the importance of these Proceedings as a medium for the publication of cutting-edge developments and compact state-of-the art overviews in the field of powder diffraction.

The last EPDIC conference. EPDIC 11, was attended by about 350 participants (nearly 60 coming from outside Europe) and, as for previous EPDIC editions, current hot research topics and the newest developments of powder diffraction were presented. Particular emphasis was laid on direct and reciprocal space methods in powder diffraction structure 
determination, the 'charge flipping' algorithm, nanomaterials and total seattering analysis, to mention only a few of the topics treated in the conference.

Another important aspect was the search for new or improved forms of organization of the conference to make it more effective and attractive. Following the general trend towards the organization of small focused meetings, EPDIC workshops either preceding or following the main meeting have been organized during the last conferences. There is an increasing interest in this form of education and exchange of knowledge and expertise that also helps to promote scientific collaboration through the formation of personal links. At EPDIC 9 in Prague there was only one workshop, already three took place in Geneva at EPDIC 10, and now six workshops took place in Warsaw at EPDIC 11. For the future, a point deserving further attention would be the analysis of the circumstances that recommend treating a topic as a workshop rather than as a normal microsymposium.

Since EPDIC 3 in Vienna, it is a tradition to reward one young scientist, who contributed significantly to the field of powder diffraction, with the Young Scientist Award.

It was an unpleasant situation for the EPDIC Committee during each selection procedure of award candidates to exclude candidates not meeting the age limit (35 years) but having remarkable contributions to the field. The EPDIC committee therefore welcomes with great satisfaction the introduction of the EPDIC Award for Distinguished Scientist, which was awarded to Juan Rodríguez-Carvajal during EPDIC 11.

The next EPDIC Conference - EPDIC 12 - will be held in Darmstadt in 2010 as a joint meeting with the 26th European Crystallographic Meeting, ECM 26.

Looking back to the eleven EPDIC Conferences (see listing of previous conferences on the following page) and their impact on the field it can be concluded that the EPDIC Conference has developed as the leading conference dedicated exclusively to all aspects of powder diffraction.

Herbert Göbel

Munich

May 2009 


\section{EPDIC Conferences: The History and the Future}

Previous EPDIC Conferences

EPDIC 1, Munich, Germany. 14-16 March 1991. Chairman: Dr. H.E. Göbel

EPDIC 2, Enschede, The Netherlands. 30 July-1 August 1992. Chairman: Dr. T.W. Ryan

EPDIC 3, Vienna, Austria. 25-28 September 1994. Chairman: Prof. A. Preisinger

EPDIC 4, Chester, UK. 10-14 July 1995. Chairman: Dr. R.J. Cernik

EPDIC 5, Parma, Italy. 25-28 May 1997. Chairman: Prof. G. Artioli

EPDIC 6, Budapest, Hungary. 22-25 August 1998. Chairman: Prof. T. Ungár

EPDIC 7, Barcelona, Spain. 20-23 May 2000. Chairman: Prof. J. Rius

EPDIC 8, Uppsala, Sweden. 23-26 May 2002. Chairman: Dr. I.G.R. Tellgren

EPDIC 9, Prague, Czech Republic. 2-5 September 2004. Chairman: Prof. R. Kužel

EPDIC 10, Geneva, Switzerland. 1-4 September 2006. Chairman: Dr. R. Cerný

EPDIC 11, Warsaw, Poland. 19-22 September 2008. Chairman: Prof. B. Palosz

Forthcoming EPDIC Conference

EPDIC 12, Darmstadt, Germany. 27-30 August 2010. Chairman: Prof. H. Fuess 


\section{Editorial Notes}

The number of papers in these Proceedings is 73. The total number of papers published in the Proceedings of the preceding EPDIC conferences ranges from 88 to about 190 .

The subdivision of the papers over the sections has been largely maintained as for preceding EPDIC proceedings. Only very minor adjustments, to adapt the subsections to the submitted papers, have been performed.

Reviewing the eleven editions of the EPDIC Proceedings, the ratios of the numbers of papers on developments in the methods and techniques of powder diffraction and those on applications of powder diffraction methods to specific classes of materials are found to be $1.0,0.7,0.5,1.0,0.9,0.5,0.7,0.7,0.8,0.5$ and, for the current proceedings, 0.8

As for the EPDIC10 Proceedings, a strict refereeing procedure was adopted for the Proceedings of EPDIC11. Each contribution was considered by at least one referee. The referees were, to a large extent, participants of EPDIC11. A few (in this sense) external referees were contacted as well. We thank all referees for their efforts and time spent on the manuscripts. We did not correct the English used, apart from minor corrections in a few papers.

We also thank Mrs Hilda David and Mrs Maritta Dudek (Max Planck Institute for Metals Research, Dept of Prof. Dr Ir. E.J. Mittemeijer, Stuttgart, Germany) for final technical corrections and invaluable help during the preparation of the required material for the publisher.

We sincerely hope that these Proceedings will be a useful collection of papers outlining the newest developments in the field of Powder Diffraction.
B. Palosz
J. Rius
U. Welzel
Warsaw
Barcelona
Stuttgart

May 2009 



\section{European Powder Diffraction Conference Award for Young Scientists}

\section{Sponsored by PANalytical B.V. (Almelo, The Netherlands)}

The EPDIC Award for Young Scientists is assigned at each EPDIC Conference and honours outstanding scientific achievement by young scientists in the field of powder diffraction. The award winner is invited to present a plenary talk at the next European Powder Diffraction Conference. The award has a value of 1,000 Euro.

The EPDIC Committee of each EPDIC Conference decides who is the winner. Short proposals of candidates containing descriptions of the scientific contributions to be assessed should be addressed to any member of the EPDIC Committee who is asked to make names public within this committee. As a rule, an age limit of 35 applies to the candidates. 



\title{
European Powder Diffraction Conference Award for Distinguished Powder Diffractionists
}

\author{
Sponsored by Bruker AXS (Karlsruhe, Germany)
}

The EPDIC Award for Distinguished Powder Diffractionists was assigned for the first time during the EPDIC-11 Conference for outstanding results and/or continued, important contributions to the field of powder diffraction. The award winner is invited to present a plenary talk at the next European Powder Diffraction Conference. The award has a value of 1,000 Euro.

The EPDIC Committee of each EPDIC Conference decides who is the winner. Short proposals of candidates containing descriptions of the scientific contributions to be assessed should be addressed to any member of the EPDIC Committee who is asked to make names public within this committee. 



\section{EPDIC Award for "Distinguished Powder Diffractionists"}

\section{Introductory speech by the Chairman of the EPDIC Committee}

On behalf of the EPDIC Committee, I am pleased to announce Dr. Juan Rodriguez-Carvajal as recipient of the Award for "Distinguished Powder Diffractionists" in its EPDIC 11 edition.

Dr. Rodriguez-Carvajal, simply Juan for his friends, was born in Sevilla (1953) and studied Physics first at the University of Sevilla and later at the University of Barcelona where he performed his doctorate. This was at the end of the seventies, beginning of the eighties. At that time I was also beginning my scientific career at the Crystallography Department and still remember the friendly and sometimes not so friendly discussions between the Director of the Department (the late Prof. Manuel Font-Altaba) and Juan regarding the possibilities of powder diffraction and the Rietveld method in relation to single-crystal diffraction. In retrospective, I think that both were right in many of their arguments. After several contracts in different University Faculties and Engineer Schools in Barcelona, Juan joined the CSIC (Institut de Ciència de Materials de Barcelona) but soon moved to the ILL (Institut LaueLangevin) where he worked as a physicist from 1988 to 1994. There, he was co-responsible for different diffractometers. Later on, he worked as "Physicien Chercheur" at "Laboratoire Léon Brillouin, Centre d'Etudes de Saclay" from 1994 to 2006, and recently, again at ILL, he was appointed Leader of the Diffraction Group of that Institute.

Juan has been recognised with this Award mainly because of three major merits clearly demonstrated during his 28 years of professional activity in the field of powder diffraction and crystallography.

(1) The first merit is that by means of powder diffraction techniques, he has made an exceptional contribution to the study of structural and magnetic aspects of strongly correlated oxides. His scientific activity has represented a decisive step forward for the advanced analysis of both structural and magnetic diffracted intensities of complex materials. His ability to extract information from diffraction patterns of complex materials has led him to collaborate with an extraordinarily large number of groups all around the world, investigating a large variety of materials through profuse collaborations with many different groups and diffraction scientists.

(2) There is no doubt that the most outstanding contribution of Juan to the scientific and diffraction communities is the "FullProf computer program for crystallographic and magnetic studies". From 1988, the successive developments of this software package have allowed the crystallographic community to address an increasing number of features, aspects and problems related with the information included in X-ray and neutron diffraction patterns. At present, FullProf is a world-wide used program going from laboratory to synchrotron Xray diffraction, and from neutron reactors to spallation sources and constitutes an 
extraordinary tool with a huge impact on the scientific community, and also on the instrumental development.

(3) A third important characteristic of Juan is his willingness to share his scientific expertise with everybody asking him for help and advice, either about scientific matters or on questions related to FullProf. In this way, an extraordinary number of powder diffractionists have benefited from his expertise.

His CV can be summarised as follows:

1) Research and Teaching experience in Condensed Matter Physics and Crystallography for 28 years.

2) Expertise in:

(i) Powder and single crystal X-ray and neutron scattering;

(ii) Symmetry analysis, crystallography and magnetism;

(iii) Oxides presenting remarkable properties: superconductivity, giant magnetoresistance, charge, spin \& orbital ordering;

(iv) Computer programming and data analysis;

(v) Neutron diffraction instrumentation.

Due to his expertise he has also been member of many scientific institutions and committees.

3) Publications \& computer programs:

209 regular papers in journals (36 in Physical Review B, 5 Rapid Comm.; 5 PRLs), 103 papers in journals resulting from proceedings, 59 reports/book/proceedings contributions and about 220 communications in meetings. Total number of article citations exceeds 6900 . The current h-index is 38 .

Author of FULLPROF, one of the most frequently used powder diffraction computer programs in the world. (currently 2040 citations of the article Physica B 192, 55 (1993) plus 2795 direct citations of the use of the program)

4) Supervisor of 8 doctoral theses, 18 stages and post-does and invited to more than 60 international events (courses and conferences).

To finish this short introduction, the members of the EPDIC Committee want to express their congratulations to Juan on receiving this award as well as their sincere appreciation for his contribution to the development of powder diffraction.

J. Rius

Barcelona

September 2008 


\section{Table of Contents}

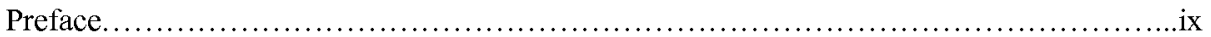

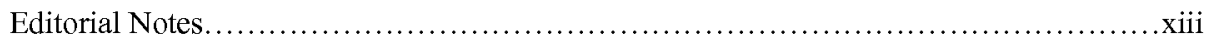

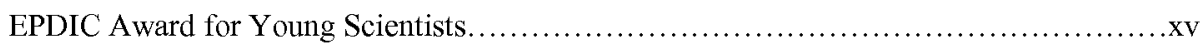

EPDIC Award for Distinguished Powder Diffractionists.................................

EPDIC Award for Distinguished Powder Diffractionists - Introductory Speech.............xix

\section{VOLUME I}

\section{The EPDIC11 Award for Young Scientists Lecture}

\section{A. L. Goodwin}

The crystallography of flexibility: Local structure and dynamics in framework materials.

\section{METHOD DEVELOPMENT AND APPLICATION}

\section{I.1 Determination of Crystal Structure}

R. Oishi, M. Yonemura, A. Hoshikawa, T. Ishigaki, K. Mori, S. Torii, T. Morishima, T. Kamiyama

New approach to the indexing of powder diffraction patterns using topographs.

\section{Y. I. Yakimov, E. S. Semenkin, I. S. Yakimov}

Two-level genetic algorithm for a full-profile fitting of X-ray powder patterns....

R. B. von Dreele

Characterization of proteins by powder diffraction

J. A. Doebbler, R. B. von Dreele

Macromolecular powder diffraction: Structure solution via molecular replacement...

\section{A. S. Wills}

Indexing magnetic structures and crystallographic distortions from powder diffraction:

Brillouin zone indexing. 


\section{I.2 Qualitative and Quantitative Phase Analysis}

R. Kleeberg

State-of-the-art and trends in quantitative phase analysis of geological and

raw materials.

P. S. Whitfield, L. D. Mitchell

The effects of particle statistics on Rietveld analysis of cement.

B. Peplinski, A. N. Fitch, A. Evans, R. M. Ibberson, D. M. Többens, L. M. D. Cranswick, I. Dörfel, F. Emmerling, R. Matschat

Structural characterization of a coarse-grained transparent silicon carbide powder by a combination of powder diffraction techniques.

\section{I.3 Analysis of Microstructure and Macrostress}

\section{I.3.1 Residual Stresses}

\section{A. Denks, T. Manns, Ch. Genzel, B. Scholtes}

An experimental approach to the problem of transforming stress distributions from the LAPLACE- into real space.

\section{I.3.2 Line Broadening Analysis}

R. Delhez, A. C. Vermeulen

A practical vision on Line Profile Analysis today and in the years to come .77

K. Beyerlein, A. Cervellino, M. Leoni, R. L. Snyder, P. Scardi

Debye equation versus Whole Powder Pattern Modelling: Real versus reciprocal space modelling of nanomaterials.

J. Martinez-Garcia, M. Leoni, P. Scardi

Diffraction contrast factor of dislocations: The case of scheelite $\mathrm{CaWO}$

A. Leineweber, E. J. Mittemeijer, A. C. Lawson, J. A. Roberts, J. A. Valdez, W. S. Kreher

Thermally induced microstrain broadening in polycrystalline hexagonal zinc....

A. Leineweber, T. Gressmann, M. Nikolussi, E. J. Mittemeijer

The $h k l$ dependences of microstrain and of macrostress-induced macrostrain; a comparison for intrinsically extremely anisotropic cementite, $\mathrm{Fe}_{3} \mathrm{C}$ 


\section{I.4 Texture}

A. Roatta, A. Fourty, R. E. Bolmaro

A primer on whole through processing simulation understanding of deformation and annealing textures in low carbon steels

R. E. Bolmaro, R. A. Renzetti, M. J. R. Sandim, H. R. Z. Sandim, J. W. Signorelli,

M. Ferrante

ECAP of Fe. Experiments and simulations of the in-elbow textures

A. Fourty, H.-G. Brokmeier, R. Martino, R. E. Bolmaro

Experiments and simulation evaluation in quartz veins textures in the

Guamanes shear belt, Córdoba Pampean Ranges, Central Argentina.

T. Kryshtab, A. Kryvko

The use of extinction phenomenon for investigation of textured thin film microstructure

\section{I.5 In-situ and Non-ambient Measurements}

B. Hinrichsen, R. E. Dinnebier, M. Jansen

On the intensity distribution within Debye-Scherrer rings. What is different in

high pressure experiments? Part I: Theory.

B. Hinrichsen, R. E. Dinnebier, M. Jansen

On the intensity distribution within Debye-Scherrer rings. What is different in high pressure experiments? Part II: Practical Application. ...

\section{INSTRUMENTAL}

\section{Z. Matěj, L. Nichtová, R. Kužel}

Coplanar grazing exit X-ray diffraction on thin polycrystalline films

J. te Nijenhuis, M. Gateshki, M. J. Fransen

Possibilities and limitations of X-ray diffraction using high-energy X-rays on a laboratory system

G. Kimmel, D. Mogityanski

Advantages and disadvantages of fast XRPD measurement by using image-plate and rotating anode source. 


\section{H. Toraya}

Diffracted-beam analyzer with multiple single crystals for high resolution parallel-beam X-ray diffraction.

M. A. Kojdecki, W. Mielcarek, K. Prociów, J. Warycha

Comparison of two standards for powder X-ray diffractometry

V. Saengsuwan, W. Klysubun, T. Bovornratanaraks, S. Rugmai

Synchrotron beamline optics for X-ray powder diffraction under high-pressure conditions at the Siam Photon Laboratory

S. Vratislav, M. Dlouhá, L. Kalvoda, M. Dráb

Texture research of metals and rocks on the KSN-2 neutron diffractometer.

M. J. Styles, D. P. Riley, J. Christoforidis, S. Olsen

Modular in-situ reaction chamber design for time resolved diffraction..... .201

\section{SOFTWARE}

P. S. Dubinin, I. S. Yakimov, O. E. Piksina, Y. I. Yakimov, A. N. Zaloga

RETRIEVE - a system for XRPD phase and structure analysis.

A. J. Florence, J. Bardin, B. Johnston, N. Shankland, T. A. N. Griffin, K. Shankland

Structure determination from powder data: Mogul and CASTEP.

H. Xu, Ch. M. Weeks, R. H. Blessing

Powder Shake-and-Bake Method.

K. Shankland, T. A. N. Griffin, J. van de Streek, J. C. Cole, N. Shankland, A. J. Florence, W. I. F. David

Large-scale distributed computing for accelerated structure solution .227 


\section{VOLUME II}

\section{MATERIALS}

\section{IV.1 Thin Layers}

L. Nichtová, R. Kužel, Z. Matěj, J. Šicha, J. Musil

Time and thickness dependence of crystallization of amorphous magnetron deposited $\mathrm{TiO}_{2}$ thin films.

M. Quaas, H. Wulff, O. Ivanova, C. A. Helm

Formation of nickel hydrides in reactive plasmas

J. Sheng, U. Welzel, E. J. Mittemeijer

Interdiffusion and stress development in $\mathrm{Ni}$ - $\mathrm{Cu}$ thin film diffusion couples.

\section{IV.2 Nanocrystalline and Amorphous Materials}

G. Dercz, L. Pajak, K. Prusik, R. Pielaszek, J. J. Malinowski

Nanocrystalline $\mathrm{MgO}$ powder materials prepared by sol-gel studied by

$\mathrm{X}$-ray diffraction and electron microscopy....

\section{IV.3 Metals and Alloys}

\section{IV.3.1 General}

E. Yu. Filatov, K. V. Yusenko, E. S. Vikulova, P. E. Plyusnin, Yu. V. Shubin XRD investigation and thermal properties of $\left[\operatorname{Ir}\left(\mathrm{NH}_{3}\right)_{6}\right]\left[\mathrm{Co}\left(\mathrm{C}_{2} \mathrm{O}_{4}\right)_{3}\right] \cdot \mathrm{H}_{2} \mathrm{O}$ and $\left[\mathrm{Co}\left(\mathrm{NH}_{3}\right)_{6}\right]\left[\operatorname{Ir}\left(\mathrm{C}_{2} \mathrm{O}_{4}\right)_{3}\right]$ - precursors for $\mathrm{Co}_{0.50} \mathrm{Ir}_{0.50}$ .263

K. V. Yusenko, I. V. Korolkov, S. A. Martynova, S. A. Gromilov $\mathrm{Ru}-\mathrm{Re}, \mathrm{Ru}-\mathrm{Os}$, and $\mathrm{Re}-\mathrm{Os}$ solid solutions - preparation under mild conditions, powder XRD investigation and phase diagram analysis.

P. Ari-Gur, G. Kimmel, J. W: Richardson, A. Huq

Application of neutron powder diffraction for the study of non-stoichiometric

$\mathrm{Ni}_{2} \mathrm{MnGa}$ based alloys. 


\section{IV.3.2 Microstructure, Stress and Texture}

V. E. Danilchenko, V. E. Iakovlev

Structural - phase state of maraging alloys.

H. J. Krzton, W. Pilarczyk

Rietveld quantitative phase analyses in iron alloys processed by mechanical alloying method.

A.V. Druker, C. Sobrero, J. Malarria, U. Garbe,H.-G. Brokmeier, R. E. Bolmaro

Effect of texture heterogeneities on the shape memory properties of

rolled Fe-Mn-Si SMA.

T. Goryczka

Texture and structure of grain boundary in Ni-Ti strip produced by twin

roll casting technique.

S. Popović, Ž. Skoko, G. Štefanić

Temperature dependence of microstructure of $\mathrm{Al}-\mathrm{Ag}-\mathrm{Zn}$ alloys....

A. M. Venter, C. P. la Grange, F. Hofmann, T-S. Jun, J. Belnoue, P. R. van Heerden, A. Evans, A. M. Korsunsky

Synchrotron investigations of non-uniformly shaped shot-peened samples.

\section{IV.4 Minerals and Inorganics}

\section{IV.4.1 Structural Changes, In-situ and Non-Ambient Investigations}

H. Boysen, I. Kaiser-Bischoff, M. Lerch, S. Berendts, A. Börger, D. M. Trots,

M. Hoelzel, A. Senyshyn

Structures and properties of variously doped Mayenite investigated by neutron

and synchrotron powder diffraction.

O. A. Bulavchenko, S. V. Cherepanova, S. V. Tsybulya

In situ XRD investigation of $\mathrm{CO}_{3} \mathrm{O}_{4}$ reduction.

J. Darul

Thermal instability of the tetragonally distorted structure of copper-iron materials....

A. Senyshyn, J. M. Engel, I. D. H. Oswald, L. Vasylechko, M. Berkowski

Powder diffraction studies of pressure-induced instabilities in orthorhombic $\mathrm{LnGaO}_{3}$..... .341 
J. Zabicky, G. Kimmel, E. Goncharov, F. Guirado

Magnesium titanate phases from xerogels by hot stage X-ray powder diffractometry....

A. F. Gualtieri, M. Lassinantii Gualtieri, C. Meneghini

In situ synchrotron powder diffraction study of the thermal decomposition of cement-

asbestos: Preliminary results

D. Jansen, F. Goetz-Neunhoeffer, J. Neubauer, W.-D. Hergeth, R. Haerzschel

In-situ XRD investigations of the influence of PDADMAC on ettringite formation in cement systems

\section{IV.4.2 Determination of Crystal Structure; Structure Refinement}

A. Dobrowolska, E. Zych

Structural and spectroscopic properties of $\mathrm{BaHfO}_{3}$ : Eu - the issue of the dopant

location in the host lattice...

N. V. Tarakina, T. A. Denisova, L. G. Maksimova, Y. V. Baklanova, A. P. Tyutyunnik, I. F. Berger, V. G. Zubkov, G. van Tendeloo

Investigation of stacking disorder in $\mathrm{Li}_{2} \mathrm{SnO}_{3}$

B. Gawel, W. Surga, W. Lasocha

$\mathrm{X}$-ray diffraction studies of transition metal molybdates.

W. Lasocha, M. Grzywa, M. Oszajca

Molybdates of p-bromoanilinium - synthesis and crystal structure of new catalytic materials.

R. A. Snellings, A. F. Gualtieri, J. Elsen

The Rietveld structure refinement of an exceptionally pure sample of clinoptilolite from Ecuador and its $\mathrm{Na}-, \mathrm{K}$-, and $\mathrm{Ca}$-exchanged forms

N. V. Tarakina, V. G. Zubkov, I. I. Leonidov, A. P. Tyutyunnik, L. L. Surat,

J. Hadermann, G. van Tendeloo

Crystal structure of the group of optical materials $\mathrm{Ln}_{2} \mathrm{MeGe}_{4} \mathrm{O}_{12}(\mathrm{Me}=\mathrm{Ca}, \mathrm{Mn})$.

H. Wang, H. Ehrenberg, A. Senyshyn, R. Schierholz, J.-C. Jaud, H. Fuess

Structural investigation on the $(1-\mathrm{x}) \mathrm{Pb}\left(\mathrm{Mg}_{1 / 3} \mathrm{Nb}_{2 / 3}\right) \mathrm{O}_{3}-\mathrm{xPbTO}_{3}(\mathrm{x}=0 ; 0.21)$

solid solution using powder diffraction 


\section{IV.4.3 Determination of Magnetic Structure, Magnetic Materials}

W. Nowicki

Synthesis, structure and magnetic properties of Fe-doped tetragonal $\mathrm{Li}_{0.95} \mathrm{Mn}_{2.05} \mathrm{O}_{4}$

\section{IV.4.4 Microstructure, Phase Analysis}

\section{A. Leineweber, M. Leoni}

Simulation of layer-faulting in $\mathrm{Nb} 2 \mathrm{Co} 7$ intermetallic compound using DIFFaX + ...

A. Argüelles, M. Leoni, J. A. Blanco, C. Marcos

Structure and microstructure of $\mathrm{Mg}$-vermiculite.

A. Sanz, J. Bastida, M. A. Kojdecki, A. Caballero, F. J. Serrano

Evolution of size and shape of mullite crystallites in triaxial porcelains

G. Cozzi, J. Bastida, A. Alvarez-Larena, M. A. Kojdecki, P. Pardo

Crystallite size of kaolinites as indicator of different geochemical types of bauxite.

S. Seufert, C. Hesse, F. Goetz-Neunhoeffer, J. Neubauer

Discrimination of bassanite and anhydrite III dehydrated from gypsum at different temperatures.

P. Piszora, J. Nawrocki, J. Darul, W. Nowicki, A. Evans

High resolution X-ray powder diffraction measurements of the wet rust from the drinking water distribution system.

B. Peplinski, C. Adam, M. Michaelis, G. Kley, F. Emmerling, F.-G. Simon

Reaction sequences in the thermo-chemical treatment of sewage sludge ashes revealed by X-ray powder diffraction - A contribution to the European project SUSAN.

J. A.G. Carrió, I. C. A. Dutra, M. C. Terence, R. Toledo, D. R. dos Santos

Quantitative analysis of clay materials and thermal treated bricks...

B. Simionescu, M. Aflori, M. Olaru

Mineral composition and stone conservation of cultural heritage building materials studied by PXRD

T. Zaremba

Anisotropic grain growth of bismuth titanate in molten salt fluxes. 


\section{IV.5 Organic Materials}

D. Jehnichen, D. Pospiech, S. Ptacek, K. Eckstein, P. Friedel, A. Janke,

C. M. Papadakis

Nanophase-separated diblock copolymers: Structure investigations on

PPMA- $b$-PMMA using X-ray scattering methods.

J. B. van Mechelen, R. Peschar, H. Schenk,

Structure and polymorphism of trans mono-unsaturated triacylglycerols 491

Author Index xxxi 

IV. MATERIALS

IV.1 Thin Layers 



\section{Time and thickness dependence of crystallization of amorphous magnetron deposited $\mathrm{TiO}_{2}$ thin films}

\section{Nichtová ${ }^{1, *}$, R. Kužel ${ }^{1}$, Z. Matěj ${ }^{1}$, J. Šícha ${ }^{2}$, J. Musil ${ }^{2}$}

${ }^{1}$ Department of Condensed Matter Physics, Faculty of Mathematics and Physics, Charles University in Prague, 12116 Praha 2, Czech Republic

${ }^{2}$ Department of Physics, Faculty of Applied Sciences, University of West Bohemia in Pilsen, Pilsen, Czech Republic

" nichtova@email.cz

Keywords: crystallization, titanium dioxide, in-situ measurements

Abstract. Titanium dioxide films have many remarkable properties, for example photocatalytic activity and hydrophilicity. However, these properties depend significantly on the crystallinity, phase composition and microstructure of the films. In this study, crystallization of amorphous films with different thickness $(50-2000 \mathrm{~nm})$ deposited on silicon substrates was investigated by XRD in-situ isochronal and isothermal annealing at different temperatures and compared with the post-annealing of both amorphous and nanocrystalline films. It was found that the crystallization depends strongly on the film thickness, especially below about $500 \mathrm{~nm}$ and it is slow for very thin films. The process can be well described by the Avrami equation modified by the initial time of crystallization. The parameters of the equation depend on the film thickness.

\section{Introduction}

The applications of titanium dioxide in powder or thin film form are very broad and of high interest. The films are used as ultra thin film coatings, nanostructured membranes, in electrochemistry and electrocatalysis, in microoptics and electrooptics, medicine, cosmetics and in photocatalysis. These applications particularly depend on the layers properties (stability, transparency, photoconductivity, photoactivity, etc.) and their microstructure (surface area, pore size distribution, particle size, crystallographic structure). The films can be prepared by several techniques but the magnetron deposition is favourable from the point of view of mechanical durability required for practical applications. Depending on the deposition conditions, the films can be prepared as amorphous or nanocrystalline.

For good photocatalytical properties and good hydrophilicity after UV irradiation, the crystalline or nanocrystalline form seems to be necessary. This can be obtained either by annealing of amorphous films or by appropriate selection of deposition parameters. Nanocrystalline anatase is supposed to have higher photocatalytic activity than other titania phases [1]. 
Microstructure and crystallization of amorphous and nanocrystalline magnetron deposited films were studied recently, for example in [2]. It was found that the amorphous $\mathrm{TiO}_{2}$ films can be crystallized at approximately $250{ }^{\circ} \mathrm{C}$ and their crystallite size was then immediately above $100 \mathrm{~nm}$. The films with the thickness below $200 \mathrm{~nm}$ crystallized at higher temperatures $\left(350^{\circ} \mathrm{C}\right)$. Tensile residual stresses have been found in crystallized films and relaxed after annealing at $500{ }^{\circ} \mathrm{C}$. Fiber texture was usually weak and of the (100) type for thicker films (above $1500 \mathrm{~nm}$ ) and 101 for thinner films.

Studies of the deposited nanocrystalline films with different thickness [3] showed that rutile phase grows on the interface with the substrate and it is replaced by anatase with increasing distance from the substrate. This was confirmed by coplanar grazing incidence depth profiling. Therefore thinner nanocrystalline films contained mainly rutile while in thicker films anatase was dominating. The stresses in as-deposited nanocrystalline films were small $(<$ $100 \mathrm{MPa}$ ) but complicated (triaxial, possible gradients) and the texture was not always fibre and it was inclined with respect to the surface. Unlike the annealed amorphous films, the crystallite size of the nanocrystalline films remains rather small (below $10 \mathrm{~nm}$ ) up to higher temperatures. Some increase of crystallites can be detected above $400{ }^{\circ} \mathrm{C}$ but rapid growth is observed only above $600{ }^{\circ} \mathrm{C}$. Transformation of anatase into rutile starts at about $700{ }^{\circ} \mathrm{C}$.

In this contribution, the results of in-situ measurements of a set of titanium dioxide thin films with different thickness are presented. Both isochronal and isothermal annealing was performed.

\section{Experimental conditions}

$\mathrm{TiO}_{2}$ films were sputtered by dual magnetron equipped with two $\mathrm{Ti}(99.5)$ targets of $50 \mathrm{~mm}$ in diameter and supplied by a dc-pulsed Advanced Energy Pinnacle Plus $+5 \mathrm{~kW}$ power supply unit operating in bipolar asymmetric mode at repetition frequency $\mathrm{f}_{\mathrm{r}}=100 \mathrm{kHz}$ and duty cycle $\tau / T=0.5$; here $\tau$ and $T$ are the length of pulse and the period of pulses. Films were deposited on unheated silicon $(100)$ substrates $(15 \times 10 \times 1 \mathrm{~mm})$ at substrate to target distance $\mathrm{d}_{\mathrm{s}-\mathrm{t}}=100 \mathrm{~mm}$, total working pressure $\mathrm{p}_{\mathrm{T}}=0.5 \mathrm{~Pa}$, average pulse discharge current $\mathrm{I}_{\mathrm{da} 1,2}=1.5 \mathrm{~A}$ and average pulse power density $\mathrm{W}_{\mathrm{da}} \approx 20 \mathrm{Wcm}^{-2}$. Further details on dual magnetron system are given elsewhere [4].

Two identical sets of samples with different thikness were measured: $48 \mathrm{~nm}, 100 \mathrm{~nm}, 130$ $\mathrm{nm}, 180 \mathrm{~nm}, 300 \mathrm{~nm}, 440 \mathrm{~nm}, 630 \mathrm{~nm}$ and $800 \mathrm{~nm}$, respectively.

The room temperature measurements were performed mainly on Philips (Panalytical) X'Pert MRD in parallel beam setup, $2 \square$ scans with the angles of incidence $0.5-1.5^{\circ}$ with a parallel plate collimator placed in the diffracted beam and the Goebel mirror inserted in the primary beam. The measurements of residual stresses were carried out by using a polycapillary and Eulerian cradle by the $\sin ^{2} \psi$ method for several different peaks. In-situ high-temperature measurements were performed in MRI high-temperature chamber with both direct and radiant heating. The films on Si substrate were fixed on the top of PtRh strip heater with a silver paste. Temperature was measured by the thermoucouple fixed to the bottom of the strip. Calibration of actual temperature on the film surface was done by using measurement of $\mathrm{Ag}$ and $\mathrm{Al}$ layer on the same substrates. Both materials, $\mathrm{Al}$ in particular, have rather high thermal expansion coefficients and consequently the peak positions are very sensitive to the changes of temperature. The films were heated primarily by radiant heater. The temperatures of the films were about $8-10^{\circ}$ higher than the measured one, in the temperature ranges used. 

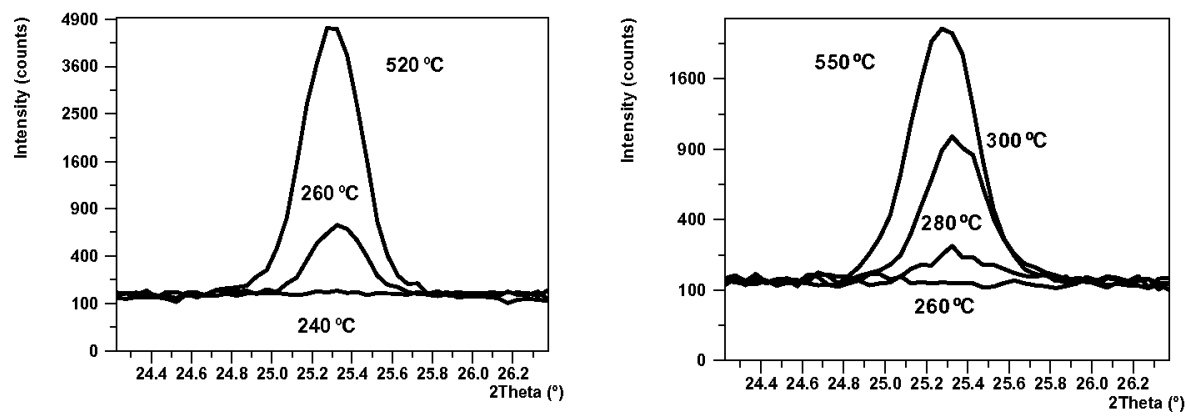

Figure 1. Evolution of XRD line profiles 101 of anatase with temperature for two thinnest films (100 nm, right; $48 \mathrm{~nm}$, left).

Below, the temperatures after the correction are shown. Parallel beam geometry with the Goebel mirror, collimator and point proportional detector were applied, respectively, and the angle of incidence of $1^{\circ}$ was used for all the in-situ HT measurements.

Each time after setting the temperature, alignment of the specimen was checked by measurement of primary beam and specimen position was corrected if necessary. Even though the parallel beam is not sensitive to the specimen displacement error, the displacement can result in a parallel shift of the beam spot which should be in the specimen centre. The alignment took always less than 3 minutes.

For in-situ measurements only selected diffraction peaks were measured. They were fitted with the Pearson VII function and the peak positions, integrated intensities and integral breadths were determined. Total pattern fitting was used for the evaluation of the whole diffraction patterns taken at room temperature. More details about the method can be found in $[5,6]$.

\section{Results}

\section{Isochronal annealing}

Isochronal annealing experiments started always at $180{ }^{\circ} \mathrm{C}$ and were performed with a step of $20^{\circ} \mathrm{C}$. Only $(101)$ peak of anatase was measured and two subsequent scans were taken at one temperature (12 minutes each, 30 minutes at the temperature with alignment check). They confirmed the results found previously on the post-annealed samples, i.e. that higher temperature is necessary for crystallization of very thin films. The present study allowed to make more specific conclusions. Some results are shown on figure 1. The crystallization temperatures found in this experiment could be called "temperatures of fast crystallization" when, of course, the definition of "fast" is somewhat arbitrary. This fast crystallization of the order of minutes appeared at $220^{\circ} \mathrm{C}$ for thicker films ( $300 \mathrm{~nm}$ and more) and were higher for the thin films $\left(240^{\circ} \mathrm{C}\right.$ for $180 \mathrm{~nm}, 260^{\circ} \mathrm{C}$ for $100 \mathrm{~nm}, 280^{\circ} \mathrm{C}$ for $48 \mathrm{~nm}$ thick, respectively).

\section{Isothermal annealing}

Time dependences of crystallization were measured at slightly lower temperatures $\left(180^{\circ} \mathrm{C}\right.$, $215^{\circ} \mathrm{C}$ ) than was the temperature of "fast" crystallization previously found on the postannealed samples. As it has been found, the crystallization occurs as well but it is very slow and it takes several days for thinner films. 
This allowed us to perform long time experiment and to follow the crystallization dependences in detail even with laboratory equipment. The following anatase peaks were watched: $101,103,004,112$, for two samples also 200 and 116 . However, it must be noted that only 101,004 and 200 were strong enough for obtaining statistically good data. Normalized intensities of 101 peak for different film thickness are shown on figure 2. The normalization was performed with respect to the maximum value reached for each sample after full crystallization.

It was found, that the process can be well described by the modified Avrami equation [7, 8] that is applied to the integrated intensities $I$ of the diffraction peaks, $\left.I=1-\exp \left[-b\left(t-t_{0}\right)^{n}\right)\right]$. Examples of the fit of this equation with three free parameters $-b, t_{0}, n$ to experimental data is shown on figure 3 . The fits were very good for all thicker samples, for some samples thinner than about $200 \mathrm{~nm}$, the intensities during the early stage of crystallization were somewhat above the values expected from the Avrami equation. The parameters of the Avrami equation fitted to the experimental data for the films of different thickness show clear dependence on film thickness. On figure 4, these dependences for the initial time of crystallization $t_{0}$ and exponent $n$ are shown. The initial time is related to the first appearance of any diffracted intensity above the background level at the peak position but was obtained by the leastsquares fits of the equation to the full intensity vs. time curve. The exponent was in the range $2-2.5$ and slightly increased with the film thickness (figure 5). These low values indicate two dimensional character of the crystallite growth [7]. The initial time $t_{0}$ of crystallization (nonzero intensity) increases nearly exponentially with the decreasing thickness while the slope $b$ (effective rate constant) increased significantly for the thicker films. Typical time necessary for the crystallization of the whole film volume varied from several hours for the thicker layers to about ten days for the thinnest films, for the mentioned temperatures.

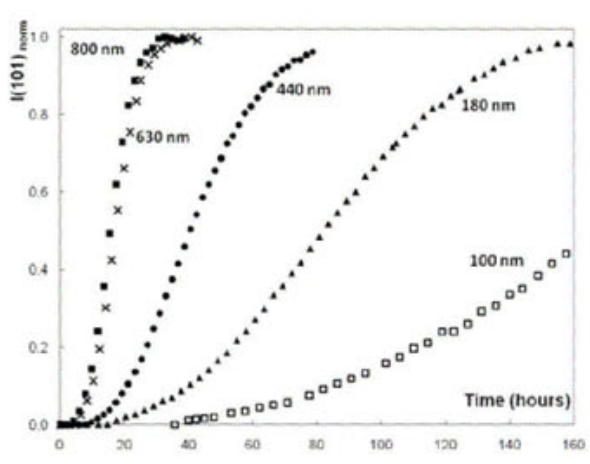

a)

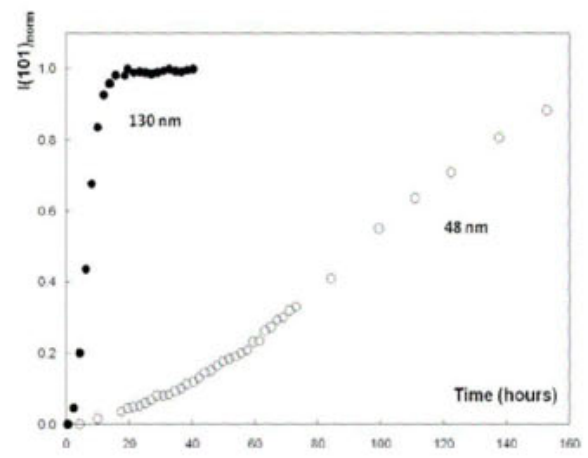

b)

Figure 2. Evolution of normalized integrated intensity of 101 peak of anatase with time at $180{ }^{\circ} \mathrm{C}$ (a) and $215^{\circ} \mathrm{C}(\mathrm{b})$, respectively for different film thickness as indicated in the graph. 


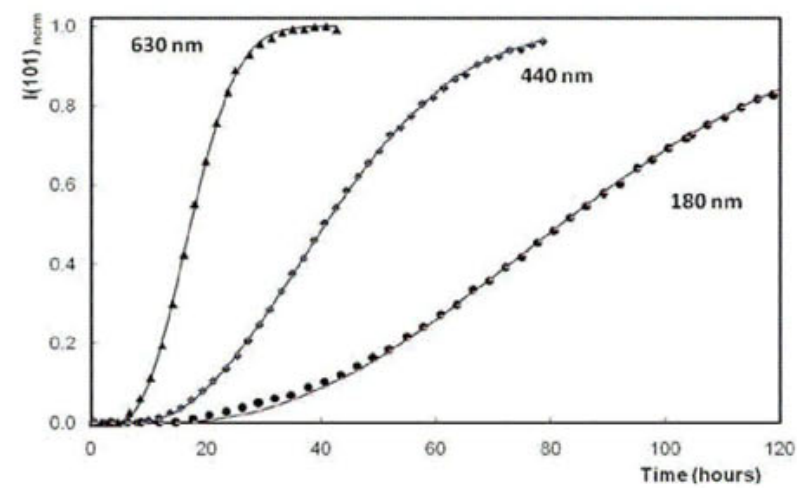

Figure 3. Dependence of normalized integrated intensity of 101 peak on annealing time at $180{ }^{\circ} \mathrm{C}$ (dots) and fitted with modified Avrami equation (line) for several films with different thickness.

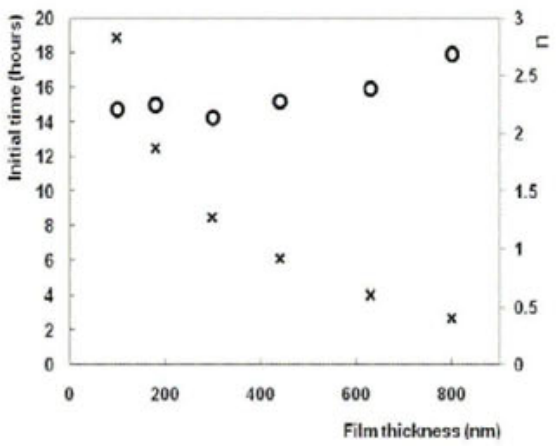

Figure 4. Dependence of parameters of the modified Avrami equation on the film thickness (initial time of crystallization - left axis, crosses; exponent $n-$ right axis, circles).

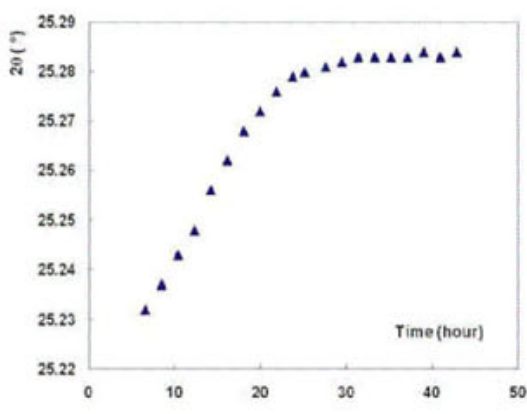

Figure 5. Dependence of the peak position for 101 reflection of anatase and the film $630 \mathrm{~nm}$ thick. Annealing temperature was $180^{\circ} \mathrm{C}$.

Weak texture was changing during the crystallization. At the beginning, the crystallites with the $(001)$ orientation were developed. However, after complete crystallization, the texture was weak except the very thin films (below $100 \mathrm{~nm}$ ). Significant shifts of diffraction peaks with the temperature were observed and tensile residual stresses were confirmed by the $\sin ^{2} \psi$ method for different diffraction peaks on the samples after annealing at room temperature. The stresses significantly decrease with the increasing film thickness. Studies of texture and stress changes are still under way. Line profile analysis indicated the growth of relatively large crystallites $(100 \mathrm{~nm})$ already at the beginning of crystallization unlike the films which were deposited as nanocrystalline with the crystallite size of 5-10 nm which remained nanocrystalline to relatively high temperatures $\left(600^{\circ} \mathrm{C}\right)[5]$. Line widths did not change significantly and systematically throughout the whole crystallization process from the very beginning of the diffraction measurable peak. This means that there is no indication of significant number of nanocrystals any time during the crystallization. 
Studies of the film thickness dependence of crystallization are surprisingly quite rare but they always indicate an increase of the crystallization temperature with decreasing thickness [e.g. $9, \mathrm{Sb}_{70} \mathrm{Te}_{30}$ films]. The explanations of the effect are not unique and based on epitaxial growth [Be-ferrite, 10] or thickness dependent interfacial energy [11]. Our investigations in this field continue.

\section{Summary}

By in-situ high temperature XRD measurements it was confirmed that the crystallization of amorphous $\mathrm{TiO}_{2}$ thin films depends strongly on the film thickness and it is rather slow for the films thinner than about $400 \mathrm{~nm}$. Evolution of the integrated intensities of anatase diffraction peaks with annealing time follows the Avrami equation modified by the introduction of initial time of crystallization. This time increases rapidly with decreasing thickness of the film. The exponent of the Avrami equation may indicate two-dimensional character of crystallite growth. Crystallites seem to be quite large already from the beginning of crystallization. Both the behavior of XRD line broadening and exponent of the Avrami equation indicate continuous nucleation with 2D growth, i.e. gradual increase of number of fast growing grains. Variation of texture and residual stress during crystallization was observed.

\section{References}

1. Gaho, L. \& Zhang, Q, 2001, Scr. Mater., 44, 1195.

2. Kužel, R., Nichtová, L., Matěj, Z., Heřman, D., Šícha, J. \& Musil, J., 2007, Z. Kristallogr. Suppl., 26, 247.

3. Kužel, R., Nichtová, L., Matěj, Z., Heřman, D., Šícha, J. \& Musil, J., 2007, Z. Kristallogr. Suppl., 26, 241.

4. Musil, J., Heřman, D. \& Š́ícha, J., 2006, J. Vac. Sci. Technol. A, Vol. 24, (3), 521.

5. Kužel, R., Nichtová, L., Matěj, Z., Šícha, J. \& Musil, J., 2008, Z. Kristallogr., in press.

6. Matěj, Z., Nichtová, L. \& Kužel, R., 2008, Materials Structure in Chemistry, Biology, Physics and Technology, 15, (1), 46,

open acces at http://www.xray.cz/ms.

7. Avrami, M.J., 1939, J. Chem. Phys., 7, 1103.

8. Avrami, M.J., 1941, J. Chem. Phys., 8, 212.

9. Her, Y-C. \& Hsu, Y-S., 2008, J. Non-Cryst. Sol, 354, 3129.

10. Cho, T.S., Doh, S.J., Je, J.H. \& Noh, D.Y., 1999, J. Appl. Phys., 86, 1958.

11. Xiaoqian, W., Luping, S. Chong, C.T., Rong, Z. \& Koon, L.H., 2007, Jpn. J. Appl. Phys., 46, 2211.

Acknowledgements. The work is supported by the Grant Agency of the Czech Republic under number 106/06/0327, Grant Agency of Charles University (258200) and also as a part of the research plans MSM 0021620834 and MSM 4977751302 financed by the Ministry of Education of the Czech Republic. 


\section{Formation of nickel hydrides in reactive plasmas}

\section{Quaas ${ }^{1}$, H. Wulff ${ }^{1, *}$, O. Ivanova ${ }^{2}$, C. A. Helm²}

${ }^{1}$ University of Greifswald, Institute of Biochemistry, Felix-Hausdorff-Strasse 4, D-17487 Greifswald, Germany,

${ }^{2}$ University of Greifswald, Institute of Physics, Felix-Hausdorff-Strasse 6, D-17487 Greifswald, Germany

*wulff@echemie.uni-greifswald.de

Keywords: nickel hydrides, plasma, X-ray diffractometry, X-ray reflectometry, kinetics

Abstract. $20 \mathrm{~nm}$ thick nickel films were exposed to argon-hydrogen microwave plasma using different negative bias voltages to study the hydride formation. The formed nickel hydride films were investigated by grazing incidence X-ray diffractometry (GIXD) to control the phase formation. Thickness and density were determined by X-ray reflectometry (XR). The effect of hydrogen plasma depends on the applied negative bias voltage. Without external bias voltage no chemical reaction occurs. At negative substrate voltages $(-10 \mathrm{~V},-25 \mathrm{~V}$, $50 \mathrm{~V},-75 \mathrm{~V}$ ) a hexagonal $\mathrm{Ni}_{2} \mathrm{H}$ phase is formed in a first quick reaction step. In subsequent plasma chemical reactions this $\mathrm{Ni}_{2} \mathrm{H}$ is transformed into cubic $\mathrm{NiH}$. The reaction rate of the $\mathrm{NiH}$ formation increases with increasing negative bias voltage. The kinetic processes are discussed using a modified isoconversional kinetic analysis which confirms competitive reactions during the $\mathrm{NiH}$ formation.

\section{Introduction}

Since many years various metal-hydride systems have been studied. Metal hydrides find a wide range of applications of which the most prominent is the reversible hydrogen storage. This is of great importance in view of possible future hydrogen economy in which hydrogen would replace fossil fuels as an energy carrier. Most binary metal hydrides are synthesized by a solid gas reaction between the metal and hydrogen. While some metals and intermetallic compounds easily take up hydrogen, other form hydrides only under high hydrogen pressure up to $10^{9} \mathrm{~Pa}$. Nickel forms hydrides only under high hydrogen pressure (>0.5 GPa) $[1,2]$. The p-T phase diagram of Ni-H in temperatures up to $630 \mathrm{~K}$ and pressures up to $1.8 \mathrm{GPa}$ of gaseous hydrogen was given by Ponyatovskiis group [1]. With rising temperature the difference between the formation and decomposition pressure decreases and disappears at $\mathrm{T}=540$ $\mathrm{K}$. The decomposition pressure at room temperature is $\leq 0.3 \mathrm{GPa}$. The nickel hydrides formed at high pressures were shown to have close-packed metal sublattices with f.c.c. structures, in which hydrogen occupies octahedral interstitial positions. Irodova et al. [3] confirmed the existence of $\mathrm{NiH}_{\mathrm{x}}$ with $\mathrm{x}=1.05$ and $\mathrm{a}_{0}=3.740 \AA$ by neutron diffraction at $\mathrm{T}=$ $120 \mathrm{~K}$. Oesterreicher et al. [4] describe high pressure formed nickel hydrides $\mathrm{NiH}_{\mathrm{x}}$ with $x=$ 
0.6 to $x=0.7$ and Zhebelev et al. [5] the f.c.c. structure of $\mathrm{NiD}_{0.75}\left(\mathrm{a}_{0}=3.720 \AA\right)$ synthesized at high pressure. Khodyrev et al. [6] reported on structure and composition of a hexagonal nickel hydride phase formed when $\mathrm{Ni}$ is sputtered in hydrogen. Hemenger and Weik $[7,8]$ have fabricated hexagonal metallic nickel films. The hexagonal lattice parameters reported there are $\mathrm{a}_{0}=2.622 \AA$, and $\mathrm{c}_{0}=4.320 \AA$. Ni is listed as crystallising in the h.c.p. modification (SG 194). The density was calculated to $7.58 \mathrm{~g}^{-\mathrm{cm}^{-3}}$. The growth conditions are extremely critical for the formation of the h.c.p. structure, which was found to be unstable at room temperature.

A specific challenge in plasma science is the investigation of chemical reactions in solid surface layers as a response to external plasma parameters.

In the focus of this paper are compounds produced from metallic nickel and hydrogen during and after argon-hydrogen plasma exposure using different negative bias voltage. It is difficult to directly modify the behaviour of the neutral particles, but charged particles can be controlled by changing the local electric field, and this is the basis of the bias method. Negative substrate bias accelerates positive ions $\left(\mathrm{Ar}^{+}, \mathrm{Ar}_{2}{ }^{+}, \mathrm{Ar}^{2+}, \mathrm{ArH}^{+}\right)$towards the Ni films. The flux and energy of all positive charged particles bombarding the nickel substrate is modified in steps of $-25 \mathrm{~V}$.

The plasma included reactions were investigated using grazing incidence X-ray diffraction (highly asymmetric Bragg case) and X-ray reflectometry.

\section{Experimental}

Thin pure Ni coatings $\left(20 \mathrm{~nm}\right.$ ) were deposited on Si substrates with $800 \mathrm{~nm} \mathrm{SiO}_{2}$ and $1 \mathrm{~nm}$ $\mathrm{Cr}$ buffer layers. These films were treated in a microwave plasma source with $10 \mathrm{secm} \mathrm{Ar} /$ $10 \mathrm{secm} \mathrm{H}_{2}$ gas flow without substrate heating. Plasma power and gas pressure were kept constant at $700 \mathrm{~W}$ and $50 \mathrm{~Pa}$, respectively. The substrate bias was varied in steps of $25 \mathrm{~V}$ from $0 \mathrm{~V}$ to $-100 \mathrm{~V}$. Within 15 minutes the energy influx by the $\mathrm{Ar} / \mathrm{H}_{2}$ plasma implements a substrate temperature of about $450 \mathrm{~K}$, independent of the used substrate voltage. The samples were characterised during and after plasma exposure by GIXD (asymmetric Bragg case, incidence angle $\omega=0.5^{\circ}$ ) regarding position, profile and intensities of observed Bragg angles and XR regarding thickness, density and roughness. Both GIXD and XR were performed on a Siemens D 5000 diffractometer equipped with a special reflectometry sample stage for the reflectometry measurements and a special parallel beam attachment (parallel plate collimator) for diffractometry investigations. $\mathrm{Cu} \mathrm{K}_{\alpha}$ radiation $(40 \mathrm{kV}, 40 \mathrm{~mA})$ was used.

\section{Results and discussion}

Table 1 gives an overview on the formed nickel hydride phases during argon-hydrogen plasma exposure. Plasma treatment without substrate voltage does not form a new phase, but a clear shift in the peak positions of the Ni reflections to smaller angles can be observed as well an increase of the peak maximum intensity accompanied by a smaller FWHM. The peak shift can be associated with lattice strain induced by external plasma forces which could be explained by the insertion of a small amount of hydrogen. Antonov [1] reports on the existence of $\mathrm{NiH}_{\mathrm{x}}$ with $\mathrm{x}=0.01$ at room temperature up to a hydrogen pressure of $0.4 \mathrm{GPa}$. At higher pressure an abrupt increase in hydrogen solubility from $\mathrm{x}=0.01$ to $\mathrm{x} \approx 1$ is observed. The observed shifts are remarkably different compared to the Ni reflections shift in an argon- 
oxygen plasma. $\mathrm{Ar} / \mathrm{O}_{2}$ plasma causes besides the formation of $\mathrm{NiO}$ a shrinking of the $\mathrm{Ni}$ unit cell which can be explained by the formation of vacancies activated by ion bombardment [9].

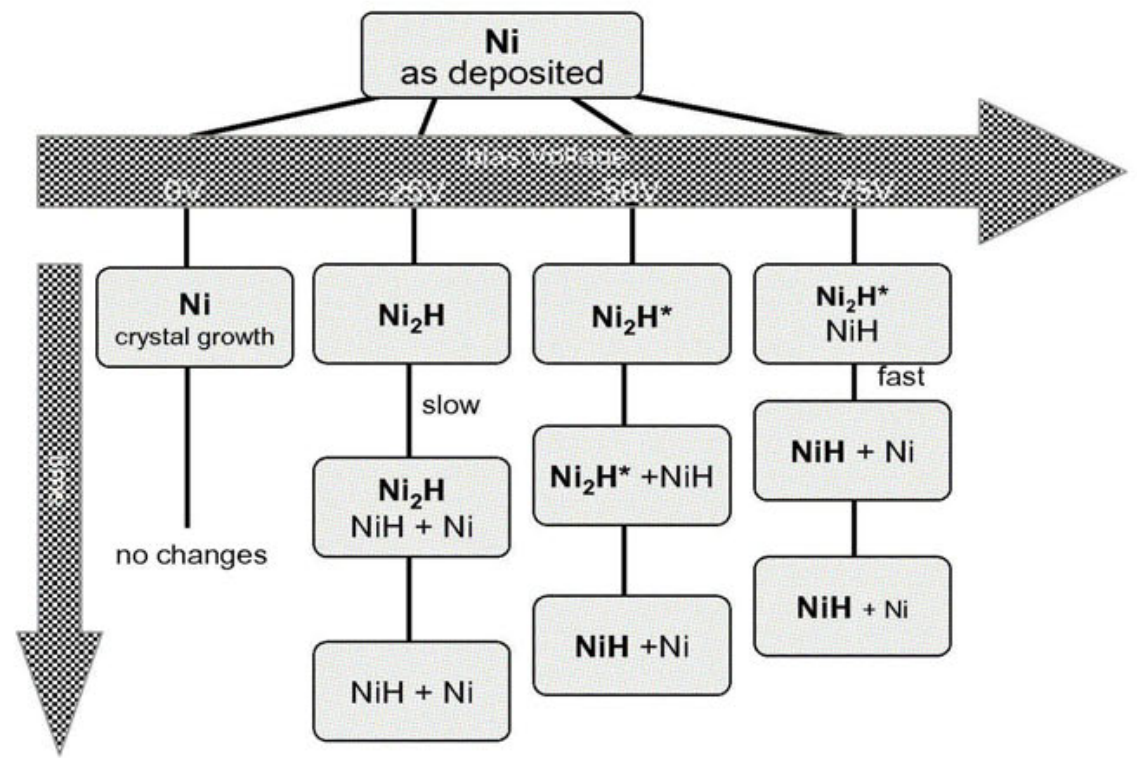

Figure 1. Results of hydrogen plasma treatment.

$\mathrm{Ar} / \mathrm{H}_{2}$-plasma treatment with applied negative bias voltage changes the metallic nickel films into a hexagonal $\mathrm{Ni}_{2} \mathrm{H}_{\mathrm{x}}$ phase. Figure 2 shows part of the $\mathrm{X}$-ray patterns of films treated at different bias voltages. Independent of the used bias voltage the phase transformation takes place during the first 15 minutes of plasma exposure. Increasing bias $(-10 \mathrm{~V}$ to $-50 \mathrm{~V})$ decreases drastically the intensity of the $\mathrm{Ni}_{2} \mathrm{H}_{\mathrm{x}} 002$ reflections. The X-ray pattern of the $-25 \mathrm{~V}$ sample corresponds to the crystal structure of $\mathrm{Ni}_{2} \mathrm{H}$ described by Khodyrev et al. [6].The calculated density is $7.45 \mathrm{~g} \cdot \mathrm{cm}^{-3}$. However, XR measurements of the plasma treated hexagonal $\mathrm{Ni}_{2} \mathrm{H}_{\mathrm{x}}$ phase results in densities of about $8.2 \mathrm{~g} \cdot \mathrm{cm}^{-3}$ and $8.91 \mathrm{~g} \cdot \mathrm{cm}^{-3}$ for the as deposited nickel films (identically with the density of bulk nickel). The film thicknesses grow about 20 $\%$ independent on the bias voltage. The volume of unit cells keeps constant in the margin of error at about $26.2 \AA^{3}$. The measured density of the plasma treated nickel hydrides is significantly higher than the calculated value for $\mathrm{Ni}_{2} \mathrm{H}$ [6] and the calculated density of h.c.p. nickel $[7,8]$. The measured density of the h.c.p. $\mathrm{Ni}_{2} \mathrm{H}_{\mathrm{x}}$ can be explained using the following assumptions. The f.c.c. $\mathrm{Ni}\left(\mathrm{a}_{0}=3.524 \AA\right)$ is transformed into in a triple hexagonal cell. The atomic positions change to

$\begin{array}{lllll}\mathrm{Ni}(1) & 0 & 0 & 0 & \mathrm{SOF}=1 \\ \mathrm{Ni}(2) & 1 / 3 & 2 / 3 & 1 / 3 & \mathrm{SOF}=1 \\ \mathrm{Ni}(3) & 2 / 3 & 1 / 3 & 2 / 3 & \mathrm{SOF}=1\end{array}$

with $\mathrm{Z}=3, \mathrm{a}_{0}=2.411 \AA, \mathrm{c}_{0}=6.103 \AA$ and $\mathrm{V}_{\mathrm{UC}}=32.817 \AA^{3}$. This is in agreement with the space group P3ml (No. 156) (Wyckoff $1 \mathrm{a}, 1 \mathrm{~b}, 1 \mathrm{c}$ ). Replacing $80 \%$ of the Ni (1) position by 
$\mathrm{H}$ atoms, the density decreases to $8.2 \mathrm{~g} \cdot \mathrm{cm}^{-3}$. The Ni replacement by $\mathrm{H}$ in $\mathrm{Ni}$ (1) is in accordance with the measured X-ray patterns. The detected lattice constants are $\mathrm{a}_{0}=2.656(3) \AA$ and $\mathrm{c}_{0}=4.30(2) \AA$. The clearly smaller c-axis is caused by the replacement/substitution of the $\mathrm{Ni}$-atoms by the smaller $\mathrm{H}$-atoms.

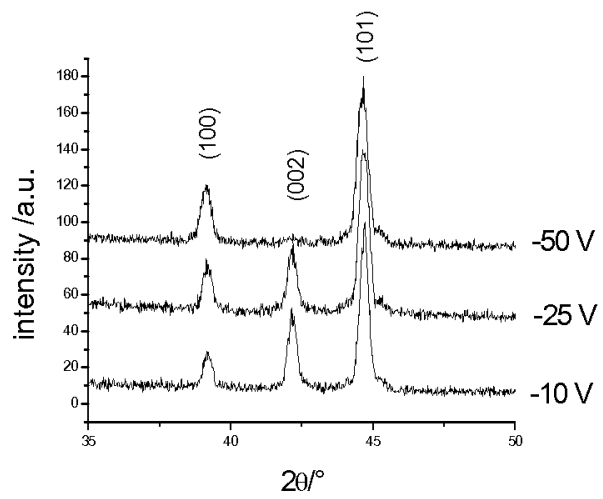

Figure 2a. X-ray pattern of $\mathrm{Ni}_{2} \mathrm{H}$ after 15 minutes plasma exposure at different substrate voltages.

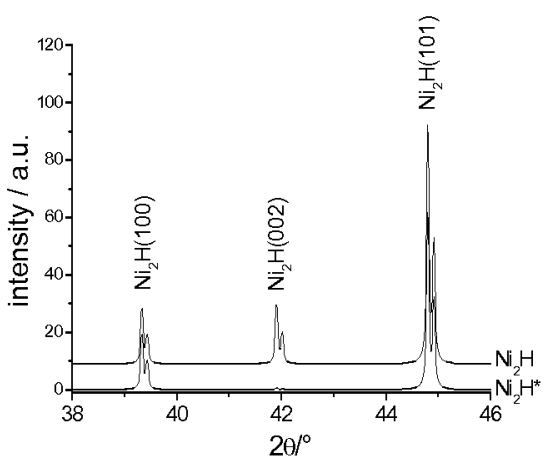

Figure $2 b$. Simulated $X$-ray pattern

(1) $\mathrm{Ni}(2) \mathrm{z}=0.25, \mathrm{Ni}(3) z=0.75$

(2) $\mathrm{Ni}(2) \mathrm{z}=0.4, \mathrm{Ni}(3) \mathrm{z}=0.68$.

From the density measurements we can draw the conclusion that the formed nickel hydride should be described by the formula $\mathrm{Ni}_{2.2} \mathrm{H}_{0.8}(\mathrm{Z}=3)$ or $\mathrm{NiH}_{0.36}$. Taking into account this chemical composition and the experimental measured density we can calculate the molecular volume $\mathrm{V}_{\mathrm{NiH}}$ for $\mathrm{NiH}_{0.36}$. The ratio $\mathrm{V}_{\mathrm{NiH}} / \mathrm{V}_{\mathrm{Ni}}=1.20$ with $\mathrm{V}_{\mathrm{Ni}}$ the molecular volume of pure metallic nickel. This is in excellent agreement with the measured increase of the film thickness.

The ion impact and the resulting collision cascades cause an amount of structural reordering in the Ni films. Negative bias induces an increasing separation of the hydrogen planes (Ni (1) in the model) from the atomic nickel layers $\mathrm{Ni}(2)$ and $\mathrm{Ni}$ (3). The Ni layers close ranks in $\mathrm{z}$ from 0.25 and 0.75 to 0.4 and 0.68 , respectively. By shifting of the z-positions of $\mathrm{H}, \mathrm{Ni}(1)$, $\mathrm{Ni}(2)$ and $\mathrm{Ni}(3)$ we can simulate the X-ray pattern as observed in the experiments (figures $2 \mathrm{a}, 2 \mathrm{~b})$. The hexagonal nickel hydride phases characterised by the disappearance of the 002 reflection are labelled $\mathrm{Ni}_{2} \mathrm{H}^{*}$. Bias voltage $-75 \mathrm{~V}$ produces directly (that is in the first ten minutes) a new nickel hydride, the f.c.c. $\mathrm{NiH}_{\mathrm{x}}$ with a lattice constant of $\mathrm{a}_{0}=3.725 \AA$.

$\mathrm{X}$-ray patterns show that continued plasma exposure changes the phase composition in the nickel films. At each operating bias voltage we find a decomposition of the hexagonal $\mathrm{Ni}_{2} \mathrm{H}_{\mathrm{x}}$ phase, but the decomposition rate is strongly influenced by the bias voltage (figure 3a). Vice versa the $\mathrm{NiH}$ formation increases with increasing negative bias voltage. Up to $-75 \mathrm{~V}$ the decomposition products are f.c.c. NiH besides metallic f.c.c. Ni. Figure $3 \mathrm{~b}$ shows the decomposition and formation of the nickel hydride phases at $-75 \mathrm{~V}$ bias voltages. The unit cell volume of the f.c.c NiH phase is higher than that of pure Ni. For metals of group VIII, the hydrogen volume is practically incompressible and is about $2.7 \AA^{3}[10]$. Taking this into 
account, the composition of the f.c.c nickel hydride can be specified from the unit cell volumes as $\mathrm{NiH}_{0.73}$. This value corresponds to the high pressure phases described in [4].

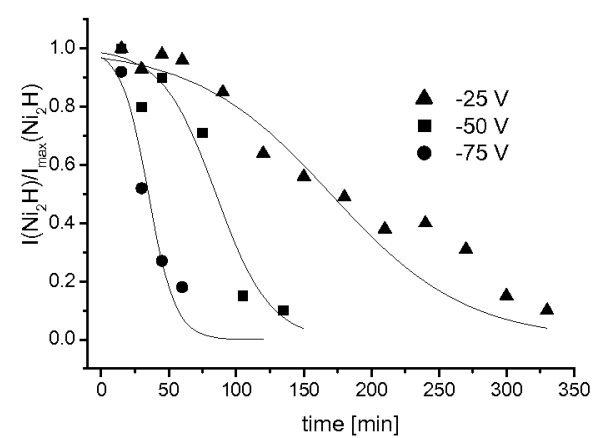

Figure 3a. Decomposition rate of $\mathrm{Ni}_{2} \mathrm{H}$ (defined as the normalized integral intensity of $\mathrm{Ni}_{2} \mathrm{H} 100$ reflection) at different bias voltages.

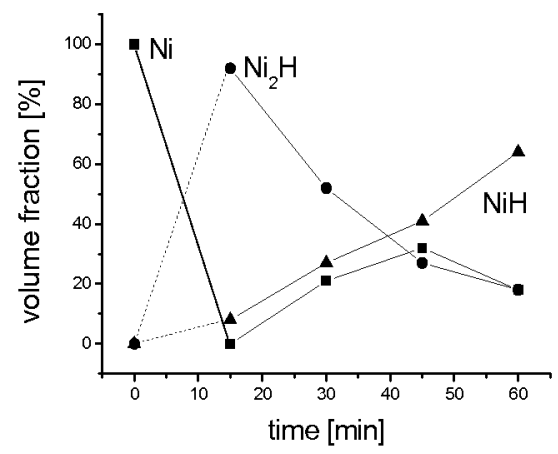

Figure 3b. Change in phase composition during plasma exposure at bias voltage of $-75 \mathrm{~V}$.

The macroscopic kinetics of these processes shown in figure $3 \mathrm{~b}$ is complex as it includes information about simultaneously occurring multiple steps as demonstrated in the following chemical equations:

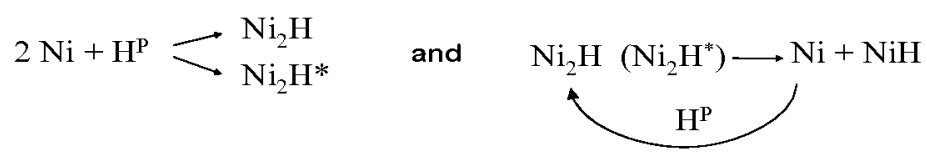

Isoconversional methods [11] allow complex (i.e. multi step) processes to be detected via a variation of the activation energy $\mathrm{E}_{\mathrm{A}}$ with $\alpha$ (extend of conversion). Conversely, independence of $E_{A}$ on $\alpha$ is a sign of a single-step process. From the diffractometry measurements $\alpha$ can be calculated from the integral intensity of $\mathrm{Ni}_{2} \mathrm{H}_{\mathrm{x}}$ reflection at the time $\mathrm{t}$ divided by the maximum $\mathrm{Ni}_{2} \mathrm{H}_{\mathrm{x}}$ intensity (see also figure 3a). In the classical isoconversional analysis $\alpha$ is measured time dependent at different temperatures $\mathrm{T}$ and $\mathrm{E}_{\mathrm{A}}$ is determined with respect to equation (1)

$$
-\ln t_{\alpha, i}=\ln \left[\frac{A_{\alpha}}{g(\alpha)}\right]-\frac{E_{A, \alpha}}{R T_{i}} \longrightarrow \ln t_{\alpha, i}=\ln \left[\frac{A_{\alpha}}{g(\alpha)}\right]-\frac{E_{A, \alpha}}{R T_{i}}
$$

In plasma chemical processes bias voltages $\mathrm{U}_{\text {bias }}$ influence the energy flux to the substrate. Hence we can take bias voltage analogue to the temperature in the classical isoconversional theory. Therefore, the slope from $\ln (\mathrm{t})$ vs. $\mathrm{U}_{\text {bias }}$ with $\mathrm{t}$ the time necessary to reach a certain value of $\alpha$ corresponds to a "plasma activation energy" $E_{A}{ }^{\text {(plasma) }}$. As shown in figure 4 the activation energy depends on the conversion rate that means the kinetics of the $\mathrm{NiH}_{0.73}$ formation results from a competition reaction. 


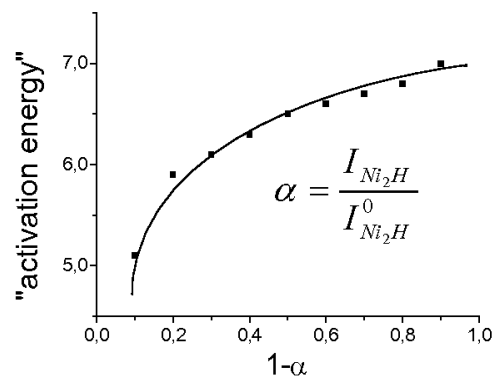

Figure 4. "Activation energy" of the degradation of $\mathrm{Ni}_{2} \mathrm{H}$, caused by different bias voltages.

\section{Conclusions}

Two different nickel hydride compounds were formed under $\mathrm{Ar} / \mathrm{H}_{2}$ plasma exposure at low pressure of $50 \mathrm{~Pa}$ and temperatures of $500 \mathrm{~K}$ using different bias voltages. These phases are stable at normal pressure and temperatures up to $750 \mathrm{~K}$. The hydrogen incorporation takes place discontinuously. h.c.p. nickel hydride $\left(\mathrm{NiH}_{0.38}\right)$ is formed in a first quick reaction step. The kinetics of the f.c.c. $\mathrm{NiH}_{0.73}$ formation results from a competition reaction.

\section{References}

1. Antonov, V.E., 2002, J. Alloys Comp., 330-332, 110.

2. Baranowski, B. \& Filipek, S.M., 2005, J. Alloys Comp., 404, 2.

3. Irodova, A.V., Glazkov, V.P., Somenkov, V.A, Shil'shtein, S.Sh. Antonov, V.E. \& Pontyatovski, E.G., 1988, Sov. Phys. Crystallogr., 33, 453.

4. Oesterreicher, H., Clinton, J. \& Bitter, H., 1976, Mat. Res. Bull., 11, 1241.

5. Zhebelev, V.P., Somenkov, V.A., Ponyatovski, E.G., Shil'shtein, S.Sh. \& Belash, I.T., 1978, Izvestiya Akademii Nauk SSSR, Neorganicheskie Materialy, 14 1620 .

6. Khodyrev, Yu.P., Baranova, R.V., Imamov, R.M. \& Semiletov, S.A., 1978, Kristallografiya, 23, 724.

7. Hemenger, P. \& Weik, H., 1965, Acta Cryst., 19, 690.

8. Weik, H. \& Hemenger, P., 1965, Bull. Am. Phys. Soc., 10, 1140.

9. Quaas, M., Wulff, H., Ivanova, O. \& Helm, C.A., 2008, Surf. Interface Anal., 40, 552 .

10. Somenkov, V.A., Glazkov, V.P., Irodova, A.V. \& Shil'shtein, S.Sh., 1987, J. Less-Common Metals, 129, 171.

11. Vyazovkin, S. \& Sbirrazzuoli N., 2006, Macromol. Rapid Commun., 27, 1515.

Acknowledgements. Financial support for this work was provided by Deutsche Forschungsgemeinschaft. (SFB TR 24). We thank Dr. K. Ellmer (Helmholtz centre Berlin) for preparation of the Ni-films. 


\title{
Interdiffusion and stress development in $\mathrm{Ni}-\mathrm{Cu}$ thin film diffusion couples
}

\section{J. Sheng, U. Welzel*, E. J. Mittemeijer}

Max Planck Institute for Metals Research, Heisenbergstrasse 3, D-70569 Stuttgart, Germany

*u.welzel@mf.mpg.de

Keywords: residual stress, diffusion, metal thin films, X-ray diffraction

\begin{abstract}
Thin film Ni-Cu diffusion couples (individual layer thicknesses: $50 \mathrm{~nm}$ ) have been prepared by direct-current magnetron sputtering on silicon substrates. The microstructural development and the stress evolution during diffusion annealing have been investigated employing ex-situ and in-situ X-ray diffraction, transmission electron microscopy and $\mathrm{Au}$ ger-electron spectroscopy (in combination with sputter-depth profiling). Annealing at relatively low temperatures $\left(175^{\circ} \mathrm{C}\right.$ to $350^{\circ} \mathrm{C}$ ) for durations up to about 100 hours results in considerable diffusional intermixing. In addition to thermal stresses due to mismatch of the coefficients of thermal expansion of layers and substrate, tensile stress contributions in the sublayers arise during diffusion anneals. The obtained stress data are discussed in terms of possible mechanisms of stress generation.
\end{abstract}

\section{Introduction}

While the stress state in a diffusion couple is altered due to diffusion $[1,2]$, the stress state and its evolution, in turn, influences the diffusion process. The effect of stress on diffusion is well known for the case of a hydrostatic state of stress: the diffusivity is influenced via the so-called activation volume. The case of a non-hydrostatic stress state, however, has received much less attention both theoretically and, in particular, experimentally. Pioneering theoretical work in this field is due to Cahn and Larché [3]. It followed that stress gradients can act as driving forces for diffusion. Thin films can exhibit very high residual, internal stresses and large stress gradients. Such stresses can be due to the film growth, to a mismatch of the film(s) and the substrate upon cooling or heating, due to their thermal expansion coefficients being different, or due to thermally activated processes as annihilation of crystal defects, grain growth, diffusion and/or phase transformations. During thermal treatment of thin films, usually more than one mechanism influencing the stress state is operative: As an example, interdiffusion in a layered structure is often accompanied by the annihilation of crystal defects and by grain growth. Above all, plastic accommodation of strains may occur if the yield limit is exceeded. In spite of its scientific and technological importance, systematic investigations of the effect of interdiffusion on residual (internal) stresses in thin films are rare [47]. In the present work, diffusion and stresses in the binary thin-film system $\mathrm{Ni} / \mathrm{Cu}$ has been investigated. The $\mathrm{Cu}-\mathrm{Ni}$ system exhibits miscibility at all compositions above about $350^{\circ} \mathrm{C}$ and absence of intermediate or ordered phases in $\mathrm{Ni}-\mathrm{Cu}$ alloys. A miscibility gap occurs 
below about $350^{\circ} \mathrm{C}$. The interdiffusion and stress evolution in thin bi-layer (individual layer thickness: $50 \mathrm{~nm}$ ) diffusion couples of $\mathrm{Cu}$ and $\mathrm{Ni}$ have been investigated employing Augerelectron spectroscopy in combination with sputter-depth profiling and ex-situ and in-situ Xray diffraction measurements. It has been found that upon annealing at relatively low temperatures $\left(125^{\circ} \mathrm{C}\right.$ to $\left.300^{\circ} \mathrm{C}\right)$ for durations up to 100 hours considerable diffusional intermixing, accompanied by considerable stress changes, occurs.

\section{Experimental details}

Polycrystalline thin-film Ni-Cu bilayer diffusion couples were prepared by successive deposition of $\mathrm{Cu}$ and $\mathrm{Ni}$ on $\mathrm{Si}(100)$ wafers of thickness $500 \mu \mathrm{m}$ covered with amorphous $\mathrm{SiO}_{2}$ and $\mathrm{Si}_{3} \mathrm{~N}_{4}$ diffusion barrier layers (each $50 \mathrm{~nm}$ thick) by $\mathrm{DC}$ magnetron sputtering. The $\mathrm{Ni}$ and $\mathrm{Cu}$ sublayers each had a thickness of $50 \mathrm{~nm}$. To decrease the defect density in the $\mathrm{Cu}$ sublayer, a pre-annealing treatment was applied to some of the specimens: The $\mathrm{Cu}$ sublayer was annealed at $500^{\circ} \mathrm{C}$ for 3 hours and cooled back to $30^{\circ} \mathrm{C}$ after its deposition without breaking the vacuum (called 'pre-annealed' condition in the following). Thereafter, the Ni layer was deposited. The substrate temperature increased slightly during the deposition at ambient temperature due to the atomic bombardment, but did not exceed $40^{\circ} \mathrm{C}$. Concentration-depth profiles were obtained by using ion sputtering combined with Auger Electron Spectroscopy (AES) in a JEOL JAMP 7830F field emission Auger microprobe.

For in-situ temperature-dependent X-ray diffraction measurements, a Bruker D8 Diseover diffractometer operating with $\mathrm{CuK} \alpha$ radiation in parallel-beam geometry was used. A domed hot stage DHS900 (Anton Paar) was mounted onto the Eulerian cradle and calibrated [8]. The specimens were heated and cooled in steps of $25^{\circ} \mathrm{C}$. Since both the $\mathrm{Ni}$ and the $\mathrm{Cu}$ sublayers were strongly $\{111\}$-fibre textured, the residual (internal) stresses were determined employing the crystallite group method (CGM) (see, for example, [9]). Each stress measurement comprising scans of the diffraction angle at two tilt angles $\left(\chi=0^{\circ}\right.$ and $\chi=70.53^{\circ}$, where $\chi$ is the inclination of the diffraction vector with respect to the specimen surface normal) took about $25 \mathrm{~min}$.

\section{Results and discussion}

\section{Diffusional intermixing}

The concentration-depth profiles of both $\mathrm{Ni}$ and $\mathrm{Cu}$ for normally deposited (ND) and preannealed (PA) Ni-Cu diffusion couples annealed at temperatures of $175^{\circ} \mathrm{C}$ and $225^{\circ} \mathrm{C}$ are shown in figure 1 . The depth profiles for the normally deposited specimens after annealing at $225^{\circ} \mathrm{C}$ for 32 hours (see figure 1(a)) show a change of the concentration slopes at the position of the initial interface and the emergence of a plateau region of considerable Ni concentration in the initially pure $\mathrm{Cu}$ sublayer. Whereas the change of the slope at the position of the original interface is due to volume diffusion across the initial interface, the plateau region on the $\mathrm{Cu}$-rich side of the couple is the result of $\mathrm{Ni}$ diffusion through $\mathrm{Cu}$ grain boundaries accompanied with sideways $\mathrm{Ni}$ diffusion from the $\mathrm{Cu}$ grain boundaries through the $\mathrm{Cu}$ grains. No significant plateau of $\mathrm{Ni}$ in the initially pure $\mathrm{Cu}$ sublayer of the pre-annealed specimens is formed upon annealing at even $225^{\circ} \mathrm{C}$ (figure 1(b)). The absence of the plateau is a consequence of the drastically reduced grain-boundary density in the pre-annealed $\mathrm{Cu}$ sublayer: 
The in-plane crystallite size of the pre-annealed $\mathrm{Cu}$ sublayer, determined by single-line broadening analysis [10] (at $\chi=70.53^{\circ}$ ) is $80 \mathrm{~nm}$, whereas an in-plane crystallite size of 20 $\mathrm{nm}$ is obtained for the normally-deposited $\mathrm{Cu}$ sublayer. A significant $\mathrm{Cu}$ concentration occurs at the surface of the bilayer specimen, whereas the $\mathrm{Cu}$ concentration in the plateau region of the $\mathrm{Ni}$ layer remains small upon annealing. Thus, sideways $\mathrm{Cu}$ diffusion from the $\mathrm{Ni}$ grain boundaries through the $\mathrm{Ni}$ grains is very limited. This is likely a consequence of the concentration-dependence of the interdiffusion coefficient in the $\mathrm{Cu} / \mathrm{Ni}$ system which is lower at a higher concentration of $\mathrm{Ni}$ (cf., for example, Ref. [11]).
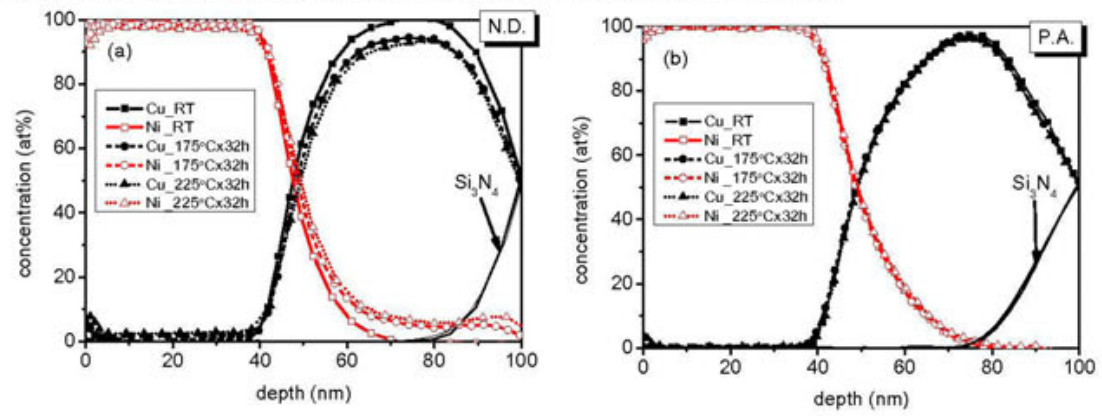

Figure 1. Depth profiles of (a) normally deposited (N.D.) and (b) pre-annealed (P.A.) Ni(top)$\mathrm{Cu}$ (bottom) bilayer diffusion couples after isothermal annealing.

\section{Solid solution formation}

According to the binary phase-diagram of the $\mathrm{Cu}-\mathrm{Ni}$ system, no intermetallic or ordered phases occur, but a miscibility gap exists below about $350^{\circ} \mathrm{C}$ [12]. X-ray diffraction patterns of the as-deposited $\mathrm{Ni}-\mathrm{Cu}$ diffusion couples show reflections of only pure $\mathrm{Cu}$ and pure $\mathrm{Ni}$ with strong $\{111\}$ fibre textures. The diffraction patterns corresponding to the diffractionangle ranges of the $\mathrm{Cu}$ and $\mathrm{Ni} 111$ reflections of both normally deposited and pre-annealed specimens annealed at $300^{\circ} \mathrm{C}$ for different durations are shown in figure 2(a) and figure 2(b), respectively. A shoulder developed at the high-angle side of the $\mathrm{Cu} 111$ reflection, for a normally deposited specimen, indicating a Cu-rich volume fraction of the diffusion couple after annealing at $300^{\circ} \mathrm{C}$ for only 4 hours, which increases in intensity upon continued annealing. For the same annealing temperature, but much larger annealing times, only a small hump developed at the high-angle side of the $\mathrm{Cu} 111$ reflection for the pre-annealed specimen. This can be understood considering that diffusional intermixing is much less pronounced in the pre-annealed specimen containing a low grain boundary density in the preannealed $\mathrm{Cu}$ sublayer (cf. section Diffusional intermixing); the emergence of a separate intensity maximum at the high-angle side of the $\mathrm{Cu} 111$ reflection for the normally-deposited specimen indicates the formation of a substantial amount of $\mathrm{Cu}$-rich solid solution, due to the intermixing enhancement caused by the relatively fast diffusion along the $\mathrm{Cu}$ grain boundaries in the initially $\mathrm{Cu}$-rich part of the couple.

\section{Stress evolution}

For the in-situ heat treatment the complete temperature cycle consists of three segments: heating from $30^{\circ} \mathrm{C}$ (ambient temperature in the diffractometer) to the annealing temperature, isothermal annealing, and cooling back to ambient temperature. The evolution of the internal, 

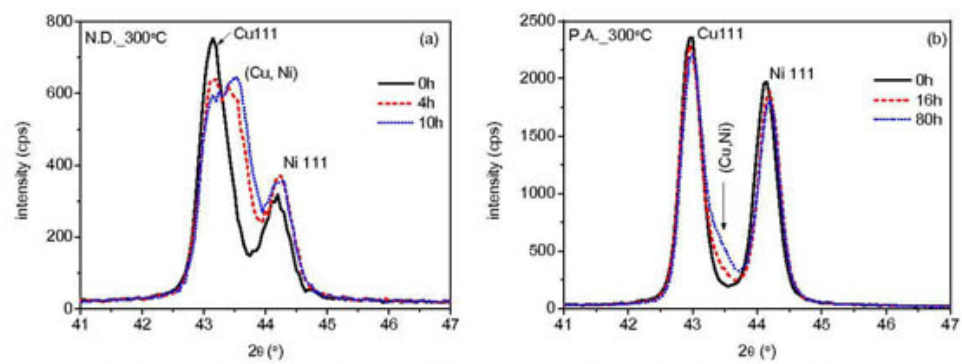

Figure 2. Solid solution formation in (a) normally deposited (N.D.) and (b) pre-annealed (P.A.) Ni/Cu bilayers during isothermal annealing at $300^{\circ} \mathrm{C}$.

mechanical stresses in the $\mathrm{Ni}$ and $\mathrm{Cu}$ sublayers during the three segments are shown in figures 3(a)-(d) for an annealing temperature of $225^{\circ} \mathrm{C}$. A fully elastic accommodation of the thermal strain during heating and cooling as imposed by the (thick) substrate (in the following called 'thermal stress/strain') would result in linear dependencies of the stresses of the individual layers on temperature.

Table 1. Thermoelastic slopes ( $\Xi$ ) for the normally deposited $\mathrm{Ni}$-Cu bilayer on a $\mathrm{Si}_{3} \mathrm{~N}_{4} / \mathrm{SiO}_{2} / \mathrm{Si}(100)$ substrate during cooling: Comparison of calculated and experimental values (elastic data and coefficients of thermal expansion taken from [13] and [14], respectively).

\begin{tabular}{|c|c|c|c|c|c|}
\hline \multicolumn{4}{|c|}{$\Xi$ calc. $(\mathrm{MPa} / \mathrm{K})$} & \multicolumn{2}{c|}{$\Xi$, expt. } \\
\cline { 1 - 4 } polycrystalline untextured & perfectly $\{111\}$-fibre textured & \multicolumn{1}{c}{ (N.D. specimen) $(\mathrm{MPa} / \mathrm{K})$} \\
\hline $\mathrm{Ni}$ & $\mathrm{Cu}$ & $\mathrm{Ni}$ & $\mathrm{Cu}$ & $\mathrm{Ni}$ & $\mathrm{Cu}$ \\
\hline-3.11 & -2.85 & -4.16 & -3.79 & -4.37 & -3.85 \\
\hline
\end{tabular}

The thermoelastic slopes $\Xi\left(\Xi=\Delta \sigma_{/ / /} / \Delta\right.$ T, i.e. stress changes per $\left.K\right)$ can be estimated from the coefficients of thermal expansion of the films and the substrate and the so-called biaxial moduli of the films. Here, the theoretical extremes for the biaxial moduli are given by the cases of randomly oriented and perfectly $\{111\}$-fibre textured polycrstals and have been gathered in table 1 (cf. Appendix of Ref. [8]). Considering the strength and the sharpness of the textures of both sublayers, it seems reasonable to assume that the values for perfectly $\{111\}$-fibre texture polycrystals are appropriate estimates of reality for the present thin film systems. Whereas the stress changes in the sublayers of the pre-annealed specimen exhibit thermoelastic behaviour during both heating and cooling, this is only the case for the cooling segment for the sublayers of the normally-deposited specimen. After subtraction of the developing thermal stress, for both sublayers, significant tensile stress contributions occur during heating. Thus, apart from thermal misfit as a source of strains, other stressgenerating/relaxing mechanisms must be operative: The relaxation of compressive thermal stresses due to plastic yielding and the generation of tensile stresses upon heating a layer sputter-deposited at ambient temperature due to the thermally activated compaction of material (as a consequence of grain growth [15] can be considered as possible sources of stress generation). It is very likely that grain growth (and, possibly, defect annihilation) in both sub-layers is the dominant sources of tensile stress contributions developing during heating of the normally deposited layers. For the pre-annealed specimens, the crystallite sizes obtained by single-line broadening analysis are considerably larger prior to the diffusion anneal 
(ND: $25 \mathrm{~nm}$ for $\mathrm{Cu}, 15 \mathrm{~nm}$ for $\mathrm{Ni}$; PA: $80 \mathrm{~nm}$ for $\mathrm{Cu}, 30 \mathrm{~nm}$ for $\mathrm{Ni}$; all measured at $\chi=70.5^{\circ}$ ). This makes plausible that distinct grain growth does not occur during heating of the preannealed specimen and that a purely thermoelastic behavior occurs during both heating and cooling.
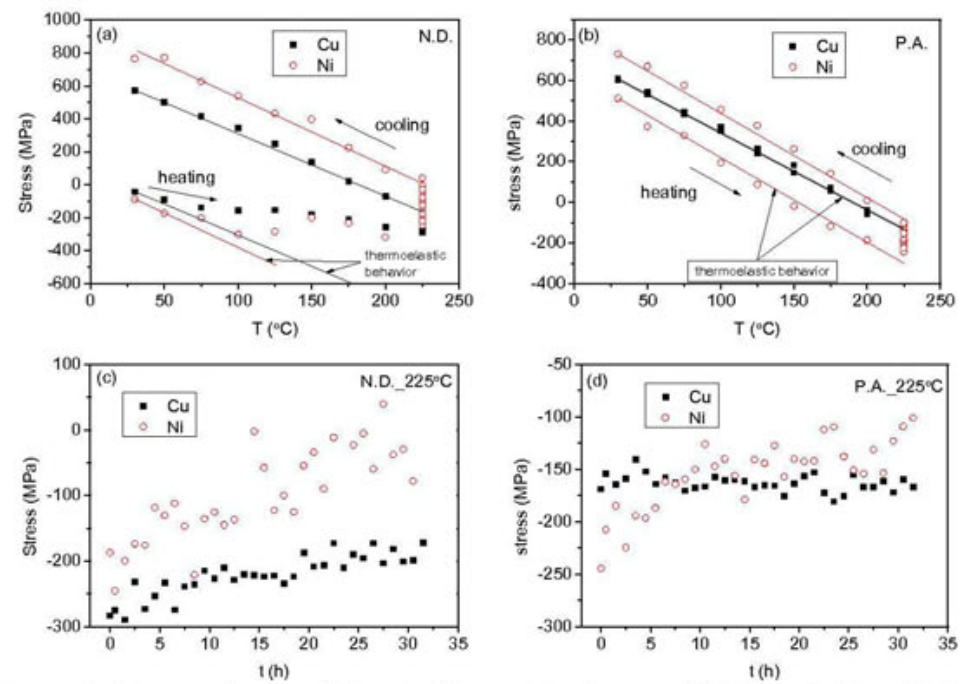

Figure 3. Stress evolution of (a) and (c) normally deposited (N.D.) and (b) and (d) pre-annealed (P.A.) $\mathrm{Ni} / \mathrm{Cu}$ bilayers during the heating and cooling cycle between $30^{\circ} \mathrm{C}$ and $225^{\circ} \mathrm{C}$ and during the isothermal annealing for 32 hours.

During isothermal annealing at $225^{\circ} \mathrm{C}$, the stresses of the $\mathrm{Ni}$ and $\mathrm{Cu}$ sublayers become less compressive except for the case of the pre-annealed $\mathrm{Cu}$ sublayer, which does stay constant. The following mechanisms leading to stress change upon isothermal annealing can be conceived: (i) interdiffusion (differences in the molar volumes and differences in the atomic fluxes of the diffusing species), (ii) (continued) grain growth, (iii) excess vacancy annihilation and shrinkage of grain-boundary voids, and (iv) plastic deformation (yielding; creep). Whereas mechanisms (ii) and (iii) invariably generate tensile stresses, mechanism (iv) always leads to stress relaxation (i.e. a decrease of the stress level). For the normally deposited specimen, the stress of the Ni sublayer becomes less compressive (but does not change the sign) due to mechanisms (i)-(iii); mechanism (iv) is less likely due to the small stress level of Ni sublayer. The stress of the Cu sublayer of the normally deposited specimen becomes less compressive, but the stress change with time for the $\mathrm{Cu}$ sublayer is smaller than for the $\mathrm{Ni}$ sublayer, which is possibly the result of a competition between different sources of compressive stress generation (mechanism (i): the net flux of $\mathrm{Ni}$ atoms diffused into $\mathrm{Cu}$ sublayer) and tensile stress generation (i.e. mechanisms (ii) and (iv), and the difference in molar volumes of $\mathrm{Ni}$ and $\mathrm{Cu}\left(\mathrm{V}_{\mathrm{Ni}}=6.59 \mathrm{~cm}^{3} ; \mathrm{V}_{\mathrm{Cu}}=7.11 \mathrm{~cm}^{3}\right)$. The stress of the pre-annealed $\mathrm{Cu}$ sublayer remains constant because neither grain growth nor significant diffusion into this Cu sublayer occurs. The stress change in the Ni sublayer for the pre-annealed specimen is likely due to (marginal) grain growth during annealing. 


\section{Conclusions}

1) Annealing of Ni-Cu thin film (sublayer thickness: $50 \mathrm{~nm}$ ) diffusion couples at temperatures between about $225^{\circ} \mathrm{C}-300^{\circ} \mathrm{C}$ results in considerable diffusional intermixing.

2) Diffusional intermixing is accompanied by the formation of a $(\mathrm{Cu}, \mathrm{Ni})$ solid solution along the (columnar) grain boundaries in the initially $\mathrm{Cu}$-rich part of the diffusion couple.

3) Upon annealing, tensile stress contributions are generated in the $\mathrm{Ni}$ and $\mathrm{Cu}$ sublayers except for the case of a pre-annealed $\mathrm{Cu}$ sublayer, for which only very limited interdiffusion occurs due to a low grain-boundary density. For normally-deposited specimens and, likely, the Ni layer in pre-annealed specimens, grain growth is a major source of tensile stress.

\section{References}

1. Beke, D.L., Szabó, I.A., Erdélyi, Z. \& Opposits, G., 2004, Mater. Sci. Eng. A, Structural Materials, 387, 4.

2. Opposits, G., Szabó, S., Beke, D.L., Guba, Z. \& Szabó, I.A., 1998, Scr. Mater., 39, (7), 977.

3. Larché, F.C. \& Cahn, J.W., 1985, Acta Met., 33, (3), 331.

4. Welzel, U., Lamparter, P., Leoni, M. \& Mittemeijer, E.J., 2000, Mater. Sci. Forum, 347-349, 405.

5. Welzel, U., Lamparter, P. \& Mittemeijer, E.J., 1999, Mater: Res. Soc. Symp. Proc., 562,147 .

6. Kuru, Y., Wohlschlögel, M., Welzel, U. \& Mittemeijer, E.J., 2008, Thin Solid Films, 516, (21), 7615.

7. Chakraborty, J., Welzel, U. \& Mittemeijer, E.J., 2008, J. Appl. Phys., 103, (11), 113512 .

8. Wohlschlögel, M., Welzel, U., Maier, G. \& Mittemeijer, E.J., 2006, J. Appl. Cryst., 39, 194.

9. Welzel, U., Ligot, J., Lamparter, P., Vermeulen, A.C. \& Mittemeijer, E.J., 2005, J. Appl. Cryst., 38, 1.

10. De Keijser, T.H., Langford, J.I., Mittemeijer, E.J. \& Vogels, A.B.P., 1982, J. Appl. Cryst., 15, 308.

11. Levasseur, J. \& Philibert, J., 1967, CR. Acad. Sci. Paris, 264, 277.

12. Subramanian, P.R., Chakrabarti, D.J. \& Laughlin, D.E., 1994, Monograph Series on Alloy Phase Diagrams, 10, 276.

13. Landolt-Börnstein, 1979, Numerical Data and Functional Relationships in Science and Technology III/29a 1.2.1., p. 12.

14. Touloukian, Y.S., 1975, Thermal Expansion: Metallic Elements and Alloys , p. 77.

15. Kuru, Y., Wohlschlögel, M., Welzel, U. \& Mittemeijer, E.J., 2008, J. Appl. Cryst., 41,428 .

Acknowledgements. The authors thank Mr F. Thiele and Dr G. Richter for specimen preparation and Mr B. Siegle, Dr L.P.H. Jeurgens and Dr J.Y. Wang for Auger-electron spectroscopy measurements and assistance during data evaluation. 
IV.2 Nanocrystalline and Amorphous Materials 



\title{
Nanocrystalline $\mathrm{MgO}$ powder materials prepared by sol-gel studied by X-ray diffraction and electron microscopy
}

\author{
G. Dercz ${ }^{1, *}$, L. Pająk ${ }^{1}$, K. Prusik ${ }^{1}$, R. Pielaszek ${ }^{2}$, \\ J. J. Malinowski ${ }^{3}$
}

${ }^{1}$ University of Silesia, Institute of Materials Science, Bankowa 12, Katowice 40-007, Poland

${ }^{2}$ Polish Academy of Sciences, Institute of High Pressure Physics (UNIPRESS), Sokolowska 29/37, Warszawa 01-142, Poland

${ }^{3}$ Institute of Chemical Engineering Polish Academy of Sciences, Baltycka 5, Gliwice 44-100, Poland

* grzegorz.dercz@us.edu.pl

Keywords: X-ray diffraction, Electron microscopy, Nanostructures, Sol-gel processes

\begin{abstract}
Materials with $\mathrm{MgO}$ nanocrystallites were prepared by sol-gel synthesis. Alcogels were dried either supercritically at $538 \mathrm{~K}$ or in supercritical $\mathrm{CO}_{2}$. Conventional drying was also applied. Structure studies were performed by X-ray diffraction, scanning and transmission electron microcopies. Nitrogen adsorption/desorption measurements were also performed. Calcination of dry gels at $723 \mathrm{~K}$ under vacuum yielded $\mathrm{MgO}$ nanocrystallites. In the studied materials the presence of the amorphous phase was observed. It is also stated that $\mathrm{MgO}$ nanocrystallites are immersed in an amorphous matrix.

Morphology of supercritically dried samples is similar; rough surface of powder particles is observed. Powder particles of conventionally dried sample are more compact. Size of $\mathrm{MgO}$ crystallites is generally similar for all samples and equal to about $7 \mathrm{~nm}$ despite the drying conditions of alcogels. The specific surface area and the volume of mesopores are the greatest for the sample supercritically dried at $538 \mathrm{~K}$.
\end{abstract}

\section{Introduction}

$\mathrm{MgO}$ is a highly ionic crystalline solid with interesting properties such as electrical insulation, high temperature stability and chemical inertness. It is of simple stoichiometry and crystal structure and it can be prepared in widely variable particle sizes and shapes [1]. $\mathrm{MgO}$ has many applications in catalysis, adsorption and in the synthesis of refractory ceramics [25]. Catalytic properties of $\mathrm{MgO}$ powders depend mainly on the crystallite size and specific surface area; structure defects and acid-basic site concentration also play an important role [6]. $\mathrm{MgO}$ as a support of catalysts is of interest because it has the ability to avoid sintering and evaporation of metal atoms. 
The chemical and physical properties required for catalytic application can now be defined at the beginning of the sol-gel synthesis procedure. Moreover this procedure is widely used in the preparation of the components for optical fibers, glasses, supports, ceramic materials, electronic semiconductors, etc.

The technique developed by H.M. Rietveld $[7,8]$ enriched by many others scientists appeared to be very valuable for structural analyses of nearly all classes of crystalline materials.

The aim of the present work is the presentation of the results of structure analysis of nanocrystalline $\mathrm{MgO}$ powder material and the comparison with those previously obtained for similar materials [9] prepared by sol-gel synthesis.

\section{Material and research methodology}

A magnesium methoxide solution (Aldrich, $8.96 \mathrm{wt} \%$ in methanol), methanol (POCh), and toluene (POCh) were used as precursors in the sol-gel synthesis. The details of the synthesis methods for preparing nanoscale magnesium oxide by supercritical drying are described by Utamapanya in [5]. The wet gels were conventionally aged for 3 days. After aging, alcogels were dried either supercritically at $538 \mathrm{~K}$ (sample labelled as Aero) or in supercritical $\mathrm{CO}_{2}$ (sample labelled as Aero- $\mathrm{CO}_{2}$ ). Additionally, the conventional drying procedure was applied to prepare the xerogel sample (sample labelled as Xero). The xerogel sample was prepared as follows. After aging alcogel was loosened for a few days to allow slow evaporation of the solvents. Next, the alcogel was vacuum-dried at room temperature for 12 hours. Finally, the sample was heated at $60^{\circ} \mathrm{C}$ until constant weight was obtained i.e. about 48 hours. Nanoparticles of magnesium oxide were obtained by heat treatment of magnesium hydroxide aerogels/xerogel samples at $723 \mathrm{~K}$ under dynamic vacuum using the following conditions: ramp from room temperature to $723 \mathrm{~K}$ at $0.5 \mathrm{~K} / \mathrm{min}$ and hold at $723 \mathrm{~K}$ for 5 hours. The whole process of heat treatment took about 15 hours.

X-ray diffraction patterns were collected on an X-Pert Philips diffractometer equipped with a secondary curved graphite monochromator. Morphology of materials was analyzed using SEM (JEOL JSM-6480) and TEM (JEOL 3010) techniques. Nitrogen adsorption-desorption isotherms measured at $77 \mathrm{~K}$ with a Micromeritics ASAP 2000 instrument were used to obtain the value of the specific surface area, $S_{\mathrm{BET}}$ and the total volume of mesopores, $V_{P}$.

\section{Results and discussion}

SEM images presented in figure 1 show the morphology of the powder particles. Surface roughness of Aero- $\mathrm{CO} 2$ and Aero samples is distinct and quite similar whereas the powder particles of Xero sample are more compact. These differences in powder morphology are the result of the different alcogel drying conditions. TEM images with the electron diffraction pattern shown in figure 2 indicate that the powder particles of Aero sample are built of MgO nanocrystallites which are immersed in an amorphous matrix. Similar observations were obtained for previously studied Aero- $\mathrm{CO} 2$ and Xero samples [9]. The above conclusions are confirmed by the HRTEM image in figure 3 . The electron microcopy results are in good correlation with the ones of the X-Ray Diffraction (XRD) studies. The Rietveld refinement plot for the Aero sample (experimental and calculated X-ray diffraction patterns and residual profile at the bottom) is presented in figure 4 . Similar plots were obtained for the other sam- 
ples. Broadened diffraction lines of periclase, the only crystalline form of magnesium oxide, are observed.
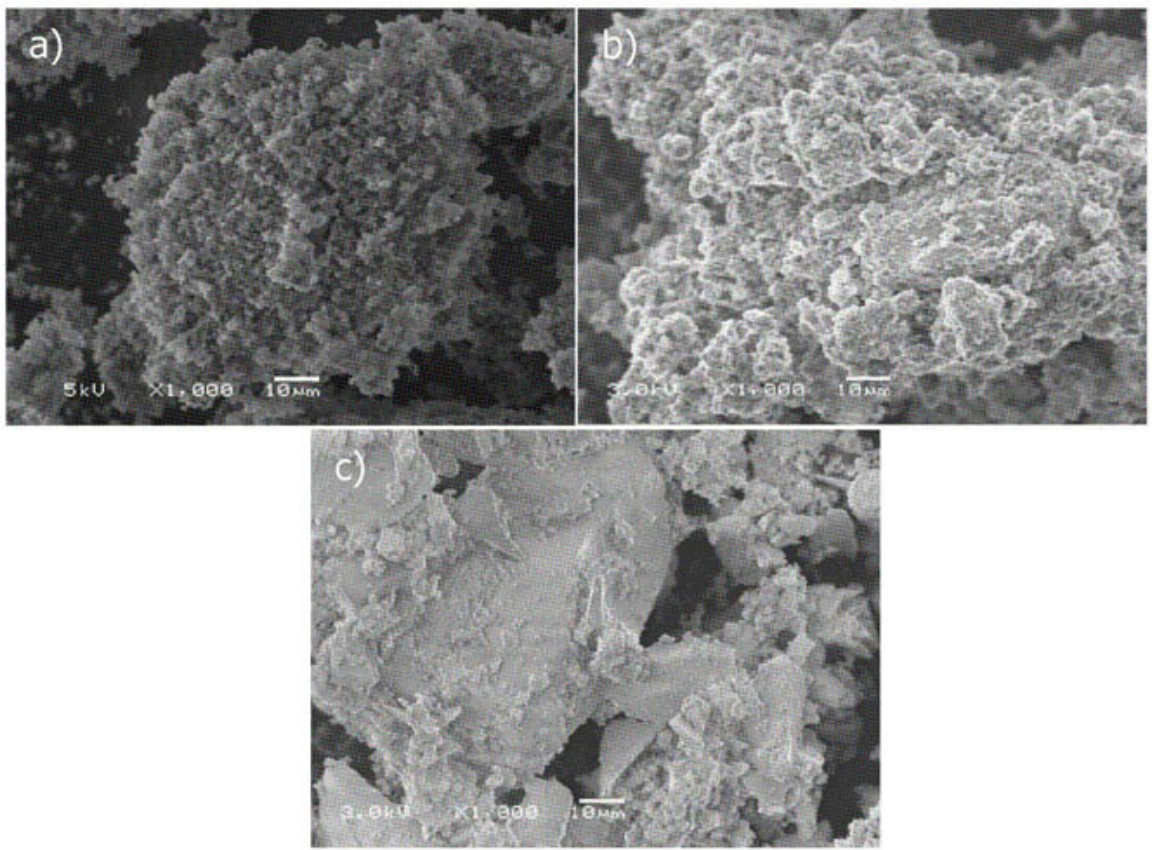

Figure 1. SEM images of Aero- $\mathrm{CO}_{2}($ a), Aero (b) and Xero (c) samples.
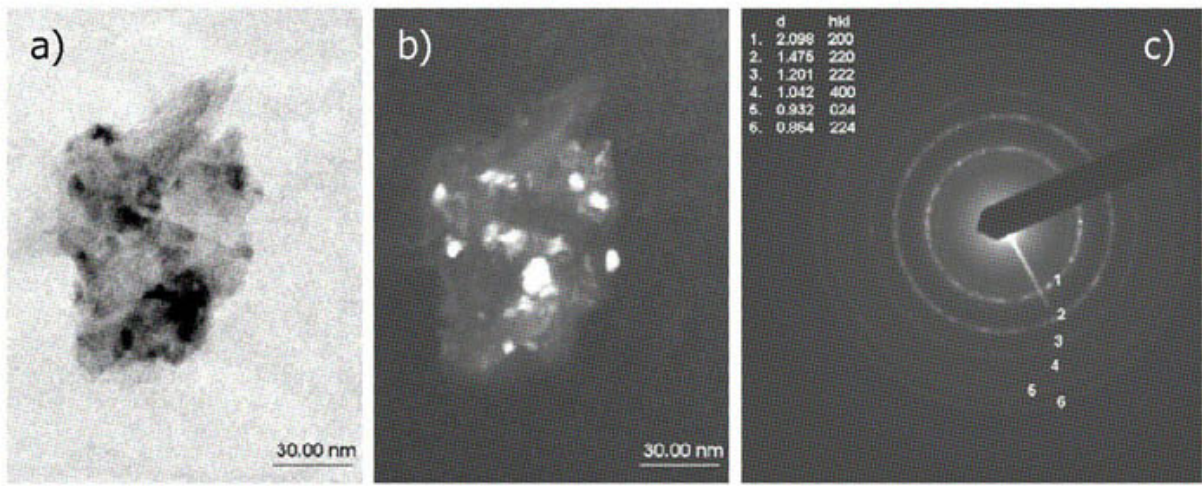

Figure 2. TEM images of Aero sample: bright-field (a), dark-field (b) and electron diffraction pattern (c). 
The $R_{w p}$ and $S$ values obtained during Rietveld refinement are of the order of $8.5 \%$ and 1.8 , respectively. They seem to be satisfactory owing to the nanosize of $\mathrm{MgO}$ crystallites and due to the presence of the amorphous phase. Lattice parameters of $\mathrm{MgO}$ phase for the studied samples, determined by the Rietveld refinement procedure, appeared to be slightly bigger than the ones found in the ICDD file.

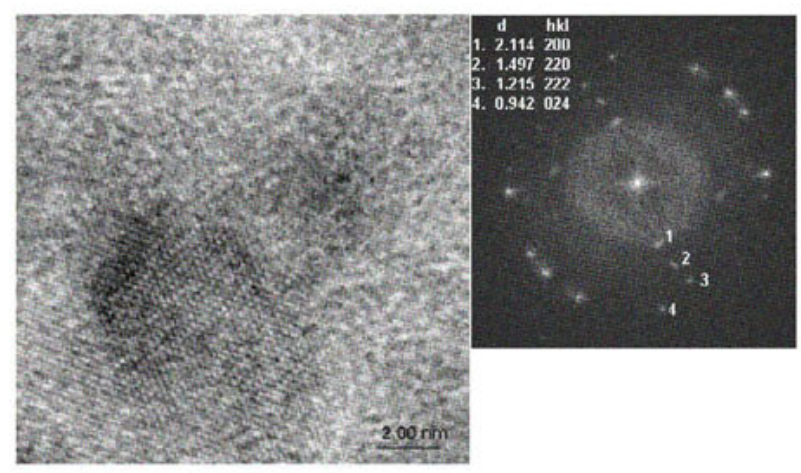

Figure 3. HRTEM image of Aero- $\mathrm{CO}_{2}$ sample with electron diffraction pattern.

The crystallite sizes and lattice distortions were analyzed using the Williamson-Hall method [10]. The Toraya PRO-FIT procedure [11] was applied for the determination of the fullwidth at the half maximum parameter (FWHM) of individual diffraction lines. For this procedure the selected line profile was a Pearson VII function. This function was also used for the Rietveld refinement. Similar plots were obtained for the other studied samples. Analysis of the Williamson-Hall plots (not presented here) indicated that the main contribution to diffraction line broadening comes from the small crystallite sizes.

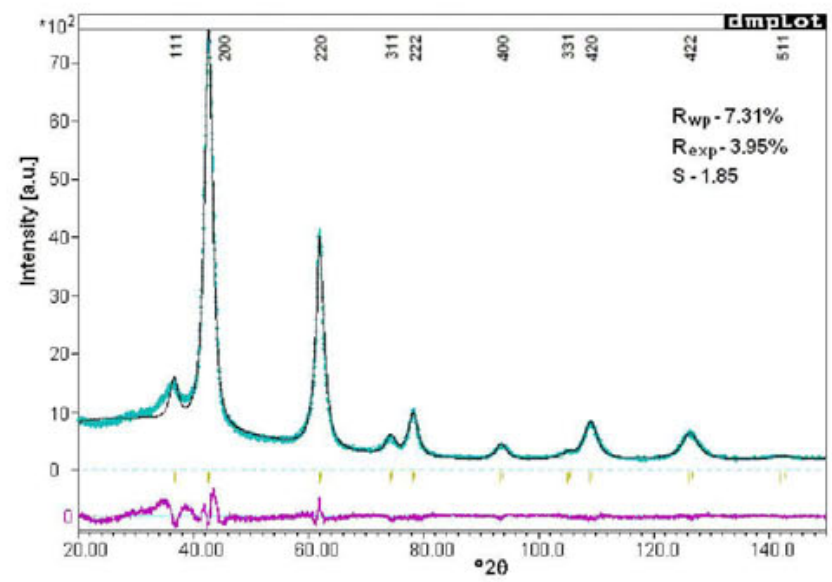

Figure 4. Rietveld refinement plot for Aero sample. 
The crystallite size distribution shown in figure 5 was determined with the $\mathrm{FW}(1 / 5) /(4 / 5) \mathrm{M}$ procedure proposed by R. Pielaszek [12]. The width measurement of the same diffraction line at $1 / 5$ and $4 / 5$ of the peak maximum (FW $1 / 5 \mathrm{M}$ and $\mathrm{FW} 4 / 5 \mathrm{M}$, respectively) allows determining the average crystallite size $\langle R\rangle$ and the dispersion of sizes $\sigma$. These parameters enable the determination of the gamma crystallite size distribution. The dispersion parameter $\sigma$, is a measure of the crystallite size polydispersity and is defined by equation $\sigma=\sqrt{\left\langle R^{2}\right\rangle-\langle R\rangle^{2}}$

The values of $\langle R\rangle$ and $\sigma$ parameters obtained by the Pielaszek procedure and the average crystallite sizes estimated by the Williamson-Hall method are presented in table 1. A good agreement between the results of both approaches is observed.

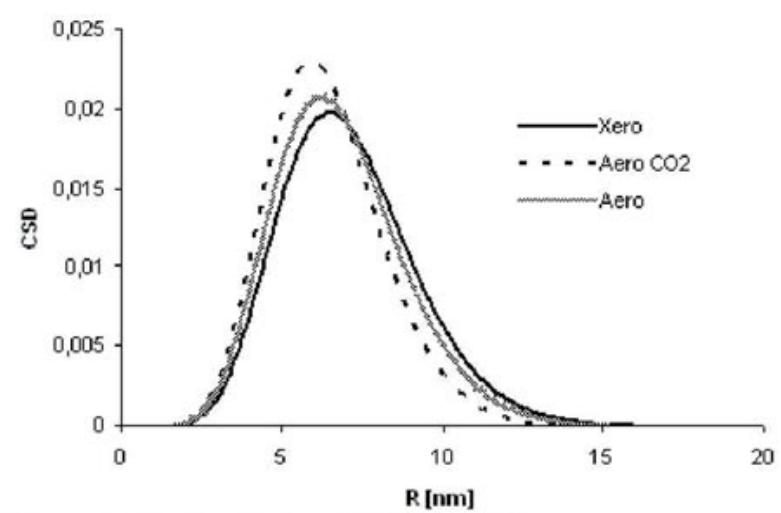

Figure 5. Size distribution of $\mathrm{MgO}$ crystallites.

The porosity characterization of the studied $\mathrm{MgO}$ materials was performed by nitrogen adsorption/desorption measurements. The specific surface area $S_{\mathrm{BET}}$ was determined by using the BET equation and the total mesopore volume $V_{\mathrm{p}}$ was calculated according to the $\mathrm{BJH}$ method from the desorption branch (Table 2). The specific surface area $\mathrm{S}_{\mathrm{BET}}$ of Aero- $\mathrm{CO}_{2}$, Aero and Xero samples before the heat treatment were $440 \mathrm{~m}^{2} / \mathrm{g}, 325 \mathrm{~m}^{2} / \mathrm{g}$ and $690 \mathrm{~m}^{2} / \mathrm{g}$, respectively.

Table 1. Lattice $\left(a_{o}\right)$, crystallite size $(D,\langle R\rangle, \sigma)$ and lattice distortion $(\langle\Delta a / a\rangle)$ parameters.

\begin{tabular}{|l|l|l|l|l|l|l|}
\hline \multirow{3}{*}{ Sample } & \multicolumn{2}{|l|}{$a_{\mathrm{o}}$, lattice parameter } & \multicolumn{3}{l|}{ Williamson-Hall } & \multicolumn{2}{l|}{ FW $\frac{1}{5} / \frac{4}{5} \mathrm{M}$} \\
\cline { 2 - 7 } & $\begin{array}{l}\text { Rietveld } \\
{[\mathrm{nm}]}\end{array}$ & $\begin{array}{l}\text { ICDD } \\
{[\mathrm{nm}]}\end{array}$ & $\begin{array}{l}D \\
{[\mathrm{~nm}]}\end{array}$ & $\begin{array}{l}<\Delta a / a> \\
{[\%]}\end{array}$ & $\begin{array}{l}<R> \\
{[\mathrm{nm}]}\end{array}$ & $\begin{array}{l}\sigma \\
{[\mathrm{nm}]}\end{array}$ \\
\hline Xero & $0.4226(1)$ & 0.4213 & $7.5(9)$ & $0.11(2)$ & $7.1(6)$ & $2.1(2)$ \\
\hline Aero- $\mathrm{CO}_{2}$ & $0.4227(1)$ & 0.4213 & $6.0(9)$ & $0.09(2)$ & $6.5(6)$ & $1.8(2)$ \\
\hline Aero & $0.4229(1)$ & 0.4213 & $7.2(9)$ & $0.09(2)$ & $6.9(6)$ & $2.0(2)$ \\
\hline
\end{tabular}


Table 2. Porosity parameters.

\begin{tabular}{|l|l|l|l|l|l|}
\hline \multicolumn{2}{|l|}{ Aero-CO $\mathrm{CO}_{2}$} & \multicolumn{2}{l|}{ Aero } & Xero \\
\hline$S_{\mathrm{BET}}\left[\mathrm{m}^{2} / \mathrm{g}\right]$ & $V_{\mathrm{p}}\left[\mathrm{cm}^{3} / \mathrm{g}\right]$ & $S_{\mathrm{BET}}\left[\mathrm{m}^{2} / \mathrm{g}\right]$ & $V_{\mathrm{p}}\left[\mathrm{cm}^{3} / \mathrm{g}\right]$ & $S_{\mathrm{BET}}\left[\mathrm{m}^{2} / \mathrm{g}\right]$ & $V_{\mathrm{p}}\left[\mathrm{cm}^{3} / \mathrm{g}\right]$ \\
\hline 238 & 0.49 & 293 & 0.86 & 138 & 0.45 \\
\hline
\end{tabular}

The specific surface area and the total volume of mesopores is the greatest for the Aero sample dried supercritically at $538 \mathrm{~K}$. The lowest specific surface area was obtained for Xero sample dried conventionally. Differences in specific surface areas are the result of different drying conditions of alcogels. The calcination process is the same for the studied samples and gives a similar size of $\mathrm{MgO}$ crystallites.

\section{Conclusions}

Apart from $\mathrm{MgO}$ nanocrystallites the studied powder materials contain also an amorphous phase. MgO nanocrystallites are immersed in an amorphous matrix.

Both X-ray diffraction analysis and TEM observations show that the size of MgO crystallites is quite similar for all studied materials and equal to about $7 \mathrm{~nm}$ despite different drying conditions of the alcogels. Generally the similar size of $\mathrm{MgO}$ crystallites in these materials is the result of the calcination processes which are the same for all samples.

The specific surface area and the total volume of mesopores is the greatest for the Aero sample dried supercritically at $538 \mathrm{~K}$. Different morphology of powder particles of the studied $\mathrm{MgO}$ materials, differences in the specific surface areas and in mesopore volumes are the result of different drying conditions of alcogels.

\section{References}

1. Morris, R.M. \& Klabunde, K.J., 1983, Inorg. Chem., 22, 682.

2. Choudhary, V.R., Rane, V.H. \& Gadre, R.V., 1994, J. Catal., 145, 300.

3. Rajagopalan, S., Koper, S., Decker, S. \& Klabunde, K.J., 2002, Chem. Eur. J., 8, 2602.

4. Xu, B.Q., Wie, J.H., Wang, H.Y., Sun, K.Q. \& Zhu, Q.M., 2001, Cat. Today, 68, 217.

5. Utamapanya. S., Klabunde. K.J. \& Schlup, J.R., 1991, Chem. Mater., 3, 175.

6. Lucas, E., Decker, S., Khaleel, A., Seitz, A., Fultz, S., Ponce, A., Li, W., Carnes, C. \& Klabund, K.J., 2001, Chem. Eur. J., 7, 2505.

7. Rietveld, H.M., 1969, J. Appl. Cryst., 3, 65.

8. Young, R.A., 1993, The Rietveld Method (Oxford University Press).

9. Dercz, G., Pająk, L., Prusik, K., Pielaszek, R., Malinowski, J.J. \& Pudło, W., 2007, Solid State Phenomena, 130, 203.

10. Williamson, G.K. \& Hall, W.H., 1953, Acta Metall., 1, 22.

11. Toraya H., 1986, J. Appl. Cryst., 19, 440.

12. Pielaszek, R., 2004, J. Alloys and Comp., 382, 128. 
IV.3 Metals and Alloys

IV.3.1 General 



\section{$\mathrm{XRD}$ investigation and thermal properties of $\left[\operatorname{Ir}\left(\mathrm{NH}_{3}\right)_{6}\right]\left[\mathrm{Co}\left(\mathrm{C}_{2} \mathrm{O}_{4}\right)_{3}\right] \cdot \mathrm{H}_{2} \mathrm{O}$ and $\left[\mathrm{Co}\left(\mathrm{NH}_{3}\right)_{6}\right]\left[\operatorname{Ir}\left(\mathrm{C}_{2} \mathrm{O}_{4}\right)_{3}\right]$ - precursors for $\mathrm{Co}_{0.50} \mathrm{Ir}_{0.50}$}

\section{E. Yu. Filatov ${ }^{1,2, *}$, K. V. Yusenko ${ }^{1}$, E. S. Vikulova ${ }^{2}$, P. E. Plyusnin ${ }^{1,2}$, Yu. V. Shubin ${ }^{1,2}$}

${ }^{1}$ Nikolaev Institute of Inorganic Chemistry SB RAS, 630090 Novosibirsk, Lavrentyev Ave.3, Russia

2 Novosibirsk State University, 630090 Novosibirsk, Pirogova str. 2, Russia

*decan@ehe.nsk.su

Keywords: cobalt, iridium, double complex salt, diffraction, nanosized alloy

Abstract. Double complex salts (DCS) $\quad\left[\mathrm{Ir}\left(\mathrm{NH}_{3}\right)_{6}\right]\left[\mathrm{Co}\left(\mathrm{C}_{2} \mathrm{O}_{4}\right)_{3}\right] \cdot \mathrm{H}_{2} \mathrm{O} \quad(1)$ and $\left[\mathrm{Co}\left(\mathrm{NH}_{3}\right)_{6}\right]\left[\operatorname{Ir}\left(\mathrm{C}_{2} \mathrm{O}_{4}\right)_{3}\right](2)$ have been synthesized by crystallization from aqueous solutions. The compounds have been characterized by single crystal and powder X-ray diffraction, IR spectroscopy, DTA and elemental analysis. Thermal decomposition of the title compounds has been studied in $\mathrm{He}$ and $\mathrm{H}_{2}$ atmospheres. Final products of thermolysis of the prepared compounds in hydrogen atmosphere are ultrafine powders of equimolar solid solutions $\left(\operatorname{Ir}_{0.5} \mathrm{M}_{0.5}\right)$.

\section{Introduction}

Studies in the field of novel heterometallic functional materials are highly topical. Heterometallic compounds can be used as precursors of new polymetallic phases [1, 2], molecular magnets [3], catalysts [4]. Heterometallic compounds can serve as functional materials with unique magnetic, thermal and adsorption properties as well as precursors for oxide and metallic phases [5]. There are multiple approaches to preparation of polymetallic compounds belonging to different chemical classes. Concerning the preparation of nanosized bimetallic materials containing platinum group metals, the most promising precursors are double complex salts. The salts with oxalate anions are particularly advantageous, as they have pronounced reductive properties. Synthesis of DCS simultaneously containing a platinum family metal and a first raw transition metal combined with oxalate as the ligand is of interest, as these compounds can serve as precursors of corresponding nanocrystalline alloys. Such materials can be used for development of novel catalytic systems having reduced content of noble metals $[6,7,8,9]$. 
Solid solutions and intermetallic compounds containing platinum metals have been demonstrated to exhibit high electrocatalytic performance and superior magnetic characteristics. The DCS containing both a platinum group metal and a transition metal are attracting to researchers as precursors of bimetallic materials of various composition and structure $[1,10]$.

Bimetallic phase $\mathrm{Co}_{0.50} \mathrm{Ir}_{0.50}$ was prepared by thermal decomposition of $\left[\operatorname{Ir}\left(\mathrm{NH}_{3}\right)_{6}\right]\left[\mathrm{Co}\left(\mathrm{C}_{2} \mathrm{O}_{4}\right)_{3}\right] \cdot \mathrm{H}_{2} \mathrm{O}$ or $\left[\mathrm{Co}\left(\mathrm{NH}_{3}\right)_{6}\right]\left[\operatorname{Ir}\left(\mathrm{C}_{2} \mathrm{O}_{4}\right)_{3}\right]$. The processes of the thermal decomposition in helium and hydrogen atmospheres were studied in detail.

\section{Experimental section}

\section{General}

Starting compounds $-\left[\operatorname{Ir}\left(\mathrm{NH}_{3}\right)_{6}\right] \mathrm{Cl}_{3}, \quad\left[\mathrm{Co}\left(\mathrm{NH}_{3}\right)_{6}\right] \mathrm{Cl}_{3}, \quad \mathrm{~K}_{3}\left[\mathrm{Co}\left(\mathrm{C}_{2} \mathrm{O}_{4}\right)_{3}\right] \cdot 3 \mathrm{H}_{2} \mathrm{O}$ and $\mathrm{K}_{3}\left[\operatorname{Ir}\left(\mathrm{C}_{2} \mathrm{O}_{4}\right)_{3}\right]$ - were prepared by literature methods $[6,11,12]$. Total $\mathrm{Ir}+\mathrm{Co}$ content was determined gravimetry after reduction in $\mathrm{H}_{2}$ atmosphere. Thermogravimetric analysis (TG) was performed on a Netzsch STA 409 PC Luxx instrument equipped with a QMS 100 Series mass-spectrometer (platinum crucible, He or air stream $14 \mathrm{~cm}^{3} / \mathrm{min}$, heating rate 10.0 $\mathrm{K} / \mathrm{min}$ ). Infrared spectra were recorded in $\mathrm{KBr}$ pellets on Scimitar FTS 2000. X-ray powder diffraction data for the complexes and thermolysis products were registered on a DRON$\mathrm{RM} 4$ diffractometer $\left(\mathrm{R}=192 \mathrm{~mm}, \mathrm{CuK}_{\alpha}\right.$ radiation, graphite monochromator) in $2 \theta$ range $5^{\circ}$ $120^{\circ}$ at room temperature. The refinement of lattice parameters was performed by the full profile technique with PowderCell 2.4 program [13] using all diffraction data. Crystallite sizes of metal phases were determined by Fourier decomposition of profiles of single diffraction peaks, and with the Scherrer equation (WINFIT 1.2.1 [14]). Single crystal diffraction data were collected on a BRUKER X8 APEX CCD diffractometer at room temperature ( $\mathrm{MoK}_{\alpha}, \lambda=0.7107 \AA$, graphite monochromator). The structures were solved by the standard heavy-atom method and refined in anisotropic approximation. The SHELX97 program package [15] was used in all computations. Scanning electron microscopy study of the samples was made on a JEOL JSM 6700F device equipped with a dispersive X-Ray analyzer EX-23000BU (EDS) operating at $15 \mathrm{kV}$.

\section{Synthesis of $\left[\mathrm{Ir}\left(\mathrm{NH}_{3}\right)_{6}\right]\left[\mathrm{Co}\left(\mathrm{C}_{2} \mathrm{O}_{4}\right)_{3}\right] \cdot \mathrm{H}_{2} \mathrm{O}(1)$}

Equimolar amounts of starting $\left[\operatorname{Ir}\left(\mathrm{NH}_{3}\right)_{6}\right] \mathrm{Cl}_{3}$ and $\mathrm{K}_{3}\left[\mathrm{Co}\left(\mathrm{C}_{2} \mathrm{O}_{4}\right)_{3}\right] \cdot 3 \mathrm{H}_{2} \mathrm{O}$ (the latter taken in slight excess) were dissolved in a small volume of water $(10-15 \mathrm{ml})$. Precipitation was achieved by combination of the solutions at $60{ }^{\circ} \mathrm{C}$. The combined solutions immediately yielded the precipitate of $\left[\operatorname{Ir}\left(\mathrm{NH}_{3}\right)_{6}\right]\left[\mathrm{Co}\left(\mathrm{C}_{2} \mathrm{O}_{4}\right)_{3}\right] \cdot \mathrm{H}_{2} \mathrm{O}$ as a light-green powder. After 15 min it was collected on a glass filter, washed with water and acetone and dried in air. The product obtained was characterized with a number of physical chemical methods. Elemental analysis for metals was made by reduction of precise weighs of the salts in a hydrogen stream.

Yield $98 \%$. Anal. Calc. for $\mathrm{C}_{6} \mathrm{H}_{20} \mathrm{~N}_{6} \mathrm{O}_{13} \mathrm{IrCo}$ ( $\left.\mathrm{Ir}+\mathrm{Co}\right)$, 39.53; C, 11.34; H, 3.17; N, 13.23 . Found: (Ir+Co), 39.6(2), C, 11.6(4); H, 2.9(4); N, 13.3(4). Crystal data for compound (1) $\mathrm{C}_{6} \mathrm{H}_{20} \mathrm{~N}_{6} \mathrm{O}_{13} \mathrm{IrCo}, \mathrm{M}=635.41$, space group $\mathrm{P} 1, \mathrm{a}=7.5630(2), \mathrm{b}=9.6046(3), \mathrm{c}=11.8116(4)$ $\AA, \alpha=84.7950(10), \beta=87.6100(10), \gamma=71.7670(10)^{\circ}, \mathrm{V}=811.47(4) \AA^{3}, \mathrm{~T}=293(2) \mathrm{K}, \mathrm{Z}$ $=2, \mathrm{~d}_{\text {calc }}=2.527, \mathrm{~d}_{\exp }=2.53 \pm 0.01 \mathrm{~g} / \mathrm{cm}^{3}$, crystal size $0.12 \times 0.10 \times 0.09 \mathrm{~mm}, \mathrm{R}_{1}=0.0149$, $\mathrm{wR}_{2}=0.0355$ for 7491 reflections with $\mathrm{I}>2 \sigma(\mathrm{I})$. 


\section{Synthesis of $\left[\mathrm{Co}\left(\mathrm{NH}_{3}\right)_{6}\right]\left[\operatorname{Ir}\left(\mathrm{C}_{2} \mathrm{O}_{4}\right)_{3}\right](2)$}

The compound $\left[\mathrm{Co}\left(\mathrm{NH}_{3}\right)_{6}\right]\left[\operatorname{Ir}\left(\mathrm{C}_{2} \mathrm{O}_{4}\right)_{3}\right]$ was prepared similarly to the technique described for $\left[\mathrm{Ir}\left(\mathrm{NH}_{3}\right)_{6}\right]\left[\mathrm{Co}\left(\mathrm{C}_{2} \mathrm{O}_{4}\right)_{3}\right] \cdot \mathrm{H}_{2} \mathrm{O}$.

Yield 98 \%. Anal. Calc. for $\mathrm{C}_{6} \mathrm{H}_{18} \mathrm{~N}_{6} \mathrm{O}_{12} \mathrm{IrCo}$ : (Ir+Co), 40.68; C, 11.67; H, 2.94; N, 13.61 . Found: (Ir+Co), 40.5(2), C, 11.8(4); H, 2.9(4); N, 13.5(4). Crystal data for compound (2) $\mathrm{C}_{6} \mathrm{H}_{18} \mathrm{~N}_{6} \mathrm{O}_{12} \mathrm{IrCo}, \mathrm{M}=617.39$, space group $\mathrm{P}-1, \mathrm{a}=7.4030(4), \mathrm{b}=9.7055(6), \mathrm{c}=11.7530(6)$ $\AA, \alpha=84.615(2), \beta=88.117(2), \gamma=69.8680(10)^{\circ}, \mathrm{V}=789.36(8) \AA^{3}, \mathrm{~T}=293(2) \mathrm{K}, \mathrm{Z}=2$, $\mathrm{d}_{\text {calc }}=2.598, \mathrm{~d}_{\mathrm{cxp}}=2.60 \pm 0.01 \mathrm{~g} / \mathrm{cm}^{3}$, crystal size $0.12 \times 0.10 \times 0.09 \mathrm{~mm}, \mathrm{R}_{1}=0.0294, \mathrm{wR}_{2}=$ 0.0789 for 4582 reflections with $\mathrm{I}>2 \sigma(\mathrm{I})$.

Detailed information on the crystal structures is available from the Fachinformationszentrum Karlsruhe, 76344 Eggenstein-Leopoldshafen, Germany, on quoting depository numbers CSD-419315 for (1) and 420455 for (2).

\section{Results and discussion}

\section{Thermal analysis in helium atmosphere.}

Thermal decomposition of $\left[\operatorname{Ir}\left(\mathrm{NH}_{3}\right)_{6}\right]\left[\mathrm{Co}\left(\mathrm{C}_{2} \mathrm{O}_{4}\right)_{3}\right] \cdot \mathrm{H}_{2} \mathrm{O}$ was studied with DTA combined with analysis of the composition of evolving gases. Figure 1 presents T, TG, DSC and MS - EGA plots for the $\left[\mathrm{Ir}\left(\mathrm{NH}_{3}\right)_{6}\right]\left[\mathrm{Co}\left(\mathrm{C}_{2} \mathrm{O}_{4}\right)_{3}\right] \cdot \mathrm{H}_{2} \mathrm{O}$ salt. Within $40-205^{\circ} \mathrm{C}$ the compound evolves $\mathrm{H}_{2} \mathrm{O}$ and $\mathrm{CO}_{2}$ affording a pink product, which was assigned with the formula $\left[\operatorname{Ir}\left(\mathrm{NH}_{3}\right)_{6}\right]\left[\mathrm{Co}\left(\mathrm{C}_{2} \mathrm{O}_{4}\right)_{2}\right] \cdot 1 / 2 \mathrm{C}_{2} \mathrm{O}_{4}$ on the basis of TG, DSC, IR and MS-EGA data. This composition of the intermediate product is indirectly supported by IR spectroscopy data. The IR spectrum of the starting compound manifests peaks characteristic of the cation $\left[\operatorname{Ir}\left(\mathrm{NH}_{3}\right)_{6}\right]^{2+}\left(\delta_{\mathrm{s}}\left(\mathrm{NH}_{3}\right) 1365 \mathrm{~cm}^{-1}\right)$ and the anion $\left[\mathrm{Co}\left(\mathrm{C}_{2} \mathrm{O}_{4}\right)_{3}\right]^{3-}\left(\mathrm{v}(\mathrm{Co}-\mathrm{O})+\delta_{\mathrm{s}}(\mathrm{O}=\mathrm{C}-\mathrm{O}) 820\right.$ $\left.+802 ; \mathrm{v}(\mathrm{Co}-\mathrm{O}) 565 \mathrm{~cm}^{-1}\right)$. In the IR spectrum of the intermediate product the lines corresponding to the vibrations within the cation have been preserved, while the peaks corresponding to the vibrations of the anion have been altered in positions and intensities $\left(\mathrm{v}(\mathrm{Co}-\mathrm{O})+\delta_{\mathrm{s}}(\mathrm{O}=\mathrm{C}-\mathrm{O}) 785 ; \mathrm{v}(\mathrm{Co}-\mathrm{O})\right.$ at $565 \mathrm{~cm}^{-1}$ are absent $)$. Decomposition ends at $550{ }^{\circ} \mathrm{C}$ and is accompanied by evolution of $\mathrm{NH}_{3}, \mathrm{~N}_{2}, \mathrm{H}_{2} \mathrm{O}, \mathrm{CO}_{2}, \mathrm{CO}$ and formation of $\mathrm{Ir}$ mixed with $\mathrm{CoO}$. The process can be represented by the following scheme:

$$
\begin{aligned}
& {\left[\mathrm{Ir}\left(\mathrm{NH}_{3}\right)_{6}\right]\left[\mathrm{Co}\left(\mathrm{C}_{2} \mathrm{O}_{4}\right)_{3}\right] \cdot \mathrm{H}_{2} \mathrm{O} \rightarrow\left[\mathrm{Ir}\left(\mathrm{NH}_{3}\right)_{6}\right]\left[\mathrm{Co}\left(\mathrm{C}_{2} \mathrm{O}_{4}\right)_{2}\right]\left(\mathrm{C}_{2} \mathrm{O}_{4}\right)_{0.5}+\mathrm{CO}_{2}+\mathrm{H}_{2} \mathrm{O}} \\
& {\left[\mathrm{Ir}\left(\mathrm{NH}_{3}\right)_{6}\right]\left[\mathrm{Co}\left(\mathrm{C}_{2} \mathrm{O}_{4}\right)_{2}\right]\left(\mathrm{C}_{2} \mathrm{O}_{4}\right)_{0.5} \rightarrow \mathrm{Ir}+\mathrm{CoO}+\mathrm{N}_{2}+\mathrm{NH}_{3}+\mathrm{H}_{2} \mathrm{O}+\mathrm{CO}_{2}+\mathrm{CO} .}
\end{aligned}
$$

Quite expectedly, decomposition of the anhydrous $\left[\mathrm{Co}\left(\mathrm{NH}_{3}\right)_{6}\right]\left[\operatorname{Ir}\left(\mathrm{C}_{2} \mathrm{O}_{4}\right)_{3}\right]$ begins at higher temperature $\left(300{ }^{\circ} \mathrm{C}\right)$ than that of its hydrated counterpart. Except for this, the process is identical to thermolysis of the hydrated complex $\left[\operatorname{Ir}\left(\mathrm{NH}_{3}\right)_{6}\right]\left[\mathrm{Co}\left(\mathrm{C}_{2} \mathrm{O}_{4}\right)_{3}\right] \cdot \mathrm{H}_{2} \mathrm{O}$.

In both cases, final products of thermal decomposition in helium atmosphere are the mixtures of $\mathrm{Ir}$ and $\mathrm{CoO}$.

\section{Products of thermal decomposition of the complexes in hydrogen atmosphere.}

All prepared compounds were subjected to decomposition in hydrogen atmosphere. Relatively fast $(0.5$ hour $)$ heating of the samples of the complexes $\left[\operatorname{Ir}\left(\mathrm{NH}_{3}\right)_{6}\right]\left[\mathrm{Co}\left(\mathrm{C}_{2} \mathrm{O}_{4}\right)_{3}\right] \cdot \mathrm{H}_{2} \mathrm{O}$ and $\left[\mathrm{Co}\left(\mathrm{NH}_{3}\right)_{6}\right]\left[\operatorname{Ir}\left(\mathrm{C}_{2} \mathrm{O}_{4}\right)_{3}\right]$ to $550{ }^{\circ} \mathrm{C}$ and further exposure of thermolysis products to this temperature during one hour results in formation of structurally 


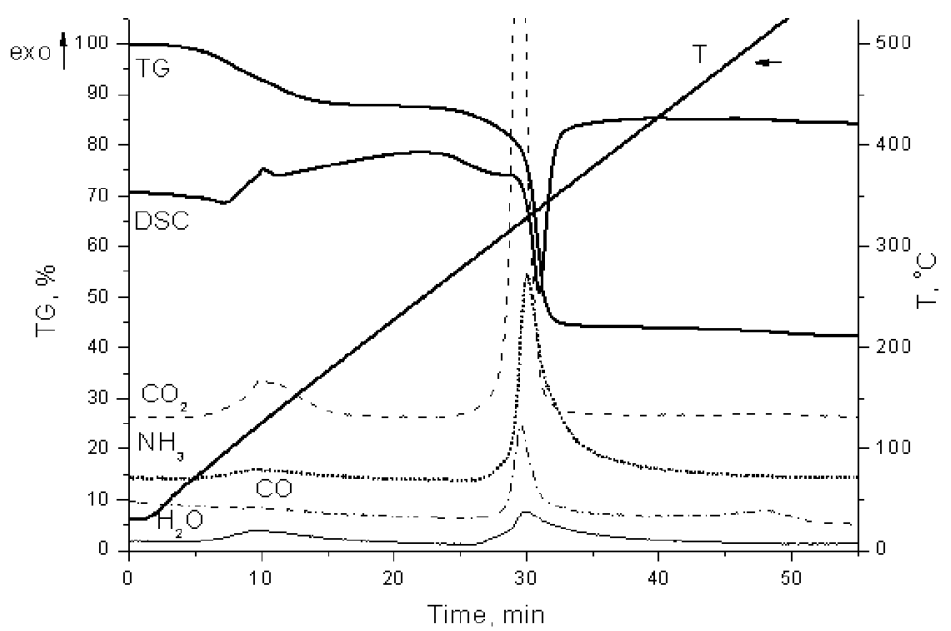

Figure 1. Gas evolution plots (DSC-MS-EGA) for the salt $\left[\mathrm{Ir}\left(\mathrm{NH}_{3}\right)_{6}\right]\left[\mathrm{Co}\left(\mathrm{C}_{2} \mathrm{O}_{4}\right)_{3}\right] \cdot \mathrm{H}_{2} \mathrm{O}$ in helium atmosphere.

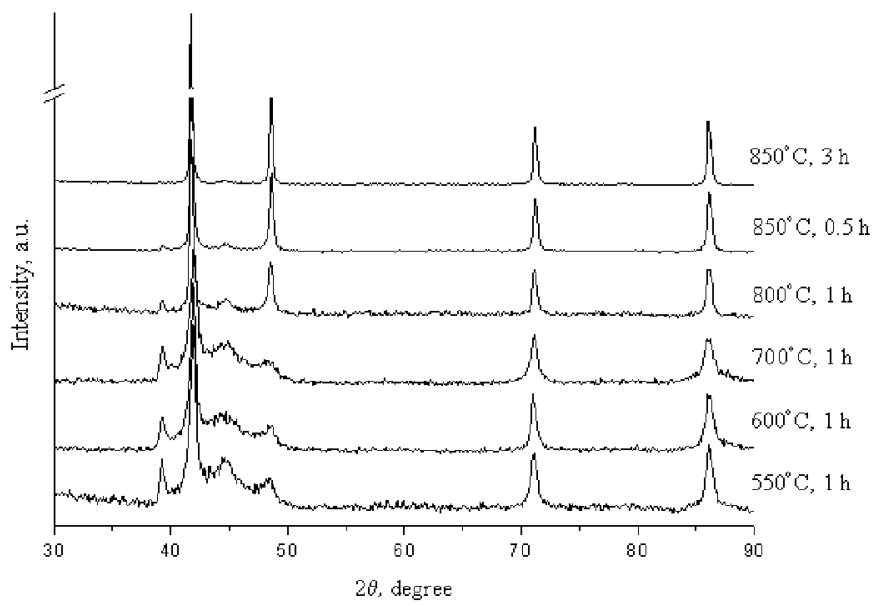

Figure 2. Diffraction patterns of the products of thermolysis of the DCS $\left[\operatorname{Ir}\left(\mathrm{NH}_{3}\right)_{6}\right]\left[\mathrm{Co}\left(\mathrm{C}_{2} \mathrm{O}_{4}\right)_{3}\right] \cdot \mathrm{H}_{2} \mathrm{O}$ in hydrogen atmosphere under different conditions.

similar products. The products were examined with powder XRD and EDS. The crystal structure of the developed phases can be represented as close packing with planar defects stacking faults $[16,17,18]$. 
Diffraction patterns of the products can be attributed to alloys of iridium with cobalt of the face-centered cubic (FCC) structure with coherent insertion of the hexagonal closely packed (HCP) fragments. Further examination of these phases will be the subject of a separate study.

Temperature rise and increased exposure time diminishes the number of packing defects. Decreased number of inclusions of the HCP fragments is manifested in the diffraction patterns in lowered intensities of the reflections at 39.7 and $45.7^{\circ}$ in $2 \theta$ (figure 2). The positions of the reflections of the FCC lattice remain constant, while relative intensity of the peak 002 increases, and the ratio $\mathrm{I}_{002} / \mathrm{I}_{111}$ approaches 0.5 . Finally, when the thermolysis product of the salt $\left[\mathrm{Ir}\left(\mathrm{NH}_{3}\right)_{6}\right]\left[\mathrm{Co}\left(\mathrm{C}_{2} \mathrm{O}_{4}\right)_{3}\right] \cdot \mathrm{H}_{2} \mathrm{O}$ was kept for $2 \mathrm{~h}$ at $850^{\circ} \mathrm{C}$, a powder of the solid solution $\mathrm{Ir}_{0.5} \mathrm{CO}_{0.5}$ was obtained, which has the $\mathrm{FCC}$ lattice with the parameter $a=$ 3.710 (3) A practically identical to that reported in [19, N 65-8951]. This value is also in a good agreement with experimental dependence between the unit cell parameter of the solid solution and composition of this system (figure 3). The composition of all samples was confirmed by energy dispersive X-ray spectroscopy (EDS).

It is noteworthy that the shape of the metallic particles inherits the habitus of the original crystals of the DCS. However, subsequent scans with enhanced resolution revealed that the particles (obtained at $550^{\circ} \mathrm{C}$ ) are built from smaller blocks with the sizes of $\sim 30 \mathrm{~nm}$.

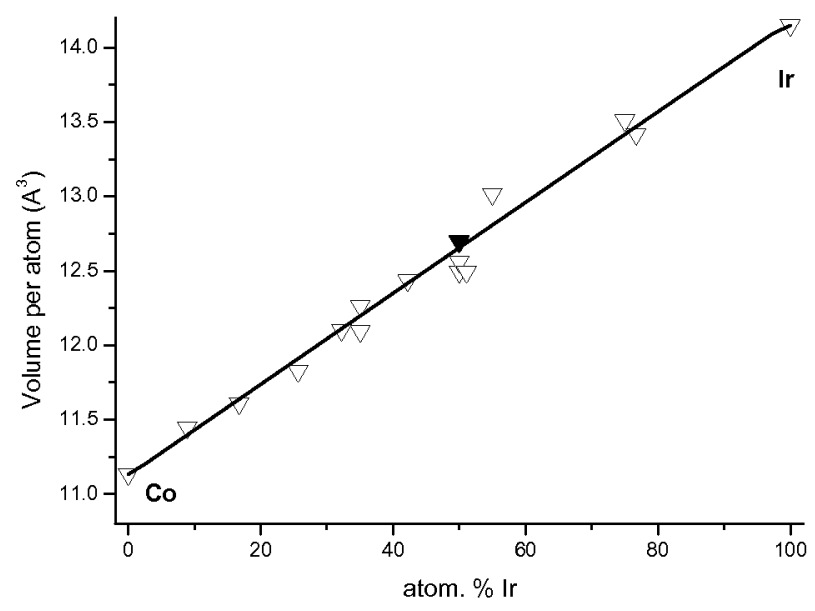

Figure 3. Calibrations curve presenting dependence of atomic volume on the composition of the solid solution. Open triangles - literature data, filled triangle - this work.

\section{Summary}

Novel double complex salts containing iridium in the cation and cobalt in the anion, or vice versa, have been prepared. It has been demonstrated that the compounds can be used as single-source molecular precursors of nanosized alloys: thermal decomposition of the compounds in helium and hydrogen results in formation of ultrafine bimetallic powders having equimolar metal ratio. 


\section{References}

1. Zadesenets, A.V., Filatov, E.Yu., Yusenko, K.V., Shubin, Yu.V., Korenev, S.V. \& Baidina, I.A., 2008, Inorganica Chimica Acta, 361, 199.

2. Min, S.H., Hoon, H.J., Bok, L.Y., Seop, K.W., Youngmee, K., Sung-Jin, K. \& Hwi, H.N., 2006, Chem. Comm., 12, 1292.

3. Parent, A.R., Vedachalam, S., Landee, C.P. \& Turnbull, M.M., 2008, J. Coord. Chem., 61, 93.

4. Ragaini, F., Cenini, S., Fumagalli, A. \& Crotti, C., 1992, J. Organomet. Chem., 428, 401 .

5. Chung, Y.-M., \& Rhee, H.-K., 2004, J. Colloid Interf. Sci., 271, 131.

6. Shubin, Yu.V., Korenev, S.V., Yusenko, K.V., Korda, T.M. \& Venediktov, A.B., 2002, Russ. Chem. Bull., 51, 39.

7. Silvestre-Albero, J., Serrano-Ruiz, J.C. \& Sepúlveda-Escribano, A., 2005, Appl. Catal. A: General, 292, 244.

8. Ito, S., Suwa, Y., Kondo, S., Kameoka, S., Tomishige, K. \& Kunimori, K., 2003, Catal. Commun., 4, 499.

9. Yang, H., Coutanceau, C. \& Léger, J.-M., 2005, J. Electroanal. Chem., 576, 305.

10. Yusenko, K.V., Filatov, E.Yu., Vasil'chenko, D.B. et al., 2007, Z. für Krist. Suppl., 26, 289.

11. Brauer, Georg, Handbook of Preparative Inorganic Chemistry, (New York: Academic), 1963-65. 2v.

12. Chernyaev, I.I., 1964, Synthesis of complex compounds of platinum group metals, Nauka, Moscow.

13. Kraus, W. \& Nolze, G., 2000, PowderCell 2.4, Program for the representation and manipulation of crystal structures and calculation of the resulting X-ray powder patterns (Berlin: Federal Institute for Materials Research and Testing).

14. Krumm, S., 1994, Materials Science Forum, 228-231, 183.

15. Sheldrick, G.M., 1997, SHELX97. Release 97-I (Göttingen: Univ. of Göttingen).

16. Paterson, M.S., 1952, J. Appl. Phys., 23, 805.

17. Ustinov, A.I., Olikhovskaya, L.A. \& Shmyt'ko, I.M., 2000, Crystallography Reports, 45, 365.

18. Guinier, A., 1956, Theorie et technique de la radiocristallographie (Paris: Dunod).

19. PCPDFWin, 1997, Ver. 1.30, JCPDS ICDD, Swarthmore, PA, USA.

Acknowledgements. This work was financially supported by the program of Presidium of RAS (project № 8-17), Interdisciplinary project of fundamental research SB RAS N112, and by RFBR grants 08-03-00603-a and NSh-636.2008.3. The authors are grateful to Prof. A.B. Venediktov for synthesizing the starting $\left[\mathrm{Ir}\left(\mathrm{NH}_{3}\right)_{6}\right] \mathrm{Cl}_{3}$. 


\title{
$\mathrm{Ru}-\mathrm{Re}, \mathrm{Ru}-\mathrm{Os}$, and $\mathrm{Re}-$ Os solid solutions - preparation under mild conditions, powder XRD investigation and phase diagram analysis
}

\author{
K. V. Yusenko", I. V. Korolkov, S. A. Martynova, \\ S. A. Gromilov
}

Nikolaev Institute of Inorganic Chemistry SB RAS, 630090 Novosibirsk, Lavrentyev Ave.3, Russia

*yusenko@che.nsk.su

Keywords: platinum metals, solid solutions, phase diagrams

Abstract. Ru - Re, Ru - Os, and Re - Os bimetal solid solutions were prepared by the thermal decomposition of bimetal compounds $\left[\mathrm{M}^{1}\left(\mathrm{NH}_{3}\right)_{5} \mathrm{Cl}\right]\left[\mathrm{M}^{2} \mathrm{Cl}_{6}\right]$ and $\left[\mathrm{M}^{1}\left(\mathrm{NH}_{3}\right)_{5} \mathrm{Cl}_{2}\left[\mathrm{M}^{2} \mathrm{Cl}_{6}\right] \mathrm{Cl}_{2}\left(\mathrm{M}^{1}=\mathrm{Ru}, \mathrm{Os} ; \mathrm{M}^{2}=\mathrm{Re}, \mathrm{Os}\right)\right.$ under $\mathrm{H}_{2}$. Precursors were prepared as described previously for the compounds of like composition. The preparation at $500^{\circ} \mathrm{C}$ gave homogeneous hcp bimetal solid solutions with the crystallite dimensions of about $100 \AA$. An additional annealing of the samples during $5 \mathrm{~h}$ at $800^{\circ} \mathrm{C}$ had no essential effect on the unit cell parameters but the crystallite size was doubled. The dependence of the atomic volumes $(V / Z)$ on the composition of the $\mathbf{R u}-\mathbf{R e}, \mathbf{R u}-\mathbf{O s}$, and $\mathbf{R e}-\mathbf{O s}$ bimetal solid solutions is depicted in the current report. The dependences are nearly linear with a negative deflection no more than $0.5 \%$. Equilibrium binary phase diagrams of the given metal systems were calculated by the CALPHAD method based on the ideal solution model. The bimetal powders obtained are nearly equilibrium and are prepared on 1/5 melting points for pure metals. It gives possibilities for the preparation of nano-dimensional solid solutions of high melting metals for further catalytic and material applications.

\section{Introduction}

The control of the size, shape and phase composition of nanocrystalline materials is a key point in current nano-scientific research. Synthetic chemical methods have proved the efficiency for the production of nano-crystals with a tight size distribution and composition. Ru, Re, and Os bulk and nano-scale solid solutions show thermal and chemical stability and good mechanical properties $[1,2]$. The metals with the hexagonal close-packed (hcp) structures as well as Re and Os have extremely high melting points. The given metals form the continuous hop solid solutions but reaching the equilibrium is hindered because of the high melting 
points. Therefore their solid solutions are difficult to be prepared and no detail data on crystallographic and physical properties of the hep solid solutions are available.

A modern way for the preparation of the high melting solid solutions is the thermal decomposition of the bimetal cluster and coordination compounds [see, for example, 3 and 4]. The thermal decomposition gives metal phases under mild conditions, and nano-dimensional phases are usually formed. We have previously used the thermal decomposition of bimetal precursors for the preparation of hep - fcc (face centered cubic) solid solutions. The main purpose of the present work is the preparation of the solid solutions of the most high melting metal systems, Ru - Re, Ru - Os, and Re - Os, by the thermal decomposition of the double complex salts (DCS) in soft conditions and their characterization by powder X-ray diffraction (PXRD) and scanning electron microscopy (SEM). Besides, binary phase and Retgers diagrams were analyzed.

\section{Experimental section}

PXRD analysis of polycrystals of the initial precursors was performed on a STADI-P STOE diffractometer ( $\mathrm{CuK \alpha}$-radiation, positional sensitive detector, the range of $2 \theta=2-120^{\circ}$, at a step of $0.02^{\circ}$, with accumulation time $2040 \mathrm{~s}$ /point). XRD patterns of the thermolysis products were recorded on a DRON-SEIFERT-RM4 diffractometer ( $\mathrm{Cu} K_{\mathrm{\alpha}}$-radiation, Ni-filter, the range of $2 \theta=30-165^{\circ}$, room temperature). A polycrystalline sample was slightly ground with hexane in an agate mortar, and the resulting suspension was deposited on the polished side of a standard quartz sample holder, and a smooth thin layer being formed after drying. Indexing of the diffraction patterns was carried out using data for pure metals and compounds reported in the PDF database [5]. The unit cell parameters were refined by the full-profile technique within the whole diffraction range with the POWDERCELL 2.4 software [6]. Crystallite sizes of the metal phases were calculated by Fourier analysis of the single diffraction peaks and by the Scherrer equation using WINFIT 1.2.1 software [7]. Silicon powder was taken as an external standard $\left(\mathrm{a}=5.4309 \AA\right.$, FWHM $\left.2 \Theta=0.1^{\circ}\right)$. SEM study of the samples was made on a JEOL JSM $6700 \mathrm{~F}$ device equipped with a dispersive X-ray analyzer EX-23000BU operating $15 \mathrm{kV}$.

The metal powders of $\mathrm{M}_{0.5}^{1} \mathrm{M}^{2}{ }_{0.5}$ and $\mathrm{M}_{0.67}^{1} \mathrm{M}^{2}{ }_{0.33}$ were obtained after the thermal decomposition of two types of DCS, $\left[\mathrm{M}^{1}\left(\mathrm{NH}_{3}\right)_{5} \mathrm{Cl}\right]\left[\mathrm{M}^{2} \mathrm{Cl}_{6}\right]$ (Type I) and $\left[\mathrm{M}^{1}\left(\mathrm{NH}_{3}\right)_{5} \mathrm{Cl}\right]_{2}\left[\mathrm{M}^{2} \mathrm{Cl}_{6}\right] \mathrm{Cl}_{2}$ (Type II) $\left(\mathrm{M}^{1}=\mathrm{Ru}, \mathrm{Os} ; \mathrm{M}^{2}=\mathrm{Re}, \mathrm{Os}\right.$ ), in $\mathrm{H}_{2}$ or He atmosphere at $500-650{ }^{\circ} \mathrm{C}$ (detailed preparatory conditions see in Table 1), respectively. In addition, two bimetal powders, namely $\mathrm{Ru}_{0.15} \mathrm{Re}_{0.75}$ [8] and $\mathrm{Ru}_{0.33} \mathrm{Re}_{0.67}$ [9], were analyzed as final products of the thermal decomposition of $\left[\mathrm{Ru}\left(\mathrm{NH}_{3}\right)_{5} \mathrm{Cl}_{2}\left[\mathrm{Re}_{6} \mathrm{~S}_{8}(\mathrm{CN})_{6}\right]\right.$ and $\left[\mathrm{Ru}\left(\mathrm{NH}_{3}\right)_{5} \mathrm{Cl}\right]\left(\mathrm{ReO}_{4}\right)_{2}$, respectively. Their precursors were prepared by crystallization from water solutions with high yields $(85-95$ $\%$ ) according to the equations:

$$
\begin{gathered}
{\left[\mathrm{M}^{1}\left(\mathrm{NH}_{3}\right)_{5} \mathrm{Cl}\right] \mathrm{Cl}_{2}+\left(\mathrm{NH}_{4}\right)_{2}\left[\mathrm{M}^{2} \mathrm{Cl}_{6}\right] \rightarrow\left[\mathrm{M}^{1}\left(\mathrm{NH}_{3}\right)_{5} \mathrm{Cl}\right]\left[\mathrm{M}^{2} \mathrm{Cl}_{6}\right] \downarrow+2 \mathrm{NH}_{4} \mathrm{Cl}} \\
2\left[\mathrm{M}^{1}\left(\mathrm{NH}_{3}\right)_{5} \mathrm{Cl}\right] \mathrm{Cl}_{2}+\left(\mathrm{NH}_{4}\right)_{2}\left[\mathrm{M}^{2} \mathrm{Cl}_{6}\right] \rightarrow\left[\mathrm{M}^{1}\left(\mathrm{NH}_{3}\right)_{5} \mathrm{Cl}\right]_{2}\left[\mathrm{M}^{2} \mathrm{Cl}_{6}\right] \mathrm{Cl}_{2} \downarrow+2 \mathrm{NH}_{4} \mathrm{Cl}
\end{gathered}
$$

All solid solutions, their preparatory conditions, the hexagonal unit cell parameters and the atomic volumes $(V / Z)$ known for $\mathrm{Ru}-\mathrm{Re}, \mathrm{Ru}-\mathrm{Os}$ and $\mathrm{Re}-\mathrm{Os}$ are listed in Table 1. 


\section{Results and discussion}

The precursors of like composition (Types I and II) are isostructural $[9,10]$. The unit cell parameters for $\left[\mathbf{R u}\left(\mathbf{N H}_{3}\right)_{5} \mathbf{C l}\right]_{2}\left[\mathbf{R e C l}_{6}\right] \mathrm{Cl}_{2}$ are $\mathrm{a}=11.3087(6), \mathrm{b}=8.0143(4), \mathrm{c}=13.4500(6)$ $\AA, \beta=99.765(2)^{\circ}, Z=2, \mathrm{C} 2 / \mathrm{m}$; for $\left[\mathbf{R u}\left(\mathbf{N H}_{3}\right)_{5} \mathbf{C l}_{2}\left[\mathbf{O s C l}_{6}\right] \mathbf{C l}_{2} \mathrm{a}=11.266(2), \mathrm{b}=7.974(1), \mathrm{c}\right.$ $=13.448(2) \AA, \beta=99.601(2)^{\circ}, \mathrm{Z}=2, \mathrm{C} 2 / \mathrm{m}$; for $\left[\mathbf{R u}\left(\mathbf{N H}_{3}\right)_{5} \mathbf{C l}\right]\left[\mathbf{R e C l}_{6}\right] \mathrm{a}=11.633(2), \mathrm{b}=$ 8.351(1), $\mathrm{c}=15.296(3) \AA, \beta=90.863(2)^{\circ}, \mathrm{Z}=4, \mathrm{P} 2_{1} / \mathrm{m}$; for $\left[\mathbf{R u}\left(\mathbf{N H}_{3}\right)_{5} \mathbf{C l}\right]\left[\mathbf{O s C l}_{6}\right] \mathrm{a}=$ 11.593(2), $\mathrm{b}=8.318(1), \mathrm{c}=15.234(3) \AA, \beta=90.707(2)^{\circ}, \mathrm{Z}=4, \mathrm{P} 2_{1} / \mathrm{m}$; and for $\left[\mathrm{Os}\left(\mathrm{NH}_{3}\right)_{5} \mathbf{C l}\right]\left[\mathrm{ReCl}_{6}\right] \mathrm{a}=11.645(3), \mathrm{b}=8.3788(2), \mathrm{c}=15.277(4), \beta=91.029(6)^{\circ}, \mathrm{Z}=4$, $\mathrm{P} 2_{1} / \mathrm{m}$. All the phases listed are built of the discrete complex cations $\left[\mathrm{M}^{1}\left(\mathrm{NH}_{3}\right)_{5} \mathrm{Cl}\right]^{2+}$, the complex anions $\left[\mathrm{M}^{2} \mathrm{Cl}_{6}\right]^{2-}$ and $\mathrm{Cl}^{-}$anions (Type II). Geometric characteristics of the coordination polyhedra are not virtually different from those for $\left[\mathrm{M}^{1}\left(\mathrm{NH}_{3}\right)_{5} \mathrm{Cl} \mathrm{Cl}_{2}\right.$ and $\left(\mathrm{NH}_{4}\right)_{2}\left[\mathrm{M}^{2} \mathrm{Cl}_{6}\right]$. In the crystal structures, the hexagonal closed-packed layers may be obtained.

All the prepared metal phases are homogeneous and have the hep structures. The dependence of $V / Z$ on the composition for the $\mathbf{R u}-\mathbf{R e}, \mathbf{R u}-\mathbf{O s}$ and $\mathbf{R e}-\mathbf{O s}$ bimetal solid solutions are depicted in figure 1 . Our data confirmed the literature data $[8,9,11-13]$. Dependences are nearly linear with the negative deflection by no more than $0.5 \%$. The $\mathrm{V} / \mathrm{Z}$ dependences can be fitted the third-order polynomials. For the $\mathrm{Ru}-$ Os system the dependence is following: $\mathrm{V} / \mathrm{Z}=13.58+0.39 * \mathrm{X}_{\mathrm{Os}}-0.14 * \mathrm{X}_{\mathrm{Os}}{ }^{2}+0.16^{*} \mathrm{X}_{\mathrm{Os}}{ }^{3} ;$ for $\mathrm{Ru}-\mathrm{Re}: \mathrm{V} / \mathrm{Z}=13.57+1.12^{*} \mathrm{X}_{\mathrm{Re}}-$ $0.21 * \mathrm{X}_{\mathrm{Re}}{ }^{2}+0.22 * \mathrm{X}_{\mathrm{Re}}{ }^{3}$; for $\mathrm{Re}-\mathrm{Os}: \mathrm{V} / \mathrm{Z}=13.97+0.48^{*} \mathrm{X}_{\mathrm{Re}}-0.44^{*} \mathrm{X}_{\mathrm{Re}}{ }^{2}-0.20^{*} \mathrm{X}_{\mathrm{Re}}{ }^{3}$; where $\mathrm{X}_{\mathrm{i}}$ is $i$-component molar shape in binary solution. The phases prepared by melting and the thermal decomposition of the bimetal compounds lie on the one line, it means that prepared solid solutions are nearly equilibrium. PXRD patterns give the dimensions of about $100-150 \AA$. for bimetal solid solutions. According to the SEM imaging, the particles obtained repeat the initial precursor crystalline form and consist of the nearly cubic conglomerates. For example, the PXRD patterns and SEM image for $\mathbf{R u}_{0,5} \mathbf{R e}_{0,5}$ solid solution prepared from $\left[\mathrm{Ru}\left(\mathrm{NH}_{3}\right)_{5} \mathrm{Cl}\right]\left[\mathrm{ReCl}_{6}\right]$ at 500 and $800{ }^{\circ} \mathrm{C}$ under $\mathrm{H}_{2}$ atmosphere are presented in figure 2 . The synthesis at $500{ }^{\circ} \mathrm{C}$ gives the solid solution with the crystallite dimensions of about 100 $\AA$. The additional annealing of the sample during $5 \mathrm{~h}$ at $800^{\circ} \mathrm{C}$ has no an essential effect on the unit cell parameters but the crystallite size is doubled.

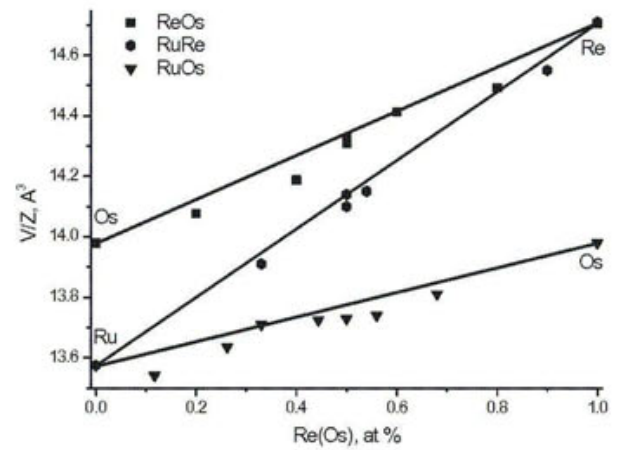

Figure 1. V/Z dependence for $\boldsymbol{R} \boldsymbol{u}-\boldsymbol{R} \boldsymbol{e}, \boldsymbol{R} \boldsymbol{u}-\boldsymbol{O}$ s and $\boldsymbol{R} \boldsymbol{e}-\boldsymbol{O}$ s hep solid solutions. 
Table 1. Crystallographic characteristics and preparatory conditions for prepared and known phases in $R u-R e, R u-O s$, and $O s-R e$ systems.

\begin{tabular}{|c|c|c|c|}
\hline $\begin{array}{l}\text { Composition } \\
\text { Reference }\end{array}$ & Preparatory conditions & $\begin{array}{c}a \\
c, \AA \\
c\end{array}$ & $V / Z, \AA^{3}$ \\
\hline $\begin{array}{c}\mathrm{Re} \\
{[\mathrm{PDF}, \text { №5-702] }}\end{array}$ & & $\begin{array}{l}2,7609(4) \\
4,4576(4)\end{array}$ & 14,715 \\
\hline $\begin{array}{c}\text { Os } \\
{[\mathrm{PDF}, \text { №6-662] }}\end{array}$ & & $\begin{array}{l}2,7341(4) \\
4,3197(4)\end{array}$ & 13,98 \\
\hline $\begin{array}{c}\mathrm{Ru} \\
{[\mathrm{PDF}, \text { №6-663] }}\end{array}$ & & $\begin{array}{l}2,7058(4) \\
4,2819(4)\end{array}$ & 13,575 \\
\hline $\begin{array}{l}\mathrm{Ru}_{0,67} \mathrm{Re}_{0,33} \\
\text { Our result }\end{array}$ & $\begin{array}{c}\text { Thermal decomposition of }\left[\mathrm{Ru}\left(\mathrm{NH}_{3}\right)_{5} \mathrm{Cl}\right]_{2}\left[\mathrm{ReCl}_{6}\right] \mathrm{Cl}_{2} \\
650^{\circ} \mathrm{C}, \mathrm{H}_{2}, 5 \mathrm{~h}\end{array}$ & $\begin{array}{l}2,724(2) \\
4,329(3) \\
\end{array}$ & 13,91 \\
\hline $\begin{array}{l}\mathrm{Ru}_{0,50} \mathrm{Re}_{0,50} \\
\text { Our result }\end{array}$ & $\begin{array}{l}\text { Thermal decomposition of }\left[\mathrm{Ru}\left(\mathrm{NH}_{3}\right)_{5} \mathrm{Cl}\right]\left[\mathrm{ReCl}_{6}\right] \\
500^{\circ} \mathrm{C}, \mathrm{He}, 1 \mathrm{~h}\end{array}$ & $\begin{array}{l}2,738(2) \\
4,355(3)\end{array}$ & 14,14 \\
\hline $\begin{array}{l}\mathrm{Ru}_{0,50} \mathrm{Re}_{0,50} \\
\text { Our result }\end{array}$ & $\begin{array}{l}\text { Thermal decomposition of }\left[\mathrm{Ru}\left(\mathrm{NH}_{3}\right)_{5} \mathrm{Cl}\right]\left[\mathrm{ReCl}_{6}\right] \\
500^{\circ} \mathrm{C}, \mathrm{H}_{2}, 1 \mathrm{~h}\end{array}$ & $\begin{array}{l}2,735(2) \\
4,354(3)\end{array}$ & 14,10 \\
\hline $\begin{array}{c}\mathrm{Ru}_{0,46} \mathrm{Re}_{0,54} \\
{[\mathrm{PDF}, 65-7948]}\end{array}$ & $\begin{array}{l}\text { Annealing of metal powder mixture } \\
1200{ }^{\circ} \mathrm{C} \text {, vacuum, } 100 \mathrm{~h} \\
\end{array}$ & $\begin{array}{l}2,737(1) \\
4,361(1) \\
\end{array}$ & 14,15 \\
\hline $\begin{array}{l}\mathrm{Ru}_{0,33} \mathrm{Re}_{0,67} \\
{[9]}\end{array}$ & $\begin{array}{l}\text { Thermal decomposition of }\left[\mathrm{Ru}\left(\mathrm{NH}_{3}\right)_{5} \mathrm{Cl}\right]\left(\mathrm{ReO}_{4}\right)_{2} \\
600^{\circ} \mathrm{C}, \mathrm{H}_{2}, 1 \mathrm{~h}\end{array}$ & $\begin{array}{l}2,745(5) \\
4,389(6)\end{array}$ & 14,33 \\
\hline $\begin{array}{l}\mathrm{Ru}_{0,25} \mathrm{Re}_{0,75} \\
{[8]}\end{array}$ & $\begin{array}{l}\text { Thermal decomposition of }\left[\mathrm{Ru}\left(\mathrm{NH}_{3}\right)_{5} \mathrm{Cl}\right]_{2}\left[\mathrm{Re}_{6} \mathrm{~S}_{8}(\mathrm{CN})_{6}\right] \cdot 3 \mathrm{H}_{2} \mathrm{O} \\
600^{\circ} \mathrm{C}, \mathrm{H}_{2}, 1 \mathrm{~h}\end{array}$ & $\begin{array}{l}2,756(2) \\
4,414(3) \\
\end{array}$ & 14,52 \\
\hline $\begin{array}{c}\mathrm{Ru}_{0,10} \mathrm{Re}_{0,90} \\
{[\mathrm{PDF}, 65-8227]}\end{array}$ & $\begin{array}{l}\text { Annealing of metal powder mixture in vacuum, } \\
1500^{\circ} \mathrm{C} \text {, vacuum, } 125 \mathrm{~h} \text {, }\end{array}$ & $\begin{array}{l}2,753(5) \\
4,432(6)\end{array}$ & 14,55 \\
\hline $\begin{array}{c}\mathrm{Ru}_{0,88} \mathrm{O}_{s_{0,12}} \\
{[11]} \\
\end{array}$ & Melting in vacuum, $2000 \mathrm{C}$ & $\begin{array}{l}2,705(1) \\
4,274(1)\end{array}$ & 13,54 \\
\hline $\begin{array}{l}\mathrm{Ru}_{0,67} \mathrm{Os}_{0,3} \\
\text { Our result } \\
\end{array}$ & $\begin{array}{c}\text { Thermal decomposition of }\left[\mathrm{Ru}\left(\mathrm{NH}_{3}\right)_{5} \mathrm{Cl}\right]_{2}\left[\mathrm{OsCl}_{6}\right] \mathrm{Cl}_{2} \\
650^{\circ} \mathrm{C}, \mathrm{H}_{2}, 5 \mathrm{~h}\end{array}$ & $\begin{array}{l}2,714(2) \\
4,297(3)\end{array}$ & 13,71 \\
\hline $\begin{array}{l}\mathrm{Ru}_{0,74} \mathrm{Os}_{0,26} \\
{[11]}\end{array}$ & Melting in vacuum, $2000 \mathrm{C}$ & $\begin{array}{l}2,710(1) \\
4,288(1)\end{array}$ & 13,64 \\
\hline $\begin{array}{l}\mathrm{Ru}_{0,56} \mathrm{Os}_{0,44} \\
{[11]} \\
\end{array}$ & Melting in vacuum, $2000 \mathrm{C}$ & $\begin{array}{l}2,716(1) \\
4,297(1) \\
\end{array}$ & 13,73 \\
\hline $\begin{array}{l}\mathrm{Ru}_{0,5} \mathrm{Os}_{0,5} \\
\text { Our result }\end{array}$ & $\begin{array}{c}\text { Thermal decomposition of }\left[\mathrm{Ru}\left(\mathrm{NH}_{3}\right)_{5} \mathrm{Cl}\right]\left[\mathrm{OsCl}_{6}\right] \\
600 \mathrm{C}, \mathrm{H}_{2}, 1 \mathrm{~h} \\
\end{array}$ & $\begin{array}{l}2,716(2) \\
4,302(3) \\
\end{array}$ & 13,74 \\
\hline $\begin{array}{l}\mathrm{Ru}_{0,5} \mathrm{Os}_{0,5} \\
{[12]}\end{array}$ & Melting in vacuum, $2000 \mathrm{C}$ & $\begin{array}{l}2,719(1) \\
4,302(1)\end{array}$ & 13,77 \\
\hline $\begin{array}{l}\mathrm{Ru}_{0,32} \mathrm{O}_{5,68} \\
{[11]} \\
\end{array}$ & Melting in vacuum, $2000 \mathrm{C}$ & $\begin{array}{l}2,721(1) \\
4,308(1) \\
\end{array}$ & 13,81 \\
\hline $\begin{array}{c}\mathrm{Re}_{0.2} \mathrm{O}_{0.8} \\
{[13]} \\
\end{array}$ & Melting in vacuum, $2000 \mathrm{C}$ & $\begin{array}{l}2.740(1) \\
4.330(1) \\
\end{array}$ & 14.078 \\
\hline $\begin{array}{c}\operatorname{Re}_{0.4} \mathrm{Os}_{0.6} \\
{[13]}\end{array}$ & Melting in vacuum, $2000 \mathrm{C}$ & $\begin{array}{l}2.745(1) \\
4.349(1) \\
\end{array}$ & 14.188 \\
\hline $\begin{array}{l}\mathrm{Re}_{0.5} \mathrm{Os}_{0.5} \\
\text { Our result }\end{array}$ & $\begin{array}{l}\text { Thermal decomposition of }\left[\mathrm{Os}\left(\mathrm{NH}_{3}\right)_{5} \mathrm{Cl}\right]\left[\mathrm{ReCl}_{6}\right] \\
500^{\circ} \mathrm{C}, \mathrm{H}_{2}, 1 \mathrm{~h}\end{array}$ & $\begin{array}{l}2.753(4) \\
4.366(6)\end{array}$ & 14.328 \\
\hline $\begin{array}{l}\mathrm{Re}_{0.6} \mathrm{Os}_{0.4} \\
{[13]}\end{array}$ & Melting in vacuum, $2000 \mathrm{C}$ & $\begin{array}{l}2.754(1) \\
4.389(1)\end{array}$ & 14.412 \\
\hline $\begin{array}{c}\mathrm{Re}_{0.8} \mathrm{Os}_{0.2} \\
{[13]}\end{array}$ & Melting in vacuum, $2000 \mathrm{C}$ & $\begin{array}{l}2.754(1) \\
4.412(1) \\
\end{array}$ & 14.493 \\
\hline
\end{tabular}




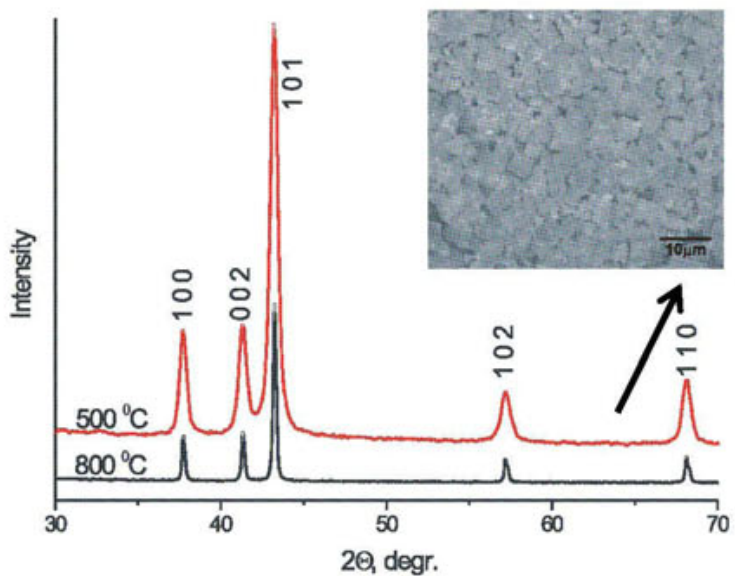

Figure 2. PXRD patterns and SEM image for $R u_{0,5} R_{0,5}$ solid solution, prepared by thermal decomposition of $\left[\mathrm{Ru}\left(\mathrm{NH}_{3}\right)_{5} \mathrm{Cl}\right][\mathrm{ReCl}]_{6}$.

Equilibrium binary phase diagrams of the given metal systems were calculated by CALPHAD method [14]. Solidus and subsolidus lines were calculated by the following equations:

$$
\begin{gathered}
G_{A}^{\circ s} \quad R T \ln x_{s} \quad x_{s}^{2} E=G_{A}^{o l}+R T \ln x_{l}+x_{l}^{2} L ; \\
G_{B}^{\circ s}+R T \ln \left(1-x_{s}\right)+\left(1-x_{s}\right)^{2} E=G_{B}^{o l}+R T \ln \left(1-x_{l}\right)+\left(1-x_{l}\right)^{2} L,
\end{gathered}
$$

where $x_{s}$ and $x_{l}$ are the A component shapes in solid and liquid phases, respectively; $G_{i}^{o s}$ and $G_{i}^{o l}$ are the standard Gibbs energies of $\mathrm{A}$ or $\mathrm{B}$ in solid and liquid phases: $G_{i}^{\circ s}=H_{i}^{\circ s}-T S_{i}^{\circ s}$ and $G_{i}^{\circ l}=H_{i}^{\circ l}-T S_{i}^{\circ l}$; E and $\mathrm{L}$ are the mixing parameters for solid and liquid phases. According to the ideal solution model $\mathrm{E}=\mathrm{L}=0$. Standard enthalpy $(\mathrm{kJ} / \mathrm{mol})$ and melting temperatures $(\mathrm{K})$ were the following: $\mathrm{Ru}-35.6,2523$; Os - 74.2, 3306; $\mathrm{Re}-48.8,3453[15,16]$. Calculated and experimantal phase diagrams for the $\mathrm{Ru}-\mathrm{Re}$ and $\mathrm{Ru}-$ Os systems are depicted in figure 3 . Very good agreement has been obtained between experimental phase diagrams $[11,13,17]$ and calculated those based on the ideal solution model.

\section{Conclusion}

The obtained bimetal powders are nearly equilibrium and were prepared under soft conditions. Preparatory temperatures were about $1 / 5$ melting points for pure metals. It gives perspective possibilities for the preparation of the nano-dimensional solid solutions of high melting metals for catalytic and material applications. Isostructurality of the precursors of like composition gives a chance to prepare their solid solutions that provides preparatory ways for synthesis of more complex metal solid solutions. 

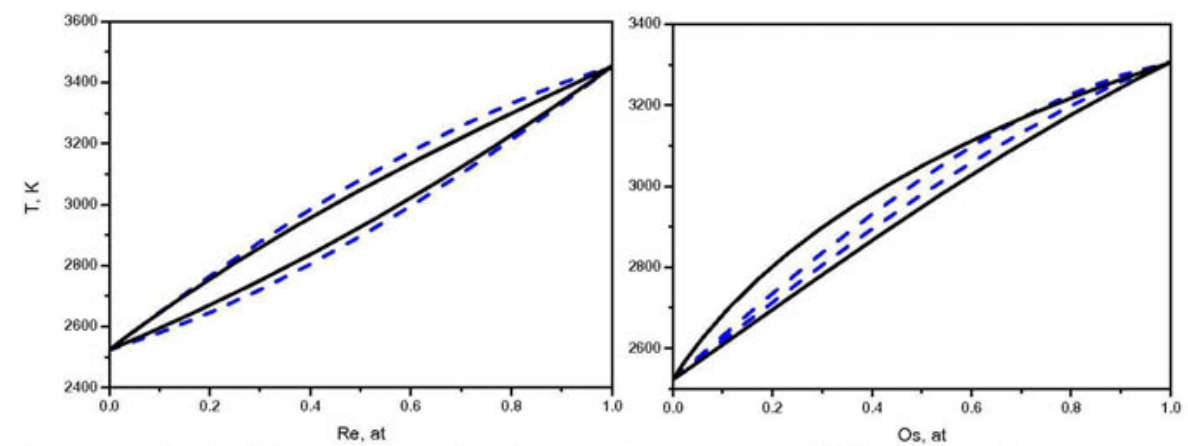

Figure 3. Calculated phase diagrams based on an ideal solution model (firm line) and experimental phase diagrams (dotted line) for $\mathrm{Ru}-\mathrm{Re}$ (left) and $\mathrm{Ru}-\mathrm{Os}$ (right) systems.

\section{References}

1. Huang, J., Yang, H., Huang, Q., Tang, Y., Lu, T. \& Akins, D. L., 2004, J. Electrochem. Soc., 151, A1810.

2. Sayre, E.D., 1994, Evol. Refract. Met. Alloys, Proc. Symp., (TMS Annu. Meet.).

3. Chotisuwan, S., Wittayakun, J., Lobo-Lapidus, R.J. \& Gates, B.C., 2007, Catal. Lett., $115,99$.

4. Michelot, B., Ouali, A., Blais, M.J., Guerin, M. \& Kappenstein, C., 1988, New J. Chem., 12, 293.

5. Powder Diffraction File. Alphabetical Index. Inorganic Phases, JCPDS, 1983 (International Centre for Diffraction Data, Pennsylvania, USA).

6. Kraus, W. \& Nolze, G., 2000, PowderCell 2.4, Program for the Representation and Manipulation of Crystal Structures and Calculation of the Resulting X-ray Powder Patterns (Berlin: Federal Institute for Materials Research and Testing).

7. Krumm, S., 1996, Mater. Sci. Forum, 228-231, 183.

8. Yusenko, K., Shusharina, E., Baidina, I. \& Gromilov, S., 2007, Acta Crystallogr. Sect. A: Found. Crystallogr., A63 (Supplement), 158.

9. Shubin, Yu.V., Filatov, E.Yu., Baidina, I.A., Yusenko, K.V., Zadesenetz, A.V. \& Korenev, S.V., 2006, J. Struct. Chem., 47, 1103.

10. Martynova, S.A., Yusenko, K.V., Korol'kov, I.V. \& Gromilov S.A., 2007, Russ. J. Coord. Chem., 33, 530.

11. Savitskii, E.M., Tylkina, M.A. \& Polyakova, V.P., 1962, Russ. J. Inorg. Chem., 7, 224.

12. Rudy, E., Kieffer, B. \& Froehlich, H., 1962, Z. Metallkd., 53, 90.

13. Tylkina, M.A., Polyakova, V.P. \& Savitskii, E.M., 1962, Russ. J. Inorg. Chem., 7, 754. 
14. Kaufman, L. \& Bernstein, H., 1970, Computer Calculation of Phase Diagrams (N. York: Acad. Press).

15. Arblaster, J.W., 1996, Platinum Metals Rev., 40, 62.

16. Arblaster, J.W., 1996, CALPHAD: Comput. Coupling Phase Diagrams Thermochem., 20, 343.

17. Tylkina, M.A., Polyakova, V.P. \& Savitskii, E.M., 1962, Russ. J. Inorg. Chem., 7, 755.

Acknowledgement. The authors are grateful to M.V. Yusenko (Universität Heidelberg, Germany) for helpful discussions. 



\title{
Application of neutron powder diffraction for the study of non-stoichiometric $\mathrm{Ni}_{2} \mathrm{MnGa}$ based alloys
}

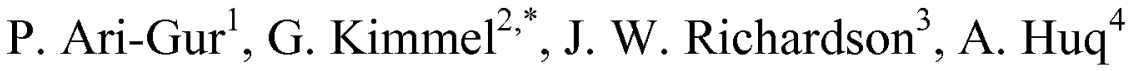 \\ ${ }^{1}$ Western Michigan University (WMU), Kalamazoo 49008, United States. \\ ${ }^{2}$ Ben Gurion University of the Negev, Beer-Sheva 84105, Israel. \\ 3 Argonne National Laboratory (ANL), Argonne, IL 60439, United States \\ ${ }^{4}$ Oak Ridge National Laboratory (ORNL), Oak Ridge, TN 37831, United States \\ *kimmel@bgu.ac.il
}

Keywords: Ni-Mn-Ga alloys, neutron diffraction, powder diffraction, thermal treatment

\begin{abstract}
Ni}_{2} \mathrm{MnGa}$ has been the focus of multiple studies due to its unique ferromagnetic, magnetostrictive, thermal and magnetically controlled shape memory properties. At room temperature, the alloy has a Heusler alloy $\mathrm{L}_{1}$ type structure. A martensitic phase transition occurs upon cooling. It is highly desirable to develop and study the properties of alloys that exhibit the MSM effect at ambient conditions. In Ni-Mn-Ga compounds the exchange between $\mathrm{Mn}$ and $\mathrm{Ga}$ reduces the degree of order which makes neutron diffraction more sensitive to the crystallographic characterization and an ideal tool to study the effect of different thermal treatments on the structure. In this work the crystallography of several nonstoichiometric polycrystalline alloys synthesized and thermally treated were studied at several temperatures using pulsed neutron source at Argonne National Laboratory. The alloys were $\mathrm{Ni}_{2} \mathrm{Mn}_{1+\mathrm{x}} \mathrm{Ga}_{1-\mathrm{x}}$ compositions with $\mathrm{x}=-0.08,0.0,0.08$ and 0.16 . The sample were arc melted pellets and were studied as-cast and after several combinations of thermal treatments including isothermal annealing at 900 and $700{ }^{\circ} \mathrm{C}$ and cooling to room temperatures. It was found that the $\mathrm{Mn}$ rich non stoichiometric alloys $(\mathrm{x}=0.08$ and 0.16$)$ are unstable at $700{ }^{\circ} \mathrm{C}$ and fast cooling from $900{ }^{\circ} \mathrm{C}$ is essential in order to retain a homogeneous solid solution. These single-phase structures were found to have high level of chemical order, for that reason, ordering treatment is not necessary.
\end{abstract}

\section{Introduction}

One important group of shape memory alloys is the ferromagnetic $\mathrm{Ni}_{2} \mathrm{MnGa}$ intermetallic compound. At room temperature, the compound has a Heusler alloy $\mathrm{L}_{1}$ type structure $[1,2]$, with Curie temperature $376 \mathrm{~K}$ [2]. A martensitic phase transition occurs upon cooling at 202 $\mathrm{K}$, transforming $\mathrm{Ni}_{2} \mathrm{MnGa}$ from cubic to lower symmetry structure [2]. The chemical ordering of $\mathrm{Ni}_{2} \mathrm{MnGa}$ is affected by heat treatment $[3,4]$. The martensitic transformation and the Curie temperatures are also very sensitive to the exact composition. By shifting from 
stoichiometry, two types of shape-memory alloys, with martensitic transformation in desired temperatures have been achieved. (a) The Curie and martensitic transformation are at the same temperature [5] and (b) only the martensitic transformation temperature is changed and the Curie temperature remains as in the stoichiometric compound [6]. The second type of materials which are promising for ultra fast actuators [7] are obtained by raising the $\mathrm{Mn}$ concentration at the expense of Ga [6]. Fortunately, a wide range of solubility was found in the system $\mathrm{Ni}_{2} \mathrm{Mn}_{1+\mathrm{x}} \mathrm{Ga}_{1-\mathrm{x}}:-0.4 \leq \mathrm{x} \leq 0.4[8]$.

The present work is an exploration of the stability of the phases in this range of $\mathrm{Mn} / \mathrm{Ga}$ concentration, using a wide range of thermal annealing of various samples. We used neutron diffraction (ND) to study their structure. There are several reasons for selecting ND over Xray diffraction (XRD) for powders of Ni-Mn-Ga alloys. (i) Due to the negative amplitude of neutron scattering length of Mn, ND provides a larger contrast between Mn and Ga resulting in better sensitivity to chemical ordering than XRD. (ii) In XRD the diffraction is mostly from the surface which may include Mn oxide and other irregularities. (iii) Powders for XRD must be very fine, with different structure than large or single crystals [9].

\section{Experimental description}

Samples of stoichiometric and non-stoichiometric Ni-Mn-Ga alloys were cast under protective atmosphere in an arc-melting furnace. The samples were marked as Dm-n, where $\mathrm{m}$ denotes the composition and $\mathrm{n}$ the heat treatment conditions as described in the tables 1 and 2. The samples were cast as polycrystalline pellets and used for ND measurements.

Table 1. Composition of $\mathrm{Ni}_{2} \mathrm{Mn}_{1+x} \mathrm{Ga} a_{1-x}$.

\begin{tabular}{|c|c|c|c|c|}
\hline Sample & $\mathrm{Ni}$ & $\mathrm{Mn}$ & $\mathrm{Ga}$ & $\mathrm{Mf}[6] \mathrm{K}$ \\
\hline D1-n & 2.0 & 1.0 & 1.0 & 143 \\
\hline D2-n & 2.0 & 1.08 & 0.92 & 290 \\
\hline D3-n & 2.0 & 1.16 & 0.84 & 320 \\
\hline D4-n & 2.0 & 0.92 & 1.08 & - \\
\hline
\end{tabular}

Neutron powder diffraction data were collected at the General Purpose Powder Diffractometer (GPPD) at the Intense Pulsed Neutron Source (IPNS), Argonne National Laboratory. Neutron Intensities were detected as a function of time of flight and the collected data was focussed to 1 bank $\left(2 \theta=125^{\circ}\right)$ for analysis. The data were analysed by GSAS [10] and FullProf/Winplotr [11] software, including Rietveld refinement for selected structures. 
Table 2. Thermal treatments.

\begin{tabular}{|l|l|l|l|l|}
\hline Sample & Designation & $1^{\text {st }}$ treatment & $2^{\text {nd }}$ treatment & $3^{\text {rd }}$ treatment \\
\hline Dm-1 & Homogenization & $\begin{array}{l}900^{\circ} \mathrm{C} 10 \mathrm{~h} \\
\text { cooling at } \\
\text { cold argon }\end{array}$ & none & none \\
\hline Dm-2 & Ordering & $900^{\circ} \mathrm{C} 10 \mathrm{~h}$ & $\begin{array}{l}700^{\circ} \mathrm{C} 10 \mathrm{~h} \\
\text { cooling to } \mathrm{RT}\end{array}$ & none \\
\hline Dm-3 & As cast & none & none & none \\
\hline Dm-4 & Ageing & $900^{\circ} \mathrm{C} 10 \mathrm{~h}$ & $\begin{array}{l}700^{\circ} \mathrm{C} 10 \mathrm{~h} \\
\text { cooling to } \mathrm{RT}\end{array}$ & $\begin{array}{l}700^{\circ} \mathrm{C} 36 \mathrm{~h} \\
\text { cooling to } \mathrm{RT}\end{array}$ \\
\hline
\end{tabular}

\section{Results and discussion}

Four different samples with varying composition of $\mathrm{Mn} / \mathrm{Ga}$ were investigated and for each sample three different types of heat treatment were used.

Sample D1: In the stoichiometric sample $\left(\mathrm{Ni}_{2} \mathrm{MnGa}\right)$ the single phase was found only in the As Cast sample. In other samples small amounts of other phases (not identified) were detected but the majority fraction of each sample consisted of $\mathrm{L} 2_{1}$ Heusler alloy type structure.

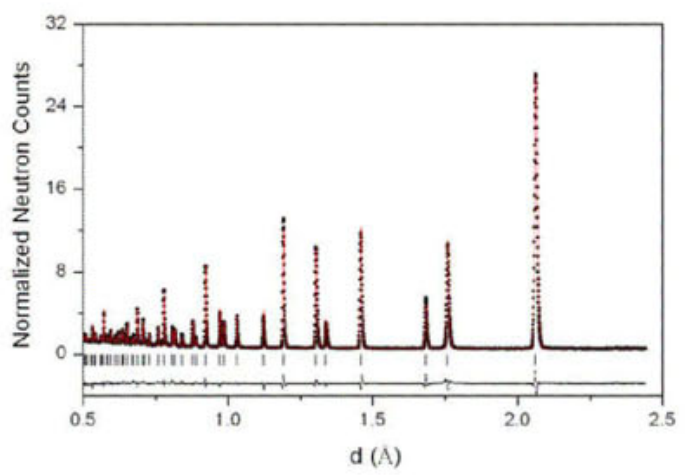

Figure 1. Refined neutron powder diffraction profile for as cast sample of $\mathrm{Ni}_{2} \mathrm{MnGa}$ using $\mathrm{L2}_{1}$ Heusler alloy structure. Shown are observed pattern (dot), best Rietveld fit profile (line), peak positions and finally at the bottom difference curve between observed and calculated profiles.

Sample D2: Sample D2 has a nominal composition $\mathrm{Ni}_{2} \mathrm{Mn}_{1+\mathrm{x}} \mathrm{Ga}_{1-\mathrm{x}}(\mathrm{x}=.08)$, at four different treatments (as described in table 1). The diffractograms shown in figure 2 are from bottom to top are: Calculated Heusler type (cubic); as cast; homogenized; ordered and aged. It can be seen that except the "aged" sample with extended treatment at $700{ }^{\circ} \mathrm{C}$, remainder samples 
showed a majority cubic $\mathrm{L1}_{2}$. The sample treated at $900^{\circ} \mathrm{C}$ and $10 \mathrm{~h} 700{ }^{\circ} \mathrm{C}$ ("ordered") was a pure Heusler type.

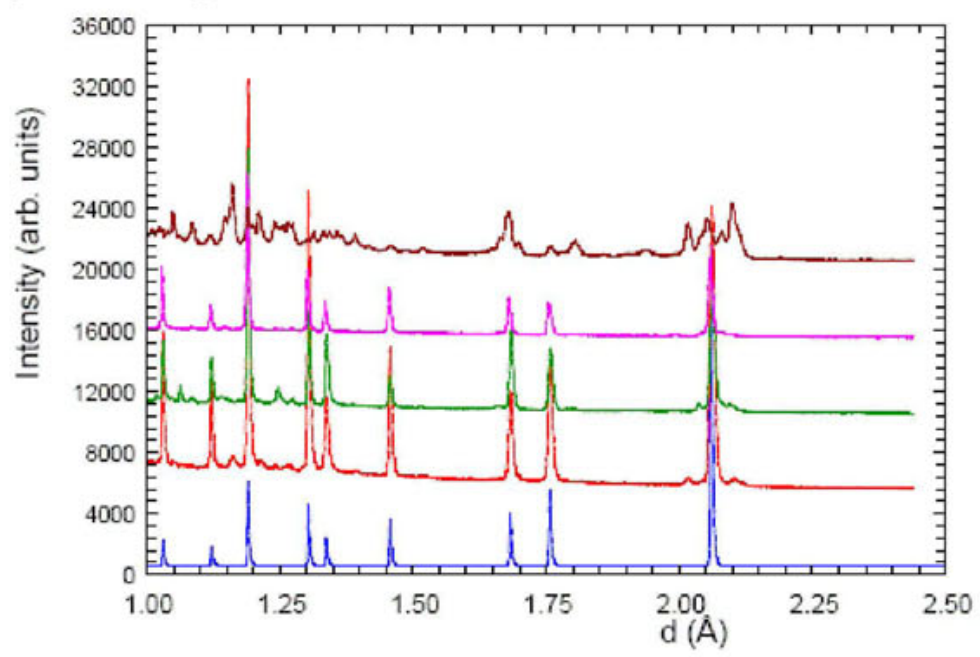

Figure 2. ND patterns of $M n_{1+x} G a_{l-x}(x=0.08)$ at $300 \mathrm{~K}$.

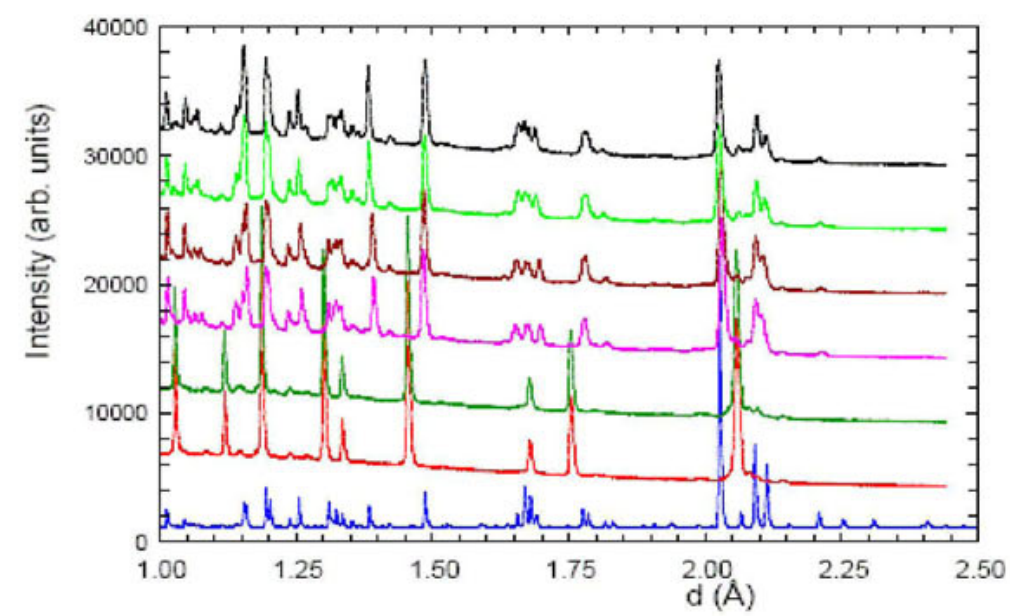

Figure 3. LT diffractograms of D2-3 sample. From bottom to top: Calculated $7 M$ martensite, and ND scans at $300,250,170,150,80$ and $20 \mathrm{~K}$.

To study the martensitic phase transformation neutron diffraction data was collected for sample D2-2 at several different temperatures. Figure 3 shows these low temperature ND patterns. The martensitic transformation $\mathrm{M}_{\mathrm{f}}$ for $\mathrm{Ni}_{2} \mathrm{Mn}_{1.08} \mathrm{Ga}_{0.92}$ is expected below $290 \mathrm{~K}$. Although it was transform to martensitic phase, at $250 \mathrm{~K}$ it was still cubic. From $170 \mathrm{~K}$ and 
lower temperatures, the structure gradually transform into a layered $7 \mathrm{M}$ martensite [12]. The absolute identification of $7 \mathrm{M}$ structure is shown in figure 3 .

Sample D3: Room temperature neutron patterns of sample D3: $\mathrm{Ni}_{2} \mathrm{Mn}_{1+\mathrm{x}} \mathrm{Ga}_{1-\mathrm{x}}(\mathrm{x}=.16)$, at four different treatments (table 1) show significant difference from the remaining samples. We find that only heat treatment of $10 \mathrm{~h}$ at $900{ }^{\circ} \mathrm{C}$ followed by fast cooling, results in an almost pure NM martensite, all other treatments failed to form a single phase. The other samples include NM martensite (major phase), cubic $\mathrm{L}_{2}$ type and unidentified segregated phases. The diffractograms from bottom to top shown in figure 4 are: Calculated nonmodulated (NM) martensite; as cast; homogenized; ordered and aged.

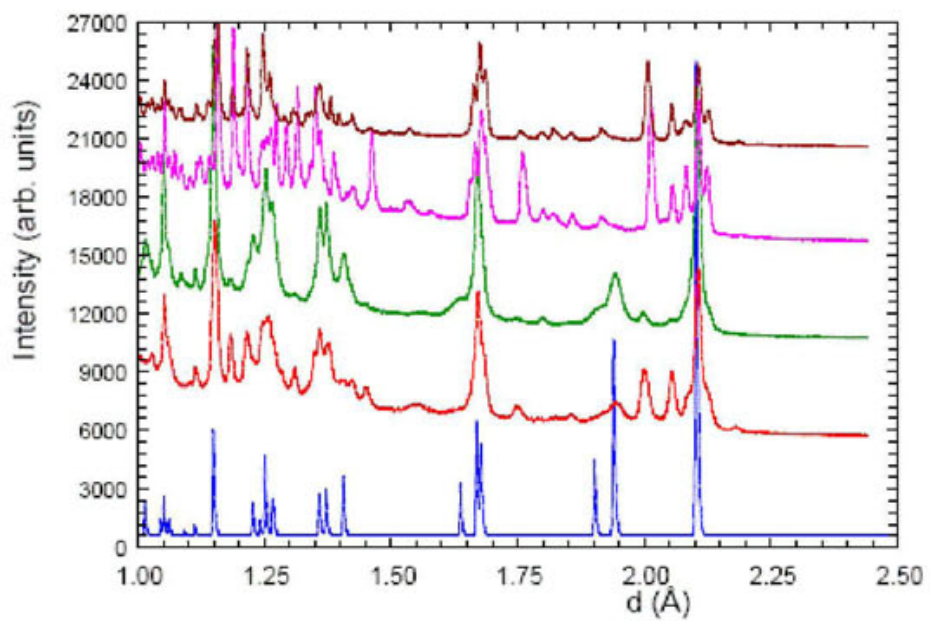

Figure 4. Neutron diffractograms of $M n_{1+x} G a_{l-x}(x=0.16)$ at $300 \mathrm{~K}$.

Sample D4: The sample D4 with excess of gallium was the only one where the extended $700{ }^{\circ} \mathrm{C}$ treatment resulted in a single Heusler structure.

\section{Summary and conclusions}

The ND was an efficient tool to figure out the best thermal treatment for each composition. It definitely shows that the structure is strongly dependent on the thermal annealing process. All single phase structures were found with high level of chemical order, in agreement with previous published results [8]. Therefore, ordering treatment is not necessary. The stability of the solid solutions of the Mn-rich non-stoichiometric $\mathrm{Ni}_{2} \mathrm{MnGa}$ alloys ( $\mathrm{x}=0.08$ and 0.16 ) are unstable at $700{ }^{\circ} \mathrm{C}$ and fast cooling from $900{ }^{\circ} \mathrm{C}$ is necessary to retain a homogeneous solid solution. The stability and instability of the non-stoichiometric $\mathrm{Ni}_{2} \mathrm{MnGa}$ alloys should be investigated in more details. 


\section{References}

1. Hames, F.A., 1960, J. Appl. Phys., 31, 307.

2. Webster, P.J., Ziebeck, K.R.A., Town, S.L. \& Peak, M.S., 1984, Phil. Mag. B, 49, 295.

3. Sholtys, J., 1975, Acta Phys. Pol. A., 46, 386.

4. Sholtys, J., 1975, Acta Phys. Pol. A., 47, 521.

5. Albertini, F., Canepa, F., Cirafici, S., Franceschi, E.A., Napoletano, M., Paoluzi, A., Pareti, L. \& Solzi, M., 2004, J. of Magn. Magn. Mater., 272-276, 2111.

6. Ullakko, K., Ezer, Y., Sozinov, A., Kimmel, G. \& Lindroos, V.K., 2001, Scripta Mater., 44, 475.

7. Suorsa, I., Pagounis, E.,\&, Ullakko, K., 2004, J. of Magn. Magn. Mater., 272-276, 2029.

8. Overholder, R.W., Wuttig, M. \& Neumann, D.A., 1999, Scripta Mater., 40, 1095.

9. Wanga, Y.D., Ren, Y., Nie, Z.H., Liu, D.M., Zuo, L., Choo, H., Li, H., Liaw, P.K., Yan, J.Q., McQueeney, R.J., Richardson, J.W. \& Huq, A., 2007, J. Appl. Phys., 101,63530 .

10. Larson, A. \& von Dreele, 2000, General Structure Analysis System (GSAS), Los Alamos National Laboratory Report LAUR 86-748.

11. Rodriguez-Carvajal, J., 1997, Fullprof, Program for Rietveld refinement, Laboratories Léon Brillouin (CEA-CNRS), Saclay, France.

12. Brown, P.J., Crangle, J., Kanomata, T., Matsumoto, M., Neumann, K-U., Ouladdiaf B. \& Ziebeck, K.R.A., 2002, J. Phys.: Condens. Matter, 14, 10159 
IV.3.2 Microstructure, Stress and Texture 



\section{Structural - phase state of maraging alloys}

\section{E. Danilchenko, V. E. Iakovlev*}

G.V. Kurdyumov Institute for Metal Physics National Academy of Sciences (IMP), Vernadsky Blvd. 36, Kyiv UA03680

"zvik83@mail.ru

Keywords: X-ray diffraction, martensitic transformation, structure, diffusion

Abstract. Surface lamination of $\gamma$-solid solution on tempering of two-phase Fe-28wt.\%Ni$2 \mathrm{wt} . \% \mathrm{Ti}-2 \mathrm{wt} . \% \mathrm{Al}$ alloy during the reverse $\alpha-\gamma$ transition was studied using X-ray diffraction in single crystal specimens. Selective iron oxidation in the subsurface layer was observed to enrich the suboxide layer with the unoxidized component.

\section{Introduction}

Heat treatment of maraging steels and metastable phase-hardened iron-nickel alloys includes heating within the $(\gamma+\alpha)$ phase interval. The heating is necessary both for producing the intermetallic phases on tempering [1,2] and for initiating the reverse $\alpha-\gamma$ transition $[3,4]$. These processes in iron-nickel-based alloys are accompanied by the redistribution of the alloying elements between the $\gamma$ - and $\alpha$-phases.

This work deals with X-ray study of structural and compositional changes in single crystals of the maraging alloys caused by heating within the $(\gamma+\alpha)$ phase interval.

\section{Experiment}

The test material was Fe-28wt.\%Ni-2wt.\%Ti-2wt.\%Al alloys, which were used for the fabrication of stamps and press molds [4]. Single crystal specimens (diameter 0.5-0.7 mm) for Xray study in rotating chamber RKV-86M1 were cut from induction-melted ingots having grain size of 3 to $8 \mathrm{~mm}$. The ingots were austenitic after quenching from $1000-1100^{\circ} \mathrm{C}$ in cold water, but the subsequent keeping at room temperature for several hours led to the isothermal martensitic transformation. The $\gamma$-phase was stabilized by heating the ingot to the onset of the solid solution decomposition $\left(520-580^{\circ} \mathrm{C}\right)$ [2] immediately after quenching. The austenite and martensitic lattice parameters were measured from the angular positions of the relevant diffraction reflections in the diffraction patterns with respect to the reference specimen (iron powder). The tempering was studied in specimens pre-held in the $\gamma$-state at $600^{\circ} \mathrm{C}$ for $100 \mathrm{~h}$. The specimens at room temperature contained $85 \%$ martensite. The reverse transition occurred when heating in salt bath at $400-500^{\circ} \mathrm{C}$. The transition kinetics and the amount of martensite were controlled by magnetometry. Selected area X-ray diffraction patterns were taken on a KAMEBAX spectrometer. 


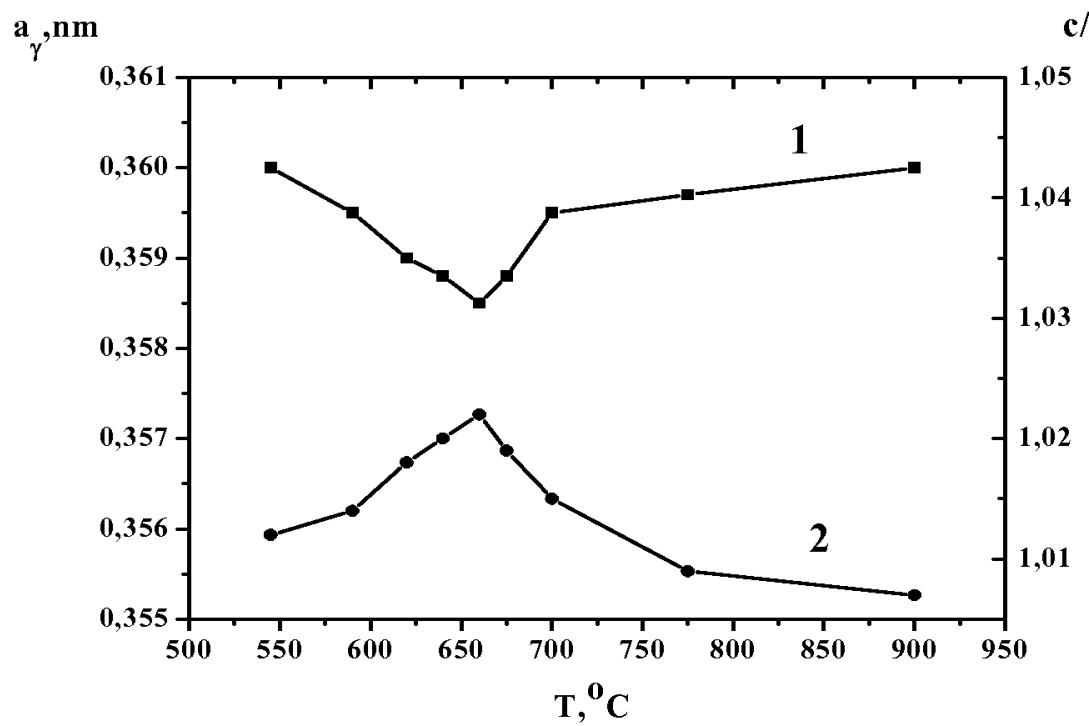

Figure 1. Change of crystal lattice parameter of austenite $\alpha_{j}(1)$ and degree of the cla ratio of martensite (2) as function of austenite tempering temperature. Tempering time for each temperature is two hours.

Nickel content of the $\gamma$-solid solution was estimated from the previously built concentration dependences of fcc-lattice parameter of binary Fe-Ni alloys. With this purpose, a set of single crystal specimens containing from 25 to $100 \% \mathrm{Ni}$ were prepared. The alloys containing 25 and $28 \% \mathrm{Ni}$ were two-phase $(\gamma+\alpha)$ at room temperature. Thus, their lattice parameters $\alpha_{\gamma}$ corresponded to the retained austenite. The lattice parameters of the fcc-crystals were similar to those of Fe-Ni polyerystals [5].

\section{Results and discussion}

Variation of the lattice parameter $\alpha_{\gamma}$ of austenite was non-monotonic as a result of austenite ageing. Change of $\alpha_{\gamma}$ curve crosses the minimum point at $660^{\circ} \mathrm{C}$ (figure 1, curve 1). In accordance with curve 1 parameters of martensitic lattice, formed in aged austenite after cooling in liquid nitrogen, such as c, a and ratio c/ $\alpha$. changed (figure 1, curve 2). Unit cell's volume of martensite remained constant during the changed parameters.

It is possible to explain such changes of lattice parameters of martensite and austenite based on the model that assumes coherence between lattices of metastable particles $\mathrm{Ni}_{3} \mathrm{Ti}$ with fcclattice $[7,11]$.

The tempering of the fresh quenched alloy reduced the parameter $\mathrm{c}_{\alpha}$ and the $\mathrm{c} / \mathrm{a}$ ratio of the martensite lattice even at room temperature. At temperatures between 100 and $300^{\circ} \mathrm{C}$, the parameter $\mathrm{c}_{\alpha}$ reduced further, and the reverse $\alpha-\gamma$ transition occurred during tempering at 
$400^{\circ} \mathrm{C}$. The reduction in $\mathrm{c}_{\alpha}$ and $\mathrm{c} / \mathrm{a}$ is associated with the loss of coherence between martensite and retained austenite lattices, as well as with the formation of a $\mathrm{Ni}_{3} \mathrm{Ti}$-type phase and the subsequent depletion of the $\alpha$-solid solution in the alloying elements due to the formation of inter-metallic compounds and the nickel redistribution between the $\gamma$ - and $\alpha$-phases [6-8].

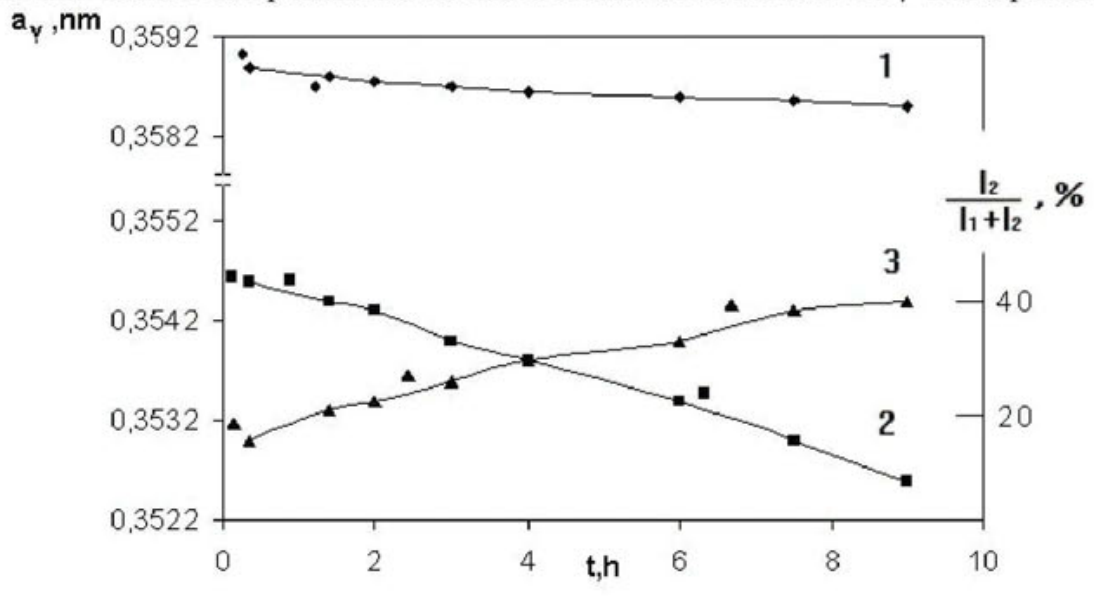

Figure 2. Change of the lattice parameter of retained (1) and reversed (2) austenite, ratio of intensity of the (200) reflection of reversed austenite $I_{2}$ to the total reflection intensity $I_{1}+I_{2}$ of reversed and retained austenite (3), $I_{1}$ - retained austenite.

Tempering of the two-phase $(\gamma+\alpha)$ specimens at $520^{\circ} \mathrm{C}$ for several minutes shifted the martensitic reflections having high third indices toward higher Bragg angles, i.e., toward the reference reflections. The retained austenite reflections were blurred at the side of low angles, and weak first-order satellite reflections appeared at the blurriness. Longer tempering at $520^{\circ} \mathrm{C}$ (for $10 \mathrm{~h}$ ) or at higher tempering temperature $\left(600^{\circ} \mathrm{C}\right)$ attenuated and eliminated the latter reflections, and the retained austenite reflections started blurring at the opposite side, with second-order satellite reflections appearing subsequently at the blurriness. Holding at $600^{\circ} \mathrm{C}$ increased the Bragg angles and intensities of the satellite reflections. The Bragg angles of the retained austenite reflections also increased.

Comparison of $\alpha_{\gamma}$ for $\mathrm{Fe}-28 \mathrm{wt} . \% \mathrm{Ni}-2 \mathrm{wt} . \% \mathrm{Ti}-2 \mathrm{wt} . \% \mathrm{Al}$ alloy (figure 2, curve 1) with $\alpha_{\gamma}$ of the binary alloys [5] demonstrated that the long holding at $600^{\circ} \mathrm{C}$ for $1000 \mathrm{~h}$ before the $\gamma-\alpha$ transition did not deplete the austenite in titanium and aluminum, atoms of which were larger than that of iron. $\alpha_{\gamma}$ of the former alloy was $0.3590 \mathrm{~nm}$ and for Fe-28wt.\%Ni alloy it was $0.3582 \mathrm{~nm}$ [5]. The decrease in $\alpha_{\gamma}$ of the retained austenite during tempering (figure 2, curve 1) can be attributed to the increase in the nickel concentration from 50 to $55 \%$ [5], and further diffusion of titanium and aluminum from the $\gamma$-solid solution. The latter process suggests that the martensitic $\gamma-\alpha$ transition changes the structure and regenerates the tempering processes due to the hardening phase of the retained austenite. Indeed, the aluminum and titanium diffusion from the initial $\gamma$-solid solution virtually ceased after one hour of tempering at $600^{\circ} \mathrm{C}$ with pre-aging [8]. Further keeping at this temperature up to $1000 \mathrm{~h}$ did not change the lattice parameter of the austenite. 
The appearance of the satellite first- and second-order reflections indicated the recovery of the initial austenite phases having an fec-lattice, and larger and smaller lattice parameters, respectively. The disappearance of the first-order satellites and the appearance of the secondorder ones with increasing tempering tune and temperature were accounted for by the permanent shifting of the first-order reflections, which were temporally superposed with the strong reflections of the retained austenite. These satellites were produced by the reversed austenite that is formed during the reverse $\gamma-\alpha$ transition.

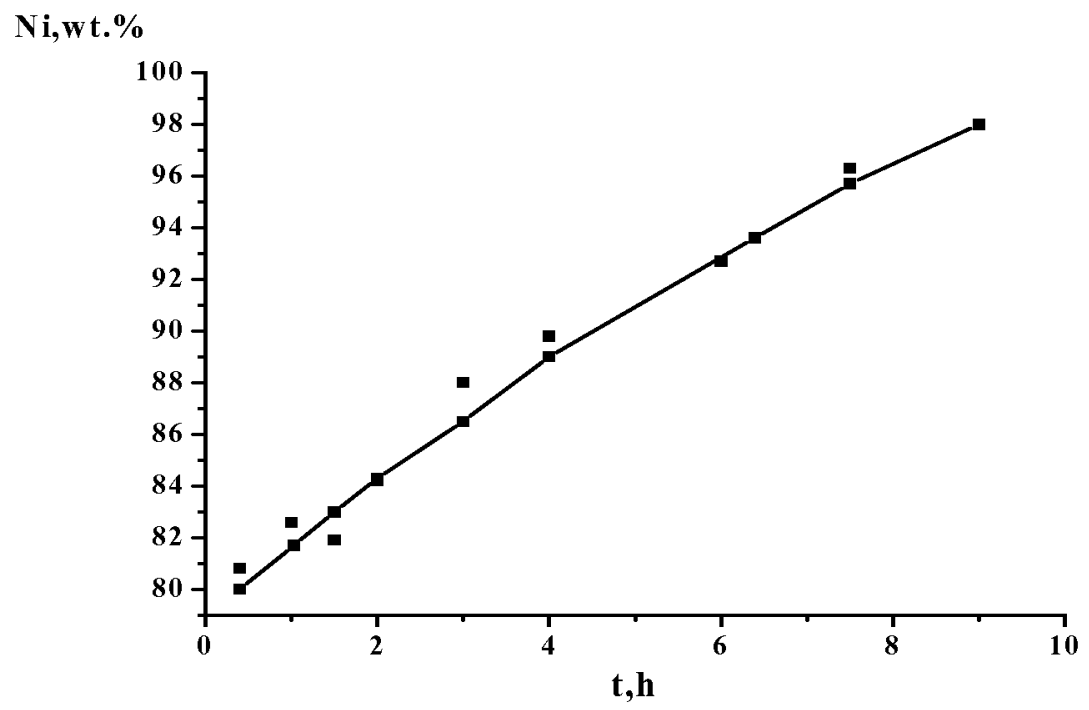

Figure 3. Nickel concentration change of reversed austenite as time function of isothermal tempering at $600^{\circ} \mathrm{C}$.

The lattice parameter of the reversed austenite, which was initially higher than that of the retained austenite, declined with increasing time of tempering at $600^{\circ} \mathrm{C}$. After one hour of tempering, the parameter was already considerably smaller than for the retained austenite (figure 2, curve 2). Each austenite reflection split into two reflections having different intensities. The amount of the reversed austenite increased with declining lattice parameter (figure 2 , curve 3 ).

The splitting of the austenite reflections, which indicates a decomposition of the $\gamma$-solid solution, was observed when heating of the $(\gamma+\alpha)$-specimens, and was not observed during keeping of $\gamma$-specimens at $600^{\circ} \mathrm{C}$ for $1000 \mathrm{~h}$. This rule was applicable for heating in air, in pyrophyllite environment, in salt bath, and in sealed quartz ampoules at $10^{-3}$ torr. The splitting was the strongest in air, i.e., in an oxidizing environment. Splitting pattern and intensity ratios in ampoules were similar to these parameters for air only after 5-8 times longer tempering. 
The shift and change in intensity of the satellites is probably explained by the permanent increase of nickel concentration in the reversed austenite. The decrease and subsequent increase of the Bragg angles triggered by the non-monotonic variation of the fcc-lattice parameter of the iron-nickel alloys with increasing nickel concentration: the parameter $\alpha_{\gamma}$, increased until nickel concentration of $41 \%$ was reached, and then it considerably decreased [5]. The calculation of nickel concentration in the reversed austenite from the parameter $\alpha_{\gamma}$ demonstrated that tempering enriched fec-lattice in nickel up to above $90 \%$ (figure 3 ). The concentrations of titanium and aluminum were not taken into account.

The enrichment of the reversed austenite in nickel during the reverse $\alpha-\gamma$ transition and isothermal holding of metastable austenitic alloys and maraging steels was reported earlier [3]. However, the large enrichment obtained in this work can hardly be accounted for by only the nickel redistribution between the $\gamma$ - and $\alpha$-phases.

The major cause of the enrichment of the $\gamma$-solid solution in nickel was deduced by analyzing the microstructure and layer-by-layer phase composition data of the tempered specimens.

The superficial layer was removed by abrasion, chemical etching, and electropolishing. The intensities of the nickel-rich austenite reflections decreased starting from a depth of $20 \mu \mathrm{m}$, and only retained austenite reflections were observed at a depth of $40 \mu \mathrm{m}$. The metallographic data taken from a tilt section demonstrated the presence of an oxide layer with welldeveloped cracks on the surface. $\mathrm{Fe}_{2} \mathrm{O}_{3}-$ and $\mathrm{Fe}_{3} \mathrm{O}_{4}$ - type oxides were revealed by X-ray diffraction. The relative intensity $\mathrm{I} / \mathrm{I}_{0}$ of the $\{200\}$ austenite reflection decreased with increasing depth in various ways for each of the oxides. For $\mathrm{Fe}_{2} \mathrm{O}_{3}, \mathrm{I} / \mathrm{I}_{0}$ dropped to zero at the depth of $24 \mu \mathrm{m}$, whereas for $\mathrm{Fe}_{3} \mathrm{O}_{4}$ it only started to decrease at this depth.

Oxides of other alloying additions were not revealed by X-ray diffraction because of the low concentrations. X-Ray probe concentration depth profiling demonstrated that, at depths of $>50 \mu \mathrm{m}$, the nickel-to-iron ratios were close to those for the mean composition, whereas at the depth of 5-8 $\mu \mathrm{m}$ from the oxide/matrix boundary, this ratio was more than 5:1. Still higher nickel concentrations were observed immediately at the surface.

The data proves that the $\gamma$-solid solution is laminated at the surface. The appearance of continuous reflection zones of polycrystalline oxides alongside with the splitting of the single crystal reflections allowed the austenite lamination to be attributed to the surface oxidation.

The surface oxidation was selective because the specific heats of the formation of iron and nickel oxides differ 4-6 times [9]. The scale was formed by the accretion of two layers. The upper layer grew by the diffusion of iron cations to the surface, and the inner layer grew by the inward oxygen diffusion along the lattice defects [10]. The diffusions of iron into the oxide layer and nickel into the suboxide layer enriched the latter layer in nickel and depleted it of iron.

As we mentioned above, the lamination of the $\gamma$-solid solution was observed only while heating two-phase alloys containing large amounts of the martensite (>20\%). Thus, the diffusion was enhanced by the phase hardening, and the mechanism and kinetics of the reverse $\alpha-\gamma$ transition at the surface differed from these in the bulk.

Electron microscopic study showed that, along with the coarse-grained $\gamma$-phase having the orientation of the retained austenite, a fine-grained lath $\gamma$-phase structure having a large number of orientations formed on rapid heating before tempering [3]. The presence of the martensite/fine-grained phase boundary enhanced the diffusion and considerably changed the composition. 
The data proves that diffusion may be enhanced even on the martensite/coarse-grained reversed lath austenite boundary. This is the only valid explanation of the $\gamma$-solid solution lamination because the lattice of the nickel-rich intervals had the orientation of the retained austenite.

\section{Conclusion}

Heating two-phase Fe-28wt.\%Ni-2wt.\%Ti-2wt.\%Al alloy during the reverse $\alpha-\gamma$ transition causes the surface lamination of the $\gamma$-solid solution. The surface of the quenched alloy is enriched in nickel because of the nickel redistribution between the $\gamma$ - and $\alpha$-phases during the $\alpha-\gamma$ transition, selective iron oxidation, and increase in the concentration of the unoxidized component in the suboxide layer.

\section{References}

1. Perkas, M.D. \& Kardonskii, V.M., 1970, High-Strength Maraging Steels, [in Russian], Moscow, Metallurgiya.

2. Samsonov, Yu.I., Kokorin, V.V. \& Oleinik, A.S.,1973, Aging in Two-Phase State as a Method of Obtaining High Strength and Plasticity Steels, [in Russian], Kiev, Znanie.

3. Malyshev, A.K., Sagaradze, V.V., Sorokin, I.P. et al., 1980, Phase Hardening of Iron-Nickel Austenitic Alloys, [in Russian], Moscow, Nauka.

4. Gorbach, V.G., Samsonov, Yu.I., Strochak, N.N. \& Avetisyan, Yu.A., 1974, Metastable Austenitic Steels as a Material for Complex-Shape Parts, [in Russian], Preprint IMF AN UkrSSR, No 74, p. 12, Kiev.

5. A.E. Vol., 1962, Structure and Properties of Binary Metallic Systems, 2, [in Russian], Moscow, Fizmatgiz.

6. Abrachaman, J.K. \& Paskover, J.S., 1969, The Transformation and Structure of FeNi-Ti Alloys, Trans. Met. Soc. AIME, 245, No 4.

7. Koval Yu.N. \& Kokorin, V.V., 1975, The tetragonality of carbon-free martensite, Fiz. Met. Metalloved., 39, No 5.

8. Danilchenko V.E. \& Okhrimenko, V.A., 1987, Structural transformation of Ni28Ti2A12 alloy on aging, Fiz. Met. Metalloved., 63, No 2.

9. Key, J. \& Lebi, T., 1962, Tables of Physical and Chemical Constants, [Russian translation], Moscow, Fizmatgiz.

10. O.Kubashevski and B. Gopkins, 1968, Oxidation of Metals and Alloys, [Russian translation], Moscow, Metallurgiya.

11. Abraham, J.K., Jackson, J.K. \& Leonard, L., 1986, X-ray Study of the Aging Process in an Austenite Fe-31Ni-3,5Ti Alloy, Trans. ASM, 61, 233. 


\title{
Rietveld quantitative phase analyses in iron alloys processed by mechanical alloying method
}

\author{
H. J. Krzton ${ }^{1, *}$, W. Pilarczyk ${ }^{2}$ \\ ${ }^{1}$ Institute for Ferrous Metallurgy, Gliwice (Poland) \\ 2 Silesian University of Technology, Gliwice (Poland) \\ *hkrzton@imz.pl
}

Keywords: mechanical alloying, Fe-C alloys, Rietveld method, quantitative phase analysis

\begin{abstract}
Two kinds of iron alloys have been mechanically alloyed: one with $6.67 \mathrm{wt} \% \mathrm{C}$ and second with $0.4 \mathrm{wt} \% \mathrm{C}$. A formation of cementite $\mathrm{Fe}_{3} \mathrm{C}$ and an amorphous phase have been detected in the first group of alloys (Fe-6.67 wt \% C) and some fraction of $\alpha$-Fe has been present up to 150 hours of milling. In alloys of the second group $(\mathrm{Fe}-0.4 \mathrm{wt} \% \mathrm{C}$ ) only $\alpha$-Fe has been observed together with amorphous component. Quantitative phase analysis has been applied to follow the changes in powders' constitution after different times of milling.
\end{abstract}

\section{Introduction}

For more than twenty years, mechanical alloying has become an important technique to produce equilibrium and non-equilibrium phases from elemental and pure powders [1]. This solid-state reaction method enables the production of supersaturated solid solutions, intermetallic phases, crystalline, quasi crystalline and amorphous alloys, also those which cannot be processed by standard techniques $[2,3]$. Using different type of mills, the particles of starting powder mixtures are subjected to severe plastic deformations, with accompanying diffusion processes and momentary, local increase of temperature [4,5]. The yield of the mechanical alloying technique depends on the type of a mill, a value of ball to powder ratio (BPR), the grain sizes of a mixture particles, time of milling, temperature of the process and on the atmosphere [6,7]. The final product of mechanical alloying process usually differs from the same material, processed using one of the standard techniques, mostly because of the nanocrystalline structure influencing some properties as e.g. ductility and formability [8]. Different Fe-based alloys have been processed applying mechanical alloying technique (e.g. AISI M2 [9], 316L [10] steels), including Fe-C system. Mechanical alloying technique is a very suitable method for processing carbides, especially in Fe-C system, because the formation of carbides by standard techniques is very difficult. Moreover, this special kind of system plays an important role as a simple model system in examining the processes of formation of metal-metaloid alloys. There have been several research studies on processing nanocrystalline iron carbides as $\mathrm{Fe}_{3} \mathrm{C}$ [11], $\mathrm{Fe}_{5} \mathrm{C}_{2}$ [12] or supersaturated solid solutions [13]. 
The presence of amorphous phase in this system has been reported in two research studies, for 17 at. $\%$ of $\mathrm{C}[14]$ and for 90 at. \% of C [15]. The proof for the existence of the amorphous component has been obtained from TEM techniques [16,17]. So far, in its application to phase analysis, X-ray diffraction has been used only as a qualitative tool to identify the products of mechanical alloying process. No effort has been done until now to quantify the phases - products of mechanical alloying process, and also to detect and to calculate the content of amorphous phase using X-ray diffraction. The aim of this study has been to determine the quantitative relations among the products of mechanical alloying process after different times of milling. The Rietveld method $[18,19]$ in its application to quantitative phase analysis $[20,21]$, e.g. SIROQUANT ${ }^{\mathrm{TM}}$ software $[22,23]$, gives the possibility to follow the phase changes in both qualitative and quantitative ways.

\section{Experimental procedure}

The elemental $\alpha$-Fe (purity of $99.5 \mathrm{wt} \%$, grain size $25 \mu \mathrm{m}$ ) and graphite $\mathrm{C}$ (purity of $99.99 \mathrm{wt} \%$, grain size $30 \mu \mathrm{m}$ ) powders have been used to make starting mixtures with chemical composition Fe- 0.4 wt \% C and Fe-6.67 wt \% C, respectively. Six mixtures of every composition have been prepared to cover six different total times of milling. The total mass of material in each mixture has been $10 \mathrm{~g}$. Mechanical alloying has been performed in a high-energy shaker mill 8000 SPEX CertiPrep 8000 Mixer/Mill, using tool steel container and six stainless steel balls as the milling media, two balls of diameter of $12 \mathrm{~mm}$ and four balls of diameter of $6 \mathrm{~mm}$. The ball-to-powder mass ratio (BPR) has been fixed to 2:1 for both type of mixtures. The applied low value of BPR in conjunction with local heating (which can be responsible for e.g. recovery process in the particles of iron powder) have affected - lowered - the level of strains introduced into the powder's particles and have slowed down the formation of cementite. The milling has been carried out in argon atmosphere, with ten minutes' breaks after every hour of milling, to avoid overheating of the powders. Six total times of milling have been applied: 25, 50, 75, 100, 125 and 150 hours. The $\mathrm{X}$-ray studies have not been done on as-milled powders, but after some time to decrease the level of strains once again. The products of milling have been characterized by X-ray diffraction using Philips PW 1140 diffractometer with $\mathrm{Co}_{\alpha}$, radiation $(35 \mathrm{kV}, 30 \mathrm{~mA})$ and a diffracted beam, graphite monochromator. The XRPD data have been collected from $30^{\circ}$ to $150^{\circ} 2 \theta$, in steps of $0.05^{\circ} 2 \theta$ and a counting time of 10 seconds per step. The starting angle of the measured range has been chosen as $30^{\circ} 2 \theta$, because in the case of Co radiation applied, all known iron carbides and other kinds of phases in $\mathrm{Fe}-\mathrm{C}$ system (i.e. martensite) have no diffraction lines below this angle. Next, the original samples have been spiked, using corundum $\alpha-\mathrm{Al}_{2} \mathrm{O}_{3}$ as a spike phase in amount of $15 \mathrm{wt} \%$, and then measured in the same experimental conditions as mentioned above. The identification of phases has been done according to Powder Diffraction File of International Centre for Diffraction Data. The quantifications of the phases, including amorphous phase, have been done using the Rietveld method applied in SIROQUANT ${ }^{\mathrm{TM}}$ software. The background intensities have been removed from the experimental data in the first step of analysis. Then, the refinement procedure has been started, using the following parameters: instrument zero parameter, phase scale factors, unit cell parameters, half width parameters of diffraction lines, pseudo-Voigt line shape, microabsorption correction. 


\section{Results and discussion}

The diffraction pattern of mixture of Fe- $0.4 \mathrm{wt} \% \mathrm{C}$ type, after $150 \mathrm{~h}$ of milling is shown in the fig.1. There are only the diffraction lines of $\alpha$-Fe which can be seen in the pattern.

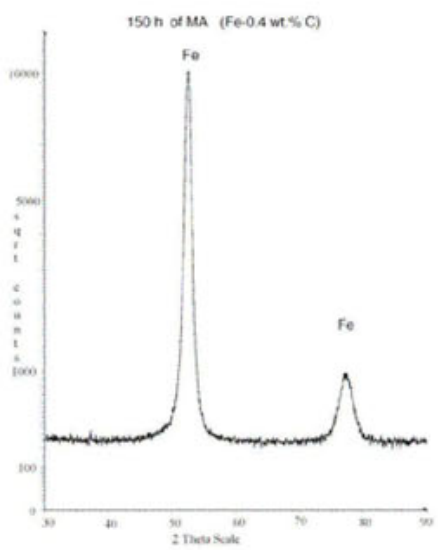

Figure 1. The diffraction pattern of $\mathrm{Fe}-0.4 \mathrm{wt} \% \mathrm{C}$ mixture of type after $150 \mathrm{~h}$ of milling.
Table 1. The results of quantitative phase analysis of $\mathrm{Fe}-0.4 \mathrm{wt} \% \mathrm{C}$ mixtures after different times of milling. Results given at wt $\%$.

\begin{tabular}{|c|c|c|}
\hline $\begin{array}{c}\text { Milling } \\
\text { Time }[\mathrm{h}]\end{array}$ & $\begin{array}{c}\text { Amorphous } \\
\text { component }\end{array}$ & $\alpha$-Fe \\
\hline 25 & 23.4 & 76.6 \\
\hline 50 & 7.2 & 92.8 \\
\hline 75 & 11.5 & 88.5 \\
\hline 125 & 7.3 & 92.7 \\
\hline 150 & 5.9 & 94.1 \\
\hline
\end{tabular}

The results of quantitative phase analysis of Fe- 0.4 wt $\%$ C mixtures after $25 \mathrm{~h}, 50 \mathrm{~h}, 75 \mathrm{~h}$, $125 \mathrm{~h}$ and $150 \mathrm{~h}$ of milling are given in the table 1 . The highest fraction of amorphous component, namely $23.4 \mathrm{wt} \%$, has been obtained in the mixture after $25 \mathrm{~h}$ of milling. Then, the fraction of amorphous phase has been reduced to the value of $7.2 \mathrm{wt} \%$ ( $50 \mathrm{~h}$ of milling). After $75 \mathrm{~h}$ of milling, there is a small rise of content of amorphous phase to $11.5 \mathrm{wt} . \%$. The observed small rise of amorphous component is the result of successive energy transfer among the powder particles, balls and walls of the container. As a final result, the value of $5.9 \mathrm{wt} \%$ of amorphous phase, has been obtained after $150 \mathrm{~h}$ of milling.

For the second type of Fe-C mixtures $(\mathrm{Fe}-6.67 \mathrm{wt} \% \mathrm{C})$, the comparison of the state of the powder mixtures after $25 \mathrm{~h}$ and $150 \mathrm{~h}$ of milling can be easily done (fig. 2). In the diffraction pattern of the mixture after $25 \mathrm{~h}$, a very initial stage of processing a crystalline $\mathrm{Fe}_{3} \mathrm{C}$ can be observed (no calculations could be made for that sample). The longest time of milling, i.e. $150 \mathrm{~h}$, results in producing a significant content of $\mathrm{Fe}_{3} \mathrm{C}$ with some fraction of $\alpha$-Fe still present. An example of the Rietveld refinement of the experimental data, measured of $150 \mathrm{~h}$ of milling sample, is shown in the fig. 3. In the diffraction pattern, the sharp lines of a spike phase of $\alpha-\mathrm{Al}_{2} \mathrm{O}_{3}$ can be seen. As in the Fe- $0.4 \mathrm{wt} \% \mathrm{C}$ mixtures, the highest fraction of amorphous phase at level of $40 \mathrm{wt} . \%$, has been obtained after $25 \mathrm{~h}$ of milling (table 2). After $50 \mathrm{~h}$ of milling, the content of amorphous phase has been equal to $40.3 \mathrm{wt} \%$ with the presence of $\alpha$-Fe at the level of $32.5 \mathrm{wt} \%$ and $\mathrm{Fe}_{3} \mathrm{C}-27.2 \mathrm{wt} . \%$. The prolongation of the milling time has resulted in increasing of the fraction of $\mathrm{Fe}_{3} \mathrm{C}(49.2 \mathrm{wt} \%$ and $60.0 \mathrm{wt} . \%$ after $100 \mathrm{~h}$ and $150 \mathrm{~h}$ of milling time, respectively) with simultaneous decreasing of content of amorphous 
phase (30.9 wt $\%$ and $24.1 \mathrm{wt} \%$, respectively) and $\alpha$-Fe (19.9 wt $\%$ and $15.9 \mathrm{wt} \%$, respectively).

Table 2. The results of quantitative phase analysis of $\mathrm{Fe}-6.67 \mathrm{wt} \% \mathrm{C}$ mixtures after different times of milling. Results given at $w t \%$.

\begin{tabular}{|c|c|c|c|}
\hline Milling Time [h] & Amorphous component & $\alpha$-Fe & $\mathrm{Fe}_{3} \mathrm{C}$ \\
\hline 10 & 11.4 & 88.6 & - \\
\hline 25 & 40.8 & 59.2 & - \\
\hline 50 & 40.3 & 32.5 & 27.2 \\
\hline 100 & 30.9 & 19.9 & 49.2 \\
\hline 150 & 24.1 & 15.9 & 60.0 \\
\hline
\end{tabular}
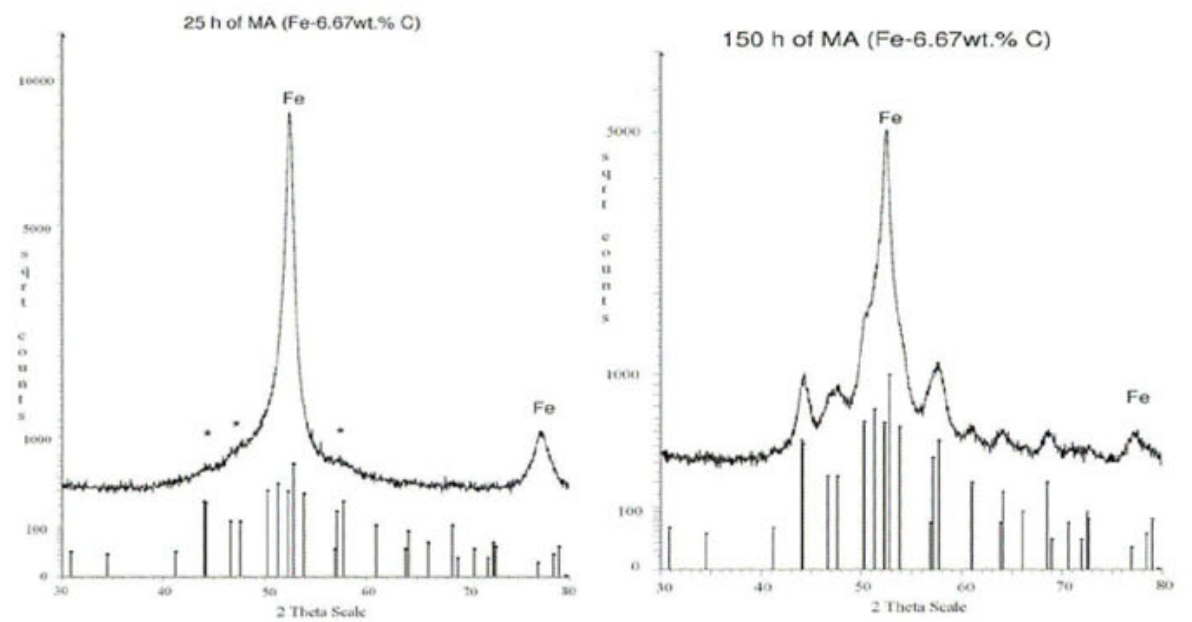

Figure 2. The diffraction patterns of two mixtures of Fe-6.67 wt \% C type after $25 \mathrm{~h}$ of milling (the left pattern) and after $150 \mathrm{~h}$ of milling (the right pattern). The vertical bars indicate the standard positions of the diffraction lines of cementite. The stars in the left diffraction pattern mark the positions of very weak, broadened lines of cementite at early stage of its formation. 

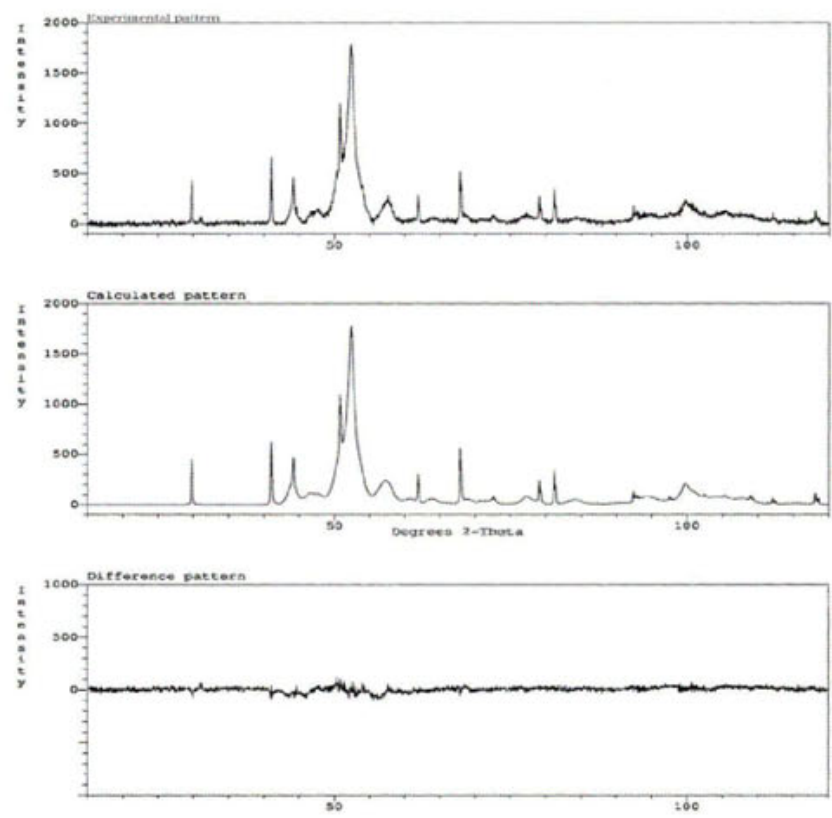

Figure 3. Experimental and calculated patterns of $\mathrm{Fe}-6.67 \mathrm{wt} \% \mathrm{C}$ mixture after $150 \mathrm{~h}$ of milling. The difference plot is given in the bottom window. The sharp diffraction lines belong to $\alpha-\mathrm{Al}_{2} \mathrm{O}_{3}$ added as a spike phase.

\section{Concluding remarks}

The formation of an amorphous phase as an initial stage of mechanical alloying process in both $\mathrm{Fe}-0.4 \mathrm{wt} \% \mathrm{C}$ and $\mathrm{Fe}-6.67 \mathrm{wt} \% \mathrm{C}$ systems has been observed. During MA process, the diffusion of $\mathrm{C}$ atoms is much higher than in traditional $\mathrm{Fe}-\mathrm{C}$ based alloys. The high level of solubility is achieved by introducing strains into the particles of iron powder. This leads to serious changes in the local order with increasing number of $\mathrm{C}$ atoms. As the next step, with a sufficient number of $\mathrm{C}$ atoms, the process of ordering, which leads to a formation of cementite, can start (fig. 2) and is followed by the expanding of the size of carbide's crystallites which can be observed in the diffraction patterns as sharpening of the diffraction lines of cementite. In $\mathrm{Fe}-0.4 \mathrm{wt} \% \mathrm{C}$ powders, no diffraction lines of cementite $\mathrm{Fe}_{3} \mathrm{C}$ has been seen in the diffraction patterns up to 150 hours of milling. After 150 hours of milling, the final content of the amorphous phase has been equal to the expected value of cemetite in this system. In the Fe- $6.67 \mathrm{wt} \% \mathrm{C}$ type of powder, after 25 hours of milling the early stage of formation of $\mathrm{Fe}_{3} \mathrm{C}$ has been noticed. Next, the prolongation of milling time has resulted in lowering the fractions of $\alpha$-Fe and the amorphous phase with simultaneous increase of $\mathrm{Fe}_{3} \mathrm{C}$ content. The presence of strains causes the increase of the intensity of the background which can influ- 
ences the systematic error, concerned with quantifying the amorphous component. In the presented research work, some efforts have been done to minimize this error, as applying low value of BPR and examining not as-milled powders. Moreover, the local heating which takes place during the MA process, helps the recovery processes to decrease the lattice defects.

\section{References}

1. Suryanarayana, C., 2001, Prog. Mater. Sci., 46, 1.

2. Koch, C.C., 1997, Nanostruct. Mater., 9, 13.

3. Ward, T.S., Chen, W., Schoenitz, M., Dave, R.N. \& Dreizin, E.L., 2005, Acta Mater., 53, 2909.

4. Joardar, J., Pabi, S.K. \& Murty B.S., 2004, Scripta Mater., 50, 1199.

5. Vasil'ev, L.S. \& Lomayeva, S.F., 2004, J. Mater. Sci., 39, 5411.

6. Suryanarayana, C., Ivanov, E. \& Boldyrev, V.V., 2001, Mater. Sci. Eng., A 304306, 151.

7. Suryanarayana, C. \& Koch C.C., 2000, Hyperfine Interact., 130, 5.

8. Ravers, J., 1999, Nanostruct. Mater., 11 (4), 513.

9. Matteazzi, P. \& Wolf, F., 1998, Mater. Sci. Eng. , A 246, 235.

10. Krztoń, H., Kolesnikow, D., Paduch, J. \& Molenda, R., 2007, J. Achievem. Mater. Manufact. Eng., 21, 73.

11. Nowosielski, R. \& Pilarczyk, W., 2005, J. Mater. Process. Technol., 162-163, 373.

12. He, Z., Zhong, M., Shen, W. \& Zhang Z., 2003, J. Shanghai Univ., 7 (1), 84.

13. Xu, S., Zhang, J., Zhong, M., Chen, H., Zhang Z. \& He, Z., 2005, J. Shanghai Univ., 9 (6), 550.

14. Miura, H., Omuro, K. \& Ogawa H., 1995, Mat. Trans. JIM, 36, 263.

15. Tanaka, T., Motoyama, M., Ishihara, K.N. \& Shingu, P.H., 1995, Mat. Trans. JIM, 36, 276.

16. Nowosielski, R. \& Pilarczyk, W., 2007, Int. J. Microstruct. Mater. Proper., $2,2$.

17. Hidaka, H., Tsuchiyama, T. \& Takaki, S., 2001, Scripta Mater., 44, 1503.

18. Rietveld, H.M., 1967, Acta Crystallogr., 22, 151.

19. Rietveld H.M., 1969, J. Appl. Crystallogr., 2, 65.

20. Hill, R.J. \& Howard, C.J., 1987, J. Appl. Crystallogr., 20, 467.

21. Bish, D.L. \& Howard S.A., 1988, J. Appl. Crystallogr., 21, 86.

22. Taylor J.C., 1991, Powder Diffr. 6, 2.

23. SIROQUANT ${ }^{\mathrm{TM}}$ Quantitative XRD Software, User's Guide and Reference, Ver. 2.5 for Windows, 2000. 


\title{
Effect of texture heterogeneities on the shape memory properties of rolled $\mathrm{Fe}$ - Mn-Si SMA
}

\author{
A.V. Druker ${ }^{1}$, C. Sobrero ${ }^{2}$, J. Malarría ${ }^{2}$, U. Garbe $^{3}$, \\ H.-G. Brokmeier ${ }^{3}$, R. E. Bolmaro ${ }^{2, *}$
}

${ }^{1}$ Fac. de Cs. Ex., Ingeniería y Agrimensura, U.N.R. Pellegrini 250, 2000 Rosario, Argentina

2 Inst. de Física Rosario, CONICET-UNR. 27 de febrero 210 bis, 2000 Rosario, Argentina

${ }^{3}$ Institute of Materials Research, GKSS-Research Centre, Max-Planck-Strasse 3, D-21502 Geesthacht, Germany

*bolmaro@ifir-conicet.gov.ar

Keywords: Shape Memory Alloys, Textures, Phase Transformations, Martensitic Transformations

\begin{abstract}
We have investigated an Fe-30Mn-4Si shape memory alloy to clarify the effect, on the bulk texture, of the shear layers resulting from two different thermo-mechanical treatments. XR analysis has shown the existence of texture heterogeneity through the rolled sheet's thickness, due to the effect of friction between sheet and rolls. Neutron diffraction revealed that textured layers on the sheet's surface affect the whole volume. The texture found on the surface of the sheet rolled at $600^{\circ} \mathrm{C}$ is the most favourable for the $\gamma \rightarrow \varepsilon$ martensitic transformation which is the origin of the shape memory effect. Comparing these results with those obtained on sheets rolled at room temperature, we found that shear deformation gradients produce changes in the bulk material texture. Tensile tests initially induce the martensitic transformation in those grains favourably oriented. As a result, these favourable orientations disappear in the remnant austenite.
\end{abstract}

\section{Introduction}

The shape memory effect (SME) is a special thermo-mechanical behaviour which implies the recuperation of apparent plastic deformation; in ferrous alloys, it is due to a stress induced $\gamma$ (austenite, fcc) $\rightarrow \varepsilon$ (martensite, hcp) martensitic transformation, and the inverse transformation obtained by heating to a temperature over the $\mathrm{A}_{\mathrm{f}}[1]$. The shape change should be reversible if the applied deformation does not involve plastic slip and if the martensitic variants creating the forward and reverse transformation are equivalent. Fe-Mn-Si-(Cr-Ni) materials are the ferrous shape memory alloys of higher commercial impact. Due to a low stacking fault energy (SFE), compositions between $28-33 \% \mathrm{Mn}$ and $4-6 \% \mathrm{Si}$, maintain the stability of austenite at room temperature and facilitate the formation of stress induced $\mathrm{M}_{\varepsilon}$ without plastic deformation. When the temperature increases, SFE [2,3] also increases, affecting certain properties related to the martensitic transformation. This transformation consists in the 
movement of one Schockley a/6 $\langle 112\rangle$ partial dislocation on alternating $\{111\}$ planes of austenite, changing the stacking sequence by a (111)[112] slip. There are three equivalent slip directions that produce an HCP structure with the same orientation. When the transformation is stress induced, the selective movement of one variant is activated, resulting in the shape change. It means that, among other important factors, the $\gamma \rightarrow \varepsilon$ martensitic transformation is strongly dependent on crystallographic orientation. Research on Fe-Mn-Si single crystals [4], has demonstrated that shape recovery is suppressed if the stress is applied in the $<001\rangle$ direction, but it is almost perfect when the stress occurs in the $<414>$ direction. So, it is expected that a particular preferred orientation or texture, can contribute to good shape recovery properties.

Rolling introduces texture in metallic alloys $[5,6]$. When a metal is obligated to pass through a channel, inevitably the deformation will produce displacement and velocity heterogeneities, which implies gradients in deformation, structure, texture and properties [7]. Some authors $[8,9]$ reported the existence of a correlation between the characteristic parameter $\Delta$ (average relation between thickness and length in the plastic zone) and the surface texture, for different forming processes, temperatures and metals and alloys with different SFE. In addition to roll gap, friction plays a special role in creating shear deformation. These effects are the largest at the roll surfaces, producing, when geometry and/or friction effects are present, a surface texture different than that in the interior. Truszkowski et al $[10,11]$ produced large shear texture gradients in rolled copper and aluminium. Yet, for several low SFE alloys, brass and silver, they could produce only small texture variations through the thickness, for the same rolling condition $[10,12]$. Low SFE promotes twinning as a deformation mode, and as explained by Duggan et al [10], twinning followed by shear banding and slip gives rise to the low-SFE, large-strain rolling texture $\{110\}<112>$ plus $\{110\}<001>$ and a minor $\{111\}<u v w>$ component. In these low SFE materials, a $\{100\}<110>$ shear component twins to $\{122\}<411>$. Following Hutchinson [13], this structure then rotates to $\{100\}<001>$ or $\{111\}<u v w>$. Thus, twinning of the components, formed under heterogeneous conditions followed by slip, produces final textures similar to homogeneous rolling. This situation does not occur in high SFE metals and alloys being the open question whether the results are due to the micromechanics of the deformation or to different contact surface properties and interaction between rolls and samples.

In this work we used X-rays and neutron diffraction to investigate the depth of shear layers in $1 \mathrm{~mm}$ thickness $\mathrm{Fe}-31 \mathrm{Mn}-4 \mathrm{Si}$ sheets rolled at $600^{\circ} \mathrm{C}$ and annealed at $650^{\circ} \mathrm{C}$. Based on the data, we rationalize how those layers affect the bulk texture and analyze how the most favourable textures influence the SME.

\section{Experimental procedures}

The Fe-31Mn-4Si (wt. \%) alloy was melted in an arc furnace, homogenized at $1000^{\circ} \mathrm{C}$ for 24 $\mathrm{h}$ and rolled at $1000^{\circ} \mathrm{C}$ to $1.7 \mathrm{~mm}$. It was then reduced $35 \%$ on several rolling passes at $600^{\circ} \mathrm{C}$ and $20^{\circ} \mathrm{C}$, to a thickness of $1 \mathrm{~mm}$. Finally, the rolled material was annealed at $650^{\circ} \mathrm{C}$ for $20 \mathrm{~min}$. Samples were mechanically and electrolytically polished, using an 80/20 (vol. \%) acetic/ perchloric acid solution. X-ray pole figures were determined using $\mathrm{Cu}-\mathrm{K} \alpha_{1} / \mathrm{K} \alpha_{2}$ lines in a Philips X-pert pro MPD goniometer. Neutron pole figures were measured at Tex-2 goniometer, GKSS, Geesthacht, Germany. Results were analyzed by standard texture and orientation distribution function calculations.

Microstructure was observed by optical microscopy and tensile tests were performed in an 
Instron 1362 testing machine, at a strain rate of $2 \times 10^{-4} \mathrm{~s}^{-1}$. The reverse transformation was obtained by heating at $600{ }^{\circ} \mathrm{C}$ for $15 \mathrm{~min}$, i.e. above the $\mathrm{A}_{\mathrm{f}}$ temperature, in $\mathrm{Ar}$ atmosphere to avoid oxidation.

\section{Results and discussion}

\section{Texture measurements}

Figure 1 shows the $\{111\}$ pole figure of austenite, as determined by $\mathrm{X}$-ray and neutron diffraction measurements, on Fe-31Mn-4Si sheets rolled under the same $\Delta$ conditions at different temperatures, and annealed at $650^{\circ} \mathrm{C}$ for $20^{\prime}$. Rolling at room temperature, produces a complete stress induced martensitic transformation in the material, with a typical HCP rolling texture. It has transformed back to austenite during annealing, thus maintaining the typical brass rolling components. The neutron diffraction data (figure 1c) indicates a stronger texture than that observed with X-rays (figure 1a). On the other hand, when the sheets are rolled at temperatures over the $\mathrm{A}_{\mathrm{f}}$, the pole figures measured by neutrons show a marked difference with those obtained by using X-rays. The sheet rolled at $600^{\circ} \mathrm{C}$ has a combination of the brass texture components, $\{111\}<112>$, plus a shear texture component $\{100\}<110>$ (near ideal $<441>$ ), on the surface. This alloy experiences an increase in SFE with temperature, and, as it behaves like high-SFE metals, twinning does not occur. Figure 2 is an optical micrograph, which shows a microstructure that is fully twinned. Pole figures measured by neutron diffraction on this sheet (figure 1d), show the contribution of the shear surface components, giving rise to a diminished brass texture, with a weak $\{100\}<110>$ component.

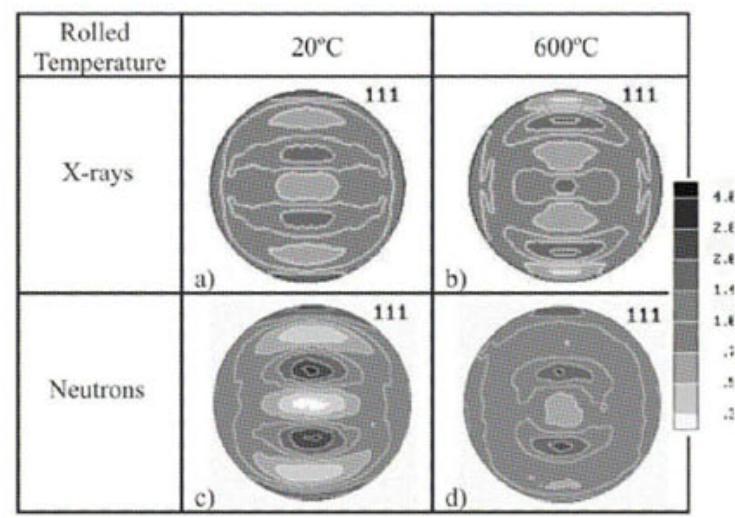

Figure 1. \{111\} pole figures of the sheets rolled at different temperatures after annealing at $650^{\circ} \mathrm{C}$ for $20 \mathrm{~min}$, as measured by $X$-rays and neutron diffraction.

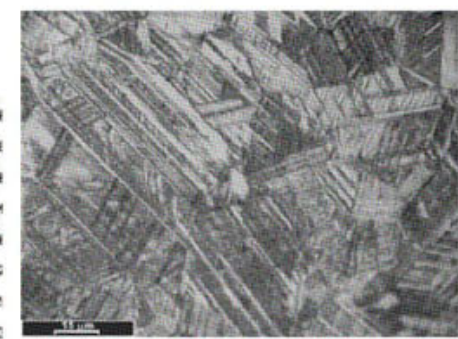

Figure 2. Microstructure of the twinned alloy.

To clarify the effect of deformation heterogeneities in the sheet rolled at $600^{\circ} \mathrm{C}$ and annealed at $650^{\circ} \mathrm{C}$ for $20 \mathrm{~min}$, we performed X-ray analysis at increasing depths bellow the surface in contact with the rolls. This condition, among the studied cases, presented the best shape recovery. For measuring the textures at increasing depth we took advantage of an apparent disadvantage of $\mathrm{Cu} \mathrm{X}$-ray radiation-Fe alloys interaction, that of the high fluorescence and 


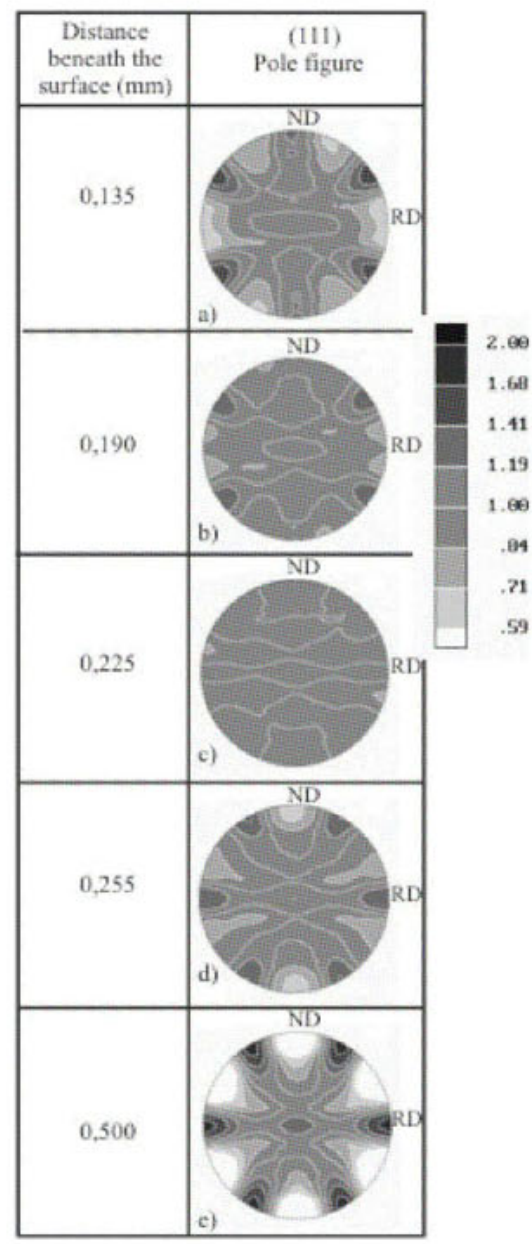

Figure 3. $\{111\}$ pole figures measured at increasing depths beneath the surface of the sheet rolled at $600^{\circ} \mathrm{C}$ and annealed. consequent large absorption effects. By careful mechanical, and further electrolytic polishing by using an 80/20 (vol. \%) acetic /perchloric acid solution, we could take texture data from the very first $5-10 \mu \mathrm{m}$ after each thinning procedure. Figure 3 summarizes the results, showing $\{111\}$ austenitic pole figures. Figure $3 \mathrm{a}$, taken at 0.135 $\mathrm{mm}$ below the surface, shows a typical FCC shear texture. At $0.19 \mathrm{~mm}$ (figure $3 \mathrm{~b}$ ) and 0.225 $\mathrm{mm}$ (figure $3 \mathrm{c}$ ), we observed a gradual rotation of the shear texture, and at $0.255 \mathrm{~mm}$ (figure 3d) the pole figure is that of a classic rolled FCC brass texture, which is maintained all through to the sheet centre (figure 3e). Inverse pole figures calculated at the sheet surface and centre (figure 4) show a slight textural difference due to deformation heterogeneities. The preferential orientation on the surface is $\{100\}<110>--$ near the $<441>$ orientation--, which activates one of the $\{111\}<112>$ systems responsible of martensitic transformation. On the other hand, there are a majority of grains oriented at $\{110\}<112>$ at the sheet centre. This texture gradient influences the SME because it affects the number of grains favourable oriented for the $\gamma \rightarrow \varepsilon$ martensitic transformation. The contribution of all those components can be integrated creating an average inverse pole figure (figure 5a). Figure 5 also shows the inverse pole figure obtained from neutron diffraction (figure $5 \mathrm{c}$ ) and the same figures corresponding to the sheet rolled at room temperature (figure $5 \mathrm{~b}$ and $5 \mathrm{~d}$, respectively). The figures obtained from the neutron diffraction study, are slightly weaker than those calculated from the weighted average of the different textures measured by X-ray diffraction at different depths. Even averaging different depths' textures the averaging process is not as good as the one performed by neutron diffraction technique.

\section{Texture influence on the shape memory effect}

We have evaluated the SME in the various textured sheets, which were obtained by rolling and annealing at $650^{\circ} \mathrm{C}$. Samples were tensile tested to $3 \%$ permanent deformation, and then heat treated at $600^{\circ} \mathrm{C}$ for $20 \mathrm{~min}$. The thermal treatment reverses the martensitic transformation. Before testing, after testing and after the heat treatment, the lengths were measured 
between two indentation marks made with a Shimadzu micro hardness tester on the sample surface. Results, summarized in Table 1, were calculated as

$$
\operatorname{SME}(\text { in percent })=\frac{l_{1}-l_{2}}{l_{1}-l_{0}} \cdot 100
$$

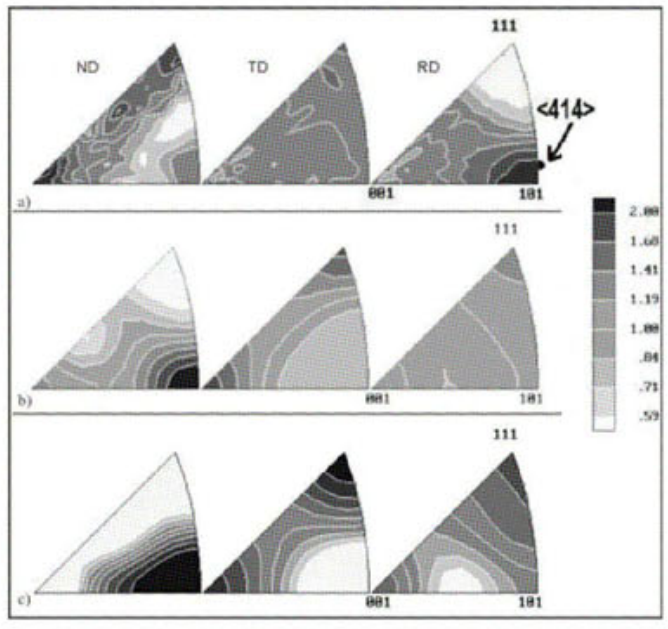

Figure 4. Inverse pole figures of the surface (a) and the centre (b) of the sheet rolled at $600^{\circ} \mathrm{C}$ and annealed, and c) from the specimen surface after tensile deformation.

Figure 5. Inverse pole figures of the austenite corresponding to: a) sheet rolled at $600^{\circ} \mathrm{C}$, b) sheet rolled at room temperature, averaging all X-ray measurements; c) sheet rolled at $600^{\circ} \mathrm{C}$, d) sheet rolled at room temperature, with the inverse pole figures obtained from neutron diffraction data.

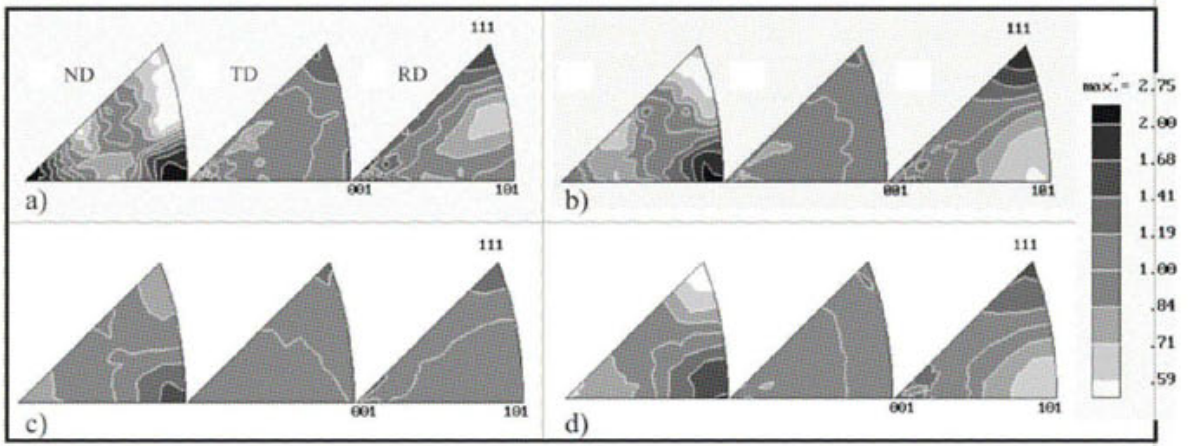

Table 1. SME results, where $\boldsymbol{\sigma}_{7 \rightarrow \varepsilon}$ is the stress that induces the martensitic transformation and $\boldsymbol{S M E}$ is the percent of deformation recovery.

\begin{tabular}{|c|c|c|}
\hline Sample & $\sigma_{\gamma \leftrightarrow \varepsilon}(\mathrm{MPa})$ & SME (\%) \\
\hline Rolled at $20^{\circ} \mathrm{C}$ and annealed at $650^{\circ} \mathrm{C}$ & 680 & 35 \\
\hline Rolled at $600^{\circ} \mathrm{C}$ and annealed at $650^{\circ} \mathrm{C}$ & 490 & 55 \\
\hline
\end{tabular}


In a previous work [6] we measured the inverse pole figures of the austenite, before and after tensile deformation. The texture component in the rolling direction near the $\langle 441>$ ideal, observed on the surface of the sheets rolled at $600^{\circ} \mathrm{C}$ (figure 4a), disappeared when the samples were deformed in tension. The reorientation of the component (figure $4 \mathrm{c}$ ) would be produced by a martensitic transformation. It should be noted that these sheets have shown the largest SME, compared to those rolled at $20^{\circ} \mathrm{C}$, with the same annealing conditions.

\section{Concluding remarks}

Mechanical processing, like rolling, may produce heterogeneous deformations in materials, through geometrical effects and friction between the sheet and rolls. As a consequence, $\mathrm{X}$ ray analyses have shown texture heterogeneities through the thickness of rolled sheets. Neutron diffraction, as well as the calculation of the average of textures measured at different depths beneath the surface, reveals that this textured layer affects the material's bulk behaviour. The surface texture of the alloy processed at $600^{\circ} \mathrm{C}$ is the most favourable for activation of the $\gamma \rightarrow \varepsilon$ martensitic transformation that produces the shape memory effect. Without a favourable surface texture, there is a very little SME in the alloy rolled at room temperature. As texture intensities are low, not above 3 m.r.o., it might be presumptuous to completely attribute differences in SME to this textural variation. However, it is interesting to note that the favourable orientations disappeared from the retained austenite when the $600^{\circ} \mathrm{C}$ rolled material was deformed in tension.

\section{References}

1. Sato, A., Chishima, E., Soma, K. \& Mori, T., 1982, Acta Metall., 30, 1117.

2. Murakami, M., Otsuka, H., Suzuki, H.G. \& Masuda, S., 1986, in Proc. of Int. Conf. on Martensitic Transformation (ICOMAT-86), Japan Inst. Met., p. 985.

3. Allain, S., Chateau, J., Bouaziz, O., Migot, S. \& Guelton, N., 2004, Mat. Sci. Eng. A, 387-389, 158.

4. Sato, A., Chishima, E., Yamaji, Y. \& Mori, T., 1984, Acta Metall., 32, 539.

5. Matsumura, O., Furusako, S., Furukawa, T. \& Otsuka, H., 1996, ISIJ Int., 36, 1103.

6. Druker, A., Sobrero, C., Brokmeier, H.-G., Malarría, J. \& Bolmaro, R., 2006, Mat. Sci. Eng. A, 481-482, 578.

7. Lee, C.S. \& Duggan, B.J., 1991, Metall. Trans., 22A, 11, 2637.

8. Backofen, W.A., 1972, Deformation Processing (Reading, MA: Addison Wesley Publishing Co.), p. 143.

9. Mathur, P.S. \& Backofen, W.A., 1973, Metall. Trans., 4, 643.

10. Truszkowski, W., Krol, J. \& Major, B., 1980, Metall. Trans., 11A, 749.

11. Truszkowski, W., Krol, J. \& Major, B., 1982, Metall. Trans., 13A, 665.

12. Truszkowski, W., Krol, J. \& Major, B., 1982, in Proc. 6th Conf. on Textures of Materials, ISIJ, Tokio, p. 39.

13. Hutchinson, W.B., Duggan, B.J. \& Hatherly, M., 1979, Met. Technol., 32, 1392. 


\title{
Texture and structure of grain boundary in Ni-Ti strip produced by twin roll casting technique
}

\section{T. Goryczka}

University of Silesia, Institute of Materials Science, Bankowa 12, Katowice 40-007, Poland goryczka@us.edu.pl

Keywords: texture, X-ray diffraction, EBSD, Ni-Ti alloy

\begin{abstract}
Combination of the X-ray and electron back scattered diffraction techniques was applied for characterization of the $\mathrm{Ni}$ - Ti shape memory alloy produced by twin roll casting. The Ni-Ti alloy revealed presence of the one-step reversible martensitic transformation. The transformation occurs between the B2 parent phase and the B19' monoclinic martensite. Columnar as well as fine grains formed at the surface were preferentially oriented along $<100\rangle,\langle 110>$ and $\{100\}<001>$ textural components. As a consequence of the high texture about $40 \%$ of grain boundaries were coincident. Especially, $\Sigma 5, \Sigma 13$ a and $\Sigma 17$ a boundaries were formed.
\end{abstract}

\section{Introduction}

The twin roll casting technique (TRC) appeared to be useful for shape memory alloy production [1]. In addition to economical advantages of this technique unique properties of the polycrystalline strip can be obtained. It combines rapid solidification together with coldrolling. In practice, the strip is ready to be used in the as-cast state without additional thermomechanical treatment, which is needed after conventional casting and rolling to obtain sheets with the shape memory effect [2-4]. This technique is especially useful for group of shape memory alloys developed from the binary Ni-Ti alloy. The main advantage of the TRC technique is preferential grains growth. Appropriate grains orientation, resulting from the crystallography of the martensitic transformation, can increase the shape memory effect [5].

Application of the X-ray diffraction technique for structure studies allows receiving general information from a large area. Thus, texture or structural changes occurring during martensitic transformation can be well characterized. However, particular information about individual grain orientation, which is essential for the shape memory effect, cannot be received from the X-ray diffraction. Thus, in the presented work for the studies of the grain orientation at the surface as well as in the cross-section of the Ni-Ti shape memory alloy, the X-ray diffraction and electron back scattered diffraction (EBSD) were combined. The obtained results allowed for carrying full characterization of the grains formation in the Ni-Ti alloys produced using the TRC. 


\section{Experimental data}

A Ni-Ti shape memory alloy, with chemical composition close to equiatomic, was produced applying twin roll casting technique. A strip was obtained in dimensions: $45 \mathrm{~mm}$ width, 29 $\mathrm{cm}$ long and about $298 \mu \mathrm{m}$ thick. Details of the manufacturing process can be found at [6].

Temperatures of the martensitic transformation were determined using differential scanning calorimeter Perkin-Elmer DSC-7 (DSC). The DSC heating/cooling curves were measured at a rate of $10 \mathrm{deg} / \mathrm{min}$.

An INEL X-ray diffractometer, equipped with a curve position sensitive detector CPS 120 and a cooling/heating attachment, was used to determine the sequence of the martensitic transformation. The Philips diffractometer (PW 1130) with the texture goniometer was applied for registering incomplete pole figures. The sample was fixed in such way that the RD was parallel to the length of the strip.

Microstructure of the strip was observed using an electron scanning microscope JEOL SEM 6448 operating at $20 \mathrm{kV}$ and equipped with an EBSD Nordlys detector. In order to measure the grain orientation in the martensite or the parent phase states, a heating attachment was used. Dependently on a grain size the EBSD maps were collected with scanning distance from 0.2 to $0.6 \mu \mathrm{m}$. Reconstruction of the grains and analysis of their orientation were done using HKL CHANNEL 5 package software.

\section{Results and discussion}

\section{Martensitic transformation}

The studied strip shows the reversible martensitic transformation B2 $\Leftrightarrow B 19$ ' (fig. 1a). Only one maximum appeared on cooling as well as heating curve. During cooling the parent phase starts to transform into the martensite at $40^{\circ} \mathrm{C}$. This transformation is completed at $35^{\circ} \mathrm{C}$. During the reverse transformation the parent phase starts to form at almost $58^{\circ} \mathrm{C}$ and finishes at about $70^{\circ} \mathrm{C}$. In order to confirm the structural changes the X-ray diffraction patterns were collected over the thermal range of the transformation (fig. 1b).
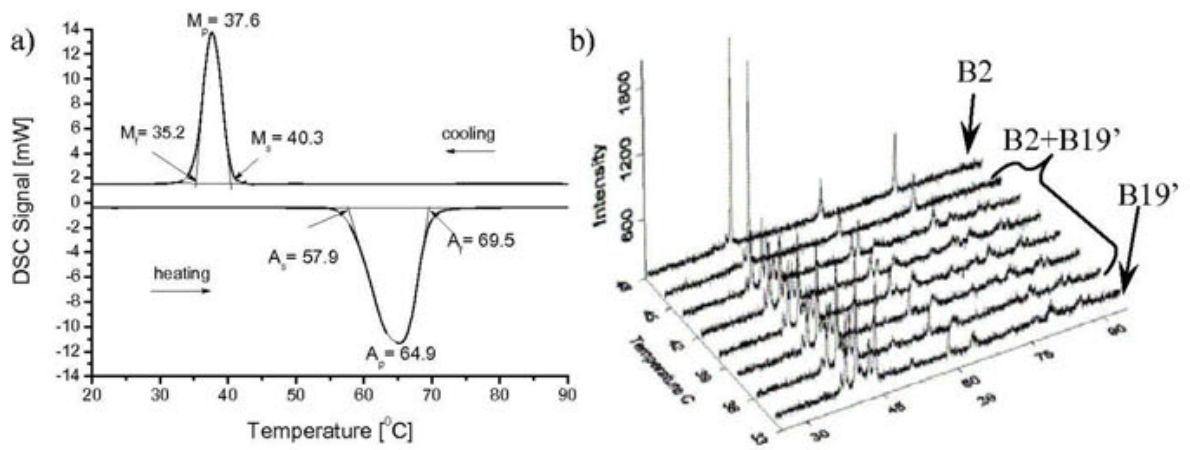

Figure 1. DSC cooling/ heating curves (a) and set of X-ray diffraction patterns registered during cooling (b). 
The X-ray diffraction pattern taken at $48^{\circ} \mathrm{C}$ reveals position and intensity of diffraction lines characteristic for the B2 parent phase. During cooling the intensity of these lines vanishes. Simultaneously, new diffraction lines appear. Finally, the X-ray diffraction pattern measured at $33^{\circ} \mathrm{C}$ represents only diffraction lines belonging to the $\mathrm{B} 19^{\prime}$ monoclinic martensite.

\section{Texture and structure of grain boundary}

Relation of the crystallographic directions between the parent phase and the martensite lattices was described by Michal and Sinclair [7] who determined the crystal structure of the B19'martnesite. They showed that the $[100]_{\mathrm{B} 2}$ direction refers to $[100]_{\mathrm{B} 19},[011]_{\mathrm{B} 2}$ to $[010]_{\mathrm{B} 19^{\prime}}$ and $[0-10]_{\mathrm{B} 2}$ to $[001]_{\mathrm{B} 19^{\prime}}$, respectively. Basing on this, indexes of calculated pole figures $\{100\},\{110\}$ and $\{211\}$ were given in the notation of the B2 lattice (fig. 2). It can be seen that the poles clasterized around a strong fibre $\langle 100\rangle$ and $\{100\}<001>$ sheet texture component. The maximum of the pole density was 37 for the $\{100\}$ pole figure. Weak diffused and incomplete ring at angle of 45 degrees from the centre of the $\{100\}$ pole figure proved presence of the $\langle 110\rangle$ additional fibre texture component.
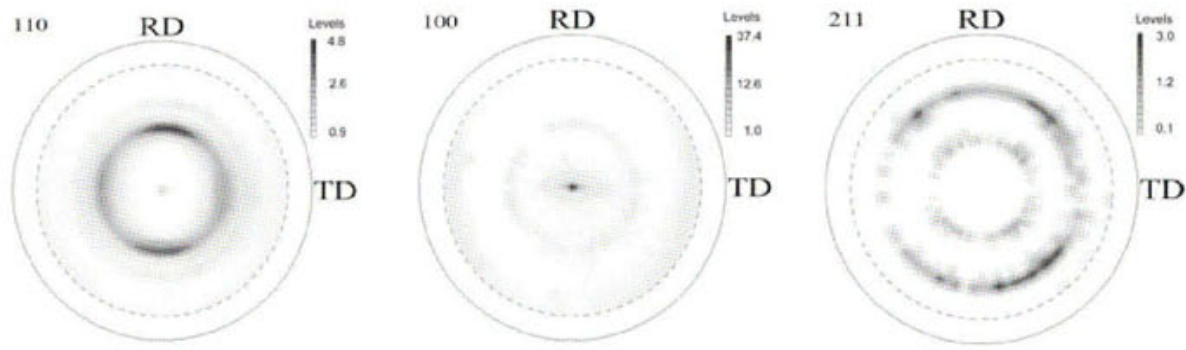

Figure 2. The pole figures measured using X-ray diffraction.

From the measured pole figures the orientation distribution function (ODF) was calculated using the computer program LaboTex. The obtained fractions of the preferentially oriented grain along $\langle 100\rangle,\langle 110\rangle$ and $\{100\}<001\rangle$ were as follows: $43,8 \%, 3,8 \%$ and $21,7 \%$, respectively. Almost $70 \%$ of grains were preferentially oriented. This strong texture is a result of the rapid solidification combined with cold rolling which occurs during strip casting. Liquid alloy is ejected into the space between two rolls rotating in opposite directions. On the surface of the both rolls liquid alloy began to solidify. The solidification front goes from the both surfaces - keeping the contact with the rotating rolls - to the inside of the forming strip. In consequence, the strip consists of two symmetrical parts, which are joined by an interface. During casting the high cooling rate forces the directional grain growth, which increases the number of preferentially oriented grains.

However, the $\mathrm{X}$-ray diffraction does not give detailed information about the individual grain orientation and correlation of grain boundaries that is an important factor for the shape memory effect. In order to receive full characterization of grains formation during the twin roll casting, additional studies were carried out using electron scanning microscope equipped with EBSD detector. In order to collect electron back scattered patterns (EBSP) for the B2 parent phase the sample was heated up to $85^{\circ} \mathrm{C}$. Kikuchi patterns were collected from the representative places on the surface as well as in the cross-section parallel to the length of the 
strip. Figure $3 \mathrm{a}$ shows reconstructed inverse orientation map with a color code obtained for the surface. Mainly, orientation along $\langle 100\rangle$ and $\langle 110\rangle$ direction was stated. Also, it can be seen that two zones of the grains are formed: "A" with relatively long grains and "B" with fine equiaxial grains. The aspect ratio, which is a measure of the grain shape, was calculated as a rate of lengths of a major and minor axis of the fitted ellipse. The length of the long grains formed in zone " $\mathrm{A}$ " was 13 times higher than their width. The obtained results indicate that the long grains are formed in shape of columns which grow at surface perpendicularly to the length of the strip.

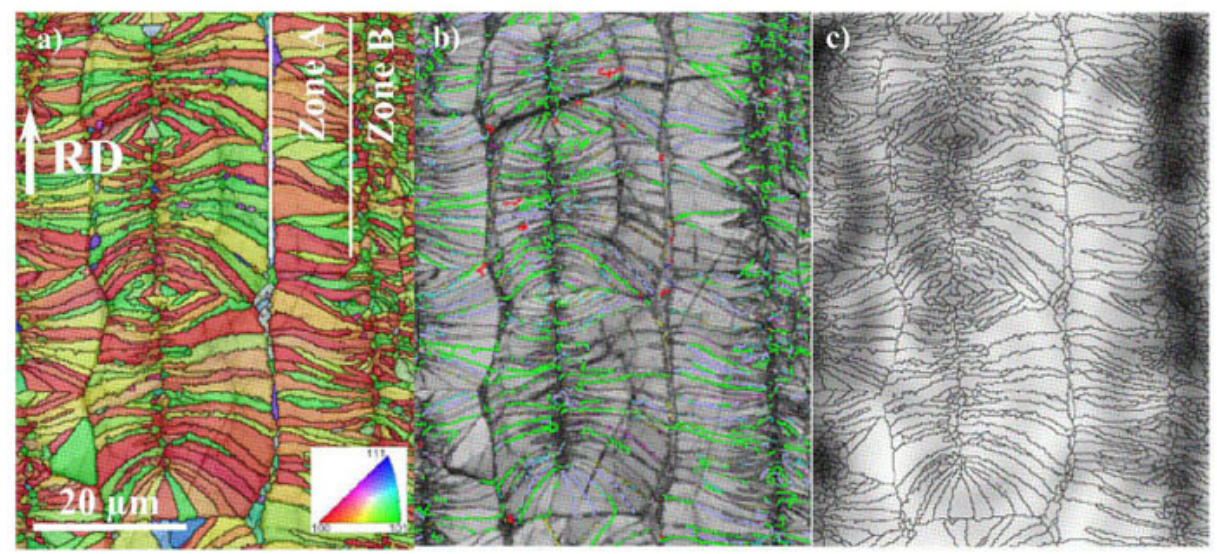

Figure 3. Orientation map (a), CSL boundary map (b) and strain contouring map (c) calculated for surface of the strip.

One of the advantages of the preferentially oriented grains is the possibility to obtain high number of coincidence site lattice parameters (CSL). This parameter describes a superlattice which can be created from sites of the crystal lattices of two neighboring grains. The CSL parameter is usually described by the reciprocal density of the coincident lattice sites $(\Sigma)$. The condition is that both neighboring grains must have an appropriate misorientation angle. Figure $3 \mathrm{~b}$ shows a map with the reconstructed grain boundaries with overlapped CSL boundaries (colored lines). Almost $40 \%$ of the all boundaries were coincident. The most significant contributions: $16 \%, 5 \%, 6 \% \quad 3.2 \%, 3.5 \%$ and $2 \%$ were calculated for the following identified boundaries: $\Sigma 5, \Sigma 13 \mathrm{a}, \Sigma 17 \mathrm{a}, \Sigma 25 \mathrm{a}, \Sigma 29 \mathrm{a}$ and $\Sigma 37 \mathrm{a}$, respectively. The presence of $\Sigma 5$ boundaries can be very helpful for the martensite formation. The reason is the correlation between two grains rotated around $[100]_{\mathrm{B} 2}$ direction of 36.9 degrees. Similar dependence can be found in $\Sigma 13$ a boundary with the rotation angle of 22.6 degrees. It means that $\{110\}_{\mathrm{B} 2}$ plane is parallel to the surface of the strip. Furthermore, also the $[110]_{\mathrm{B} 2}$ direction is parallel to the length of the strip. Both, the plane and the direction are dominant in formation of the B19' lattice. During transformation from the parent phase to the martensite a coordinate atom shuffle appears in the $\{110\}_{\mathrm{B} 2}$ plane and $\langle 110\rangle_{\mathrm{B} 2}$ direction. Also in NiTi shape memory alloys the martensitic transformation can be triggered by applying external stress. Its directional application along the length of the strip can result in obtaining higher degree of the shape recovery. 
It has been known that in shape memory alloy two sources of internal strain can be distinguished. The first comes as a result of the martensitic plate formation during the phase transformation. This strain can be compensated by formation of specific orientation of the martensitic plates - self-accommodating plates. The second originates in grain misorientation and is a characteristic property of the material surface. Measurement of an individual grain orientation enables calculating and estimating the extent of deformation, or strain, in the obtained map. During calculation the maximum misorientation between any 2 points in a grain is determined and then referred to mean misorientation values inside the grain. Figure $3 \mathrm{c}$ shows the calculated strain contouring map overlapped on the reconstructed grain boundary. The black contour indicates maximum of the strain level -2.5 , whereas the white one 0 . It can be seen that in the shorter grain, formed in the "B" zone, the level of strain reached the maximum. The long columnar grains were almost free from any strain.

In order to get information about orientation of grains which extended from the surface to the interface of the strip the EBSD measurements were carried out on the cross-section perpendicular to the length of the strip. The orientation map (fig. 4a) reveals grain formation on the cross-section of the strip in previously discussed zones: "A" and "B". Calculation of the aspect ratio (about 1,5) done for grains at the cross-section of the zone " $\mathrm{A}$ " proves that the grains are only slightly elongated. The aspect ratio

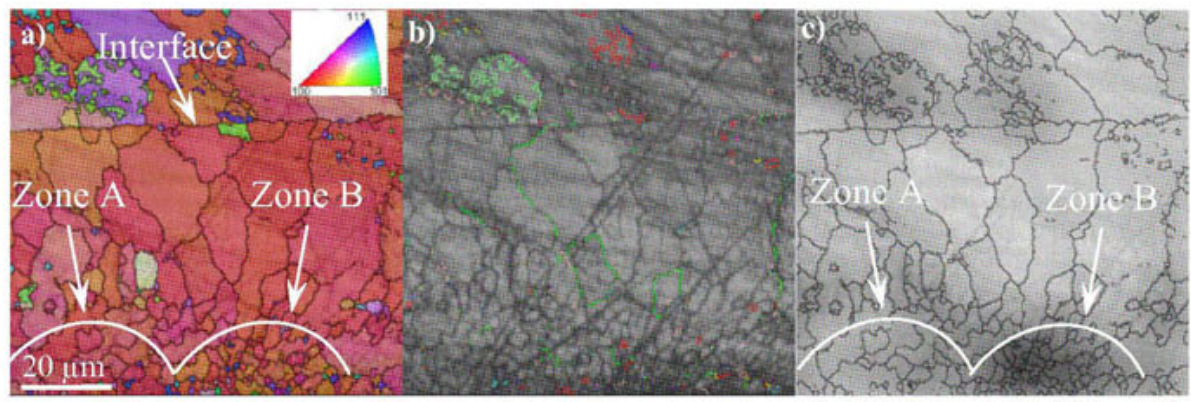

Figure 4. Orientation map (a), CSL boundary map (b) and strain contouring map (c) calculated for perpendicular cross-section of the strip.

calculated for the grains formed in the zone "B" was 1 and it confirmed their equiaxial shape. Going from the surface of the strip in the direction of the interface, under grains, which form both zones long columnar grains extend to the centre of the strip. Their average length was about $23 \mu \mathrm{m}$. Set of the pole figures calculated for the cross-section is shown in figure $5 \mathrm{a}$. ND is direction normal to the strip surface. It can be seen that grains are tilted in the direction of the strip head. Moreover, the pole densities, marked as the arcs at the $\{110\}$ pole figure, clustered around the $<100\rangle$ fibre texture component. This strong texture ensures presence of the $\{110\}_{\mathrm{B} 2}$ plane spreaded around the normal to surface. Such orientation can support and increase the shape memory effect.

Figure $4 \mathrm{~b}$ shows the map of the reconstructed CSL boundaries. Also $\Sigma 5$ and $\Sigma 3$ can be distinguished. However, the amount of the total coincident boundaries was lower than it was observed for the surface and its value did not exceed $15 \%$. Figure $4 \mathrm{c}$ reveals strain 
contouring. The highest strain appeared in the equiaxial grains formed in the zone "B" and similarly to the value calculated for the surface reached level of 2.5 .
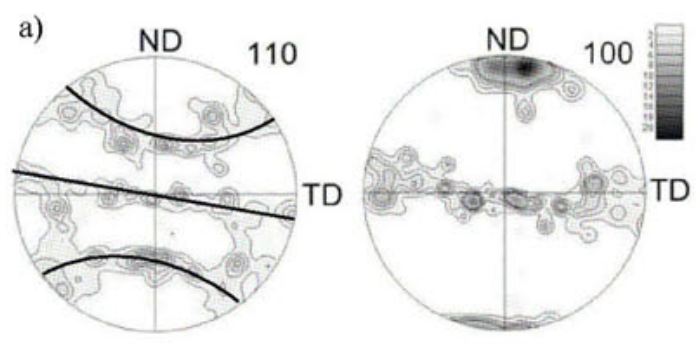

b)

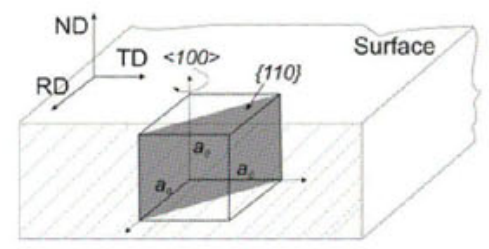

Figure 5. Pole figures calculated from EBSD (a) and fibre texture of $\langle 100\rangle$ axis normal to the surface (b).

\section{Conclusions}

Results obtained form combination of both applied diffraction methods allowed to conclude the following:

- The strip reveals strong texture - only $30 \%$ of grains at the surface were randomly oriented.

- The strongest textural component in the cross-section was $<100>$ fibre.

- High number of the coincident boundaries (about $40 \%$ ), especially $\Sigma 5$, can be helpful for improving the shape memory effect.

- The martensitic transformation in the Ni-Ti strip is reversible and occurs in the temperature range of $35.2^{\circ} \mathrm{C}-69.5^{\circ} \mathrm{C}$ between the $\mathrm{B} 2$ phase and the $\mathrm{B} 19^{\prime}$ 'martensite.

\section{References}

1. Dalle, F., Vermaut, Ph., Portier, R., Dezellus, A., Plaindoux, P. \& Ochin, P., 2003, Mat. Sci. Eng., A 346, 320.

2. Miyazaki, S., Ohmi, Y., Otsuka, K. \& Suzuki, Y., 1982, J. de Phys., 43, C4-255.

3. Stróż, D., Bojarski, Z., Ilczuk, J., Lekston, Z. \& Morawiec ,H., 1991, J. Mater. Sci., 26, 1741 .

4. Liu, Y., Xie, Z.L., van Humbeeck, J. \& Delaey, L., 1999, Acta Metall., 47, 645.

5. Chang, S.H. \& Wu, S.K., 2004, Scr. Mater., 50, 937.

6. Goryczka, T. \& Ochin, P., 2005, J. Mat. Proces. Tech., 162-163, 178.

7. Michal, G.M. \& Sinclair, R., 1981, Acta Cryst., B37, 1803.

Acknowledgements. Dr Patrick Ochin (ICMPE CNRS, France) is acknowledged for the support during the strip manufacturing. Studies were financially supported from the project no. N N507 458733 funded by the Polish Ministry of Science and High Education. 


\section{Temperature dependence of microstructure of Al-Ag-Zn alloys}

S. Popović ${ }^{1, *}, \check{Z}$. Skoko ${ }^{1}$, G. Štefanić ${ }^{2}$

${ }^{1}$ Physics Department, Faculty of Science, University of Zagreb, P.O.B. 331, 10002 Zagreb, Croatia

${ }^{2}$ Ruđer Bošković Institute, P.O.B. 180, 10002 Zagreb, Croatia

* spopovic@phy.hr

Keywords: aluminium-based ternary alloys, X-ray powder diffraction, microstructure, phase transitions

Abstract: Al-rich Al-Ag-Zn alloys, quenched to RT from a temperature, $T_{\mathrm{t}}$, higher than the solid-solution temperature, $T_{\mathrm{ss}}$, contained GP zones, as proved by XRD and TEM. GP zones grew during ageing, remaining fcc and coherent with the Al-rich $\alpha$-phase (fcc). In parallel, metastable precipitates, $\varepsilon^{\prime}$ (hep), were formed by discontinuous nucleation and by a direct transition from GP zones. The unit-cell parameters of $\varepsilon^{\prime}$ and of the equilibrium phase, $\varepsilon$ (hcp), formed in the alloys slowly cooled from $T_{\mathrm{t}}$ to RT, depended on the solute content. The quenched and prolongedly aged alloys, being two-phase systems, $\alpha+\varepsilon^{\prime}$, were studied in situ at high temperature. The following was observed: an initial lattice strain annealing, an anisotropy of thermal expansion of $\varepsilon^{\prime}$, a gradual dissolution of $\varepsilon^{\prime}$ in the $\alpha$-phase above $500 \mathrm{~K}$, the formation of solid solution. $T_{\mathrm{ss}}$ depended on the alloy composition. On cooling, a temperature hysteresis was noticed in reversal changes. The dependence of unit-cell parameters of $\varepsilon^{\prime}$ on temperature during cooling was different from that during heating. After the heating and cooling cycle, unit-cell parameters of the precipitates were close to those of the equilibrium phase, $\varepsilon$.

\section{Introduction}

The physical properties and microstructure of the Al-rich alloys depend on the composition and applied heat treatment. Much of the knowledge achieved on the Al-alloys, and on the the $\mathrm{Al}-\mathrm{Zn}$ alloys in particular, is collected in a monograph edited by Löffler [1]. In both Al-Ag and Al-Zn Al-rich alloys Guinier-Preston (GP) zones are formed upon quenching. The precipitation sequence observed on ageing in Al-Ag alloys is spherical GP zones $\rightarrow \gamma^{\prime}$ (hcp) $\rightarrow \gamma$ (hcp) and that found in Al-Zn alloys is spherical - ellipsoidal GP zones $\rightarrow$ rhombohedral $\alpha_{\mathrm{R}}^{\prime} \rightarrow \alpha^{\prime} \rightarrow \beta$ [1-3]. Precipitation in ternary alloys may be too complex to be interpreted in terms of that in binary alloys.

$\mathrm{Al}-\mathrm{Ag}-\mathrm{Zn}$ alloys have not been studied much, even though that is a system in which similar precipitates are formed in a wide composition interval. Bates and Gould showed that the precipitation sequence was GP zones $\rightarrow \varepsilon^{\prime}$ (hcp) $\rightarrow \varepsilon$ (hcp) and that the $\alpha$-phase was strained 
around GP zones [4]. Kähkönen [5] studied the formation of metastable precipitates, $\varepsilon^{\prime}$, by SAXS. Popović and Passoja [6] and Passoja et al. [7] followed precipitation processes in several Al-Ag-Zn alloys upon quenching by XRD and TEM. Lyon et al. [8] studied anomalous SAXS in Al-Ag-Zn alloys with comparison to Al-Zn alloys. The present work is an extension of the previous studies [6,7], with the aim to check the influence of a prolonged ageing of the quenched alloys on microstructure. Also, the microstructure of the same alloys was followed by in situ XRD from RT to $T_{\mathrm{t}}$ and back to RT, with comparison to that in the slowly cooled alloys.

\section{Experimental}

The alloys, prepared from elements of purity $5 \mathrm{~N}$, had an electron to atom ratio of 2.9 (table 1). The chosen $\mathrm{Ag}$ to $\mathrm{Zn}$ ratios might promote a certain stoichiometry of precipitates, e.g. $\mathrm{Al}_{2} \mathrm{AgZn}$ or $\mathrm{AlAgZn}$ for alloy 3. The melted alloys were cast into copper moulds and homogenized at $T_{\mathrm{t}}=820 \mathrm{~K}$. Small amounts of powdered alloys, wrapped in a thin perforated Al foil, were treated at $T_{\mathrm{t}}$, quenched in water at RT $(293 \mathrm{~K})$ and immediately aged at $T_{\mathrm{a}}=$ $420 \mathrm{~K}$. Also, powdered alloys were slowly furnace cooled to RT over a week.

XRD patterns were recorded by a Philips diffractometer (proportional counter monochromatized $\mathrm{CuK \alpha}$ radiation, high-temperature attachment). The method for minimization of systematic errors in unit-cell parameters $a(\alpha), a(\mathrm{GPZ}), a\left(\varepsilon^{\prime}, \varepsilon\right)$ and $c\left(\varepsilon^{\prime}, \varepsilon\right)$ was described previously [6,9]. For the $\alpha$-phase, diffraction lines at highest Bragg angles were utilized, while diffraction lines of precipitates appeared only at low/medium Bragg angles, that influencing the accuracy of obtained values. Samples for TEM were thermally treated in the same way as those for XRD.

The alloys that had been quenched from $T_{\mathrm{t}}$ to RT, aged at $T_{\mathrm{a}}$ for 50 days and then aged for 38 years at RT, were studied in situ from RT to $T_{\mathrm{t}}$ and back to RT. A platinum strip (in fact, a Pt alloy) served as a sample holder and a heater; diffraction lines of Pt were used to calibrate the angular scale, utilizing its thermal expansion coefficient $\left[10(2) \times 10^{-6} / \mathrm{K}\right.$, measured by the same diffractometer].

Table 1. Composition of alloys and unit-cell parameter a( $\alpha$ ) at RT of the slowly-cooled (a-phase in equilibrium with precipitates $\varepsilon$ ) and as-quenched ( $\alpha$-phase containing $G P$ zones) alloys at $R T$; the measured unit-cell parameter of pure Al was $4.0492(1)$ A at $R T$.

\begin{tabular}{|c|c|l|l|c|c|}
\hline Alloy & $\mathrm{Ag} / \mathrm{at} \%$ & $\mathrm{Zn} / \mathrm{at} \%$ & $\mathrm{Ag} / \mathrm{Zn}$ & $\begin{array}{c}\text { Slowly-cooled } \\
\text { alloys } \\
a(\alpha / \varepsilon)) / \AA\end{array}$ & $\begin{array}{c}\text { As-quenched } \\
\text { alloys } \\
a(\alpha / \mathrm{GPZ}) / \AA\end{array}$ \\
\hline 1 & 4.44 & 1.11 & 4.00 & $4.0491(1)$ & $4.0478(2)$ \\
\hline 2 & 4.00 & 2.00 & 2.00 & $4.0488(1)$ & $4.0467(2)$ \\
\hline 3 & 3.33 & 3.33 & 1.00 & $4.0483(1)$ & $4.0455(1)$ \\
\hline 4 & 2.50 & 5.00 & 0.50 & $4.0476(2)$ & $4.0440(2)$ \\
\hline 5 & 1.43 & 7.15 & 0.20 & $4.0463(2)$ & $4.0428(2)$ \\
\hline
\end{tabular}




\section{Results and discussion}

\section{Slowly-cooled and quenched-aged alloys}

The unit-cell parameter $a(\alpha)$ for the slowly cooled and as-quenched alloys are given in table 1. $a(\alpha)$ decreased with the solute content, this being mainly influenced by $\mathrm{Zn}$, as its atomic radius is smaller than that of $\mathrm{Al}$. A fraction of $\mathrm{Zn}$ remained solved in the slowly cooled alloys. The unit-cell parameters of the equilibrium phase, $\varepsilon$ (hcp), formed in the slowlycooled alloys, are given in table 2 . These parameters decreased as the solute $(\mathrm{Zn})$ content increased. If extrapolated to $5 \mathrm{at} \%$ solute (i.e. to zero at $\% \mathrm{Zn}), a(\varepsilon)$ and $c(\varepsilon)$ were close to the parameters of the $\gamma$-phase in Al-Ag alloys [1]. For alloy 3, $a(\varepsilon)$ was almost equal to the spacing $d_{\alpha 110}$.

Table 2. Unit-cell parameters of the $\varepsilon$-phase in slowly-cooled alloys and of the $\varepsilon$-phase of alloys aged at $T_{a}$ after quenching, measured at RT.

\begin{tabular}{|c|l|l|l|l|l|c|}
\hline Alloy & $a(\varepsilon) / \AA$ & $c(\varepsilon) / \AA$ & $c(\varepsilon) / a(\varepsilon)$ & $a\left(\varepsilon^{\prime}\right) / \AA$ & $c\left(\varepsilon^{\prime}\right) / \AA$ & $c\left(\varepsilon^{\prime}\right) / a\left(\varepsilon^{\prime}\right)$ \\
\hline 1 & $2.875(1)$ & $4.562(1)$ & 1.587 & $2.867(2)$ & $4.557(2)$ & 1.589 \\
\hline 2 & $2.872(1)$ & $4.553(1)$ & 1.585 & $2.867(2)$ & $4.545(2)$ & 1.585 \\
\hline 3 & $2.862(1)$ & $4.526(1)$ & 1.581 & $2.860(2)$ & $4.510(2)$ & 1.577 \\
\hline 4 & $2.854(1)$ & $4.501(1)$ & 1.577 & $2.854(2)$ & $4.474(3)$ & 1.568 \\
\hline 5 & $2.835(1)$ & $4.450(1)$ & 1.570 & $2.839(2)$ & $4.412(3)$ & 1.554 \\
\hline
\end{tabular}

The quenched and aged (at $T_{\mathrm{a}}$ ) alloys showed the precipitation sequence GP zones (fec) $\rightarrow \varepsilon^{\prime}$ (hcp) $\rightarrow \varepsilon$ (hcp). GP zones and initial precipitates, $\varepsilon^{\prime}$, are shown in figure 1 . A line of zero contrast through the GP zones indicated strained lattice around the zones. For alloys with $\mathrm{Ag} / \mathrm{Zn}>1 \mathrm{GP}$ zones were spheres; for alloys with $\mathrm{Ag} / \mathrm{Zn} \leq 1 \mathrm{GP}$ zones were also cubes and ellipsoids. GP zones exhibited broad XRD lines; an example is shown in figure 2. GP zones diffraction lines sharpened with ageing due to their growth, from 3 or 4 to $6 \mathrm{~nm}$ for alloys 1 and 2 or to 8 to $9 \mathrm{~nm}$ for alloys 3,4 and 5; these values were obtained from XRD broadening and by TEM. The misfit $[a(\mathrm{GPZ})-a(\alpha)] / a(\alpha)$ in the as-quenched alloys changed from $-0.4 \%$ for alloy 1 and to $-2 \%$ for alloy 5 due to the increased $\mathrm{Zn}$ content in the zones. During ageing, the misfit decreased due to a diffusion of $\mathrm{Zn}$ away from the zones.

The metastable phase, $\varepsilon^{\prime}$, is analogous to $\gamma^{\prime}$ in Al-Ag alloys. The orientation relationship between $\varepsilon^{\prime}$ or $\varepsilon$ platelets and the $\alpha$-phase was: $\varepsilon^{\prime}, \varepsilon\{001\}\left\|\alpha\{111\} ; \varepsilon^{\prime}, \varepsilon[110]\right\| \alpha[110]$. Diffraction lines of the $\alpha$-phase split in two components on ageing, the high angle component corresponded to the as-quenched alloy, and the low-angle component to the slowly-cooled alloy. The former component (contained mainly GP zones) transformed to the latter (having $\varepsilon^{\prime}$ precipitates) on ageing. The separation between the components was small $\left(\approx 0.3^{\circ}\right.$ at $2 \Theta \approx$ $138^{\circ}$ ). That effect was interpreted as the discontinuous precipitation of $\varepsilon^{\prime}$. Similar effect was observed in $\mathrm{Al}-\mathrm{Cu}[10]$ and $\mathrm{Al}-\mathrm{Zn}$ [2] alloys (where the separation increased with the $\mathrm{Zn}$ content). 


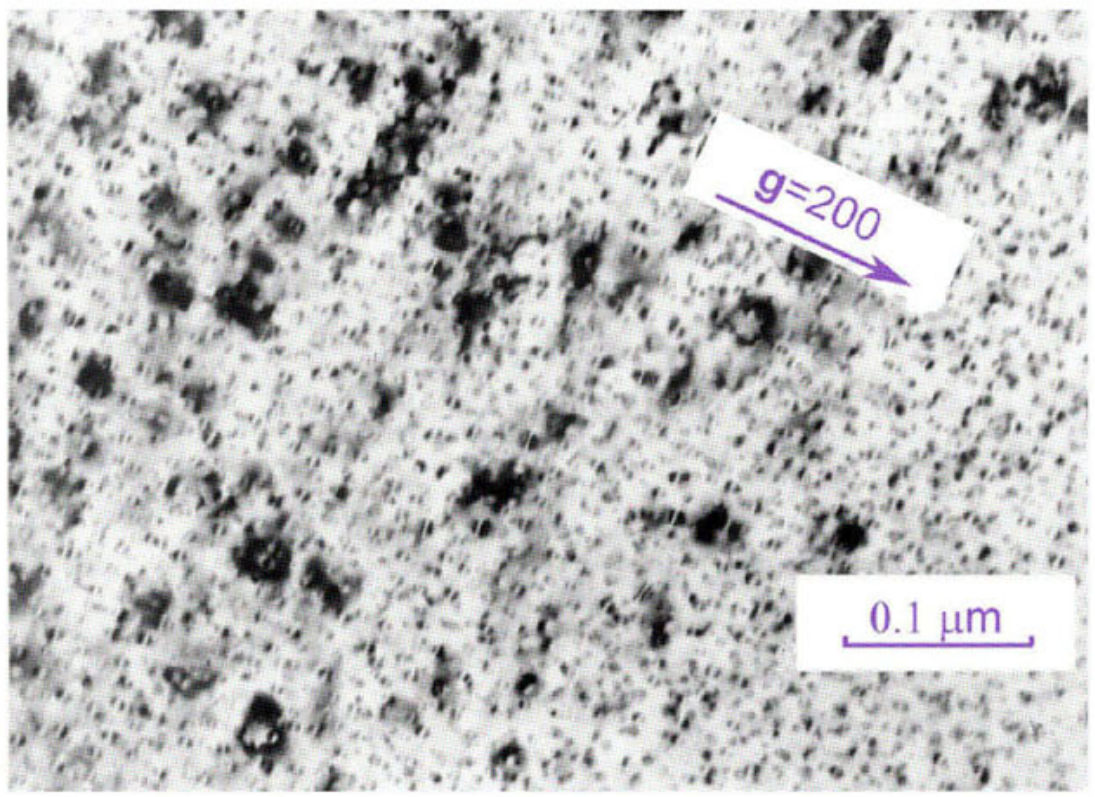

Figure 1. GP zones and initial precipitates $\varepsilon^{\prime}$ in as-quenched alloy 4.

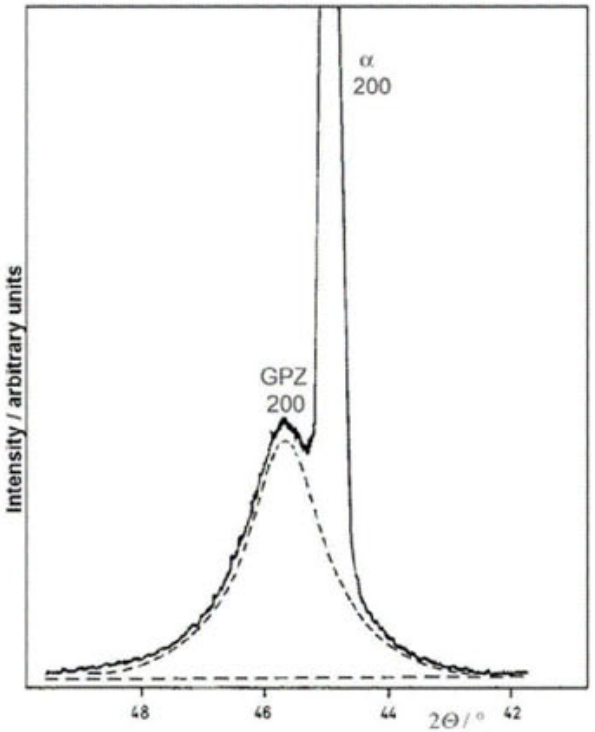

Figure 2. $\alpha 200$ and GP zones 200 diffraction lines of alloy 3, aged at $T_{a}$ for 8 hourss after quenching. 
The alloys 1 and 2 showed rather sharp $\varepsilon^{\prime}$ diffraction lines 100,002 and 101 after 2 days of ageing. As ageing proceeded other $\varepsilon^{\prime}$ diffraction lines appeared not changing their positions. The unit-cell parameters of $\varepsilon^{\prime}$ are given in table 2 ; it was found that $a(\varepsilon)>a\left(\varepsilon^{\prime}\right)>d_{\alpha 110}, c(\varepsilon)$ $>c\left(\varepsilon^{\prime}\right)$. For alloy $3, \varepsilon^{\prime}$ diffraction lines 100 and 200 were rather sharp, but those with $l \neq 0$ consisted of a sharp component and a broad one. That effect was attributed to $\varepsilon^{\prime}$ precipitates of two different sizes with similar unit-cell parameters given in table 2. If followed: $c(\varepsilon)>$ $c\left(\varepsilon^{\prime}\right) ; a(\varepsilon) \approx a\left(\varepsilon^{\prime}\right) \approx d_{\alpha, 110}$, this meaning a partial coherence between the $\alpha$ and $\varepsilon^{\prime}$-phases. Diffraction lines of $\varepsilon^{\prime}$ in alloy 3 did not move as ageing proceeded. The alloys 4 and 5 showed rather sharp $\varepsilon^{\prime}$ diffraction lines at an initial ageing. After a prolonged ageing, $\varepsilon^{\prime}$ diffraction lines consisted of a sharp component and a broad one, that effect being more pronounced for alloy 5 than for alloy 4 . For the broad component $d_{\varepsilon^{\prime} 100} \approx d_{\varepsilon 100}$; for the sharp one $d_{\varepsilon^{\prime} 100} \approx \sqrt{3} / 2 d_{\alpha 100}$. The sharp component moved to the broad one on ageing, the latter did not move, but sharpened. After a prolonged ageing, $a(\varepsilon) \approx a\left(\varepsilon^{\prime}\right)<d_{0110}, c(\varepsilon)>c\left(\varepsilon^{\prime}\right)$ (table 2 ). The sharp component of $\varepsilon^{\prime}$ diffraction lines for alloys 3,4 and 5 corresponded to the discontinuously nucleated $\varepsilon^{\prime}$, while the broad component resulted from a direct transition of GP zones into $\varepsilon^{\prime}$. After ageing at $620 \mathrm{~K}$ and slow cooling to RT, these alloys showed only sharp diffraction lines of precipitates, close to those of $\varepsilon$.

\section{In situ XRD study of quenched and aged alloys at high temperature}

As mentioned above, the microstructure of the quenched and aged (at $T_{\mathrm{a}}$ ) alloys was different from that of the slowly-cooled alloys. Crystal lattice of the $\alpha$-phase was strained around the $\varepsilon^{\prime}$ platelets, that had very variable sizes. Diffraction patterns of the alloys that had been prolongedly stored at RT, remained rather unchanged, except for small sharpening of diffraction lines. In experiments with these alloys at high temperature a special attention was paid to alloys 3,4 , and 5. Parts of XRD patterns of the alloy 4 at selected temperatures are shown in figure 3. As the temperature of the alloys increased above RT, a slight sharpening of diffraction lines of both $\alpha$ - and $\varepsilon^{\prime}$-phases was observed due to strain annealing. At the same time, a gradual shift of diffraction lines took place due to thermal expansion. Thermal expansion coefficient of $\varepsilon^{\prime}$ was slightly smaller along the $a$-axis $\square a$ ) $=23(3) \tilde{x}_{1}^{\square} \square$ than along the $c$-axis $\square c)=26(3) \times 10^{-6} / \mathrm{K}$, measured in the interval from RT to $500 \mathrm{~K}$. Thermal expansion of the $\alpha$-phase was similar to that of $\varepsilon^{\prime}$ along the $a$-axis. Above $\approx 500 \mathrm{~K}$ a gradual dissolution of $\varepsilon^{\prime}$ in the $\alpha$-phase took place, manifesting in an enhanced decrease of $\varepsilon^{\prime}$ diffraction intensities. At the same time, the shift of $\varepsilon^{\prime}$ diffraction lines toward smaller Bragg angles was accelerated. The solution of $\mathrm{Zn}$, from the $\varepsilon^{\prime}$ precipitates, in the $\alpha$-phase compensated or even reversed the shift of its diffraction lines due to thermal expansion. The solid-solution temperature, $T_{\mathrm{ss}}$, was estimated from the extrapolation of $\varepsilon^{\prime}$ diffraction intensities to zero. $T_{\mathrm{ss}}$ depended on the alloy composition, amounting $\approx 720,700$ and $680 \mathrm{~K}$ for alloys 3,4 and 5 , resp. The dissolution of $\varepsilon^{\prime}$ was followed by crystal grain coarsening of the $\alpha$-phase.

In the cooling run, the alloys exhibited a temperature hysteresis in reversal changes. The precipitation started at a temperature lower for 10 to $20 \mathrm{~K}$ than that at which precipitates dissolved on heating. The precipitation of $\varepsilon^{\prime}$ on cooling caused fragmentation of $\alpha$-phase grains. The dependence of the unit-cell parameters of $\varepsilon^{\prime}$ on temperature during cooling was 


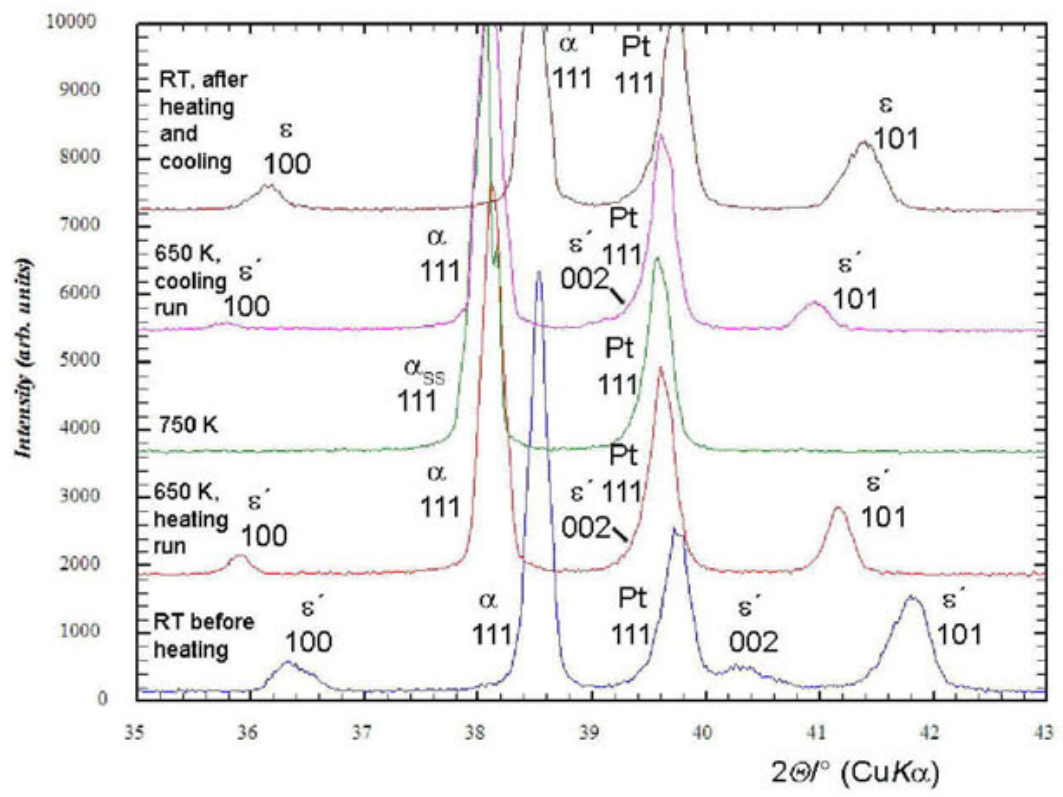

Figure 3. Characteristic parts of XRD patterns of the alloy 4 at selected temperatures.

little different from that during heating. After a completion of the heating and cooling cycle, the unit-cell parameters of the precipitates were very close to those of the equilibrium phase, $\varepsilon$. Diffraction lines of the $\alpha$-phase were slightly shifted toward smaller Bragg angles, in comparison to their position before heating; that may mean a smaller fraction of $\mathrm{Zn}$ remained solved in the $\alpha$-phase. Diffraction lines of precipitates formed in alloys 3, 4 and 5, after a completion of the heating and cooling cycle, did not show composite profiles any more.

\section{References}

1. Löffler, H., 1995, Structure and Structure Development in Al-Zn Alloys (Berlin: Akademie Verlag).

2. Popović, S. \& Gržeta, B., 1999, Croatica Chemica Acta, 72, 621.

3. Skoko, Ž. \& Popovié, S., 2006, Fizika A, 15, 61.

4. Bates, S. R. \& Gould, W. R., 1971, Advances in X-ray Analysis, 14, 146.

5. Kähkönen, H. A., 1971, J. Appl. Cryst., 4, 396.

6. Popović, S. \& Passoja, D.E., 1971, J. Appl. Cryst., 4, 427.

7. Passoja, D.E., Popović, S. \& Barrand, P., 1974, Met. Trans., 5, 715.

8. Lyon, O, Hoyt, J.J., Pro, R., Davis, B.E.C., Clark, B., de Fontaine, D. \& Simon, J.P.,1985, J. Appl. Cryst., 18, 480.

9. Popovié, S., 1985, Cryst. Res. Technol., 20, 552.

10. Popović, S., Passoja, D.E. \& Barrand, P., 1970, Fizika, 2, 231. 


\title{
Synchrotron investigations of non- uniformly shaped shot-peened samples
}

\author{
A. M. Venter ${ }^{1, *}$, C. P. la Grange ${ }^{2}$, F. Hofmann ${ }^{3}$, T-S. Jun ${ }^{3}$, \\ J. Belnoue ${ }^{3}$, P. R. van Heerden ${ }^{1}$, A. Evans ${ }^{4}$, \\ A. M. Korsunsky ${ }^{3}$
}

${ }^{1}$ Necsa Limited, PO Box 582, Pretoria 0001, South Africa

${ }^{2}$ Denel Land Systems, South Africa

${ }^{3}$ Oxford University, Department of Engineering Science, United Kingdom

${ }^{4}$ Previously from the ESRF, France, now with PSI, Switzerland

*andrew.venter@necsa.co.za

Keywords: X-ray synchrotron diffraction, residual strain, shot peening

\begin{abstract}
We report results from X-ray synchrotron residual strain investigations of conically shaped 17-4PH stainless steel samples treated to different shot-peen intensities. The residual strains are compared to the as-manufactured condition as well as a sample coldwater quenched. The magnitudes of the induced residual strains in the shot-peened samples are in relation to the peen intensities. Investigations on slices cut from the bulk samples indicate a underlying material thickness dependence.
\end{abstract}

\section{Introduction}

Shot peening is an important surface impact treatment widely used in industry to improve the performance of metallic parts subjected to fatigue loading, contact (fretting), stress corrosion and other damage mechanisms by inducing beneficial compressive residual stresses in the surface region [1-3]. Residual stresses induced by shot peening are not limited to the material surface regions, but may extend to depths of up to $1 \mathrm{~mm}$ into steel, depending on the shotpeening intensity and the material properties. The conditioning process is quantified using the ALMEN calibration method where a flat reference plate is simultaneously shot blasted in parallel to the sample being treated [4]. This verification procedure however does not take into account differing material geometries, surface properties and residual stresses existing in practical specimens due to their manufacture. Direct verification of the extent of the beneficial compressive residual stresses induced through the shot-peening process has become essential due to the occurrence of premature fatigue failures through cracking of such geometries in practical use. It is suspected that the shot peening does not occur homogeneously in samples of varying thickness and shape. We report results from an X-ray synchrotron radiation investigation performed at the ID31 beam line of the European Synchrotron Radiation Facility to elucidate the residual strains in conically shaped samples and sectioned slices. 


\section{Experimental}

Samples of this study are conically shaped as shown in figure 1. The samples are manufactured from 17-4PH, a precipitation hardened stainless steel, with chemical composition given in table 1. Over and above its corrosion resistance it has desirable qualities, such as its inability to decarburise, making it conducive to fatigue fracture resistance, its ease of heat treatment and ability to transform to a carbon-lean martensitic structure when air cooled. Furthermore, it can be machined to near net-shape dimensions before age-hardening which can assist in reducing residual stress due to machining.
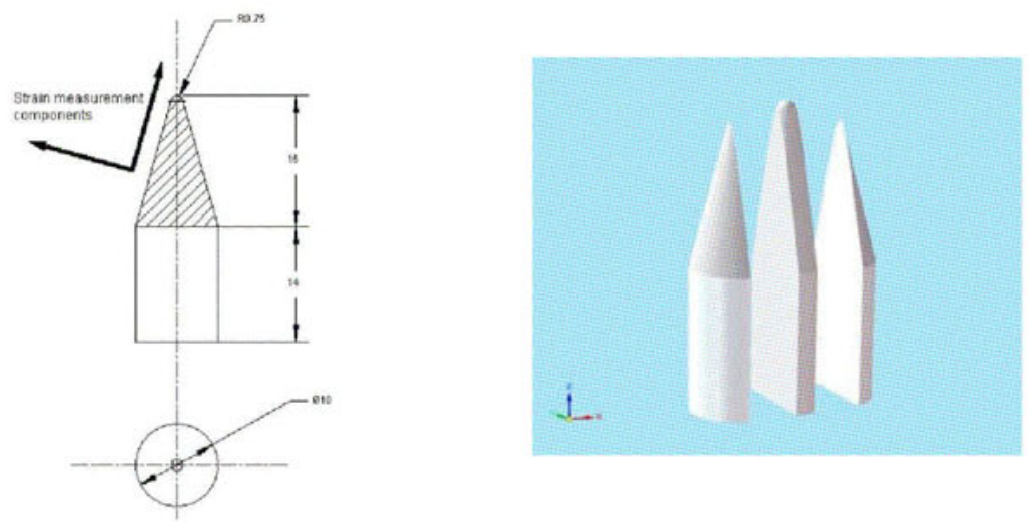

Figure 1. Schematic showing the conical sample shapes and dimensions. The figure on the right shows the positions where the slices were extracted by wire EDM.

The material [5] of this study was produced by air-melt and electroslag refinement (remelting), followed by forging to size. The manufacturer controls the presence of delta ferrite to values typically between 3 to 5 percent by controlling the chromium-to-nickel ratio. The material of this study had no observable delta ferrite. After machining the samples were treated at $1040 \pm 15^{\circ} \mathrm{C}$ in a salt bath from where they were air cooled to below $30^{\circ} \mathrm{C}$, followed by aging at $480^{\circ} \mathrm{C}$ for one hour from where they were air cooled, except for one sample that was cold-water quenched. The latter option was explored as there are indications that steels quenched from elevated temperatures without undergoing phase changes can have residual stresses induced [6]. All samples were de-scaled chemically. All of these processes should minimise residual stress contributions. The samples were then shot-peen treated in accordance to the ALMEN procedure [5] to intensities $3 \mathrm{~A}, 6 \mathrm{~A}$ and $16 \mathrm{~A}$ : Reference to respective resultant ALMEN A strip (0.051 \pm 0.001 inch thickness) curvatures $0.003,0.006$ and 0.016 inches, each approximating being the optimum intensities for material thicknesses of 2 , 5 and $10 \mathrm{~mm}$. The conical sample shapes of this study thus allows for the direct verification of this due to its underlying diameters. The sample identification and shot peen treatment is summarised in table 2. The microstructure of sample $\mathrm{C} 0$ was analysed to be a low-carbon precipitation hardened martensite with a hardness of 46 Rockwell ' $C$ ' with limited nonmetallic inclusion content. Its micrograph is shown in figure 2. 
Table 1. Chemical composition of 17-4PH (precipitation hardened) stainless steel in weight \%.

\begin{tabular}{|l|c|c|c|c|c|c|c|c|}
\hline $\begin{array}{l}\text { Chemical } \\
\text { composition }\end{array}$ & $\mathrm{Cr}$ & $\mathrm{Ni}$ & $\mathrm{Cu}$ & $\mathrm{C}$ & $\mathrm{Mn}$ & $\mathrm{P}$ & $\mathrm{S}$ & $\mathrm{Si}$ \\
\hline Typical & $15-17.5$ & $3-5$ & $3-5$ & $\begin{array}{c}0.07 \\
\max \end{array}$ & $\begin{array}{c}1.00 \\
\max \end{array}$ & $\begin{array}{c}0.04 \\
\max \end{array}$ & $\begin{array}{l}0.03 \\
\max \end{array}$ & $1.0 \max$ \\
\hline Sample C0 & 16.01 & 4.74 & 3.84 & 0.058 & 0.62 & 0.033 & 0.006 & 0.36 \\
\hline
\end{tabular}

Table 2. Description of conical samples investigated in this study.

\begin{tabular}{|c|c|}
\hline Sample designation & Sample treatment \\
\hline C0 & As manufactured \\
\hline $\mathrm{C} 1$ & Cold water quenched \\
\hline $\mathrm{CP} 1$ & Shot peen treated at intensity 3A \\
\hline $\mathrm{CP} 2$ & Shot peen treated at intensity 6A \\
\hline $\mathrm{CP} 3$ & Shot peen treated at intensity $16 \mathrm{~A}$ \\
\hline
\end{tabular}

High spatial resolution residual strain investigations focused within the first $2 \mathrm{~mm}$ from the surface were done non-destructively down to $50 \mu \mathrm{m}(25 \mu \mathrm{m}$ probe volume displacements). As the ID31 instrument is equipped with an analyser crystal the occurrence of pseudo-strain aberrations, normally associated with partially filled gauge volumes such as when entering surfaces or due to sample geometries having strong curvature (the conical samples of this study), are negated. Investigations primarily focussed on slices $2 \mathrm{~mm}$ in thickness, as shown in figure 1, wire EDM cut from the bulk samples. This approach was adopted with the initiating investigations to ensure access to large radial depths as it was perceived that it would not be possible in the bulk samples due to the still limited penetration depths of high energy Xrays into steel. Measurements on the slices were done in transmission geometry that facilitated constant sample volume illumination throughout except the near surface regions.

Residual strain investigations from the (211) Bragg reflection were observed at $\sim 10^{\circ}$ scattering angle with the $0.206 \AA \mathrm{X}$-ray wavelength. As a compromise between available time and investigation points envisaged, measurement times of $20 \mathrm{~s}$ per measurement position rendered strain accuracies within $50 \mu$ strain in conjunction with a gauge volume of $50 \mu \mathrm{m} \times 0.5 \mathrm{~mm} \times$ $50 \mu \mathrm{m}^{3}$. The $50 \mu \mathrm{m}$ dimension was always set parallel to the sample surface. The strain components in-plane (parallel to the shot-peened surface) and normal (perpendicular to the shotpeened surface) were measured. Line scans focussed on the strain dependences close to the surface to depths of $4 \mathrm{~mm}$ along three measurement lines respectively 3,8 and $13 \mathrm{~mm}$ from the sample tip as shown in figure 3 . 


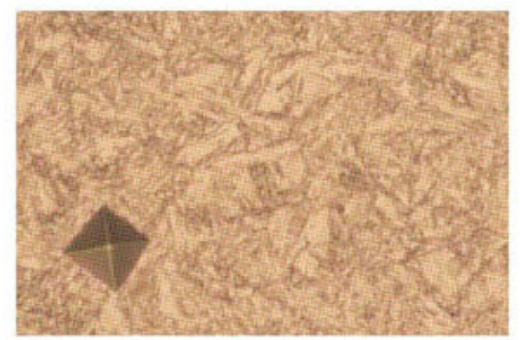

Figure 2. Micrograph of sample CO (The Vickers micro-hardness indentation serves as magnification

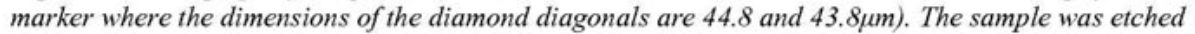
with Kalling's reagent $\left(5 \mathrm{~g} \mathrm{CuCl}_{2}, 100 \mathrm{ml} \mathrm{HCl}\right.$ and $100 \mathrm{ml}$ ethanol).
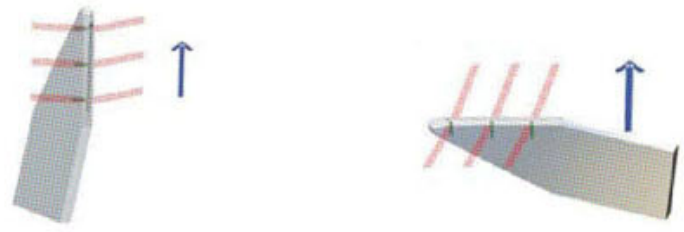

Figure 3. Sample orientations and locations of strain measurements (dotted lines) respectively along lines 3,8 and $13 \mathrm{~mm}$ from the sample tips. The red "rays" represent the X-ray beam trajections with the blue arrows indicating the strain component measured. in-plane (left) and normal (right).

\section{Results and discussion}

Within the experimental error, minimal residual strains are observed in the reference and cold-water quenched samples, with no obvious surface aberration contributions resulting from the partially submerged probe volumes in the near surface region.

Shot-peened bulk samples:

- The in-plane components of strains are compressive with the maximum strains at the surface where after they fall off with depth to zero after approximately $1 \mathrm{~mm}$.

- The normal components of strains are tensile with the maximum strains at the surface where after they fall off with depth to zero after approximately $1 \mathrm{~mm}$.

- The magnitudes of the surface strains increase with the shot peen intensity. In sample CP3, peened to intensity $16 \mathrm{~A}$, some surface strain relaxation is evident as well as the extent of the plastic region extending to at least $2 \mathrm{~mm}$ in depth. 


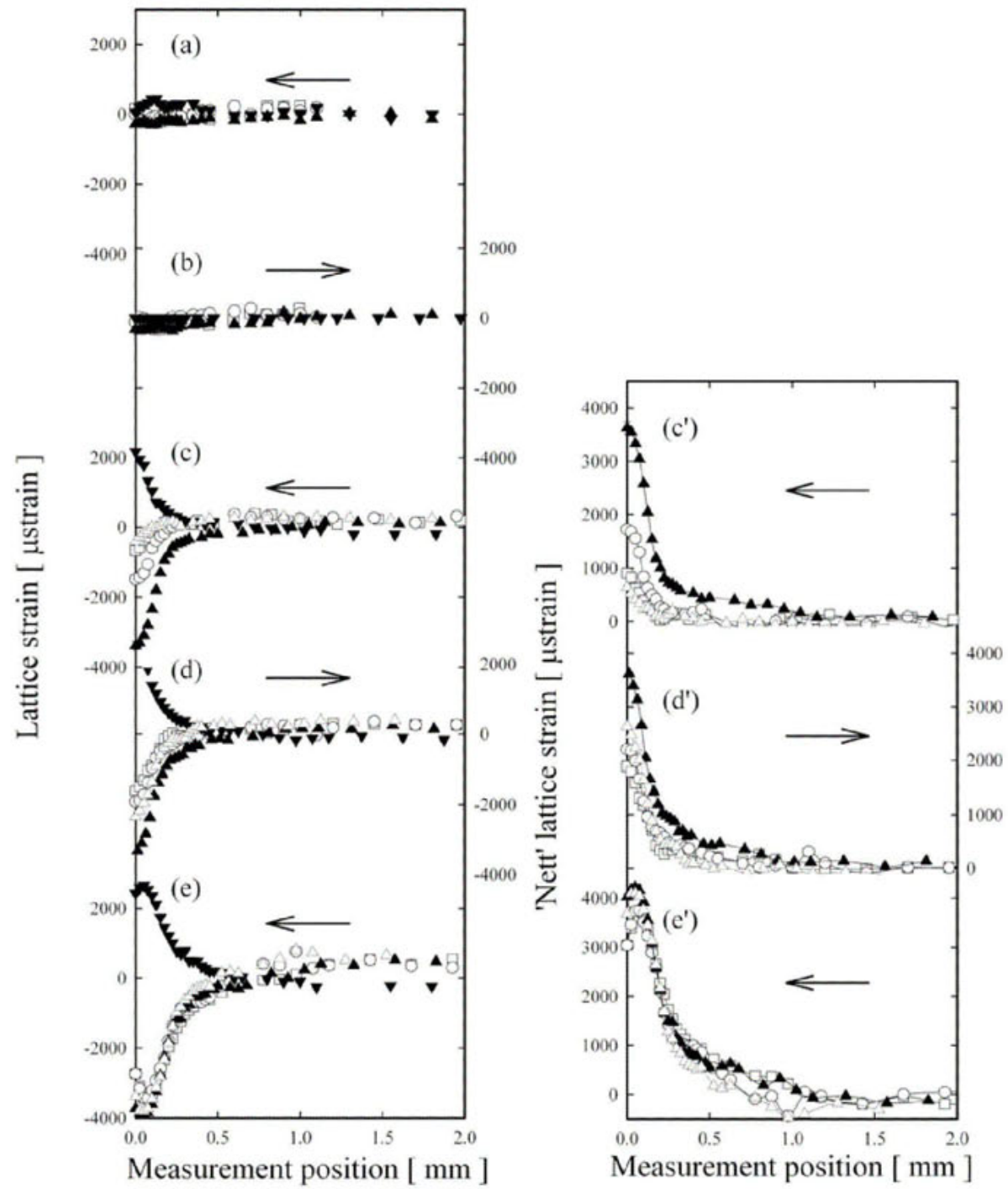

Figure 4. Residual strain behaviours observed in the conical samples along measurement lines shown in figure 3. Results depict: (a) As-manufactured reference C0; (b) Cold water-quenched sample C1; (c) Shot-peen intensity 3A, CP1; (d) Shot-peen intensity 6A, CP2; (e) Shot-peen intensity 16A, CP3. In each curve the open symbols represent the in-plane strain component measured in the slices along lines respectively $3 \mathrm{~mm}(4), 8 \mathrm{~mm}$ () and $13 \mathrm{~mm}$ from the tip (O), with the filled symbols representing results from the solid samples obtained from measurement lines $3 \mathrm{~mm}$ from the tip: ( $\mathbf{\Delta})$ in-plane strain component and ( $\boldsymbol{\nabla}$ ) normal strain component. The curves on the right show the 'nett' strains determined from each line measurement by subtraction of the measured profile from its far field linear part extrapolated from $1 \mathrm{~mm}$ from the surface to the surface [3]. 
The investigations as function of underlying material thickness were only performed in the slices. These results though indicate substantial strain relaxation between the measurements at different lines from the tips in the slices as compared to the solid samples:

- A dependence of the extent of relaxation on underlying material thickness is evident in sample treated to intensity $3 \mathrm{~A}$. The strain relaxation is most severe at $3 \mathrm{~mm}$ from the tip (underlying material thickness approximately $2 \mathrm{~mm}$ ).

- Strain relaxation is prevalent in sample peened to intensity $6 \mathrm{~A}$, but does not have such a large variation with underlying material thickness as in the case of $3 \mathrm{~A}$.

- No obvious strain relaxation is observed in sample peened to intensity $16 \mathrm{~A}$ except very close to the surface.

Sectioning of the conical samples in order to produce slices involves redistribution of stresses and the corresponding elastic strains within the sample. The transition from axisymmetric to thin section sample causes more severe redistribution in the regions where the original sample diameter is smaller, i.e. closer to the cone tip. The extraction of the 'nett' strains also demonstrates however that in the samples peened to intensities $3 \mathrm{~A}$ and $6 \mathrm{~A}$ some relaxation of plastic strains also take place during sectioning. The origin of this phenomenon requires careful modelling of the stress state within the sample and will be addressed in a separate publication.

\section{References}

1. James, M.N., Hughes, D.J., Chen, Z., Lombard, H., Hattingh, D.G., Asquith, D., Yates, J.R. \& Webster, P.J., 2007, Engineering Failure Analysis, 14, (2), 384.

2. King, A., Steuwer, A., Woodward, C., \& Withers, P.J., Mat. Sci. and Eng. A, 2006, 435436, 12 .

3. Zhang, S.Y., Venter, A.M., Vorster, W.J.J., \& Korsunsky, A.M., 2008, J. Strain Anal. Eng. Design, 43, 229.

4. Wood, W.G., 1982, Metals Handbook $9^{\text {th }}$ Edition, Volume 5, Surface Cleaning. Finishing, and Coating, $2^{\text {nd }}$ printing 1987 (Metals Park, Ohio 44073:American Society for Metals), p. 138.

5. Private communication, Special Alloys and Metallurgical Services.

6. Dieter, G.E., 2000, Engineering Design - A Materials and Processing Approach, $3^{\text {rd }}$ edition (Mc Graw-Hill International Editions, Mechanical Engineering Series), p. 445.

Acknowledgements. The National Research Foundation of South Africa under grant GUN 65435 and the European Union access to large facilities are acknowledged for mobility and subsistence support, as well as the scientific committee of the ESRF for awarding beam time under project award MA449. 
IV.4 Minerals and Inorganics

IV.4.1 Structural Changes, In-situ and NonAmbient Investigations 



\title{
Structures and properties of variously doped Mayenite investigated by neutron and synchrotron powder diffraction
}

\author{
H. Boysen ${ }^{1, *}$, I. Kaiser-Bischoff ${ }^{1}$, M. Lerch ${ }^{2}$, S. Berendts ${ }^{2}$,

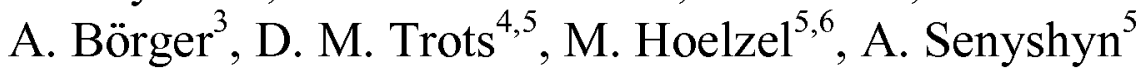

${ }^{1}$ LMU, Department f. Geo. und Umweltwissenschaften, Sektion Kristallographie, Theresienstr. 41, 80333 München, Germany

2 TU Berlin, Institut für Chemie, Straße des 17. Juni 135, 10623 Berlin, Germany

${ }^{3}$ TU Braunschweig, Institut für Physikalische und Theoretische Chemie, Hans-SommerStraße 10, 38106 Braunschweig, Germany

${ }^{4}$ HASYLAB, Notkestrasse 85, 22607 Hamburg, Germany

5 TU Darmstadt, Institute of Materials Science, Petersenstr. 23, 64287 Darmstadt, Germany

${ }^{6}$ TUM, Forschungsneutronenquelle FRM-II, 85747 Garching, Germany

*boysen@Imu.de

Keywords: mayenite, disorder, diffusion, anharmonic refinement, Fourier methods

\begin{abstract}
Mayenite $\left(\mathrm{Ca}_{12} \mathrm{Al}_{14} \mathrm{O}_{33}\right)$ is a promising new ionic conductor. This is related to its particular crystal structure, consisting of a calcium-aluminate framework, comprising 32 of the 33 oxygen anions. The remaining "free" oxygen is distributed over 1/6 of large cages in the framework and may diffuse through large openings between adjacent cages. The structure is heavily disordered involving displacements of Ca cations and the presence of extra anions like $\mathrm{O}_{2}^{-}, \mathrm{O}_{2}{ }^{2-}, \mathrm{O}^{-}$and $\mathrm{OH}^{-}$, which are released at high temperatures under vacuum conditions. The "free" oxygen has been substituted by nitrogen, opening up possibilities for a pure nitrogen ionic conductor. Material properties can be modified by doping on the cation sites. Detailed structure determinations with respect to disorder and ionic diffusion were carried out by neutron and X-ray synchrotron powder diffraction. At high temperatures diffusion of oxygen proceeds via a jump-like process involving exchange of "free" oxygen with framework oxygen, coupled to relaxations of the $\mathrm{Ca}$ ions. In contrast, nitrogen diffuses as $\mathrm{NH}_{2}^{-}$via an interstitial process. Fe doping leads to samples free of extra anions, larger disorder and promises higher ionic conductivities at high temperatures.
\end{abstract}

\section{Introduction}

Mayenite, of nominal composition $\mathrm{Ca}_{12} \mathrm{Al}_{14} \mathrm{O}_{33}$, known as a major component of calcium aluminate cements, has recently attracted renewed attention for modern technological applications, e.g. as transparent conductive oxide, as catalyst for the combustion of volatile organic compounds or as oxygen ionic conductor. These properties can be related to its particu- 
lar crystal structure (space group $I \overline{4} 3 d, a=11.98 \AA$ ) $[1,2]$ consisting of a calciumaluminate framework comprising 32 of the 33 oxygen anions. The remaining "free" oxygen is distributed over $1 / 6$ of large cages in the framework and is thought to diffuse through large openings between adjacent cages. The true structure is heavily disordered $[3,4]$ involving displacements of $\mathrm{Ca}$ cations and the presence of extra anion species $\mathrm{O}_{2}^{-}, \mathrm{O}_{2}{ }^{2-}, \mathrm{O}^{-}$and $\mathrm{OH}^{-}$. The extra-framework oxygen can be exchanged by other anions opening up possibilities for new anionic conductors. Material properties can further be modified by doping on the cation sites. Here we present detailed structure determinations related to the disorder and diffusion properties as a function of composition and temperature by high temperature neutron and $\mathrm{X}$ ray powder diffraction experiments. The results demonstrate that rather fine structural details, as revealed by anharmonic contributions to the Debye-Waller factor and by difference Fourier methods, can reliably be deduced from the high quality powder data nowadays available at modern neutron and synchrotron sources. Information about the amount of hydrogen in the samples can be drawn from the incoherent scattering contribution to the background.

\section{Experimental}

Pure oxygen mayenite samples were synthesized via a solid-state route. Nitrogen doped samples with $0.55,1.00$ and $1.27 \mathrm{wt}-\% \mathrm{~N}$ were obtained from these by ammonolysis, iron doped samples with 0.1 and $2.5 \mathrm{~mol}-\% \mathrm{Fe}$ via a sol-gel route. Neutron measurements were carried out at the powder diffractometer SPODI at the FRM2 (Garching) using a wavelength of $\lambda=1.548 \AA$ and $\mathrm{a} \mathrm{Nb}$ vacuum resistance furnace up to $1050^{\circ} \mathrm{C}$. Synchrotron $\mathrm{X}$-ray measurements were done at instrument B2 at HASYLAB (Hamburg) with $\lambda=0.49324 \AA$ and a graphite furnace up to $900{ }^{\circ} \mathrm{C}$. The data were analysed by the Rietveld method including anharmonic Debye-Waller factors and by difference Fourier methods with JANA2000 [5]. All samples contained small amounts of side phases whose weight fractions were determined from the refinements and are summarized in table 1.

Table 1. Phase analysis of samples.

\begin{tabular}{|c|l|l|l|}
\hline $\mathrm{O}$ (pure) & $4.98(11) \% \mathrm{Ca}_{3} \mathrm{Al}_{2} \mathrm{O}_{6}$ & $\mathrm{Fe}(2.5)$ & $5.47(10) \% \mathrm{CaAl}_{2} \mathrm{O}_{4}$ \\
\hline $\mathrm{O} / \mathrm{N}(0.55)$ & $5.71(14) \% \mathrm{Ca}_{3} \mathrm{Al}_{2} \mathrm{O}_{6}$ & $\mathrm{~N}(1.27)$ & $6.28(10) \% \mathrm{CaAl}_{2} \mathrm{O}_{4}$ \\
\hline
\end{tabular}

\section{Results}

\section{O-Mayenite}

The results for pure O-mayenite have already been published in [3] and are briefly summarized here for comparison. The as synthesized sample shows an excess of oxygen with composition $\mathrm{Ca}_{12} \mathrm{Al}_{14} \mathrm{O}_{33.5}$, which is explained by the presence of extra species $\mathrm{O}_{2}^{-}, \mathrm{O}_{2}^{2-}, \mathrm{O}^{-}$ and/or $\mathrm{OH}^{-}$. Two of these, peroxide and hydroxide groups, could be identified by difference Fourier synthesis as shown in figure 1(left). This assignment, i.e. ignoring other minima and maxima, is validated by "correct" $\mathrm{O}-\mathrm{O}$ and $\mathrm{O}-\mathrm{H}$ distances, respectively. Note that the $\mathrm{O}_{2}{ }^{2-}$ anion is oriented along the larger extension of the cage, while $\mathrm{OH}^{-}$point towards the neighbouring $\mathrm{Ca}$. The sample becomes stoichiometric at high temperatures under the vacuum conditions of the used furnace. The loss of $\mathrm{H}$ is evidenced by a reduction of the incoherent background (see below). Above ca. $900^{\circ} \mathrm{C}$ atomic displacement parameters (ADP's) become extremely large $\left(U_{\text {iso }}\right.$ around $0.3 \AA^{2}$ ) indicating the delocalization and diffusion of the free oxygen. Difference Fourier maps (calculated with empty cage) show two maxima located 
off-centre of the cage (figure 1, right). The additional maxima represent split Ca positions, also located off the $\overline{4}$ axis running vertically through the cage. It is then straight forward to assume that $\mathrm{Ca}$ atoms are shifted away from their normal positions when the cage is occupied by an $\mathrm{O}$ ion. This means that $\mathrm{Ca}-\mathrm{O}$ bonds are formed instantaneously (the distances indicated by the arrows in figure 1 (right) agree with the normal Ca-O bond distances of 2.4 $\AA$ ), i.e. the "free" oxygen is not "loosely bound" as anticipated in the early literature and the diffusion has to be considered as a coupled Ca-O process. Since no continuous density could be found between adjacent cages and from further indications of transiently occupied metastable positions it can be concluded, that oxygen diffuses via an exchange with framework oxygen, i.e. an interstitialcy process. All these findings are in excellent agreement with theoretical ab initio calculations [6].
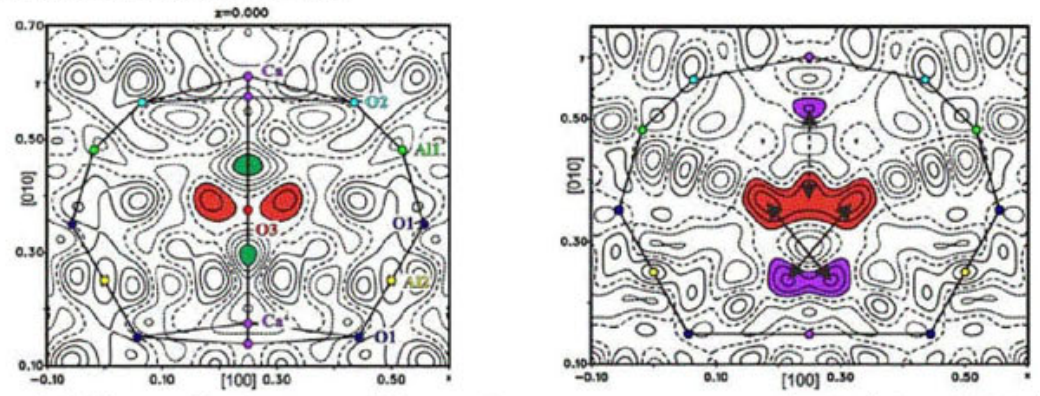

Figure 1. Difference Fourier maps of $O$-mayenite at room temperature after inclusion of $O$ in the centre of the cage and split Ca position (left) and at $1050^{\circ} \mathrm{C}$ after refinement with empty cage (right).

\section{O/N-Mayenite}

The formal replacement of $1 \mathrm{O}^{2-}$ by $2 / 3 \mathrm{~N}^{3--}$ leads to a composition $\mathrm{Ca}_{12} \mathrm{Al}_{14} \mathrm{O}_{32} \mathrm{~N}_{0.67}$, i.e. $1 / 9$ of cage positions should be occupied. In the first sample $(0.55 \mathrm{wt} . \% \mathrm{~N})$ only part of the oxygen was substituted by nitrogen. Again there is over-occupancy of the cage position, probably due to additional species $\mathrm{NH}^{2-}$ and/or $\mathrm{NH}_{2}{ }^{\circ}$, but only a slight decrease with temperature. A concomitant decrease of the background indicates the loss of some but not all hydrogen. The multitude of different species did not allow a clear assignment. However, in contrast to Omayenite, a continuous density distribution between adjacent cages was found from anharmonic refinements at high temperatures. This is interpreted as an interstitial diffusion process of $\mathrm{N}$, probably in the form of $\mathrm{NH}_{2}$-groups (see below). Further details can be found in [7].

\section{N-Mayenite}

The sample with $1.00 \mathrm{wt} . \% \mathrm{~N}$ was measured only at room temperature. Difference Fourier maps revealed residual positive and negative densities (figure 2). The highlighted ones may be assigned to $\mathrm{N}$ and $\mathrm{H}$, respectively. Refinement resulted in a ratio 1:2 of the occupancies and "correct" $\mathrm{N}-\mathrm{H}$ distances. Therefore part of the nitrogen is present as $\mathrm{NH}_{2}$-groups, supporting the assumption of interstitially diffusing amide anions in $\mathrm{O} / \mathrm{N}$-mayenite (see above). The sample with 1.27 wt.\% $\mathrm{N}$ also shows an over-occupancy, which decreases to stoichiometric values above $700{ }^{\circ} \mathrm{C}$. The almost complete loss of hydrogen is again confirmed by a reduction of the incoherent background. Very large $U_{\text {iso }}$ 's are again found at high temperatures (e.g. $0.25 \AA^{2}$ at $900{ }^{\circ} \mathrm{C}$ ), but, unfortunately, no clear conclusions about the un- 
derlying diffusion properties of the remaining $\mathrm{N}^{3-}$-ions could be drawn so far from neither anharmonic refinements nor difference Fourier analyses.
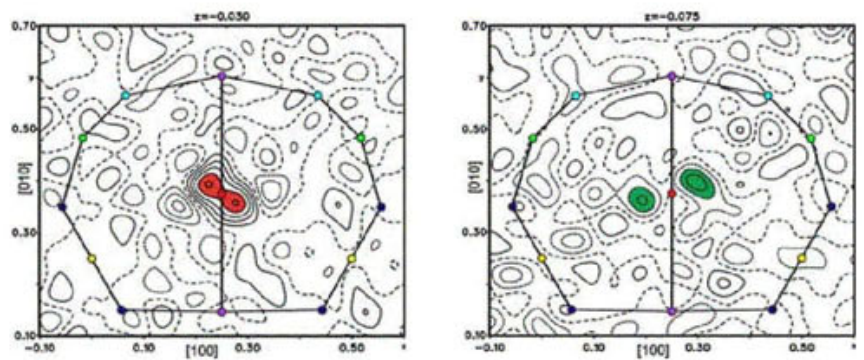

Figure 2. Difference Fourier maps of $\mathrm{N}$-mayenite at room temperature at two sections through minima and maxima of highlighted residual peaks (after inclusion of nominal amount of $N$ in the cage).

\section{Fe-Mayenite}

The sol-gel synthesized sample doped with $2.5 \mathrm{~mol}-\% \mathrm{Fe}$ (which replaces $\mathrm{Al}$ ) shows a stoichiometric composition, more or less (see below) independent of temperature, i.e. it contains no appreciable amounts of additional species. A very low content of hydrogen is confirmed by the much lower background as compared to the other samples (see figure 4 below). The $\mathrm{O}$ density is considerably smeared out already at room temperature (figure 3 , left) and not really detectable above $900{ }^{\circ} \mathrm{C}$ (figure 3 , right), confirmed by an unrealistically large $U_{\text {iso }}$ of about $0.5 \AA^{2}$ (which also implies large uncertainties on the refined occupancies). This complete smearing, i.e. the extremely low local densities prohibited so far to determine reliable diffusion path ways. The sample with $0.1 \mathrm{~mol} . \% \mathrm{Fe}$ still contained hydrogen and behaved similar to pure O-mayenite. This means that there must be a certain threshold above which $\mathrm{Fe}$ inhibits the inclusion of unwanted extra anions.
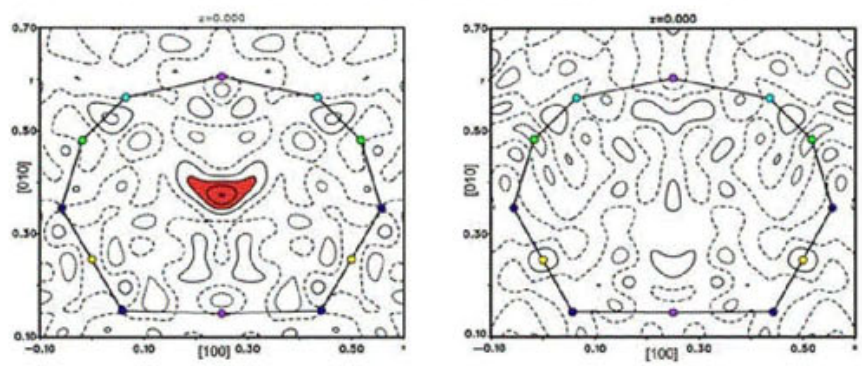

Figure 3. Difference Fourier maps of Fe-mayenite at room temperature (left) and at $1050^{\circ} \mathrm{C}$ (right), after refinement with empty cage.

\section{Estimation of hydrogen content}

The strong incoherent scattering cross section of protons leads to a large background in neutron powder diagrams. By proper scaling with the scale factor of the Bragg intensities it is possible to determine at least approximately the amount of hydrogen in the sample. However, this requires to consider carefully other sources of background such as thermal diffuse scattering, multiple scattering, etc. This has been done before for the simple case of glassy carbon [8] and will be developed further for more demanding cases like this. Here we use only some qualitative arguments to estimate the gain and loss of hydrogen.

Figure 4 shows a comparison of three samples. Apart from the clearly lower hydrogen content of the Fe doped sample (see above) one may notice the different form of the low angle 
part between $\mathrm{O}$ - and $\mathrm{N}$-mayenite. This might be related to the different bond states of hydroxide and imide/amide, respectively, but this has to be explored further more carefully.

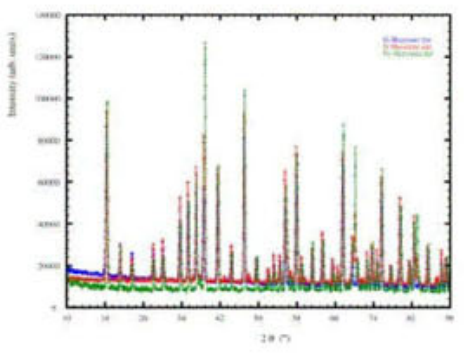

Figure 4. Comparison of neutron powder diagrams of $O$ (blue)-, $N$ (red)- and Fe (green)- mayenite at room temperature. The data were normalized to the first (mayenite) peak. Larger intensity differences are due to texture effects of the $\mathrm{Nb}$ peaks arising from the furnace.

\section{Thermal expansion}

The thermal expansion of four samples has been followed by synchrotron measurements at instrument B2, whose OBI image plate allows rapid data collection. The results of heating/cooling cycles are shown in figure 5. The lattice constants of all samples show "normal" linear increase up to about $400^{\circ} \mathrm{C}$. At higher temperatures expansion increases more strongly. For O-mayenite this is due to the loss of extra anion species in agreement with previous reports that both $\mathrm{OH}^{-}$and active oxygen species $\left(\mathrm{O}_{2}{ }^{-}, \mathrm{O}^{-}\right)$, contract the unit cell when they are clathrated into the mayenite cages. Then, as expected, after cooling to room temperature the lattice constant of O-mayenite is larger than initially. At ambient conditions those of the Ndoped samples increase with nitrogen content and Fe-mayenite also has a larger lattice constant. These findings can simply be explained by larger ionic radii. However, in contrast to $\mathrm{O}$-mayenite, thermal expansion in $\mathrm{N}$-mayenite samples is retarded between $800-900^{\circ} \mathrm{C}$, due to loss of nitrogen, which is not recovered and consequently leads to smaller lattice constants at room temperature after cooling. In $\mathrm{O} / \mathrm{N}$-mayenite the smaller losses of extra anions lead to an almost reversible behaviour. That of Fe-mayenite is similar, but less pronounced, as in $\mathrm{N}$ mayenite, which might be due a possible change of the Fe oxidation state.
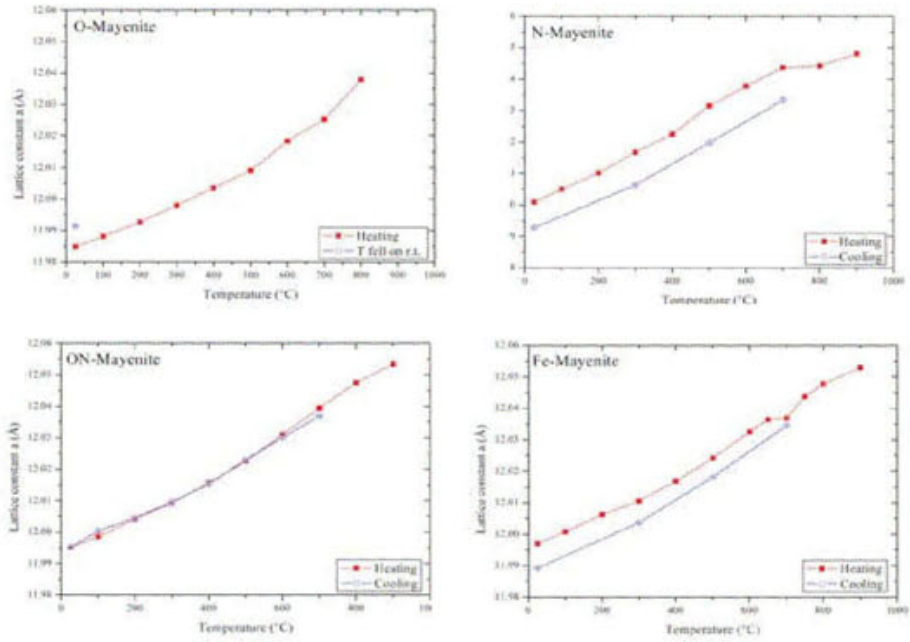

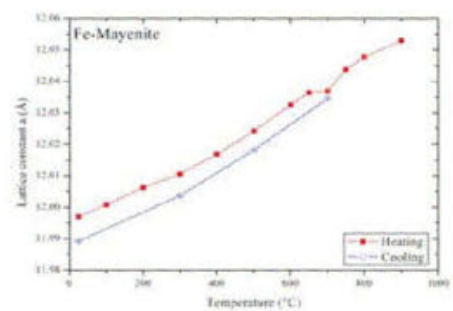

Figure 5.

Temperature dependence of lattice constants of $\mathrm{O}-\mathrm{O} / \mathrm{N}-, \mathrm{N}-$ and $\mathrm{Fe}$ mayenite during heating and cooling (only one point for $\mathrm{O}$ mayenite due to a failure of the furnace). 


\section{Discussion and conclusions}

O-Mayenite synthesized via a solid-state route contains extra anions, of these $\mathrm{OH}^{-}$and $\mathrm{O}_{2}{ }^{-}$ could be substantiated from neutron powder diffraction data. The excess anions are lost at high temperatures under vacuum conditions. This change is irreversible as evidenced by larger lattice constant after heating/cooling. Oxygen diffusion proceeds via a jump-like process involving exchange of "free" oxygen with framework oxygen, coupled to relaxations of the $\mathrm{Ca}$ ions. Test refinements proved that no nitrogen is incorporated into the framework of $\mathrm{N}$ doped samples. Possible excess anions are imide and/or amide, $\mathrm{NH}_{2}^{-}$was substantiated. Again they are lost at high temperatures and the process is irreversible under vacuum conditions. Nitrogen diffuses (probably) as $\mathrm{NH}_{2}^{-}$via an interstitial process. This may be related to the larger radius and lower valence if compared with $\mathrm{O}^{2-}$ exhibiting interstitialcy behaviour. The situation for pure $\mathrm{N}^{3-}$ is not clear at the moment. Of technical importance is the observation that the incorporation of extra anions is suppressed by doping with iron. Fe-mayenite shows large disorder already at room temperature and extreme delocalisation at high temperatures. This points towards very high anionic conductivity (to be verified).

The results presented in this paper demonstrate the power of neutron powder diffraction for the elucidation of disorder and diffusion effects in crystals. However, it has to be admitted that very subtle details, like e.g. an unambiguous detection of intermediate metastable positions during the diffusion processes or of the very low local densities at high temperature, requires even higher quality single crystal data. It should also be noted that attempts to disentangle the various species by combined neutron and synchrotron refinements did not lead to unambiguous results, because they were very sensitive to the oxidation state used for the different ions in the X-ray case. Hence, extreme care should be taken when trying to deduce occupancies from $\mathrm{X}$-ray data.

\section{References}

1. Büssem, W. \& Eitel, A., 1936, Z. Kristallogr., 95, 175.

2. Bartl, H. \& Scheller, T., 1970, N. Jb. Miner. Mh., 35, 547.

3. Boysen, H., Lerch, M., Stys, A. \& Senyshyn, A., 2007, Acta Cryst. B, 63, 675.

4. Palacios, L., De La Torres, A., Bruque, S., Garcia-Munoz, J., Garcia-Granda, S. Sheptyakov, D. \& Aranda, M.A.G., 2007, Inorganic Chemistry, 46, 4167.

5. Petricek, V., Dusek M. \& Palatinus, L., 2000, Jana2000, the crystallographic computing system, Institute of Physics, Praha, Czech Republic.

6. Sushko, P.V., Shluger, A.L., Hayashi, K., Hirano, M. \& Hosono, H., 2006, Phys. Rev. B, 73, 014101-1-10.

7. Boysen, H., Kaiser-Bischoff, I. \& Lerch, M., 2008, Diffusion Fundam., 8, 2.1.

8. Boysen, H., 1985, J. Appl. Cryst.,18, 320.

Acknowledgements. This work was supported by the DFG within the priority program SPP 1136 under BO 1199/2 and LE 781/10. The diffractometer SPODI is operated in the frame of BMBF grant no. 03F47DAR. We thank Anne Kristin Paland and Prof. K.D. Becker (Braunschweig) for their help to prepare the Fe doped samples. 


\title{
In situ XRD investigation of $\mathrm{Co}_{3} \mathrm{O}_{4}$ reduction
}

\author{
O. A. Bulavchenko ${ }^{1, *}$, S. V. Cherepanova ${ }^{1,2}$, \\ S. V. Tsybulya ${ }^{1,2}$
}

${ }^{1}$ Boreskov Institute of Catalysis, pr. akad. Lavrentieva 5, Novosibirsk 630090, Russian Federation

2 Novosibirsk State University, Pirogova 2, Novosibirsk 630090, Russian Federation isizy@catalysis.ru

Keywords: in situ XRD, staking faults, reduction, cobalt oxide, alumina, Fischer-Tropsch catalysts

Abstract. In situ X-ray diffraction (XRD) method was used to investigate the $\mathrm{CO}_{3} \mathrm{O}_{4}$ reduction in catalyst supported on $\gamma-\mathrm{Al}_{2} \mathrm{O}_{3}$ and oxide monophase sample. Initial structure of $\mathrm{Co}_{3} \mathrm{O}_{4}$ in both samples is similar and contains vacancies in the cation positions. In spite of this reduction process of $\mathrm{CO}_{3} \mathrm{O}_{4}$ is different for supported and monophase samples as has been shown by in situ XRD. In monophase sample the cobalt oxide is reduced to metallic cobalt (hep) directly. On the contrary the two-stage reduction is observed for supported sample: $\mathrm{Co}_{3} \mathrm{O}_{4}$ is reduced to $\mathrm{CoO}$ and then to metallic $\mathrm{Co}$ (fcc). In both cases nanoparticles of metallic cobalt contains high concentration of stacking faults.

\section{Introduction}

The Fischer-Tropsch synthesis (FTS) is one of the processes producing synthetic liquid fuel and valued chemical compounds from syngas (i.e. $\mathrm{CO}$ and $\mathrm{H}_{2}$ ) [1]. The supported metallic catalysts are widely used in FTS. Cobalt based catalysts are suited to produce high yields of long alkanes in FTS. Catalysts are activated by reduction of $\mathrm{Co}_{3} \mathrm{O}_{4}$ in hydrogen. It is important to understand how the reduction conditions influence on the structural parameters of catalyst.

Investigation of phase composition and structure of reduced catalyst by ex situ methods is difficult because nanoparticles of metallic cobalt are oxidized in the air. Information concerning the phase composition and the crystallite size during reduction was obtained by in situ X-ray powder diffraction experiments.

We have researched supported and monophase nanocrystalline $\mathrm{Co}_{3} \mathrm{O}_{4}$ samples to compare its behavior during reduction. 


\section{Experimental}

\section{Preparation}

Monophase sample was prepared by calcination of cobalt carbonate at $300^{\circ} \mathrm{C}$ for $4 \mathrm{~h}$.. Supported catalyst was prepared by the incipient wetness impregnation of $\gamma-\mathrm{Al}_{2} \mathrm{O}_{3}$ by cobalt nitrate aqueous solution with further calcination at $250^{\circ} \mathrm{C}$ for $4 \mathrm{~h}$.

\section{$\mathrm{X}$-ray diffraction}

The X-ray diffraction experiments were carried out on the Siemens D-500 diffractometer with $\mathrm{Cu} \mathrm{K}_{\alpha}$ radiation. In situ XRD experiments were performed using the reactor chamber [2] developed in Boreskov Institute of Catalysis. Reagent gas was pure hydrogen at the pressure of $1 \mathrm{bar}$ and the flow of $600 \mathrm{~cm}^{3} / \mathrm{min}$. The samples were heated at the rate of $25^{\circ} / \mathrm{min}$. Diffraction patterns were recorded by scanning in the range $2 \theta=15-70^{\circ}$ at the rate of $0.6^{\circ} \mathrm{min}$. XRD measurements were carried out at the room temperature in air and at the temperature little lower than temperature of beginning of $\mathrm{Co}_{3} \mathrm{O}_{4}$ reduction (detected by $\mathrm{XRD}$ ) in the hydrogen atmosphere.

Structural refinement was carried out with use of software [3]. Unit cell and structure (atom coordinates, occupancies, temperature factors) parameters were refined using peaks positions and areas correspondingly.

Average sizes of coherently scattering domains (CSD) were calculated with use of Scherrer equation.

Simulation of the diffraction patterns of the disordered metallic cobalt was carried out with use of software [4,5]. The X-ray diffraction patterns are calculated on the basis of statistical model of one-dimensional (1D) disordered crystal.

\section{Results and discussion}

\section{Initial structure of $\mathrm{Co}_{3} \mathrm{O}_{4}$}

$\mathrm{CO}_{3} \mathrm{O}_{4}$ has spinel-type structure (space group $\mathrm{Fd} 3 \mathrm{~m}$, crystal chemical formula $\mathrm{Co}^{2+} \mathrm{Co}_{2}{ }^{3+} \mathrm{O}_{4}$, where $\mathrm{Co}^{2+}$ ions occupy the tetrahedral positions $8 \mathrm{a}(1 / 8,1 / 8,1 / 8), \mathrm{Co}^{3+}$ ones are in the octahedral positions $16 \mathrm{~d}(1 / 2,1 / 2,1 / 2))$.

Results of the refinement of the initial $\mathrm{Co}_{3} \mathrm{O}_{4}$ structure are represented in the Table. These data show that in the both samples $\mathrm{Co}_{3} \mathrm{O}_{4}$ has similar structure namely it contain considerable quantity of vacancies both in tetrahedral and octahedral positions (the occupation of the positions is less than 1). A lack of cations in the samples is observed that means that the condition of a charge neutrality formally is not met. Presumably, compensation of the excess negative charge occurs via partial transition $\mathrm{Co}^{2+}$ in $\mathrm{Co}^{3+}$ and presence at samples residual carbonate and nitrate groups. The calculated average size of CSD is about $140 \AA$ for both samples

\section{Reduction of $\mathrm{Co}_{3} \mathrm{O}_{4}$}

For the determination of the minimal temperature of reduction the X-ray diffraction in situ has been carried out. For monophase sample the minimal temperature of $\mathrm{Co}_{3} \mathrm{O}_{4}$ reduction is about of $190^{\circ} \mathrm{C}$. The temperature was fixed when metallic cobalt (hep) peaks appeared (CoO peaks were not detected). For supported sample the temperature of the $\mathrm{CO}_{3} \mathrm{O}_{4}$ reduction be- 
Table 1. The crystalline structure of $\mathrm{Co}_{3} \mathrm{O}_{4}$ under various conditions.

\begin{tabular}{|l|l|l|l|l|}
\hline \multirow{2}{*}{ Sample } & \multicolumn{2}{|l|}{$\mathrm{Co}_{3} \mathrm{O}_{4}$} & \multicolumn{2}{l|}{$\mathrm{Co}_{3} \mathrm{O}_{4} / \gamma \mathrm{Al}_{2} \mathrm{O}_{3}$} \\
\cline { 2 - 5 } & $\begin{array}{l}\text { Initial struc- } \\
\text { ture }\end{array}$ & $\begin{array}{l}\text { Structure } \\
\text { before reduc- } \\
\text { tion }\end{array}$ & $\begin{array}{l}\text { Initial struc- } \\
\text { ture }\end{array}$ & $\begin{array}{l}\text { Structure } \\
\text { before reduc- } \\
\text { tion }\end{array}$ \\
\hline $\begin{array}{l}\text { Filling the tetrahe- } \\
\text { dral position }\end{array}$ & 0.77 & 0.79 & 0.80 & 0.89 \\
\hline $\begin{array}{l}\text { Filling the octahedral } \\
\text { position }\end{array}$ & 0.81 & 0.84 & 0.72 & 0.91 \\
\hline $\begin{array}{l}\text { Filling non-spinel } \\
\text { octahedral position }\end{array}$ & 0 & 0 & 0 & 0.08 \\
\hline
\end{tabular}

ginning is about of $180^{\circ} \mathrm{C}$. It was revealed on appearance of $\mathrm{CoO}$ peaks. In this case metallic cobalt (fcc) reflexes appeared only at the further increase in temperature up to $260^{\circ} \mathrm{C}$.

A series of the experiments for the refinement of $\mathrm{Co}_{3} \mathrm{O}_{4}$ structure before the reduction beginning has been spent. For this purpose at the temperature lower on $10^{\circ}$ than the minimal temperature of reduction the X-ray diffraction patterns in the angle interval $2 \theta=30-50^{\circ}$ were recorded until there are no changes in the intensities i.e. until structure becomes stable at this temperature. Only after that recording XRD patterns was carried out in the angle range $2 \theta=15-70^{\circ}$.

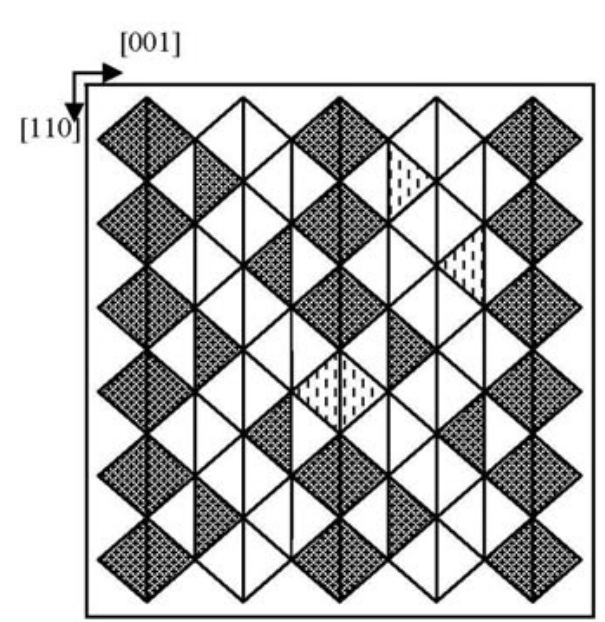

a

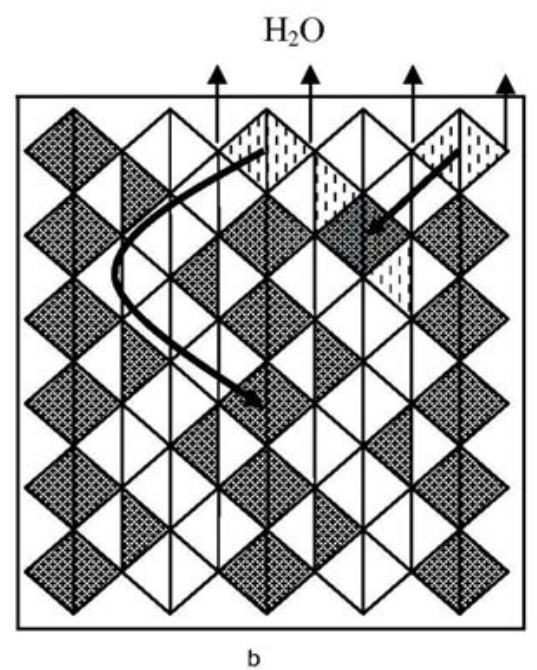

b

Figure 1. a) Initial structure: vacancies in octahedral and tetrahedral positions. b) Structure before complete reduction: the ions migrate to the vacancies and non-spinel positions. 
Structure refinement has shown that in both cases $\mathrm{Co}_{3} \mathrm{O}_{4}$ is also defective before reduction. The increase in occupation of the octahedral and tetrahedral positions is observed. Also occupation of the additional «non-spinel» positions $16 \mathrm{c}(0,0,0)$ (table) is detected.

It is possible to guess the structural mechanism of $\mathrm{Co}_{3} \mathrm{O}_{4}$ reduction (figure 1): 1) excess oxygen ions leave $\mathrm{Co}_{3} \mathrm{O}_{4}$ under the influence of hydrogen and temperature, 2) cations fill vacancies and additional «non-spinel» octahedral positions using channels. Presence of cobalt ions in «non-spinel» positions means filling of two adjacent octahedrons jointed by edges, i.e. $\mathrm{CoO}$ structure fragments appear in the $\mathrm{Co}_{3} \mathrm{O}_{4}$ structure both in monophase and supported samples. Nevertheless, in monophase sample appearance of crystal $\mathrm{CoO}$ phase is not observed and $\mathrm{Co}_{3} \mathrm{O}_{4}$ is reduced directly to metallic cobalt (hcp).

\section{Structure of cobalt particles forming during reduction from $\mathrm{Co}_{3} \mathrm{O}_{4}$}

Metallic cobalt with hep and fec structures is formed as a result of reduction of the model and supported samples correspondingly. Particles of metallic cobalt of hep and fec structures have high concentration of stacking faults (SF) i.e. disordering of layer sequence $\mathrm{AB}$ $\mathrm{ABAB} \ldots$ and $\mathrm{ABCABC} \ldots$, respectively. It is observed as the anisotropic peak broadening on the X-ray diffraction patterns (figure $2 \mathrm{f}, 3 \mathrm{f}$ ).

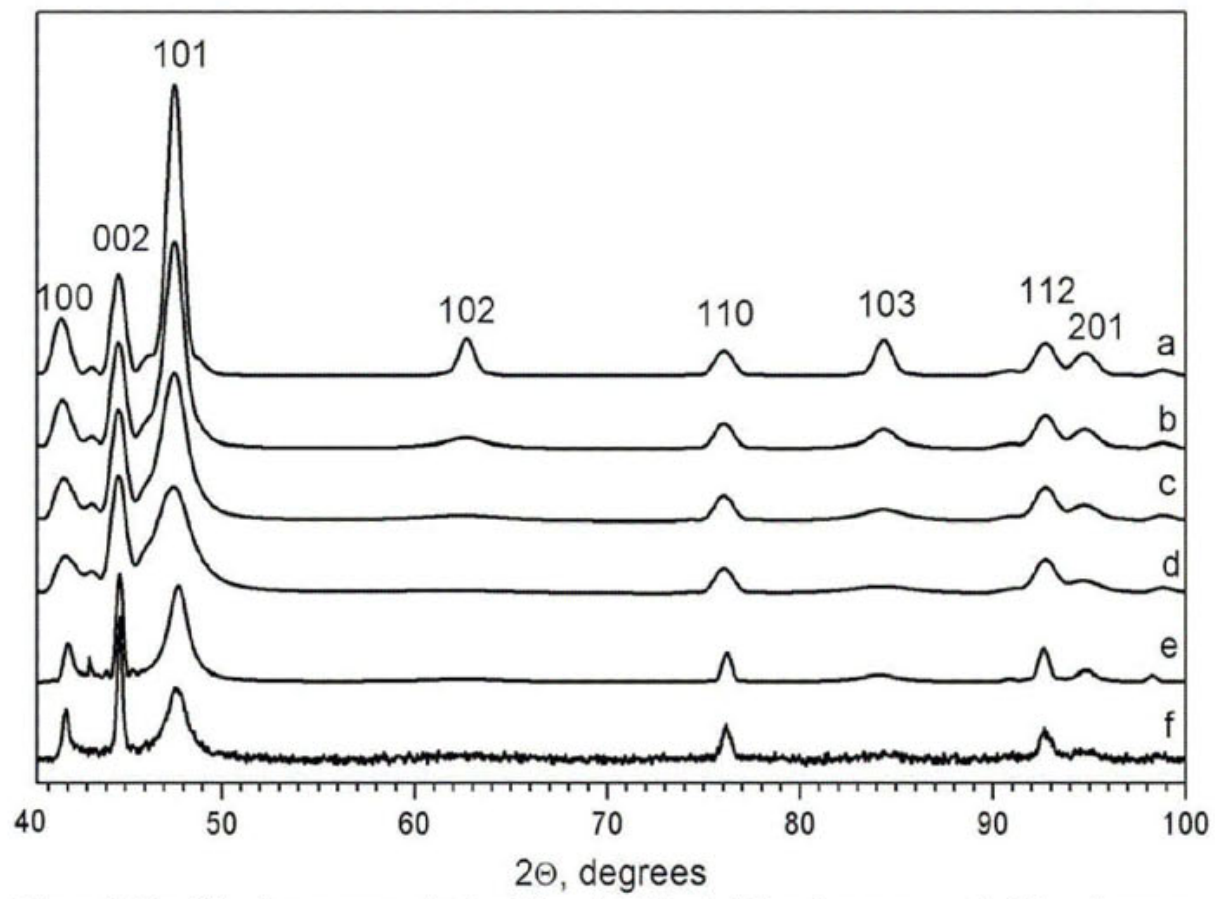

Figure 2. The diffraction patterns simulated for cobalt (hcp) with various average size(D) and concentration of staking faults $(\alpha) \cdot a) D=100 \AA, \alpha=0, b) D=100 \AA, \alpha=0.1, c) D=100 \AA, \alpha=0.2, d) D=$ $100 \AA, \alpha=0.3, e) D=200 \AA, \alpha=0.2, f)$ experimental data. 


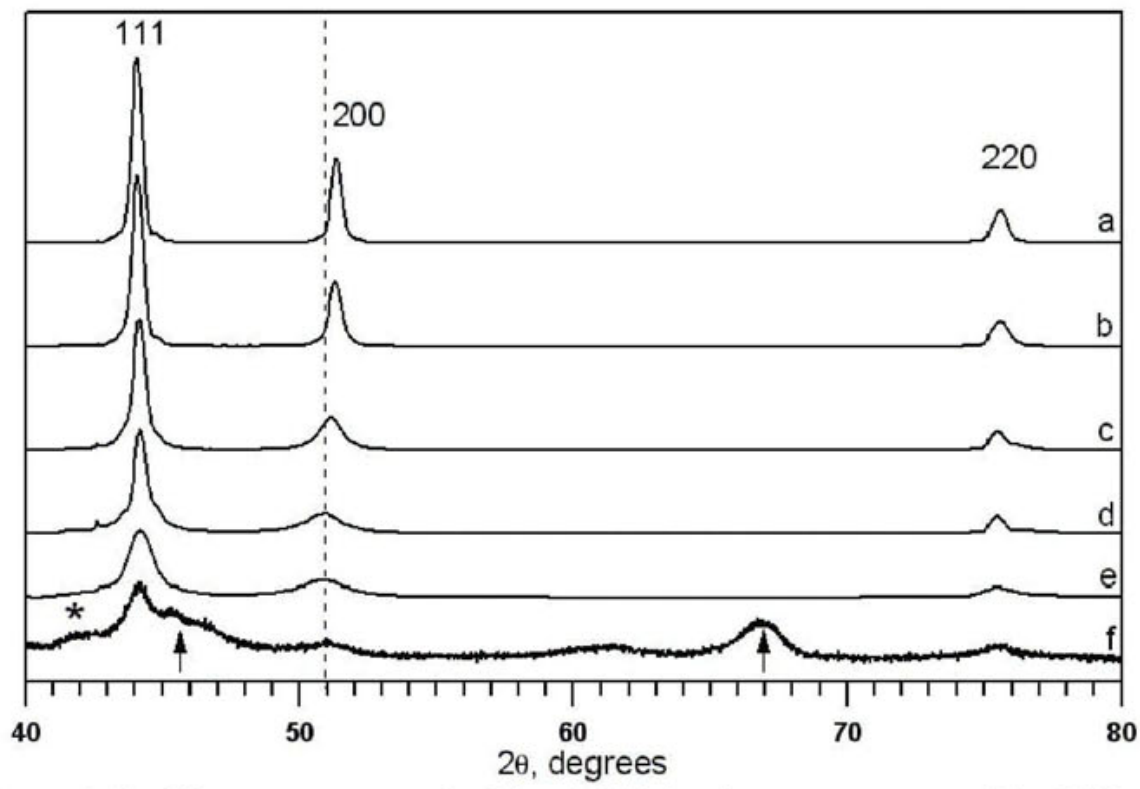

Figure 3. The diffraction patterns simulated for cobalt ( $f c c)$ with various average size (D) and different concentration of staking faults $(\alpha)$. a) $D=200 \AA, \alpha=0, b) D=200, \AA \alpha=0.01, c) D=200 \AA, \alpha=0.05$, d) $D=200 \AA, \alpha=0.1$, e) $D=100 \AA, \alpha=0.1, f$ experimental data, where arrows point at peaks of $\gamma$ $\mathrm{Al}_{2} \mathrm{O}_{3}$, asterisk-peak of $\mathrm{CoO}$.

We simulated XRD patterns for metallic cobalt (hep and fec) with various concentration of $\mathrm{SF}$ (figure 2,3). One can see that increase in concentration of SF in hep structure remain $002,110,112$ and 004 peaks without changes. At the same time 100,101, 102, 103 and 201 peaks become wider. Moreover, 100 peak become less wider in comparison with 102 and 103 peaks. Simulation of the XRD patterns has shown that the experimental diffraction pattern approximately corresponds to the hep structure with SF concentration of about $20 \%$.

For fcc structure increase in SF concentration leads to broadening of 111, 200 peaks and to shifting of 200 towards to smaller angles. Beside of metallic cobalt the supported sample contains $\mathrm{CoO}$ and $\gamma-\mathrm{Al}_{2} \mathrm{O}_{3}$. There are several overlapped peaks in the region of 111 peak of metallic cobalt. So the main criterion for determination of SF concentration was position of shifted 200 peak. Simulation has shown that metallic cobalt in supported sample has fcc structure with SF concentration of about $10 \%$.

\section{Conclusion}

Investigation of $\mathrm{Co}_{3} \mathrm{O}_{4}$ reduction in monophase and supported samples by in situ X-ray diffraction has been carried out. Initial structures of both samples are defective. They contain vacancies in octahedral and tetrahedral positions. Reduction of $\mathrm{Co}_{3} \mathrm{O}_{4}$ in both cases begins at temperature of about $180-190^{\circ} \mathrm{C}$. In both cases the structural rebuilding before reduction 
consists in decreasing the cationic vacancies and partial filling the octahedral hollows in spinel structure i.e. in formation of $\mathrm{CoO}$ clusters in structure $\mathrm{CO}_{3} \mathrm{O}_{4}$. However further process of $\mathrm{Co}_{3} \mathrm{O}_{4}$ reduction in the monophase and supported samples proceeds through different stages and leads to different products. So in the supported sample the $\mathrm{CO}_{3} \mathrm{O}_{4}$ reduction takes place in two steps: the first step - $\mathrm{Co}_{3} \mathrm{O}_{4}$ is reduced to $\mathrm{CoO}$, the second step - $\mathrm{CoO}$ is reduced to metallic cobalt (fcc). For monophase sample $\mathrm{Co}_{3} \mathrm{O}_{4}$ is reduced to metallic $\mathrm{Co}$ (hcp) in one stage. Nanoparticles of metallic cobalt in both cases have very high concentration of staking faults. The simulation of the X-ray diffraction patterns has shown that $\mathrm{Co}(\mathrm{hcp})$ and $\mathrm{Co}(\mathrm{fcc})$ structures contains staking faults with concentration of about 0.2 and 0.1 correspondingly.

\section{References}

1. Storch, H.H., Golumbic, N. \& Anderson, R.B., 1951, The Fisher-Tropsch and Related Syntheses (New York: Yohn Wiley \& Sons Inc.).

2. Vishnevskii, A.L., Molchanov, V.V., Kriger, T.A. \& Plyasova, L.M., 1994, in International Conference on Powder Diffraction and Crystal Chemistry, June (St. Petersburg), p.206.

3. Tsybulya, S.V., Cherepanova, S.V. \& Soloviyova L.P., 1996, J. Struct. Chem., 34, 332.

4. Tsybulya, S.V., Cherepanova, S.V. \& Kryukova, G.N., 2004, in Diffraction Analysis of the Microstructure of the Materials, edited by E.J. Mittemeijer \& P. Scardi (Berlin: Springer), pp.93-124.

5. Cherepanova S.V. \& Tsybulya, S.V., 2004, in Proceedings of EPDIC 8, edited by Y. Andersson, E.J. Mittemeijer \& U. Welzel (Switzerland: TTP Ldt), pp.87-90.

Acknowledgements. This study was supported by the Russian Foundation of Basic Research, grant No. 08-03-00964a and the project No.17 of the program No. 27 of fundamental researches of presidium of RAS. 


\title{
Thermal instability of the tetragonally distorted structure of copper-iron materials
}

\section{J. Darul}

Faculty of Chemistry, Adam Mickiewicz University, Grunwaldzka 6, PL-60780 Poznań, Poland

jola@amu.edu.pl

Keywords: $\mathrm{CuFe}_{2} \mathrm{O}_{4}$, powder diffraction, structural transition

\begin{abstract}
Polycrystalline $\mathrm{CuFe}_{2} \mathrm{O}_{4}$ samples have been successfully prepared using a simple combustion method starting from citrate-nitrate precursors. All the ferrite samples studied were prepared at the same temperatures $\left(300,600,900^{\circ} \mathrm{C}\right)$, but one series was slowly cooled from $900^{\circ} \mathrm{C}$ to room temperature, and another was quenched in solid $\mathrm{CO}_{2}$. The nature of the structural transition in inverse spinels has been investigated by the synchrotron XRD technique. Slowly cooled samples are subjected to a diffusionless order-disorder transformation, due to the modified of the orientation of the Jahn-Teller distortions, and to the migration of $\mathrm{Cu}^{2+}$ ions from octahedral to tetrahedral sites This phenomenon transforms the tetragonally distorted spinel structure $\left(F 4_{1} / d d m\right)$ into a cubic lattice $(F d \overline{3} m)$. It occurs at temperatures from $\sim 360^{\circ} \mathrm{C}$ to $\sim 420^{\circ} \mathrm{C}$.
\end{abstract}

\section{Introduction}

Copper ferrite is known to exist in tetragonal and cubic structure. Since $\mathrm{Cu}^{2+}$ is a Jahn-Teller ion (JT), it gives the anomalous favorable properties and also exhibits phase transition from tetragonal to cubic, depending on the temperature. The JT transition is argued to be orderdisorder in character, but its exact nature is still not fully understood [1-4]. $\mathrm{CuFe}_{2} \mathrm{O}_{4}$ can be described as a cubic close-packed arrangement of oxygen ions, with $\mathrm{Cu}^{2+}$ and $\mathrm{Fe}^{3+}$ ions at two different crystallographic sites. From a structural standpoint, the formula unit of stoichiometric ferrite can be written as: $\left(\mathrm{Cu}_{\mathrm{x}}{ }^{2+} \mathrm{Fe}_{1-\mathrm{x}}{ }^{3+}\right)_{\mathrm{A}}\left[\mathrm{Cu}_{1-\mathrm{x}}{ }^{2+} \mathrm{Fe}_{1+\mathrm{x}}{ }^{3+}\right]_{\mathrm{B}} \mathrm{O}_{4}$, where (A) denotes tetrahedral sites, [B] represents octahedral sites [2-3]. The parameter of inversion, $x$, is equal to 0 for inversion spinels, and to 1 , when the spinel is normal. The temperature of the orderdisorder transformation depend on the content of octahedral cupric ions and on the nonstoichiometry [5]. When the spinel is synthesized using classical ceramic technologies (sufficiently high temperature treatment of the initial oxides of the metal cations) with strict stoichiometry, it has a tetragonal structure of hausmannite type with crystal cell parameters $a$ $=8.20 \AA$ and $c=8.60 \AA ; c / a \sim 1.05$. The $c / a$ ratio can be changed via decreasing the copper concentration, or alternatively by heat treatment temperature and cooling rate [2]. Part of the 
$\mathrm{Cu}^{2+}$ ions can be frozen in tetrahedral sites when the ferrites are quenched in air from above $400^{\circ} \mathrm{C}$. The resulting ferrites show a smaller tetragonal distortion since a great proportion of the cupric ions are located on tetrahedral sites. Moreover, quenching treatments in air from temperature higher than $750^{\circ} \mathrm{C}$ lead to the formation of oxygen-deficient spinel ferrites, the nonstoichiometry arising from the reduction of some $\mathrm{Cu}^{2+}$ ions to $\mathrm{Cu}^{+}$ions $[2,6-7]$. Some authors [2-3] consider that the $\mathrm{Cu}^{+}$ions are located in the intersitial sites (i.e., sites normally not occupied in the spinel structure). Recently, it has been found, that $\mathrm{Cu}$-ferrite films can be stabilized in either tetragonal or cubic phase even at room temperature depending on deposition conditions and post-deposition heat treatment; the samples annealed in air at high temperatures $\left(>800^{\circ} \mathrm{C}\right)$ and slowly cooled to room temperature were tetragonal, while those rapidly quenched in liquid nitrogen were cubic [8-9].

In this work, bulk copper ferrites samples were prepared by a simple combustion method using citrate-nitrate precursors. A citrate process as an alternative synthesis route has been successfully employed to synthesize such materials with improved properties for specific applications, such as magnetic powder for massive storage devices [10].

\section{Experimental details}

Powder samples with nominal composition $\mathrm{CuFe}_{2} \mathrm{O}_{4}$ were prepared by the combustion method. The stoichiometric quantities of starting materials, viz., $\mathrm{Cu}\left(\mathrm{NO}_{3}\right)_{2} \cdot 6 \mathrm{H}_{2} \mathrm{O}$, $\mathrm{Fe}\left(\mathrm{NO}_{3}\right)_{3} \cdot 9 \mathrm{H}_{2} \mathrm{O}$, (Merck) and $\mathrm{C}_{6} \mathrm{H}_{8} \mathrm{O}_{7} \cdot \mathrm{H}_{2} \mathrm{O}$, were dissolved in distilled water. The mixed citrate-nitrate solution was heated at $120^{\circ} \mathrm{C}$, with continuous stirring. After the evaporation of excess of water, a highly viscous gel was obtained. Ultimately, the powder was sintered in air at different temperatures $\left(300,600,900^{\circ} \mathrm{C}\right)$ for $5 \mathrm{~h}$. After heating, the preparations were either cooled slowly (SC) to room temperature or rapidly quenched (QC) in solid $\mathrm{CO}_{2}$. The compounds formation and crystallinity of the materials were identified by XRD patterns, which were recorded on a Bruker D8 Advance diffractometer, with $\mathrm{CuK} \alpha$ radiation. Finally, investigations on the temperature phase transition for product formed as a result of slow cooling of $\mathrm{CuFe}_{2} \mathrm{O}_{4}$ sample were carried out at the synchrotron beamline B2 at HASYLAB (DESY, Hamburg). The diffractometer was equipped with capillary furnace (STOE) and the on-site readable image-plate detector OBI. The polycrystalline samples placed in quartz capillaries of diameter $0.3 \mathrm{~mm}$ were heated and cooled at the temperature range from RT to $500^{\circ} \mathrm{C}$. The wavelength, determined by calibration using a NIST silicon standard, was $0.493421 \AA$. Analysis of the XRD data was undertaken with a full-pattern fitting procedure based on the Rietveld method. Structure refinement was performed using FullProf program [11].

\section{Results and discussion}

The XRD studies show that the slow cooled and quenched in solid $\mathrm{CO}_{2}$ samples displayed different thermal behavior (figure 1). In spite of the fact that a quench cooling process usually leads to the cubic structure of $\mathrm{CuFe}_{2} \mathrm{O}_{4}[2-3,12]$, from structural analysis results based on the powder diffraction data we confirmed that the rapidly quenched sample showed a dominant cubic phase $(76.4 \%)$ with a minor tetragonal component $(23.6 \%)$. The slowly cooled sample crystallizes in the tetragonal system and at room temperature it could be indexed as a single-phase tetragonal spinel with space group $\mathrm{I}_{1} /$ amd. The unit cell with lattice constants 
$a=5.8111(5) \AA$ and $\mathrm{c}=8.6888(9) \AA$ consists of four $\mathrm{CuFe}_{2} \mathrm{O}_{4}$ units. It is, however, much more informative to consider the crystal lattice as a tetragonally distorted spinel structure with the non-standard face-centred space group $F 4_{1} / d d m$. In that case the unit cell is doubled, with $a_{\mathrm{s}}$ $=8.2387(5) \AA$ and $c / a_{s}=1.055$, containing eight $\mathrm{CuFe}_{2} \mathrm{O}_{4}$, and the copper ions occupy the octahedral sites of the spinel lattice, leading to a structural formula close to $\left(\mathrm{Fe}^{3+}\right)_{\mathrm{A}}\left[\mathrm{Cu}^{2+} \mathrm{Fe}^{3+}\right]_{\mathrm{B}} \mathrm{O}_{4}$, corresponding to the equilibrium distortion at room temperature.

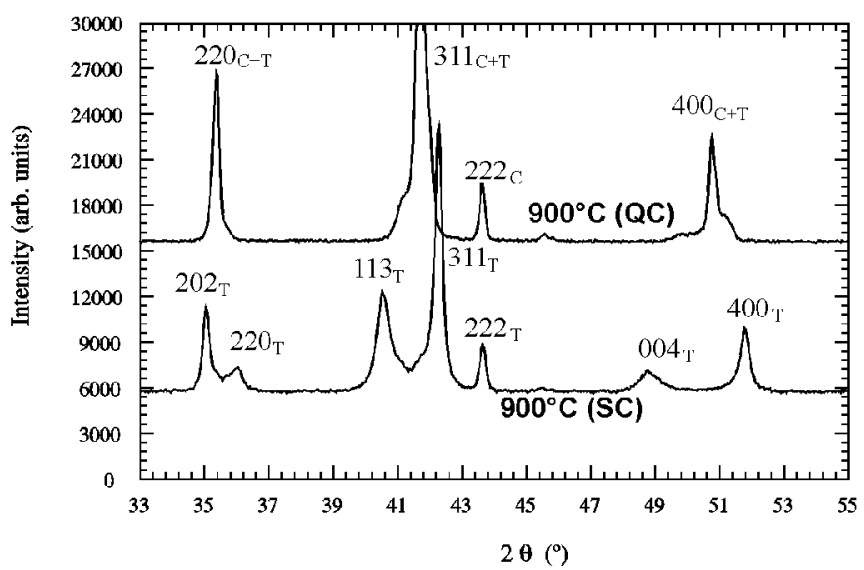

Figure 1. X-ray diffraction patterns of samples $\mathrm{CuFe}_{2} \mathrm{O}_{4}$ obtained by slow cooling (SC) or by rapid quenching in $\mathrm{CO}_{2}(Q C)$, measured at room temperature.

A $\quad$ B

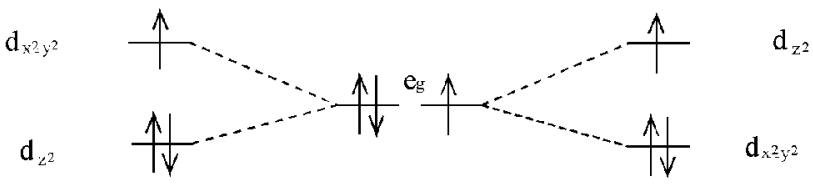

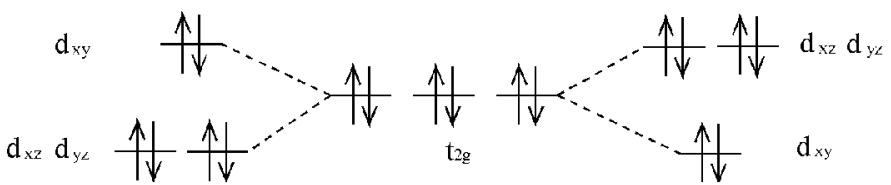

Figure 2. Orbital splitting of the d-energy levels within the cooper ion ( $\left.d^{9}\right)$ diagram showing the two possible Jahn-Teller distortion in an octahedral complex.

The tetragonal distortion in the $\mathrm{CuFe}_{2} \mathrm{O}_{4}$ compounds is attributed to the cooperative distortion that is driven by the octahedrally coordinated $\mathrm{Cu}^{2+}\left(3 \mathrm{~d}^{9}\right)[2,6,13]$. The electron configu- 
ration of $\mathrm{Cu}^{2+}$ located at octahedral sites is $\left(\mathrm{t}_{2 g}^{6}\right) \mathrm{e}_{g}^{3}$. While six electrons with antiparallely aligned spins occupy the triplet, three electrons on the orbital $\mathrm{e}_{\mathrm{g}}$ can be distributed in two ways (double degenerancy) see figure 2 . The first alternative (figure $2 \mathrm{~B}$ ) leads to an axially flattened octahedron $(c / a<1)$; the second one (figure $2 \mathrm{~A}$ ) causes elongating of the octahedron $(c / a>1)$ e.g., $\mathrm{CuFe}_{2} \mathrm{O}_{4}$.

Investigations of the thermal phase transition of the product formed as a result of slow cooling of copper ferrite sample were carried out at the synchrotron beamline B2 at HASYLAB (DESY, Hamburg).

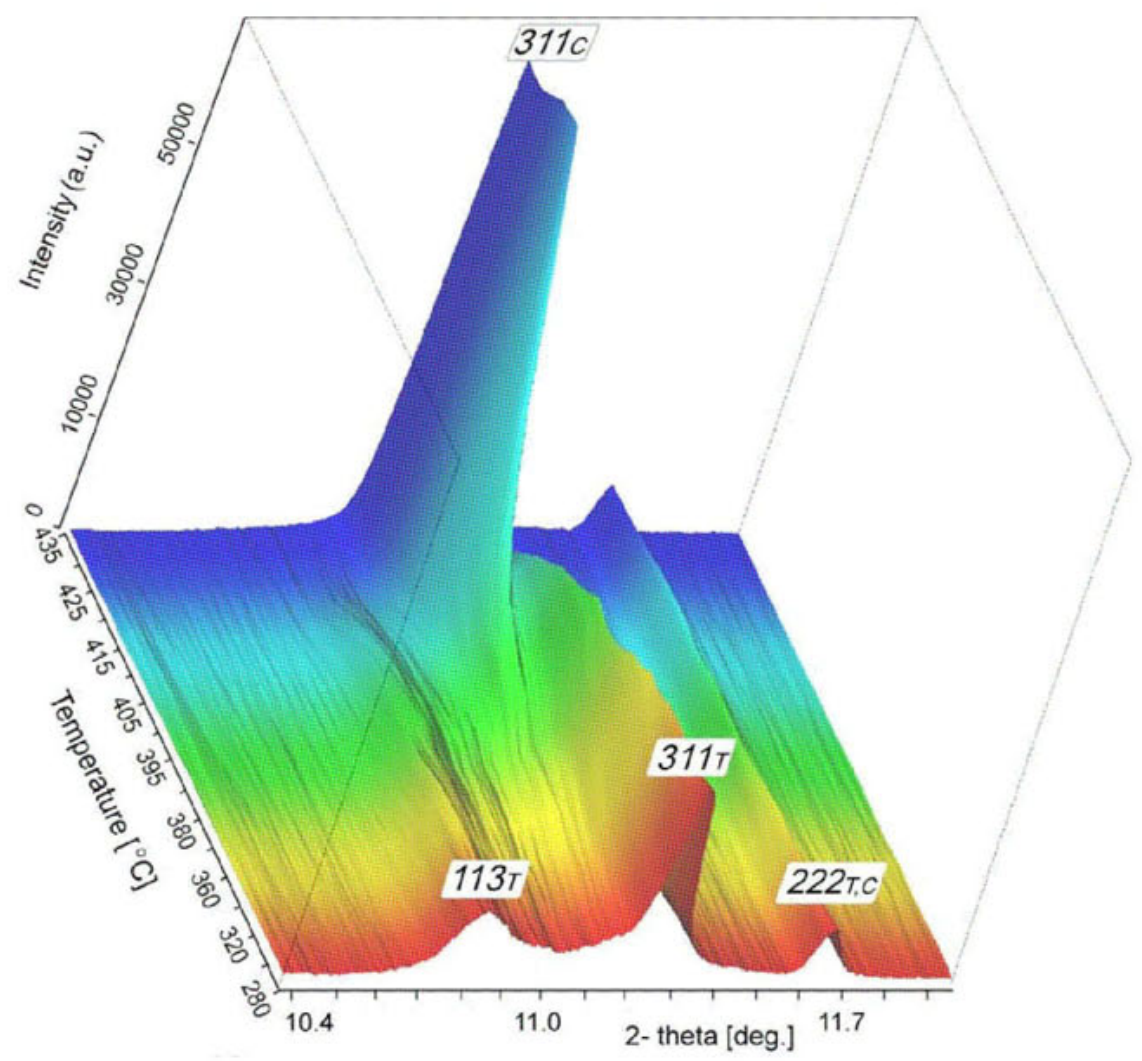

Figure 3. Temperature evolution of the X-ray diffraction patterns for $\mathrm{CuFe}_{2} \mathrm{O}_{4}(\mathrm{SC})$. Data were measured at beamline B2, Synchrotron DESY HASYLAB, Hamburg. The shift of reflections $113_{T}, 311_{T}$ at the tetragonal $\rightarrow$ cubic phase transition can clearly be observed. For the graphic representation the program EVA (DIFFRAC plus BASIC Evaluation Package, Bruker AXS) was used.

The synchrotron powder diffraction scans (figure 3) reveal that the structural phase transition at approximately $\sim 340^{\circ} \mathrm{C}$ to $\sim 420^{\circ} \mathrm{C}$ is manifested by the shift and disappearance of the reflections $113_{\mathrm{T}}, 311_{\mathrm{T}}$ (tetragonally distorted spinel, $F 4_{1} / d d m$ ) and appearance of a new re- 
flection $311_{\mathrm{C}}$ (cubic spinel, $F d \overline{3} \mathrm{~m}$ ) in the investigated temperature range. Evidence is given that above $420^{\circ} \mathrm{C}$ the JT-distortion disappears in the structure.

Annealing treatment can modify the cationic distribution in the spinel lattice. Structural analysis results indicated that above $350^{\circ} \mathrm{C}$ copper ions partially occupy the tetrahedral sites and the structural $\left(F 4_{1} / d d m\right) \rightarrow(F d \overline{3} m)$ phase transition appears. On heating the sample, the copper ions migrate to tetrahedral sites (figure $4 \mathrm{~A}$ ) and the structural formula of the ferrite changes to $\left(\mathrm{Cu}_{\mathrm{x}}{ }^{2+} \mathrm{Fe}_{1-\mathrm{x}}{ }^{3+}\right)_{\mathrm{A}}\left[\mathrm{Cu}_{1-\mathrm{x}}{ }^{2+} \mathrm{Fe}_{1+\mathrm{x}}{ }^{3+}\right]_{\mathrm{B}} \mathrm{O}_{4}$. The resulting decrease in $\mathrm{Cu}^{2+}$ ions in the octahedral sites gives rise to a less distorted crystalline structure. The redistribution $\mathrm{Cu}^{2+}$ ions in the spinel lattice is revealed by the variation of the tetragonal distortion ( $\mathrm{c} / a$ of the unit cell). For the slow cooled sample, the c/a ratio of the tetragonal spinel phase is reported in figure $4 \mathrm{~A}$ as a function of temperature. In this case, the $c / a$ ratio diminishes as the temperature increases up to $415^{\circ} \mathrm{C}$. In particular, $\mathrm{Cu}^{2+}$ intersite cationic migration is also correlated with the decrease in the $c / a$ ratio. More precisely, the migration of $\mathrm{Cu}^{2+}$ ions from octahedral to tetrahedral sites is responsible for this decrease. It has been shown that placed in the octahedral sites $\mathrm{Cu}^{2+}$ ions migrate to the tetrahedral sites. Such migration occurs above $350^{\circ} \mathrm{C}$ (figure 4B).
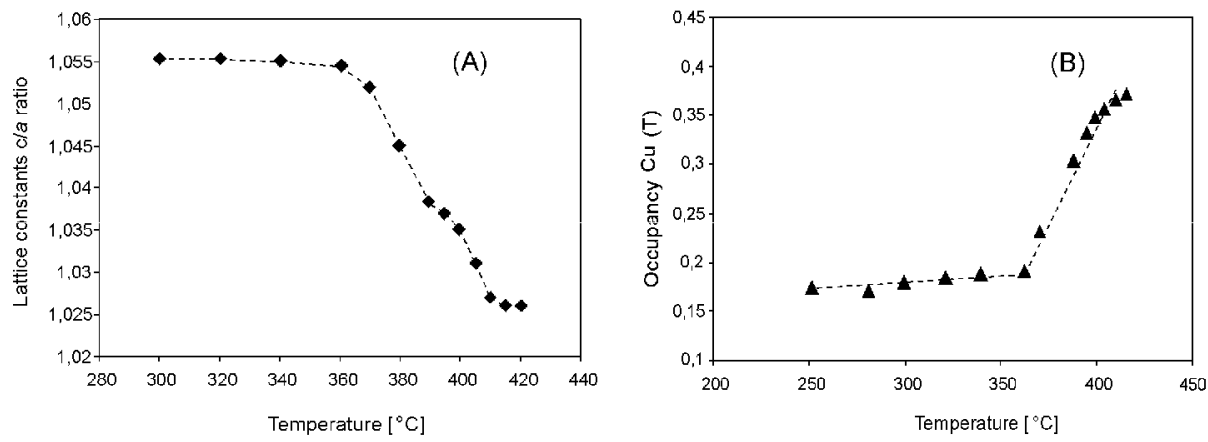

Figure 4. Evolution of the cla ratio for the tetragonally distorted spinel phase $\mathrm{CuFe}_{2} \mathrm{O}_{4}(\mathrm{SC})$ as a function of temperature $(A)$, and the occupancy of $\mathrm{Cu}^{2+}$ at the spinel tetrahedral sites $(B)$. Dashed lines are guides for the eyes.

\section{Concluding remarks}

Polycrystalline $\mathrm{CuFe}_{2} \mathrm{O}_{4}$ samples have been successfully prepared by a simple combustion method using citrate-nitrate precursors. All the ferrite samples studied were prepared at the same temperatures, but one series was slowly cooled from $900^{\circ} \mathrm{C}$ to room temperature, and another was quenched from $900^{\circ} \mathrm{C}$. The most striking effect observed in this experiment was the dependence of the reaction products on the preparation conditions. The SC and QC sample displayed different thermal behavior. The nature of the structural transition in inverse spinels has been investigated using the synchrotron XRD technique. Slow cooled samples are subjected to order-disorder transformation, due to the migration $\mathrm{Cu}^{2+}$ from octahedral to tetrahedral sites. This phenomenon transforms the tetragonally distorted spinel structure 
$\left(F 4_{1} / d d m\right)$ into a cubic lattice $(F d \overline{3} \mathrm{~m})$. The tetragonal $\rightarrow$ cubic phase transition occurs in the temperature range from $\sim 360^{\circ} \mathrm{C}$ to $\sim 420^{\circ} \mathrm{C}$. The resulting cubic lattice cannot be stabilized at room temperature by quenching. Annealing can modify the cationic distribution in spinel lattice - it has been shown that placed in the octahedral sites $\mathrm{Cu}$ ions migrate to the tetrahedral positions. The resulting decrease in copper ions in the octahedral sublattice gives rise to a less distorted crystalline structure, and above $420^{\circ} \mathrm{C}$ the structural JT-distortion disappears.

\section{References}

1. Selvan, R. K., Krisham V., Augustin, Ch.O., Bertagnolli, H., Kim, Ch.S. \& Gedanken, A., 2008, Chem. Mater., 20, 429.

2. Tang, X.X., Manthiram, A. \& Goodenough, J.B., 1989, J. Solid State Chem., 79, 250.

3. Villette, C., Tailhades, Ph. \& Rousset, A., 1995, J. Solid State Chem., 117, 64.

4. Gajbhiye, N.S., Balaji, G., Bhattacharyya S. \& Ghafari, M., 2004, Hyperfine Interact., 156-157, 57.

5. Ohnishi, H. \& Teranishi, T., 1961, J. Phys. Soc. Jpn., 16, 35.

6. Ata-Allah, S.S., 2004, J. Solid State Chem., 177, 4443.

7. d'Huysser, A., Lerebours-Hannoyer, R., Lenglet, M. \& Bonnelle, J.P., 1981, J. Solid State Chem., 39, 246.

8. Desai, M., Prasad, S., Venkataramani, N., Samajdar, I., Nigam, A.K. \& Krishnan, R., 2002, J. Appl. Phys., 91, 2220.

9. Desai, M., Prasad, S., Venkataramani, N., Samajdar, I., Nigam, A.K. \& Krishnan, R., 2002, J. Magn. Magn. Mater., 246, 266.

10. Verma, S. \& Joy, P.A., 2008, Mater. Res. Bull. in press, doi: 10.1016/j.materresbull.2008.01.023.

11. Rodriguez-Carvajal, J., 2001, Newslett. IUCr Commission Powd. Diffr., 26, 12.

12. Tailhades, Ph., Villette, C., Rousset, A., Kulkarni, G.U., Kannan, K.R., Rao, C.N.R. \& Lenglet, M., 1998, J. Solid State Chem., 141, 56.

13. Falvello, L.R., 1997, J. Chem. Soc., Dalton Trans., 23, 4463.

Acknowledgements. The synchrotron measurements at DESY-HASYLAB were supported by the IA-SFS-Contract No. RII3-CT-2004-506008 of European Commission. The author would like to thank Dr. D. Trots from Hasylab, for his assistance during experiments. 


\section{Powder diffraction studies of pressure- induced instabilities in orthorhombic $\mathrm{LnGaO}_{3}$}

\section{A. Senyshyn ${ }^{1,2, *}$, J. M. Engel ${ }^{1,3}$, I. D. H. Oswald ${ }^{4,5}$, L. Vasylechko ${ }^{2}$, M. Berkowski ${ }^{6}$}

${ }^{1}$ Materialwissenschaft, Technische Universität Darmstadt, Petersenstraße 23, 64287 Darmstadt, Germany

2 Lviv Polytechnic National University, Bandera St. 12, 79013 Lviv, Ukraine

3 Institut für Werkstoffwissenschaft, Technische Universität Dresden, Helmholtzstraße 7, 01069 Dresden, Germany

${ }^{4}$ School of Chemistry, University of Edinburgh, King's Buildings, Mayfield Road, Edinburgh EH9 3JZ, United Kingdom

${ }^{5}$ European Synchrotron Radiation Facility, BP 220, F-38043 Grenoble, France

${ }^{6}$ Institute of Physics Polish Academy of Sciences, Al. Lotników 32/46, 02-668 Warsaw, Poland

* anatoliy.senyshyn@gmail.com

Keywords: perovskite, rare-earth, gallate, powder diffraction, high pressure, $\mathrm{LnGaO}_{3}$, $\mathrm{RGaO}_{3}$, bulk modules, pressure medium, phase transition

Abstract. Pressure evolution of $\mathrm{LaGaO}_{3}, \mathrm{CeGaO}_{3}, \mathrm{PrGaO}_{3}, \mathrm{NdGaO}_{3}, \mathrm{La}_{0.75} \operatorname{Pr}_{0.25} \mathrm{GaO}_{3}$ and $\mathrm{La}_{0.63} \mathrm{Nd}_{0.37} \mathrm{GaO}_{3}$ crystal structures was studied using powder diffraction with synchrotron radiation at ambient temperature in a broad pressure range. Structural instabilities corresponding to phase transformations have been observed in all studied compositions and it was found that $P_{\mathrm{c}}$ of these transitions depends on the deformation of the perovskite structure. The effect of the pressure medium on the phase transition, bulk modulus and compressibilities of $\mathrm{LaGaO}_{3}, \mathrm{CeGaO}_{3}, \mathrm{PrGaO}_{3}, \mathrm{NdGaO}_{3}, \mathrm{La}_{0.75} \mathrm{Pr}_{0.25} \mathrm{GaO}_{3}$ and $\mathrm{La}_{0.63} \mathrm{Nd}_{0.37} \mathrm{GaO}_{3}$ has been discussed in brief.

\section{Introduction}

The exploration of materials at high pressure gives better understanding of physical processes occurred in the material and stays as an alternative tool for probing the repulsive part of the interatomic potential between atoms and its influence on packing and bonding. In that context structural changes occurring in perovskite-type materials at high pressures encourage considerable attention and any systematics in the high-pressure behaviour of perovskite materials becomes very attractive [1]. Thus perovskite type rare-earth gallates $\mathrm{LnGaO}_{3}$, 
whose crystal structures and their thermal evolution were systematically studied in broad temperature and composition ranges [2], seem to be well-suited model objects for highpressure diffraction studies, i.e. a good link between in-situ temperature- and pressuredependent structural properties can be established. At ambient conditions all $\mathrm{LnGaO}_{3}$ are isostructural ( $\mathrm{Pbnm}$ space group, $\mathrm{GdFeO}_{3}$ type of structure, $\mathrm{OR}$ ) and undergo a transformation to $\mathrm{LaAlO}_{3}$ structure type $(R-3 c$ space group, $\mathrm{RH})$ at elevated temperatures, where the temperature of the phase transformation depends linearly on the tolerance factor $t=\left\{r_{L n}+r_{O}\right\} / \sqrt{2}\left\{r_{G a}+r_{O}\right\}$. In the case of in $\mathrm{LnGaO}_{3}$ only the ionic radii $r_{\mathrm{Ln}}$ varies upon rare-earth substitution and, hence, a perovskite distortion can be analysed as function of $r_{\mathrm{Ln}}$ only $[3,4]$. It is worth to mention that the high-pressure phase of the first representative La$\mathrm{GaO}_{3}$ has the same symmetry as the phase found at high temperatures (RH) [5-7], which is in accordance with [1] corresponding to a higher compressibility of the $\mathrm{GaO}_{6}$ octahedra compared to the $\mathrm{LaO}_{12}$ polyhedra.

Thus the main aim of the current study was to answer the following questions:

- Does the same high-pressure OR-to-RH phase transformation occur in $\mathrm{LnGaO}_{3}$ (excluding lanthanum) and how isomorphic rare-earth substitution influences those transformations?

- What is the behaviour of bulk modules and coefficients of lattice contraction upon rareearth substitution?

- Is there any influence of type of pressure medium on pressure-driven structural changes in rare-earth gallium perovskites?

\section{Experimental}

In order to get an answer on the issues addressed, six samples have been chosen for highpressure structural studies: four orthogallates $\mathrm{LaGaO}_{3}, \mathrm{CeGaO}_{3}, \mathrm{PrGaO}_{3}$ and $\mathrm{NdGaO}_{3}$, whose perovskite lattice deformation increases smoothly with increasing periodic numbers of the rare-earth elements and two solid solutions: the $\mathrm{La}_{0.75} \mathrm{Pr}_{0.25} \mathrm{GaO}_{3}$ one and the $\mathrm{La}_{0.63} \mathrm{Nd}_{0.37} \mathrm{GaO}_{3}$ whose magnitude of perovskite distortion is very close to one of cerium gallate (see table 1). All samples except cerium gallate were grown either by Czhochralski or floating zone technique, whilst $\mathrm{CeGaO}_{3}$ was synthesized by solid state reaction method at $1470 \mathrm{~K}$ in argon.

Table 1. Linear moduli and parameters of a $2^{\text {nd }}$ order Birch-Murnaghan equation.

\begin{tabular}{|l|l|l|l|l|l|l|}
\hline Compound & $r_{\mathrm{n} n}, \AA$ & $V, \AA^{3}$ & $\mathrm{~K}, \mathrm{GPa}$ & $\beta_{3}, \mathrm{GPa}^{-1}$ & $\beta_{0}, \mathrm{GPa}^{-1}$ & $\beta_{\mathrm{c}}, \mathrm{GPa}^{-1}$ \\
\hline $\mathrm{LaGaO}_{3}{ }^{*, \#}$ & 1.216 & $353.1(2)$ & $193(2)$ & $-0.0076(2)$ & - & $-0.0198(4)$ \\
\hline $\mathrm{La}_{0.75} \mathrm{Pr}_{0.25} \mathrm{GaO}_{3}{ }^{\#}$ & 1.213 & $235.2(1)$ & $173(3)$ & $-0.0113(2)$ & $-0.0062(5)$ & $-0.0139(2)$ \\
\hline $\mathrm{CeGaO}_{3}{ }^{\mathrm{S}}$ & 1.196 & $233.61(7)$ & $177(3)$ & $-0.0113(2)$ & $-0.0077(3)$ & $-0.0137(7)$ \\
\hline $\mathrm{La}_{0.63} \mathrm{Nd}_{0.37} \mathrm{GaO}_{3}{ }^{\mathrm{S}}$ & 1.196 & $234.0(4)$ & $178(6)$ & $-0.0106(3)$ & $-0.0064(9)$ & $-0.0137(9)$ \\
\hline $\mathrm{PrGaO}_{3}{ }^{\mathrm{s}}$ & 1.179 & $231.4(3)$ & $182(5)$ & $-0.0075(5)$ & $-0.0060(4)$ & $-0.0120(9)$ \\
\hline $\mathrm{NdGaO}_{3}{ }^{\mathrm{S}}$ & 1.163 & $231.0(6)$ & $189(8)$ & $-0.0063(3)$ & $-0.0068(2)$ & $-0.0100(2)$ \\
\hline
\end{tabular}

* Data for high-pressure $\left(\mathrm{LaAlO}_{3}\right.$ structure type) modification are given; ethanol-methanol" or $\mathrm{N}_{2}{ }^{5}$ were used as a pressure media.

In-situ X-ray diffraction experiments were carried out at the high-pressure beamline ID 27 of European Synchrotron Radiation Facility (Grenoble, France). The "White spectra" X-rays 
from two phased undulators were monochromatised to a wavelength of $\lambda=0.3738 \AA$ using channel-cut $\mathrm{Si}(111)$ monochromator and the monochromatic X-ray beam was focused down to $10 \times 10 \mu \mathrm{m}^{2}$ using Kirkpatrick-Baez mirrors.

Membrane driven diamond-anvil cells with culet faces of $600 \mu \mathrm{m}$ diameter were used for experiment. Depending on the sample either methanol-ethanol mixture or nitrogen were used as a pressure medium (see table 1). The samples were loaded into gasket holes (stainless steel foil of $10 \mu \mathrm{m}$ thickness) with $100 \mu \mathrm{m}$ diameter together with pressure medium and small single-crystalline ruby chip. The ruby fluoreseence technique was applied to determine the pressure.

The Debye-Scherrer rings were collected with MAR345 image plate detector $(230 \mathrm{~mm}$ in effective diameter, pixel size $100 \times 100 \mu \mathrm{m}^{2}$ ) placed at $424 \mathrm{~mm}$ distance from the sample. The accessible angular range was limited by the aperture of diamond-anvil cell to ca. $23^{\circ}$ in 20. The obtained two-dimensional patterns were corrected for spatial distortions and nonlinear features in the background with subsequent integration to yield diffraction pattern in the conventional form, i.e. intensity versus $2 \theta$ angle, using the software FIT2D. More than 140 diffraction patterns have been collected during experiment. The data evaluation has been performed using the Rietveld method and full profile decomposition technique (Le-Bail) implemented into GSAS and FullProf program packages, while the single profile decomposition was carried out using WinCSD software.

\section{Results and discussion}

In current work the lanthanum gallate was used as a reference. Among all rare-earth gallates this material possesses the smallest perovskite cell distortion and its behaviour with respect to temperature and pressure has been studied extensively [2,3,5-7]. Thus it is well known from the literature that $\mathrm{LaGaO}_{3}$ undergoes OR-to-RH phase transformation which can be either temperature- (ca. $420 \mathrm{~K}$ ) or pressure- (ca. $2.2 \mathrm{GPa}$ ) driven. In our experiment the first diffraction pattern has been collected at $3.5 \mathrm{GPa}$ and a splitting of the characteristic Bragg reflections reveals the RH-type structure can be unambiguously assigned at this pressure. A further increase of pressure resulted in simple compression of the crystal structure to a maximum pressure of $14.7 \mathrm{GPa}$. Lattice parameters contract almost linearly with pressure and the obtained linear compressibilities $\beta_{L}=\partial L / \partial P$ for $a$ - and $c$-directions of the RH lattice $\left(\beta_{\text {a }}\right.$ and $\beta_{\mathrm{c}}$ ) are listed in table 1 together with bulk modulus, $K$.

The splitting of the characteristic Bragg reflections on the diffraction pattern of $\mathrm{La}_{0.75} \mathrm{Pr}_{0.25} \mathrm{GaO}_{3}$ at the first pressure point $(P=0.85 \mathrm{GPa})$ revealed the OR-type structure, which remained stable up to ca. $9.9 \mathrm{GPa}$. A further pressure increase to 10.26(4) GPa resulted in a distinct change in the diffraction pattern: OR (111) reflection vanishes, whereas significant broadening occurred for OR $(020)+(112)+(200)$ triplet together with noticeable redistribution of intensity within $(040)+(224)+(400)$ triplet. The observed changes in X-ray diffraction patterns, measured cell parameters, peak intensities and systematic absences are consistent with $R-3 c$ symmetry.

For rare-earth gallates with higher distortion of the perovskite structure the phase transition was expected to occur at higher pressures. Therefore nitrogen was used as a pressure medium instead of ethanol-methanol mixture. Very similar anomalies to those observed in $\mathrm{La}_{0.75} \mathrm{Pr}_{0.25} \mathrm{GaO}_{3}$, i.e. vanishing of (111) reflection, broadening of $(020)+(112)+(200)$ triplet 
and redistribution of intensities within $(040)+(224)+(400)$ triplet, were detected for $\mathrm{CeGaO}_{3}$ at ca. $9.6 \mathrm{GPa}$, for $\mathrm{La}_{0.63} \mathrm{Nd}_{0.37} \mathrm{GaO}_{3}$ at ca. $12.3 \mathrm{GPa}$, for $\mathrm{PrGaO}_{3}$ at ca. $24 \mathrm{GPa}$ and for $\mathrm{NdGaO}_{3}$ at ca. $38 \mathrm{GPa}$. Selected examples are shown in figure 1.
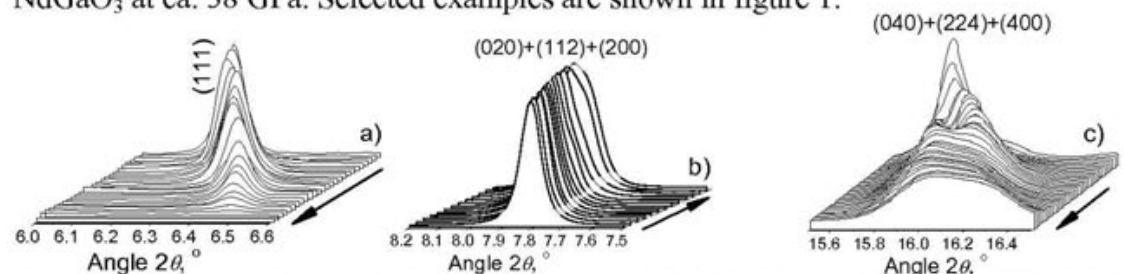

Figure 1. Pressure evolution of relative intensities for OR (111) Bragg reflection in $\mathrm{NdGaO}_{3}($ a), $(020)+(112)+(200)$ triplet in $\mathrm{La}_{0.50} \mathrm{Pr}_{0.50} \mathrm{GaO}_{3}$ (b) and $(040)+(224)+(400)$ triplet in $\mathrm{CeGaO}_{3}(\mathrm{c})$. Direction of pressure increase is shown by arrow, points of pressure instabilities are shown in yellow.

The resemblance of structural anomalies in $\mathrm{La}_{0.75} \mathrm{Pr}_{0.25} \mathrm{GaO}_{3}$ to those observed in $\mathrm{CeGaO}_{3}$, $\mathrm{La}_{0.63} \mathrm{Nd}_{0.37} \mathrm{GaO}_{3}, \mathrm{PrGaO}_{3}$ and $\mathrm{NdGaO}_{3}$ can be explained by possible OR-to- $\mathrm{RH}$ transition. Significant broadening of the peaks right after pressure-induced instability (similar to that reported by Angel et al. [7] for $\mathrm{La}_{1-x} \mathrm{Nd}_{x} \mathrm{GaO}_{3}$ ) occurred in these compositions and it can be an additional indicator of phase transformation. Unfortunately such a broadening significantly embrangles the data treatment of high-pressure phases and their symmetries require additional verification, which seems to be not possible on the basis of current data.
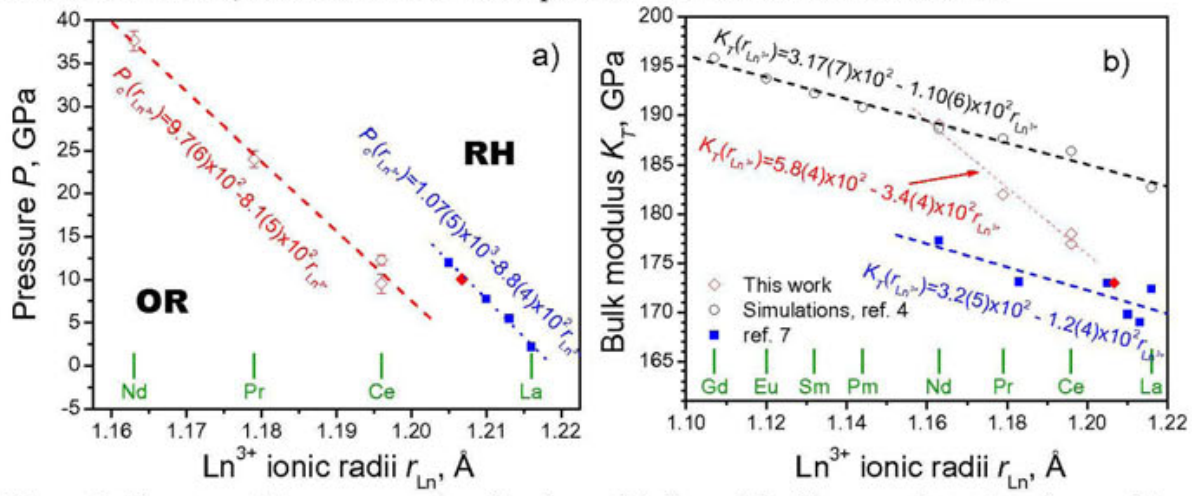

Figure 2. Phase transition pressure a) and isothermal bulk modulus b) versus distortion of perovskite lattice (in terms of $\mathrm{Ln}^{3+}$ ionic radii). Diamonds - current study, squares - ref. [7]. Solid and open points correspond to datasets using methanol-ethanol and nitrogen as a pressure media. Lines denote results of linear fit.

In compositions with a high degree of perovskite distortion the instabilities occur at rather higher pressures (diamonds in fig. 2a) with almost linear decrease in the row $\mathrm{NdGaO}_{3}-$ $\mathrm{PrGaO}_{3}-\mathrm{CeGaO}_{3}$. The pressure instability in $\mathrm{La}_{0.63} \mathrm{Nd}_{0.37} \mathrm{GaO}_{3}$ has been found very close to those of $\mathrm{CeGaO}_{3}$ (thus indicating its insensitivity to the kind of rare-earth) and in this context one would expect the phase transformation in $\mathrm{La}_{0.75} \mathrm{Pr}_{0.25} \mathrm{GaO}_{3}$ at much lower pressures than in $\mathrm{La}_{0.63} \mathrm{Nd}_{0.37} \mathrm{GaO}_{3}(12.3 \mathrm{GPa})$ and $\mathrm{CeGaO}_{3}(9.6 \mathrm{GPa})$. Hence, the diffraction experiment on $\mathrm{La}_{0.75} \mathrm{Pr}_{0.25} \mathrm{GaO}_{3}$ showed the structural instability at $9.9 \mathrm{GPa}$, which is very close to $\mathrm{La}_{0.63} \mathrm{Nd}_{0.37} \mathrm{GaO}_{3}$ and $\mathrm{CeGaO}_{3}$. Bearing in mind the fact that lanthanum gallate undergoes 
OR-to-RH phase transition at ca. 2.2 GPa [5-7], the $P_{\mathrm{c}}\left(r_{\mathrm{In}}\right)$ dependence becomes complicated and requires more detailed consideration.

Thus Angel et al. [7] studied systematically the pressure behaviour of compositions within the pseudobinary system $\mathrm{LaGaO}_{3}-\mathrm{NdGaO}_{3}$ using single crystal X-ray diffraction. Much better quality of structural information was obtained from this experiment in comparison to powder diffraction using synchrotron radiation, which enables careful analysis of cationanion bond lengths and anisotropic displacement parameters. The pressure-induced phase transformations occurred in the $\mathrm{LaGaO}_{3}$-rich end of the pseudobinary system $\mathrm{LaGaO}_{3}$ $\mathrm{NdGaO}_{3}$ (squares in fig. 2a) and the $P_{c}$ linearly increases with the decrease of rare-earth ionic radius $r_{\mathrm{Ln}}$ (which correspond to increase the distortion of perovskite cell). Two lines describing a linear fit to the $P_{c}\left(r_{\mathrm{Ln}}\right)$ data from the current study and from [7] display almost the same slope, but a significant onset $\Delta P_{c} \approx 8.6 \mathrm{GPa}$. On the other hand the phase transition in $\mathrm{La}_{0.75} \mathrm{Pr}_{0.25} \mathrm{GaO}_{3}$ fits perfectly into the behaviour observed for $\mathrm{La}_{1 . \mathrm{x}} \mathrm{Nd}_{\mathrm{x}} \mathrm{GaO}_{3}$ solid solutions.

It is well known that studies of ferroelastic materials at high pressures are difficult as they are highly sensitive to deviatoric stresses. In experiments of such kind it is essential to use pressure-transmitting media that approximate as closely as possible hydrostatic conditions. The use of different soft solids, liquids and gases (often as supercritical fluids) as pressure media at suitable pressure ranges is the matter of discussion since many years. Nevertheless it is already established that the type of pressure-transmitting medium can influence the phase transitions in inorganic compounds [8-11]. As it can be seen from table 1 the methanol-ethanol mixture was used as a pressure-transmitting medium for studies of $\mathrm{La}_{0.75} \mathrm{Pr}_{0.25} \mathrm{GaO}_{3}$ similar to $\mathrm{La}_{1 . x} \mathrm{Nd}_{\mathrm{x}} \mathrm{GaO}_{3}$ solid solutions [7], whereas for other compositions the nitrogen was cryogenically loaded. Thus observed discrepancies can be attributed to the deteriorated hydrostaticity of nitrogen pressure medium when compared to ethanol-methanol one which is in good agreement with [8].

It has to be mentioned that the use of different pressure media probably influences the isothermal bulk modules as well (fig. 2b, table 1). Good agreement has been noticed between $\mathrm{La}_{0.75} \mathrm{Pr}_{0.25} \mathrm{GaO}_{3}$ and $\mathrm{La}_{1 . x} \mathrm{Nd}_{\mathrm{x}} \mathrm{GaO}_{3}$ solid solutions [7]. The observed tendency (slope of $K_{\mathrm{T}}\left(r_{\mathrm{Ln}}\right)$ line) coincide fairly well with the results of semiclassical simulations $[2,4]$, whereas bulk modules in $\mathrm{NdGaO}_{3}, \mathrm{PrGaO}_{3}, \mathrm{CeGaO}_{3}$ and $\mathrm{La}_{0.63} \mathrm{Nd}_{0.37} \mathrm{GaO}_{3}$ display much higher slopes of $K_{\mathrm{T}}\left(r_{\mathrm{Ln}}\right)$ and agree with data of Angel et al. [7] around $r_{\mathrm{Ln}}=1.213 \AA$ $\left(\mathrm{La}_{0.75} \mathrm{Pr}_{0.25} \mathrm{GaO}_{3}\right)$. The parameters describing contraction of lattice parameters in $\mathrm{La}_{0.75} \mathrm{Pr}_{0.25} \mathrm{GaO}_{3}, \mathrm{CeGaO}_{3}, \mathrm{La}_{0.63} \mathrm{Nd}_{0.37} \mathrm{GaO}_{3}, \mathrm{PrGaO}_{3}$ and $\mathrm{NdGaO}_{3}$ are listed in table 1. As for $\mathrm{LaGaO}_{3}$ the anisotropic lattice compression can be fairly described as linear. Similar to thermal expansion coefficients the linear compressibility follows the conditions $\beta_{\mathrm{c}}>\beta_{\mathrm{a}}>\beta_{\mathrm{b}}$. The increase of perovskite distortion leads to the increase of linear compressibility in $a$ - and $c$-directions of OR lattice, whereas the linear compressibility in $b$-direction remains almost constant.

\section{Concluding remarks}

It has been confirmed that the high-pressure form of lanthanum gallate belongs to $\mathrm{LaAlO}_{3}$ type of structure at least below $14.7 \mathrm{GPa}$. In $\mathrm{La}_{0.75} \mathrm{Pr}_{0.25} \mathrm{GaO}_{3}, \mathrm{CeGaO}_{3}, \mathrm{La}_{0.63} \mathrm{Nd}_{0.37} \mathrm{GaO}_{3}$, $\mathrm{PrGaO}_{3}$ and $\mathrm{NdGaO}_{3}$ the similar high-pressure structures were observed (which is in good agreement with [1] assuming the modest fluctuations in compressibility of $\mathrm{LnO}_{12}$ and $\mathrm{GaO}_{6}$ 
polyhedra upon rare-earth substitution), but the significant broadening of Bragg peaks right after the phase transformation makes their determination with powder diffraction a cumbersome task. The observed phase transitions display an obvious relationship to the perovskite cell deformation but at the same time $P_{\mathrm{c}}$ was found highly dependent on the type of pressure medium, either ethanol-methanol or nitrogen. Taking into account that the studied compositions belong to the group of ferroelastic materials, this fact can be explained by their sensitivity to non-hydrostatic conditions. The analysis of the obtained bulk modulus reveals that the ethanol-methanol mixture supplies better hydrostatic conditions in comparison to cryogenically loaded nitrogen, which is in good agreement with gigahertz ultrasonic interferometry measurements [8].

Nevertheless considering only compositions measured with a ethanol-methanol mixture it has been found that the phase transition in $\mathrm{La}_{0.75} \mathrm{Pr}_{0.25} \mathrm{GaO}_{3}$ fits well into the behaviour observed for $\mathrm{La}_{1-\mathrm{x}} \mathrm{Nd}_{\mathrm{x}} \mathrm{GaO}_{3}(\mathrm{x}=0.00,0.06,0.12$ and 0.20$)$ [7]. Therefore one can assume the linear dependence of $P_{\mathrm{c}}$ on the deformation of perovskite structure (similar to temperaturedriven Pbnm-R-3c phase transformations [2]) and not only on the $x$ composition in the pseudobinary system $\mathrm{LaGaO}_{3}-\mathrm{NdGaO}_{3}$.

\section{References}

1. Angel, R.J., Zhao, J. \& Ross N.L., 2005, Phys. Rev. Lett., 95, 025503.

2. Vasylechko, L., Senyshyn, A. \& Bismayer, U., 2008, in Handbook on the Physics and Chemistry of Rare-earths, edited by K. Gschneider, J. Bunzli \& V. Pecharsky (Amsterdam: Elsevier), 39, chapter 242, p. 113-295.

3. Vasylechko, L., Pivak, Ye., Senyshyn, A., Savytskii, D., Berkowski, M., Borrmann, H., Knapp, M. \& Paulmann, C., 2005, J. Solid State Chem., 178, 270.

4. Senyshyn, A., Ehrenberg, H., Vasylechko, L., Gale, J.D. \& Bismayer U., 2005, J. Phys.: Condens. Matter, 17, 6217.

5. Kennedy, B.J., Vogt, T., Martin C.D., Parise J.B. \& Hriljac, J.A., 2001, J. Phys.: Condens. Matter, 13, L925.

6. Shibasaki, T., Furuya, T., Kuwahara, J., Takahashi, Y., Takahashi H. \& Hashimoto, T., 2005, J. Therm. Analys. Calor., 81, 575.

7. Angel, R.J., Zhao, J., Ross, N.L., Jakeways, C.V., Redfern, S.A.T. \& Berkowski, M., 2007, J. Solid State Chem., 180, 3408.

8. Angel, R.J., Bujak, M., Zhao, J., Gatta G.D. \& Jacobsen, S.D., 2007, J. Appl. Cryst., 40, 26 .

9. Goni, A.R., Zhou, T., Schwarz, U., Kremer, R.K. \& Syassen, K., 1996, Phys. Rev. Lett., 77, 1079.

10. Mezouar, M., Libotte, H., Deputier, S., Le Bihan, T. \& Hausermann, D., 1999, Phys. Stat. Solidi B, 211, 395.

11. Resela, R., Oehzelt M., Shimizu, K., Nakayama, A. \& Takemura, K., 2004, Sol. State Comm., 129, 103.

Acknowledgements. This work was partially supported by the German Federal Ministry of Education and Research (BMBF project 03FU7DAR, WTZ Grant UKR-04/007), Ukrainian Ministry of Education and Science (project "Segnet"). 


\title{
Magnesium titanate phases from xerogels by hot stage X-ray powder diffractometry
}

\author{
J. Zabicky ${ }^{1}$, G. Kimmel ${ }^{1,}{ }^{*}$, E. Goncharov ${ }^{1}$, F. Guirado ${ }^{2}$ \\ ${ }^{1}$ Ben-Gurion University of the Negev, PO.B 653, Beer-Sheva 84105, Israel \\ ${ }^{2}$ Universitat Rovira i Virgili (URV), Av. Paisos Catalans 26, Tarragona 43007, Spain \\ *kimmel@bgu.ac.il
}

Keywords: magnesium titanate, HT XRPD, Rietveld refinement, nanocrystalline oxides

\begin{abstract}
Coprecipitated xerogel precursors of nanocrystalline magnesium titanates, with $\mathrm{Mg}: \mathrm{Ti}$ stoichiometric ratio varying from 1:1 to 2:1, were subjected to thermal treatment at constant temperature in the range from 550 to $1200{ }^{\circ} \mathrm{C}$, in air, using a hot-stage X-ray powder diffractometer. The kinetics during the first hour showed dependence on temperature and the $\mathrm{Mg}$ : Ti stoichiometric ratio. At low temperatures, for compositions near 2:1, a single nonstoichiometric metastable nanocrystalline qandilite-like phase is formed; however, when the $\mathrm{Mg}$ content is lowered a solubility limit is reached, below which a nonstoichiometric qandilite, of fixed composition depending on the temperature, is in equilibrium with stoichiometric geikielite. The limit moves to higher Mg contents as the temperature rises. In the approximate $900-1000{ }^{\circ} \mathrm{C}$ range the metastable qandilite-like phases decompose into geikielite and periclase. From $1100^{\circ} \mathrm{C}$ on stoichiometric qandilite is obtained in equilibrium with geikielite. At low temperatures nanocrystalline qandilite is formed much faster than nanocrystalline geikielite, probably owing to the isotropic chemical structure of both the amorphous xerogels and qandilite. A phase diagram is proposed for the metastable nanocrystalline phases formed at low temperatures in the composition range of the present study.
\end{abstract}

\section{Introduction}

The $\mathrm{MgO}-\mathrm{TiO}_{2}$ ceramic system includes three stoichiometric magnesium titanate phases, the structure of which has been determined by X-ray diffraction and neutron diffraction [1-3]: Geikielite (magnesium metatitanate, $\mathrm{MgTiO}_{3}$, rhombohedral of ilmenite type), forms above $600{ }^{\circ} \mathrm{C}$ and is stable from room temperature to its melting point [4]; qandilite (magnesium orthotitanate, $\mathrm{Mg}_{2} \mathrm{TiO}_{4}$, cubic of inverse spinel type), forms above $1150^{\circ} \mathrm{C}$ and undergoes a low-temperature transition to a tetragonal phase $[5,6]$; and karrooite $\left(\mathrm{MgTi}_{2} \mathrm{O}_{5}\right.$, orthorhombic of pseudobrookite type), forms above $500^{\circ} \mathrm{C}$ and shows slight deviations from stoichiometry as a function of temperature due to disordering in the ionic occupation $[1,2,7]$. The thermodynamic properties of the magnesium titanates were correlated with the crystalline structure, unit cell parameters and order-disorder. Although below $900^{\circ} \mathrm{C}$ qandilite should decompose into periclase $(\mathrm{MgO})$ and geikielite, this has not been observed due to kinetic limitations, and only a transition to the tetragonal form of $\mathrm{Mg}_{2} \mathrm{TiO}_{4}$ takes place [2].

Mechanically mixed magnesia and titania xerogels followed by low-temperature thermal annealing usually showed partial yields of magnesium titanates [8]. Coprecipitated xerogels 
afforded varied results, depending on their stoichiometric composition and the temperature of thermal annealing, $T$. Straightforward results were achieved for $\mathrm{Mg}: \mathrm{Ti}=1: 1$ atomic ratio, yielding nanocrystalline geikielite at about $600^{\circ} \mathrm{C}$ [9]. After $3 \mathrm{~h}$ at $600{ }^{\circ} \mathrm{C}$, coprecipitated xerogels with $\mathrm{Mg}: \mathrm{Ti}=x: 1,2<x \leq 4$, yielded a mixture of nanocrystalline periclase and qandilite; for $\mathrm{Mg}: \mathrm{Ti}=1: x, 2<x \leq 4$, the product was a mixture of nanocrystalline karrooite and titania (mainly rutile with some anatase) $[10,11]$. After $3 \mathrm{~h}$ at $600^{\circ} \mathrm{C}$, xerogels with $\mathrm{Mg}: \mathrm{Ti}=$ $x: 1, \sim 1.3 \leq x \leq 2$, yielded a single nonstoichiometric qandilite-like phase, with the same cationic content as the precursor; if $1<x \leq \sim 1.3$ geikielite and nonstoichiomeric qandilite with $\mathrm{Mg}: \mathrm{Ti}=\sim 1.3: 1$ were formed; as $T$ raised the lower limit for single phase formation raised, until it became $\sim 2$ at $850-900{ }^{\circ} \mathrm{C}[10,12]$. The unit cell parameters of the qandilite-like phases were nearly independent of their composition [11]. An analogous behavior was observed for $\mathrm{Mg}: \mathrm{Ti}=1: x, 1<x \leq 2$, yielding metastable karrooite phases $[10,13,14]$, with unit cell parameters strongly dependent on composition. The nanocrystalline qandilite-like product with $\mathrm{Mg}: \mathrm{Ti}=1.75: 1$, after a primary annealing of $3 \mathrm{~h}$ at $600^{\circ} \mathrm{C}$, was subjected to a secondary annealing for $3 \mathrm{~h}$ at constant $T$ up to $1500^{\circ} \mathrm{C}$; inception of decomposition into geikielite and periclase was observed at $800{ }^{\circ} \mathrm{C}$, at $900-1100{ }^{\circ} \mathrm{C}$ extensive decomposition took place, leaving only traces of qandilite, and from $1300{ }^{\circ} \mathrm{C}$ upward the conventional equilibrium mixture of qandilite and geikielite was obtained [11].

The present work discusses the crystal structures derived from coprecipitated xerogel precursors with atomic ratio $\mathrm{Mg}: \mathrm{Ti}=x: 1,1 \leq x \leq 2$, during the first hour of thermal annealing at constant $T$, from 550 to $1300^{\circ} \mathrm{C}$, on the hot stage of an X-ray diffractometer.

\section{Experimental}

Synthesis was carried out with best quality laboratory reagents (Aldrich, Alfa, Fluka, Merck). Toluene solutions of magnesium and titanium metallorganics, with atomic ratios $\mathrm{Mg}$ :Ti from 1:1 to 2:1 were hydrolyzed with superheated steam, by the sol-gel method described elsewhere [15]. Water and most of the organic residuals left after the sol-gel process were eliminated by heating the xerogels in air for about $3 \mathrm{~h}$ at $400{ }^{\circ} \mathrm{C}$; trace organic residues were burned in situ while heating the samples in air to the temperature of the run.

High temperature X-ray powder diffractograms were obtained with a Siemens D5000 diffractometer (Bragg-Brentano parafocusing geometry and vertical $\theta-\theta$ goniometer). Nifiltered $\mathrm{Cu}-\mathrm{K} \beta$ radiation $(30 \mathrm{~mA}, 40 \mathrm{kV})$ and a Braun position-sensitive detector were used. The sample holder was an Anton-Paar HTK10 Pt ribbon heating stage, in static air. A mull was prepared with a small amount of the xerogel and a few drops of a special lacquer (Anton Paar), and was evenly spread on the Pt ribbon. After placing the thermal shield of the sample holder, the Pt ribbon was heated to $400{ }^{\circ} \mathrm{C}$ at a slow rate $\left(1^{\circ} \mathrm{C} / \mathrm{s}\right)$, to burn up the lacquer, and then to $T$ at $6 \%$ s. The first diffractogram of a run was recorded within about 6 min after $T$ was reached, and the others in succession every $6 \mathrm{~min}$, scanning the range $15^{\circ} \leq 2 \theta \leq 65^{\circ}$, at an angular rate of $10 \% \mathrm{~min}$. The Pt diffraction lines usually appeared in the diffractograms. The diffraction lines of the various phases showed a shift to lower $2 \theta$ angles as compared with the corresponding reported powder diffractograms [16], due to thermal expansion taking place on the hot stage. The diffractograms were processed with FullProf software [17]. 


\section{Results and discussion}

Four crystal structures were met in the present study: $\mathrm{NaCl}$ type (i), inverse spinel (qandilitelike) low-temperature structure (ii), stoichiometric qandilite (iii) and geikielite (iv). All diffraction patterns of the titanates showed that the powder was randomly oriented. However, the Pt ribbon was highly oriented presenting week 111 and strong 200 lines. Examples of refined structures are given in Table 1. The two structures (i+ii) are coherent and form a single quasi-amorphous phase below $700{ }^{\circ} \mathrm{C}$ at all concentrations other than $\mathrm{Mg}: \mathrm{Ti}=1: 1$. Structure (ii) is found at all concentrations below some temperature depending on the time of formation, $t$ and the $\mathrm{Mg}$ : Ti ratio. A pure (ii) structure was found for $\mathrm{Mg}: \mathrm{Ti}=2: 1$ at all temperatures, after $t \leq 15 \mathrm{~min}$. Structures (iii) and (iv) are phases in equilibrium. The structures formed from the xerogel start as cubic solid solutions. It seems that the factor controlling the structure in the early stage is grain size. After longer $t$ or at higher $T$, when the grain size crosses some threshold, the stoichiometric compounds segregate forming the structures of the equilibrium phase.

Table 1. Crystal data.

\begin{tabular}{|c|c|c|c|c|}
\hline $\begin{array}{l}\text { Structure } \\
\text { code }\end{array}$ & $\begin{array}{l}\text { Space } \\
\text { group }\end{array}$ & Cell parameters $[\AA]$ & $\begin{array}{l}\text { Linear expan- } \\
\text { sion coef. }\left[\mathrm{K}^{-1}\right]\end{array}$ & Atomic positions \\
\hline \multirow{4}{*}{$\begin{array}{l}\text { i } \\
\text { ii and iii }\end{array}$} & $\mathrm{Fm}-3 \mathrm{~m}$ & $\mathrm{a}=4.25$ at $550^{\circ} \mathrm{C}$ & - & M 4(a); O 4(b) \\
\hline & $\mathrm{F} \mathrm{d}-3 \mathrm{~m}$ & $\mathrm{a}=8.49$ at $600^{\circ} \mathrm{C}$ & $7.62 \times 10^{-6}$ & $\operatorname{Mg} 8(\mathrm{a}) ; \mathrm{Mg}, \mathrm{Ti}$ \\
\hline & & $\mathrm{a}=8.544$ at $1200^{\circ} \mathrm{C}$ & & $16(\mathrm{~d}) ; \mathrm{O} 32(\mathrm{e})$ \\
\hline & & & & 0.26 \\
\hline \multirow[t]{3}{*}{ iv } & $\mathrm{R}-3$ & $\mathrm{a}=5.093 ; \mathrm{c}=14.034$ at $800^{\circ} \mathrm{C}$ & a: $5.42 \times 10^{-6}$ & $\operatorname{Mg} 6$ (c) 0.1665 ; \\
\hline & & $\mathrm{a}=5.119 ; \mathrm{c}=14.121$ at $1200^{\circ} \mathrm{C}$ & c: $9.16 \times 10^{-6}$ & Ti 6 (c) 0.445 \\
\hline & & & & O 18 (f) 0.32 , \\
\hline
\end{tabular}

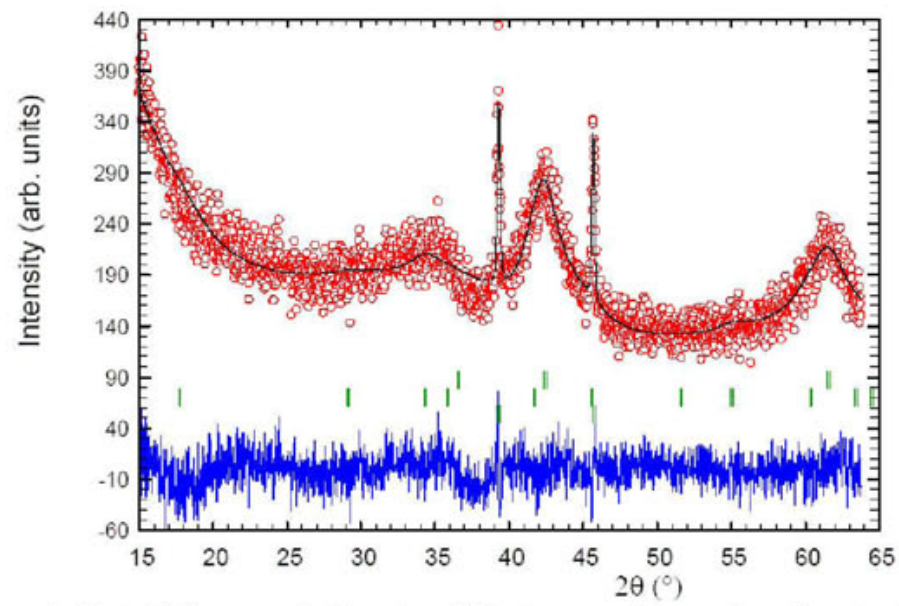

Figure 1. Rietveld diagram of a hot-stage diffractogram of magnesium titanate with $\mathrm{Mg}: T i=2: 1$, after $6 \min$ at $575^{\circ} \mathrm{C}$. 
Figure 1 shows a Rietveld diagram of an early stage of formation of $\mathrm{Mg}_{2} \mathrm{TiO}_{4}(t=6 \mathrm{~min})$. The diffractogram includes a major structure (i) of the $\mathrm{NaCl}$ type, a minor structure (ii) of inverse spinel type and the Pt ribbon. The diffraction lines are severely broadened (grain size $\sim 5 \mathrm{~nm}$ ), with excellent agreement between calculated and observed data. Figure 2 shows a Rietveld diagram of early stage formation of $\mathrm{MgTiO}_{3}(t=72 \mathrm{~min})$. The diffractogram includes a quasi-amorphous phase based on a single titanate qandilite-like structure (ii) and the Pt ribbon.

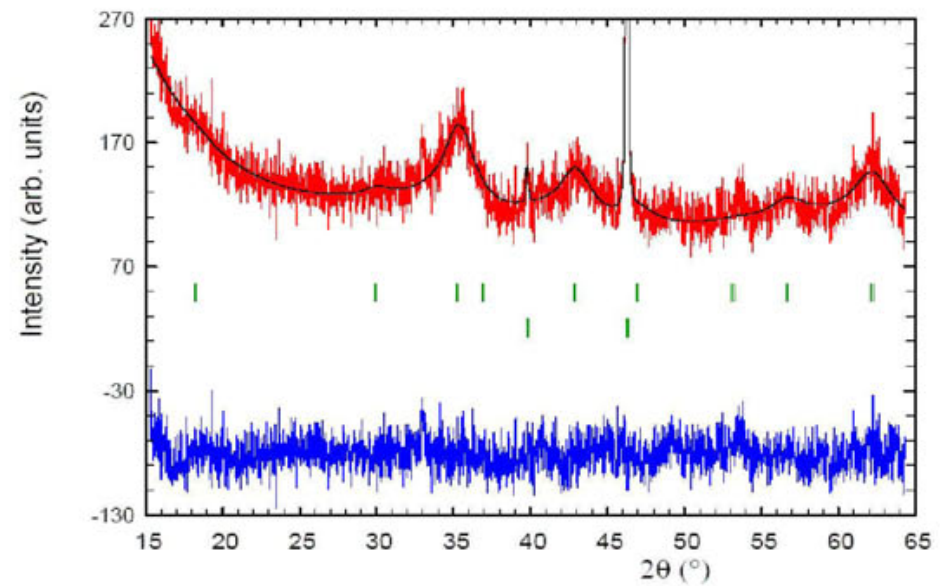

Figure 2. Rietveld diagram of hot-stage diffractogram of magnesium titanate with Mg:Ti $=1: 1$, after $72 \min$ at $600{ }^{\circ} \mathrm{C}$.

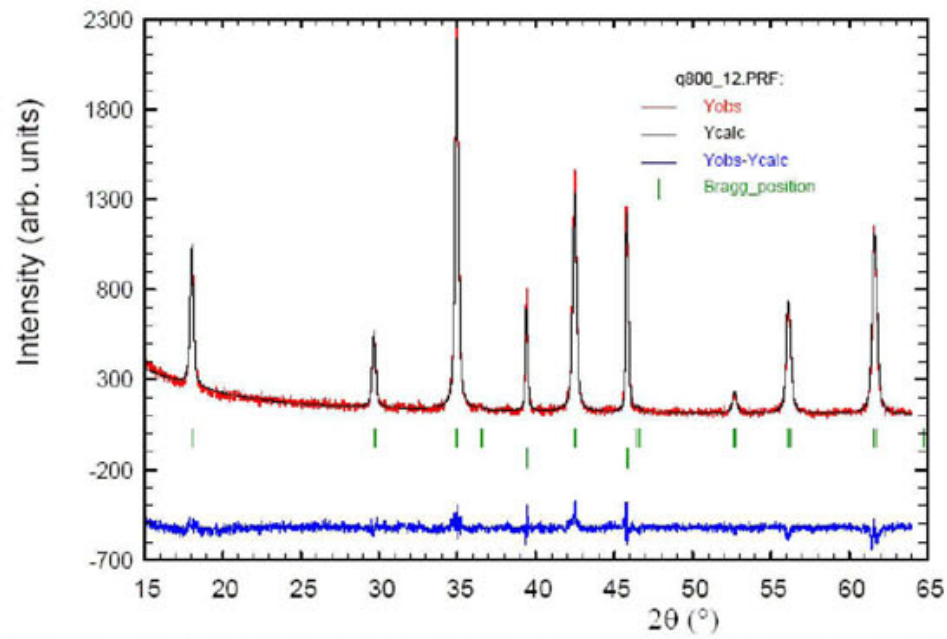

Figure 3. Rietveld diagram of hot-stage diffractogram of magnesium titanate with $\mathrm{Mg}: T i=2: 1$, after 72 min at $800{ }^{\circ} \mathrm{C}$. The sample is a well crystallized form of structure (ii). 
Figure 3 shows a Rietveld diagram of an intermediate stage of formation of $\mathrm{Mg}_{2} \mathrm{TiO}_{4}(t=72$ $\mathrm{min}$ ). The diffractogram includes a single qandilite-like structure (ii) and the Pt ribbon. In contrast to the equilibrium structure (iii) at much higher $T$, the qandilite-like structure is initially formed and stays as a dominant structure from 700 up to $1000{ }^{\circ} \mathrm{C}$. Small amount of geikielite appear only at 900 and $1000{ }^{\circ} \mathrm{C}$ after annealing for $t \geq 30 \mathrm{~min}$. At lower temperatures the initial structure is the $\mathrm{NaCl}$ (i) type. Figure 4 shows the early $(t=6 \mathrm{~min})$ phase development for xerogel with $\mathrm{Mg}: \mathrm{Ti}=1: 1$ composition. Until $650^{\circ} \mathrm{C}$ structure (ii) is dominant. Structure (iv), geikielite, starts appearing at $700{ }^{\circ} \mathrm{C}$. At $800{ }^{\circ} \mathrm{C}$ a single phase with structure (iv) is observed. At $650^{\circ} \mathrm{C}$ geikielite starts appearing only at $t \geq 30 \mathrm{~min}$. In the present study the structure (ii) was a single structure as long as it was annealed in the hot stage. It should be noticed that after several hours the xerogel is totally transforms into geikielite. $[11,13]$. Figure 5 shows the early $(\mathrm{t}=6 \mathrm{~min})$ phase development for xerogel with $\mathrm{Mg}: \mathrm{Ti}=1.4: 1 \mathrm{com}$ position. Until $700{ }^{\circ} \mathrm{C}$ the structure (ii) is dominant. Geikielite (iv) starts appearing at 800 ${ }^{\circ} \mathrm{C}$. Until $1000{ }^{\circ} \mathrm{C}$ non-equilibrium structure (ii) still exists. At $1100{ }^{\circ} \mathrm{C}$ the equilibrium state riches with structures (iii) and (iv).

\section{Summary and conclusions}

Xerogels with Mg:Ti $=x: 1,2 \geq x>1$, at $T<700{ }^{\circ} \mathrm{C}$, yield after a short annealing time $(t)$ products with cubic structure of NaCl-type. After a longer $t$ or at higher temperatures the inverse spinel type - structure (ii) - appears. Geikielite appears later and needs higher temperatures (until $1000^{\circ} \mathrm{C}$ ). It seems that grain size is the controlling factor. In very small grains ( $5 \mathrm{~nm}$ and less), the NaCl-type (i) is found as a major phase. When the grain size reaches $\sim 10 \mathrm{~nm}$ the inverse spinel (iii) is formed at all concentrations other than 1:1. Above some grain size geikielite begins to segregate from non-stoichiometric phases, according to the equilibrium phase diagram. In the present study, a state of equilibrium was attained only at high temperatures.

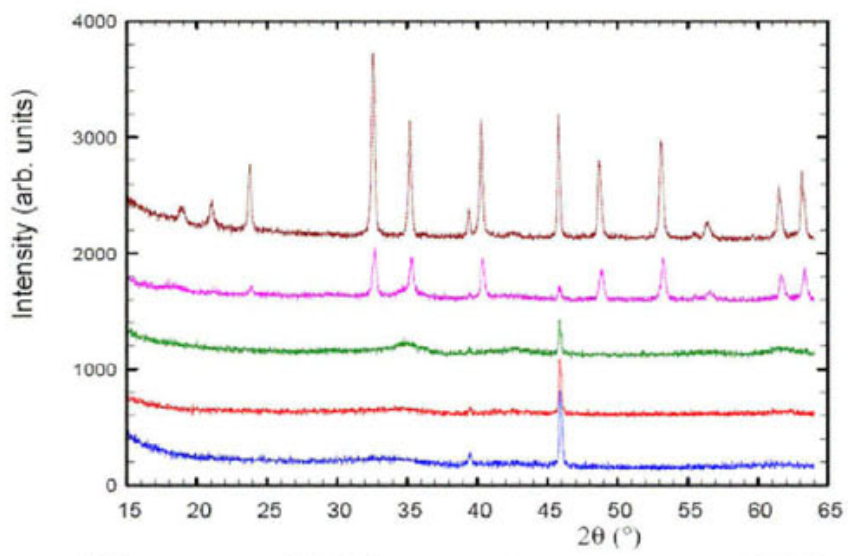

Figure 4. Hot-stage diffractometery of $\mathrm{MgTiO}_{3}$ composition at temperatures 550 (bottom), 600, 650 , $700,800^{\circ} \mathrm{C}$ (top) after $6^{\prime}$ heating. 


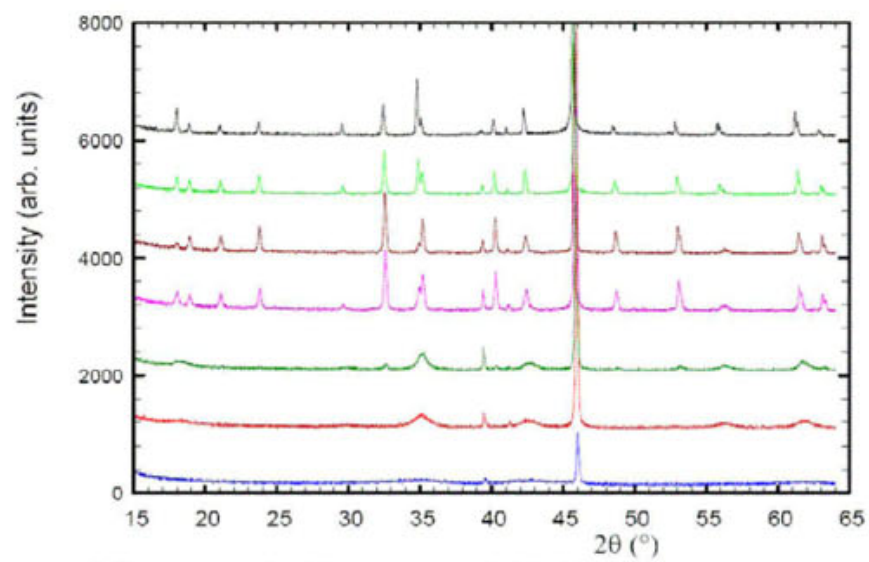

Figure 5. Hot-stage diffractometry for $\mathrm{Mg}$ titanate with $\mathrm{Mg}: \mathrm{Ti}=1.42$, at temperatures 550 (bottom), $700,800,900,1000,1100,1300{ }^{\circ} \mathrm{C}$ (top) after 6 ' heating.

\section{References}

1. Shindo, J., 1980, J. Cryst. Growth, 50,839.

2. Wechsler, B.A. \& Navrotsky, A., 1984, J. Solid. State Chem., 55, 165.

3. Wechsler, B.A. \& Von Dreele, R.B., 1989, Acta Cryst., B45, 542.

4. Swanson, H.E., Gilfrich, N.T. \& Ugrinic, G.M., 1955, Nat. Bur. Stand., Circ. 5, No. 539, p. 43.

5. De Grave, E, De Sitter, J. \& Vandenberghe, R., 1975, Appl. Phys., 7, 77.

6. Delamoye P. \& Michel, A., 1969, Compt. Rend. Acad. Sci., Ser. C, 269, 837.

7. Lind, M.D. \& Housley, R.M., 1972, Science, 175, 521.

8. Zabicky, J., Kimmel, G., Goncharov, E. \& Hazan, N., 1999, J. Metast. Nanocryst. Mater., 2-6, 191.

9. Zabicky, J., Zingerman, D., Shneck, R. \& Manor, E., 1996, Nanostruct. Mater., 7, 527.

10. Zabicky, J., Frage, N., Kimmel, G., Hazan, N., El-Fahel, H., Goncharov, E., Manor, E. \& Shneck, R., 1997, Phil. Mag. B, 76, 605.

11. Kimmel, G. \& Zabicky, J., 1999, Adv. X-Ray Anal., 42, 238.

12. Kimmel, G. \& Zabicky, J., 1998, Mater. Sci. Forum, 278-281, 624.

13. Kimmel, G., Zabicky, J. \& Ari-Gur, P., 1999, Acta Cryst. , A55, 580.

14. Zabicky, J., Kimmel, G., Goncharov, E. \& Dayan, D., 2001, Mater. Sci. Forum, 378-383, 741.

15. Zabicky, J., Zevin, L., Simon, E., Shneivais, A., Sason, U., Abramovich, L., Ondracek, G., Schüller, M. \& Fredel, M., 1993, Nanostruct. Mater., 3, 77.

16. International Centre for Diffraction Data, Powder Diffraction Files, Cards No. 4802, 6-494, 25-1157 \& 43-1022 (PA, Newton Square).

17. Rodrigez-Carvajal, J., 1997, Fullprof, Program for Rietveld refinement, Laboratories Léon Brillouin ,CEA-CNRS, (France, Saclay). 


\title{
In situ synchrotron powder diffraction study of the thermal decomposition of cement-asbestos: Preliminary results
}

\author{
A. F. Gualtieri ${ }^{1, *}$, M. Lassinantti Gualtieri ${ }^{1}$, \\ C. Meneghini ${ }^{2}$ \\ ${ }^{1}$ Department of Earth Sciences, University of Modena and Reggio Emilia, Italy \\ ${ }^{2}$ Physics Department, University of Roma Tre, Italy \\ *alessandro.gualtieri@unimore.it
}

Keywords: asbestos, chrysotile, cement-asbestos, in situ synchrotron powder diffraction

\begin{abstract}
The elimination of asbestos-containing materials like cement-asbestos, is an environmental priority. An industrial process for the safe recovery of cement-asbestos slates was recently developed and permits the thermal transformation of asbestos fibres into non-fibrous crystalline phases in a tunnel kiln. Optimisation of the process requires knowledge of the reaction dynamics. Here, time-resolved synchrotron powder diffraction was used to follow the thermal transformation of cement-asbestos. The use of a closed capillary as sample holder allowed to closely resemble the atmospheric conditions found in the industrial reactor. In this preliminary work, we describe the reaction sequence which undergoes cementasbestos during its thermal decomposition. The excellent time resolution of the collected data allowed the observation of meta-stable phases at non-ambient conditions.
\end{abstract}

\section{Introduction}

Asbestos minerals have been extensively used since the beginning of the last century. Since the early 50's, it is known that inhalation of asbestos fibres may cause lung diseases. For this reason, asbestos is now banned in many countries worldwide. Nevertheless, products manufactured before the ban of asbestos minerals may still be found in the environment. An example is cement-asbestos slates. With time, the risk of fibre dispersion in air increases due to progressive degradation of the slates. The cement-matrix in non-aged (i.e. cement-asbestos not subjected to degradation conditions) cement-asbestos generally contain typical Portland cement phases such as portlandite and CSH. With time, carbonation of these phases invariably takes place. Hence, cement-asbestos materials generally contain calcite as a major phase. Due to the risk of fibre dispersion, the destiny of asbestos-containing materials is to be removed and dumped in controlled waste plants. However, it is common feeling that this should not be the ultimate solution due to the risk of fibre dispersion in air during dumping and storage [1]. A promising alternative is the thermal transformation of asbestos containing 
materials to non-hazardous minerals which may be eventually recycled. An industrial process for the direct annealing of sealed packages of cement-asbestos slates at a temperature of ca. $1250^{\circ} \mathrm{C}$ for at least $16 \mathrm{~h}$ using a tunnel kiln was recently developed and patented [2].

To optimize the temperature cycle of the novel industrial process, it is important to gain a full understanding of the transformation sequence. To this aim, the reaction path of commercial cement-asbestos slates during a temperature ramp up to $1200^{\circ} \mathrm{C}$ was studied by in situ high temperature X-ray powder diffraction (HT-XRPD) using an in-house instrument [3]. Although this previous work provided a relatively detailed description of the events occurring during firing of this system, the limited time resolution offered by the in-house instrument may was not adequate to obtain a complete picture of the temperature-induced reaction pathway, e.g. appearance and disappearance of metastable phases. An alternative experimental setup which offer considerable higher time resolution and hence, the possibility to observe metastable phases, is the use of a synchrotron radiation source in combination with an imaging plate (IP) detector.

This contribution reports the preliminary results of the in situ high temperature synchrotron powder diffraction study of the thermal transformation of cement-asbestos. An important detail in the experimental setup used in the present work is that the sample is sealed in a capillary. Opposed to previous in situ investigations using an in-house instrument [3], the partial pressure of $\mathrm{CO}_{2}$ gradually increases in the capillary as a result of decomposition of calcite. These conditions are more closely resembling the ones found in the designed industrial reactor [2].

\section{Experimental}

Data for the In situ High Temperature Synchrotron Powder Diffraction (HT-SPD) were collected at beamline BM08 (GILDA) at the European synchrotron radiation facility (ESRF), Grenoble (France) using a sample of commercial cement-asbestos. The sample was enclosed in a quartz capillary with a diameter of $0.5 \mathrm{~mm}$, and mounted on a spinned goniometer head. A parallel beam Debye geometry was used. The wavelength was $0.68888 \AA$, determined using FIT2D and the NIST SRM $640 \mathrm{~b}$ Si standard with $\mathrm{a}=5.43094$ (4) at $25^{\circ} \mathrm{C}$. A selected portion of the diffraction rings were collected using an imaging plate (IP) detector working in the translating mode. The IP slit size was $2 \mathrm{~mm}$ and the IP detector was mounted perpendicular the incoming beam at a distance of about $277.2 \mathrm{~mm}$ from the sample. The sample temperature was regulated by a hot air stream generated by a heating gun placed vertically under the capillary. The sample was heated from 25 to $900{ }^{\circ} \mathrm{C}$ using a temperature gradient of $3.8^{\circ} \mathrm{C} / \mathrm{min}$. Following a $10 \mathrm{~min}$ isotherm at $900^{\circ} \mathrm{C}$, the sample was cooled down to 300 ${ }^{\circ} \mathrm{C}$ with a rate of $5^{\circ} \mathrm{C} / \mathrm{min}$. The temperature of the experiment was monitored with a thermocouple positioned about half a millimetre below the sample. The image stored in the IP detector was recovered using a Fuji BAS2500 laser scanner ( 16 bit/pixel with a minimum pixel size of $50 \times 50 \mathrm{~mm}$ ). Data were extracted using original software available at BM08 and powder patterns were extracted in $10 \mathrm{~min}$ slices which corresponds to data every $38{ }^{\circ} \mathrm{C}$ during heating and $50^{\circ} \mathrm{C}$ during cooling. Mineralogical identification was performed using X'Pert Highscore Plus (PANalytical, version 2.1) with a PDF2 reference data base implemented in the software. Quantitative phase analyses of the crystalline fraction in the sample was per- 
formed using the in situ data and Rietveld refinements which were carried out with GSAS [4] with its graphical interface EXPGUI [5].

Scanning electron microscope (SEM) images of specimens of the sample before and after the HT-SPD experiment were collected using a Philips XL 30 instrument.

\section{Results and discussion}

Figure 1 shows a selected $2 \theta$ range collected in situ during heating up to $900{ }^{\circ} \mathrm{C}$ and subsequent cooling down to $300^{\circ} \mathrm{C}$. The compositions of the crystalline fraction, as determined by the Rietveld refinements, of cement-asbestos before the in situ experiment at $25^{\circ} \mathrm{C}$, at 900 ${ }^{\circ} \mathrm{C}$ and after cooling to $300{ }^{\circ} \mathrm{C}$ are shown in table 1 . The agreement factors are: $\mathrm{Rwp}=7.8$ $8.5 \%, \mathrm{Rp}=5.5-6.2 \%$. The non-treated sample shows calcite, clinochrysotile, C2S $\left(2 \mathrm{CaO} \times \mathrm{SiO}_{2}\right)$, from the anhydrous cement, as well as minor quartz and dolomite $\left(\mathrm{CaMg}\left(\mathrm{CO}_{3}\right)_{2}\right)$ as filler components. This composition is similar to that found by Dias et al. [6]. Unfortunately, the amorphous fraction could not be quantified as such analyses require the addition of an internal standard which may interfere with the high-temperature reactions under study and render difficult phase identification due to possible peak overlap. However, earlier papers report that cement-asbestos generally contain ca $25-30 \mathrm{wt} \%$ amorphous material before and after thermal transformation, respectively [3]. At $900{ }^{\circ} \mathrm{C}$, the major event is the recrystallization of $\mathrm{C} 2 \mathrm{~S}$, accompanied by minor residual calcite.

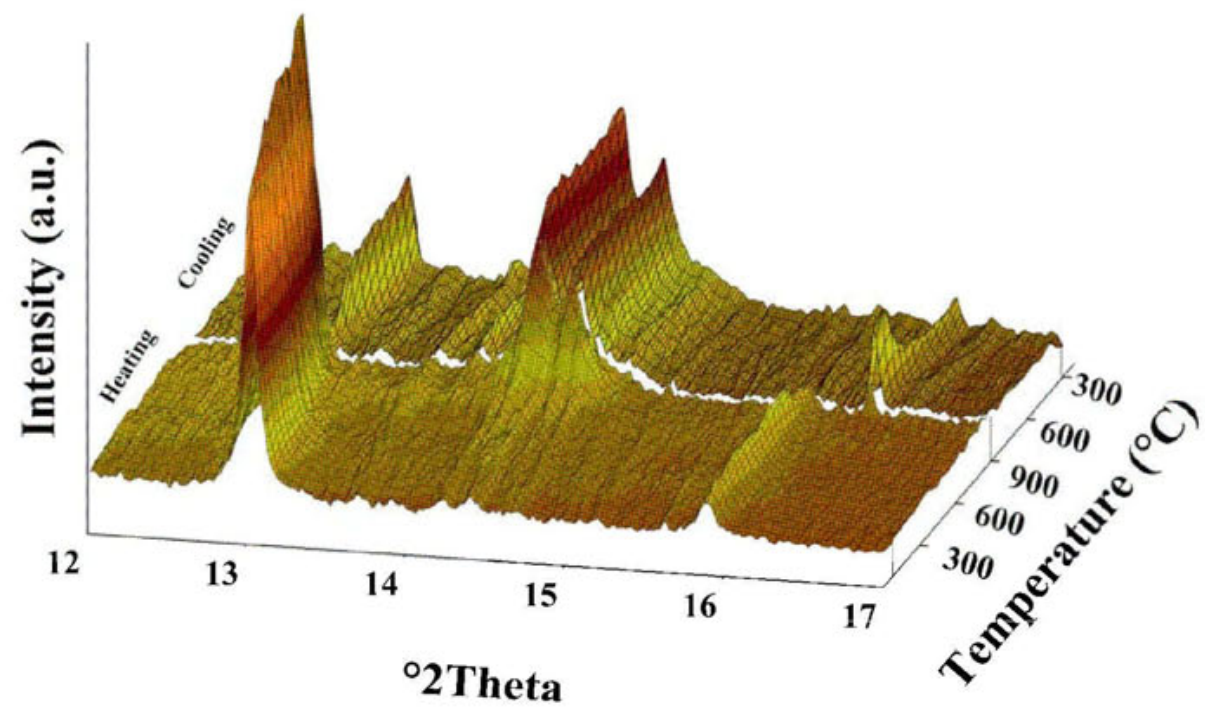

Figure 1 . Selected ${ }^{\circ} 2 \theta$ range of powder patterns collected in situ during heating up to $900{ }^{\circ} \mathrm{C}$ and subsequent cooling down to $300^{\circ} \mathrm{C}$, showing important temperature-induced changes in the mineralogical composition. 
Table 1. The mineralogical composition of cementasbestos before the in situ HT-SPD experiment at 25 ${ }^{\circ} \mathrm{C}$, at $900{ }^{\circ} \mathrm{C}$ and after cooling to $300^{\circ} \mathrm{C}$.

\begin{tabular}{|l|l|l|l|}
\hline \multirow{2}{*}{ Phase } & \multicolumn{3}{|c|}{ Weight (\%) } \\
\cline { 2 - 4 } & $25{ }^{\circ} \mathrm{C}$ & $900{ }^{\circ} \mathrm{C}$ & $300{ }^{\circ} \mathrm{C}$ \\
\hline chrysotile & $10.6(9)$ & - & - \\
\hline$\beta$-larnite & $9.1(4)$ & $78.9(4)$ & $75.8(5)$ \\
\hline calcite & $73.5(2)$ & $4.5(5)$ & $8.5(5)$ \\
\hline dolomite & $0.8(1)$ & - & - \\
\hline quartz & $6(2)$ & - & - \\
\hline forsterite & - & $1.3(7)$ & $5.2(9)$ \\
\hline anhydrite & - & $3.1(7)$ & $1.3(2)$ \\
\hline lime & - & $5.8(2)$ & $4.1(4)$ \\
\hline rankinite & - & $3.3(9)$ & $3(1)$ \\
\hline magnesite & - & - & $1.2(6)$ \\
\hline wollastonite & - & $3.0(8)$ & $0.8(6)$ \\
\hline
\end{tabular}
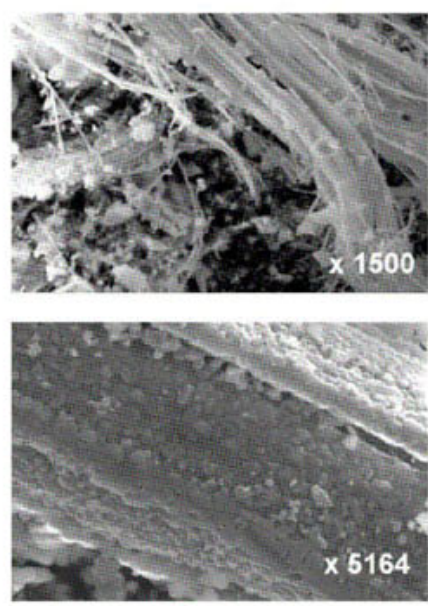

Figure 2. SEM image of the cementasbestos sample before (a) and after (b) the in situ HT-SPD experiment.

Chrysotile, quartz and dolomite decompose with temperature. Figure 2 shows SEM images of the raw (a) and cement-asbestos sample (b) after the in situ HT-SPD experiment. See a thorough description in [3]. The $\mathrm{C} 2 \mathrm{~S}$ polymorph observed in this system is monoclinic $\beta$ larnite which is stable in the investigated temperature range [7]. C2S recrystallization was due to the combination of large fraction of lime $(\mathrm{CaO})$ after calcite decomposition $\left(\mathrm{CaCO}_{3} \leftrightarrow \mathrm{CaO}+\mathrm{CO}_{2}\right)$ and $\mathrm{SiO}_{2}$, possibly present in the original amorphous phase, and to a lesser extent made available by the decomposition of quartz and chrysotile. Lime has not been totally consumed for the formation of $\mathrm{C} 2 \mathrm{~S}$ and is still present at $900{ }^{\circ} \mathrm{C}$. In [3] the authors showed that lime crystallized in cement-asbestos during thermal treatment combines with silica and metals (e.g. magnesium, iron, aluminum) to form typical clinker phases such as $\mathrm{C} 2 \mathrm{~S}$, ferrite $\left(\mathrm{Al}_{2} \mathrm{Ca}_{4} \mathrm{Fe}_{2} \mathrm{O}_{10}\right)$ and merwinite $\left(\mathrm{Ca}_{3} \mathrm{Mg}\left(\mathrm{SiO}_{4}\right)_{2}\right)$. Only $\mathrm{C} 2 \mathrm{~S}$ was observed here, possibly due to a lower maximum temperature compared to previous experiments [3]. The excess of lime with respect to $\mathrm{SiO}_{2}$ likely prompted the crystallization of rankinite $\mathrm{Ca}_{3} \mathrm{Si}_{2} \mathrm{O}_{7}$ (C3S2), and wollastonite $\mathrm{CaSiO}_{3}(\mathrm{CS})$, observed at $900{ }^{\circ} \mathrm{C}$. The wollastonite polymorph observed here is actually the triclinic 1A [8]. Other newly-formed phases are forsterite and anhydrite. Forsterite is one of the earlier products of the chrysotile decomposition. In fact, chrysotile decomposes at around $650{ }^{\circ} \mathrm{C}$ followed by a prompt crystallization of enstatite and forsterite [9]. Forsterite is stable throughout the heating cycle, whereas enstatite decomposes upon further temperature increase. The crystallization of minor anhydrite $\left(\mathrm{CaSO}_{4}\right)$ is possibly due to the decomposition of ettringite [10] which has not been identified in the original sample due to its poor crystallinity. The sample cooled down to $300^{\circ} \mathrm{C}$ exhibits more or less the composition of the high temperature sample. However, the calcite content has increased and magnesite has formed. These phases witness a re-carbonation process of the sample due to 
the presence of residual $\mathrm{CO}_{2}$ inside the capillary. Calcite may have formed at expenses of poorly crystalline anhydrite and lime and partly $\mathrm{C} 2 \mathrm{~S}$ as they all show a decrease in their relative weight fraction back to $300{ }^{\circ} \mathrm{C}$. Wollastonite is also apparently not stable during cooling as its weight fraction reduces to about $1 \%$.The complete reaction path from the in situ experiment is reported in figure 3 which shows the temperature ranges of all identified crystalline phases in the sample. The thickness of the bars representing each phase varies according to the intensity of the diffraction peaks.

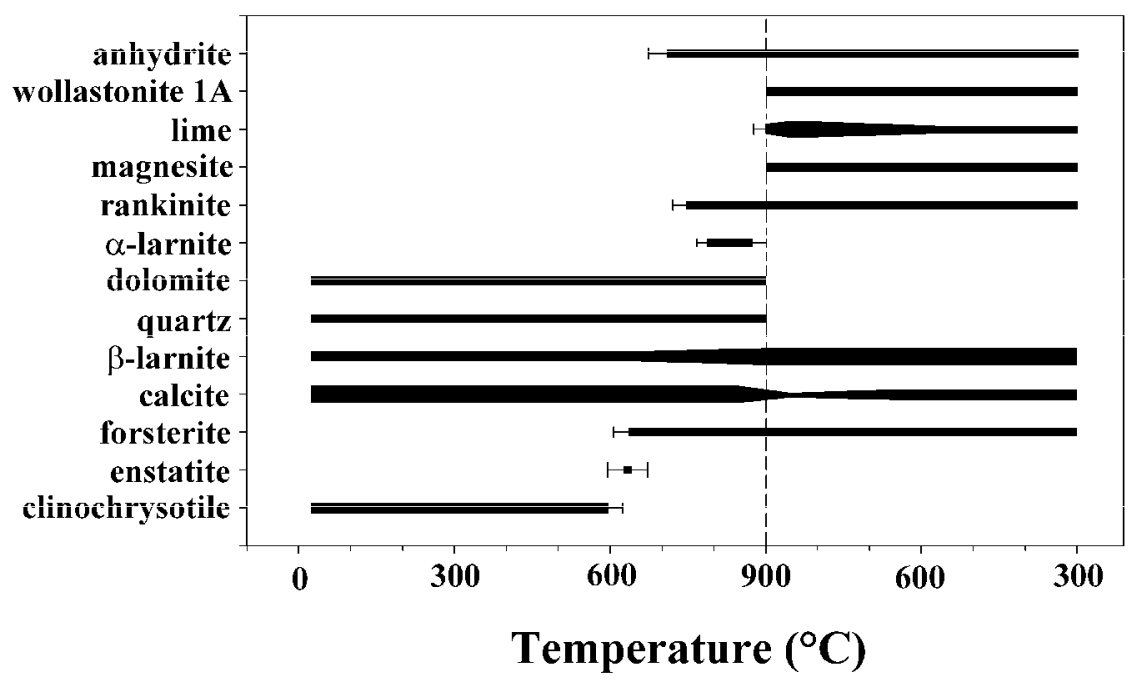

Figure 3. The evolution of the phase assemblage of the cement-asbestos system during the thermal cycle $25-900-300^{\circ} \mathrm{C}$.

Besides the reactions described above, the picture shows the presence of two metastable phases in the system, enstatite and $\alpha$-larnite (C2S). The peaks of monoclinic $\alpha$-larnite $\left(\alpha_{\mathrm{L}}^{\prime}\right)$, one of the high temperature polymorphs of $\mathrm{C} 2 \mathrm{~S}$ have been observed in a narrow temperature range $\left(800-900{ }^{\circ} \mathrm{C}\right)$ which is consistent with literature data [7]. Calcite decomposes in the range $850-900{ }^{\circ} \mathrm{C}$. This reaction is concomitant with the crystallization of lime which combines with silica to form C2S. Partial calcite recrystallization is observed during cooling (see table 1). This observation is explained by the high partial pressure of $\mathrm{CO}_{2}$ in the closed capillary due to decomposition of calcite which allows for re-carbonation of lime during cooling. This process is considered beneficial for two reasons: i) The emission of $\mathrm{CO}_{2}$, which is considered a serious threat to the environment, is reduced; ii) For recycling of thermally treated cement-asbestos in e.g. stoneware tile mixtures, residual lime in the transformation product is detrimental for the rheology of the mixture slurry, due to the formation of calcium hydroxide [11]. 


\section{Conclusions}

This paper reports the preliminary results of the in situ HT-SPD study of the thermal transformation of cement-asbestos. A more detailed description of the results will be published elsewhere [12]. For the experiment, the sample was sealed in a closed capillary in order to mimic the conditions in the real industrial process where the packs of slates are sealed in a tunnel kiln. Calcite, the major phase in aged cement-asbestos, decomposes in the range 850$900{ }^{\circ} \mathrm{C}$ to lime and $\mathrm{CO}_{2}$. Consequently, the partial pressure of $\mathrm{CO}_{2}$ gradually increases in the closed system. A large fraction of the newly crystallized lime combines with silica to form $\mathrm{C} 2 \mathrm{~S}$. Due to the high partial pressure of $\mathrm{CO}_{2}$, calcite recrystallizes during cooling by a recarbonation process of e.g. residual lime. Hence, a part $(4 \mathrm{wt} \%)$ of the initial fraction of calcite is recovered.

\section{References}

1. Paglietti, F., Zamengo, L., Polizzi, S., Giangrasso, M. \& Fasciani G., 2002,. in: Atti del Congresso L'Industria e l'amianto I nuovi materiali e le nuove tecnologie a dieci anni dalla Legge 257/1992, Rome 26-28 November 2002, 229-249.

2. Gualtieri, A.F. \& Zanatto, I., 2007, European Patent Nr. EP07425495.

3. Gualtieri, A.F., Cavenati, C., Zanatto, I., Meloni, M., Elmi, G. \& Lassinantti Gualtieri, M., 2008, J. Hazard. Mater., 152, 563.

4. Larson, A.C. \& von Dreele, R.B., 1994, “GSAS Generalized structure analysis system” Laur 86-748, Los Alamos National Laboratory, Los Alamos, New Mexico.

5. Toby, B.H., 2001, J. Appl. Cryst., 34, 210.

6. Dias, C.M.R., Cincotto, M.A., Savastano Jr., H. \& John, V.M., 2008, Cement Concrete Comp., 30, 255.

7. Taylor, H.F.W., 1990, Cement Chemistry (Academic Press Harcourt Brace Jovanovich, Publishers) p. 475.

8. Swamy, V. \& Dubrovinsky, L.S., 1997, Geochim. Cosmochim. Acta. 61, 1181.

9. Cattaneo, A., Gualtieri, A.F. \& Artioli, G., 2003, Phys. Chem. Miner., 30, 177.

10. Hall C., Barnes P., Billimore A.D., Jupe A.C., \& Turrillas X., 1996, J. Chem. Soc., Faraday Trans., 92, (12), 2125.

11. Gualtieri, A.F. \& Tartaglia, A., 2000, J. Eur. Cer. Soc., 20, 1409.

12. Gualtieri A.F., Lassinantti Gualtieri M. \& Meneghini C., 2008, Powder Diffr., in press.

Acknowledgements. The synchrotron experiment at beamline BM08 (ESRF, exp. ref. no. 08-02/641 BM08) was granted by the Italian institutions CNR, INFM and INFN. This work is part of a general project granted by ZETADI s.r.1. (Ferno, Italy) within a research contract with the Earth Sciences Dept., The University of Modena and Reggio Emilia (Italy). 


\title{
In-situ XRD investigations of the influence of PDADMAC on ettringite formation in cement systems
}

\author{
D. Jansen ${ }^{1, *}$, F. Goetz-Neunhoeffer ${ }^{1}$, J. Neubauer ${ }^{1}$, \\ W.-D. Hergeth ${ }^{2}$, R. Haerzschel ${ }^{2}$ \\ ${ }^{1}$ GeoZentrum Nordbayern, Mineralogy, University of Erlangen, Germany \\ ${ }^{2}$ Wacker Polymer Systems GmbH \& Co. KG, Burghausen, Germany \\ djan@geol.uni-erlangen.de
}

Keywords: OPC, PDADMAC, Ettringite, lattice parameters

\begin{abstract}
Since polymers are known to be responsible for the emergence of important properties and the microstructure in mortar systems, our focus was on the influence of the polymer PDADAC on cement hydration. The examinations were focused specifically on the formation of the phase ettringite which is generated during the hydration of Ordinary Portland Cement (OPC). The investigations of the formation of ettringite in the presence of PDAMAC were carried out by means of X-ray diffraction analysis in combination with Rietveld refinement. During hydration of OPC in the presence of low PDADMAC concentrations no significant changes of the ettringite lattice parameters could be determined. After synthesis of pure ettringites only a very small increase of the lattice parameter $a$ occurs, likely due to chloride incorporation.
\end{abstract}

\section{Introduction}

Cementitious building materials generally are modified and optimized by polymers [1]. Polymers fulfil different tasks during the hardening of the mortars and confer decisive properties on the hardened mortar, as the microstructures of mortar systems are influenced by the presence of polymers $[2,3,4]$.

Since many dry mortar systems are based on Portland cements, the influences of polymers on the hydration of cements are a topic of great interest $[5,6,7,8,9]$. During the hydration of Portland cements, we see the formation of the crystalline phases portlandite, ettringite, and of an amorphous C-S-H gel [10]. Determination of the lattice parameters of ettringite during hydration offers one possibility to determine the influence of polymers on the hydration behaviour of cements.

In the performed investigations we investigated a CEM I 52.5 R Ordinary Portland Cement (OPC). The aim of this work was to investigate changes in the lattice parameters of the ettringites formed due to a possible incorporation of parts of the polymer. 


\section{Experimental section}

Firstly, investigation was made of the influence of PDADMAC on ettringite formation during hydration of the CEM I 52.5 R. Secondly ettringites were synthesized from suspension [11] with and without PDADMAC added. We chose to set the amount of polymer at 1 ma.$\%$, due to the amount of cement, and at $1 \mathrm{ma} .-\%$ in the suspensions, due to the expected amount of the synthesized ettringite. This concentration of the polymer is typical for business applications. The polymer was dissolved respectively in water used for mixing with cement for in-situ X-ray investigations and in water used for dissolution of the chemicals for the syntheses. The structure of PDADMAC is shown in figure 1.

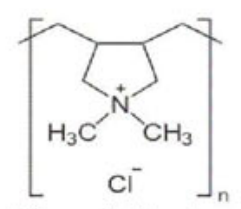

Figure 1. Structure of PDADMAC.

The selected CEM I 52.5 R was analyzed by Rietveld refinement. To this end the cement was ground to a particle size below $30 \mu \mathrm{m}$. To achieve an accurate quantitative determination of the phases in the 12-phase composite, selective dissolution experiments were performed as described in Refs. $[12,13]$. The residues and the original CEM I $52.5 \mathrm{R}$ were investigated five times. The structural and phase-relevant data obtained from the residues were used for the quantitative analysis of the cement.

The following measurement conditions at a Siemens D5000 diffractometer were applied:

Range: $10^{\circ}-70^{\circ} 2 \theta$; Step: $0.02^{\circ} 2 \theta$; Time/Step: $3 \mathrm{~s}$; Radiation: Copper $\mathrm{K} \alpha$; Generator: $30 \mathrm{~mA}, 40 \mathrm{kV}$; Divergence slit: $0.5^{\circ}$; Detector Slit: $0.2 \mathrm{~mm}$

The information obtained from the accurate analysis of the CEM I 52.5 R used was then used for establishing a refinement project for the cement during hydration. Lattice parameters and crystallite sizes of the clinker phases from the refinement of the cement were employed. Additionally the structures of ettringite and portlandite were added to the refinement project. The phase evolution in the cement pastes and especially the lattice parameters of the ettringites were investigated in-situ [14] by X-ray diffraction at a temperature of $23{ }^{\circ} \mathrm{C}$. The samples were prepared as follows:

- Mixing of the cement with water/water-polymer solution for $60 \mathrm{~s}$,

- Preparation of the pastes into a special sample holder.

- $\quad$ Covering of the pastes with a $7 \mu \mathrm{m}$ Kapton polymide film.

- Measurements were started about $2 \mathrm{~min}$ after the addition of water.

After preparation, the samples were investigated for 22 hours, recording 48 ranges. The two systems investigated (namely, with and without polymer) were measured three times respectively. For the in-situ XRD investigations of the hydration of the CEM I $52.5 \mathrm{R}$, the following measurement conditions at a Siemens D5000 diffractometer, equipped with a SolX detector, were employed:

Range: $7^{\circ}-36^{\circ} 2 \theta$; Step: $0.02^{\circ} 2 \theta$; Time/Step: $0.7 \mathrm{~s}$; Radiation: Copper $\mathrm{K} \alpha$; Generator: $30 \mathrm{~mA}, 40 \mathrm{kV}$; Divergence slits: $0.5^{\circ}$; Detector Slit: $0.2 \mathrm{~mm}$ 
Pure ettringite was synthesized using sucrose with and without PDADMAC three times respectively. The synthesized ettringites were dried under defined atmosphere [11]. The dried ettringites were subjected to X-ray diffraction analysis five times for each synthesis. The lattice parameters of the synthesized, dried ettringites were determined by Rietveld refinement from X-ray patterns obtained employing a Siemens D5000 diffractometer equipped with a scintillation counter. The measurements were done for a diffraction angle range from $7^{\circ}$ to $70^{\circ}$. The same conditions as for the quantitative analysis of the pure cement were employed (see above).

\section{Results and discussion}

The selected OPC was analyzed by calibrated Rietveld refinement. Lattice parameters of cubic and orthorhombic $\mathrm{C}_{3} \mathrm{~A}$ were calculated from Rietveld refinements of XRD patterns of the residue of the salicylic-methanol extraction of the OPC. Belite and $\alpha^{\prime}-\mathrm{C}_{2} \mathrm{~S}$ parameters were taken from the refinement of the residue of the $\mathrm{KOH}$ extraction. The mineralogical composition determined for the selected OPC is shown in table 1.

Table 1. Quantitative phase composition of the CEMI $52.5 \mathrm{R}$.

\begin{tabular}{|c|c|c|}
\hline Phase & ma.-\% & Standard deviation [ma.-\%] \\
\hline Alite & 60 & 2 \\
\hline Belite & 14 & 1 \\
\hline$\alpha$ - $\mathrm{C}_{2} \mathrm{~S}$ & 10 & 1 \\
\hline $\mathrm{C}_{3} \mathrm{~A}_{\text {cubic }}$ & 4 & 1 \\
\hline $\mathrm{C}_{3} \mathrm{~A}_{\text {orthorhombic }}$ & 3 & 1 \\
\hline $\mathrm{C}_{4} \mathrm{AF}$ & 2 & 0.5 \\
\hline Gypsum & 1 & 0.5 \\
\hline Bassanite & 1 & 0.5 \\
\hline Anhydrite & 2 & 0.5 \\
\hline Calcite & 2 & 0.5 \\
\hline Quartz & $<$ determination limit & \\
\hline Arcanite & 1 & 0.5 \\
\hline
\end{tabular}

Figure 2 shows an example of the refined XRD patterns of the cement hydration after $3 \mathrm{~h}$. The values for the GOF of the refinements were all situated between 1.1 and 1.2, and were strongly dependent on the fit of the major cement phase alite. 


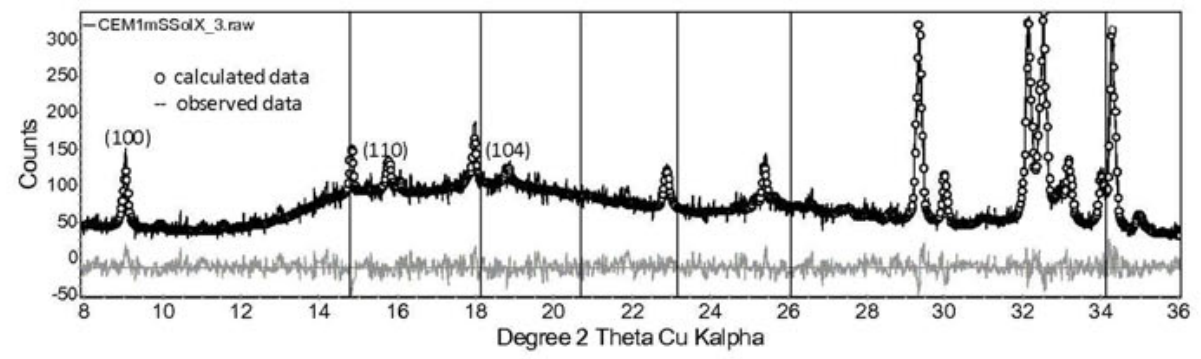

Figure 2. Rietveld refinement of the hydrating CEMI 52.5 R (hydration time: 3 hours).

The values for the lattice parameters of the ettringites formed in the cement pastes were determined. Figures 3 and 4 show the results of the determination during $22 \mathrm{~h}$ of hydration. The plotted values are mean values calculated from three measurements.

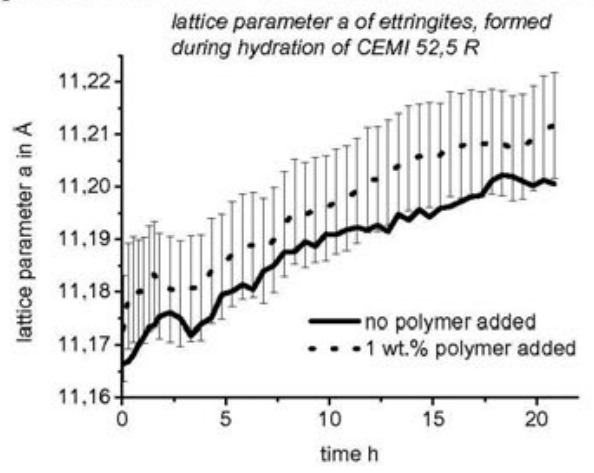

Figure 3. Development of lattice parameter a of ettringite within $22 \mathrm{~h}$ of hydration of the OPC without polymer (continuous line) and with 1 ma.-\% PDADMAC (dotted line).

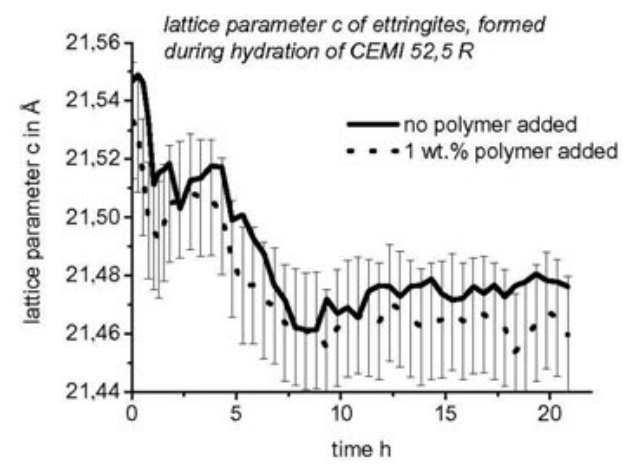

Figure 4. Development of lattice parameter $c$ of ettringite within $22 \mathrm{~h}$ of hydration of the OPC without polymer (continuous line) and with 1 ma.-\% PDADMAC (dotted line). 
The error bars shown in figures 3 and 4 characterize the precision of the lattice parameter determination. The errors determined for the system without polymer added are comparable to the errors for the systems with polymer added; the corresponding error bars have been omitted for the sake of clarity.

The lattice parameters $a$ and $c$ of the ettringites formed during hydration of the CEM I 52.5 R with and without polymer do not show marked differences within the error bars. Yet, two distinct trends for the lattice parameters can be observed. While the lattice parameter $a$ of the ettringites formed tends to increase during hydration, the lattice parameter $c$ tends to decrease. These results are in accordance with other research results already published [15].

The synthesized ettringites from three syntheses were subjected to X-ray diffraction analysis five times. Figure 5 shows an example of a refinement of the synthesized ettringites. In all refinements a very good fit was achieved.

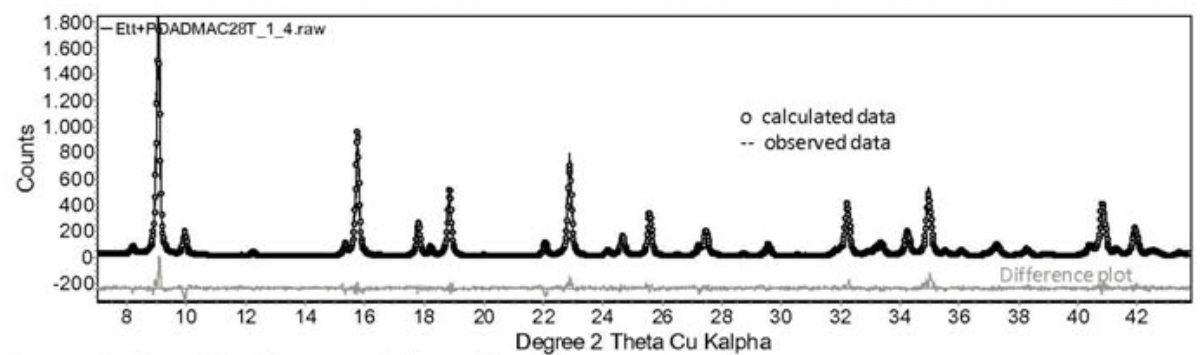

Figure 5. Rietveld refinement of the synthesized ettringites.

Figure 6 gathers the arithmetic mean lattice parameters of ettringite obtained from 15 measurements with and without PDADMAC addition during synthesis.

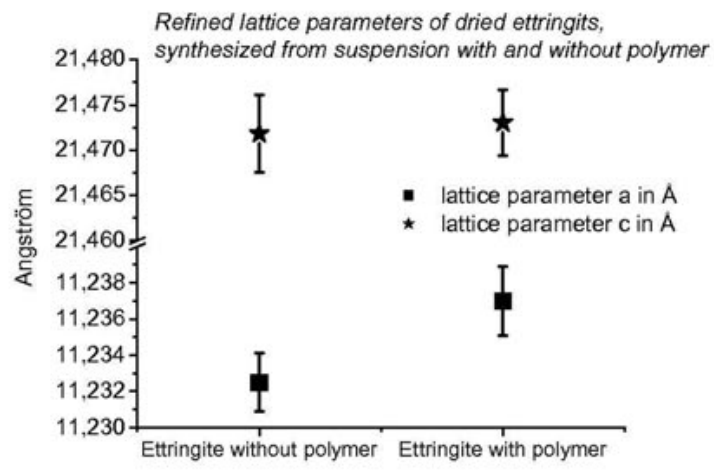

Figure 6. Lattice parameters of the synthesized ettringites.

No difference could be detected between the lattice parameter $c$ of ettringites synthesized in the presence of the polymer and the lattice parameter $c$ of ettringites synthesized in the absence of the polymer. However, the lattice parameter $a$ of the ettringites synthesized in the presence of the polymer is distinctly larger than the lattice parameter $a$ of ettringites synthe- 
sized in the absence of the polymer. This marginal increase is definitely too small to be caused by the incorporation of parts of the polymer chains in the channel of the ettringite structure. A change in the anionic composition (e.g. chloride) of the synthesis solution caused by the polymer is more likely.

These results demonstrate that the addition of PDADMAC does not affect the ettringite lattice parameters. Further data for the hydration of OPC with respect to quantitative phase development with polymer addition have to be evaluated to show whether or not PDADMAC influences OPC hydration.

\section{References}

1. Schulze, J. \& Killermann, O., 2001, Cem. Concr. Res., 31, 357.

2. Dimmig-Osburg, A., Pietsch, I. \& Pakusch, J., 2006, ZKG International, 5, 72.

3. Jenni, A., Holzer, L., Zurbriggen, R. \& Herwegh, M., 2005, Cem. Concr. Res., 35, 35.

4. Kim, J.-H., Robertson, R.E. \& Naaman, A.E., 1999, Cem. Concr. Res., 29, 407.

5. Atkins, K.M., Edmonds, R.N. \& Majumdar, A.J., 1991, J. Mater. Sci., 26, 2372.

6. Chandra, S. \& Flodin, P., 1987, Cem. Concr. Res., 17, 875.

7. Georgescu, M., Puri, A., Coarna, M., Voicu, G. \& Voinitchi, D., 2002, Cem. Concr. Res., 32, 1269.

8. Mansur, A.A.P., Santos, D.B. \& Mansur, H.S., 2007, Cem. Concr. Res., 37, 270.

9. Silva, D.A. \& Monteiro, P.J.M., 2006, Cem. Concr. Res., 36, 1501.

10. Bensted, J., 1991, World Cement, pp. 27-32.

11. Goetz-Neunhoeffer, F., Neubauer, J. \& Schwesig, P., 2006, Cem. Concr. Res., 36, 65.

12. Gutteridge, W.A., 1979, Cem. Concr. Res., 9, 319.

13. Struble, L., 1985, Cem. Concr. Res., 15, 631.

14. Hesse, C., Degenkolb, M., Gäberlein, P., Götz-Neunhoeffer, F., Kutschera, M., Neubauer, J. \& Schwarz, V., 2007, GDCh-Monographie, 37, 213.

15. Neubauer, J., Götz-Neunhoeffer, F., Schmitt, D., Degenkolb, M. \& Holland, U., 2006, Tagungsbericht IBAUSIL, Weimar, 16, 1-0375. 
IV.4.2 Determination of Crystal Structure; Structure Refinement 



\title{
Structural and spectroscopic properties of $\mathrm{BaHfO}_{3}: \mathrm{Eu}$ - the issue of the dopant location in the host lattice
}

\author{
A. Dobrowolska, E. Zych*
}

University of Wroclaw, Faculty of Chemistry, 14 F. Joliot - Curie, 50-383 Wroclaw, Poland

*zych@wchuwr.pl

Keywords: Barium hafnate, luminescence, site-selective spectroscopy, defects

Abstract. $\mathrm{BaHfO}_{3}$ : Eu powders containing $0-10 \%$ of the dopant with respect to Hf were synthesized with the classic ceramic method at $1400^{\circ} \mathrm{C}$. X-ray diffraction analysis proved that up to $10 \%$ of the Eu concentration the product is crystallographically pure and crystallizes in a cubic perovskite-type structure. Detailed structural measurements showed that the size of the unit cell decreases up to the Eu concentration of 3\% and increases when the dopant content is further increased. Spectroscopic analysis gave evidence that three different symmetry sites of $\mathrm{Eu}^{3+}$ exist in $\mathrm{BaHfO}_{3}: \mathrm{Eu}(5 \%)$ powders. It is postulated that two of them results from simple substitution of the $\mathrm{Ba}^{2+}$ and $\mathrm{Hf}^{4+}$ ion sites and the third one results from ( $\mathrm{Eu}_{\mathrm{Hf}}-\mathrm{Eu}_{\mathrm{Ba}}$ ) pair formation. Only one of the sites, giving emission peaking at $595.6 \mathrm{~nm}$ is fully centrosymmetric, although positions of both metal ions in the host material possess inversion symmetry.

\section{Introduction}

Barium hafnate $\left(\mathrm{BaHfO}_{3}\right)$ was found to be an attractive host lattice for new X-ray phosphors $[1,2,3]$. Due to high effective atomic number and high density $\left(64.58\right.$ and $8.5 \mathrm{~g} / \mathrm{cm}^{3}$, respectively) [3] barium hafnate containing materials have distinctly higher photofraction and absorption coefficient in the range of medical X-rays [4] compared to today commercial phosphors used in planar imaging and computed tomography based on $\mathrm{Gd}_{2} \mathrm{O}_{2} \mathrm{~S}(57.72,7.34$ $\mathrm{g} / \mathrm{cm}^{3}$, respectively). Till now luminescence and radioluminescence properties of $\mathrm{BaHfO}_{3}$ were studied mostly on Ce-doped compositions, which appeared to have efficient and fast decaying radioluminescence, which parameters are important for computed tomography and positron emission tomography $[3,5,6,7]$.

So far there was not much interest in Eu-activated $\mathrm{BaHfO}_{3}$. Emission of $\mathrm{Eu}^{3+}$ ion is relatively slow as its $\mathrm{f} \rightarrow \mathrm{f}$ transitions are forbidden. Electric dipole induced luminescence of $\mathrm{Eu}^{3+}$ has usually decay time in the order of 1-1.5 ms and appears when the ion is placed in the site having no inversion symmetry. Magnetic dipole transitions, observed only when $\mathrm{Eu}^{3+}$ occupies a centrosymmetric site, produce emissons with even longer decay, typically above $4 \mathrm{~ms}$. Such a long luminescence is not appropriate for dynamic medical imaging but is quite ac- 
ceptable for planar imaging, also in its digital version with electronic recording of the signal from the phosphor [14].

In this paper we concentrate on structural and spectroscopic characterization of Eu-activated $\mathrm{BaHfO}_{3}$ prepared in the oxidizing atmosphere of air. One of the issues we will try to elucidate is whether the dopant tends to replace $\mathrm{Ba}^{2+}$ or $\mathrm{Hf}^{4+}$ ions, which is further connected with possible charge compensation schemes, quite numerous in every case. Moreover, the possibility of $\left(\mathrm{Eu}_{\mathrm{Hf}}^{\prime}-\mathrm{Eu}_{\mathrm{Ba}}\right)$ pair formation should not be overlooked, as such a pair would not require any additional structural defects to balance the incompatibility of the $3+$ charge of the dopant ion and the $2+$ and $4+$ charges of the sites offered by the host.

\section{Materials and methods}

Barium hafnate $\left(\mathrm{BaHfO}_{3}\right)$ powders with the concentration of europium varying in the range of 0-10 at.\% were prepared using ceramic method with $\mathrm{BaCO}_{3}(>99 \%), \mathrm{HfO}_{2}(99.9 \%)$, and $\mathrm{Eu}_{2} \mathrm{O}_{3}(99.99 \%)$ as substrates. It was arbitrary assumed that Eu substitutes $\mathrm{Hf}$ in the host lattice and all calculations were performed accordingly. The starting materials were mixed and ground in an alumina mortar with acetone as a wetting medium. After drying the mixture was heated in air at $1400^{\circ} \mathrm{C}$ for $4 \mathrm{~h}$. Following cooling the material was again pulverized by additional grinding and re-heated at the same conditions. To find the best temperature of synthesis a separate series of powders containing $1 \%$ of $\mathrm{Eu}$ was prepared at different temperatures in the range of $1000-1700^{\circ} \mathrm{C}$.

The X-ray diffraction (XRD) patterns were measured with IRYS diffractometer using $\mathrm{Cu} \mathrm{K}_{0.1}$ radiation $(\lambda=1.54056 \AA)$ in the range of $2 \theta=10-120$ degree with the step $\Delta \theta=0.05^{\circ}$. High resolution XRD spectra were recorded with the step $\Delta \theta=0.01^{\circ}$ for the most intensive diffraction lines located around $30.3^{\circ}, 43.3^{\circ}$ and $53.7^{\circ}$ to monitor their detailed positions as a function of Eu concentration. Photoluminescence and excitation spectra as well as decay kinetics were recorded using FSL 920 spectrofluorimeter from Edinburgh Instruments. Experiments were performed at room and liquid nitrogen temperature (RT and $\mathrm{LN}_{2}$, respectively). Photoluminescence and excitation spectra were taken with a $0.25 \mathrm{~nm}(\mathrm{RT})$ and $0.1 \mathrm{~nm}$ $\left(\mathrm{LN}_{2}\right)$ resolution. The latter were corrected for the incident light intensity. The RT survey excitation spectrum was recorded with the emission monochromator slits set to $3 \mathrm{~nm}$. For excitation spectra recorded at $\mathrm{LN}_{2}$ temperature this parameter was set to $0.2 \mathrm{~nm}$ which allowed for semi site selective analysis. Decay kinetics measurements were performed using the instrument dedicated Xe flash lamp $(60 \mathrm{~W})$ as an excitation source. For these experiments excitation and emission monochromators slits were set to $0.2 \mathrm{~nm}$.

\section{Structural analysis}

Seeking the optimal conditions of the materials preparation we performed synthesis of a series of samples $\mathrm{BaHfO}_{3}: \mathrm{Eu}(1 \%)$ at different temperatures in the range of $1000-1700^{\circ} \mathrm{C}$ and monitored the variations in XRD patterns. The results are presented in figure 1. While for all samples the main structural phase was that of cubic $\mathrm{BaHfO}_{3}$, only for materials synthesized in the range of temperatures of $1200-1400^{\circ} \mathrm{C}$ no foreign lines were detected $[8,9]$. Basing on these results, for further analysis we decided to synthesize all materials at $1400^{\circ} \mathrm{C}$. Figure 2a shows X-rays diffraction spectra for $\mathrm{BaHfO}_{3}$ powders with different concentration of the activator. Independently on the $\mathrm{Eu}^{3+}$ ion content the XRD patterns do not contain any 
cubic $\mathrm{BaHfO}_{3}$-irrelevant diffraction lines, which proves that up to the concentration of $10 \%$ Eu dissolves in the barium hafnate host lattice and does not lead to another structural phase.

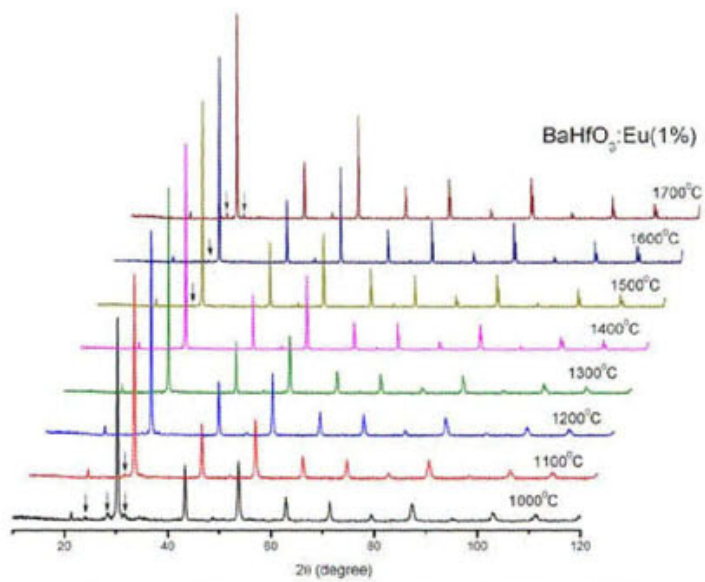

Figure 1. X-ray diffraction patterns for $\mathrm{BaHfO}_{3}: \mathrm{Eu}(1 \%)$ powders prepared at different temperatures. Arrows indicate lines of unidentified foreign phases.

(a)

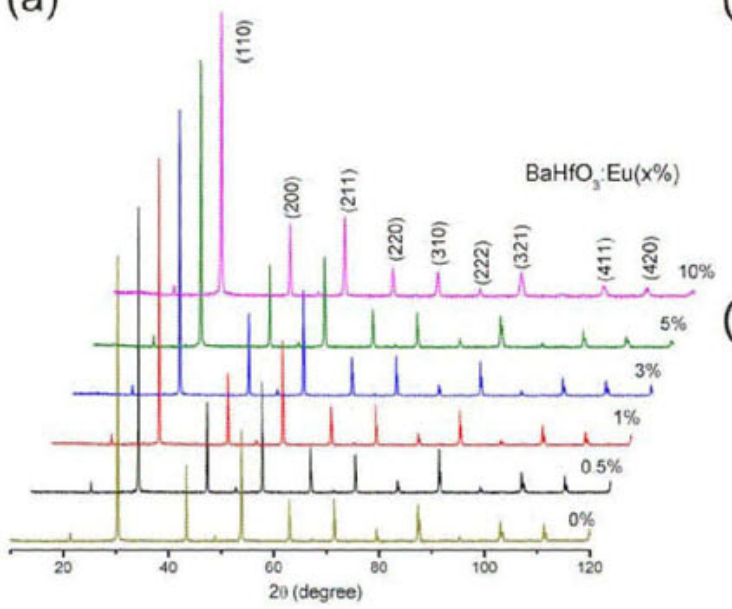

(b)

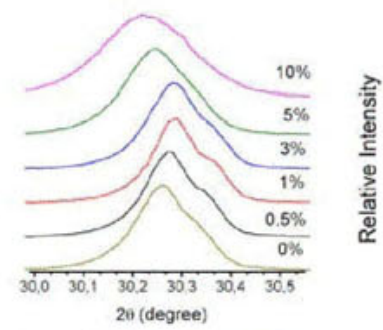

(c)

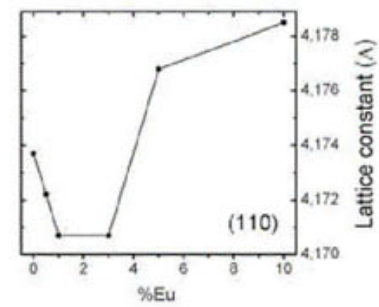

Figure 2. a) XRD patterns for $\mathrm{BaHfO}_{3}$ powders with different content of $\mathrm{Eu}, \mathrm{b}$ ) variation in the position of the (110) diffraction line of $\mathrm{BaHfO}_{3}: \mathrm{Eu}(x \%)$ powders, $c$ ) relation between the cubic lattice constant and concentration of the $\mathrm{Eu}^{3+}$ ion in $\mathrm{BaHfO}_{3}$.

Figure $2 \mathrm{~b}$ presents high resolution X-rays diffraction spectra for powders with different content of Eu in the range of angles where the most significant diffraction line appears. Results for other strong lines are very similar. It is immediately seen that the position of the diffraction line shifts to higher angels for the Eu content in the range of $0-3 \%$ and goes back to 
smaller angles in the range of 3-10\% of Eu. Clearly, the Vegard's law [10] is not obeyed in that case. Even more, it looks that contrary processes occur for lower Eu concentrations (0$3 \%$ ) and for higher Eu contents (5-10\%). The changes in position of diffraction lines reflect variations in the size of the unit cell. Figure $2 \mathrm{c}$ shows how the perovskite cubic unit cell constant changes with concentration of Eu raising from $0 \%$ up to 10\%. Clearly, up to 3\% the unit cell shrinks and for heavier doping it again expands.

Comparison of ionic radii of $\mathrm{Eu}^{3+}(0.947 \AA), \mathrm{Ba}^{2+}(1.61 \AA)$ and $\mathrm{Hf}^{4+}(0.71 \AA)$ [11] indicates that introduction of the Eu dopant into the barium hafnate varies the unit cell size due to significant differences in the size of the relevant ions. However, from the simple comparison of the sizes we cannot definitely judge about what should happen with the size of the unit cell when $\mathrm{Eu}^{3+}$ replaces $\mathrm{Ba}^{2+}$ and what would occur when the dopant goes to the positions of $\mathrm{Hf}^{4+}$. We have to be aware that whichever of the two cases happen charge compensation will be required. Consequently, quite a few different possibilities can be anticipated. Below, using the Kröger-Vink notation $[12,13]$, we present only those which seems to be the most probable: (1) $2 \mathrm{Eu}_{\mathrm{Ba}}+\mathrm{O}_{\mathrm{i}}^{\prime \prime}$, (2) $2 \mathrm{Eu}_{\mathrm{Ba}}+\mathrm{V}_{\mathrm{Ba}}^{\prime \prime}$, (3) $2 \mathrm{Eu}_{\mathrm{Hf}}^{\prime}+\mathrm{V}_{\mathrm{O}}^{\prime \prime}$, (4) $2 \mathrm{Eu}_{\mathrm{Hf}}+\mathrm{Ba}_{\mathrm{i}}$. Yet, we cannot ignore the option of (5) $\left(\mathrm{Eu}_{\mathrm{Ba}}-\mathrm{Eu}_{\mathrm{Hf}}^{\prime}\right)$ pair formation, which would need no additional chargecompensating defect as we already mentioned in introduction.

Analyzing the various possibilities we can easily realize that from the variation of the unit cell size, which we found with high resolution XRD experiments, we cannot undeniably conclude which of the two metal sites offered by the host lattice is indeed entered by $\mathrm{Eu}^{3+}$ ion. However, from the different changes of the unit cell size for lower range of Eu concentrations compared to the higher Eu contents we can anticipate that the dopant may preferentially enter one of the two sites when the concentrations are low (up to 3\%) and the other one when they are higher (above 3\%). Consequently, by inference we can further conclude that $\mathrm{Eu}^{3+}$ enters the $\mathrm{BaHfO}_{3}$ lattice substituting both $\mathrm{Ba}^{2+}$ and $\mathrm{Hf}^{4+}$, although presumably with opposite preferences for low and high total Eu concentrations. This in turn would give the ratio of the populations of the two sites, $\mathrm{Eu}_{\mathrm{Ba}}^{\cdot}$ and $\mathrm{Eu}_{\mathrm{Hf}}^{\prime}$, dependent on the overall Eu concentration. As we shall see later, further going conclusions will be possible from the spectroscopic data.

\section{Luminescence spectroscopy}

Local positions of $\mathrm{Ba}^{2+}$ and $\mathrm{Hf}^{4+}$ ions in the $\mathrm{BaHfO}_{3}$ host lattice are centrosymmertric [8]. In the case of the former ion the coordination number is as high as 12 and for the latter one it is 6. If $\mathrm{Eu}^{3+}$ entering the host preserves the centrosymmetric environment only magnetic dipole induced transitions, with strict selection rules of $\Delta \mathrm{J}=0, \pm 1$ and $0 \rightarrow 0$ transition forbidden, would occur in excitation and emission spectra. In the case of $\mathrm{Eu}^{3+}$ ion basically only ${ }^{7} \mathrm{~F}_{0} \rightarrow{ }^{5} \mathrm{D}_{1}$ in excitation and ${ }^{5} \mathrm{D}_{0} \rightarrow{ }^{7} \mathrm{~F}_{1}$ and ${ }^{5} \mathrm{D}_{1} \rightarrow{ }^{7} \mathrm{~F}_{0,1,2}$ in emission spectra would be partially allowed and thus recordable with a standard instrumentation. However, any distortion of the purely centrosymmetric surrounding would relax the selection rules leading to an increase of the number of observed transitions as well as to the rise of their intensities $[14,15,16,17]$. Figure 3 presents RT excitation and luminescence spectra of $\mathrm{BaHfO}_{3}: \mathrm{Eu}(5 \%)$. Emission was recorded upon excitation into the charge transfer (CT) band of $\mathrm{Eu}^{3+}$ and the excitation spectrum was measured monitoring the $595.6 \mathrm{~nm}$ emission with a relatively broad slit (low resolution) of the analyzing monochromator. Hence, these spectra are not site selective. The excitation spectrum consists of a broad CT band peaking around $260 \mathrm{~nm}$ and numerous but 
very week lines located in three regions of wavelengths: 350 - $420 \mathrm{~nm}, 450-475 \mathrm{~nm}$ and 520 $-540 \mathrm{~nm}$. The low intensity of all the $\mathrm{f} \rightarrow \mathrm{f}$ related excitation lines is a good indication that the symmetry around the activator is high. On the other hand, the high number of the $f \rightarrow f$ transitions seen in the excitation spectrum makes it evident that the local environment of $\mathrm{Eu}^{3+}$ is not centrosymmetric, as not only the magnetic dipole induced transitions occur. These conclusions get support from the analysis of the luminescence spectrum seen in figure 3 (red line). It clearly contains a number of lines, which are not allowed upon the selection rules of magnetic dipole transitions. Consequently we have to accept that the $\mathrm{Eu}^{3+}$ environment is not centrosymmetric. Nevertheless, the two most significant luminescent lines are clearly due to magnetic dipole induced transitions and this accords with the earlier conclusion that although the $\mathrm{Eu}^{3+}$ surrounding is not purely centrosymmetric the distortion around the centre of inversion is not very significant.

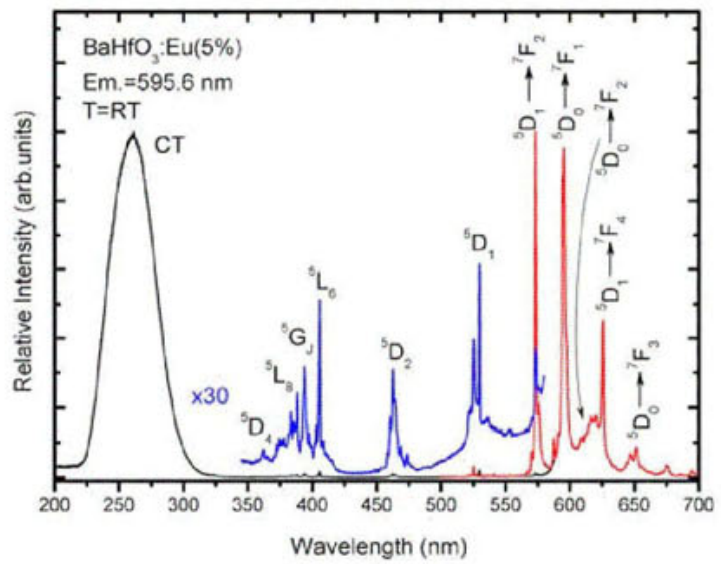

Figure 3. Room temperature excitation (black and blue lines) and emission (red line) spectra of the $\mathrm{BaHfO}_{3}: \mathrm{Eu}(5 \%)$ powder.

The relatively reach emission spectrum tells to raise a question if there is only one type of $\mathrm{Eu}^{3+}$ ions present in the material. To track this problem we decided to record a series of excitation spectra for selected emission lines at the reduced temperature of liquid nitrogen and using higher resolution. The results of the semi site-selective spectroscopy measurements are presented in figure 4 (excitation spectra) and figure 5 (luminescence spectra). In the case of excitation spectra especially important information can be deduced from the region of the ${ }^{7} \mathrm{~F}_{0} \rightarrow{ }^{5} \mathrm{D}_{2}$ hypersensitive, electric dipol transitions $(455-475 \mathrm{~nm})$ and from the part where the ${ }^{7} \mathrm{~F}_{0} \rightarrow{ }^{5} \mathrm{D}_{1}$ magnetic dipole induced transitions occur $(520-531 \mathrm{~nm})$. 

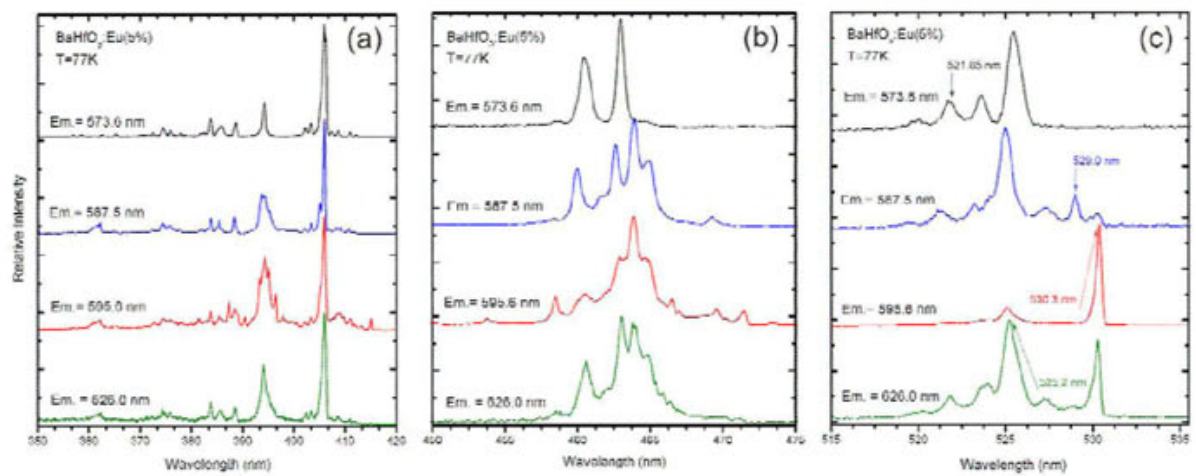

Figure 4. Semi-site- selective excitation spectra of $\mathrm{BaHfO}_{3}: \mathrm{Eu}(5 \%)$. The monitored emission wavelengths are indicated in the figure.

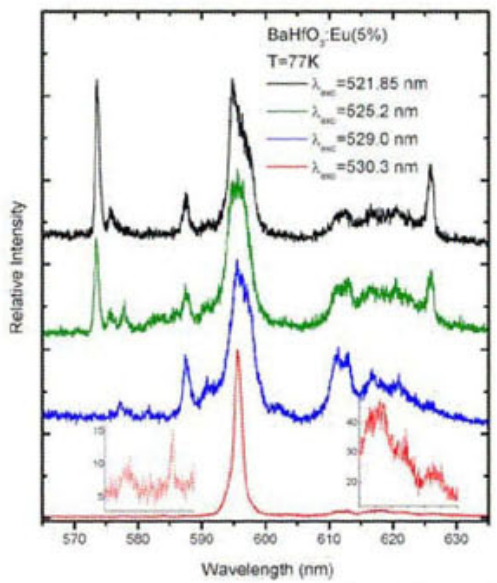

Figure 5. Emission spectra of $\mathrm{BaHfO}_{3}: \mathrm{Eu}(5 \%)$ upon semi-selective excitation. The stimulation wavelengths are indicated in the figure.

Comparing the excitation spectra it appears clear that they differ significantly attesting the existence of $\mathrm{Eu}^{3+}$ ions with different symmetry of their local surrounding. Emission spectra obtained upon stimulation of the material into selected excitation lines also differ considerably. All these significant differences could be recorded despite we did not use laser spectroscopy that is we could not excite the different $\mathrm{Eu}^{3+}$ ions fully selectively. Analysis of the excitation spectra presented in figure 4 allows concluding that at least three of them, for the $573.6 \mathrm{~nm}, 587.5 \mathrm{~nm}$ and $595.6 \mathrm{~nm}$ emissions differ in such a way that they have to result from the presence of three different $\mathrm{Eu}^{3+}$ sites in the $\mathrm{BaHfO}_{3}: \mathrm{Eu}(5 \%)$ material. Yet, the fourth excitation spectrum of the $626 \mathrm{~nm}$ luminescence seems to consist of features already seen in the other three spectra. Indeed, luminescence spectra presented in figure 5 prove that three different emissions, upon excitation with $521.85 \mathrm{~nm}, 529.0 \mathrm{~nm}$ and $530.3 \mathrm{~nm}$ radiation, can clearly be distinguished. However, the luminescence excited into the $525.2 \mathrm{~nm}$ line 
contains only features, which can be found in the other three spectra and therefore cannot be treated as resulting from a separate (fourth) $\mathrm{Eu}^{3+}$ symmetry site.

An interesting spectral characteristic shows the emission excited with $530.3 \mathrm{~nm}$ radiation. Namely, it consists of basically one luminescence line peaking at $595.6 \mathrm{~nm}$. At shorter and longer wavelengths we can find some satellite components but they are of extremely low intensity, and are hardly recordable, indeed. The position of this luminescence indicates that it results from ${ }^{5} \mathrm{D}_{0} \rightarrow{ }^{7} \mathrm{~F}_{1}$ transition and the lack of other components tells to treat this emission as coming from $\mathrm{Eu}^{3+}$ positioned in a perfectly centrosymmetric site. In the case of the other two $\mathrm{Eu}^{3+}$ sites, which we identified above, the inversion symmetry has to be broken due to slight distortion because the components which do not come from the magnetic dipole induced transitions show relatively higher intensities.

Since the host lattice offers only two sites for the $\mathrm{Eu}^{3+}$ ion entering the host, finding three spectroscopically different symmetry sites may be confusing at first. Basing on the presently available results we presume that two of these sites results from the substitution of both $\mathrm{Ba}^{2+}$ and $\mathrm{Hf}^{4+}$ ions giving a positively charged $\mathrm{Eu}_{\mathrm{Ba}}$ site and $\mathrm{Eu}_{\mathrm{Hf}}^{\prime}$ site possessing a negative net charge. Since the host does not formally offer a third site for the dopant we hypothesize that the third spectroscopic site of $\mathrm{Eu}^{3+}$ ion results from the $\left(\mathrm{Eu}_{\mathrm{Hf}}^{\prime}-\mathrm{Eu}_{\mathrm{Ba}}\right.$ ) pair formation. To confirm or deny this idea further spectroscopic experiments will have to be performed. We believe that an analysis of a concentration dependence of the luminescence spectra will shed more light on this problem.

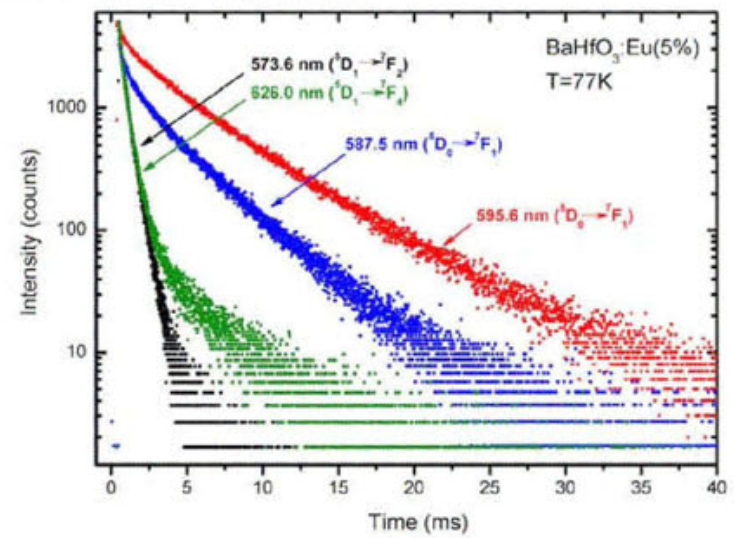

Figure 6. Luminescence decay traces of the main emission lines of the $\mathrm{BaHfO}_{3}: \mathrm{Eu}(5 \%)$ powder .

To better elucidate the problem of the various luminescent features observed in BaH$\mathrm{fO}_{3}: \mathrm{Eu}(5 \%)$ powders we recorded the decay traces of the emission lines located at $573.6 \mathrm{~nm}$, $587.5 \mathrm{~nm}, 595.6 \mathrm{~nm}$ and $626.0 \mathrm{~nm}$. The results are presented in figure 6. It is clear that the $573.6 \mathrm{~nm}$ and $626.0 \mathrm{~nm}$ emissions have common origin. A tail seen in the decay of the 626 $\mathrm{nm}$ luminescence results from an overlapping constituent of a longer decay. A closer examination of the energy levels of $\mathrm{Eu}^{3+}$ tells to assign both this components $(573.6 \mathrm{~nm}$ and 626.0 $\mathrm{nm}$ ) to the radiative relaxation of the ${ }^{5} \mathrm{D}_{1}$ level. The decay of the $587.5 \mathrm{~nm}$ and $595.6 \mathrm{~nm}$ emissions are clearly longer, which is expected as they must necessarily result from a radiative relaxation of the ${ }^{5} \mathrm{D}_{0}$ level of $\mathrm{Eu}^{3+}$. The time constants of these two emissions are $3.4 \mathrm{~ms}$ 
$(587.5 \mathrm{~nm})$ and $5.1 \mathrm{~ms}(595.6 \mathrm{~nm})$, which values are characteristic for $\mathrm{Eu}^{3+}$ ion in a highsymmetry environment $[14,17]$. Also, it is reasonable that the value for the $595.6 \mathrm{~nm}$ luminescence is longer as we already concluded that this emission results from $\mathrm{Eu}^{3+}$ ion located in a purely centrosymmetric position. Hence, analysis of the results of XRD measurements, excitation and luminescence spectra as well as decay kinetics leads to quite a consistent picture, with the main conclusion that $\mathrm{Eu}^{3+}$ ions enters both, $\mathrm{Ba}^{2+}$ and $\mathrm{Hf}^{4+}$ positions.

\section{Conclusions}

$\mathrm{Eu}^{3+}$ activated $\mathrm{BaHfO}_{3}$ was prepared with ceramic method at $1400{ }^{\circ} \mathrm{C}$. Up to $10 \%$ of $\mathrm{Eu}$ content no foreign phase was detected with XRD technique. Results of spectroscopic experiments gave evidence that $\mathrm{Eu}^{3+}$ ion enters both $\mathrm{Ba}^{2+}$ and $\mathrm{Hf}^{4+}$ sites. XRD spectra suggests that the relative population of both sites $\left(\mathrm{Eu}_{\mathrm{Hf}}^{1}\right.$ and $\left.\mathrm{Eu}_{\mathrm{Ba}}\right)$ depends on the overall concentration of Eu. The material containing 5\% of the dopant possesses three spectroscopically different sites with clearly different luminescence and excitation spectra. We suppose that the third $\mathrm{Eu}^{3+}$ site is connected with the formation of $\left(\mathrm{Eu}_{\mathrm{Hf}}^{\prime}-\mathrm{Eu}_{\mathrm{Ba}}\right)$ pairs.

\section{References}

1. Lambert, P.M., Jarrold, G.S. \& Trauernicht, D.P., 1997, US Patent \# 5698857.

2. Lambert P.M., Jarrold, G.S. \& Bryan P.S., 1998, US Patent \# 5786600.

3. Van Loef, E.V., Higgins, W.M., Glodo, J., Brecher, C., Lempicki, A., Venkataramani, V.S., Moses, W.W., Derenzo, S.E. \& Shah, K.S., 2007, IEEE T. Nucl. Sci. 54 (3), 741 .

4. http://physics.nist.gov/cgi-bin/Xcom/xcom2

5. Dole, L. \& Venkataramani, V.S., 1992, US Patent \# 5124072.

6. Venkataramani, V.S., Loureiro, S.M. \& Rane, M.V., 2004, US Patent \# 6706212 B2.

7. Villanueva-Ibañez, M., Le Luyer, C., Parola, S., Dujardin, C. \& Mugnier, J., 2005, Opt. Mat., 27, 1541.

8. Maekawa, T., Kurosaki, K. \& Yamanaka, S., 2006, J. Alloy Compd., 407, 44.

9. PDF $\# 00-022-0084$

10. Denton, A.R. \& Ashcroft, N.W., 1991, Phys. Rev. A, 43, 3161.

11. Shannon, R.D., 1976, Acta Cryst. A, 32, 751.

12. Kröger, F.A. \& Vink, H.H., 1956, Solid State Physics, edited by F. Sietz \& D. Turnbull (San Diego, CA: Academic Press).

13. Bridge, F., Davies, G., Robertson, J. \& Stoneham, A.M., 1990, J. Phys.: Condens. Matter, 2, 2875.

14. Blasse, G. \& Grabmaier, B.C., 1994, Luminescent Materials (Springer-Verlag).

15. Derenzo, S.E., Moses, W.W., Weber, M.J., West, Z.C., 1994, Mater. Res. Soc. Symp. Proc. 348, 39.

16. Shionoya, S. \& Yen, W.M., 1990, Phosphors Handbook (CRC Press LLC).

17. Zych, E., 2002, J.Phys. : Condens. Mater, 14, 5637.

Acknowledgements. The financial support from the Minister of Science and Higher Education under grant \# N205 024 31/1207 is greatly acknowledged. 


\section{Investigation of stacking disorder in $\mathrm{Li}_{2} \mathrm{SnO}_{3}$}

\section{N. V. Tarakina ${ }^{1, *}$, T. A. Denisova ${ }^{1}$, L. G. Maksimova ${ }^{1}$, Y. V. Baklanova ${ }^{1}$, A. P. Tyutyunnik ${ }^{1}$, I. F. Berger ${ }^{1}$, V. G. Zubkov' ${ }^{1}$, G. van Tendeloo ${ }^{2}$}

${ }^{1}$ Institute of Solid State Chemistry, Ural Branch of Russian Academy of Sciences, Pervomayskaya 91, Ekaterinburg, 620041 Russia

${ }^{2}$ EMAT, University of Antwerp, Groenenborgerlaan 171, Antwerp, Belgium

•tarakina@ihim.uran.ru

Keywords: diffuse scattering, stacking disorder, microstructure, powder diffraction, electron diffraction

Abstract. A crystal structure investigation of the low temperature $\mathrm{Li}_{2} \mathrm{SnO}_{3}$ modification has been carried out. X-ray, neutron powder and electron diffraction data showed that this compound crystallizes in a monoclinic unit cell with parameters: $a=5.3033(2) \AA, b=$ 9.1738(3) $\AA, c=10.0195(2) \AA, \beta \sim 100.042(2)^{\circ}$ and has stacking disorder along the $c$-axis. Simulation of diffraction patterns with different stacking faults mainly reveal the presence of rotational stacking faults with a probability of about $40 \%$.

\section{Introduction}

Lithium salts $\mathrm{Li}_{2} \mathrm{MO}_{3}(\mathrm{M}=\mathrm{Sn}, \mathrm{Ti}, \mathrm{Zr}, \mathrm{Mo}$, $\mathrm{Pd}$, etc. $)$ are candidate materials for $\mathrm{Li}^{+}$ion conductors and solid breeders in a fusion reactor [1-3]. $\mathrm{Li}_{2} \mathrm{SnO}_{3}$ can also be used as a precursor for the synthesis of $\mathrm{XSnO}_{3}(\mathrm{X}=\mathrm{Zn}, \mathrm{Co}, \mathrm{Ni})$ [4]. It has also been shown that, depending on the sintering conditions, the reactivity of $\mathrm{Li}_{2} \mathrm{SnO}_{3}$ is different [5]. As we will demonstrate, the reason for this could lie in the complex structural features of the compound.

According to the literature, $\mathrm{Li}_{2} \mathrm{SnO}_{3}$ can be synthesized in two modifications. Lowtemperature (LT) $\mathrm{Li}_{2} \mathrm{SnO}_{3}$ is formed at temperatures below $800{ }^{\circ} \mathrm{C}$ and crystallizes in S.G. $\mathrm{C} 2 / m[6,7]$. High-temperature (HT) $\mathrm{Li}_{2} \mathrm{SnO}_{3}$ is formed at $1000^{\circ} \mathrm{C}$ and also has a monoclinic unit cell (S.G. $C 2 / c$ ) with lattice parameters: $a=5.2889(2) \AA, b=9.1872(3) \AA, c=$ $10.0260(2) \AA, \beta=100.348(2)^{\circ}[8]$. Both modifications have a NaCl-related structure in which the oxygen atoms form a distorted cubic closed-packed network with octahedral cavities occupied by $\mathrm{Li}^{+}$and $\mathrm{Sn}^{4+}$ cations. Ordering of the metals along the [111] direction of the cubic body diagonal results in the appearance of two types of layers: one consists only lithium atoms $\left(\mathrm{Li}_{3}\right)$ and another - lithium and tin atoms $\left(\mathrm{LiSn}_{2} \mathrm{O}_{6}\right)$ (figure 1). The main difference between the HT and LT modifications lies in a different stacking of the $\mathrm{LiSn}_{2} \mathrm{O}_{6}$ layers. In spite of a lot of data from the literature the question about the exact crystal structure of the LT modification of $\mathrm{Li}_{2} \mathrm{SnO}_{3}$ is not clear yet. It was proposed in [6] and [7] that $\mathrm{LT}-\mathrm{Li}_{2} \mathrm{SnO}_{3}$ 
could just be a defect modification of the HT phase, and that the phase transition would correspond to a "healing" of the defects in the disordered structure.

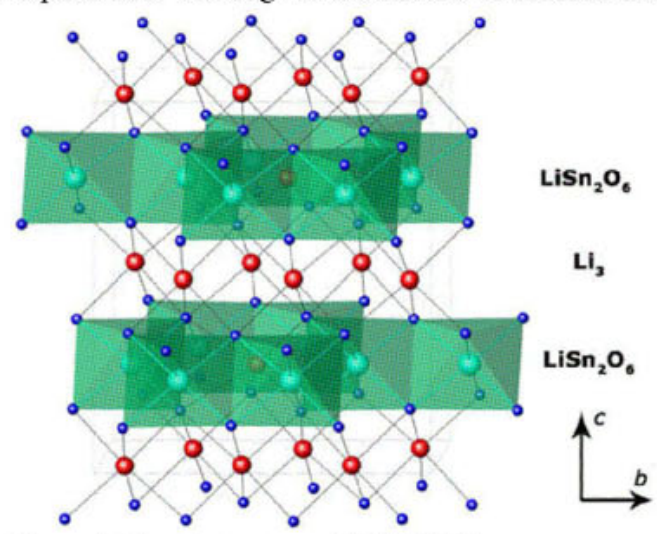

Figure 1. Crystal structure of $\mathrm{HT}-\mathrm{Li}_{2} \mathrm{SnO}_{3}$.

During our investigation of the interaction processes between lithium salts $\mathrm{Li}_{2} \mathrm{MO}_{3}(\mathrm{M}=\mathrm{Zr}$, $\mathrm{Sn}, \mathrm{Ti}$ ) with evaporated water in an air environment and acid water solutions it has been shown that an extent of the exchange of $\mathrm{Li}^{+}$ions for hydrogen, realized through the formation of $\mathrm{Li}_{2-\mathrm{x}} \mathrm{H}_{\mathrm{X}} \mathrm{SnO}_{3}$, depends on the sintering condition of the $\mathrm{Li}_{2} \mathrm{MO}_{3}$ precursors. For $\mathrm{Li}_{2} \mathrm{SnO}_{3}$ annealed at $700{ }^{\circ} \mathrm{C}$ the extent of the exchange of $\mathrm{Li}^{+}$ions for hydrogen $(x)$ is about 1.8 , whereas for precursors annealed at $900-1100{ }^{\circ} \mathrm{C}$ the exchange $x$ is $\leq 0.3$ [5]. In order to clear out the origin of this difference in $\mathrm{Li}^{+}$exchange, a structural investigation of $\mathrm{Li}_{2} \mathrm{SnO}_{3}$ has been carried out.

\section{Experimental}

$\mathrm{Li}_{2} \mathrm{SnO}_{3}$ has been prepared by the solid-state reaction in air from a stoichiometric mixture of $\mathrm{SnO}_{2}(99.9 \%)$ and $\mathrm{Li}_{2} \mathrm{CO}_{3}(99.9 \%)$. The mixture was placed in platinum crucibles and annealed at temperatures up to $700{ }^{\circ} \mathrm{C}$ during $48 \mathrm{~h}$.

X-ray powder diffraction (XRD) data were collected at room temperature on a transmission STADI-P (STOE, Germany) diffractometer equipped with a linear mini-PSD detector, using $\mathrm{Cu} \mathrm{K} \alpha_{1}$ radiation in the $2 \theta$ range $2^{\circ}$ to $120^{\circ}$ with a step of $0.02^{\circ}$. Polycrystalline silicon ( $a=$ $5.43075(5) \AA$ ) was used as external standard. The phase purity of the sample was checked by comparing XRD patterns with those in the PDF2 database (Powder diffraction file, ICDD, USA), release 2006. Neutron powder diffraction data (ND) were collected at room temperature using the $7 \mathrm{~A}$ diffractometer at the IWW $2 \mathrm{M}$ reactor (Zarechny) in the $2 \theta$ range $2^{\circ}$ to $120^{\circ}$ with a step of $0.02^{\circ}, \lambda=1.5323 \AA$. For the transmission electron microscopy (TEM) study the sample was crushed in ethanol. A drop of this dispersion was put on a copper grid covered with a holey carbon film. Electron diffraction (ED) patterns were obtained using a Philips CM20 operated at $200 \mathrm{kV}$.

The XRD and ND simulations were performed using the DISCUS software package [9] in order to model stacking disorder along the $c$-axis. 


\section{Results and discussion}

$\mathrm{X}$-ray and neutron powder data yielded for $\mathrm{Li}_{2} \mathrm{SnO}_{3}$ the unit cell parameters $a=5.3033(2) \AA$, $b=9.1738(3) \AA, c=10.0195(2) \AA, \beta \sim 100.042(2)^{\circ}$. The reflection conditions $(h k l: h+k=2 n$; $h 0 l: h=2 n, l=2 n ; 0 k l: k=2 n ; h k 0: h+k=2 n)$ indicate a $C$-centered lattice, with possible space groups $C c$ and $C 2 / c$. The crystal structure of the LT-phase was refined using XRD and ND data simultaneously with the program package GSAS [10].

The intensity of some Bragg reflections and Warren falls in the powder patterns could not be refined in an ideal model with space group $\mathrm{C} 2 / \mathrm{c}$. In some previous works the fit of observed and calculated data has been improved by introducing $\mathrm{Me}^{+} / \mathrm{Me}^{4+}$ exchange between the atomic sites in $\mathrm{Me}^{+} \mathrm{Me}^{4+}{ }_{2} \mathrm{O}_{6}$ layers $[8,11]$. Li/Sn exchange resulted in a slightly improved fit but the obtained R-factors were still quite high. The refinement converged with $w R p=$ $7.20(14.58) \%, R p=5.35(10.46) \%, D w d=0.246(0.079), R\left(F^{2}\right)=26.03(22.07) \%$, for neutron powder diffraction data and for X-ray powder diffraction data in brackets, respectively.

In order to clear out the origin of the imperfections, ED experiments were performed along different major zone axes of $\mathrm{Li}_{2} \mathrm{SnO}_{3}$. In the [100]* diffraction pattern intense lines of diffuse scattering have been observed along $c^{*}$ (figure 2).

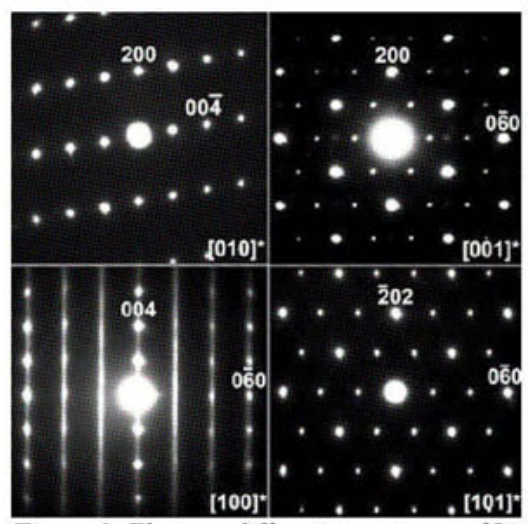

Figure 2. Electron diffraction patterns of $\mathrm{Li}_{2} \mathrm{SnO}_{3}$ along the main zone axes.

Clear maxima in these patterns appear for the reflection series $(00 l),(06 l)$. This indicates a stacking disorder of $\mathrm{LiSn}_{2} \mathrm{O}_{6}$ slabs shifted over $\left[\begin{array}{lll}u & 0 & 0\end{array}\right]$ and $\left[\begin{array}{lll}u & 1 / 6 & 0\end{array}\right]$. Analysis of the crystal structure allows us to propose that $u$ can take on two values: 0 and $1 / 2$. Thus, a random stacking of $\mathrm{LiSn}_{2} \mathrm{O}_{6}$ slabs shifted either over $\left[\begin{array}{lll}1 / 2 & 0 & 0\end{array}\right]$ or $\left[\begin{array}{lll}1 / 2 & 1 / 6 & 0\end{array}\right]$ or $\left[\begin{array}{lll}0 & 1 / 6 & 0\end{array}\right]$ can cause the appearance of diffuse intensity along $c^{*}$.

These three displacement vectors of the (001) layers coincide with possible stacking schemes in $\mathrm{Me}_{2}{ }^{+} \mathrm{Me}^{4+} \mathrm{O}_{3}$-type compounds proposed by Lang [6]. Displacement over the [ $\left[\begin{array}{lll}0 & 1 / 6 & 0\end{array}\right]$ vector is the intrinsic translation relating $\mathrm{LiSn}_{2} \mathrm{O}_{6}$ slabs in compounds with S.G. C2/c (IIItype according to Lang). If there is no displacement along the $\boldsymbol{b}$-direction, a shift over [1/2 0 0 ] will lead to the formation of S.G. C2/m (I-type according to Lang). A shift of several layers over the $[1 / 21 / 60]$ vector is equivalent to a rotation over $120^{\circ}$ and therefore the stacking results in the appearance of a 3-fold screw axis along the c-axis. Diffuse scattering observed 
in X-ray, neutron powder and ED diffraction patterns can therefore appear either due to one of these stacking displacements or due to a superposition of them.

In order to check this assumption, we performed simulations of X-ray and neutron powder diffraction data with the DISCUS software package [9]. The unit cell of the $\mathrm{HT}-\mathrm{Li}_{2} \mathrm{SnO}_{3}$ modification with S.G. $C 2 / c$ was taken as a starting model for a defect structure simulation. Stacking of large, flat layers ( $a b$ planes) along the $00 l$ direction have been considered. The step in reciprocal space along the $c^{*}$ direction was taken as $1 /$ (number of layers) in order to match the spacing of the Fourier transform calculation. A random distribution of stacking faults was considered. The instrumental broadenings have been simulated by Gaussian resolution functions with a full width at half maximum (FWHM) of $0.1^{\circ}$ and $0.5^{\circ}$ for X-ray and neutrons, respectively.

It has been shown that the number of layers stacked along the $c$-axis should be not less than 500 in order to average the stacking faults in the crystal and get an appropriate model. In our

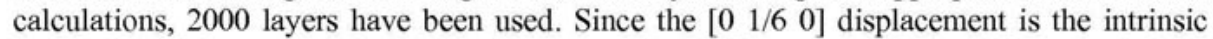
translation of the proposed model only two stacking shifts were applied. Different probabilities of stacking faults from 0 to $50 \%$ with a step of $5 \%$ have been verified.

The best fit with the experimental patterns has been found for the model with a displacement over $\left[\begin{array}{lll}1 / 2 & 1 / 6 & 0\end{array}\right]$ with a probability of $45 \%$ (figure 3 ). However, in spite of the real improvement of the Warren fall shapes, the intensity of some Bragg reflections still does not fit perfectly. It is important to mention that there were no significant changes in the diffraction patterns with probabilities of stacking in the range from $30 \%$ to $45 \%$ which could be an indication of the presence of some other kind of disorder in the system. Attempts to introduce extra displacement vectors or take another starting model did not give better solutions. For example, the introduction of a shift over $\left[\begin{array}{lll}1 / 2 & 0 & 0\end{array}\right]$ did not significantly improve the model, since it only causes a decrease in intensity of the $(h h l)$ and $(-h h l)$ reflections.

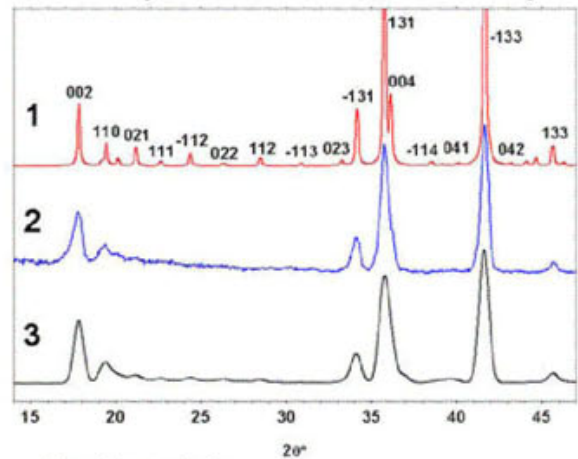

Neutron data

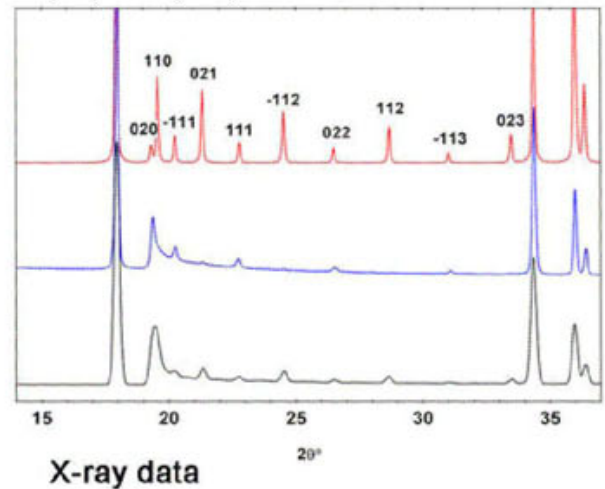

X-ray data

Figure 3. Enlargement between $2 \theta=14^{\circ}-47(37)^{\circ}$ of the $\mathrm{ND}$ and $\mathrm{XRD}$ patterns of $\mathrm{Li}_{2} \mathrm{SnO}_{3}(1)$ simulated, S.G. C2/c without stacking faults; (2) experimental; (3) simulated, S.G. C2/c with a probability of stacking faults of $45 \%$.

Attempts to take a unit cell with half of the $c$-axis (S.G. $C 2 / m$ ) as a starting model gave approximately the same result. In order to correctly simulate the positions of the Bragg reflections it was necessary to introduce a $\left[\begin{array}{lll}0 & 1 / 6 & 0\end{array}\right]$ displacement between the stacking layers. A 
concentration of stacking faults of $30 \%$ with a translation over [1/2 $1 / 60]$ has to be introduced in order to model the powder diffraction profile in the $C 2 / \mathrm{m}$ model.

In spite of the incompleteness for the description of some Bragg reflection intensities the model based on S.G. C2/c gives the better fit and will be considered in the subsequent discussion. The $120^{\circ}$ rotational stacking faults which introduce the appearance of a 3-fold rotation axis in short range order along the $c$-axis can explain the presence of some extra spots on the ED patterns (figure 4).

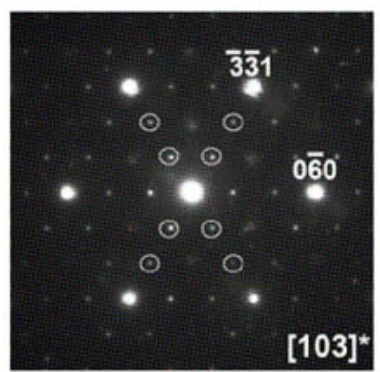

Figure 4. ED pattern collected from $\mathrm{Li}_{2} \mathrm{SnO}_{3}$ along the [103]* direction. The reflections related to three different unit cells are marked by circles.

We can assume that if the concentration of rotational defects is quite high and the crystal is sufficiently thick, three sets of reflections related to three different unit cells should appear on the ED with equal intensities. Although the simulations model the experimental data quite well, $\mathrm{Li}_{2} \mathrm{SnO}_{3}$ appears to be more heterogeneous than implied by our model.

Analyzing the data from the literature, it may be assumed that an additional kind of disorder in the system is related to the formation of a domain structure in the $a b$ plane [12-15].

Still, for such type of disorder, the information, one can obtain from powder diffraction data and ED, is not enough to make a final conclusion. HREM studies could give a definite answer; however, crystals of the considered phase were very sensitive to the electron beam and clear images have not been obtained.

Since extending of annealing time of the sample with fixed temperature results in a decrease of stacking faults it is believed that the observed disorder appears due to slow kinetics of ordering both in the $a b$ plane and along the $c$-direction. A similar conclusion was drawn in [12] for $\mathrm{Li}_{2} \mathrm{MnO}_{3}$ oxide. The possibility to stabilize an imperfect state increases the reactivity of $\mathrm{Li}_{2} \mathrm{SnO}_{3}$ and allows almost complete exchange of $\mathrm{Li}^{+}$ions for hydrogen.

\section{Concluding remarks}

A crystal structure investigation of the $\mathrm{LT}-\mathrm{Li}_{2} \mathrm{SnO}_{3}$ modification has been carried out. It has been shown that this compound crystallizes in a monoclinic unit cell with parameters: $a=$ 5.3033(2) $\AA, b=9.1738(3) \AA, c=10.0195(2) \AA, \beta \sim 100.042(2)^{\circ}$. However, the main feature of $\mathrm{LT}-\mathrm{Li}_{2} \mathrm{SnO}_{3}$, by which it differs from the HT- $\mathrm{Li}_{2} \mathrm{SnO}_{3}$ modification is related to the shortrange structure of the concerned phase. Diffraction experiments and DISCUS simulations of defect models reveal presence of stacking disorder along the $c$-axis. The possibility of easier stabilization of such an imperfect state could be explained by the slow kinetics of ordering both in the $a b$ plane and along the $c$-direction. However, in order to make a final conclusion and confirm the proposed defect model, first-principles calculations are desirable. 


\section{References}

1. Miller, J.M., Hamilton, H.B. \& Sullevan, J.D., 1994, J. of Nuclear Mat., 215, 877.

2. Antidormi, R., Proust, E. \& Roux, N., 1995, Fusion Technology, 28, 519.

3. Moritani, K. \& Moriyama, H., 1997, J. of Nuclear Mat., 248, 132.

4. Kovacheva, D. \& Petrov, K., 1998, Solid State Ionics, 109, 327.

5. Denisova, T.A., 2007, International Scientific Journal for Alternative Energy and Ecology ISJAE, 3, 39.

6. Lang, G.V., Z. Anorg. Allg. Chem., 1966, 348, 246.

7. Trömel, Von M. \& Hauck, J., 1970, Z. Anorg. Allg. Chem., 373, 8.

8. Hodeau, J.L., Marezio, M., Santoro, A. \& Roth, R.S., 1982, J. Solid State Chemistry, 45, 170.

9. Proffen, T. \& Neder, R.B., 1997, J. Appl. Cryst., 30, 171.

10. Larson, A.C. \& Von Dreele, R.B., 2000, General Structure Analysis System-GSAS (Los Alamos National Laboratory Report LAUR 86-748).

11. Panin, R.V., Krasanova, N.R., Abakumov, A.M., Antipov, E.V., Van Tendeloo, G. \& Schnelle, W., 2007, J. Solid State Chemistry, 180, 1566.

12. Breger, J., Jiang, M., Dupre, N., Meng, Y.S., Shao-Horn, Y., Ceder, G. \& Grey, C.P., 2005, J. Solid State Chemistry, 178, 2575.

13. Meng, Y.S., Ceder, G., Grey, C.P., Yoon, W.-S., Jiang, M., Breger, J. \& Shao-Horn, Y., 2005, Chem. Mater., 17, 2386.

14. Kim, J.-S., Johnson, C.S., Vaughey, J.T. \& Thackeray, M.M., 2004, Chem. Mater., 16, 1996.

15. Hinuma, Y., Meng, Y.S., Kang, K. \& Ceder, G., 2007, Chem. Mater., 19, 1790.

Acknowledgements. This work has been supported by the Russian Foundation for Basic Research grants (no. 06-08-00847), by the Belgium Science Policy, by the Council for Grants of the President of the Russian Federation for Support of Young Scientists (grant no. MK-84.2007.3) and for Support of Leading Scientific Schools (grant no. NSh-1170.2008.3). We also thank Prof. R.B. Neder for help with using the DISCUS software package. 


\section{X-ray diffraction studies of transition metal molybdates}

\section{B. Gawet ${ }^{1}$, W. Surga ${ }^{3}$, W. Lasocha ${ }^{1,2, *}$}

${ }^{1}$ Faculty of Chemistry, Jagiellonian University. ul. Ingardena 3, 30-060 Kraków, Poland

${ }^{2}$ Institute of Catalysis and Surface Chemistry, Polish Academy of Sciences, ul. Niezapominajek 8, 30-239 Kraków, Poland

${ }^{3}$ Institute of Chemistry, Jan Kochanowski University, ul. Świętokrzyska 15G, 25-406 Kielce, Poland

* lasocha@chemia.uj.edu.pl

Keywords: powder diffraction, molybdates, X-ray structure determination

Abstract. Methods of synthesis of copper dimolybdate trihydrate and cobalt trimolybdate pentahydrate are described below. Crystal structures of these compounds have been solved by $\mathrm{X}$-ray powder diffraction methods. The space group of $\mathrm{CuMo}_{2} \mathrm{O}_{7} \cdot 3 \mathrm{H}_{2} \mathrm{O}(\mathrm{I})$ is $P$ - 1 with lattice parameters $a=8.678(7) \AA, b=9.289(4) \AA, c=6.064(5) \AA, \alpha=90.72(9)^{\circ}, \beta=$ $95.99(5)^{\circ}, \gamma=97.42(8)^{\circ}$, and for $\mathrm{CoMo}_{3} \mathrm{O}_{10} \cdot 8 \mathrm{H}_{2} \mathrm{O}$ (II) the space group is $P 2_{1} / c$ with lattice parameters $a=12.072(3) \AA, b=19.833(2) \AA, c=7.624(9) \AA, \beta=107.58(5)^{\circ}$. Results on the crystal structure determination and the Rietveld refinement of both compounds are presented.

\section{Introduction}

Polyoxometalates (POM) chemistry has attracted intense interest because of their potential applications in catalysis, biomedicine and battery industry [1-4]. Some of them exhibit also photochromic activity [5]. Thanks to wide structural diversity of polyoxometalates some of their structural as well as physical and chemical properties can be modified. We have obtained in our lab new layered dimolybdate built up of tetrameric moieties $\left[\mathrm{Mo}_{4} \mathrm{O}_{14}\right]^{4-}$ linked by $\left[\mathrm{Cu}_{2}\left(\mathrm{H}_{2} \mathrm{O}\right)_{6}\right]^{4+}$ species (compound $\mathbf{I}$ ), and a new fibrillar trimolybdate with $\left[\mathrm{Co}\left(\mathrm{H}_{2} \mathrm{O}\right)_{6}\right]^{2+}$ as counterion (compound II).

\section{Experimental}

\section{Synthesis}

All reagents were used as received from commercial supplier (POCH Gliwice).

Compound I: Molybdenum oxide was added to a boiling suspension of $\mathrm{CuCO}_{3}$ in water until saturation was reached. The reaction mixture was filtered to remove the unreacted $\mathrm{MoO}_{3}$. After few weeks a blue-green precipitate was obtained [6]. This precipitate can be kept in 
mother solution for few years without visible changes. However, the compound changes very quickly in the air upon dehydration.

Compound II: It was synthesised in the same way using $\mathrm{CoCO}_{3}$ instead of $\mathrm{CuCO}_{3}$.

\section{X-ray powder diffraction investigations}

X-ray powder diffraction patterns for both compounds were collected on a Philips X'pert Pro diffractometer in transmission mode with focusing mirror and 1-D PSD detector. The compounds were finely grounded in the mortar and loaded into capillaries ( $1 \mathrm{~mm}$ diameter) with mother solution to avoid dehydration and to minimise texture. The patterns were collected at $25^{\circ} \mathrm{C}$ using $\mathrm{CuK} \alpha$ radiation (counting time: $9 \mathrm{~s} /$ step; $2 \theta$ ranges: $8-95^{\circ}$ for $\mathbf{I}$ and $5-80^{\circ}$ for $\mathbf{I I}$ ).

\section{Structure solution}

Powder diffraction patterns were indexed using the PROSZKI [7] package. For compound I the best solution was triclinic with unit cell parameters $a, b, c, \alpha, \beta, \gamma: 8.678(7) \AA, 9.289(4)$ $\AA, 6.064(5) \AA, 90.72(9)^{\circ}, 95.99(5)^{\circ}, 97.42(8)^{\circ}, V=745.32(8) \AA^{3}, \mathrm{~F}_{20}=67, \mathrm{SG}: P-1$ (2). Compound II is monoclinic with $a, b, c, \beta=12.072(3) \AA, 19.833(2) \AA, 7.624(9) \AA$, $107.58(5)^{\circ}, V=719.20(6) \AA^{3}, Z=2, F_{20}=25$, SG: $P 2_{1} / c(14)$. The initial structure models were found by direct methods (Expo2004 [8]) (I) and by global optimisation methods (FOX program [9]) (II). Subsequent Rietveld refinements were carried out using the JANA2000 program [10]. Initial profiles parameters were obtained by fitting the observed pattern according to the Le Bail method [11]. The refinement procedure included scale, zero-point, background, preferred orientation, lattice parameters, atomic positions, occupations and isotropic displacement parameters. Final $R_{\mathrm{wp}}$ and $\chi^{2}$ values are $0.0883,2.34$ (for $\mathbf{I}$ ) and $0.159,3.48$ (for II), respectively. The final Rietveld plots are shown in figure 1 and the atomic coordinates are listed in tables 1 and 2 . Relevant interatomic distances in $\AA$ (with $\sigma \approx 0.03 \AA$ ) are for $\mathbf{I}$ :
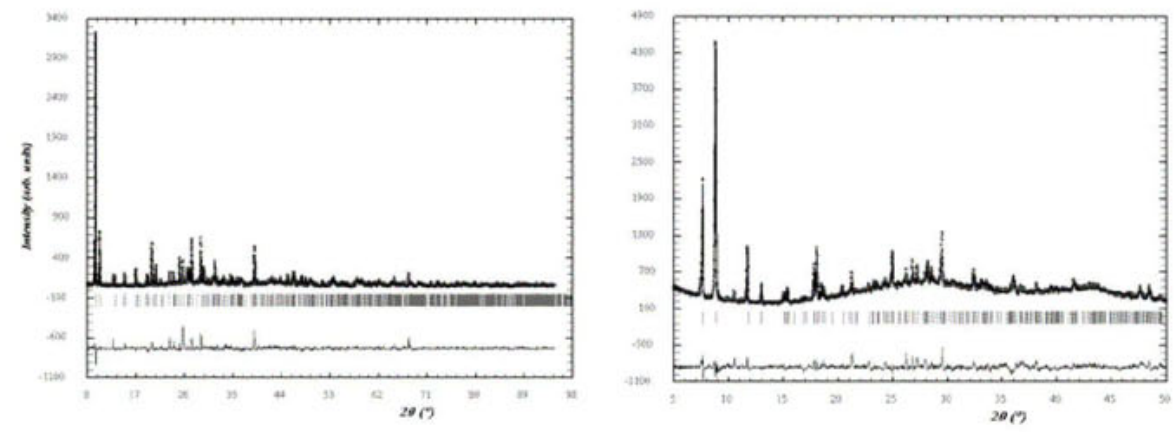

Figure 1. Final Rietveld plots for $\boldsymbol{I}$ (left) and for $\boldsymbol{I I}$ (right). 
Table 1. Atomic coordinates for $\mathrm{CuMo}_{2} \mathrm{O}_{7} \cdot 3 \mathrm{H}_{2} \mathrm{O}(\boldsymbol{I})$ (w indicates oxygen in water molecules).

\begin{tabular}{|c|c|c|c|c|}
\hline atom & $\mathrm{x} / \mathrm{a}$ & $\mathrm{y} / \mathrm{b}$ & $\mathrm{z} / \mathrm{c}$ & $\mathrm{U}_{\mathrm{iso}}\left[\AA^{2}\right]$ \\
\hline $\mathrm{Mo} 1$ & $0.415(4)$ & $0.347(1)$ & $0.110(6)$ & $0.018(5)$ \\
\hline $\mathrm{Mo} 2$ & $0.397(6)$ & $0.385(2)$ & $0.432(2)$ & $0.044(10)$ \\
\hline $\mathrm{Cu} 1$ & $0.535(2)$ & $0.404(5)$ & $0.857(8)$ & $0.046(13)$ \\
\hline $\mathrm{O} 1$ & $0.606(8)$ & $0.356(8)$ & $0.569(4)$ & $0.04(6)$ \\
\hline $\mathrm{O} 2$ & $0.567(2)$ & $0.157(7)$ & $0.621(7)$ & $0.01(4)$ \\
\hline $\mathrm{O} 3$ & $0.636(6)$ & $0.371(2)$ & $0.249(10)$ & $0.03(2)$ \\
\hline $\mathrm{O} 4$ & $0.820(6)$ & $0.381(1)$ & $0.639(5)$ & $0.01(2)$ \\
\hline $\mathrm{O} 5$ & $0.010(3)$ & $0.361(7)$ & $0.844(8)$ & $0.037(11)$ \\
\hline $\mathrm{O} 6 \mathrm{~W}$ & $0.115(4)$ & $0.300(3)$ & $0.526(4)$ & $0.01(4)$ \\
\hline $\mathrm{O} 7 \mathrm{~W}$ & $0.925(7)$ & $0.154(6)$ & $0.899(11)$ & $0.02(4)$ \\
\hline $\mathrm{O} 8 \mathrm{~W}$ & $0.925(5)$ & $0.391(3)$ & $0.187(9)$ & $0.02(2)$ \\
\hline $\mathrm{O} 9$ & $0.379(11)$ & $0.135(1)$ & $0.052(3)$ & $0.01(4)$ \\
\hline $\mathrm{O} 10$ & $0.224(8)$ & $0.338(3)$ & $0.973(9)$ & $0.02(3)$ \\
\hline
\end{tabular}

Table 2. Atomic coordinates for $\mathrm{CoMo}_{3} \mathrm{O}_{10} \cdot 8 \mathrm{H}_{2} \mathrm{O}(\boldsymbol{I})$ ( $w$ indicates oxygen in water molecules).

\begin{tabular}{|c|c|c|c|c|}
\hline atom & $\mathrm{x} / \mathrm{a}$ & $\mathrm{y} / \mathrm{b}$ & $\mathrm{z} / \mathrm{c}$ & $\mathrm{U}_{\mathrm{iso}}\left[\AA^{2}\right]$ \\
\hline $\mathrm{Co} 1$ & $0.724(6)$ & $0.992(9)$ & $0.977(8)$ & $0.03(2)$ \\
\hline $\mathrm{Mo} 1$ & $0.865(9)$ & $0.353(6)$ & $0.940(3)$ & $0.08(4)$ \\
\hline $\mathrm{Mo} 2$ & $0.998(7)$ & $0.253(7)$ & $0.266(2)$ & $0.04(3)$ \\
\hline $\mathrm{Mo3}$ & $0.739(1)$ & $0.245(4)$ & $0.115(2)$ & $0.04(3)$ \\
\hline $\mathrm{O} 1 \mathrm{w}$ & $0.630(2)$ & $0.051(1)$ & $0.089(6)$ & $0.02(1)$ \\
\hline $\mathrm{O} 2 \mathrm{w}$ & $0.794(3)$ & $0.956(4)$ & $0.227(4)$ & $0.02(1)$ \\
\hline $\mathrm{O} 3 \mathrm{w}$ & $0.851(11)$ & $0.059(7)$ & $0.019(2)$ & $0.02(1)$ \\
\hline $\mathrm{O} 4 \mathrm{w}$ & $0.648(2)$ & $0.031(3)$ & $0.731(7)$ & $0.02(1)$ \\
\hline $\mathrm{O} 5 \mathrm{w}$ & $0.596(8)$ & $0.927(11)$ & $0.942(12)$ & $0.02(1)$ \\
\hline $\mathrm{O} 6 \mathrm{w}$ & $0.810(6)$ & $0.930(9)$ & $0.863(8)$ & $0.02(1)$ \\
\hline $\mathrm{O} 7$ & $0.973(8)$ & $0.415(5)$ & $0.033(3)$ & $0.04(3)$ \\
\hline $\mathrm{O} 8$ & $0.874(5)$ & $0.186(4)$ & $0.206(4)$ & $0.03(2)$ \\
\hline $\mathrm{O} 9$ & $0.630(9)$ & $0.182(4)$ & $0.010(2)$ & $0.04(3)$ \\
\hline $\mathrm{O} 10$ & $0.740(6)$ & $0.406(4)$ & $0.878(7)$ & $0.04(3)$ \\
\hline $\mathrm{O} 11$ & $0.852(9)$ & $0.317(1)$ & $0.176(4)$ & $0.03(2)$ \\
\hline $\mathrm{O} 12$ & $0.998(9)$ & $0.274(5)$ & $0.017(3)$ & $0.03(2)$ \\
\hline $\mathrm{O} 13$ & $0.748(7)$ & $0.266(1)$ & $0.872(3)$ & $0.03(2)$ \\
\hline $\mathrm{O} 14$ & $0.118(4)$ & $0.190(4)$ & $0.288(5)$ & $0.04(3)$ \\
\hline $\mathrm{O} 15$ & $0.646(7)$ & $0.316(3)$ & $0.082(2)$ & $0.04(3)$ \\
\hline $\mathrm{O} 16$ & $0.131(1)$ & $0.294(3)$ & $0.367(7)$ & $0.04(3)$ \\
\hline $\mathrm{O} 17 \mathrm{w}$ & $0.707(3)$ & $0.845(7)$ & $0.393(1)$ & $0.05(5)$ \\
\hline $\mathrm{O} 18 \mathrm{w}$ & $0.552(8)$ & $0.743(7)$ & $0.480(3)$ & $0.01(2)$ \\
\hline
\end{tabular}


Mo1-O1: 2.00, Mo1-O2: 2.01, Mo1-O2: 2.31, Mo1-O4: 2.01, Mo1-O9: 1.88, Mo1-O10: 1.86, Mo2-O1: 2.14, Mo2-O2: 2.00, Mo2-O2: 2.40, Mo2-O3: 1.87, Mo2-O4: 2.00, Mo2-O5: 1.86, Cu1-O5: 1.93, Cu1-O6: 2.32, Cu1-O7: 2.30, Cu1-O8: 2.30, Cu1-O8: 2.30, Cu1-O10: 1.81 ; and for II: Co1-O1: 1.95, Co1-O2: 1.96, Co1-O3: 1.98, Co1-O4: 1.97, Co1-O5: 1.96, Co1-O6: 2.01, Mo1-O7: 1.71, Mo1-O8: 2.10, Mo1-O10: 1.70, Mo1-O11: 1.94, Mo1-O12: 2.28, Mo1-O13: 2.26;, Mo2-O8: 2.10, Mo2-O11: 2.28, Mo2-O12: 1.86, Mo2-O12: 2.09, Mo2-O14: 1.71, Mo2-O16: 1.60, Mo3-O9: 1.73, Mo3-O11: 2.09, Mo3-O13: 2.10, Mo3O13: 1.86 , Mo3-O15: 1.50 .

\section{Results and discussion}

\section{Crystal structures}

The structure of $\mathrm{CuMO}_{2} \mathrm{O}_{7} \cdot 3 \mathrm{H}_{2} \mathrm{O}$ (I) consists of 1D stairs-like anions (figure 2). Each "step" consists of 4 distorted octahedra which are connected by edges (figures $2 \mathrm{a}$ and $3 \mathrm{a}$ ).

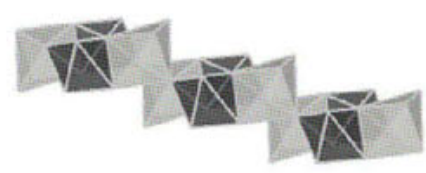

(a)

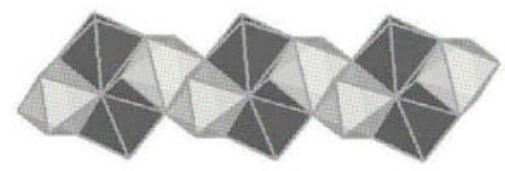

(b)

Figure 2. $\left[\mathrm{Mo}_{2} \mathrm{O}_{7}{ }^{2-}\right]_{\infty}$ molybdate chain in compound I. Black and gray octahedra represent Mol $-\mathrm{O}_{6}$ and $\mathrm{Mo} 2-\mathrm{O}_{6}$ octahedra, respectively.

Compound I contains 2D layers which consist of infinite dimolybdate chains linked by dimers $\left[\mathrm{Cu}_{2} \mathrm{O}_{4}\left(\mathrm{H}_{2} \mathrm{O}\right)_{6}\right]^{4+}$. Dimers are formed by pairs of $\mathrm{CuO}_{6}$ octahedra connected to each other by edges. Copper-oxygen dimers are connected to dimolybdate chains by corners (figu-

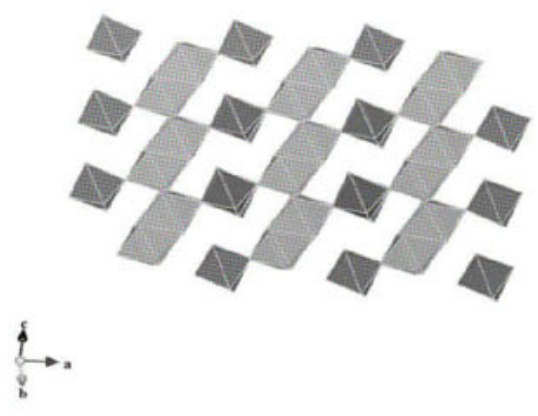

(a)

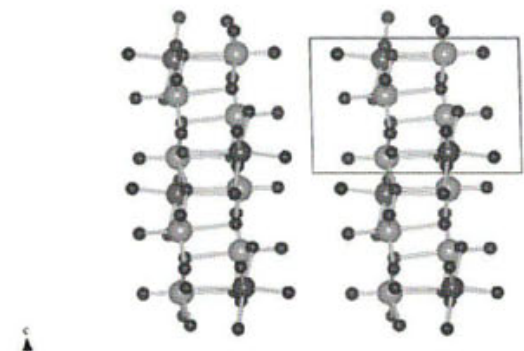

(b)

Figure 3. (a) Infinite layers in the structure of $\mathrm{CuMo}_{2} \mathrm{O}_{7} \cdot 3 \mathrm{H}_{2} \mathrm{O}$, black octahedra $-\left[\mathrm{CuO}_{2}\left(\mathrm{H}_{2} \mathrm{O}\right)_{4}\right]$, grey octahedra $-\mathrm{MoO}_{6}$. (b) parallel layers seen along [100]. 
re 3). The anion of $\mathbf{I}$ is the same as in $\mathrm{Ag}_{2} \mathrm{Mo}_{2} \mathrm{O}_{7}$ [12]. In this kind of dimolybdates tetramer units (built of four $\mathrm{MoO}_{6}$ octahedra), similar to those observed in tetramolybdates, exist. However, in our case the linking of tetramers is different than in tetramolybdates, resulting in different stochiometry, typical for dimolybdates.

The structure of II $\mathrm{CoMo}_{3} \mathrm{O}_{10} \cdot 8 \mathrm{H}_{2} \mathrm{O}$ consists of infinite zigzag anionic chains, presented in figure 4. The chains are constructed of $\mathrm{Mo}_{3} \mathrm{O}_{10}{ }^{2-}$ trimers built of distorted $\mathrm{MoO}_{6}$ octahedra connected to each other by edges and corners. Each octahedron has two short, two medium and two long molybdenum-oxygen bonds. The short distances in each $\mathrm{MoO}_{6}$ octahedron correspond to the two terminal oxygen atoms. Pairs of octahedra (black) form infinite ribbon with formula $\mathrm{Mo}_{2} \mathrm{O}_{8}$ to which $\mathrm{MoO}_{6}$ octahedra (grey) are attached alternately to both sides, forming infinite zig-zag chains with formula $\left[\mathrm{Mo}_{3} \mathrm{O}_{10}{ }^{2}{ }_{\infty}\right]_{\infty}$.

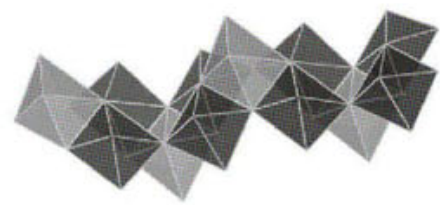

Figure 4. Infinite $\left[\mathrm{MoO}_{3} \mathrm{O}_{10}{ }^{2-}\right]_{\infty}$ zigzag chain, observed in $\mathrm{CoMo}_{3} \mathrm{O}_{10} \cdot 8 \mathrm{H}_{2} \mathrm{O}$.
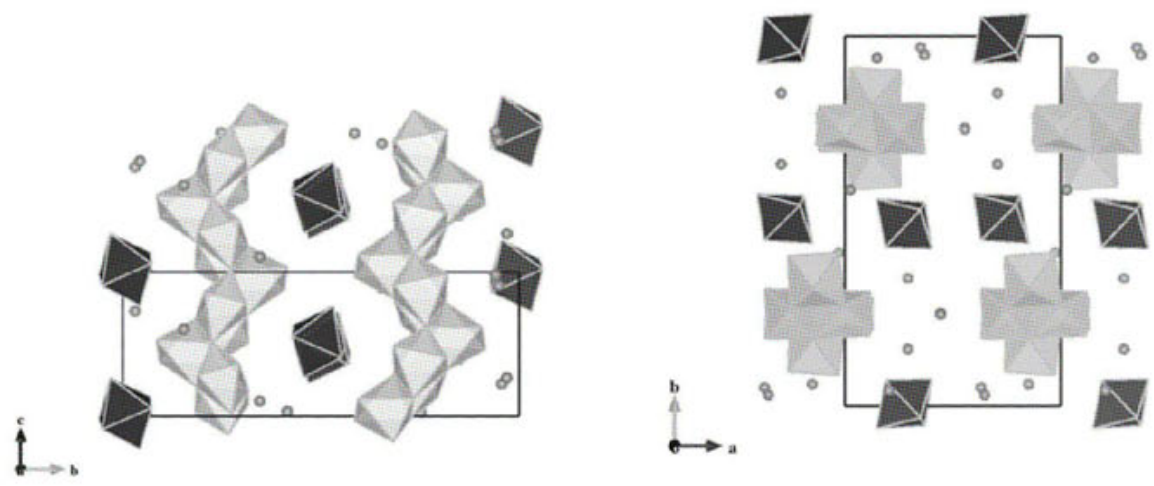

Figure 5. Packing in the unit cell of compound $\boldsymbol{I}$ (left - view along a axis, right - view along $c$ axis). black octahedra $-\left[\mathrm{Co}\left(\mathrm{H}_{2} \mathrm{O}\right)_{6}{ }^{2+}\right]$, grey octahedra $-\mathrm{MoO}_{6}$, small circles represent water molecules. 
Compound II consists also of $\left[\mathrm{Co}\left(\mathrm{H}_{2} \mathrm{O}\right)_{6}{ }^{2+}\right]$ octahedra and water molecules (see figure 5) which separate trimolybdate chains. They are connected to $\left[\mathrm{MO}_{3} \mathrm{O}_{10}{ }^{2-}\right]_{\infty}$ anions by a set of hydrogen bonds.

\section{Concluding remarks}

The crystal structures of: $\mathrm{CuMo}_{2} \mathrm{O}_{7} \cdot 3 \mathrm{H}_{2} \mathrm{O}(\mathbf{I})$, and $\mathrm{CoMo}_{3} \mathrm{O}_{10} \cdot 8 \mathrm{H}_{2} \mathrm{O}$ (II) were determined by X-ray powder diffraction methods. First of them (I), contains unusual 2D layers formed by dimolybdate chains linked by $\left[\mathrm{Cu}_{2} \mathrm{O}_{4}\left(\mathrm{H}_{2} \mathrm{O}\right)_{6}\right]$ octahedra. The second one (II), is a typical polymeric trimolybdate, built of $1 \mathrm{D}$ molybdate chains, $\left[\mathrm{Co}\left(\mathrm{H}_{2} \mathrm{O}\right)_{6}{ }^{2+}\right]$ octahedra and water molecules which separate the chains. Both compounds were investigated due to their potent applications as catalysts or battery materials. These properties will be the subject of our future investigations. [Supplementary crystallographic data for both compounds have been deposited in ICSD database (FIZ, Karlsruhe)].

\section{References}

1. Waters, T., O'Hair, R.A.J. \& Wedd, A.G., 2003, J. Am. Chem. Soc., 125, 3384.

2. Hill, C.L., Weeks, M.S. \& Schinazi, R.F., 1990, J. Med. Chem, 33, 2767.

3. Begam, K.M., Michael, M.S. \& Prabaharan, S.R.S., 2008, J. Solid State Electrochem., 12, 971 .

4. Pope, M.T. \& Muller, A., 1991, Angew. Chem. Int. Ed. Engl., $30,34$.

5. Yamase, T. \& Tomita, K., 1990, Inorg. Chim. Acta, 169, 147.

6. Surga et. al., 1998, Thermochimica Acta, 317,193.

7. Lasocha, W. \& Lewiński, K., 1994, J. Appl. Cryst. 27, 437.

8. Altomare, A., Burla, M.C., Camalli, M., Carrozzini, B., Cascarano, G.L., Giacovazzo, C., Guagliardi, A., Moliterni, A.G.G., Polidori, G., Rizzi, R., 1999, J. Appl. Cryst. 32, 339.

9. Favre-Nicolin, V. \& Cerny, R., 2002, J. Appl. Cryst., 35, 734.

10. Petricek, V., Dusek, M. \& Palatinus, L., 2000, Jana2000, the crystallographic computing system, Institute of Physics, Praha, Czech Republic.

11. Le Bail, A, Duroy, H, \& Fourquet, J.L, 1988, Math. Res. Bull., $23,447$.

12. Gatehouse, B.M. \& Leverett, P., 1976, J. Chem. Soc., Dalton Transition, Inorg. Chem., No 14, 1316.

Acknowledgements. The support of the Polish Ministry of Higher Education (grant 1T09A 07730) is kindly acknowledged. 


\section{Molybdates of p-bromoanilinium - synthesis and crystal structure of new catalytic materials}

W. Lasocha $^{1,2, *}$, M. Grzywa ${ }^{1,3}$, M. Oszajca ${ }^{2}$

${ }^{1}$ Institute of Catalysis and Surface Chemistry, PAS, ul. Niezapominajek 8, 30-239 Kraków, Poland

${ }^{2}$ Faculty of Chemistry, Jagiellonian University, ul. R. Ingardena 3, 30-060 Kraków, Poland

${ }^{3}$ University Ulm, Inorganic Chemistry II, Albert-Einstein-Allee 11, $89081 \mathrm{Ulm}$, Germany

*lasocha@chemia.uj.edu.pl

Keywords: molybdates, structure determination, Rietveld method

Abstract. The crystal structures of two new p-bromoanilinium molybdates have been determined by the X-ray powder diffraction method. Both compounds crystallize in monoclinic system; p-bromoanilinium pentamolybdate (I) $\mathrm{a}=28.691(4), \mathrm{b}=5.6865(4), \mathrm{c}=14.386(2) \AA$, $\beta=113.22(2)^{\circ}, V=2157.0(5) \AA^{3}$, space group $C 2 / c(15)$, and $p$-bromoanilinium trimolybdate (II) $\mathrm{a}=15.490(2), \mathrm{b}=7.589(1), \mathrm{c}=17.874(3) \AA, \beta=101.54(1)^{\circ}, \mathrm{V}=2058.7(4) \AA^{3}$, space group $\mathbf{P} 2 / \mathrm{c}(14)$. Synthesis and the results of crystal structure determination and thermal decomposition studies are presented in this paper. Crystal structure determination was performed from powder diffraction data, using direct methods and global optimisation techniques.

\section{Introduction}

Molybdenum compounds are interesting as prospective candidates for various applications in catalysis (hydrodesulfurisation, selective oxidation of methanol to formaldehyde, propene to acrolein and acrylonitrile), optoelectronics and anticancer therapy [1]. They can be also used as corrosion inhibitors, pigments, smoke suppressants ( $\mathrm{MoO}_{3}$, molybdates), lubricants $\left(\mathrm{MoS}_{2}\right)$ and components of ceramics $\left(\mathrm{MoSi}_{2}\right)$ [2]. It is also possible to obtain very fine powders (nanoparticles) of $\mathrm{MoO}_{3}, \mathrm{NiO}, \mathrm{CuO}, \mathrm{ZnO}$ or $\mathrm{MoC}_{\mathrm{x}}$ and $\mathrm{MoN}_{\mathrm{y}}$ through thermal decomposition of some molybdates [3, 4]. Recently, we have focused our attention on fibrillar or layered materials with organic cations. Organic components with proper substituents can be used for modification of the properties of the synthesised compounds. Layered or fibrillar materials based on polymolybdate anions offer interesting chemical properties and unusual architecture. The synthesis of a desired molybdate with specifically tailored properties is, however, still a very challenging task. 


\section{Experimental}

\section{Synthesis of p-bromoanilinium molybdates}

Molybdic acid $(1.80 \mathrm{~g}, 0.01 \mathrm{M})$ was dissolved in hot water $(150 \mathrm{ml})$. To the boiling solution $\mathrm{p}$ bromoaniline $(3.44 \mathrm{~g}, 0.02 \mathrm{M})$ was added and the solution was heated for more than 12 hours. Subsequently, a white-gray precipitate of $\left(\mathrm{BrC}_{6} \mathrm{H}_{4} \mathrm{NH}_{3}\right)_{2} \mathrm{Mo}_{3} \mathrm{O}_{10}$ was obtained. The precipitate was filtered off, washed with a small amount of water-propanol mixture and dried in air. When, in similar conditions, hydrochloric acid $(1.75 \mathrm{ml}, 35 \%)$ was added to the solution of pbromoaniline and $\mathrm{H}_{2} \mathrm{MoO}_{4},\left(\mathrm{BrC}_{6} \mathrm{H}_{4} \mathrm{NH}_{3}\right)_{2} \mathrm{Mo}_{5} \mathrm{O}_{16}$ was obtained.

\section{Elemental analysis, PXRD, thermal decomposition and SEM studies}

For PXRD studies, portions of the samples were powdered and placed into a standard sample holder. The PXRD patterns were collected using a Philips X' Pert Pro MPD diffractometer. Details of data collection are listed in table 1.

The thermal decompositions in air of the investigated molybdates were studied in situ by $\mathrm{X}$ ray diffraction. Measurements were performed at a temperature range from 25 to $700^{\circ} \mathrm{C}$, in $2 \theta$ range $4.0^{\circ}-60^{\circ}$, step size $0.025^{\circ} 2 \Theta$, time $1.5 \mathrm{~s} / \mathrm{step}$. The $\mathrm{X}$-ray radiation source was a $\mathrm{Cu}$ tube. The heating rate was $5^{\circ} \mathrm{C} / \mathrm{min}$. Once the appropriate temperature was reached the sample was heated for 30 minutes. The VT-XRPD investigations were carried out on a Siemens D5005 diffractometer equipped with an XRK 900 (Anton Paar) reaction chamber. Evolutions of the diffraction patterns measured during the thermal decompositions in air of $\left(\mathrm{BrC}_{6} \mathrm{H}_{4} \mathrm{NH}_{3}\right)_{2} \mathrm{Mo}_{5} \mathrm{O}_{16}$ and $\left(\mathrm{BrC}_{6} \mathrm{H}_{4} \mathrm{NH}_{3}\right)_{2} \mathrm{Mo}_{3} \mathrm{O}_{10}$ are presented in figure 1 and 2, respectively.

Scanning microscopy studies were performed using a JEOL JSM-5410 microscope and Hitachi S-4700 with EDS microanalysis in the Scanning Microscopy Laboratory of Biological and Geological Science of Jagiellonian University. SEM images of $\left(\mathrm{BrC}_{6} \mathrm{H}_{4} \mathrm{NH}_{3}\right)_{2} \mathrm{Mo}_{5} \mathrm{O}_{16}$ and $\left(\mathrm{BrC}_{6} \mathrm{H}_{4} \mathrm{NH}_{3}\right)_{2} \mathrm{Mo}_{3} \mathrm{O}_{10}$ are presented in figure 3 .

Table 1.Crystal and experimental data for $\left(\mathrm{BrC}_{6} \mathrm{H}_{4} \mathrm{NH}_{3}\right)_{2} \mathrm{Mo}_{5} \mathrm{O}_{16}$ and $\left(\mathrm{BrC}_{6} \mathrm{H}_{4} \mathrm{NH}_{3}\right)_{2} \mathrm{Mo}_{3} \mathrm{O}_{10}$.

\begin{tabular}{|l|l|l|}
\hline Compound & $\left(\mathrm{BrC}_{6} \mathrm{H}_{4} \mathrm{NH}_{3}\right)_{2} \mathrm{Mo}_{5} \mathrm{O}_{16}$ & $\left(\mathrm{BrC}_{6} \mathrm{H}_{4} \mathrm{NH}_{3}\right)_{2} \mathrm{Mo}_{3} \mathrm{O}_{10}$ \\
\hline $\begin{array}{l}\text { percentage of C, } \mathrm{N}, \mathrm{H}, \\
\text { found/(calculated) }[\%]\end{array}$ & $13.43(13.32), 2.56(2.59)$, & $\begin{array}{l}18.30(18.16), 3.78(3.53), \\
1.90(1.78)\end{array}$ \\
\hline $2 \theta$ range, step [ $\left.{ }^{\circ}\right]$, time/step & $4.0-80.0,0.02,18$ & $4.5-60^{\circ}, 0.02,270$ \\
\hline $\mathrm{M} / \mathrm{g} \cdot \mathrm{mol}^{-1}$, density $\left[\mathrm{g} / \mathrm{cm}^{3}\right]$ & $1081.76,3.03$ & $793.88,2.564$ \\
\hline Space group & $\mathrm{C} 2 / \mathrm{c}(15)$ & $\mathrm{P} 2{ }_{1} / \mathrm{c}(14)$ \\
\hline $\mathrm{a} / \AA$ & $28.691(4)$ & $15.490(2)$ \\
\hline $\mathrm{b} / \AA$ & $5.6865(4)$ & $7.589(1)$ \\
\hline
\end{tabular}




\begin{tabular}{|l|l|l|}
\hline $\mathrm{c} / \AA$ & $14.386(2)$ & $17.874(3)$ \\
\hline$\beta /{ }^{\circ}$ & $113.22(2)$ & $101.54(1)$ \\
\hline $\mathrm{V} / \AA^{3}$ & $2157.0(5)$ & $2058.7(4)$ \\
\hline $\mathrm{Z}$ & 4 & 4 \\
\hline $\mathrm{F}_{30}$ & 42.95 & 134.00 \\
\hline $\mathrm{M}_{30}$ & 17.17 & 36.43 \\
\hline $\begin{array}{l}\text { Number of observations and } \\
\text { reflections }\end{array}$ & $3440 / 711$ & $2776 / 582$ \\
\hline Restraints (distances, angles) & $32(22,10)$ & $33(20,13)$ \\
\hline $\begin{array}{l}\text { Ratio restraints/diffraction } \\
\text { data }- \text { end of refinement [\%] }\end{array}$ & $20 \%$ & $10 \%$ \\
\hline $\mathrm{R}_{\mathrm{p}}[\%]$ & & 7.25 \\
\hline \begin{tabular}{l}
$\mathrm{R}_{\mathrm{wp}}[\%]$ \\
\hline
\end{tabular} & 10.8 & 10.10 \\
\hline
\end{tabular}

\section{Obtained results}

Figures 1 and 2 show the evolution of the diffraction patterns measured during the thermal decompositions in air of $\left(\mathrm{BrC}_{6} \mathrm{H}_{4} \mathrm{NH}_{3}\right)_{2} \mathrm{Mo}_{5} \mathrm{O}_{16}$ and $\left(\mathrm{BrC}_{6} \mathrm{H}_{4} \mathrm{NH}_{3}\right)_{2} \mathrm{Mo}_{3} \mathrm{O}_{10}$, respectively. According to VT-XRPD investigations, the decomposition processes are similar. The initial phases are stable up to $200^{\circ} \mathrm{C}$, beyond which decomposition processes were observed. At a temperature of $300^{\circ} \mathrm{C}$ the organic part was burned out and amorphous phases were obtained. Similar behavior - amorphisation of the samples - was observed in the case of the thermal decomposition of $\mathrm{Co}, \mathrm{Ni}, \mathrm{Cu}$ and $\mathrm{Zn}$ trimolybdates [3] and anilinium trimolybdate [4]. It is very possible that at this temperature nanoparticles of $\mathrm{MoO}_{3}$ were created (very broad peaks in the pattern).

Formation of a new crystalline phase starts at a temperature of $400^{\circ} \mathrm{C}$. Comparison of the obtained patterns with the PDF-4+ database [5] indicates that during the thermal decompositions of $\left(\mathrm{BrC}_{6} \mathrm{H}_{4} \mathrm{NH}_{3}\right)_{2} \mathrm{Mo}_{5} \mathrm{O}_{16}$ and $\left(\mathrm{BrC}_{6} \mathrm{H}_{4} \mathrm{NH}_{3}\right)_{2} \mathrm{Mo}_{3} \mathrm{O}_{10}$ in air, molybdenum oxide(VI) $\mathrm{MoO}_{3}$ was created. This phase is stable up to a temperature of $700^{\circ} \mathrm{C}$. 


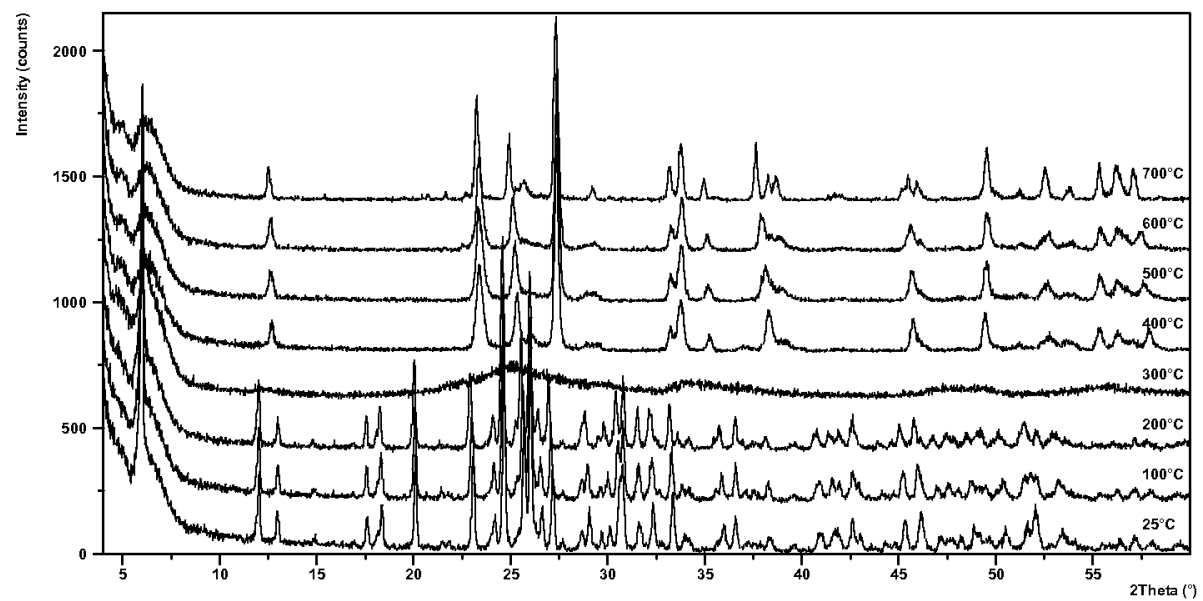

Figure 1. Evolution of the X-ray diffraction pattern during decomposition of $\left(\mathrm{Br}-\mathrm{C}_{6} \mathrm{H}_{4} \mathrm{NH}_{3}\right)_{2} \mathrm{Mo} \mathrm{O}_{5} \mathrm{O}_{16}$.

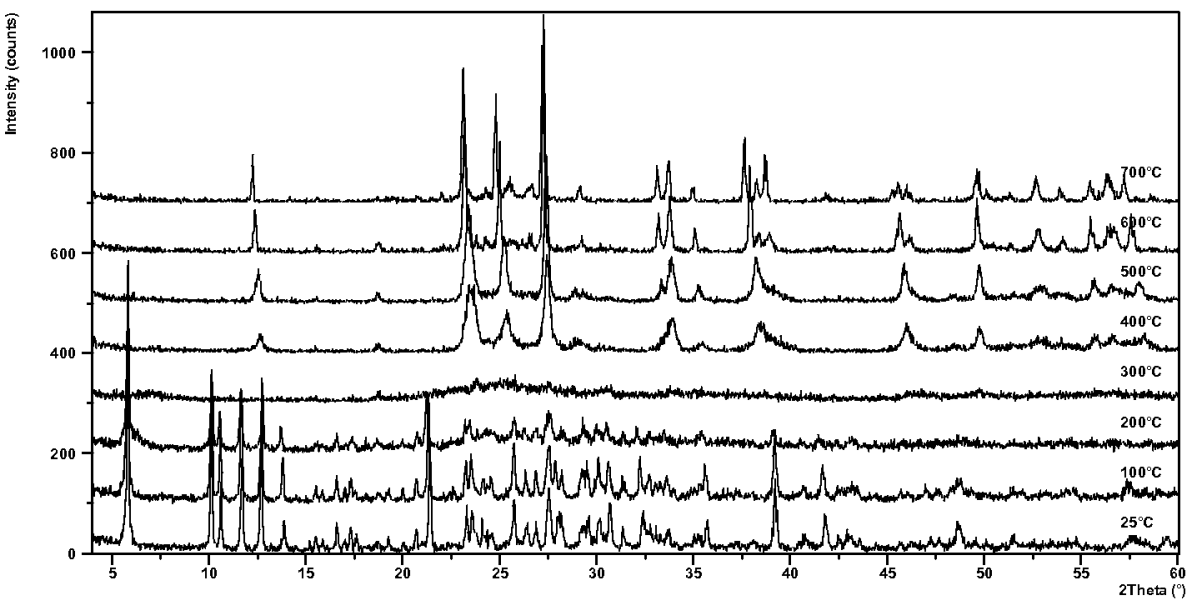

Figure 2. Evolution of the $\mathrm{X}$-ray diffraction pattern during decomposition of $\left(\mathrm{BrC}_{6} \mathrm{H}_{4} \mathrm{NH}_{3}\right)_{2} \mathrm{Mo}_{3} \mathrm{O}_{10}$.

As shown in figure 3, $\left(\mathrm{BrC}_{6} \mathrm{H}_{4} \mathrm{NH}_{3}\right)_{2} \mathrm{Mo}_{5} \mathrm{O}_{16}$ forms large, massive plates, while $\left(\mathrm{BrC}_{6} \mathrm{H}_{4} \mathrm{NH}_{3}\right)_{2} \mathrm{Mo}_{3} \mathrm{O}_{10}$ is a fibre-like substance. 


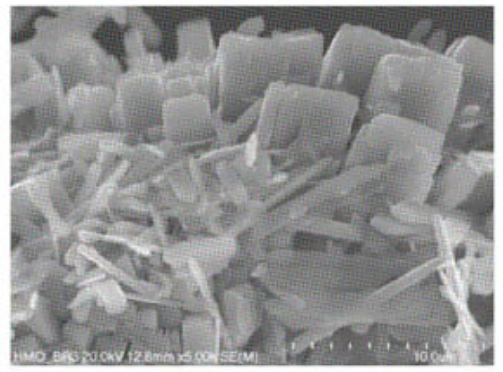

(a)

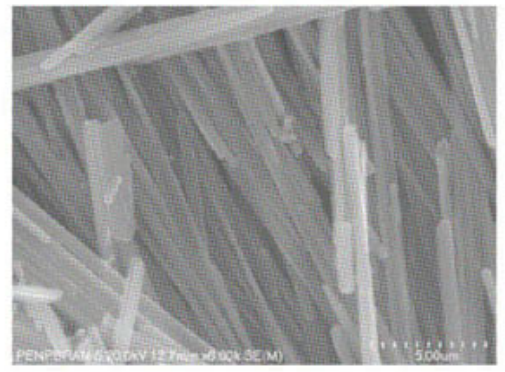

(b)

Figure 3. SEM images of (a) $\left(\mathrm{BrC}_{6} \mathrm{H}_{4} \mathrm{NH}_{3}\right)_{2} \mathrm{Mo}_{5} \mathrm{O}_{16}$ and (b) $\left(\mathrm{BrC}_{6} \mathrm{H}_{4} \mathrm{NH}_{3}\right)_{2} \mathrm{Mo}_{3} \mathrm{O}_{10}$.

\section{Crystal structure study and discussion}

The lattice parameters and space groups were determined using the PROSZKI package [6]. Positional parameters of molybdenum atoms were found using the EXPO2004 program [7]. The crystal structure models were completed by the FOX program using the global optimization method [8]. Final refinements were performed using the XRS-82 Rietveld system [9] and the Jana2000 program [10]. Weak geometric constraints were applied during the refinement process. The Rietveld refinement plots for $\left(\mathrm{BrC}_{6} \mathrm{H}_{4} \mathrm{NH}_{3}\right)_{2} \mathrm{Mo}_{5} \mathrm{O}_{16}$ and $\left(\mathrm{BrC}_{6} \mathrm{H}_{4} \mathrm{NH}_{3}\right)_{2} \mathrm{Mo}_{3} \mathrm{O}_{10}$ are presented in figure 4 and 5 , respectively.

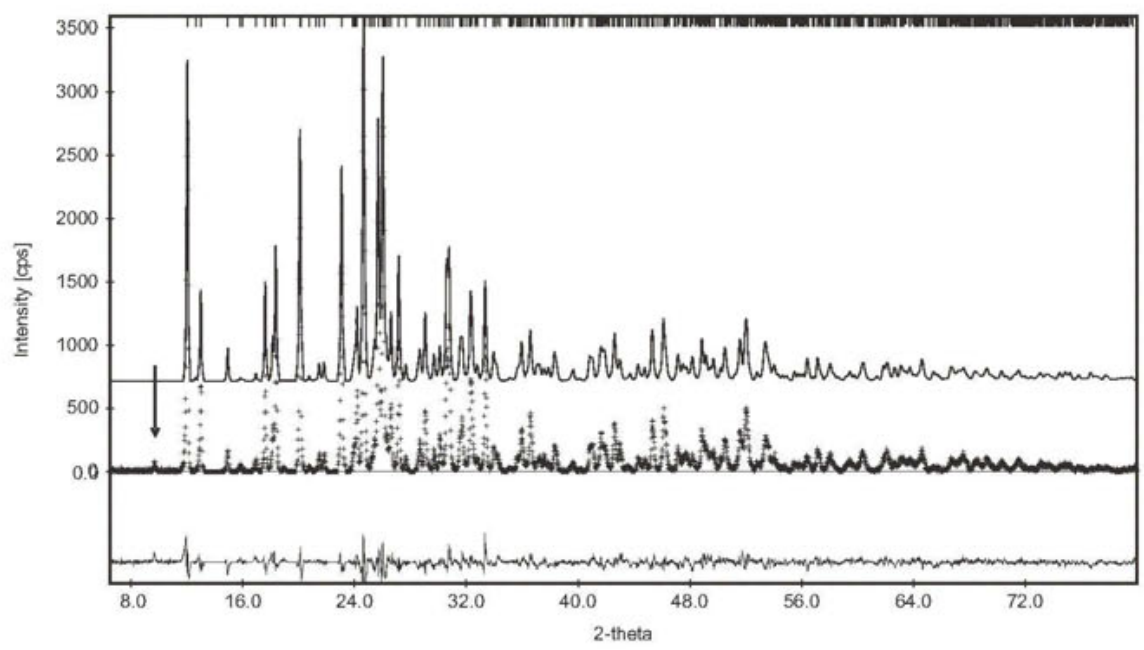

Figure 4. Rietveld refinement plots for $\left(\mathrm{BrC}_{6} \mathrm{H}_{4} \mathrm{NH}_{3}\right)_{2} \mathrm{Mo}_{5} \mathrm{O}_{16}$ curves from top to bottom are: calculated, observed and difference patterns, respectively. To increase stability of Rietveld refinement the strongest, very asymmetric peak (200) was excluded from calculations, line at 9.60 is the strongest line from hexagonal $\mathrm{MoO} 3$ (indicated by arrow). 


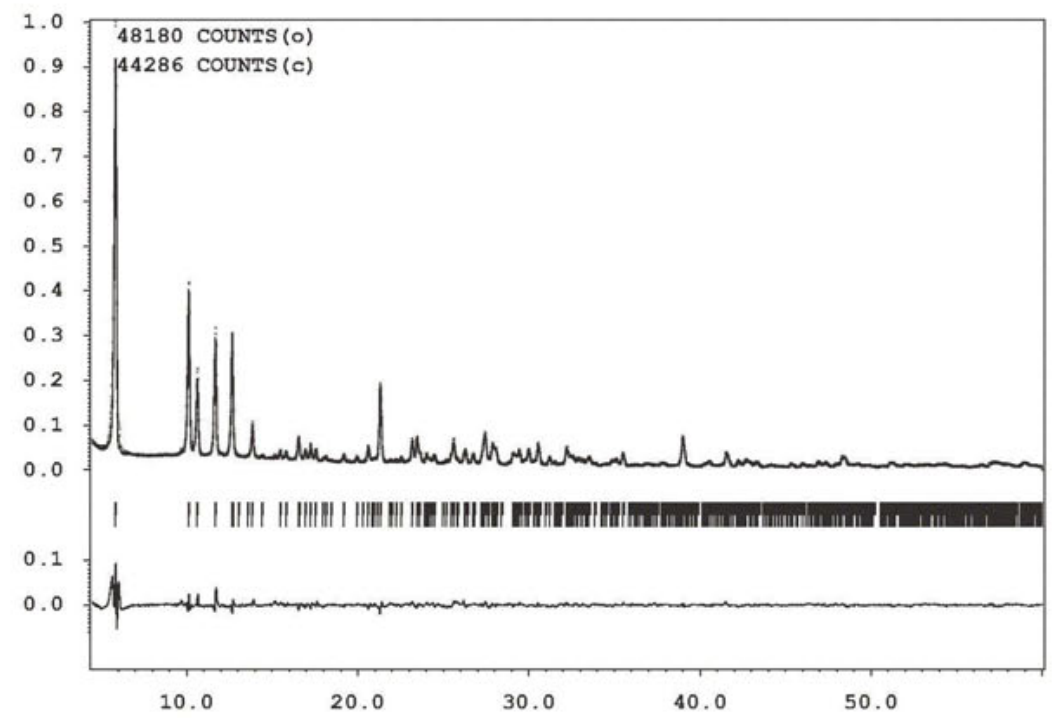

Figure 5. Rietveld refinement plots for $\left(\mathrm{BrC}_{6} \mathrm{H}_{4} \mathrm{NH}_{3}\right)_{2} \mathrm{Mo}_{3} \mathrm{O}_{10}$.

The crystal structure of $\left(\mathrm{BrC}_{6} \mathrm{H}_{4} \mathrm{NH}_{3}\right)_{2} \mathrm{Mo}_{5} \mathrm{O}_{16}$ consists of infinite layers parallel to (100), built from $\mathrm{MoO}_{6}$ octahedra. There are organic cations (p-bromoanilinium) between the layers. Packing of molecules in the unit cell and Mo-O chains in $\left(\mathrm{BrC}_{6} \mathrm{H}_{4} \mathrm{NH}_{3}\right)_{2} \mathrm{Mo}_{5} \mathrm{O}_{16}$ are depicted in figure 6.

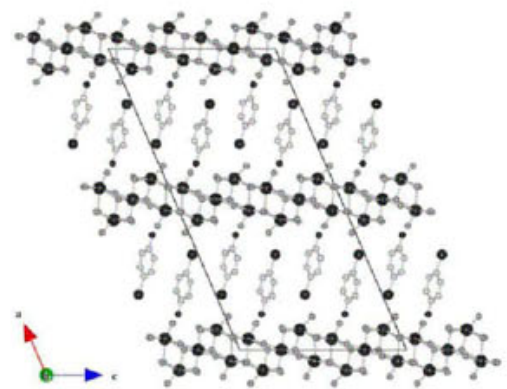

(a)

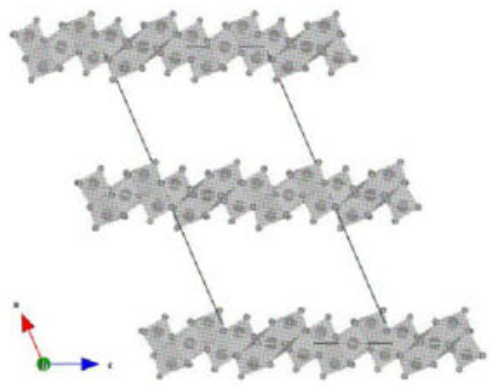

(b)

Figure 6. (a) Packing of molecules in the unit cell of $\left(\mathrm{BrC}_{6} \mathrm{H}_{4} \mathrm{NH}_{3}\right)_{2} \mathrm{Mo}_{5} \mathrm{O}_{16}$

(b) infinite $\mathrm{Mo}_{5} \mathrm{O}_{16}{ }^{2-}$ layers in polyhedral representation.

The crystal structure of $\left(\mathrm{BrC}_{6} \mathrm{H}_{4} \mathrm{NH}_{3}\right)_{2} \mathrm{Mo}_{3} \mathrm{O}_{10}$ consists of infinite $\mathrm{Mo}_{3} \mathrm{O}_{10}{ }^{2 \cdot}$ anionic chains running along the $[010]$ direction. The chains are separated by p-bromoaniline cations. Within the chains distorted octahedral of $\mathrm{MoO}_{6}$ are connected by edge- and corner-sharing. Pack- 
ing of molecules in the unit cell and Mo-O chains in $\left(\mathrm{BrC}_{6} \mathrm{H}_{4} \mathrm{NH}_{3}\right)_{2} \mathrm{Mo}_{3} \mathrm{O}_{10}$ are depicted in figure 7 .

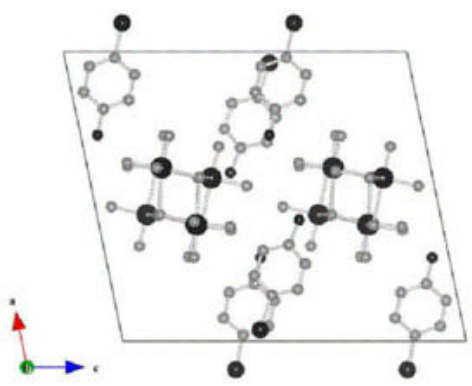

(a)

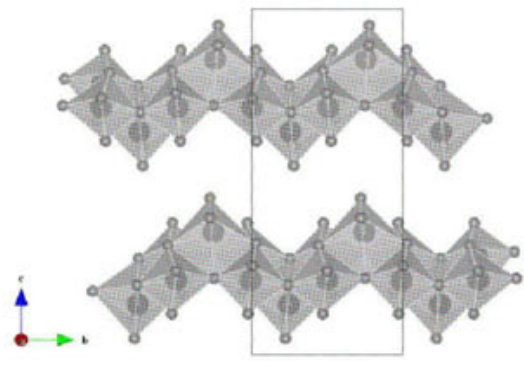

(b)

Figure 7. (a) Packing of molecules in the unit cell of $\left(\mathrm{BrC}_{6} \mathrm{H}_{4} \mathrm{NH}_{3}\right)_{2} \mathrm{Mo}_{3} \mathrm{O}_{10}$

(b) infinite $\mathrm{Mo}-\mathrm{O}$ chains in $\left(\mathrm{BrC}_{6} \mathrm{H}_{4} \mathrm{NH}_{3}\right)_{2} \mathrm{Mo}_{3} \mathrm{O}_{10}$ (polyhedral representation).

Mo-O distances in $\left(\mathrm{BrC}_{6} \mathrm{H}_{4} \mathrm{NH}_{3}\right)_{2} \mathrm{Mo}_{5} \mathrm{O}_{16}$ and $\left(\mathrm{BrC}_{6} \mathrm{H}_{4} \mathrm{NH}_{3}\right)_{2} \mathrm{Mo}_{3} \mathrm{O}_{10}$ are collected in table 2 .

Table 2. Mo-O distances (and symmetry codes for atoms) in $\left(\mathrm{BrC}_{6} \mathrm{H}_{4} \mathrm{NH}_{3}\right)_{2} \mathrm{Mo}_{5} \mathrm{O}_{16}$ and $\left(\mathrm{BrC}_{6} \mathrm{H}_{4} \mathrm{NH}_{3}\right)_{2} \mathrm{Mo}_{3} \mathrm{O}_{10}$.

\begin{tabular}{|l|l|l|l|}
\hline Mo-O distance & $\left(\mathrm{BrC}_{6} \mathrm{H}_{4} \mathrm{NH}_{3}\right)_{2} \mathrm{Mo}_{5} \mathrm{O}_{16}$ & Mo-O distance & $\left(\mathrm{BrC}_{6} \mathrm{H}_{4} \mathrm{NH}_{3}\right)_{2} \mathrm{Mo}_{3} \mathrm{O}_{10}$ \\
\hline Mo1-O8 & $1.74(5)$ & Mo1-O2 & $1.700(4)$ \\
\hline Mo1-O7 & $1.79(7) ; \mathrm{x}, \mathrm{y}-1, \mathrm{z}$ & Mo1-O5 & $1.80(2)$ \\
\hline Mo1-O4 & $1.89(4)$ & Mo1-O6 & $1.930(5)$ \\
\hline Mo1-O3 & $1.99(6) ;-\mathrm{x}+2,-\mathrm{y}-1,-\mathrm{z}+1$ & Mo1-O3 & $1.99(3)$ \\
\hline Mo1-O4 & $2.44(7) ;-\mathrm{x}+2,-\mathrm{y}-1,-\mathrm{z}+1$ & Mo1-O1 & $1.99(8)$ \\
\hline Mo1-O2 & $2.51(6) ;-\mathrm{x}+2,-\mathrm{y},-\mathrm{z}-1$ & Mo1-O4 & $2.500(9)$ \\
\hline Mo2-O6 & $1.73(8)$ & Mo2-O7 & $1.70(2)$ \\
\hline Mo2-O5 & $1.75(5)$ & Mo2-O8 & $1.90(8)$ \\
\hline Mo2-O4 & $1.99(8)$ & Mo2-O4 & $2.25(6) ; \mathrm{x}, 1+\mathrm{y}, \mathrm{z}$ \\
\hline Mo2-O3 & $2.06(4)$ & Mo2-O1 & $2.262(9) ; \mathrm{x}, 1+\mathrm{y}, \mathrm{z}$ \\
\hline Mo2-O1 & $2.30(7)$ & Mo2-O3 & $2.42(6) ; \mathrm{x}, 1+\mathrm{y}, \mathrm{z}$ \\
\hline Mo2-O7 & $2.36(7)$ & Mo2-O6 & $2.57(5) ; \mathrm{x}, 1+\mathrm{y}, \mathrm{z}$ \\
\hline
\end{tabular}




\begin{tabular}{|l|l|l|l|}
\hline Mo3-O2 & $1.86(7)$ & Mo3-O9 & $1.70(2)$ \\
\hline Mo3-O2 & $1.86(7) ;-\mathrm{x}+2, \mathrm{y},-\mathrm{z}+3 / 2$ & Mo3-O10 & $1.80(2)$ \\
\hline Mo3-O1 & $1.96(4)$ & Mo3-O1 & $1.803(8) ; \mathrm{x}, 1+\mathrm{y}, \mathrm{z}$ \\
\hline Mo3-O1 & $1.96(4) ;-\mathrm{x}+2, \mathrm{y},-\mathrm{z}+3 / 2$ & Mo3-O6 & $1.929(7) ; \mathrm{x}, 1+\mathrm{y}, \mathrm{z}$ \\
\hline Mo3-O3 & $2.24(5)$ & Mo3-O4 & $2.08(4)$ \\
\hline Mo3-O3 & $2.24(5) ;-\mathrm{x}+2, \mathrm{y},-\mathrm{z}+3 / 2$ & Mo3-O3 & $2.50(4)$ \\
\hline
\end{tabular}

\section{Conclusions}

Two new compounds belonging to the family of p-bromoanilinium molybdates were obtained and characterised by chemical analysis, VT-XRPD and SEM methods. Their crystal structures were solved by powder diffraction methods.

\section{References}

1. Ullmann's Encyclopedia of Industrial Chemistry, sixth ed., 2002, (New York: Wiley $\mathrm{VCH})$.

2. Mitsui, S., Ogata, A., Yanagie, H., Kasano, H., Hisa, T., Yamase, T. \& Eriguchi, M., 2006, Biomedicine \& Pharmacotherapy, 60, 353.

3. Surga, W., Lasocha, W. \& Hodorowicz, S., 1998, Thermochimica Acta, 317, 193.

4. Grzywa, M. \& Lasocha, W., 2008, Journal of Thermal Analysis and Calorimetry.

5. PDF-4+, The International Centre for Diffraction Data, 2007.

6. Lasocha, W. \& Lewiński, K., 1994, PROSZKI - a system of programs for powder diffraction data analysis, J. Appl. Cryst., 27, 437.

7. Altomare, A., Caliandro, R., Camalli, M., Cuocci, C., Giacovazzo, C., Moliterni, A.G.G. \& Rizzi, R., 2004, Automatic structure determination from powder data with EXPO2004, J. Appl. Cryst., 37, 1025.

8. Favre-Nicolin, V. \& Cerny, R., 2002, J. Appl. Cryst., 35, 734.

9. Baerlocher, Ch., 1992, XRS-82, The X-ray Rietveld System, Institut für Kristallographie, ETH Zürich, Switzerland.

10. Petricek, V., Dusek, M. \& Palatinus, L., 2000, Jana2000, The Crystallographic Computing System, Institute of Physic, Praha, Czech Republic.

Acknowledgements. The support of the Polish Ministry of Science and Higher Education (grant 1T09A 07730) is gratefully acknowledged. 


\title{
The Rietveld structure refinement of an exceptionally pure sample of clinoptilolite from Ecuador and its $\mathrm{Na}-, \mathrm{K}-$, and $\mathrm{Ca}-$ exchanged forms
}

\author{
R. A. Snellings ${ }^{1, *}$, A. F. Gualtieri ${ }^{2}$, J. Elsen $^{1}$ \\ ${ }^{1}$ Katholieke Universiteit Leuven, Leuven, Belgium \\ ${ }^{2}$ Universita di Modena and Reggio Emilia, Modena, Italy \\ * ruben.snellings@ees.kuleuven.be
}

Keywords: clinoptilolite, Ecuador, XRPD, synchrotron, Rietveld method

\begin{abstract}
The crystal structures of a highly pure, sedimentary clinoptilolite and the Na-, K-, and Ca-exchanged forms were refined by the Rietveld method using X-ray diffraction data collected with both conventional and synchrotron sources and different instrumental geometries. The refined structures showed that the clinoptilolite framework is not sensitive to cation exchange with $\mathrm{Na}^{+}, \mathrm{K}^{+}$or $\mathrm{Ca}^{2+}$. The $\mathrm{Ml}$ extraframework cation site is occupied by $\mathrm{Na}$, $\mathrm{Ca}$, and $\mathrm{Ba}, \mathrm{M} 2$ by $\mathrm{Ca}, \mathrm{M} 3$ by $\mathrm{K}$, and $\mathrm{M} 4$ by $\mathrm{Mg}$. The $\mathrm{M} 1$ site occupancy significantly influences the configuration of extraframework water molecules.
\end{abstract}

\section{Introduction}

Clinoptilolite [HEU] is a monoclinic zeolite belonging to the group of tabular zeolites. The framework is built up out of $(\mathrm{Si}, \mathrm{Al})_{10} \mathrm{O}_{20}$ units arranged in sheets. The sheets are connected in the $(010)$ plane to form a three-dimensional channel system consisting of A channels built up of flattened ten-membered rings and $\mathrm{B}$ channels formed by regular eight-membered rings running along the c-axis. The $\mathrm{C}$ channels are oriented along the $a$ axis and consist of regular eight-membered rings [1-3]. Clinoptilolite is distinguished from heulandite by a framework $\mathrm{Si} / \mathrm{Al}>4$.

Extraframework cation sites are situated on the (010) mirror plane (figure 1). Most sites can be occupied only partially, due to incompatibilities with other cation and water positions. The M1 site in cage II, formed by channel $\mathrm{A}$ and $\mathrm{C}$, is preferentially occupied by Na and often also contains $\mathrm{Ca}$. The M2 site in cage $\mathrm{I}$, formed by channel B and $\mathrm{C}$, usually contains $\mathrm{Ca}[1,4]$. The M3 site near the centre of the eight-membered ring at the intersection of the A and $\mathrm{C}$ channel shows cation framework distances suitable for $\mathrm{K} . \mathrm{Mg}$ is situated in the centre of the cage II (M4) coordinated only to $\mathrm{H}_{2} \mathrm{O}$ molecules [5].

Few crystal structure refinements of very fine-grained $(2-20 \mu \mathrm{m})$ natural sedimentary clinoptilolite are reported in the literature [6] due to the presence of inseparable sample impurities such as clay minerals and feldspars. 
Here, a comparison is made between Rietveld crystal structures of natural, K-, Na-, and Caexchanged clinoptilolite refined from data collected at various conventional and synchrotron sources.

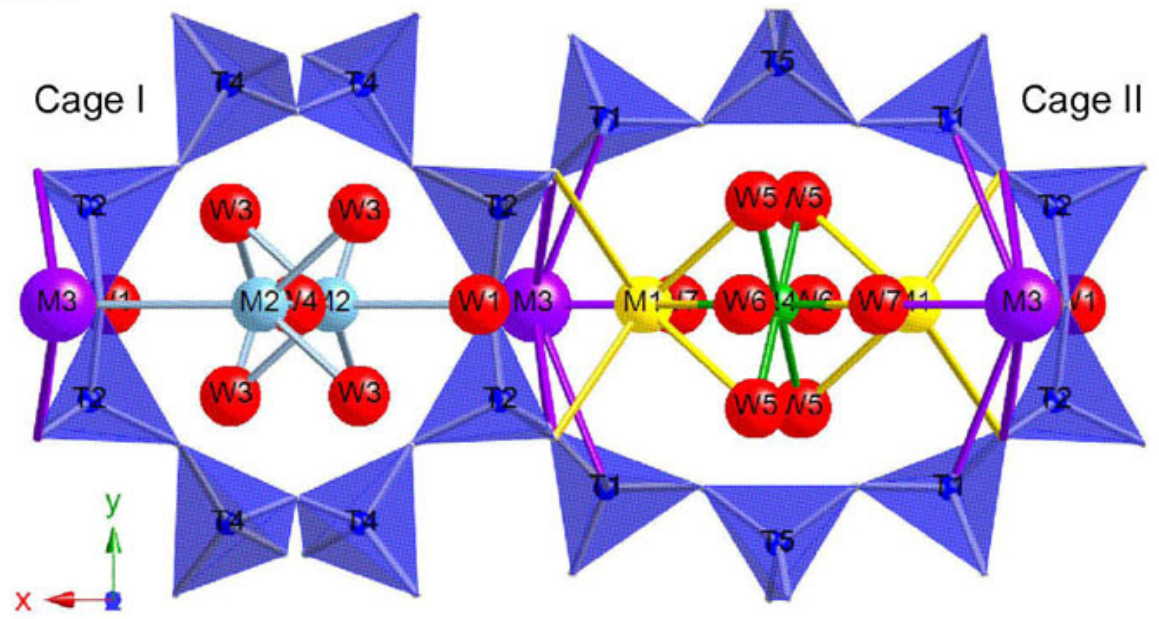

Figure 1. Overview of the clinoptilolite structure projected along the $c^{*}$-axis; extraframework cations and water molecules are shown in cage I, at the intersection of the B and C channel, and cage II, at the intersection of the A and C channel.

\section{Experimental}

The clinoptilolite studied here contains only ca. $1 \mathrm{wt} \%$ quartz as impurity. This specimen allowed for a direct structure refinement based on laboratory and synchrotron diffraction data. The sample was taken along the Rio Ayampe in the vicinity of the village of Guale and occurred as a series of white to greenish tuff layers situated within the volcanoclastic Cayo Formation (Ecuador) [7]. The clinoptilolite sample was exchanged to $\mathrm{Na}, \mathrm{Ca}$, and $\mathrm{K}$ by immersion in regularly renewed nearly saturated solutions of the corresponding acetates for 20 days at $333 \mathrm{~K}$. The crystal chemistry was determined by X-ray Fluorescence (XRF) spectroscopy and thermal analysis (TG) on bulk samples. X-ray powder diffraction data were collected at lab sources for all samples, and at the synchrotron Italian beamline BM08 at ESRF for the natural and Na-exchanged samples. The in-house data were collected with a Philips PW 1830, Bragg-Brentano geometry, $\mathrm{Cu} \mathrm{K} \alpha$ radiation, and with a Panalytical X'Pert Pro diffractometer with a $\theta / \theta$ geometry, $\mathrm{Cu} \mathrm{K} \alpha$ radiation, and a Real Time Multiple Strip (RTMS) detector.

Addition of NIST SRM $640 \mathrm{Si}$ standard allowed the refinement of absolute lattice parameters. Structure models were taken from the literature [5,6]; atomic coordinates and population parameters were refined in the first stages, only $\mathrm{Si}$ was used as zeolite framework cation, finally thermal parameters were refined. Site occupancies were cross-checked with crystal chemistry. The Rietveld refinements were conducted with both EXPGUI-GSAS $[8,9]$ and Topas Academic ${ }^{\circledR}[10]$ packages. 
Table 1. Clinoptilolite crystal chemistry of natural and exchanged samples are reported and compared to the chemistry obtained from Rietveld structure refinements on X-ray diffraction patterns collected at different equipment and source types.

\begin{tabular}{|c|c|c|}
\hline $\begin{array}{l}\text { Sample } \\
\text { Equipment }\end{array}$ & $\begin{array}{l}\text { Extraframework cation and water } \\
\text { content from XRF and } \mathrm{TG} \text { based on } \\
\mathrm{Al}_{7.10} \mathrm{Si}_{28.90} \mathrm{O}_{72} \text { framework }\end{array}$ & $\begin{array}{l}\text { Extraframework cation and water } \\
\text { content from the Rietveld refinement } \\
\text { based on } \mathrm{Si}_{36} \mathrm{O}_{72} \text { framework }\end{array}$ \\
\hline $\begin{array}{l}\text { Natural } \\
\text { X'Pert }\end{array}$ & & $\mathrm{Na}_{0.92} \mathrm{~K}_{1.28} \mathrm{Ca}_{1.91} \mathrm{Ba}_{0.04} \mathrm{Mg}_{0.67} \cdot 23.53 \mathrm{H}_{2} \mathrm{O}$ \\
\hline $\begin{array}{l}\text { Natural } \\
\text { PW1830 }\end{array}$ & $\mathrm{Na}_{0.92} \mathrm{~K}_{1.31} \mathrm{Ca}_{1.95} \mathrm{Ba}_{0.05} \mathrm{Mg}_{0.63} \cdot 22.15 \mathrm{H}_{2} \mathrm{O}$ & $\mathrm{Na}_{0.92} \mathrm{~K}_{1.25} \mathrm{Ca}_{2.16} \mathrm{Ba}_{0.07} \mathrm{Mg}_{0.65} \cdot 24.04 \mathrm{H}_{2} \mathrm{O}$ \\
\hline $\begin{array}{l}\text { Natural } \\
\text { ESRF BM08 }\end{array}$ & & $\mathrm{Na}_{0.92} \mathrm{~K}_{1.31} \mathrm{Ca}_{1.97} \mathrm{Ba}_{0.05} \mathrm{Mg}_{0.61} \cdot 23.25 \mathrm{H}_{2} \mathrm{O}$ \\
\hline $\begin{array}{l}\mathrm{Na} \\
\text { exchanged } \\
\mathrm{X}^{\prime} \text { Pert }\end{array}$ & $\mathrm{Na}_{3.97} \mathrm{~K}_{0.98} \mathrm{Ca}_{0.91} \mathrm{Ba}_{0.03} \mathrm{Mg}_{0.41} \cdot 23.29 \mathrm{H}_{2} \mathrm{O}$ & $\mathrm{Na}_{3.11} \mathrm{~K}_{1.26} \mathrm{Ca}_{1.51} \mathrm{Mg}_{0.72} \cdot 23.16 \mathrm{H}_{2} \mathrm{O}$ \\
\hline $\begin{array}{l}\mathrm{Na} \\
\text { exchanged }\end{array}$ & & $\mathrm{Na}_{3.09} \mathrm{~K}_{1.36} \mathrm{Ca}_{1.58} \mathrm{Mg}_{0.67} \cdot 23.01 \mathrm{H}_{2} \mathrm{O}$ \\
\hline $\begin{array}{l}\mathrm{Ca} \\
\text { exchanged } \\
\text { X'Pert }\end{array}$ & $\mathrm{Na}_{0.60} \mathrm{~K}_{1.22} \mathrm{Ca}_{2.00} \mathrm{Ba}_{0.04} \mathrm{Mg}_{0.61} \cdot 23.13 \mathrm{H}_{2} \mathrm{O}$ & $\mathrm{Na}_{0.64} \mathrm{~K}_{1.31} \mathrm{Ca}_{2.36} \mathrm{Ba}_{0.08} \mathrm{Mg}_{0.67} \cdot 24.23 \mathrm{H}_{2} \mathrm{O}$ \\
\hline $\begin{array}{l}\mathrm{K} \text { exchanged } \\
\mathrm{X}^{\prime} \text { 'Pert }\end{array}$ & $\mathrm{Na}_{0.45} \mathrm{~K}_{4.39} \mathrm{Ca}_{1.02} \mathrm{Ba}_{0.02} \mathrm{Mg}_{0.42} .21 .64 \mathrm{H}_{2} \mathrm{O}$ & $\mathrm{K}_{4.38} \mathrm{Ca}_{1.48} \mathrm{Mg}_{0.58} \cdot 20.48 \mathrm{H}_{2} \mathrm{O}$ \\
\hline
\end{tabular}

\section{Results}

$\mathrm{XRF}$ results indicated that the cation exchange was not complete (table 1). Exchange of $\mathrm{K}$ and $\mathrm{Mg}$ cations was reported least successful [6], respectively owing to the high density of framework bonds (7) of $\mathrm{K}$ in $\mathrm{M} 3$, and to the large effective diameter of the water solvation sphere of $\mathrm{Mg}$ (M4) in clinoptilolite. The final residuals of the Rietveld refinements were situated between 0.01 and 0.07 for $R_{p}, 0.02$ and 0.10 for $R_{w p}$, and 0.04 and 0.08 for $R\left(F^{2}\right)$. Refined cell parameters, refinement statistics, framework and extraframework atomic coordinates, population and thermal parameters, bond distances, and T-O-T and O-T-O angles can be obtained from the authors upon request. In general, differences between refined models are modest compared to variability in structure models proposed in the literature.

The overall crystal chemistry determined from XRD showed very good agreement with XRF and TG bulk chemistry when a mixed occupancy of Ml by $\mathrm{Na}, \mathrm{Ca}$, and $\mathrm{Ba}$ was assumed (table 1). In the $\mathrm{Na}$ and $\mathrm{K}$ exchanged forms, incorporation of $\mathrm{Na}$ in $\mathrm{M} 2$ is suggested from comparison of crystal chemistry with population parameters. Diffraction experiments cannot differentiate between statistical and dynamical disorder in extraframework cation positions [11]. This resulted in relatively large thermal parameters, and in some cases, in a significantly wider spread of extraframework site fractional coordinates than expected from esd. (table 2). 
Table 2. Space group, cell parameters, extraframework cation site coordinates, population and thermal parameters. The estimated standard deviation values are reported between parentheses.

\begin{tabular}{|c|c|c|c|c|c|c|c|}
\hline Site & $\begin{array}{l}\text { Natural } \\
\text { X'Pert }\end{array}$ & $\begin{array}{l}\text { Natural } \\
\text { PW } 1830\end{array}$ & $\begin{array}{l}\text { Natural } \\
\text { ESRF }\end{array}$ & $\begin{array}{l}\mathrm{Na} \\
\mathrm{X}^{\prime} \text { Pert }\end{array}$ & $\begin{array}{l}\mathrm{Na} \\
\text { ESRF }\end{array}$ & $\begin{array}{l}\mathrm{Ca} \\
\mathrm{X}^{\prime} \text { Pert }\end{array}$ & $\begin{array}{l}\mathrm{K} \\
\mathrm{X}^{\prime} \text { Pert }\end{array}$ \\
\hline Space & $C 2 / m$ & $C 2 / m$ & $C 2 / m$ & $C 2 / m$ & $C 2 / m$ & $C 2 / m$ & $C 2 / m$ \\
\hline$a(\AA)$ & $17.684(2)$ & $17.684(2)$ & $17.684(2)$ & $17.684(2)$ & $17.684(2)$ & $17.664(2)$ & $17.6879(12$ \\
\hline$b(\AA)$ & $17.965(2)$ & $17.965(2)$ & $17.965(2)$ & $17.957(2)$ & $17.957(2)$ & $17.932(2)$ & $17.9503(13$ \\
\hline$c(\AA)$ & $7.4124(7)$ & $7.4124(7)$ & $7.4124(7)$ & $7.4134(5)$ & $7.4134(5)$ & $7.4045(7)$ & $7.4143(4)$ \\
\hline \multicolumn{8}{|c|}{ ( } \\
\hline$x$ & $0.139(2)$ & $0.128(2)$ & $0.134(2)$ & $0.130(2)$ & $0.128(2)$ & $0.137(2)$ & $0.129(2)$ \\
\hline$z$ & $0.657(5)$ & $0.654(5)$ & $0.645(4)$ & $0.676(3)$ & $0.680(4)$ & $0.668(6)$ & $0.671(5)$ \\
\hline Occ. (Na) & 0.23 & 0.23 & 0.23 & $0.78(2)$ & $0.77(2)$ & 0.16 & \\
\hline Occ. (Ca) & 0.135 & 0.135 & 0.135 & & & $0.195(14)$ & \\
\hline Occ. (Ba) & $0.010(4)$ & $0.015(3)$ & $0.012(4)$ & & & 0.016 & \\
\hline Occ. $(\mathrm{K})$ & & & & & & & $0.458(11)$ \\
\hline $\mathrm{U}_{\text {iso }}$ & $0.045(11)$ & $0.056(11)$ & $0.054(11)$ & $0.095(10)$ & $0.11(1)$ & $0.082(14)$ & $0.095(10)$ \\
\hline \multicolumn{8}{|l|}{ M2 $(y=0.5)$} \\
\hline$x$ & $0.037(2)$ & $0.0314(13)$ & $0.0315(14)$ & $0.039(2)$ & $0.032(2)$ & $0.0326(14)$ & $0.037(2)$ \\
\hline$z$ & $0.197(4)$ & $0.202(3)$ & $0.208(3)$ & $0.243(3)$ & $0.230(4)$ & $0.209(3)$ & $0.235(4)$ \\
\hline Occ. (Ca) & $0.342(9)$ & $0.405(7)$ & $0.356(9)$ & $0.377(8)$ & $0.396(10)$ & $0.394(9)$ & $0.369(9)$ \\
\hline $\mathrm{U}_{\text {iso }}$ & $0.092(11)$ & $0.063(8)$ & $0.038(9)$ & $0.062(10)$ & $0.070(9)$ & $0.064(9)$ & $0.079(11)$ \\
\hline \multicolumn{8}{|l|}{ M3 $(y=0.5)$} \\
\hline$x$ & $0.246(2)$ & $0.247(2)$ & $0.240(2)$ & $0.242(2)$ & $0.238(2)$ & $0.240(2)$ & $0.238(5)$ \\
\hline$z$ & $0.041(6)$ & $0.052(4)$ & $0.012(7)$ & $0.037(5)$ & $0.058(4)$ & $0.046(4)$ & $0.030(10)$ \\
\hline Occ. $(\mathrm{K})$ & $0.32(2)$ & $0.313(11)$ & $0.33(2)$ & $0.31(2)$ & $0.34(2)$ & $0.33(2)$ & $0.36(6)$ \\
\hline $\mathrm{U}_{\text {iso }}$ & $0.084(13)$ & $0.050(11)$ & $0.072(14)$ & $0.068(14)$ & $0.065(11)$ & $0.051(11)$ & $0.087(13)$ \\
\hline \multicolumn{8}{|c|}{ M4 $(x, y=0 ; z=0.5)$} \\
\hline Occ. (Mg) & $0.33(4)$ & $0.32(2)$ & $0.31(2)$ & $0.36(3)$ & $0.34(4)$ & $0.34(4)$ & $0.29(5)$ \\
\hline $\mathrm{U}_{\text {iso }}$ & $0.10(3)$ & $0.09(3)$ & $0.17(4)$ & $0.15(3)$ & $0.13(3)$ & $0.16(4)$ & $\begin{array}{l}0.15(4) \\
\text { M3 }^{\prime}(y=0)\end{array}$ \\
\hline$x$ & & & & & & & $0.314(8)$ \\
\hline$z$ & & & & & & & $0.067(14)$ \\
\hline Occ. $(\mathrm{K})$ & & & & & & & $0.27(6)$ \\
\hline $\mathrm{U}_{\text {iso }}$ & & & & & & & $0.11(2)$ \\
\hline
\end{tabular}

\section{Discussion and conclusions}

$\mathrm{T}-\mathrm{O}$ distances indicate that some $(\mathrm{Si}, \mathrm{Al})$ ordering is present in the clinoptilolite framework. As concluded by previous workers $[4,5,10]$, the $\mathrm{Al}$ content seems largest in the $\mathrm{T} 2$ tetrahedron, which bridges the $\mathrm{T}_{10} \mathrm{O}_{20}$ layers and is closely associated with $\mathrm{M} 3$. Here, the clinoptilolite framework was not found very sensitive to cation exchange.

In all refinements, the coordination sphere of M1 $(\sim 2.8 \AA)$ was significantly larger than for M2 ( 2.55 $\AA)$, with Ca residing in the site with smaller coordination distances [5]. 


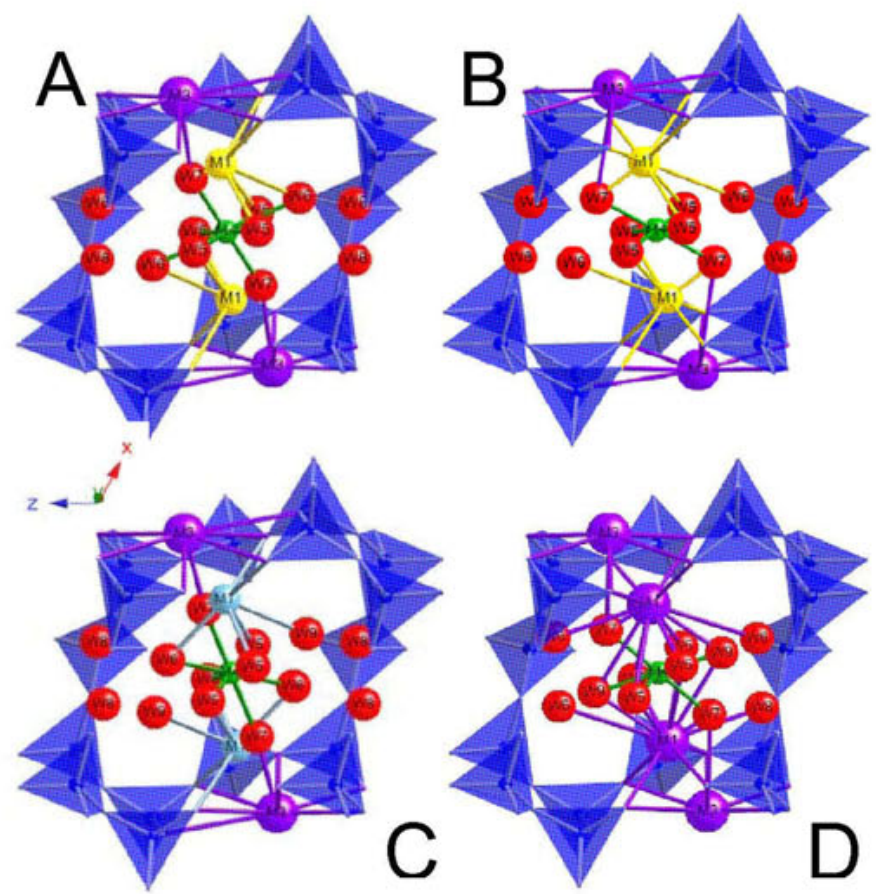

Figure 2. The influence of extraframework cation content on the extraframework configuration is shown. Different configurations of cage II extraframework cations (M1, M3, and M4) and water molecules (W5-W9) present in the natural clinoptilolite (A), Na-exchanged clinoptilolite (B), Caexchanged clinoptilolite $(C)$, and $K$-exchanged clinoptilolite are depicted.

Higher occupancy of M1 in Na- and K-exchanged samples induced a positional shift towards the centre of cage I. Simultaneously, coordinating water molecules were situated at larger distances. The M3 site can be found at the intersection of the A and C channel (figure 2). This site is preferentially occupied by $\mathrm{K}$, which fits into the rather large coordination sphere (3.1 $\AA$ ). For K-exchanged clinoptilolite an additional M3' cation site, close to M3, was found and assigned to $\mathrm{K}$. This M3' site was also previously reported for K-exchanged heulandite [12]. The Mg (M4) is six-fold coordinated by water molecules (W5, W6 or W9, and W7). Distortion of the ideal coordination is largest for Na- and $\mathrm{K}$-exchanged samples.

All water molecule sites present, except W9, can be related to earlier studies, although a mixture of different published models fitted best the data. The W9 site has not been published elsewhere. This site can be seen as a split of the W6 site. W9 was introduced for the coordination of the larger extraframework cations in M1. It can also be coordinated to M4. 


\section{References}

1. Merkle, A.B. \& Slaughter, M., 1968, Am. Mineral., 53, 1138.

2. Breck, D.W., 1974, Zeolite Molecular Sieves (New York: Wiley).

3. Armbruster, T. \& Gunter, M.E., 2001, in Natural Zeolites: Occurrence, Properties, Applications, edited by D.L. Bish \& D.W. Ming (Washington: Mineralogical Society of:America), pp. 1-57.

4. Alberti, A., 1972, Tscher. Miner. Petrog., 18, 129.

5. Koyama, K. \& Takeuchi, Y., 1977, Z. Kristallogr., 145, 216.

6. Cappelletti, P., Langella, A. \& Cruciani, G., 1999, Eur. J. Mineral., 11, 1051.

7. Machiels, L., Morante, F., Snellings, R., Calvo, B., Canoira, L., Paredes, C. \& Elsen, J., 2008, Appl. Clay Sci., 42, 180.

8. Larson, A.C. \& von Dreele, R.B., 2000, GSAS (report LAUR 86-748)

9. Toby, B.H., 2001, J. Appl. Crystallogr., 34, 210.

10. Coelho, A.A., 2007, http://members.optusnet.com.au/alancoelho/.

11. Liebau, F., 1985, Structural Chemistry of Silicates (Berlin: Springer).

12. Yang, P. \& Armbruster, T., 1996, J. Solid State Chem., 123, 140. 


\section{Crystal structure of the group of optical materials $\mathrm{Ln}_{2} \mathrm{MeGe}_{4} \mathrm{O}_{12}(\mathrm{Me}=\mathrm{Ca}, \mathrm{Mn})$}

\section{N. V. Tarakina ${ }^{1, *}$, V. G. Zubkov ${ }^{1}$, I. I. Leonidov ${ }^{1}$, A. P. Tyutyunnik ${ }^{1}$, L. L. Surat ${ }^{1}$, J. Hadermann ${ }^{2}$, G. van Tendeloo ${ }^{2}$}

${ }^{1}$ Institute of Solid State Chemistry, Ural Branch of the Russian Academy of Sciences, 91 Pervomayskaya str., Ekaterinburg, 620041 Russia

2 EMAT, University of Antwerp, Groenenborgerlaan 171, 2020 Antwerp, Belgium

* tarakina(a)ihim.uran.ru

Keywords: rare-earth luminescent materials, crystal structure, germanates, X-ray powder diffraction

Abstract. The crystal structure of the group of optical materials $\mathrm{Ln}_{2} \mathrm{MeGe}_{4} \mathrm{O}_{12}, \mathrm{Ln}=\mathrm{Eu}$, $\mathrm{Gd}, \mathrm{Dy}-\mathrm{Lu}, \mathrm{Y} ; \mathrm{Me}=\mathrm{Ca}, \mathrm{Mn}$ and of the solid solution $\left(\mathrm{Y}_{1-x} \mathrm{Er}_{x}\right)_{2} \mathrm{CaGe}_{4} \mathrm{O}_{12}(x=0-1)$, promising materials for photonics, has been studied in detail. The crystal structure of all compounds exhibit two alternating layers: one formed by $\mathrm{Ln}$ and $\mathrm{Me}$ atoms and another by cyclic $\left[\mathrm{Ge}_{4} \mathrm{O}_{12}\right]^{8-}$ anions.

\section{Introduction}

One of the well-known problems of modern photonics is the practical use of the far- and mid-IR ranges. Therefore synthesis of new compounds allowing up- and down emission conversion is one of the main goals of materials science. Recently, it was found that the new group of optical materials $\mathrm{Ln}_{2} \mathrm{CaGe}_{4} \mathrm{O}_{12}, \mathrm{Ln}=\mathrm{Gd}, \mathrm{Ho}, \mathrm{Er}, \mathrm{Yb}$ and $\mathrm{Y}(\Delta E=4.95(5) \mathrm{eV})$ shows luminescence under continuous laser pumping at $\lambda=976 \mathrm{~nm}$ [1-3]. The crystal structure and the optical and luminescence properties of these rare-earth tetrametagermanates were discussed earlier [2-5]. This work concentrates on crystal structure features of the concerned compounds and takes into consideration a broader range of germanates, $\mathrm{Ln}_{2} \mathrm{MeGe}_{4} \mathrm{O}_{12}, \mathrm{Ln}=\mathrm{Eu}, \mathrm{Gd}, \mathrm{Dy}-\mathrm{Lu}, \mathrm{Y} ; \mathrm{Me}=\mathrm{Ca}, \mathrm{Mn}$ and the solid solution $\left(\mathrm{Y}_{1-x} \mathrm{Er}_{x}\right)_{2} \mathrm{CaGe}_{4} \mathrm{O}_{12}(x=0-1)$.

\section{Experiment}

The $\mathrm{Ln}_{2} \mathrm{MeGe}_{4} \mathrm{O}_{12}, \mathrm{Ln}=\mathrm{Eu}, \mathrm{Gd}, \mathrm{Dy}-\mathrm{Lu}, \mathrm{Y} ; \mathrm{Me}=\mathrm{Ca}, \mathrm{Mn}$ samples and the $\left(\mathrm{Y}_{1-x} \mathrm{Er}_{x}\right)_{2} \mathrm{CaGe}_{4} \mathrm{O}_{12}(x=0$ - 1) solid solution were prepared by solid-state reaction in air from stoichiometric mixtures of $\mathrm{Ln}_{2} \mathrm{O}_{3}(\mathrm{Ln}=\mathrm{Eu}, \mathrm{Gd}, \mathrm{Dy}, \mathrm{Ho}, \mathrm{Er}, \mathrm{Tm}, \mathrm{Yb}, \mathrm{Lu}, \mathrm{Y}), \mathrm{CaCO}_{3}(99.9 \%)$, $\mathrm{Mn}_{2} \mathrm{O}_{3}(99.9 \%)$ and $\mathrm{GeO}_{2}(99.9 \%)$ at $T=1100^{\circ} \mathrm{C}$ as described in [1]. 
X-ray powder diffraction (XRD) patterns were collected at room temperature on a transmission STADI-P (STOE, Germany) diffractometer equipped with a linear mini-PSD detector, using $\mathrm{Cu} \mathrm{K \alpha} \alpha_{1}$ radiation in the $2 \theta$ range $2^{\circ}$ to $120^{\circ}$ with a step of $0.02^{\circ}$. Polycrystalline silicon $(a=5.43075(5) \AA)$ was used as external standard. The phase purity of the samples was checked by comparing their XRD patterns with those in the Powder diffraction file - PDF2 database (ICDD, USA, release 2007). The crystal structure has been refined with the GSAS [6] program suite using $\mathrm{X}$-ray powder diffraction data.

For the transmission electron microscopy (TEM) studies the samples were crushed in ethanol. A drop of this suspension was put on a copper grid covered with a holey carbon film.

The energy dispersive X-ray (EDX) spectra and electron diffraction (ED) patterns were obtained using a Philips CM20 operated at $200 \mathrm{kV}$, equipped with an Oxford INCA system.

\section{Results and discussion}

The XRD and ED patterns have been indexed in the S.G. P4/nbm (N. 125), $Z=2$. Selected area diffraction patterns viewed along the [100] and [001] zone axes and the XRD pattern are shown in figure 1. EDX analyses of all compounds were made to check the purity of the samples. The obtained compositions were in good agreement with the nominal compositions.

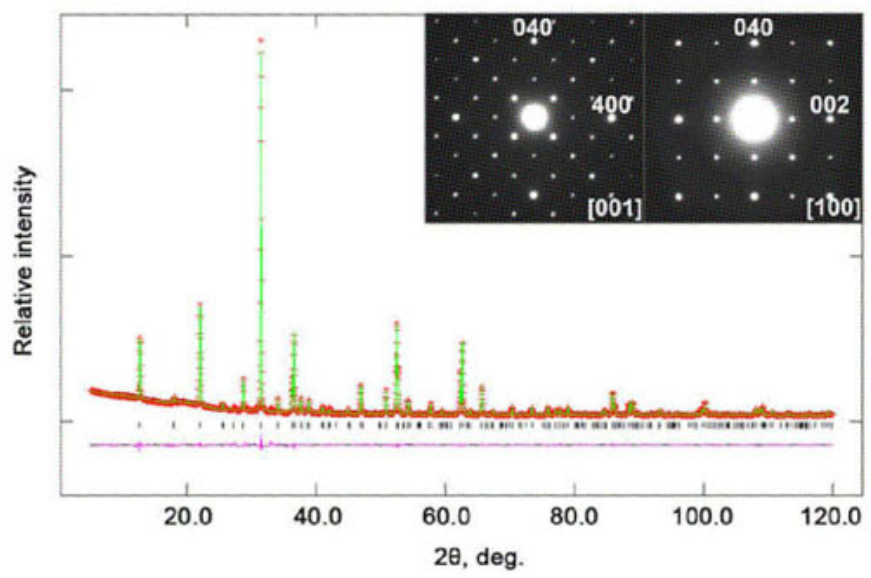

Figure 1. Observed (crosses), calculated (solid line) and difference (bottom) powder diffraction pattern of $\mathrm{Ho}_{2} \mathrm{CaGe}_{4} \mathrm{O}_{12}\left(\mathrm{Ln}_{2} \mathrm{MeGe}_{4} \mathrm{O}_{12}, \mathrm{Ln}=\mathrm{Eu}, \mathrm{Gd}, \mathrm{Dy}-\mathrm{Lu}, \mathrm{Y} ; \mathrm{Me}=\mathrm{Ca}, \mathrm{Mn}\right)$. Inset: Selected area electron diffraction patterns of concerned germanates recorded along [001]* and [100]*.

The variation in the unit cell parameters of the $\mathrm{Ln}_{2} \mathrm{MeGe}_{4} \mathrm{O}_{12}, \mathrm{Ln}=\mathrm{Eu}, \mathrm{Gd}$, Dy- $\mathrm{Lu}, \mathrm{Y} ; \mathrm{Me}=$ $\mathrm{Ca}, \mathrm{Mn}$ for the different rare-earth elements is shown in the figure 2 (b). The structure of $\mathrm{Y}_{2} \mathrm{CaGe}_{4} \mathrm{O}_{12}[1]$ was used as starting model for the crystal structure refinement. The peak profiles were fitted with a pseudo-Voigt function, $I(2 \theta)=x * L(2 \theta)+(1-x) * G(2 \theta)$ (where $L$ and $G$ are the Lorentzian and Gaussian part, respectively). The angular dependence of the peak width was described by the relation $(F W H M)^{2}=U \operatorname{tg}^{2} \theta+V \operatorname{tg} \theta+W$, where $F W H M$ is the full line width at half maximum. The background level was described as a combination 
of fifteenth Chebyshev polynomials. In total, 49 parameters for each pattern were refined. The complete details of the refinement, refined atomic coordinates, temperature parameters and $\mathrm{R}$-values are given in [2,3]. The crystal structure of the $\mathrm{Ln}_{2} \mathrm{MeGe}_{4} \mathrm{O}_{12}, \mathrm{Ln}=\mathrm{Eu}, \mathrm{Gd}$, Dy$\mathrm{Lu}, \mathrm{Y} ; \mathrm{Me}=\mathrm{Ca}, \mathrm{Mn}$ can be described as consisting of alternating layers of cations and tetracycles $\left[\mathrm{Ge}_{4} \mathrm{O}_{12}\right]$ (figure 3). Calcium, manganese and $\mathrm{Ln}$ atoms are located at the octahedral $4 f$ site $(0,0,1 / 2)$ in a $0.5 / 0.5$ ratio; the $2 b$ site $(1 / 4,1 / 4,1 / 2)$ is fully occupied with Ln atoms. An attempt to refine the occupancy of the Ln sites showed that within standard deviation the $\mathrm{Ca}(\mathrm{Mn}) / \mathrm{Ln}$ occupancy of the $4 f$ position is equal to $0.5 / 0.5$ for all concerned compounds.
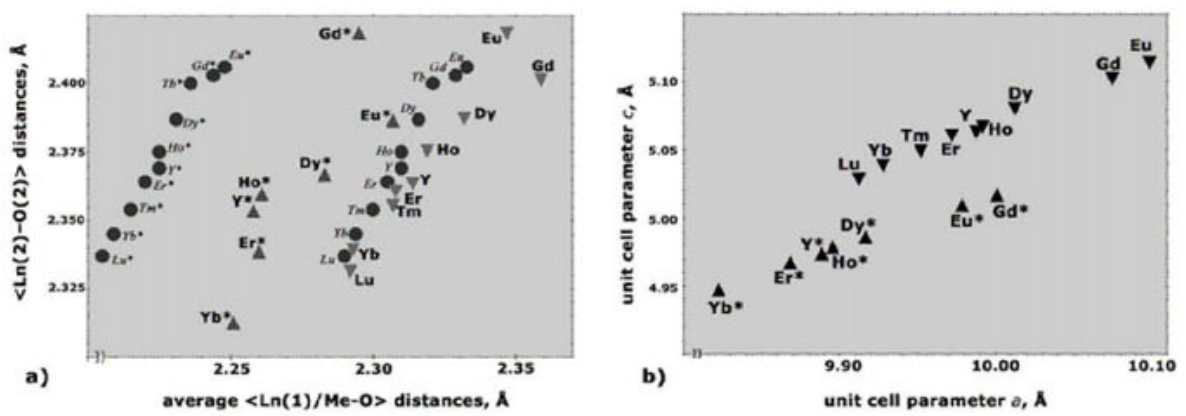

Figure 2. a) Correlation between average distances in octahedral $(\mathrm{Ln}(1) / \mathrm{Me}-\mathrm{O})$ and antiprism (Ln(2)$O(2))$ : black circles- theoretical values for $\mathrm{Ln}_{2} \mathrm{CaGe}_{4} \mathrm{O}_{12}, \mathrm{Ln}=\mathrm{Eu}, \mathrm{Gd}, \mathrm{Dy}$-Lu, Y; with *-note for manganese compounds [11]; grey triangles - experimental value for $\mathrm{Ln}_{2} \mathrm{CaGe}_{4} \mathrm{O}_{12}, \mathrm{Ln}=\mathrm{Eu}, \mathrm{Gd}$, $D y-L u, Y$; with *-note for manganese compounds; b) The variation of unit cell parameters of the $\mathrm{Ln}_{2} \mathrm{MeGe}_{4} \mathrm{O}_{12}, \mathrm{Ln}=\mathrm{Eu}, \mathrm{Gd}, \mathrm{Dy}-\mathrm{Lu}, \mathrm{Y} ; \mathrm{Me}=\mathrm{Ca}, \mathrm{Mn}$ for the different rare-earth elements (upturned triangles -for calcium compounds; triangles with * note - for manganese compounds).

The octahedra occupied by $\mathrm{Ln}$ and $\mathrm{Ca}(\mathrm{Mn})$ atoms are elongated along the distances to the $\mathrm{O}(1)$ atoms and shortened along the distances to the $\mathrm{O}(2)$ atoms. This is a specific feature of this type of structure $[1-5,7-12]$. The average $\mathrm{Ln}(1) / \mathrm{Ca}(\mathrm{Mn})-\mathrm{O}$ distances in octahedra and the $\operatorname{Ln}(2)-O(2)$ distances in square antiprisms are shortened with decreasing of the crystal radii of the rare-earth element, figure $2 \mathrm{a}$. However, a linear dependence is not strict for cations with bigger crystal radius. For example, substitution of $\mathrm{Gd}\left(r\left(\mathrm{Gd}^{3+(\mathrm{VI})}\right)=1.078 \AA\right)[13]$ allows stabilization of the $\mathrm{Gd}_{2} \mathrm{Ca}\left(\mathrm{GeO}_{3}\right)_{4}$ structure; however, the octahedra are heavily distorted. Attempts to obtain $\mathrm{Nd}_{2} \mathrm{Ca}\left(\mathrm{GeO}_{3}\right)_{4}$ were not successful at all probably due to the relatively big size of $\mathrm{Nd}$ atoms $\left(r\left(\mathrm{Nd}^{3+(\mathrm{VI})}\right)=1.123 \AA\right.$ ). As an indirect evidence to prove it, the existence of the $\mathrm{CeMn}_{2} \mathrm{Ge}_{4} \mathrm{O}_{12}$ compound, in which Mn atoms occupy only the octahedral $4 f$ site while $\mathrm{Ce}^{4+}$ atoms $\left(r\left(\mathrm{Ce}^{4+(\mathrm{VII})}\right)=1.11 \AA\right)$ occupy only $2 b$ site can be considered [4].

Germanium atoms are located at the tetrahedral $8 k$ site $(x / a, 1 / 4,0)$. The difference between the $\mathrm{Ge}-\mathrm{O}(1)$ and $\mathrm{Ge}-\mathrm{O}(2)$ bond lengths in $\mathrm{GeO}_{4}$ tetrahedra ranges from $0.055 \AA$ (for $\mathrm{Y} / \mathrm{Ca}$ ) to $0.086 \AA$ (for $\mathrm{Gd} / \mathrm{Ca}$ ). In [5] and [14] it has been shown that $\mathrm{Ge}_{4} \mathrm{O}_{12}$ groups in cyclogermanates consist of $\mathrm{O}(2) \mathrm{GeO}(2)$ groups which are connected to each other by means of $\mathrm{Ge}-\mathrm{O}(1)-$ Ge bridge bonds. For all compounds the germanium tetracycle is considerably distorted and rigid; the bond angles for the $\mathrm{O}(2) \mathrm{GeO}(2)$ groups vary from 121.0 to $122.5^{\circ}$ and for the Ge$\mathrm{O}(1)$-Ge from 126.5 to $127.5^{\circ}$. The distortion is associated with the $\mathrm{O}(2)-\mathrm{O}(2)$ distances, 
which form the local environment of a $\mathrm{Ln}$ atom in a square antiprism. For calcium com pounds, as the $\mathrm{Ln}$ radius decreases, both the $\mathrm{Ln}-\mathrm{O}(2)$ distances and the lengths of the $\mathrm{O}(2)$ $\mathrm{O}(2)$ edges of the square antiprism decrease. The length of the edge lying in the $a b$ plane in $\mathrm{Yb}_{2} \mathrm{Ca}\left(\mathrm{GeO}_{3}\right)_{4}(2.82 \AA)$ and $\mathrm{Lu}_{2} \mathrm{Ca}\left(\mathrm{GeO}_{3}\right)_{4}(2.81 \AA)$ is exactly equal to the sum of the crystal

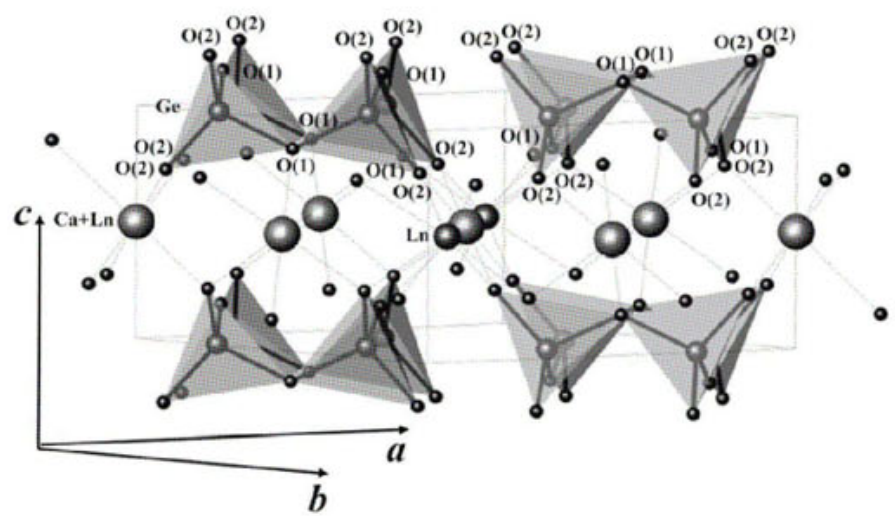

Figure 3. Crystal structure of $\mathrm{Ln}_{2} \mathrm{MeGe}_{4} \mathrm{O}_{12}, \mathrm{Ln}=\mathrm{Eu}, \mathrm{Gd}, \mathrm{Dy}-\mathrm{Lu}, \mathrm{Y} ; \mathrm{Me}=\mathrm{Ca}, \mathrm{Mn}$.

radii of two oxygen atoms. This means that the four $\mathrm{O}(2)$ atoms which form the face of the square antiprism in the $a b$ plane are in close contact. Thus, the tetracycles in $\mathrm{Ln}_{2} \mathrm{Ca}\left(\mathrm{GeO}_{3}\right)_{4}$ $(\mathrm{Ln}=\mathrm{Gd}, \mathrm{Ho}, \mathrm{Er}, \mathrm{Y}, \mathrm{Dy}, \mathrm{Tm})$ can be considered as isolated and rigid, whereas in $\mathrm{Yb}_{2} \mathrm{Ca}\left(\mathrm{GeO}_{3}\right)_{4}$ and $\mathrm{Lu}_{2} \mathrm{Ca}\left(\mathrm{GeO}_{3}\right)_{4}$ they form two-dimensional networks.

A similar behavior is observed for the manganese cyclogermanates, but with some particular features caused by a smaller radius of $\mathrm{Mn}^{2+}(r(\mathrm{VI})=0.97 \AA)$. First of all they display a heavier distortion of the octahedra and a large deviation of the $c / a$ parameter from 1 (figure $2 b$ ).

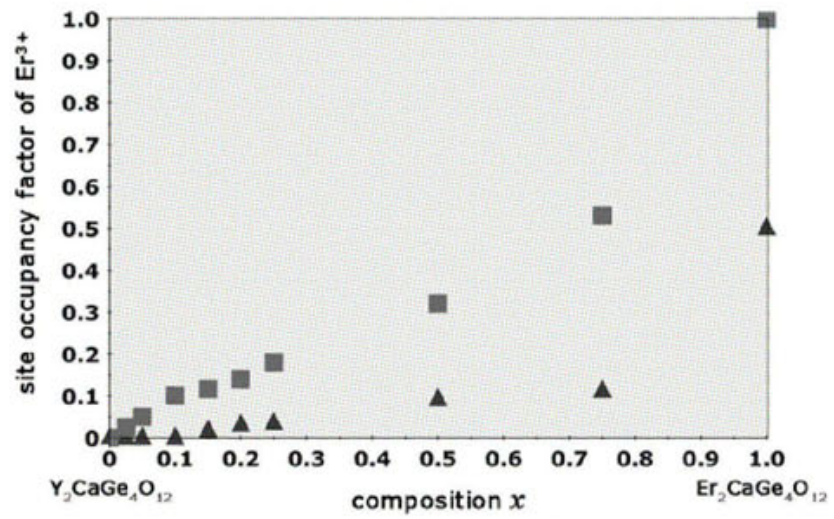

Figure 4. Dependence of site occupancies of $\mathrm{Er}^{3+}$ on composition $x(x=0-1)$ for the $\mathrm{Er}_{x} \mathrm{Y}_{2-x} \mathrm{CaGe}_{4} \mathrm{O}_{12}$ solid solution (triangles - six-coordinated site; squares - eight-coordinated site). 
Meanwhile the tendency in the change of the $O(2)-O(2)$ distances remains the same as for calcium tetragermanates; the germanium tetracycle is considerably distorted and rigid. Only in case of $\mathrm{Yb}_{2} \mathrm{Mn}\left(\mathrm{GeO}_{3}\right)_{4}$ the formation of two-dimensional networks was observed.

In order to determine a tendency according to which rare earth elements occupy six- or eightcoordinated sites in the structure the solid solution $\mathrm{Er}_{\mathrm{x}} \mathrm{Y}_{2-\mathrm{x}} \mathrm{CaGe}_{4} \mathrm{O}_{12}$ was first synthesized. A smooth change of the unit cell parameters and volume with $x$ has been observed in the whole range of solid solution. The refinement procedure reveals that Er atoms mainly occupy the $2 b$ sites with antiprismatic configuration and less the octahedral $4 f$ site (figure 4 ).

Thus, the crystal structure of the $\mathrm{Ln}_{2} \mathrm{MeGe}_{4} \mathrm{O}_{12}, \mathrm{Ln}=\mathrm{Eu}, \mathrm{Gd}$, Dy-Lu, Y; $\mathrm{Me}=\mathrm{Ca}, \mathrm{Mn}$, which can be considered as a two-sublattice system composed of polyatomic anions (tetracycles $\left[\mathrm{Ge}_{4} \mathrm{O}_{12}\right]^{8-}$ ) weakly bound to each other and a "heavy" cationic sublattice, determines their optical properties.

\section{Concluding remarks}

A group of optical materials $\mathrm{Ln}_{2} \mathrm{MeGe}_{4} \mathrm{O}_{12}, \mathrm{Ln}=\mathrm{Eu}, \mathrm{Gd}$, Dy-Lu, Y; $\mathrm{Me}=\mathrm{Ca}, \mathrm{Mn}$ and the solid solution $\left(\mathrm{Y}_{1-x} \mathrm{Er}_{x}\right)_{2} \mathrm{CaGe}_{4} \mathrm{O}_{12}$ with $\mathrm{x}=0$ - 1 have been synthesized and their crystal structure studied in detail. An isovalent substitution of cations in the two-sublattice crystal structure allows the tuning of optical properties. The synthesis of the isostructural series of manganese-containing compounds $\mathrm{Ln}_{2} \mathrm{MnGe}_{4} \mathrm{O}_{12}, \mathrm{Ln}=\mathrm{Eu}, \mathrm{Gd}, \mathrm{Dy}, \mathrm{Ho}, \mathrm{Er}, \mathrm{Yb}, \mathrm{Y}$ allows revealing new structural features of tetragermanates and also the extension of the total number of promising optical matrices containing $\left[\mathrm{Ge}_{4} \mathrm{O}_{12}\right]^{8-}$ groups.

\section{References}

1. Yamane, H., Tanimura, R., Yamada, T., Takahashi, J., Kajiwara, T. \& Shimada, M., 2006, J. Solid State Chem., 179, 289.

2. Zubkov, V.G., Leonidov, I.I., Tyutyunnik, A.P., Tarakina, N.V., Surat, L.L., Baklanova, I.V., Perelyaeva, L.A. \& Bamburov, V.G., 2008, Dokl. Phys.l Chem., 30, 418.

3. Zubkov, V.G., Leonidov, I.I., Tyutyunnik, A.P., Tarakina, N.V., Baklanova, I.V., Perelyaeva, L.A. \& Surat, L.L., 2008, Phys. Solid State, 50, 1699.

4. Taviot-Gueho, C., Leone, P., Palvadeau, P. \& Rouxel, J., 1999, J. Solid State Chem., $143,145$.

5. Baran, E.J., Wagner, C.C., Lavat, A.E. \& Cascales, C., 1997, J. Raman Spectrosc., 28, 927.

6. Larson, A.C. \& Von Dreele, R.B., 2004, General Structure Analysis System (GSAS) (Los Alamos: Nat. Lab.).

7. Tordjman, I., Martin, C., \& Durif, A., 1967, Bull. Soc. Fr. Miner. Cristallogr., 90, 293.

8. Cavero-Ghersi, C. \& Durif, A., 1975, J. Appl. Crystallogr., 8, 562.

9. Averbuch-Pouchot, M.T. \& Durif, A., 1983, Acta Crystallogr., Sect. C: Cryst. Struct. Commun., 39, 811.

10. Averbuch-Pouchot, M.T., 1996, Eur. J. Solid State Inorg. Chem., 33, 15. 
11. Slobodin, B.V., Surat, L.L., Zubkov, V.G., Tyutyunnik, A.P, Berger, I.F., Perelyaeva, L.A., Shein, I.R.,. Ivanovskii, A.L., Kuznetsov, M.V., Shulgin, B.V., Solomonov, V.I., Svensson, G., Forslund, B .\& Sayagués M.J., 2005, Phys. Rev. B: Condens. Matter, 72, 155205.

12. Zubkov, V.G., Surat, L.L., Tyutyunnik, A.P, Berger, I.F., Tarakina, N.V., Slobodin, B.V., Zhuravlev, N.A., Perelyaeva, L.A., Baklanova, I.V., Shein, I.R.,. Ivanovskii, A.L., Kuznetsov, M.V., Denisova, T.A., Shulgin, B.V., Ishchenko, A.V., Tcherepanov, A.N., Svensson, G., Forslund, B. \& Skripkin, M. Yu., 2008, Phys. Rev. B: Condens. Matter, 77174113.

13. Shannon, R.D., 1976, Acta Crystallogr., Sect. A: Found. Crystallogr., 32, 751.

14. Sitarz, M., Handke, M. \& Otto, H.H., 2002, Vib. Spectrosc., 29, 45.

Acknowledgements. This work was supported by the Russian Foundation for Basic Research under Grant No. 07-03-00143, the Councils for Grants of the President of Russia for Support of Young Scientists (Grant No. MK-84.2007.3) and for Support of Leading Scientific Schools (Grant NSh-1170.2008.3), the Program of the Presidium RAS for Basis Research No.18 and the Belgian Science Policy. Nadezda Tarakina wants to thank CKP "Electron microscopy" (Institute of Metal Physics, Ekaterinburg) for technical support. 


\section{Structural investigation on the $(1-x) \mathrm{Pb}\left(\mathrm{Mg}_{1 / 3} \mathrm{Nb}_{2 / 3}\right) \mathrm{O}_{3}-x \mathrm{PbTiO}_{3}(x=0$; $0.21)$ solid solution using powder diffraction}

\section{H. Wang ${ }^{1, *}$, H. Ehrenberg ${ }^{1,2}$, A. Senyshyn ${ }^{1,3}$, R. Schierholz ${ }^{1}$, J.-C. Jaud ${ }^{1}$, H. Fuess ${ }^{1}$}

${ }^{1}$ Institute for Materials Science, Darmstadt University of Technology, Petersenstr. 23, D-64287 Darmstadt, Germany

${ }^{2}$ Institute for Complex Materials, IFW Dresden, Helmholtzstr.20, D-01069 Dresden, Germany

${ }^{3}$ Forschungsneutronenquelle Heinz Maier-Leibnitz (FRM II), Technische Universität München, Lichtenbergstr. 1, D-85747 Garching b. München, Germany

*saliencas@hotmail.com

Keywords: powder diffraction, Rietveld method, electron diffraction, PMN-PT

Abstract. A structural investigation of the complex perovskite ferroelectrics $(1-x) \mathrm{Pb}\left(\mathrm{Mg}_{1 / 3} \mathrm{Nb}_{2 / 3}\right) \mathrm{O}_{3}-x \mathrm{PbTiO}_{3}$ (= PMN-PT) was carried out by X-ray and neutron powder diffraction. Qualitative phase analysis using Rietveld method combined with electron diffraction was performed for compositions in the PMN-rich region. A ferroelectric state of a rhombohedral symmetry $(R 3 \mathrm{mH})$ gradually developed with the input of titanate to cubic PMN $(P m 3 m)$, as further evidenced by hysteresis loops measured and domain contrast observed in dark field imaging. Intrinsic local structure due to atomic mismatch seems to be the main reason for the complexity of this disordered system.

\section{Introduction}

Ferroelectric materials exhibit high dielectric permittivity and piezoelectricity and are widely used as transducers and electronic components. Compounds actually used are complex mixed-ion perovskite, such as relaxor ferroelectrics PMN-PT, that exhibit superior piezoelectric and electromechanical properties over a wide range of composition compared with commercial $\mathrm{PbZr}_{1-x} \mathrm{Ti}_{x} \mathrm{O}_{3}[1,2]$. PMN prototype relaxors showed the existence of two components - an isotropic spherical glass matrix and anisotropic ferroelectric (FE) nanoclusters [3,4]. Also, $\mathrm{Pb}$ nuclei are displaced in the spherical glass matrix at $290 \mathrm{~K}$ $\left(T_{C}=210 \mathrm{~K}\right)$, but there is no preferential frozen orientation or magnitude of displacement. Neutron diffraction revealed a drastic increase of the volume and correlation lengths of polar nanoregions (PNRs) below $200 \mathrm{~K}$ [5]. Two different atomic displacements $\delta_{c . m}$ (caused by 
soft-mode condensation) and $\delta_{\text {shitt }}$ (a uniform displacement of PNRs along their polar direction) were proposed below the Burns temperature of a PMN crystal by neutron diffuse scattering [6]. Neutron diffraction of PMN crystals reveals a strong lattice distortion and depth dependence in the surface region over a length scale of $100 \mu \mathrm{m}$ [7]. These phenomena indicate that PMN is an incipient FE, also found in PMN-PT crystals for $0<x<0.21$ from dielectric and domain studies [8,9]. This "hidden" transition nature (seen in unpoled samples but not as clearly as in the poled samples) was enhanced by an $E$ field [8] associated with the appearance of polar microdomains. Since the nanodomain size is much smaller than the coherence length of diffraction radiation, scattered waves from individual nanodomains coherently superimpose during diffraction, thus, significant broadening of the reflection is expected. Consequently, the observed tetragonal-phase nanotwins with a domain size about $10 \mathrm{~nm}$ by transmission electron microscopy in PMN-PT $(x=0.33)$ [10] appear to be the monoclinic $M_{C}$ phase in low-resolution diffraction \& polarized light microscopy.

Recent neutron and X-ray scattering studies on PMN-PT crystals $(x<0.25)$ in zero E-field found no long-range structural distortion in the bulk at very low temperatures, therefore this new phase is referred as unkown phase- $X[11,12]$. High-energy XRD results of PZN-PT crystals also show that skin effects in distinct outer layers $(10-50 \mu \mathrm{m})$ are present in all samples [13]. Recently a $R$ phase was found to develop in both the outer layer and crystal interior with increasing Ti content, seen in a PMN-PT $(x=0.27)$ crystal [12]. The polarization of FE nanoclusters and boundary conditions near the surface are believed to play an essential role in the surface distortion. Addition of Ti tends to decrease chemical ordered domain size and increase the ferroelectrically active polar domains in the PMN-PT system. Powder neutron diffraction studies [14] of PMN-PT $(0.25 \leq x \leq 0.39)$ with compositions across the morphotropic phase boundary construct a phase diagram showing stability regions of monoclinic $M_{B}$, monoclinic $M_{C}$, tetragonal and cubic phases. Due to the existence of unkown $X$ phase in the PMN-rich side, the interactions between the cationic ordered chemical domains and the growing polar domains would be highly interesting. As presented above, great attention was paid to microstructures related to special properties, but the true symmetry is still under controversy [11-14]. To clarify the relevance of structural aspects in physical mechanisms for such complex systems, which is critical for their applications, we aim to clarify the basic structure using neutron powder diffraction.

\section{Experimental procedure}

Well-grown PMN-PT $(x=0,0.21)$ crystals $[15]$ were treated by grinding into fine powder in an agate mortar and annealed at about $900 \mathrm{~K}$. The structure of powder samples was first recorded to be a pure phase in glass capillaries with well-match amount by X-ray measurement with transmission geometry (Mo wavelength $0.7093 \AA$ ). The overall measuring time for one sample was 12 hours to have very good counting statistics over the angular range $3-50^{\circ}$ (in 2theta) while rotating the samples during data collection. Constant wavelength neutron powder diffraction (NPD) experiments were carried out on the neutron powder diffractometer SPODI at FRM II, Garching, Germany [16]. The wavelength of $1.5482 \AA$ was obtained at $155^{\circ}$ take off angle from a (551) reflection of composite Ge monochromator. Two dimensional powder diffraction patterns were collected using a multidetector consisting of $80{ }^{3} \mathrm{He}$ vertical position-sensitive ( $300 \mathrm{~mm}$ effective height) tubes. The powdered sample was contained into a thin wall $(0.15 \mathrm{~mm}$ thickness) vanadium can, 
which was further mounted into a top-loading closed cycle refrigerator and cooled down to 3 $\mathrm{K}$. Data sets were recorded at $300 \mathrm{~K}$ and $3 \mathrm{~K}$ in a 2 theta range of $0-155^{\circ}$ with $\Delta 2 \theta$ steps of $0.04^{\circ}$. The full profile Rietveld method implemented in the FullProf suite of programs was applied to analyze powder patterns [17]. The peak profile shape was described by a modified Thompson-Cox-Hastings pseudo-Voigt function. The background of the diffraction pattern was fitted using a linear interpolation between selected data points in non-overlapping regions. The scale factor, lattice parameters, fractional coordinates of atomic sites and their isotropic displacement parameters, zero angular shift, profile shape parameters and half width (Caglioti) parameters were refined. For PMN it was found that the refinement of anisotropic thermal displacement parameters for $\mathrm{Nb} / \mathrm{Mg}$ sites improved significantly the fitting, whereas the displacements of $\mathrm{Pb}$ and $\mathrm{O}$ for PMN-PT $(0.21)$ were described by $2^{\text {nd }}$ rank tensors.
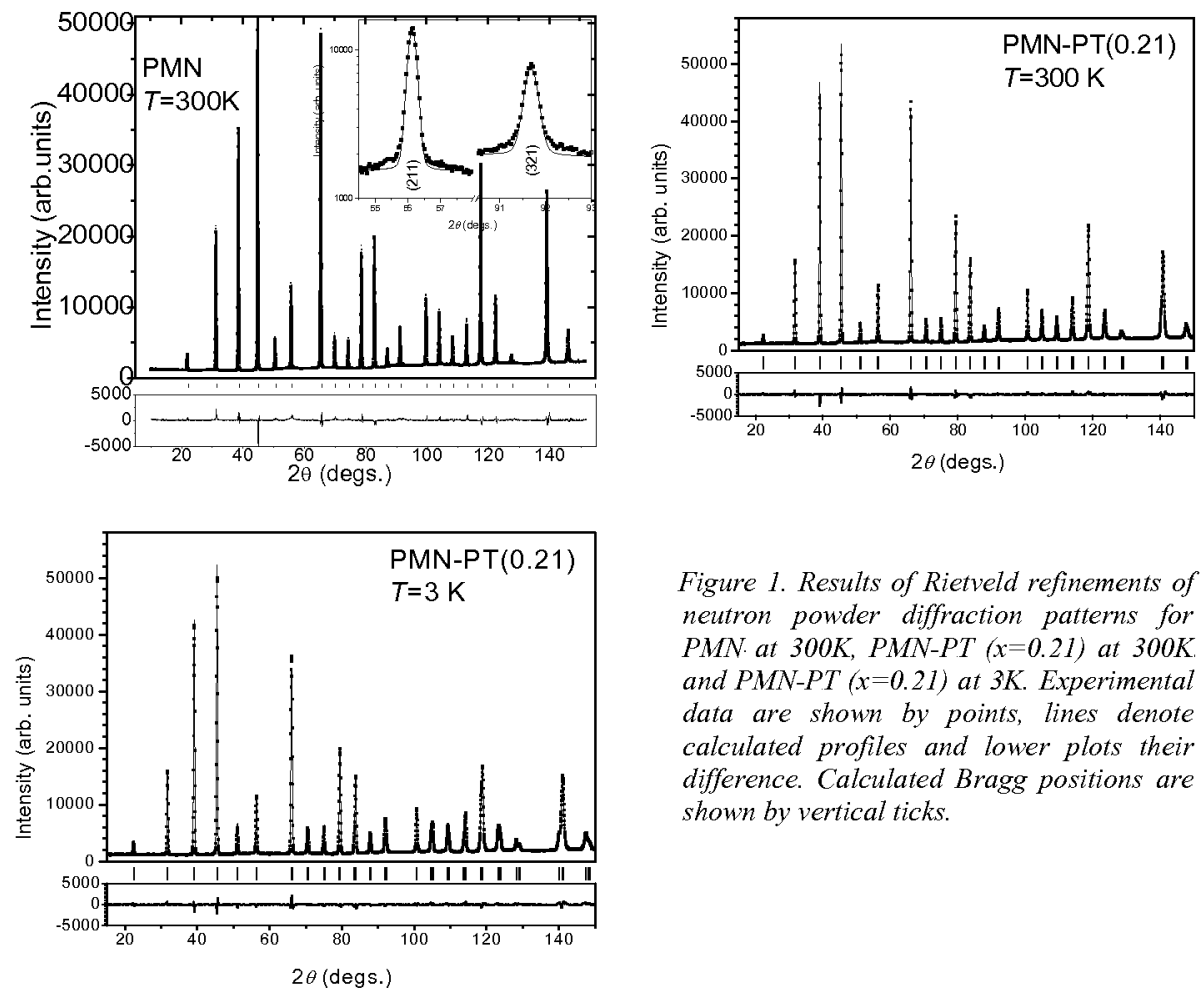

Figure 1. Results of Rietveld refinements of neutron powder diffraction patterns for $P M N$ at $300 K, P M N-P T(x=0.21)$ at $300 K$ and PMN-PT $(x=0.21)$ at $3 K$. Experimental data are shown by points, lines denote calculated profiles and lower plots their difference. Calculated Bragg positions are shown by vertical ticks.

Crystal samples for transmission electron microscopy were prepared by the procedure of sawing, polishing, dimpling, and ion milling [18]. Transmission electron microscopy was performed with a Philips CM20 operating at $200 \mathrm{kV}$ for imaging and selected area electron diffraction (SAED) and at $80 \mathrm{kV}$ for convergent-beam electron diffraction (CBED) with a double-tilt holder. The hysteresis (P-E) loops were obtained using a Sawyer-Tower circuit at $1 \mathrm{~Hz}$ and at different temperatures. 


\section{Results and discussion}

The well fitted neutron diffraction patterns of pure PMN and PMN-PT $(x=0.21)$ are shown in figure 1. The final refined atomic coordinates and isotropic displacement parameters (except $\mathrm{Pb}$ and $\mathrm{O}$ for $x=0.21$ ) for the two samples are reported in table 1 . In these two samples, mixed cation site occupancies of $\mathrm{Mg}^{2+} / \mathrm{Nb}^{5+} / \mathrm{Ti}^{4+}$ were calculated from their nominal compositions. For the structural study of pure PMN, we assumed a cubic symmetry with space group $P m-3 m$. In the early stages of PMN refinements, constraints on the cubic model were introduced initially with ideal sites. The ambient cubic structure of PMN seems even more complicated than the normal perovskite one due to the appearence of diffuse scattering shown in figure 1 (inset), seen also e.g. reference [7,19], where the decrease of intensity of diffuse scattering with the addition of $\mathrm{Ti}$ was observed. It seems to be the reason why the ideal cubic model slightly mismatched without involving the local structure characteristics.

Table 1. Final refined coordinates and displacement parameters $\left(A^{2}\right)$.

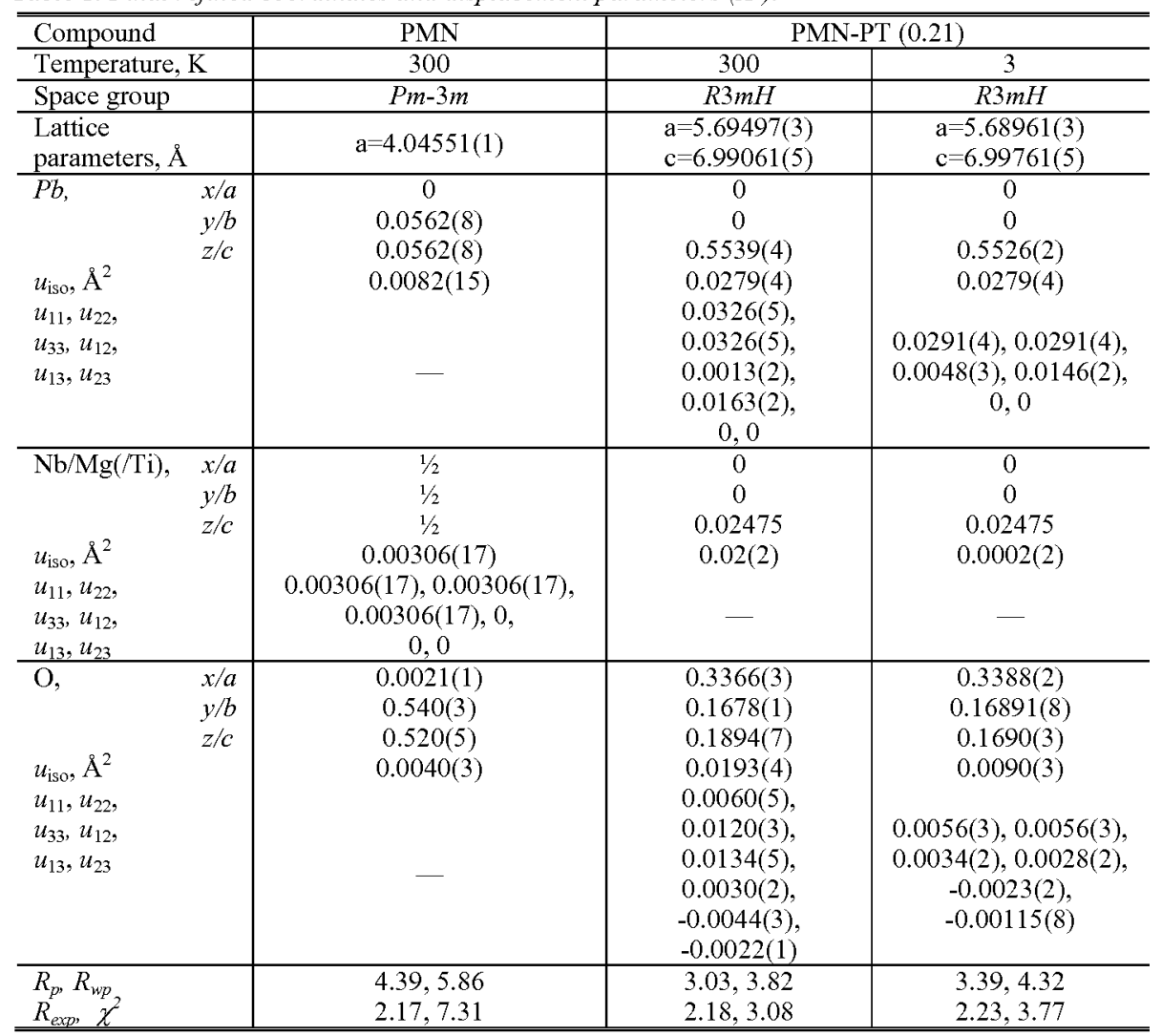

For PMN-PT $(x=0.21)$ the effort to refine its crystal structure in a cubic model failed, whereas fairly promising results were obtained using a rhombohedral one. Therefore, at 3 
and $300 \mathrm{~K}$, we used space group setting $R 3 \mathrm{mH}$. In the asymmetric unit of the structure of the rhombohedral phase during the Rietveld refinement, $\mathrm{Pb}^{2+}$ and $\mathrm{Nb}^{5+} / \mathrm{Ti}^{4+} / \mathrm{Mg}^{2+}$ ions occupy $3(a)$ sites at $(0,0, z)$ and $\mathrm{O}^{2-}$ at the $9(b)$ site at $(2 x, x, z)$.

To understand the effects visible in the diffraction pattern of samples with different compositions, we performed transmission electron microscopy and electron diffraction. As seen in figure 2, domain contrast was found in dark field image close to [01-1] incidence with intersecting domains in [100] and [011] and inner contrast within the domains. The SAED shows superlattice reflections of type $1 / 21 / 21 / 2$, probably due to chemical ordering [20]. The First Order Laue-Zone in the [1-11] CBED Pattern shows a deviation from cubic symmetry, by revealing only symmetry $\mathrm{m}$ instead of $3 \mathrm{~m}$.
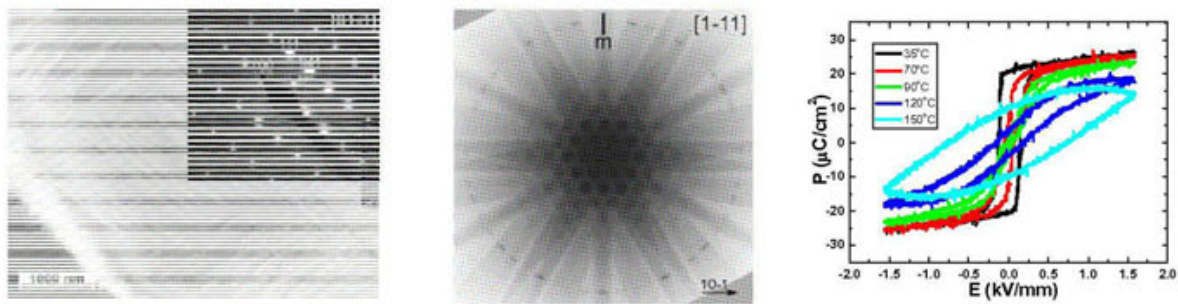

Figure 2. Left: Dark field image and corresponding SAED [01-1]c zone axis for PMN-PT crystal $x=0.21$. Middle: $\langle 111\rangle$ incidence CBED-pattern recorded at $80 \mathrm{kV}$ on a crushed sample revealing only mirror symmetry. Right: Hysteresis loops of PMN-PT $(x=0.21)$ crystal.

Recently, the transformation of spontaneous ferroelectric to paraelectric state was evidenced for unkown Rhombohedral $X$ or Cubic $X$ phase $[8,12]$ of the phase diagram associated with two branches. The further study of the hysteresis loops across the transition temperature (about $340 \mathrm{~K})$ for PMN-PT $(x=0.21)$ crystals were performed. The square shape of P-E loop was recorded at $35^{\circ} \mathrm{C}$ well below the transition temperature, which shows the existence of the ferroelectric phase at $300 \mathrm{~K}$. Hysteresis loops became slim and then nonlinear at elevated temperatures with a phase transition at about $70^{\circ} \mathrm{C}$. It was well considered during Rietveld refinement for the PMN end to PMN-PT $(x=0.21)$ which supposed the phase evolution of a cubic to rhombohedral one. A detailed structure analysis carried out for these materials revealed the development of a ferroelectric state. For the whole series of ferroelectric to paraelectric phase, qualitative phase analysis using Rietveld method and electron diffraction are further performed for compositions in the two temperature regions which will be dedicated to the phase identification and structure stability of PMN-PT solid solution.

\section{Concluding remarks}

The structure changes of PMN-PT solid solution have been investigated and the structure refinements were based on neutron powder diffraction data at $300 \mathrm{~K}$ and $3 \mathrm{~K}$ by the full pattern Rietveld analysis. The two powders of PMN and PMN-PT $(x=0.21)$ prepared from crystals were not isostructural, but exist in the space groups $P m 3 m$ and $R 3 m H$, respectively. Both hysteresis loops and domain contrast from electron diffraction show the reliability of the presented structural models. A comparison between these two series shed light on the structural development with different symmetries, even though a close resemblance exists in the phase diagram. 


\section{References}

1. Smolensky, G.A., 1981, Ferroelectrics and Related Materials (New York: Gordon and Breach).

2. Service, R.F., 1997, Science, 275, 1878.

3. Blinc, R., Laguta, V.V.\& Zalar B., 2003, Phys. Rev. Lett., 91, 247601.

4. Blinc, R., Laguta, V.V., Zalar, B. \& Banys J., 2006, J. Mater. Sci., 41, 27.

5. Jeong, I.K., Darling, T.W., Lee, J.K., Proffen, T., Heffner, R.H., Park, J.S., Hong, K.S., Dmowski,W. \& Egami, T., 2005, Phys. Rev. Lett., 94, 147602.

6. Hirota, K., Ye, Z.-G., Wakimoto, S., Gehring, P.M. \& Shirane, G., 2002, Phys. Rev.

7. $B, \mathbf{6 5}, 104105$.

8. Conlon, K.H., Luo, H., Viehland, D., Li, J.F., Whan, T., Fox, J.H., Stock, C. \& Shirane, G., 2004, Phys. Rev. B, 70, 172204.

9. Wang, H., Pan, X., Lin, D., Luo, H., Yin, Z. \& Elouadi, B., 2007, Appl. Phys. Lett., 90, 252902.

10. Tu, C.-S.,Chien, R.R.,Wang, F., Schmidt, V.H. \& Han, P.,2004, Phys. Rev. B, 70, 220103.

11. Wang H., Zhu J., Lu N., Bokov A. A., Ye Z. G., and Zhang X. W., 2006, Appl. Phys. Lett. 89, 042908.

12. Noheda, B. \& Cox, D.E., 2006, Phase Transit., 79, 5.

13. Xu, G.,Viehland, D., Li, J.F., Gehring, P. \& Shirane, G., 2003, Phys. Rev. B, 68, 212410.

14. Xu G., Hiraka H., Shirane G. \& Ohwada, K., 2004, Appl. Phys. Lett., 84, 3975.

15. Singh, A.K., Pandey, D. \& Zaharko, O., 2006, Phys. Rev. B, 74, 024101.

16. Luo, H., Shen, G., Wang, P., Le, X. \& Y in, Z., 1997, J. Inorg. Mater., 12, 768.

17. Hölzel M., Senyshyn A., Gilles R.,Boysen H., Fuess H.,2007, Neutron News 18, 23.

18. Roisnel, T. \& Rodríquez-Carvajal, J., 2001, Mater. Sci. Forum, 378-381, 118.

19. Schmitt, L.A., Schönau, K.A., Theissmann, R., Fuess, H., Kungl, H., \& Hoffmann, M.J., 2007, J. Appl. Phys., 101, 074107.

20. De Mathan, N., Husson, E., Calvarin, G., Gavarri, J.R., Hewat, A.W. \& Morell, A., 1991, J. Phys. Condens. Matter., 3, 8159.

21. Miao, S., Zhu, J., Zhang, X. \& Cheng, Z.-Y., 2002, Phys. Rev. B, 65, 052101.

Acknowledgements. This work is supported by the European project "Network of excellence" program "FAME: Functionalised Advanced Materials Engineering of Hybrids and Ceramics" and Deutsche Forschungsgemeinschaft SFB 595 "Electrical fatigue of functional materials". The neutron powder diffractometer SPODI is operated in the frame of the "Instrumentation at large-scale facilities for condensed matter research" program of the Bundesministerium fuer Bildung und Forschung under grant No. 03FU7DAR. 
IV.4.3 Determination of Magnetic Structure, Magnetic Materials 



\section{Synthesis, structure and magnetic properties of Fe-doped tetragonal $\mathrm{Li}_{0.95} \mathrm{Mn}_{2.05} \mathrm{O}_{4}$}

\section{W. Nowicki}

Department of Materials Chemistry, Faculty of Chemistry, Adam Mickiewicz University, Grunwaldzka 6, PL-60780 Poznan, Poland waldek@amu.edu.pl

Keywords: Lithium-iron-manganese oxides, neutron and X-ray powder diffraction, crystal structure refinement, magnetic properties

Abstract. This work presents results of measurements of X-ray powder diffraction, neutron powder diffraction and magnetic susceptibility of lithium deficient $\mathrm{Li}_{0.95} \mathrm{Mn}_{2.05-\mathrm{x}} \mathrm{Fe}_{\mathrm{x}} \mathrm{O}_{4}$ $(0.0 \leq \mathrm{x} \leq 0.1)$ spinel samples. Partial substitution of $\mathrm{Fe}^{3+}$ ions restrains the Jahn-Teller effect, owing to the reduction of $\mathrm{Mn}^{3+} / \mathrm{Mn}^{4+}$ ratio in tetragonal $\mathrm{Li}_{0.95} \mathrm{Mn}_{2.05} \mathrm{O}_{4}$. Magnetic susceptibility of a series $\mathrm{Li}_{0.95} \mathrm{Mn}_{2.05-\mathrm{x}} \mathrm{Fe}_{\mathrm{x}} \mathrm{O}_{4}$ compounds, with the $\mathrm{Fe}^{3+}$ increasing from $\mathrm{x}=0.0-0.1$, showing the antiferromagnetic ordering at low temperature. The Néel point increases from $11 \mathrm{~K}$ to $27 \mathrm{~K}$.

\section{Introduction}

The Li-Mn-O system is one of the main candidates for the cathode materials of rechargeable lithium batteries. In particular, the spinel $\mathrm{LiMn}_{2} \mathrm{O}_{4}$ has several advantages such as low cost and lower toxicity compared to $\mathrm{LiCOO}_{2}$ and $\mathrm{LiNiO}_{2}$ [1-5]. However, stoichiometric lithium manganese oxide shows unacceptably large capacity fade on cycling and exhibits a phase transition $(F \mathrm{~d} 3 \mathrm{~m} \rightarrow F$ ddd) at around room temperature $(290 \mathrm{~K})$ due to Jahn-Teller distortion of trivalent $\mathrm{Mn}$ ions. In general raising of the manganese valence stabilizes the cubic structure even below room temperature. The average manganese valence in stoichiometric $\mathrm{LiMn}_{2} \mathrm{O}_{4}$ is 3.5 , the lithium ions occupying the tetrahedral sites, and a $1: 1$ mixture of $\mathrm{Mn}^{3+}$ and $\mathrm{Mn}^{4+}$ ions randomly distributed over the octahedral positions in the spinel lattice $(F \mathrm{~d} 3 \mathrm{~m})$. Even a small deficiency of lithium can dramatically involve essential changes in the crystal structure (e.g. $\mathrm{Li}_{0.95} \mathrm{Mn}_{2.05} \mathrm{O}_{4}$ obtained by quenching shows a tetragonally distorted spinel lattice, with $\mathrm{c} / \mathrm{a}=0.98$, s.g. $F 4_{1} / \mathrm{ddm}[6]$ ). The body-centred unit cell $I 4_{1}$ amd has been replaced by the face-centred $F 4_{1} / \mathrm{ddm}$ with $a_{F}=a_{I} \sqrt{2}$ which is more convenient to demonstrate the degree of tetragonal distortion for the spinel type structure.

One way to stabilize the cubic spinel structure and to inhibit the phase transition effect, is achieved by adding other $3 d$-cations to the spinel lattice. The purpose of this work is an at- 
tempt to modify the structure and magnetic properties of lithium-deficient manganese oxide, $\left(\mathrm{Li}_{0.95} \mathrm{Mn}_{2.05} \mathrm{O}_{4}\right)$ by doping with $\mathrm{Fe}^{3+}$ ions.

\section{Experimental details}

Series of compounds with the $\mathrm{Li}_{0.95} \mathrm{Mn}_{2.05-\mathrm{x}} \mathrm{Fe}_{\mathrm{x}} \mathrm{O}_{4}(0.0 \leq \mathrm{x} \leq 0.1)$ stoichiometry have been obtained by solid state reaction of $\mathrm{Li}_{2} \mathrm{CO}_{3}$ with the manganese oxide or iron-manganese oxide precursors. The samples underwent a successive thermal treatment in air, at $700^{\circ} \mathrm{C}$ and $800^{\circ} \mathrm{C}$ for $4 \mathrm{~h}$. After heating, the preparations were either cooled slowly to the room temperature during $24 \mathrm{~h}$ (series SC), or rapidly quenched in the solid $\mathrm{CO}_{2}$ (series Q). The powder $\mathrm{X}$-ray diffraction patterns of the substituted oxides were obtained using an X-ray diffractometer (Bruker D8 Advance), with copper $\mathrm{K}_{\alpha}$ radiation. XRD patterns were recorded at the room temperature, in the range $2 \theta=15^{\circ}-120^{\circ}$ by step scanning, using $2 \theta$ increments of $0.01^{\circ}$ and fixed counting time of $5 \mathrm{~s} / \mathrm{step}$. Neutron diffraction data were collected on the multicounter high-resolution diffractometer E9 installed at the BER II reactor at the Helmholth Zentrum für Materialien und Energie (Berlin), using plane (511) of a Ge monochromator. The incident neutron wavelength was $1.79801(3) \AA$. About $3 \mathrm{~g}$ of sample was encapsulated into vanadium container. The full diffraction patterns were measured in the $2^{\circ} \leq 2 \theta \leq$ $150^{\circ}$ range with $0.07^{\circ}$ steps at $300 \mathrm{~K}$. The magnetometric measurements were made with a DC-magnetometr/AC-susceptometer MagLab 2000 System (Oxford Instruments Ltd.). The $\mathrm{Li}_{0.95} \mathrm{Mn}_{2.05-\mathrm{x}} \mathrm{Fe}_{\mathrm{x}} \mathrm{O}_{4}$ powders of average mass about $100 \mathrm{mg}$ were held in gelatine capsules and fitted to the standard sample holder of the magnetometer. The magnetization dependence on magnetic field $\mathrm{M}(\mathrm{H})$ was measured in the range of $0.2-5 \mathrm{~T}$ at two extreme temperatures namely $4.2 \mathrm{~K}$ and $320 \mathrm{~K}$. The filed cooling was steeped with the step size of $0.2 \mathrm{~T}$. The magnetization as a function of temperature was measured in two sequences. Samples were cooled to $4 \mathrm{~K}$ in absence of magnetic field, next the field of $0.1 \mathrm{~T}$ was applied, and the temperature increased up to $320 \mathrm{~K}$ (so called zero field cooling - ZFC mode). After the sequence was completed, the sample was cooled in the applied magnetic field (filed cooling $-\mathrm{FC}$ mode) from $320 \mathrm{~K}$ to $4 \mathrm{~K}$. Measurements were performed with $2 \mathrm{~K}$ step, and the average warming/cooling rate was below $1 \mathrm{~K} / \mathrm{min}$. The phase identification and phase analysis were performed using the program PowderCell [7]. The structure refinements were performed using the program FullProf [8].

\section{Results and discussion}

The series compounds with the $\mathrm{Li}_{0.95} \mathrm{Mn}_{2.05-\mathrm{x}} \mathrm{Fe}_{\mathrm{x}} \mathrm{O}_{4}(0.0 \leq \mathrm{x} \leq 0.1)$ stoichiometry, formed as a result of slow cooling technique, give nearly stoichiometric $\mathrm{LiMn}_{2} \mathrm{O}_{4}$ with the admixture of manganese oxides, (figure1, series SC). The value of unit cell constant of the $\mathrm{LiMn}_{2} \mathrm{O}_{4} \mathrm{spinel}$ phase type increases from $8.2479(2) \AA$ to $8.2523(3) \AA$. The precise determination of lattice constants of the cubic unit cell for the similar solid solution series $\mathrm{LiMn}_{2} \mathrm{O}_{4} / \mathrm{LiFe}_{5} \mathrm{O}_{8}$ reveals that the replacement of $\mathrm{Mn}^{3+}$ with $\mathrm{Fe}^{3+}$, having equal ionic radii, causes the increase of the spinel unit cell volume [9]. The other crystalline phases determined by X-ray diffraction are cubic bixbyite $\left(\mathrm{Mn}_{2} \mathrm{O}_{3}\right.$, s.g. Ia3, [10]), and tetragonal hausmannite $\left(\mathrm{Mn}_{3} \mathrm{O}_{4}\right.$, s.g. $F 4_{1} / \mathrm{ddm}$, $c / a=1.16,[11])$, the refined amounts are between $0.25 \%-3.5 \%$, and $3.6 \%-4.4 \%$ respectively (see table 1 , series $\mathrm{SC}$ ). The samples of an identical composition, $\mathrm{Li}_{0.95} \mathrm{Mn}_{2.05-\mathrm{x}} \mathrm{Fe}_{\mathrm{x}} \mathrm{O}_{4}$ $(0.0 \leq \mathrm{x} \leq 0.1)$, obtained by quenching the products of thermal treatment, crystallize in the 
tetragonal space group $F 4_{1} / \mathrm{ddm}$, except the last compound in the series, described in cubic space group $F \mathrm{~d} 3 \mathrm{~m}$, (figure 1, series Q). Two different tetragonal spinel phases, ("flat" unit cell with $c / a<1$, and "elongated" unit cell with $c / a>1$ ) are observed for quenched samples. Lattice parameters of all spinel phases are presented in table 1 . The increase of $\mathrm{Fe}^{3+}$ ions content gradually inhibits Jahn-Teller effect, and the existence of tetragonally flattened spinel structure $(c / a<1)$, or the tetragonally elongated spinel structure $(c / a>1)$, depending on the amount of $\mathrm{Mn}^{3+}$ ions on tetrahedral and octahedral sites respectively. These results are consistent with those reported by Piszora [12].
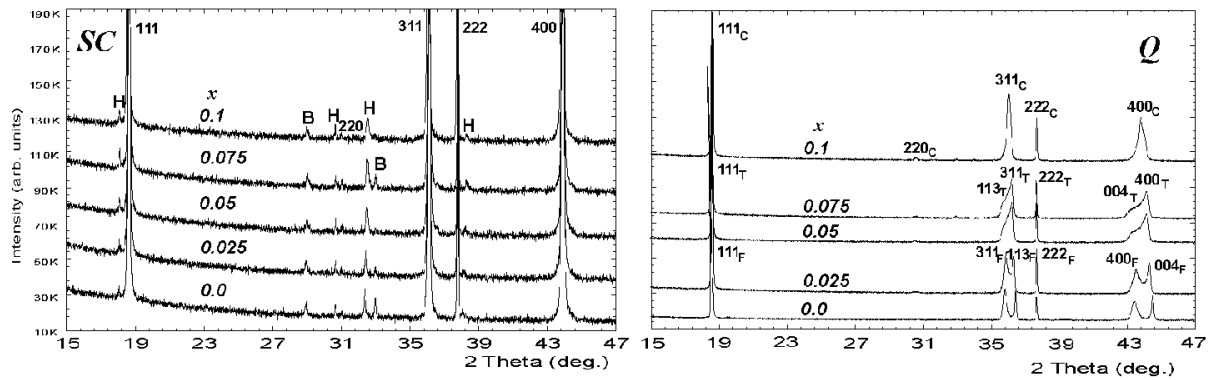

Figure 1. X-ray powder diffraction patterns of a series $\mathrm{Li}_{0.95} \mathrm{Mn}_{2.05-x} \mathrm{Fe}_{x} \mathrm{O}_{4}(0.0 \leq x \leq 0.1)$, prepared by slow cooling technique (series SC), and obtained by quenching preparations (series Q), recorded at room temperature.

Series $\mathrm{SC}: \mathrm{H}$-hausmannite, $\left(\mathrm{Mn}_{3} \mathrm{O}_{4}\right) ; \mathrm{B}$ - bixbyite, $\left(\mathrm{Mn}_{2} \mathrm{O}_{3}\right)$.

Series $Q: C$ - cubic phase; $T$-tetragonal phase c $/ a>1 ; F$-tetragonal phase with $c / a<1$.

Table 1. Unit cell parameters calculated from X-ray powder diffraction data of the tetragonal $\left(\mathrm{FH}_{\mathrm{l}} / \mathrm{ddm}\right)$ and cubic $(\mathrm{Fd} 3 \mathrm{~m})$ spinel phases and additional phases, (weight percent) in samples $\mathrm{Li}_{0.95} \mathrm{Mn}_{2.0 \mathrm{~S}_{-} \mathrm{Fe}} \mathrm{Fe}_{x} \mathrm{O}_{4}$, obtained by quenching, $(Q)$ and slow cooling, $(\mathrm{SC})$ techniques.

\begin{tabular}{|c|c|c|c|c|c|c|c|}
\hline \multirow{2}{*}{$\begin{array}{l}\text { Composition of } \\
\mathrm{Li}_{0.95} \mathrm{Mn}_{2.05-\mathrm{x}} \mathrm{He}_{\mathrm{x}} \mathrm{O}_{4} \\
(0.0<\mathrm{x}<0.1)\end{array}$} & \multicolumn{4}{|c|}{ Series $Q$} & \multicolumn{3}{|c|}{ Series SC } \\
\hline & s. $g$. & $a, b[\AA]$ & $c^{\prime}[\AA]$ & $c / a$ & s. $g$. & $a[\AA]$ & $\begin{array}{l}\mathrm{B}-\mathrm{Mn}_{2} \mathrm{O}_{3}[\%] \\
\mathrm{H}-\mathrm{Mn}_{3} \mathrm{O}_{4}[\%]\end{array}$ \\
\hline $\mathrm{Li}_{0.95} \mathrm{Mn}_{2.05} \mathrm{O}_{4}$ & $F 4_{1}^{\prime} d d m$ & $8.3275(2)$ & $8.1445(4)$ & 0.978 & $F d \overline{3} m$ & $8.2479(2)$ & В $0.6, \mathrm{H} 3.6$ \\
\hline $\mathrm{Li}_{0.05} \mathrm{Mn}_{2.025} \mathrm{Fe}_{0025} \mathrm{O}_{4}$ & $F 4_{1} / \mathrm{ddm}$ & $8.3050(5)$ & $8.1905(2)$ & 0.986 & $F d \overline{3} m$ & $8.2497(6)$ & В 0.3 , H 3.7 \\
\hline $\mathrm{Li}_{0.99} \mathrm{Mn}_{2.0} \mathrm{Fe}_{0.05} \mathrm{O}_{4}$ & $F 4 d d m$ & $8.2139(3)$ & $8.3566(6)$ & 1.017 & $F d \overline{3} m$ & $8.2513(5)$ & В 0.7, H 4.4 \\
\hline $\mathrm{Li}_{0.95} \mathrm{Mn}_{1.075} \mathrm{Fe}_{0075} \mathrm{O}_{4}$ & $F 4_{l} / d d m$ & $8.2065(3)$ & $8.3709(6)$ & 1.020 & $F d \overline{3} m$ & $8.2525(9)$ & B 0.35, H 3.9 \\
\hline $\mathrm{Li}_{0.95} \mathrm{Mn}_{1.95} \mathrm{Fe}_{0.1} \mathrm{O}_{4}$ & $F d 3 m$ & \multicolumn{2}{|c|}{$8.2669(2)$} & 1.000 & $F d \overline{3} m$ & $8.2523(3)$ & B 0.25, H 3.8 \\
\hline
\end{tabular}

The forward scattering amplitudes of atoms for X-rays increase linearly with the number of electrons in the atom. Therefore, localization of light atoms in the presence of heavy ones can be very poor. Furthermore, it is not possible to distinguish between neighbouring elements in a compound. Neutron powder diffraction experiments enable the location of $\mathrm{Li}^{+}$ in the A and B spinel positions, and the atomic distribution of the Fe and Mn ions. On the other hand, the resolution achieved in the neutron powder diffraction in comparison lower, and the wide instrumental FWHM does not allow to observe many details, i.e. splitting 
peaks. Combining X-ray and neutron patterns is more useful to determine the precise distribution of ions in given pure phase. This reveals figure 2, showing an example of the Rietveld refinements of the $\mathrm{Li}_{0.95} \mathrm{Mn}_{1.95} \mathrm{Fe}_{0.1} \mathrm{O}_{4}$, calculated with different diffraction techniques. The structure refinement by the Rietveld method was carried out to determine the cation distribution over tetrahedral and octahedral sites. The distribution of cations in $\mathrm{Li}_{0.95} \mathrm{Mn}_{1.95} \mathrm{Fe}_{0.1} \mathrm{O}_{4}$ compound, can be expressed as $\left(\mathrm{Li}_{7.608} \mathrm{Fe}_{0.399}\right)_{\mathrm{Tet}}\left[\mathrm{Mn}_{15.602} \mathrm{Fe}_{0.402}\right]_{\mathrm{oct}} \mathrm{O}_{32}$, and shows the absence of manganese ions in the tetrahedral spinel sublattice. A more detailed report on the structure refinement, based on the X-ray and neutron powder diffraction experiments will be published before long.

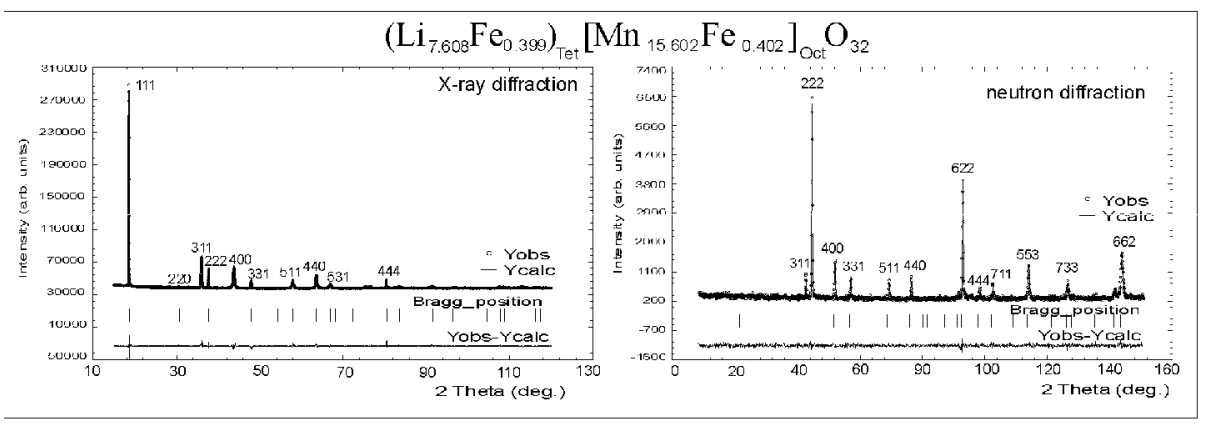

Figure 2. Observed, calculated and difference profiles from the Rietveld analysis of X-ray and neutron powder diffraction data of sample $\mathrm{Li}_{0.95} \mathrm{Mn}_{1.95} \mathrm{Fe}_{0.1} \mathrm{O}_{4}$, (series $\mathrm{Q}$ ), at the temperature $300 \mathrm{~K}$.

Determination of magnetic structure appears to be essential, because the samples of solid solutions $\mathrm{LiMn}_{2-\mathrm{x}} \mathrm{Fe}_{\mathrm{x}} \mathrm{O}_{4}$ system reveal different magnetic ordering [13]. Figure 3, part $\mathrm{A}$ presents the evolution of the magnetic susceptibility values, in the temperature range of $4.2 \mathrm{~K}-320 \mathrm{~K}$. A divergence between the zero-field-cooled (ZFC) and field-cooled (FC) data upon cooling, may be observed below $50 \mathrm{~K}$. With increasing $\mathrm{Fe}^{3+}$ substitution, the maximum value of susceptibility increases. The magnetic field dependence of the magnetization, measured at $4.2 \mathrm{~K}$ and $320 \mathrm{~K}$, for samples of $\mathrm{Li}_{0.95} \mathrm{Mn}_{2.05-\mathrm{x}} \mathrm{Fe}_{\mathrm{x}} \mathrm{O}_{4}$ series, is shown in figure 3 , part B. With the increasing $\mathrm{x}$, a "hysteresis" appears, suggesting the "ferrimagnetic" behaviour of the iron-containing lithium-manganese oxides, which could be explained by the occurrence of $\mathrm{Fe}^{3+}$ in the tetrahedral spinel sites [14]. Figure 4 shows the relation between $\mathrm{Fe}^{3+}$ content and the values of Weiss constant, $\theta$, obtained by extrapolation to $\chi^{-1}=0$. The Weiss temperatures vary from $\theta=-62 \mathrm{~K}$, for the $\mathrm{Li}_{0.95} \mathrm{Mn}_{2.05} \mathrm{O}_{4}$, to $\theta=-28 \mathrm{~K}$, for $\mathrm{Li}_{0.95} \mathrm{Mn}_{1.95} \mathrm{Fe}_{0.1} \mathrm{O}_{4}$. The Néel point increases from $T_{N}=11 \mathrm{~K}$ to $T_{\mathrm{N}}=27 \mathrm{~K}$. 


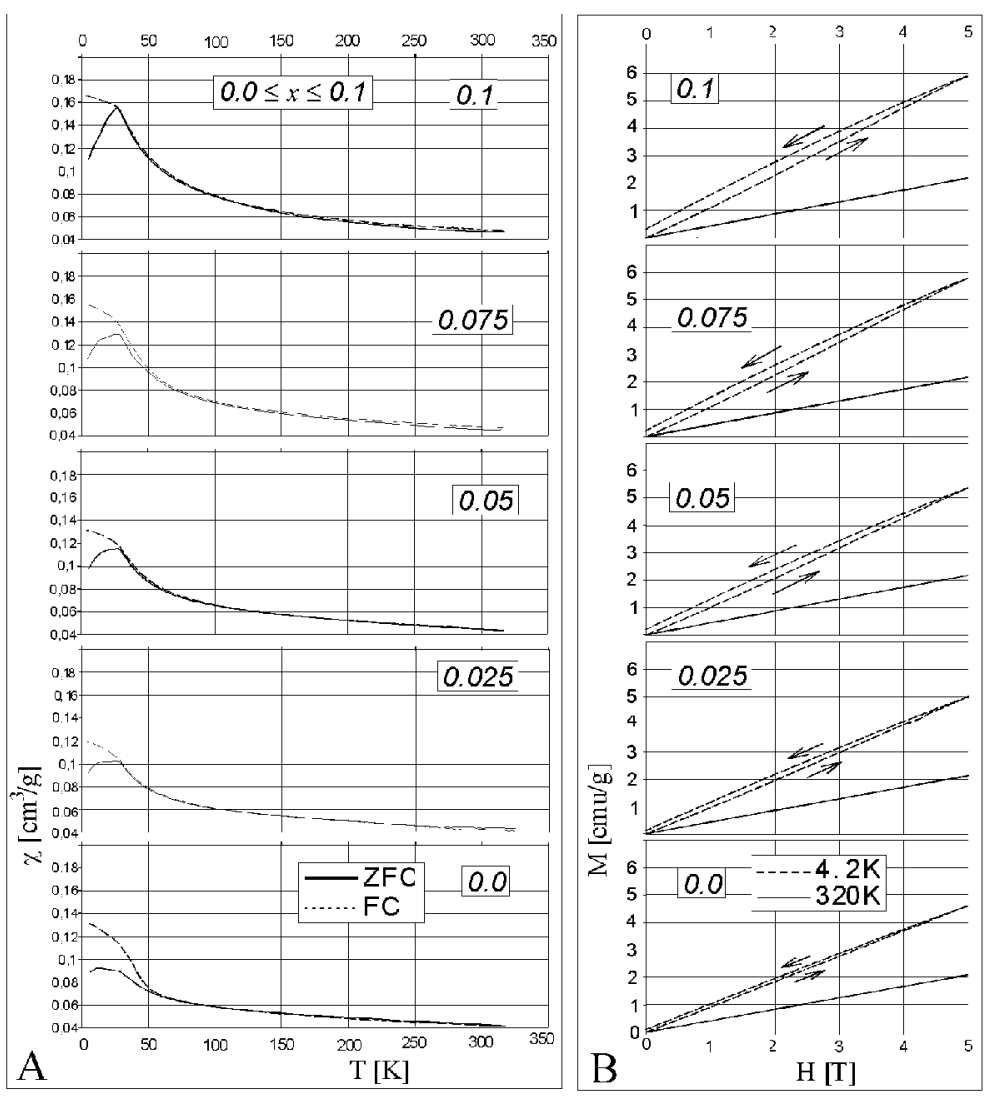

Figure 3. Part'A, magnetic susceptibility of a series $\mathrm{Li}_{0.95} \mathrm{Mn}_{2.05-x} \mathrm{Fe}_{x} \mathrm{O}_{4}(0.0 \leq x \leq 0.1)$, plotted as a function of temperature (series $Q$ ). Direct current susceptibility measurements reveal, upon cooling, $a$ divergence between zero-field-cooled $(Z F C)$ and field-cooled $(F C)$ data. Part $\boldsymbol{B}$, the magnetic field dependence of magnetization, recorded at $4.2 \mathrm{~K}$ and $320 \mathrm{~K}$.
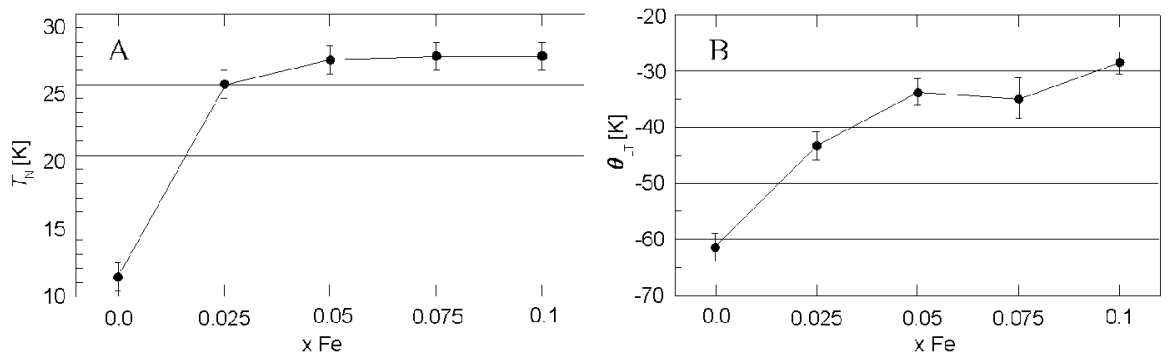

Figure 4. The Néel temperature, $T_{N},(\boldsymbol{A})$, and the Weiss constant, $\theta,(\boldsymbol{B})$, as a function of $\mathrm{Fe}^{3+}$ content in $\mathrm{Li}_{0.95} \mathrm{Mn}_{2.05-x} \mathrm{Fe}_{x} \mathrm{O}_{4}$, (series $\mathrm{Q}$ ). 


\section{Concluding remarks}

Stoichiometry and synthesis conditions play important roles in the formation different products of $\mathrm{Li}_{0.95} \mathrm{Mn}_{2.05-\mathrm{x}} \mathrm{Fe}_{\mathrm{x}} \mathrm{O}_{4}(0.0 \leq \mathrm{x} \leq 0.1)$ system. The cubic and tetragonally distorted spinel oxides may be observed when the sintering products are quenched rapidly to the room temperature. The metastable, distorted crystalline phases decompose during slow cooling. Substitution with $\mathrm{Fe}^{3+}$ ions reduces the total concentration of $\mathrm{Mn}^{3+}$, suppressing the JahnTeller effect, and stabilizes the cubic spinel structure. Low temperature neutron powder diffraction experiments on the series $\mathrm{Li}_{0.95} \mathrm{Mn}_{2.05-\mathrm{x}} \mathrm{Fe}_{\mathrm{X}} \mathrm{O}_{4}$ are required to confirm the changes of the Néel temperature obtained from the macroscopic susceptibility measurements.

\section{References}

1. Shukla, A.K.\& Kumar, T.P., 2008, Curr. Sci., 94, (3), 314.

2. Tarascon, J.M. \& Armand, M., 2001, Nature, 414, 359.

3. Tarascon, J.M., McKinnon, W.R., Coowar, F., Bowmer, T.N., Amatucci, G. \& Guyomard, D., 1994, J. Electrochem. Soc., 141, (6), 1421.

4. Tarascon, J.M. \& Guyomard, D., 1993, Electrochim. Acta 38, (9), 1221.

5. Xia, Y., Takeshige, H., Noguchi, H. \& Yoshio, M., 1995, J. Power Sources, 56, 61.

6. Nowicki, W., Darul, J., Piszora, P., Baehtz, C. \& Wolska, E., 2005, J. Alloys Comp., 401,55 .

7. Kraus, W. \& Nolze, G., 1998, PowderCell 2.3.

8. Rodriguez-Carvajal, J., 2001, Newslett. IUCr Commission Powd. Diffr., 26, 12.

9. Wende, C. \& Langbein, H., 2006, Cryst. Res. Technol., 1, 18.

10. Geller, S., 1971, Acta Cryst., B27, 821.

11. Aminoff, G., 1926, Z. Kristallogr., 64, 475.

12. Piszora, P., 2006, Chem. Mater., 18, 4802.

13. Wolska, E., Stempin K. \& Krasnowska-Hobbs O., 1997, Solid State Ionics, 101$103,527$.

14. Wolska, E., Nowicki W., Darul J., Piszora P. \& Knapp M., 2004, Appl. Crystallogr. Proc. XIX Conf., 412.

Acknowledgements. This research project has been supported by the European Commission under the $6^{\text {th }}$ Framework Programme through the Key Action: Strengthening the European Research Area, Research Infrastructures. Contract No. RII-CT-2003-505925 (NMI3). 
IV.4.4 Microstructure, Phase Analysis 



\title{
Simulation of layer-faulting in $\mathrm{Nb}_{2} \mathrm{Co}_{7}$ intermetallic compound using DIFFaX+
}

\author{
A. Leineweber ${ }^{1,}$, M. Leoni ${ }^{2}$ \\ 1 Max Planck Institute for Metals Research, Stuttgart, Germany \\ ${ }^{2}$ University of Trento - Department of Materials Engineering and Industrial Technologies, \\ Trento, Italy \\ *a.leineweber@mf.mpg.de
}

Keywords: intermetallic compounds, line-profile analysis, layer faulting

Abstract. The crystal structure and the fault structure of the intermetallic $\mathrm{Nb}_{2} \mathrm{CO}_{7}$ phase were determined using DIFFaX + refinements on the basis of synchrotron powder-diffraction data. The crystal structure was confirmed to be of the monoclinic $\mathrm{Zr}_{2} \mathrm{Ni}_{7}$ type. The layer faulting can be understood as partial occurrence of structural features of the $\mathrm{Yb}_{2} \mathrm{Ag}_{7}$-type structure, which contains the same "triple layers" as the $\mathrm{Zr}_{2} \mathrm{Ni}_{7}$-type structure does, but stacked in a different fashion.

\section{Introduction}

The binary $\mathrm{Nb}$-Co system [1] contains, among other phases, the intermetallic $\mathrm{Nb}_{2} \mathrm{Co}_{7}$ compound with the recently identified crystal structure described in ref. [2]. For an intermetallic, $\mathrm{Nb}_{2} \mathrm{CO}_{7}$ exhibits an unusually high plastic deformability $[3,4]$. Consequently, the question arises, whether there is a relation between the actual crystal structure and the high deformability. The X-ray powder-diffraction patterns used of $\mathrm{Nb}_{2} \mathrm{CO}_{7}$ for structural studies exhibit strong line broadening, which can be ascribed to faulting with respect to the layered structure of $\mathrm{Nb}_{2} \mathrm{CO}_{7}$. Such defects are expected to play a role in the deformability of this phase, but the traditional Rietveld approach is not able to handle fully the diffraction phenomena associated with faulting. Therefore, DIFFaX + refinements are provided here, which depart from the layered nature of the material and from previous qualitative considerations concerning the character of the faulting [2]. The results provide useful inside into the defect structure of the material.

\section{Crystal structure and faulting model}

Structure/microstructure analyses were conducted on a $\mathrm{Nb}_{2} \mathrm{CO}_{7}$ powder sample containing tiny amounts of the cubic $\mathrm{C} 15-\mathrm{NbCO}_{2}$ Laves phase (equilibrium phase with $\mathrm{Nb}_{2} \mathrm{Co}_{7}$ in a twophases region at the final annealing temperature of $900^{\circ} \mathrm{C}$ [1]). An X-ray powder-diffraction pattern recorded on the powder diffractometer installed at the ID31 beamline of the ESRF synchrotron (Grenoble, F) using a wavelength of $0.400094 \AA$ was available for the specimen. 
The average (defect free) crystal structure of $\mathrm{Nb}_{2} \mathrm{CO}_{7}$ was previously identified [2] to be similar to monoclinic $\mathrm{Zr}_{2} \mathrm{Ni}_{7}[5,6]$, figure 1a. Within the crystal structure one can identify triple-layers parallel to (001) with a complex internal atomic structure: an inner Kagométype layer $(K A)$ of " $\mathrm{CO}_{3}$ " sandwiched by two outer "close-packed" layers $(C L)$ " $\mathrm{NbCO}_{2}$ " (with $\mathrm{Nb}$-Co ordering), leading to a layer composition of $\mathrm{Nb}_{2} \mathrm{CO}_{7}$ corresponding also to the overall phase composition. The triple layers $(C L-K A-C L)$ by themselves ideally exhibit an hexagonal layer symmetry, but in view of the final crystal structure, a centred rectangular unit cell with basis vectors $\mathbf{a}$ and $\mathbf{b}$ should be considered, identical to the lattice-basis vectors of the monoclinic crystal structure (figure 1b).

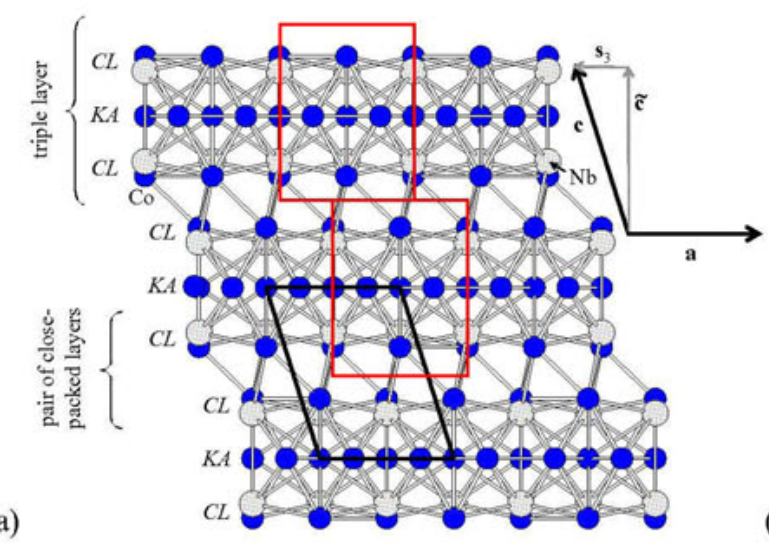

(b)

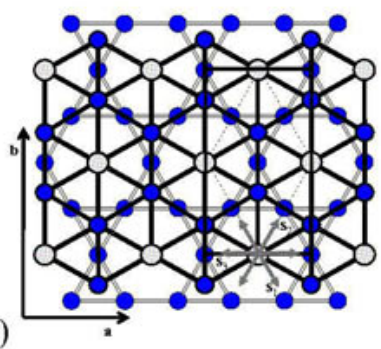

Figure 1. (a) Ideal $\mathrm{Zr}_{2} \mathrm{Ni}_{7}$-type crystal structure of $\mathrm{Nb}_{2} \mathrm{Co}_{7}$ (view along [010]) with monoclinic unit cell according to ref. [6]). In red (online version): layer unit cells. (b) View of triple layer along - $\tilde{\mathbf{c}}$, grey: shift vectors $\pm \mathbf{s}_{m}(m=1,2,3)$.

Upon stacking the layers perpendicular to the (001)-layer planes, the outer close-packed $C L$ layers of the triple layers $C L-K A-C L$ come into direct contact. These $C L-C L$ contacts simply correspond to contacts between close-packed layers. Due to the $\mathrm{Nb}$-Co ordering in the $C L$ layers, two adjacent triple layers are shifted with respect to each other by stacking vectors $\mathbf{R}$ $=\tilde{\mathbf{c}} \pm \mathbf{s}_{m}(m=1,2,3)$ with $\mathbf{s}_{1}=\mu(\mathbf{a}+\mathbf{b}) / 2, \mathbf{s}_{2}=\mu(\mathbf{a}-\mathbf{b}) / 2, \mathbf{s}_{3}=-\mu \mathbf{a}$ (cf. figure $\left.1 \mathrm{~b}\right)$, with $\tilde{\mathbf{c}}$ lying perpendicular to the layer plane, and with the shift vector $\mathbf{s}_{m}$ lying within the layer plane, and with $\mu$ corresponding ideally to $1 / 3$.

Different stacking sequences can be realised by different periodic sequences of stacking vectors [6]. The ideal stacking corresponding to the $\mathrm{Zr}_{2} \mathrm{Ni}_{7}$-type structure is realised by occurrence of one single shift vector, e.g. $+\mathbf{s}_{3}\left(\right.$ or $\left.-\mathbf{s}_{3}\right)$ whereas the closely related orthorhombic $\mathrm{Yb}_{2} \mathrm{Ag}_{7}$ structure can be realised by alternate occurrence of $+\mathbf{s}_{3}$ and $-\mathbf{s}_{3}$.

The above-mentioned powder-diffraction pattern of $\mathrm{Nb}_{2} \mathrm{CO}_{7}$ exhibits several features suggesting that extended defects are present in the phase's crystal structure:

(a) $0 k l$ and $5 k l$ reflections are narrow, whereas other reflections $h k l$ are broadened.

(b) some unindexed intensity maxima remain. 
These features can be understood as a consequence of an irregular stacking of the triple layers [2]. The most obvious stacking error is that among the $+\mathbf{s}_{3}$ shift vectors also other shift vectors $\pm \mathbf{s}_{m}$ occur. In particular the narrowness of the $0 \mathrm{kl}$ reflections indicates that the shift vector $-\mathbf{s}_{3}$ occurs as well besides the predominant $+\mathbf{s}_{3}$ vector (or $+\mathbf{s}_{3}$ besides $-\mathbf{s}_{3}$ ), because it leaves the projection of the crystal structure along a (responsible for the $0 \mathrm{kl}$ reflections) unaffected. Additional occurrence of the shift vectors $\pm \mathbf{s}_{1,2}$ appears to be less important, because this would broaden the $0 \mathrm{kl}$ reflections, which is not observed. These interpretations are further confirmed by the narrowness of $5 \mathrm{kl}$ reflections, which is expected if for the shift vectors $\pm \mathbf{s}_{3}=\mp 0.4 \mathrm{a}$ holds. The latter relation is, in fact, independently suggested by the values of the monoclinic lattice parameters. The occurrence of the unindexed intensity maxima can be ascribed to low-angle cut-offs of diffuse intensity bands parallel to $\mathbf{c}^{*}$ expected due to the faulting; these bands can by indexed by two indices $h k$.

\section{$\mathrm{DIFFaX}+$ refinement}

In order to confirm the crystal structure model and the faulting model derived in ref. [2] on a largely qualitative basis, the $\mathrm{X}$-ray powder-diffraction pattern (considering diffraction data from $2 \theta=3-21^{\circ}$ ) of the $\mathrm{Nb}_{2} \mathrm{Co}_{7}$ sample were used as a basis for refinement of the parameters of a crystal- and fault-structure model in a Rietveld-like fashion using the DIFFaX+ software [7], v. $2.400 \mathrm{~b} 13$, cf. figure 2.

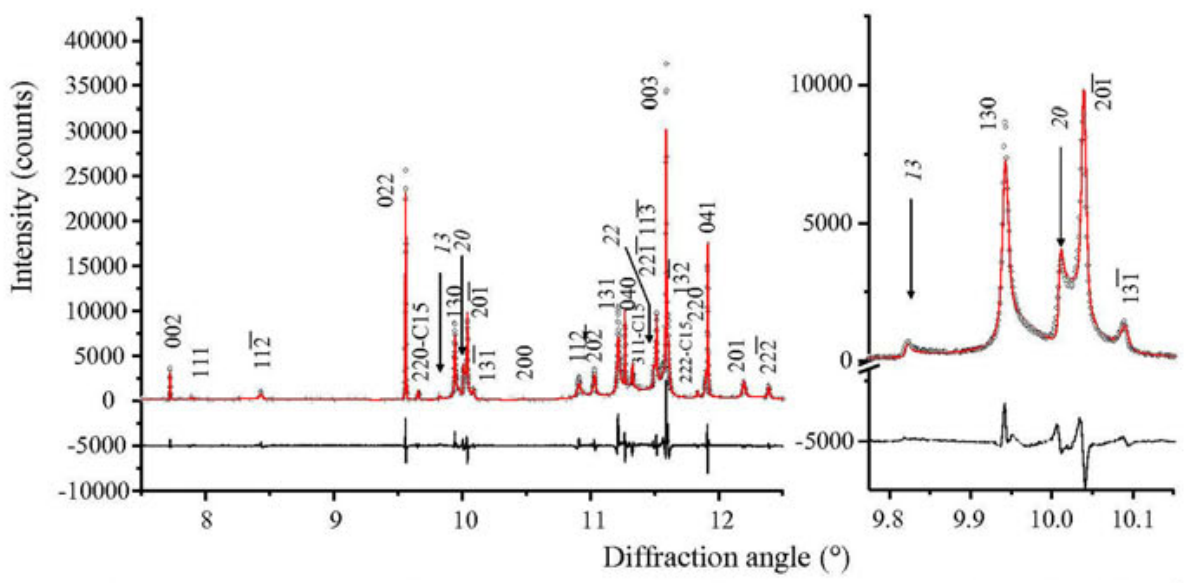

Figure 2. Synchrotron powder-diffraction pattern of the $\mathrm{Nb}_{2} \mathrm{Co}_{7}$ sample (wavelength $0.400094 \mathrm{~A}$ ) together with calculated profile (red in online version) from DIFFaX+ refinements, and difference curve. Bragg-reflection indices hkl are indicated by regular numbers, low-angle cut-offs of diffuseintensity rods parallel $c^{*}$ are indicated by the indices hk pertaining to that band in italic letters. The right part corresponds to an enlarged section of the left part.

DIFFaX + is based on the recursive formula by Treacy et al. [8] and has been designed to deal with complex faulted and modular structures. The structure is described as a sequence of $N$ different layers types. Each layer type can be described either in terms of a layer-unit cell and a shift vector $\mathbf{s}$, or it can be derived from another layer e.g. by a symmetry operation. 
The diffraction pattern is obtained on the basis of kinematical diffraction theory, considering a statistical sequence of layers, stacked along $\tilde{\mathbf{c}}$ on the basis of independent layer-transition probabilities. The instrumental profile contribution is taken into account through a pseudoVoigt function.

In the present case the instrumental profile contribution was determined on the basis of the narrow $0 \mathrm{kl}$ reflections. Additional reflections due to $\mathrm{C} 15-\mathrm{NbCO}_{2}$ were accounted for by separate pseudo-Voigt like peaks. $N=4$ structurally identical triple layers, labeled $A, B, C, D$, as considered in section "Crystal structure and faulting model" were employed to model the crystal structure of $\mathrm{Nb}_{2} \mathrm{CO}_{7}$. Two of these layers are associated with a shift vector of $+\mathbf{s}_{3}=$ $-\mu \mathbf{a}(A, C)$ and two with $-\mathbf{s}_{3}=+\mu \mathbf{a}(B, D)$. The transition probabilities between each possible pair of layers are refined as parameters. Since there are $4 \times 4=16$ pairs of layers and considering the restriction that starting from a given layer (say $A$ ) the probabilities for transition into layers $A-D$ must sum up to 1 , altogether 12 independent transition probabilities result.

\section{Results and discussion}

The DIFFaX + refinement was performed by sequentially refining parameters pertaining to different more or less independent aspects of the diffraction pattern. In order to achieve reasonably short computation times per refinement cycle, parameters pertaining to background and instrumental profile were held fixed after convincing values had been obtained. In later cycles, lattice parameters, zero-point error, fractional coordinates and the atomic displacement parameters of the different atoms (isotropic) were refined to reasonable values, confirming the $\mathrm{Zr}_{2} \mathrm{Ni}_{7}$-type structure for $\mathrm{Nb}_{2} \mathrm{Co}_{7}$ derived in ref. [2]. Finally, emphasis was put on the transition probabilities, which were refined together with the overall scale factor of $\mathrm{Nb}_{2} \mathrm{CO}_{7}$. The transition probabilities can be summarised in the following transition matrix:

$$
\mathbf{p}=\left(\begin{array}{llll}
0.971 & 0.014 & 0.015 & 0.000 \\
0.000 & 0.000 & 1.000 & 0.000 \\
0.030 & 0.162 & 0.707 & 0.102 \\
0.000 & 0.311 & 0.058 & 0.630
\end{array}\right),
$$

where the component $p_{L L}$, corresponds to the probability that a layer $L$ is followed by a layer $L^{\prime}$, with $L, L^{\prime} \in\{A, B, C, D\}$. E.g. the probability $p_{C B}$ is $16.2 \%$. The refined structure model successfully predicts the line-profile features in the powder-diffraction patterns mentioned in section "DIFFaX+ refinement", cf. figure 2, leading to acceptable residuals: $R_{w p}=12.5 \%$ and $R_{p}=9.6 \%$ ).

The Eigenvector associated with the Eigenvalue of 1 of the transition matrix p, contains the probabilities for occurrence of layers A-D, which are $40.3 \%, 10.2 \%, 38.8 \%, 10.7 \%$. A more instructive view of the random structure of the investigated $\mathrm{Nb}_{2} \mathrm{CO}_{7}$ can be constructed on the basis of $\mathbf{p}$ and of random numbers. Such a model structure is shown in figure 3 . This model structure demonstrates the occurrence of longer blocks of the monoclinic $\mathrm{Zr}_{2} \mathrm{Ni}_{7}$ structure. These blocks are characterized by occurrence of the same shift vectors $+\mathbf{s}_{3}$ or $-\mathbf{s}_{3}$ (containing thus only A and C, or B and D layers, indicated by "+" or "_" in figure 3). Occasional sections of alternate shift vectors $\left(+-+-\ldots\right.$ ) correspond to local (orthorhombic) $\mathrm{Yb}_{2} \mathrm{Ag}_{7}$-type stacking (see section "Crystal structure and faulting model"). 
Individual layers can, furthermore, be characterized as stacked "cubic" or "hexagonal" (-like) in a Jagodzinski-type fashion, analogous to the nomenclature for close-packed structures: A layer receives a " $c$ " if the shift vectors towards that layer and to the next layer are the same, and an " $h$ " if the shift vectors towards that layer and to the next layer are opposite. An ideal $\mathrm{Zr}_{2} \mathrm{Ni}_{7}$-type structure would contain only " $c$ " layers, whereas an $\mathrm{Yb}_{2} \mathrm{Ag}_{7}$-type structure would contain only " $h$ " layers. By straightforward analysis of the transition probabilities one obtains that $78 \%$ of all layers should be " $c$ ", the remaining ones should be " $h$ ".

It should be noted that the multiple use of a combination of a shift vector and of a layer ( $A$, $C)$ and $(B, D))$ is required to model complicated stacking sequences. The independent transition probabilities associated with the different layers, then allow that e.g. layers $A$ and $C$ assume different roles in the stacking sequences. This is e.g. required to model independently occurrence of twinning and of deformation faults. Test calculations were performed in order to elucidate whether fits of similar quality could be achieved with model structures containing 3 instead of 4 layers. Slightly but significantly poorer residuals were obtained. However, these fits give similar proportions of " $c$ " layers like refinements with 4 layers. This suggests that the result concerning the proportion of " $c$ " and " $h$ " layers is robust. However, it is questionable whether the obtained probability matrix is unique, i.e. unique beyond the obvious possibility for e.g. the mutual exchange the roles of layers $\mathrm{A}$ and $\mathrm{C}$ and of $\mathrm{B}$ and $\mathrm{D}$, which would lead to a "transformed" transition matrix.

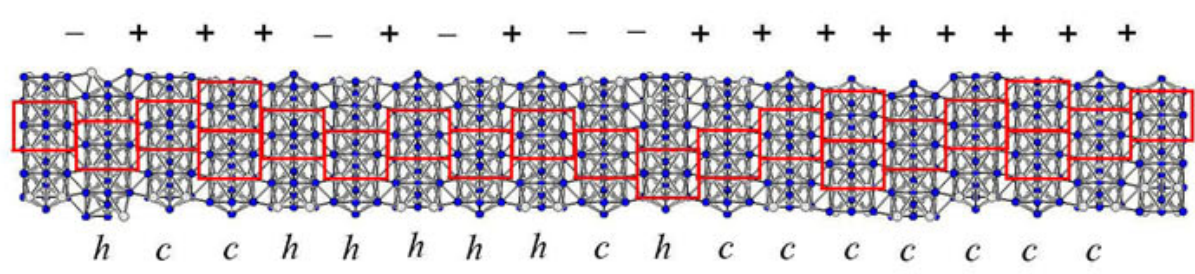

Figure 3. Model structure of $\mathrm{Nb}_{2} \mathrm{Co}_{7}$ obtained on the basis of the refined transition probabilities from eq. (1) and random numbers. Boxes indicate the orthorhombic layer-unit cell. The signs at the top pertain to the shift vector $\pm_{\mathbf{s}_{3}}$ and the letters at the bottom refer to "cubic" and "hexagonal" (-like) stacking around the considered triple layer (Jagodzinski-like nomenclature). Note that with respect to figure la the present structure drawing has been rotated $90^{\circ}$ around the viewing direction.

\section{Conclusion}

The crystal and defect structure of a $\mathrm{Nb}_{2} \mathrm{CO}_{7}$ intermetallic phase was obtained by DIFFaX+ refinement of synchrotron powder-diffraction data. The resulting model is able to reproduce the different features of the powder-diffraction pattern quantitatively. In particular the refinement confirms that:

$$
\mathrm{Nb}_{2} \mathrm{Co}_{7} \text { is based on the monoclinic } \mathrm{Zr}_{2} \mathrm{Ni}_{7} \text {-type structure }
$$

faulting occurs in layer stacking, which can be understood as partial occurrence of structural $\mathrm{Yb}_{2} \mathrm{Ag}_{7}$-type features, containing the same "triple layers" as the $\mathrm{Zr}_{2} \mathrm{Ni}_{7}$ type structure, but stacked in a different fashion. 
The main quantitative result of the refinement is the transition matrix containing the 16 transition probabilities between the four different layers. This transition matrix can be used to derive illustrative model structures. Even more, the matrix components can also provide the probability of complex structural features like the proportion of certain types of layer stacking sequences.

The easiness of changing the stacking sequence in $\mathrm{Nb}_{2} \mathrm{CO}_{7}$ suggests a low stacking-fault energy between the triple layers, i.e. where close packed " $\mathrm{NbCO}_{2}$ " layers are in contact. Dislocation dissociation may also easily occur at these contacts. These two aspects suggest that the layer-type structure of $\mathrm{Nb}_{2} \mathrm{CO}_{7}$ has a close relation with the easy plastic deformability of this phase [3]. For further details, see also [2].

\section{References}

1. Stein, F., Jiang, D., Palm, M., Sauthoff, G., Grüner, D. \& Kreiner, G., 2008, Intermetallics, 16, 785 .

2. Leineweber, A., Stein, F., Grüner, D. \& Dinnebier, R. et al., 2009. In preparation.

3. Siggelkow, L., Burkhardt, U., Kreiner, G., Palm, M. \& Stein, M., 2008, Mater. Sci. Eng. A, 497, 174.

4. Stein, F., Palm, M., Frommeyer, G., Jain, P., Kumar, K.S., Siggelkow, L., Grüner, D., Kreiner, G. \& Leineweber, A., 2008, Proc. MRS Fall Meeting, accepted.

5. Eshelman, F.R. \& Smith, J.F., 1972, Acta Cryst. B, 28, 1594.

6. Parthé, E. \& Lemaire, R., 1975, Acta Cryst. B, 31, 1879.

7. Leoni, M., Gualtieri, A.F. \& Roveri, N., 2004, J. Appl. Cryst., 37, 166.

8. Treacy, M.M.J., Newsam, J.M. \& Deem, M.W., 1991, Proc. Roy. Soc. Lond. A, 433, 499 .

Acknowledgements. This work has been performed within the framework of the InterInstitutional Research Initiative "The Nature of Laves Phases" funded by the Max Planck Society. The authors thank Prof. Robert Dinnebier for measurement of the powderdiffraction data at ID31 and for valuable discussions. 


\section{Structure and microstructure of Mg-vermiculite}

\section{A. Argüelles ${ }^{1}$, M. Leoni ${ }^{2,}$, J. A. Blanco ${ }^{1}$, C. Marcos $^{3}$}

${ }^{1}$ Dpto. Física, Universidad de Oviedo, C/ Calvo Sotelo, s/n, 33007, Oviedo, Spain

${ }^{2}$ Department of Materials Engineering and Industrial Technologies, University of Trento, via Mesiano, 77, 38100, Trento, Italy

${ }^{3}$ Dpto. Geología e Instituto de Organometálica Enrique Moles, Universidad de Oviedo, C/ Jesús Arias de Velasco, s/n, 33005, Oviedo, Spain

*Matteo.Leoni@unitn.it

Keywords: Mg-vermiculite, clay structure, stacking faults, DIFFaX+

Abstract. A specimen of Mg-vermiculite from Santa Olalla (Spain) has been investigated. Powder diffraction data has been simulated and an experimental pattern refined by means of the DIFFaX + software. The best modelling is obtained assuming the mineral to possess bidimensional periodicity instead of a tridimensional one: the structure can be described as a disordered sequence of two different types of layers, differing in the arrangement of the interlayer cations.

\section{Introduction}

Mg-vermiculite has been the subject of mineralogical, physical and chemical studies for several decades. This hydrous phyllosilicate is nowadays finding new interesting applications in environmental science [1] and nanoscience [2].

From a structural point of view, vermiculite is a 2:1 clay: a T-O-T unit (formed by the assembly of an octahedral and two tetrahedral layers) is present, with an interlayer filled with $\mathrm{Mg}^{2+}$ hydrated cations (see figure 1). The crystalline structure of vermiculite has been theoretically and experimentally investigated by several groups (see e.g. [3-5]), but the most widely cited model is still that proposed by Shirozu and Bailey [6], which does not deal with disorder, because of its simplicity. The presence of defects such as stacking faults makes the diffraction analysis of this material a hard task.

Traditionally, the refinement of a structure from powder diffraction data is accomplished by means of the Rietveld method. Existing refinement software, however, assumes the structure to be periodic and the presence of defects is at most considered through a variational approach. Extensive amount of faults, leading to strong diffuse effects on the pattern, cannot be handled. An alternative approach has been proposed, where the recursive description of the stacking proposed by Treacy et al. [7] is employed. 


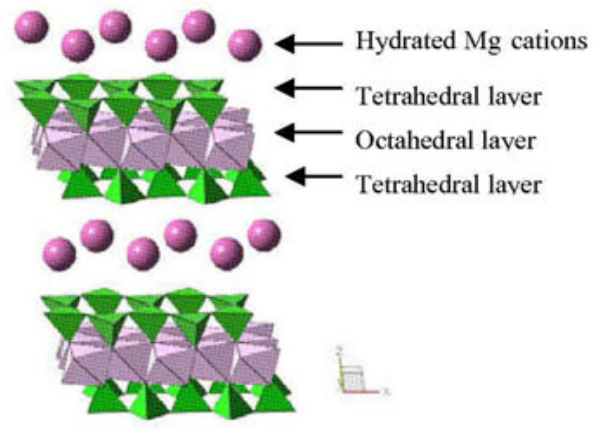

Figure 1. Schematic drawing of Mg-vermiculite structure.

In the present paper, a preliminary structural refinement by using the DIFFaX + software [8], implementing a refinement routine based on the formula of Treacy et al., has been undertaken. The literature model proposed by de la Calle et al. [9] has been taken as the starting point. Experimental data have been obtained by X-ray diffraction (XRD) in transmission mode. As a result, DIFFaX+ software has allowed deal with such faulted structure in an easy and fast way, refining the structure of $\mathrm{Mg}$-vermiculite from Santa Olalla with that model proposed by de la Calle $e t$ al.

\section{Experimental results and discussion}

A powder sample was prepared by milling some flakes of natural $\mathrm{Mg}$-vermiculite from Santa Olalla (Huelva, Spain).

A morphological study was conducted both by Scanning Electron Microscopy (SEM) and by Laser Diffraction using, respectively, a FEI Quanta 400 microscope and a FRITSCH MicroTec XT laser particle sizer.

X-ray powder diffraction data were collected on an INEL XRD RG3000 diffractometer operating in Debye-Scherrer capillary transmission mode. The system is equipped with a cobalt tube and a primary beam Johannson-type monochromator.

The crystalline and defect structure refinement was performed by means of the DIFFaX+ software v. 2.400 .

\section{Morphological study}

Figure 2 shows the morphology of the particles in the milled sample of natural $\mathrm{Mg}$ vermiculite sample: the maximum size is around $80 \mu \mathrm{m}$. Although particles show a heterogeneous morphology, a platelet-like habit is observed, as expected for a lamellar phyllosilicate. 


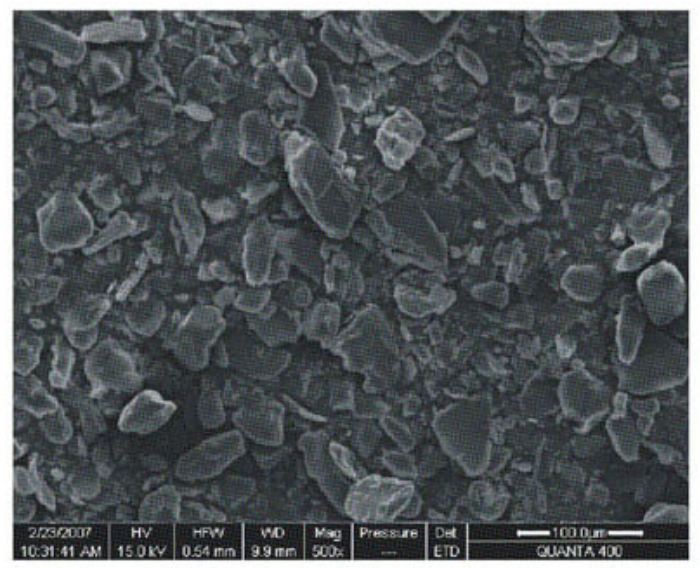

Figure 2. SEM micrograph (500x) of Mg-vermiculite powder sample from Santa Olalla (Spain).

The particle size distribution was determined by laser diffraction. An effective particle diameter was calculated, as the diameter of the sphere that gives the same diffraction signal as the actual particle [10]. The granulometric curve obtained by laser scattering is shown in figure 3. A multimodal distribution is apparent, the main fractions having an average of about 10 and $55 \mu \mathrm{m}$, respectively.

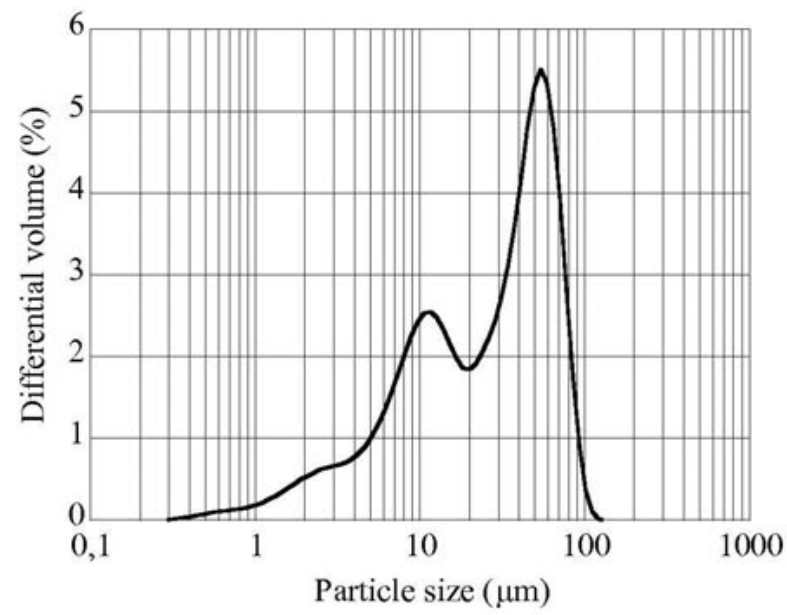

Figure 3. Particle size distribution of Mg-vermiculite obtained by laser diffraction.

\section{Structural refinement}

The most accredited reference for vermiculite structure is probably the work of Shirozu and Bailey [6] where the mineral is described as being monoclinic and ordered. It seems, however, that this model can just partly justify the observed features in the X-ray powder diffrac- 
tion pattern of the mineral. An alternative, not widely accepted by the scientific community, was proposed by de la Calle et al. [9], who described vermiculite as semi-ordered. According to those authors, the structure is made of a stacking of two types of layers, A and B (see below), differing in the position of the interlayer hydrated cation. Moreover, the relative translations of those layers along the crystallographic $y$-axis are not unique.

In the present work, the model of de la Calle et al. was assumed as the starting point for the analysis. Owing to the irregular nature of the stacking, the Rietveld method is not appropriate for the structural refinement. Moreover, due to the layered character of this material, preferred orientation (in particular $00 l$ fibre texture, i.e. enhancement of the signal from basal reflections) is highly probably in a powder specimen, further contributing to render a fullprofile analysis quite difficult [11]. For this reason, techniques specially devised for the analysis of faulted structures need being used.

In this case, the DIFFaX + software, based on a recursive description of the stacking has been employed [8]. This methodology has been already tested on clay systems and proven to be effective for the modelling of complex layered systems [12]. XRD data collected in capillary transmission mode was undertaken, to limit the effect of preferred orientation.

To test the relative efficiency of the ordered and semi-ordered models to describe the structure of vermiculite, different stacking configurations were simulated and compared (cf. table 1 and figure 4).

Two types of layers (named, respectively, A and B) were considered, differing only in the position of the interlayer hydrated cation relative to the silicate surface layer. One modelling attempt has been made supposing a translation of $\pm 1 / 2$ along the $y$-axis (translation mode in table 1).

Table 1. Information of the different stacking models for Mg-vermiculite: type of stacking mode, spatial translations, probabilities of the sequences and reliability factor.

\begin{tabular}{|l|c|c|c|c|c|c|c|c|c|}
\hline $\begin{array}{c}\text { Description of } \\
\text { the stacking } \\
\text { mode }\end{array}$ & \multicolumn{2}{|c|}{$\begin{array}{c}\text { Translation from one layer to } \\
\text { graphic y-axis }\end{array}$} & \multicolumn{2}{|c|}{ Probabilities of the sequence } & \multicolumn{2}{|c|}{$\begin{array}{c}\mathrm{R}_{\mathrm{wp}} \\
(\%)\end{array}$} \\
\cline { 2 - 11 } & $\begin{array}{l}\mathrm{t}_{\mathrm{yA} \rightarrow} \\
\mathrm{A}\end{array}$ & $\mathrm{t}_{\mathrm{yA} \rightarrow \mathrm{B}}$ & $\mathrm{t}_{\mathrm{yB} \rightarrow \mathrm{A}}$ & $\mathrm{t}_{\mathrm{yB} \rightarrow \mathrm{B}}$ & $\alpha_{\mathrm{AA}}$ & $\alpha_{\mathrm{AB}}$ & $\alpha_{\mathrm{BA}}$ & $\alpha_{\mathrm{BB}}$ & \\
\hline Random & $+1 / 3$ & $+1 / 3$ & $-1 / 3$ & $-1 / 3$ & $1 / 2$ & $1 / 2$ & $1 / 2$ & $1 / 2$ & 6.6 \\
\hline Segregated & $+1 / 3$ & $+1 / 3$ & $-1 / 3$ & $-1 / 3$ & 0.75 & 0.25 & 0.25 & 0.75 & 7.2 \\
\hline Translation & $+1 / 2$ & $+1 / 2$ & $-1 / 2$ & $-1 / 2$ & 0.416 & 0.584 & 0.584 & 0.416 & 12.3 \\
\hline Alternated & 0.34 & 0.34 & -0.32 & -0.33 & 0.416 & 0.584 & 0.584 & 0.416 & 6.5 \\
\hline
\end{tabular}




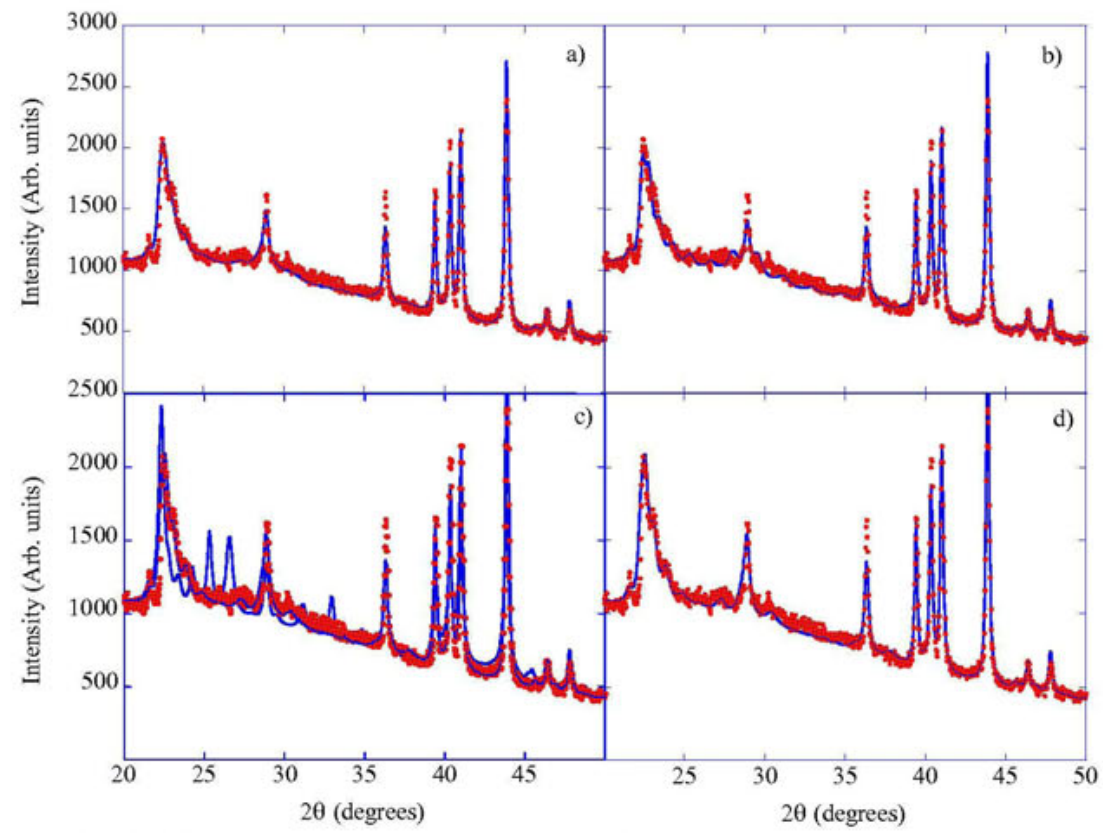

Figure 4. Experimental (dots) and calculated (solid line) X-ray diffraction patterns: random mode (4a), segregated mode $(4 b)$, translation mode $(4 c)$ and alternated mode $(4 d)$. Refined probabilities corresponding to the case presented in $4 d$ are listed in table 1. See the text for more details.

The other models consider a translation of $\pm 1 / 3$, as usually admitted in vermiculites. Different sequences of the stacking have been considered by changing the probabilities of layer to layer transition. In particular, the random mode corresponds to a random alternation of $\mathrm{A}$ and B type layers, while the segregated one corresponds to a sequence favouring one type of layer being followed by a layer of the same type. In all cases a refinement was tried. The best match between experimental and calculated diffraction patterns was obtained for the alternated model. In that case, the translation and probability parameters describing the sequence were both refined, obtaining a $\mathrm{R}_{\mathrm{wp}}$ factor of $6.45 \%$ (see table 1 ). The probability of a layer being followed for other of the same type has been found to be 0.416 , a bit lower than in a totally random model.

In figure 4 , all calculated patterns obtained for the different stacking modes described in table 1 , are shown and compared to the experimental pattern. It should be noted that the (02) reflection (aka ( $02 l$ ) diffraction band) between $21^{\circ}$ and $25^{\circ} 2 \theta$ is the most affected by the stacking mode parameters. The best refinement is obtained with a value of approximately $\pm 1 / 3$ for translations along the $y$-axis and almost equal transition probabilities for the various sequences.

A defective structure, with a small tendency to alternation of two similar types of layers seems to be the most suitable model for describing the stacking mode of this Mg-vermiculite from Santa Olalla (Spain) as has been obtained from DIFFaX+ refinements. Further investi- 
gations coupling powder X-ray diffraction and 1D Fourier analysis are in progress and the results will be presented elsewhere [13].

\section{Concluding remarks}

A powder specimen of Mg-vermiculite from Santa Olalla (Spain) was analysed by X-ray powder diffraction. The structure was modelled by means of the DIFFaX + software. A semiordered arrangement is confirmed to be the more plausible for this Mg-vermiculite. The parameters describing such stacking mode have been refined leading to a model that confirms that proposed by de la Calle et al., a preliminary difference being the uneven layer transition probabilities. The refined model is not totally random but certain tendency to alternation has been found. Further investigations using synchrotron powder X-ray diffraction are in progress to further validate this picture.

\section{References}

1. Huang, H.C., Lee, J.F., Chao, H.P., Yeh, P.W., Yang, Y.F. \& Liao, W.L, 2005, J. Colloid Interface Sci., 286, 127.

2. Matějka, V., Šupová-Křístková, M., Kratošová, G. \& Valášková, M., 2006, J. Nanosci. Nanotechnol., 6, (8), 2482.

3. Weiss, Z. \& Durovic, S., 1980, Acta Crystallogr., A36, 633.

4. de la Calle, C., Dubernat, J., Suquet, H. \& Pezerat, H., 1980, Bull. Mineral., 103, 419.

5. de la Calle, C., Suquet, H. \& Pezerat, H., 1985, Clay Miner., 20, 221.

6. Shirozu, H. \& Bailey, S.W., 1966, Am. Mineral., 51, 1124.

7. Treacy, M.M.J., Newsam, J.M. \& Deem, M.W., 1991, Proc. R. Soc. Lond., A 433, 499.

8. Leoni, M., Gualtieri, A.F. \& Roveri, N., 2004, J. Appl. Crystallogr., 37, 166.

9. de la Calle, C., Suquet, H. \& Pons, C.H., 1988, Clays Clay Miner., 36, (6), 481.

10. Konert, M. \& Vandenberghe, J., 1997, Sedimentology, 44, (3), 523.

11. Martínez Blanco, D., Gorría, P., Blanco, J.A., Pérez, M.J. \& Campo, J., 2008, J. Phys. Condens. Mater, $20,335213$.

12. Gualtieri, A.F., Ferrari, S., Leoni, M., Grathoff, G., Hugo, R., Shatnawi, M., Paglia, G. \& Billinge, S., 2008, J. Appl. Crystallogr., 41, 402.

13. Argüelles, A., Leoni, M., Pons, C.H., de la Calle, C., Blanco, J.A. \& Marcos, C., 2009, J. Appl. Crystallogr., in preparation.

Acknowledgements. The authors wish to acknowledge the Institut des Sciences de la Terre d'Orléans (France) for diffraction measurements in transmission geometry. The financial support from FEDER and the Spanish MICINN (FROMER MEC) grant number MAT200806542-C04-03 is greatly acknowledged. Special thanks to both C. de la Calle and C. H. Pons for helping us in the X-ray experiments and for stimulating discussions. 


\title{
Evolution of size and shape of mullite crystallites in triaxial porcelains
}

\author{
A. Sanz ${ }^{1}$, J. Bastida ${ }^{1, *}$, M. A. Kojdecki ${ }^{2}$, A. Caballero ${ }^{3}$, \\ F. J. Serrano ${ }^{1}$ \\ ${ }^{1}$ Departamento de Geologia, Universidad de Valencia. 46100 Burjasot (Valencia), Spain \\ ${ }^{2}$ Instytut Matematyki i Kryptologii, Wojskowa Akademia Techniczna, 00-908 Warszawa, \\ Poland \\ 3 Instituto de Cerámica y Vidrio (CSIC), Campus Universidad Autónoma de Madrid, \\ $\mathrm{C}^{\circ}$ Valdelatas SN, 2809, Madrid, Spain \\ *bastida@uv.es
}

Keywords: Mullite, crystallite, size, shape, strain, X-ray Diffraction

\begin{abstract}
A microstructural X-ray diffraction analysis involving prevalent crystallite shape and volume weighted crystallite size for mullite of two formulations of triaxial whiteware porcelain at different temperatures has been performed. The difference between formulations is the addition of petalite. Prism approximation for crystallites shows a preferential growth of crystallites for the formulation with petalite addition.
\end{abstract}

\section{Introduction, materials and aim}

Porcelain is one of the most complex ceramics materials. Primarily composed of clay, feldspar and quartz, they are called "triaxial" whitewares and fired at about $1300^{\circ} \mathrm{C}$ to obtain a mixture of glass and crystalline phases, where mullite is the main appearing and growing crystalline phase. $[1,2]$

Primary raw materials used in commercial whitewares include kaolins, ball clays, soda feldspars, potash feldspars, nepheline syenite and quartz. Other raw materials are also used as secondary: glass frits, petalite, bone ash, talc, zircon, and calcium carbonate[3, 4].

Potash feldspars (microcline and orthoclase) have been the most commonly used fluxes in porcelains. Potash feldspars rarely are pure, and usually appear in assemblages containing also other feldspars as albite or other plagioclases. Nepheline - syenite has replaced feldspars in many commercial body formulations, reducing firing temperature and increasing the alkali level in the glass phase [5]. The present paper deals with substitution of potash feldspar by petalite (lithium feldspar) to test the effect of the alkaline elements (K versus $\mathrm{Li}$ ) in the flux

Microstructural analysis from X-ray diffraction involving shape and size modellization [6] has been used in the analysis of mullite in kaolin-alumina refractories [9] and of mullite produced from single phase gels [10]. 
This paper concerns the crystallite size and shape of mullite by firing "triaxial" compositions $[1,2]$ of porcelain. Influence of firing temperature and different compositions concerning flux characteristics will be observed.

The studied porcelain compositions are in the range of those of industrial porcelains [3]. The two studied compositions are: $14 \%$ quartz, 53\% kaolin, 33\% K-feldspar (for MGS composition) and $14 \%$ quartz, $53 \%$ kaolin, 33\% Li-feldspar (for MGC composition). Table 1 shows the chemical composition of both formulations.

Table 1. Chemical composition of samples. Expressed as oxides and Lost on Ignition percentages.

\begin{tabular}{|l|c|c|c|c|c|c|c|c|c|c|c|}
\hline Sample & L.O.I. & SiO2 & Al2O3 & K2O & Na2O & Li2O & CaO & MgO & Fe2O3 & TiO2 & P2O5 \\
\hline MGC & 8,07 & 63,52 & 24,55 & 1,07 & 1,72 & 0,24 & 0,22 & 0,03 & 0,17 & 0,065 & 0,38 \\
\hline MGS & 7,78 & 62,90 & 24,41 & 3,44 & 0,88 & 0,0 & 0,16 & 0,02 & 0,17 & 0,055 & 0,0 \\
\hline
\end{tabular}

The samples were subjected to four different firing cycles as shown in table 2, in an electric kiln. These firing cycles correspond to normal values in an industrial porcelain process [5].

Table 2. Firing cycles (heating ratio: $2^{\circ} \mathrm{C} / \mathrm{min}$.; standing at maximal temperature: $180 \mathrm{~min}$ ).

\begin{tabular}{|c|c|c|c|}
\hline Cycle & $\mathbf{T} \max \left({ }^{\circ} \mathbf{C}\right)$ & $\mathbf{t}(\mathbf{m i n})$ & Fired Samples \\
\hline $\mathbf{1}$ & 1270 & 815 & MGC1, MGS1 \\
\hline $\mathbf{2}$ & 1300 & 830 & MGC2, MGS2 \\
\hline $\mathbf{3}$ & 1320 & 840 & MGC3, MGS3 \\
\hline $\mathbf{4}$ & 1340 & 860 & MGC4, MGS4 \\
\hline
\end{tabular}

\section{Methods}

Fired samples were milled in a Fritsch Pulverisette 9 vibrating cup mill (WC coated steel cup, ring and cylinder) to a grain size passing the 230 sieve (standard ASTM -8E11-81).

X-ray diffraction patterns were obtained in a Bruker D5005 diffractometer with graphite monochromator, $1^{\circ}$ divergence slit, $1^{\circ}$ antiscatter slit, and with a $0,5 \mathrm{~mm}$ width of receiving slit, running under the Diffrac-Plus System. Table 3 shows the angular ranges and scanning conditions for fast and slow diffraction patterns.

Standard line profiles corresponding to mullite Bragg angles were simulated on the basis of a description of lines from the data of the reference pattern used as line broadening standard (LaB6, NIST reference material 660a ) obtained in the same experimental conditions than for the analyzed mullites. 
Table 3. Angular ranges and scanning conditions used to obtain diffraction patterns.

\begin{tabular}{|c|c|c|c|c|}
\hline PATTERN & REFLECTION & RANGE $\left({ }^{\circ} 2 \theta\right)$ & STEP $\left({ }^{\circ} 2 \theta\right)$ & TIME (s) \\
\hline FAST (identification) & General & $2-72$ & 0,025 & 4 \\
\hline \multirow{4}{*}{$\begin{array}{l}\text { SLOW (micro structural } \\
\text { analysis) }\end{array}$} & 110 & $15-18$ & \multirow{4}{*}{0,01} & \multirow{4}{*}{25} \\
\hline & 120,210 & $24-28$ & & \\
\hline & $001,220,111$ & $30-36$ & & \\
\hline & 121 & $40-42$ & & \\
\hline
\end{tabular}

Crystallite size and shape were obtained by the Kojdecki method $[6,7]$. In this method several experimental peaks (separated or partially overlapping in groups of two or three) are selected for further simultaneous analysis. Computations are repeated with several models of crystallites (prism, cylinder and sphere) to achieve approximately the smallest-possible discrepancy between a system of experimental line profiles and a corresponding system of line profiles simulated with refined model parameters. The discrepancy is computed, for each model of crystallite, using a weighted Euclidean norm.

A recent review of line profile analysis methods, including this one, is provided by Mittemeijer and Welzel [8].

SEM observations of fired samples, previously treated with HF $10 \%$, were made using an Hitachi S-4100 microscope.

\section{Results}

Figures 1-2 show examples of the obtained experimental analysed X-ray diffraction peaks for mullite from MGC and MGS samples.

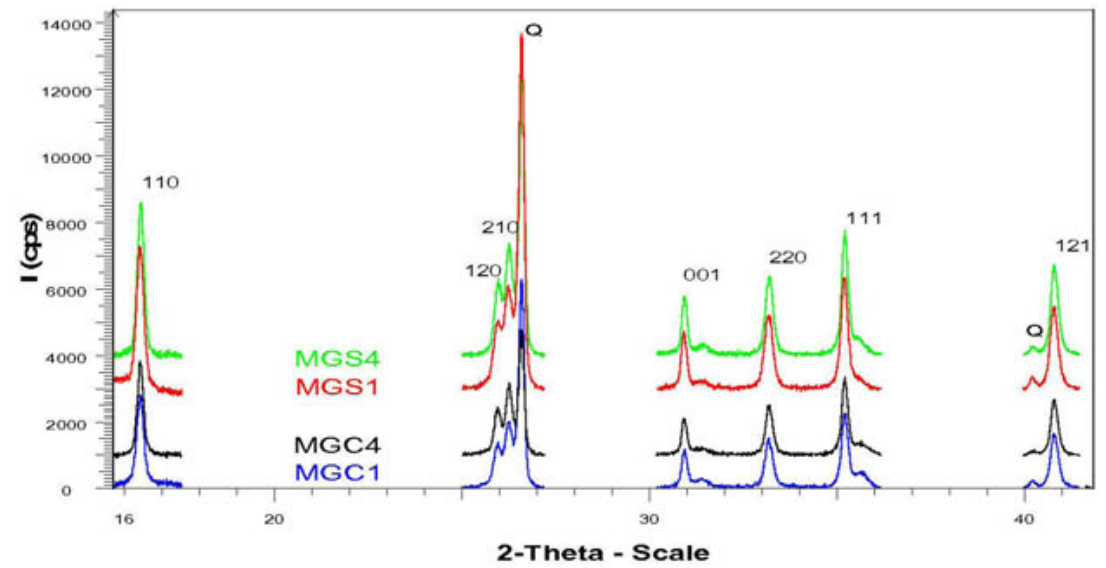

Figure 1. Studied X-ray diffraction peaks obtained from MGC1-4 and MGS1-4 samples in different firing cycles. 


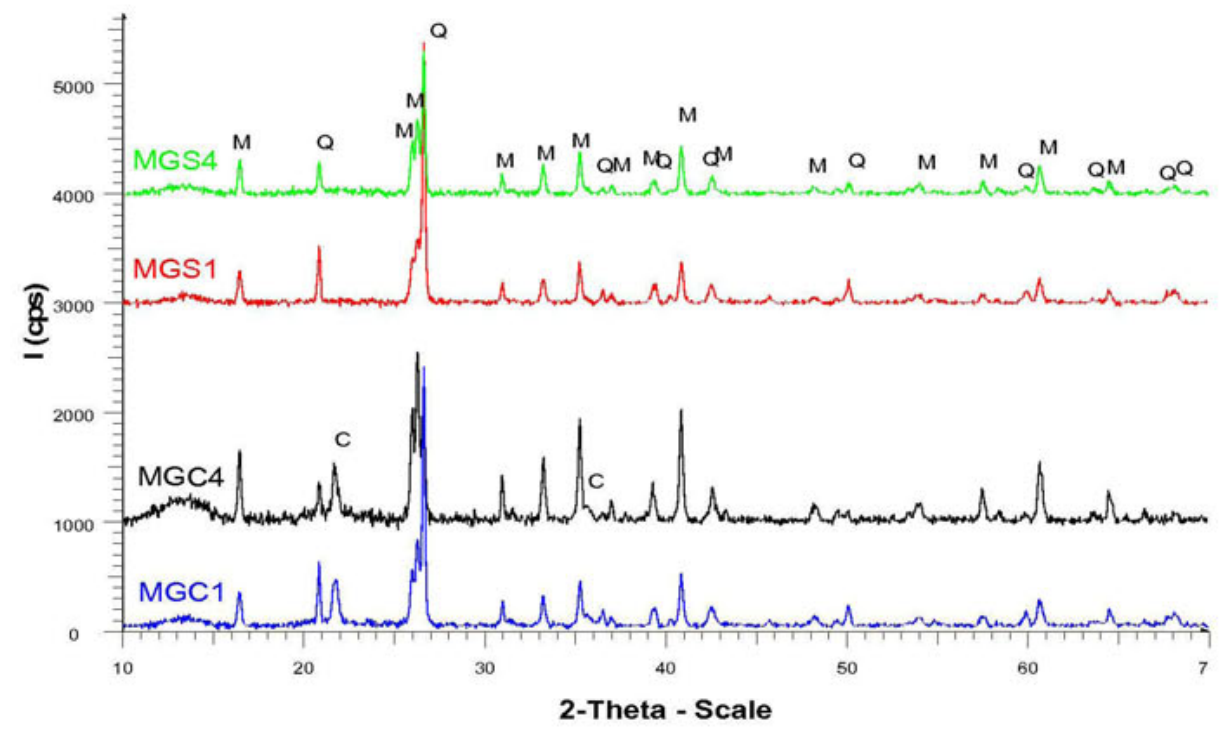

Figure 2. General powder X-ray diffractogram from MGC1-4 and MGS1-4 samples. M: mullite; Q: quartz; C: Cristobalite.

Table 4 includes the obtained shape and size data for modelization for prism crystallite shape. A and B are the dimensions of prism base and $\mathrm{C}$ is the height. So C/A means the relation of shape of prism. $\mathrm{V}^{1 / 3}$ is the standardized size and means the edge of the volume equivalent cube. It would be seen that parameter C/A is almost constant in MGS samples but not in MGC samples, where the higher size the higher C/A value. So there is a preferred crystallite growth from 1300 to $1330^{\circ} \mathrm{C}$ firings in the studied petalite containing formulations.

Other shapes evaluated by Kojdecki method (cylinder and sphere) have been refused because of their higher root mean square error [6] in calculated parameters.

Table 4. Parameters of size and shape for the prism approximation.

\begin{tabular}{|c|c|c|c|c|c|}
\hline Sample & $\left.\mathbf{A} \mathbf{( 1 0}^{-\mathbf{1 0}} \mathbf{m}\right)$ & $\mathbf{B}\left(\mathbf{1 0}^{-\mathbf{1 0}} \mathbf{m}\right)$ & $\left.\mathbf{C ~ ( 1 0 ^ { - 1 0 }} \mathbf{m}\right)$ & $\mathbf{C} / \mathbf{A}$ & $\mathbf{V}^{\mathbf{1 / 3}}$ \\
\hline MGC1 & 442 & 450 & 762 & 1,724 & 533 \\
\hline MGC2 & 387 & 395 & 1010 & 2,610 & 536 \\
\hline MGC3 & 488 & 496 & 1180 & 2,418 & 659 \\
\hline MGC4 & 690 & 704 & 2196 & 3,183 & 1022 \\
\hline MGS1 & 441 & 450 & 913 & 2,070 & 566 \\
\hline MGS2 & 428 & 436 & 836 & 1,953 & 538 \\
\hline MGS3 & 503 & 512 & 772 & 1,535 & 584 \\
\hline MGS4 & 414 & 422 & 753 & 1,819 & 508 \\
\hline
\end{tabular}


The difference of crystallite growth evolution between MGC and MGS compositions is in agreement with the observed difference concerning morphological evolution, where the greater development of mullite crystals is clearly shown in MGC samples at highest temperature, as shown in figure 3. In addition SEM pictures show the bigger size of crystals in MGC. As can be seen in figure 4, dimensions A and B of prisms show sizes similar to those calculated by Kojdecki method (table 4) for crystallites.

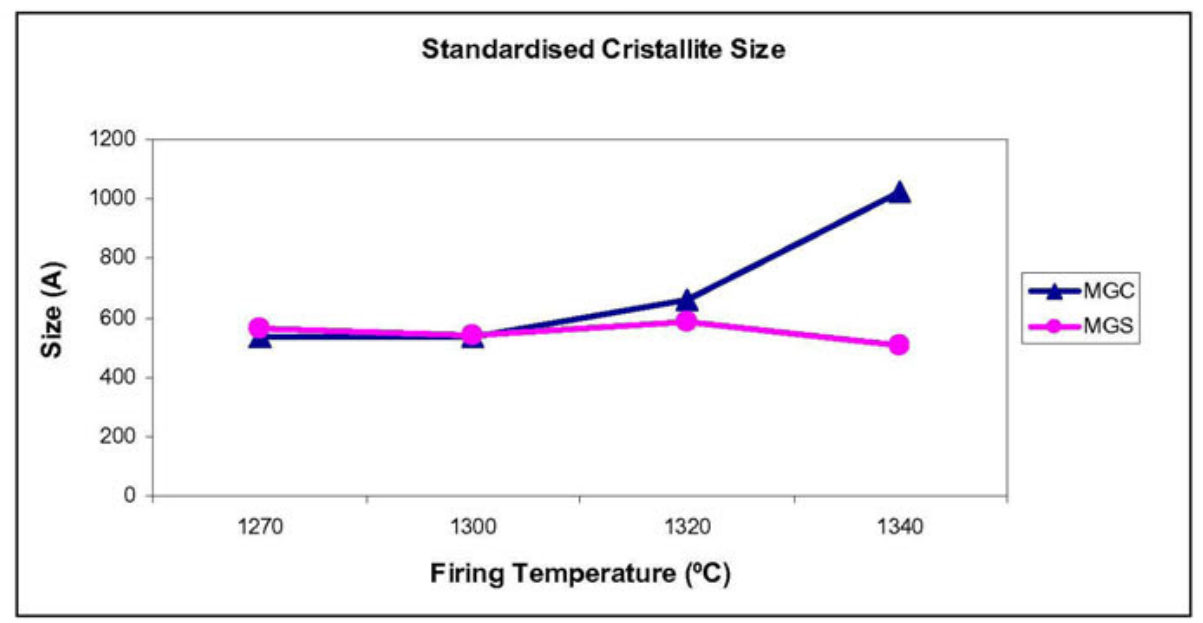

Figure 3. Standardized crystallite size $\left(V^{1 / 3}\right)$.
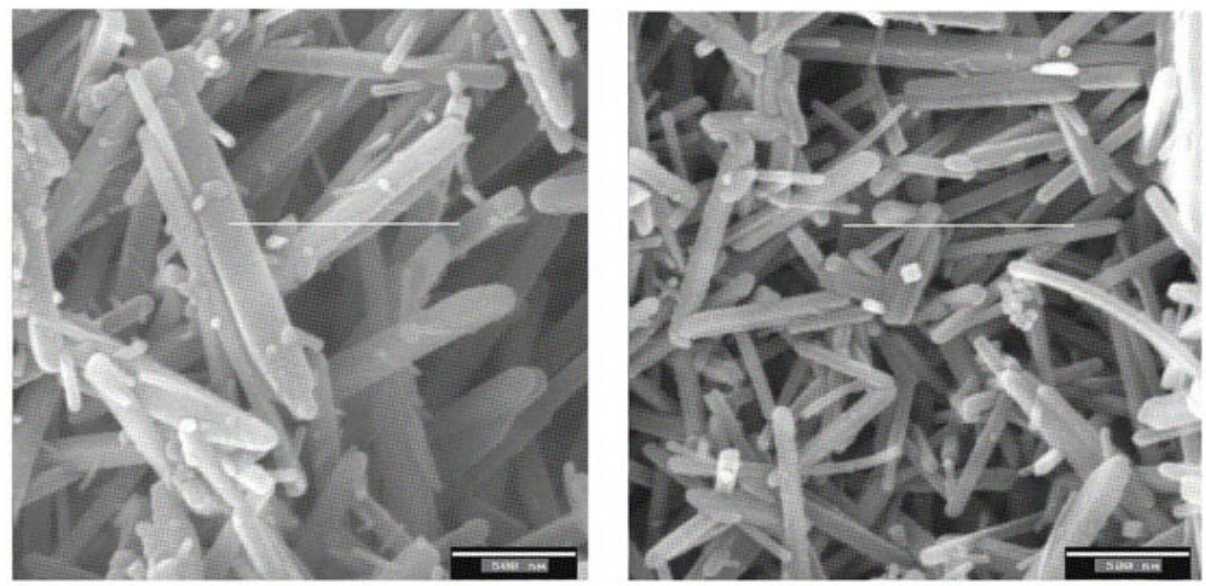

Figure 4. MGC4 (left) and MGS4 (right) SEM images. 


\section{Conclusions}

There is a significant difference in crystallite growth between MGC and MGS samples as firing temperature increases. These values show the effect of petalite addition instead of potash feldspar. In samples obtained from potash feldspar formulation (MGS) there is no size growth for mullite crystallites as firing temperature increases, while in samples from petalite formulations the higher temperature the bigger size of mullite crystallites have been produced. The different conditions of flux with petalite, due to total alkaline content, make possible the crystallite evolution with temperature.

Moreover different features have been found for crystallite growth in this case (high flux content) at the highest temperature respect to that found in simple sintering, with clear decrease for $\mathrm{C} / \mathrm{A}$ at $1300^{\circ} \mathrm{C}$ [9], or reactive sintering where slight increases are found for mullites obtained from $3: 2$ gels in the range $1300-1500^{\circ} \mathrm{C}[10]$.

\section{References}

1. Norton, F.H., 1988, Fine Ceramics:Technology and Applications (New York: McGraw-Hill Inc.).

2. Kingery, W.D., 1976, Introduction to Ceramics (New York: Wiley).

3. Dinsdale, A., 1986, Pottery Science (Ed. Ellis Horwood Ltd).

4. Carty, W.M. \& Sanapati, U., 1988, J. Am. Ceram. Soc., 81, (1), 3.

5. Singer, F., 1963, Industrial Ceramics (Ed. Springer).

6. Kojdecki, M.A., 2001, Computational test of effective algorithms, Mater. Sci. Forum, 378-381, 12.

7. Kojdecki, M.A., 2004, approximate estimations of contributions to pure X-ray diffraction line profiles from crystallite sizes, shapes and strains by analysing peaks widths, Mater. Sci. Forum, 443-444, 107.

8. Mittemeijer, E.J. \& Welzel, U., 2008, "The "state of the art" of the diffraction analysis of crystallite size and lattice strain", Z. Kristallogr., 223, 552.

9. Kojdecki, M.A., Bastida, J., Serrano, F.J. \& Clausell, J.V., 2001, "Sizes and shapes of crystallites in mullites produced by thermal treatment of a Kaolin - Alumina mixture", Mater. Sci. Forum, 378-381, 747.

10. Kojdecki, M.A., Ruiz de Sola E., Serrano F.J., Delgado Pinar V., Raventós M., \& Esteve V.J., 2007, "Microstructural evolution of mullites produced from singlephase gels", J. Appl. Cryst., 40, 260.

Acknowledgements. This research was supported by the Generalitat Valenciana project: No GV02-527. 


\title{
Crystallite size of kaolinites as indicator of different geochemical types of bauxite
}

\author{
G. Cozzi ${ }^{1}$, J. Bastida ${ }^{2, *}$, A. Álvarez-Larena ${ }^{3}$, \\ M. A. Kojdecki ${ }^{4}$, P. Pardo ${ }^{2,5}$
}

${ }^{1}$ Servicio Geológico Minero Argentino (SEGEMAR). (1650) San Martín, Buenos Aires, Argentina.

${ }^{2}$ Departamento de Geologia, Universidad de Valencia. 46100 Burjasot (Valencia), Spain

${ }^{3}$ Servicio de Difracción de Rayos X, Universidad Autónoma de Barcelona. 08193 Cerdanyola del Vallès (Barcelona), Spain.

${ }^{4}$ Instytut Matematyki i Kryptologii, Wojskowa Akademia Techniczna. 00-908 Warszawa, Poland

${ }^{5}$ Instituto de Ciencia de Materiales. Universidad de Valencia. 46980 Paterna (Valencia), Spain

•bastida@uv.es

Keywords: Kaolinite, crystallite size, strain, bauxite, Voigt function

\begin{abstract}
A simple microstructural X-ray diffraction analysis using the Voigt function method reveals differences between bauxite deposits in the Iberian Range - Catalanides area (NE, Spain). The highest values of apparent crystallite sizes, measured by volume weighted Dv values from 001 kaolinite reflection, have been found in bauxites of type 1 .
\end{abstract}

\section{Introduction and materials}

Classification of Iberian and Catalanides Ranges (NE, Spain) bauxites in four types was firstly stated by Combes (1969). These bauxitic rocks are made mainly of kaolinite, iron oxides, and incidentally aluminium hydroxides. They were formed from clayey mother rocks of the early Cretaceous by a ferrallitic "in situ" alteration of type 2 and type 3 bauxites, with an occasional additional karstic evolution in allocthonous deposits of type 1 bauxites and a subsequent sedimentary reworking in type 4 bauxites [1]. This classification and ore model were basically maintained in subsequent papers referred in [2].

Apparent crystallite size values (from the Scherrer formula) for kaolinites in bauxites from NE Spain were found in the ranges 500-1430, 800-1600 and 714-1500 $\AA$ for the Pyrenees, Catalanides and Maestrazgo areas, respectively [3], showing maximum and minimum values for the Catalanides and the Pyrenees. The called type 1 bauxites were formed from the less evoluted 2 and 3 types, according to geochemical considerations [4]. Type 1 bauxites correspond to bauxites "sensu stricto", according to Ki geochemical parameter of Bardossy [5] and their powder patterns show the lowest FWHM values [9]. The Voigt function simplified method of microstructural analysis by powder X-ray diffraction [6] as shown in [7] has been used previously to analyze reference kaolinite samples [8,9]. Apparent 
crystallite sizes obtained by this method for 001 reflection of kaolinite were compared to results of high magnification FESEM measurements of crystallite size in kaolinites of the Iberian Massif (Spain), and a quite good correlation $\left(\mathrm{r}^{2}=0.9246\right)$ was found [10]. Significant differences in FWHM values of 001 reflection of kaolinites from different bauxite types of NE Spain were observed [9]. The Voigt function method is used here to obtain results from kaolinites of different genetic systems in bauxites from NE Spain.

\section{Methods}

Mineralogical analysis of whole rocks was obtained using back pressed powdered bauxite samples $(<50 \mu \mathrm{m})$ of the studied whole samples. A Philips XPERT diffractometer (PW3020, with $\mathrm{Cu}$ anode, graphite monochromator, working at $40 \mathrm{kV}, 50 \mathrm{~mA}$, and sample holder spinning) was used for mineralogical analysis of whole rock sample and for microstructural analysis of kaolinite 001 reflections on oriented aggregates of the clay fraction $(<2 \mu \mathrm{m}$, separated by decantation of a suspension of the powdered $<50 \mu \mathrm{m}$ material and drying on a glass slide holder).

Used primary optics: 0.04 rad Soller slits, $1^{\circ}$ divergence slit, $15 \mathrm{~mm}$ beam mask. Secondary optics: $1^{\circ}$ anti-scatter slit; $0.10 \mathrm{~mm}$ receiving slit and 0.04 rad Soller slit, graphite monochromator and scintillation counter. Data collection was performed by step seanning using $0.02^{\circ} 2 \theta$ step size and $1 \mathrm{~s}$ counting time from 5 to $65^{\circ} 2 \theta$ for the general identification and the same step $0.02^{\circ} 2 \theta$ and $20 \mathrm{~s}$ counting time from 11 to $13.2^{\circ} 2 \theta$ for microstructural analysis.

Instrumental line profiles were obtained from the $\mathrm{LaB}_{6}$ standard (NIST SRM660a) using the same experimental conditions. The microstructural analysis of the 001 reflections was performed with the simplified method of the Voigt function [7]. The 001 pure line profile was derived by deconvolution of the instrumental profile from the experimental one, and the fitting carried out with the Winfit software available at http://www.ccp14.ac.uk/ ccp/ccp14/ftp-mirror/krumm /Software/. Estimation of contents of the different minerals in whole rock samples was obtained by the reference intensity method [11] implemented in the High Score Plus v. 2.0a package of PANalytical.

Image collection and measurement of crystallite thicknesses of kaolinites were performed by means of a field emission scanning electron microscope (FESEM) Hitachi 4100 employing the method for sample preparation and crystallite thickness measurement provided by the above referred method [10] working with an accelerating voltage of $30 \mathrm{kV}$. The microstructural study was carried out analysing the 001 peak of kaolinite. Other higher order peaks of kaolinite suffer from severe overlapping (figure 1).

\section{Results}

Only the mineralogical compositions of the two samples of type 4 bauxite are clearly different from the rest (low kaolinite and high calcite contents) (table 1). On the other hand, the microstructural analysis (table 2) gets a better discrimination between type 1 and the other types (figure 2). Simultaneous increase of kaolinite content and associated "crystallinity" is frequently but not necessarily found (see samples 11 and 12 of type 2 in figure 2). Samples of type 1 have greater crystallite sizes except for the sample Nr. 5 (figure 3). In this case the smaller size is related to the pisolithic texture of the sample that contains 


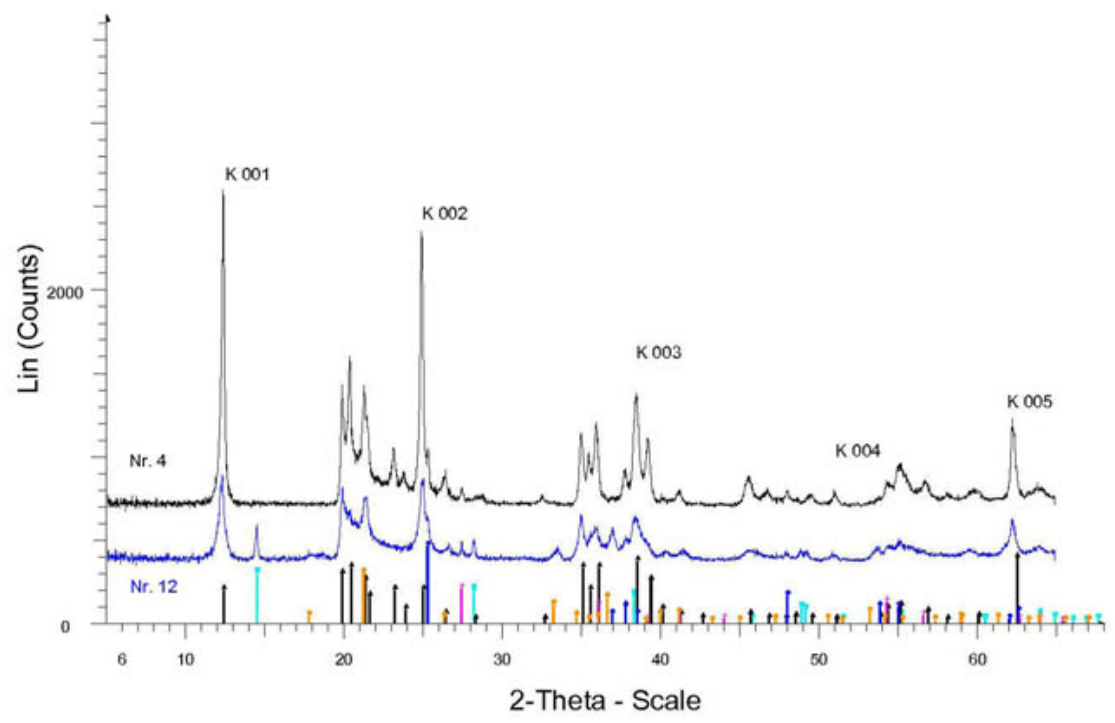

Figure 1. XRD patterns of whole rocks (samples 4 and 13) with (00l) kaolinite peaks labeled. Potential minerals present in samples according to Table 1 are included as bars; kaolinite - black triangle, bohemite - blue square, rutile - magenta cross, anatase - blue diamond, goethite-orange circle.

some allochtonous type 3 material, not evolved. Results from FESEM images (figure 4) are correlated with those obtained by X-ray diffraction, according to previous results observed for other samples [14]. For example, the sizes determined by X-ray diffraction and by FESEM are, for sample Nr. 1, 55 and $41 \mathrm{~nm}$, and, for sample Nr.14, 25 and $18 \mathrm{~nm}$, respectively. Concerning the strain, the greatest values are observed for samples of types 2 and 3 . However, this behaviour can not be established as usual, since some samples (10, 12, 13 , and 21) show values as low as those of type 1 samples.

\section{Concluding remarks}

The diffraction analysis of the microstructure of materials shows a continuous growing in tools and in scope. New and more powerful methods are being developed and more complicated problems are approached. However, sometimes older and simpler procedures are effective. In this paper, the microstructural analysis carried out with the Voigt method using only the 001 reflection of the kaolinite allows to discriminate different geochemical types of bauxite in powdered whole samples, where no clear mineralogical differences were found. The greater apparent crystallite size in kaolinite for the type 1 of bauxite is related to a longer crystallite growth during the ferrallitic alteration and subsequent processes, in agreement with the geological features of the bauxite deposits. On the other hand, the higher values for the e strain parameter have been generally found for bauxite types 2 and 3 . 


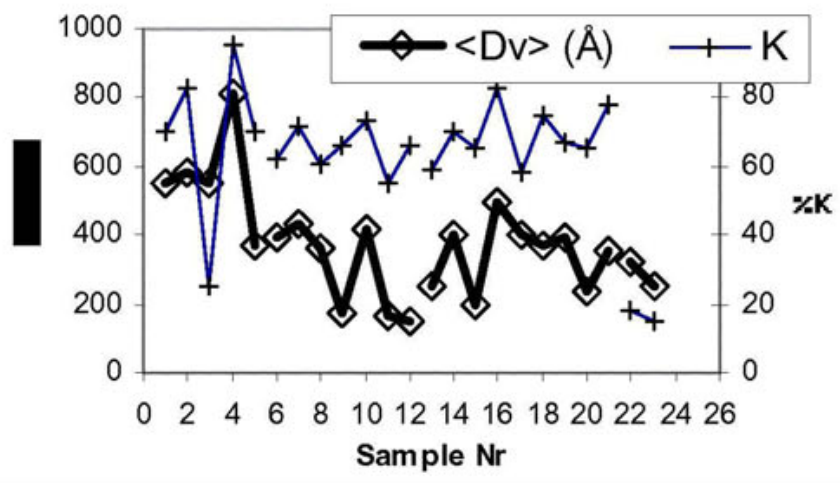

Figure 2. Contents in whole rocks and $\langle D v>$ values for kaolinite.

Table 1. Mineralogical normalized analysis (\%). Mineral codes: $K=$ kaolinite, $I /$ Mi $=$ illite-muscovite, Gib $=$ gibbsite, Dia =diaspore, Boh=boehmite, $\mathrm{He}=$ hematite, $\mathrm{Mg}=$ magnetite, Go $=$ goehtite, $\mathrm{Ru}=$ rutile, Ana = anatase, $C a=$ calcite,$Q z=$ quartz, ? = traces of other components: $c=$ chlorites, $s=$ smectites.

\begin{tabular}{|c|c|c|c|c|c|c|c|c|c|c|c|c|c|c|}
\hline $\begin{array}{c}\text { Sample } \\
\mathrm{Nr}\end{array}$ & Type & K & $\mathrm{I} / \mathrm{Mi}$ & Gib & Dia & Boh & $\mathrm{He}$ & Mgn & Goe & $\mathrm{Ru}$ & Ana & $\mathrm{Ca}$ & Qz & Others \\
\hline 1 & \multirow{5}{*}{1} & 70 & & & & & 7 & 1 & 9 & 4 & 3 & 6 & & \\
\hline 2 & & \begin{tabular}{|l|}
83 \\
\end{tabular} & & & & & 5 & & 6 & 3 & 3 & & & \\
\hline 3 & & \begin{tabular}{|l|}
25 \\
\end{tabular} & & 53 & 8 & 4 & 5 & & & 2 & 2 & 1 & & \\
\hline 4 & & 95 & & & & & & & & 2 & 3 & & & \\
\hline 5 & & 70 & & & & & 10 & & 11 & 4 & 5 & & & \\
\hline 6 & \multirow{7}{*}{2} & \begin{tabular}{|l|}
62 \\
\end{tabular} & & 9 & & 4 & 10 & & 7 & 4 & 4 & & & \\
\hline 7 & & \begin{tabular}{|l|}
72 \\
\end{tabular} & & & & & 6 & & 15 & 3 & 4 & & & \\
\hline 8 & & \begin{tabular}{|l|}
61 \\
\end{tabular} & & & & & 9 & & 18 & 4 & 8 & & & \\
\hline 9 & & \begin{tabular}{|l|}
66 \\
\end{tabular} & & 10 & & & 7 & & 9 & 3 & 5 & & & \\
\hline 10 & & \begin{tabular}{|l|}
73 \\
\end{tabular} & & & & & 5 & & 12 & 4 & 6 & & & \\
\hline 11 & & \begin{tabular}{|l|}
55 \\
\end{tabular} & & & & 10 & 17 & & 9 & 4 & 5 & & & \\
\hline 12 & & \begin{tabular}{|l|}
66 \\
\end{tabular} & & & & 12 & & & 13 & 3 & 6 & & & \\
\hline 13 & \multirow{9}{*}{3} & 70 & & & & & 5 & & 15 & 5 & 5 & & & s? \\
\hline 14 & & \begin{tabular}{|l|}
59 \\
\end{tabular} & & & 14 & & 9 & & 11 & 3 & 4 & & & \\
\hline 15 & & \begin{tabular}{|l|}
65 \\
\end{tabular} & & & & & 8 & & 16 & 5 & 6 & & & s?, c? \\
\hline 16 & & \begin{tabular}{|l|}
83 \\
\end{tabular} & & & & & & & 8 & 4 & 5 & & & $\mathrm{~s} ?, \mathrm{c} ?$ \\
\hline 17 & & \begin{tabular}{|l|}
58 \\
\end{tabular} & & & & & 21 & & 12 & 5 & 4 & & & \\
\hline 18 & & \begin{tabular}{|l|}
75 \\
\end{tabular} & & & & & 9 & & 8 & 4 & 4 & & & \\
\hline 19 & & \begin{tabular}{|l|}
67 \\
\end{tabular} & & & & & 11 & & 13 & 4 & 5 & & & s? \\
\hline 20 & & \begin{tabular}{|l|}
65 \\
\end{tabular} & & & & & 16 & & 12 & 3 & 4 & & & \\
\hline 21 & & \begin{tabular}{|l|}
78 \\
\end{tabular} & & & & & 4 & & 10 & 4 & 4 & & & \\
\hline 22 & \multirow{2}{*}{4} & 18 & & & & & & & 3 & 4 & $<1$ & 65 & 10 & \\
\hline 23 & & 15 & 5 & & & & & & 3 & 4 & 1 & 72 & & \\
\hline
\end{tabular}


Table 2. Microstructural analysis of 001 reflection of kaolinite. $R \%=$ residual of profile fitting, $2 \theta=$ position $\left({ }^{\circ} 2 \theta\right), N=$ normalised peak area $\left(\right.$ cps $\left.^{\circ}{ }^{\circ} 2 \theta\right), H=$ height of the peak (cps), FWHM = full width at half maximum $(2 \theta), \beta=$ integral breadth $(2 \theta), \phi=$ line aspect ratio, $\langle D v\rangle=$ apparent volumeweigthed crystallite size $(\AA), e=$ strain.

\begin{tabular}{|c|c|c|c|c|c|c|c|c|c|c|}
\hline $\begin{array}{c}\text { Sample } \\
\mathrm{Nr}\end{array}$ & Type & $\mathrm{R} \%$ & $2 \theta$ & $\mathrm{N}$ & $\mathrm{H}$ & FWHM & $\beta$ & $\phi$ & $<\mathrm{Dv}\rangle$ & e \\
\hline 1 & \multirow{5}{*}{1} & 91,87 & 12,413 & 7001 & 23691 & 0,218 & 0,296 & 0,73648 & 553 & 0,0055 \\
\hline 2 & & 94,09 & 12,411 & 7418 & 33579 & 0,148 & 0,221 & 0,66968 & 586 & 0,0019 \\
\hline 3 & & 95,15 & 12,396 & 1561 & 5871 & 0,189 & 0,266 & 0,71052 & 553 & 0,0041 \\
\hline 4 & & 90,98 & 12,418 & 7467 & 29309 & 0,195 & 0,255 & 0,76470 & 815 & 0,0055 \\
\hline 5 & & 95,95 & 12,306 & 5671 & 11546 & 0,38 & 0,491 & 0,77393 & 371 & 0,0115 \\
\hline 6 & \multirow{7}{*}{2} & 95,11 & 12,321 & 8132 & 16635 & 0,382 & 0,489 & 0,78118 & 391 & 0,0118 \\
\hline 7 & & 95,37 & 12,324 & 5161 & 11200 & 0,362 & 0,461 & 0,78524 & 433 & 0,0113 \\
\hline 8 & & 95,65 & 12,311 & 6773 & 12958 & 0,409 & 0,523 & 0,78202 & 363 & 0,0127 \\
\hline 9 & & 95,72 & 12,314 & 7888 & 13400 & 0,383 & 0,589 & 0,65025 & 172 & 0,0037 \\
\hline 10 & & 95,93 & 12,356 & 6447 & 12991 & 0,393 & 0,496 & 0,79233 & 417 & 0,0125 \\
\hline 11 & & 97,58 & 12,322 & 3338 & 5294 & 0,413 & 0,631 & 0,65451 & 162 & 0,0046 \\
\hline 12 & & 97,88 & 12,303 & 4306 & 6515 & 0,428 & 0,661 & 0,64750 & 150 & 0,0037 \\
\hline 13 & \multirow{9}{*}{3} & 95,83 & 12,317 & 6228 & 12406 & 0,396 & 0,502 & 0,78884 & 401 & 0,0125 \\
\hline 14 & & 97,24 & 12,302 & 3500 & 6503 & 0,388 & 0,538 & 0,72118 & 249 & 0,0094 \\
\hline 15 & & 97,5 & 12,317 & 8216 & 14401 & 0,388 & 0,571 & 0,679509 & 197 & 0,0068 \\
\hline 16 & & 95,58 & 12,329 & 4507 & 9686 & 0,374 & 0,465 & 0,804301 & 499 & 0,0123 \\
\hline 17 & & 96,16 & 12,317 & 4580 & 8934 & 0,407 & 0,513 & 0,793372 & 404 & 0,0130 \\
\hline 18 & & 96,12 & 12,348 & 4699 & 9870 & 0,365 & 0,476 & 0,766806 & 367 & 0,0107 \\
\hline 19 & & 95,97 & 12,312 & 7245 & 13745 & 0,419 & 0,527 & 0,795066 & 396 & 0,0135 \\
\hline 20 & & 98,06 & 12,33 & 4712 & 9659 & 0,331 & 0,488 & 0,678278 & 233 & 0,0057 \\
\hline 21 & & 96,02 & 12,35 & 5297 & 11866 & 0,334 & 0,446 & 0,748878 & 357 & 0,0092 \\
\hline 22 & \multirow{2}{*}{4} & 96,49 & 12,314 & 6631 & 11808 & 0,436 & 0,562 & 0,775800 & 320 & 0,0133 \\
\hline 23 & & 97,56 & 12,324 & 3109 & 5515 & 0,414 & 0,564 & 0,734042 & 251 & 0,0107 \\
\hline
\end{tabular}

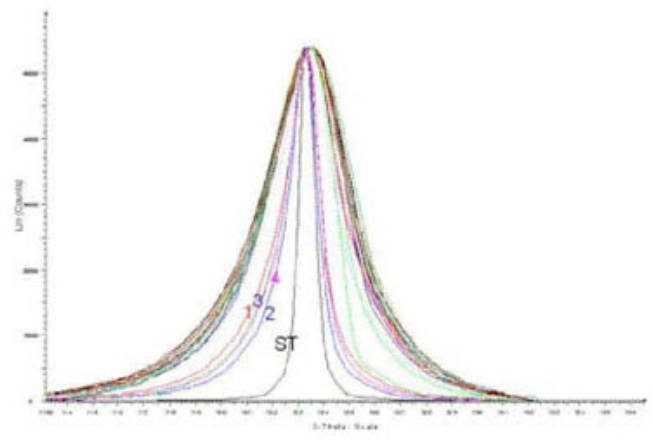

Figure 3. XRD patterns of 001 kaolinite peaks from clay $(<2 \mu \mathrm{m})$ fractions (height normalized representation). 

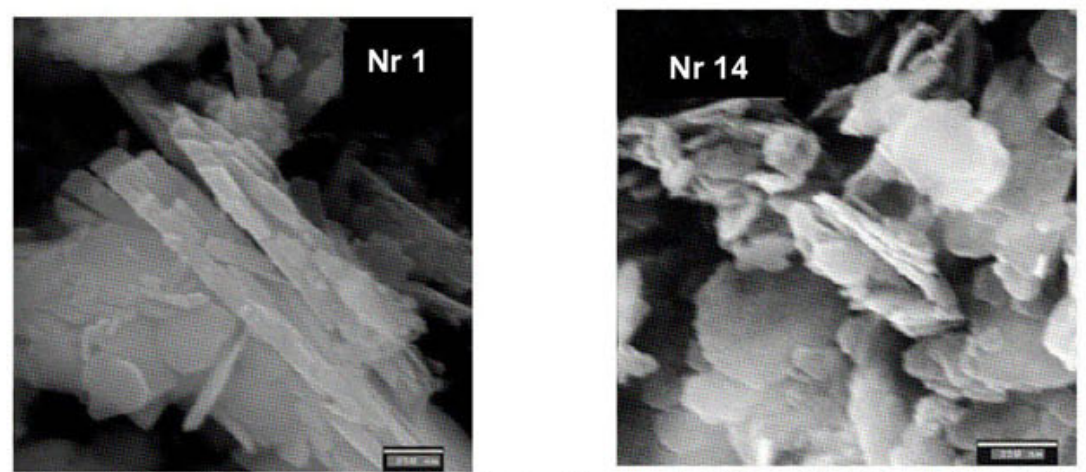

Figure 4. FESEM images at high magnification for samples $N^{\circ} 1$ and $N^{\circ} 14$ (scale bar is $250 \mathrm{~nm}$ ).

\section{References}

1. Combes, P.J., 1969, Recherches sur la genése des bauxites dans le Nord-Est de l'Espagne, Le Languedoc et L'Ariége (France), Tesis Doctoral, Univ. Montpellier, Mém. Centre d'Etudes et Rech. Geol. et Hydrogéol, Montpellier.

2. Cozzi, G., Bastida, J., Alvarez Larena, A., Martinez, S. \& Pardo, P., 2005, GeoTemas, $8,42$.

3. La Iglesia, A. \& Ordoñez, S., 1990, Bol. Soc. Españ. Mineral, 13, 81.

4. Ordóñez, S., Fort, R. \& Bustillo, M., 1990, Estudios Geol., 46, 373.

5. Valeton, I., 1972, Bauxites. Developments in Soil Science, 2 (Amsterdam: Elsevier).

6. Langford, J.I., 1978, J. Appl. Cryst., 11, 10.

7. De Keijser, T.H., Mittemeijer, E.J. \& Rozendaal, H.C.F., 1983, J. App. Cryst., 16, 309.

8. Serrano, F.J., Sanz, A., Esteve, V., Bastida, J. \& Amigo, J.M., 1995, XRD line broadening of kaolinites from Teruel (Spain), in Applied Crystallography, (Morawiec \& Strotz, Editors) (Praga: World Scientfic Press), pp. 367-371.

9. Pardo, P., Bastida, J., Kojdecki, M., Ibañez, R. \& Zbick, M., 2007, Z. Kristallogr. Suppl., 26, 549.

10. Clausell, J.V., Bastida, J., Serrano, J., Pardo, P. \& Huertas, F.J., 2007, App. Clay Sci., 37, 127.

11. Hubbard, C.R. \& Snyder, R., 1988, RIR, Powder Diffr., 3, (2), 74.

Acknowledgements. Supported by GV02-52 project (Generalitat Valenciana, Spain) and ISSB project (IP 026661, NMP2-CT-2006-026661, EEC $6^{\text {th }}$ F.) 


\section{Discrimination of bassanite and anhydrite III dehydrated from gypsum at different temperatures}

\section{S. Seufert", C. Hesse, F. Goetz-Neunhoeffer, J. Neubauer}

Geozentrum Nordbayern, Mineralogy, University of Erlangen-Nuernberg, Schlossgarten 5a, 91054 Erlangen, Germany

* sese(a)geol.uni-erlangen.de

Keywords: OPC, sulphate carrier, Rietveld refinement

Abstract. Technical OPC normally contains mixed sulphate carriers in varying amounts. Gypsum (calcium sulphate dihydrate, $\mathrm{CaSO}_{4} \cdot 2 \mathrm{H}_{2} \mathrm{O}$ ) is added to the clinker before the milling process and dehydrates partially to bassanite (calcium sulphate hemihydrate, $\mathrm{CaSO}_{4} \cdot 0.5$ $\left.\mathrm{H}_{2} \mathrm{O}\right)$ and anhydrite $\left(\mathrm{CaSO}_{4}\right)$. The dehydration temperature and humidity affects the resulting calcium sulphates. Anhydrite exists in different polymorphs (anhydrite I-III) of which anhydrite III shows the highest reactivity in contact with $\mathrm{H}_{2} \mathrm{O}$. Due to different kinetics of the calcium sulphate phases, which strongly influence hydration, it is very important to be able to characterize the sulphate carrier composition in the cement and in applications with high sulphate content. This study could prove that it is possible to quantify bassanite and anhydrite III in samples of dehydrated gypsum by Rietveld refinement of XRD data especially in mixtures consisting of more than one calcium sulphate modification.

\section{Introduction}

Bassanite as well as anhydrite III are metastable phases in the system $\mathrm{CaSO}_{4}-\mathrm{H}_{2} \mathrm{O}$ [1]. Figure 1 shows the similarity of the crystal structures of bassanite and anhydrite III described by Bezou. Both structures consist of a crystal frame of $\mathrm{Ca}^{2+}$ ions and $\mathrm{SO}_{4}{ }^{2-}$ tetrahedral with channels of $0.2 \mathrm{~nm}$ diameters. Those channels are partially filled with water in case of bassanite and empty in case of anhydrite III [2]. Bassanite is formed from gypsum at temperatures from $45^{\circ} \mathrm{C}$ to $200{ }^{\circ} \mathrm{C}$ due to the loss of 1.5 mole $\mathrm{H}_{2} \mathrm{O}$ per formula unit. Under ambient conditions exclusively the $\beta$-modifications of bassanite will be formed. Different polymorphic modifications of bassanite $(\alpha, \beta, \beta)$ depending on synthesis conditions were reported [3]. 

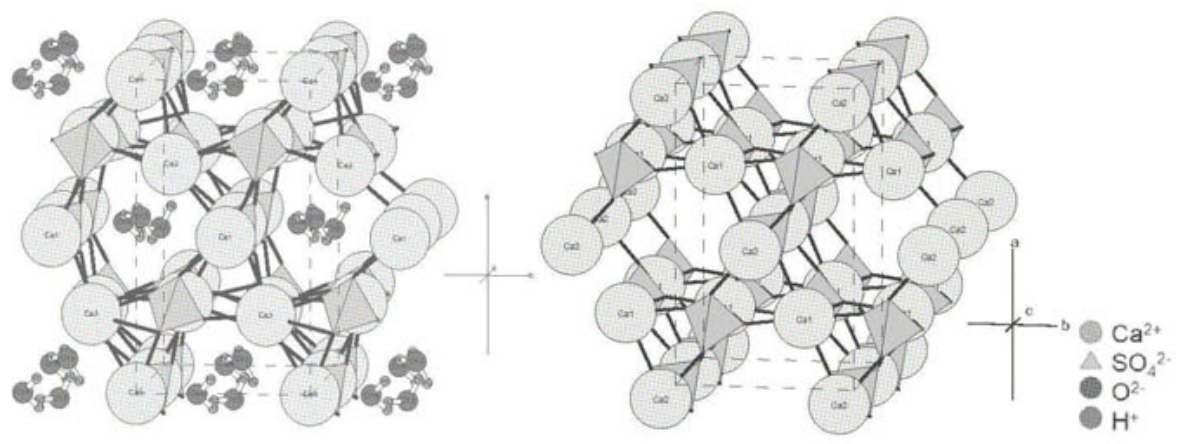

Figure 1. Bassanite (left) and anhydrite III (right) structure [2].

Heating at ambient conditions above temperatures of $100{ }^{\circ} \mathrm{C}$ leads to a loss of the remaining 0.5 mole $\mathrm{H}_{2} \mathrm{O}$ per formula unit and therefore to the formation of $\mathrm{H}_{2} \mathrm{O}$-free $\beta$-anhydrite III [4]. In the present investigations thermogravimetric methods and XRD techniques using the Rietveld method were combined to carry out an accurate discrimination between the $\beta$ modifications of bassanite and anhydrite III that may be used in dry mortars. Objective was the setup of an analytical method for use in quality control during production of cements and sulphates.

\section{Materials and methods}

The dehydration of high purity gypsum (calcium sulphate dihydrate, Fluka, $99.9 \%$ purity) was performed in chamber furnaces at $85^{\circ} \mathrm{C}$ up to $550{ }^{\circ} \mathrm{C}$ in air. The initial high purity gypsum was weighed in corundum crucibles of $60 \mathrm{ml}$ size and dehydrated for 16 to 120 hours (dehydration time was dependant on dehydration temperature see table 1).

Table 1. Sample names, dehydration time and dehydration temperature.

\begin{tabular}{|l|l|l|}
\hline Sample Name & Time of dehydration & Temperature of dehydration \\
\hline Gypsum_85 & $120 \mathrm{~h}$ & $85( \pm 5)^{\circ} \mathrm{C}$ \\
\hline Gypsum_120 & $120 \mathrm{~h}$ & $120( \pm 5)^{\circ} \mathrm{C}$ \\
\hline Gypsum_180 & $16 \mathrm{~h}$ & $180( \pm 5)^{\circ} \mathrm{C}$ \\
\hline Gypsum_250 & $16 \mathrm{~h}$ & $250( \pm 5)^{\circ} \mathrm{C}$ \\
\hline Gypsum_380 & $16 \mathrm{~h}$ & $380( \pm 5)^{\circ} \mathrm{C}$ \\
\hline Gypsum_550 & $16 \mathrm{~h}$ & $550( \pm 5)^{\circ} \mathrm{C}$ \\
\hline
\end{tabular}

For the dehydrated gypsum samples the loss on ignition was determined. To determine the experimental loss on ignition a reference gypsum sample was dehydrated at $800{ }^{\circ} \mathrm{C}$ and weighed out. The resulting value for the experimental loss on ignition value was $20.7 \mathrm{wt} \%$. With this value all samples dehydrated at lower temperatures were corrected. Assuming that at temperatures of $85^{\circ} \mathrm{C}$ and above the $\mathrm{H}_{2} \mathrm{O}$ is chemically bound to calcium sulphate hemi- 
hydrate (with exactly 0.5 mole water), the amount of bassanite in the dehydrated samples could be recalculated by using equation (1).

$$
m_{\text {Bassanite }}=m_{\mathrm{H}_{2} \mathrm{O}}+m_{\mathrm{H}_{2} \mathrm{O}} \cdot \frac{M\left(\mathrm{CaSO}_{4}\right)}{0.5 \mathrm{M}\left(\mathrm{H}_{2} \mathrm{O}\right)}
$$

$m_{\mathrm{H}_{2} \mathrm{O}}=$ Mass of tared chemically bound $\mathrm{H}_{2} \mathrm{O}$ in bassanite

$m_{\mathrm{H}_{2} \mathrm{O}} \cdot \frac{\mathrm{M}\left(\mathrm{CaSO}_{4}\right)}{0.5 \mathrm{M}\left(\mathrm{H}_{2} \mathrm{O}\right)}=$ Mass of chemically bound calcium sulphate in Bassanite

$\mathrm{M}\left(\mathrm{CaSO}_{4}\right)=136.1576 \mathrm{~g} / \mathrm{mole} ; 0.5 \mathrm{M}\left(\mathrm{H}_{2} \mathrm{O}\right)=9.00767 \mathrm{~g} / \mathrm{mole}$

To avoid any reaction of the dehydrated gypsum samples with air humidity, the powders were instantly transferred to small press-on lid pots, sealed with parafilm and stored in an exiccator above silica gel. Preparation of the dehydrated gypsum samples for X-ray investigations was carried out at $7 \%$ relative humidity in a glove box. The sample was prepared in a D5000 sample holder and covered by Kapton brand X-ray film to prevent further transformation reactions (figure 2). The preparation was performed in the glove box to minimize reactions due to ambient humidity.

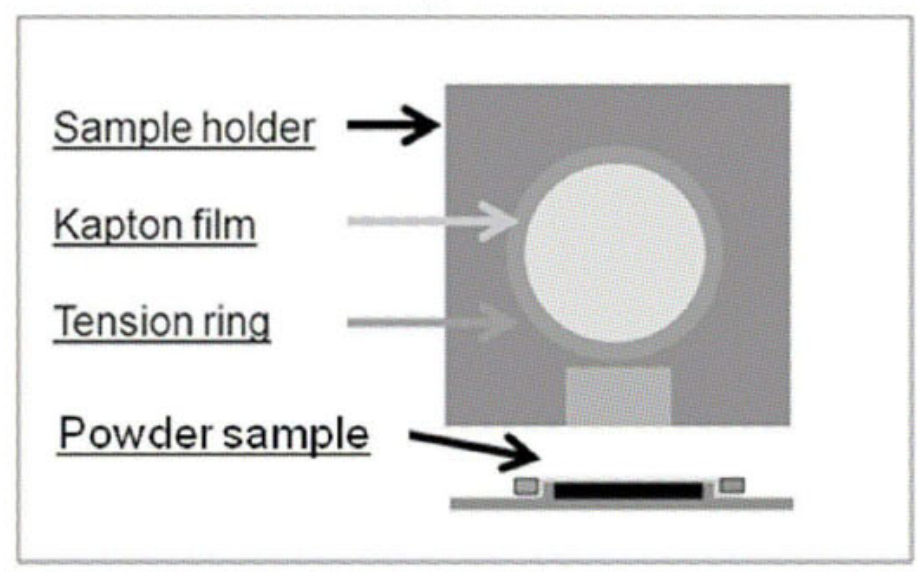

Figure 2. Scheme of D5000 sample holder covered with Kapton film.

Phase composition of the samples was examined at room temperature by quantitative $\mathrm{X}$-ray powder diffraction (XRPD) with a Siemens D5000 diffractometer with $\mathrm{CuK} \alpha . \mathrm{X}$-ray radiation, secondary monochromator and a scintillation detector. The scanning range was $10^{\circ}$ $60^{\circ}$ and the scanning time was 9 seconds in total. All samples were measured at an ambient temperature of $22^{\circ} \mathrm{C}\left( \pm 2{ }^{\circ} \mathrm{C}\right)$. Rietveld refinement was performed using the structural models (ICSD) of all occurring calcium sulphate phases and the TOPAS software (V 3.0 Bruker AXS). Additionally the structures data for bassanite and anyhdrite III from Christensen et al. [7] were applied. Table 2 shows the used structure data for Rietveld refinement. 
Table 2. Structures used for Rietveld refinement.

\begin{tabular}{|l|l|l|l|}
\hline Phase & ICDD-PDF-No. & ICSD-No. & Author [ref.] \\
\hline gypsum & $33-0311$ & 92567 & Schofield [5] \\
\hline bassanite & $41-0224$ & 79529 & Bezou et al. [2], Christensen et al. [7] \\
\hline anhydrite III & $41-0224$ & 79527 & Bezou et al. [2], Christensen et al. [7] \\
\hline anhydrite II & $37-1496$ & 16382 & Kirfel [6] \\
\hline
\end{tabular}

Refined parameters were: scale factor, zero displacement, background as Chebychev polynomial of 5th grade, crystallite size and lattice parameters for anhydrite III and anhydrite II. Lattice parameters of bassanite were kept fix in order to achieve stable refinement results. Especially in samples with a low bassanite content (calculated from loss on ignition) fixing of the lattice parameters of bassanite is crucial. Since Kapton film was used to cover the samples during measurement it was necessary to correct the background with a manually generated peak phase for the Kapton film.

\section{Results}

All investigated powders consisted of at least two different calcium sulphates. Small amounts of less reactive anhydrite II were found in all dehydrated gypsum samples even at lower temperatures. With the chosen refinement method it was possible to discriminate highly reactive anhydrite III from bassanite in diffraction patterns measured under applicationoriented conditions at room temperature. Figure 3 and 4 show the refined data set of gypsum dehydrated at $180^{\circ} \mathrm{C}$ with high anhydrite III content refined with different structures for bassanite and anhydrite III from two different authors [2,7]. The differences in Rietveld results by exchanging the structures of bassanite and anhydrite III respectively were smaller than the assumed error. In figure 3 and 4 the discrimination of the two phases is visible due to slightly different peak positions of bassanite and anhydrite III. 


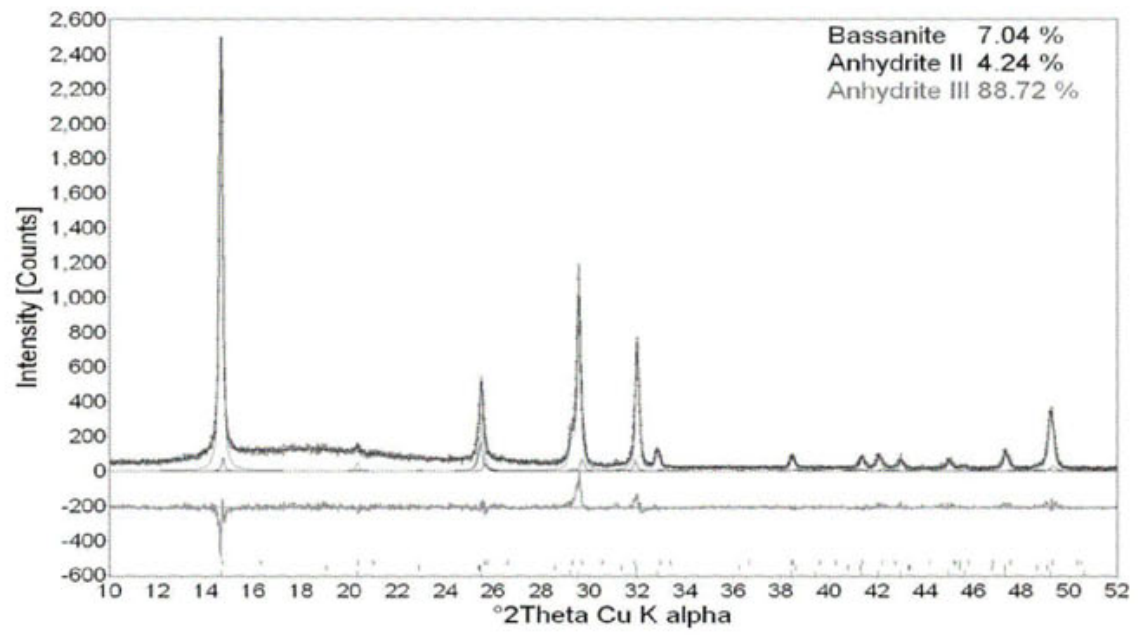

Figure 3. Rietveld refinement of dehydrated gypsum at $180^{\circ} \mathrm{C}$ with refined structures of Bassanite[7], Anyhdrite III [7] and Anhydrite II [6]. $R_{\text {Bragg-Bassanite }}=7.7 \%, R_{\text {Bragg-Anhydrite II }}=2.1 \%, R_{\text {Bragg-Anhydrite III }}$ $=7.6 \%, R_{w p}=14.4 \%$.

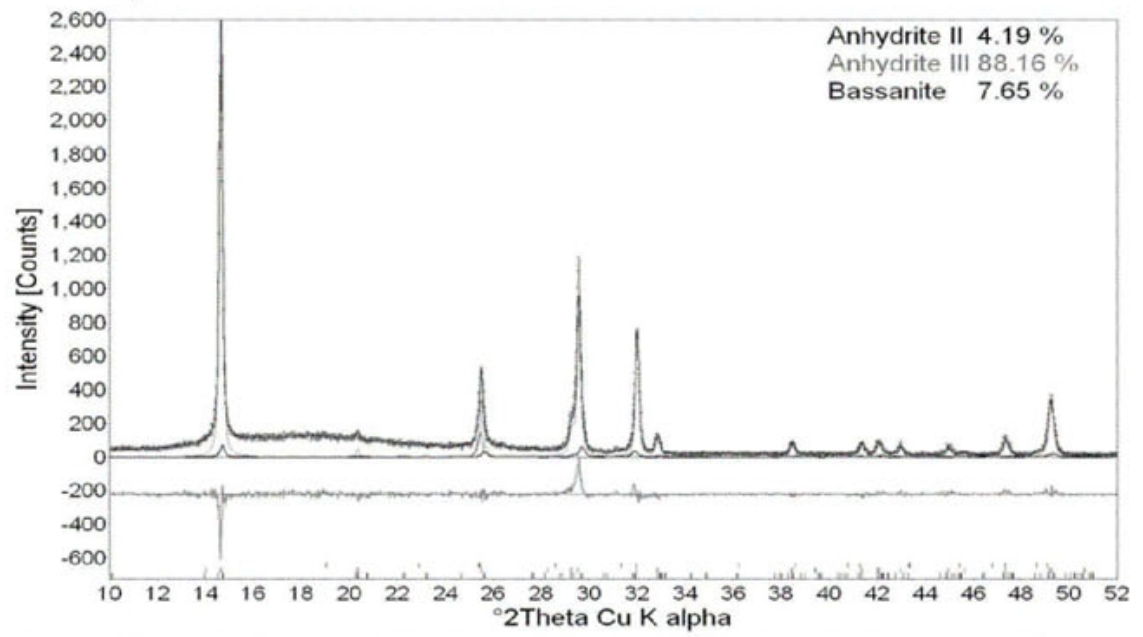

Figure 4. Rietveld refinement of dehydrated gypsum at $180^{\circ} \mathrm{C}$ with refined structures of Bassanite[2], Anyhdrite III [2] and Anhydrite II [6]. $R_{\text {Bragg-Bassanite }}=6.8 \%, R_{\text {Bragg-Anhydrite II }}=2.9 \%, R_{\text {Bragg-Anhydrite III }}$ $=7.9 \%, R_{w p}=14.6 \%$.

The determined loss on ignition of the dehydrated gypsum samples showed increasing anhydrite III content at the expense of bassanite with increasing temperature. Anhydrite III is formed at temperatures of $120{ }^{\circ} \mathrm{C}$, and transformed to anhydrite II at higher temperatures $\left(>180^{\circ} \mathrm{C}\right)$. Table 3 shows the results of Rietveld refinement. In case of bassanite the calculated content from loss on ignition (LOI) are comparable to the results from the Rietveld 
refinement. Only for the sample of gypsum dehydrated at $120^{\circ} \mathrm{C}$ it can be assumed that an early rehydration process of anhydrite III to bassanite is responsible for the different results of Rietveld refinement compared to the calculated bassanite content from loss of ignition.

Table 3. Results of Rietveld refinement compared to calculated bassanite content from LOI.

\begin{tabular}{|c|c|c|c|c|}
\hline \multirow{2}{*}{$\begin{array}{c}\text { Dehydration } \\
\text { Temperature }\end{array}$} & \multicolumn{2}{|c|}{$\begin{array}{c}\text { bassanite } \\
\text { [wt } \% \text { ] }\end{array}$} & $\begin{array}{c}\text { anhydrite III } \\
\text { [wt\%] }\end{array}$ & $\begin{array}{c}\text { anhydrite II } \\
\text { [wt \%] }\end{array}$ \\
\cline { 2 - 5 } & $\begin{array}{c}\text { (calculated } \\
\text { from LOI) }\end{array}$ & $\begin{array}{c}6 \% \text { rel. } \\
\text { humidity }\end{array}$ & $6 \%$ rel. humidity & $6 \%$ rel. Humidity \\
\hline $85{ }^{\circ} \mathrm{C}$ & 95.7 & 95.2 & 0.6 & 4.2 \\
\hline $120^{\circ} \mathrm{C}$ & 11.0 & 20.9 & 75.8 & 3.3 \\
\hline $180^{\circ} \mathrm{C}$ & 6.0 & 7.6 & 88.2 & 4.2 \\
\hline $250^{\circ} \mathrm{C}$ & 3.8 & 3.2 & 73.2 & 23.6 \\
\hline $380^{\circ} \mathrm{C}$ & 2.1 & 2.3 & 16.7 & 81.0 \\
\hline $550^{\circ} \mathrm{C}$ & 0.0 & 0.0 & 0.0 & 100.0 \\
\hline
\end{tabular}

\section{Conclusion}

A discrimination of bassanite and anhydrite III in the same powder via Rietveld refinement can be accomplished in application-oriented XRD patterns. Moreover, the investigations showed the hygroscopic character of dehydrated calcium sulphates and the need of storage under exclusion of humidity, especially in case of anhydrite III. High humidity causes an instant transformation of anhydrite III to bassanite. Rietveld refinement could be utilized to differentiate calcium sulphates with very similar crystalline structure and to measure in-situ transformation processes by rehydration in pure calcium sulphates. Discrimination of anhydrite III and bassanite was performed in powders of high purity. The determination of anhydrite III besides bassanite in dry mortar mixtures containing $20 \mathrm{Ma} \%$ and more sulphate is possible by Rietveld refinement. In cement applications with lower sulphate content the discrimination via Rietveld refinement could be more difficult. Furthermore, other investigations showed that a rehydration of anhydrite III to bassanite even through the Kapton film of the sample holder can not be avoided.

\section{References}

1. Murat, M. \& Foucault, M., 1977, Sulphates de Calcium et Materiaux Derivés, Colloq. Intern. de la RILEM, Saint Rémy les Chevreuse, France, 546.

2. Bezou, C., Nonat, A. et al., 1995, J. Solid State Chem., 117 (1), 165.

3. Powell, D.A., 1958, Nature, 182, 792.

4. Bensted, J. \& Prakash, S., 1968, Nature, 219, 60.

5. Schofield, P.F., Wilson, C.C., Knight, K.S. \& Stretton, I.C., 2000, Z. Kristallogr., $215,707$.

6. Kirfel, A. \& Will, G., 1980, Acta Crystallogr. B, 36, 2881.

7. Nørlund Christensen A., Olesen M., Cerenius Y. \& Jensen T.R., 2008, Chem. Mater., 20, 2124. 


\title{
High resolution X-ray powder diffraction measurements of the wet rust from the drinking water distribution system
}

\author{
P. Piszora ${ }^{1, *}$, J. Nawrocki ${ }^{1}, J$ Darul ${ }^{1}$, W. Nowicki ${ }^{1}$, \\ A. Evans ${ }^{2}$ \\ ${ }^{1}$ Faculty of Chemistry, Adam Mickiewicz University, Grunwaldzka 6, \\ PL-60780 Poznań, Poland \\ ${ }^{2}$ European Synchrotron Radiation Facility (ESRF), Grenoble 38043, France \\ *pawel@amu.edu.pl
}

Keywords: corrosion, green rust

\begin{abstract}
The composition and the crystal structure of corrosion scales from steel pipes have been analysed. High concentrations of green-rust-type phases have been detected in samples mounted on the axis of diffractometer as suspension in native water. This type of sample loading combined with measurements at the high-resolution powder-diffraction beamline (ID31/ESRF) has proven useful in the phase determination of the studied corrosion products.
\end{abstract}

\section{Introduction}

The presence of corrosion scales can significantly affect water quality in drinking water distribution systems. The intensity of corrosion depends first of all on the quality of water flowing through the system. Corrosion produced lining is also a good environment for the growth of micro-organisms. The presence of microorganisms on the surface of the corrosion deposits is the most significant source of odour in water supplies and a strict correlation between corrosion and micro-organism growth processes could be observed.

Details about preparation of the corrosion products for X-ray diffraction (XRD) measurements have been omitted in many papers [1,2]. It can be seen that the analytical results are very similar when measurements are not preceded by a special sample treatment, and, in most cases, $\mathrm{Fe}_{3} \mathrm{O}_{4}, \alpha$-FeOOH and $\gamma$-FeOOH are the only observed crystal phases [1-5]. However, observation of the intermediate and metastable corrosion products requires a special sample treatment. Important intermediate phases in the corrosion of iron are for example green rusts (GRs) [6-8]. GRs are layered double hydroxides composed of positively charged layers and charge balancing anions located in the interlayer region. GRs with brucite-type layers containing Fe(II) and Fe(III) can have anions such as sulphate, chloride, carbonate and also water filling the interlayers. The crystal structures of GRs depend on the type of interlayer anion $[8-10]$. 
It was expected that GR phases could be detected by XRD simply by covering a flat sample with glycerol $[8,11,12]$ or, alternatively, to subject it to additional sample treatment by freeze-drying [13]. However, detailed research showed the progressive sample decomposition during measurement, even for samples covered with glycerol [14]. Unfortunately, also the freeze-drying procedure could alter the structure of the corrosion products [15]. Sample drying, even in inert atmosphere, can result in the product decomposition due to removal of the interlayer water. Therefore, even complex sample treatments before XRD analysis can not guarantee the stabilisation of the rust sample.

The rapid reaction of GRs with atmospheric oxygen renders it difficult to identify these compounds in natural and engineered systems $[4,5,16]$. By studying the oxidation products of synthetic GRs, some authors suggested that GRs might be important precursors of other iron compounds found in corrosion products and soil $[17,18]$. However, it is necessary to demonstrate their presence in a natural environment to substantiate their importance.

Request for loading the sample as slurry is not only specific of rust samples. Recently, some attempts have been taken to investigate by XRD solution-fine powder reactions [19] or polycrystalline slurry of proteins [20]. Our work has shown that an analogous treatment is necessary to study corrosion products by XRD.

\section{Materials and methods}

\section{Materials}

Two samples (S31 and S17) from a wide survey concerning the corrosion of pipes in two distribution systems of a large agglomeration were analysed. Scales were studied

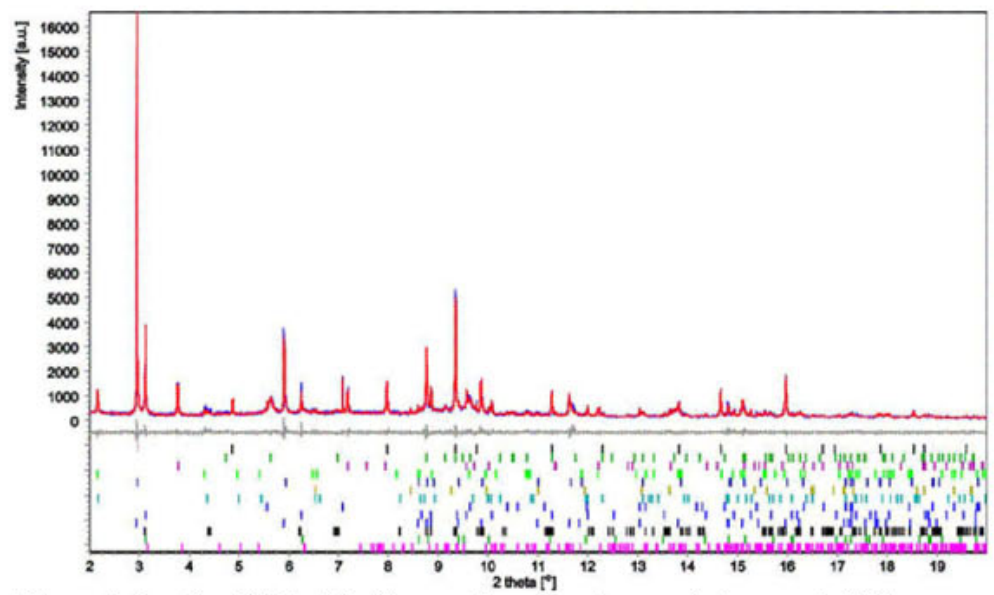

Figure 1. Results of Rietveld refinement for corrosion product - sample S17.

both before and after drying, and goethite, magnetite and lepidocrocite were identified as the primary constituents of the dried samples. However, high concentrations of GR phases were detected in the samples mounted on the axis of the diffractometer as a suspension in native water. 


\section{Sample preparation and data collection}

Small quantities of rust-in-water suspension were ground with a pestle in an agate mortar, and introduced as slurry into $0.5 \mathrm{~mm}$ diameter glass capillaries. X-ray powder diffraction data were collected on the high-resolution X-ray powder diffractometer (beamline ID31 at ESRF) selecting X-rays of $0.41274(6) \AA$ wavelength from the white undulator source. Data were collected for half an hour, normalized against monitor counts and detector efficiencies, and rebound into steps of $2 \theta=0.001^{\circ}$. Samples were introduced in the glass capillaries to avoid degradation of the air-sensitive phases, and the capillaries were rotated during measurement to minimise the effect of preferential orientation.

\section{Results}

\section{Rietveld refinement results}

Rietveld refinement was performed using the Topas package [21]. The starting structure models were taken from the following papers: magnetite $\left(\mathrm{Fe}_{3} \mathrm{O}_{4}\right)$ [22]; goethite $(\alpha-\mathrm{FeOOH})$ [23]; lepidocrocite $(\gamma-\mathrm{FeOOH})$ [24]; green rust II with $\mathrm{SO}_{4}{ }^{2-}$ anion, denoted as GR2_SO4 $\left(\mathrm{Fe}_{6}(\mathrm{OH})_{12}\left(\mathrm{SO}_{4}\right)\left(\mathrm{H}_{2} \mathrm{O}\right)_{8}\right)$ [7]; green rust I with $\mathrm{Cl}^{-}$anion, denoted as GR1_Cl $\left(\mathrm{Fe}_{6}(\mathrm{OH})_{10} \mathrm{Cl}\left(\mathrm{H}_{2} \mathrm{O}\right)_{3}\right)$ [25]; siderite $\left(\mathrm{FeCO}_{3}\right)$ [26]; green rust I with $\mathrm{CO}_{3}{ }^{2-}$ anion, denoted as GR1_CO3 $\left(\mathrm{Fe}_{6}(\mathrm{OH})_{12}\left(\mathrm{CO}_{3}\right)\left(\mathrm{H}_{2} \mathrm{O}\right)_{3}\right)$ [27]; quartz $\left(\mathrm{SiO}_{2}\right)$ [28]; akaganéite $(\beta-\mathrm{FeOOH})$ [29]; chukanovite $\mathrm{Fe}_{2}\left(\mathrm{CO}_{3}\right)(\mathrm{OH})_{2}$ [30]. For each crystal phase, the atomic displacement parameters were constrained to be equal for all atoms because of the multi-phase character of the sample. The peak shape profile parameters were well fitted with a pseudo-Voigt function for the $\mathrm{LaB}_{6}$ standard and as such fixed for all phases of the corrosion sample. Background was corrected using a Chebychev polynomial function. The relatively flat background suggests that no amorphous phase is present in the investigated samples. Crystallite size and strain contributions to the broadening were refined. Because the real morphology of crystallites reveals significant anisotropy, the presented crystallite size could be treated as a tentative only. The crystallite size without standard deviation (Table 1) refers to phases for which it was fixed without refinement, however with great care to get the best profile fit. The obtained low final $\mathrm{R}$ values (footnotes of Table 2) prove the proper choice of initial crystal structures. The results of the phase analysis are presented in Table 2 . The refined lattice parameters and the crystallite size for each crystal phase could be useful for modelling both long-term biotic and abiotic corrosion behaviour. For instance, goethite, used to be treated as one of the final corrosion products, has nanosize crystallites $(25 \mathrm{~nm})$, which suggests that is not a final product but an intermediate in the succession of corrosion processes.

On the basis of data from literature, three types of iron(II,III) hydroxysalts (GRs) can be distinguished. However, further division into six GR phases improves the final $\mathrm{R}$ values. To the best of the authors' knowledge, it is for the first time that this type of discontinuity in anion exchange in GR interlayer has been observed by XRD. The structure and composition of green rusts depend upon the specific anions they incorporate. Two GR phases (GR2_SO4_(1) and GR2_SO4_(2) ) containing non-planar $\mathrm{SO}_{4}{ }^{2-}$ ions were distinguished by differences in cell parameters (Table 1. sample S17). The brucite-like layers of GRs carry positive charge and the inter-layers are constituted of different amounts of anions and water molecules. These small differences in chemical composition have resulted in significant differences in the respective crystal structures (e.g. GR1_CO3_(1) and GR1_CO3_(2) ). 
Table 1.Corrosion products, crystal size and cell parameters obtained from the Rietveld refinement.

\begin{tabular}{|c|c|c|c|c|c|c|c|}
\hline \multirow{2}{*}{$\begin{array}{l}\text { phase } \\
\text { Sample S31 }\end{array}$} & \multirow[t]{2}{*}{$\%$} & \multirow{2}{*}{$\begin{array}{l}\text { Space } \\
\text { group }\end{array}$} & \multirow{2}{*}{$\begin{array}{c}\text { Crystallite } \\
\text { size }(\mathrm{nm})\end{array}$} & \multicolumn{4}{|c|}{ Lattice parameters } \\
\hline & & & & $a(\AA)$ & $b(\AA)$ & $c(\AA)$ & $\beta\left(^{\circ}\right)$ \\
\hline Magnetite & $21.13(14)$ & $F d 3 m$ & $365(32)$ & $8.4024(1)$ & & & \\
\hline Goethite & $48.37(32)$ & Pbnm & $24.96(55)$ & $4.6252(3)$ & $9.9909(6)$ & $3.0308(2)$ & \\
\hline Lepidocrocite & $1.459(65)$ & Amam & $235(84)$ & $3.8782(7)$ & $12.535(2)$ & $3.0718(9)$ & \\
\hline GR2_SO4_(1) & $0.200(14)$ & $P 3 m 1$ & $106(21)$ & $5.527(6)$ & & $10.974(6)$ & \\
\hline GR1_Cl_(2) & $2.287(95)$ & $R 3 m$ & $26.8(14)$ & $3.201(1)$ & & $23.71(1)$ & \\
\hline Siderite & $3.326(40)$ & $R 3 c$ & $115.3(46)$ & $4.7210(3)$ & & $15.406(1)$ & \\
\hline Quartz & $1.196(55)$ & $P 3_{1} 21$ & 1700 & $4.9136(2)$ & & $5.4053(3)$ & \\
\hline GR1 CO3 (1) & $7.25(31)$ & $R 3 m$ & 3600 & $3.1763(1)$ & & $22.7143(8)$ & \\
\hline GR1_Cl_(1) & $0.414(35)$ & $R 3 m$ & $225(27)$ & $3.1998(4)$ & & $24.015(3)$ & \\
\hline Akaganeite_( ) & $1.530(53)$ & $I 2 / m$ & $72(11)$ & $10.948(5)$ & $3.001(2)$ & $10.785(5)$ & $89.42(5)$ \\
\hline GR1_CO3_(2) & $5.96(27)$ & $R 3 m$ & $226.3(69)$ & $3.1767(2)$ & & $22.757(2)$ & \\
\hline Chukanovite & $6.88(11)$ & $P 12_{1} / a 1$ & $24.6(16)$ & $12.549(8)$ & $9.400(7)$ & $3.259(2)$ & $101.44(6)$ \\
\hline \multicolumn{8}{|l|}{ Sample S17 } \\
\hline Magnetite & $18.05(23)$ & $F d 3 m$ & $292(31)$ & $8.4029(1)$ & & & \\
\hline Goethite & $26.01(67)$ & Pbnm & $25.5(21)$ & $4.6256(6)$ & $9.984(2)$ & $3.0310(3)$ & \\
\hline Lepidocrocite & $4.51(12)$ & Amam & $136.7(88)$ & $3.8757(2)$ & $12.5331(6)$ & $3.0717(2)$ & \\
\hline GR2_SO4_(1) & $4.27(30)$ & $P 3 m 1$ & $349(51)$ & $5.507(1)$ & & $10.968(1)$ & \\
\hline$\overline{\text { GR1_Cl_(2) }}$ & $10.05(19)$ & $R 3 m$ & $88.39(68)$ & $3.1968(5)$ & & $23.924(1)$ & \\
\hline Siderite & $1.242(82)$ & $R 3 c$ & $91(32)$ & $4.748(1)$ & & $15.327(5)$ & \\
\hline GR2_SO4_(2) & $1.73(16)$ & $P 3 m 1$ & $349(51)$ & $5.473(2)$ & & $10.860(2)$ & \\
\hline Quartz & $1.897(98)$ & $P 3_{1} 21$ & 10000 & $4.9137(2)$ & & $5.4053(3)$ & \\
\hline GR1_CO3_(1) & $6.18(26)$ & $R 3 m$ & $424(45)$ & $3.17565(8)$ & & $22.7093(7)$ & \\
\hline GR1_Cl_(1) & $15.35(47)$ & $R 3 m$ & 10000 & $3.20256(5)$ & & $24.0484(5)$ & \\
\hline Akaganeite_( ) & $3.13(11)$ & $I 2 / m$ & 61.4 & $10.815(5)$ & $2.990(1)$ & $10.696(4)$ & $90.29(4)$ \\
\hline GR1_CO3_(2) & $2.49(18)$ & $R 3 m$ & $26.9(27)$ & $3.199(2)$ & & $22.67(1)$ & \\
\hline Chukanovite & $5.09(19)$ & $P 12_{1} / a 1$ & 20.0 & 12.396 & 9.407 & 3.2152 & 97.78 \\
\hline
\end{tabular}

Values without a standard deviation were not refined.

S31 Global R-values: $\mathrm{R}_{\exp }: 9.84 \quad \mathrm{R}_{\mathrm{wp}}: 10.15 \quad \mathrm{R}_{\mathrm{p}}: 7.87 \quad \mathrm{GOF}: 1.03$

S17 Global R-values: $\mathrm{R}_{\exp }: 5.37 \mathrm{R}_{\mathrm{wp}}: 7.97 \quad \mathrm{R}_{\mathrm{p}}: 6.05 \quad$ GOF $: 1.49$ 


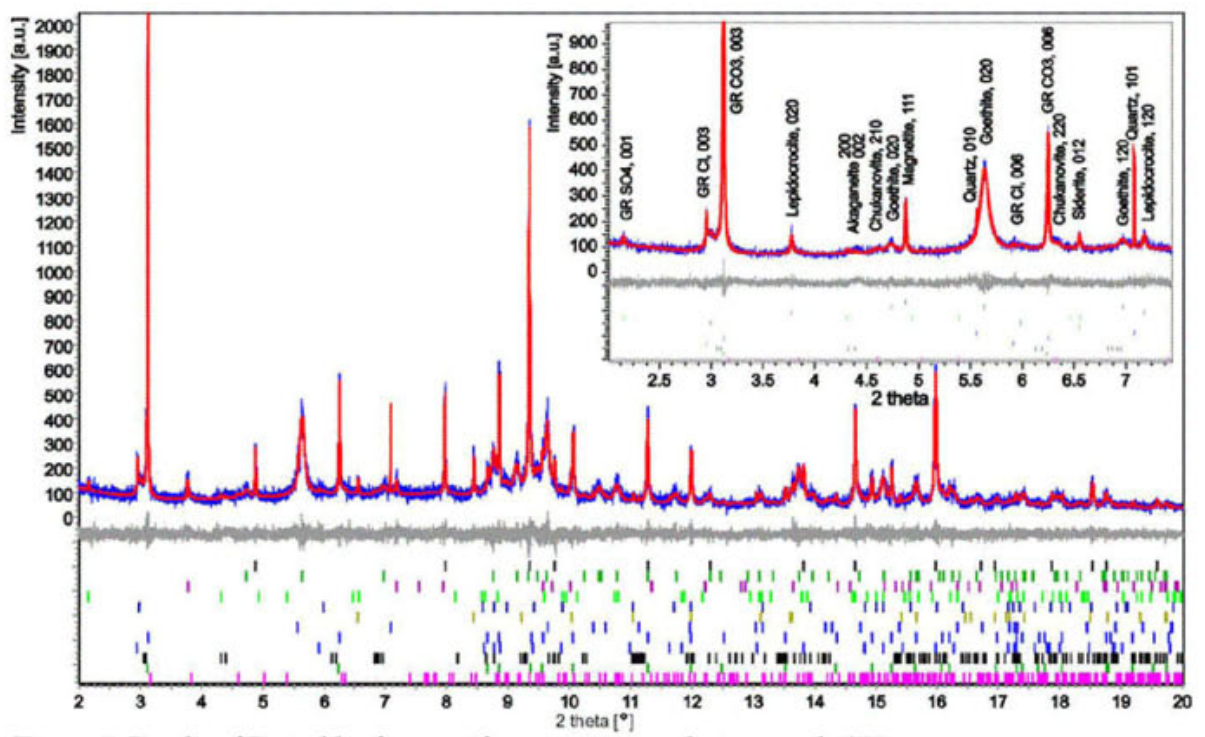

Figure 2. Results of Rietveld refinement for corrosion product-sample S31.

\section{Concluding remarks}

Our synchrotron diffraction studies have shown important differences between the corrosion products of the two different water distribution systems. The most important difference is the GR_CO3 / GR_Cl ratio. This phase ratio is proposed as a possible indicator of the water chlorination efficiency; however, further research is still needed before it can be technologically applied.

In the present work we have demonstrated that the sample preparation plays a key role in Xray measurements of very labile systems. Moreover, the crystal structures of the corrosion products were refined on the basis of the synchrotron data using the Rietveld method. The authors believe that the obtained results give further insight into the real mechanism of corrosion processes and, consequently, contribute to their better understanding.

\section{References}

1. Badan, B., Magrini, M. \& Ramous, E., 1991, J. Mater. Sci., 26, 1951.

2. Taseva, V., Dobrevsky, I., Nenov, V., Dimitrova, N., Bonev, B., Grudeva, V. \& Novakova, A., 2000, Mater. Corros., 51, 811.

3. Frateur, I., Deslouis, C., Kiene, L., Levi, Y. \& Tribollet, B., 1999, Wat. Res., 33, 1781.

4. Gismelseed, A., Elzain, M., Yousif, A., Al Rawas, A., Al-Omari, I. A., Widatallah, H. \& Rais A., 2004, Hyperfine Interact., 156/157, 487.

5. Lin, J., Ellaway, M. \& Adrien, R., 2001, Corros. Sci., 43, 2065. 
6. Refait, Ph., Abdelmoula, M. \& Génin, J.-M.R., 1998, Corros. Sci., 40, 1547.

7. Simon, L., François, M., Refait, P., Renaudin, G., Lelaurain, M. \& Génin, J.-M.R., 2003, Solid State Sci., 5, 327.

8. Hansen, H.C.B., 1989, Clay Miner., 24, 663.

9. McGill, I.R., McEnaney, B. \& Smith, D.C., 1976, Nature, 259, 200.

10. Evans, D.G. \& Slade, R.C.T., 2006, Structural aspects of layered double hydroxides in Layered Double Hydroxides, Ed. Duan, X. \& Evans, D.G. (Berlin/Heidelberg: Springer).

11. Zegeye, A., Huguet, L., Abdelmoula, M., Carteret, C., Mullet, M. \& Jorand, F., 2007, Geochim. Cosmochim. Acta, 71, 5450.

12. Zegeye, A., Ruby, Ch. \& Jorand, F., 2007, Geomicrobiol. J., 24, 51.

13. Williams, A.G.B. \& Scherer, M.M., 2001, Environ. Sci. Technol., 35, 3488.

14. Mazeina, L., Navrotsky, A. \& Dyar ,D., 2008, Geochim. Cosmochim. Acta, 72, 1143.

15. Greffié, C., Amouric, M. \& Parron, C., 2001, Clay Miner., 36, 381.

16. Zhang, Z., Stout, J.E., Yu, V.L. \& Vidic, R., 2008, Water Res., 42, 129.

17. Refait, Ph., Abdelmoula, M., Génin J.-M.R. \& Sabot, R., 2006, C. R. Geosci., 338, 476.

18. Ponamperuma, F.N., 1972, Adv. Agron., 24, 173.

19. Warr, L.N., 2003, J. Appl. Cryst., 36, 948.

20. Von Dreele, R.B., 2006, J. Appl. Cryst., 39, 124.

21. Bruker AXS, 2003, TOPAS V2.1: General profile and structure analysis software for powder diffraction data - User's Manual, (Bruker AXS, Karlsruhe, Germany).

22. Fleet, M.E., 1981, Acta Crystallogr., B37, 917.

23. Verdonck, L., Hoste, S., Roelandt, F.F. \& van der Celen, G.P., 1982, J. Mol. Structure, 79, 273.

24. Ewing, F.J., 1935, J. Chem. Phys., 3, 420.

25. Allmann, R. \& Donnay, J.D.H., 1969, Amer. Mineral., 54, 296.

26. Graf, D.L., 1961, Amer. Mineral. 46, 1283.

27. Aissa, R., Francois, M., Ruby, C., Fauth, F., Medjahdi, G., Abdelmoula, M. \& Génin, J.-M.R., 2006, J. Phys. Chem. Solids, 67, 1016.

28. Glinnemann, J., King, H.E., Schulz, H., Hahn, T., La Placa, S.J. \& Dacol, F., 1992, Z. Kristallogr. 198, 177.

29. Ståhl, K., Nielsen, K., Jiang, J., Lebech, B., Hanson, J.C., Norby, P. \& van Lanschot, J., 2003, Corros. Sci., 45, 2563.

30. Pekov, I.V., Perchiazzi, N., Merlino, S., Kalachev, V.N., Merlini, M. \& Zadov, A.E., 2007, Eur. J. Mineral. 19, 891.

Acknowledgements. We acknowledge the European Synchrotron Radiation Facility for provision of synchrotron radiation facilities and we would like to thank Dr Michela Brunelli for assistance in using beamline ID31. 


\title{
Reaction sequences in the thermo-
} chemical treatment of sewage sludge ashes revealed by X-ray powder diffraction - A contribution to the European project SUSAN B. Peplinski*, C. Adam, M. Michaelis, G. Kley, F. Emmerling, F.-G. Simon

BAM Federal Institute for Materials Research and Testing, Richard-Willstätter-Str. 11, D-12489 Berlin, Germany

* burkhard.peplinski@bam.de

Keywords: XRD, phosphorus recovery, sewage sludge ash, urban mining, fertilizers

\begin{abstract}
The sequence of reactions accompanying the thermochemical treatment of an ironand aluminium-bearing sewage sludge ash was ascertained by investigating two systematic series of samples. The ash was thermochemically treated in a lab-scale rotary furnace after mixing it with a chlorine-donor, either $\mathrm{CaCl}_{2}$ or $\mathrm{MgCl}_{2}$. Within each of these two sample series only a single process parameter, the reaction temperature, was varied, namely between 350 and $1050^{\circ} \mathrm{C}$. It was found, that among the numerous crystalline phases present in the raw ash only quartz and hematite continue to exist after thermochemical treatments carried out at $1050^{\circ} \mathrm{C}$, whereas all other components undergo at least one decomposition-recrystallization cycle. Some of the components re-crystallize even several times. It was proved that the restructuring of the calcium- and phosphorus-bearing mineral phases proceeds via the formation of chlorspodioside, $\mathrm{Ca}_{2} \mathrm{PO}_{4} \mathrm{Cl}$. The influence of the type of chlorine-donor on the final product was elucidated in detail and - to the best of our knowledge - for the first time crystalline $\mathrm{AlPO}_{4}$ was found in a sewage sludge ash and its decomposition was investigated, too.
\end{abstract}

\section{Introduction}

In the EU consumption of phosphorus (P) fertilizers exceeds 1.2 million tons of $\mathrm{P}$ per year. However, $\mathrm{P}$ is a non-renewable resource, becoming scarce and should be saved by applying recycling technologies to P-bearing waste streams. One of the major carriers of $\mathrm{P}$ is sewage sludge. In the EU more than 10 million tons (dry mass) of sewage sludge are produced annually, which contain about 0.3 million tons phosphorus. The European project SUSAN (Sustainable and Safe Re-use of Municipal Sewage Sludge for Nutrient Recovery) bundles the research efforts of seven partners from four countries, aiming at the development of a sustainable and safe strategy for phosphorus recovery from sewage sludge for agricultural utili- 
sation using a two-step thermal treatment including mono-incineration of sewage sludge and subsequent thermochemical treatment of the resulting ash. Essential aspects of the thermochemical treatment are the addition of a chlorine-donor to the ash (mainly for the removal of heavy metals) and the transformation of all phosphorus-bearing components into such mineral phases that are bio-available (available for plants).

To gain insight into the sequence of reactions proceeding during the thermochemical treatment of an iron- and aluminium-bearing sewage sludge ash X-ray powder diffraction (XRD) was applied to two systematic series of samples produced in a laboratory-scale equipment using two chlorine-donors, $\mathrm{CaCl}_{2}$ or $\mathrm{MgCl}_{2}$, alternatively. Within each series of samples only a single process parameter, the reaction temperature, was varied.

\section{Samples}

The not-yet thermochemically treated raw ash used for this investigation was produced at Slibverwerking Noord-Brabant (NL), the largest mono-incineration facility for sewage sludge in Europe. The chemical composition of the ash is given in table 1. The amount of chlorine-donor $\left(\mathrm{CaCl}_{2}\right.$ or $\mathrm{MgCl}_{2}$ for the first and second sample series, respectively) added to

Table 1. Mass fraction of the main chemical components in the raw ash prior to the thermochemical treatment, according to $X$-ray fluorescence analysis $(X R F)$.

\begin{tabular}{|c|r|r|r|r|r|r|r|r||c|c|}
\hline \multicolumn{10}{|c|}{ mass fractions $/ \%$} \\
\hline $\mathrm{SiO}_{2}$ & $\mathrm{Al}_{2} \mathrm{O}_{3}$ & $\mathrm{Fe}_{2} \mathrm{O}_{3}$ & $\mathrm{MnO}$ & $\mathrm{MgO}$ & $\mathrm{CaO}$ & $\mathrm{Na}_{2} \mathrm{O}$ & $\mathrm{K}_{2} \mathrm{O}$ & $\mathrm{P}_{2} \mathrm{O}_{5}$ & $\mathrm{Zn}$ & $\mathrm{Cl}$ \\
\hline \hline 21.6 & 10.8 & 16.3 & 0.15 & 2.85 & 15.7 & 1.04 & 0.99 & 21.3 & 0.22 & $<0.01$ \\
\hline
\end{tabular}

the ash prior to the thermal treatment was chosen in such a way, that the mass fraction of chlorine was $150 \mathrm{~g} \mathrm{Cl} / \mathrm{kg}$ raw ash. The quartz glass reactor of the rotary furnace was continuously flushed with air. The retention time at the nominal treatment temperature was 60 min. The chlorine content of some of the thermochemically treated ashes is given in table 2 . Further details of the thermochemical treatment are described elsewhere [1].

\section{Data collection and data analysis}

Samples analysed by XRD were pulverized and homogenized in a vibratory disc mill using a tungsten carbide grinding set. XRD measurements were performed on a Bruker-AXS D-5000 diffractometer in Bragg-Brentano geometry using an $1.0 \mathrm{~mm}$ aperture, a $0.1 \mathrm{~mm}$ receiving slit, a sample spinner ( 0 or $15 \mathrm{rpm})$, a curved graphite monochromator in the diffracted beam and a scintillation counter. Data were collected in the $2 \Theta$-range from $5^{\circ}$ to $140^{\circ}$ in steps of $0.02^{\circ}$ for $40 \mathrm{~s}$ per step, using copper $\mathrm{K} \alpha_{1,2}$ radiation with $40 \mathrm{kV}$ x $30 \mathrm{~mA}$. Selected samples were re-measured using cobalt $\mathrm{K} \alpha_{1,2}$ radiation. Diffraction patterns collected with $\mathrm{Cu}-$ and Co-radiation were found to be identical if represented as 'intensity versus $d$-value curves'. Rietveld analyses [2] were carried out using the program package BGMN [3] which employs the fundamental parameter approach. 


\section{Results and discussion}

Figure 1 summarizes the results of the phase identification for the first sample series, for which $\mathrm{CaCl}_{2}$ had been used as a chlorine-donor. Calcite, which is added to the sewage sludge during the incineration process for flue gas desulfurisation, free lime, most probably the decomposition product of limestone, as well as portlandite, $\mathrm{Ca}(\mathrm{OH})_{2}$, disappear during the thermochemical treatment already at temperatures below $400^{\circ} \mathrm{C}$, while anhydrite, $\mathrm{CaSO}_{4}$, is stable to at least $900^{\circ} \mathrm{C}$. Starting at about $500^{\circ} \mathrm{C}$ chlorspodiosite, $\mathrm{Ca}_{2} \mathrm{PO}_{4} \mathrm{Cl}$, is formed, presumably as a product of the reaction of $\mathrm{CaCl}_{2}$ with whitlockite, $\mathrm{Ca}_{3-\mathrm{x}}\left(\mathrm{Mg}, \mathrm{Fe}^{2+}\right)_{\mathrm{x}}(\mathrm{PO} 4)_{2}$.

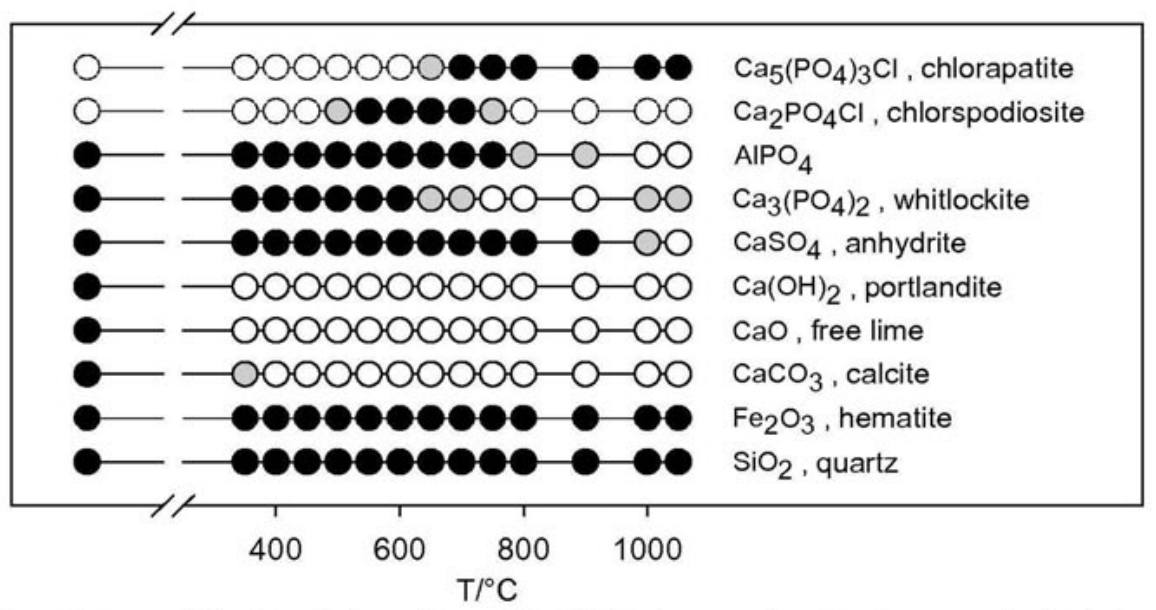

Figure 1. Some of the mineral phases detected by XRD in the raw ash and in the same ash after being thermochemically treated with the chlorine-donor $\mathrm{CaCl}_{2}$ at temperatures between 350 and $1050^{\circ} \mathrm{C}$.

- = phase detected by XRD, $\bigcirc=$ phase not detected by XRD, • = phase just (dis)appearing.

A similar reaction has previously been described [4]. Between $750^{\circ} \mathrm{C}$ and $950^{\circ} \mathrm{C}$ whitlockite is no longer detected. At about $750^{\circ} \mathrm{C}$ the formation of chlorapatite, which has the nominal composition $\mathrm{Ca}_{5}\left(\mathrm{PO}_{4}\right)_{3} \mathrm{Cl}$, starts. Whereas chlorspodiosite is an intermediate species which disappears with decreasing concentration of chlorine in the air-flushed reactor (see table 2),

Table 2. Mass fractions of chlorine in the ash thermochemically treated in the air-flushed furnace as a function of the treatment temperature (in ${ }^{\circ} \mathrm{C}$ ) and of the type of chlorine-donor used, according to XRF.

\begin{tabular}{|c|c|c|c|c|c|}
\hline \multirow{2}{*}{$\begin{array}{c}\text { chlorine- } \\
\text { donor }\end{array}$} & \multicolumn{5}{|c|}{ mass fraction of chlorine $/ \mathrm{wt} \%$} \\
\cline { 2 - 6 } & $400^{\circ} \mathrm{C}$ & $600^{\circ} \mathrm{C}$ & $700^{\circ} \mathrm{C}$ & $800^{\circ} \mathrm{C}$ & $1000^{\circ} \mathrm{C}$ \\
\hline $\mathrm{CaCl}_{2}$ & 8.4 & 7.9 & 6.6 & 3.4 & 2.6 \\
\hline $\mathrm{MgCl}_{2}$ & 8.2 & 5.7 & 4.1 & 1.6 & 0.7 \\
\hline
\end{tabular}

chlorapatite is stable up to the maximum reaction temperature of $1050^{\circ} \mathrm{C}$ where it is the dominant phosphorus-bearing phase in the diffraction pattern. Another step towards the understanding of the phase formation processes in sewage sludge ashes is the finding of 
crystalline, although disordered $\mathrm{AlPO}_{4}$ - which to the best of our knowledge - has not been described before in such ashes. This component appears to be considerably more stable under the given reaction conditions than whitlockite, see figures 1-2.

Figure 2 summarizes the results of the phase identification for the second sample series, for which $\mathrm{MgCl}_{2}$ had been used as a chlorine-donor. All reactions described for the first sample series occur here too. Besides these, several additional reactions take place leading to the formation of at least four magnesium-bearing phases. Farringtonite, a magnesium phosphate with the nominal composition $\mathrm{Mg}_{3}\left(\mathrm{PO}_{4}\right)_{2}$, is first detected at about $750^{\circ} \mathrm{C}$, whereas

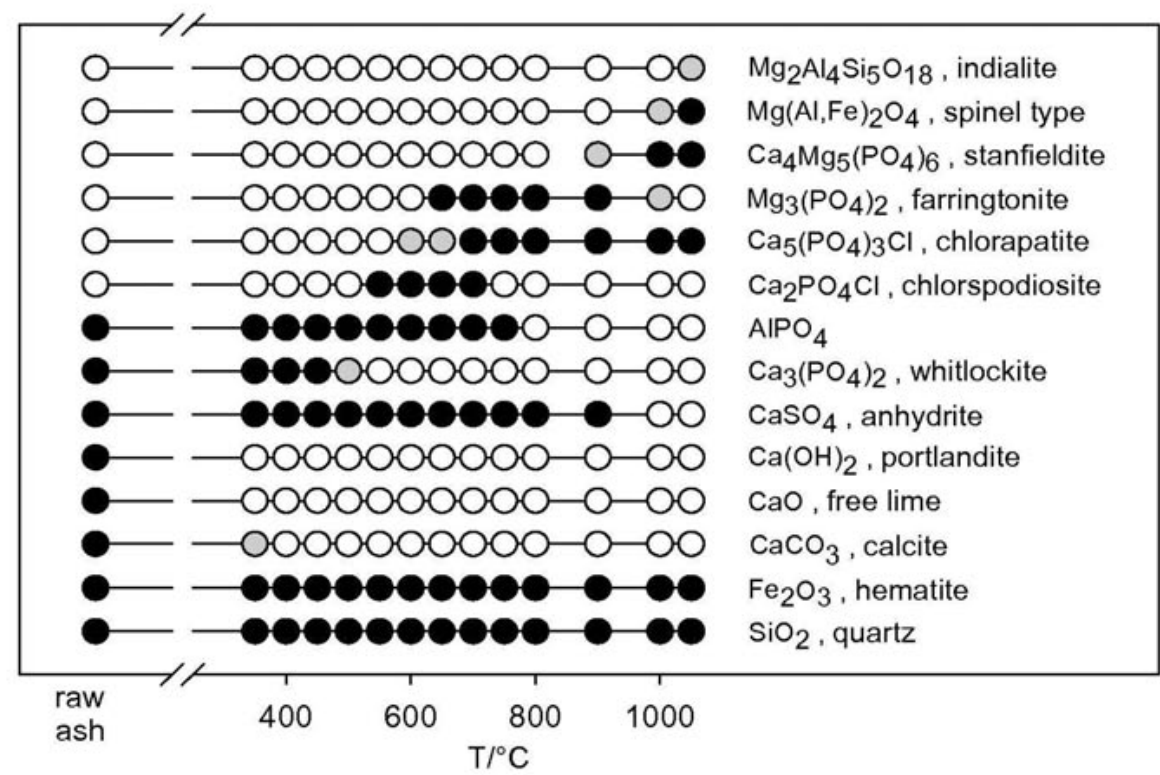

Figure 2. Some of the mineral phases detected by XRD in the raw ash and in the same ash after being thermochemically treated with the chlorine-donor $\mathrm{MgCl}_{2}$ at temperatures between 350 and $1050^{\circ} \mathrm{C}$.

- = phase detected by $X R D, \mathrm{O}=$ phase not detected by $X R D, \bullet=$ phase just (dis)appearing.

stanfieldite, a mixed (Mg,Ca)-phosphate with the nominal composition $\mathrm{Ca}_{4} \mathrm{Mg}_{5}\left(\mathrm{PO}_{4}\right)_{6}$, forms only above $850^{\circ} \mathrm{C}$, presumably on the expense of the former which gradually disappears between 900 and $1050^{\circ} \mathrm{C}$. In samples treated at $1000^{\circ} \mathrm{C}$ or $1050^{\circ} \mathrm{C}$, two aluminium- and magnesium-bearing compounds are detected: the spinel type $\mathrm{Mg}(\mathrm{Al}, \mathrm{Fe})_{2} \mathrm{O}_{4}$ as well as $\mathrm{Mg}_{2}(\mathrm{Al}, \mathrm{Fe})_{4} \mathrm{SiO}_{18}$ (indialite or cordierite). They are presumably formed through the reaction of the magnesium component released upon the decomposition of the chlorine-donor with aluminium oxide originating from the thermal decomposition of the aluminium phosphate. Following the procedure previously described [5] the outcome of the phase identification was validated, see figure $3-5$, and in addition phase quantification was carried out. Table 3 summarizes some of the results. They show that the mass fraction of chlorspodioside exceeds $40 \mathrm{wt} \%$ in the ash treated at $650^{\circ} \mathrm{C}$. Another important result is that using $\mathrm{MgCl}_{2}$ instead of $\mathrm{CaCl}_{2}$ as a chlorine-donor leads to a reduction of the mass fraction of chlorapatite from 41.9 


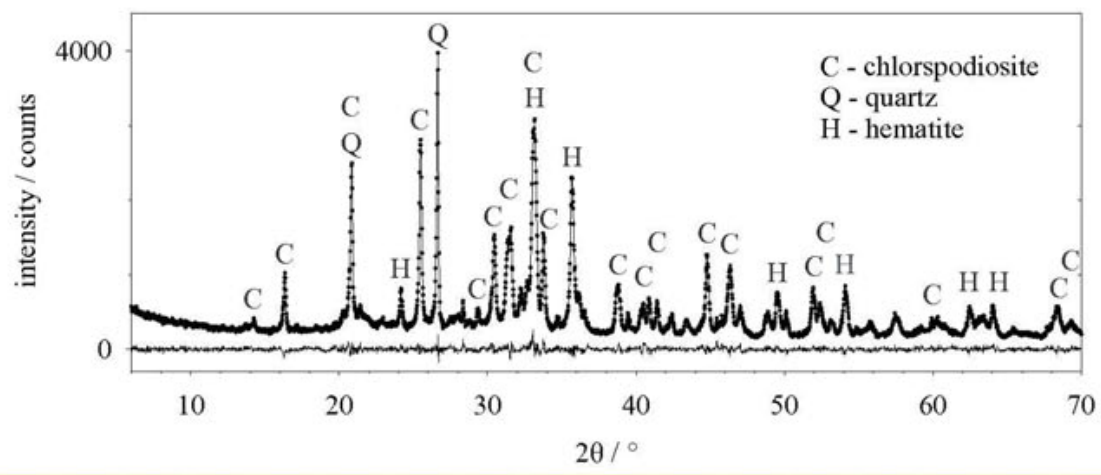

Figure 3. Rietveld plot for the diffraction pattern of the ash treated with the chlorine-donor $\mathrm{CaCl}_{2}$ at $650^{\circ} \mathrm{C}, \mathrm{Rwp}=6.11 \mathrm{Rexp}=4.74 \mathrm{GoF}=1.29$ for $L A L=6^{\circ}$ and $H A L=70^{\circ}$.

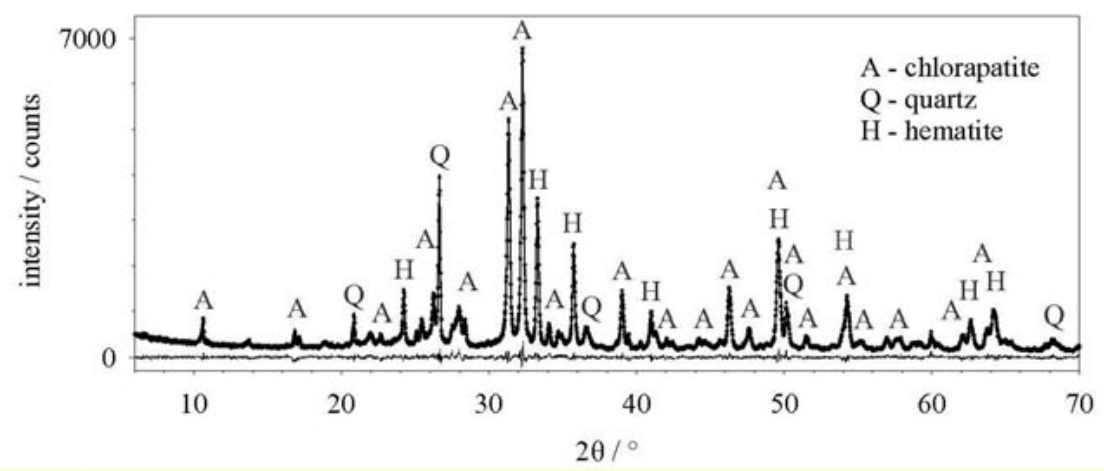

Figure 4. Rietveld plot for the diffraction pattern of the ash treated with the chlorine-donor $\mathrm{CaCl}_{2}$ at $1000^{\circ} \mathrm{C}, \mathrm{Rwp}=6.39 \operatorname{Rexp}=4.77 \mathrm{GoF}=1.34$ for $L A L=6^{\circ}$ and $H A L=70^{\circ}$.

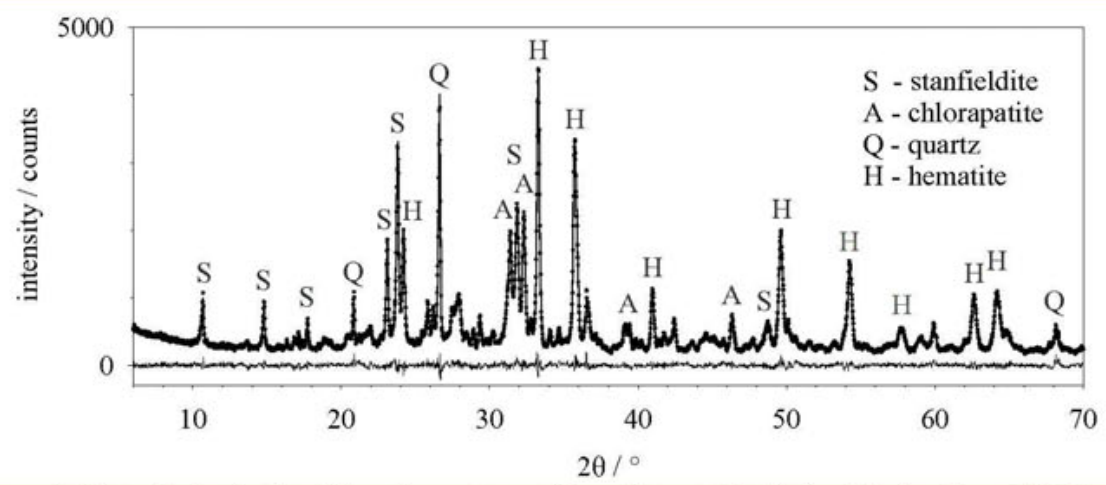

Figure 5. Rietveld plot for the diffraction pattern of the ash treated with the chlorine-donor $\mathrm{MgCl}_{2}$ at $1000^{\circ} \mathrm{C}, R w p=6.10 \operatorname{Rexp}=4.49 \mathrm{GoF}=1.36$ for $L A L=6^{\circ}$ and $H A L=70^{\circ}$. 
Table 3. Phase quantification for a sewage sludge ash thermochemically treated under three different conditions; results from Rietveld analyses of the diffraction patterns displayed in figures 3-5; numbers in parentheses are estimated standard deviations (esd) calculated by the Rietveld program.

\begin{tabular}{|c|c|c|c|c|c|c|c|}
\hline \multirow{3}{*}{$\begin{array}{l}\text { chlorine- } \\
\text { donor }\end{array}$} & \multirow{3}{*}{$\begin{array}{l}\text { tempe- } \\
\text { rature } \\
{ }^{\circ} \mathrm{C}\end{array}$} & \multicolumn{6}{|c|}{ weight fraction $/ \%$} \\
\hline & & \multicolumn{6}{|c|}{ phosphorus-bearing phases } \\
\hline & & $\begin{array}{c}\text { chlor- } \\
\text { spodiosite }\end{array}$ & $\begin{array}{l}\text { chlor- } \\
\text { apatite }\end{array}$ & $\begin{array}{l}\text { whit- } \\
\text { lockite }\end{array}$ & $\begin{array}{l}\text { farring- } \\
\text { tonite }\end{array}$ & $\begin{array}{l}\text { stan- } \\
\text { fieldite }\end{array}$ & $\mathrm{AlPO}_{4}$ \\
\hline $\mathrm{CaCl}_{2}$ & 650 & $41.3(6)$ & $4.6(3)$ & $4.2(3)$ & 0 & 0 & $\sim 8$ \\
\hline $\mathrm{CaCl}_{2}$ & 1000 & 0 & $41.9(5)$ & $5.7(2)$ & 0 & 0 & $\sim 2$ \\
\hline $\mathrm{MgCl}_{2}$ & 1000 & 0 & $15.6(5)$ & $5.2(3)$ & $4.4(2)$ & $29.3(4)$ & $\sim 0$ \\
\hline
\end{tabular}

to $15.6 \mathrm{wt} \%$, i.e. to about just one third. This finding corresponds with the XRF results given in table 2 showing that the chlorine content of these samples is reduced from $2.6 \mathrm{wt} \%$ to 0.7 wt $\%$, i.e. to about one third, too.

\section{Summary}

In the European project SUSAN ('Sustainable and Safe Re-use of Municipal Sewage Sludge for Nutrient Recovery') a sustainable and safe strategy for phosphorus recovery from sewage sludge was investigated. The key technology was a two-step thermal treatment including mono-incineration of sewage sludge and a subsequent thermochemical treatment of the resulting ashes targeting on the production of phosphorus fertilisers. Within this framework phase formation processes accompanying the thermochemical treatment of sewage sludge ashes were elucidated in detail by applying X-ray powder diffraction - in combination with other analytical techniques - to systematic sample series prepared from iron- and aluminiumbearing raw ashes using either $\mathrm{CaCl}_{2}$ or $\mathrm{MgCl}_{2}$ as a chlorine-donor for the thermochemical treatment. It was found that the reaction of the phosphorous bearing-phases contained in the raw ash with either of the two chlorine donors proceeds via the formation of an intermediate species, namely chlorspodioside, $\mathrm{Ca}_{2} \mathrm{PO}_{4} \mathrm{Cl}$. It was proved that the phase composition of the final products was strongly influenced by the type of chlorine donor used. The main Pspecies in the $\mathrm{CaCl}_{2}$-treated ashes was chlorapatite whereas mainly stanfieldite was formed when using $\mathrm{MgCl}_{2}$ as Cl-donor. For the first time crystalline aluminium phosphate was detected in sewage sludge ashes and its decomposition was investigated as well.

\section{References}

1. Adam, C. et al., 2007, Materials Transactions, 48, 3056.

2. Young R.A., 1995, The Rietveld Method, International Union of Crystallography Monographs on Crystallography (Oxford University Press).

3. Bergmann, J., http://www.bgmn.de, distributed by GE Inspection Technologies.

4. Mackay, A.L., 1953, Miner. Mag., 30, 166.

5. Peplinski, B. et al., 2006, Z. Kristallogr. Suppl., 23, 29. 


\title{
Quantitative analysis of clay materials and thermal treated bricks
}

\author{
J. A. G. Carrió ${ }^{1, *}$, I. C. A. Dutra ${ }^{1}$, M. C. Terence ${ }^{1}$, \\ R. Toledo ${ }^{2}$, D. R. dos Santos ${ }^{2}$
}

${ }^{1}$ Presbyterian University Mackenzie - UPM, CEP 01302-907, São Paulo, SP, Brazil

${ }^{2}$ Universidade Estadual do Norte Fluminense Darcy Ribeiro - UENF, CEP 28013-620, Campos dos Goytacazes, RJ, Brazil

*jgcarrio@mackenzie.br

Keywords: clay minerals, bricks, soil fractions, FT-IR, Rietveld

\begin{abstract}
In this work we present a mineralogical analysis of natural clay deposits from Rio de Janeiro State, Brazil. The samples were sieved to prepare a homogeneous powder representative of the natural soil. This powder was extruded to form bricks and submitted to a firing process in a furnace with controlled heating and cooling rates. Brick samples were prepared with firing temperatures varying from $300^{\circ} \mathrm{C}$ to $1200^{\circ} \mathrm{C}$. X-ray powder diffraction data allowed quantitative analysis by the Rietveld method using the refinement program GSAS. The Rietveld analysis showed that the ceramic high resistance after treatment at $1200^{\circ} \mathrm{C}$ is associated with the formation of mullite, cristobalite and hematite phases. In contrast to the analysis of the bulk material, Rietveld quantitative analysis for the clay, silt and sand fractions revealed quantifiable concentrations of kaolinite, illite, gibbsite, goethite, quartz, anorthoclase and muscovite. The effect of the thermal treatment in the clay structural properties was also analyzed by infra-red spectroscopy.
\end{abstract}

\section{Introduction}

A large quaternary sediment basin situated in the northern Rio de Janeiro State, Brazil, supplies raw material for the production of ceramic materials, mainly bricks and roof tiles, which is an important economical activity in this region. Several studies have been carried out in order to characterize the raw material from this sediment-hosted clay deposit. A quantitative mineralogical analysis for the natural soil (not separated) using the Rietveld method was previously reported [1], where the results indicate kaolinite as the main phase (about $86 \%$ in mass), followed by quartz and gibbsite. The environmental impact of the gas emitted during firing of these clay materials was also evaluated [2].

In the present work, we used the Rietveld method to perform a structural analysis of ceramic samples produced from natural soil fired at $1200^{\circ} \mathrm{C}$. Furthermore, the Rietveld method was also used to analyze the raw material, which was separated into the three granulometric fractions: Clay, silt and sand. This procedure is suitable for the identification of minor crystalline 
phases, which could not be detected in the natural soil, hence allowing a better quantitative analysis.

\section{Experimental}

The material collected at the mining site was dried, grounded, and passed through a sequence of sieves yielding a homogeneous powder with particle size smaller than $74 \mu \mathrm{m}$. Only $5 \%$ of the original mass was rejected in this procedure, indicating that samples were quite representative of the original raw material. This powder was separated according to its particle size in three granulometric fractions, named clay (the fraction with grain sizes below $2 \mu \mathrm{m}$ ), silt (grain sizes between 2 and $60 \mu \mathrm{m}$ ) and sand (above $60 \mu \mathrm{m}$ ). The separation was performed following the Brazilian ABNT technical standards [3]. Brick samples of the raw material (not separated soil) were also prepared by mixing the powder with water and extruding at $36 \mathrm{MPa}$. The result was a rectangular bar with a $20 \times 10 \mathrm{~mm}$ cross-section, which was cut into bricks $100 \mathrm{~mm}$ long. After drying at room temperature in air for two weeks, the residual water was removed at $110^{\circ} \mathrm{C}$ for $24 \mathrm{~h}$. The samples were then thermally treated at different temperatures: $300,400,500,600,700,800,850,900,950,1000,1050,1100$ and $1200^{\circ} \mathrm{C}$, using a heating rate of $2^{\circ} \mathrm{C} / \mathrm{min}$, constant temperature for $3 \mathrm{~h}$, and cooling rate of $1.5^{\circ} \mathrm{C} / \mathrm{min}$. Powder diffraction data were collected at room temperature with a Seifert URD65 diffractometer, equipped with a diffracted beam monochromator. The experimental conditions for the non-treated samples were $6^{\circ} \leq 2 \theta \leq 60^{\circ}, \Delta 2 \theta=0.02^{\circ}$, counting time $5 \mathrm{~s}$ and $\mathrm{CoK} \alpha$ radiation. For the determination of the crystalline volume fraction of the bricks treated at different temperatures, their diffraction patterns were collected with $3^{\circ} \leq 2 \theta \leq 75^{\circ}, \Delta 2 \theta=0.03^{\circ}$, counting time $3 \mathrm{~s}$ and $\mathrm{CuK} \alpha$ radiation. Two different $\mathrm{X}$-ray sources were used due to a necessary substitution of the X-ray tube in this period. Of course, direct comparison was done only for diffractograms obtained under the same experimental conditions.

The brick bending rupture tension was determined with a universal testing machine using the three-point loading test with a crosshead speed of $0.1 \mathrm{~mm} / \mathrm{min}$, following the ABNT standard procedure [4]. The bending strength $B S$ was calculated using the relation $B S=3 P L / 2 b d^{2}$, where $P$ is the maximum breaking load, $L$ is the distance between the sample supports, $b$ is the brick width and $d$ is the brick thickness.

\section{Results}

$\mathrm{X}$-ray diffraction patterns show a typical evolution as a function of the firing temperature (see, for instance, reference [2]). For the natural powder soil and for brick samples treated up to $400^{\circ} \mathrm{C}$ the diffractograms were very similar, suggesting a large fraction of kaolinite, and small quantities of illite, gibbsite, goethite and quartz. Between 400 and $500^{\circ} \mathrm{C}$ the kaolinite transforms to a non-crystalline phase, metakaolin, according to the reaction $\mathrm{Al}_{2} \mathrm{Si}_{2} \mathrm{O}_{5}(\mathrm{OH})_{4} \rightarrow \mathrm{Al}_{2} \mathrm{Si}_{2} \mathrm{O}_{7}+2 \mathrm{H}_{2} \mathrm{O}$ while the other crystalline phases remain unchanged up to $950^{\circ} \mathrm{C}$. Treatments at $1000^{\circ} \mathrm{C}$ or higher cause the sample recrystallization, with the formation of mullite, cristoballite and hematite phases. 


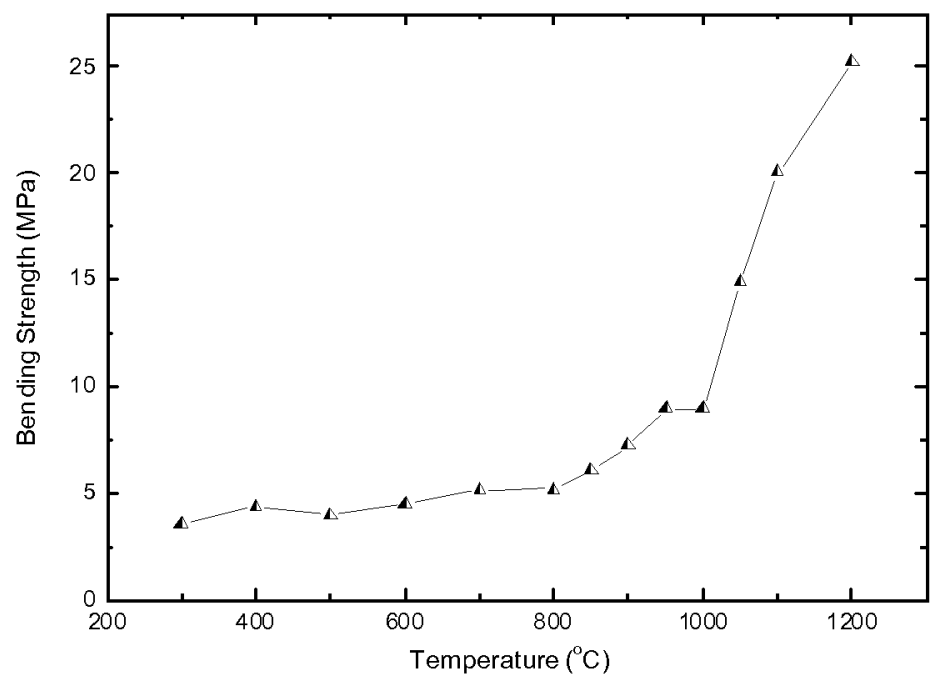

Figure 1. Bending Strength for bricks samples fired at different temperatures.

The bricks bending strength as a function of the firing temperature is shown in figure 1 . The rupture tension presents a strong increase from 8.9 to $25.2 \mathrm{MPa}$ in the temperature range from $1000^{\circ} \mathrm{C}$ to $1200^{\circ} \mathrm{C}$. This behaviour suggests that the recrystallization occurs during a sintering process, leading to the improvement of the ceramic performance. The Rietveld Method was applied to study the mineralogical composition of the brick fired at $1200^{\circ} \mathrm{C}$, which has the best ceramic properties. The refinement was performed using the program GSAS [5] with profile function 4 and without refining preferred orientation. Furthermore, the natural soil was separated in three granulometric fractions and, from this separation procedure, it was determined that the clay fraction correspond to $56.1 \%$ of the total soil mass, while the silt fraction corresponds to $40.1 \%$ and sand to $3.8 \%$. The mineralogical composition of the clay fraction was also analyzed using GSAS. The asymmetric line broadening for the three main clay phases was refined through parameters Sxxx and ptec of profile function 4, which indicate anisotropic strain and crystallite size broadening respectively [5]. The sublattice anisotropic broadening (sfec) parameter, typically due to stacking faults, stayed invariable at its default value during the refinement. The parameter eta indicates a Lorentzian asymmetric broadening for kaolinite. The refinement results are presented in figure 2 , and the corresponding crystalline phases and parameters are presented in tables 1 and 2 . A comparison between the diffractograms in figure 2 , and between the mineralogical compositions results in tables 1 and 2 shows the drastic changes that occur upon heating. Furthermore, the Rietveld results for the silt and sand fractions are resumed in table 3, and revealed the presence of some minor phases, as muscovite and anorthoclase, undetected in the complete soil powder. Differences in the compositions are due to the process of separation in fractions. 

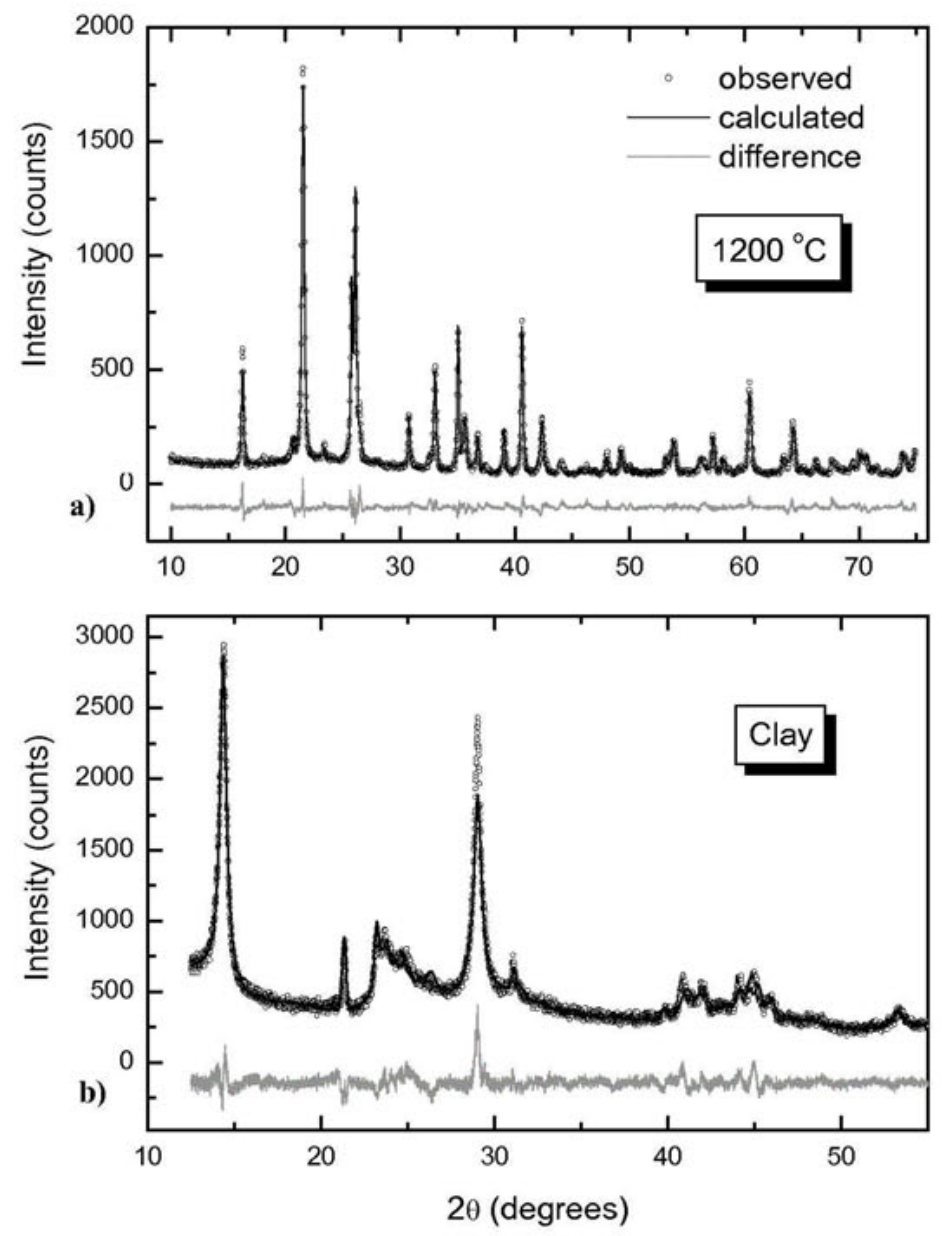

Figure 2. X-ray powder diffractogram and Rietveld calculated diffraction patterns for a) brick fired at $1200^{\circ} \mathrm{C}$; b) soil clay fraction. 
Table 1. Crystalline phases, lattice parameters and weight fractions for the brick fired at $1200{ }^{\circ} \mathrm{C}$. $R_{w p}=9.52 \% \quad R_{p}=7.15 \% \quad R_{\text {Bragg }}=6.65 \% \quad \chi^{2}=1.019$

\begin{tabular}{|l|l|l|l|l|l|}
\hline Phase & Mullite & Cristobalite_a & Cristobalite_b & Quartz & Hematite \\
\hline Space group & P b a m & P 41 21 2 & F d -3 m & P 32 2 1 & R -3 c \\
\hline a $(\AA)$ & $7.5265(4)$ & $5.48(1)$ & $7.1299(8)$ & 4.933 & 5.027 \\
\hline b $(\AA)$ & $7.6842(4)$ & $5.48(1)$ & $7.1299(8)$ & 4.933 & 5.027 \\
\hline c ( $)$ ( $)$ & $2.8810(1)$ & $6.23(2)$ & $7.1299(8)$ & 5.430 & 13.822 \\
\hline$\alpha($ deg.) & 90.000 & 90.000 & 90.000 & 90.000 & 90.000 \\
\hline$\beta($ deg.) & 90.000 & 90.000 & 90.000 & 90.000 & 90.000 \\
\hline$\gamma($ deg.) & 90.000 & 90.000 & 90.000 & 120.000 & 120.000 \\
\hline Wt. Fraction & $0.7640(9)$ & $0.115(2)$ & $0.071(2)$ & $0.029(1)$ & $0.021(1)$ \\
\hline
\end{tabular}

Table 2. Crystalline phases, lattice parameters and weight fractions for the clay fraction. $R_{w p}=6.44 \% \quad R_{p}=5.12 \% \quad R_{\text {Bragg }}=2.98 \% \quad \chi^{2}=2.04$

\begin{tabular}{|l|l|l|l|l|l|}
\hline Phase & Kaolinite & Illite & Gibbsite & Quartz & Goethite \\
\hline Space group & C1 & C 1 2/c 1 & P 1 21/n 1 & P 32 2 1 & P n m a \\
\hline a $(\AA)$ & $5.128(5)$ & $5.137(3)$ & $8.710(3)$ & 4.921 & 9.957 \\
\hline b $(\AA)$ & $8.797(3)$ & $8.62(1)$ & $5.031(1)$ & 4.921 & 3.026 \\
\hline c $(\AA)$ & $7.368(2)$ & $20.4(1)$ & $9.741(1)$ & 5.390 & 4.594 \\
\hline$\alpha($ deg.) & $95.89(4)$ & 90.000 & 90.000 & 90.000 & 90.000 \\
\hline$\beta($ deg.) & $103.76(7)$ & $94.8(3)$ & $94.64(4)$ & 90.000 & 90.000 \\
\hline$\gamma($ deg.) & $90.4(1)$ & 90.000 & 90.000 & 120.000 & 90.000 \\
\hline Wt. Fraction & $0.3629(9)$ & $0.434(3)$ & $0.177(2)$ & $0.0115(9)$ & $0.014(5)$ \\
\hline
\end{tabular}

Table 3. Crystalline phases and weight fractions for the silt $\left(R_{w p}=12.61 \% \quad R_{p}=9.37 \% \quad R_{\text {Bragg }}=\right.$ $\left.8.99 \% \quad \chi^{2}=6.140\right)$ and for the sand $\left(R_{w p}=11.64 \% \quad R_{p}=8.56 \% \quad R_{\text {Bragg }}=9.67 \% \quad \chi^{2}=4.961\right)$.

\begin{tabular}{|c|c|c|c|c|c|c|c|}
\hline \multirow{2}{*}{ SILT } & Phase & Quartz & Anorthoclase & Muscovite & Kaolinite & Gibbsite & Goethite \\
\cline { 2 - 7 } & $\begin{array}{c}\text { Weight } \\
\text { Fraction }\end{array}$ & $0.302(2)$ & $0.180(2)$ & $0.365(4)$ & $0.114(2)$ & $0.026(1)$ & $0.012(1)$ \\
\hline \multirow{2}{*}{ SAND } & Phase & \multicolumn{2}{|c|}{ Quartz } & \multicolumn{2}{|c|}{ Kaolinite } & Gibbsite \\
\cline { 2 - 7 } & $\begin{array}{c}\text { Weight } \\
\text { Fraction }\end{array}$ & \multicolumn{2}{|c|}{$0.34(2)$} & \multicolumn{2}{|c|}{$0.559(6)$} & $0.105(5)$ \\
\hline
\end{tabular}




\section{Concluding remarks}

The composition of the clay fraction of natural soil, mainly illite, kaolinite and gibbsite, is clearly different from the composition of fractions silt and sand. The highest amount of kaolinite was found in the sand fraction, with about $2.13 \mathrm{wt} \%$ (absolute value). Quartz is mainly present in the fractions silt and sand. For the brick treated at $1200{ }^{\circ} \mathrm{C}$, the refinement with GSAS profile function 4 indicate a well crystallized sample with no significant preferential orientation and a composition of mainly mullite and cristobalite, with about $76 \%$ and $19 \%$ respectively. This composition results from a recrystallization during the sintering process, which leads to the improvement of the ceramic performance with a strong increase of the rupture tension.

\section{References}

1. Manhães, R.S.T., Auler, L.T., Sthel, M.S., Alexandre, J., Massunaga, M.S.O., Carrió, J.G., dos Santos, D.R., da Silva, E.C., Garcia-Quiroz, A. \& Vargas, H., 2002, Appl. Clay Sci., 21, 303.

2. Toledo, R., dos Santos, D.R., Faria Jr., R.T., Carrió J.G., Auler, L.T. \& Vargas, H., 2004, Appl. Clay Sci., 27, 151.

3. ABNT, Brazilian Association of Technical Standards, 1984, NBR 7181: Granulometric fractions, Rio de Janeiro.

4. ABNT - Brazilian Association of Technical Standards, 1984, NBR 9451: Standard testing method for bending rupture tension of fired ceramic floor tile. Rio de Janeiro.

5. Larson, A.C. \& Von Dreele, R.B., (2004), General Structure Analysis System (GSAS), Los Alamos National Laboratory Report LAUR 86-748.

Acknowledgements: The authors acknowledge the financial support from Brazilian Agencies: Mackpesquisa, CAPES and FAPERJ. 


\title{
Mineral composition and stone conservation of cultural heritage building materials studied by PXRD
}

\author{
B. Simionescu ${ }^{1}$, M. Aflori ${ }^{2, *}$, M. Olaru ${ }^{2}$ \\ ${ }^{1}$ University of Oviedo, Spain \\ 2 "Petru Poni" Institute of Macromolecular Chemistry, Iasi, Romania \\ *maflori@icmpp.ro
}

Keywords: powder diffraction, phase identification, mineral composition, databases

\begin{abstract}
For the present study two types of rock, from Spain and Romania, selected by regional significance, abundance and importance were characterized by powder X-ray diffraction (PXRD). The samples were prepared by casting the same amount of a silsesquioxanebased polymer obtained through sol-gel approach and radical polymerization of 3(trimethoxysilyl)propyl methacrylate (TMSPMA) onto powder stones. Due to the distinct stones composition, the different polymer behaviour as regarding to these samples was evidenced through PXRD techniques. Conclusions concerning acid resistance, stone conservation and durability were revealed by using Match! Software and IUCr/COD/AMCSD database.
\end{abstract}

\section{Introduction}

The physico-chemical analytical techniques employed in the study of building materials provide very accurate qualitative and quantitative results on the alteration features, both related to the alteration layers and to the bulk chemistry of the exposed stone. The study of dolomitic sandstones evidenced the presence of weathering products inside the pores of sheltered parts after exposure to weathering conditions [1]. If a high amount of soluble salts is present in the stone structure, the local porosity is altered. Thus, the pore etching on the rain-washed side will allow pollutants to react on a greater surface area, and therefore will speed up the deterioration process. The petrography of the building material may also have an influence on the local porosity [2].

For the present study - two types of rocks, from Spain and Romania, were selected. The first type of rock is a bright white micritic dolomitic stone, typical from the Spanish region of Asturias, called Laspra (L) [3]. Important parts of Santa Maria del Naranco and San Miguel de Lillo churches, both listed in UNESCO's World Heritage List since 1985, were built by using $\mathrm{L}$ as building material. Due to the high level of $\mathrm{L}$ usage over the centuries, there is no quarry any more and in consequence, a small block of L arising from the Oviedo's cathedral portico was extracted for this study. The second selected stone is coming from Romania and it can be described as being a bioclastic oolitic stone, named Repedea (R). This type of stone 
can be found in the east of Romania, along the Moldavian platform. Dobrovat monastery, one of the most important monasteries situated in the north-eastern part of Ro-mania, was built from $\mathrm{R}$ limestone.

The TMSPMA polymer was obtained by a combination of sol-gel and radical polymerization reactions, a common method for manufacturing mesoporous materials for stone conservation. Such type of products polymerize within the stone pores by means of a sol-gel process, thereby strengthening the material. Their low viscosity allows them to penetrate deeply into the porous material after polymerization, a process that occurs upon contact with environmental moisture, by the formation of a stable gel with a silicon-oxygen backbone. In the conservation product synthesis, the sol-gel transition occurs in the presence of a surfactant, an approach that provides an efficient means of avoiding cracking of the gel while it is drying inside the stone [4].

\section{Experimental}

\section{Sample characterization}

The samples were carefully grounded in an agate mortar in order to eliminate as far as possible any particle size effects during the measurements. XRD patterns were recorded with a D8 Advance Bruker AXS diffractometer (Bragg-Bretagno geometry) equipped with a scintillation detector. X-rays were generated using a $\mathrm{CuK} \alpha$ source with an emission current of $40 \mathrm{~mA}$ and a voltage of $36 \mathrm{kV}$. A $0.6 \mathrm{~mm}$ fixed divergence slit was used for all samples to improve the signal-to-noise ratio. Scans were collected over the range $5-70^{\circ} 2$ th using a step size of $0.01^{\circ}$ and a count time of $0.5 \mathrm{~s} / \mathrm{step}$. The instrument calibration was carefully made by using the provided SRM1976 corundum sample.

The phase identification was first obtained by using the Match! Software version 1.9 together with IUCr/COD/AMCSD database. Match! performs a semi-quantitative analysis of the sample using the "Reference Intensity Ratio method" (RiR-method). The quantity of the phase in the phase mixture was measured by using the profile fitting of the complete pattern (for all accepted phases). Raw data processing included stripping of the alpha-2-radiation, data smoothing, background subtraction, peak searching, profile fitting and zero-point correction (specimen displacement) for errors. Quartz was the internal standard for all minerals.

\section{Mixture preparation}

Through radical polymerization of TMSPMA, that contains both methacrylate and silica phases, new hybrid materials can be obtained, in which the silica phase is dispersed in the form of domains with typical sizes of nanometers [5]. The complete process that develops the final silica phase takes place in three steps: the hydrolysis of the alcoxide groups of TMSPMA to form silanols, the condensation of the previously formed silanols to polymerize into silica polymers, and the aggregation of partially condensed silica macromolecules to build up the network [6]. The synthesis of the hybrid composite of polymethacrylate type implies the radical polymerization of TMSPMA in the presence of 2,2'-azobis(2methylpropionitrile) and subsequent sol-gel reaction in the presence of dodecylamine. The schematic structure of the hybrid composite is presented in figure 1. Stone-polymer samples were prepared by mixing the same amount of TMSPMA with powder stones and dried at vacuum at $30^{\circ} \mathrm{C}$. 


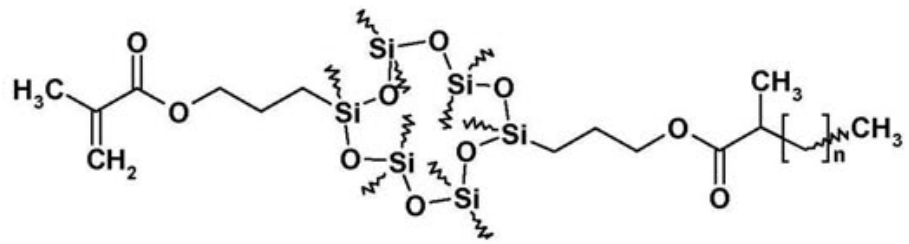

Figure 1. The structure of TMSPMA hybrid composite.

\section{Results and discussions}

\section{$\mathrm{L}$ and $\mathrm{R}$ mineral composition}

The PXRD peaks of L (figure 2) indicate the presence of four phases: dolomite, calcite, ankerite and quartz. Dolomite (D) and calcite (C) are the most abundant minerals in case of $\mathrm{L}$, followed by ankerite (A) and a small amount of quartz (Q) (table 1). The peaks of R (figure 3) indicate the presence of calcite $(\mathrm{C})$, magnesian calcite $(\mathrm{MC})$, quartz $(\mathrm{Q})$ and aragonite (Ar).

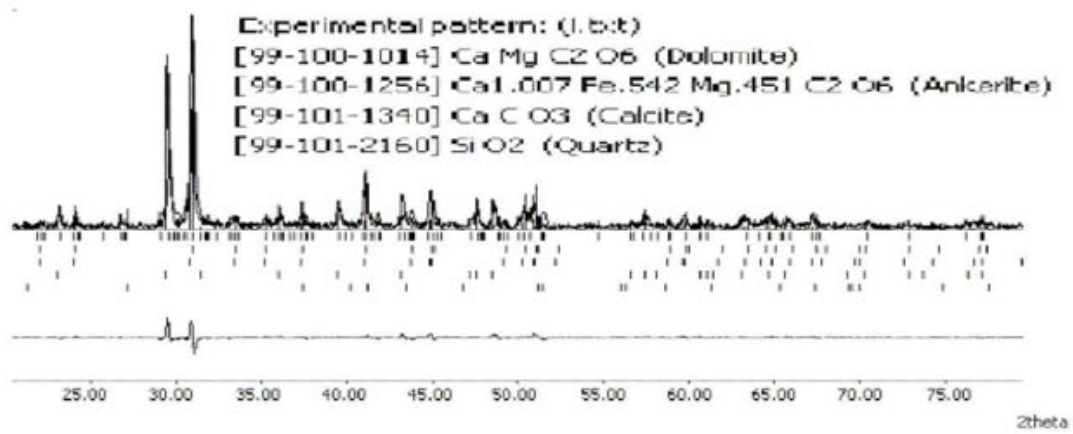

Figure 2. PXRD patterns of $L$ sample using Match! $R_{p}=51 \%$.

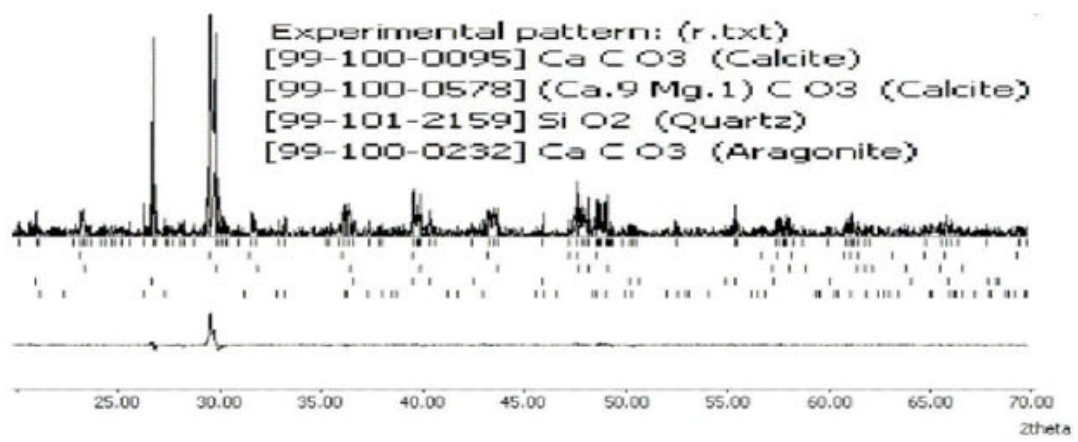

Figure 3. PXRD patterns of $R$ sample using Match! $R_{p}=61 \%$. 
Table 1. PXRD measurements results (wt \%).

\begin{tabular}{|c|c|c|c|c|c|c|c|c|}
\hline Sample & $\mathbf{A}$ & $\mathbf{Q}$ & $\mathbf{C}$ & $\mathbf{D}$ & $\mathbf{M c}$ & $\mathbf{A r}$ & $\mathbf{C a C l}_{\mathbf{2}}$ & $\mathbf{M g C l}_{\mathbf{2}}$ \\
\hline L untreated & 11 & 4 & 53 & 32 & & & & \\
\hline R untreated & & 20 & 27 & & 36 & 17 & & \\
\hline L+TMSPMA & 22 & 8 & 18 & 17 & & & 28 & 7 \\
\hline R+TMSPMA & & 29 & 19 & & 28 & 13 & 6 & 5 \\
\hline
\end{tabular}

The difference between the experimental data and the calculated profile studied by using Match! Software (figures 2, 3) appears on the bottom line, and the Bragg reflections are represented as vertical marks. The first row is represented by the experimental data, and the rest are the Bragg reflections of the selected mineral.

\section{TMSPMA characterization}

The X-ray diffraction patterns (figure 4) revealed the presence of three peaks, one sharp and two diffuse, which is somewhat different from the typical diffractograms of polysilsesquioxanes that exhibit two diffuse but rather intense peaks [7]. This behaviour excludes the formation of cage structures only (caged silsesquioxanes are normally crystallizable) and indicates the co-existence of crystalline and amorphous units, although crystallinity is not high. Also, in X-ray diffraction spectra, the peaks which appear at small Braggs' angles are particularly important for the elucidation of the nanocomposite structure of sol-gel materials. Such diffraction peaks stem from the silica clusters, which are linked via the non-hydrolyzable Si-C bond to the organic phase of the organic/inorganic hybrid network.

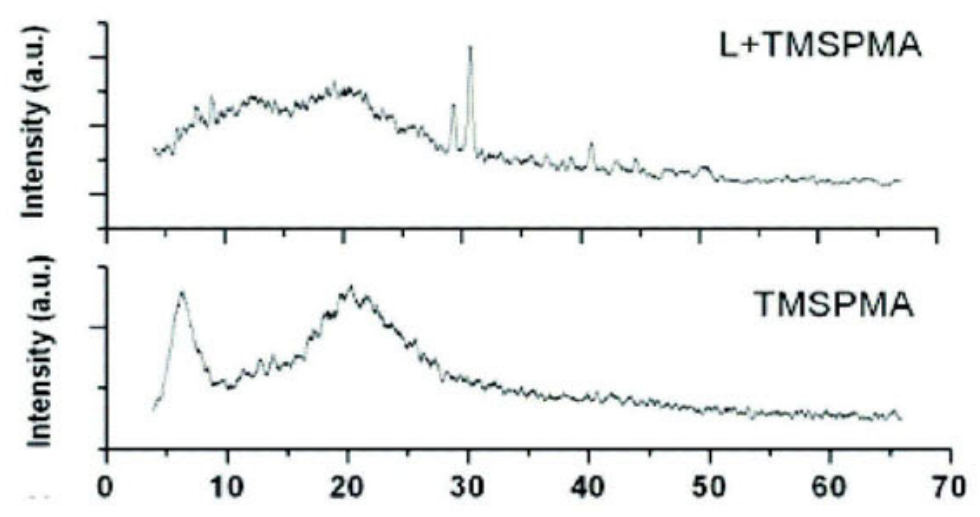

Figure 4. PXRD patterns of TMSPMA and L+TMSPMA samples.

\section{Characterization of L+TMSPMA and R+TMSPMA mixtures}

Due to the different stones compositions, the polymer addition has different effects. The structure of $\mathrm{R}$ is not affected by polymer addition, while $\mathrm{L}$ adopts a distinct amorphous configuration evidenced at angles from 5 to $25^{\circ}$ (figures 5,6 ). In both cases, a decrease of the relative intensities and peak areas is evidenced, more drastically in case of L+TMSPMA 
mixture. The modification of cell parameters for L+TMSPMA (different cards from same database) is attributed to the existence of nanoscale order domains in polymer structure.

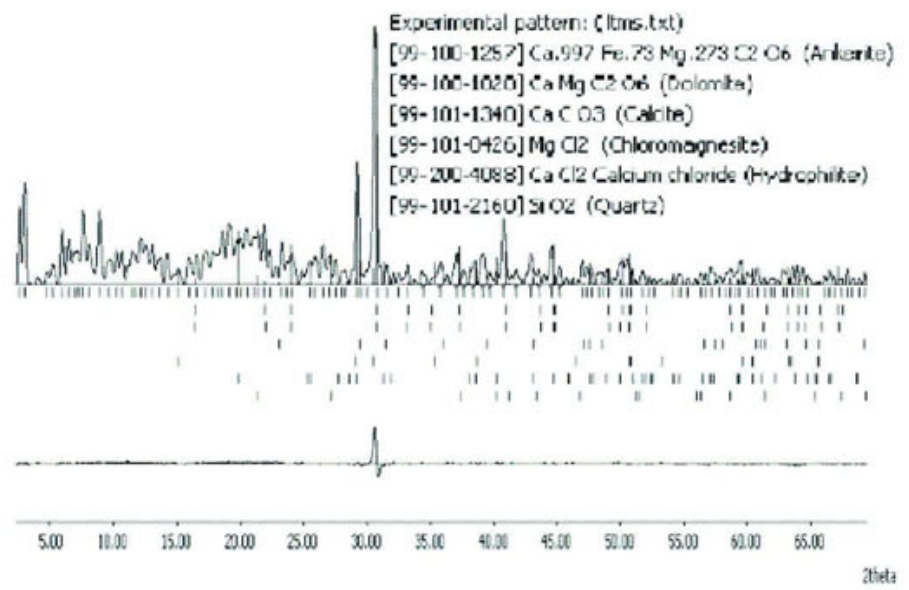

Figure 5. $P X R D$ patterns of $L+T M S P M A$ sample using Match! in the $2 \theta=5-70^{\circ}$ range, $R_{p}=45 \%$.

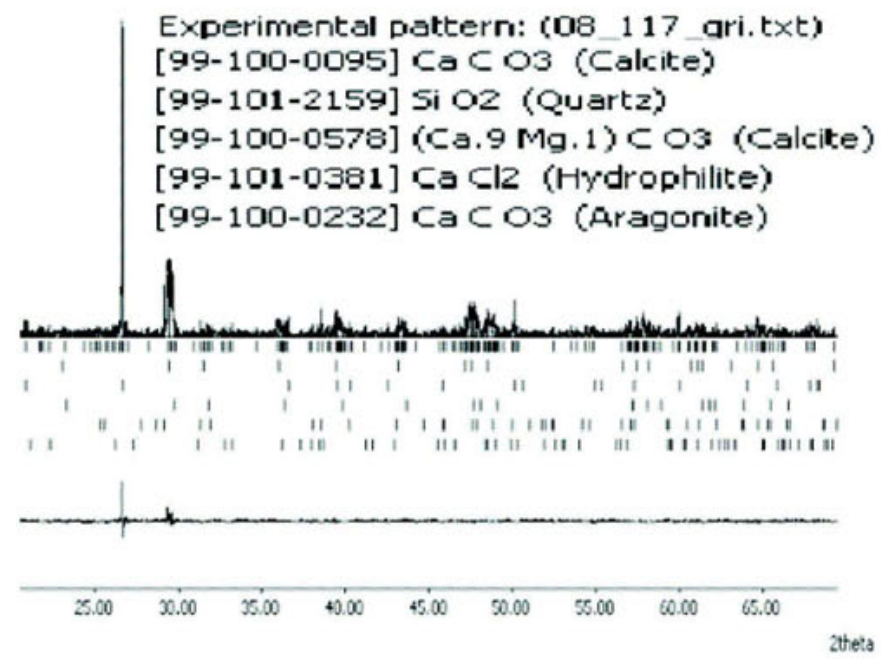

Figure 6. $P X R D$ patterns of $R+T M S P M A$ sample using Match! in the $2 \theta=20-70^{\circ}$ range, $R_{p}=50 \%$.

As a consequence of the presence of an acid medium $(\mathrm{pH}=5)$, a dramatically reduction of dolomite and calcite weights is evidenced for $\mathrm{L}$ (table 1), this behaviour being attributed to the reaction of $\mathrm{Ca}^{2+}$ and $\mathrm{Mg}^{2+}$ with hydrochloric acid, with the formation of $\mathrm{CaCl}_{2}$ and 
$\mathrm{MgCl}_{2}$ The percent of reaction products is of $35 \%$ for $\mathrm{L}+\mathrm{TMSPMA}$, as compared to the value of $11 \%$ for $\mathrm{R}+\mathrm{TMSPMA}$. This behaviour can be correlated with the high $\mathrm{L}$ stone porosity and high $\mathrm{Ca}^{2+}$ and $\mathrm{Mg}^{2+}$ concentrations $[3,8]$ as regarding to $\mathrm{R}$ one. It can be concluded that $\mathrm{R}$ is acid-resistant, a valuable information for the ranking of stones durability under the action of acid rains. The relative areas and intensities of the diffraction peaks in the XRD spectra can be related to the minerals crystallites size and stones degrees of crystallinity.

\section{Concluding remarks}

The relationship between intrinsic properties, such as mineralogy and microstructure, and functional and durability properties of stone and rocks used as construction materials is revealed. The PXRD technique is a valuable method to decide the applicability of a new conservation material for a certain type of stone. The conservation process of stone samples with the product under investigation modifies the carbonated area and is more efficient for Repedea. An additional examination of the contact angle, permeability and swelling behaviour of the products cast on stones surfaces is required, taking into account that such polymeric structures could function as water repellents and as crack-free conservation products for porous stones.

\section{References}

1. Sweevers, H., Delalieux, F. \& van Grieken, R., 1998, Atm. Env., 32, 733.

2. Lindqvist , J.E., Åkesson, U. \& Malaga, K., 2007, Mat. Characterization, 58, 1183.

3. Ruiz de Argandoda, V.G., Rodriguez, R.A., Celorio, C., Susirez del Rio, L.M, Calleja, L. \& Llavona, J., 1999, Phys. Chem. Earth A, 24, 633.

4. Scherer, G.W. \& Wheeler, G.S., 2009, Key Eng. Mater., 391, 1.

5. Jackson, C.L., Bauer, B.J., Nakatani, A.I. \& Barnes, J.D., 1996, Chem. Mater., 8, 727.

6. Brinker, C.J., Keefer, K.D., Schaefer D.W. \& Ashley C.S., 1982, J. Non-Cryst. Sol$i d s, \mathbf{4 8}, 47$.

7. Kaneko, Y. \& Iyi, N., 2007, Z. Kristallogr., 222, 656.

8. Grossi, C.M. \& Murray, M., 1999, Const. Build. Mater., 13, 101.

Acknowledgements. The author Dr Magdalena Aflori thanks to ICDD members and especially to Dr Timothy Fawcett for helpful discussions and support during the 2008 ICDD clinics and EPDIC 11 events. 


\title{
Anisotropic grain growth of bismuth titanate in molten salt fluxes
}

\section{T. Zaremba}

Silesian University of Technology, Department of Chemistry and Inorganic Technology, ul. B. Krzywoustego 6, 44-100 Gliwice, Poland teresa.zaremba@polsl.pl

Keywords: bismuth titanate, molten salt, X-ray diffraction (XRD), scanning electron microscopy (SEM)

\begin{abstract}
Bismuth titanate $\left(\mathrm{Bi}_{4} \mathrm{Ti}_{3} \mathrm{O}_{12}\right)$ powders were prepared by the molten salt synthesis method in the presence of $\mathrm{NaCl}-\mathrm{KCl}$ fluxes, using a mixed $\mathrm{Bi}_{2} \mathrm{O}_{3}-\mathrm{TiO}_{2}$ mixture as the starting materials. The effect of temperature and duration of heating on the morphology and structure of $\mathrm{Bi}_{4} \mathrm{Ti}_{3} \mathrm{O}_{12}$ powders was studied. The morphology of $\mathrm{Bi}_{4} \mathrm{Ti}_{3} \mathrm{O}_{12}$ particles changes during the formation and growth processes. Finally, the aggregated particles change to discrete, plate-like ones and their size increased with heating temperature. The intensities of reflections, emerging from the (001) planes increase.
\end{abstract}

\section{Introduction}

Bismuth titanate $\left(\mathrm{Bi}_{4} \mathrm{Ti}_{3} \mathrm{O}_{12}\right)$ is a suitable material for applications as high temperature transducers, capacitors and sensors, due to its high Curie temperature and high piezoelectric coefficient [1-4]. However, its layered structure and low crystal symmetry leads to difficulty in polarizing the conventional polycrystalline $\mathrm{Bi}_{4} \mathrm{Ti}_{3} \mathrm{O}_{12}$ ceramics. It is desirable to obtain $\mathrm{Bi}_{4} \mathrm{Ti}_{3} \mathrm{O}_{12}$ ceramics with textured microstructure since improved properties can be achieved in textured ceramics compared to ceramics with randomly oriented grain structure. $\mathrm{Bi}_{4} \mathrm{Ti}_{3} \mathrm{O}_{12}$ particles with plate-like morphology are preferred templates for fabricating textured $\mathrm{Bi}_{4} \mathrm{Ti}_{3} \mathrm{O}_{12}$ ceramics [5]. Molten salt synthesis (MSS) is a suitable method for synthesizing oxide powders with anisotropic particle morphologies [6-8].

In the molten salt method, the reactant oxide or carbonate mixture is heated with salt above its melting point. Usually, mixed salts are used to lower the melting point. The solubilities of starting and product oxides in salts are usually quite small, so that liquid and solid phases coexist during the process $[6,9]$.

In the present study, bismuth titanate powders were prepared in the presence of chloride fused salts. The equimolar mixture of sodium chloride and potassium chloride was used. The eutectic temperature for this kind of chloride flux is $650^{\circ} \mathrm{C}$.

\section{Experimental}

Stoichiometric amounts of $\mathrm{Bi}_{2} \mathrm{O}_{3}$ (purity 99.4\%, $\mathrm{POCH}$, Poland) and $\mathrm{TiO}_{2}$ - rutile (purity 99.5\%, POLICE, Poland) powders were weighed and mixed in isopropyl alcohol employing 
an agate mortar and pestle for $1 \mathrm{~h}$. The eutectic mixture of $43.94 \% \mathrm{NaCl}-56.06 \% \mathrm{KCl}$ (by weight) was prepared in the same way as described above. Equal weight mixtures of salts and component oxides were used in this experiment.

The mixture of the precursors and salts was dried at $120^{\circ} \mathrm{C}$ for $2 \mathrm{~h}$ for complete removal of isopropyl alcohol, placed in a Pt crucible and heated in a sealed alumina crucible (to prevent salt evaporation) at temperatures between $700^{\circ} \mathrm{C}$ and $1100^{\circ} \mathrm{C}$ for a desired time period. After firing the chlorides were removed from the products by washing with hot deionized water several times until the filtrates gave no reaction with silver nitrate solution. The powders were finally dried at $100^{\circ} \mathrm{C}$ for $2 \mathrm{~h}$. The phase structure of the powders was analyzed by an Xray diffractometer (XRD-3003 TT, Seifert) using Ni-filtered $\mathrm{CuK}_{\alpha}$ radiation. The phase structure $\mathrm{Bi}_{4} \mathrm{Ti}_{3} \mathrm{O}_{12}$ was confirmed using the PDF card No. 80-2143 (ICDD). The powders were gold-coated and then the grain size and morphology were investigated by scanning electron microscope (BS 340, Tesla).

\section{Results and discussion}

Figs. 1 and 2 show the XRD patterns of $\mathrm{Bi}_{4} \mathrm{Ti}_{3} \mathrm{O}_{12}$ crystals prepared at different temperatures. At $700^{\circ} \mathrm{C}$, the phase $\mathrm{Bi}_{12} \mathrm{TiO}_{20}$ co-existed with $\mathrm{Bi}_{4} \mathrm{Ti}_{3} \mathrm{O}_{12}$. Pure crystalline $\mathrm{Bi}_{4} \mathrm{Ti}_{3} \mathrm{O}_{12}$ was obtained after growing at $800^{\circ} \mathrm{C}$ for 15 minutes. Increasing the temperature to $1100^{\circ} \mathrm{C}$, the intensities of the $00 l$ diffraction lines were increased compared to the corresponding powder spectrum. These results indicate that during sample preparation for X-ray diffraction characterization, $\mathrm{Bi}_{4} \mathrm{Ti}_{3} \mathrm{O}_{12}$ crystals with platelet morphology may align with (001) planes parallel to the flat specimen holder.

Fig. 3 shows morphology and size of $\mathrm{Bi}_{4} \mathrm{Ti}_{3} \mathrm{O}_{12}$ crystals prepared: (a) at $700^{\circ} \mathrm{C}$, (b) at $800^{\circ} \mathrm{C}$, (c) at $900^{\circ} \mathrm{C}$ and (d) at $1000^{\circ} \mathrm{C}$ for 240 minutes. The synthesizing temperature could significantly influence the growth rate and crystallization habit of the $\mathrm{Bi}_{4} \mathrm{Ti}_{3} \mathrm{O}_{12}$ particles. Between $700^{\circ}$ and $800^{\circ} \mathrm{C}$ aggregate particles were formed. The powder synthesized at $700^{\circ} \mathrm{C}$ was composed of fine particles. The size of the primary particles increased and their shape changed from lumpy to plate-like with increasing temperature. Above $800^{\circ} \mathrm{C}$ discrete platelike particles with increased particle size were formed. On the other hand, the effect of heating time on morphology and particle size is smaller (figs 4-5). The degree of aggregation decreased with increasing preparing temperature. These results suggest that the molten salt method is useful not only for producing powders with anisotropy, but also for controlling the aggregation states of the powders. 


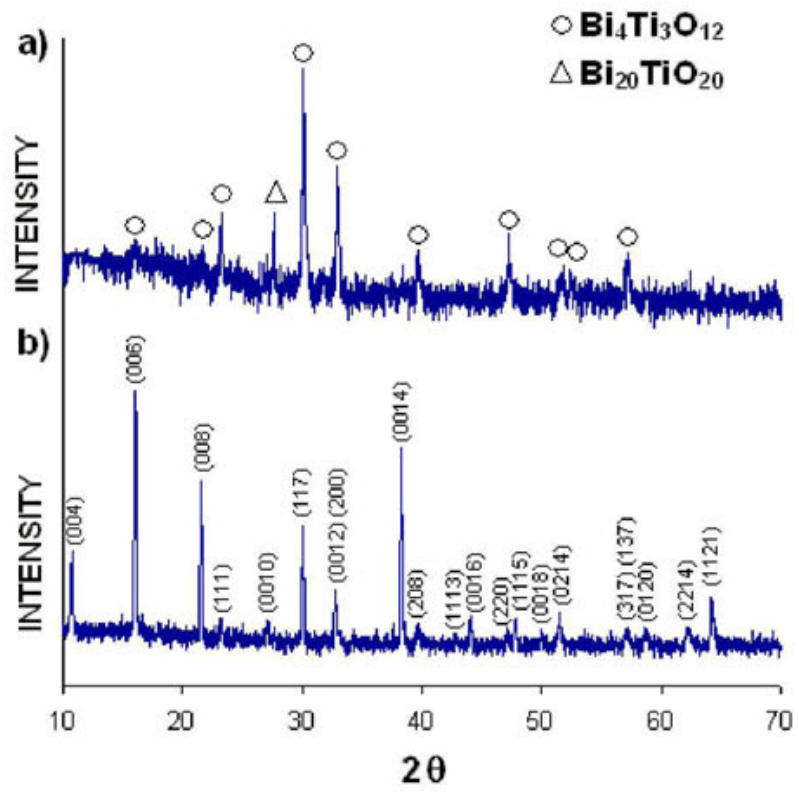

Figure 1. XRD patterns of $\mathrm{Bi}_{4} \mathrm{Ti}_{3} \mathrm{O}_{12}$ powders obtained at (a) $700^{\circ}$ and (b) $1000^{\circ} \mathrm{C}$ for $15 \mathrm{~min}$.

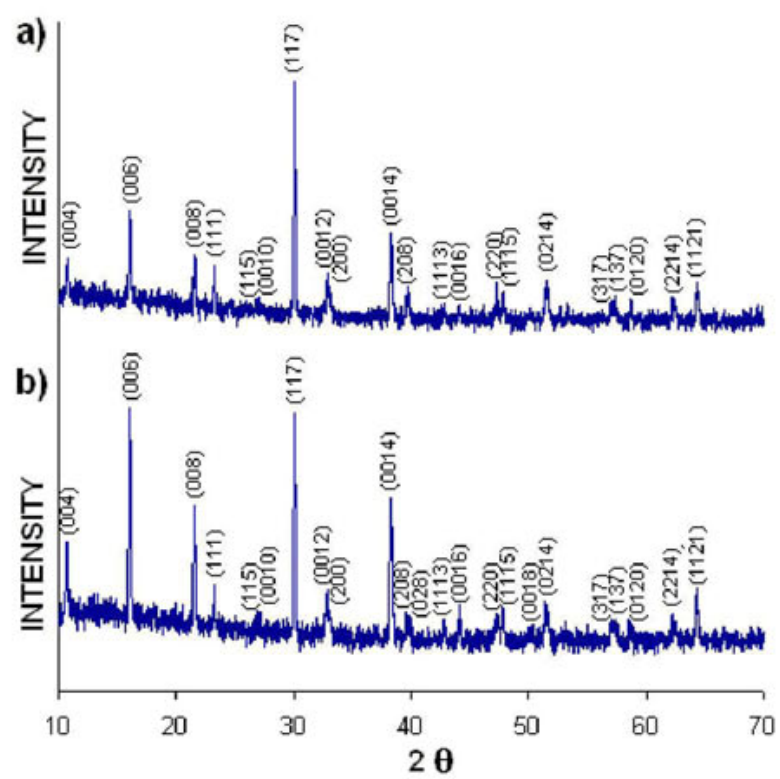

Figure 2. XRD patterns of $\mathrm{Bi}_{4} \mathrm{Ti}_{3} \mathrm{O}_{12}$ powders obtained at $900^{\circ} \mathrm{C}$ for (a) 30 and (b) $240 \mathrm{~min}$. 
a)

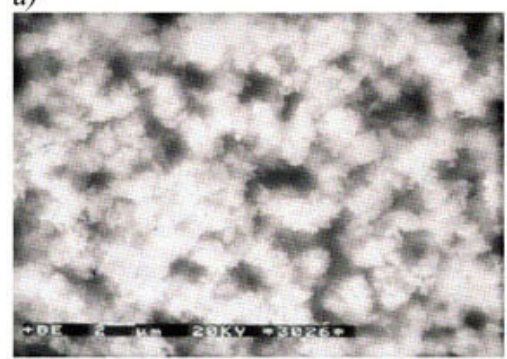

c)

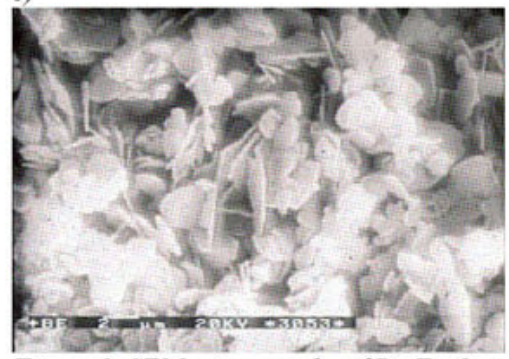

b)

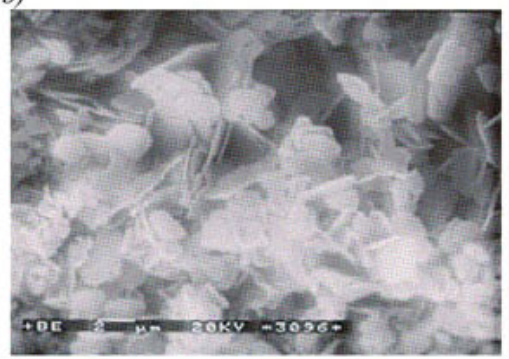

d)

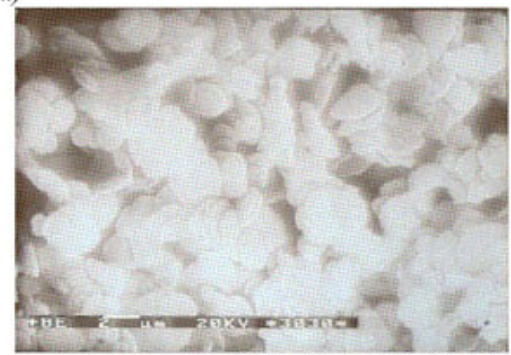

Figure 3. SEM micrographs of Bi $4 \mathrm{Ti}_{3} \mathrm{O}_{12}$ powders obtained at (a) $700^{\circ} \mathrm{C}$, (b) $800^{\circ} \mathrm{C}$, (c) $900^{\circ} \mathrm{C}$ and (d) $1000^{\circ} \mathrm{C}$ for $240 \mathrm{~min}$; magn. $x 9000$.

a)

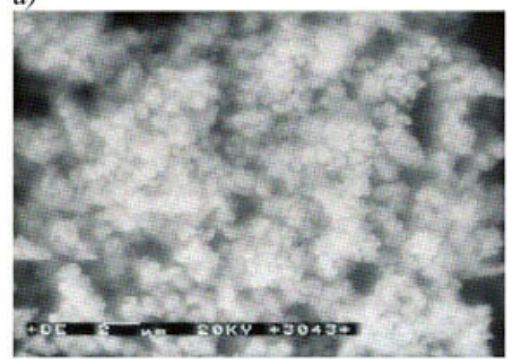

c)

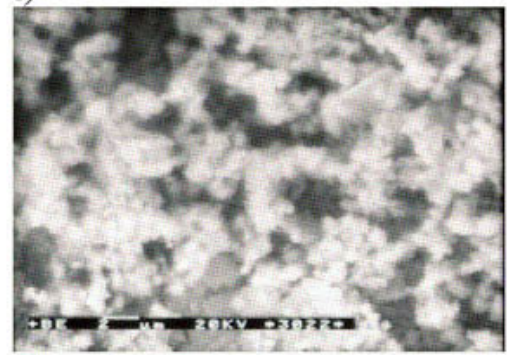

b)

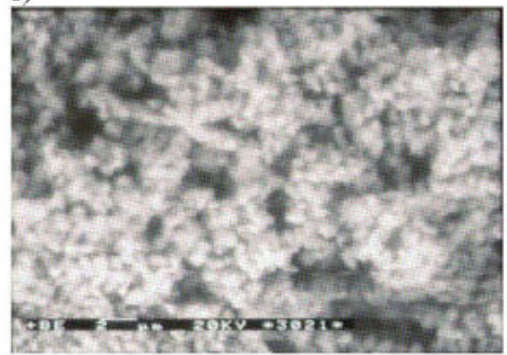

d)

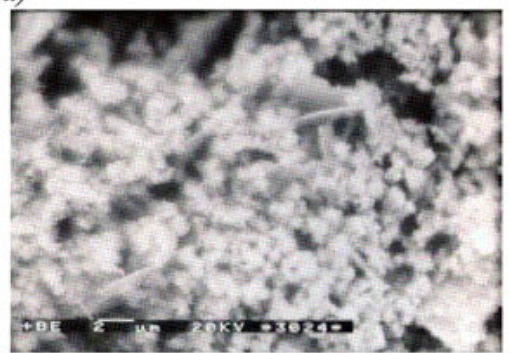

Figure 4. SEM micrographs of Bi $4{ }_{4} i_{3} \mathrm{O}_{12}$ powders obtained at $700^{\circ} \mathrm{C}$ for (a) 15 , (b) 30 , (c) 60 and (d) 120 min; magn. $x 9000$. 
a)

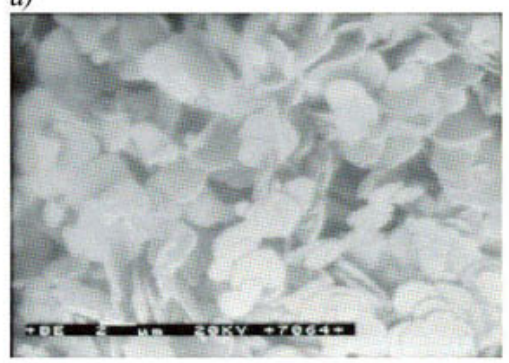

c)

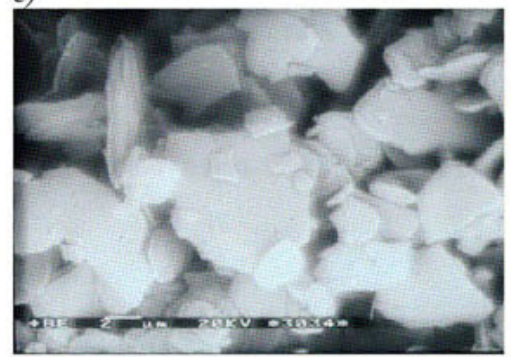

b)

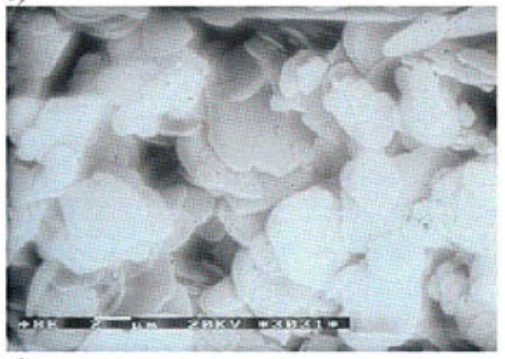

d)

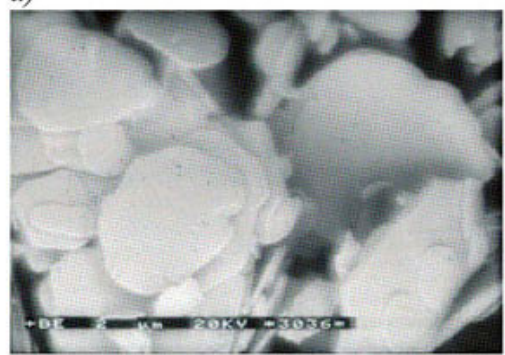

Figure 5. SEM micrographs of $\mathrm{Bi}_{4} \mathrm{Ti}_{3} \mathrm{O}_{12}$ powders obtained at $1100^{\circ} \mathrm{C}$ for (a) 15 , (b) 30 , (c) 60 and (d) 120 min; magn. $x 9000$.

\section{Conclusions}

Anisotropic-shaped bismuth titanate $\left(\mathrm{Bi}_{4} \mathrm{Ti}_{3} \mathrm{O}_{12}\right)$ crystals were obtained by the molten salt synthesis method in $\mathrm{NaCl}-\mathrm{KCl}$. It was found that the temperature affects the phase composition, the shape and the size of the formed crystals. Pure crystalline $\mathrm{Bi}_{4} \mathrm{Ti}_{3} \mathrm{O}_{12}$ was formed at $800^{\circ} \mathrm{C}$. Much larger crystals were grown at $1100^{\circ} \mathrm{C}$. Scanning electron microscopy and Xray diffraction techniques revealed the relationship of crystal size and crystalline orientation; the intensities of reflections emerging from $(00 l)$ planes increase with increasing crystal size.

\section{References}

1. Kong, L.B., Ma, J., Zhu, W. \& Tan, O.K., 2001, Mater. Lett., 51, 108.

2. Yang, Q., Li, Y., Yin, Q., Wang, P. \& Cheng, Y.B., 2003, J. Eur. Ceram. Soc., 23, 161.

3. Pookmanee, P., Uriwilast, P. \& Phanichpant, S., 2004, Ceram. Int., 30, 1913.

4. Patwardhan, J.S. \& Rahaman, M.N., 2004, J. Mater. Sci., 39, 133.

5. Kan, Y., Jin, X., Wang, P., Li, Y., Cheng, Y.B. \& Yan, D., 2003, Mater. Res. Bull., 38, 567 . 
6. Kimura, T. \& Yamaguchi, T., 1987, in Advances in Ceramics, vol. 21, Ceramic Powder Science, edited by K.S. Mazdiyashi, J.W. McCauley \& R.A. Haber (Westerville, OH: The American Ceramic Society, Inc.), pp. 169-177.

7. Kimura, T. \& Yamaguchi, T., 1983, in Ceramic Powders, edited by P. Vincenzini (Amsterdam: Elsevier), pp. 555-564.

8. Kimura, T. \&Yamaguchi, T., 1983, Ceram. Int., 9, 13.

9. Thirumal, M., Jain, P. \& Ganguli A.K., 2001, Mater. Chem. Phys., 70, 7.

Acknowledgements. The author wishes to thank Teresa Buczek for SEM work. 
IV.5 Organic Materials 



\title{
Nanophase-separated diblock copolymers: Structure investigations on PPMA- $b$ - PMMA using X-ray scattering methods
}

\author{
D. Jehnichen ${ }^{1, *}$, D. Pospiech ${ }^{1}$, S. Ptacek ${ }^{1}$, K. Eckstein ${ }^{1}$, \\ P. Friedel $^{1}$, A. Janke ${ }^{1}$, C. M. Papadakis ${ }^{2}$ \\ ${ }^{1}$ Leibniz Institute of Polymer Research, Hohe Str. 6, D-01069 Dresden, Germany \\ ${ }^{2}$ Physikdepartment E13, Technische Universität München, James-Franck-Str. 1, \\ D-85747 Garching, Germany \\ *djeh@ipfdd.de
}

Keywords: diblock copolymers, nanophase separation, nanostructured surfaces, morphology characterisation, phase diagram

Abstract. Diblock copolymers show phase separation on the sub-micrometer scale thus giving rise to specially ordered morphologies both in bulk and thin films. One important question is to find out relationships between bulk morphology (determined mainly by molecular parameters, i.e., the block ratio, molar mass, thermal history), and morphologies in thin films, which are strongly influenced by film parameters and film thickness related to bulk domain spacings. PPMA- $b$-PMMA diblock copolymers with hexagonally close-packed cylinders in the bulk and varied molar masses but identical molar composition were chosen for this study. Thin films with thicknesses below and above the bulk domain spacing were prepared and examined using different X-ray scattering methods using grazing incidence techniques. Additionally, the experimentally found phase behaviour of the diblock copolymers was compared with the calculated phase diagram.

\section{Introduction}

Nanostructured materials based on diblock copolymers have got a vigorous interest due to the opportunity to exploit their microphase/nanophase separation behaviour for developing materials with nanostructures in bulk and films or at surfaces [1,2,3]. Because of the immiscibility of the blocks, block copolymers show self-organization and are able to form regular nanostructures on nm-length scales. The Flory-Huggins interaction parameter $\chi$, molar mass, polydispersity $M_{\mathrm{w}} / M_{\mathrm{n}}$ as well as the block length ratio $M_{\mathrm{w} 1} / M_{\mathrm{w} 2}$ control the phase separation in general, the type of ordered phases, as well as their thermal behaviour $[4,5]$. In thin films, the total molar mass has been recognized as important parameter determining the orientation of the nanostructure relative to the film surface $[6,7]$. 
This work aims to clarify the phase behaviour of poly(n-pentyl methacrylate- $b$-methyl methacrylate) diblock copolymers (PPMA- $b$-PMMA) in bulk and thin films. To analyse the morphology in confined dimensions, thin films on Si wafer were prepared by dip-coating in two different film thicknesses $f$, smaller and slightly bigger than the bulk domain spacings $d_{\text {bulk. }}$ In thin films with $f<d_{\text {bulk, }}$, lying (parallel) morphologies are expected to be suppressed due to confinement effects $[8,9]$. The phase behaviour in thin films was detected by AFM, Xray reflectometry (XR) and grazing-incidence small-angle X-ray scattering (GISAXS).

\section{Experimental}

Synthesis: PPMA-b-PMMA diblock copolymers (scheme 1) via sequential living anionic polymerization in THF [10]. Samples characterised by low polydispersities of $M_{\mathrm{w}} / M_{\mathrm{n}} \sim 1.1$. The sample characteristics are given in table 1.

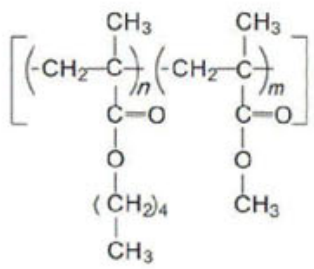

Scheme 1. Simplified chemical structure of PPMA-b-PMMA diblock copolymers.

Table 1. Chemical characterisation and bulk morphology results of used diblock copolymers.

\begin{tabular}{|c|c|c|c|c|c|c|}
\hline $\begin{array}{c}\text { sample } \\
\text { name }\end{array}$ & $\begin{array}{c}\text { sample com- } \\
\text { position }\end{array}$ & $\begin{array}{c}\text { volume } \\
\text { fraction } \\
\phi_{\text {PPMA }}\end{array}$ & $\begin{array}{c}\text { molar ratio } \\
\text { PPMA / PMMA } \\
\text { (mol/mol) }\end{array}$ & $\begin{array}{c}\text { bulk } \\
\text { morpho } \\
\text { logy }^{3+4)}\end{array}$ & $\begin{array}{c}\text { domain } \\
\text { spacing }^{3)} \\
d_{\text {bulk }}(\mathrm{nm})\end{array}$ & $\begin{array}{c}\text { unit cell }^{3)} \\
\boldsymbol{a}_{\text {hex }}(\mathrm{nm})\end{array}$ \\
\hline $\mathbf{1}$ & $\begin{array}{c}\mathbf{6 4 . 1 P M 2 0 . 2} \\
(\mathbf{8 4 . 3 )}\end{array}$ & 0.78 & $67.1 / 32.9$ & $\begin{array}{c}\text { cylin- } \\
\text { ders }\end{array}$ & 41.9 & 48.4 \\
\hline $\mathbf{2}$ & $\begin{array}{c}\mathbf{5 1 . 5 P M 1 6 . 2} \\
(\mathbf{6 7 . 7})\end{array}$ & 0.78 & $67.1 / 32.9$ & $\begin{array}{c}\text { cylin- } \\
\text { ders }\end{array}$ & 35.4 & 40.9 \\
\hline
\end{tabular}

${ }^{1)}$ molar mass by SEC (in kDa), $M_{\mathrm{w}}$ in brackets (P: PPMA, M: PMMA); ${ }^{2)}{ }^{1} \mathrm{H}-\mathrm{NMR} ;{ }^{3)} \mathrm{SAXS} ;{ }^{4)} \mathrm{TEM}$.

Mean field calculations: Phase diagram calculations and determination of spinodals by random phase approximation as shown in figure 1 based on data summarised in table 1 [11].

Thin film preparation: On $\mathrm{Si}(111)$ wafers by dip-coating from polymer solutions (1 or $2 \mathrm{wt} \%$ in THF) at room temperature with different withdrawal speeds.

$X$-ray Scattering / SAXS: Temperature-dependent small-angle X-ray scattering (T-SAXS) at the Soft Condensed Matter Beamline A2 (HASYLAB at DESY Hamburg); oven experiments with heating/cooling rates of $3 \mathrm{~K} / \mathrm{min}$.

$X$-ray Scattering / GISAXS: Grazing-incidence small-angle X-ray scattering at the Beamline BW4 (HASYLAB at DESY Hamburg). 2D-pattern within incidence angles $\alpha_{\mathrm{i}}\left(0.03 \ldots 0.30^{\circ}\right)$, close to the critical angles $\alpha_{c}$ of the polymer film (e.g., for pure PMMA and $\lambda=0.138 \mathrm{~nm}: \alpha_{c}$ $\left.\approx 0.148^{\circ}\right)$ and of the substrate $\left(\mathrm{SiO}_{\mathrm{x}}, \alpha_{c} \approx 0.20^{\circ}\right)$.

AFM measurements were performed using a Nanoscope IIIa Microscope Dimension 3100 scanning force microscope (Digital Instruments) in tapping mode. 


\section{Results and discussion}

Two PPMA- $b$-PMMA diblock copolymers with the same volume fraction $\phi_{\text {PPMA }} \sim 0.78$ both having hexagonal morphology in the bulk were chosen. Based on the sequence of reflections present in the scattering curves, the morphology in bulk can be classified as hexagonally close-packed cylinders (hcp; figure 1, see also table 1).

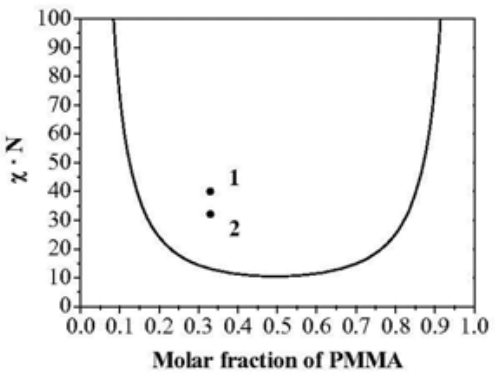

Figure 1. PPMA-b-PMMA diblock copolymer samples chosen for this study and their position in the phase diagram.

The spinodal (black line) represents diblock copolymers with a polydispersity of $M_{w} / M_{n}=1.0$.

For illustration, results of a typical SAXS experiment in the temperature range of $30-200{ }^{\circ} \mathrm{C}$ are shown in figure 2 . The first heating run is characteristic for the temperature-dependent behaviour of the as-synthesised samples, showing the formation of an ordered morphology above $T_{\text {g.PMMA }}\left(\sim 115^{\circ} \mathrm{C}\right)$. During the cooling run the morphology does not change. The morphology is stable even up to $\sim 300{ }^{\circ} \mathrm{C}$. Special temperature ranges determined by weakly positive as well as negative coefficients of linear expansion are quite well visible by changed positions of the strongest reflections. Phenomena like this will be investigated in more detail in extended T-dependent bulk and film experiments in the near future.

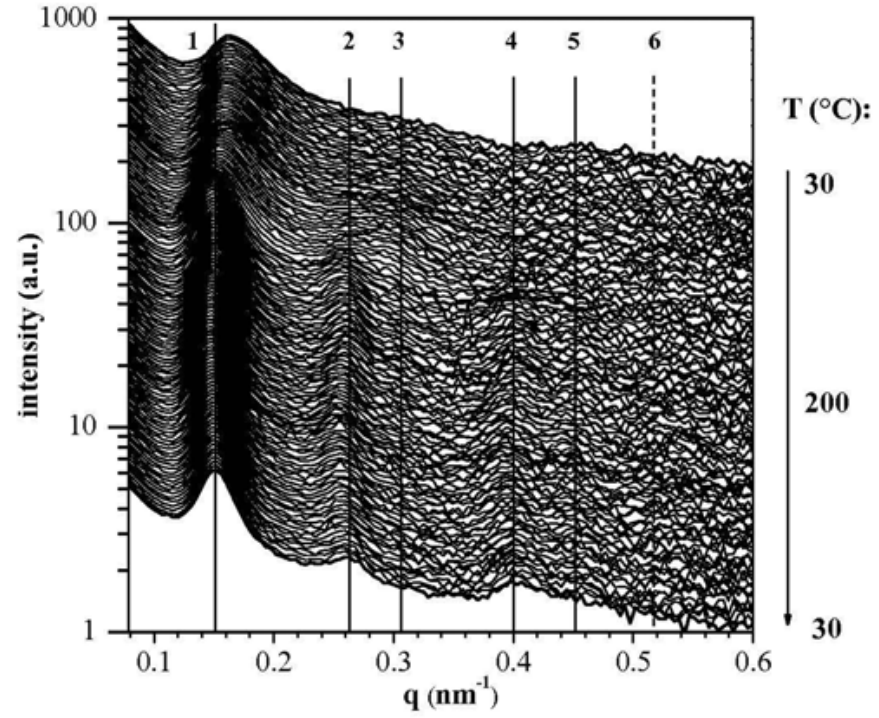

Figure 2. Temperaturedependent SAXS curves of the cylindrical sample 1 having an hcp unit cell with $\mathrm{a}_{\text {hex }}=48.4 \mathrm{~nm}$.

Reflection assignment: $1-\mathrm{d}_{(100)}=41.9 \mathrm{~nm}, 2-$ $\mathrm{d}_{(110)}, 3-\mathrm{d}_{(200)}, 4-\mathrm{d}_{(210)}$, $5-\mathrm{d}_{(300)} ;$ expected: 6 $d_{(220)}$. The corresponding reflection ratios are $1: \sqrt{3}: 2: \sqrt{7}: 3: \sqrt{12}$.

Single curves are shifted vertically. 
The dip-coating conditions chosen permitted to produce films with desired thicknesses smaller and little bigger than $d_{\text {bulk }}$, confirmed by data of both XR and ellipsometry. For all samples, GISAXS and AFM measurements were performed and the results are compared in figures 3 and 4 . Ordered morphologies lateral $\left(q_{\|}\right)$as well as parallel $\left(q_{\perp}\right)$ to the surface were found in confining thin film geometries. The results are summarised in table 2 . It seems to be typical for laterally nanostructured films of PPMA- $b$-PMMA diblock copolymers that there is only a single peak in the scattering profiles along $q_{\|}$. That means, primarily only one repeating distance ( $d$-spacing) can be derived from the single maximum position. For a reliable characterisation of the thin film morphology, GISAXS maps have to be taken and discussed together with results from AFM and transmission SAXS of bulk samples.

Table 2. Results of thin film investigations (samples annealed $48 \mathrm{~h}$ at $140^{\circ} \mathrm{C}$ before measurement).

\begin{tabular}{|c|c|c|c|c|c|c|}
\hline & \multicolumn{2}{|c|}{ film thickness } & \multicolumn{2}{|c|}{ film morphology } & \multicolumn{2}{|c|}{ domain spacings ${ }^{1)}$} \\
\hline & $\begin{array}{l}\text { ellipsom } \\
f_{\text {ell }}(\mathrm{nm})\end{array}$ & $\begin{array}{c}X R \\
f_{\mathrm{XR}}(\mathrm{nm})\end{array}$ & $A F M$ & GISAXS & $\begin{array}{c}A F M \\
d_{\mathrm{AFM}}(\mathrm{nm})\end{array}$ & $\begin{array}{l}\text { GISAXS } \\
d_{\mathrm{X}}(\mathrm{nm})\end{array}$ \\
\hline \multirow[t]{2}{*}{1} & $\sim 25$ & 26.7 & standing $M$ cylinders & $\begin{array}{l}\text { standing cyl- } \\
\text { inders (hcp) }\end{array}$ & $\sim 45$ & 41.8 \\
\hline & $\sim 50$ & 48.6 & lying curved $\mathrm{M}$ cylinders & lying cylinders & $\sim 46$ & 43.6 \\
\hline \multirow[t]{2}{*}{2} & $\sim 20$ & 17.5 & $\begin{array}{l}\text { lying curved M cyl., few } \\
\text { single standing cyl. }\end{array}$ & lying cylinders & $\sim 36$ & 35.3 \\
\hline & $\sim \mathbf{5 0}$ & 49.0 & lying curved $\mathrm{M}$ cylinders & lying cylinders & $\sim 37$ & $35.7^{2)}$ \\
\hline
\end{tabular}

${ }^{1)} d_{X}$ values from GISAXS out-of-plane peak in $\log (I) v s . q_{\|}$at $q_{\perp} \approx q_{\text {Yoneda }} ;{ }^{2)}$ broad distributed.

Within the sample set, figure $3 a$ (sample 1 25) shows the best lateral periodic demixing. The periodicity fits to hep cylinder morphology. The film has a thickness significantly smaller than $d_{\text {bulk }}$ (see figure 2), which is expected to hamper lying cylinder structures. AFM examination indeed reveals circular dots with locally hexagonal order with a distance of $\sim 45 \mathrm{~nm}$. This surface pattern can be attributed to hep arrangement of standing cylinders. Expecting almost the same $d$-spacing in the film than in the bulk, the lateral correlation peak should correspond to a very similar value in the case of standing cylinders. Then, the peak in the GISAXS map can be assigned as (100) reflection of the hexagonal morphology. For comparison, in a fictive case of lying cylinders, the correlation peak would be expected to correspond to the cylinder-cylinder distance (i.e. the $a$-axis of the unit cell) at $\sim 48.4 \mathrm{~nm}$ based on the SAXS result. GISAXS gives a value of $41.8 \mathrm{~nm}$, which is close to the bulk result and confirms the presence of standing cylinders. The arrangement of cylinders in films is determined by the ratio of the film thickness and the domain spacing of the hexagonal lattice [12]. Both GISAXS and AFM consistently indicated the existence of standing cylinders.

A thicker film (sample 1 50) displays a lateral correlation as well (figure 3b). The film thickness is slightly larger than $d_{\text {bulk, }}$, so one can assume that inside the film only one "layer" of hexagonally staggered lying cylinders is arranged, which should give rise to a correlation peak corresponding to the (100) reflection of a deranged hexagonal unit cell. This was indeed observed. The out-of-plane peak in the GISAXS map corresponds to a slightly enlarged $a$ axis $(\sim 50.4 \mathrm{~nm}$ compared to $\sim 48.4 \mathrm{~nm})$. 

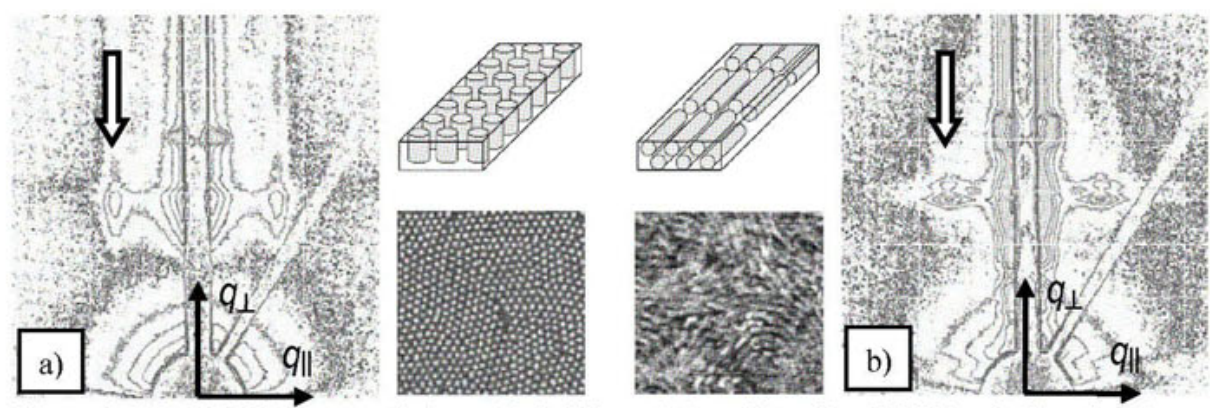

Figure 3. 2D-GISAXS pattern of a) sample 1 25 consisting of standing PMMA cylinders (hcp in extended areas) (normal to the surface); b) sample 1 50 consisting of short lying bent PMMA cylinders (parallel to each other in limited areas). Lateral correlation peaks assigned by arrows.

For illustration see pictograms. Corresponding AFM images are in between (phase contrast, $1 \times 1 \mu m^{2}$ ).
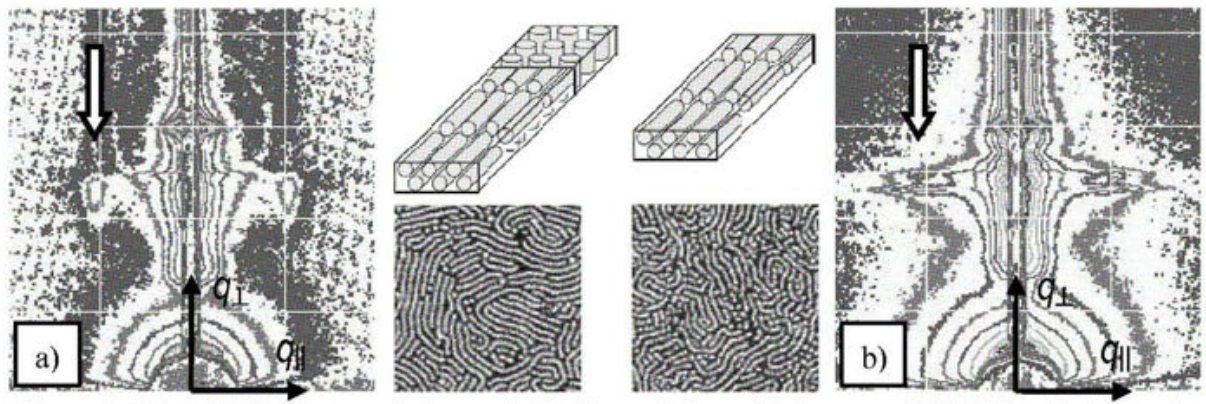

Figure 4. 2D-GISAXS pattern of a) sample 2 20 consisting of lying curved PMMA cylinders and a small amount of standing cylinders; b) sample 2 50 consisting of lying curved PMMA cylinders.

For sample 2 20 (with lower molar mass than sample 1, but same composition) a morphology with hep cylinders would be expected, too. Contrary, figure 4a shows a mixed morphology with lying, curved cylinders and only few standing cylinders. This gives rise to the conclusion that for the development of standing cylinder morphologies under the preparation conditions chosen the condition $f / d \sim 1$ is not sufficient. It seems necessary to have a much lower film thickness than $d_{\text {bulk. }}$.

Figure $4 \mathrm{~b}$ reflects the results for sample $\mathbf{2} \sim \mathbf{5 0}$. From the $f / d$ ratio the formation of lying cylinder morphology can be expected. Both GISAXS and AFM results confirm this expectation, showing lying cylinder morphology. However, the broadly distributed out-of-plane peak found in the GISAXS map (not displayed) suggests a slightly disturbed surface morphology, which results in a broader distribution of repeating distances between cylinders and more strongly bent cylinders. The AFM image in figure $4 \mathrm{~b}$ resembles this conclusion.

\section{Summary and conclusions}

The systematic search for laterally ordered and demixed morphologies is an essential precondition to obtain surfaces with regions of different properties, which can serve as template 
for selective tethering of different species (e.g., nanoparticles). In all cases, the dip-coated thin films formed nanostructured surfaces. The periodicities of nanostructures in thin films were comparable to the periodicities obtained in bulk samples. The bulk structure (hcp cylinders) was partly disordered in the thin films. The arrangement of cylinders (standing or lying) was governed by the film thickness dependent on the molar mass of the block copolymer. Vertically standing cylinders were formed for film thicknesses $f$ smaller than $d_{\text {bulk }}$ only in the case of the sample with higher molar mass. Significantly thicker films $(f \gg d)$ were not suited for laterally structured surfaces with desired pattern in this class of diblock copolymers. An open question is whether or not the films obtained by dip-coating and postannealing are in thermodynamic equilibrium. This has to be clarified in future investigations. In summary it can be noted that the combination of AFM and GISAXS is advisable in order to uniquely determine the thin film morphology. GISAXS offers the possibility to acquire structural information inside the film both in normal (i.e. along the film normal) and lateral (i.e. in the film plane) direction and averages over a large sample area. Otherwise, AFM images represent mostly a surface image with detailed information about a smaller scan area.

\section{References}

1. Hamley, I.W., 2003, Nanotechnology, 14, R39.

2. Lazzari, M. \& López-Quintela, M.A., 2003, Adv. Mater., 15, 1583.

3. Park, C., Yoon, J. \& Thomas, E.L., 2003, Polymer, 44, 6725.

4. Leibler, L., 1980, Macromolecules, 13, 1602.

5. Benoit, H. \& Hadziioannou, G., 1988, Macromolecules, 21, 1449.

6. Busch, B., Posselt, D., Smilgies, D.-M., Rheinländer, B., Kremer, F. \& Papadakis, C.M., 2003, Macromolecules, 36, 8717; 2006, Macromolecules, 39, 3098 (erratum).

7. Potemkin, I.I., Busch, P., Posselt, D., Smilgies, D.-M. \& Papadakis, C.M., 2007, Macromol. Rapid Commun., 28, 579.

8. Jehnichen, D., Pospiech, D., Keska, R., Ptacek, S., Janke, A., Funari, S.S., Timmann, A. \& Papadakis, C.M., J. Nanostruct. Polym. Nanocomp., (acc. 09/2008).

9. Fasolka, M.J. \& Mayes, A.M., 2001, Annu. Rev. Mater. Res., 31, 323.

10. Kęska, R., Pospiech, D., Eckstein, K., Jehnichen, D., Ptacek, S., Häußler, L., Friedel, P., Janke, A. \& Voit, B., 2006, J. Nanostruct. Polym. \& Nanocomp., 2, 43.

11. Friedel, P., John, A., Netz, R.R., Pospiech, D. \& Jehnichen, D., 2002, Macromol. Theory Simul., 11, 785; John, A., Friedel, P., Pospiech, D., Jehnichen, D. \& Kunert, C., 2004, Macromol. Theory Simul., 13, 702.

12. Russell, T.P., Menelle, A., Anastiadis, S.H., Satija, S.K. \& Majkrzak, C.F., 1991, Macromolecules, 24, 6263.

Acknowledgements. We thank all unmentioned colleagues of the IPF, participating in synthesis and characterization of the polymers. We gratefully acknowledge financial support by and participation in EU NoE "NANOFUN-POLY" and the German Science Foundation. We are grateful to the HASYLAB staff of the Beamlines A2 and BW4. 


\section{Structure and polymorphism of trans mono-unsaturated triacylglycerols}

\section{J. B. van Mechelen ${ }^{*}$, R. Peschar, H. Schenk}

University of Amsterdam, HIMS/FNWI/Crystallography, Valckenierstraat 65, 1018 XE Amsterdam, The Netherlands

*j.b.vanmechelen@uva.nl

Keywords: powder diffraction, trans mono-unsaturated triacylglycerols, $\beta$ polymorph, $\beta$ ' polymorph, TAG

Abstract. Crystal structures of $\beta$ and $\beta^{\prime}$ polymorphs of trans mono-unsaturated and related trisaturated triacylglycerols, including a novel type of $\beta^{\prime}$ polymorph, have been solved from high resolution synchrotron and laboratory X-ray powder diffraction data by direct space methods. The influence of replacement of a saturated chain by a trans mono-unsaturated chain is discussed.

\section{Introduction}

Appropriately chosen blends of fats determine the physical properties of many food products like bakery products and table spreads. Soft natural oils often have to be hardened to give a blend the desired properties. Hydrogenation is a widespread applied hardening treatment with an undesired side effect: the (partial) isomerization of cis double bonds in trans double bonds, for example transformation of oleic acid (cis-9-octadecanoic acid; O) in elaidic acid (trans-9-octadecanoic acid; E). Trans fatty acids, commonly regarded as a health risk [1], supposedly replace their saturated analogues in the solid state but in fact little is known about their actual packing.

In absence of single crystals, X-ray powder diffraction (XRPD) turned out to be a realistic but challenging alternative to obtain crystal structures for a better understanding of the polymorphic stability and phase-transition behavior of trans mono-unsaturated triacylglycerols (TAGs), and related saturated ones.

\section{Experimental}

Samples of 1,3-dipalmitoyl-2-elaidoylglycerol (PEP) and 1,2-dipalmitoyl-3-elaidoylglycerol (PPE) have been obtained from Larodan Fine Chemicals $\mathrm{AB}$ (Malmö, Sweden) as $\beta-2$ powders. 1,3-dipalmitoyl-2-stearoylglycerol (PSP), 1,2-dipalmitoyl-3-stearoylglycerol (PPS) and 1-palmitoylglycerol-2,3-distearoyl (PSS) have been obtained from Unilever Research Laboratories (Vlaardingen, The Netherlands): PPS and PSS as $\beta$-2 powder, whereas from PSP a $\beta$ 2 powder as well as $\beta_{1}{ }_{1}-2$ powder was obtained. The $\beta^{\prime}{ }_{1}-2$ polymorphs of PEP, PPE, PPS and PSS were made by crystallization from the melt in a capillary. The $\beta_{1}{ }_{1}-2$ PSS transformed into a new, higher-melting $\beta^{\prime}$ polymorph (the $\beta_{0}^{\prime}-2$ ) in several weeks during storage at $295 \mathrm{~K}$. 
All samples have been prepared in glass capillaries. The structures of all solved polymorphs (five $\beta$-2, four $\beta^{\prime}{ }_{1}$-2 and one $\beta_{0}{ }_{0}-2$ polymorph) have been determined using high resolution XRPD data collected at various instruments (synchrotron ESRF: BM16, BM01B, ID31; inhouse: PANalytical X'pert Pro $\alpha 1$ ). All details on the data collection, structure solution and refinement have been published in two papers in Acta B, 2008 [2,3]. Melting points and phase transition points have been obtained using a PANalytical X'pert Pro MPD diffractometer that was equipped with an Oxford Cryostream Compact (Abbington, England) for temperature control. Temperature-resolved XRPD data have been collected in 1 min scans at a heating rate of $0.5 \mathrm{Kmin}^{-1}$. DSC measurements have been performed using a Linkam DSC600 (Linkam Scientific Instruments Ltd, Tadworth, England).

\section{Indexing, structure solution and refinement}

Dominant zones and peak overlap are severe problems in indexing of TAG powder patterns and make the chance of a successful indexing with standard indexing programs small [4,5]. We have been able to index the patterns of the solved structures with our own developed program LSQDETC [6] that uses a systematic search and with the program McMaille [7] in Monte Carlo mode both applying a limited solution space. Chekcell [8] was used for unit cell verification, unit cell refinement and space group selection. The $\beta-2$ structures have been solved in space group $P-1$. The most probable space group for the $\beta^{\prime}$ structures had to be selected in a trial and error procedure with FOX [9]. For the structure solution process FOX was used in parallel tempering mode. The $\beta_{1}{ }_{1}-2$ structures have been solved in space group 12 with two molecules in the asymmetric unit and the new PSS polymorph that is given the name $\beta^{\prime}{ }^{-}-2$ in space group $C 2 / c$. In all cases continuous critical guidance of the developing structural model was necessary to avoid getting locked in a local minimum with an unrealistic molecule conformation. GSAS [10] was used for structure refinement. Distance, angle and planar restraints had to be applied to stabilize the refinement. In figure 1 the Rietveld refinement of PEP $\beta^{\prime}{ }_{1-2}$ is given as an example of the series. A summary of the refined results of the $\beta-2$ and the $\beta^{\prime}-2$ structures has been published elsewhere [2,3]. Because TAGs usually do not diffract significantly beyond the fingerprint area (6 - $3 \AA$ ) the angle range included in the refinement is limited accordingly at the high angle side.

\section{Results and discussion}

The molecules of all polymorphs crystallize in seat-facing pairs (insert figure 1) with different molecule conformations. All $\beta-2$ polymorphs crystallize in P-1 with molecules in a [1-3] conformation: sn- 1 and sn-3 are packed parallel, sn-2 points in the opposite direction and in line with sn-1. The molecules are packed in flat double chain length layers (2-packs) (top figure 2). The $\beta_{1}^{\prime}-2$ polymorphs crystallize in I2 with 2 independent molecules in the asymmetric unit. Symmetric molecules (PSP, PEP) have a [1-2] conformation and asymmetric molecules (PPS, PPE) a [2-3] conformation. The molecule pairs have a characteristic $\beta^{\prime}$ bend in the middle and fill the unit cell with two 2-packs with the bend pointing in the same direction (middle figure 2). The new $\beta_{0}^{\prime}$-2PSS polymorph crystallizes in $C 2 / c$ and differs from the $\beta_{1}^{\prime}$-2 in having an opposite direction of the bend in the two 2-packs (bottom figure 2). Replacement of $\mathrm{S}$ by $\mathrm{E}$ does not influence the molecule packing. 


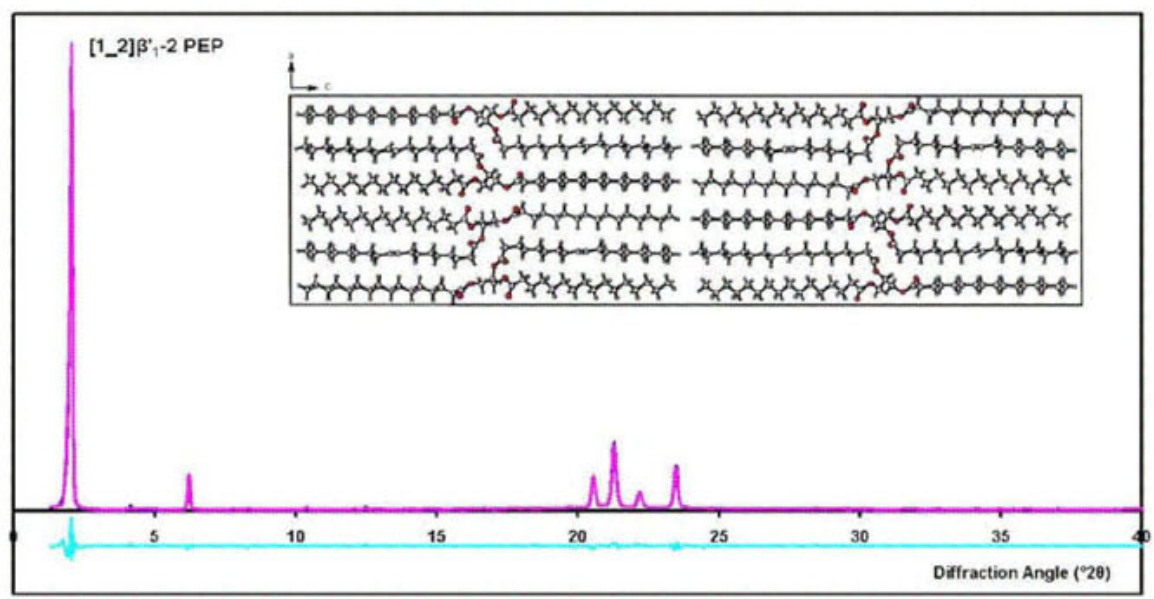

Figure 1. Rietveld refinement result of PEP $\beta_{1}^{\prime}{ }^{-2}\left(\mathrm{Cu} \mathrm{Ka} a_{1}\right.$ radiation, $N_{\text {refl. }}=664, N_{\text {ref. param. }}=986$, $\left.R_{p}=0.061, R_{w p}=0.086\right)$. The insert shows the pair wise seat facing packing of molecules.

Table 1. Phase transitions (K), melting temperatures $(K)$ and "life time" $(<>$, in minutes) of polymorphs during time and temperature resolved XRPD using a heating rate of $0.5 \mathrm{Kmin}^{-1}$.

\begin{tabular}{|c|c|c|c|c|c|c|c|}
\hline TAG & $\begin{array}{c}\alpha \text { to } \\
\beta_{2}^{\prime}{ }_{2}-2\end{array}$ & $\begin{array}{c}\beta_{2-2}^{\prime} \\
\text { lifetime' }\end{array}$ & $\begin{array}{c}\beta_{{ }_{2}-2}^{\prime} \\
\text { to } \beta^{\prime}{ }_{1}-2\end{array}$ & $\begin{array}{c}\beta_{{ }_{1}-2} \\
\text { 'lifetime' }\end{array}$ & $\beta_{{ }_{1}-2}^{\prime}$ & $\beta_{0}^{\prime}-2$ & $\beta-2$ \\
\hline PEP & 303 & $<18>$ & 312 & $<34>$ & 329 & - & 327 \\
\hline PSP & 317 & $<4>$ & 319 & $<48>$ & 343 & - & 339 \\
\hline PPE & 304 & $<14>$ & 311 & $<18>$ & 320 & - & 320 \\
\hline PPS & 321 & $<4>$ & 323 & $<18>$ & 332 & - & 338 \\
\hline PSS & 323 & $<14>$ & 330 & $<14>$ & 337 & 339 & 339 \\
\hline
\end{tabular}

Melting points and phase transition points determined by Temperature-resolved XRPD are listed in table 1. Additional information about phase transitions has been obtained from DSC measurements with the same temperature profile. The DSC trace clearly shows an exotherm related to the $\beta_{2}^{\prime}-2$ to $\beta_{1}^{\prime}-2$ transition, in contrast to mono-acid trisaturated TAGs like SSS [3]. Although the molecule packing is not influenced by replacement of S by $\mathrm{E}$, the melting point decreases considerably (table 1). In the time resolved XRPD experiment an E extends the "life time" of the unstable $\beta_{2}^{\prime}-2$ polymorph (table 1). The DSC signal of $\beta-2$ stable samples shows melting of $\alpha$ preceding the crystallization of $\beta_{2}^{\prime}-2$. The two $\beta_{1}^{\prime}-2$ stable samples PEP and PSP do not show such an $\alpha$ melt. This may be indicative for a different packing of 
chains in the $\alpha$ vs. $\beta_{2}^{\prime}-2$ polymorphs of $\beta$-2 stable samples (PPE, PPS, PSS) and a similar packing in $\beta_{2}^{\prime}$-2 stable samples (PEP, PSP). Another difference between these two groups is the stepped methyl end-plane in $\beta$-2 stable samples but a smooth methyl end-plane in $\beta_{2}^{\prime}$-2 stable samples. This explains the unusual lower-melting $\beta$-2 polymorph of PEP and PSP. All major peaks in the diffraction patterns can be explained as dominant zones that correlate with the packing of the acyl chains.

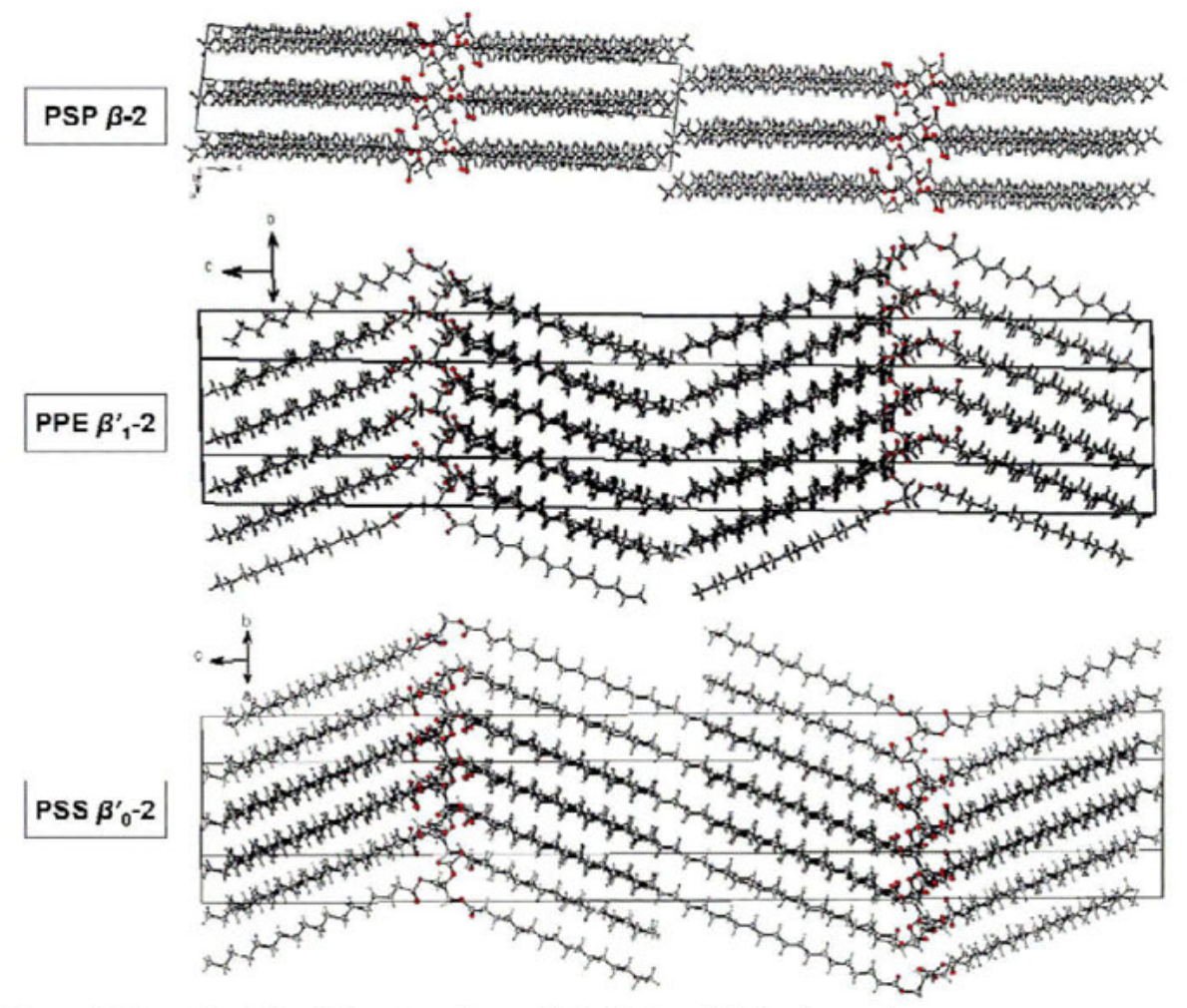

Figure 2. Examples of the different packings of $\beta-2, \beta_{1}^{\prime}-2$ and $\beta_{v^{\prime}}-2$ polymorphs.

\section{Conclusions}

Using synchrotron as well as high resolution laboratory XRPD data, crystal structures have been determined by direct space methods of the $\beta-2$ and $\beta_{1}^{\prime}-2$ polymorphs of PEP, PSP, PPS and PPE, and the $\beta$-2 as well as the novel $\beta_{0}^{\prime}-2$ polymorph of PSS. The structure solution was a challenging process as it was severely hampered by dominant zones and peak overlap. The structures provide an explanation for the unusual, lower melting point of the $\beta$-2 polymorph compared to that of the $\beta_{2}^{\prime}-2$ of PSP and PEP. The results show that replacement of a saturated $\mathrm{S}$ chain by a trans mono-unsaturated $\mathrm{E}$ chain does not change the crystal structure at the given resolution but influences physical parameters. 


\section{References}

1. 2004, EFSA Journal 81, 1.

2. Van Mechelen, J.B., Peschar, R. \& Schenk, H., 2008, Acta Cryst. B64, 240.

3. Van Mechelen, J.B., Peschar, R. \& Schenk, H., 2008, Acta Cryst. B64, 249.

4. Van Mechelen, J.B., Peschar, R. \& Schenk, H., 2006, Acta Cryst. B62, 1121.

5. Van Mechelen, J.B., Peschar, R. \& Schenk, H., 2006, Acta Cryst. B62, 1131.

6. Peschar, R., Etz, A., Jansen, J. \& Schenk, H., 2002, in Structure determination from powder diffraction data, edited by W.I.F. David, K. Shankland, L.B. McCusker \& Ch Baerlocher, Chapter 10.

7. Le Bail, A., 2004, Powder Diffr. 19, 249.

8. Laugier, J. \& Bochu, B., 2001, http//www.inpg.fr/LMPG.

9. Favre-Nicolin, V. \& Črný, R., 2002, J. Appl. Cryst. 35, 734.

10. Larson, A.C. \& Von Dreele, R.B., 1987, General Structure Analysis System, Los Alamos National Laboratory rep. no. LA-UR-86-748.

Acknowledgements. The authors thank Unilever Research for the PSP samples. The authors acknowledge the ESRF (Grenoble, France) for providing the facilities to perform the synchrotron diffraction experiments and they thank W. van Beek (BM01B), O. Masson (BM16) and I. Margiolaki (ID31) for their valuable help during the experimental sessions. The authors also thank E. Dova, K. Goubitz, R.B. Helmholdt, E. Sonneveld and M.M Pop (all UvA) for their help in data collection during the experimental sessions at the ESRF. Shell Research and Technology Centre Amsterdam is acknowledged for making the DSC cell available. The investigations have been supported by the Netherlands Foundation for Chemical Research (NWO/CW) with financial aid from the Netherlands Technology Foundation (STW; project 790.35.405). The members of the User Committee of this project are thanked for stimulating discussions and continuous interest. 



\section{Author Index}

Volume I: pp. 1-232

Volume II: pp. 233-495

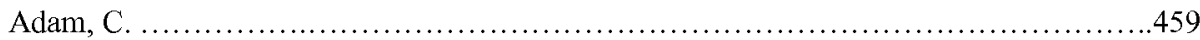

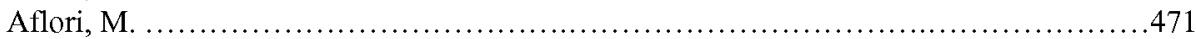

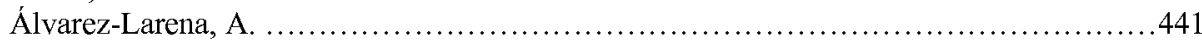

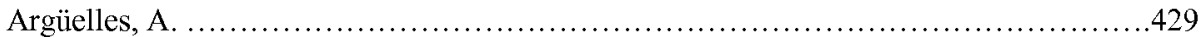

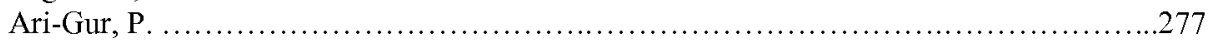

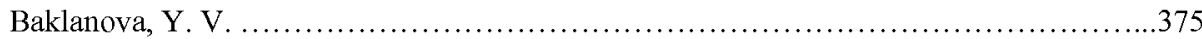

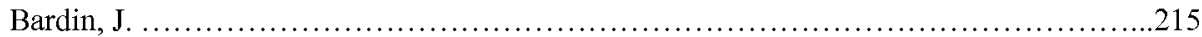

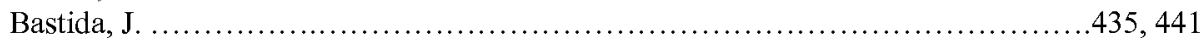

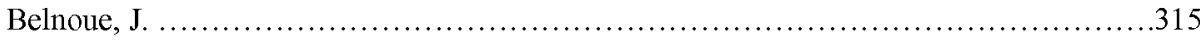

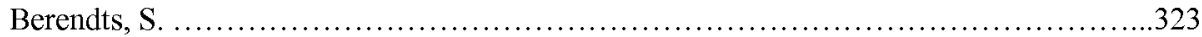

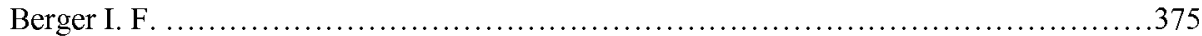

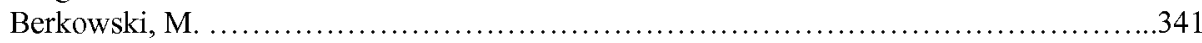

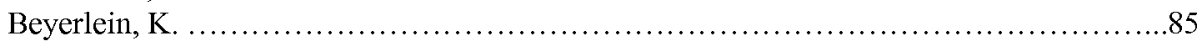

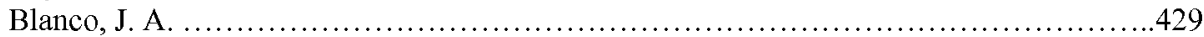

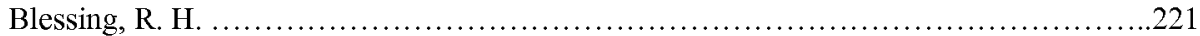

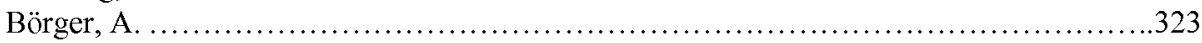

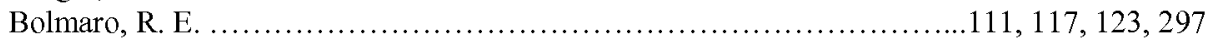

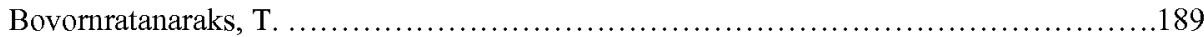

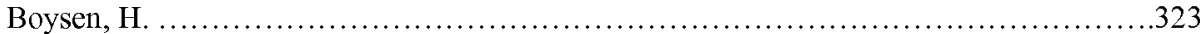

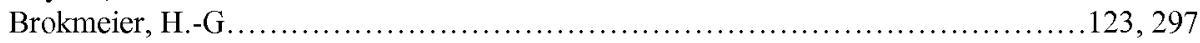

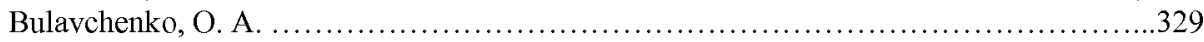

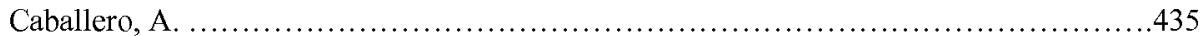

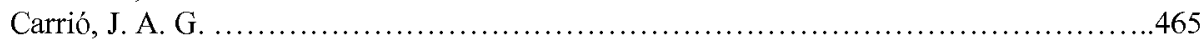

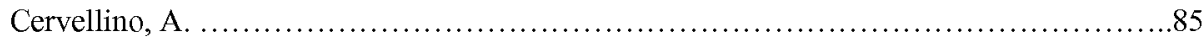

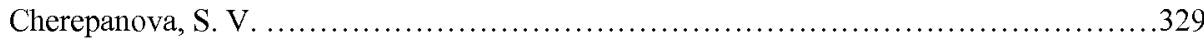

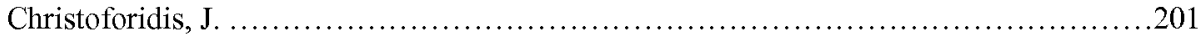

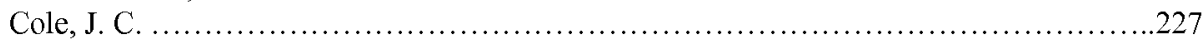

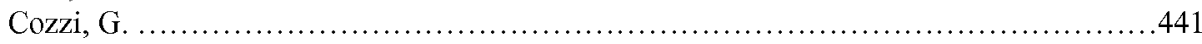

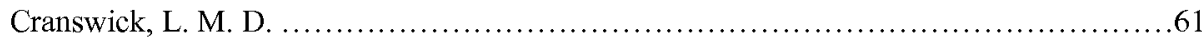

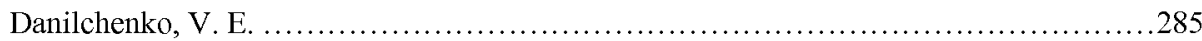

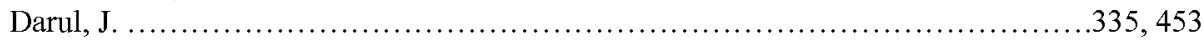

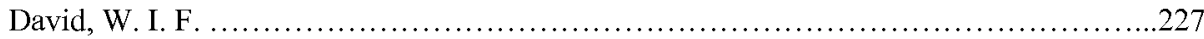

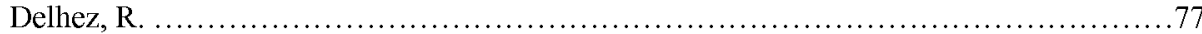

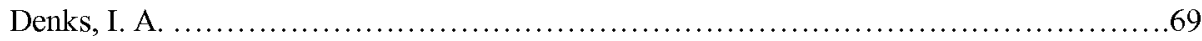

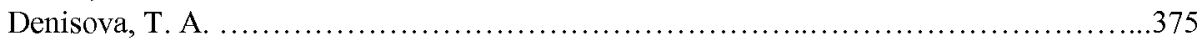

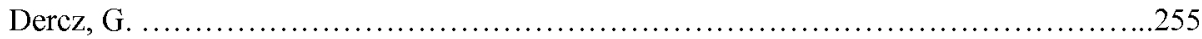

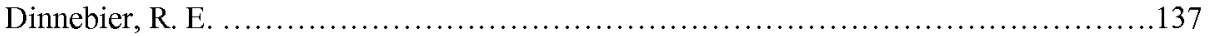




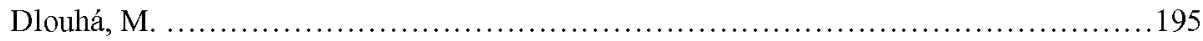

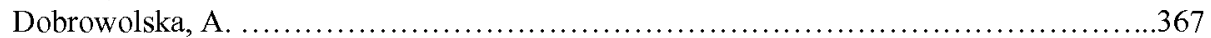

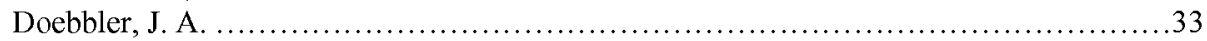

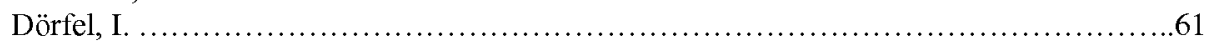

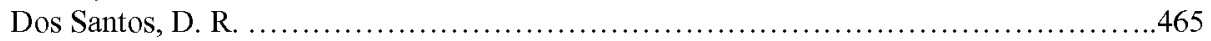

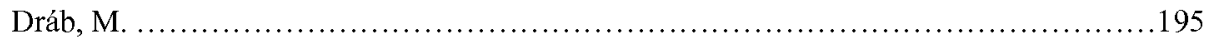

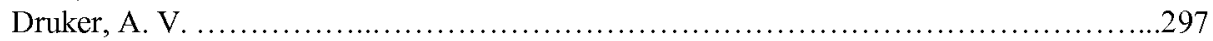

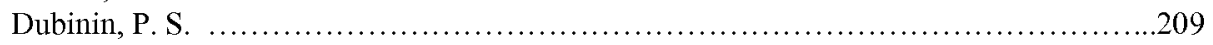

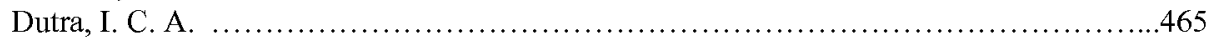

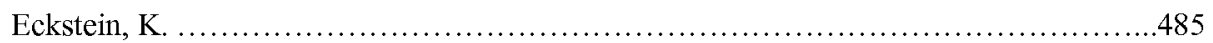

Ehrenberg, H. ........................................................... 407

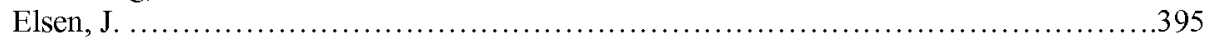

Emmerling, F. ......................................................... 61, 459

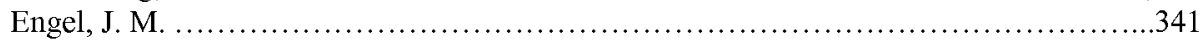

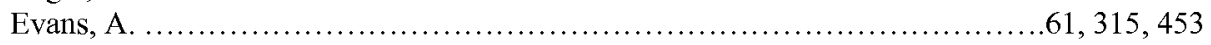

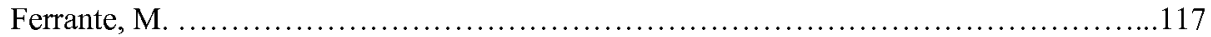

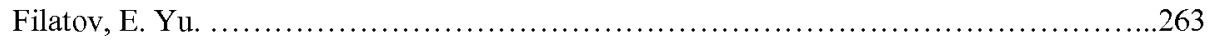

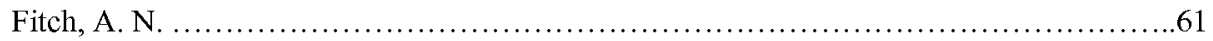

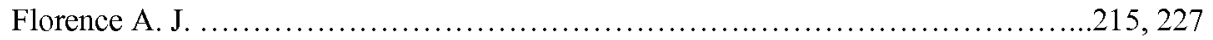

Fourty, A. ............................................................111, 123

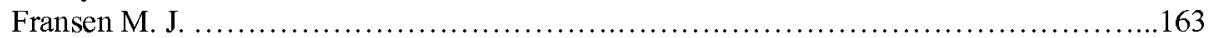

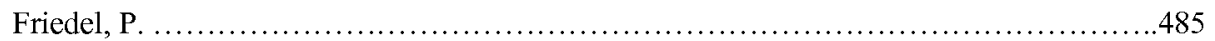

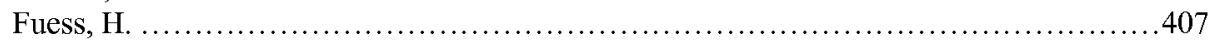

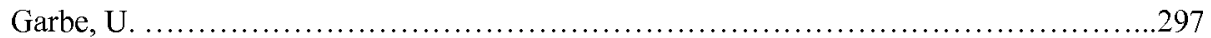

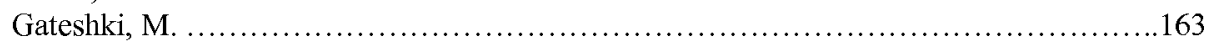

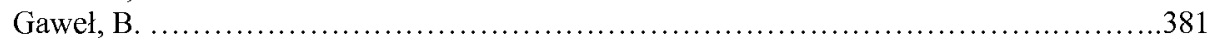

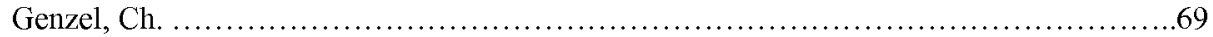

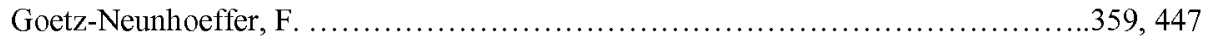

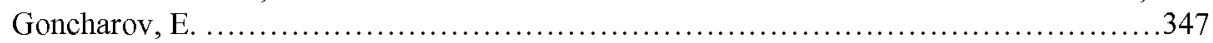

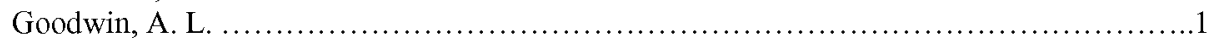

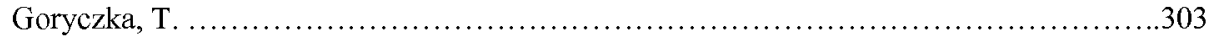

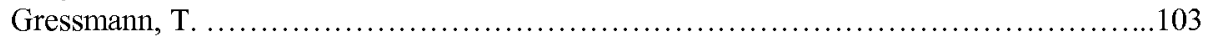

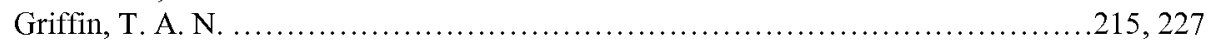

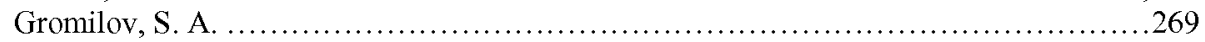

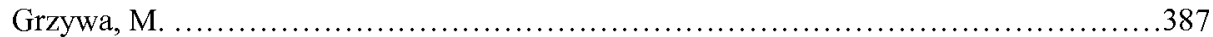

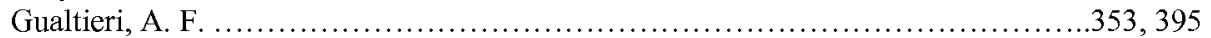

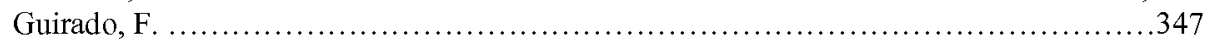

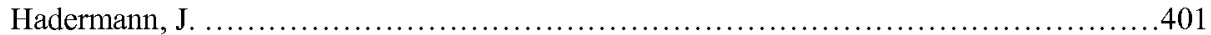

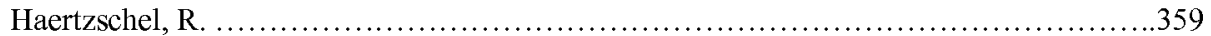

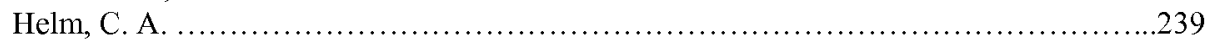

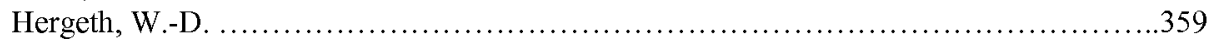

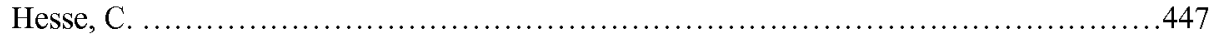

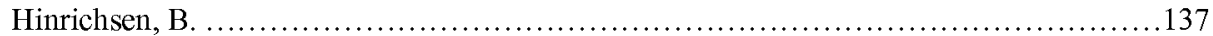


Hoelzel, M. ........................................................ 323

Hofmann, F. . . . . . . . . . . .

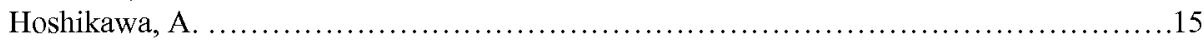

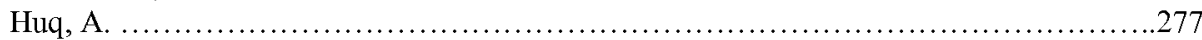

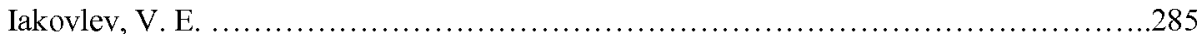

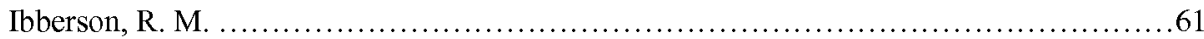

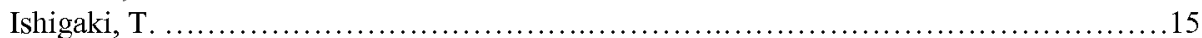

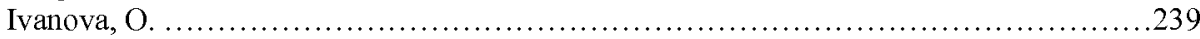

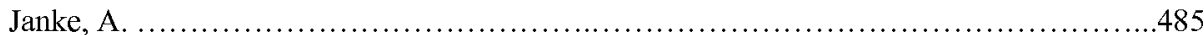

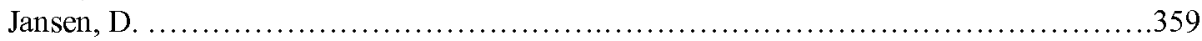

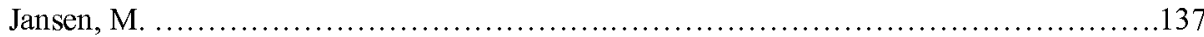

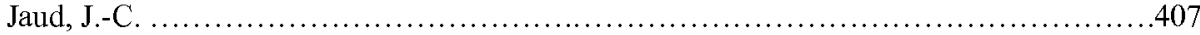

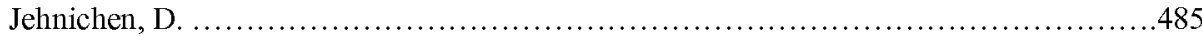

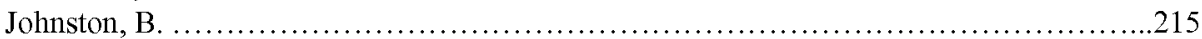

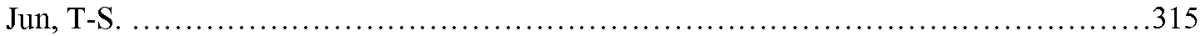

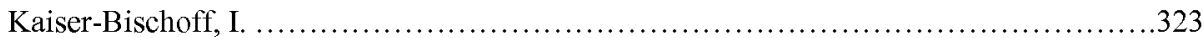

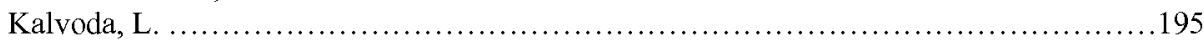

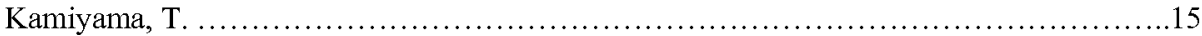

Kimmel, G. ........................................................171, 277, 347

Kleeberg, R. ....................................................................

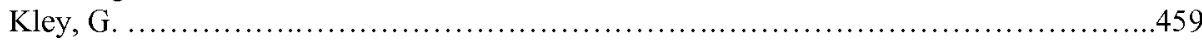

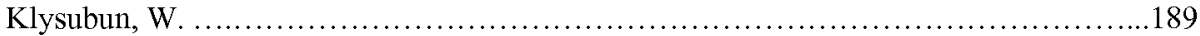

Kojdecki, M. A. .......................................................183, 435, 441

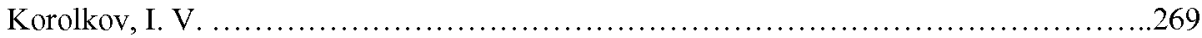

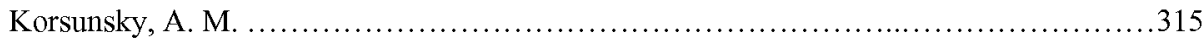

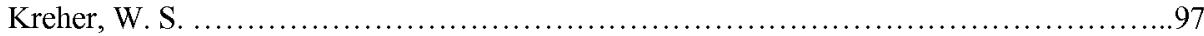

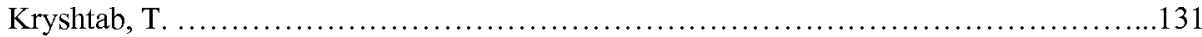

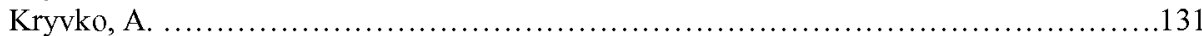

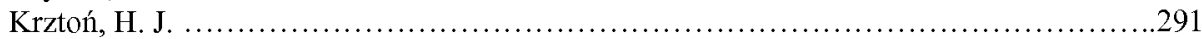

Kužel, R. .........................................................157, 233

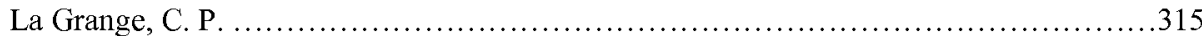

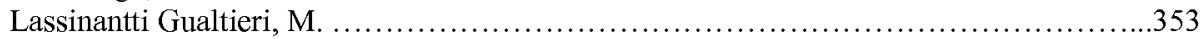

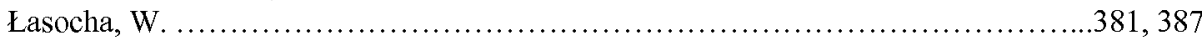

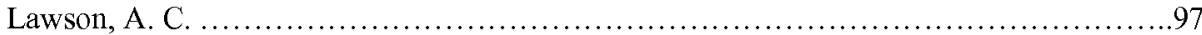

Leineweber, A. ..................................................... 97, 103, 423

Leoni, M. .......................................................... 85, 91, 423, 429

Leonidov, I. I. .............................................................. 401

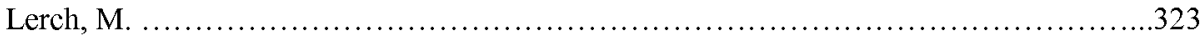

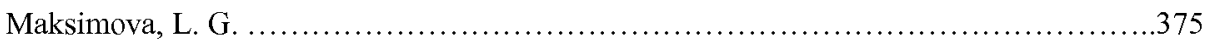

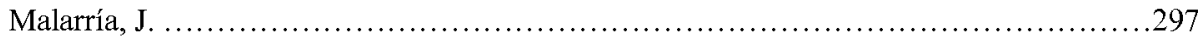

Malinowski, J. J. ..........................................................255

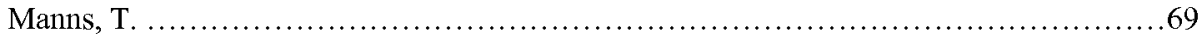




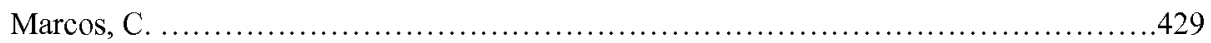

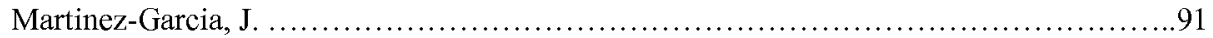

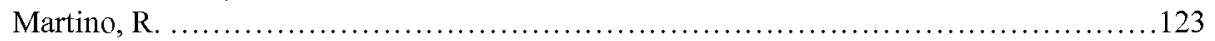

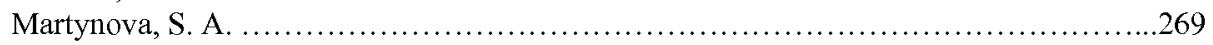

Matěj, Z. ........................................................... 157, 233

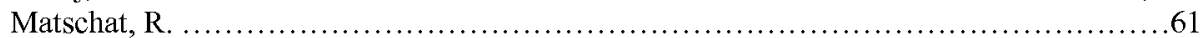

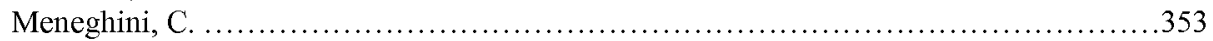

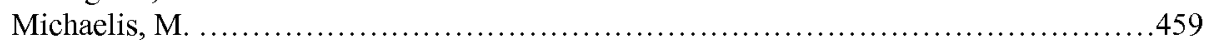

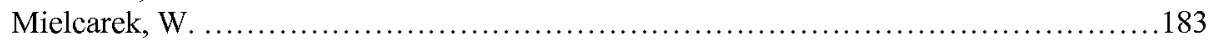

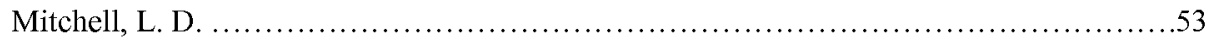

Mittemeijer, E. J. ..............................................99, 103, 245

Mogilyanski, D. .......................................................... 171

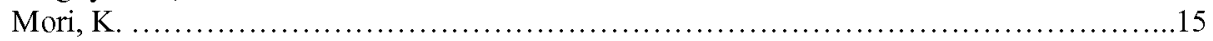

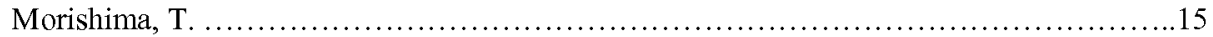

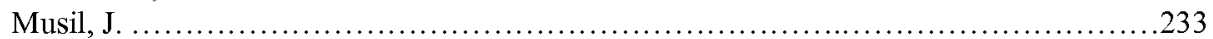

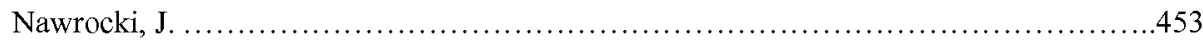

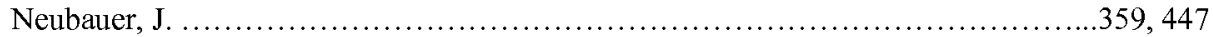

Nichtová, L. .................................................... 157, 233

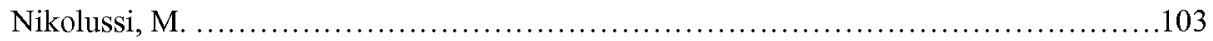

Nowicki, W. ........................................................415, 453

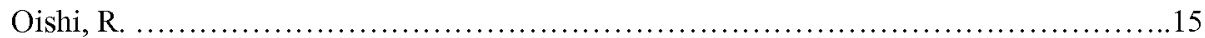

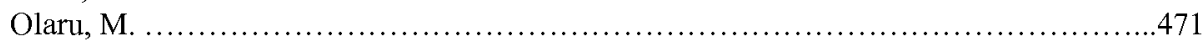

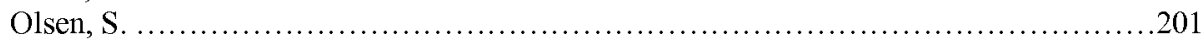

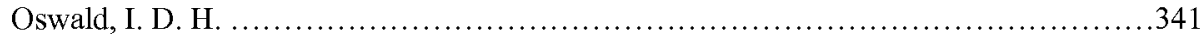

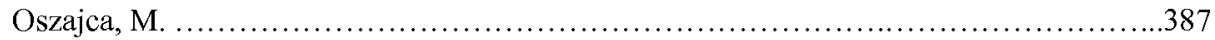

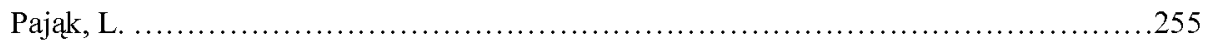

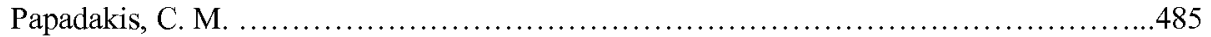

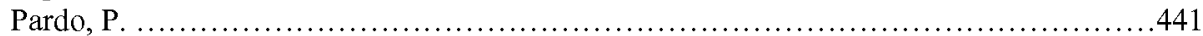

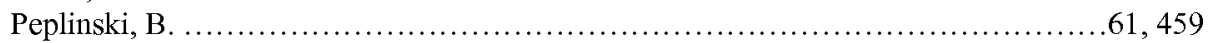

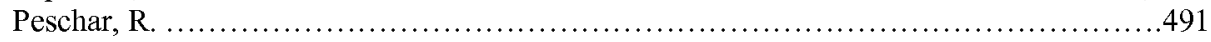

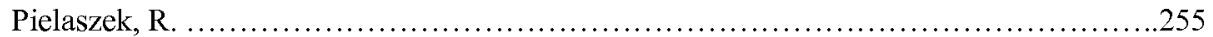

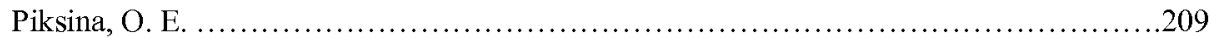

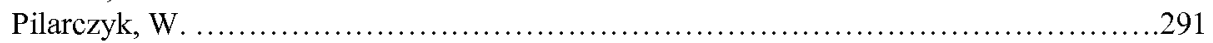

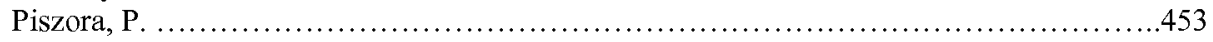

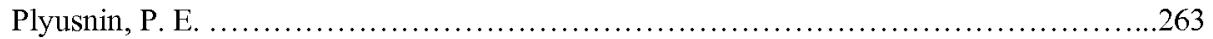

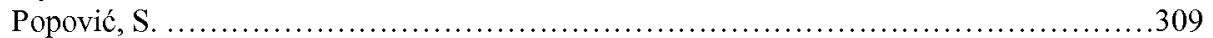

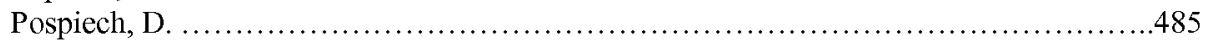

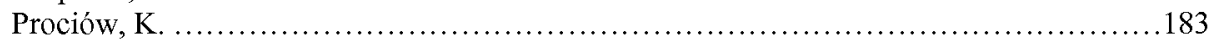

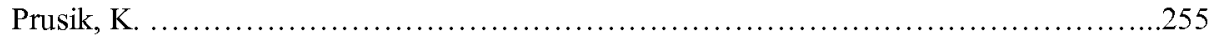

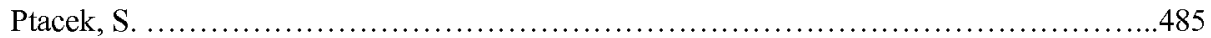

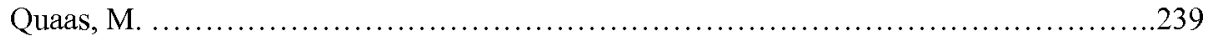




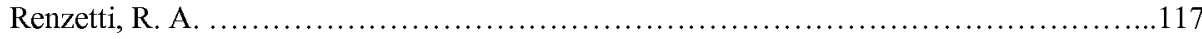

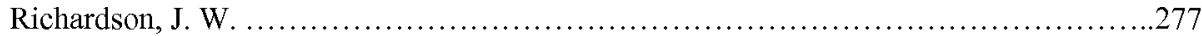

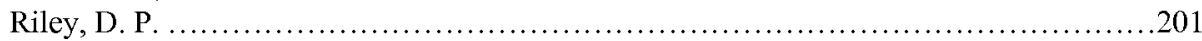

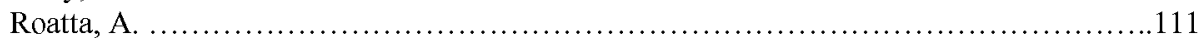

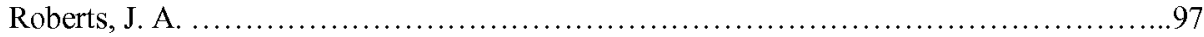

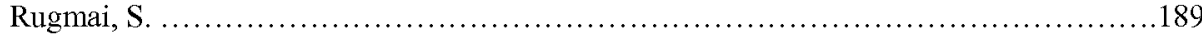

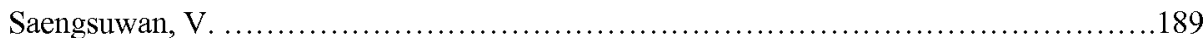

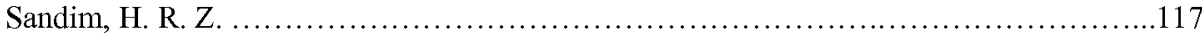

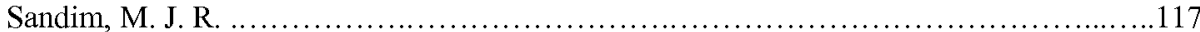

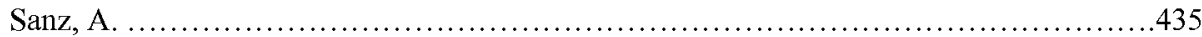

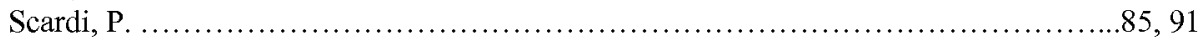

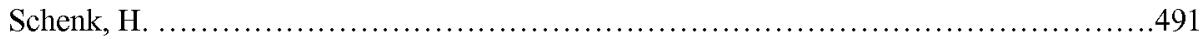

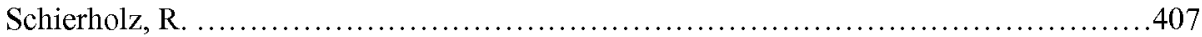

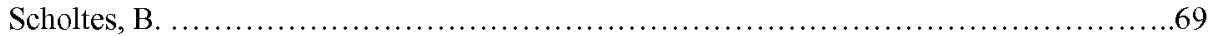

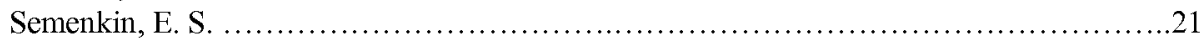

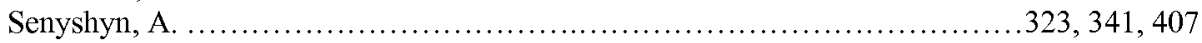

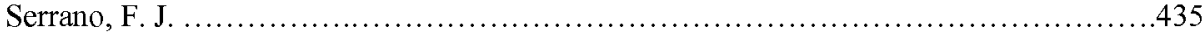

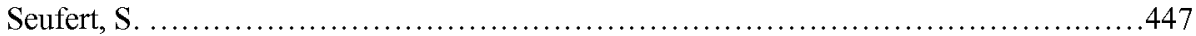

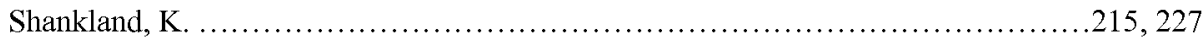

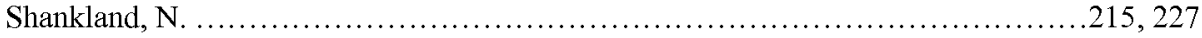

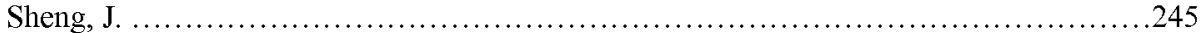

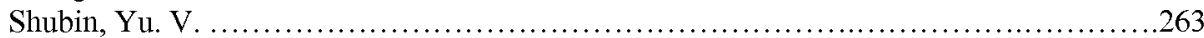

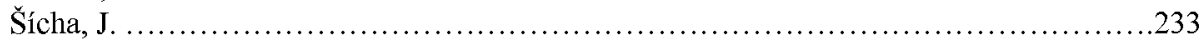

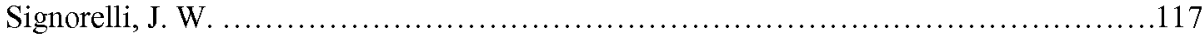

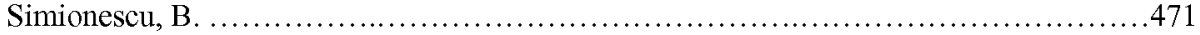

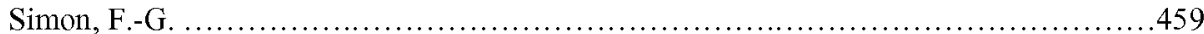

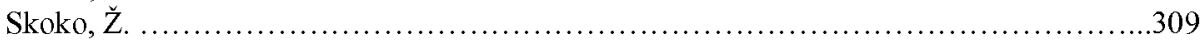

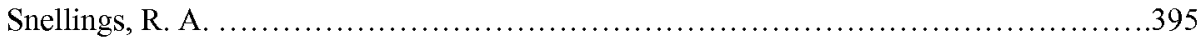

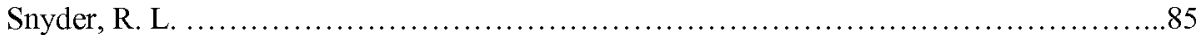

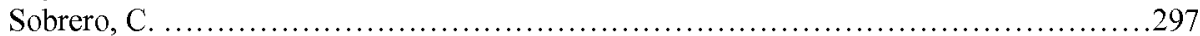

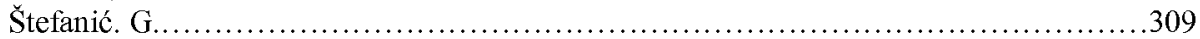

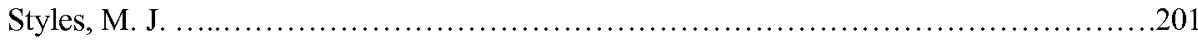

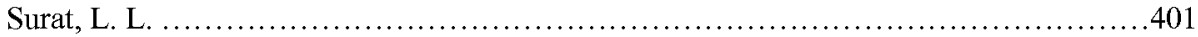

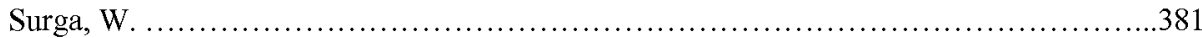

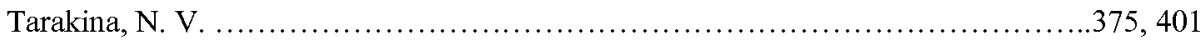

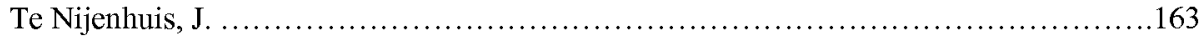

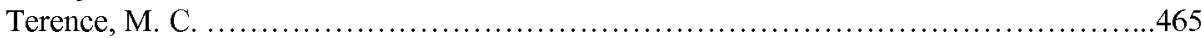

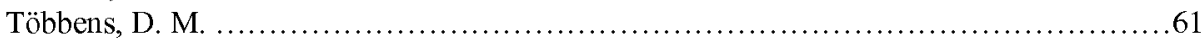

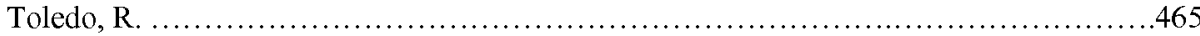

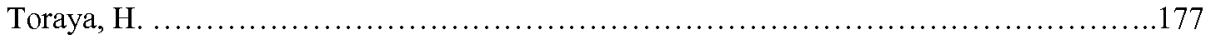

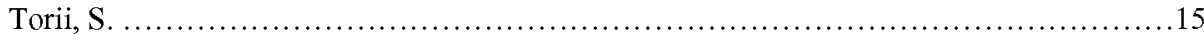

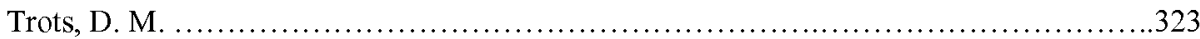

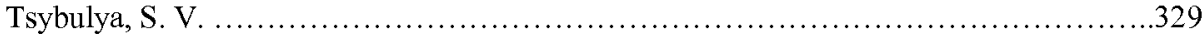

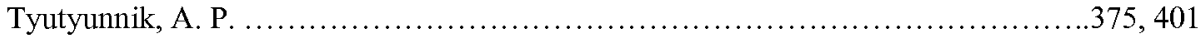




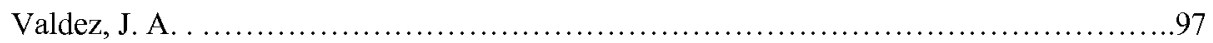

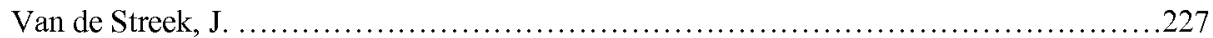

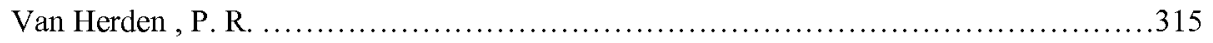

Van Mechelen, J. B. ........................................................491

Van Tendeloo, G. .................................................. 375,401

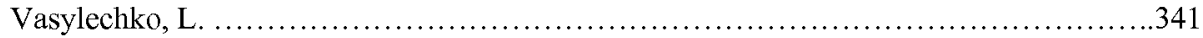

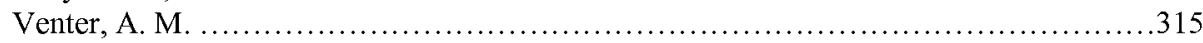

Vermeulen, A. C. ........................................................... 77

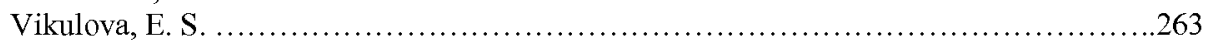

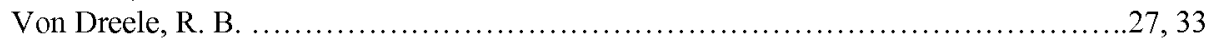

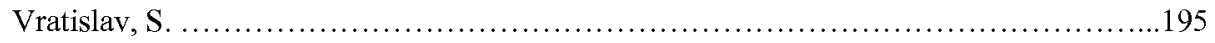

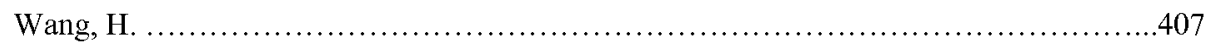

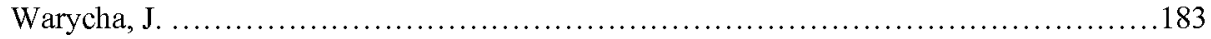

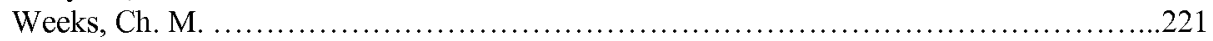

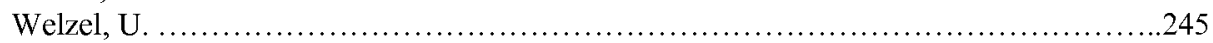

Whitfield, P. S. . . . . . . . . . . . .

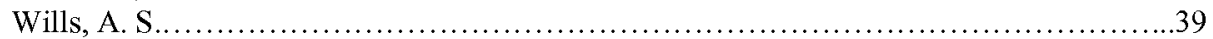

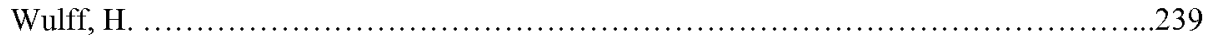

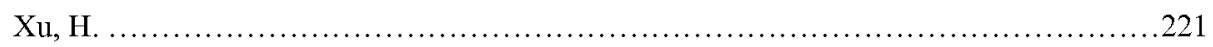

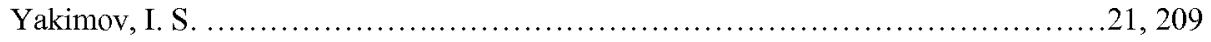

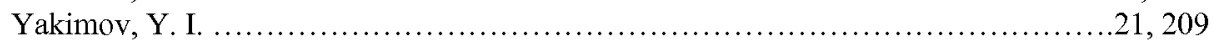

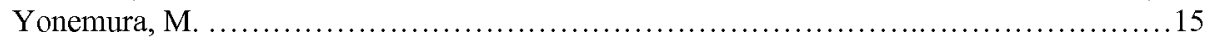

Yusenko, K. V. ......................................................263, 269

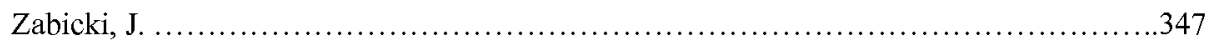

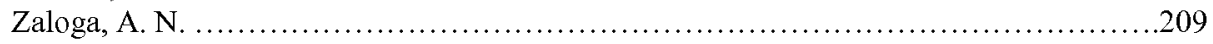

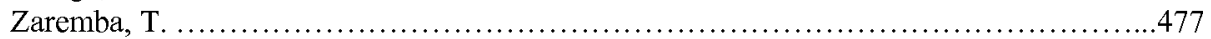

Zubkov, V. G. ..................................................... 375, 401

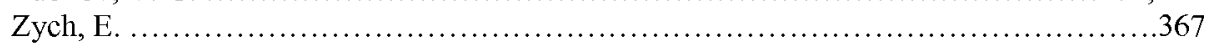



